\title{
Ökonomische Analyse \\ forstlicher Bestandesbehandlung
}

\author{
Dissertation \\ zur Erlangung des Doktorgrades (Dr. forest.) \\ der Fakultät für Forstwissenschaften und Waldökologie \\ der Georg-August-Universität Göttingen
}

vorgelegt von

Roman Koster

geboren am 08. September 1987 in Bad Honnef

Göttingen, Oktober 2020 
1. Gutachter: Prof. Dr. Bernhard Möhring

2. Gutachter: Prof. Dr. Christian Ammer

Tag der mündlichen Prüfung: 11. September 2020 


\section{Vorwort und Danksagung}

Die vorliegende Dissertation entstand im Rahmen meiner dreijährigen Tätigkeit als wissenschaftlicher Mitarbeiter der Abteilung Forstökonomie an der Georg-August-Universität Göttingen. Neben vielfältigen Aufgaben im Bereich von Lehre und Verwaltung bearbeitete ich das Teilvorhaben 2 „Ökonomische Bewertung“ im Verbundforschungsprojekt „Neue Generation dynamischer Ertragstafeln (dyn-ET)“. Gefördert durch das Bundesministerium für Ernährung und Landwirtschaft (BMEL) über die Fachagentur für Nachwachsende Rohstoffe e. V. (FNR) erfolgte die Bearbeitung in Kooperation mit der Nordwestdeutschen Forstlichen Versuchsanstalt (NW-FVA).

Das Projekt der Erstellung, Bewertung und Analyse neuer forstlicher Ertragstafeln, die auf heutigen Standortsverhältnissen beruhen und aktuelle Waldbaukonzepte einbeziehen, reizte mich von Beginn an. Die tabellarische, kompakte und verallgemeinerte Darstellung von Wachstum und Nutzung unserer Hauptbaumarten sah und sehe ich als fundamentale Grundlage einer nachhaltigen Forstwirtschaft an - im Widerspruch dazu steht meines Erachtens die Nutzung der etablierten, leider aber auch quantitativ überholten Ertragstafeln des mittleren 20. Jahrhunderts. Teil des Projekts zu sein, welches sich dieser Herausforderung stellte und mir die Arbeit an der Seite ausgewiesener Expertinnen und Experten ermöglichte, erfüllte mich mit Stolz und bescherte mir lehrreiche Jahre an der Schnittstelle zwischen Waldwachstumskunde, Ertragskunde, Waldbau und Forstökonomie. Ich bin sehr glücklich, dass ich dieses Themenfeld auch in meiner Dissertation aufgreifen konnte und danke allen Beteiligten.

Mein besonderer Dank gilt zunächst meinem Doktorvater Prof. Dr. Bernhard Möhring - er schickte mich auf den Weg, gab mir Raum und Zeit für inhaltliche Exkurse und führte mich schließlich mit den richtigen Fragen zum angestrebten Ziel. Die vielen Diskussionen zu später Stunde wirken noch heute in mir nach und so manches Sprichwort wird mich wohl noch in einigen Lebenssituationen begleiten. Vielen Dank Herr Möhring, für diese vielfältige Weiterbildungszeit, auf die ich gerne zurückschaue.

Prof. Dr. Christian Ammer übernahm die Zweitbetreuung meines Promotionsvorhabens, wodurch mein Ziel einer Dissertation an der Schnittstelle zwischen Waldbau und Forstökonomie möglich wurde. Ich danke Ihnen vielmals, Herr Ammer, für den fachlichen Input in langen Diskussionen und den wohltuenden Zuspruch.

Prof. Dr. Carola Paul war nicht nur Drittbetreuerin meines Promotionsvorhabens, sondern Beraterin, Motivatorin, Krisenmanagerin, Gastgeberin und Freundin. Es erfüllt mich mit Stolz, 
Deine ersten Jahre an der Georg-August-Universität als Teil eines wunderbaren Teams miterlebt haben zu dürfen und ich hoffe, dass der Kontakt nie abbricht. Vielen Dank für alles, Carola. Jasper Fuchs wurde während meiner Zeit in der Abteilung Forstökonomie nicht nur zum überaus geschätzten Kollegen, sondern für mich persönlich auch zum Freund, der zum Gelingen dieser Arbeit in großem Maße beitrug. Die unzähligen und stets produktiven Diskussionen sowie die gemeinsamen Tage vor unseren Modellen werde ich sehr vermissen - sollte mich mein beruflicher Weg jemals in die Wissenschaft zurückführen, dann unter anderem deinetwegen, Jasper. Ich hoffe sehr, dass unsere Namen noch öfters über gemeinsamen Artikeln stehen und danke Dir ganz besonders für Deine Hilfe.

Volker von Groß hat mich mit seiner Hilfe zu jeder Tages- und Nachtzeit so manches Mal vor der Verzweiflung bewahrt und diese Dissertation profitierte an einigen Stellen von seinem Drang zur Lösungsfindung. Ich danke Dir und freue mich sehr, Volker, dass auch Dein Weg in der Wissenschaft als Teil eines überragenden Teams vielversprechend weitergeht.

Dieses Team wird nicht zuletzt geprägt von Dr. Kai Husmann, der mich durch die gesamte Zeit an der Georg-August-Universität Göttingen fachlich und freundschaftlich begleitete. Ihm verdanke ich viele R-Skills und zahlreiche methodische Hilfestellungen. Ich danke Dir, Kai, für Dein stets offenes Ohr und die niemals verschwindende Ruhe.

Franziska Pfaar, Jonathan Fibich und Fritz Hofheinz haben mir als studentische Hilfskräfte im Projekt bzw. Jonathan auch mit seiner Bachelorarbeit sehr geholfen. Ich danke Euch, liebe Franziska, lieber Jonathan, lieber Fritz, für Euren Fleiß und die Unterstützung.

Dr. Hilmar von Bodelschwingh, PD Dr. Matthias Albert, Dr. Nora Koster, Jasper Fuchs, Johannes Wildberg, Franziska Pfaar, Olaf Hoyer und Robert Schlachta danke ich für die Geduld beim Lesen dieser Arbeit, die konstruktiven Anmerkungen und die vielen nützlichen Hinweise. Ihr habt zum letzten Schliff dieses Werkes beigetragen, wofür ich Euch unglaublich dankbar bin.

Besonders danken möchte ich darüber hinaus Johannes Wildberg und Fabian Böttcher für den gemeinsamen Weg durch die Promotion, die schnelle Hilfe in jeder Lage und die Motivation in schwierigen Phasen. Vielen Dank, lieber Johannes, lieber Fabian, für so manches Bier zu später Stunde, viele leckere Abendessen und den immer freien Schlafplatz. Auf das wir uns nicht aus den Augen verlieren und ihr mich bald besuchen kommt.

Darüber hinaus danke ich natürlich auch allen anderen Kolleginnen und Kollegen der Abteilung Forstökonomie, der Abteilung Forstökonomie und nachhaltige Landnutzungsplanung sowie der Nordwestdeutschen Forstlichen Versuchsanstalt. 
Zuletzt möchte ich den beiden Menschen danken, die mir das alles überhaupt ermöglicht haben. Meine Frau, Dr. Nora Koster, war immer für mich da, hatte stets ein offenes Ohr, wurde niemals müde im Aufmuntern und hielt mir uneingeschränkt den Rücken frei. Ich danke Dir für alles, Nora, und blicke voller Freude auf unsere gemeinsame Zeit in Göttingen sowie das Wertvollste, was ich aus dieser Zeit mitnehme - unsere Tochter. Dir, liebe Juna, danke ich von ganzem Herzen für den Sonnenschein in meinem Leben, die wunderbaren Momente zwischen den Stunden am Schreibtisch und das frühe Verständnis für „Papa muss arbeiten!“‘. 



\section{Inhaltsverzeichnis}

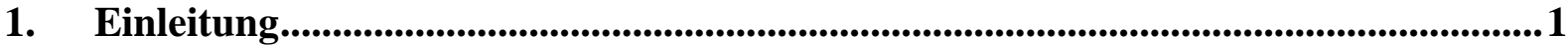

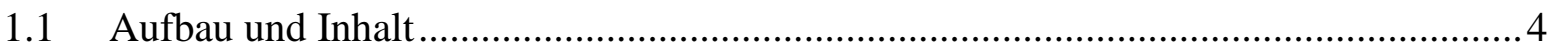

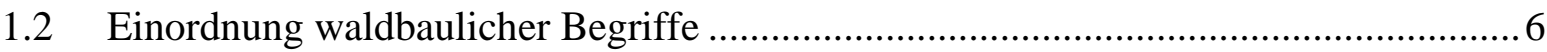

2. Ökonomie der forstlichen Bestandesbehandlung .....................................................13

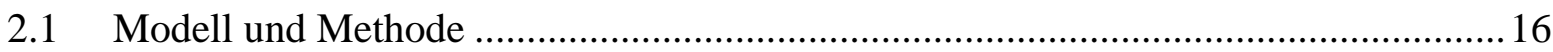

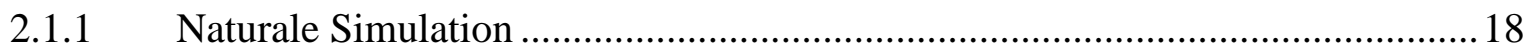

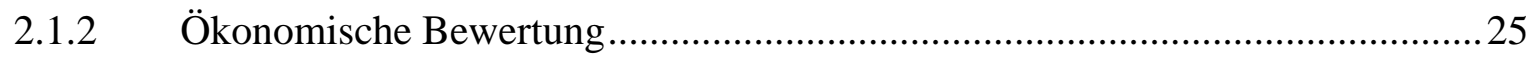

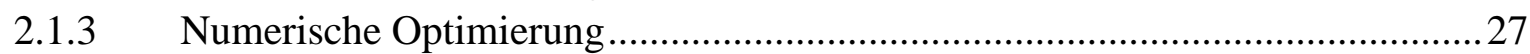

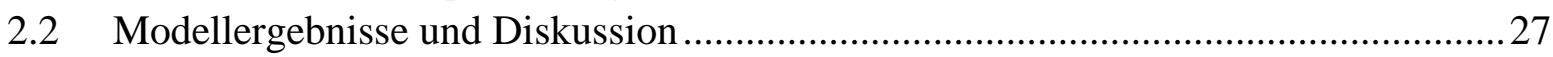

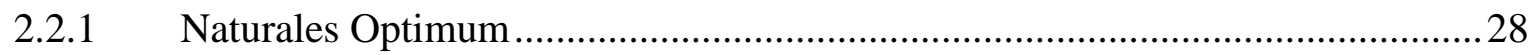

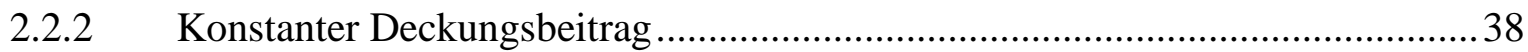

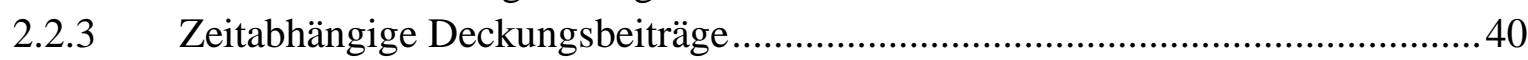

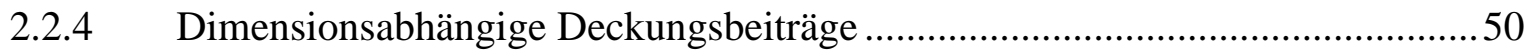

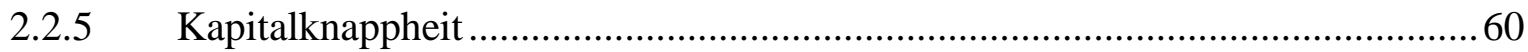

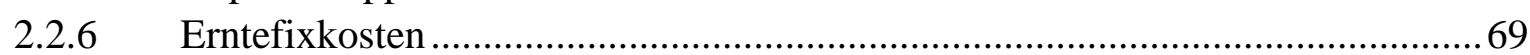

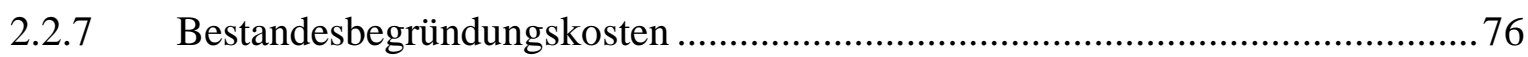

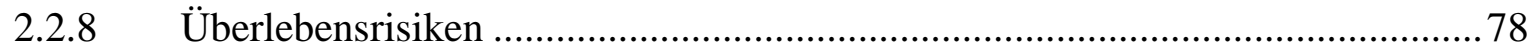

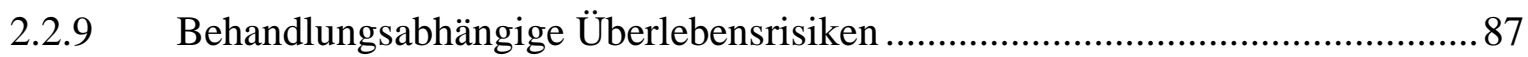

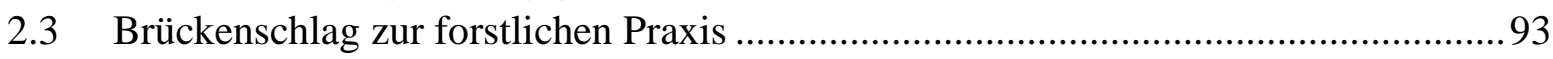

2.4 Kritische Methoden- und Modellwürdigung ......................................................... 101

3. Ertragstafeln - forstliche Wachstums- und Nutzungsmodelle ................................... 107

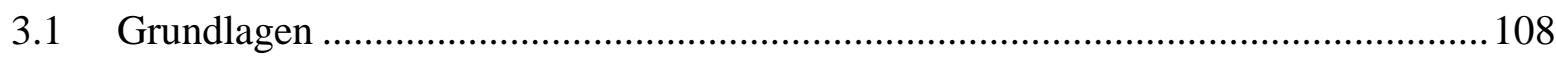

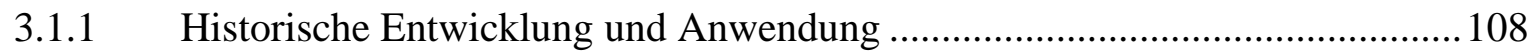

3.1.2 Bonitierung und ertragskundliche Grundbeziehungen .................................... 116

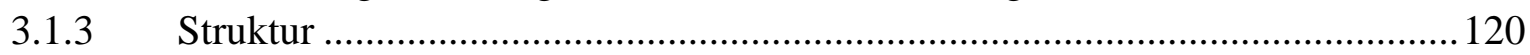

3.2 Praxisbefragung zur gegenwärtigen und zukünftigen Verwendung......................... 123

3.2.1 Methodik und Charakterisierung der Befragten ........................................... 123

3.2.2 Anwendungsgebiete forstlicher Ertragstafeln ............................................... 124

3.2.3 Räumliche Einsatzschwerpunkte etablierter Ertragstafelwerke ....................... 125

3.2.4 Stärken und Schwächen etablierter Ertragstafelwerke ................................... 127

3.2.5 Einschätzungen zur zukünftigen Verwendung forstlicher Ertragstafeln .......... 128

3.3 Neue Generation dynamischer Ertragstafeln (dyn-ET) ...................................... 130

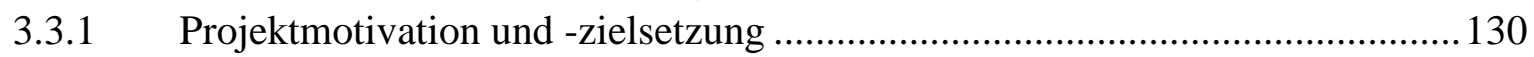

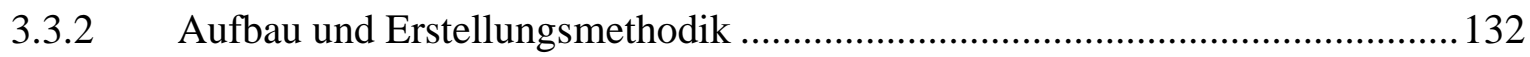

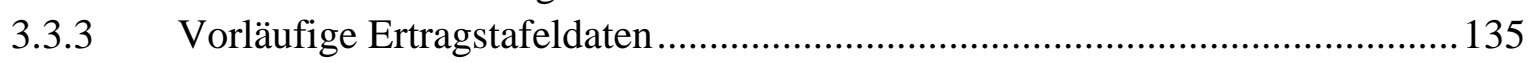

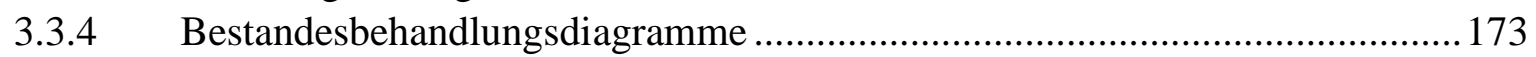

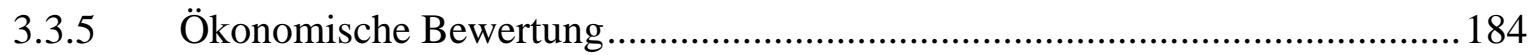

3.3.6 Sensitivitätsanalysen und Brückenschlag zur forstlichen Praxis ......................219

3.3.7 Kritische Methoden- und Modellwürdigung ..............................................245

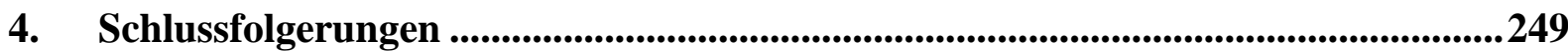

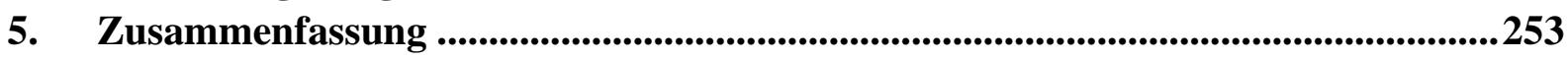

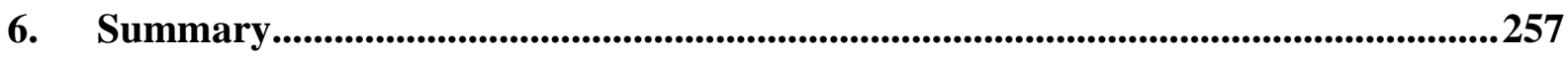

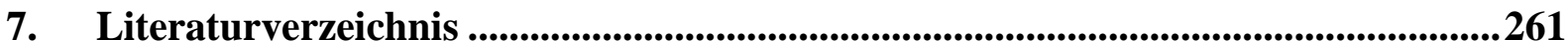

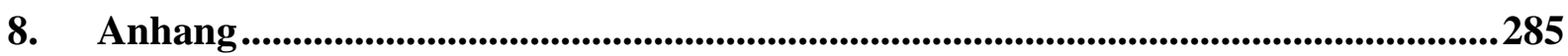





\section{Abbildungsverzeichnis}

Abbildung 1: Ablaufdiagramm des R-basierten Optimierungsmodells........................... 17

Abbildung 2: Volumenzuwachs eines konkurrenzlos wachsenden Einzelbaums.............. 19

Abbildung 3: Einzelbaumvolumenzuwachs im Alter 50 a......................................... 21

Abbildung 4: $\quad$ Bestandeskapazitätsgrenze (max. Stammzahl). ........................................... 21

Abbildung 5: $\quad$ Laufenden Bestandesvolumenzuwachs im Alter 50 a...............................22

Abbildung 6: Alters- und stammzahlabh. laufender Bestandesvolumenzuwachs..............23

Abbildung 7: Ablaufdiagramm des Analyserahmens. ..................................................26

Abbildung 8: $\quad$ Stammzahl im nat. Optimum ohne Folgebestand. ......................................29

Abbildung 9: Bestandesvolumenzuwachs im nat. Optimum ohne Folgebestand. ..............30

Abbildung 10: Gesamtwuchsleistung und Vorrat im naturalen Optimum ohne Folge-

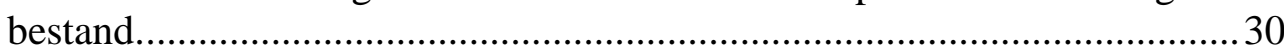

Abbildung 11: Flächendurchschnittlicher Einzelbaumvolumenzuwachs und flächenabh. Grenzvolumenzuwachs je Einzelbaum im Alter 20 a............................. 32

Abbildung 12: Flächendurchschnittlicher Einzelbaumvolumenzuwachs und flächenabh. Grenzvolumenzuwachs je Einzelbaum im Alter 80 a................................ 33

Abbildung 13: Stammzahl im naturalen Optimum mit Folgebestand................................. 36

Abbildung 14: Bestandesvolumenzuwachs im nat. Optimum mit Folgebestand................. 37

Abbildung 15: Gesamtwuchsleistung und Vorrats im naturalen Optimum mit Folge-

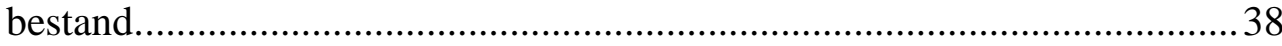

Abbildung 16: Bestandeswertzuwachs bei konstantem Deckungsbeitrag. ..........................39

Abbildung 17: Zeitabhängiger Deckungsbeitrag je Festmeter.......................................... 42

Abbildung 18: Stammzahl bei zeitabhängiger DB-Steigerung. ......................................... 43

Abbildung 19: Bestandesvolumenzuwachs bei zeitabhängiger DB-Steigerung..................44

Abbildung 20: Gesamtwuchsleistung und Vorrat bei zeitabhängiger DB-Steigerung......... 45

Abbildung 21: Disparitätskurven der relativen Gesamtwuchs- und Gesamtwertleistung bei zeitabhängiger DB-Steigerung. ........................................................ 46

Abbildung 22: Wertfaktoren bei zeitabhängiger DB-Steigerung........................................ 48

Abbildung 23: Dimensionsabhängiger Deckungsbeitrag je Festmeter. …...........................52

Abbildung 24: Stammzahl bei dimensionsabh. DB-Steigerung........................................53

Abbildung 25: Bestandesvolumenzuwachs bei dimensionsabh. DB-Steigerung................54

Abbildung 26: Gesamtwuchsleistung und Vorrat bei dimensionsabh. DB-Steigerung.......54

Abbildung 27: Einzelbaumvolumina bei dimensionsabhängiger DB-Steigerung. ...............55

Abbildung 28: Disparitätskurven bei dimensionsabhängiger DB-Steigerung. ....................56

Abbildung 29: Wertfaktoren bei dimensionsabhängiger DB-Steigerung. ...........................59

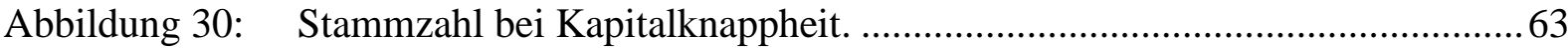




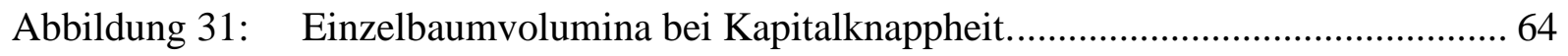

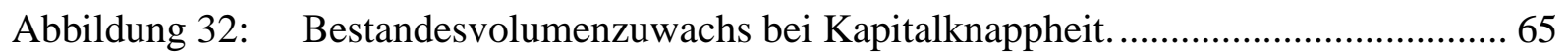

Abbildung 33: Gesamtwuchsleistung und Vorrat bei Kapitalknappheit. ........................... 66

Abbildung 34: Stammzahl bei zeitabhängiger DB-Steigerung und mittlerem

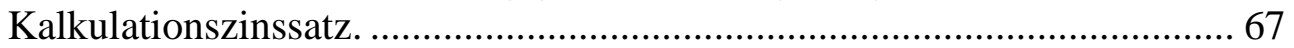

Abbildung 35: Wertfaktoren bei mittlerem und hohem Kalkulationszinssatz im Vergleich zu den zinsfreien Optima. ...................................................... 68

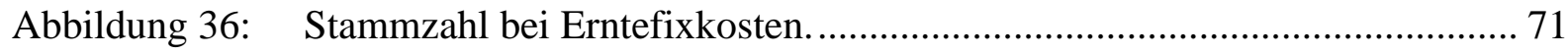

Abbildung 37: Gesamtwuchsleistung und Vorrat bei Erntefixkosten. ............................... 72

Abbildung 38: Bestandesvolumenzuwachs bei Erntefixkosten....................................... 73

Abbildung 39: Stammzahl bei Bestandesbegründungskosten.......................................... 76

Abbildung 40: Gesamtwuchsleistung und Vorrat bei Bestandesbegründungskosten.......... 78

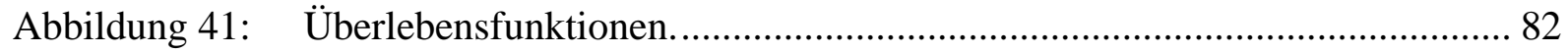

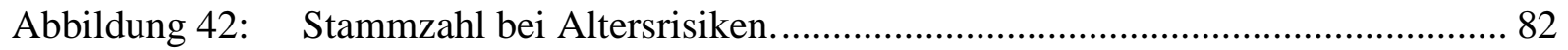

Abbildung 43: Bestandesvolumenzuwachs bei Altersrisiken.......................................... 83

Abbildung 44: Gesamtwuchsleistung und Vorrat bei Altersrisiken. ................................... 84

Abbildung 45: Überlebensrisikostärke in Abh. vom Stückvolumen im Alter 50 a. ........... 88

Abbildung 46: Stammzahl bei behandlungsabh. Altersrisiken.......................................... 89

Abbildung 47: Einzelbaumvolumina bei behandlungsabh. Altersrisiken. ......................... 90

Abbildung 48: Bestandesvolumenzuwachs bei behandlungsabh. Altersrisiken.................. 91

Abbildung 49: Gesamtwuchsleistung und Vorra bei behandlungsabh. Altersrisiken. ........ 92

Abbildung 50: Stammzahlentwicklungen der abschnittsübergreifenden Zusammen-

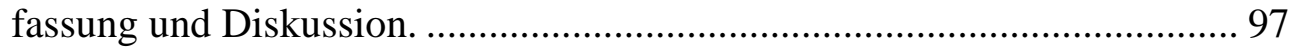

Abbildung 51: Durchschn. Erfolgsgrößen der abschnittsübergreifenden Zusammenfassung und Diskussion. .......................................................................... 99

Abbildung 52: Tabellenköpfe etablierter Fichten-Ertragstafelwerke. …........................... 122

Abbildung 53: Anwendungsgebiete forstlicher Ertragstafeln........................................ 125

Abbildung 54: Räumliche Zuordnung forstlicher Ertragstafelwerke. .............................. 126

Abbildung 55: Stärken gegenwärtig verwendeter forstlicher Ertragstafelwerke............... 127

Abbildung 56: Schwächen gegenwärtig verwendeter forstlicher Ertragstafelwerke......... 128

Abbildung 57: Gesamtwuchsleistung der Baumart Buche (mäßige Hochdurchforstung) gemäß dyn-ET (unveröff.).

Abbildung 58: Laufender Bestandesvolumenzuwachs der Baumart Buche (mäßige Hochdurchforstung) gemäß dyn-ET (unveröff.)...

Abbildung 59: Gesamtwuchsleistung der Baumart Buche (mäßige Durchforstung) gemäß dyn-ET (unveröff.) und Schober (1967).

Abbildung 60: Oberhöhe der Baumart Buche (mäßige Durchforstung) gemäß dyn-ET (unveröff.) und Schober (1967). 
Abbildung 61: Laufender Bestandesvolumenzuwachs der Baumart Buche (mäßige Durchforstung) gemäß dyn-ET (unveröff.) und Schober (1967).

Abbildung 62: Vorrat der Baumart Buche (mäßige Durchforstung) gemäß dyn-ET (unveröff.) und Schober (1967)

Abbildung 63: Volumenzuwachsraten der Baumart Buche (mäßige Durchforstung) gemäß dyn-ET (unveröff.) und Schober (1967).

Abbildung 64: Ausscheidendes Bestandesvolumen der Baumart Buche (mäßige Durchforstung) gemäß dyn-ET (unveröff.) und Schober (1967)............... 144

Abbildung 65: Stammzahl (verbleibender Bestand) der Baumart Buche (mäßige Durchforstung) gemäß dyn-ET (unveröff.) und Schober (1967).

Abbildung 66: Stückvolumenrelationen der Baumart Buche (mäßige Durchforstung) gemäß dyn-ET (unveröff.) und Schober (1967).

Abbildung 67: Durchmesser des Grundflächenmittelstamms (verbleibender Bestand) der Baumart Buche (mäßige Durchforstung) gemäß dyn-ET (unveröff.) und Schober (1967).

Abbildung 68: Durchmesser des Grundflächenmittelstamms (ausscheidender Bestand) der Baumart Buche (mäßige Durchforstung) gemäß dyn-ET (unveröff.) und Schober (1967).

Abbildung 69: Durchschn. Bestandesvolumenzuwachs der Baumart Buche (mäßige Durchforstung) gemäß dyn-ET (unveröff.) und Schober (1967).

Abbildung 70: Normalvorrat der Baumart Buche (mäßige Durchforstung) gemäß dynET (unveröff.) und Schober (1967).

Abbildung 71: Gesamtwuchsleistung der Baumart Buche getrennt nach Behandlungsvarianten gemäß dyn-ET (unveröff.).

Abbildung 72: Laufender Bestandesvolumenzuwachs der Baumart Buche getrennt nach Behandlungsvarianten gemäß dyn-ET (unveröff.).

Abbildung 73: Vorrat der Baumart Buche getrennt nach Behandlungsvarianten gemäß dyn-ET (unveröff.).

Abbildung 74: Volumenzuwachsraten der Baumart Buche getrennt nach Behandlungsvarianten gemäß dyn-ET (unveröff.).

Abbildung 75: Ausscheidendes Bestandesvolumen der Baumart Buche getrennt nach Behandlungsvarianten gemäß dyn-ET (unveröff.).

Abbildung 76: Durchmesser des Grundflächenmittelstamms (verbleibender Bestand) der Baumart Buche getrennt nach Behandlungsvarianten gemäß dyn-ET (unveröff.).

Abbildung 77: Durchmesser des Grundflächenmittelstammes (ausscheidender Bestand) der Baumart Buche getrennt nach Behandlungsvarianten gemäß dyn-ET (unveröff.).

Abbildung 78: Stückvolumenrelationen der Baumart Buche getrennt nach Behandlungsvarianten gemäß dyn-ET (unveröff.)................................ 155

Abbildung 79: Gesamtwuchsleistung der Baumart Fichte (mäßige Hochdurchforstung) gemäß dyn-ET (unveröff.). 
Abbildung 80: Laufender Bestandesvolumenzuwachs der Baumart Fichte (mäßige Hochdurchforstung) gemäß dyn-ET (unveröff.)

Abbildung 81: Gesamtwuchsleistung der Baumart Fichte (mäßige Durchforstung) gemäß dyn-ET (unveröff.) und Wiedemann (1936/42)...

Abbildung 82: Oberhöhe der Baumart Fichte (mäßige Durchforstung) gemäß dyn-ET (unveröff.) und Wiedemann (1936/42).

Abbildung 83: Laufender Bestandesvolumenzuwachs der Baumart Fichte (mäßige Durchforstung) gemäß dyn-ET (unveröff.) und Wiedemann (1936/42). .. 160

Abbildung 84: Vorrat der Baumart Fichte (mäßige Durchforstung) gemäß dyn-ET (unveröff.) und Wiedemann (1936/42).

Abbildung 85: Volumenzuwachsraten der Baumart Fichte (mäßige Durchforstung) gemäß dyn-ET (unveröff.) und Wiedemann (1936/42).

Abbildung 86: Ausscheidendes Bestandesvolumen der Baumart Fichte (mäßige Durchforstung) gemäß dyn-ET (unveröff.) und Wiedemann (1936/42). .. 163

Abbildung 87: Stammzahl (verbleibender Bestand) der Baumart Fichte (mäßige Durchforstung) gemäß dyn-ET (unveröff.) und Wiedemann (1936/42). .. 163

Abbildung 88: Stückvolumenrelationen der Baumart Fichte (mäßige Durchforstung) gemäß dyn-ET (unveröff.) und Wiedemann (1936/42).

Abbildung 89: Durchmesser des Grundflächenmittelstamms (verbleibender Bestand) der Baumart Fichte (mäßige Durchforstung) gemäß dyn-ET (unveröff.) und Wiedemann (1936/42).

Abbildung 90: Durchmesser des Grundflächenmittelstamms (ausscheidender Bestand) der Baumart Fichte (mäßige Durchforstung) gemäß dyn-ET (unveröff.) und Wiedemann (1936/42).

Abbildung 91: Durchschn. Bestandesvolumenzuwachs der Baumart Fichte (mäßige Durchforstung) gemäß dyn-ET (unveröff.) und Wiedemann (1936/42)... 166

Abbildung 92: Normalvorrat der Baumart Fichte (mäßige Durchforstung) gemäß dynET (unveröff.) und Wiedemann (1936/42).

Abbildung 93: Gesamtwuchsleistung der Baumart Fichte getrennt nach Behandlungsvarianten gemäß dyn-ET (unveröff.).

Abbildung 94: Laufender Bestandesvolumenzuwachs der Baumart Fichte getrennt nach Behandlungsvarianten gemäß dyn-ET (unveröff.).

Abbildung 95: Vorrat der Baumart Fichte getrennt nach Behandlungsvarianten gemäß dyn-ET (unveröff.).

Abbildung 96: Volumenzuwachsraten der Baumart Fichte getrennt nach Behandlungsvarianten gemäß dyn-ET (unveröff.).

Abbildung 97: Ausscheidendes Bestandesvolumen der Baumart Fichte getrennt nach Behandlungsvarianten gemäß dyn-ET (unveröff.).

Abbildung 98: Durchmesser des Grundflächenmittelstamms (verbleibender Bestand) der Baumart Fichte getrennt nach Behandlungsvarianten gemäß dyn-ET (unveröff.). 
Abbildung 99: Durchmesser des Grundflächenmittelstammes (ausscheidender Bestand) der Baumart Fichte getrennt nach Behandlungsvarianten gemäß dyn-ET (unveröff.).

Abbildung 100: Stückvolumenrelationen der Baumart Fichte getrennt nach Behandlungsvarianten gemäß dyn-ET (unveröff.)................................ 172

Abbildung 101: Bestandesbehandlungsdiagramm der Baumart Buche. 180

Abbildung 102: Bestandesbehandlungsdiagramm der Baumart Buche mit ausgewählten Oberhöhen-Stammzahl-Entwicklungen.

Abbildung 103: Bestandesbehandlungsdiagramm der Baumart Fichte.

Abbildung 104: Bestandesbehandlungsdiagramm der Baumart Fichte mit ausgewählten Oberhöhen-Stammzahl-Entwicklungen.

Abbildung 105: Ablaufdiagramm der ökonomischen Bewertung.

Abbildung 106: Baumartenspezifische, dimensionsabhängige Deckungsbeiträge.

Abbildung 107: Gesamtwertleistung der Baumart Buche (mäßige Hochdurchforstung) gemäß dyn-ET (unveröff.).

Abbildung 108: Laufender Bestandeswertzuwachs der Baumart Buche (mäßige Hochdurchforstung) gemäß dyn-ET (unveröff.).

Abbildung 109: Gesamtwertleistung der Baumart Buche (mäßige Durchforstung) gemäß dyn-ET (unveröff.) und Schober (1967).

Abbildung 110: Laufender Bestandeswertzuwachs der Baumart Buche (mäßige Durchforstung) gemäß dyn-ET (unveröff.) und Schober (1967).

Abbildung 111: Abtriebswert der Baumart Buche (mäßige Durchforstung) gemäß dynET (unveröff.) und Schober (1967).

Abbildung 112: Wertzuwachsraten der Baumart Buche (mäßige Durchforstung) gemäß dyn-ET (unveröff.) und Schober (1967).

Abbildung 113: Durchschnittlicher waldb. Überschuss der Baumart Buche (mäßige Durchforstung) gemäß dyn-ET (unveröff.) und Schober (1967).

Abbildung 114: Durchschnittlicher Abtriebswert der Baumart Buche (mäßige Durchforstung) gemäß dyn-ET (unveröff.) und Schober (1967).

Abbildung 115: Brutto-Bodenrente der Baumart Buche (mäßige Durchforstung) gemäß dyn-ET (unveröff.) und Schober (1967).

Abbildung 116: Vornutzungswerte der Baumart Buche (mäßige Durchforstung) gemäß dyn-ET (unveröff.) und Schober (1967).

Abbildung 117: Gesamtwertleistung der Baumart Buche getrennt nach Behandlungsvarianten gemäß dyn-ET (unveröff.)

Abbildung 118: Laufender Bestandeswertzuwachs der Baumart Buche getrennt nach Behandlungsvarianten gemäß dyn-ET (unveröff.).

Abbildung 119: Wertzuwachsraten der Baumart Buche getrennt nach Behandlungsvarianten gemäß dyn-ET (unveröff.).

Abbildung 120: Durchschnittlicher waldbaulicher Überschuss der Baumart Buche getrennt nach Behandlungsvarianten gemäß dyn-ET (unveröff.). 
Abbildung 121: Brutto-Bodenrente der Baumart Buche getrennt nach Behandlungsvarianten gemäß dyn-ET (unveröff.).

Abbildung 122: Gesamtwertleistung der Baumart Fichte (mäßige Hochdurchforstung) gemäß dyn-ET (unveröff.).

Abbildung 123: Laufender Bestandeswertzuwachs der Baumart Fichte (mäßige Hochdurchforstung) gemäß dyn-ET (unveröff.).................................... 208

Abbildung 124: Gesamtwertleistung der Baumart Fichte (mäßige Durchforstung) gemäß dyn-ET (unveröff.) und Wiedemann (1936/42)....

Abbildung 125: Laufender Bestandeswertzuwachs der Baumart Fichte (mäßige Durchforstung) gemäß dyn-ET (unveröff.) und Wiedemann (1936/42). .. 211

Abbildung 126: Abtriebswert der Baumart Fichte (mäßige Durchforstung) gemäß dynET (unveröff.) und Wiedemann (1936/42).

Abbildung 127: Wertzuwachsraten der Baumart Fichte (mäßige Durchforstung) gemäß dyn-ET (unveröff.) und Wiedemann (1936/42).

Abbildung 128: Durchschn. waldbaulicher Überschuss der Baumart Fichte (mäßige Durchforstung) gemäß dyn-ET (unveröff.) und Wiedemann (1936/42)... 213

Abbildung 129: Durchschnittlicher Abtriebswert der Baumart Fichte (mäßige Durchforstung) gemäß dyn-ET (unveröff.) und Wiedemann (1936/42)... 213

Abbildung 130: Brutto-Bodenrente der Baumart Fichte (mäßige Durchforstung) gemäß dyn-ET (unveröff.) und Wiedemann (1936/42).

Abbildung 131: Vornutzungswerte der Baumart Fichte (mäßige Durchforstung) gemäß dyn-ET (unveröff.) und Wiedemann (1936/42).

Abbildung 132: Gesamtwertleistung der Baumart Fichte getrennt nach Behandlungsvarianten gemäß dyn-ET (unveröff.).

Abbildung 133: Laufender Bestandeswertzuwachs der Baumart Fichte getrennt nach Behandlungsvarianten gemäß dyn-ET (unveröff.).

Abbildung 134: Wertzuwachsraten der Baumart Fichte getrennt nach Behandlungsvarianten gemäß dyn-ET (unveröff.).

Abbildung 135: Durchschnittlicher waldbaulicher Überschuss der Baumart Fichte getrennt nach Behandlungsvarianten gemäß dyn-ET (unveröff.).

Abbildung 136: Brutto-Bodenrente der Baumart Fichte getrennt nach Behandlungsvarianten gemäß dyn-ET (unveröff.).

Abbildung 137: Sensitivitätsanalyse der Baumart Buche (Deckungsbeitrag).................... 224

Abbildung 138: Sensitivitätsanalyse der Baumart Fichte (Deckungsbeitrag)..................... 226

Abbildung 139: Disparitätskurven der Baumart Buche bei einer dimensionsabhängiger DB-Steigerung gemäß der Referenz-DB-Variante aus Abschnitt 3.3.5 ... 228

Abbildung 140: Disparitätskurven der Baumart Buche bei einer dimensionsabhängiger DB-Steigerung gemäß der DB-Variante 6 .

Abbildung 141: Disparitätskurven der Baumart Fichte bei einer dimensionsabhängiger DB-Steigerung gemäß der Referenz-DB-Variante aus Abschnitt 3.3.5.... 229

Abbildung 142: Disparitätskurven der Baumart Fichte bei einer dimensionsabhängiger DB-Steigerung gemäß der DB-Variante 6 . 
Abbildung 143: Sensitivitätsanalyse der Baumart Buche (Kalkulationszinssatz). .............230

Abbildung 144: Sensitivitätsanalyse der Baumart Fichte (Kalkulationszinssatz)...............231

Abbildung 145: Sensitivitätsanalyse der Baumart Buche (Überlebensrisikostärke)............234

Abbildung 146: Sensitivitätsanalyse der Baumart Fichte (Überlebensrisikostärke)............235

Abbildung 147: Sensitivitätsanalyse der Baumart Buche (Wechselwirkung zwischen

Deckungsbeitragsfunktion und Kalkulationszinssatz)............................240

Abbildung 148: Sensitivitätsanalyse der Baumart Fichte (Wechselwirkung zwischen

Deckungsbeitragsfunktion und Kalkulationszinssatz).

Abbildung 149: Sensitivitätsanalyse der Baumart Buche (Wechselwirkung zwischen Deckungsbeitragsfunktion, Kalkulationszinssatz und Überlebensrisikostärke).

Abbildung 150: Sensitivitätsanalyse der Baumart Fichte (Wechselwirkung zwischen Deckungsbeitragsfunktion, Kalkulationszinssatz und Überlebensrisikostärke).

Abbildung 151: Mailanfrage der Praxisbefragung zur gegenwärtigen und zukünftigen Verwendung forstlicher Ertragstafeln.

Abbildung 152: Gesamtwuchsleistung der Baumart Buche (starke Hochdurchforstung) gemäß dyn-ET (unveröff.).

Abbildung 153: Laufender Bestandesvolumenzuwachs der Baumart Buche (starke Hochdurchforstung) gemäß dyn-ET (unveröff.).

Abbildung 154: Gesamtwuchsleistung der Baumart Buche (gestaffelte Hochdurchforstung) gemäß dyn-ET (unveröff.).

Abbildung 155: Laufender Bestandesvolumenzuwachs der Baumart Buche (gestaffelte Hochdurchforstung) gemäß dyn-ET (unveröff.).

Abbildung 156: Gesamtwuchsleistung der Baumart Buche (starke Durchforstung) gemäß dyn-ET (unveröff.) und Schober (1967).

Abbildung 157: Oberhöhe der Baumart Buche (starke Durchforstung) gemäß dyn-ET (unveröff.) und Schober (1967).

Abbildung 158: Laufender Bestandesvolumenzuwachs der Baumart Buche (starke Durchforstung) gemäß dyn-ET (unveröff.) und Schober (1967)..............295

Abbildung 159: Vorrat der Baumart Buche (starke Durchforstung) gemäß dyn-ET (unveröff.) und Schober (1967).

Abbildung 160: Volumenzuwachsraten der Baumart Buche (starke Durchforstung) gemäß dyn-ET (unveröff.) und Schober (1967).

Abbildung 161: Ausscheidendes Bestandesvolumen der Baumart Buche (starke Durchforstung) gemäß dyn-ET (unveröff.) und Schober (1967).

Abbildung 162: Stammzahl (verbleibender Bestand) der Baumart Buche (starke Durchforstung) gemäß dyn-ET (unveröff.) und Schober (1967).

Abbildung 163: Stückvolumenrelationen der Baumart Buche (starke Durchforstung) gemäß dyn-ET (unveröff.) und Schober (1967) 
Abbildung 164: Durchmesser des Grundflächenmittelstamms (verbleibender Bestand) der Baumart Buche (starke Durchforstung) gemäß dyn-ET (unveröff.) und Schober (1967).

Abbildung 165: Durchmesser des Grundflächenmittelstamms (ausscheidender Bestand) der Baumart Buche (starke Durchforstung) gemäß dyn-ET (unveröff.) und Schober (1967).

Abbildung 166: Durchschnittlicher Bestandesvolumenzuwachs der Baumart Buche (starke Durchforstung) gemäß dyn-ET (unveröff.) und Schober (1967)... 299

Abbildung 167: Normalvorrat der Baumart Buche (starke Durchforstung) gemäß dyn-ET (unveröff.) und Schober (1967). 300

Abbildung 168: Vorrat der Baumart Buche getrennt nach Behandlungsvarianten gemäß dyn-ET (unveröff.). 300

Abbildung 169: Gesamtwuchsleistung der Baumart Fichte (starke Hochdurchforstung) gemäß dyn-ET (unveröff.).

Abbildung 170: Laufender Bestandesvolumenzuwachs der Baumart Fichte (starke Hochdurchforstung) gemäß dyn-ET (unveröff.)

Abbildung 171: Gesamtwuchsleistung der Baumart Fichte (starke Durchforstung) gemäß dyn-ET (unveröff.) und Wiedemann (1936/42).

Abbildung 172: Oberhöhe der Baumart Fichte (starke Durchforstung) gemäß dyn-ET (unveröff.) und Wiedemann (1936/42).

Abbildung 173: Laufender Bestandesvolumenzuwachs der Baumart Fichte (starke Durchforstung) gemäß dyn-ET (unveröff.) und Wiedemann (1936/42)... 303

Abbildung 174: Vorrat der Baumart Fichte (starke Durchforstung) gemäß dyn-ET (unveröff.) und Wiedemann (1936/42).

Abbildung 175: Volumenzuwachsraten der Baumart Fichte (starke Durchforstungn) gemäß dyn-ET (unveröff.) und Wiedemann (1936/42).

Abbildung 176: Ausscheidendes Bestandesvolumen der Baumart Fichte (starke Durchforstung) gemäß dyn-ET (unveröff.) und Wiedemann (1936/42). .. 305

Abbildung 177: Stammzahl (verbleibender Bestand) der Baumart Fichte (starke Durchforstung) gemäß dyn-ET (unveröff.) und Wiedemann (1936/42). .. 305

Abbildung 178: Stückvolumenrelationen der Baumart Fichte (starke Durchforstung) gemäß dyn-ET (unveröff.) und Wiedemann (1936/42).

Abbildung 179: Durchmesser des Grundflächenmittelstamms (verbleibender Bestand) der Baumart Fichte (starke Durchforstung) gemäß dyn-ET (unveröff.) und Wiedemann (1936/42).

Abbildung 180: Durchmesser des Grundflächenmittelstamms (ausscheidender Bestand) der Baumart Fichte (starke Durchforstung) gemäß dyn-ET (unveröff.) und Wiedemann (1936/42).

Abbildung 181: Durchschn. Bestandesvolumenzuwachs der Baumart Fichte (starke Durchforstung) gemäß dyn-ET (unveröff.) und Wiedemann (1936/42). .. 307

Abbildung 182: Normalvorrat der Baumart Fichte (starke Durchforstung) gemäß dyn-ET (unveröff.) und Wiedemann (1936/42). 
Abbildung 183: Stammzahl (ausscheidender Bestand) der Baumart Fichte (mäßige Durchforstung) gemäß dyn-ET (unveröff.) und Wiedemann (1936/42)....308

Abbildung 184: Vorrat der Baumart Fichte getrennt nach Behandlungsvarianten gemäß dyn-ET (unveröff.).

Abbildung 185: Gesamtwertleistung der Baumart Buche (starke Hochdurchforstung) gemäß dyn-ET (unveröff.).

Abbildung 186: Laufender Bestandeswertzuwachs der Baumart Buche (starke Hochdurchforstung) gemäß dyn-ET (unveröff.).

Abbildung 187: Gesamtwertleistung der Baumart Buche (gestaffelte Hochdurchforstung) gemäß dyn-ET (unveröff.).

Abbildung 188: Laufender Bestandeswertzuwachs der Baumart Buche (gestaffelte Hochdurchforstung) gemäß dyn-ET (unveröff.).

Abbildung 189: Gesamtwertleistung der Baumart Buche (starke Durchforstung) gemäß dyn-ET (unveröff.) und Schober (1967).

Abbildung 190: Laufender Bestandeswertzuwachs der Baumart Buche (starke Durchforstung) gemäß dyn-ET (unveröff.) und Schober (1967).

Abbildung 191: Abtriebswert der Baumart Buche (starke Durchforstung) gemäß dyn-ET (unveröff.) und Schober (1967).

Abbildung 192: Wertzuwachsraten der Baumart Buche (starke Durchforstung) gemäß dyn-ET (unveröff.) und Schober (1967).

Abbildung 193: Durchschnittlicher waldbaulicher Überschuss der Baumart Buche (starke Durchforstung) gemäß dyn-ET (unveröff.) und Schober (1967)...315

Abbildung 194: Durchschnittlicher Abtriebswert der Baumart Buche (starke Durchforstung) gemäß dyn-ET (unveröff.) und Schober (1967).

Abbildung 195: Brutto-Bodenrente der Baumart Buche (starke Durchforstung) gemäß dyn-ET (unveröff.) und Schober (1967).

Abbildung 196: Vornutzungswerte der Baumart Buche (starke Durchforstung) gemäß dyn-ET (unveröff.) und Schober (1967).

Abbildung 197: Gesamtwertleistung der Baumart Fichte (starke Hochdurchforstung) gemäß dyn-ET (unveröff.).

Abbildung 198: Laufender Bestandeswertzuwachs der Baumart Fichte (starke Hochdurchforstung) gemäß dyn-ET (unveröff.).

Abbildung 199: Gesamtwertleistung der Baumart Fichte (starke Durchforstung) gemäß dyn-ET (unveröff.) und Wiedemann (1936/42).

Abbildung 200: Laufender Bestandeswertzuwachs der Baumart Fichte (starke Durchforstung) gemäß dyn-ET (unveröff.) und Wiedemann (1936/42)....318

Abbildung 201: Abtriebswert der Baumart Fichte (starke Durchforstung) gemäß dyn-ET (unveröff.) und Wiedemann (1936/42).

Abbildung 202: Wertzuwachsraten der Baumart Fichte (starke Durchforstung) gemäß dyn-ET (unveröff.) und Wiedemann (1936/42).

Abbildung 203: Durchschnittlicher waldbaulicher Überschuss der Baumart Fichte (starke Durchforstung) gemäß dyn-ET (unveröff.) und Wiedemann (1936/42)....321 
Abbildung 204: Durchschnittlicher Abtriebswert der Baumart Fichte (starke Durchforstung) gemäß dyn-ET (unveröff.) und Wiedemann (1936/42).

Abbildung 205: Brutto-Bodenrente der Baumart Fichte (starke Durchforstung) gemäß dyn-ET (unveröff.) und Wiedemann (1936/42).

Abbildung 206: Vornutzungswerte der Baumart Fichte (starke Durchforstung) gemäß dyn-ET (unveröff.) und Wiedemann (1936/42).

Abbildung 207: Sensitivitätsanalyse der Baumart Buche (Deckungsbeitragsfunktion) mit den DB-variantenabhängig optimalen Umtriebszeiten.

Abbildung 208: Sensitivitätsanalyse der Baumart Fichte (Deckungsbeitragsfunktion) mit den DB-variantenabhängig optimalen Umtriebszeiten.

Abbildung 209: Sensitivitätsanalyse der Baumart Buche (Kalkulationszinssatz) mit den DB-variantenabhängig optimalen Umtriebszeiten. 325

Abbildung 210: Sensitivitätsanalyse der Baumart Buche (Kalkulationszinssatz) mit den DB-variantenabhängig optimalen Umtriebszeiten. 


\section{Tabellenverzeichnis}

Tabelle 3-1: Institutionelle und räumliche Tätigkeitszuordnung der befragten Anwenderinnen und Anwender forstlicher Ertragstafeln.

Tabelle 3-2: $\quad$ Vergleich ausgewählter Ertragstafeldaten der Baumart Buche (mäßige Hochdurchforstung) gemäß dyn-ET (unveröff.).

Tabelle 3-3: $\quad$ Vergleich ausgewählter Ertragstafeldaten der Baumart Buche (mäßige Durchforstung) gemäß dyn-ET (unveröff.) und Schober (1967).

Tabelle 3-4: $\quad$ Vergleich ausgewählter Ertragstafeldaten der Baumart Buche gemäß dyn-ET (unveröff.); unterschiedliche Behandlungsvarianten.

Tabelle 3-5: $\quad$ Vergleich ausgewählter Ertragstafeldaten der Baumart Fichte (mäßige Hochdurchforstung) gemäß dyn-ET (unveröff.).

Tabelle 3-6: $\quad$ Vergleich ausgewählter Ertragstafeldaten der Baumart Fichte (mäßige Durchforstung) gemäß dyn-ET (unveröff.) und Wiedemann (1936/42).... 161

Tabelle 3-7: $\quad$ Vergleich ausgewählter Ertragstafeldaten der Baumart Fichte gemäß dyn-ET (unveröff.); unterschiedliche Behandlungsvarianten.

Tabelle 3-8: $\quad$ Koeffizienten der Isolinien-Funktionen (SDMD).

Tabelle 3-9: $\quad$ Absolute und relative Wurzel der mittleren Fehlerquadratsumme als Gütemaße für die Regressionsanalyse d. Isolinien-Funktionen (SDMD)..177

Tabelle 3-10: Baumartenabh. Bestandesbegründungs- und Bestandespflegekosten........ 185

Tabelle 3-11: $\quad$ Baumartenabh. Leitsortiment-, Industrie- sowie Brennholzpreise. ............ 187

Tabelle 3-12: Koeffizienten der Chapman-Richards-Deckungsbeitragsfunktion............. 188

Tabelle 3-13: Vergleich ausgew. ökonomischer Bewertungsergebnisse der Baumart Buche (mäßige Hochdurchforstung) gemäß dyn-ET (unveröff.).

Tabelle 3-14: Vergleich ausgewählter ökonomischer Bewertungsergebnisse (mäßige Durchforstung) gemäß dyn-ET (unveröff.) und Schober (1967).

Tabelle 3-15: Vergleich ausgewählter ökonomischer Bewertungsergebnisse der Baumart Buche gemäß dyn-ET (unveröff.).

Tabelle 3-16: $\quad$ Vergleich ausgew. ökonomischer Bewertungsergebnisse der Baumart Fichte (mäßige Hochdurchforstung) gemäß dyn-ET (unveröff.).

Tabelle 3-17: Vergleich ausgewählter ökonomischer Bewertungsergebnisse (mäßige Durchforstung) gemäß dyn-ET (unveröff.) und Wiedemann (1936/42)....210

Tabelle 3-18: Vergleich ausgewählter ökonomischer Bewertungsergebnisse der Baumart Fichte gemäß dyn-ET (unveröff.)..

Tabelle 3-19: Koeffizienten der Chapman-Richards-Funktion für den DB-Variantenvergleich der Baumart Buche.

Tabelle 3-20: Koeffizienten der Chapman-Richards-Funktion für den DB-Variantenvergleich der Baumart Fichte.

Tabelle 3-21: Normierte Gini-Koeffizienten im Vergleich der waldbaulichen Behandlungsvarianten gemäß dyn-ET (unveröff.)................................227

Tabelle 8-1: $\quad$ Preismatrizen der Stammholzsortimente. 
Tabelle 8-2: $\quad$ Ausgewählte Daten der vorl. Ertragstafeltabelle Buche, mäßige Hochdurchforstung, Oberhöhenbonität 24 m gemäß dyn-ET (unveröff.)......... 326

Tabelle 8-3: $\quad$ Ausgewählte Daten der vorl. Ertragstafeltabelle Buche, mäßige Hochdurchforstung, Oberhöhenbonität 28 m gemäß dyn-ET (unveröff.)......... 327

Tabelle 8-4: $\quad$ Ausgewählte Daten der vorl. Ertragstafeltabelle Buche, mäßige Hochdurchforstung, Oberhöhenbonität 32 m gemäß dyn-ET (unveröff.)......... 328

Tabelle 8-5: Ausgewählte Daten der vorl. Ertragstafeltabelle Buche, mäßige Hochdurchforstung, Oberhöhenbonität 36 m gemäß dyn-ET (unveröff.)......... 329

Tabelle 8-6: Ausgewählte Daten der vorl. Ertragstafeltabelle Buche, mäßige Hochdurchforstung, Oberhöhenbonität 40 m gemäß dyn-ET (unveröff.)......... 330

Tabelle 8-7: $\quad$ Ausgewählte Daten der vorl. Ertragstafeltabelle Buche, starke Hochdurchforstung, Oberhöhenbonität 24 m gemäß dyn-ET (unveröff.).

Tabelle 8-8: $\quad$ Ausgewählte Daten der vorl. Ertragstafeltabelle Buche, starke Hochdurchforstung, Oberhöhenbonität 28 m gemäß dyn-ET (unveröff.)......... 332

Tabelle 8-9: Ausgewählte Daten der vorl. Ertragstafeltabelle Buche, starke Hochdurchforstung, Oberhöhenbonität 32 m gemäß dyn-ET (unveröff.)......... 333

Tabelle 8-10: Ausgewählte Daten der vorl. Ertragstafeltabelle Buche, starke Hochdurchforstung, Oberhöhenbonität 36 m gemäß dyn-ET (unveröff.)......... 334

Tabelle 8-11: Ausgewählte Daten der vorl. Ertragstafeltabelle Buche, starke Hochdurchforstung, Oberhöhenbonität 40 m gemäß dyn-ET (unveröff.)......... 335

Tabelle 8-12: Ausgewählte Daten der vorl. Ertragstafeltabelle Buche, gestaffelte Hochdurchforstung, Oberhöhenbon. 24 m gemäß dyn-ET (unveröff.). ... 336

Tabelle 8-13: Ausgewählte Daten der vorl. Ertragstafeltabelle Buche, gestaffelte Hochdurchforstung, Oberhöhenbon. 28 m gemäß dyn-ET (unveröff.). ... 337

Tabelle 8-14: Ausgewählte Daten der vorl. Ertragstafeltabelle Buche, gestaffelte Hochdurchforstung, Oberhöhenbon. 32 m gemäß dyn-ET (unveröff.). ... 338

Tabelle 8-15: Ausgewählte Daten der vorl. Ertragstafeltabelle Buche, gestaffelte Hochdurchforstung, Oberhöhenbon. 36 m gemäß dyn-ET (unveröff.). ... 339

Tabelle 8-16: Ausgewählte Daten der vorl. Ertragstafeltabelle Buche, gestaffelte Hochdurchforstung, Oberhöhenbon. 40 m gemäß dyn-ET (unveröff.). ... 340

Tabelle 8-17: $\quad$ Ausgewählte Daten der vorl. Ertragstafeltabelle Fichte, mäßige Hochdurchforstung, Oberhöhenbonität 27 m gemäß dyn-ET (unveröff.)......... 341

Tabelle 8-18: Ausgewählte Daten der vorl. Ertragstafeltabelle Fichte, mäßige Hochdurchforstung, Oberhöhenbonität 31 m gemäß dyn-ET (unveröff.)......... 342

Tabelle 8-19: Ausgewählte Daten der vorl. Ertragstafeltabelle Fichte, mäßige Hochdurchforstung, Oberhöhenbonität 35 m gemäß dyn-ET (unveröff.).

Tabelle 8-20: Ausgewählte Daten der vorl. Ertragstafeltabelle Fichte, mäßige Hochdurchforstung, Oberhöhenbonität 39 m gemäß dyn-ET (unveröff.)......... 344

Tabelle 8-21: Ausgewählte Daten der vorl. Ertragstafeltabelle Fichte, mäßige Hochdurchforstung, Oberhöhenbonität 43 m gemäß dyn-ET (unveröff.)......... 345

Tabelle 8-22: Ausgewählte Daten der vorl. Ertragstafeltabelle Fichte, starke Hochdurchforstung, Oberhöhenbonität 27 m gemäß dyn-ET (unveröff.)......... 346 
Tabelle 8-23: Ausgewählte Daten der vorl. Ertragstafeltabelle Fichte, starke Hochdurchforstung, Oberhöhenbonität 31 m gemäß dyn-ET (unveröff.).

Tabelle 8-24: Ausgewählte Daten der vorl. Ertragstafeltabelle Fichte, starke Hochdurchforstung, Oberhöhenbonität 35 m gemäß dyn-ET (unveröff.).

Tabelle 8-25: Ausgewählte Daten der vorl. Ertragstafeltabelle Fichte, starke Hochdurchforstung, Oberhöhenbonität 39 m gemäß dyn-ET (unveröff.). 349

Tabelle 8-26: Ausgewählte Daten der vorl. Ertragstafeltabelle Fichte, starke Hochdurchforstung, Oberhöhenbonität 43 m gemäß dyn-ET (unveröff.).......... 350 



\section{Variablen- und Einheitenverzeichnis}

A

$a$

$a_{\eta}$

a

$\alpha$

$b_{0}, \ldots, b_{6}$

$\beta_{1}, \beta_{2}$

$C$

$\gamma_{1}, \gamma_{2}, \gamma_{3}$

$D_{g}$

$D_{100}$

$\delta_{1}, \ldots, \delta_{4}$

$\widehat{E}$

$€$

$e$

Efm

$\varepsilon$

F

$f(\ldots)$

$\mathrm{Fm}$

G

GWL

GWertL

$H$

$H_{100}$

$h$ (Index)

ha

$i$
Fläche je Einzelbaum

durchschnittlicher Überschuss der forstlichen Produktion bzw. Brutto-Bodenrente (Annuität)

durchschnittlicher waldbaulicher Überschuss der forstlichen Produktion (nachhaltige Betriebsklasse)

Jahr bzw. Jahre

.... Risikoart-Parameter der Überlebensfunktion (Abschnitte 2.2.8 und 3.3.6)

Parameter der Isolinien-Funktionen (Abschnitt 3.3.4)

.Parameter der Volumenzuwachsfunktionen (Abschnitt 2.1.1)

Bestandesbegründungskosten

Parameter der dimensionsabhängigen Deckungsbeitragsfunktion

(Abschnitt 2.2.4)

Durchmesser des Grundflächenmittelstammes

Durchmesser der 100 stärksten Bäume eines Bestandes bzw. je Hektar

Parameter der dimensionsabhängigen Deckungsbeitragsfunktion zur monetären Bewertung forstlicher Ertragstafeln

(Abschnitt 3.3.5)

Erwartungswert

Euro

Eulersche Zahl

Erntefestmeter

Ertragsniveau (unteres, mittleres, oberes) nach Assmann und Franz (1963, S. 1 f.)

Erntefixkosten

Funktion (in Abhängigkeit von)

Festmeter ( $m^{3}$ Holzvolumen)

Bestandesgrundfläche

Gesamtwuchsleistung

Gesamtwertleistung

Höhe

Bestandesoberhöhe

(mittlere Höhe der 100 stärksten Bäume des Bestandes bzw. je Hektar) hoch/hohe $(\mathrm{r} / \mathrm{s})$

Hektar $\left(10.000 \mathrm{~m}^{2}\right)$ Iterationsschritt des Optimierungsmodells (Abschnitt 2.1) 
j

$K_{S}$

$\overline{K_{S}}$

$k$

konst.

$L$

$\lambda$

$M_{t}$

$m$ (Index)

$\mathrm{m}$

$\max$

N

$n$ (Index)

nat

$\mathbb{N}$

P

$p$

$\varpi$

$\psi$

$r$

$R S$

RMSE

$\mathbb{R}$

S

S

$\sigma_{t}$

$T$

t

$\tau$

V

$V_{B}$

$v_{B}$

$\overline{v_{B}}$
Einzelbaum-Laufvariable (Analysebaum) laufende Risikokosten durchschnittliche Risikokosten Einzelbaum-Laufvariable (von $j$ beeinflusster Baum; Element von $L$ ) konstant Menge der von $j$ beeinflussten Bäume Vornutzungsvolumen (ausscheidendes Bestandesvolumen) ..Vornutzungsstammzahl zum Zeitpunkt $t\left(\sigma_{t} \cdot N_{t-1}\right)$ $\mathrm{mittel} / \mathrm{mittlere}(\mathrm{r} / \mathrm{s})$ Meter maximal(e/er/es) Stammzahl niedrig(e/er/es) natural Menge der natürlichen Zahlen

Deckungsbeitrag (im Sinne eines erntekostenfreien Holzerlöses) Deckungsbeitragsveränderung jährlich-konstante Deckungsbeitragssteigerungsrate Bonitätssystem (langsames, mittleres, schnelles) nach Wenk et al. (1984, S. 3) . Kalkulationszinssatz relativer Baumabstand . Root Mean Square Error (Wurzel der mittleren Fehlerquadratsumme) Menge der reellen Zahlen Überlebenswahrscheinlichkeit unbedingte Ausfallwahrscheinlichkeit Vornutzungsvektor mit den Vornutzungsanteilen $\sigma_{1}, \ldots, \sigma_{t}$ . Endnutzungszeitpunkt, Umtriebszeit Alter, Zeitpunkt zeitliche Laufvariable Volumen verbleibendes Bestandesvolumen (Vorrat) Bestandesvolumenzuwachs durchschnittlicher Bestandesvolumenzuwachs 
$V_{E}$

$v_{E}$

$\overline{v_{E}}$

$V_{E}$

$V_{\eta}$

$W_{B}$

$w_{B}$

$\overline{w_{B}}$

$W_{E}$

$w_{E}$

$\overline{w_{E}}$

$W_{\eta}$

$\varphi$

$\Phi$

Z
Einzelbaumvolumen laufender Einzelbaumvolumenzuwachs durchschnittlicher Einzelbaumvolumenzuwachs . Normalvorrat (nachhaltige Betriebsklasse) . Normalvorrat (nachhaltige Betriebsklasse) . Bestandeswert, Abtriebswert Bestandeswertzuwachs durchschnittlicher Bestandeswertzuwachs Einzelbaumwert laufender Einzelbaumwertzuwachs durchschnittlicher Einzelbaumwertzuwachs durchschnittlicher Abtriebswert (nachhaltige Betriebsklasse) relative Deckungsbeitragsreduktion bei Kalamitätsnutzung absolute Deckungsbeitragsreduktion bei Kalamitätsnutzung Zwangsnutzung (kalamitätsbedingt) 



\section{Abkürzungsverzeichnis}

aussch.

ausscheidend

Abb.

Abbildung

Abs.

Absatz

Anm. d. A.

Anmerkung(en) des Autors

B4, B2b

Sortimentsbezeichnungen

abh.

abhängig

BHD

Brusthöhendurchmesser (Baumdurchmesser in einer Höhe von 1,3 m über dem Grund)

bspw.

beispielsweise

BMEL

bzw.

Bundesministerium für Ernährung und Landwirtschaft beziehungsweise

ca.

c. p. circa ceteris paribus (unter ansonsten gleichen Bedingungen)

DB Deckungsbeitrag

DDR

Df. Durchforstung

d. h. das heißt

dim.abh. dimensionsabhängig durchschn. durchschnittlich(e/er/es) dyn-ET Forschungsprojekt: "Neue Generation dynamischer Ertragstafeln" (Nordwestdeutsche Forstliche Versuchsanstalt und Abteilung Forstökonomie der Georg-August-Universität Göttingen)

ET Ertragstafel

etc. et cetera

et al. et alii, et aliae, et alia (und andere)

EStG Einkommensteuergesetz EStDV Einkommensteuer-Durchführungsverordnung

f. auf der nächsten Seite

ff. auf den nächsten Seiten forstl. forstlich(e/er/es)

FNR Fachagentur Nachwachsende Rohstoffe e.V. Projektträger des Bundesministeriums für Ernährung und Landwirtschaft 
ggf. gegebenenfalls

ggü. gegenüber

GWL Gesamtwuchsleistung

H/D Höhe/Durchmesser (vgl. H/D-Verhältnis, H/D-Wert oder auch Schlankheitsgrad: Verhältnis aus Baumhöhe [m] und Baumdurchmesser [m])

i. d. R. in der Regel

i. V.m. in Verbindung mit

$\mathrm{IL}$ Industrieholz-lang inkl. inklusive insb. insbesondere IS Industrieholz-kurz/Industrieschichtholzholz jährl. . jährlich(e/er/es)

Kalk.zinssatz Kalkulationszinssatz Kalk.zins. konstant konst. laufend(e/er/es)

lfd. maximal(e/er/es)

$\max$. mittlere $(\mathrm{r} / \mathrm{s})$

mittl. Nummer

$\mathrm{Nr}$. Nordwestdeutsche Forstliche Versuchsanstalt

NW-FVA Seite(n)

S. Stand Density Management Diagrams (Bestandesbehandlungsdiagramme)

schw.

S. 0 . $\operatorname{schwach}(e)$

$\operatorname{sog}$. siehe oben

st. sogenannte $(\mathrm{s} / \mathrm{r})$ $\operatorname{stark}(\mathrm{e})$

Steig. Steigerung

Tab. Tabelle

u. a. unter anderem Übers. d. A. Übersetzung(en) des Autors unveröff. unveröffentlicht verbl. verbleibend vgl. vergleiche z. B. zum Beispiel 
Z-Baum

Zukunfts- oder auch Zielbaum (ausgewählte, markierte und geförderte Bäume des Endbestandes)

2SLS Two-Stage Least Squares (Verfahren der simultanen Regressionsanalyse)

waldb. waldbaulich(e/er/es) 

"Forestry is basically the regeneration and harvest of interdependently growing trees."

Coordes (2016, S. 174)

\section{Einleitung}

Grundlage der Waldbewirtschaftung und damit auch der hier getätigten ökonomischen Analyse forstlicher Bestandesbehandlung bildet stets das Wachstum von Waldbeständen. Dieses wird über die Gesamtheit der einzelnen Bäume des Bestandes determiniert und stellt nach Gadow (2003, S. 1) eine „komplexe Reaktion der Waldbäume auf unterschiedliche externe Einflüsse“ dar - natürliche und anthropogene.

Die natürlichen Einflussfaktoren, wie z. B. das Klima und der Boden (anorganische Umgebung), bilden dabei die standörtlichen Rahmenbedingungen (vgl. Gadow 2003, S. 3 ff.; Röhrig et al. 2006, S. 53 ff.; Albert und Schmidt 2010; Skovsgaard und Vanclay 2013). Während sie in Kapitel 2 als konstant angesehen werden und damit der Einfluss auf die optimale Bestandesbehandlung vernachlässigt wird, finden die Auswirkungen unterschiedlicher Standortsgüten über die Oberhöhenbonitäten der Ertragstafeln (indirekt) Eingang in die ökonomischen Analysen von Kapitel 3.

Im Fokus der vorliegenden Arbeit stehen aber die durch anthropogene, waldbauliche Handlungen beeinflussbaren, wachstumsbestimmenden Konkurrenzverhältnisse zwischen den Bäumen (vgl. Gadow 2003, S. 91 ff.; Röhrig et al. 2006, S. 214 ff.; Pretzsch 2009, S. 291 ff.) sowie ihr Einfluss auf den ökonomischen Erfolg der forstlichen Produktion. Denn nach der standortspezifischen Auswahl und Begründung eines Waldbestandes, ist das waldbauliche Handeln maßgeblich durch die Entnahme von Bäumen zur Regulation der Bestandesdichte gekennzeichnet (Wippermann 2005, S. 1; Fuchs 2018, S. 1). Newton (1997, S. 251) beschreibt diesbezüglich „stand density management“ als den Prozess der Steuerung des Ressourcenwettbewerbs (Stammzahl und deren räumliche Anordnung je Bestandesfläche) zur Erreichung festgelegter Ziele. In Anlehnung an Long (1985) bezeichnen Patrício und Nunes (2017, S. 866; Übers. d. A.) die Durchforstung gar als die ,wahrscheinlich einflussreichste Tätigkeit, die ein Waldbauer zwischen aufeinanderfolgenden Regenerationsperioden ausüben kann“. Dabei findet nach Utschig (2002, S. 336) stets eine Abwägung zwischen flächen- (z. B. Volumenleistung und Wertleistung) und einzelbaumbezogenen (z. B. Dimension, Qualität und Vitalität) Zielen statt. 
Die vorliegende Arbeit agiert somit an der Schnittstelle von Waldwachstumskunde, Waldbau, Ertragskunde und Forstökonomie. Unter Waldwachstumskunde wird dabei in Anlehnung an Pretzsch (2009, S. 1; Übers. d. A.) die Auseinandersetzung „,mit den Veränderungen der Struktur und Zusammensetzung des Waldes im Laufe der Zeit, einschließlich seines Verhaltens als Reaktion auf anthropogene und natürliche Störungen“ verstanden. Sie bildet die Grundlage für Waldbau und Ertragskunde, welche den klaren Bezug zu anthropogenen Handlungen haben und nach Zingg (2005) bspw. im angelsächsischen Raum unter „,silviculture“ zusammengefasst werden. In dieser Arbeit wird eine begriffliche Trennung angestrebt und unter Waldbau in Anlehnung an Ammer und Puettmann (2009, S. 91) zunächst allgemein die „Steuerung von Waldökosystemen anhand vorgegebener Ziele des Waldeigentümers und der Gesellschaft [...]“ verstanden. Während diese Ziele mannigfaltig sein können, wird mit der Ertragskunde die Produktion und Nutzung von Holzvolumen und damit das wirtschaftliche Element waldbaulichen Handelns in den Fokus gerückt (vgl. Zingg 2005). Die effiziente Gestaltung dieser Holznutzung ist schließlich (u. a.) Aufgabe der Forstökonomie. Diese wird hier im engeren Sinn als forstliche Betriebswirtschaftslehre und damit in Anlehnung an Speidel (1984, S. 11) als „Lehre von der Zweckmäßigkeit wirtschaftlichen Handelns im Forstbetrieb“ verstanden. Im Einklang mit Speidels „Neoklassische[m] Programm der Forstökonomik“ (Oesten und Roeder 2012b, S. 201 ff.) werden Instrumente der allgemeinen Betriebswirtschaftslehre genutzt, um im Zuge der forstlichen Produktion über den planvollen und zweckmäßigen Einsatz knapper Ressourcen zu entscheiden (Wirtschaftlichkeits- bzw. Rationalprinzip; Oesten und Roeder 2012b, S. 202) ${ }^{1}$. Zahlreiche Besonderheiten der forstlichen Produktion (vgl. z. B. Stinglwagner et al. 2005, S. 314; Möhring 2010) erschweren dabei die Anwendung dieser Instrumente und rechtfertigen damit zugleich die fortlaufende Forschung, Lehre und Anwendung der Forstökonomie. Möhring (2010) zählt zu den Besonderheiten der forstlichen Produktion u. a. die extreme Langfristigkeit der forstlichen Produktion, die bereits erwähnten räumlichen und zeitlichen Abhängigkeiten zwischen den einzelnen Bäumen eines Bestandes (Konkurrenz) sowie die Standortabhängigkeit des Waldwachstums inkl. der daraus folgenden biotischen und abiotischen Einflussgrößen (Ri-

\footnotetext{
${ }^{1}$ Dieses Verständnis der Forstökonomie ordnet sich in die grundlegende und vielzitierte Definition von Robbins (1932, S. 15; Übers. d. A.) ein, der die Ökonomie als Wissenschaft bezeichnete, „die das menschliche Verhalten als eine Beziehung zwischen Zielen und knappen Mitteln mit alternativen Verwendungsmöglichkeiten“" untersucht. Die Knappheit entsteht nach Bergen et al. (2013, S. 1), da die menschlichen Bedürfnisse nach Ressourcen und Gütern deren Verfügbarkeit übersteigen - Gegenstand der Ökonomik ist demnach die Milderung dieser Knappheit durch planvolles Handeln.
} 
siken). Elementar für die Analysen dieser Arbeit sind zudem die von Möhring (2010) aufgeführte Doppelfunktion der Bäume als Produktionsmittel (bis zum Zeitpunkt der Ernte) und Produkt (nach der Ernte) sowie die Tatsache, dass der Volumenzuwachs in einem Waldbestand in der Regel mit einer (monetär) zusätzlich positiv wirkenden Zunahme der Einzelbaumdimension einhergeht. Nicht zuletzt unterliegt die forstliche Produktion zahlreichen Restriktionen, ,in deren Zentrum in der Regel die Forderung nach Nachhaltigkeit steht“" (Möhring 2010, S. 347).

Die aus den Besonderheiten der forstlichen Produktion resultierende Komplexität macht es nach Wippermann (2005, S. 2) „unmöglich, allgemein gültige waldbauliche Vorgaben zu entwickeln“ - vielmehr sei die „Kenntnis der qualitativen Eigenschaften der optimalen Lösung des Bestandesbehandlungsproblems“ von Bedeutung. In diesem Sinne und im Einklang mit der oben aufgeführten Definition der Forstökonomie wird unter der ökonomischen Analyse forstlicher Bestandesbehandlung die Gesamtheit aller Analysen verstanden, die zum Verständnis einer planvollen und zweckmäßigen Gestaltung der forstlichen Bestandesbehandlung beitragen (vgl. Oesten und Roeder 2012b, S. 202). Im Sinne eines Brückenschlags zwischen Theorie und forstlicher Praxis (vgl. Möhring 2009) ist es das übergeordnete Ziel dieser Arbeit, rationale Entscheidungen im Rahmen der forstlichen Bestandesbehandlung sowie die ökonomischen Konsequenzen ausgewählter waldbaulicher Handlungsalternativen aufzuzeigen. Methodisch werden dafür zwei unabhängige Ansätze mit entsprechenden Teilzielen genutzt und in separaten Kapiteln bearbeitet:

- In Kapitel 2 erfolgt die numerische Optimierung eines stark vereinfachten forstlichen Produktionsmodells vor dem Hintergrund verschiedener Prämissen (theoretischer Analysrahmen) - gesucht wird die jeweils optimale Bestandesbehandlung.

- In Kapitel 3 werden alternative Bestandesbehandlungsvarianten forstlicher Ertragstafeln (praxisnahe Wachstums- und Nutzungsmodelle) vor dem Hintergrund gleicher Prämissen ökonomisch bewertet und miteinander verglichen - gesucht wird die optimale bzw. vorteilhafte Variante der forstlichen Produktion.

In beiden Fällen bewegt sich die Arbeit im klassischen Spannungsfeld der Modellbildung, welches Möhring (1994, S. 1, Fußnote1) in Anlehnung an Oesten (1986, S. 78 f.) als „methodologisches Dilemma“ bezeichnet: Die Modelle liefern demnach „,vereinfachende Abbilder realer Tatbestände“ bei gleichzeitig angestrebter „Strukturgleichheit bzw. Strukturähnlichkeit zwischen dem Realsystem und dem Modellsystem“ (Möhring 1994, S. 1, Fußnote 1). Sie sind „Kondensate von Wahrnehmungen, Bewertungen und Erklärungsversuchen, deren Güte sich erst dann erweist, wenn mit ihrer Hilfe Systemverhalten in befriedigender Weise vorausgesagt 
werden konnte“" (Detten 2018, S. 191). Die Modellbildung dieser Arbeit dient demnach dazu, Entscheidungsträgerinnen und Entscheidungsträger mit den ökonomischen Konsequenzen ausgewählter waldbaulicher Handlungen bzw. der Anwendung forstlicher Ertragstafeln vertraut zu machen (vgl. Gadow 2003, S. 144) und gleichzeitig rationale (optimale) Entscheidungen im Rahmen der forstlichen Bestandesbehandlung aufzuzeigen (vgl. Möhring 1994, S. 5). Sie ordnet sich damit in die normative (vgl. Möhring 1994, S. 5 f. und S. 9 ff.; Gerst 2015, S. 3 f. i. V. m. Sieben und Schildbach 1990, S. 1) bzw. präskriptive (Bamberg et al. 2019, S. 3 ff.) Entscheidungstheorie ein, welche die Frage nach rationalen Entscheidungen unter gegebenen Prämissen beantwortet (Bamberg et al. 2019, S. 5).

Optimal bezieht sich dabei stets auf das Ziel der Produktion von Holz für die energetische und stoffliche Nutzung. Ökonomische Bewertung meint mit Bezug auf dieses Ziel die Inwertsetzung forstlicher Bestandesbehandlung für innerbetriebliche Entscheidungssituationen (vgl. auch Oesten und Roeder 2012a, S. 181 ff.). Aus den zahlreichen Waldfunktionen (vgl. BWaldG 1975, § 2; Röhrig et al. 2006, S. 27 f. nach Kuusela 1994 und Oesten und Roeder 2012a, S. 15) bzw. Funktionen einer nachhaltigen Forstwirtschaft (vgl. Spellmann 2010, S. 102 nach MCPFE 2003), greift die vorliegende Arbeit damit ausschließlich die Nutz- bzw. Produktionsfunktion heraus. Dies stellt keine Wertung gegenüber anderen Ökosystemleistungen (vgl. Millennium Ecosystem Assessment; MEA 2005), sondern lediglich den Ansatz einer Komplexitätsreduktion dar. Mit dem Ziel, das Verständnis für einen effizienten Einsatz knapper Ressourcen zu steigern (vgl. Möhring 2009, 2010), sieht der Autor die Arbeit zudem nicht im Widerspruch zu den forstwirtschaftlichen Herausforderungen rund um Klimawandel und Naturschutz (vgl. Lindner et al. 2010; Hanewinkel et al. 2013; Nabuurs et al. 2013; Hickler et al. 2014; Seidl et al. 2014; Schulze und Ammer 2015; Ammer et al. 2017), sondern vielmehr als Beitrag zur verantwortungsvollen und nachhaltigen Bewirtschaftung unserer Wälder.

\subsection{Aufbau und Inhalt}

Die vorliegende Arbeit gliedert sich anhand der genannten Ziele in einen theoretischen Analysrahmen (Kapitel 2) und einen davon unabhängigen, ertragstafelbasierten Praxisteil (Kapitel 3). Die Kapitel sind dabei eigenständig aufgebaut - inhaltliche Einleitung, methodische Beschreibung sowie Präsentation und Diskussion der Ergebnisse erfolgen jeweils separat.

Durch die schrittweise Einführung von Modellprämissen (Abschnitt 2.1.2) wird die Komplexität eines stark vereinfachten forstlichen Produktionsmodells (Abschnitt 2.1.1) in Kapitel 2 systematisch erhöht. Die numerisch ermittelten Optima (Abschnitt 2.1.3) der forstlichen Produktion geben Aufschluss über die Wechselwirkungen zwischen waldwachstumskundlichen 
Grundbeziehungen, waldbaulichen Handlungen und den resultierenden ökonomischen Zielgrößen (Abschnitt 2.2). Die zentralen Ergebnisse werden abschnittsübergreifend zusammengefasst und ermöglichen in Anlehnung an Möhring (2009) einen Brückenschlag zur forstlichen Praxis (Abschnitt 2.3). Abschnitt 2.4 zeigt mit einer kritischen Methoden- und Modellwürdigung schließlich die Grenzen des theoretischen Analyserahmens auf und verweist auf alternative Forschungsansätze.

In Kapitel 3 skizziert Abschnitt 3.1 zunächst die historische Entwicklung, diskutiert die ertragskundlichen Grundlagen und beschreibt Struktur sowie exemplarische Anwendungsgebiete forstlicher Ertragstafeln. Die Ergebnisse einer eigenständig durchgeführten Praxisbefragung zeigen in Abschnitt 3.2 die gegenwärtige Nutzung forstlicher Ertragstafeln und verdeutlichen die methodischen und inhaltlichen Ansprüche an zukünftige Ertragstafelwerke. Das Verbundforschungsprojekt „Neue Generation dynamischer Ertragstafeln (dyn-ET)“ hat die Erstellung solcher Ertragstafelwerke zum Ziel - Abschnitt 3.3 stellt das Projekt vor (Abschnitt 3.3.1), beschreibt Aufbau und Erstellungsmethodik (Abschnitt 3.3.2) und analysiert vorläufige Ertragstafeldaten ausgewählter Baumarten (Abschnitte 3.3.3 und 3.3.4). Darauf aufbauend erfolgt in Abschnitt 3.3.5 deren ökonomische Bewertung anhand eines nachvollziehbaren und auf realistischen Eingangsdaten basierenden Bewertungsmodells. Sowohl auf naturaler als auch auf ökonomisch bewerteter Ebene erfolgen Vergleichsanalysen zu in der Forstpraxis etablierten Ertragstafelwerken sowie zwischen den alternativen waldbaulichen Behandlungsalternativen. Abschnitt 3.3.6 analysiert schließlich die Wertrelationen der waldbaulichen Behandlungsalternativen anhand der Veränderung relevanter Treiber (Deckungsbeitragsfunktion, Kalkulationszinssatz und Überlebensrisikostärke) sowie deren Wechselwirkungen und ermöglicht damit einen Brückenschlag zur forstlichen Praxis.

Die abschließenden Schlussfolgerungen verknüpfen die zentralen Erkenntnisse der eigentlich unabhängigen Kapitel 2 und 3, leiten allgemeine Handlungsempfehlungen ab und zeigen weitergehenden Forschungsbedarf auf.

Da diese Arbeit u. a. im Zuge des drittmittelfinanzierten Verbundforschungsprojekts „Neue Generation dynamischer Ertragstafeln (dyn-ET)“ entstand, sind Teile der in Kapitel 3 gezeigten Ergebnisse ebenfalls Bestandteil der Projektzwischenberichte bzw. werden im Zuge des Projektabschlussberichts präsentiert. Auf wechselseitige Verweise zu selbst verfassten Inhalten wird an den entsprechenden Stellen bewusst verzichtet. 
Ausdrücklich erwähnt seien an dieser Stelle drei studentische Abschlussarbeiten, die im Rahmen des dyn-ET-Projekts und unter enger fachlicher Betreuung durch den Autor in der Abteilung Forstökonomie der Georg-August-Universität Göttingen verfasst wurden: Jasper Fuchs schrieb seine Masterarbeit über die „Ökonomie der Einzelbaumentnahme“ und trug damit zum tiefgehenden Verständnis von Grenznutzen und Grenzkosten der forstlichen Bestandesbehandlung bei. Seine Ergebnisse und Recherchen sind Grundlage einiger Ausführungen des Kapitels 2. Volker von Groß schrieb seine Masterarbeit über die „Auswirkungen der Anwendung einer neuen Generation forstlicher Ertragstafeln auf betriebliche Planungs- und Entscheidungsgrößen“. Die Ergebnisse seiner Abschlussarbeit sowie zahlreiche darüber hinaus von ihm im Rahmen des dyn-ET-Projekts getätigte Analysen trugen zur umfassenden Analyse der neuen Generation dynamischer Ertragstafeln in Abschnitt 3.3 bei. Jonathan Fibich führte im Rahmen seiner Bachelorarbeit einen „Vergleich verschiedener Fichtenertragstafeln anhand ertragskundlicher und ökonomischer Kenngrößen“ durch. Gemeinsam mit einem für das dyn-ET-Projekt verfassten Berichts zu forstlichen Ertragstafeln fanden seine Ergebnisse Eingang in den Abschnitt 3.1. Die Abschlussarbeiten werden an entsprechender Stelle zitiert, wobei inhaltliche Übereinstimmungen und sprachliche Nähen auch an anderer Stelle vorkommen und der engen studentischen Betreuung durch den Autor dieser Arbeit geschuldet sind - seinem Geist entsprangen Motivation, Zielsetzungen und Methodik der genannten Abschlussarbeiten.

\subsection{Einordnung waldbaulicher Begriffe}

Unter dem Begriff der forstlichen Bestandesbehandlung werden in dieser Arbeit ausgewählte waldbauliche Handlungen im Rahmen der Bewirtschaftung eines Waldbestandes verstanden. Solch ein (Wald-)Bestand ist nach Röhrig et al. (2006, S. 349) der „Teil eines Waldes, der sich durch unterschiedliche Zusammensetzung oder verschiedene Alter der Bäume unterscheidet, räumlich gegeneinander abzugrenzen ist und über eine gewisse Mindestgröße verfügt“. Als „waldbauliche Behandlungseinheit“ (Ammer und Puettmann 2009, S. 91) bildet der Bestand die Analyseeinheit dieser Arbeit, für die ,einheitliche Management-Entscheidungen“ (Gadow 2005, S. 31) getroffen werden. Damit entsprechen die Analysen dieser Arbeit (überwiegend ${ }^{2}$ ) dem Modell des aussetzenden Betriebes - es wird die Vorteilhaftigkeit der Bestandesbehand-

\footnotetext{
${ }^{2}$ In den Abschnitten 3.3.3 und 3.3.5 werden im Rahmen der Analyse forstlicher Ertragstafeln ergänzend auch zwei Kennzahlen einer nachhaltigen Betriebsklasse aufgezeigt.
} 
lung aus Sicht eines Investors analysiert und diese nicht in Bezug zum forstbetrieblichen Kontext, mit daraus resultierenden Restriktionen, wie z. B. der nachhaltigen betrieblichen Liquidität, gesetzt (Wippermann 2005, S. 18 f. i. V. m. Hultkrantz 1991).

Innerhalb dieser Analyseeinheit wird die forstliche Bestandesbehandlung von der Gesamtheit aller waldbaulichen Handlungen abgegrenzt, indem nur ausgewählte Eingriffe zwischen der Bestandesbegründung und der Endnutzung des Bestandes analysiert und im Folgenden begrifflich eingeordnet werden. Ausgeschlossen ist demnach die vor der Bestandesbegründung stattfindende und nach Schall und Ammer (2013, S. 382; Übers. d. A.) ,strategisch grundlegendstes Managemententscheidung“ - die Baumartenwahl. Sie bestimmt nicht nur über Ertrag, Dauer und Störungsanfälligkeit der forstlichen Produktion (Schall und Ammer 2013), sondern beeinflusst den gesamten Bestandesaufbau und damit auch „Struktur und Funktionsweise des Waldökosystems“، (Röhrig et al. 2006, S. 49). Die Wahl der Baumart erfolgt entsprechend forstbetrieblicher Zielsetzungen und möglichst angepasst an standörtliche Gegebenheiten (vgl. Röhrig et al. 2006, Kapitel 2), wobei dieser Aspekt insbesondere durch den fortschreitenden Klimawandel nochmal mehr in den Fokus wissenschaftlicher Untersuchungen (vgl. u. a. Bolte et al. 2009; Schou et al. 2015; Yousefpour und Hanewinkel 2016; Albert et al. 2017; Paul et al. 2019a; Paul et al. 2019b) und praktischer Waldbaurichtlinien bzw. -empfehlungen (vgl. z. B. ForstBW 2014; HMUKLV 2018; LWF 2019; NLF 2019) gerückt ist. Kapitel 2 analysiert die Ökonomie forstlicher Bestandesbehandlung losgelöst von einer konkreten Baumart und erst ab dem Moment der Bestandesbegründung. Kapitel 3 geht zwar auf Ertragstafeln unterschiedlicher Baumarten ein, stellt aber keine direkten quantitativen Vergleiche zwischen diesen her.

Nach der zielkonformen und standortangepassten Baumartenwahl beginnt die Bestandesentwicklung mit der Begründung einer bestimmten Anzahl von Bäumen oder Sämlingen bzw. Samen (Ausgangsstammzahl), welche für die Konkurrenzverhältnisse innerhalb des Bestandes entscheidend ist (vgl. Coordes 2014a, S. 11 ff.). Die Bestandesbegründung kann durch Samenabfall, Samenanflug, Stockausschlag, Wurzelbrut oder Absenker (natürlich) und/oder in Form von Saat oder Pflanzung (künstlich) erfolgen (vgl. Röhrig et al. 2006, Kapitel 3). Für diese Arbeit spielt es dabei keine Rolle, ob auf der Fläche zuvor bereits Waldbestände gestockt haben (Verjüngung im engeren Sinne, vgl. Röhrig et al. 2006, S. 75) oder bspw. landwirtschaftliche Flächen zu Wald umgewandelt werden (Aufforstung, vgl. Röhrig et al. 2006, S. 75). Die Bestandesbegründung wird somit als Verjüngung im weiteren Sinne verstanden und bezeichnet den Zeitpunkt des planmäßigen Beginns einer Bestandesentwicklung. Im Modell des Kapitels 2 
sowie bei den Ertragstafelanalysen in Kapitel 3 wird dieser Zeitpunkt einheitlich durch das Alter 0 a aller Bäume charakterisiert, auch wenn gewisse Altersunterschiede sowohl bei natürlicher als auch bei künstlicher Bestandesbegründung stets gegeben sind. Die für die Konkurrenzsituation innerhalb des Bestandes ebenfalls ausschlaggebende, und primär durch die Bestandesbegründung determinierte, räumliche Anordnung der Bäume (vgl. Coordes 2014a, S. 11 ff.), findet keinen Eingang in die Analysen dieser Arbeit. Notwendige Pflegemaßnahmen bis zur gesicherten Verjüngung werden (sofern vorhanden) abweichend von Röhrig et al. (2006, S. 188 ff.) der Bestandespflege (siehe unten) zugeordnet, um der finanzmathematisch korrekten Verbuchung damit verbundener Kosten gerecht zu werden.

Abweichend von Röhrig et al. (2006, Kapitel 4) findet bei den waldbaulichen Maßnahmen zwischen Bestandesbegründung und Endnutzung in dieser Arbeit eine Unterteilung zwischen Maßnahmen ohne und mit der Entnahme von Bestandesvolumen aus dem Waldbestand (Nutzung) statt. Während Röhrig et al. (2006, Kapitel 4) die Bestandespflege im weiteren Sinne definiert und darunter Läuterungen, Durchforstungen, Ästungen sowie Düngungen zusammenfasst, wird der Begriff in dieser Arbeit enger gefasst. Unter Bestandespflege im engeren Sinne werden folglich alle rein investiven waldbaulichen Maßnahmen zur Förderung der Bestandeszielerreichung ohne Holznutzung verstanden. Dazu zählen Pflegemaßnahmen zur Sicherung der Bestandesbegründung (vgl. Röhrig et al. 2006, S. 188 ff.), die Jungwuchspflege bis zum Eintritt des Bestandesschlusses sowie die Läuterung (vgl. Röhrig et al. 2006, S. 209 und 260 ff.). Auch wenn Läuterung und Durchforstung in Bezug auf Charakter und Zielsetzung ähnlich sind (siehe Vornutzung), sind Läuterungen regelmäßig durch negative Deckungsbeiträge gekennzeichnet (Röhrig et al. 2006, S. 210), weshalb das geerntete Bestandesvolumen oftmals im Bestand verbleibt und meist flächen- bzw. arbeitsstundengebundene Kosten entstehen ${ }^{3}$. Weitere, in dieser Arbeit jedoch nicht behandelte, investive Bestandespflegemaßnahmen sind die Wertästung totasterhaltender Baumarten zur Steigerung des astfreien Holzvolumens (vgl. Röhrig et al. 2006, S. 313 ff.) sowie die Düngung zur Erhaltung oder langfristigen Verbesserung des Standortspotentials (vgl. Röhrig et al. 2006, S. 318 ff.).

\footnotetext{
${ }^{3} \mathrm{Im}$ forstlichen Produktionsmodell von Kapitel 2 sowie bei der ökonomischen Bewertung forstlicher Ertragstafeln (Abschnitt 3.3.5) ergeben sich teils negative Deckungsbeiträge für die Vornutzungsvolumina bzw. ausscheidenden Bestandesvolumina. Es entstehen somit mengengebundene Kosten und es wird an diesen Stellen auf eine Unterscheidung zwischen Vornutzungen mit negativem und Vornutzungen mit positivem Deckungsbeitrag verzichtet.
} 
Vornutzungen werden in dieser Arbeit einerseits von Bestandespflegemaßnahmen (s. o.) und andererseits in Anlehnung an Röhrig et al. (2006, S. 210) von Endnutzungen abgegrenzt. Allerdings erfolgt die Abgrenzung schärfer und es werden aufgrund der ausschließlichen Analyse von Altersklassenwäldern jegliche Baumentnahmen vor Entnahme des letzten Baums eines Bestandeskollektivs, und damit ohne nachfolgende Verjüngung, der Vornutzung zugeordnet. Zielstärkennutzungen können somit, anders als bei Röhrig et al. (2006, S. 300 ff.), sowohl Teil der Vornutzungen als auch Teil der Endnutzung sein. Generell wird eine Verjüngung des Folgebestands vor der Endnutzung ausgeschlossen (vgl. auch Coordes 2014a, S. 21). Vornutzungen oder (in dieser Arbeit synonym dazu verwendet) Durchforstungen (vgl. Röhrig et al. 2006, S. 210 ff. und 266 ff.) sind somit durch die Entnahme und Nutzung von Bestandesvolumen vor der Umtriebszeit gekennzeichnet (ausscheidendes Bestandesvolumen). Es werden durch die Entnahme einzelner Bäume „Wuchsräume“ geschaffen, die in der Folge durch die entsprechenden Nachbarbäume genutzt werden (Röhrig et al. 2006, S. 210). Nach Coordes (2014a, S. 22) wirken Vornutzungen somit in gleicher Weise wie eine Verringerung der Ausgangsstammzahl (siehe oben), wenn auch mit umso geringerer Effektstärke, je später der Eingriff erfolgt. Welche Gründe für die Entnahme von Bäumen vor der Endnutzung sprechen und wie sie sich in Abhängigkeit der Prämissen verändern, ist Analysebestandteil des Kapitels 2. Vorweggenommen sei an dieser Stelle nur, dass das von Röhrig et al. (2006, S. 210) formulierte Ziel der „Steigerung des Wertzuwachses des Bestandes“ zu kurz gefasst ist. Es vernachlässigt unter anderem die, vor dem Hintergrund von Kapitalknappheit vorteilhafte, vorzeitige Realisierung von Durchforstungseinnahmen (vgl. Klemperer 1996, S. 242 ff.).

Röhrig et al. (2006, S. 268) charakterisiert klassische Durchforstungen im Sinne der Durchforstungsversuche der Deutschen Forstlichen Versuchsanstalten anhand von Art, Stärke und zeitlicher Abfolge:

Die Durchforstungsart stellt dabei die qualitative Beschreibung einer Vornutzung dar, d. h. die Charakterisierung, welche Bäume eines Bestandes entnommen werden. In den klassischen Durchforstungsversuchen der Deutschen Forstlichen Versuchsanstalten (vgl. Assmann 1961, S. 208 ff.) wurden diesbezüglich Nieder- von Hochdurchforstungen unterschieden. Während bei Niederdurchforstungen „,von unten her“ eingegriffen wird, finden hochdurchforstungsartige Eingriffe vorrangig im „herrschenden Bestand zur besonderen Pflege bestimmter Stämme unter grundsätzlicher Schonung eines Teiles der beherrschten Bäume“"statt (Röhrig et al. 2006, S. 268). Bei der Erstellung der neuen Generation dynamischer Ertagstafeln (siehe Abschnitt 3.3.2) verbirgt sich hinter den Behandlungsvarianten der „Hochdurchforstung“ eine 
Kombination aus Z-Baum-orientierter Auslesedurchforstung und einer Hochdurchforstung in den Zwischenfeldern (interne dyn-ET-Projektkommunikation i. V. m. Albert et al. 2019). Z-Baum-orientierte Auslesedurchforstung bezeichnet dabei die regelmäßige Entnahme von Bedrängern (überlappende Kronen; vgl. Albert et al. 2019) der Z-Bäume (altersunabhängig konstante Anzahl; vgl. Albert et al. 2019). Eine Abgrenzung zwischen verschiedenen Durchforstungsarten und den damit verbundenen ertragskundlichen Auswirkungen erfolgt im Kapitel 3 (vgl. auch Röhrig et al. 2006, S. 276 ff.). Zur Quantifizierung der Durchforstungsart wird dort (trotz bekannter Schwächen bei der Charakterisierung von Z-Baum-orientierten Auslesdurchforstungen; siehe Fußnote 65 auf Seite 136) die Relation zwischen den Stückvolumina von ausscheidendem und verbleibendem Bestand genutzt (vgl. Stückvolumenrelation in Abschnitt 3.3.3 oder harvest quotient in Meilby und Nord-Larsen 2012). Da in dem stark vereinfachten Optimierungsmodell in Kapitel 2 alle Bestände als homogen, das heißt als Summe identischer Bäume, verstanden werden, spielt die Durchforstungsart dort keine Rolle (siehe Abschnitt 2.4; vgl. Coordes 2014b).

Die Durchforstungsstärke oder Vornutzungsintensität beschreibt die (mengenmäßige) Stärke eines Vornutzungseingriffs. Sie kann nach Röhrig et al. (2006, S. 270 ff.) einerseits verbal beschrieben werden (vgl. „Arten und Grade klassischer Durchforstungen“ in Röhrig et al. 2006, S. 273 nach Verein Deutscher Forstlicher Versuchsanstalten 1902 und Wiedemann 1935). Andererseits kann die Durchforstungsart über Bestandeskennzahlen quantifiziert werden (vgl. z. B. Zielkreisflächen nach Wiedemann 1951, S. 62, der natürliche Bestockungsgrad nach Assmann 1961, S. 210 oder die relative Vornutzungsstärke in Abschnitt 2.1.1). Elementarer Bestandteil der Analysen in Kapitel 3 sind die ertragskundlichen Unterschiede und ökonomischen Auswirkungen verschiedener Durchforstungsstärken, die in Ertragstafelwerken bspw. über die Behandlungsvarianten mäßige, starke und gestaffelte (vgl. Wiedemann 1937, Röhrig et al. 2006, S. 282 f.) Durchforstung unterschieden werden.

Meist sind Durchforstungsstärke und -turnus bzw. Vornutzungsintervall wechselseitig miteinander verknüpft - längere Durchforstungsturnusse, d. h. mehr Zeit zwischen zwei Eingriffen, bedingen i. d. R. höhere Durchforstungsintensitäten, d. h. stärkere Eingriffe, und vice versa (Röhrig et al. 2006, S. 276). Der Zeitpunkt im Bestandesleben, an dem die Holzvolumenentnahme, wird als Vornutzungszeitpunkt (Durchforstungsalter) bezeichnet. Die Vornutzungszeitpunkte beeinflussen den ökonomischen Erfolg der forstlichen Bestandesbehandlung zum einen direkt, über die Vorteilhaftigkeit früherer Zahlungseingänge unter Kapitalknappheit (siehe Abschnitt 2.2.5). Trotz einem in der Realität ggf. über mehrere Wochen bis Monate verlaufenden 
Zeitintervall zwischen erster Baumentnahme und den tatsächlichen Finanztransaktionen, wird die gesamte Maßnahme in forstlichen Produktionsmodellen i. d. R. in einer gedachten Sekunde vollzogen und somit auch verbucht. In forstlichen Ertragstafeln gelistetes ausscheidendes Bestandesvolumen (siehe Abschnitt 3.1.3) wird bspw. auch zu einem Zeitpunkt (i. d. R. alle 5 oder 10 Jahre) quantifiziert und somit bewertet, obwohl es sich in der forstlichen Praxis auch um Volumina einer oder mehrerer Durchforstungen im Zeitintervall davor (oder danach) handeln kann. Zum anderen hat der Zeitpunkt des letzten Eingriffs (i. V. m. der Durchforstungsstärke) Einfluss auf das Sturmwurfrisiko (vgl. u. a. Lohmander und Helles 1987, Donis et al. 2018) und damit auf die Überlebenswahrscheinlichkeit eines Waldbestandes. Dieser indirekte Einfluss auf die ökonomische Erfolgsgröße wird in Kapitel 2 zwar aus Gründen der Modellvereinfachung nicht berücksichtigt, aber in den Abschnitten 2.2.9 und 2.4 diskutiert.

Wie bereits erwähnt, erfolgt eine scharfe Abgrenzung zwischen Vor- und Endnutzung - diese wird als Entnahme der letzten Bäume eines Bestandeskollektivs und damit Einleitung der folgenden Bestandesentwicklung (erneute Bestandesbegründung) definiert. Die Arbeit stellt damit gleichaltrige Waldbestände (Altersklassenwald) in den Fokus der Analyse. Dies stellt keine Wertung gegenüber anderen Betriebsformen (vgl. Röhrig et al. 2006, S. 337 ff.) dar, sondern dient der vereinfachten ökonomischen Analyse in Kapitel 2 und steht im Einklang mit der Analyse der forstlichen Ertragstafeln in Kapitel 3. Dennoch sind die ökonomischen Erkenntnisse dieser Arbeit in großen Teilen auch auf andere Betriebsformen und ungleichaltrige Bestandesstrukturen (Dauerwald) übertragbar (siehe Abschnitt 2.4). 



\section{2. Ökonomie der forstlichen Bestandesbehandlung}

Eine der bereits erwähnten Besonderheiten der forstlichen Produktion stellt die Tatsache dar, dass die Bäume eines Waldbestandes Produktionsmittel und Produkt zugleich sind (Möhring 2010). Aus ökonomischer Sicht sind sie somit Investitions- und Finanzierungsobjekt zugleich (vgl. Coordes 2014a, S. 146 ff.; Möhring 1994, S. 44; Möhring und Dieter 2020) - sie binden Kapital zur Umwandlung in Vermögen (Investition; Thommen und Achleitner 2012, S. 554), solange sie im Bestand gehalten werden und liefern Zahlungsrückflüsse und damit Kapital zur Investition an anderer Stelle (Finanzierung; Thommen und Achleitner 2012, S. 553), sobald sie geerntet werden. Für einen rational entscheidenden und den Nutzen maximierenden Forstbetrieb (vgl. Homo oeconomicus in z. B. Dichtl und Issing 1987, S. 822 f.) in einer Welt knapper Ressourcen stellt sich somit stets die Frage nach den optimalen Erntezeitpunkten von Bäumen bzw. Waldbeständen und damit der optimalen Allokation von Fläche bzw. Wuchsraum ${ }^{4}$ und Kapital (vgl. Möhring 2009). Die durch einen Baum oder Bestand genutzte Fläche (bzw. der eingenommene Wuchsraum) kann von keinem zweiten Baum oder Bestand genutzt werden ${ }^{5}$ und eine bereits gebundene Kapitaleinheit kann zeitgleich nicht alternativ investiert werden.

Pertz (1983; in Verbindung mit Samuelson 1976) ordnet die Frage nach der optimalen Allokation von Fläche und Kapital, welche für die optimale Nutzungsdauer bzw. Umtriebszeit eines gleichaltrigen Reinbestandes seit Faustmann $(1849$; 1995) beantwortet ist, der neoklassischen Wirtschaftstheorie $^{6}$ zu. Die auf der Faustmann-Formel aufbauende Methode der Maximierung des Bodenreinertrags wurde seither vielfach bestätigt, angewendet, ergänzt und abgewandelt vgl. die umfassenden Literaturanalyse von Newman (2002) anlässlich des 150sten Jahrestages

\footnotetext{
${ }^{4}$ Der Begriff Wuchsraum wird in Anlehnung an die Waldwachstumskunde (vgl. z. B. Gadow 2003, S. 91) verwendet, um im Folgenden die Abhängigkeit des Baumwachstums von einem dreidimensionalen, unter- und überirdisch zur Verfügung stehenden Raum zu verdeutlichen. Wird von der Fläche als knappe Ressource gesprochen, ist damit immer auch der (zumindest in Teilen) davon abhängige Wuchsraum gemeint. Andersherum ragt der Wuchsraum eines Baumes aber ggf. über die ihm zur Verfügung stehende Fläche hinaus (Pretzsch 2009, S. 291 ff.), weshalb die Quantifizierung des Wuchsraums über ein Flächenmaß (siehe z. B. Abschnitt 2.2.1) stets als vereinfachende Annahme angesehen werden muss.

${ }^{5}$ Vernachlässigt wird hierbei die sogenannte Nischen-Komplementarität als Mischungseffekt, d. h. die Konkurrenzminderung in Mischbeständen durch die Ausnutzung der gleichen Fläche bzw. des gleichen Wuchsraums durch unterschiedliche Ansprüche verschiedener Baumarten (vgl. Pretzsch et al. 2013b und Morin et al. 2011).

${ }^{6}$ „Während in der klassischen Schule der Wert eines Gutes als objektiv und dem Gut immanent betrachtet wurde (objektive Wertlehre), betonen die Neoklassiker, dass der Wert eines Gutes durch den Nutzen, den dieses Gut dem Verbraucher zur Befriedigung seiner Bedürfnisse stiftet, bestimmt wird (subjektive Wertlehre)“"(Oesten und Roeder 2012b, S. 197, Fußnote 23).
} 
der Faustmann-Formel. Beispielhaft seien hier nur Ohlin $(1921$; 1995) mit einer der ersten Lösungen des Maximierungsproblems, Samuelson (1976) mit seinem viel beachteten Aufsatz zur komparativ-statischen Umtriebszeitoptimierung, Hartman (1976) mit seiner Erweiterung des Faustmann-Ansatzes um Nichtholzprodukte, Clarke und Reed (1989) mit der Einbeziehung von Unsicherheiten sowie Chang (1998) mit seinem generalisierten Faustmann-Modell genannt. Unabhängig von Faustmann $(1849 ; 1995)$ entwickelte Pressler $(1860 ; 1995)$ eine grenzverzinsungsbasierte Entscheidungsregel zur Bestimmung des optimalen Abtriebs- bzw. Ersatzzeitpunkts, welche die Bedingung erster Ordnung bei der Bestimmung der optimalen Umtriebszeit über die Faustmann-Formel darstellt (Chang und Deegen 2011 i. V. m. Johansson und Löfgren 1985, S. 79 f.). Diese zentralen Erkenntnisse forstökonomischen Handelns werden oft unter dem sogenannten Faustmann-Pressler-Ohlin-Theorem (FPO-Theorem) zusammengefasst (Johansson und Löfgren 1985, S. 74 ff.). Insbesondere nach Samuelson (1976) wurden die ursprünglich von Faustmann $(1849 ; 1995)$ berücksichtigen (aber nicht optimierten) Vornutzungen (Durchforstungen) in zahlreichen ökonomischen Analysen zur Vereinfachung nicht integriert bzw. bei der Optimierung ebenfalls als konstant angesehen (Coordes 2014a, S. 36 und Tahvonen 2016). Dies unterschlägt zwar die teils immense Bedeutung von Teilentnahmen des Bestandes vor dessen Endnutzung für die forstliche Produktion (u. a. Klemperer 1996, S. 242 f. und Tahvonen et al. 2013) und reduziert die Anwendung des FPO-Theorems auf die reine Plantagenwirtschaft (Tahvonen 2016), ermöglicht aber die analytische Lösung der optimalen forstlichen Flächen- und Kapitalallokation in einfachster Form.

Der optimale Abtriebs- bzw. Ersatzzeitpunkt $T$ eines gleichaltrigen Waldbestandes ohne Vornutzungen ergibt sich demnach aus der Gleichheit von Grenznutzen und Grenzkosten des Bestandeserhalts für ein weiteres Jahr (Johansson und Löfgren 1985, S. 80 i. V. m. Pressler 1860; 1995 und Ohlin 1921; 1995; vgl. u. a. auch Möhring 2009). Es gilt:

$$
\begin{gathered}
w_{B}(T, T+1)=W_{B}(T) \cdot r+a, \\
T \in \mathbb{N}^{\geq 0},
\end{gathered}
$$

mit dem laufenden Bestandeswertzuwachs $w_{B}$ zwischen den Jahren $T$ und $T+1$, dem Bestandeswert $W_{B}$ (Abtriebswert), dem Kalkulationszinssatz $r$ und der Brutto-Bodenrente $a$ (Annuität). Das heißt, dass die forstliche Produktion eines Bestandes solange für ein weiteres Jahr aufrecht erhalten wird, wie sein Wertzuwachs in diesem Zeitschritt die alternative Verwendung des in ihm gebundenen Kapitals $\left(W_{B}(T) \cdot r\right)$ zuzüglich der Opportunitätskosten für die alternative Verwendung der Fläche durch einen Folgebestand $a$ überwiegt (vgl. u. a. Chang 1984; Klemperer 1996, S. 38 ff.). Dabei kann, muss aber nicht, vom identischen Ersatz des aktuellen 
Bestandes ausgegangen werden - die Flächenopportunität kann auch durch die Brutto-Bodenrente einer anderweitigen Nutzung, bspw. durch eine andere Baumart, repräsentiert werden (Möhring 2009).

Analog kann die Optimalitätsbedingung [2-1] nach $r$ umgestellt werden:

$$
r=\frac{w_{B}(T, T+1)-a}{W_{B}(T)}
$$

Diese als Pressler`sches Weiserprozent (vgl. Pressler 1860; 1995 und z. B. Möhring 1994, S. 51 ff.; Möhring 2001, 2009) bekannte interne Grenzverzinsung der Investition des Bestandeserhalts sinkt über dem Alter und entspricht im Optimum der Grenzverzinsung der alternativen Kapitalverwendung (vgl. auch Klemperer 1996, S. 121. ff.). Der Erhalt eines Bestandes für ein weiteres Jahr ist demnach vorteilhaft, bei einem vergleichsweise hohen laufenden Bestandeswertzuwachs, einer vergleichsweise niedrigen Flächenopportunität und einem vergleichsweise geringen Bestandeswert (Möhring 2009).

Werden bei der Optimierung der Altersklassenwaldbewirtschaftung zusätzlich Vornutzungen (Durchforstungen) berücksichtigt, sind Erweiterungen bzw. Anpassungen des klassischen FPOTheorems notwendig. Neben der Einbeziehung des Folgebestandes (Interbestandes-Opportunität) zur Bestimmung des optimalen Endnutzungszeitpunkts des Bestandes (s. o.), spielen dabei auch Intrabestandes-Opportunitäten (Wuchsraumopportunitäten), d. h. Grenznutzen der alternativen Flächen- bzw. Wuchsraumverwendungen innerhalb eines Bestandes, eine entscheidende Rolle (Coordes 2014c; Fuchs 2018, S. 53 f.). Das Optimierungsproblem der Vornutzung lässt sich dabei in die Ökonomie der Nutzung nachwachsender Ressourcen einordnen (vgl. Fishery-Model; u. a. Schaefer 1954; Clark 2010, S. 233 ff.; Borchert 2002, S. 14 ff.) und wurde auf unterschiedlichen methodischen Wegen analysiert: Es lassen sich Ansätze auf der Bestandesebene (vgl. u. a. Näslund 1969; Pearse 1967; Schreuder 1971; Clark und Pree 1979; Cawrse et al. 1984; Betters et al. 1991; Borchert 2002; Hyytiäinen und Tahvonen 2002; Wippermann 2005; Möhring 2010; Halbritter und Deegen 2015; Halbritter 2020) von Ansätzen auf Ebene des Einzelbaums (vgl. u. a. Heshmatol Vaezin et al. 2009; Härtl et al. 2010; Degenhardt 2011; Coordes 2014a, 2014b; Fuchs 2018) unterscheiden. Gemeinsam haben alle die deutlich erhöhte Komplexität gegenüber der reinen Endnutzungsoptimierung, da alle Durchforstungszeitpunkte mit der jeweiligen Durchforstungsintensität und -art sowie der Endnutzungszeitpunkt simultan bestimmt werden müssen (vgl. Johansson und Löfgren 1985, S. 78; Hyytiäinen und Tahvonen 2002; Wippermann 2005, S. 40 f.; Klemperer 1996, S. 242 ff.). 


\subsection{Modell und Methode}

Anhand eines stark vereinfachten forstlichen Produktionsmodells (Modellumsetzung in R; R Core Team 2019) wird im Folgenden die Ökonomie der forstlichen Bestandesbehandlung in einem homogenen Altersklassenwald analysiert. In Anlehnung an Möhring (2009) werden die Kriterien der optimalen Bestandesbehandlung anhand des theoretischen Analyserahmens leicht verständlich und mit Bezug zur forstlichen Praxis dargelegt. Dazu wird die Komplexität, ausgehend vom Fall der maximalen Volumenproduktion, durch schrittweise Erweiterung der Modellprämissen erhöht - die dadurch bedingten Veränderungen der optimalen Bestandesbehandlung dienen der systematischen Analyse im Sinne eines Variantenstudiums. Es wird bewusst auf ein analytisches Optimierungsverfahren verzichtet und stattdessen an entsprechender Stelle auf existierende Lösungsansätze in der Literatur ${ }^{7}$ zurückgegriffen bzw. diese durch Analogieschluss erweitert. Die Optima werden im Sinne eines heuristischen Verfahrens ${ }^{8}$ numerisch hergeleitet, grafisch dargestellt und i. V. m. den jeweiligen Optimalitätsbedingungen diskutiert.

Das verwendete Optimierungsmodell (Abbildung 1) gliedert sich in die drei ineinandergreifenden bzw. aufeinander aufbauenden Modellbestandteile

- naturale Simulation der forstlichen Produktion (Abschnitt 2.1.1),

- ökonomische Bewertung (Abschnitt 2.1.2) und

- $\quad$ numerische Optimierung (Abschnitt 2.1.3).

Die Optimierungsfunktion erhält als Nutzereingaben die Startwerte und Grenzen (Nebenbedingungen) der Entscheidungsvariablen

- Vornutzungsvektor $\boldsymbol{\sigma}$ (Vornutzungsanteile $0 \leq \sigma_{t}<1$ für 0 a $<t<100$ a) ${ }^{9}$ und

- Endnutzungszeitpunkt $T(0 \mathrm{a}<T \leq 100 \mathrm{a})^{10}$,

mit $\sigma_{t} \in \mathbb{R}$ und $t, T \in \mathbb{N}$.

${ }^{7}$ Besonders sei an dieser Stelle bereits auf das umfassende Werk von Coordes (2014a) hingewiesen, welches die optimale Durchforstung über den Faustmann-Ansatz analysiert.

${ }^{8}$ Heuristische Verfahren sind nach Degenhardt (2011, S. 13) „Techniken zur Suche nach guten (nahezu optimalen) Lösungen für komplexe Optimierungsprobleme in möglichst kurzer Zeit“. Vorteile dieser Verfahren sind, dass „,kaum Kenntnisse über das Verhalten des Systems erforderlich“ sind und „Voraussetzungen wie Stetigkeit, Differenzierbarkeit, Konvexität" nicht erfüllt sein müssen (Degenhardt 2011, S. 13).

${ }^{9}$ Unabhängig vom Endnutzungszeitpunkt $T$ hat der Vornutzungsvektor modellbedingt immer 99 Einträge - alle $\sigma_{t}$ für $t \geq T$ sind für die forstliche Produktion im spezifischen Fall allerdings irrelevant.

${ }^{10}$ Das Alter 100 a wird somit als modell-maximales Alter $t_{\max }$ definiert. 
Zusätzlich zu den Nebenbedingungen der Entscheidungsvariablen wird die maximale Anzahl an gewünschten Iterationsschritten $i$ festgelegt (siehe Abschnitt 2.1.3).

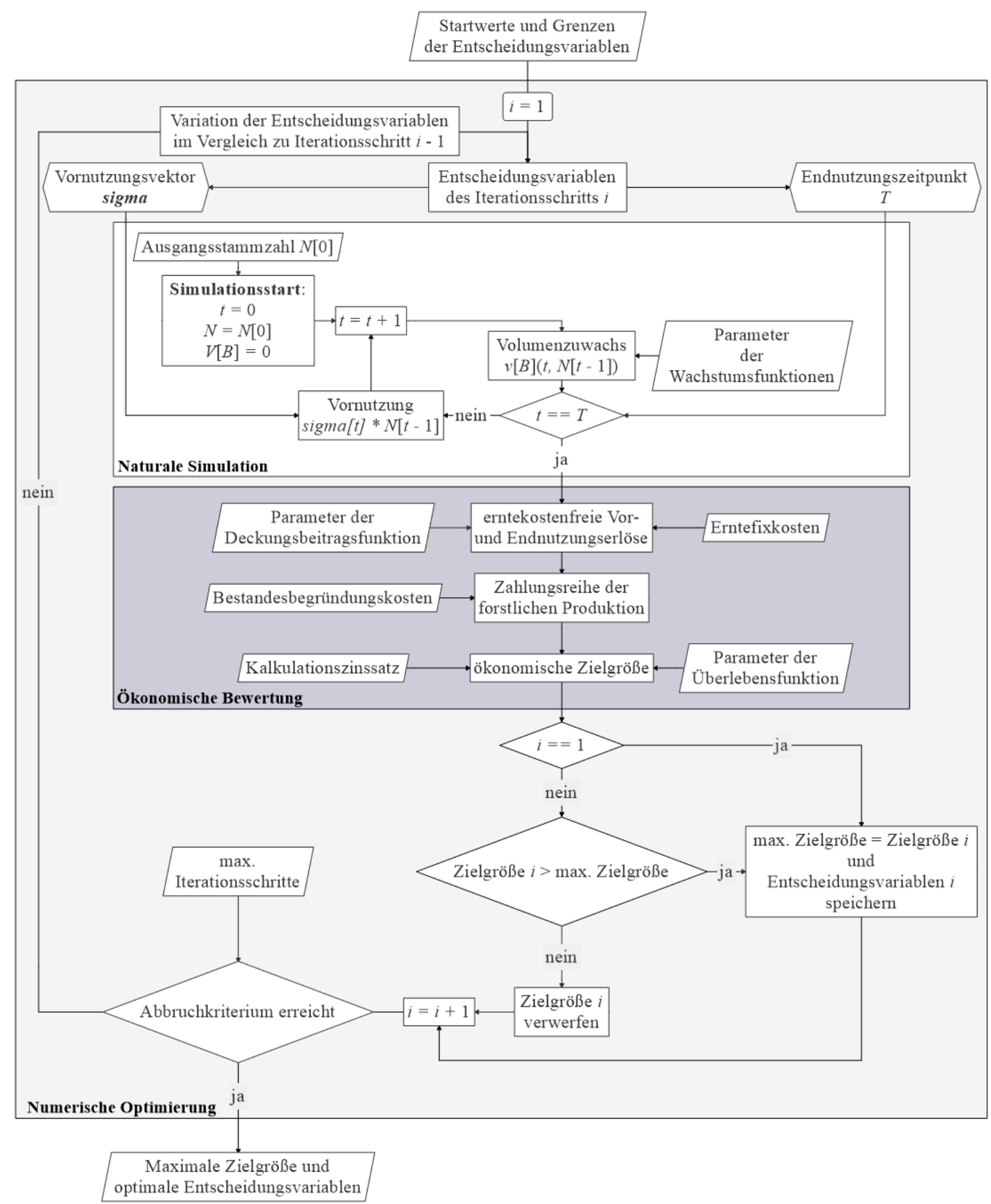

Abbildung 1: Ablaufdiagramm des R-basierten Optimierungsmodells. Dargestellt in unterschiedlichen Grautönen sind die ineinandergreifenden Modellbestandteile Naturale Simulation (Abschnitt 2.1.1), Ökonomische Bewertung (Abschnitt 2.1.2) und Numerische Optimierung (Abschnitt 2.1.3). Parallelogramm: Nutzungseingabe und Datenausgabe, Rechteck: Prozessschritt, Raute: Entscheidung; $V[B]$ : Vorrat (verbleibender Bestand), $v[B]$ : fld. Volumenzuwachs; $N$ : Stammzahl, sigma: Vornutzungsvektor, sigma $[t]$ : Vornutzungsanteil zum Zeitpunkt $t, i$ : Iterationsschritt, $t$ : Alter bzw. Zeitpunkt, T: Endnutzungszeitpunkt. 
Mit dem ersten Iterationsschritt $i=1$ startet die naturale Simulation mit den entsprechend gewählten Startwerten der Entscheidungsvariablen und einer fest vorgegebenen, natural-optimalen Ausgangsstammzahl ${ }^{11} N_{0}=2.500 \mathrm{ha}^{-1}$. Die naturale Simulation durchläuft die Bestandesentwicklung entsprechend der Wachstumsfunktionen des Produktionsmodells (Abschnitt 2.1.1) chronologisch vom Alter $t=0$ a (Bestandesbegründung) bis $t=T$ (Endnutzung) in diskreten 1-Jahres-Schritten. In Abhängigkeit vom Bestandesalter $t$ und der Stammzahl $N_{t-1}$ wächst das Bestandesvolumen $V_{B}$ zwischen den Jahren $t-1$ und $t$ um den Bestandesvolumenzuwachs $v_{B}\left(t, N_{t-1}\right)$ zu (frühestens ab $t=1 \mathrm{a}$ ). Ab dem Alter $t=1$ a sind schematische ${ }^{12}$ Vornutzungen möglich. Eine Verjüngung des Folgebestandes vor dem Endnutzungszeitpunkt $T$, d. h. der Ernte der letzten Bäume des Bestandes, ist dabei ausgeschlossen ungleichaltrige Bäume stehen also niemals in einem Bestand (Altersklassenwald). Ist der Endnutzungszeitpunkt $T$ erreicht, erfolgt die Übergabe der naturalen Bestandesentwicklungsdaten an das ökonomische Bewertungsmodell (Abschnitt 2.1.2). Die Zielgröße der ökonomischen Bewertung ist dabei abhängig von den jeweiligen Modellprämissen (siehe Abbildung 7). Der Optimierungsalgorithmus speichert die Zielgröße im ersten Iterationsschritt $(i=1)$ bzw. prüft eine mögliche Verbesserung dieser Zielgröße gegenüber des bisherigen Maximums bei $i>1$ und speichert das mögliche neue Optimum in Verbindung mit den entsprechenden Entscheidungsvariablen ab. Solange keines der Abbruchkriterien des Optimierungsalgorithmus erfüllt ist erfolgt ein neuer Iterationsschritt $i=i+1$. Andernfalls wird das gefundene Maximum mit den dazugehörigen Entscheidungsvariablen ausgegeben (Abbildung 1).

\subsubsection{Naturale Simulation}

Der naturale Bestandesvolumenzuwachs des Produktionsmodells wird durch drei funktionale Zusammenhänge abgebildet:

1. Altersabhängiger, degressiver Einzelbaumvolumenzuwachs im konkurrenzlosen Zustand (solitär wachsender Einzelbaum),

2. lineare Abnahme des Einzelbaumvolumenzuwachses bei steigender Stammzahl (d. h. steigender Konkurrenz) im Bestand und

3. altersabhängige Erhöhung der Konkurrenz durch eine regressive Entwicklung der maximalen Stammzahl im Bestand (Bestandeskapazitätsgrenze).

\footnotetext{
${ }^{11}$ Es wird als konstante Ausgangsstammzahl die Stammzahl gewählt, die den modell-maximalen Bestandesvolumenzuwachs im ersten Zeitschritt ermöglicht.

${ }^{12}$ Der ausscheidende Stammzahlanteil entspricht dem Anteil an ausscheidendem Bestandesvolumen.
} 
Der altersabhängige, laufende Volumenzuwachs eines konkurrenzlos (solitär) und somit maximal wachsenden Einzelbaums $v_{E \text {,solitär }}(t)$ wird definiert als

$$
v_{E, \text { solitär }}(t)=v_{E, \max } \cdot\left(1-e^{-\beta_{1} \cdot t}\right) \text {, }
$$

$t \in \mathbb{R}^{\geq 0}$,

mit $v_{E, \max }=0,04 \mathrm{Fm} \mathrm{a}^{-1}$ und $\beta_{1}=0,04 \mathrm{a}^{-1}$ (Abbildung 2).

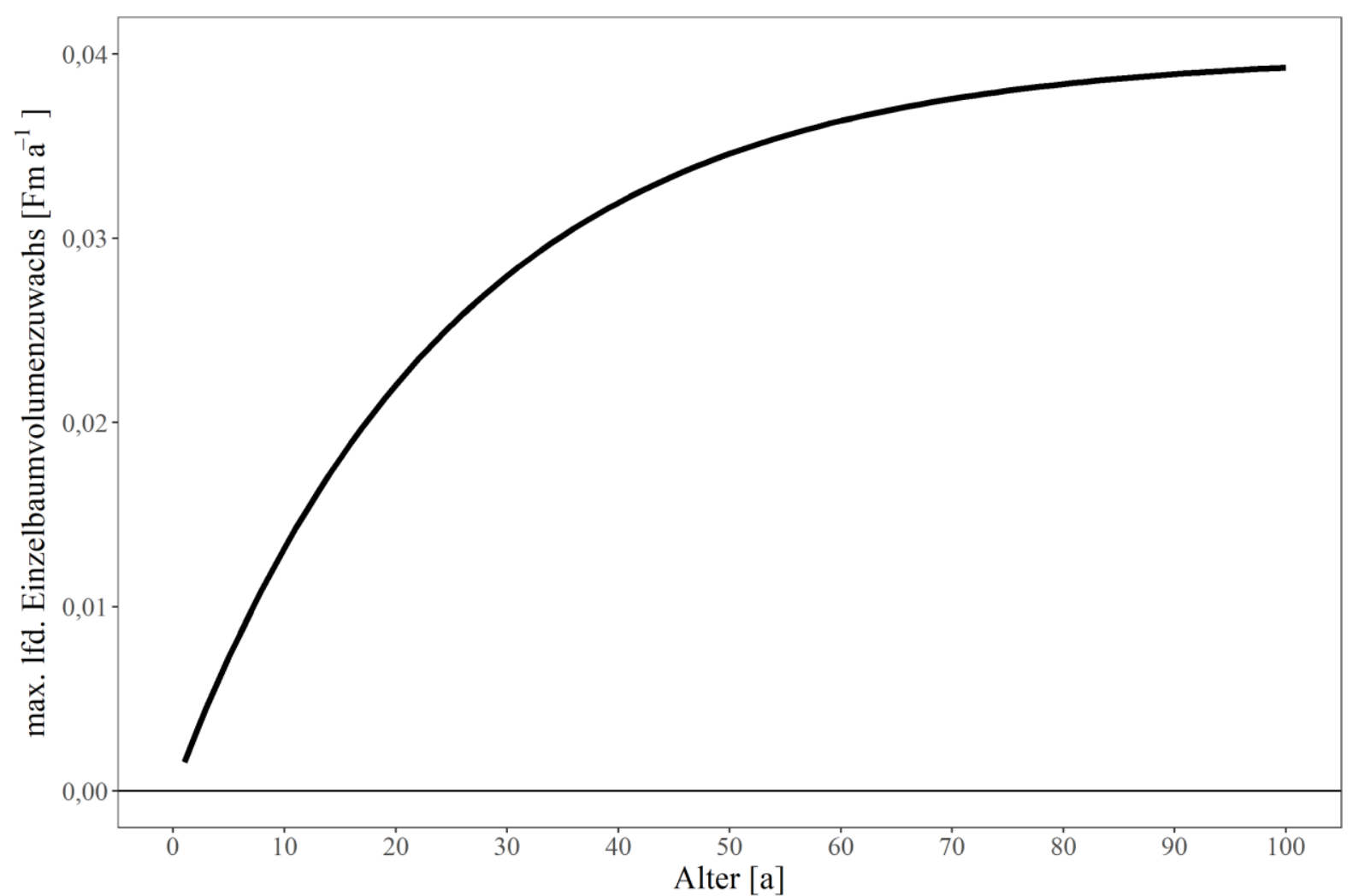

Abbildung 2: Altersabhängiger, laufender Volumenzuwachs eines konkurrenzlos wachsenden Einzelbaums (altersabhängig-maximaler laufender Einzelbaumvolumenzuwachs) gemäß Funktion [2-3]. 
Steigende Konkurrenz durch eine zunehmende Stammzahl $N$ im Bestand senkt den Einzelbaumvolumenzuwachs linear ab - je mehr Bäume sich die begrenzten Ressourcen der Bestandesfläche bzw. des Wuchsraums im gleichen Alter teilen, desto geringer ist der laufende Volumenzuwachs am Einzelbaum ${ }^{13}$ (vgl. Pretzsch 2009, S. 291 ff.). Es gilt in Verbindung mit Funktion [2-3] für den tatsächlichen Einzelbaumvolumenzuwachs $v_{E}\left(t, N_{t}\right)$ eine Abhängigkeit vom Alter $t$ sowie der entsprechenden Bestandesstammzahl $N_{t}$ :

$$
\begin{aligned}
v_{E}\left(t, N_{t}\right) & =v_{E, \text { solitär }} \cdot\left(1-\frac{N_{t}}{N_{\max }(t)}\right) \\
& =v_{E, \max } \cdot\left(1-e^{-\beta_{1} \cdot t}\right) \cdot\left(1-\frac{N_{t}}{N_{\max }(t)}\right),
\end{aligned}
$$

$$
N_{t} \in \mathbb{R}^{\geq 0} .
$$

$N_{\max }(t)$ bildet dabei die altersabhängige Bestandeskapazitätsgrenze ${ }^{14}$, bei welcher der Einzelbaumvolumenzuwachs zum Erliegen kommt. Es gilt:

$$
\begin{aligned}
& v_{E}\left(t, N_{t}\right)>0 \text { für } N_{t}<N_{\max }(t), \\
& v_{E}\left(t, N_{t}\right)=0 \text { für } N_{t}=N_{\max }(t) \text { sowie } \\
& v_{E}\left(t, N_{t}\right)<0 \text { für } N_{t}>N_{\text {max }}(t) .
\end{aligned}
$$

Abbildung 3 zeigt den linear abnehmenden Einzelbaumvolumenzuwachs exemplarisch für das Alter 50 a. Erkennbar ist der Volumenzuwachs eines konkurrenzlos (solitär) wachsenden Einzelbaums $v_{E, \text { solitär }}(50 \mathrm{a})=0,035 \mathrm{Fm} \mathrm{a}^{-1}$ am Ordinatenachsenschnittpunkt (vgl. Abbildung 2) sowie die entsprechende Bestandeskapazitätsgrenze $N_{\text {max }}(50 \mathrm{a})=1433 \mathrm{ha}^{-1}$ am Abzissenachsenschnittpunkt (vgl. Abbildung 4).

Für die Bestandeskapazitätsgrenze $N_{\max }(t)$ gilt:

$$
N_{\max }(t)=N_{0}^{\max } \cdot\left(1-e^{-\beta_{2} \cdot t}\right)
$$

mit $N_{0}^{\max }=5.000 \mathrm{ha}^{-1}$ sowie $\beta_{2}=0,025$ (Abbildung 4).

\footnotetext{
${ }^{13}$ Als wachstumsbeeinflussendes Bestandesdichtemaß wird somit unabhängig von der Einzelbaumdimension ausschließlich die Bestandesstammzahl verwendet (vgl. etablierte Bestandesdichtemaße, wie z. B. Bestandesgrundfläche, Bestandesdichteindex oder Relativer Baumabstand; Gadow 2003, S. $92 \mathrm{ff}$.).

${ }^{14}$ Vgl. Selbstdifferenzierungs- oder Selbstdurchforstungslinie (Pretzsch 2000, S. 205), Self-Thinning Rule (Pretzsch 2009, S. 399) oder Stammzahl-Durchmesser-Grenzbeziehung (Gadow 2003, S. 94 f.) nach Reineke (1933) und Yoda et al. (1963). In Anlehnung daran kann die Bestandeskapazitätsgrenze als baumartenspezifische „Tragfähigkeit des Standorts“ interpretiert werden, welche mit steigender Standortsgüte zunimmt (vgl. Pretzsch 2000, S. 206 u. 209).
} 


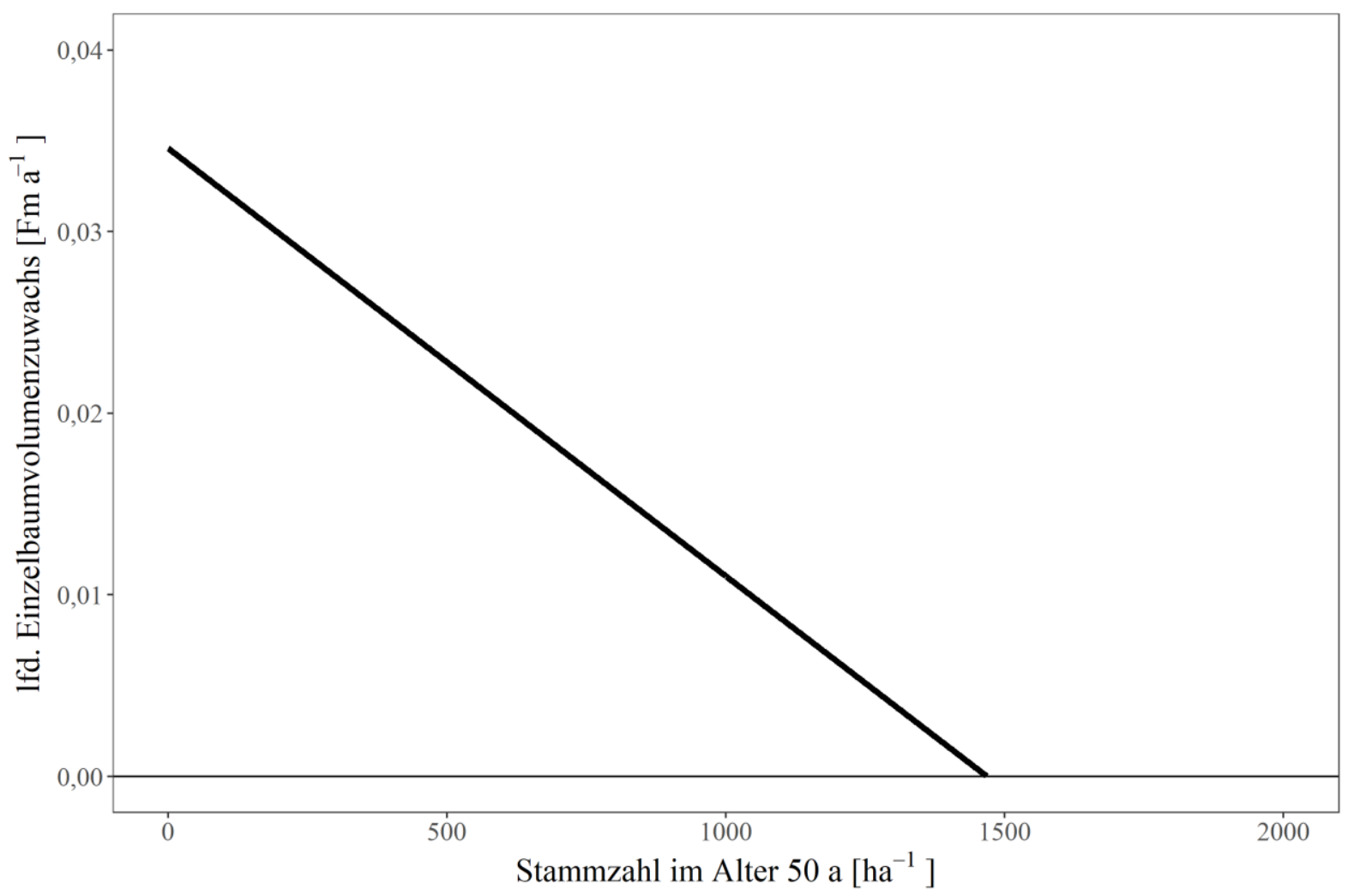

Abbildung 3: Exemplarische Darstellung des laufenden Einzelbaumvolumenzuwachses in Abhängigkeit von der Stammzahl im Alter 50 a gemäß Funktion [2-4].

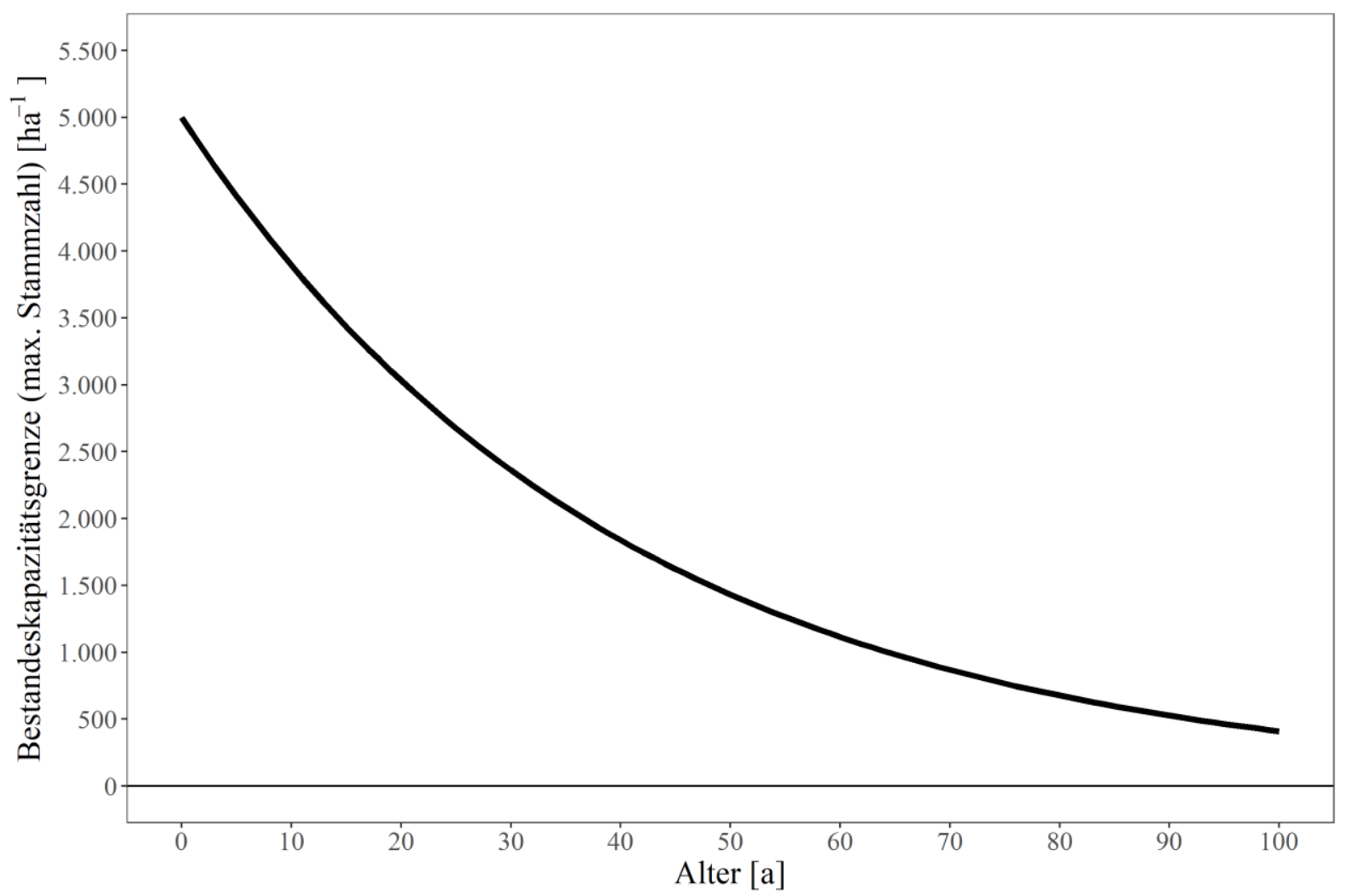

Abbildung 4: Altersabhängige Bestandeskapazitätsgrenze (max. Stammzahl) gemäß Funktion [2-5]. 
Der laufende Bestandesvolumenzuwachs $v_{B}\left(t, N_{t}\right)$ ergibt sich schließlich aus der Multiplikation des Einzelbaumvolumenzuwachses $v_{E}\left(t, N_{t}\right)$ mit der entsprechenden Stammzahl $N_{t}$ (siehe auch Abbildung 5):

$$
\begin{aligned}
v_{B}\left(t, N_{t}\right) & =N_{t} \cdot v_{E}\left(t, N_{t}\right) \\
& =N_{t} \cdot v_{E, \text { max }} \cdot\left(1-\frac{N_{t}}{N_{0}^{\max } \cdot\left(1-e^{-\beta_{2} \cdot t}\right)}\right) \cdot\left(1-e^{-\beta_{1} \cdot t}\right) .
\end{aligned}
$$

Durch die Multiplikation des Einzelbaumzuwachses mit der Stammzahl wird ein homogener Bestand impliziert, d. h. ein Waldbestand aus identischen Bäumen. Der Volumenzuwachs aller Bäume ist gleich und alle Bäume reagieren in gleicher Weise auf eine veränderte Konkurrenzsituation im Bestand. Es wird zudem eine identische Qualität sowie eine identische räumliche Ausdehnung und Anordnung der Einzelbäume unterstellt.

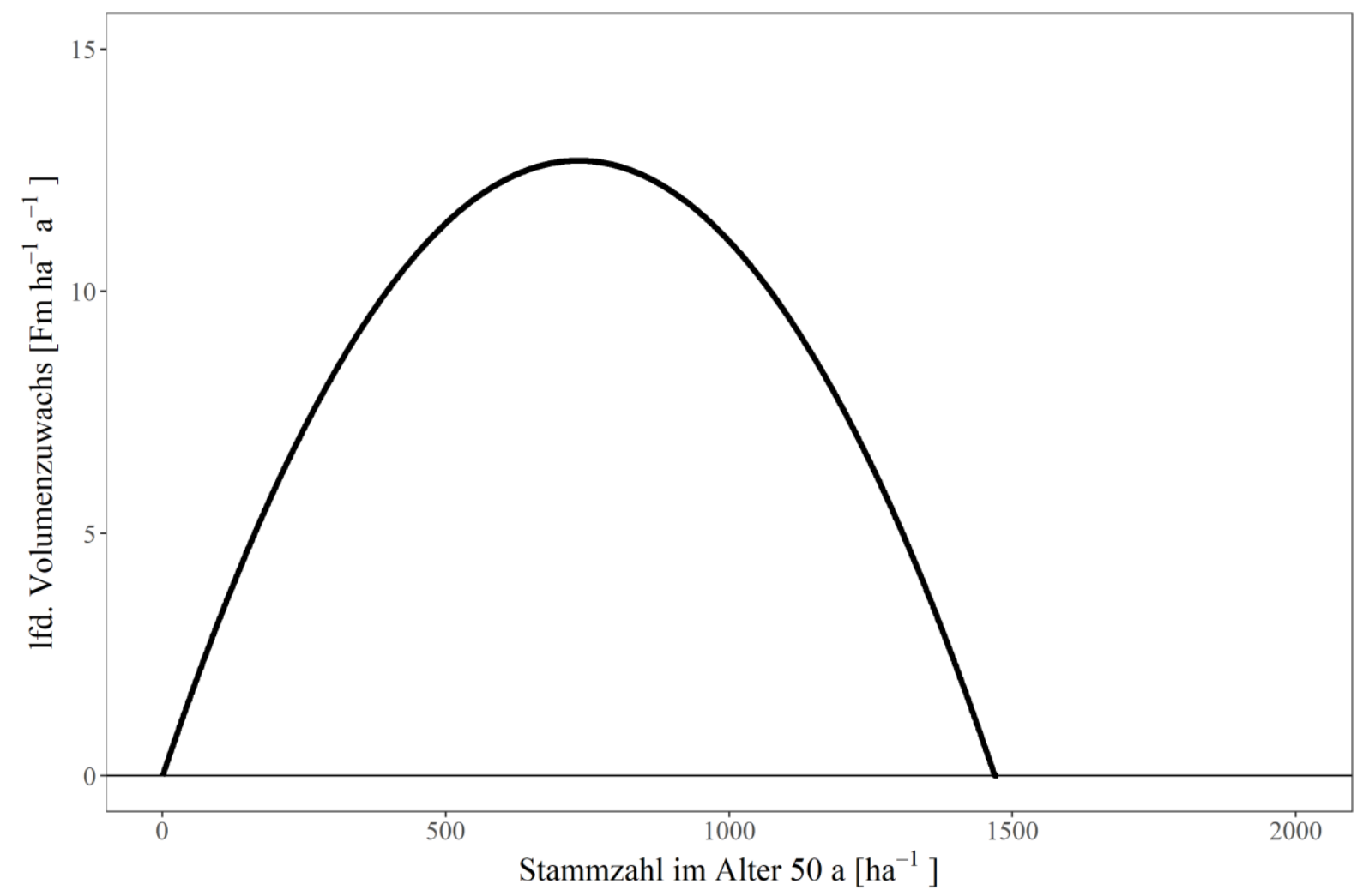

Abbildung 5: Exemplarische Darstellung des laufenden Bestandesvolumenzuwachses in Abhängigkeit von der Stammzahl im Alter 50 a gemäß Funktion [2-6].

Für das jährlich-diskrete forstliche Produktionsmodell werden die laufenden Bestandesvolumenzuwächse im Intervall $(0, T]=\{t \in \mathbb{N} \mid 0<t \leq T\}$ aus der stetigen Funktion [2-6] abgegriffen. Dabei wird der Bestandesvolumenzuwachs zeitlich rückblickend determiniert, d. h. der Bestandesvolumenzuwachs im Alter $t$ ist der Zuwachs im Zeitschritt $[t-1, t)$, wobei die zuwachsbestimmende Bestandesstammzahl nach der Vornutzung zum Vorjahreszeitpunkt $N_{t-1}$ 
abgegriffen und ins Verhältnis zur Kapazitätsgrenze $N_{\max }(t-1)$ gesetzt wird.

Funktion [2-6] verändert sich zu:

$$
\begin{aligned}
v_{B}\left(t, N_{t-1}\right) & =N_{t-1} \cdot v_{E}\left(t, N_{t-1}\right) \\
& =N_{t-1} \cdot v_{E, \max } \cdot\left(1-\frac{N_{t-1}}{N_{0}^{\max } \cdot\left(1-e^{-\beta_{2} \cdot(t-1)}\right)}\right) \cdot\left(1-e^{-\beta_{1} \cdot t}\right),
\end{aligned}
$$

$0<t \leq T$

$t, T \in \mathbb{N}$,

$N_{t} \in \mathbb{R}^{\geq 0}$

Während das Alter ganzzahlig definiert wird $\left(t, T \in \mathbb{N}^{>0}\right)$, werden aus Gründen der stabileren Optimierung Stammzahlen aus der Menge der reellen Zahlen zugelassen $\left(N \in \mathbb{R}^{\geq 0}\right.$ ), d. h. es können modellbedingt auch Baumanteile auf der Fläche stehen bzw. im Umkehrschluss Bäume anteilig geerntet werden.

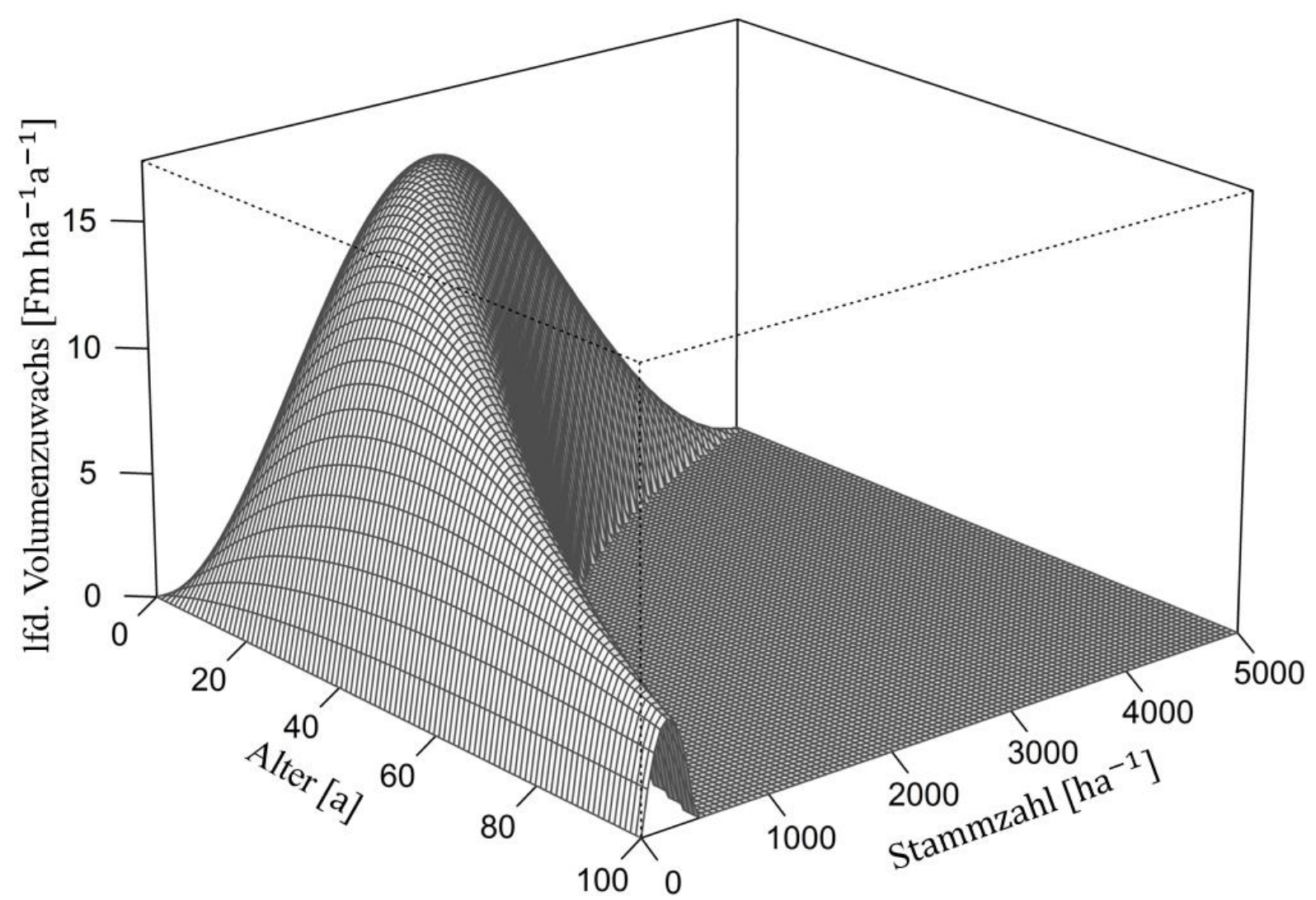

Abbildung 6: Alters- und stammzahlabhängiger laufender Bestandesvolumenzuwachs gemäß Funktion [2-7].

Die modellbedingt möglichen negativen Bestandesvolumenzuwächse $v_{B}\left(t, N_{t-1}\right)<0$ werden zudem ausgeschlossen (Abbildung 6) - eine Minderung des Bestandesvolumens findet somit niemals statt. Außerdem wird eine mögliche zeitliche Verzögerung der Wachstumsreaktionen 
auf eine veränderte Bestandesdichte (vgl. z.B. Härtl et al. 2010) nicht berücksichtigt - die verbleibenden Bäume wachsen nach einer Vornutzung stets ohne zeitlichen Verzug entsprechend der neuen Konkurrenzsituation.

Die kumulierte Summe aller Bestandesvolumenzuwächse bis zum Zeitpunkt $t$ bildet die Gesamtwuchsleistung $G W L\left(t, \boldsymbol{N}_{t-1}\right)$, wobei diese neben dem Alter $t$ vom Vektor aller vorherigen Bestandesstammzahlen $\boldsymbol{N}_{t-1}=N_{0}, \ldots, N_{t-1}$ abhängt. Es erfolgt dabei die Festlegung, dass der Volumenzuwachs $v_{B}\left(t, N_{t-1}\right)$ zwischen den Zeitpunkten $t-1$ und $t$ Bestandteil der Gesamtwuchsleistung $G W L(t)$ ist. Es gilt:

$$
\operatorname{GWL}\left(t, \boldsymbol{N}_{t-1}\right)=\sum_{\tau=1}^{t} v_{B}\left(\tau, N_{\tau-1}\right) .
$$

Die Bestandesstammzahl zum Zeitpunkt $t-1$ ist dabei neben dem Alter $t$ und der Ausgangsstammzahl $N_{0}=$ konst. $=2.500 \mathrm{ha}^{-1}$ von allen bisherigen Vornutzungen abhängig. Diese werden über den Vornutzungsvektor $\boldsymbol{\sigma}_{t-1}=\sigma_{1}, \ldots, \sigma_{t-1}$ (relative Entnahmemengen) und die jeweils vorhandene Stammzahl bzw. das jeweils vorhandene Bestandesvolumen (schematische Vornutzungen) vor der Entnahme determiniert. Die Gesamtwuchsleistung kann somit auch direkt über den Vornutzungsvektor $\boldsymbol{\sigma}_{t-1}$ (Entscheidungsvariablen des Optimierungsmodells) definiert werden:

$$
\begin{aligned}
& \quad \operatorname{GWL}\left(t, \boldsymbol{\sigma}_{t-1}, N_{0}\right)=v_{B}\left(1, N_{0}\right)+\sum_{\tau=2}^{t} v_{B}\left(\tau, \sigma_{\tau-1}, N_{0}\right), \\
& 0 \leq \sigma_{t}<1, \\
& \sigma_{t} \in \mathbb{R} .
\end{aligned}
$$

Mortalität, d. h. das natürlich bedingte Absterben von Bäumen, wird ausgeschlossen. Des Weiteren erfolgt die Festlegung, dass die Ausgangsstammzahl $N_{0}=2.500$ ha $^{-1}$ den Ausgangspunkt der forstlichen Produktion bildet, welche zum Zeitpunkt $t=0$ a durch entsprechende Bestandesbegründung hergestellt wird. Ein korrespondierendes Bestandesvolumen $V_{B}$ im Alter $t=0$ a existiert nicht - die Ausgangsstammzahl kann somit als Summe von Samen oder Sämlingen angesehen werden, die erst im Alter $t=1$ a ein messbares Volumen ${ }^{15}$ aufweisen.

\footnotetext{
${ }^{15}$ Kluppschwellen (bspw. die ausschließliche Berücksichtigung von Derbholzvolumen ab $D_{g}>7 \mathrm{~cm}$ ) existieren aus Vereinfachungsgründen nicht.
} 
Der Vornutzungsvektor $\boldsymbol{\sigma}_{T-1}=\sigma_{1}, \ldots, \sigma_{T-1}$ bildet im Folgenden zusammen mit dem Endnutzungszeitpunkt $T$ die Entscheidungs- bzw. Optimierungsvariablen des Produktionsmodells stets wird dabei die Bestandesbehandlung, als Kombination aus optimalem Vornutzungsvektor $\boldsymbol{\sigma}_{T-1}^{*}$ sowie optimalem Endnutzungszeitpunkt $T^{*}$ gesucht, welche die prämissenabhängige Zielgröße maximiert.

\subsection{2 Ökonomische Bewertung}

Die ökonomische Bewertung der forstlichen Bestandesbehandlung erfolgt in Abschnitt 2.2 aufbauend auf der naturalen Simulation mittels schrittweiser Einführung von Modellprämissen und klar strukturiertem Variantenstudium (Abbildung 7). Ausgehend vom naturalen Optimum ohne und mit Berücksichtigung eines Folgebestandes (Abschnitt 2.2.1) werden zunächst konstante (Abschnitt 2.2.2), dann zeit- (Abschnitt 2.2.3) und schließlich dimensionsabhängige (Abschnitt 2.2.4) Deckungsbeiträge zur Bewertung der Vor- und Endnutzungsvolumina ${ }^{16}$ eingeführt. Abschnitt 2.2.5 führt die Berücksichtigung von Kapitalknappheit durch die Einbeziehung unterschiedlich hoher Kalkulationszinssätze ein, wodurch die Zielgröße des durchschnittlichen Überschusses finanzmathematisch zur Annuität wird. Abschnitt 2.2.6 verdeutlicht darauf aufbauend den Einfluss von Erntefixkosten, d. h. unabhängig von der Vor- und Endnutzungsmenge auftretenden Kosten der Erntemaßnahmen. Mit der Einführung konstanter Bestandesbegründungskosten (Abschnitt 2.2.7) wird die Zielgröße schließlich zur Brutto-Bodenrente. In Abschnitt 2.2.8 werden Überlebensrisiken berücksichtigt und die Bedeutung unterschiedlicher Altersrisikostärken analysiert. Aufbauend darauf untersucht Abschnitt 2.2.9 schließlich den Einfluss behandlungsabhängiger Überlebensrisikostärken und damit die Rückkopplung zwischen waldbaulichem Handeln und Bestandesstabilität.

\footnotetext{
${ }^{16}$ Es wird auf die Unterscheidung zwischen Vorrats- und Erntefestmetern (vgl. z. B. Offer und Staupendahl 2018) verzichtet, da diese den absoluten Wert, nicht aber die hier analysierten Effekte beeinflusst.
} 


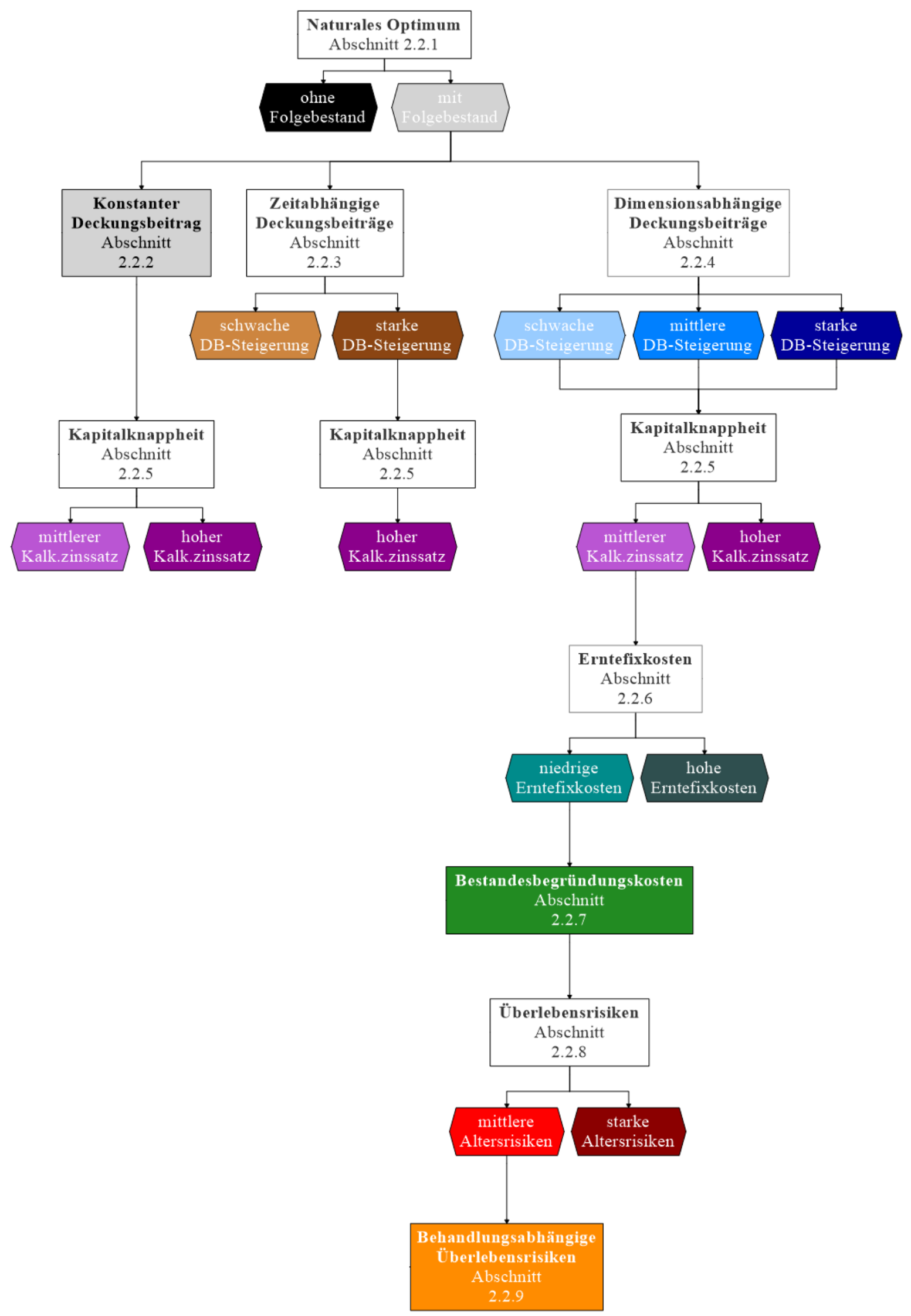

Abbildung 7: Ablaufdiagramm des Analyserahmens mit der schrittweisen Einführung von Modellprämissen gemäß der Abschnitte 2.2.1 bis 2.2.9; Rechteck: Abschnitt, Sechseck: (Prämissen-)variante inkl. der durchgehend in allen folgenden Abbildungen verwendeten variantenspezifischen Farben. 


\subsubsection{Numerische Optimierung}

Das jeweilige variantenspezifische Modelloptimum wird numerisch mittels NOMAD-Algorithmus für nicht-lineare black-box Programmierung (Audet et al. 2009) bestimmt, welcher über die im R-Paket crs (Racine et al. 2018) enthaltene snomadr-Funktion aufgerufen werden kann. Dieser Optimierungsalgorithmus benötigt keine weitergehenden Informationen über die Zielfunktion und lieferte in durchgeführten Vortests trotz der hohen, deutlich über seinem Optimalbereich liegenden, Variablenanzahl zufriedenstellende, d. h. stabile, Ergebnisse. Der hohen Startwertsensitivität sowie den zahlreichen lokalen Maxima der Zielfunktionen wird mit einem mehrstufigen Optimierungsverfahren begegnet (vgl. z. B. Degenhardt 2011). Zunächst wird die snomadr-Funktion 25-mal mit zufällig, gleichverteilten Startwerten (vgl. z. B. Rämö und Tahvonen 2017) innerhalb der spezifischen Grenzen der Entscheidungsvariablen aufgerufen, wobei jeweils 10.000 Iterationsschritte (Abbruchkriterium) zugelassen werden. Der Lösungsvektor mit dem höchsten Zielwert bildet den Startwertvektor der zweiten Optimierungsstufe, in der mit reduzierten unteren und oberen Variablengrenzen der snomadr-Funktion erneute 99.000 Iterationsschritte zugelassen werden. Das Prinzip der zweiten Optimierungsstufe wird mit dem jeweiligen Optimum der vorhergehenden Stufe weitere zwei Mal wiederholt, bis im Anschluss an das Durchlaufen der 4. Optimierungsstufe eine für diese Arbeit ausreichende Annäherung an das globale Optimum angenommen wird - eine Veränderung der Zielgröße findet dann maximal noch im Bereich von Tausendsteln statt. Dennoch auftretende, visuell in den Ergebnisgrafiken erkennbare, Schwankungen der Analysegrößen sind der bereits erwähnten hohen Anzahl an Optimierungsvariablen geschuldet - die Aussagekraft der Ergebnisse wird dadurch nicht beeinflusst.

\subsection{Modellergebnisse und Diskussion}

Die Modellergebnisse werden in den folgenden Abschnitten entsprechend der in Abbildung 7 aufgezeigten Reihenfolge präsentiert und diskutiert. Dabei wird jeweils auf die Zielfunktion als notwendige Bedingung ${ }^{17}$ der optimalen Bestandesbehandlung eingegangen und insbesondere die Veränderung gegenüber dem jeweils vorhergehenden Abschnitt durch Einführung der abschnittsspezifischen Modellprämisse erläutert. Die numerisch hergeleiteten Modellergebnisse werden in Verbindung mit den Optimalitätsbedingungen der Vor- und Endnutzung diskutiert, wobei insbesondere auf die Auswirkungen durch Einführung der jeweiligen Modellprämisse

\footnotetext{
${ }^{17}$ Zu hinreichenden Bedingungen des FPO-Theorems vgl. z. B. Johansson und Löfgren (1985, S. 74 ff.).
} 
eingegangen wird. Eine abschnittsübergreifende und forstpraktisch orientierte Zusammenfassung ausgewählter Modellergebnisse erfolgt darüber hinaus in Abschnitt 2.3. Schließlich werden die angewandte Methode, das Modell sowie alle eingeführten Modellprämissen in Abschnitt 2.4 kritisch gewürdigt.

\subsubsection{Naturales Optimum}

Ausgangspunkt aller folgenden ökonomischen Analysen bildet das naturale Optimum im Sinne der höchsten Bestandesproduktivität. Gesucht wird die Bestandesbehandlung, welche zur maximalen Volumenleistung, d. h. dem maximalen Volumenzuwachs in jedem Zeitschritt, führt.

Wird dabei zunächst die Inter-Bestandesopportunität vernachlässigt, d. h. ein potentieller Folgebestand bei der Optimierung nicht mit einbezogen, gilt es das insgesamt nutzbare Holzvolumen, d. h. die Gesamtwuchsleistung GWL, innerhalb der Modellgrenzen ( $T \leq 100$ a) und unter Veränderung des Vornutzungsvektors $\boldsymbol{\sigma}_{t}$ zu maximieren (Wippermann 2005, S. 22):

$$
\begin{aligned}
& \max _{T, \boldsymbol{\sigma}_{T-1}} \operatorname{GWL}\left(T, \boldsymbol{\sigma}_{T-1}, N_{0}\right)=\max _{T, \boldsymbol{\sigma}_{T-1}} \sum_{t=1}^{T-1} \lambda_{t}\left(\sigma_{t}, t, \boldsymbol{\sigma}_{t-1}, N_{0}\right)+V_{B}\left(T, \boldsymbol{\sigma}_{T-1}, N_{0}\right) \\
& 0 \leq \boldsymbol{\sigma}_{T-1}<1, \\
& 0 \mathrm{a}<T \leq 100 \mathrm{a} .
\end{aligned}
$$

mit dem verbleibenden Bestandesvolumen $V_{B}\left(T, \boldsymbol{\sigma}_{T}, N_{0}\right)$ zum Zeitpunkt der Endnutzung sowie den Vornutzungsvolumina $\lambda_{t}$, die neben dem jeweiligen Vornutzungsanteil $\sigma_{t}$ indirekt $^{18}$ auch vom Alter $t$, allen vorhergehenden Vornutzungsanteilen $\boldsymbol{\sigma}_{t-1}$ sowie der Ausgangsstammzahl $N_{0}$ abhängig sind:

$$
\lambda_{t}\left(\sigma_{t}, t, \sigma_{t-1}, N_{0}\right)=\sigma_{t} \cdot\left(V_{B}\left(t-1, \sigma_{t-2}, N_{0}\right)+v_{B}\left(t, \sigma_{t-1}, N_{0}\right)\right) .
$$

\footnotetext{
${ }^{18}$ Diese indirekte Abhängigkeit wird notwendig, da im Optimierungsmodell nicht die Vornutzungsvolumina selbst (vgl. z. B. Halbritter und Deegen 2015), sondern die Vornutzungsanteile als Entscheidungsvariablen dienen. Das jeweilige Vornutzungsvolumen setzt sich somit aus der multiplikativen Verknüpfung von Vornutzungsanteil und dem Bestandesvolumen vor der Vornutzung zusammen. Dieses ergibt sich aus dem verbleibenden Bestandesvolumen des vorherigen Zeitschritts und dem Volumenzuwachs im letzten Zeitschritt (Funktion [2-11]). Während bspw. Halbritter und Deegen (2015, S. 39) über die Nebenbedingungen (01) direkt definieren, dass nicht mehr genutzt wird als Bestandesvolumen vorhanden ist $(0 \leq h(t) \leq Q(t))$, wird dieser Fall hier durch $0 \leq \sigma_{t}<1$ ausgeschlossen. Durch die verwendete mathematische Formulierung wird eine Abhängigkeit deutlich, die zwar implizit immer gegeben ist (das zu einem Zeitpunkt realisierbare Vornutzungsvolumen hängt auch vom vorhandenen Bestandesvolumen und somit den vorhergehenden waldbaulichen Handlungen ab), in vielen Modellen (z. B. Halbritter und Deegen 2015) aber nicht explizit genannt wird.
} 


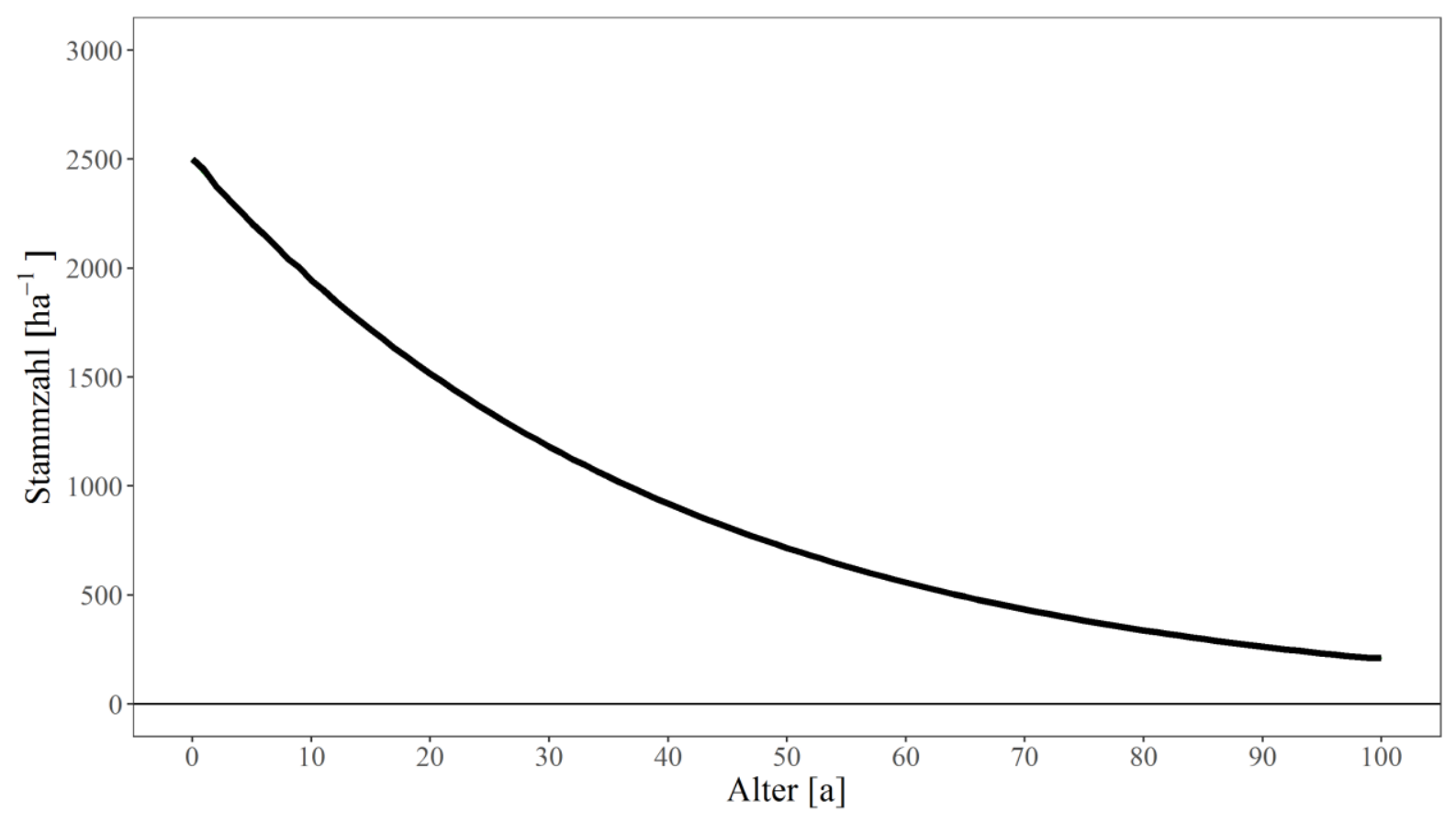

Abbildung 8: Altersabhängige Stammzahlentwicklung im naturalen Optimum ohne Folgebestand gemäß Zielfunktion [2-10].

Die forstliche Produktion beginnt per Modelldefinition im Alter $t=0 \mathrm{a}$ mit der konstant vorgegebenen Anfangsstammzahl $N_{0}=2.500 \mathrm{ha}^{-1}$ (siehe Abschnitt 2.1) und dem dadurch bedingten, maximalen Bestandesvolumenzuwachs von 2,0 $\mathrm{Fm} \mathrm{ha}^{-1} \mathrm{a}^{-1}$ im ersten Zeitschritt. In jedem folgenden Zeitschritt, d. h. nach jeweils erfolgtem laufenden Bestandesvolumenzuwachs, werden im Optimum gemäß Zielfunktion [2-10] anschließend so viele Bäume entnommen, dass erneut die zuwachsoptimale Stammzahl erreicht wird - es resultiert der optimale Stammzahlvektor $\boldsymbol{N}_{\text {nat }}^{*}$ (Abbildung 8). Dieser führt zum maximalen Bestandesvolumenzuwachs in jedem Zeitschritt - der resultierende Optimalpfad verläuft auf dem Grat der Bestandesvolumenzuwachskuppel (Abbildung 9). Maximal wächst das Bestandesvolumen im Alter $t=24$ a um 17,4 Fm ha ${ }^{-1} \mathrm{a}^{-1} \mathrm{zu}$. Bei einer maximalen Gesamtwuchsleistung (Zielgröße) und damit einem insgesamt nutzbaren Holzvolumen von $1.097 \mathrm{Fm} \mathrm{ha}^{-1}$ bis zum modell-maximalen Bestandesalter $t_{\max }=100$ a ergibt sich ein durchschnittlicher Bestandesvolumenzuwachs von 11,0 Fm ha $\mathrm{Fa}^{-1} \mathrm{a}^{-1}$. Das bis dahin verbliebene Bestandesvolumen von $320 \mathrm{Fm} \mathrm{ha}^{-1}$ wird im Zuge der Endnutzung geerntet (Abbildung 10).

Das naturale Optimum ohne Folgebestand bildet mit der modell-maximalen Gesamtwuchsleistung die Referenz für alle folgenden Abschnitte - die entsprechenden Entwicklungen über dem Alter werden in den Abbildungen jeweils durch eine bzw. zwei schwarze Referenzlinie(n) dargestellt. 


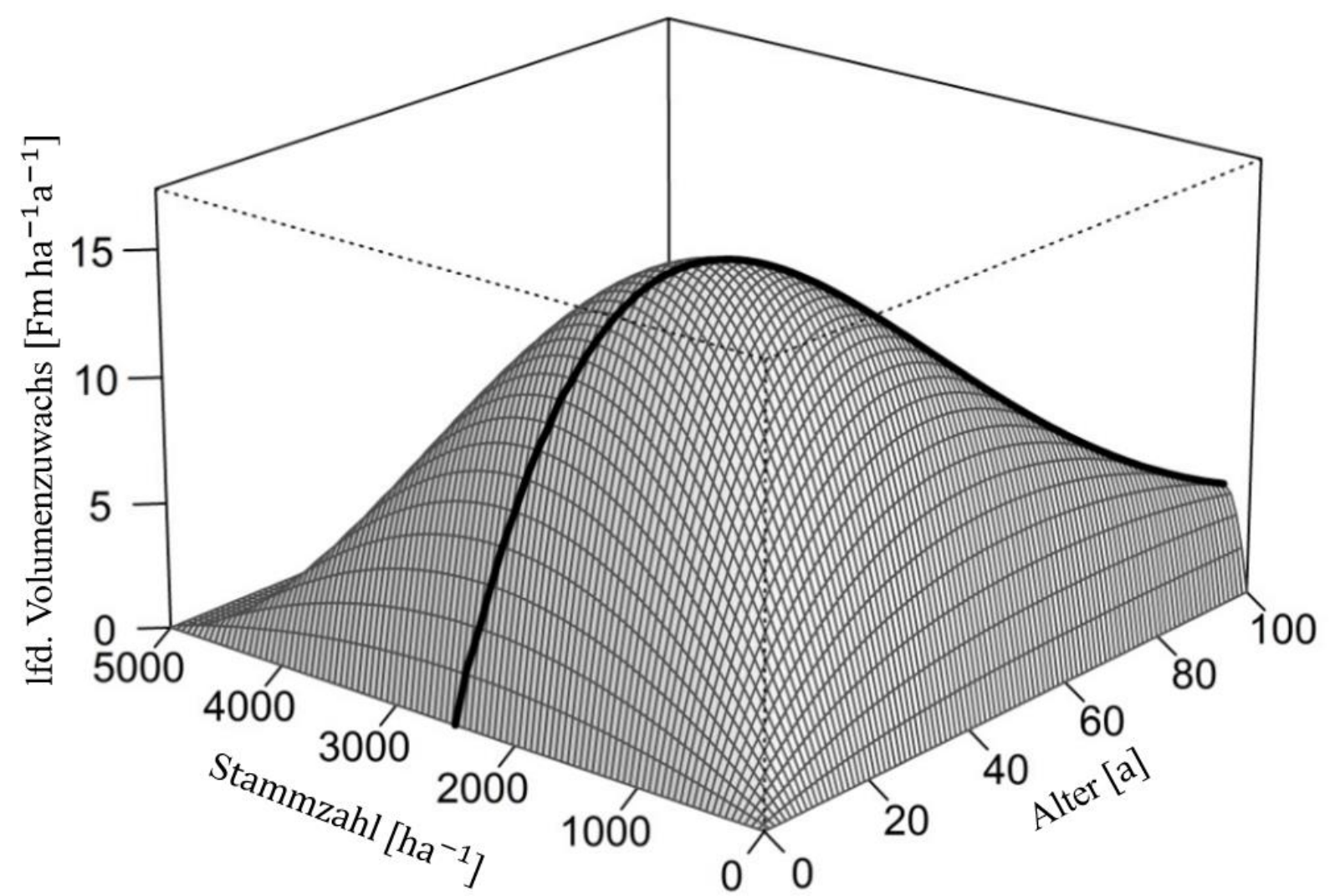

Abbildung 9: Alters- und stammzahlabhängiger laufender Bestandesvolumenzuwachs im naturalen Optimum ohne Folgebestand gemäß Zielfunktion [2-10] (schwarz; entspricht zugleich dem maximalen Bestandesvolumenzuwachs).

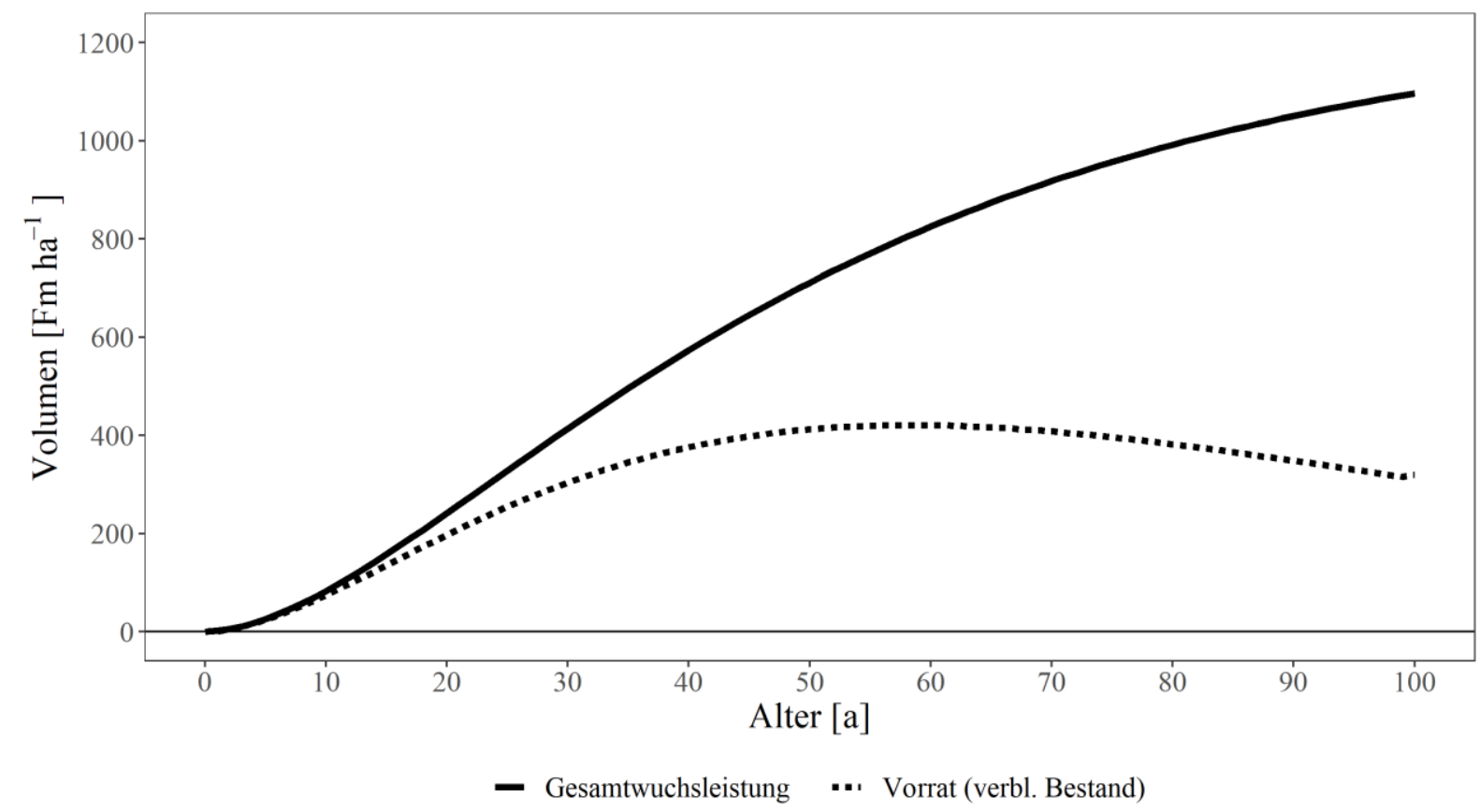

Abbildung 10: Altersabhängige Gesamtwuchsleistung und Vorratsentwicklung im naturalen Optimum ohne Folgebestand gemäß Zielfunktion [2-10]. 
Das Optimierungsproblem der forstlichen Bestandesbehandlung lässt sich zur Formulierung der Optimalitätsbedingungen hier und in allen folgenden Abschnitten, trotz später noch zu diskutierender Wechselwirkungen (vgl. u. a. Coordes 2014c), in mehrere Vornutzungsentscheidungen und eine Endnutzungsentscheidung aufteilen.

Für die Vornutzungsentscheidungen wird zunächst der Vornutzungsvektor $\boldsymbol{\sigma}_{T-1}^{*}$ gesucht, mit dem zu jedem Zeitpunkt bis zum Endnutzungszeitpunkt $T$ der maximale, laufende Bestandesvolumenzuwachs herrscht, d. h. der Bestandesvolumenzuwachsvektor $\boldsymbol{v}_{B}^{*}$ maximal ist.

Nähert man sich diesem Problem auf der Bestandesebene, muss die zur Verfügung stehende, knappe Bestandesfläche (und der damit verbundene Wuchsraum) zu jedem Zeitpunkt durch entsprechende Vornutzungen so aufgeteilt werden, dass die in Konkurrenz stehenden Einzelbäume als Gesamtkollektiv den größten Bestandesvolumenzuwachs erzielen. Das Optimum ist demnach erreicht, wenn die naturale, räumliche Grenzproduktivität in jedem Zeitschritt simultan Null ist (vgl. Halbritter und Deegen 2015, Gleichung (07) mit $r \searrow 0$ und $P=1)^{19}$, d. h. die marginale Veränderung der Stammzahl zu keiner weiteren Veränderung des Bestandesvolumenzuwachses führt.

Es gilt im Optimum für jeden Zeitpunkt:

$$
\frac{\Delta v_{B}\left(t, N_{t-1}\right)}{\Delta N_{t-1}}=0
$$

Diese Art der Grenznutzenanalyse wird im Folgenden als räumlich-zeitlich charakterisiert und von einer zeitlich-räumlichen Grenznutzenanalyse abgegrenzt, da für jeden Zeitschritt die optimale Bestandesdichte ermittelt wird und daraus implizit die zeitliche Abfolge der Einzelbaumentnahme folgt.

Durch Übertragung dieser räumlich-zeitlichen Grenznutzenanalyse von der Bestandesebene auf den Einzelbaum lässt sich auch dort eine Optimalitätsbedingung formulieren - der Bestand wird dabei als Summe seiner Einzelbäume aufgefasst (vgl. u. a. Coordes 2014a) und die Optimalitätsbedingung muss im Optimum für jeden einzelnen Baum simultan gelten. Die veränderbare Einheit bildet dabei nicht länger die Stammzahl des Bestandes, sondern die (Stand-)Fläche (und damit indirekt der Wuchsraum), die jedem einzelnen Baum altersabhängig zur Verfügung steht (vgl. u. a. Assmann 1961, S. 99 f.; Utschig 2002).

\footnotetext{
${ }^{19}$ In Anlehnung an das forstliche Produktionsmodell erfolgt die mathematische Formulierung im Gegensatz zur stetigen Analyse von Halbritter und Deegen (2015) jährlich diskret. Daher wird als Symbol der marginalen Veränderung ein $\Delta$ anstatt eines $\partial$ verwendet.
} 


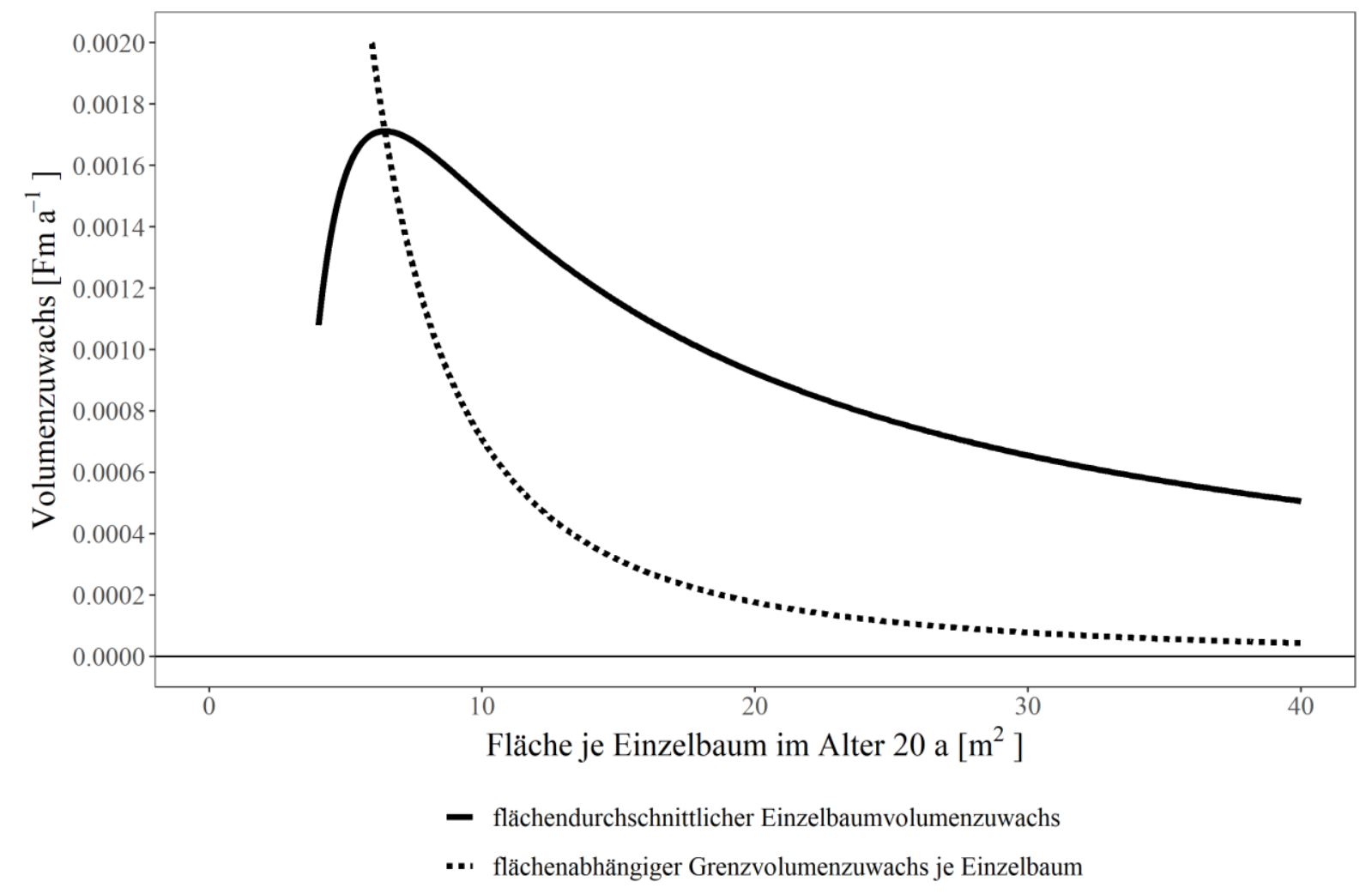

Abbildung 11: Exemplarische Gegenüberstellung von flächendurchschnittlichem Einzelbaumvolumenzuwachs und flächenabhängigem Grenzvolumenzuwachs je Einzelbaum in Abhängigkeit von der Fläche je Einzelbaum im Alter 20 a.

Im Fall des homogenen Bestandes sind die Stammzahl des Bestandes und die Fläche je Einzelbaum direkt ineinander überführbar - die Entnahme von Bäumen vergrößert die Fläche, die jedem verbleibenden Baum zur Verfügung steht, in gleichem Maße.

Bei natural-optimaler Vornutzung wird jedem Baum durch Entnahme anderer Bäume so viel Fläche $A$ zur Verfügung gestellt, dass der flächenabhängige Grenzvolumenzuwachs je Einzelbaum $\Delta v_{E}\left(t, A_{t-1}\right) \cdot\left(\Delta A_{t-1}\right)^{-1}$ dem flächendurchschnittlichen Einzelbaumvolumenzuwachs $\overline{v_{E}}\left(t, A_{t-1}\right)$ entspricht - es gilt:

$$
\begin{aligned}
& \frac{\Delta v_{E}\left(t, A_{t-1}\right)}{\Delta A_{t-1}}=\overline{v_{E}}\left(t, A_{t-1}\right), \\
& A_{t} \in \mathbb{R}^{\geq 0} .
\end{aligned}
$$

Der flächenabhängige Grenzvolumenzuwachs je Einzelbaum sinkt stark mit jeder weiteren Flächeneinheit und liegt ab der optimalen Fläche je Einzelbaum $A^{*}$ unterhalb des flächendurchschnittlichen Einzelbaumvolumenzuwachs. Bei optimaler Flächenaufteilung je Einzelbaum $A^{*}$ würde jede weitere Flächeneinheit den flächendurchschnittlichen Einzelbaumvolumenzuwachs senken. 


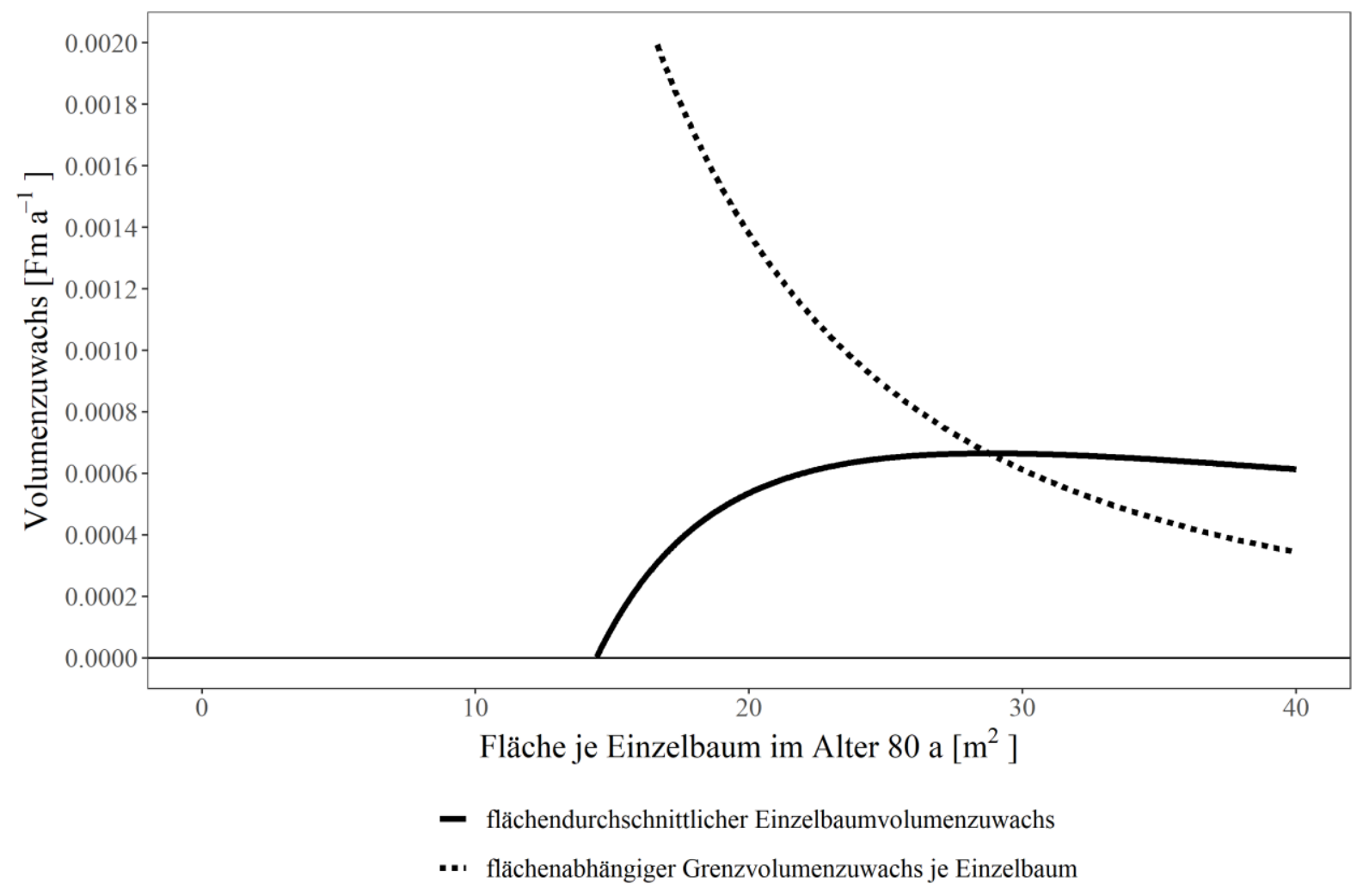

Abbildung 12: Exemplarische Gegenüberstellung von flächendurchschnittlichem Einzelbaumvolumenzuwachs und flächenabhängigem Grenzvolumenzuwachs je Einzelbaum in Abhängigkeit von der Fläche je Einzelbaum im Alter 80 a.

Abbildungen 11 und 12 zeigen die flächenabhängigen Entwicklungen von flächendurchschnittlichem Einzelbaumvolumenzuwachs und flächenabhängigem Grenzvolumenzuwachs je Einzelbaum exemplarisch für die Alter 20 und 80 a. Entsprechend der sinkenden Bestandesstammzahl (Abbildung 8) steigt die optimale Fläche je Einzelbaum über dem Alter (Schnittpunktverschiebung nach rechts).

Ein dritter Weg zur Formulierung einer Optimalitätsbedingung für die Vornutzung führt über die zeitlich-räumliche Grenznutzenanalyse am Einzelbaum. Während die beiden bisher genannten Optimalitätsbedingungen die optimale Ausnutzung der Fläche bzw. des Wuchsraums zu jedem Zeitpunkt bis zur Endnutzung und damit implizit die Erntezeitpunkte der einzelnen Bäume definieren, liefert diese zeitlich-räumliche Grenznutzenanalyse den optimalen Erntezeitpunkt eines jeden Baums und damit implizit die optimale Stammzahl zu jedem Zeitpunkt (vgl. Deegen 1997, S. 107 ff.; Coordes 2014a, S. 36 ff.; Coordes 2014c). Mit der Verwendung des Einzelbaums anstatt des Bestandes als Grundeinheit überwindet dieser Ansatz „die strukturelle Gleichförmigkeit des Faustmann-Ansatzes“ und ermöglicht u. a. die Anwendung ,auf vielseitige Problemstellungen der waldbaulichen Behandlung von Waldbeständen“ (Coordes 2014c, S. 326). Auch den intertemporalen Wechselwirkungen bei steigender Modellkomplexi- 
tät kann mit der zeitlich-räumlichen Grenznutzenanalyse besser begegnet werden (siehe Abschnitt 2.2.4). Ab Abschnitt 2.2.3 kommt in dieser Arbeit daher ausschließlich die zeitlichräumliche Grenznutzenanalyse zur Formulierung der Vornutzungs-Optimalitätsbedingungen zum Einsatz.

In Anlehnung an Fuchs (2018, S. 13 ff.) und in Übereinstimmung mit Coordes (2014a, S. 61) wird dafür die auf Pressler $(1860 ; 1995)$ beruhende Endnutzungsentscheidungsregel (vgl. FPOTheorem; Optimalitätsbedingung [2-1]) durch Analogieschluss auf die Einzelbaumebene übertragen. Für den optimalen Erntezeitpunkt eines jeden Baums gilt die Gleichheit des laufenden Einzelbaumvolumenzuwachses $v_{j}\left(t_{j}, N_{t-1}\right)$, als Grenznutzen des Baumerhalts für einen weiteren Zeitschritt, und der Summe aller durch seinen Erhalt um ein weiteres Jahr an den beeinflussten Bäumen entgehenden Volumenveränderungen $\sum \sum \Delta V_{k, j}\left(t_{k}\right)$, als Wuchsraumopportunität bzw. Grenzkosten des Baumerhalts für einen weiteren Zeitschritt (vgl. auch „Beeinflussungsrate“ bei Coordes 2014c):

$$
v_{j}\left(t_{j}, N_{t_{j}-1}\right)=\sum_{t=t_{j}}^{T} \sum_{\substack{k \in L_{t} \\ k \neq j}} \Delta V_{k, j}\left(t_{k}\right) .
$$

Für das jährlich-diskrete Modell bedeutet dies, dass der Erhalt eines jeden Baums $j$ zum Zeitpunkt $t_{j}-1$ nur solange rational ist, wie sein laufender Volumenzuwachs $v\left(t_{j}, N_{t_{j}-1}\right)$ zwischen den Zeitpunkten $t_{j}-1$ und $t_{j}$ noch die Summe aller durch den Erhalt des Baums um ein weiteres Jahr (Ernte zum Zeitpunkt $t_{j}$ statt $t_{j}-1$ ) an den von ihm beeinflussten Bäumen $k \in L_{t_{j}}$ entgehenden Volumenveränderungen $\Delta V_{k, j}$ zum Zeitpunkt ihrer jeweiligen Ernte $t_{k}$ übertrifft ${ }^{20}$. Verbleibt Baum $j$ zum Zeitpunkt $t_{j}-1$ ein weiteres Jahr im Bestand, generiert er den Volumenzuwachs $v_{j}\left(t_{j}, N_{t_{j}-1}\right)$ als zeitlichen Grenznutzen. Dem gegenüber stehen die entgehenden Volumenveränderungen $\Delta V_{k, j}$ an allen beeinflussten Bäumen $k$ (jeweils realisiert zum Zeitpunkt ihrer Ernte $t_{k}$ ) als Opportunitätskosten (Grenzkosten) des Erhalts von Baum $j$ für ein weiteres Jahr - die sogenannte Wuchsraumopportunität. Diese Wechselwirkungen des marginal verlängerten Baumerhalts werden im Folgenden als Dichteeffekt bezeichnet. Denn würde Baum $j$ bereits zum Zeitpunkt $t_{j}-1$ geerntet, so herrschte im Zeitschritt $t_{j}-1$ bis $t_{j}$ eine verminderte Dichte und damit Konkurrenz um die verfügbaren, begrenzten Ressourcen.

\footnotetext{
${ }^{20}$ Als hinreichende Optimalitätsbedingung wird hierbei (wie auch im Folgenden) die bereits überschrittene Kulmination des laufenden Volumenzuwachses (ab Abschnitt 2.2.2: Wertzuwachses) unterstellt (vgl. u. a. Johansson und Löfgren 1985, S. 75 f.; Möhring 1994, S. 137; Wippermann 2005, S. 39 f.).
} 
Alle durch Baum $j$ beeinflussten Bäume (im homogenen Modellbestand sind das alle anderen zu diesem Zeitpunkt verbliebenen Bäume des Bestandes) hätten in diesem Zeitschritt somit einen marginal erhöhten Volumenzuwachs und könnten ggf. sogar einen Teil des durch die vorgezogenen Ernte von Baum $j$ entgehenden Volumenzuwachses auf Bestandesebene kompensieren (vgl. Pretzsch 2005a).

Bei der Endnutzungsentscheidung unter Vernachlässigung eines Folgebestandes treten weder Intra- noch Inter-Bestandesopportunitäten auf. Nach Möhring (2009) wird die forstliche Produktion demnach aufrecht erhalten, solange ein positiver Grenznutzen, d. h. ein laufender Volumenzuwachs, generiert wird. Für den optimalen Endnutzungszeitpunkt $T^{*}$ gilt:

$$
v_{B}\left(T, N_{T-1}\right)=0
$$

Im jährlich-diskreten Modell ist die Ernte des Bestandes zum Zeitpunkt $T$ - 1 dann rational, wenn der folgende Bestandesvolumenzuwachs $v_{B}\left(T, N_{T-1}\right)$ zwischen den Zeitpunkten $T-1$ und $T$ negativ ist. Da dieser Punkt innerhalb der Modellgrenzen ausgeschlossen ist, dauert die forstliche Produktion bis zum modell-maximalen Bestandesalter $t_{\max }=100 \mathrm{a}$ an.

Wird hingegen die Prämisse der Vernachlässigung des Folgebestandes aufgegeben (vgl. Wippermann 2005, S. 22 ff.), treten zusätzlich zu den Intra-Bestandesopportunitäten der Vornutzung auch Inter-Bestandesopportunitäten im Zuge der Endnutzung auf. Der laufende Grenznutzen des aktuellen Bestandes (laufender Bestandesvolumenzuwachs) muss den durchschnittlichen Grenznutzen potenzieller Folgebestände (durchschnittlicher Bestandesvolumenzuwachs $^{21}$ ) überwiegen, damit der Bestandeserhalt für ein weiteres Jahr rational ist.

Es gilt somit das durchschnittlich nutzbare Holzvolumen, d. h. den durchschnittlichen Bestandesvolumenzuwachs $\overline{v_{B}}$, unter Veränderung der Bestandesbehandlung als Kombination aus Vornutzungsvektor $\boldsymbol{\sigma}_{t}$ und Endnutzungszeitpunkt $T$ zu maximieren:

$$
\begin{aligned}
& \max _{T, \boldsymbol{\sigma}_{T-1}} \overline{v_{B}}\left(T, \boldsymbol{\sigma}_{T-1}, N_{0}\right)=\max _{T, \boldsymbol{\sigma}_{T-1}} \frac{G W L\left(T, \boldsymbol{\sigma}_{T-1}, N_{0}\right)}{T}, \\
& 0 \leq \boldsymbol{\sigma}_{T-1}<1 \\
& 0 \mathrm{a}<T \leq 100 \mathrm{a} .
\end{aligned}
$$

\footnotetext{
${ }^{21}$ Damit wird (wie auch in den folgenden Abschnitten) der Ersatz des aktuellen Bestandes mit einem identischen Folgebestand impliziert, was z. B. im Rahmen eines angestrebten Baumartenwechsels keine zwangsläufige Annahme für die Anwendung der Optimalitätsbedingung darstellt (vgl. Möhring 2009).
} 
Bei gleicher natural-optimaler Stammzahlentwicklung $\boldsymbol{N}_{\text {nat }}^{*}$ verkürzt sich die forstliche Produktion vom modell-maximalen Bestandesalter zum natural-optimalen Endnutzungszeitpunkt unter Einbeziehung eines Folgebestandes $T_{\text {nat }}^{*}=43$ a (Abbildung 13).

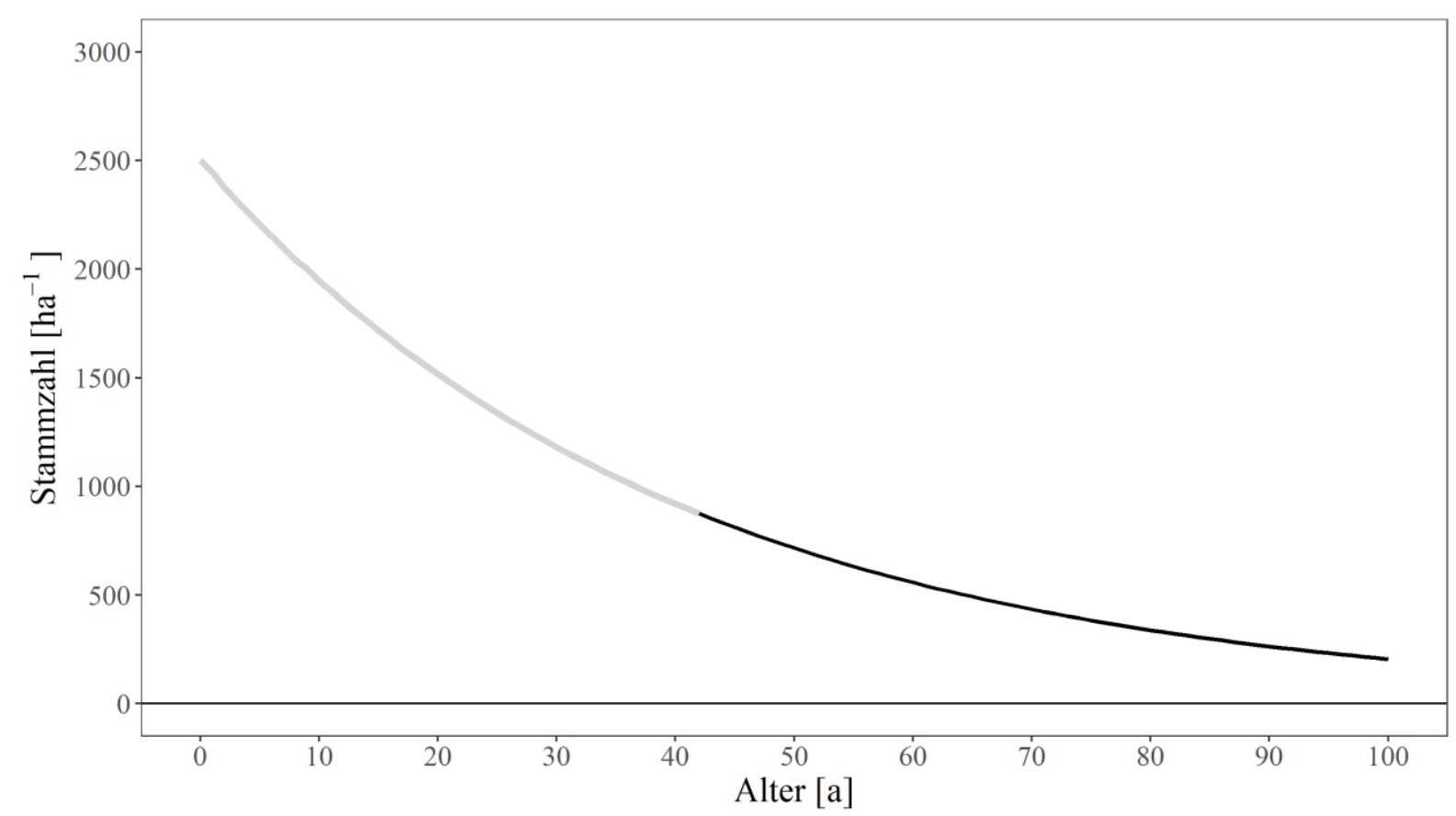

Abbildung 13: Altersabhängige Stammzahlentwicklung im naturalen Optimum mit Folgebestand (grau) gemäß Zielfunktion [2-16] im Vergleich zum naturalen Optimum ohne Folgebestand (schwarz).

Bis zum natural-optimalen Endnutzungszeitpunkt von 43 Jahren wächst der Bestand zu jedem Zeitpunkt maximal zu (Abbildung 14). Es resultiert bei einer Gesamtwuchsleistung von $617 \mathrm{Fm} \mathrm{ha}^{-1}$ ein durchschnittlicher Bestandesvolumenzuwachs und damit ein durchschnittlich nutzbares Holzvolumen (Zielgröße) von 14,4 $\mathrm{Fm} \mathrm{ha}^{-1} \mathrm{a}^{-1}(+30 \%$ ggü. der Modellvariante ohne Folgebestand). Zum Endnutzungszeitpunkt wird ein verbliebenes Bestandesvolumen von $400 \mathrm{Fm} \mathrm{ha}^{-1}$ geerntet (Abbildung 15). 
Während sich die Optimalitätsbedingungen der Vornutzung gegenüber der Variante ohne Folgebestand nicht ändert (vgl. Gleichungen [2-12], [2-13] oder [2-14]) muss der laufende Grenznutzen des Bestandes für dessen Erhalt nun den durchschnittlichen Nutzen eines Folgebestandes übertreffen. Die Optimalitätsbedingung der Endnutzung zeigt somit die Gleichheit von laufendem Bestandesvolumenzuwachs und durchschnittlichem Bestandesvolumenzuwachs (Gadow 2003, S. 153; Halbritter und Deegen 2015, Gleichung (10) ${ }^{22}$ mit $r \searrow 0$ und $P=1$ ):

$$
v_{B}\left(T, N_{T-1}\right)=\overline{v_{B}}\left(T, N_{T-1}\right)
$$

Im jährlich-diskreten Modell ist der Bestandeserhalt zum Zeitpunkt $T-1$ somit rational, wenn der laufende Bestandesvolumenzuwachs zwischen den Zeitpunkten $T-1$ und $T$ noch den durchschnittlichen Bestandesvolumenzuwachs (eines Folgebestandes) übertrifft.

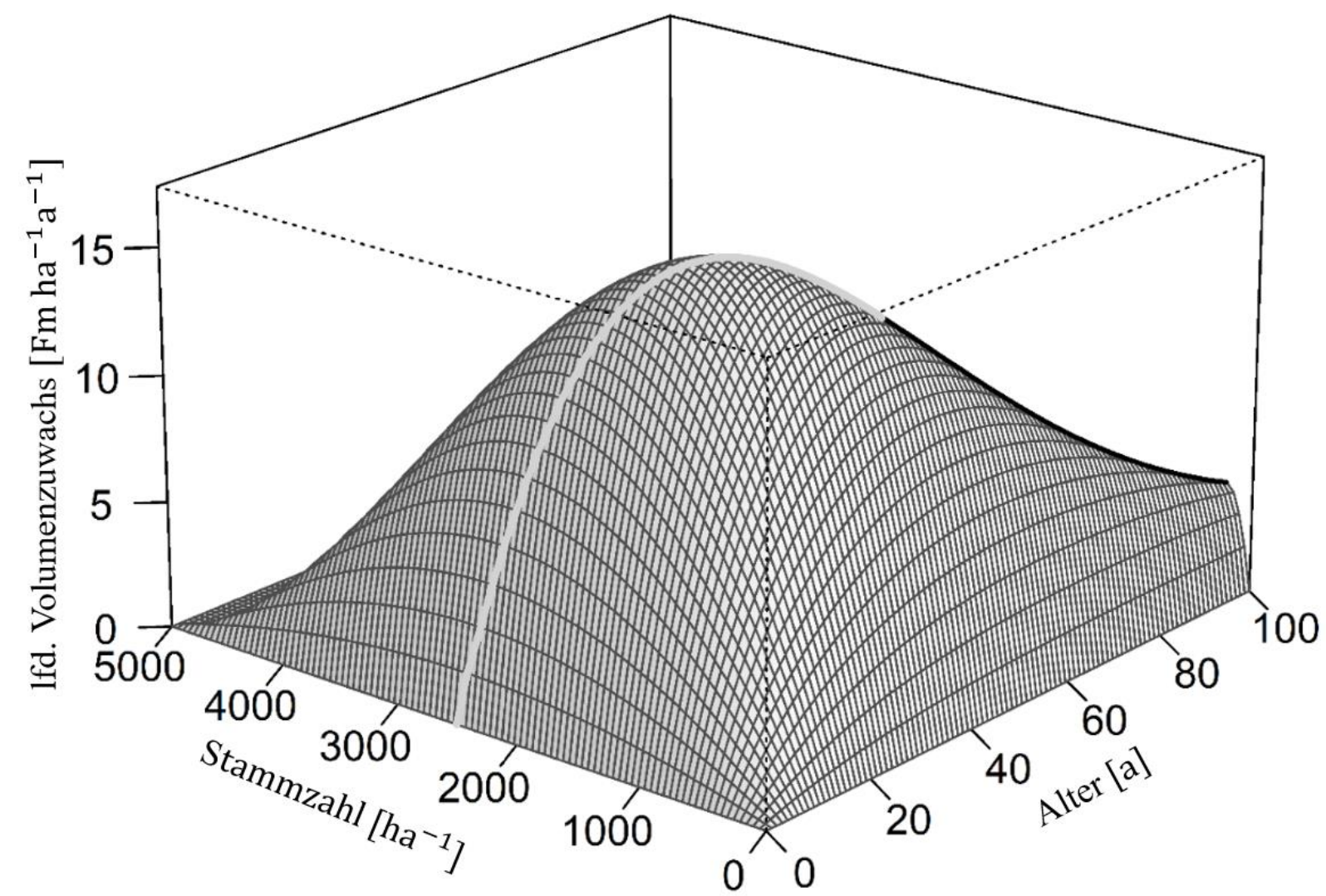

Abbildung 14: Alters- und stammzahlabhängiger laufender Bestandesvolumenzuwachs im naturalen Optimum mit Folgebestand (grau) gemäß Zielfunktion [2-16] im Vergleich zum maximalen Volumenzuwachs (schwarz).

${ }^{22}$ Der von Halbritter und Deegen (2015) in Gleichung (10) genutzte Term $r L E V$ steht für die BruttoBodenrente. Bei einem gegen Null strebenden Kalkulationszinssatz $r \searrow 0$, einem konstanten Deckungsbeitrag von $P=1$ und bei Vernachlässigung von Bestandesbegründungskosten entspricht dieser Term dem durchschnittlichen Volumenzuwachs $\bar{v}_{B}$ (vgl. Regeln von de L'Hospital; Forster 2013, S. 186 ff.; vgl. auch Möhring 1994, S. 74 f. und Pertz 1983, S. 149). 


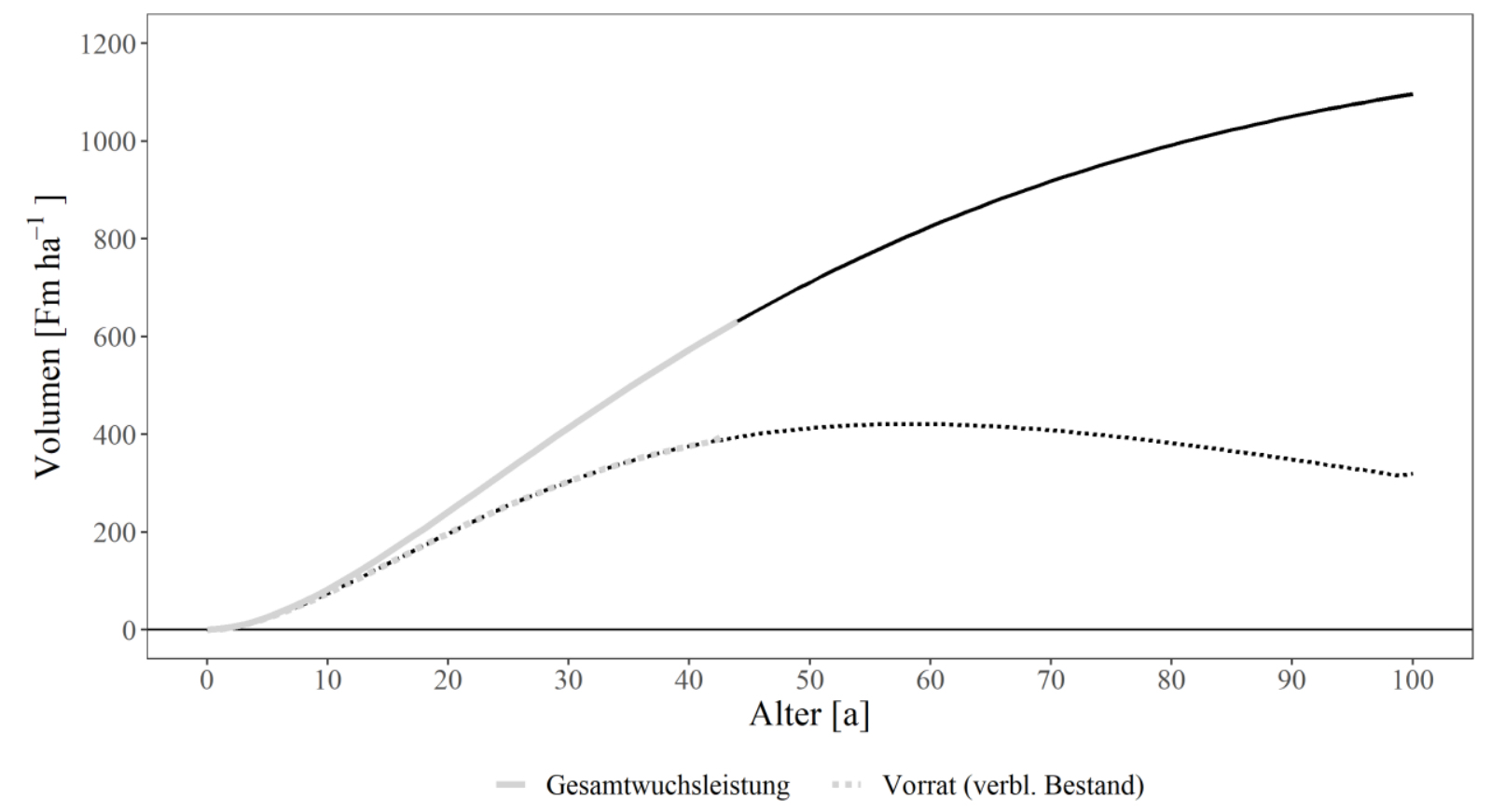

Abbildung 15: Altersabhängige Gesamtwuchsleistung und Vorratsentwicklung im naturalen Optimum mit Folgebestand gemäß Zielfunktion [2-16] im Vergleich zum naturalen Optimum ohne Folgebestand (schwarz).

\subsubsection{Konstanter Deckungsbeitrag}

Durch die Bewertung der Vornutzungsvolumina $\lambda_{t}\left(\sigma_{t}, t, \sigma_{t-1}, N_{0}\right)$ und des Endnutzungsvolumens $V_{B}\left(T, \sigma_{T-1}, N_{0}\right)$ mit einem konstanten erntekostenfreien Holzerlös $P$ (Deckungsbeitrag), ergibt sich der durchschnittliche Überschuss der forstlichen Produktion $a_{P}$ im Sinne eines Deckungsbeitrags I (vgl. Keuffel 1980, S. 57 f.) als zu maximierende Zielfunktion - es gilt:

$$
\begin{aligned}
\max _{T, \boldsymbol{\sigma}_{T-1}} & a_{P}\left(T, \boldsymbol{\sigma}_{T-1}, N_{0}, P\right) \\
& =\max _{T, \boldsymbol{\sigma}_{T-1}}\left(\sum_{t=1}^{T-1} \lambda_{t}\left(\sigma_{t}, t, \boldsymbol{\sigma}_{t-1}, N_{0}\right) \cdot P+V_{B}\left(T, \boldsymbol{\sigma}_{T-1}, N_{0}\right) \cdot P\right) \cdot \frac{1}{T}
\end{aligned}
$$

$0 \leq \boldsymbol{\sigma}_{T-1}<1$

$0 \mathrm{a}<T \leq 100 \mathrm{a}$

$P \in \mathbb{R}$.

Trotz veränderter Zielfunktion bleibt die optimale Bestandesbehandlung ggü. Abschnitt 2.2.1 unverändert, da die ökonomische Bewertung der genutzten Holzvolumina mit einem konstanten Deckungsbeitrag lediglich zu einer proportionalen Skalierung der Zielgröße führt. Aus dem maximal nutzbaren, durchschnittlichen Holzvolumen von 14,4 $\mathrm{Fm} \mathrm{ha}^{-1} \mathrm{a}^{-1}$ wird durch Multiplikation mit dem konstanten Deckungsbeitrag $P=30 € \mathrm{Fm}^{-1}$ ein durchschnittlicher Überschuss von $431 € \mathrm{ha}^{-1} \mathrm{a}^{-1}$. Das absolute Deckungsbeitragsniveau ist dabei irrelevant für die optimale Bestandesbehandlung. Aus der Volumenzuwachskuppel in Abbildung 14 entsteht 
eine Wertzuwachskuppel mit gleicher Form und veränderter Skala (Abbildung 16). Für den laufenden Bestandeswertzuwachs gilt:

$$
w_{B}\left(t, N_{t-1}, P\right)=v_{B}\left(t, N_{t-1}\right) \cdot P .
$$

Die optimale Stammzahlentwicklung $\boldsymbol{N}_{P}^{*}$ entspricht der natural-optimalen Stammzahlentwicklung (vgl. Abbildung 13) und führt zu maximalen Bestandeswert- und damit auch Bestandesvolumenzuwächsen in jedem Zeitschritt - der Optimalpfad verläuft auf dem Grat der Wertzuwachskuppel (Abbildung 16). Die maximalen laufenden und durchschnittlichen Bestandesvolumenzuwächse liegen unverändert bei $17,4 \mathrm{Fm} \mathrm{ha}^{-1} \mathrm{a}^{-1}$ und $14,4 \mathrm{Fm} \mathrm{ha}^{-1} \mathrm{a}^{-1}$. Das optimale Endnutzungsalter $T_{P}^{*}=43$ a entspricht dem Endnutzungsalter im naturalen Optimum.

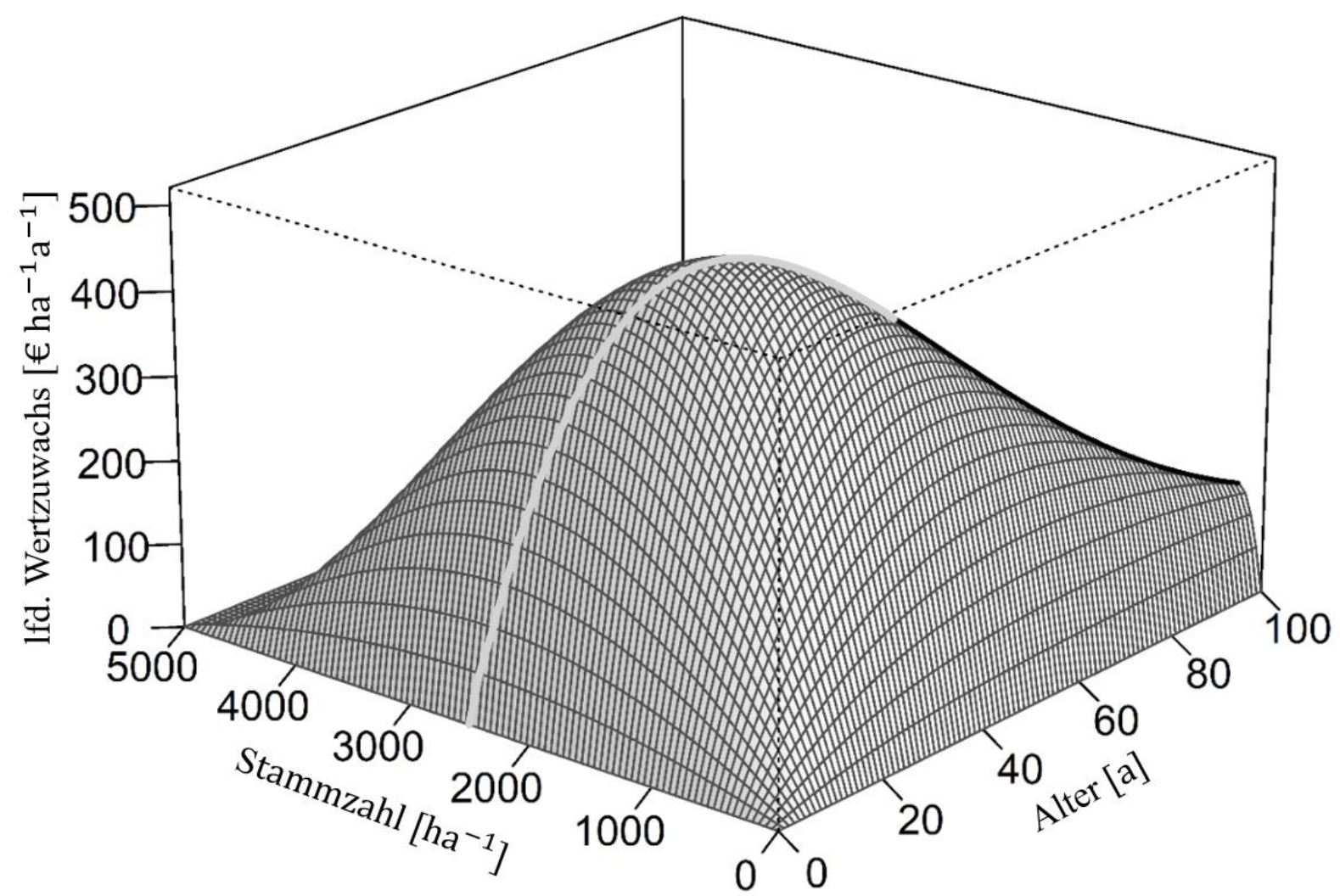

Abbildung 16: Alters- und stammzahlabhängiger laufender Bestandeswertzuwachs bei konstantem Deckungsbeitrag (grau) gemäß Zielfunktion [2-18] im Vergleich zum maximalen Wertzuwachs (schwarz).

Durch entsprechende Erweiterung der räumlich-zeitlichen Optimalitätsbedingung [2-12] wird die optimale Vornutzung auf Bestandesebene gekennzeichnet durch:

$$
\frac{\Delta w_{B}\left(t, N_{t-1}, P\right)}{\Delta N_{t-1}}=0
$$

Für eine optimale Vornutzung muss die räumliche Grenzproduktivität zu jedem Zeitpunkt Null sein - mehr und weniger Bäume im Bestand würden dessen laufenden Wertzuwachs 
$w_{B}\left(t, N_{t-1}, P\right)$ senken (vgl. auch Halbritter und Deegen 2015, Gleichung (08) mit $r=0$ und $P=$ konst.).

Die beiden alternativen Optimalitätsbedingungen der Vornutzung (siehe Abschnitt 2.2.1) können analog auf eine ökonomisch bewertete Ebene transformiert werden. Durch die Bewertung mit einem konstanten Deckungsbeitrag ändert sich lediglich die Skalierung aller Größen, nicht aber deren Abhängigkeiten und Wechselwirkungen.

Für den Einzelbaum gilt im Optimum einerseits (räumlich-zeitlich) die Gleichheit von flächenabhängigem Grenzwertzuwachs des Einzelbaums $\Delta w_{E}\left(t, A_{t-1}, P\right) \cdot\left(\Delta A_{t-1}\right)^{-1}$ und flächendurchschnittlichem Einzelbaumwertzuwachs $\overline{w_{E}}\left(t, A_{t-1}, P\right)$ :

$$
\frac{\Delta w_{E}\left(t, A_{t-1}, P\right)}{\Delta A_{t-1}}=\overline{w_{E}}\left(t, A_{t-1}, P\right)
$$

Andererseits gilt im Optimum (zeitlich-räumlich) die Gleichheit von laufendem Wertzuwachs des Einzelbaums $w_{j}\left(t_{j}, N_{t-1}, P\right)$, dem Grenznutzen des Baumerhalts, und der Summe aller durch den Erhalt des Baums um ein weiteres Jahr an den von ihm beeinflussten Bäumen entgehenden Wertveränderungen zum Zeitpunkt ihrer Realisierung $\sum \sum \Delta W_{k, j}\left(t_{k}\right)$, der Wuchsraumopportunität:

$$
w_{j}\left(t_{j}, N_{t_{j}-1}, P\right)=\sum_{t=t_{j}}^{T} \sum_{\substack{k \in L_{t_{j}} \\ k \neq j}} \Delta W_{k, j}\left(t_{k}\right) .
$$

Der optimale Endnutzungszeitpunkt $T^{*}$ wird nach entsprechender Erweiterung der Optimalitätsbedingung [2-17] durch die Gleichheit von laufendem und durchschnittlichem Bestandeswertzuwachs gekennzeichnet. Es gilt:

$$
\begin{aligned}
w_{B}\left(T, N_{T-1}, P\right) & =\overline{w_{B}}\left(T, \boldsymbol{N}_{T-1}, P\right) \\
& =a_{P}\left(T, \boldsymbol{\sigma}_{T-1}, N_{0}, P\right),
\end{aligned}
$$

mit dem durchschnittlichen Bestandeswertzuwachs $\overline{w_{B}}\left(T, N_{T-1}, P\right)$, welcher dem durchschnittlichen Überschuss der forstlichen Produktion $a_{P}\left(T, N_{0}, \sigma_{T-1}, P\right)$, d. h. der zu maximierenden Zielgröße, entspricht.

\subsubsection{Zeitabhängige Deckungsbeiträge}

Zur weiteren Komplexitätssteigerung wird in diesem Abschnitt ein zeitabhängig steigender Deckungsbeitrag eingeführt. Dieser repräsentiert eine Wertsteigerung in der Zukunft und kann in 
dem hier gewählten Beispiel einer konstanten DB-Steigerungsrate wie ein negativer Kalkulationszinssatz (vgl. Abschnitt 2.2.5) interpretiert werden ${ }^{23}$ - zukünftige Holznutzungen sind damit umso wertvoller, je weiter sie in der Zukunft liegen.

Durch die Bewertung der genutzten Holzvolumina mit dem zeitabhängigen Deckungsbeitrag $P(t)$ erweitert sich Optimalitätsbedingung [2-18] zu:

$$
\begin{aligned}
& \max _{T, \boldsymbol{\sigma}_{T-1}} a_{P(t)}\left(T, \boldsymbol{\sigma}_{T-1}, N_{0}, P(t)\right) \\
& \quad=\max _{T, \boldsymbol{\sigma}_{T-1}}\left(\sum_{t=1}^{T-1} \lambda_{t}\left(\sigma_{t}, t, \boldsymbol{\sigma}_{t-1}, N_{0}\right) \cdot P(t)+V_{B}\left(T, N_{0}, \boldsymbol{\sigma}_{T-1}\right) \cdot P(T)\right) \cdot \frac{1}{T}, \\
& \quad 0 \leq \boldsymbol{\sigma}_{T-1}<1, \\
& 0 \mathrm{a}<T \leq 100 \mathrm{a}, \\
& P \in \mathbb{R} .
\end{aligned}
$$

Es gilt den durchschnittlichen Überschuss der forstlichen Produktion $a_{P(t)}$ unter Veränderung des Vornutzungsvektors $\boldsymbol{\sigma}_{T}$ sowie des Endnutzungszeitpunkts $T$ zu maximieren.

Der zeitabhängige Deckungsbeitrag $P(t)$ wird für das Variantenstudium über die Funktion

$$
P(t)=P \cdot(1+\varpi)^{t}
$$

mit dem konstanten Deckungsbeitrag $P=30 € \mathrm{Fm}^{-1}$ (vgl. Abschnitt 2.2.2) sowie den jährlich-konstanten DB-Steigerungsraten

$$
\begin{aligned}
& \varpi_{\text {schwach }}=0,005 \text { und } \\
& \varpi_{\text {stark }}=0,015
\end{aligned}
$$

definiert (Abbildung 17).

\footnotetext{
${ }^{23}$ Die Definition des konstant steigenden DB erfolgt bewusst in Abhängigkeit von der Zeit und nicht in Abhängigkeit vom Alter. Während Zeit und Alter im forstlichen Produktionsmodell zunächst deckungsgleich sind, spielt die Unterscheidung bei der Interpretation der Modellprämisse und bei der Verwendung des durchschnittlichen Überschusses als Flächenopportunität (identischer Bestandesersatz) eine entscheidende Rolle. So erlaubt die Abhängigkeit von der Zeit die Interpretation als Wertsteigerung und damit den Brückenschlag zur Kapitalknappheit (negativer Kalkulationszinssatz) - bei der Abhängigkeit vom Alter wäre dies nicht gegeben, da mit der Endnutzung und der Begründung eines Folgebestandes auch der Deckungsbeitrag wieder auf das Ausgangsniveau fallen würde. Gleichzeitig ist mit der Zeitabhängigkeit aber auch verbunden, dass bei dem hier angenommenen identischen Ersatz des Bestandes der durchschnittliche Überschuss des aktuellen Bestandes seine Gültigkeit als Flächenopportunität verliert, da die Deckungsbeiträge von Umtriebszeit zu Umtriebszeit weiter steigen (vgl. Generalisiertes Faustmann Modell; Chang 1998). Aus Vereinfachungsgründen wird dies in der vorliegenden Arbeit allerdings vernachlässigt.
} 


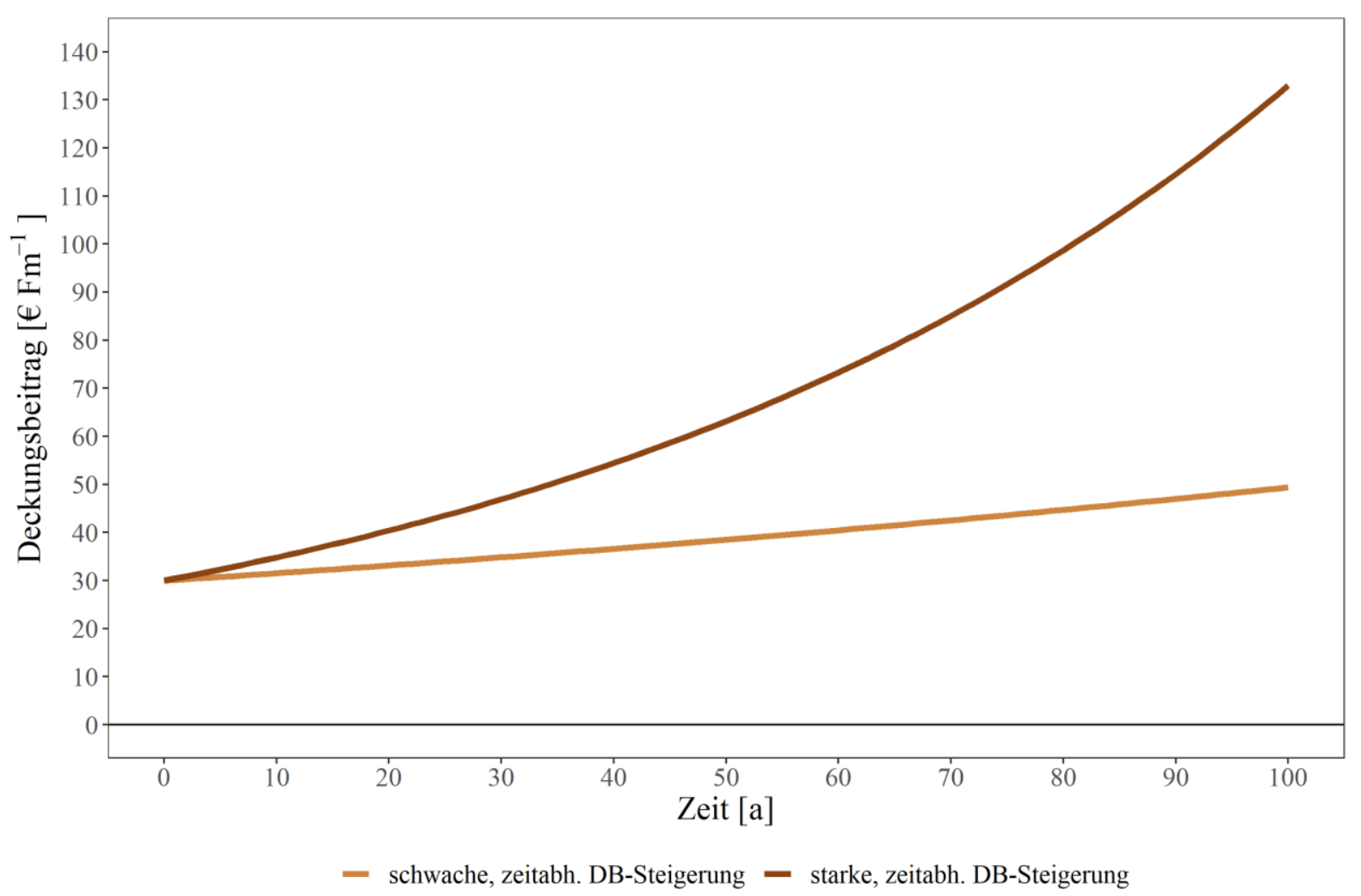

Abbildung 17: Zeitabhängiger Deckungsbeitrag (erntekostenfreier Holzerlös) je Festmeter mit schwacher (hellbraun) und starker (dunkelbraun) DB-Steigerung gemäß Deckungsbeitragsfunktion [2-25].

Damit liegen die maximalen durchschnittlichen Überschüsse (Zielgröße) erwartungsgemäß deutlich oberhalb des Optimums bei konstantem Deckungsbeitrag (vgl. Abschnitt 2.2.2): $533 € \mathrm{ha}^{-1} \mathrm{a}^{-1}(+24 \%)$ bei schwacher und $1.062 € \mathrm{ha}^{-1} \mathrm{a}^{-1}(+147 \%)$ bei starker DBSteigerung. Zur Erreichung dieser Zielgrößen weichen die optimalen Stammzahlentwicklungen erstmals vom naturalen Optimum ab (Abbildung 18). In den variantenspezifischen Optima resultieren durch mehr oder weniger starke Vornutzungen im ersten Zeitschritt zunächst Stammzahlen unterhalb der natural-optimalen Stammzahlentwicklung - bis zu einem Alter von 31 Jahren gilt:

$$
\boldsymbol{N}_{P(t), \text { stark }}^{*}<\boldsymbol{N}_{P(t), \text { schwach }}^{*}<\boldsymbol{N}_{\text {nat }}^{*}
$$

Dann überschreitet die Stammzahl der schwachen DB-Steigerung die natural-optimale Stammzahlentwicklung und ab einem Alter von 59 Jahren liegt auch die optimale Stammzahlentwicklung der starken DB-Steigerung oberhalb der naturalen Referenz.

Diese Stammzahlentwicklungen sind nicht unbedingt selbsterklärend, da unter zeitabhängig steigenden Deckungsbeiträgen frühe Eingriffe zunächst nicht sinnvoll wirken - jegliche spätere Realisierung der geernteten Holzvolumina würde höhere Deckungsbeiträge je Volumeneinheit bedingen. 


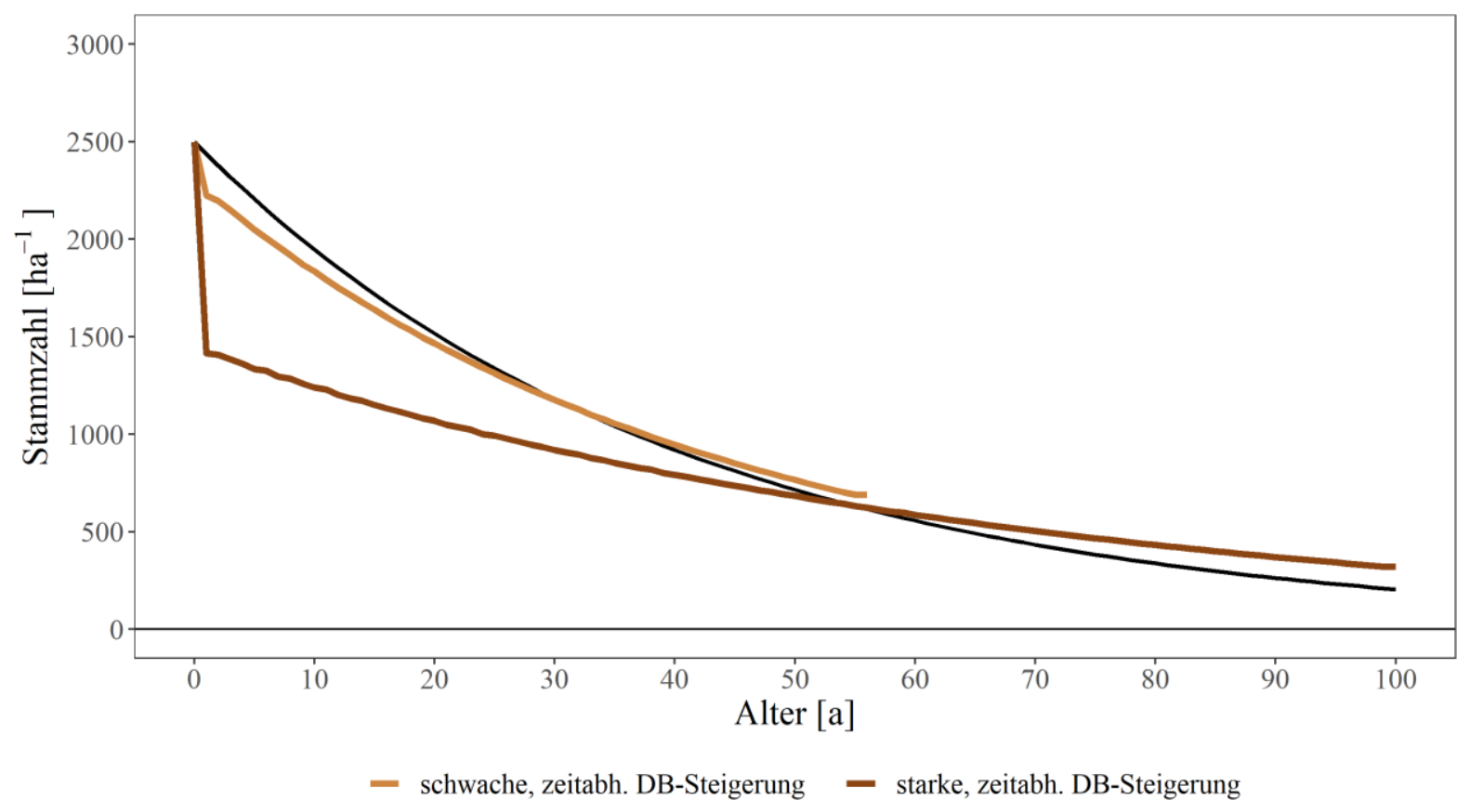

Abbildung 18: Altersabhängige Stammzahlentwicklung bei schwacher (hellbraun) bzw. starker (dunkelbraun), zeitabhängiger DB-Steigerung gemäß Zielfunktion [2-24] im Vergleich zum naturalen Optimum ohne Folgebestand (schwarz).

An dieser Stelle kommen allerdings modellbedingte Besonderheiten zum Tragen, die einer kurzen Erläuterung bedürfen: Ein vielleicht im ersten Augenblick sinnvoll erscheinender Verzicht auf jegliche Vornutzungen würde bereits nach kürzester Zeit aufgrund der dann entsprechend hohen Stammzahl und der mit der Zeit sinkenden Bestandeskapazitätsgrenze (siehe Abbildung 4) starke Volumenzuwachseinbußen bewirken (siehe Abbildung 6) - eine Stammzahlreduktion ist unabdingbar. Die Frage ist also nicht ob, sondern lediglich wann wieviele Bäume entnommen werden. Aufgrund der Modellbedingung der schematischen Vornutzungen sind mit jeder Stammzahlentnahme auch Volumenentnahmen mit gleichem Anteil verbunden. Umso später die Stammzahlentnahme demnach erfolgt, desto mehr wirkt sie einer angestrebten Volumenakkumulation (Realisierung zu späteren Zeitpunkten) entgegen. Dies gilt insbesondere vor dem Hintergurnd der Modellbedingung, dass zu Beginn der forstlichen Produktion 2.500 Bäume je Hektar ohne messbares Holzvolumen vorhanden sind. Durch die starke Stammzahlentnahme zu Beginn der forstlichen Produktion wird somit bei kleinstmöglicher Volumenreduktion (0,2 Fm ha ${ }^{-1}$ verteilt auf 276 Bäume bei schwacher DB-Steigerung bzw. 0,9 Fm ha-1 verteilt auf 1.084 Bäume bei starker DB-Steigerung) eine ggü. dem naturalen Optimum reduzierte Vornutzung in den kommenden Jahren (siehe flachere Entwicklung der braunen Linien im Vergleich zur schwarzen Referenzlinie in Abbildung 18) und damit eine größere Volumenakkumulation ermöglicht. 

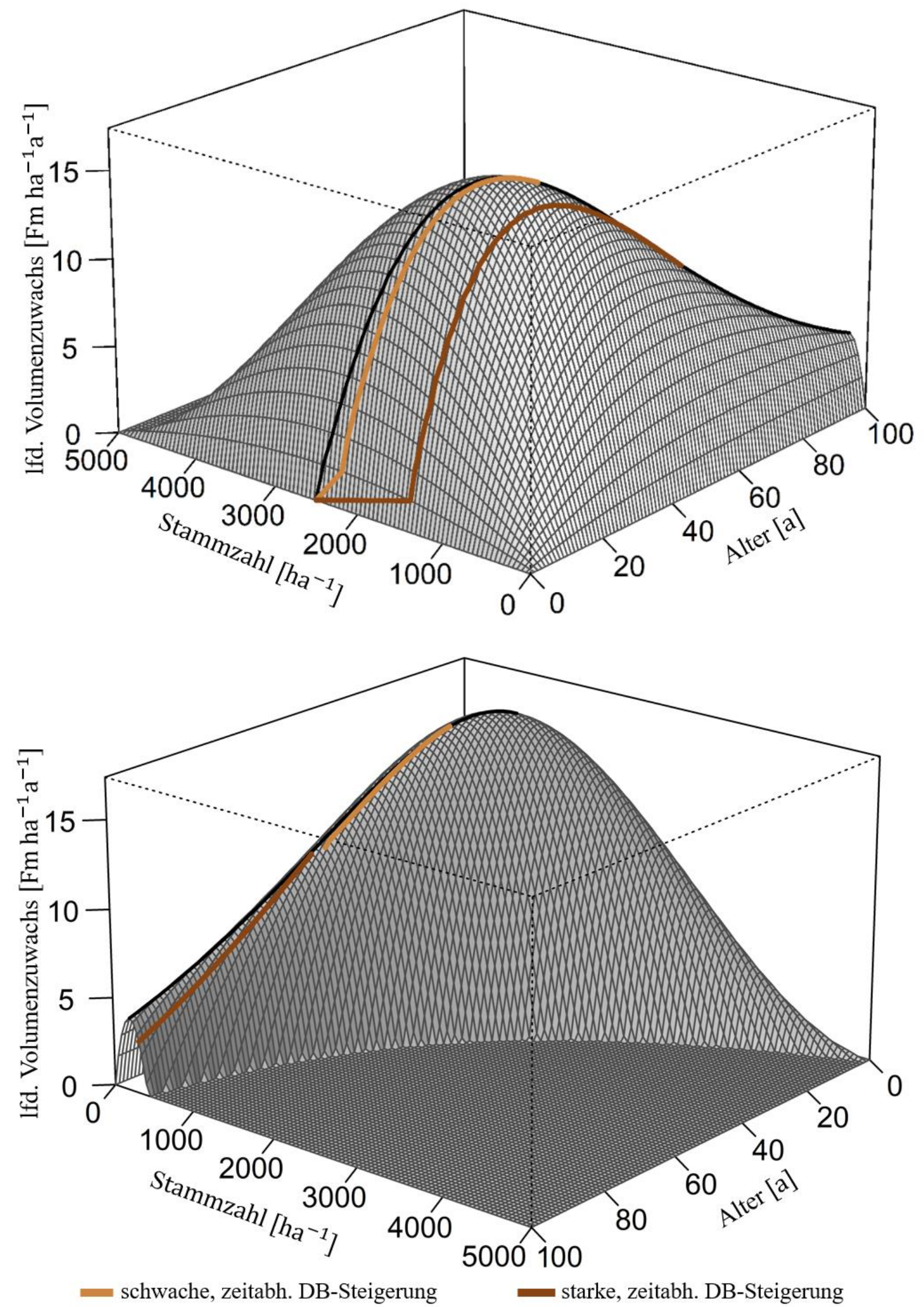

Abbildung 19: Alters- und stammzahlabhängiger laufender Bestandesvolumenzuwachs bei schwacher (hellbraun) bzw. starker (dunkelbraun), zeitabhängiger DB-Steigerung gemäß Zielfunktion [2-24] im Vergleich zum maximalen Volumenzuwachs (schwarz). Perspektivwechsel: (a) ist ggü. (b) horizontal um $180^{\circ}$ gedreht. 
Während die forstliche Produktion unter Berücksichtigung eines Folgebestandes bei schwacher DB-Steigerung im Alter $T_{P(t) \text {,schwach }}^{*}=56$ a und somit 13 Jahre später als beim Optimum mit konstantem Deckungsbeitrag endet, wird der optimale Endnutzungszeitpunkt bei starker DBSteigerung modellbedingt nicht erreicht - die forstliche Produktion endet im modell-maximalen Bestandesalter $t_{\max }=100 \mathrm{a}$.

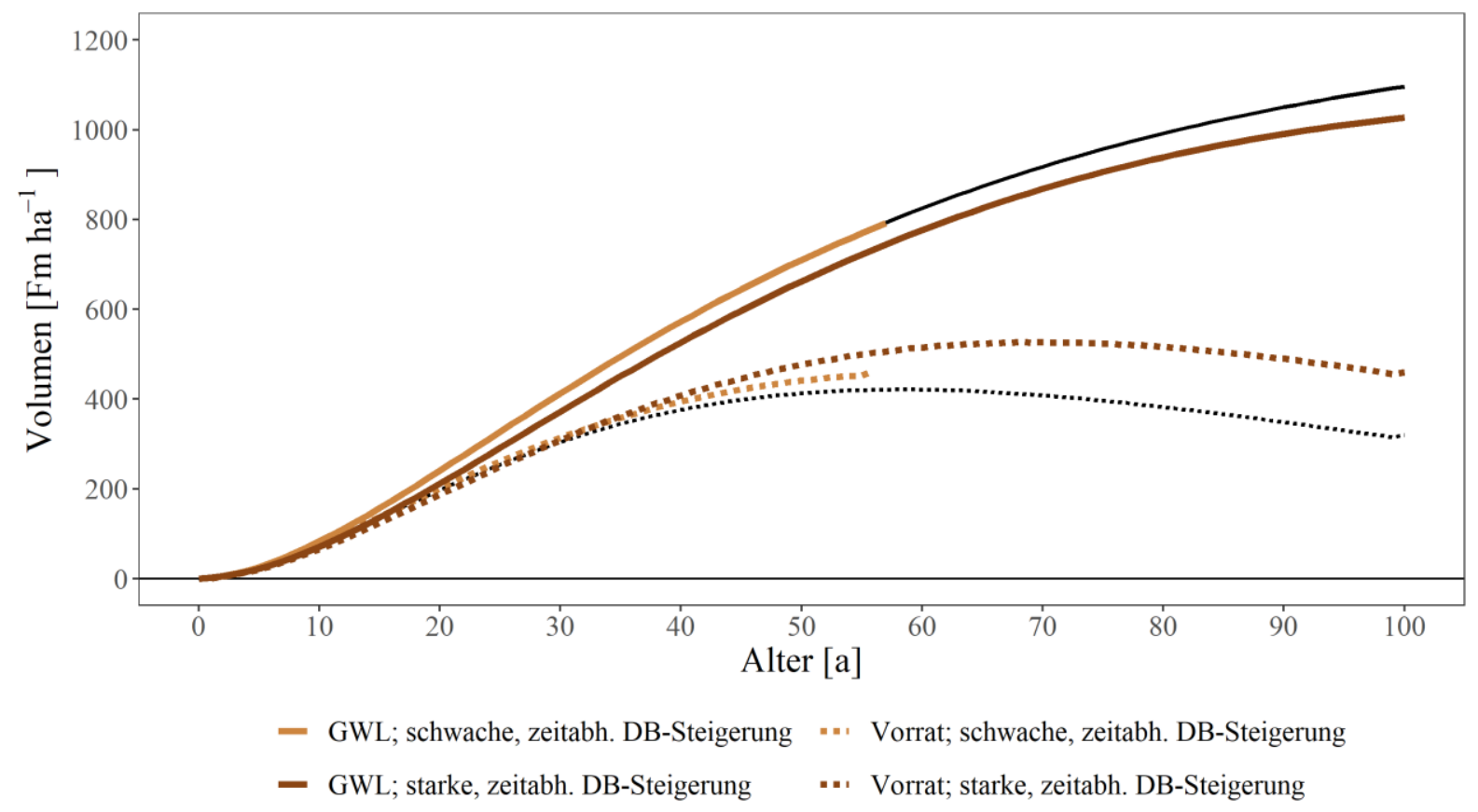

Abbildung 20: Altersabhängige Gesamtwuchsleistung und Vorratsentwicklung bei schwacher (hellbraun) bzw. starker (dunkelbraun), zeitabhängiger DB-Steigerung gemäß Zielfunktion [2-24] im Vergleich zum naturalen Optimum ohne Folgebestand (schwarz).

Aufgrund der Abweichungen von der natural-optimalen Stammzahlentwicklung wird auf Volumenzuwachs verzichtet - umso mehr, je stärker die jährliche DB-Steigerung ist (Abbildung 19). Maximal wird ein laufender Bestandesvolumenzuwachs von 17,4 $\mathrm{Fm} \mathrm{ha}^{-1} \mathrm{a}^{-1}$ (schwache DB-Steigerung im Alter 24 a) bzw. 16,0 Fm ha-1 $\mathrm{a}^{-1}$ (starke DB-Steigerung im Alter 27 a) erzielt. Es ergeben sich mit dem optimalen Endnutzungszeitpunkt bzw. modell-maximalen Bestandesalter durchschnittliche Bestandesvolumenzuwächse von 13,9 Fm ha-1 $\mathrm{a}^{-1}$ (schwache DB-Steigerung) bzw. 10,3 $\mathrm{Fm} \mathrm{ha}^{-1} \mathrm{a}^{-1}$ (starke DB-Steigerung). Bei verminderter Gesamtwuchsleistung wird bei zeitabhängig steigendem Deckungsbeitrag vermehrt Bestandesvolumen aufgebaut (Volumenakkumulation), um möglichst viel Holzvolumen zu einem späten Zeitpunkt realisieren zu können (Abbildung 20). Die Gesamtwuchsleistungen liegen zu den Endnutzungszeitpunkten mit $781 \mathrm{Fm} \mathrm{ha}^{-1}$ (schwache DB-Steigerung) bzw. 1.029 Fm ha-1 (starke DB-Steigerung) um 0,2 \% bzw. 6 \% unterhalb der entsprechenden maximalen Gesamtwuchsleistungen (vgl. schwarze Linie in Abbildung 20). Demgegenüber stehen Endnutzungsvolumina, welche die entsprechenden natural-optimalen Endnutzungsvolumina mit 
$464 \mathrm{Fm} \mathrm{ha}^{-1}$ (schwache DB-Steigerung) bzw. $459 \mathrm{Fm} \mathrm{ha}^{-1}$ (starke DB-Steigerung) um $11 \%$ bzw. 44 \% (vgl. schwarz gepunktete Linie in Abbildung 20) übersteigen.

Während sich die relative Gesamtwuchs- und die relative Gesamtwertleistung bei einem konstanten Deckungsbeitrag deckungsgleich entwickeln, wird die Gesamtwertleistung des Bestandes bei zeitabhängig steigenden Deckungsbeiträgen zu größeren Teilen erst in höheren Altern und somit bei bereits höheren Gesamtwuchsleistungen generiert. Die entstehenden Abweichungen zwischen relativer Gesamtwuchs- und relativer Gesamtwertleistung lassen sich durch Disparitätskurven (auch: Lorenz-Kurven; vgl. z. B. Gastwirth 1971; Dichtl und Issing 1987, S. 1200 f.) darstellen (Abbildung 21).

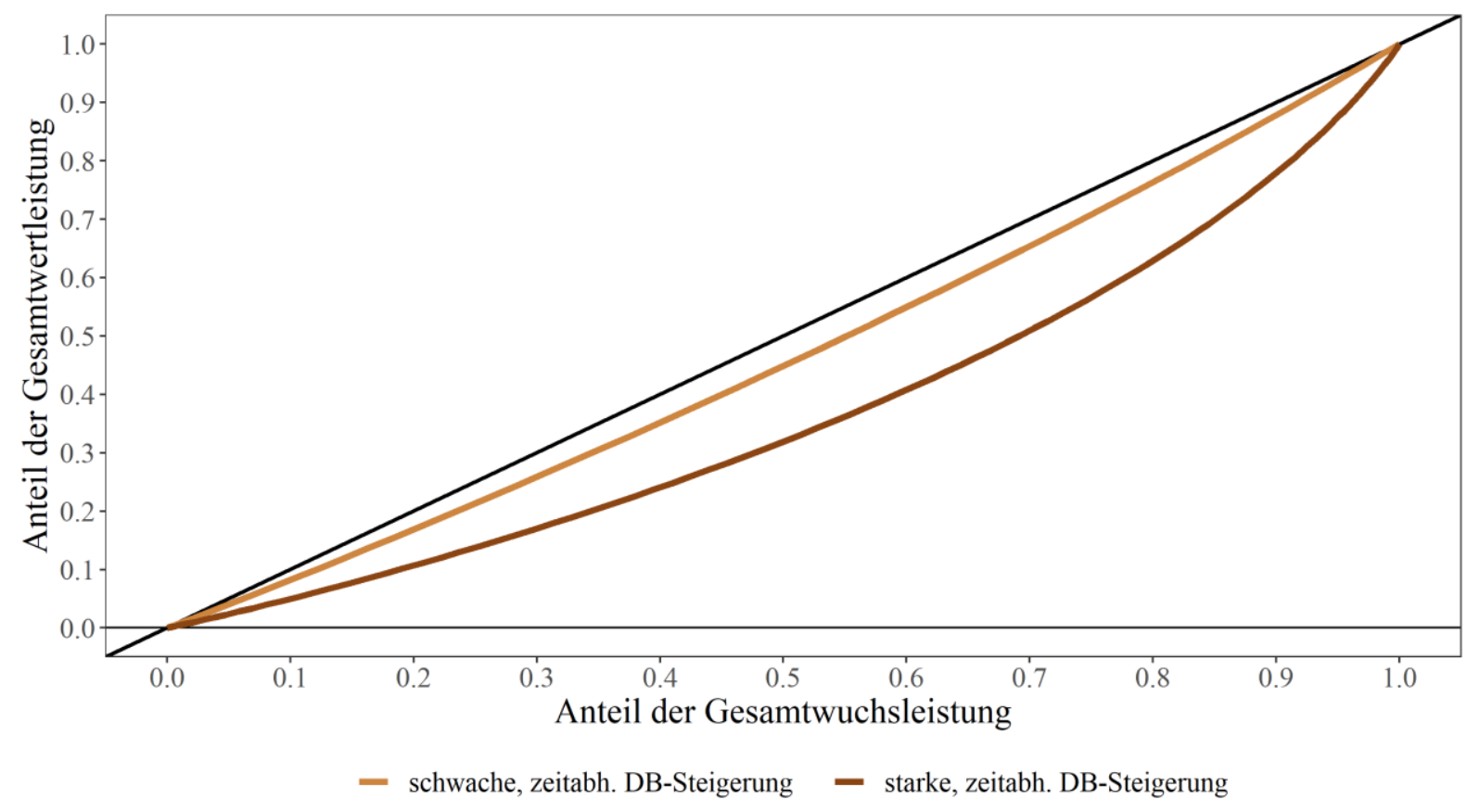

Abbildung 21: Disparitätskurven der relativen Gesamtwuchs- und Gesamtwertleistung bei schwacher (hellbraun) bzw. starker (dunkelbraun), zeitabhängiger DB-Steigerung gemäß Zielfunktion [2-24] im Vergleich zur Deckungsgleichheit von relativer Volumen- und Wertleistung bei der Bewertung mit einem konstanten Deckungsbeitrag (schwarz).

Je stärker die Deckungsbeiträge über der Zeit steigen, desto stärker ist die Abweichung zwischen relativer Gesamtwuchs- und relativer Gesamtwertleistung. Einer zu 50 \% realisierten Gesamtwuchsleistung stehen bei schwacher DB-Steigerung 45 \% der Gesamtwertleistung gegenüber - bei starker DB-Steigerung lediglich $32 \%$. Neben der grafischen Darstellung über die Lorenz-Kurven kann diese Disparität, und damit auch der Einfluss zeitabhängig steigender Deckungsbeiträge auf die optimale Bestandesbehandlung, über den sogenannten Gini-Koeffizienten (auch: Lorenzsches Konzentrationsmaß; vgl. u. a. Dorfman 1979; Dichtl und Issing 1987, S. 732 f.) als relatives Disparitätsmaß quantifiziert werden. Hierzu wird die Fläche zwischen Winkelhalbierender und Disparitätskurve in Verhältnis zur gesamten Fläche unterhalb 
der Winkelhalbierenden gesetzt. Es ergibt sich ein Wert zwischen 0 und 1, der umso größer ist, je größer die Disparität, das heißt die Abweichung zwischen relativer Gesamtwuchsleistung und relativer Gesamtwertleistung ist. Für die schwach steigenden Deckungsbeiträge (hellbraune Linie in Abbildung 21) ergibt sich ein Gini-Koeffizient von 0,07. Bei stark zeitabhängig steigenden Deckungsbeiträge (dunkelbraune Linie in Abbildung 21) liegt der Gini-Koeffizient deutlich höher bei 0,26 - der Einfluss der zeitabhängig steigenden Deckungsbeiträge auf die forstliche Bestandesbehandlung ist größer.

Die Optimalitätsbedingung (zeitlich-räumliche Grenznutzenanalyse am Einzelbaum) verändert sich durch die Einbeziehung der zeitabhängigen Deckungsbeiträge äußerlich zunächst kaum gegenüber dem Optimum bei konstantem Deckungsbeitrag (vgl. Optimalitätsbedingung [2-22] in Abschnitt 2.2.2):

$$
w_{j}\left(t_{j}, N_{t_{j}-1}, P\left(t_{j}\right)\right)=\sum_{t=t_{j}}^{T} \sum_{\substack{k \in L_{t_{j}} \\ k \neq j}} \Delta W_{k, j}\left(t_{k}\right) .
$$

Weiterhin gilt für jeden Einzelbaum $j$ im Optimum die Gleichheit von laufendem Wertzuwachs $w_{j}\left(t_{j}, N_{t_{j}-1}, P\left(t_{j}\right)\right)$ und der Summe aller durch seinen Erhalt um ein weiteres Jahr an den von ihm beeinflussten Bäumen $k \in L_{t_{j}}$ entgehenden Wertveränderungen zum Zeitpunkt der jeweiligen Realisierung $\sum \sum \Delta W_{k, j}\left(t_{k}\right)$. Beide Gleichungsseiten setzen sich aufgrund der Zeitabhängigkeit der Deckungsbeiträge aber bedeutend anders zusammen.

Für den laufenden Einzelbaumwertzuwachs $w_{j}$ (linke Gleichungsseite in [2-26]) gilt:

$$
\begin{gathered}
w_{j}\left(t_{j}, N_{t_{j}-1}, P\left(t_{j}\right)\right)=v_{j}\left(t_{j}, N_{t_{j}-1}\right) \cdot P\left(t_{j}-1\right)+ \\
V_{j}\left(t_{j}-1, N_{t_{j}-2}\right) \cdot p\left(t_{j}-1, t_{j}\right)+ \\
v_{j}\left(t_{j}, N_{t_{j}-1}\right) \cdot p\left(t_{j}-1, t_{j}\right) .
\end{gathered}
$$

Dabei beschreibt der erste Term der rechten Gleichungsseite in [2-27] die mit dem AusgangsDeckungsbeitrag bewertete Veränderung des Baumvolumens im Zeitschritt $t_{j}-1$ nach $t_{j}$, der zweite Term den durch den marginal verlängerten Baumerhalt steigenden Wert des bereits zum Zeitpunkt $t_{j}-1$ vorhandenen Einzelbaumvolumens $V_{j}$ und der dritte Term den durch den marginal verlängerten Baumerhalt steigenden Wert der Baumvolumenveränderung. Durch Zusammenfassung von Term 1 und 3 kann Gleichung [2-27] schließlich verkürzt werden zu:

$$
w_{j}\left(t_{j}, N_{t_{j}-1}, P\left(t_{j}\right)\right)=v_{j}\left(t_{j}, N_{t_{j}-1}\right) \cdot P\left(t_{j}\right)+V_{j}\left(t_{j}-1, N_{t_{j}-2}\right) \cdot p\left(t_{j}-1, t_{j}\right) .
$$


Die rechte Gleichungsseite in [2-26] (Wuchsraumopportunität) wird zusätzlich zum bereits in Abschnitt 2.2.1 beschriebenen Dichteeffekt bei marginal verlängertem Erhalt von Baum $j$ auch von einer, indirekt vom Dichteeffekt abhängigen, Wertkomponente beeinflusst. Zur Quantifizierung dieser Wertkomponente kann das im Folgenden als Wertfaktor bezeichnete Verhältnis zwischen der Wertveränderung und der reiner Volumenveränderung an allen von Baum $j$ beeinflussten Bäumen $k \in L_{t_{j}}$ genutzt werden (zur Methode der Kalkulation siehe den Exkurs auf der folgenden Seite). Neben dem bereits beschriebenen Gini-Koeffizienten wird über die Wertfaktoren somit ebenfalls der Einfluss der zeitabhängig steigenden Deckungsbeiträge auf die optimale Bestandesbehandlung quantifiziert.

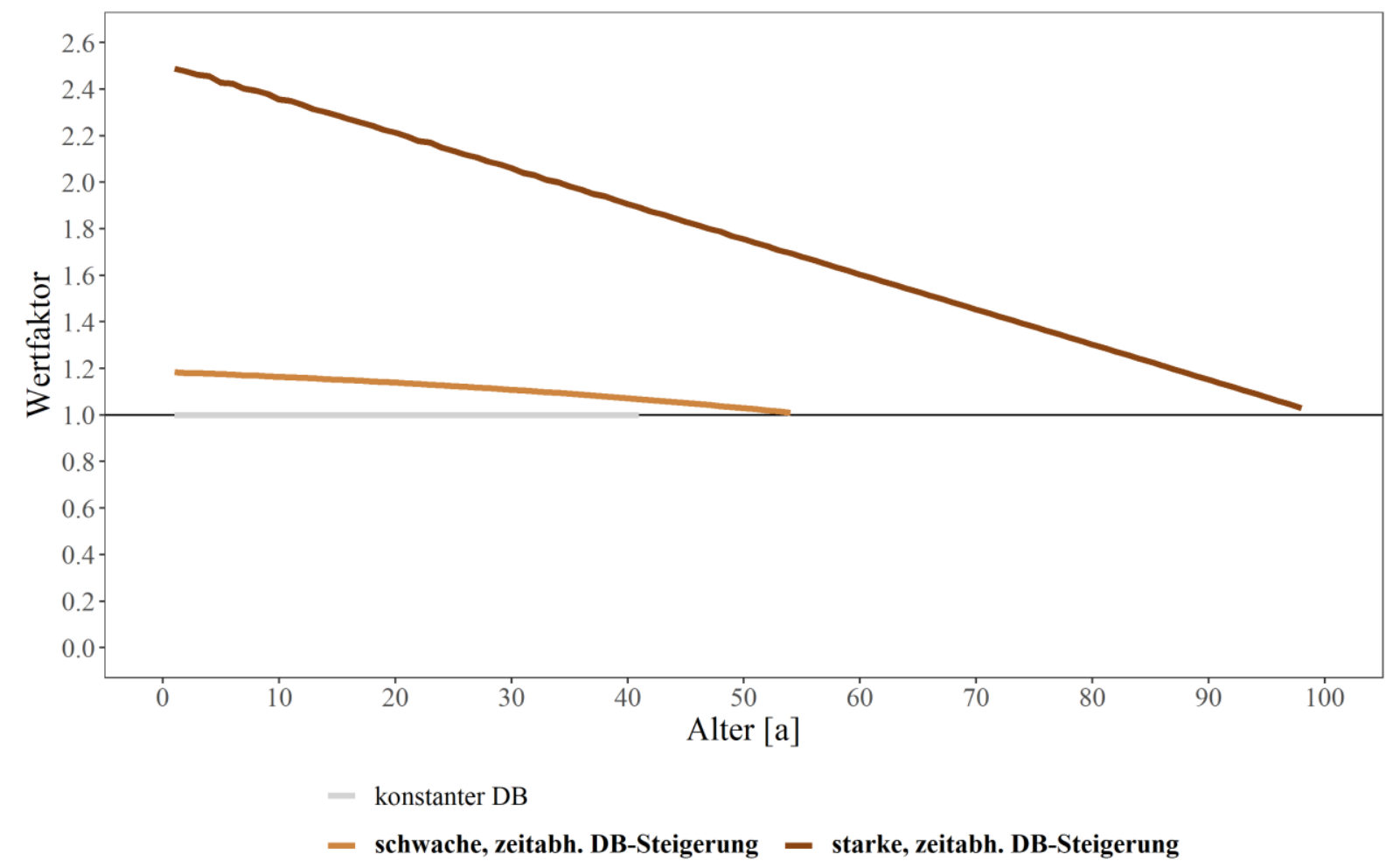

Abbildung 22: Wertfaktoren bei schwacher (hellbraun) bzw. starker (dunkelbraun), zeitabhängiger DB-Steigerung gemäß Zielfunktion [2-24] im Vergleich zum ökonomischen Optimum bei konstantem Deckungsbeitrag (grau).

Die Wertfaktoren sinken bei jährlich-konstant steigenden Deckungsbeiträgen mit zunehmendem Alter (Abbildung 22). Je später der Entscheidungszeitpunkt über den marginal verlängerten Erhalt von Baum $j$ liegt, desto geringer ist die über die Volumenveränderung hinausgehende Wertveränderung. Dies bedeutet, dass die Entwicklung der Deckungsbeiträge über der Zeit bei der Entscheidung über den Erhalt eines Baums in niedrigen Bestandesaltern eine größere Rolle spielt als in höheren Bestandesaltern. Denn je früher der Erhalt von Baum $j$ marginal verlängert wird, desto mehr Gesamtvolumenveränderung entsteht (Dichteeffekt) und desto mehr wird der 
volumengewichtete, mittlere Gesamtdeckungsbeitrag aller von Baum $j$ beeinflussten Bäume $k \in L_{t_{j}}$ aufgrund der zeitabhängigen Deckungsbeiträge verändert (Zeiteffekt).

\section{Exkurs: Berechnung der Wertfaktoren}

Für die Berechnung der Wertfaktoren bedarf es zunächst der Summe aller durch den Erhalt eines Baums $j$ um ein weiteres Jahr an den von ihm beeinflussten Bäumen $k \in L_{t_{j}}$ entgehenden Wertveränderungen $\Delta W_{k, j}$ zum Zeitpunkt der jeweiligen Realisierung $t_{k}-\mathrm{d}$. h. die Wuchsraumopportunität des Baumerhalts für ein weiteres Jahr (rechte Seite der Gleichungen [2-22] bzw. [2-26]) muss quantifiziert werden. Hierfür werden zwei alternative Ernteentscheidungen miteinander verglichen: Die Barwerte aller zukünftigen Vor- und Endnutzungen der optimalen Bestandesbehandlung werden für den Zeitpunkt $t_{j}$ summiert und mit der Summe der Barwerte aller zukünftigen Vor- und Endnutzungen bei (marginal) veränderter Vornutzung zu den Zeitpunkten $t_{j}$ und $t_{j}+1$ (d. h. bei Aufschub der Ernte von Baum $j$ um ein Jahr) ceteris paribus (c. p.) verglichen.

Wie bereits beschrieben, unterscheiden sich diese Opportunitätskosten des Erhalts von Baum $j$ für ein weiteres Jahr zwischen den Varianten mit konstantem (ausschließliche Volumenveränderung an allen beeinflussten Bäumen) und variablem (zusätzliche Wertveränderung an allen beeinflussten Bäumen) Deckungsbeitrag. Das Verhältnis aus der Wuchsraumopportunität bei zeit- oder dimensionsabhängigen (siehe Abschnitt 2.2.4) Deckungsbeiträgen und der Wuchsraumopportunität bei konstanten Deckungsbeiträgen bildet den Wertfaktor.

Der optimale Endnutzungszeitpunkt $T^{*}$ ist auch bei einem zeitabhängig steigenden Deckungsbeitrag $P(t)$ erreicht, wenn der laufende Bestandeswertzuwachs $w_{B}\left(T, N_{T-1}, P(t)\right)$ dem durchschnittlichen Bestandeswertzuwachs $\overline{w_{B}}\left(T, \boldsymbol{N}_{T-1}, P(t)\right)$ entspricht (Möhring 2009, Gleichung (6); Halbritter und Deegen 2015, Gleichung (10) mit $r \searrow 0)^{24}$; es gilt im Optimum:

$$
\begin{aligned}
w_{B}\left(T, N_{T-1}, P(t)\right) & =\overline{w_{B}}\left(T, \boldsymbol{N}_{T-1}, P(t)\right), \\
v_{B}\left(T, N_{T-1}\right) \cdot P(T)+p(T-1, T) \cdot V_{B}\left(T-1, \boldsymbol{N}_{T-2}\right) & =a_{P(t)}\left(T, \boldsymbol{N}_{T-1}, P(t)\right)
\end{aligned}
$$

\footnotetext{
${ }^{24}$ Der von Halbritter und Deegen (2015) in Gleichung (10) genutzte Term $r L E V$ steht für die BruttoBodenrente. Mit einem gegen Null strebenden Kalkulationszinssatz $r \searrow 0$ und bei Vernachlässigung von Bestandesbegründungskosten entspricht dieser Term dem durchschnittlichen Überschuss der forstlichen Produktion $\overline{w_{B}}\left(T, \boldsymbol{N}_{T-1}\right)$ im Sinne eines jährlichen Deckungsbeitrages (vgl. Regeln von de L’Hospital; Forster 2013, S. 186 ff.; vgl. auch Möhring 1994, S. 74 f. und Pertz 1983, S. 149).
} 
Analog zu Abschnitt 2.2.2 sinkt ab diesem Zeitpunkt der laufende, zeitliche Grenznutzen des Bestandeserhalts, d. h. der laufende Wertzuwachs $w_{B}\left(T, N_{T-1}, P(t)\right)$ unter den durchschnittlichen Überschuss des Folgebestandes $a_{P(t)}\left(T, \boldsymbol{N}_{T-1}, P(t)\right)$.

Der laufende Bestandeswertzuwachs $w_{B}\left(T, N_{T-1}, P(t)\right)$ setzt sich bei zeitabhängigen Deckungsbeiträgen allerdings analog zur Vornutzungs-Optimalitätsbedingung, aus dem bewerteten Volumenzuwachs $v_{B}\left(T, N_{T-1}\right) \cdot P(T)$ und dem Produkt aus der Deckungsbeitragssteigerung und dem bereits vorhandenen Bestandesvolumen $p(T-1, T) \cdot V_{B}\left(T-1, \boldsymbol{N}_{T-2}\right)$ zusammen (vgl. Halbritter und Deegen 2015, Gleichung (10)).

\subsubsection{Dimensionsabhängige Deckungsbeiträge}

In Anlehnung an forstpraktische Gegebenheiten werden die geernteten Vor- und Endnutzungsvolumina der forstlichen Produktion im Folgenden mit dimensionsabhängigen Deckungsbeiträgen $P\left(V_{E}\right)$ bewertet, wobei das mittlere Einzelbaumvolumen $V_{E}$ (Stückvolumen) als Dimensionsmaß genutzt wird. Damit wird der Tatsache Rechnung getragen, dass in der mitteleuropäischen Forstwirtschaft stärkere Baumdimensionen einerseits aufgrund veränderter Sortimentszusammensetzung und besserer technischer Verwertbarkeit i. d. R. höhere Holzerlöse erzielen (vgl. z. B. Bodelschwingh 2018, S. 59 ff.) und andererseits aufgrund von vermindertem Aufarbeitungsaufwand je Volumeneinheit (vgl. Stück-Volumen-Gesetz; Hilf 1928; 1941) geringere Holzerntekosten bedingen.

Somit gilt es, den nun auch vom mittleren Einzelbaumvolumen abhängigen durchschnittlichen

Überschuss der forstlichen Produktion $a_{P\left(V_{E}\right)}$ unter Veränderung des Vornutzungsvektors $\boldsymbol{\sigma}_{T-1}$ sowie des Endnutzungszeitpunktes $T$ zu maximieren:

$$
\begin{aligned}
& \max _{T, \boldsymbol{\sigma}_{T-1}} a_{P\left(V_{E}\right)}\left(T, \boldsymbol{\sigma}_{T-1}, N_{0}, P\left(V_{E}\right)\right) \\
& \quad=\max _{T, \boldsymbol{\sigma}_{T-1}}\left(\sum_{t=1}^{T-1} \lambda_{t}\left(\sigma_{t}, t, \boldsymbol{\sigma}_{t-1}, N_{0}\right) \cdot P\left(V_{E}\right)+V_{B}\left(T, \boldsymbol{\sigma}_{T-1}, N_{0}\right) \cdot P\left(V_{E}\right)\right) \cdot \frac{1}{T}, \\
& 0 \leq \boldsymbol{\sigma}_{T-1}<1, \\
& 0 \mathrm{a}<T \leq 100 \mathrm{a}, \\
& P \in \mathbb{R},
\end{aligned}
$$


wobei das mittlere Einzelbaumvolumen eine Funktion des Alters $t$, der Ausgangsstammzahl $N_{0}$ und des Vornutzungsvektors $\boldsymbol{\sigma}_{t-1}$ darstellt ${ }^{25}$ :

$$
V_{E}=f\left(t, \sigma_{t-1}, N_{0}\right)
$$

$$
V_{E} \in \mathbb{R}^{\geq 0}
$$

Damit erhöht sich die Komplexität des Produktionsmodells aufgrund hinzukommender intertemporaler Wechselwirkungen elementar. Wichtig ist dabei die Abgrenzung zu den bereits in den vorhergehenden Modellvarianten (Abschnitte 2.2.1 bis 2.2.3) bestehenden Wechselwirkungen. Stets sind auch bei diesen Modellvarianten die absoluten Werte der Vornutzungen sowie der Endnutzung vom geernteten Volumen und damit indirekt auch von der Entstehungsgeschichte des Bestandes abhängig (vgl. Fußnote 18 auf Seite 28) - intertemporale Wechselwirkungen bestehen also auch dort. Wertbestimmend ist allerdings jeweils nur das absolut vorhandene Bestandesvolumen zum entsprechenden Zeitpunkt, nicht aber durch welche zeitliche Abfolge von Volumenzuwachs und Vornutzung dieses Bestandesvolumen zustande kam. Im Unterschied dazu ist diese zeitliche Abfolge der Entstehungsgeschichte des Bestandes für das mittlere Einzelbaumvolumen zu einem bestimmten Zeitpunkt entscheidend. Eine starke Vornutzung mit dadurch bedingter geringerer Bestandesdichte zu einem frühen Zeitpunkt der forstlichen Produktion beeinflusst die mittlere Einzelbaumdimension zum Zeitpunkt $t$ anders, als wenn die gleiche Bestandesdichte erst durch einen Vornutzungseingriff zum Zeitpunkt $t-1$ erzielt wird.

Für das Variantenstudium werden die dimensionsabhängigen Deckungsbeiträge über dem mittleren Einzelbaumvolumen $V_{E}$ (Stückvolumen) anhand einer nichtlinearen Funktion

$$
P\left(\mathrm{~V}_{E}\right)=\gamma_{1} \cdot\left(1-e^{\gamma_{2}-\mathrm{V}_{E}}\right)+\gamma_{3},
$$

mit den drei Parametrisierungen für eine schwache, mittlere bzw. starke DB-Steigerung

$$
\begin{aligned}
& \gamma_{1, \text { schwach }}=57,826 € \mathrm{Fm}^{-1}, \gamma_{2, \text { schwach }}=-1, \\
& \gamma_{1, \text { mittel }}=64,361 € \mathrm{Fm}^{-1}, \gamma_{2, \text { mittel }}=-0,5, \\
& \gamma_{1, \text { stark }}=79,099 € \mathrm{Fm}^{-1}, \gamma_{2, \text { stark }}=0
\end{aligned}
$$

sowie $\gamma_{3}=-20 € \mathrm{Fm}^{-1}$ definiert.

\footnotetext{
${ }^{25}$ Auf die Darstellung der funktionalen Abhängigkeiten von $V_{E}$ wird im Folgenden aus Gründen der Darstellungsvereinfachung überwiegend verzichtet.
} 
Je stärker die DB-Steigerung, desto höher sind die DB-Unterschiede zwischen geringen und hohen Einzelbaumdimensionen. Dabei drehen sich die drei DB-Varianten um einen einheitlichen Deckungsbeitrag von $30 € \mathrm{Fm}^{-1}$ (vgl. Abschnitt 2.2.2) bei einem Einzelbaumvolumen von 1,0 Fm (Abbildung 23).

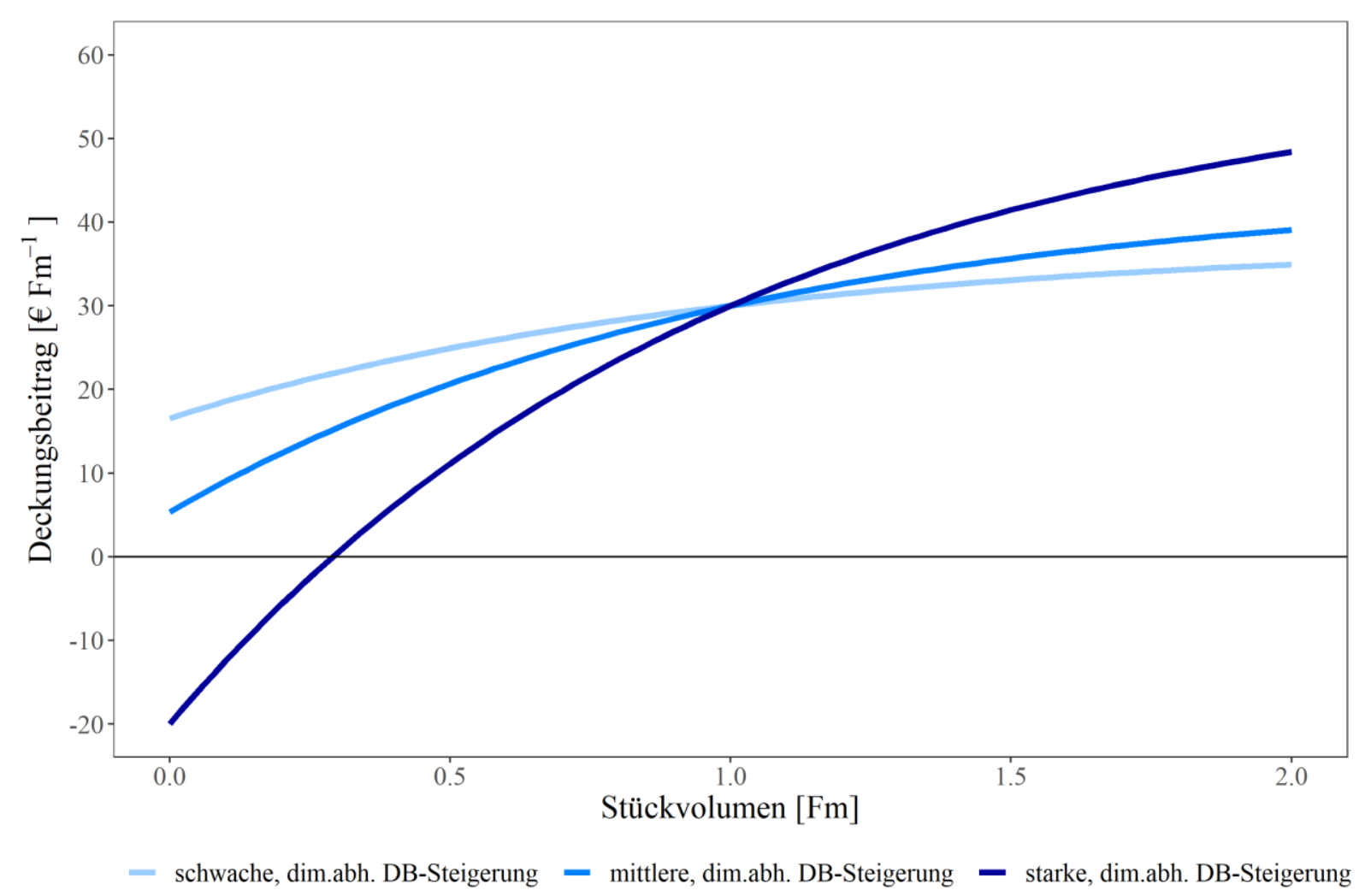

Abbildung 23: Dimensionsabhängiger Deckungsbeitrag (erntekostenfreier Holzerlös) je Festmeter bei schwacher (hellblau), mittlerer (blau) und starker (dunkelblau) DB-Steigerung gemäß Deckungsbeitragsfunktion [2-32].

Die sich ergebenden maximalen Zielgrößen der durchschnittlichen Überschüsse liegen mit 361,347 und $375 € \mathrm{ha}^{-1} \mathrm{a}^{-1}$ um 13 bis $19 \%$ unterhalb des maximalen Überschusses bei konstantem Deckungsbeitrag (431€ ha ${ }^{-1} \mathrm{a}^{-1}$; Abschnitt 2.2.2) ${ }^{26}$.

Die optimalen Stammzahlentwicklungen $\boldsymbol{N}_{P\left(V_{E}\right), \text { schwach }}^{*}, \boldsymbol{N}_{P\left(V_{E}\right), \text { mittel }}^{*}$ und $\boldsymbol{N}_{P\left(V_{E}\right) \text {,stark liegen }}^{*}$ zunächst deutlich unterhalb der natural-optimalen Stammzahlen (Abbildung 24). Variantenspezifisch starke Vornutzungseingriffe reduzieren die Ausgangsstammzahl in den ersten drei Zeitschritten auf $1.686 \mathrm{ha}^{-1}$ (schwache DB-Steigerung), $900 \mathrm{ha}^{-1}$ (mittlere DB-Steigerung) bzw. $618 \mathrm{ha}^{-1}$ (starke DB-Steigerung). Während bei schwacher DB-Steigerung auch anschließend fortlaufend Vornutzungen stattfinden, erfolgen bei mittlerer und starker DB-Steigerung weitere

\footnotetext{
${ }^{26}$ Stückvolumina unterhalb von 1,0 Fm beeinflussen die durchschnittlichen Überschüsse der forstlichen Produktion folglich mehr als die Stückvolumina oberhalb von 1,0 Fm.
} 
Eingriffe erst ab einem Alter von 35 bzw. 54 Jahren. Ab einem Alter von zirka 54 Jahren entsprechen die optimalen Stammzahlentwicklungen aller DB-Varianten annähernd dem naturalen Optimum - ab diesem Alter wird folglich der modell-maximale laufende Bestandesvolumenzuwachs generiert.

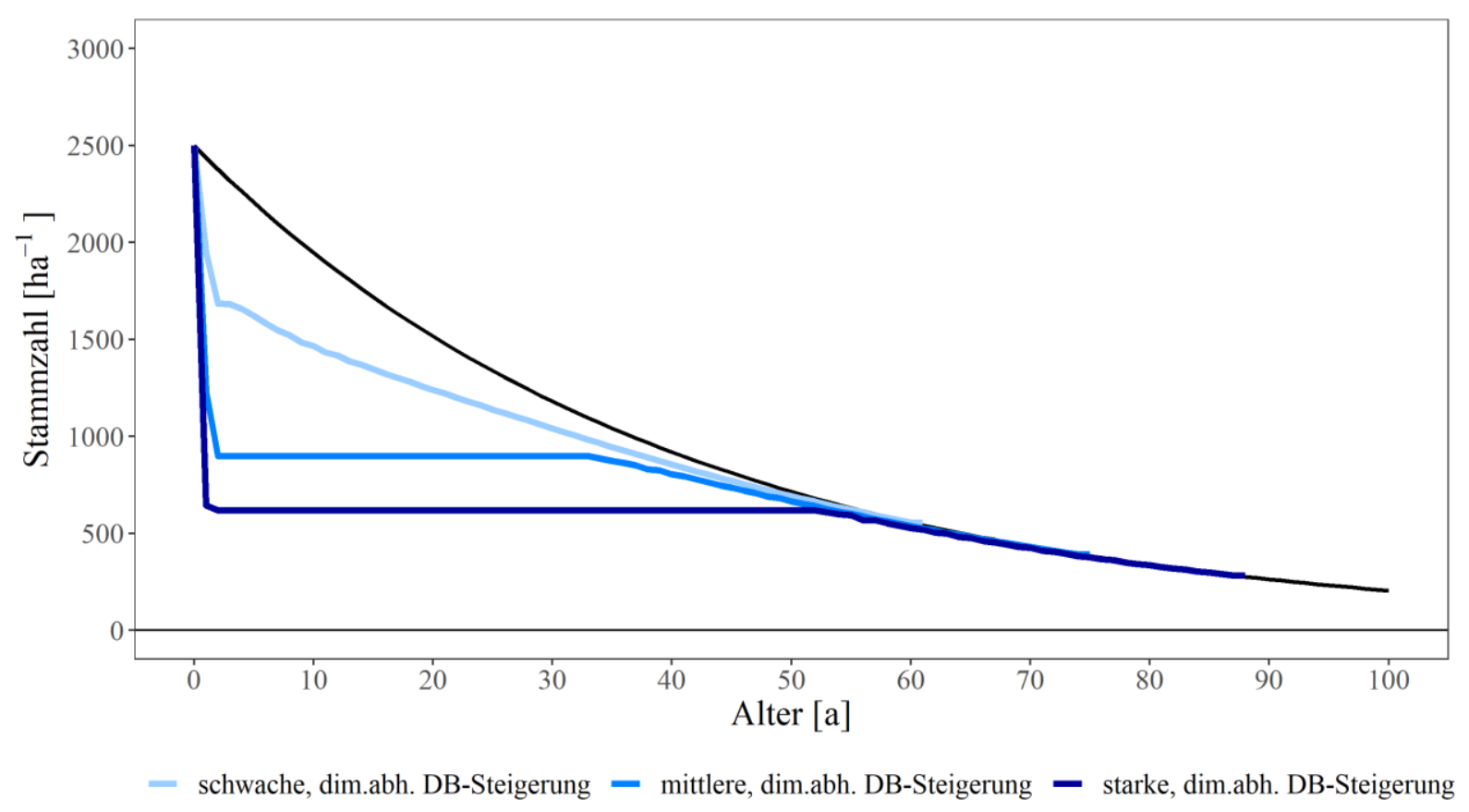

Abbildung 24: Altersabhängige Stammzahlentwicklungen bei schwacher (hellblau), mittlerer (blau) und starker (dunkelblau), dimensionsabhängiger DB-Steigerung gemäß Zielfunktion [2-30] im Vergleich zum naturalen Optimum ohne Folgebestand (schwarz).

Es wird durch die frühen Stammzahlreduktionen umso mehr auf Bestandesvolumenzuwachs verzichtet, je stärker die dimensionsabhängige DB-Steigerung ausfällt (Abbildung 25). Maximal wird ein laufender Bestandesvolumenzuwachs von 16,9 $\mathrm{Fm} \mathrm{ha}^{-1} \mathrm{a}^{-1}$ (schwache DB-Steigerung im Alter $25 \mathrm{a}$ ), 15,8 $\mathrm{Fm} \mathrm{ha}^{-1} \mathrm{a}^{-1}$ (mittlere DB-Steigerung im Alter $32 \mathrm{a}$ ) bzw. 13,3 Fm ha ${ }^{-1} \mathrm{a}^{-1}$ (starke DB-Steigerung im Alter 37 a) erzielt.

Es ergeben sich mit den optimalen Endnutzungszeitpunkten $T_{P\left(V_{E}\right) \text {,schw. }}^{*}=61 \mathrm{a}$, $T_{P\left(V_{E}\right), \text { mittel }}^{*}=75 \mathrm{a}$ bzw. $T_{P\left(V_{E}\right) \text {,stark }}^{*}=88 \mathrm{a}$ durchschnittliche Bestandesvolumenzuwächse von 13,4 Fm ha $\mathrm{Fa}^{-1}$, 11,6 Fm ha $\mathrm{Fm}^{-1}$ bzw. 9,6 Fm ha-1 $\mathrm{a}^{-1}(-7 \%,-19 \%$ bzw. - 33 \% ggü. dem Optimum bei konstantem Deckungsbeitrag). 


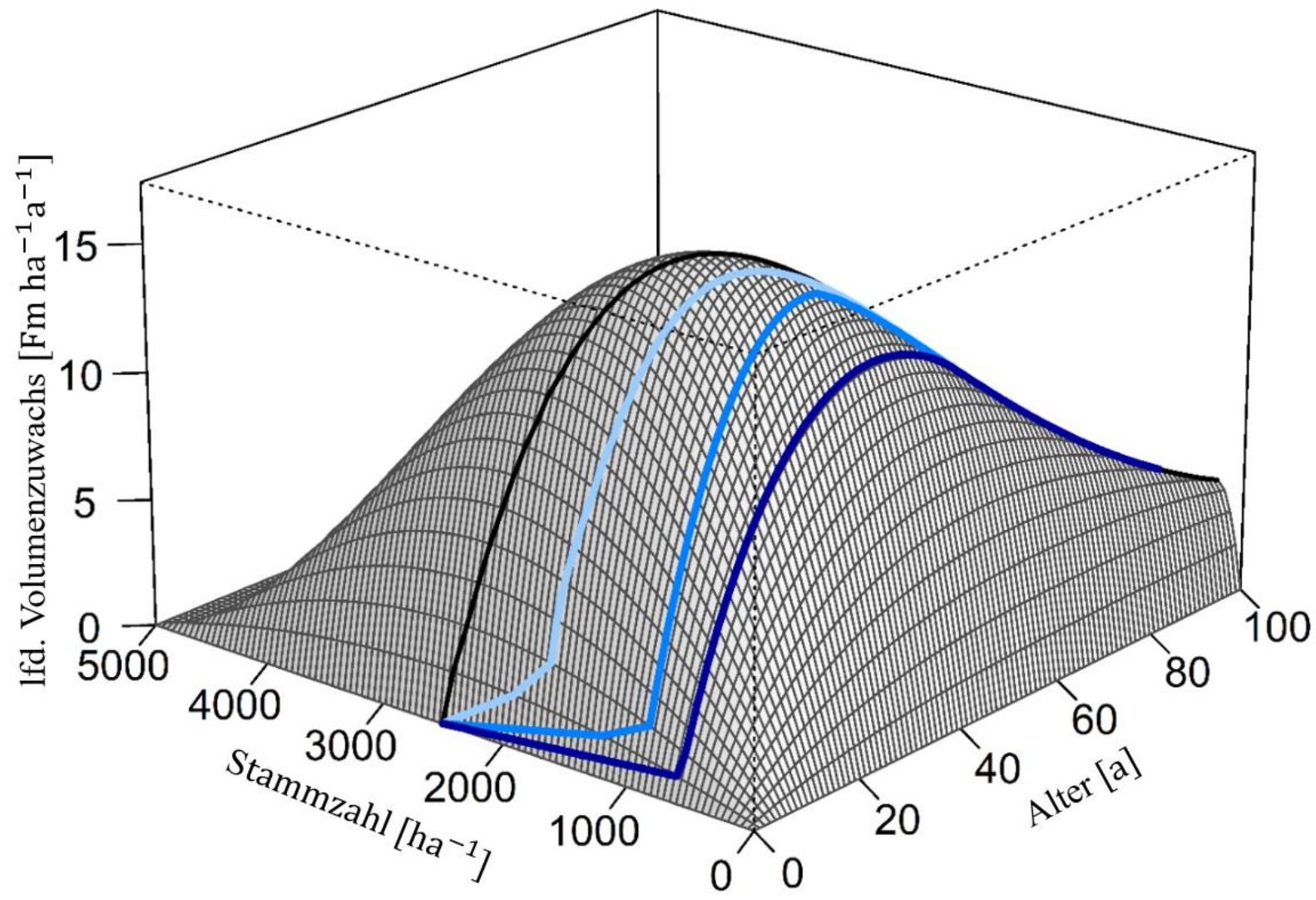

schw., dim.abh. DB-Steig.

mittl., dim.abh. DB-Steig.

st., dim.abh. DB-Steig.

Abbildung 25: Alters- und stammzahlabhängiger laufender Bestandesvolumenzuwachs bei schwacher (hellblau), mittlerer (blau) und starker (dunkelblau), dimensionsabhängiger DB-Steigerung gemäß Zielfunktion [2-30] im Vergleich zum maximalen Volumenzuwachs (schwarz).

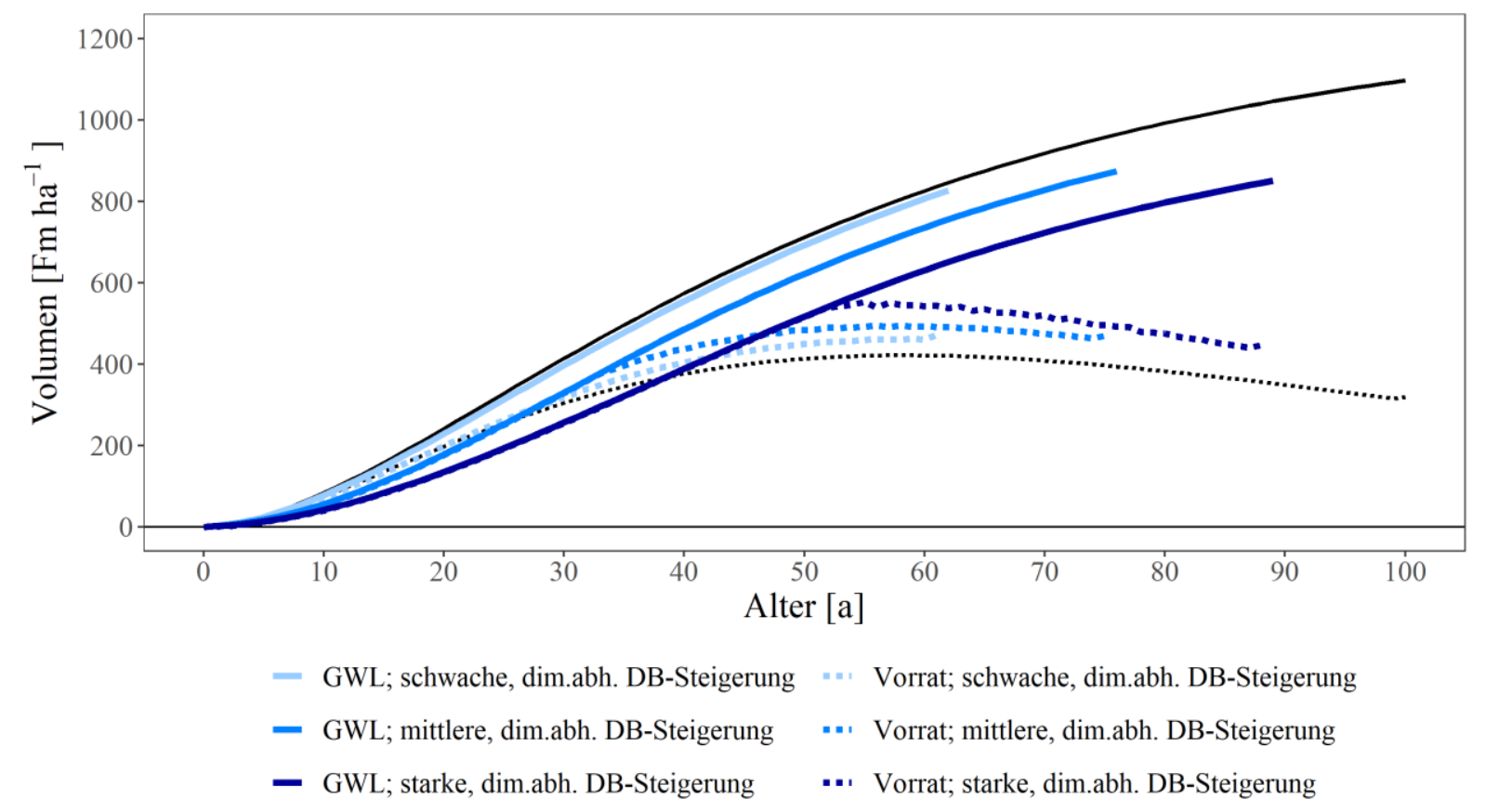

Abbildung 26: Altersabhängige Gesamtwuchsleistung und Vorratsentwicklung bei schwacher (hellblau), mittlerer (blau) und starker (dunkelblau), dimensionsabhängiger DB-Steigerung gemäß Zielfunktion [2-30] im Vergleich zum naturalen Optimum ohne Folgebestand (schwarz). 
Es resultieren höhere Vorräte bei gleichzeitig gegenüber der natural-optimalen Referenz verminderten Gesamtwuchsleistungen (Abbildung 26). In Verbindung mit den reduzierten Stammzahlen zu Beginn der forstlichen Produktion (Abbildung 24) ergeben sich teils deutlich erhöhte Einzelbaumvolumina während der gesamten forstlichen Produktion (Abbildung 27).

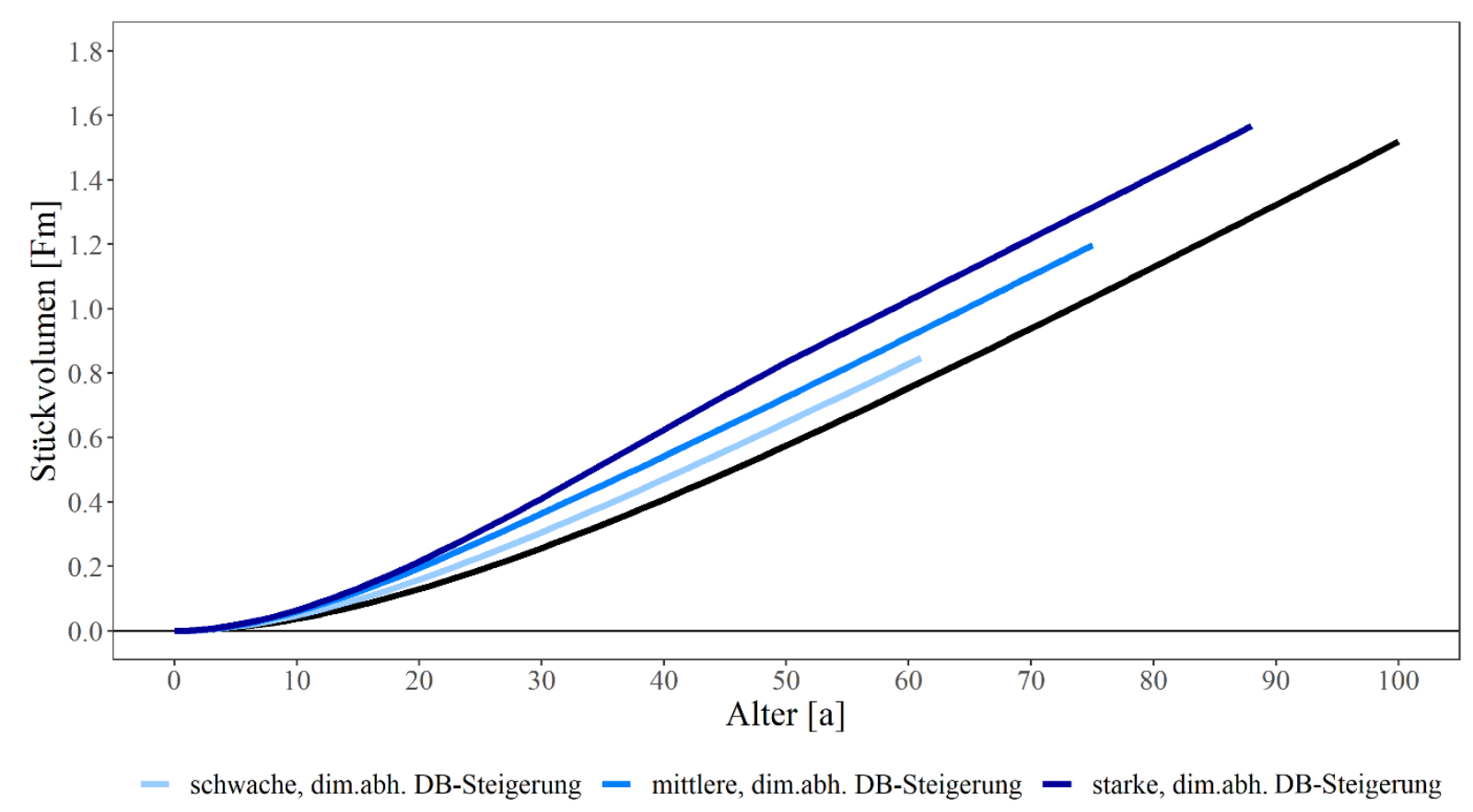

Abbildung 27: Altersabhängige Einzelbaumvolumina (Stückvolumina) bei schwacher (hellblau), mittlerer (blau) und starker (dunkelblau), dimensionsabhängiger DB-Steigerung gemäß Zielfunktion [2-30] im Vergleich zum naturalen Optimum ohne Folgebestand (schwarz).

Gegenüber der Bewertung mit einem konstanten Deckungsbeitrag erfolgt, wie auch schon beim zeitabhängigen Deckungsbeitrag (vgl. Abbildung 21 in Abschnitt 2.2.3), eine im Vergleich zur Gesamtwuchsleistung verzögerte Gesamtwertleistung (Abbildung 28). Die Disparität steigt dabei mit Zunahme der dimensionsabhängigen DB-Steigerung an. Einer zu $50 \%$ realisierten Gesamtwuchsleistung stehen bei schwacher DB-Steigerung $41 \%$, bei mittlerer DB-Steigerung $34 \%$ und bei starker DB-Steigerung lediglich $25 \%$ der Gesamtwertleistung gegenüber. Für die Disparität ist dabei nicht das absolute Niveau der Deckungsbeiträge, sondern das Spannungsverhältnis zwischen den Deckungsbeiträgen geringer und hoher Einzelbaumdimension ausschlaggebend. Aufgrund der nichtlinearen DB-Funktion kann dieses monetäre Spannungsverhältnis nicht anhand zweier Fixpunkte quantifiziert werden - vielmehr ist der dimensionsabhängige Verlauf als Ganzes und somit jedes einzelne Wertepaar der DB-Funktion entscheidend. Der Gini-Koeffizient greift dieses ganzheitliche Spannungsverhältnis und kann somit er- 
neut als Quantifizierungsmaß eingesetzt werden (siehe Abschnitt 2.2.3). Bei dimensionsabhängig steigenden Deckungsbeiträgen liegen die Gini-Koeffizient bei 0,12 (schwache DB-Steigerung), 0,22 (mittlere DB-Steigerung) und 0,35 (starke DB-Steigerung) ${ }^{27}$.

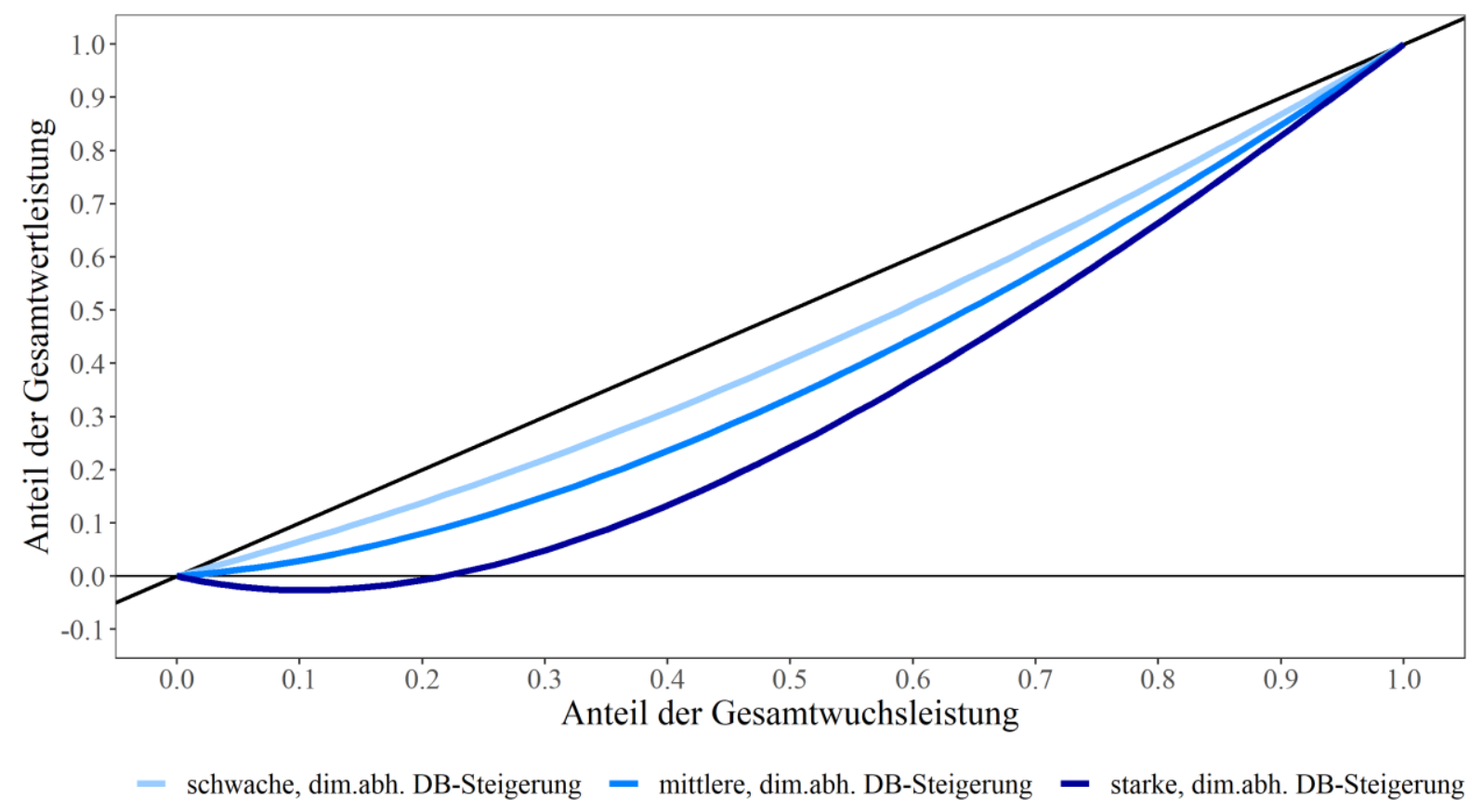

Abbildung 28: Disparitätskurven der relativen Gesamtwuchs- und Gesamtwertleistung bei schwacher (hellblau), mittlerer (blau) und starker (dunkelblau), dimensionsabhängiger DB-Steigerung gemäß Zielfunktion [2-30] im Vergleich zur Deckungsgleichheit von relativer Volumen- und Wertleistung bei Bewertung mit konstantem Deckungsbeitrag (schwarz).

Die Optimalitätsbedingung der zeitlich-räumlichen Grenznutzenanalyse am Einzelbaum bleibt im äußeren Erscheinungsbild ggü. Abschnitt 2.2.3 annähernd unverändert:

$$
w_{j}\left(t_{j}, N_{t_{j}-1}, P\left(V_{j}\right)\right)=\sum_{t=t_{j}+1}^{T} \sum_{\substack{k \in L_{t_{j}} \\ k \neq j}} \Delta W_{k, j}\left(t_{k}\right),
$$

Weiterhin gilt, dass sich der Erhalt des Einzelbaums $j$ nur so lange lohnt, wie sein laufender Wertzuwachs $w_{j}\left(t_{j}, N_{t_{j}-1}, P\left(V_{j}\right)\right)$, als Grenznutzen des Baumerhalts, die Summe aller durch seinen Erhalt um ein weiteres Jahr an den von ihm beeinflussten Bäumen $k \in L_{t_{j}}$ entgehenden

\footnotetext{
${ }^{27}$ Beim Vergleich der Gini-Koeffizienten ist die Besonderheit negativer Wertleistungen bei der Variante der starken DB-Steigerung zu beachten. Hier ermöglicht die Verwendung des normierten Gini-Koeffizienten nach Chen et al. (1982) die Vergleichbarkeit zwischen Gini-Koeffizienten nicht-negativer und (in Teilen) negativer Disparitäten.
} 
Wertveränderungen zum Zeitpunkt der jeweiligen Realisierung $\sum \sum \Delta W_{k, j}\left(t_{k}\right)$, als Grenzkosten des Baumerhalts bzw. Wuchsraumopportunität, übersteigt. Beide Gleichungsseiten setzen sich aufgrund der Dimensionabhängigkeit der Deckungsbeiträge aber erneut anders zusammen. Für den laufenden Einzelbaumwertzuwachs $w_{j}$ (linke Gleichungsseite in [2-33]) gilt:

$$
\begin{array}{r}
w_{j}\left(t_{j}, N_{t_{j}-1}, P\left(V_{j}\right)\right)=v_{j}\left(t_{j}, N_{t-1}\right) \cdot P\left(V_{j}\left(t_{j}-1, N_{t_{j}-2}\right)\right)+ \\
V_{j}\left(t_{j}-1, N_{t_{j}-2}\right) \cdot p\left(v_{j}\left(t_{j}, N_{t_{j}-1}\right)\right)+ \\
v_{j}\left(t_{j}, N_{t_{j}-1}\right) \cdot p\left(v_{j}\left(t_{j}, N_{t_{j}-1}\right)\right),
\end{array}
$$

wobei analog zu Gleichung [2-27] die Terme 1 und 3 der rechten Gleichungsseite zusammengefasst werden können und sich Gleichung [2-34] somit verkürzt zu:

$$
\begin{array}{r}
w_{j}\left(t_{j}, N_{t_{j}-1}, P\left(V_{j}\right)\right)=v_{j}\left(t_{j}, N_{t_{j}-1}\right) \cdot P\left(V_{j}\left(t_{j}, N_{t_{j}-1}\right)\right)+ \\
V_{j}\left(t_{j}-1, N_{t_{j}-2}\right) \cdot p\left(v_{j}\left(t_{j}, N_{t_{j}-1}\right)\right) .
\end{array}
$$

Der laufende Einzelbaumwertzuwachs setzt sich demnach zusammen aus der bewerteten Volumenveränderung (Term 1 der rechten Gleichungsseite in [2-35]) und der Wertveränderung des bereits zum Zeitpunkt $t_{j}-1$ vorhandenen Einzelbaumvolumens $V_{j}$ (Term 2 der rechten Gleichungsseite in [2-35]).

Die Wuchsraumopportunität $\sum \sum \Delta W_{k, j}\left(t_{k}\right)$ (rechte Gleichungsseite in [2-33]) wird aufgrund der dimensionsabhängigen Deckungsbeiträge nun zusätzlich zum Dichteeffekt vom sogenannten Dimensionseffekt beeinflusst. Dieser Dimensionseffekt beschreibt den Einfluss der durch den marginal verlängerten Erhalt von Baum $j$ verringerten Einzelbaumdimensionen aller beeinflussten Bäume $k \in L_{t_{j}}$ zum Zeitpunkt ihrer jeweiligen Realisierung und der damit verbundenen niedrigeren Deckungsbeiträge je Volumeneinheit auf die entgehenden Wertveränderungen $\Delta W_{k, j}\left(t_{k}\right)$. Denn wird Baum $j$ bereits zum Zeitpunkt $t_{j}-1$ geerntet, wird bei dimensionsabhängigen Deckungsbeiträgen nicht nur der Volumenmehrzuwachs der verbleibenden Bäume $k \in L_{t_{j}}$ (siehe Dichteeffekt) höher bewertet - vielmehr führen die dann höheren Deckungsbeiträge je Volumeneinheit auch zur höheren Bewertung des unabhängig von der marginal veränderten Vornutzung bereits vorhandenen Volumens aller Bäume $k \in L_{t_{j}}$. 
Die Wertveränderung $\Delta W_{k, j}\left(t_{k}\right)$ setzt sich folglich aus der bewerteten, dichteeffektbedingten Volumenveränderung (Term 1 der rechten Gleichungsseite in [2-36]), der dimensionseffektbedingt höheren Bewertung des bereits vorhandenen Volumens (Term 2 der rechten Gleichungsseite in [2-36]) sowie dem Produkt aus dichteeffektbedingter Volumenveränderung und dimensionseffektbedingt höheren Deckungsbeiträgen (Term 3 der rechten Gleichungsseite in [2-36]) zusammen - es gilt:

$$
\begin{array}{r}
\Delta W_{k, j}\left(t_{k}\right)=\Delta V_{k, j}\left(t_{k}\right) \cdot P\left(V_{E}\left(t_{k}, \sigma_{t_{k}-1}, N_{0}\right)\right)+ \\
V_{k}\left(t_{k}\right) \cdot p_{j}\left(\Delta V_{k, j}\left(t_{k}\right)\right)+ \\
\Delta V_{k, j}\left(t_{k}\right) \cdot p_{j}\left(\Delta V_{k, j}\left(t_{k}\right)\right)
\end{array}
$$

oder bei Zusammenführung der Terme 1 und 3 der rechten Gleichungsseite in [2-36]:

$$
\begin{array}{r}
\Delta W_{k, j}\left(t_{k}\right)=\Delta V_{k, j}\left(t_{k}\right) \cdot P_{j}\left(V_{E}\left(t_{k}, \boldsymbol{\sigma}_{t_{k}-1}, N_{0}\right)\right)+ \\
V_{k}\left(t_{k}\right) \cdot p_{j}\left(\Delta V_{k, j}\left(t_{k}\right)\right) .
\end{array}
$$

$P_{j}\left(V_{E}\left(t_{k}, \sigma_{t_{k}-1}, N_{0}\right)\right)$ steht dabei für den Deckungsbeitrag, der c. p. zum Zeitpunkt $t_{k}$ durch die marginal verzögerte Ernte von Baum $j$ entsteht.

Die Kombination aus Dichte- und Dimensionseffekt zusammengefasst, generiert der marginal verlängerte Erhalt des Baumes $j$ c. p. einen Einzelbaumwertzuwachs (Grenznutzen), dem entgehende Einzelbaumvolumenzuwächse, daraus resultierende höhere Einzelbaumvolumina und damit verbundenen höhere Deckungsbeiträge je Volumeneinheit an allen anderen, durch die Ernte des Baumes $j$ beeinflussten, Bäumen entgegen stehen (Grenzkosten).

Die resultierenden Wertfaktoren (siehe Exkurs zur Berechnung der Wertfaktoren in Abschnitt 2.2.3) sinken über dem Alter und sind umso höher, je stärker die DB-Steigerung ist (Abbildung 29). Mit zunehmender DB-Steigerung steigen die Grenzkosten eines marginal verlängerten Baumerhalts in jungen Bestandesaltern, denn die Konzentration des Volumenzuwachses auf weniger Bäume (geringere Stammzahl) und somit resultierende höhere Einzelbaumdimensionen werden umso vorteilhafter. Je später solch ein Eingriff erfolgt, desto weniger kommt der Dimensionseffekt zum Tragen und die Wertfaktoren nähern sich dem Wert 1,0 (ausschließlicher Dichteeffekt) an. 


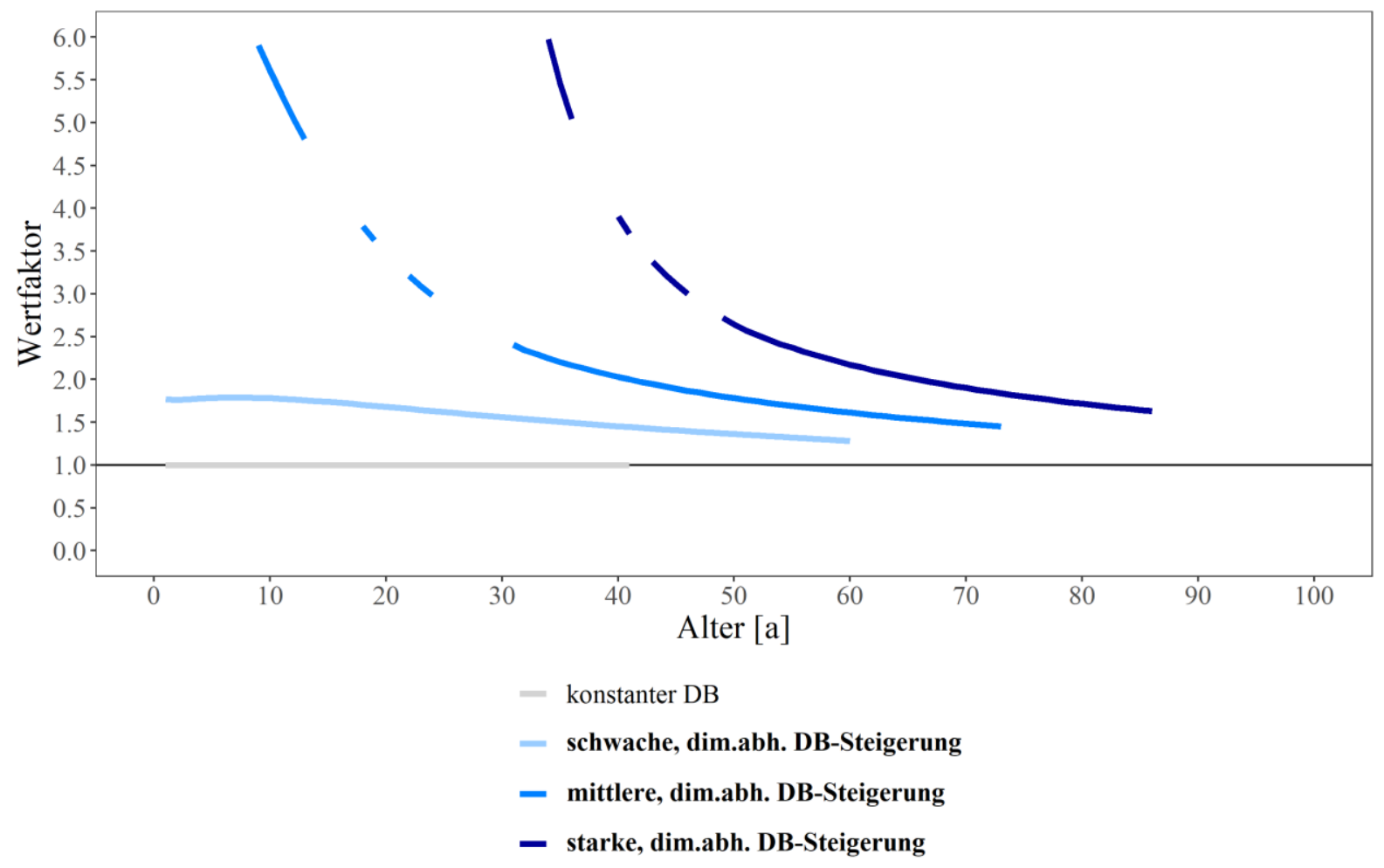

Abbildung 29: Wertfaktoren bei schwacher (hellblau), mittlerer (blau) und starker (dunkelblau), dimensionsabhängiger DB-Steigerung gemäß Zielfunktion [2-30] im Vergleich zum ökonomischen Optimum bei konstantem Deckungsbeitrag (grau). Aufgrund zeitweise unterbleibender Vornutzungen bei mittlerer und starker DB-Steigerung können die Wertfaktoren im Modell nicht für jedes Alter bestimmt werden - eine gedankliche Ergänzung der Linienverläufe ist inhaltlich zulässig.

Für die optimale Endnutzungsentscheidung gilt durch logische Erweiterung der Optimalitätsbedingung [2-29] weiterhin die Gleichheit von laufendem und durchschnittlichem Bestandeswertzuwachs bzw. durchschnittlichem Überschuss der forstlichen Produktion:

$$
\begin{aligned}
w_{B}\left(T, N_{T-1}, P\left(V_{E}\right)\right) & =\overline{w_{B}}\left(T, \boldsymbol{N}_{T-1}, P\left(V_{E}\right)\right) \\
& =a_{P\left(V_{E}\right)}\left(T, \boldsymbol{N}_{T-1}, P\left(V_{E}\right)\right),
\end{aligned}
$$

wobei sich der laufende Wertzuwachs in Anlehnung an Gleichung [2-35] ergibt als:

$$
\begin{aligned}
w_{B}\left(T, N_{T-1}, P\left(V_{E}\right)\right)=v_{B}\left(T, N_{T-1}\right) \cdot P\left(V_{E}\left(T, \boldsymbol{N}_{T-1}\right)\right)+ \\
V_{B}\left(T-1, \boldsymbol{N}_{T-2}\right) \cdot p\left(v_{E}\left(T, \boldsymbol{N}_{T-1}\right)\right) .
\end{aligned}
$$

An dieser Stelle sei (für alle Modellvarianten analog geltend) darauf hingewiesen, dass es trotz der getrennten Formulierung der Optimalitätsbedingungen von Vor- und Endnutzung zu Wechselwirkungen zwischen diesen kommt - im globalen Optimum müssen beide Optimalitätsbedingungen der forstlichen Produktion simultan erfüllt sein. Für die Vornutzungsentscheidung gilt es, den verfügbaren Wuchsraum innerhalb des Bestandes unter allen verbliebenen Bäumen 
optimal aufzuteilen und gleichzeitig müssen alle verbliebenen Bäume gemeinsam mit Blick auf die Endnutzung der Inter-Bestandesopportunität, d. h. der alternativen Nutzung der Fläche durch einen Folgebestand stand halten (Coordes 2014c). So berücksichtigt Gleichung [2-33] als notwendige Bedingung bei einer von der Endnutzung getrennten Analyse nicht, dass die Entnahme von Bäumen aus dem Bestand nur dann sinnvoll ist, wenn die verbleibenden Bäume anschließend noch den Erhalt des Bestandes für ein weiteres Jahr rechtfertigen (hinreichende Bedingung) - die Inter-Bestandesopportunität kann somit auch Vornutzungen verhindern (Coordes 2014c). Andersherum berücksichtigt Gleichung [2-38] bei einer von der Vornutzung getrennten Analyse keine mögliche Dichteveränderung im letzten Zeitschritt. So wäre es bspw. möglich, dass die Entnahme eines Baums im letzten Zeitschritt den Erhalt der restlichen Bäume durch Erhöhung der mittleren Wertzuwachsrate für einen weiteren Zeitschritt rechtfertigen würde (Coordes 2014c; Fuchs 2018, S. 54 f.). Bei der in dieser Arbeit genutzten numerischen Bestimmung der optimalen Bestandesbehandlung werden diese Wechselwirkungen selbstverständlich berücksichtigt.

\subsubsection{Kapitalknappheit}

Die bisherige Vernachlässigung der Kapitalknappheit kann konzeptionell in die Forderung nach der Maximierung des Waldreinertrags ${ }^{28}$ eingeordnet werden (Wippermann 2005, S. 23 f., Möhring 2009). Da dieser Ansatz, welchen schon Abetz (1929, S. 292) als „absurd“ bezeichnete, die Bindung von Kapital in stehendem Holzvolumen und damit den zum Output notwendigen Input ignoriert, wird ,vieles von dem, was die Maximierung des Waldreinertrags nahelegt“ nach Möhring (2009, S. 116) ,,in Anbetracht knapper Mittel im Forstbetrieb nicht realisierbar sein“. Die Einführung eines Kalkulationszinssatzes zur Berücksichtigung der Zeitpräferenz eines Entscheidungsträgers (frühere sind gegenüber späteren Zahlungseingängen vorteilhaft; vgl. Hering 2017, S. 23) steigert somit die Realitätsnähe des Modells, wobei mit dem vollkommenen Kapitalmarkt (vgl. Hering 2017, S. 33 ff.) in Anlehung an die Prämissen des FPO-Theorems (vgl. Johansson und Löfgren 1985, S. 74) das einfachste Konzept der dynamischen Investitionsrechnung zur Anwendung kommt.

\footnotetext{
${ }^{28}$ Vom Waldreinertrag trennt den hier verwendeten durchschnittlichen Überschuss an dieser Stelle lediglich die Berücksichtigung von Bestandesbegründungskosten (Einführung erst in Abschnitt 2.2.7) und allgemeinen Verwaltungskosten (im Zuge der forstlichen Bestandesbehandlung und somit in dieser Arbeit entscheidungsirrelevant).
} 
Unter der Berücksichtigung von Kapitalknappheit gilt es im Folgenden den finanzmathematisch korrekt berechneten durchschnittlichen Überschuss der forstlichen Produktion $a_{r}$ (Annuität der forstlichen Produktion) zu maximieren. Um die durch die Berücksichtigung von Kapitalknappheit entstehenden Wechselwirkungen umfassend aufzuzeigen, werden neben den Optimierungsergebnissen bei dimensionsabhängigen (Abschnitt 2.2.4) teilweise auch Optimierungsergebnisse bei konstanten (Abschnitt 2.2.2) und zeitabhängigen (Abschnitt 2.2.3) Deckungsbeiträgen aufgezeigt. Aufgrund der höchsten Realitätsnähe und der weiteren Verwendung in den folgenden Abschnitten wird die Zielfunktion allerdings nur für den Fall der dimensionsabhängigen Deckungsbeiträge $P\left(V_{E}\right)$ formuliert. Es erweitert sich die zu maximierende Zielfunktion [2-30] mit dem konstanten Kalkulationszinssatz $r$ zu (vgl. Coordes 2014a, S. 37):

$$
\begin{aligned}
& \max _{T, \boldsymbol{\sigma}_{T-1}} a_{r}\left(T, \boldsymbol{\sigma}_{T-1}, N_{0}, P\left(V_{E}\right), r\right) \\
& \qquad=\max _{T, \boldsymbol{\sigma}_{T-1}}\left(\sum_{t=1}^{T-1} \frac{\lambda_{t}\left(\sigma_{t}, t, \boldsymbol{\sigma}_{t-1}, N_{0}\right) \cdot P\left(V_{E}\right)}{(1+r)^{t}}+\frac{V_{B}\left(T, \boldsymbol{\sigma}_{T-1}, N_{0}\right) \cdot P\left(V_{E}\right)}{(1+r)^{T}}\right) \cdot \frac{r \cdot(1+r)^{T}}{(1+r)^{T}-1}, \\
& 0 \leq \boldsymbol{\sigma}_{T-1}<1, \\
& 0 \mathrm{a}<T \leq 100 \mathrm{a}, \\
& r=\text { konst., } \\
& r \in \mathbb{R}^{\geq 0},
\end{aligned}
$$

wobei der sogenannte Wiedergewinnungsfaktor $r \cdot(1+r)^{T} \cdot\left((1+r)^{T}-1\right)^{-1}$ den Kapitalwert der forstlichen Produktion (Anfangswert) in eine gleichbleibende Annuität aufteilt.

Die sich mit einem mittleren $\left(r_{m}=1,5 \%\right)$ bzw. hohen $\left(r_{h}=3,0 \%\right)$ Kalkulationszinssatz ergebenden maximalen Annuitäten der forstlichen Produktion liegen allesamt unterhalb der zinsfrei berechneten Zielgrößen der vorangegangenen Abschnitte. Bei einem konstanten Deckungsbeitrag reduzieren sich die Zielgrößen um $20 \%\left(r_{m}\right)$ bzw. $33 \%\left(r_{h}\right)$ auf 347 bzw. $290 € \mathrm{ha}^{-1} \mathrm{a}^{-1}$. Bei zeitabhängiger, starker DB-Steigerung führt die Einführung des mittleren Kalkulationszinssatzes zu einer Zielgrößenreduktion um $40 \%$ auf $641 € \mathrm{ha}^{-1} \mathrm{a}^{-1}$. Die Zielgrößen bei dimensionsabhängigen, starken DB-Steigerungen sinken um $42 \%\left(r_{m}\right)$ bzw. $66 \%$ $\left(r_{h}\right)$ auf 217 bzw. $127 € \mathrm{ha}^{-1} \mathrm{a}^{-1}$.

Bei einem konstanten Deckungsbeitrag (vgl. Abschnitt 2.2.2) bewirkt die Berücksichtigung von Kapitalknappheit zunächst einen Verzicht auf Vornutzungen in den ersten Zeitschritten (Abbildung 30 a) - erste Eingriffe finden erst ab einem Alter von $5\left(r_{m}\right)$ bzw. $6\left(r_{h}\right)$ Jahren statt. Diese unter Kapitalknappheit und dem damit verbundenen Zinseffekt zunächst nicht 
intuitiv erscheinende Bestandesbehandlung ist, wie auch schon die frühen Eingriffe bei altersabhängig steigendem Deckungsbeitrag (vgl. Abschnitt 2.2.3), modellbedingt zu erklären und steht nicht im Widerspruch zur Optimalitätsbedingung. Vielmehr ist der Verzicht auf frühe Vornutzungen auch hier auf den schematischen Vornutzungscharakter sowie die von der Ausgangsstammzahl losgelösten minimalen Bestandesvolumina in den ersten Zeitschritten zurückzuführen. Denn durch den Verzicht auf Eingriffe in den ersten Zeitschritten wird ggü. der natural-optimalen Bestandesbehandlung oder auch noch stärkeren Eingriffen kaum auf die Realisierung von Holzvolumen verzichtet. Gleichzeitig wird durch die längere Haltung höherer Stammzahlen aber die Option für anschließend deutlich stärkere Eingriffe (stärkerer Abfall der violetten Linien ggü. zinsfreien Variante in Abbildung 30 a) und damit höhere Vornutzungsvolumina zu verhältnismäßig immernoch frühen Zeitpunkten geschaffen. Trotz des Zinseffekts überwiegen die Vorteile dieser stärkeren Vornutzungen (relativ hohes Erntevolumen) zu etwas späteren Zeitpunkten die Nachteile der unterbliebenen Vornutzungen (relativ geringes Erntevolumen) in den ersten Jahren. Bei nur minimalem Verzicht auf laufende Bestandesvolumenzuwächse (Abbildung 32 a) und somit annähernd natural-optimale Gesamtwuchsleistungen $\left(-0,6 \%\left(r_{m}\right)\right.$ bzw. $-1,4 \%\left(r_{h}\right)$ ggü. dem naturalen Optimum zum jeweiligen Zeitpunkt der Endnutzung) werden die Endnutzungsvolumina (337 $\mathrm{Fm} \mathrm{ha}^{-1}\left(r_{m}\right)$ bzw. $275 \mathrm{Fm} \mathrm{ha}^{-1}\left(r_{h}\right)$; Abbildung 33 a) durch die stärkeren Vornutzungen ggü. dem naturalen Optimum vermindert. Die Endutzungsvolumina werden unter Kapitalknappheit zudem früher realisiert - die Endnutzungszeitpunkte reduzieren sich gegenüber der zinsfreien Variante um 9 bzw. 16 Jahre auf $T_{P, r[m]}^{*}=34$ a bzw. $T_{P, r[h]}^{*}=27$ a. 

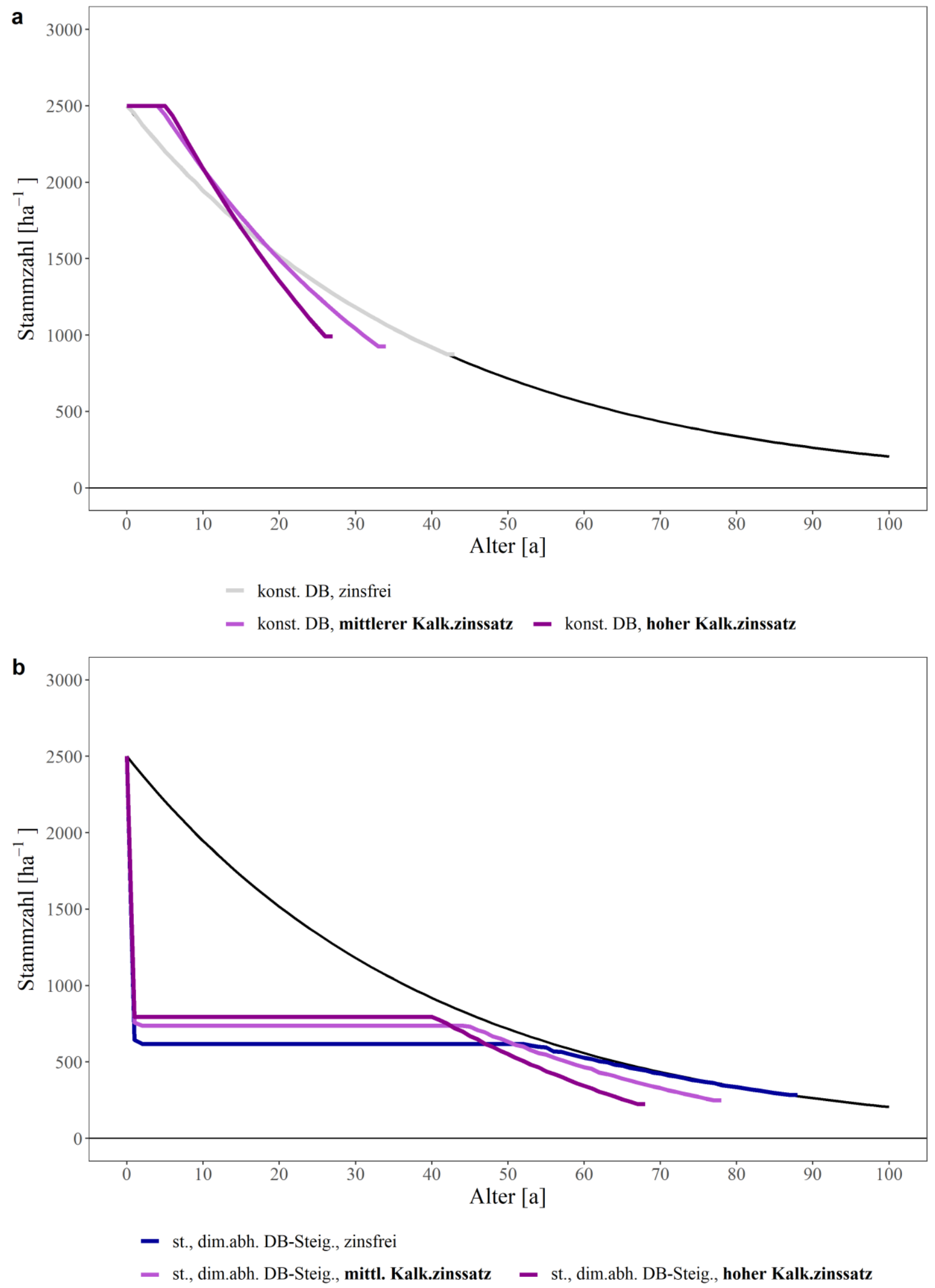

Abbildung 30: Altersabhängige Stammzahlentwicklungen bei konstantem Deckungsbeitrag (a) bzw. starker, dimensionsabhängiger DB-Steigerung (b) und jeweils mittlerem (hellviolett) bzw. hohem (dunkelviolett) Kalkulationszinssatz gemäß Zielfunktion [2-40] im Vergleich zum zinsfreien Optimum bei konstantem DB (grau) bzw. starker, dimensionsabhängiger DB-Steigerung (dunkelblau) und dem naturalen Optimum ohne Folgebestand (schwarz). 
Bei dimensionsabhängigen Deckungsbeiträgen wird die Stammzahl, wie auch schon in Abschnitt 2.2.4, zur Erzielung höherer Einzelbaumvolumina zunächst deutlich abgesenkt (Abbildung 30 b), wobei die Einführung der Kapitalknappheit diesen Effekt dämpft (vgl. Möhring 2010) - die positiven Effekte der höheren Einzelbaumvolumina können aufgrund der vorgezogenen Endnutzungszeitpunkte $\left(T_{P\left(V_{E}\right), \operatorname{stark}, r[m]}^{*}=78 \mathrm{a}\right.$ und $\left.T_{P\left(V_{E}\right), \operatorname{stark}, r[h]}^{*}=68 \mathrm{a}\right)$ nicht mehr in gleichem Maße ausgenutzt werden. Die Einzelbaumvolumina sinken ggü. der zinsfreien Variante zunächst leicht ab, um dann kurz vor der jeweiligen Endnutzung aufgrund verringerter Stammzahlen (Abbildung 30 b) anzusteigen und schließlich sogar leicht über den Einzelbaumvolumina der zinsfreien Variante zu liegen (Abbildung 31).

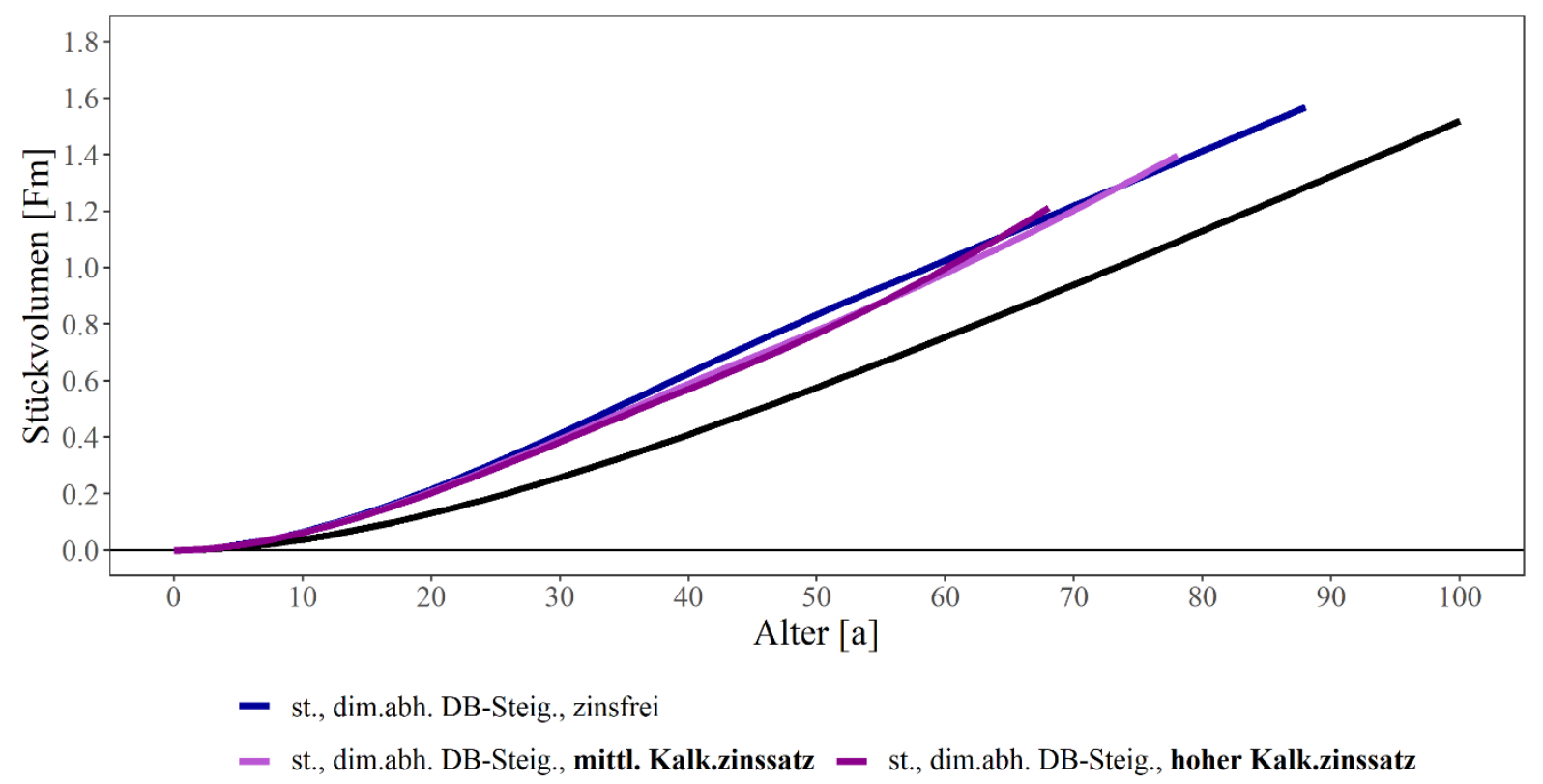

Abbildung 31: Altersabhängige Einzelbaumvolumina (Stückvolumina) bei mittlerem (hellviolett) und hohem (dunkelviolett) Kalkulationszinssatz gemäß [2-40] im Vergleich zum zinsfreien Optimum bei starker, dimensionsabhängiger DB-Steigerung (dunkelblau) und dem naturalen Optimum ohne Folgebestand (schwarz).

Die ggü. der zinsfreien Variante gestiegenen Vornutzungen in höheren Bestandesaltern sind aufgrund des Zinseffekts vorteilhaft gegenüber einem höheren Endnutzungsvolumen. Entsprechend fallen die verbleibenden Bestandesvolumina ggü. der zinsfreien Variante geringer aus (Abbildung 33 b). Aus höheren laufenden Bestandesvolumenzuwächsen (Abbildung 32 b) folgen bei früheren Endnutzungszeitpunkten durchschnittliche Bestandesvolumenzuwächse von 10,6 Fm ha $\mathrm{F}^{-1} \mathrm{a}^{-1}\left(r_{m}\right)$ bzw. 11,1 $\mathrm{Fm} \mathrm{ha}^{-1} \mathrm{a}^{-1}\left(r_{h}\right)$ was einem Plus von $11 \%$ bzw. $15 \%$ ggü. der zinsfreien Variante bei starker, dimensionsabhängiger DB-Steigerung in Abschnitt 2.2.4 entspricht. 
a

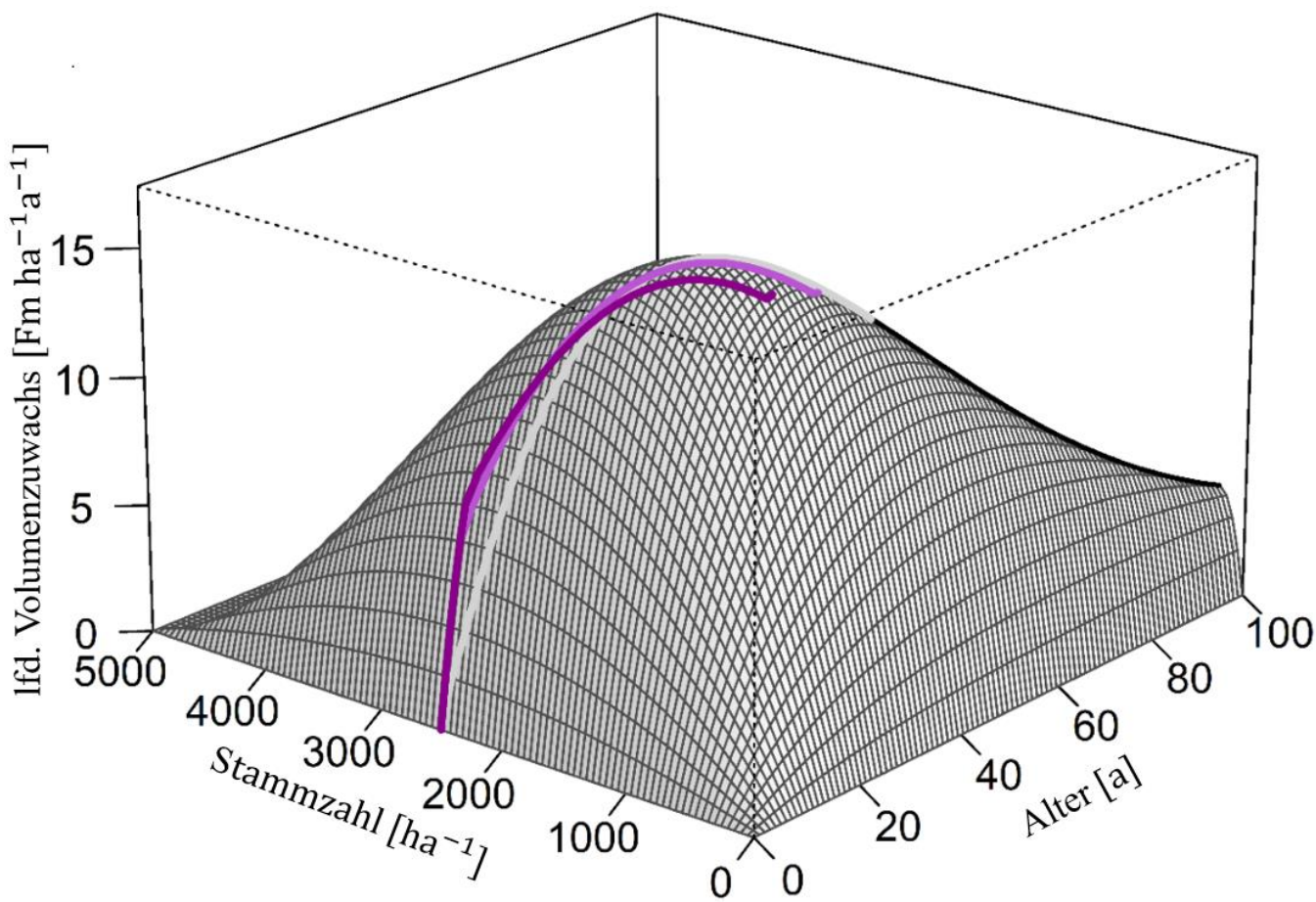

konst. DB, zinsfrei

konst. DB, mittl. Kalk.zinssatz

konst. DB, hoher Kalk.zinssatz

b

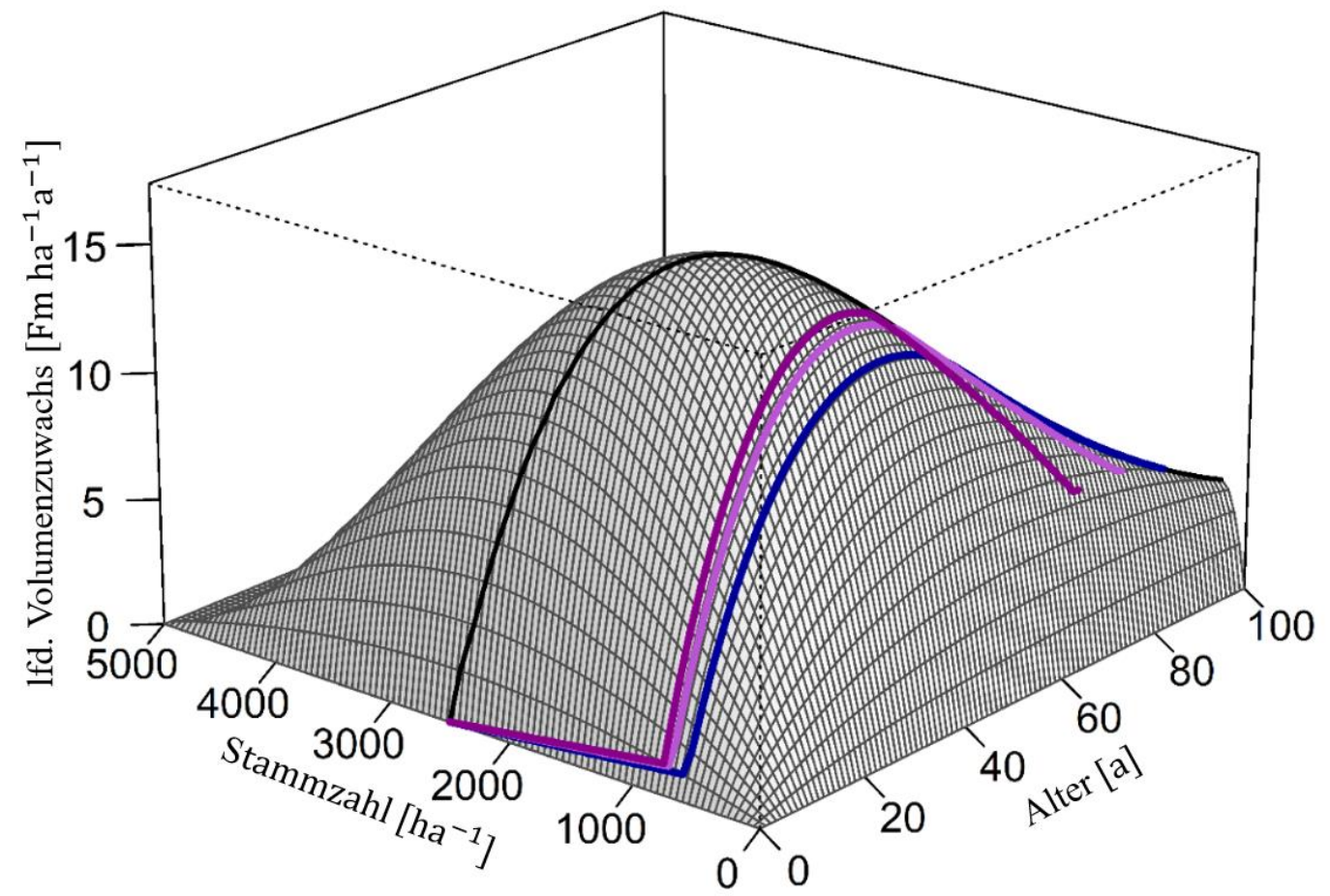

st., dim.abh. DB-Steig., zinsfrei

st., dim.abh. DB-Steig., mittl. Kalk.zinssatz

st., dim.abh. DB-Steig., hoher Kalk.zinssatz

Abbildung 32: Alters- und stammzahlabhängiger laufender Bestandesvolumenzuwachs bei konstantem Deckungsbeitrag (a) bzw. starker, dimensionsabhängiger DB-Steigerung (b) und jeweils mittlerem (hellviolett) bzw. hohem (dunkelviolett) Kalkulationszinssatz gemäß Zielfunktion [2-40] im Vergleich zum zinsfreien Optimum bei konstantem DB (grau) bzw. starker, dimensionsabhängiger DB-Steigerung (dunkelblau) und dem maximalen Volumenzuwachs (schwarz). 

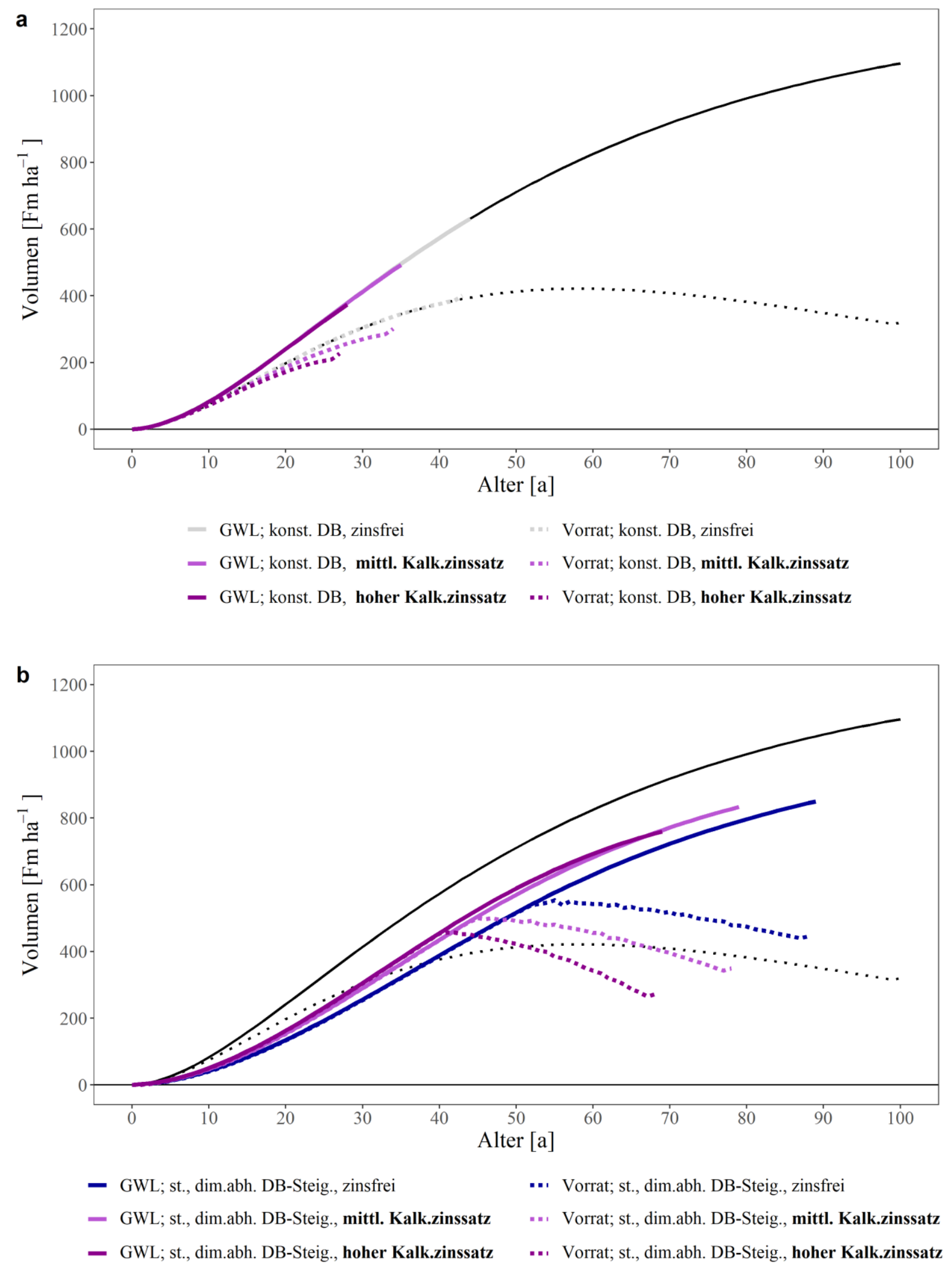

Abbildung 33: Altersabhängige Gesamtwuchsleistung und Vorratsentwicklung bei konstantem Deckungsbeitrag (a) bzw. starker, dimensionsabhängiger DB-Steigerung (b) und jeweils mittlerem (hellviolett) bzw. hohem (dunkelviolett) Kalkulationszinssatz gemäß Zielfunktion [2-40] im Vergleich zum zinsfreien Optimum bei konstantem DB (grau) bzw. starker, dimensionsabhängiger DB-Steigerung (dunkelblau) und dem naturalen Optimum ohne Folgebestand (schwarz). 
Der bereits in Abschnitt 2.2.3 erwähnte Charakter eines negativen Kalkulationszinssatzes bei jährlich-konstanter DB-Steigerung kann bestätigt werden. Die Effekte einer jährlich-konstanten DB-Steigerung um 1,5 \% aus Abschnitt 2.2.3 werden durch den Kalkulationszinssatz in Höhe von 1,5\% $\left(r_{m}\right)$ aufgehoben - die resultierende optimale Stammzahlentwicklung $\boldsymbol{N}_{P(t), \text { stark, }[\mathrm{m}]}^{*}$ entspricht dem naturalen Optimum (Abbildung 34). Der Endnutzungszeitpunkt ist ggü. dem naturalen Optimum mit Folgebestand (Abschnitt 2.2.1) allerdings deutlich (um 39 Jahre) nach hinten verschoben $\left(T_{P(t), s t a r k, r[m]}^{*}=82 \mathrm{a}\right)$. Die Einbeziehung des Zinseffekts bei der Berechnung der Inter-Bestandesopportunität führt somit trotz der hinzukommenden Kapitalopportunität zur Minderung der Grenzkosten des Bestandeserhalts.

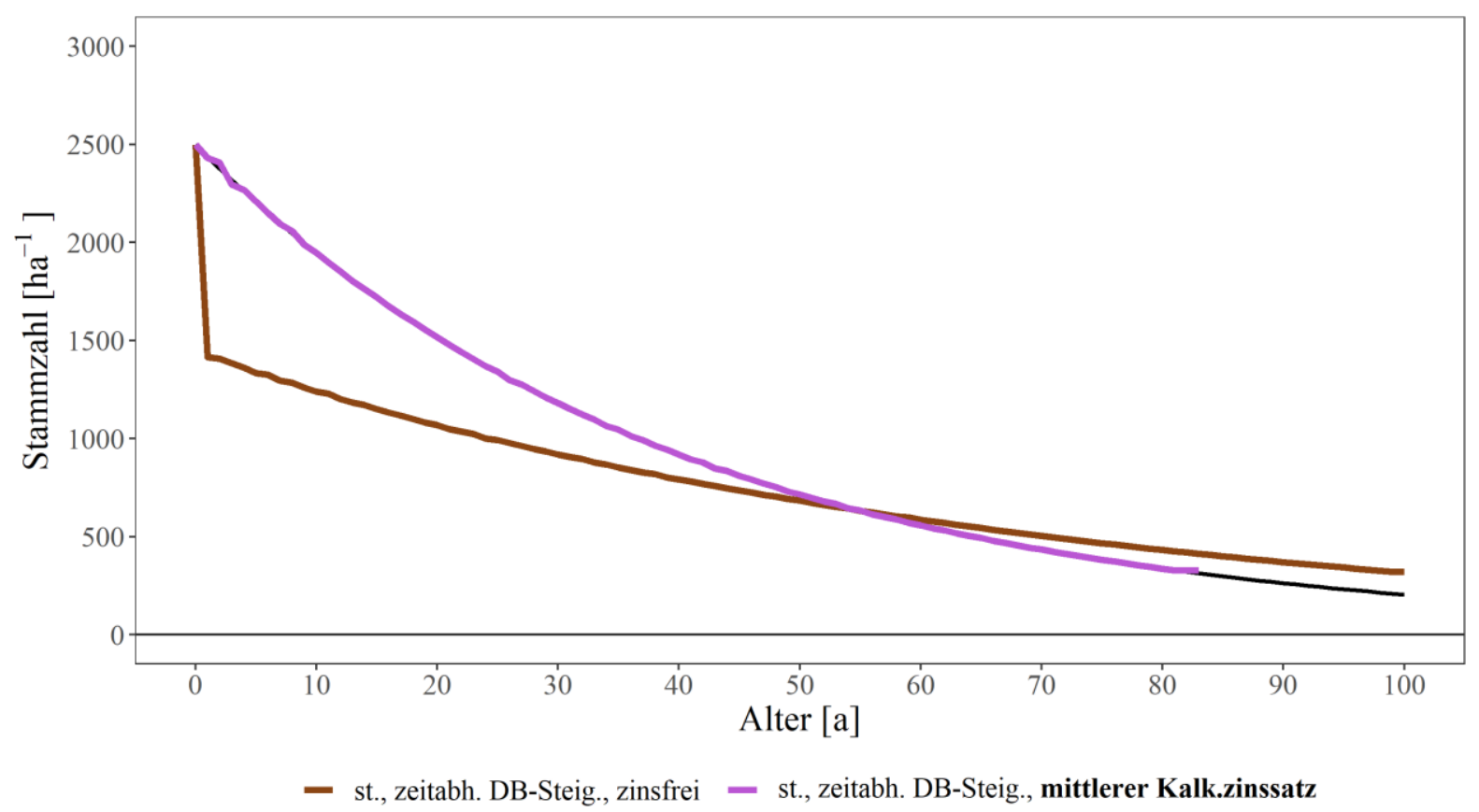

Abbildung 34: Altersabhängige Stammzahlentwicklungen bei starker, zeitabhängiger DB-Steigerung und mittlerem Kalkulationszinssatz (hellviolett) im Vergleich zum zinsfreien Optimum (dunkelbraun) und dem naturalen Optimum ohne Folgebestand.

Durch entsprechende Erweiterung der Optimalitätsbedingung [2-33] ergibt sich für die optimale Vornutzung unter Kapitalknappheit mit dimensionsabhängigen Deckungsbeiträgen:

$$
w_{j}\left(t_{j}, N_{t_{j}-1}, P\left(V_{E}\right)\right)=W_{j}\left(t_{j}-1, N_{t_{j}-2}, P\left(V_{E}\right)\right) \cdot r+\sum_{t=t_{j}}^{T} \sum_{\substack{k \in L_{t_{j}} \\ k \neq j}} \frac{\Delta W_{k, j}\left(t_{k}\right)}{(1+r)}\left(t_{\left.k^{-}-t_{j}\right)} .\right.
$$

Für den Erhalt eines Baums $j$ um ein weiteres Jahr (Ernte in $t_{j}$ statt $t_{j}-1$ ) muss dessen laufender Wertzuwachs $w_{j}\left(t_{j}, N_{t_{j}-1}, P\left(V_{E}\right)\right)$ nun zusätzlich zum Nutzenentgang der alternativen Flächen- bzw. Wuchsraumnutzung durch alle beeinflussten Bäume $k \in L_{t_{j}}$ 
(Wuchsraumopportunität) auch die Kosten der Kapitalbindung $W_{j}\left(t_{j}-1, \boldsymbol{N}_{t_{j}-2}, P\left(V_{E}\right)\right) \cdot r$ (Kapitalopportunität) überwiegen (Coordes 2014a, S. 61).

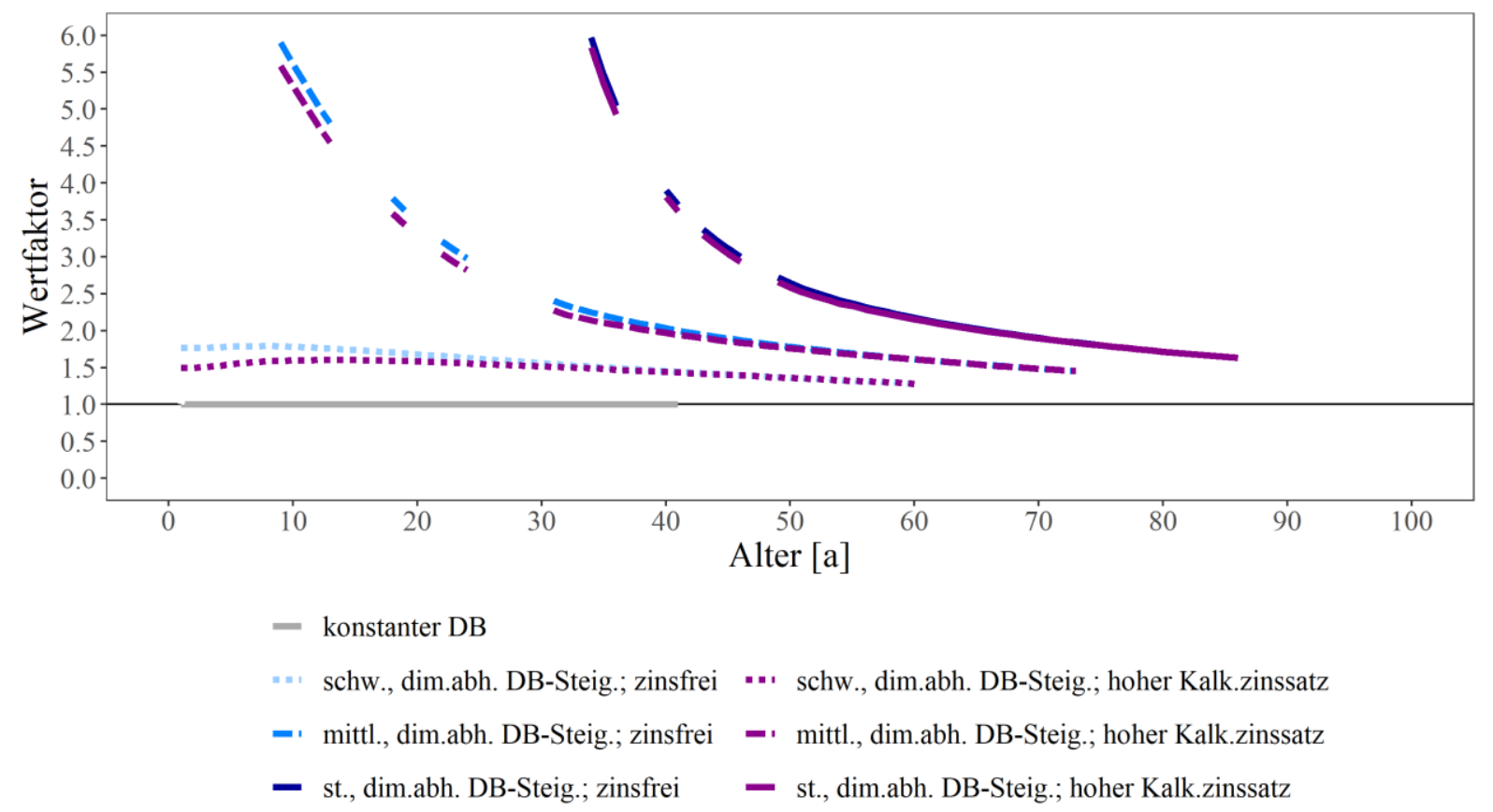

Abbildung 35: Wertfaktoren bei mittlerem (hellviolett) und hohem (dunkelviolett) Kalkulationszinssatz gemäß [2-40] im Vergleich zu den zinsfreien Optima bei schwacher (hellblau), mittlerer (blau) und starker (dunkelblau), dimensionsabhängiger DB-Steigerung und dem ökonomischen Optimum bei konstantem Deckungsbeitrag (grau). Aufgrund zeitweise unterbleibender Vornutzungen bei mittlerer und starker DB-Steigerung können die Wertfaktoren nicht für jedes Alter bestimmt werden.

Die resultierenden Wertfaktoren (siehe Exkurs zur Berechnung der Wertfaktoren in Abschnitt 2.2.3) liegen leicht unterhalb der zinsfreien Wertfaktoren aus Abschnitt 2.2.4. Je früher im Bestandesleben über den Erhalt eines Baums entschieden wird und je größer somit der zeitliche Abstand zur Realisierung der beeinflussten Bäume ist, desto mehr reduziert der Zinseffekt den Wertfaktor - der Dimensionseffekt wird gedämpft. Insgesamt sinkt mit steigendem Alter die Bedeutung der Wuchsraumopportunität für die Entscheidung über den Erhalt eines Baums für ein weiteres Jahr, bei gleichzeitig steigendem Einfluss der Kapitalopportunität.

Auch die Optimalitätsbedingung der Endnutzung verändert sich mit Blick auf die Grenznutzenanalyse des Bestandeserhalts für ein weiteres Jahr. Sie beinhaltet nun neben der Flächenopportunität in Form einer Annuität $a_{r}$ zusätzlich auch eine Kapitalopportunität in Form der jährlichen Verzinsung des Bestandesabtriebswertes (vgl. Möhring 2009 i. V.m. Pressler 1860; 1995). Es gilt:

$$
w_{B}\left(T, N_{T-1}, P\left(V_{E}\right)\right)=W_{B}\left(T-1, \boldsymbol{N}_{T-2}, P\left(V_{E}\right)\right) \cdot r+a_{r}\left(T, \boldsymbol{N}_{T-1}, P\left(V_{E}\right), r\right) .
$$


Für den Erhalt des Bestandes um ein weiteres Jahr, muss der Grenznutzen des Bestandeserhalts $w_{B}\left(T, N_{T-1}, P\left(V_{E}\right)\right)$ nun neben dem Grenznutzen eines Folgebestandes $a_{r}\left(T, \boldsymbol{N}_{T-1}, P\left(V_{E}\right), r\right)$ zusätzlich auch den Grenznutzen der alternativen Verwendung des in ihm gebundenen Kapitals $W_{B}\left(T-1, \boldsymbol{N}_{T-2}, P\left(V_{E}\right)\right) \cdot r$ überwiegen. Dabei ist es wichtig zu betonen, dass die alternative Verwendung keinesfalls nur als externe Geldanlage zu verstehen ist - vielmehr kann die interne Verzinsung jeder innerbetrieblichen Investitionsalternative als Opportunität für das im Bestand gebundene Kapital verstanden werden (vgl. z. B. Möhring 1994, S. 44 ff.).

\subsubsection{Erntefixkosten}

Aufbauend auf der Modellvariante mit dimensionsabhängigen, stark steigenden Deckungsbeiträgen (siehe Abbildung 23) und einem mittleren Kalkulationszinssatz ( $r_{m}=1,5 \%$, vgl. Abschnitt 2.2.5) werden im Folgenden Erntefixkosten eingeführt (vgl. z. B. Hyytiäinen und Tahvonen 2002; Coordes 2014a, S. 118 ff.; Tahvonen und Rämö 2016; Rämö und Tahvonen 2017). Als Erntefixkosten werden dabei maßnahmenspezifische, d. h. der forstlichen Behandlung eines Bestandes (Vor- oder Endnutzung) direkt zuordbare, mengenunabhängige Kosten bezeichnet. Während die bisher ausschließlich verwendeten Holzerntekosten die Fällung und Rückung bis zum Verkaufsort Waldstraße in Abhängigkeit vom Erntevolumen abdecken, sind die mengenunabhängigen Kosten für die Planung, Durchführung und Nachbereitung einer Erntemaßnahme bisher nicht berücksichtigt. Beispiele sind mengenunabhängige Kosten für Ausschreibung, Angebotseinholung und Verfassen der Arbeitsanweisungen, Fixkosten im Rahmen der Einweisung und Kontrolle des durchführenden Forstunternehmens sowie mengenunabhängige Kosten für Sortierung, Aushaltung und Verkauf des geernteten Holzes. Zudem sind unter Erntefixkosten bspw. auch Umsetzungs- bzw. Anfahrtskosten für Erntemaschinen (vgl. Rämö und Tahvonen 2017) zu verstehen. Abgegrenzt werden die Erntefixkosten auch von allgemeinen Verwaltungs- bzw. Fixkosten, die sich durch eine gewisse Unabhängigkeit von der forstlichen Produktion auszeichnen. Allgemeine Verwaltungskosten fallen bspw. durch die allgemeine Walderschließung, laufende Wegeinstandsetzung, laufende Gebäudeunterhaltung, die allgemeinen Kosten für die Mobilität der bewirtschaftenden Person, allgemeine Personalkosten sowie maßnahmenunabhängige Abgaben und Steuern an. 
Die unter Veränderung des Vornutzungsvektors $\boldsymbol{\sigma}_{T-1}$ sowie des Endnutzungszeitpunkts $T$ zu maximierende Zielfunktion [2-40] erweitert sich mit den Erntefixkosten $F$ zu:

$$
\begin{aligned}
& \quad \max _{T, \boldsymbol{\sigma}_{T-1}} a_{F}\left(T, N_{0}, \boldsymbol{\sigma}_{T-1}, P\left(V_{E}\right), r, F\right) \\
& \quad=\max _{T, \boldsymbol{\sigma}_{T-1}}\left(\sum_{t=1}^{T-1} \frac{\left(\lambda_{t}\left(\sigma_{t}, t, \sigma_{t-1}, N_{0}\right) \cdot P\left(V_{E}\right)-F\left(\sigma_{t}\right)\right)}{(1+r)^{t}}+\frac{\left(V_{B}\left(T, N_{0}, \boldsymbol{\sigma}_{T-1}\right) \cdot P\left(V_{E}\right)-F\right)}{(1+r)^{T}}\right) \cdot \frac{r \cdot(1+r)^{T}}{(1+r)^{T}-1}, \\
& 0 \leq \boldsymbol{\sigma}_{T-1}<1, \\
& 0 \mathrm{a}<T \leq 100 \mathrm{a}, \\
& F\left(\sigma_{t}\right)=\left\{\begin{array}{l}
F, \text { für } \sigma_{t}>0 \\
0, \text { für } \sigma_{t}=0
\end{array}\right. \\
& F \in \mathbb{R}^{\geq 0},
\end{aligned}
$$

wobei die Erntefixkosten als konstant angenommen werden und zusätzlich zur Endnutzung zu anderen Zeitpunkten auftreten, wenn der entsprechende Vornutzungsanteil größer 0 ist, d. h. eine Vornutzung stattfindet (vgl. Coordes 2014a, S. 118). Die Berücksichtigung der Erntefixkosten steigert damit die Realitätsnähe des jährlich-diskreten Modells, in welchem bisher auch die jährliche Ernte extrem geringer Holzmengen keine monetären Nachteile (sofern positiver DB) bedingte.

Um den Einfluss der Erntefixkostenhöhe auf die Bestandesbehandlung zu analysieren, werden im Variantenstudium einerseits sehr niedrige $\left(F_{n}=10 €\right.$ ha $\left.^{-1}\right)$ und andererseits extrem hohe $\left(F_{h}=500 €\right.$ ha $\left.^{-1}\right)$ Erntefixkosten genutzt.

Die resultierenden maximalen Annuitäten liegen erwartungsgemäß unterhalb der erntefixkostenfrei berechneten Zielgrößen aus Abschnitt 2.2.5. Bei einer dimensionsabhängig starken DBSteigerung und einem mittleren Kalkulationszinssatz von $r=1,5 \%$ sinken sie um $1 \%\left(F_{n}\right)$ bzw. $11 \%\left(F_{h}\right)$ auf 215 bzw. $193 € \mathrm{ha}^{-1} \mathrm{a}^{-1}$.

In beiden Fällen reduziert sich die Anzahl der Vornutzungen im Vergleich zur erntefixkostenfreien Variante aus Abschnitt 2.2.5 deutlich (Abbildung 36). Statt der bisherigen 33 jährlichen Eingriffe zwischen den Altern 45 a und 78 a finden im gleichen Zeitraum nun lediglich $7\left(F_{n}\right)$ bzw. $1\left(F_{h}\right)$ Vornutzung(en) statt, wobei sich der Endnutzungszeitpunkt bei hohen Erntefixkosten um 2 Jahre reduziert $\left(T_{F[h]}^{*}=76 \mathrm{a}\right)$. Der Endnutzungszeitpunkt bei niedrigen Erntefixkosten liegt im Vergleich zur erntefixkostenfreien Variante aus Abschnitt 2.2.5 unverändert bei $T_{F[n]}^{*}=78 \mathrm{a}$. 


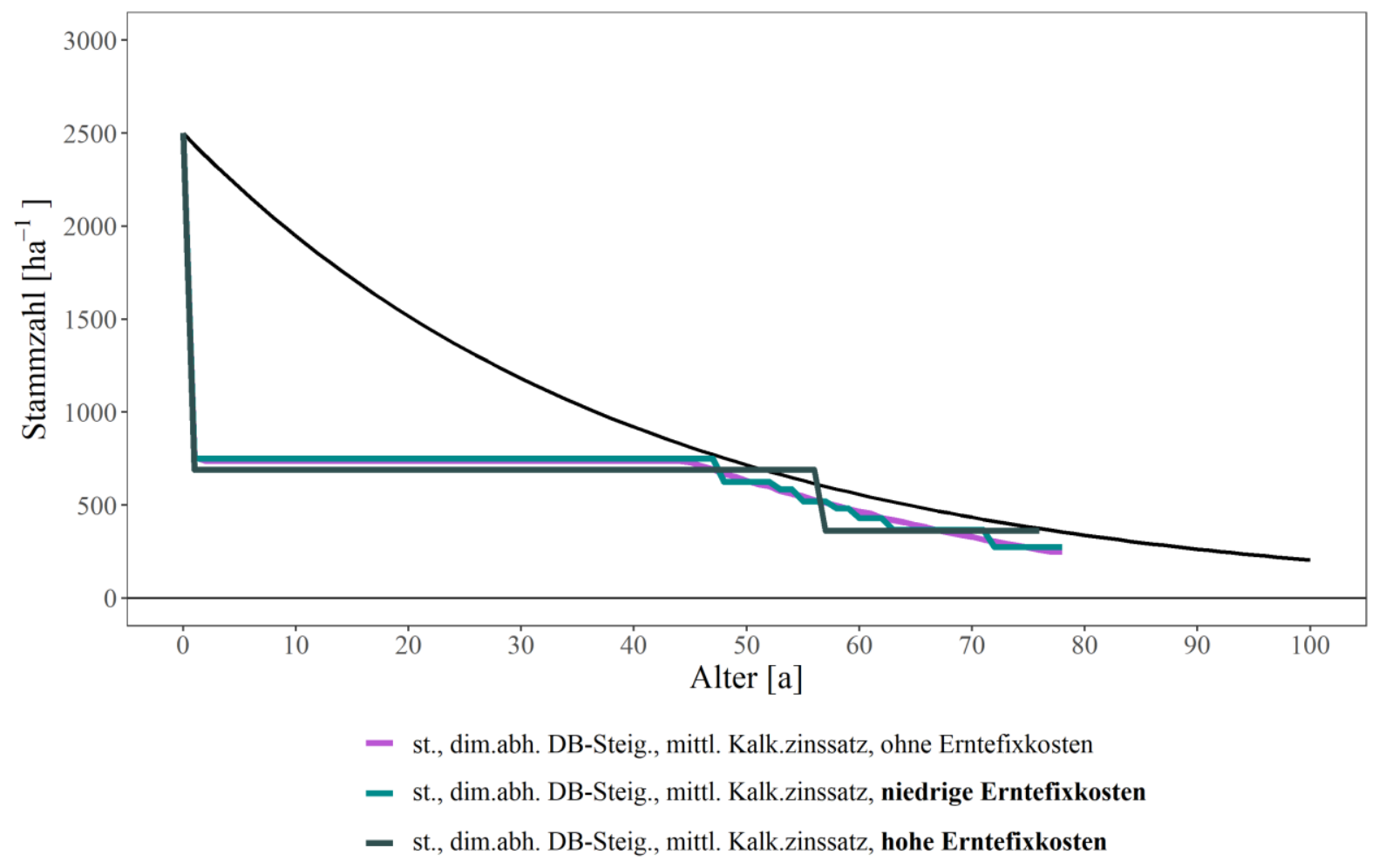

Abbildung 36: Altersabhängige Stammzahlentwicklungen bei niedrigen (hellcyan) bzw. hohen (dunkelcyan) Erntefixkosten gemäß Zielfunktion [2-43] im Vergleich zum erntefixkostenfreien Optimum bei starker, dimensionsabängiger DB-Steigerung und mittlerem Kalkulationszinssatz (hellviolett) und zum naturalen Optimum ohne Folgebestand (schwarz).

Bei nur leicht erhöhtem Bestandesvolumen zum Zeitpunkt der Endnutzungen ggü. der erntefixkostenfreien Variante aus Abschnitt 2.2.5 (383 $\mathrm{Fm} \mathrm{ha}^{-1}$ statt $349 \mathrm{Fm} \mathrm{ha}^{-1}$; Abbildung 37), steigen die Erntemengen je Vornutzung entsprechend der nun stark verminderten Häufigkeit bereits bei niedrigen Erntefixkosten deutlich an (vgl. Rämö und Tahvonen 2017 oder auch Eriksson 1999). Das mittlere Vornutzungsvolumen je Eingriff liegt bei niedrigen Erntefixkosten im Zeitraum zwischen 45 a und 78 a bei $64 \mathrm{Fm} \mathrm{ha}^{-1}$ gegenüber $14 \mathrm{Fm} \mathrm{ha}^{-1}$ bei der erntefixkostenfreien Variante. Im Fall der extrem hohen Erntefixkosten wird bei deutlich höherem Endnutzungsvolumen (489 $\mathrm{Fm} \mathrm{ha}^{-1}$ ) mit der alleinigen Vornutzung im Alter von 57 Jahren ein Erntevolumen von $301 \mathrm{Fm}$ realisiert ${ }^{29}$.

${ }^{29}$ Angesichts es äußerst hohen Vornutzungsvolumens wird an dieser Stelle nochmals betont, dass im Modell weder Qualitäts- noch erntemengenbedingte Bestandesstabilitätsaspekte berücksichtigt werden. 


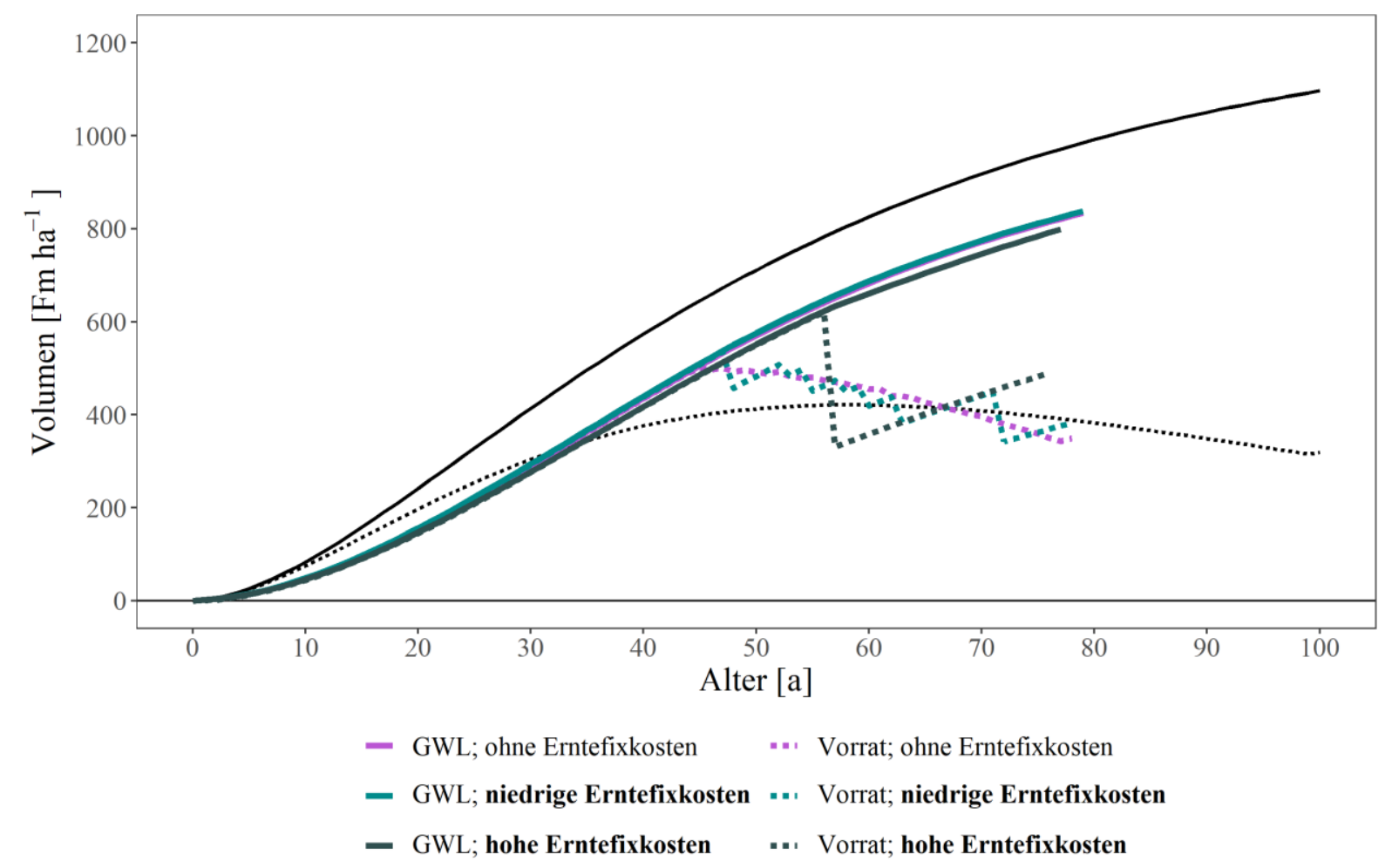

Abbildung 37: Altersabhängige Gesamtwuchsleistung und Vorratsentwicklung bei niedrigen (hellcyan) bzw. hohen (dunkelcyan) Erntefixkosten gemäß Zielfunktion [2-43] im Vergleich zum erntefixkostenfreien Optimum bei starker, dimensionsabängiger DB-Steigerung und mittlerem Kalkulationszinssatz (hellviolett) und zum naturalen Optimum ohne Folgebestand (schwarz).

Auf die laufenden Bestandesvolumenzuwächse (Abbildung 38) und damit auch die Gesamtwuchsleistungen (Abbildung 37) haben die veränderten Vornutzungen nur geringe Auswirkungen. Bei niedrigen Erntefixkosten erhöht sich sogar die Gesamtwuchsleistung bis zum Zeitpunkt der Endnutzung um $1 \%$, was einer Steigerung des durchschnittlichen Bestandesvolumenzuwachses von 10,6 auf 10,7 $\mathrm{Fm} \mathrm{ha}^{-1} \mathrm{a}^{-1}$ entspricht. Die hohen Erntefixkosten senken die Gesamtwuchsleistung leicht ab - der durchschnittlichen Bestandesvolumenzuwachs sinkt auf $10,4 \mathrm{Fm} \mathrm{ha}^{-1} \mathrm{a}^{-1}$.

Gegenüber Abschnitt 2.2.5 steigt die Komplexität der zeitlich-räumlichen Grenznutzenanalyse am Einzelbaum durch die Einbeziehung von Erntefixkosten deutlich. Eine isolierte Formulierung aller auftretenden Effekte ist aufgrund von Wechselwirkungen zwischen der marginal verzögerten Einzelbaumentnahme und den Entscheidungen über den Erhalt bzw. die Ernte aller anderen Bäume des Bestandes (in der hier gewählten Form der mathematischen Formulierung; vgl. alternativ Coordes 2014a, S. 118 ff.) nicht möglich. 


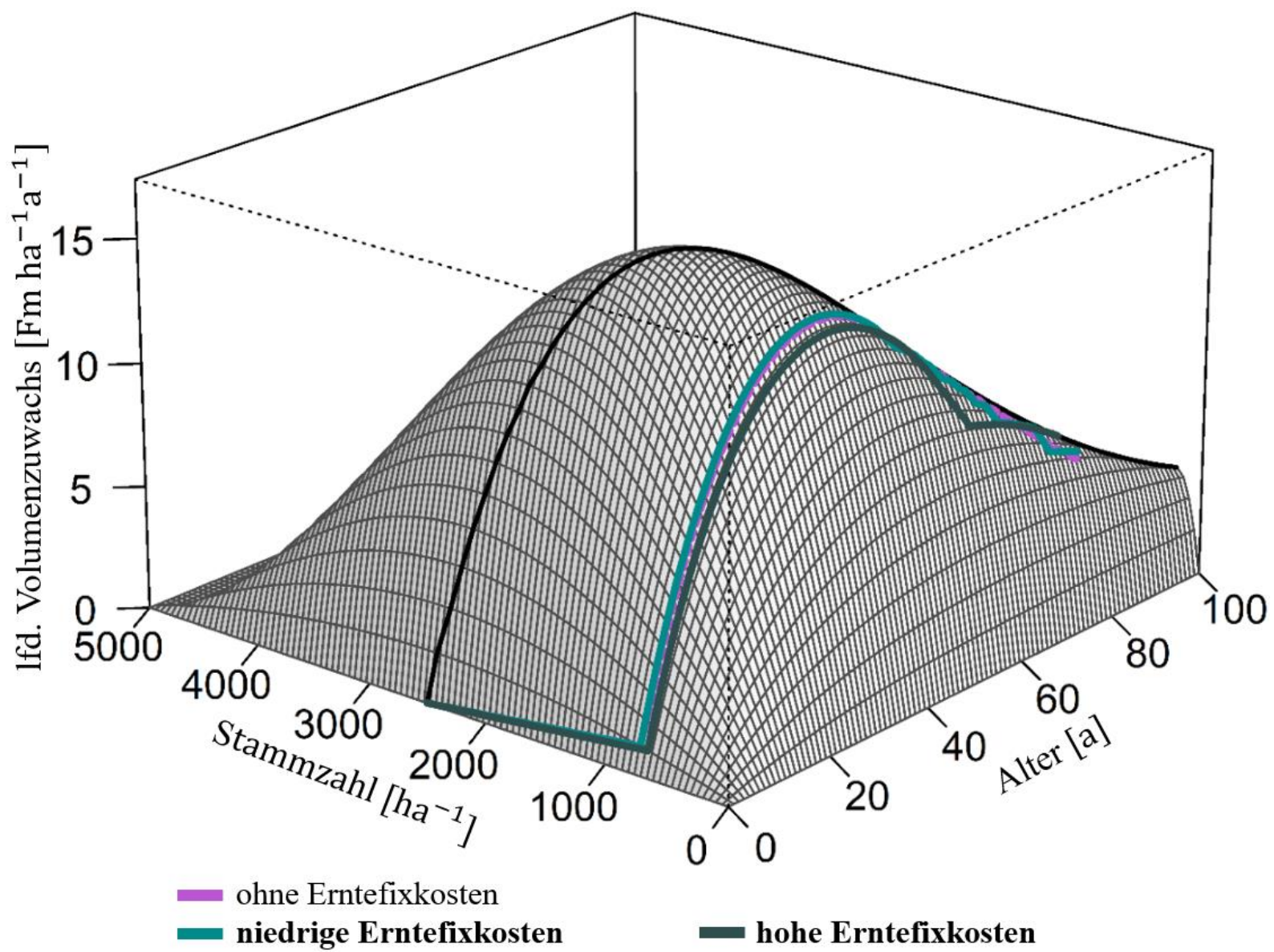

Abbildung 38: Alters- und stammzahlabhängiger laufender Bestandesvolumenzuwachs bei niedrigen (hellcyan) bzw. hohen (dunkelcyan) Erntefixkosten gemäß Zielfunktion [2-43] im Vergleich zum erntefixkostenfreien Optimum bei starker, dimensionsabängiger DB-Steigerung und mittlerem Kalkulationszinssatz (hellviolett) und dem maximalen Volumenzuwachs (schwarz).

Die zeitlich-räumliche Grenznutzenanalyse am Einzelbaum wird um einen weiteren Term ergänzt. Dieser beschreibt die durch den marginal verlängerten Baumerhalt bedingte Verschiebung der Erntefixkostenbelastung. Wird dabei von einer gleichmäßigen Verteilung der Erntefixkosten auf alle Bäume einer Erntemaßnahme $M_{t}=\sigma_{t} \cdot N_{t-1}$ ausgegangen, bedingt der marginal verlängerte Erhalt von Baum $j$ (Ernte zum Zeitpunkt $t_{j}$ statt $t_{j}-1$ ) die Einsparung des Erntefixkostenanteils $F \cdot\left(M_{t_{j}-1}\right)^{-1}$ zum Zeitpunkt $t_{j}-1$ bei hinzukommender Belastung durch den Erntefixkostenanteil $F \cdot\left(M_{t_{j}}\right)^{-1}$ zum Zeitpunkt $t_{j}$.

Ob dadurch Grenznutzen oder Grenzkosten des marginal verlängerten Baumerhalt entstehen, kann für den Einzelbaum $j$ isoliert nicht beantwortet werden und hängt maßgeblich von den Wechselwirkungen mit allen anderen Erntebäumen der Zeitpunkte $t_{j}-1$ und $t_{j} \mathrm{ab}$. In Abhängigkeit von der Anzahl der über den Baum $j$ hinausgehenden Erntebäume zu den Zeitpunkten 
$t_{j}-1$ und $t_{j}$ verändert sich dessen Erntefixkostenbelastung ${ }^{30}$. Werden bspw. zum Zeitpunkt $t_{j}-1$ insgesamt 50 und zum Zeitpunkt $t_{j}$ lediglich 10 Bäume geerntet, steigt die Erntefixkostenbelastung für Baum $j$ erheblich durch den marginal verlängerten Erhalt - es entstehen zusätzliche Grenzkosten des marginal verlängerten Baumerhalts. Im Extremfall trägt Baum $j$ die gesamten Erntefixkosten, wenn zum Zeitpunkt $t_{j}$ kein anderer Baum ausscheidet. Andersherum kann die Erntefixkostenbelastung sinken und somit ein zusätzlicher Grenznutzen des marginal verlängerten Baumerhalts entstehen, wenn zum Zeitpunkt $t_{j}$ mehr Bäume ausscheiden als zum Zeitpunkt $t_{j}-1$, der Erntefixkostenanteil für Baum $j$ also verringert wird.

Ein Grenznutzen des marginal verlängerten Erhalts von Baum $j$ wird in jedem Fall zusätzlich durch den Zinseffekt generiert. Der Aufschub der Erntefixkostenbelastung um ein Jahr bedingt eine längere Verfügbarkeit des für die Deckung des Erntefixkostenanteils notwendigen Kapitals - es entsteht ein Kapitalgrenznutzen.

Unter Berücksichtigung des zusätzlichen Terms gilt für die Vornutzungsentscheidung als Erweiterung der Optimalitätsbedingung [2-41]:

$$
\begin{gathered}
w_{j}\left(t_{j}, N_{t_{j}-1}, P\left(V_{E}\right)\right)+\left(\frac{F}{M_{t_{j}-1}} \cdot(1+r)-\frac{F}{M_{t_{j}}}\right) \\
=W_{j}\left(t_{j}-1, N_{t_{j}-2}, P\left(V_{E}\right)\right) \cdot r+\sum_{t=t_{j}}^{T} \sum_{\substack{k \in L_{t_{j}} \\
k \neq j}} \frac{\Delta W_{k, j}\left(t_{k}\right)}{(1+r)}\left(t_{\left.k^{-}-t_{j}\right)}\right) \\
\frac{F}{M_{t}}=\frac{F}{\sigma_{t} \cdot N_{t-1}}=\left\{\begin{array}{r}
F \cdot\left(M_{t}\right)^{-1}, \text { für } \sigma_{t}>0 \\
0, \text { für } \sigma_{t}=0 .
\end{array}\right.
\end{gathered}
$$

Wie bereits erwähnt, können aufgrund der Wechselwirkungen mit allen anderen Erntebäumen der Zeitpunkte $t_{j}-1$ und $t_{j}$ keine allgemeingültigen Aussagen über die Richtung der Erntezeitpunktverschiebung getroffen werden. Maßgeblich dafür ist im Einzelfall das Verhältnis zwischen dem aufgrund des marginal verlängerten Erhalts von Baum $j$ eingesparten, zinseffektbereinigten Erntefixkostenanteil $F \cdot(1+r) \cdot\left(M_{t_{j}-1}\right)^{-1}$ (Grenznutzen) und dem anstatt dessen anfallenden Erntefixkostenanteil $F \cdot\left(M_{t_{j}}\right)^{-1}$ (Grenzkosten).

\footnotetext{
${ }^{30}$ Ausdrücklich nicht berücksichtigt werden bei dieser Form der mathematischen Formulierung die Wirkungen des marginal verlängerten Baumerhalts auf die Erntefixkostenbelastung aller anderen Bäume des Bestandes und damit auch deren Erntezeitpunkte. Die Grenznutzenanalyse erfolgt stets unter ansonsten gleichen Bedingungen, d. h. ceteris paribus.
} 
Unabhängig von der Verschiebung der Einzelbaum-Erntezeitpunkte kommt es in jedem Fall zu einer erntefixkostenabhängigen Konzentration auf weniger Vornutzungen, um die Erntefixkostenbelastung auf Bestandesebene insgesamt möglichst gering zu halten (vgl. z. B. Hyytiäinen und Tahvonen 2002; Wippermann 2005, S. 81 f. u. 107; Coordes 2014a, S. 120).

In Anlehnung an die Erweiterung der Vornutzungsoptimalitätsbedingung gilt für die Endnutzungsentscheidung im Optimum:

$$
\begin{aligned}
w_{B}\left(T, N_{T-1}, P\left(V_{E}\right)\right) & +(F \cdot(1+r)-F) \\
& =W_{B}\left(T-1, \boldsymbol{N}_{T-2}, P\left(V_{E}\right)\right) \cdot r+a_{F}\left(T, \boldsymbol{N}_{T-1}, P\left(V_{E}\right), r, F\right),
\end{aligned}
$$

was sich aufgrund der nun entweder ganz (zum Zeitpunkt $T$ ) oder gar nicht $(T-1)$ auftretenden Fixkostenbelastung vereinfachen lässt zu:

$$
\begin{aligned}
w_{B}\left(T, N_{T-1}, P\left(V_{E}\right)\right) & +(F \cdot r) \\
& =W_{B}\left(T-1, N_{T-2}, P\left(V_{E}\right)\right) \cdot r+a_{F}\left(T, N_{T-1}, P\left(V_{E}\right), r, F\right),
\end{aligned}
$$

Hier existiert also ein Grenznutzen des marginal verlängerten Bestandeserhalts, der nur von der Höhe der Erntefixkosten $F$ sowie dem Kalkulationszinssatz $r$ abhängt. Ceteris paribus führt die Berücksichtigung von Erntefixkosten somit zur Verzögerung der Endnutzung (vgl. Coordes 2014a, S. 119).

Dennoch kommt es im Modell durch die Einführung extrem hoher Erntefixkosten zu einer, wenn auch sehr geringen, Verkürzung der forstlichen Produktion; bei der Einführung geringer Erntefixkosten bleibt der Endnutzungszeitpunkt unverändert. Erneut spielen dafür die bereits mehrfach diskutierten und durch die getrennte Analyse von Vor- und Endnutzung nicht zu greifenden Wechselwirkungen zwischen Vor- und Endnutzung eine erhebliche Rolle. Die Abhängigkeiten zwischen den Vornutzungseingriffen (Stärke und Zeitpunkt) und dem Endnutzungszeitpunkt, welche insbesondere durch die Einführung extremer Erntefixkosten deutlich werden, verhindern auch hier allgemeingültige Aussagen über deren Wirkungsrichtung. Wie das Variantenstudium zeigt, ist aber selbst bei sehr hohen Erntefixkosten nicht von einer erheblichen Verschiebung des Endnutzungszeitpunkts auszugehen. 


\subsubsection{Bestandesbegründungskosten}

Werden aufbauend auf der Modellvariante aus Abschnitt 2.2.6 mit dimensionsabhängig starken DB-Steigerungen, einem mittleren Kalkulationszinssatz $\left(r_{m}=1,5 \%\right)$ sowie niedrigen Erntefixkosten $\left(F_{n}=10 € \mathrm{ha}^{-1}\right)$ konstante (vgl. alternativ u. a. Coordes 2014a, S. $120 \mathrm{ff}$; Halbritter und Deegen 2015) Bestandesbegründungskosten von $C=5.000 € \mathrm{ha}^{-1}$ eingeführt, erweitert sich die zu maximierende Zielfunktion [2-43] zu:

$$
\begin{aligned}
& \max _{T, \boldsymbol{\sigma}_{T-1}} a_{C}\left(T, N_{0}, \boldsymbol{\sigma}_{T-1}, P\left(V_{E}\right), r, F, C\right) \\
& \quad=\max _{T, \boldsymbol{\sigma}_{T-1}}\left(-C+\sum_{t=1}^{T-1} \frac{\left(\lambda_{t}\left(\sigma_{t}, t, \boldsymbol{\sigma}_{t-1}, N_{0}\right) \cdot P\left(V_{E}\right)-F\left(\sigma_{t}\right)\right)}{(1+r)^{t}}+\frac{\left(V_{B}\left(T, N_{0}, \boldsymbol{\sigma}_{T-1}\right) \cdot P\left(V_{E}\right)-F\right)}{(1+r)^{T}}\right) \cdot \frac{r \cdot(1+r)^{T}}{(1+r)^{T}-1}, \\
& 0 \leq \boldsymbol{\sigma}_{T-1}<1, \\
& 0 \mathrm{a}<T \leq 100 \mathrm{a}, \\
& C=\text { konst. } \\
& C \in \mathbb{R}^{\geq 0} .
\end{aligned}
$$

Die Zielgröße wird durch die Einbeziehung der Bestandesbegründungskosten zur Brutto-Bodenrente (,jährlicher Holzproduktionswert“; vgl. Möhring et al. 2006) - sie reduziert sich ggü. der bestandesbegründungskostenfreien Variante deutlich um $49 \%$ auf $110 € \mathrm{ha}^{-1} \mathrm{a}^{-1}$.

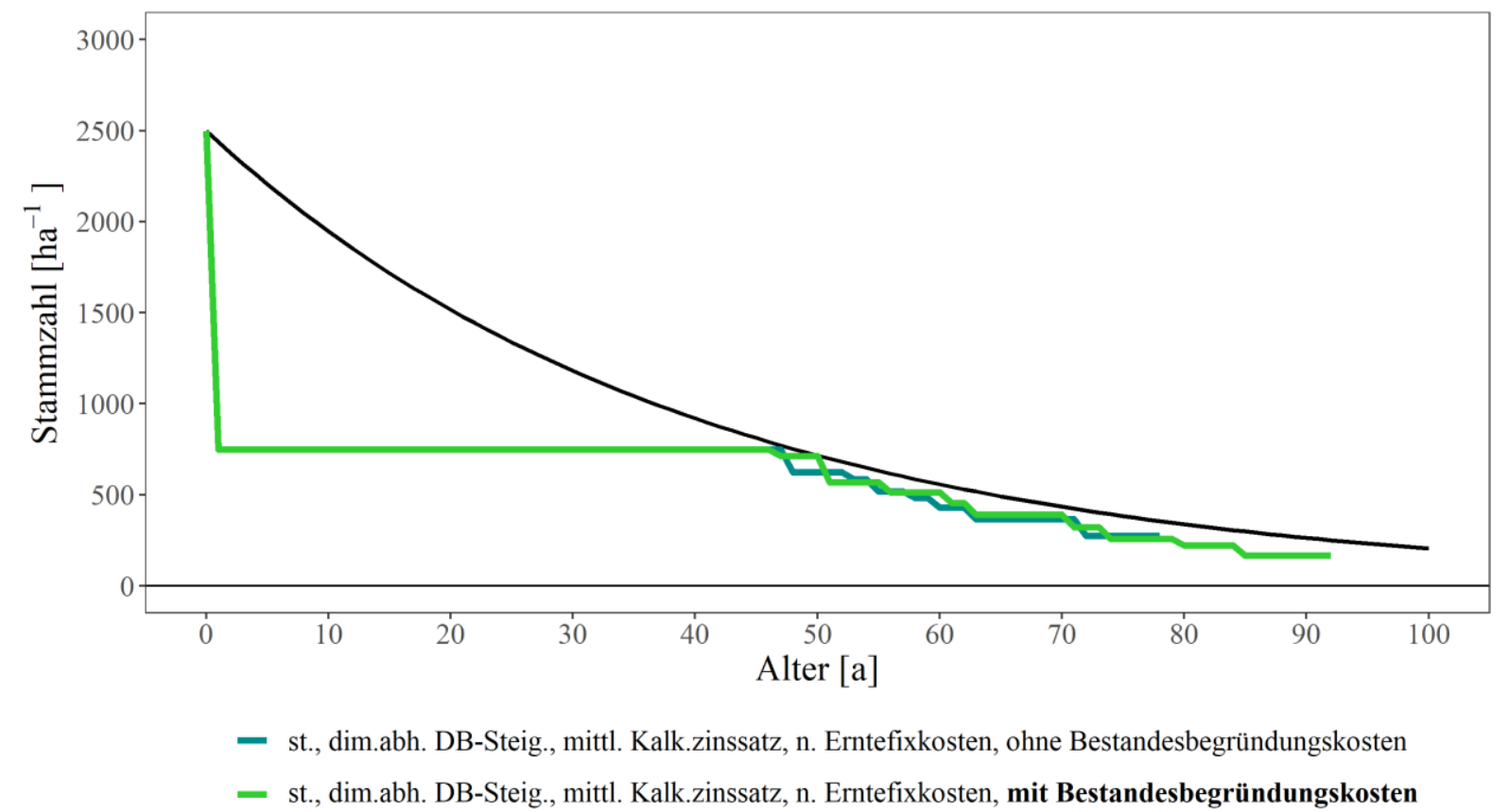

Abbildung 39: Altersabhängige Stammzahlentwicklungen bei Bestandesbegründungskosten (hellgrün) gemäß Zielfunktion [2-47] im Vergleich zum bestandesbegründungskostenfreien Optimum bei starker, dimensionsabängiger DB-Steigerung, mittlerem Kalkulationszinssatz sowie niedrigen Erntefixkosten (hellcyan) und zum naturalen Optimum ohne Folgebestand (schwarz). 
Da die Anfangsstammzahl $N_{0}$, anders als z. B. bei Halbritter und Deegen (2015), keine Entscheidungsvariable des Optimierungsmodells und zudem unabhängig von den Bestandesbegründungskosten $C$ festgelegt ist, haben die Bestandesbegründungskosten keinen direkten Einfluss auf die Vornutzung. Die Grenznutzenanalyse der Vornutzung unterscheidet sich grundsätzlich nicht von Optimalitätsbedingung [2-44] in Abschnitt 2.2.6. Indirekt aber kommt es über die Erhöhung des Endnutzungszeitpunkts $T$ (siehe unten) zu Auswirkungen auf die Vornutzungen - die optimalen Stammzahlentwicklungen weichen minimal vom Optimum aus Ab-

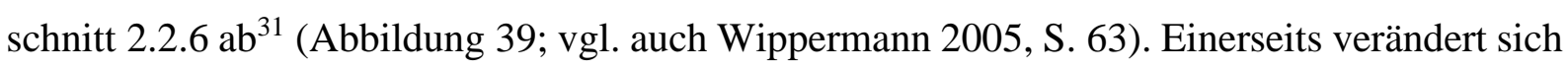
die Wuchsraumopportunität (zweiter Term der rechten Gleichungsseite in [2-44]) durch die Verschiebung der Realisationszeitpunkte eines Teils der von Baum $j$ beeinflussten Bäume. Andererseits spielen die in Abschnitt 2.2.6 diskutierten Wechselwirkungen aufgrund der Berücksichtigung von Erntefixkosten auch hier eine Rolle.

In der Optimalitätsbedingung der Endnutzungsentscheidung kommt es durch die Einbeziehung von Bestandesbegründungskosten zu einer Minderung der Brutto-Bodenrente $a_{C}$, d. h. der Flächenopportunität (zweiter Term der rechten Gleichungsseite):

$$
\begin{aligned}
w_{B}\left(T, N_{T-1}, P\left(V_{E}\right)\right) & +(F \cdot r) \\
& =W_{B}\left(T-1, \boldsymbol{N}_{T-2}, P\left(V_{E}\right)\right) \cdot r+a_{C}\left(T, \boldsymbol{N}_{T-1}, P\left(V_{E}\right), r, F, C\right) .
\end{aligned}
$$

Die Begründung des Folgebestandes wird dadurch unattraktiver, die Grenzkosten des Bestandeserhalts sinken und die Endnutzung verzögert sich - hier, mit Bestandesbegründungskosten in Höhe von $5.000 €$ ha $^{-1}$, um 14 Jahre auf $T_{C}^{*}=92$ a. Die Höhe der Bestandesbegründungskosten entscheidet dabei über die Verzögerung der Endnutzung und somit indirekt auch über die Stärke des Einflusses auf die Vornutzung.

Die Entwicklung der Gesamtwuchsleistung über dem Bestandesalter wird durch die Einführung der Bestandesbegründungskosten annähernd nicht beeinflusst (Abbildung 40) - aufgrund der verlängerten Produktionsdauer reduziert sich der durchschnittliche Bestandesvolumenzuwachs um $8 \%$ auf 9,9 $\mathrm{Fm} \mathrm{ha}^{-1} \mathrm{a}^{-1}$. Das verbleibende Bestandesvolumen (Vorrat) verläuft auf ähnlichem Niveau wie ohne Bestandesbegründungskosten und liegt zum Zeitpunkt der Endnutzung bei $290 \mathrm{Fm} \mathrm{ha}^{-1} \mathrm{a}^{-1}$.

\footnotetext{
${ }^{31}$ Nicht unerwähnt soll an dieser Stelle bleiben, dass zumindest ein Teil der geringfügigen Abweichungen zwischen den optimalen Stammzahlentwicklungen immer auch auf die bereits diskutierten lokalen Minima des umfangreichen Optimierungsproblems zurückgeführt werden kann.
} 


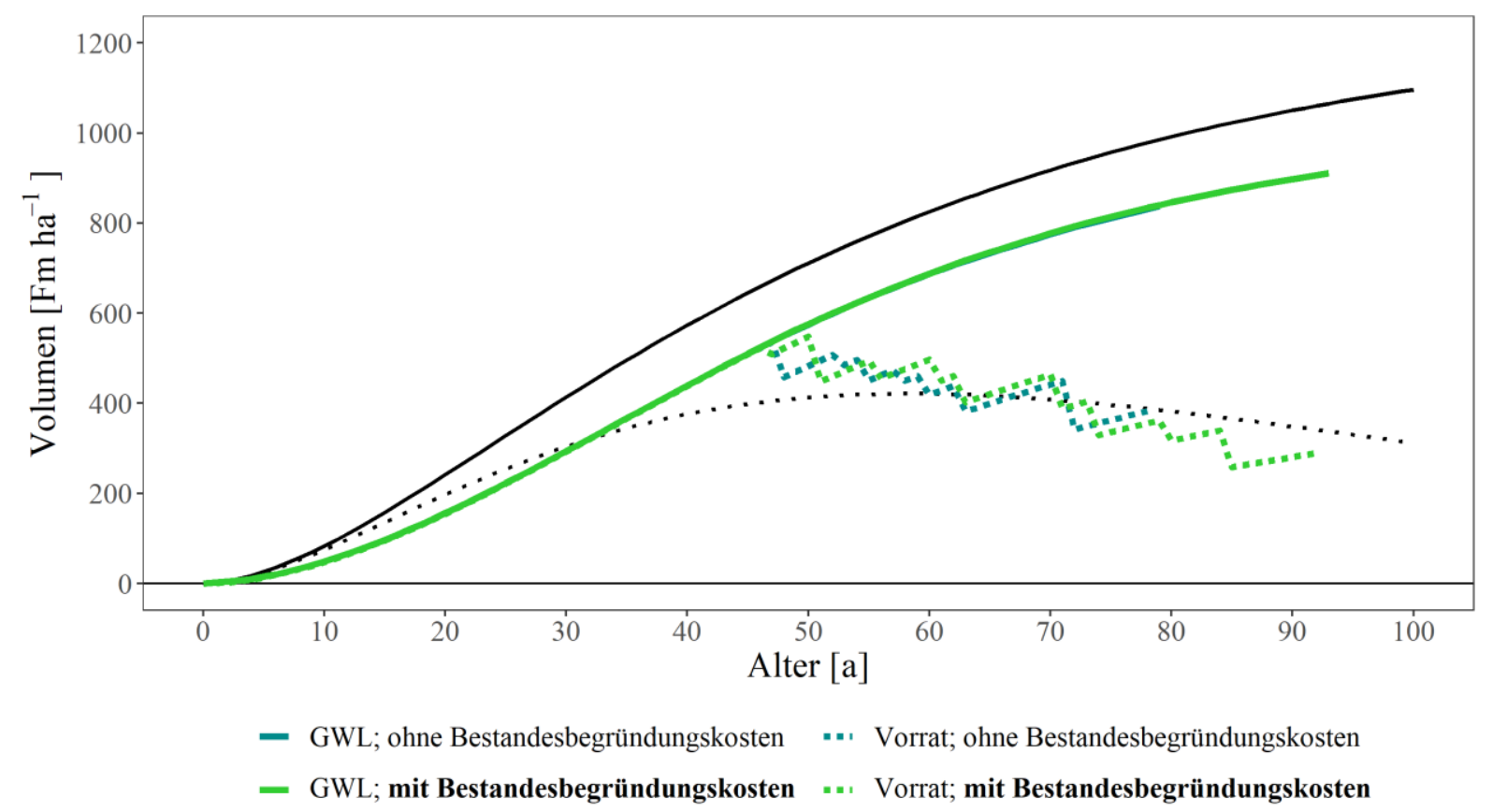

Abbildung 40: Altersabhängige Gesamtwuchsleistung und Vorratsentwicklung bei Bestandesbegründungskosten (hellgrün) gemäß Zielfunktion [2-47] im Vergleich zum bestandesbegründungskostenfreien Optimum bei starker, dimensionsabhängiger DB-Steigerung, mittlerem Kalkulationszinssatz sowie niedrigen Erntefixkosten (hellcyan) und zum naturalen Optimum ohne Folgebestand (schwarz).

\subsection{8 Überlebensrisiken}

Die Berücksichtigung von Überlebensrisiken bei der ökonomischen Analyse forstlicher Bestandesbehandlung erscheint aus vielerlei Hinsicht angebracht und sinnvoll:

- Die forstliche Produktion beruht auf natürlichen Prozessen - biotische (z. B. Insektenfraß oder Pilzbefall) und abiotische (z. B. Stürme, Frost, Dürre oder Hagel) Risiken sind fester Bestandteil der Forstwirtschaft (Möllmann und Möhring 2018).

- Die extrem langen Produktionszeiträume bedingen eine langfristige Exposition der Waldbestände ggü. den natürlichen Risikofaktoren (Staupendahl und Zucchini 2011).

- Der fortschreitende Klimawandel wird über die Veränderung der durchschnittlichen Klimabedingungen sowie der veränderten Häufigkeit und Intensität von Extremwetterereignissen die Überlebenswahrscheinlichkeiten unserer Baumarten (artspezifisch) verändern (Brandl et al. 2020; vgl. u. a. auch Seidl et al. 2011; Haarsma et al. 2013; Seidl et al. 2017).

- Überlebensrisiken senken u. a. den ökonomischen Erfolg der forstlichen Produktion, beeinflussen damit das forstliche Management (z. B. Staupendahl und Möhring 2011 oder Griess und Knoke 2013) und der fortschreitende Klimawandel wird diese Effekte weiter verstärken (vgl. u. a. Kolström et al. 2011; Hanewinkel et al. 2013). 
Risiken werden im Folgenden als „Abweichung von einem erwarteten Wert“ (Paul und Benfer 2019, S. 31) verstanden, wobei in dieser Arbeit mit den Überlebensrisiken nur negative Abweichungen einbezogen werden (vgl. Downside-Risikomaße; Beinhofer 2009, S. 74 ff.). Die Überlebensrisiken beziehen sich dabei auf den altersabhängigen Ausfall des Produktionsmittels Baum bzw. Bestand durch biotisch oder abiotisch bedingte Mortalität (Brandl und Falk 2019). Die Integration in das bestehende Modell geschieht in Form von Überlebenswahrscheinlichkeiten und der Berechnung eines Erwartungswertes ${ }^{32}$ der Zielgröße, als mit den jeweiligen Eintrittswahrscheinlichkeiten und Produktionsdauern gewichteter Mittelwert aller möglichen Brutto-Bodenrenten (vgl. z. B. Möllmann und Möhring 2017).

Durch die Einbeziehung der Überlebenswahrscheinlichkeiten existiert folglich nicht mehr nur eine mögliche Brutto-Bodenrente als jährliche Erfolgsgröße der forstlichen Produktion. Neben der planmäßigen Endnutzung kann zu jedem vorhergehenden Zeitpunkt eine kalamitätsbedingte Zwangsnutzung mit dementsprechend veränderter Brutto-Bodenrente und verkürzter Produktionsdauer eintreten - dabei fällt der Bestand entweder komplett aus (Zwangsnutzung) oder bleibt im Ganzen erhalten (Bernoulli-verteiltes Ereignis). Die Quantifizierung der Wahrscheinlichkeit für das jeweilige Eintreten einer Zwangsnutzung (Zufallsereignis) erfolgt in Anlehnung an Staupendahl und Möhring (2011) über eine altersabhängige, zweiparametrische Weibull-Überlebensfunktion $S(t)$, die nach Staupendahl (2011, S. 12) die Wahrscheinlichkeit angibt, „dass ein zufällig ausgewählter Bestand den Zeitpunkt $t$ überlebt, der kalamitätsbedingte Ausfall [des Bestandes] also erst nach $t$ eintreten wird (Überlebenswahrscheinlichkeit)“ - es gilt:

$$
\begin{aligned}
& \qquad S\left(t, S_{100}, \alpha\right)=S_{100}\left(\frac{t}{100}\right)^{\alpha}, \\
& 0 \leq S_{100} \leq 1, \\
& S_{100} \in \mathbb{R}, \\
& \alpha \in \mathbb{R}^{\geq 0},
\end{aligned}
$$

mit der Überlebensrisikostärke $S_{100}$ sowie der Überlebensrisikoart $\alpha$. Während die Überlebensrisikostärke $S_{100}$ die Wahrscheinlichkeit angibt, dass ein zufällig ausgewählter Waldbestand

\footnotetext{
${ }^{32}$ Mit der Verwendung des Erwartungswertes als Zielgröße wird eine risikoneutral entscheidende Person unterstellt - davon abweichende Risikoaversionen oder -sympathien bedürften der Berechnung des auf der individuellen Nutzenfunktion beruhenden Sicherheitsäquivalents als Zielgröße (Möhring 1994, $14 \mathrm{ff}$.$) .$
} 
ein Alter von 100 Jahren erreicht, charakterisiert die Überlebensrisikoart $\alpha$ die Form der Überlebensfunktion (Möllmann und Möhring 2017). $\alpha<1$ repräsentieren durch eine streng monoton fallende Ausfallrate sogenannte Jugendrisiken, $\alpha=1$ steht mit einer konstanten Ausfallrate für altersindifferente Überlebensrisiken und $\alpha>1$ zeigen durch steigende Ausfallraten sogenannte Altersrisiken an (Staupendahl 2011).

Die Brutto-Bodenrente der planmäßigen Nutzung entspricht der Brutto-Bodenrente aus Abschnitt 2.2.7 $a_{C}$ (Zielfunktion [2-47]):

$$
\begin{aligned}
a_{C}\left(T, N_{0},\right. & \left.\boldsymbol{\sigma}_{T-1}, P\left(V_{E}\right), r, F, C\right) \\
\quad & \left(-C+\sum_{t=1}^{T-1} \frac{\left(\lambda_{t}\left(\sigma_{t}, t, \boldsymbol{\sigma}_{t-1}, N_{0}\right) \cdot P\left(V_{E}\right)-F\left(\sigma_{t}\right)\right)}{(1+r)^{t}}+\frac{\left(V_{B}\left(T, N_{0}, \boldsymbol{\sigma}_{T-1}\right) \cdot P\left(V_{E}\right)-F\right)}{(1+r)^{T}}\right) \cdot \frac{r \cdot(1+r)^{T}}{(1+r)^{T}-1} .
\end{aligned}
$$

Die Wahrscheinlichkeit, mit der diese Brutto-Bodenrente realisiert werden kann, entspricht der Überlebenswahrscheinlichkeit bis zum planmäßigen Endnutzungszeitpunkt $S(T)$ und kann direkt mittels Überlebensfunktion [2-49] bestimmt werden.

Fällt der Bestand vorzeitig kalamitätsbedingt aus (Zwangsnutzung), verringert sich nicht nur die Produktionsdauer $T_{Z}<T$, sondern aufgrund von erhöhten Erntekosten sowie verringerten Holzerlösen auch der Deckungsbeitrag der Endnutzung (u. a. Dieter 2001). Es gilt für die Brutto-Bodenrenten der Zwangsnutzungen:

$$
\begin{aligned}
a_{Z}\left(T_{Z}, N_{0}, \boldsymbol{\sigma}_{T_{Z}-1}, P\left(V_{E}\right), r, F, C, \varphi, \Phi\right) \\
=\left(-C+\sum_{t=1}^{T_{Z}-1} \frac{\lambda_{t}\left(\sigma_{t}, t, \sigma_{t-1}, N_{0}\right) \cdot P\left(V_{E}\right)-F\left(\sigma_{t}\right)}{(1+r)^{t}}+\right. \\
\left.\frac{V_{B}\left(T_{Z}, N_{0}, \sigma_{T_{Z}-1}\right) \cdot P\left(V_{E}\right) \cdot(1-\varphi)-\Phi-F}{(1+r)^{T} Z}\right) \cdot \frac{r \cdot(1+r)^{T} Z}{(1+r)^{T} Z-1}
\end{aligned}
$$

$\varphi, \Phi \in \mathbb{R}^{\geq 0}$

Dabei mindert $\varphi$ die Deckungsbeiträge im Falle einer Kalamität relativ und $\Phi$ berücksichtigt als absoluter Reduktionsfaktor kalamitätsspezifische Erntefixkosten in Form von z. B. Flächenräumungs- und Flächenaufbereitungskosten (vgl. u. a. Möllmann und Möhring 2018).

Die Wahrscheinlichkeiten für das Eintreten der Zwangsnutzungen zum Zeitpunkt $T_{Z}$ und damit für die Realisierung einer reduzierten Brutto-Bodenrenten können als unbedingten Ausfallwahrscheinlichkeiten $s\left(T_{Z}\right)$ mittels Überlebensfunktion [2-49] bestimmt werden es gilt:

$$
s\left(T_{Z}, S_{100}, \alpha\right)=S\left(T_{Z}-1, S_{100}, \alpha\right)-S\left(T_{Z}, S_{100}, \alpha\right) .
$$


Als Brutto-Bodenrente der risikobehafteten forstlichen Produktion ergibt sich schließlich ein Erwartungswert $\hat{E}\left[a_{S}\right]$ als mit den Eintrittswahrscheinlichkeiten $S(T)$ und $s\left(T_{Z}\right)$ sowie den Produktionsdauern $\mathrm{T}$ und $T_{Z}$ gewichteter Mittelwert der Brutto-Bodenrenten $a_{C}$ und $a_{Z}$ (Staupendahl und Möhring 2011; Möllmann und Möhring 2017). Es gilt:

$$
\begin{aligned}
& \max _{T, \boldsymbol{\sigma}_{T-1}} \hat{E}\left[a_{S}\left(T, N_{0}, \boldsymbol{\sigma}_{T-1}, P\left(V_{E}\right), r, F, C, S_{100}, \alpha, \varphi, \Phi\right)\right] \\
& =\max _{T, \boldsymbol{\sigma}_{T-1}} \frac{\left(S\left(T, S_{100}, \alpha\right) \cdot T \cdot a_{C}\left(T, N_{0}, \boldsymbol{\sigma}_{T-1}, P\left(V_{E}\right), r, F, C\right)+\sum_{T_{Z}=1}^{T} s\left(T_{Z}, S_{100}, \alpha\right) \cdot T_{Z} \cdot a_{Z}\left(T_{Z}, N_{0}, \boldsymbol{\sigma}_{T_{Z}-1}, P\left(V_{E}\right), r, F, C, \varphi, \Phi\right)\right)}{\left(S\left(T, S_{100}, \alpha\right) \cdot T+\sum_{T_{Z}}^{T}{ }_{1} s\left(T_{Z}, S_{100}, \alpha\right) \cdot T_{Z}\right)} \\
& 0 \leq \boldsymbol{\sigma}_{T-1}<1, \\
& 0 \mathrm{a}<T \leq 100 \mathrm{a} .
\end{aligned}
$$

Die für den Variantenvergleich gewählten und in Abbildung 41 dargestellten Überlebensfunktionen gemäß [2-49] repräsentieren mittlere $\left(S_{100, m}=0,5\right)$ und hohe $\left(S_{100, h}=0,1\right)$ Altersri$\operatorname{siken}^{33}(\alpha=2,5)$. Bei beiden Varianten wird der Deckungsbeitrag bei kalamitätsbedingter Zwangsnutzung um $\varphi=40 \%$ vermindert. Hinzu kommt ein pauschaler Abzug je Zwangsnutzung von $\Phi=500 € \mathrm{ha}^{-1}$ (vgl. Möllmann und Möhring 2017).

Die risikobeeinflussten Zielgrößen $\hat{E}\left[a_{S}\right]$ liegen deutlich unterhalb der Brutto-Bodenrente aus Abschnitt 2.2.7. Die mittleren Altersrisiken führen zu einer Absenkung der durchschnittlichen Erfolgsgröße um $27 \%$ auf $81 € \mathrm{ha}^{-1} \mathrm{a}^{-1}$. Bei hohen Altersrisiken fällt die risikobedingte Absenkung noch größer aus - der Erwartungswert der Brutto-Bodenrente fällt ggü. der risikofreien Brutto-Bodenrente um $74 \%$ auf dann nur noch $29 € \mathrm{ha}^{-1} \mathrm{a}^{-1}$. Zur Erreichung dieser Erfolgsgrößen verändert sich die Bestandesbehandlung sowohl in der Vor- als auch in der Endnutzung. Je höher die Altersrisiken, desto stärker sind Vornutzungseingriffe durchschnittlich ab einem Alter von etwa 45 Jahren - gleichzeitig verkürzen die Altersrisiken die Produktionsdauer um 4 bzw. 8 Jahre auf $T_{S[m]}^{*}=88$ a bzw. $T_{S[h]}^{*}=84$ a (Abbildung 42). Dementsprechend wird mit zunehmendem Alter umso mehr auf laufenden Bestandesvolumenzuwachs verzichtet, je höher die Altersrisiken sind - in der Variante der extrem hohen Altersrisiken wachsen im Alter von 83 Jahren aufgrund der äußerst geringen Stammzahl (54 ha $\left.{ }^{-1}\right)$ nur noch 1,9 Fm ha ${ }^{-1} \mathrm{a}^{-1}$ zu (Abbildung 43).

\footnotetext{
${ }^{33}$ Empirische Studien (z. B. Staupendahl und Zucchini 2011; Brandl et al. 2020) zeigen für die mitteleuropäischen Hauptbaumarten eine Tendenz zu mit dem Alter steigenden Ausfallraten (Altersrisiken), wobei eine gewisse Unterschätzung von Jugendrisiken auf die Datengrundlage zurückzuführen ist (Möllmann und Möhring 2017).
} 


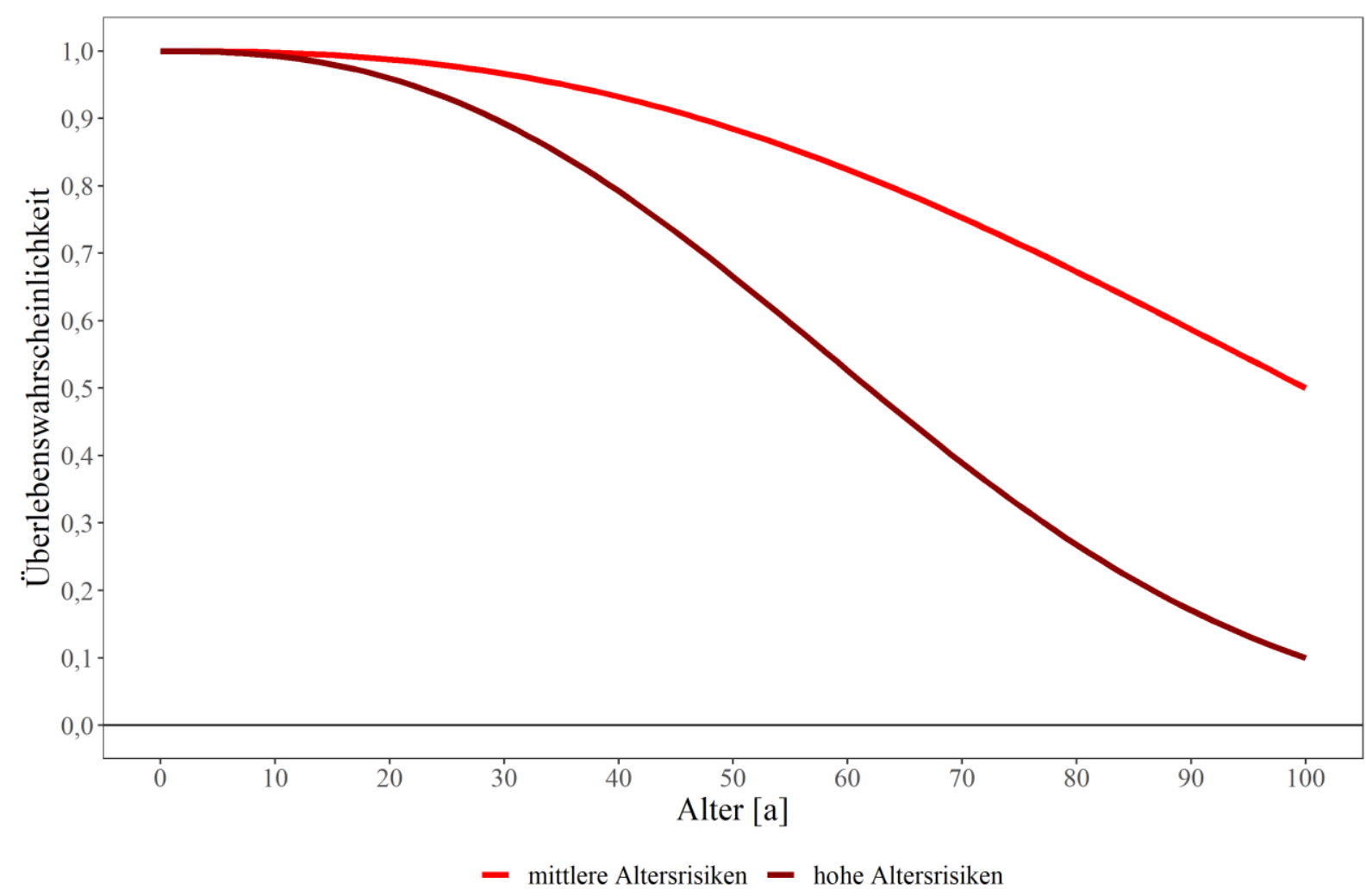

Abbildung 41: Altersabhängige Überlebenswahrscheinlichkeiten bei mittleren (hellrot) und starken (dunkelrot) Überlebensrisiken gemäß Überlebensfunktion [2-49].

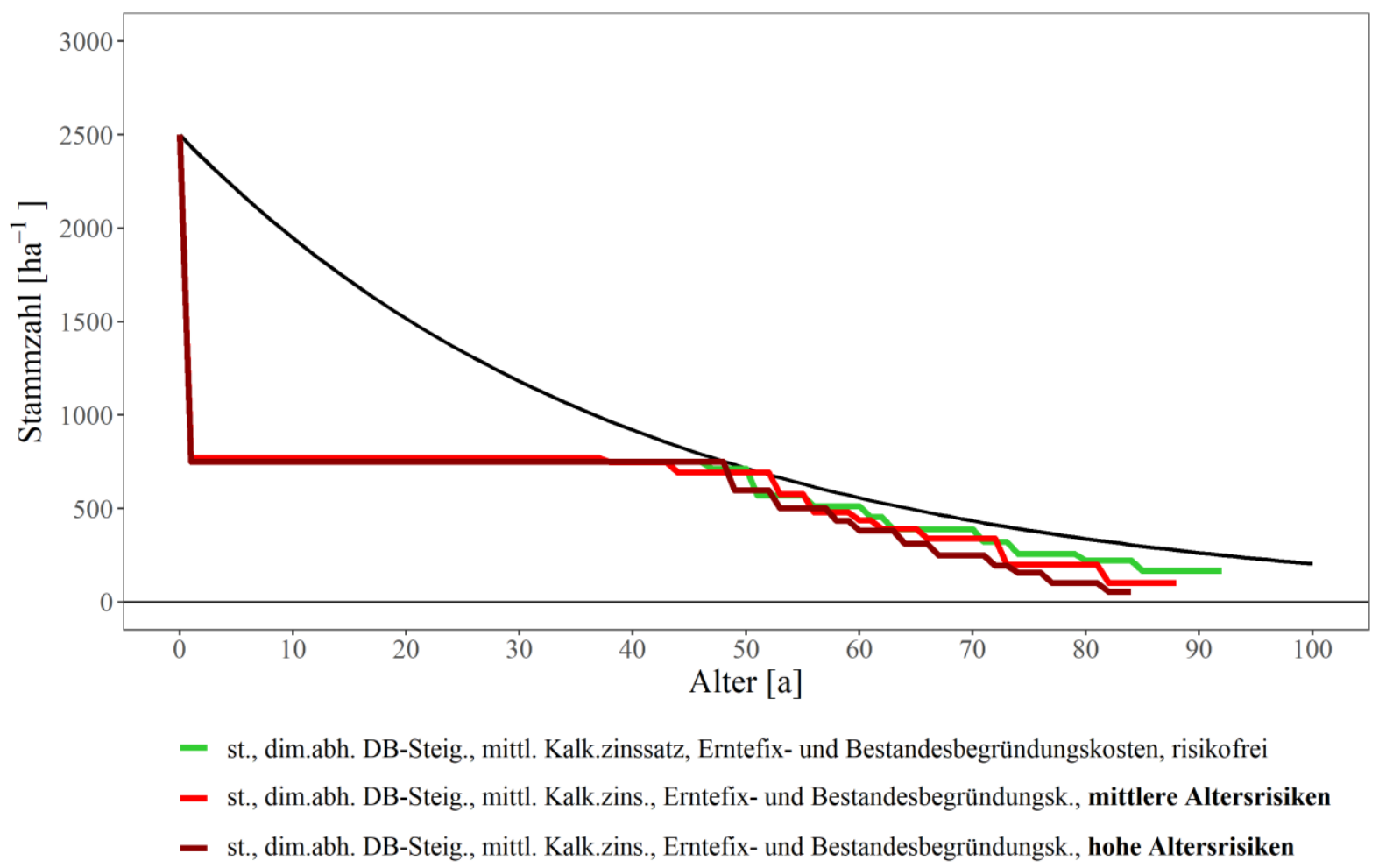

Abbildung 42: Altersabhängige Stammzahlentwicklungen bei mittleren (hellrot) bzw. hohen (dunkelrot) Altersrisiken gemäß Zielfunktion [2-53] im Vergleich zum risikofreien Optimum bei starker, dimensionsabhängiger DB-Steigerung, mittlerem Kalkulationszinssatz sowie Erntefix- und Bestandesbegründungskosten (grün) und zum naturalen Optimum ohne Folgebestand (schwarz). 


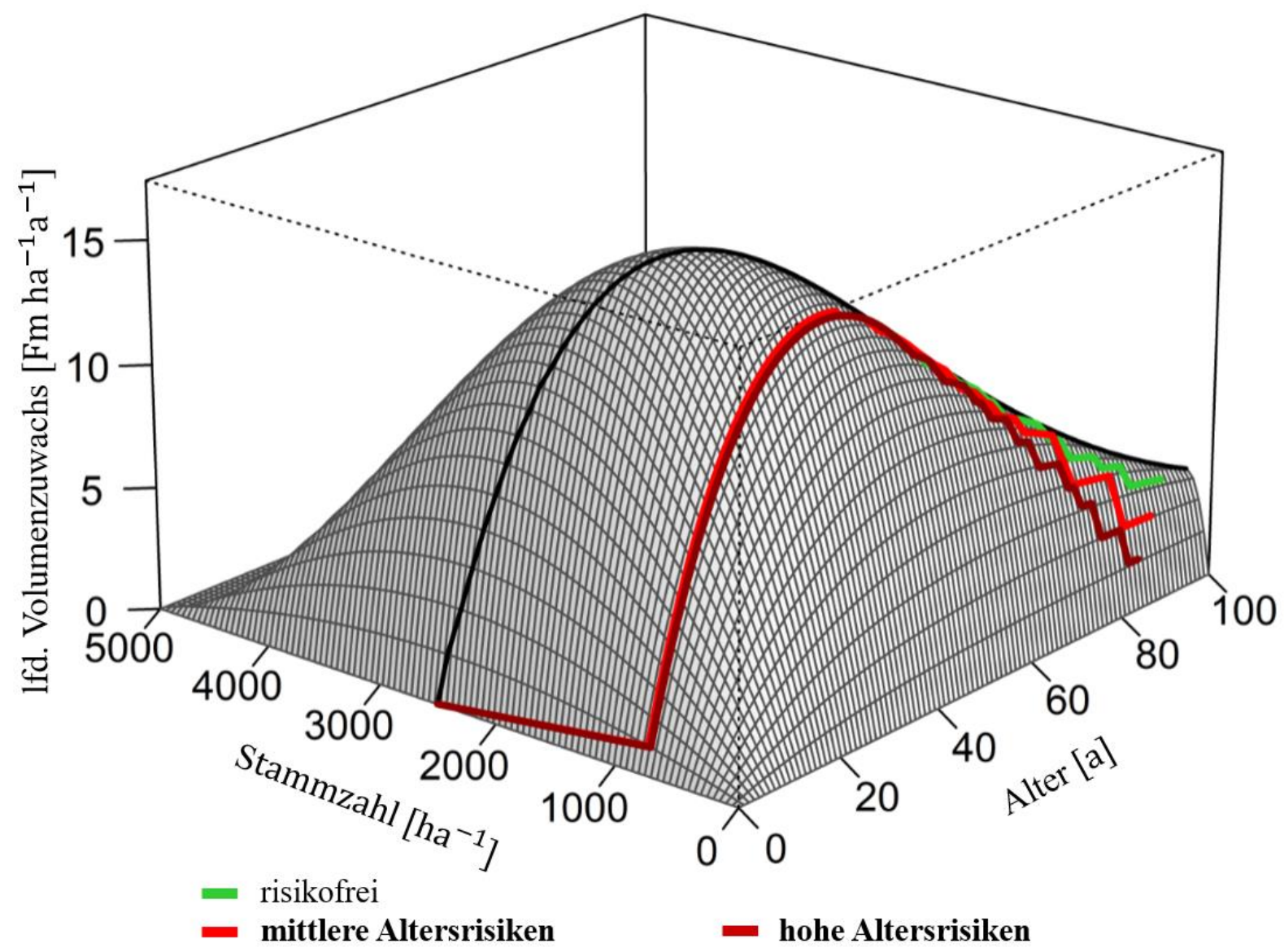

Abbildung 43: Alters- und stammzahlabhängiger laufender Bestandesvolumenzuwachs bei mittleren (hellrot) bzw. hohen (dunkelrot) Altersrisiken gemäß Zielfunktion [2-53] im Vergleich zum risikofreien Optimum bei starker, dimensionsabhängiger DB-Steigerung, mittlerem Kalkulationszinssatz sowie Erntefix- und Bestandesbegründungskosten (grün) und dem maximalen Volumenzuwachs (schwarz).

Bei dementsprechend leicht bis mäßig reduzierten Gesamtwuchsleistungen im letzten Drittel der forstlichen Produktion (Abbildung 44) steigt der durchschnittliche Bestandesvolumenzuwachs aufgrund der verkürzten Produktionsdauer bei mittleren Altersrisiken dennoch um $1 \%$ auf $10,0 \mathrm{Fm} \mathrm{ha}^{-1} \mathrm{a}^{-1}$ an bzw. sinkt bei hohen Altersrisiken nur minimal um $0,3 \%$ auf 9,8 Fm ha ${ }^{-1} \mathrm{a}^{-1} \mathrm{ab}$. Die verbleibenden Bestandesvolumina sinken in höheren Bestandesaltern deutlich (Abbildung 44) - zum jeweiligen Zeitpunkt der Endnutzung liegen die Vorräte bei $176 \mathrm{Fm} \mathrm{ha}^{-1}\left(S_{m} ;-39 \%\right.$ ggü. der risikofreien Variante) bzw. $90 \mathrm{Fm} \mathrm{ha}^{-1}\left(S_{h} ;-69 \%\right.$ ggü. der risikofreien Variante). 


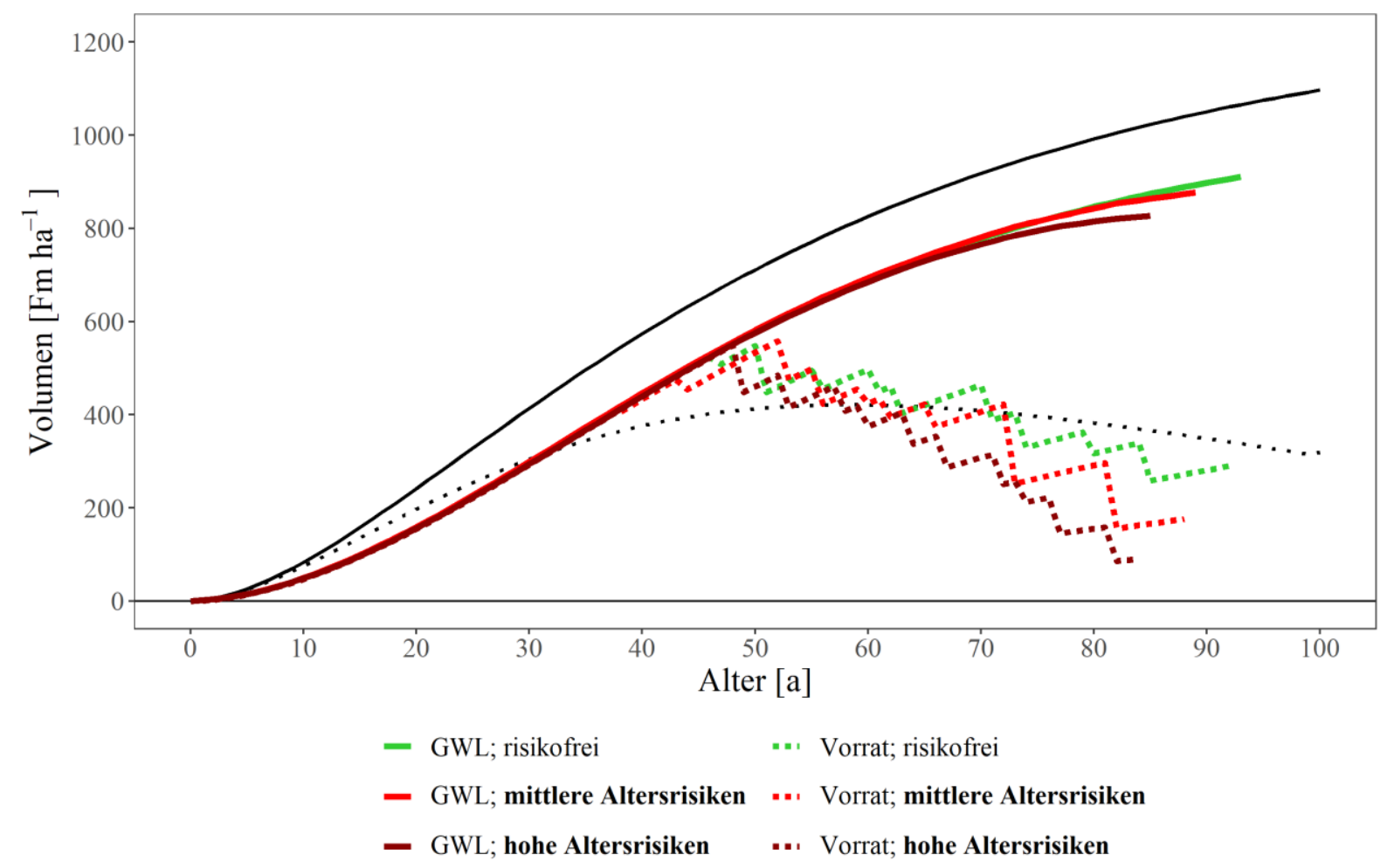

Abbildung 44: Altersabhängige Gesamtwuchsleistung und Vorratsentwicklung bei mittleren (hellrot) bzw. hohen (dunkelrot) Altersrisiken gemäß Zielfunktion [2-53] im Vergleich zum risikofreien Optimum bei starker, dimensionsabhängiger DB-Steigerung, mittlerem Kalkulationszinssatz sowie Erntefixund Bestandesbegründungskosten (grün) und zum naturalen Optimum ohne Folgebestand (schwarz).

In Anlehnung an Möllmann und Möhring (2017) erweitert sich die Optimalitätsbedingung der Endnutzung durch die Einbeziehung der Überlebensrisiken zu:

$$
\begin{aligned}
\hat{E}\left[w_{B}\left(T, N_{T-1}, P\left(V_{E}\right), S_{100}, \alpha, \varphi, \Phi\right)\right]+(F \cdot r) & \\
& =W_{B}\left(T-1, \boldsymbol{N}_{T-2}, P\left(V_{E}\right)\right) \cdot r+\hat{E}\left[a_{S}\left(T, N_{0}, \boldsymbol{\sigma}_{T-1}, P\left(V_{E}\right), r, F, C, S_{100}, \alpha, \varphi, \Phi\right)\right] .
\end{aligned}
$$

Sowohl der laufende Bestandeswertzuwachs (Teil der Grenznutzen des Bestandeserhalts) als auch die Flächenopportunität in Form der durchschnittlichen Erfolgsgröße des Folgebestandes (Teil der Grenzkosten des Bestandeserhalts) werden durch die reduzierte Eintrittswahrscheinlichkeit zukünftiger Ereignisse vermindert ${ }^{34}$. Der laufende Bestandeswertzuwachs zwischen den Jahren $T-1$ und $T$ wird nicht mit Sicherheit erreicht - mit einer gewissen Wahrscheinlichkeit (unbedingte Ausfallwahrscheinlichkeit $s(T)$ ) fällt der Bestand in diesem Zeitschritt aus und die kalamitätsbedingt verminderten Deckungsbeiträge kommen zum Tragen. Gleichzeitig

\footnotetext{
${ }^{34}$ Aufgrund der ausschließlichen Einbeziehung von Überlebensrisiken werden die zinseffektbedingten Grenznutzen und -kosten des Bestandeserhalts (zweiter Term der linken und erster Term der rechten Gleichungsseite in [2-54]) als sicher angesehen.
} 
liegt der Erwartungswert der Brutto-Bodenrente unterhalb der risikofrei berechneten BruttoBodenrente (siehe oben). Da folglich beide Seiten der Optimalitätsbedingung durch die Überlebensrisiken beeinflusst werden, fällt die Analyse der Wirkrichtung (frühere oder spätere Endnutzung) schwer. Möllmann und Möhring (2017) vollziehen die Auswirkungsanalyse daher über das Verhältnis von laufenden und durchschnittlichen Risikokosten $\left(K_{S}\right)$ - diese stellen dabei die jeweilige Differenz zwischen der risikofrei kalkulierten Größe und dem Erwartungswert dar. Optimalitätsbedingung [2-54] kann demnach umgeformt werden zu:

$$
\begin{aligned}
w_{B}\left(T, N_{T-1}, P\left(V_{E}\right)\right) & -K_{S}\left[w_{B, S}\right]+(F \cdot r) \\
= & W_{B}\left(T-1, \boldsymbol{N}_{T-2}, P\left(V_{E}\right)\right) \cdot r+a_{C}\left(T, \boldsymbol{N}_{T-1}, P\left(V_{E}\right), r, F, C\right)-\overline{K_{S}}\left[a_{S}\right],
\end{aligned}
$$

mit den laufenden $K_{S}\left[w_{B, S}\right]$ und den durchschnittlichen $\overline{K_{S}}\left[a_{S}\right]$ Risikokosten.

Das Verhältnis zwischen den laufenden und durchschnittlichen Risikokosten entscheidet über die Richtung der Endnutzungsverschiebung. Bei Gleichheit bleibt der Endnutzungszeitpunkt ggü. der risikofreien Kalkulation unverändert. Liegen die laufenden oberhalb der durchschnittlichen Risikokosten, d. h. $K_{S}\left[w_{B, S}\right]>\overline{K_{S}}\left[a_{S}\right]$, wird die linke Seite in Gleichung [2-55] ggü. dem risikofreien Optimum stärker reduziert als die rechte Gleichungsseite. Ceteris paribus wird die Ernte des Bestandes somit attraktiver und der Endnutzungszeitpunkt verschiebt sich ausgehend vom risikofreien Optimum zu niedrigeren Bestandesaltern. Andersherum verschiebt sich der Endnutzungszeitpunkt ausgehend vom risikofreien Optimum c. p. zu höheren Bestandesaltern, wenn gilt $\overline{K_{S}}\left[a_{S}\right]>K_{S}\left[w_{B, S}\right]$, d. h. wenn die durchschnittlichen oberhalb der laufenden Risikokosten liegen (Möllmann und Möhring 2017).

Dementsprechend, und auch intuitiv einleuchtend, verkürzen die im Modell eingeführten Altersrisiken c. p. die Produktionsdauer. Bei Altersrisiken liegen die laufenden Risikokosten in hohen Bestandesaltern oberhalb der durchschnittlichen Risikokosten - der risikobehafteten Produktionsphase wird ausweichend mit der Herabsetzung des Endnutzungszeitpunkts begegnet (vgl. Staupendahl und Möhring 2011; Möllmann und Möhring 2017). 
Für die Vornutzungen gilt per Analogieschluss in Anlehnung an die Erweiterung der Optimalitätsbedingung [2-54]:

$$
\begin{aligned}
\hat{E}\left[w_{j}\left(t_{j}, N_{t_{j}-1}, P\left(V_{E}\right), S_{100}, \alpha, \varphi, \Phi\right)\right]+\left(\frac{F}{M_{t_{j}-1}} \cdot(1+r)-\frac{F}{M_{t_{j}}}\right) \\
=W_{j}\left(t_{j}-1, N_{t_{j}-2}, P\left(V_{E}\right)\right) \cdot r+\sum_{t=t_{j}}^{T} \sum_{\substack{k \in L_{t_{j}} \\
k \neq j}} \frac{\hat{E}\left[\Delta W_{k, j}\left(t_{k}, S_{100}, \alpha, \varphi, \Phi\right)\right]}{(1+r){ }^{\left(t_{k}-t_{j}\right)}},
\end{aligned}
$$

wobei der Erwartungswert der Wertveränderung an einem von Baum $j$ beeinflussten Baum $k$ neben der Überlebenswahrscheinlichkeit bis zum Zeitpunkt der jeweiligen Realisierung $t_{k}$ auch vom zeitlichen Abstand zwischen $t_{j}$ und $t_{k}$ abhängt. Durch Bezug der Überlebenswahrscheinlichkeit bis zum Zeitpunkt $t_{k}$ auf die Überlebenswahrscheinlichkeit bis zum Zeitpunkt $t_{j}$ entsteht eine bedingte Überlebenswahrscheinlichkeit ${ }^{35}$ bis zum Zeitpunkt $t_{k}$, d. h. eine Wahrscheinlichkeit dafür, dass Baum $k$ seinen planmäßigen Endnutzungszeitpunkt $t_{k}$ erreicht. In Anlehnung an die Berechung des Erwartungswertes der Brutto-Bodenrente (Funktion [2-53]) kann demnach der Erwartungswert der Wertveränderung an einem von Baum $j$ beeinflussten Baum $k$ berechnet werden als mit den Eintrittswahscheinlichkeiten aller möglichen Realisationszeitpunkte $t_{j} \leq t_{Z, k} \leq t_{k}$ gewichteter Mittelwert aller möglichen, durch den marginal verlängerten Erhalt von Baum $j$ an Baum $k$ bedingten, Wertveränderungen.

Auch hier werden sowohl der laufende Wertzuwachs des Einzelbaums (Teil der Grenznutzen des Einzelbaumerhalts) als auch die Wuchsraumopportunität (Teil der Grenzkosten des Einzelbaumerhalts) durch die Überlebensrisiken beeinflusst. Analog zur Optimalitätsbedingung der Endnutzung kann auch hier eine Umformulierung mit den laufenden und durchschnittlichen Risikokosten erfolgen:

$$
\begin{aligned}
w_{j}\left(t_{j}, N_{t_{j}-1}, P\left(V_{E}\right)\right) & -K_{S}\left[w_{j, S}\right]+\left(\frac{F}{M_{t_{j}-1}} \cdot(1+r)-\frac{F}{M_{t_{j}}}\right) \\
& =W_{j}\left(t_{j}-1, \boldsymbol{N}_{t_{j}-2}, P\left(V_{E}\right)\right) \cdot r+\sum_{t=t_{j}}^{T} \sum_{\substack{k \in L_{t_{j}} \\
k \neq j}} \frac{\left(\Delta W_{k, j}\left(t_{k}\right)-\overline{K_{S}}\left[\Delta W_{k, j}\right]\right)}{\left.(1+r)^{\left(t_{k}-t_{j}\right.}\right)} .
\end{aligned}
$$

\footnotetext{
${ }^{35}$ Vgl. Satz von Bayes (Fahrmeir et al. 2011, S. 213), wonach sich Wahrscheinlichkeiten durch Bedingungen verändern können. Die Überlebenswahrscheinlichkeit bis zum Zeitpunkt $t_{j}$ stellt demnach die Bedingung dar, welche die Wahrscheinlichkeit für das Erreichen des planmäßigen Endnutzungszeitpunkts $t_{k}$ beeinflusst. Es ergibt sich eine bedingte Wahrscheinlichkeit dafür, dass Baum $k$ zum Zeitpunkt $t_{k}$ planmäßig geerntet werden kann, unter der Voraussetzung, dass Zeitpunkt $t_{j}$ erreicht wird.
} 
Überwiegen die laufenden Risikokosten die Summe der diskontierten durchschnittlichen Risikokosten ist eine frühere Ernte des Einzelbaums im Vergleich zur risikofreien Variante c. p. vorteilhaft. Da das Verhältnis zwischen den laufenden Risikokosten und der Summe der diskontierten durchschnittlichen Risikokosten nun von den bedingten Ausfallwahrscheinlichkeiten $\mathrm{zu}$ allen Realisationszeitpunkten $t_{k}$ und zudem auch vom damit wechselwirkenden Zinseffekt abhängt, sind allgemeingültige Aussagen zur Verschiebungsrichtung einzelner Erntezeitpunkte, anders als bei der Endnutzungsentscheidung, nicht möglich. Insgesamt führen die in der Modellvariante gewählten mittelstarken Altersrisiken i. V. m. dem mittleren Kalkulationzinssatz zusätzlich zur früheren Endnutzung auch zur früheren Realisierung größerer Vornutzungsmenge (vgl. z. B. Loisel 2014).

\subsubsection{Behandlungsabhängige Überlebensrisiken}

In stark vereinfachter Weise wird mit der folgenden Modellerweiterung der Tatsache Rechnung getragen, dass die Überlebenswahrscheinlichkeit eines Waldbestandes nicht nur baumarten(vgl. z. B. Staupendahl und Zucchini 2011) und standortspezifisch (vgl. z. B. Schmidt et al. 2010), sondern auch abhängig von der waldbaulichen Behandlung ist (vgl. z. B. Albrecht et al. 2012). Ziel ist es, die Komplexität der Wechselwirkungen zwischen Bestandesbehandlung und Überlebenswahrscheinlichkeit (vgl. Albrecht 2009; Deegen und Matolepszy 2015) zu reduzieren und somit eine nachvollziehbare sowie leicht interpretierbare Analyse der Einflüsse auf die optimale Bestandesbehandlung zu ermöglichen. Gesucht wird dafür zunächst ein behandlungsabhängiger Treiber der Überlebenswahrscheinlichkeit eines Waldbestandes, der ohne komplexe Erweiterung ins Modell integriert werden kann. Dafür werden aus allen biotischen und abiotischen, die Überlebenswahrscheinlichkeit eines Waldbestandes beeinflussenden, Schadfaktoren ausschließlich Stürme aufgrund ihrer zentralen Bedeutung für die europäische Forstwirtschaft (vgl. Schelhaas et al. 2003) herausgegriffen. Als die Sturmschadenswahrscheinlichkeit beeinflussender und gleichzeitig direkt behandlungsabhängiger Parameter ${ }^{36}$ wird trotz uneinheitlicher Ergebnisse empirischer Sturmschadensanalysen (vgl. z. B. Albrecht 2009, S. 135 ff.) das

\footnotetext{
${ }^{36}$ Obwohl nicht explizit ausgewiesen, erhält die Bestandeshöhe, als mit der Baumart einflussreichster Faktor für die Sturmschadenswahrscheinlichkeit (vgl. u. a. Albrecht et al. 2012), bereits über die Altersabhängigkeit der Überlebensfunktion indirekt Eingang in das Modell. Den mit der Höhe (dem Alter) steigenden Überlebensrisiken (Altersrisiken) wird in Abschnitt 2.2.8 u. a. mit vorgezogenen Endnutzungszeitpunkten begegnet (indirekte Behandlungsabhängigkeit).

Weitere, die Sturmwurfwahrscheinlichkeit maßgeblich beeinflussende, direkte Behandlungsparameter, wie z. B. die (relative) Eingriffsstärke in den Jahren vor einem Sturmereignis, die Zeit zwischen letztem Eingriff und Sturmereignis oder der Durchforstungsquotient, d. h. das Verhältnis aus ausscheidender und verbleibender Baumdimension, (vgl. Lohmander und Helles 1987; Albrecht 2009, S. 143 f.; Donis et al. 2018) bleiben unberücksichtigt.
} 
H/D-Verhältnis ${ }^{37}$ gewählt. Es wird in Anlehnung an praxiserprobte Waldbaurichtlinien (z. B. BaySF 2009; vgl. Kramer und Akça 2008, S. 34) davon ausgegangen, dass die Sturmschadenswahrscheinlichkeit mit sinkenden mittleren H/D-Verhältnissen abnimmt. Bei der im Modell als behandlungsunabhängig angenommenen Bestandeshöhenentwicklung sinkt das mittlere H/DVerhältnis mit der Zunahme des mittleren Einzelbaumvolumens.

Es erfolgt die Festlegung, dass die Überlebensrisikostärke $S_{100}$ der Überlebensfunktion [2-49] aufbauend auf dem mittleren Altersrisiko $\left(S_{100, m}=0,5 ; \alpha=2,5\right)$ aus Abschnitt 2.2.8 in Abhängigkeit vom mittleren Einzelbaumvolumen (Stückvolumen) im Alter 50 a definiert wird ${ }^{38}$. Ausgehend vom bisherigen mittleren Einzelbaumvolumen im Alter 50 a in Höhe von 0,77 Fm kann die Überlebensrisikostärke nun durch höhere Stückvolumina (bis 1,4 Fm) auf Werte von $0,5<S_{100} \leq 0,9$ reduziert werden (Abbildung 45).

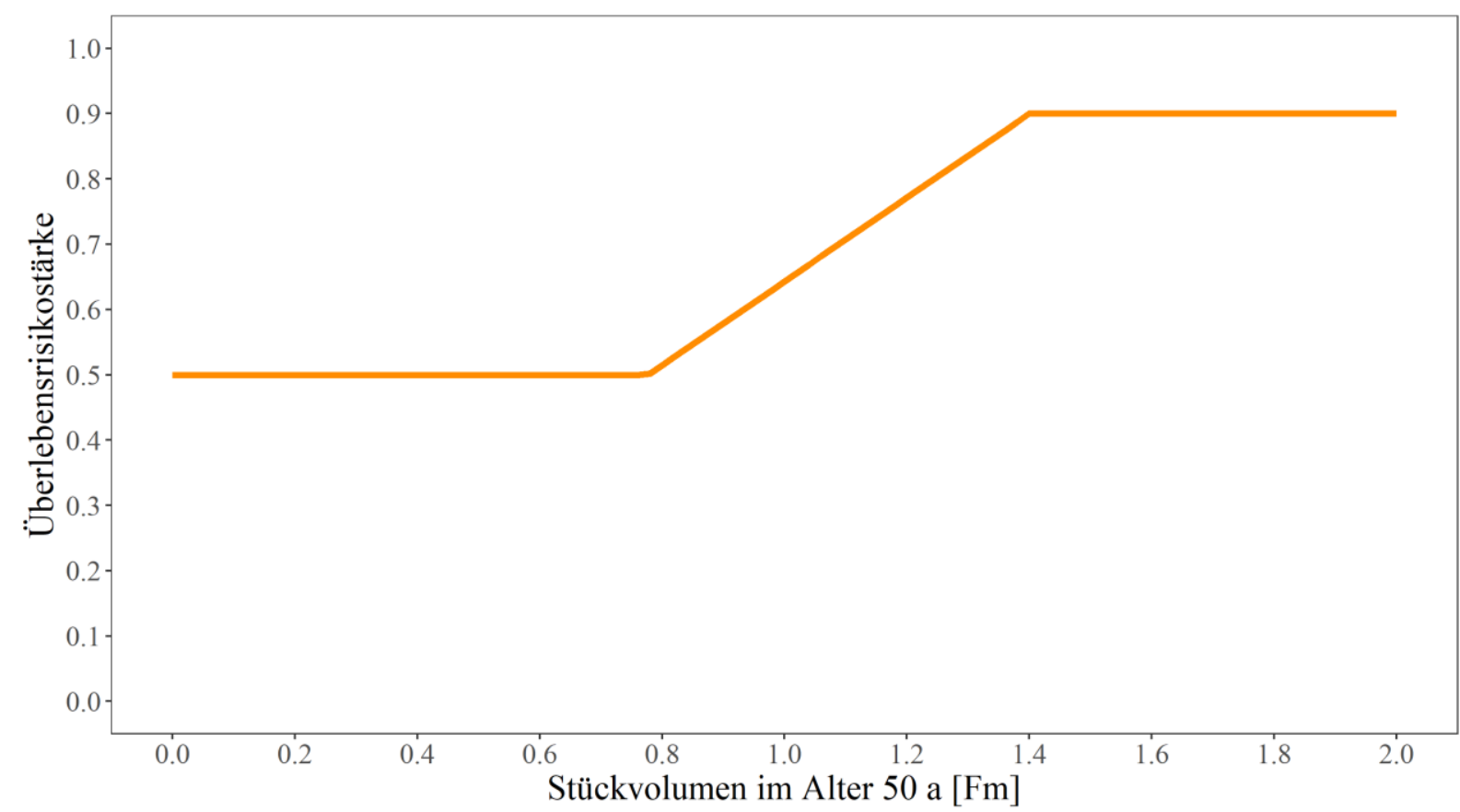

Abbildung 45: Überlebensrisikostärke (Überlebenswahrscheinlichkeit bis zum Alter 100 a) in Abhängigkeit vom Einzelbaumvolumen (Stïckvolumen) im Alter 50 a.

\footnotetext{
${ }^{37}$ Das H/D-Verhältnis wird auch als H/D-Wert oder Schlankheitsgrad bezeichnet und gibt das Verhältnis aus Baumhöhe und Baumdurchmesser in Brusthöhe (1,3 m) an (Kramer und Akça 2008, S. 34).

${ }^{38}$ Waldbauliche Eingriffe nach dem Alter 50 a haben somit keinen Einfluss auf die Überlebensrisikostärke.
} 
Zielfunktion [2-53] erweitert sich durch diese Abhängigkeit zu:

$$
\begin{aligned}
& \max _{T, \boldsymbol{\sigma}_{T-1}} \hat{E}\left[a_{S\left(V_{E}\right)}\left(T, N_{0}, \boldsymbol{\sigma}_{T-1}, P\left(V_{E}\right), r, F, C, S_{100}\left(V_{E}\left(50 \mathrm{a}, \boldsymbol{\sigma}_{49 \mathrm{a}}, N_{0}\right)\right), \alpha, \varphi, \Phi\right)\right] \\
& =\max _{T, \boldsymbol{\sigma}_{T-1}} \frac{S\left(T, S_{100}\left(V_{E}\left(50 \mathrm{a}, \boldsymbol{\sigma}_{49} \mathrm{a}, N_{0}\right)\right), \alpha\right) \cdot T \cdot a_{C}\left(T, N_{0}, \boldsymbol{\sigma}_{T-1}, P\left(V_{E}\right), r, F, C\right)}{\left(S\left(T, S_{100}\left(V_{E}\left(50 \mathrm{a}, \boldsymbol{\sigma}_{49}, N_{0}\right)\right), \alpha\right) \cdot T+\sum_{T_{Z}=1}^{T} S\left(T_{Z}, S_{100}\left(V_{E}\left(50 \mathrm{a}, \boldsymbol{\sigma}_{49}, N_{0}\right)\right), \alpha\right) \cdot T_{Z}\right)}+ \\
& \frac{\sum_{T_{Z}=1}^{T} s\left(T_{Z}, S_{100}\left(V_{E}\left(50 \mathrm{a}, \boldsymbol{\sigma}_{49}, N_{0}\right)\right), \alpha\right) \cdot T_{Z} \cdot a_{Z}\left(T_{Z}, N_{0}, \boldsymbol{\sigma}_{T_{Z}-1}, P\left(V_{E}\right), r, F, C, \varphi, \Phi\right)}{\left(S\left(T, S_{100}\left(V_{E}\left(50 \mathrm{a}, \boldsymbol{\sigma}_{49}{ }_{\mathrm{a}}, N_{0}\right)\right), \alpha\right) \cdot T+\sum_{T_{Z}=1}^{T} s\left(T_{Z}, S_{100}\left(V_{E}\left(50 \mathrm{a}, \boldsymbol{\sigma}_{49}{ }_{\mathrm{a}}, N_{0}\right)\right), \alpha\right) \cdot T_{Z}\right)},
\end{aligned}
$$

$0 \leq \sigma_{T-1}<1$

$0 \mathrm{a}<T \leq 100 \mathrm{a}$,

wobei das Einzelbaumvolumen $V_{E}\left(50 \mathrm{a}, \boldsymbol{\sigma}_{49}\right.$ a,$\left.N_{0}\right)$ und damit die Überlebensrisikostärke $S_{100}$ abhängig ist von der Bestandesbehandlungsgeschichte bis zum Alter 50 a. Die Berechnung der risikobeeinflussten Erfolgsgröße $\widehat{E}\left[a_{S\left(V_{E}\right)}\right]$ bleibt ansonsten unverändert ggü. Abschnitt 2.2.8.

Der Erwartungswert der Brutto-Bodenrente steigt ggü. Abschnitt 2.2.8 leicht um $2 \%$ auf $82 € \mathrm{ha}^{-1} \mathrm{a}^{-1}$ und bleibt damit $25 \%$ unterhalb der risikofreien Brutto-Bodenrente.

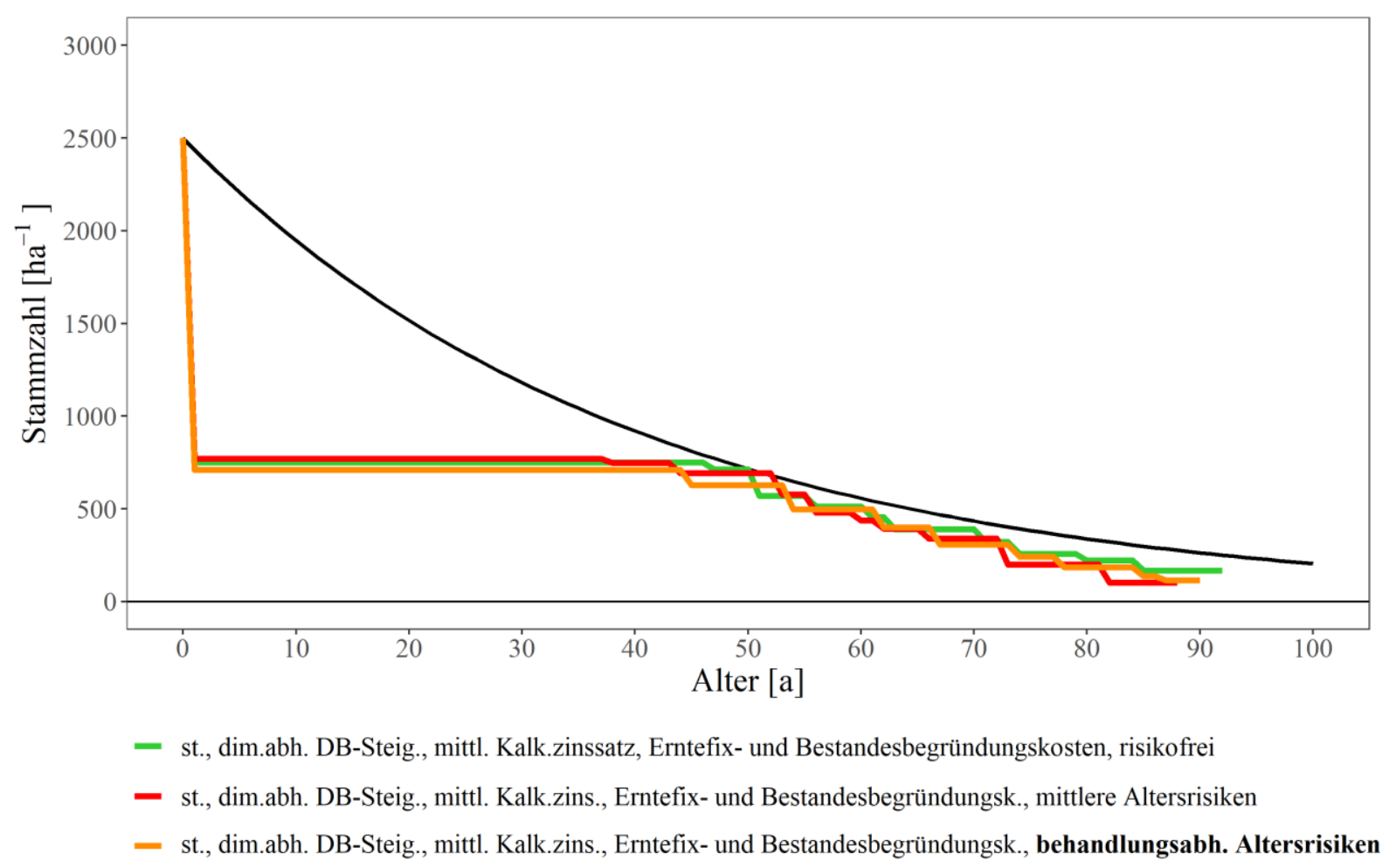

Abbildung 46: Altersabhängige Stammzahlentwicklungen bei behandlungsabhängigen Altersrisiken (orange) gemäß Zielfunktionen [2-58] im Vergleich zum Optimum bei mittleren, behandlungsunabhängigen Altersrisiken (hellrot) bzw. dem risikofreien Optimum bei starker, dimensionsabhängiger DBSteigerung, mittlerem Kalkulationszinssatz sowie Erntefix- und Bestandesbegründungskosten (grün) und zum naturalen Optimum ohne Folgebestand (schwarz). 
Erwartungsgemäß zeigt sich eine Risikoreduzierung durch Reduktion der Stammzahl in niedrigen Bestandesaltern (Abbildung 46). Das dadurch um 4 \% auf 0,8 Fm erhöhte Einzelbaumvolumen im Alter 50 a (Abbildung 47) steigert die Überlebenswahrscheinlichkeit bis zum Alter 100 a auf $S_{100}=0.51$. Diese äußerst geringe behandlungsbedingte Steigerung der Überlebenswahrscheinlichkeit wird auf vielfältige Wechselwirkungen innerhalb des Modells zurückgeführt. Die bereits im Kontext der Einführung dimensionsabhängiger Deckungsbeiträge stark abgesenkte Stammzahl (siehe Abschnitt 2.2.4) wurde durch die Berücksichtigung von Kapitalknappheit (siehe Abschnitt 2.2.5) sowie allen weiteren eingeführten Modellprämissen nur leicht gesteigert. Die mit einer weiteren, das Einzelbaumvolumen im Alter 50 a steigernde, Stammzahlreduktion in jungen Bestandesaltern verbundenen Vorteile (geringere Überlebensrisikostärke) werden durch Nachteile an anderer Stelle (z. B. den weiteren Verzicht auf Bestandesvolumenzuwachs) überwogen.

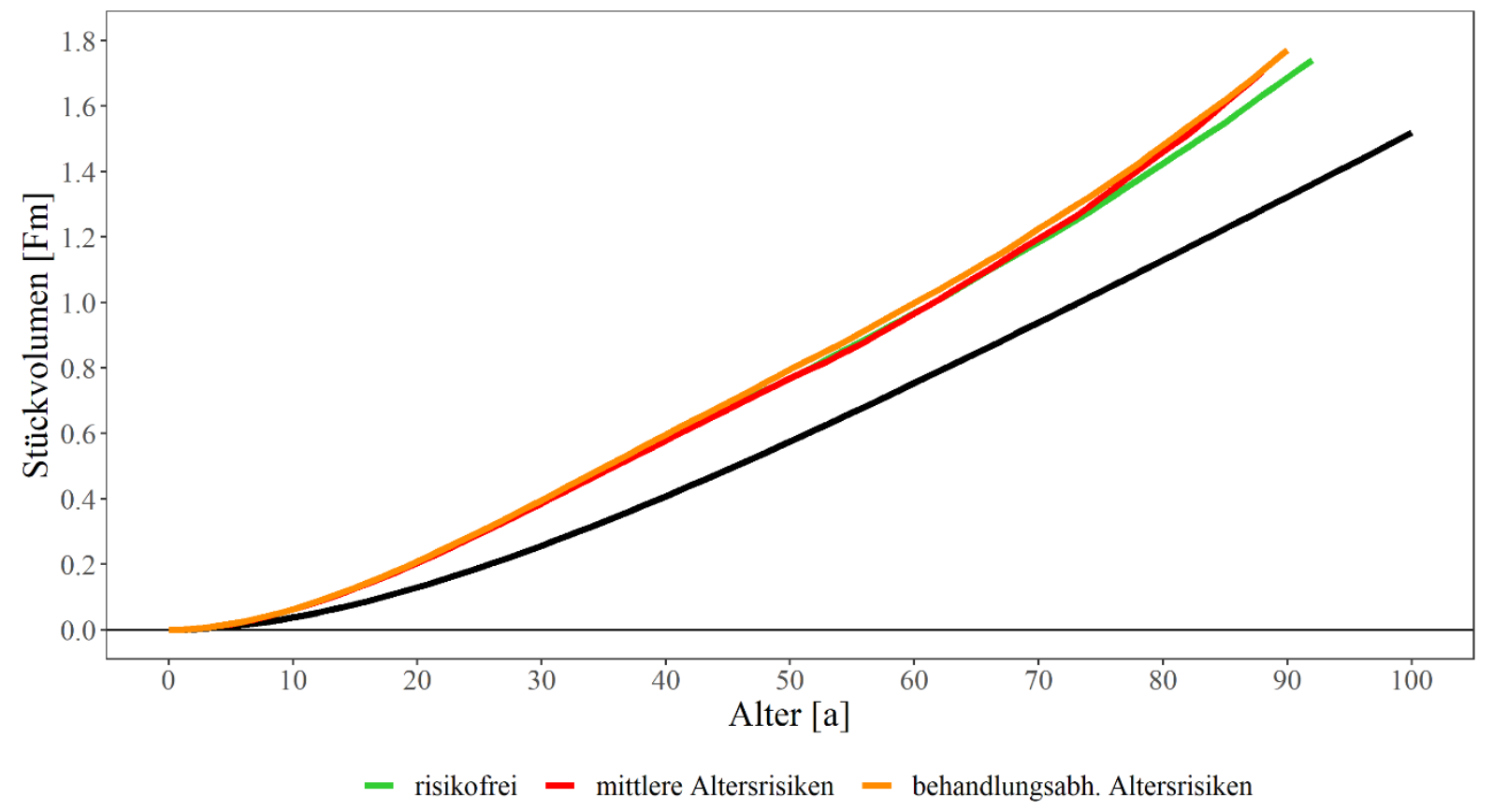

Abbildung 47: Altersabhängige Einzelbaumvolumina (Stückvolumina) bei behandlungsabhängigen Altersrisiken (orange) gemäß Zielfunktionen [2-58] im Vergleich zum Optimum bei mittleren, behandlungsunabhängigen Altersrisiken (hellrot) bzw. dem risikofreien Optimum bei starker, dimensionsabhängiger DB-Steigerung, mittlerem Kalkulationszinssatz sowie Erntefix- und Bestandesbegründungskosten (grün) und zum naturalen Optimum ohne Folgebestand (schwarz).

Bei leicht verminderten laufenden Bestandesvolumenzuwächsen in der ersten Hälfte der forstlichen Produktionsdauer (Abbildung 49) sinkt die Gesamtwuchsleistung ggü. Abschnitt 2.2.8 minimal ab - zum Zeitpunkt der um zwei Jahre nach hinten verschobenen Endnutzung $\left(T_{S\left(V_{E}\right)}^{*}=90 \mathrm{a}\right)$ beträgt sie $866 \mathrm{Fm} \mathrm{ha}^{-1}$, was einem durchschnittlichen Bestandesvolumenzuwachs von 9,6 Fm ha ${ }^{-1} \mathrm{a}^{-1}$ entspricht (-3\% ggü. Abschnitt 2.2.8). Der zunächst stärkere 
Eingriff zu Beginn der forstlichen Produktion wird durch leicht verminderte Vornutzungsmengen im letzten Drittel ausgeglichen, sodass das Bestandesvolumen zum Zeitpunkt der Endnutzung mit $205 \mathrm{Fm} \mathrm{ha}^{-1}$ um 16 \% höher liegt als das Endnutzungsvolumen bei behandlungsunabhängigen Überlebensrisiken (Abbildung 49).

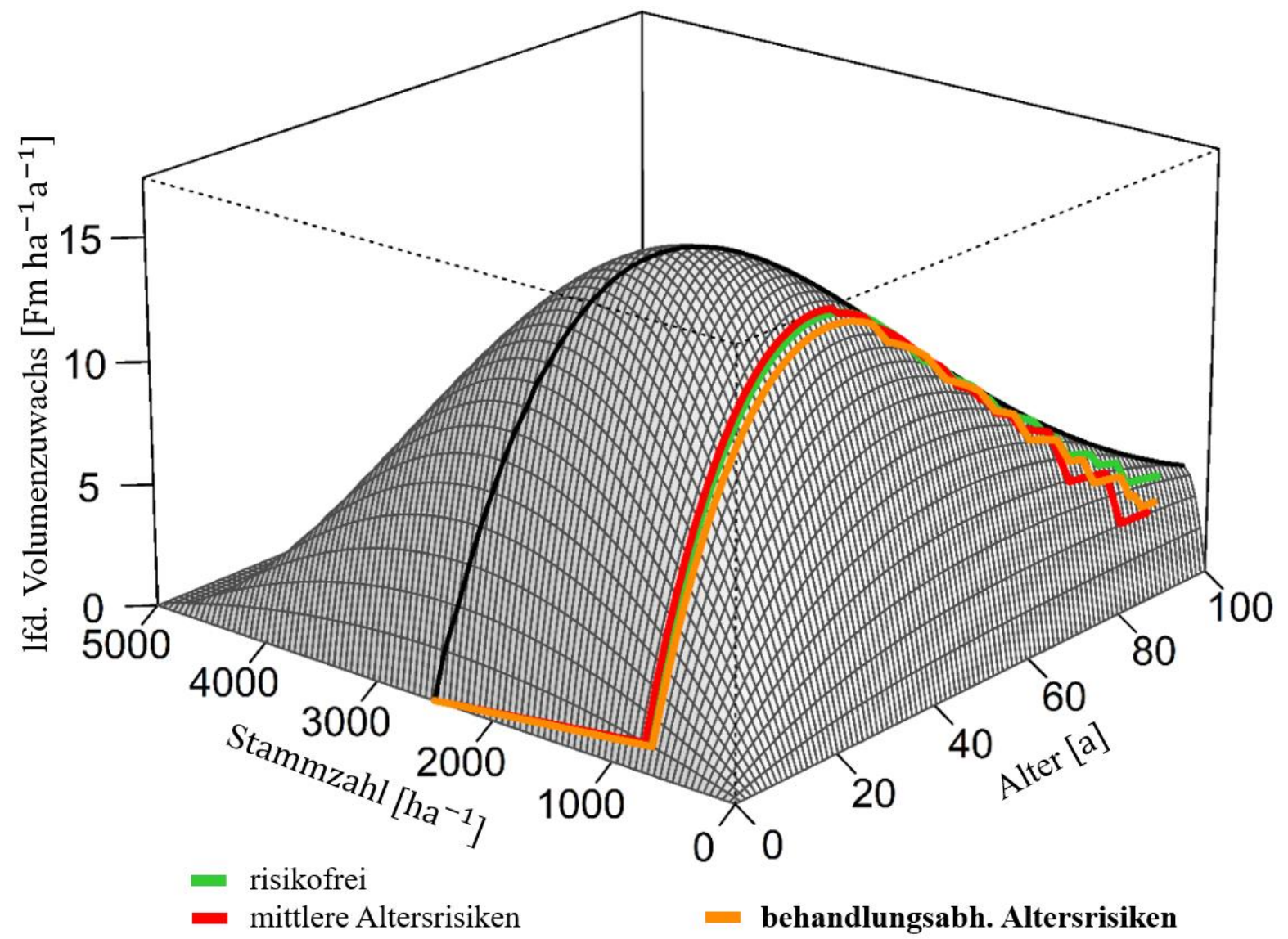

Abbildung 48: Alters- und stammzahlabhängiger laufender Bestandesvolumenzuwachs bei behandlungsabhängigen Altersrisiken (orange) gemäß Zielfunktionen [2-58] im Vergleich zum Optimum bei mittleren, behandlungsunabhängigen Altersrisiken (hellrot) bzw. dem risikofreien Optimum bei starker, dimensionsabhängiger DB-Steigerung, mittlerem Kalkulationszinssatz sowie Erntefix- und Bestandesbegründungskosten (grün) und dem modell-maximalen Bestandesvolumenzuwachs (schwarz).

Durch die Modellerweiterung der behandlungsabhängigen Überlebensrisiken ändern sich die Optimalitätsbedingungen der Vor- und Endnutzung nur minimal im Vergleich zu Abschnitt 2.2.8 - es gilt mit der ergänzten Abhängigkeit der Überlebensrisikostärke:

$$
\begin{gathered}
\hat{E}\left[w_{j}\left(t_{j}, N_{t_{j}-1}, P\left(V_{E}\right), S_{100}\left(V_{E}\left(50 \mathrm{a}, \boldsymbol{\sigma}_{49} a, N_{0}\right)\right), \alpha, \varphi, \Phi\right)\right]+\left(\frac{F}{M_{t_{j}-1}} \cdot(1+r)-\frac{F}{M_{t_{j}}}\right) \\
=W_{j}\left(t_{j}-1, N_{t_{j}-2}, P\left(V_{E}\right)\right) \cdot r+\sum_{t=t_{j}}^{T} \sum_{\substack{k \in L_{t_{j}} \\
k \neq j}} \frac{\hat{E}\left[\Delta W_{k, j}\left(t_{k}, S_{100}\left(V_{E}\left(50 \mathrm{a}, \boldsymbol{\sigma}_{49}, N_{0}\right)\right), \alpha, \varphi, \Phi\right)\right]}{(1+r)^{\left(t_{k}-t_{j}\right)}} .
\end{gathered}
$$


Weiterhin gilt für jeden Vornutzungsbaum, dass der Erwartungswert seines laufenden Wertzuwachses zuzüglich des Grenznutzen der zeitlich verzögerten Erntefixkostenbelastung die Opportunitätskosten aus alternativer Kapitalverwendung und dem Erwartungswert des Grenznutzens der alternativen Wuchsraumnutzung überwiegen muss, damit sein Erhalt für ein weiteres Jahr vorteilhaft ist. Die weiteren Modellabhängigkeiten steigern die Wechselwirkungen zusätzlich und verhindern allgemeingültige Aussagen über die Wirkungsrichtung behandlungsabhängiger Überlebensrisiken in Bezug auf die Erntezeitpunkte einzelner Bäume.

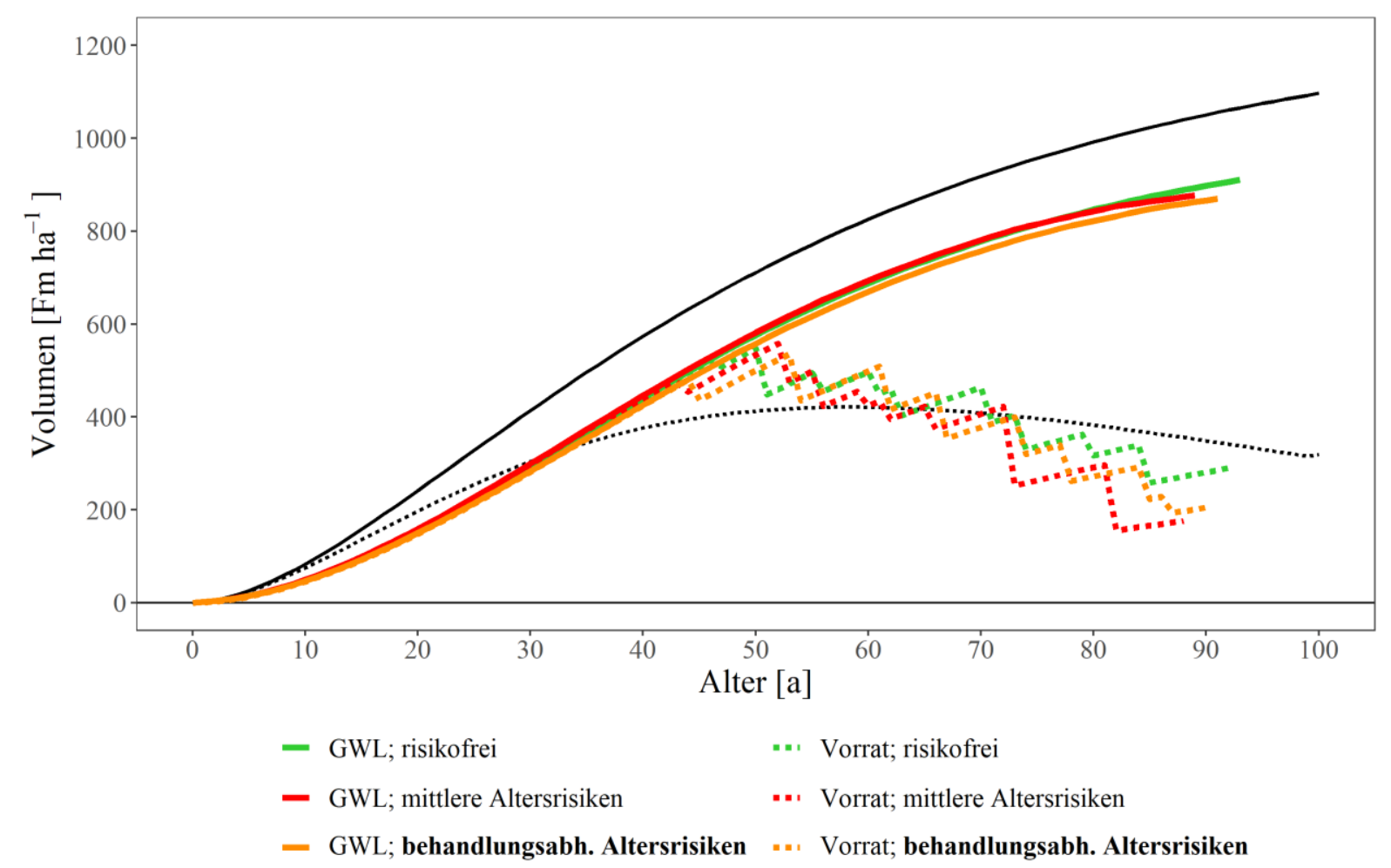

Abbildung 49: Altersabhängige Gesamtwuchsleistung und Vorratsentwicklung bei behandlungsabhängigen Altersrisiken (orange) gemäß Zielfunktionen [2-58] im Vergleich zum Optimum bei mittleren, behandlungsunabhängigen Altersrisiken (hellrot) bzw. dem risikofreien Optimum bei starker, dimensionsabhängiger DB-Steigerung, mittlerem Kalkulationszinssatz sowie Erntefix- und Bestandesbegründungskosten (grün) und zum naturalen Optimum ohne Folgebestand (schwarz).

Für die Endnutzungsentscheidung erweitert sich Optimalitätsbedingung [2-54] zu:

$$
\begin{gathered}
\hat{E}\left[w_{B}\left(T, N_{T-1}, P\left(V_{E}\right), S_{100}\left(V_{E}\left(50 \mathrm{a}, \boldsymbol{\sigma}_{49 \mathrm{a}}, N_{0}\right)\right), \alpha, \varphi, \Phi\right)\right]+(F \cdot r) \\
=W_{B}\left(T-1, \boldsymbol{N}_{T-1}, P\left(V_{E}\right)\right) \cdot r+ \\
\hat{E}\left[a_{S}\left(T, N_{0}, \boldsymbol{\sigma}_{T-1}, P\left(V_{E}\right), r, F, C, S_{100}\left(V_{E}\left(50 \mathrm{a}, \boldsymbol{\sigma}_{49} \mathrm{a}, N_{0}\right)\right), \alpha, \varphi, \Phi\right)\right] .
\end{gathered}
$$

Auch hier gilt für den optimalen Erntezeitpunkt des Bestandes weiterhin die Gleichheit vom Erwartungswert des laufenden Bestandeswertzuwachses zuzüglich des Kapitalgrenznutzens aus der verzögerten Erntefixkostenbelastung und der Summe aus Kapital- und Flächenopportunität (Erwartungswert der Brutto-Bodenrente). 


\subsection{Brückenschlag zur forstlichen Praxis}

Was bedeuten die gezeigten Ergebnisse des theoretischen Analyserahmens nun für die praktische Behandlung eines Waldbestandes? Welche Handlungsempfehlungen können trotz der Begrenztheit des Modells (siehe Abschnitt 2.4) abgeleitet werden? Antworten darauf liefert dieser Abschnitt als kompakte und auf die Übertragung in die forstliche Praxis fokussierte Zusammenfassung der vorangegangenen Analysen.

Hätte die waldbewirtschaftende Person unendlich viel Kapital und unendliche viel Fläche für weitere Waldbestände zur Verfügung, könnte sie die Bewirtschaftung des Bestandes bis zum Zeitpunkt der Volumen- oder Wertabnahme (negativer Bestandesvolumen- oder Bestandeswertzuwachs) aufrechterhalten (Wippermann 2005, S. 22; Möhring 2009). Kapital- oder InterBestandesopportunitäten bestünden nicht. Innerhalb des Bestandes würde aber dennoch Knappheit und damit Konkurrenz der Bäume um die verfügbare Fläche bzw. den verfügbaren Wuchsraum herrschen - die Standortfixierung der Bäume verhindert eine Erschließung der außerhalb des Bestandes unendlich verfügbaren Fläche. Für eine optimale Allokation der knappen Ressourcen Licht, Wasser und Nährstoffe wird jeder einzelne Baum nur solange im Bestand gehalten, wie sein zeitlicher Grenznutzen, d. h. sein laufender Wertzuwachs, den zeitlichen Grenznutzen aus der alternativen Nutzung des von ihm belegten Wuchsraums durch andere Bäume (Wuchsraum- oder Intra-Bestandesopportunitäten) überwiegt (Coordes 2014a, S. 61; Fuchs 2018, S. 13 ff.). Zu jedem Zeitpunkt muss gemäß dieser zeitlich-räumlichen Grenznutzenanalyse entschieden werden, mit welchen ${ }^{39}$ Bäumen der verfügbare Wuchsraum im nächsten Zeitschritt optimal ausgenutzt wird. Der durch die Ernte eines Baums entgehende Volumenzuwachs muss durch die dann höheren Einzelbaumvolumenzuwächse der verbleibenden Bäume kompensiert werden (Dichteeffekt). Die im Modell resultierende optimale Stammzahlentwicklung bei einem zunächst als konstant angenommenen erntekostenfreien Holzerlös (Deckungsbeitrag) entspricht der schwarzen Linie in Abbildung 50. Es wird damit in jedem Zeitschritt der modellmaximale laufende Bestandesvolumen- und damit auch Bestandeswertzuwachs erzielt - der begrenzte Wuchsraum des Bestandes wird optimal ausgenutzt. An dieser optimalen Wuchsraumausnutzung ändert sich auch nichts, wenn man die mit der Realität nicht vereinbare Annahme der unendlichen Flächenverfügbarkeit aufgibt. Die Einbeziehung eines Folgebestandes in die Optimierung der forstlichen Produktion führt allerdings zu einer deutlichen Reduktion

\footnotetext{
${ }^{39} \mathrm{Im}$ Fall des homogenen Modellbestandes reduziert sich diese Frage auf die Anzahl der identischen Bäume im Bestand.
} 
des Endnutzungszeitpunkts (graue Linie in Abbildung 50 a). Denn der Grenznutzen des aktuellen Bestandes, der laufende Bestandeswertzuwachs, muss nun den zeitlichen Grenznutzen eines Folgebestandes (Inter-Bestandesopportunität) überwiegen, um den Bestandeserhalt für ein weiteres Jahr zu rechtfertigen (Wippermann 2005, S. 22 f.; Möhring 2009). Wird bspw. von einem identischen Bestandesersatz ausgegangen, bildet der durchschnittliche Bestandeswertzuwachs bzw. der durchschnittliche Überschuss der forstlichen Produktion diesen zeitlichen Grenznutzen. Wächst der Bestandeswert laufend weniger zu als im Durchschnitt der forstlichen Produktion, sollte die bewirtschaftende Person den aktuellen durch einen nachfolgenden Bestand ersetzen.

Nun zeichnet sich die mitteleuropäische Forstwirtschaft aber u. a. dadurch aus, dass sie eben kein homogenes Gut produziert, eine Volumeneinheit Holz somit nicht unabhängig von der Baumdimension stets die gleichen Deckungsbeiträge erzielt. Höhere Baumdimensionen bedingen aufgrund der über die reine Industrie- und Brennholzproduktion hinausgehenden Zielsetzung der Säge- und Wertholzproduktion i. d. R. ${ }^{40}$ höhere Holzerlöse (vgl. z. B. Bodelschwingh 2018, S. 59 ff.). Zudem verursacht die Ernte eines Baums mit hohem Stückvolumen, unabhängig vom Holzernteverfahren, geringere Holzerntekosten je Volumeneinheit als die Ernte eines Baums geringer Dimension (vgl. Stück-Volumen-Gesetz; Hilf 1928; 1941). Die Ernte geringdimensionierter Bäume verursacht teilweise negative Deckungsbeiträge - wenn überhaupt verkaufsfähiges Holzvolumen anfällt, decken die damit erzielbaren Holzerlöse teilweise nicht die entstehenden Holzerntekosten. Die Berücksichtigung dieser Tatsache führt zu einer veränderten Wuchsraumopportunität bei der zeitlich-räumlichen Grenznutzenanalyse am Einzelbaum. Dem Grenznutzen des Baumerhalts steht insbesondere in jungen Jahren eine erhöhte Wuchsraumopportunität gegenüber, denn der von dem Baum eingenommene Wuchsraum kann alternativ zur Erhöhung des mittleren Stückvolumens und damit auch der zukünftigen Deckungsbeiträge je Volumeneinheit aller nachfolgend geernteten Bäume eingesetzt werden (Dimensionseffekt). Zur optimalen Ausnutzung der knappen Bestandesfläche sollte die bewirtschaftende Person somit die Stammzahl in jungen Bestandesaltern derart reduzieren, dass der verminderte Bestandesvolumenzuwachs wertmäßig durch höhere Deckungsbeiträge späterer Realisationszeitpunkte kompensiert wird (blaue Linie in Abbildung 50 a). Frühe Eingriffe zur Zuwachslenkung auf den Einzelbaum lohnen sich dabei

\footnotetext{
${ }^{40}$ Aufgrund heutiger Holzbearbeitungstechnologien oder der teils fehlenden Wertholzqualität sind steigende Baumdimensionen kein Garant für immer weiter steigende Holzerlöse (vgl. Sauter et al. 2016b; Kohnle und Teuffel 2020).
} 
umso mehr, je höher das monetäre Spannungsverhältnis zwischen gering- und hochdimensionierten Sortimente, das heißt je stärker die DB-Steigerung über der Einzelbaumdimension ist (vgl. Wippermann 2005, S. 112 f.). Wichtig ist dabei zu beachten, dass diese Aussage vor dem Hintergrund weiterer, von der Bestandesdichte (Stammzahl) abhängigen, aber im Modell dieser Arbeit nicht berücksichtigten, Faktoren zu relativieren bzw. anzupassen ist (vgl. Coordes 2014a, S. 99 ff.). Bei der Übertragung auf tatsächliche Baumarten sind von der bewirtschaftenden Person bspw. Qualitätsaspekte, wie die Astreinigung bei Laubhölzern oder die Starkastigkeit bei Nadelhölzern, zu berücksichtigen. Bei heterogenen Beständen spielt im Zuge der Zuwachslenkung auf weniger Einzelbäume zudem deren spezifische Werterwartung eine entscheidende Rolle für die Auswahl der verbleibenden Bäume. Mit steigendem Bestandesalter nimmt der Einfluss der dimensionsabhängig steigenden Deckungsbeiträge auf die Entscheidung über den Baumerhalt ab - die Nachteile des Verzichts auf laufenden Bestandesvolumenzuwachs werden zunehmend weniger von den dimensionsbedingten Vorteilen kompensiert, was an sinkenden Wertfaktoren über dem Alter, d. h. einer Reduktion des Dimensionseffekts, erkennbar ist (Abbildung 29). Eine einmal verpasste Chance der Zuwachslenkung auf weniger Einzelbäume ist ggf. nicht mehr aufholbar, wobei diesbezüglich insb. die artspezifischen Wuchsdynamiken der Baumarten berücksichtigt werden müssen. Damit die Vorteile der gestiegenen Einzelbaumvolumina ausgenutzt werden können, ist die bewirtschaftende Person angehalten, die Endnutzung des Bestandes zu verzögern. Die mit der Dimension steigenden Deckungsbeiträge erhöhen den laufende Bestandeswertzuwachs insb. in höheren Bestandesaltern - der zeitliche Grenznutzen des Bestandeserhalts überwiegt länger den durchschnittlichen Überschuss des potentiellen Folgebestandes ${ }^{41}$.

Wird der bewirtschaftenden Person eine Zeitpräferenz und damit die Bevorzugung früherer ggü. späterer Einnahmen unterstellt, wird bei der Optimierung der forstlichen Produktion neben der Flächen- auch die Kapitalknappheit berücksichtigt. Zahlungen in der Zukunft haben einen reduzierten Jetztwert und der durchschnittliche Überschuss der forstlichen Produktion wird finanzmathematisch als Annuität berechnet. Es erhöhen sich die Grenzkosten des Bestandesbzw. Baumerhalts um die alternative Verwendung des im Bestand bzw. Baum gebundenen Kapitals (Wippermann 2005, S. 26 ff.; Möhring 2009; Coordes 2014a, S. 61; Fuchs 2018, S. 13 ff.). Im Zuge der Vornutzungsentscheidung steigt durch die Berücksichtigung der

${ }^{41}$ Im Modell dieser Arbeit sinkt dieser zudem durch die Einführung dimensionsabhängiger Deckungsbeiträge (Abbildung 51), was den Effekt des längeren Bestandeserhalts verstärkt. 
Kapitalknappheit zudem auch die Relevanz der Realisationszeitpunkte aller durch den Erhalt eines Baums beeinflussten Bäume. Die Wuchsraumopportunität eines marginal verlängerten Baumerhalts wird umso mehr reduziert, je größer der zeitliche Abstand zwischen Ernteaufschub und Realisierung der beeinflussten Bäume ist (Zinseffekt). Dieser Zinseffekt schmälert den Dimensionseffekt und damit die Wuchsraumopportunität insbesondere in niedrigen Bestandesaltern (siehe reduzierte Wertfaktoren in Abbildung 35). Aufgrund der zunächst geringen Kapitalopportunität (geringe Baumwerte in niedrigen Altern) führt die gesunkene Wuchsraumopportunität zu reduzierten Vornutzungsmengen in niedrigen Bestandesaltern (hellviolette Linie in Abbildung 50 a). Mit steigender Kapitalopportunität (hohe Baumwerte in hohen Altern) wird der Baumerhalt zunehmend unvorteilhaft und die Vornutzungsmengen steigen, bevor die forstliche Produktion ggü. der zinsfreien Variante früher beendet wird. Entscheidend für das Ausmaß der zinseffektbedingten Veränderungen ist die Höhe des angenommenen Kalkulationszinssatzes. Dabei steht als alternative Kapitalverwendung nicht nur die externe Geldanlage zur Disposition - vielmehr kann der angesetzte Zinssatz entscheidungsorientiert auch als Rentabilität innerbetrieblicher Handlungsalternativen interpretiert werden und der bewirtschaftenden Person somit zur optimalen Allokation des im Betrieb gebundenen Kapitals verhelfen.

Erwartungsgemäß senkt die Einführung von Erntefix- und Bestandesbegründungskosten den durchschnittlichen Überschuss bzw. die Brutto-Bodenrente ab (Abbildung 51). Der durchschnittliche Bestandesvolumenzuwachs sinkt im Modell ebenfalls, was bei annähernd unveränderter Entwicklung der Gesamtwuchsleistung vor allem auf die deutlich verzögerte Endnutzung zurückzuführen ist (hellgrüne Linie in Abbildung 50 b). Diese ist im gewählten Beispiel ausschließlich auf die Einführung der Bestandesbegründungskosten zurückzuführen, da diese die Brutto-Bodenrente und damit die Opportunitätskosten des Bestandeserhalts deutlich absenken (vgl. Wippermann 2005, S. 63). Je höher die Begründungskosten eines Folgebestandes sind, desto länger wird die bewirtschaftende Person die aktuelle forstliche Produktion aufrechterhalten - den laufenden Grenznutzen folglich ausnutzen. Während die Bestandesbegründungskosten nur indirekt über die Veränderung des Endnutzungszeitpunkts Einfluss auf die Wuchsraumopportunität und somit die Grenzkosten des Einzelbaumerhalts haben, reduzieren Erntefixkosten die Anzahl der Vornutzungen erheblich (hellgrüne Linie in Abbildung 50 b; vgl. Coordes 2014a, S. 120; Rämö und Tahvonen 2017). Dem Streben nach der Vermeidung von Erntefixkosten durch das Bündeln von Einzelbaumentnahmen steht dabei stets die Abweichung vom optimalen Behandlungspfad, d. h. der Verzicht auf Bestandesvolumenzuwachs, entgegen. Steigende Erntefixkosten rechtfertigen einen immer höheren Verzicht auf Volumenzuwachs. 

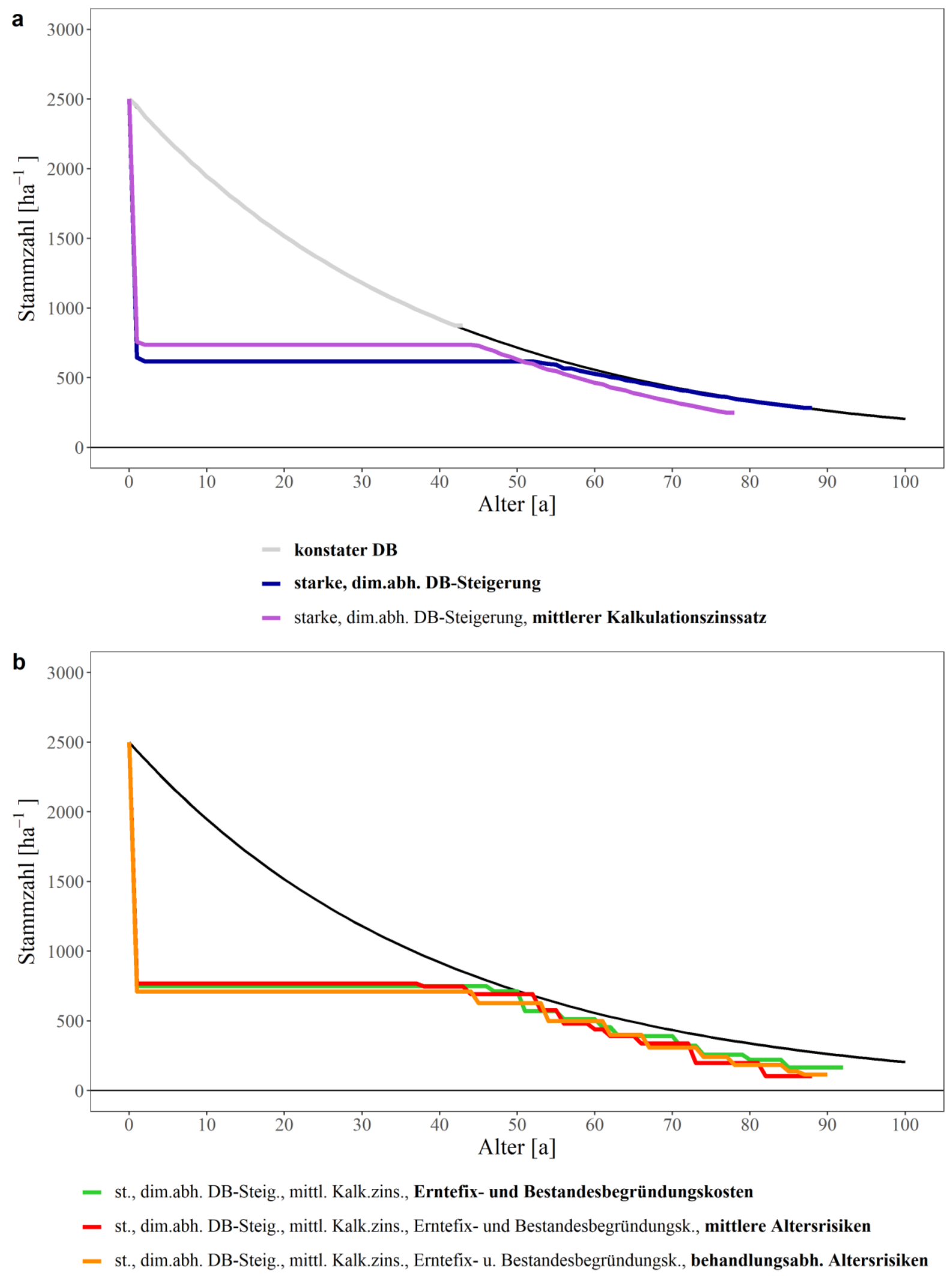

Abbildung 50: Altersabhängige Stammzahlentwicklungen der abschnittsübergreifenden Zusammenfassung und Diskussion gemäß der Abschnitte 2.2.2 (konstanter DB; (a): grau), 2.2.4 (starke, dimensionsabhängige DB-Steigerung; (a): dunkelblau), 2.2.5 (mittlerer Kalkulationszinssatz; (a): hellviolett), 2.2.7 (niedrige Erntefixkosten und Bestandesbegründungskosten; (b): grün), 2.2.8 (mittlere Altersrisiken; (b): hellrot) sowie 2.2.9 (behandlungsabhängige Altersrisiken; (b): orange) im Vergleich zum naturalen Optimum ohne Folgebestand (schwarz). 
Komplexe Wechselwirkungen zwischen den anteilsmäßig mit Erntefixkosten belasteten Bäumen verhindern allerdings allgemeingültige Aussagen über die Wirkungsrichtung der Erntefixkosten auf die Vornutzungsentscheidung am Einzelbaum.

Bezieht die bewirtschaftende Person Überlebensrisiken in den Entscheidungsprozess über die optimale Bestandesbehandlung ein, erreicht der Bestand nur mit einer gewissen (Überlebens-)Wahrscheinlichkeit den geplanten Endnutzungszeitpunkt (Möllmann und Möhring 2017). Bei vorzeitiger, kalamitätsbedingter Zwansgsnutzung reduziert sich deren Deckungsbeitrag aufgrund verminderter Holzerlöse und erhöhter Holzerntekosten. Aus den mit den jeweiligen Eintrittswahrscheinlichkeiten sowie Produktionsdauern gewichteten, möglichen BruttoBodenrenten ergibt sich als Zielgröße ein Erwartungswert der Brutto-Bodenrente, welcher unterhalb der risikofreien Variante liegt (Abbildung 51). In Abhängigkeit vom zeitlichen Verlauf der Ausfallrate (Überlebensrisikoart) verschiebt sich der Endnutzungszeitpunkt. Jugendrisiken, d. h. mit dem Alter sinkende Ausfallraten, sollte die bewirtschaftende Person mit einer Verlängerung der Produktionszeit begegnen, um den dann zunächst erneut hohen Ausfallraten des Folgebestands längstmöglich auszuweichen und gleichzeitig die Phase geringerer Ausfallraten des aktuellen Bestandes auszunutzen. Beim in Mitteleuropa vorherrschenden Schadfaktor Sturm (vgl. u. a. Schelhaas et al. 2003) steigen die Ausfallraten allerdings insb. mit der Bestandeshöhe und damit dem Alter an. In Bezug auf die Endnutzung kann die bewirtschaftende Person diesen Überlebensrisiken nur durch eine Verkürzung der Produktionsdauer begegnen (rote Linie in Abbildung 50 b). Dies leuchtet nicht nur intuitiv ein, sondern stimmt auch mit waldbaulichen Empfehlungen überein, insbesondere auf labilen Standorten unnötig hohe Bestandesbzw. Einzelbaumhöhen zu vermeiden (vgl. z. B. Albrecht 2009, S. 145; ForstBW 2014, S. 60). Dennoch auf eine längere Produktionsdauer zu setzen und damit auch höhere Risiken einzugehen, kann bspw. durch alternative gesellschaftliche Ansprüche an die forstliche Produktion (Erhaltung von Habitatbäume, Ausweisung von Prozessschutzflächen) und/oder einer anteiligen Übertragung der Risikokosten auf den Staat im Zuge forstspezifischer Regelungen der Einkommenssteuer begründet werden (Möllmann und Möhring 2018). In Bezug auf die Vornutzungsentscheidung unter Risiko sind aufgrund vielfältiger Wechselwirkungen keine allgemeingültigen Aussagen zur Verschiebung einzelner Erntezeitpunkte möglich. Insgesamt steigen jedoch die Grenzkosten des Baumerhalts bei mit dem Alter steigenden Ausfallraten ggü. den Grenznutzen des Baumerhalts an - bei Vernachlässigung behandlungsabhängiger Veränderung der Überlebenswahrscheinlichkeit ist die frühere Realisierung höherer Vornutzungsmengen in hohen Bestandesaltern vorteilhaft (rote Linie in Abbildung 50 b; vgl. z. B. Loisel 2014). 


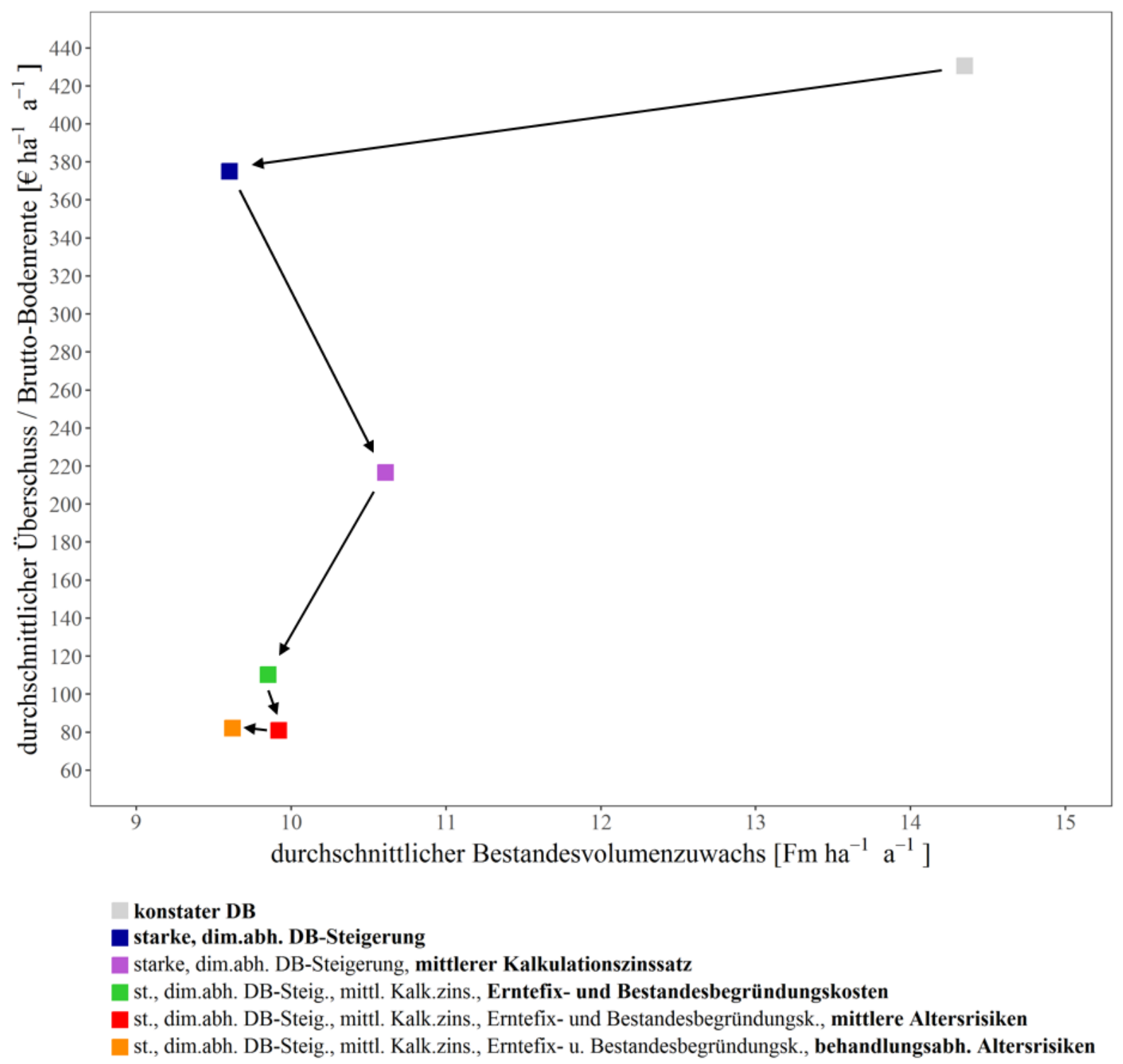

Abbildung 51: Naturale (Bestandesvolumenzuwachs) und ökonomische (Überschuss bzw. Brutto-Bodenrente) durchschnittliche Erfolgsgrößen gemäß der Abschnitte 2.2.2 (konstanter DB; (a): grau), 2.2.4 (starke, dimensionsabhängige DB-Steigerung; (a): dunkelblau), 2.2 .5 (mittlerer Kalkulationszinssatz; (a): hellviolett), 0 und 2.2.7 (niedrige Erntefixkosten und Bestandesbegründungskosten; (b): grün), 2.2.8 (mittlere Altersrisiken; (b): hellrot) sowie 2.2.9 (behandlungsabhängige Altersrisiken; (b): orange). Die Pfeile kennzeichnen die schrittweise Einführung der Modellprämissen in chronologischer Reihenfolge von oben rechts nach unten links.

Geht die waldbewirtschaftende Person von einer Risikominderung durch höhere Einzelbaumvolumina und einer damit verbundenen höheren Bestandesstabilität aus, ist eine angepasste Bestandesbehandlung in Form einer reduzierten Stammzahl zu Beginn der forstlichen Produktion sinnvoll (orange Linie in Abbildung 50 b). Es resultiert ein ggü. den behandlungsunabhängigen Überlebensrisiken leicht verminderter durchschnittlicher Bestandesvolumenzuwachs bei gleichzeitig minimal gesteigertem Erwartungswert der Brutto-Bodenrente (Abbildung 51). Die ökonomischen Auswirkungen der Risikointegration können an dieser Stelle also durch waldbauliche Anpassungsstrategien abgemildert, aber nicht ausgeglichen werden. Die nur minimal 
veränderte forstliche Bestandesbehandlung zeigt aber auch, dass andere Modellprämissen an dieser Stelle einen höheren Einfluss auf die optimale forstliche Bestandesbehandlung haben. Eine für die Risikominderung vorteilhafte weitere Reduzierung der Stammzahl hätte an anderer Stelle (z. B. Volumenzuwachs) schwerwiegendere Nachteile. Weitergehende waldbauliche Empfehlungen zur Risikominimierung, bspw. mit Blick auf die Kombination von Durchforstungsstärke, -turnus und -art, sind aufgrund der Modellgrenzen (siehe Abschnitt 2.4) nicht möglich. Albrecht (2009, S. 145) erachtet diesbezüglich gestaffelte Behandlungsprogramme für sinnvoll und verschiedene Waldbaurichtlinien bzw. -empfehlungen plädieren (bspw. bei der Fichte) für die Schaffung hoher Einzelbaumstabilitäten durch geringe Stammzahlen in der Bestandesjugend und zunehmend schwächer werdenden Eingriffen im Herrschenden bei großen Bestandeshöhen (ForstBW 2014, S. 55 ff.; vgl. auch Gadow 2003, S. 60 f.). Mit Blick auf den Einfluss des zeitlichen Abstands zwischen letzter Vornutzung und Sturmschadensereignis (vgl. u. a. Lohmander und Helles 1987; Donis et al. 2018) sind insb. bei großen Bestandeshöhen und auf labilen Standorten angepasste Vornutzungsintervalle notwendig. So empfiehlt bspw. die Richtlinie zur Bewirtschaftung von Fichten- und Fichtenmischbeständen im Bayerischen Staatswald für ältere Bestände eine umso häufigere Vornutzung mit entsprechend geringen Entnahmemengen, je instabiler der Bestand ist (BaySF 2009, S. 45). Eine indirekt risikomindernde Wirkung niedriger Stammzahlen in der Jugend und der damit verbundenen höheren Einzelbaumdurchmesser wird zudem durch die frühere Realisierung der Zieldurchmesser, d. h. einer Endnutzung bei geringerer Baumhöhe erreicht (Albrecht et al. 2017).

Abschnittsübergreifend kann festgehalten werden, dass es nicht die eine optimale forstliche Bestandesbehandlung gibt (Abbildung 50). Vielmehr ist vor dem Hintergrund der Zielsetzung und in Anbetracht bestehender bzw. von der bewirtschaftenden Person in die Entscheidung einbezogener Prämissen stets neu über die Allokation von Fläche und Kapital zu entscheiden. Ein erheblicher Verzicht auf Volumenleistung kann bspw. sinnvoll sein (Abbildung 51). Die effiziente Bestandesbehandlung ist daher nicht nur von den Zielen der waldbewirtschaftenden Person, sondern immer auch von externen, nicht direkt beeinflussbaren Faktoren abhängig. Diese zu erkennen, im Angesicht der eigenen Ziele zu validieren und dann angemessen in den Entscheidungsprozess einzubeziehen, kann als zentrale Aufgabe einer ökonomisch handelnden Entscheidungsträgerin bzw. eines ökonomisch handelnden Entscheidungsträgers angesehen werden. Adäquate Modelle können und sollten diesbezüglich eingesetzt werden, um die bekannten Zusammenhänge und Wechselwirkungen einer effizienten Bestandesbehandlung auf die konkrete Entscheidungssituation zu übertragen. 


\subsection{Kritische Methoden- und Modellwürdigung}

Der theoretische Analyserahmen dieser Arbeit bezieht sich ausschließlich auf die gewinnorientierte Rohholzerzeugung und vernachlässigt somit sämtliche andere Funktionen und Leistungen des Ökosystems Wald (vgl. z. B. Samuelson 1976 und MEA 2005, S. 26 ff.). So findet bspw. keine Analyse der Auswirkungen der Bestandesbehandlung auf andere Leistungen abseits des Holzertrags (z. B. Biodiversität) statt (vgl. Schall und Ammer 2013). Aussagen über die Optima der forstlichen Bestandesbehandlung sind stets vor diesem Hintergrund zu interpretieren und insbesondere im Rahmen einer multifunktionalen Forstwirtschaft um weitere Aspekte zu ergänzen - durch etwaige Modellerweiterungen ergeben sich andere Optima.

Die gewählte Methode des Variantenstudiums (vgl. z. B. Wippermann 2005) ermöglicht durch eine schrittweise Erweiterung der Modellprämissen die Zerlegung des Gesamtproblems in überschaubare Teilprobleme. Die Kriterien einer optimalen Bestandesbehandlung können dadurch leicht verständlich eingeführt und dennoch im Gesamtkonzept mit Bezug zur forstlichen Praxis diskutiert werden. Es wird auf vertiefende, analytische Lösungsansätze verzichtet, da diese für Teilaspekte bereits existieren (vgl. u. a. Faustmann Faustmann 1849; 1995; Pressler 1860; 1995; Ohlin 1921; 1995, Näslund 1969; Samuelson 1976; Clark und Pree 1979; Cawrse et al. 1984; Johansson und Löfgren 1985; Chang 1998; Borchert 2002; Amacher et al. 2009; Clark 2010; Chang und Deegen 2011; Coordes 2014a; Halbritter und Deegen 2015; Tahvonen 2016; Halbritter 2020), darauf aufbauend Analysen per Analogieschluss möglich sind (vgl. z. B. Möhring 2009 oder Fuchs 2018) und zudem komplexitätsbedingt an anderen Stellen Grenzen der mathematisch-allgemeingültigen Formulierung erreicht werden (vgl. z. B. Chang 1983; Coordes 2014b; Halbritter und Deegen 2015; Jacobsen et al. 2018). Die laut Möhring (2010, S. 348) mit dem gewählten Modellansatz verbundene Aufgabe der „Allgemeingültigkeit und Eleganz der algebraischen Lösung“ wird mit Blick auf die vergleichsweise leicht nachvollziehbaren Erkenntnisse bewusst in Kauf genommen.

Mit dem fiktiv gewählten und nur an forstlich-realistischen Größen orientierten Modell wird bewusst auf Bezüge zu tatsächlichen Baumarten (wie bspw. bei Wippermann 2005) verzichtet. Ziel sind keine konkreten Aussagen zur optimalen Behandlung einer bestimmten Baumart vielmehr werden baumartenunabhängige Effekte einzelner Modellprämissen aufgezeigt. Der numerische Optimierungsansatz garantiert dabei zwar nicht das Auffinden des jeweils globalen Optimums, liefert aber in Kombination mit der Vielzahl an zufällig gewählten Startpunkte genügend stabile und für die Interpretation ausreichende Ergebnisse (vgl. Wippermann 2005, 
S. 43 f.). Damit diese Effekte erkennbar sind und nicht durch modellbedingte Wechselwirkungen verschwimmen, wird die naturale Produktion so einfach wie möglich definiert. So wird der Modellbestand aus homogenen, $d$. h. vollkommen identischen und zueinander im gleichen Abstand stehenden Bäumen aufgebaut - Aussagen zu Mischungseffekten (vgl. z. B. Roessiger et al. 2016) oder Art und räumlicher Anordnung von Vornutzungseingriffen (vgl. z. B. Meilby und Nord-Larsen 2012; Halbritter 2020) sind somit nicht möglich. Der Volumenzuwachs der identischen Einzelbäume ist altersabhängig degressiv definiert, wird allein über die (stetige) Stammzahl als Bestandesdichtemaß beeinflusst und ergibt als Produkt mit der Stammzahl den laufenden Bestandesvolumenzuwachs (vgl. im Kontrast dazu bspw. Pretzsch 2010a). Der Volumenzuwachs reagiert im jährlich-diskreten Modell zudem ohne Zeitverzug auf eine vornutzungsbedingte Dichteveränderung (vgl. im Kontrast dazu z. B. Härtl et al. 2010), wodurch Wuchsdynamiken in ihrer zeitlichen Ausprägung eher überschätzt werden.

Die durch den homogenen Bestandesaufbau bedingten schematischen Vornutzungen, d. h. die Gleichheit von relativem Vornutzungsvolumen und relativer Entnahmestammzahl, führen in Kombination mit der fixierten Ausgangsstammzahl ohne messbares Holzvolumen sowie der Divergenz zwischen Verkaufseinheit (Holzvolumen; ohne Kluppschwellen) und Bestandesdichtemaß (Stammzahl) zu teils schwer interpretierbaren Teilergebnissen (siehe Abschnitte 2.2.3 und 2.2.5). An den entsprechenden Stellen werden die Modellbesonderheiten daher ausführlich erläutert und die Ergebnisse entsprechend eingeordnet.

Mit der fixierten Ausgangsstammzahl werden deren Wechselwirkungen mit den Vornutzungen einseitig definiert. So kann einer prämissenspezifischen, suboptimalen Ausgangsstammzahl nur durch eine (unterbleibende) Vornutzung im Alter 1 a begegnet werden. Angepasste Ausgangsstammzahlen (vgl. u. a. Cao et al. 2006; Coordes 2013; Halbritter und Deegen 2015), mit dementsprechend auch veränderten Bestandesbegründungskosten, sind nicht möglich.

Die Beschränkung der Analysen auf gleichaltrige Bestände (Altersklassenwald) hat in dieser Arbeit inhaltliche und methodische Gründe: Zu den inhaltlichen Gründen zählt die Anknüpfung des praxisorientierten Kapitels 3 und die Anwendung der hier gewonnenen Erkenntnisse bei der ökonomischen Analyse forstlicher Ertragstafeln, bspw. im Rahmen der Sensitivitätsanalyse in Abschnitt 3.3.6. Methodisch spricht erneut die Komplexitätsreduktion für die Beschränkung der Analyse auf den Altersklassenwald. Denn mit der Einführung dauerwaldartige Strukturen aus ungleichaltrigen Bäumen verliert das klassischen FPO-Theorem an Erklärungskraft (vgl. u. a. Heshmatol Vaezin et al. 2009; Tahvonen 2016). Der Komplexitätsgrad des Modells würde durch die Einbeziehung einer Verjüngungsdynamik, mehrerer Baumkohorten unterschiedlicher 
Stärkeklassen sowie der Aufgabe eines (endlichen) Endnutzungszeitpunkts weiter ansteigen (Tahvonen und Rämö 2016). Coordes (2016) führt diese Komplexität ungleichaltriger Bestandesstrukturen auch als Grund für die häufige Wahl numerischer Lösungsansätze an und zählt diesbezüglich beispielhaft Adams und Ek (1974), Haight und Monserud (1990) und Tahvonen (2009) auf. Für die weniger häufigen, analytischen Lösungsansätze verweist er beispielhaft auf Chang (1981), Haight (1985), Getz und Haight (1989) und Halbritter (2015). Ergänzend sei an dieser Stelle auf Hanewinkel (2002) mit seinem Vergleich ökonomischer Methoden zur Analyse gleichaltriger und ungleichaltriger Waldbausysteme, Tahvonen $(2004,2016)$ mit seinem Optimierungsansatz für mehrere Altersklassen bzw. seinen Ansätzen zur Optimierung dauerwaldartiger Bestandesstrukturen, Roessiger et al. (2016) mit ihrer Analyse der optimalen Behandlung ungleichaltriger Bergmischwälder anhand eines Matrix-Übergangsmodells, Messerer et al. (2020) mit der Untersuchung der Auswirkungen von Holzpreisschwankungen auf die Behandlung ungleichaltriger Bestände über ein Kohorten-Modell sowie Halbritter (2020) mit seiner Analyse zur Durchforstungsart in einem Bestand zweier wechselwirkender Baumkohorten unterschiedlicher Vitalität verwiesen.

Gleichzeitig ermöglicht die in dieser Arbeit verwendete zeitlich-räumliche Grenznutzenanalyse auf Einzelbaumebene in Anlehnung an Coordes (2014a) und Fuchs (2018) die vergleichsweise einfache Ausweitung auf ungleichaltrige, dauerwaldartige Bestandesstrukturen - nach Coordes (2014c, S. 326) überwindet dieser Ansatz „die strukturelle Gleichförmigkeit des FaustmannModells“ und ermöglicht damit die Übertragung auf vielfältige waldbauliche Fragestellungen. Dabei kann die Grenznutzenanalyse des Einzelbaumerhalts im Dauerwald als allgemeiner Fall der Vornutzung im Altersklassenwald angesehen werden (vgl. auch Coordes 2014c). Ohne Endnutzungszeitpunkt des Bestandes sind die Anzahl der durch den Erhalt eines Baumes beeinflussten Bäume sowie der zu berücksichtigende Zeitraum (theoretisch) unendlich. Beispielhaft für die Entscheidungsregel der Vornutzung unter Kapitalknappheit erweitert sich Optimalitätsbedingung [2-41] (siehe Abschnitt 2.2.5) zu:

$$
w_{j}\left(t_{j}, N_{t_{j}-1}, P\left(V_{E}\right)\right)=W_{j}\left(t_{j}-1, N_{t_{j}-2}, P\left(V_{E}\right)\right) \cdot r+\sum_{t=t_{j}}^{\infty} \sum_{\substack{k \in L_{t_{j}} \\ k \neq j}} \frac{\Delta W_{k, j}\left(t_{k}\right)}{(1+r)}\left(t_{\left.k^{-}-t_{j}\right)},\right.
$$

wobei die Menge der durch den Erhalt von Baum $j$ beeinflussten Bäume $k \in L_{t_{j}}$ nun auch nachfolgende Bäume enthält und die zeitliche Summe somit unendlich ist. Unter Kapitalknappheit sinkt die Bedeutung zukünftiger Wuchsraumopportunitäten aber natürlich mit zunehmendem Abstand vom Zeitpunkt $t_{j}$, wodurch eine gewisse Endlichkeit der Berücksichtigung pragmatisch erscheint. 
Die verwendeten dimensionsabhängigen Deckungsbeitragsfunktionen bilden die generelle Tendenz höherer Deckungsbeiträge bei steigender Einzelbaumdimensionen ab (vgl. z. B. Möhring (2010) oder auch die auf realistischen Eingangsdaten beruhenden Deckungsbeitragsfunktionen in Abschnitt 3.3.5). Mögliche Erlösrückgänge im Bereich hoher Einzelbaumdimensionen (vgl. bspw. Rotkernbildung bei der Baumart Buche; u. a. Knoke 2002; Schmidt et al. 2008) werden nicht berücksichtigt. Ebenfalls keine Berücksichtigung in den Deckungsbeitragsfunktionen finden Wechselwirkungen zwischen Bestandesdichte und Holzqualität (vgl. Höwler et al. 2017), welche baumartenabhängig von hoher Bedeutung für die Bewertung des Rohstoffs Holz sein können (vgl. Knoke et al. 2006; Ammer 2016) und somit bei der Interpretation der Ergebnisse bedacht werden müssen (vgl. Wippermann 2005, S. 113).

Mit der Verwendung der Kalkulationszinssatz-Varianten $r_{m}=0,015$ und $r_{h}=0,03$ wird ein vollkommener Kapitalmarkt und damit u. a. die unbegrenzte Möglichkeit zur Kapitalaufnahme und -anlage zu einem einheitlichen Zinssatz sowie rationales Entscheiden auf Grundlage vollständiger Informationen unterstellt (vgl. Samuelson 1976; Johansson und Löfgren 1985, S. 74; Wippermann 2005, S. 28 f.; Hering 2017, S. 33 ff.). Vernachlässigt werden damit u. a. der Einfluss der Liquidität (vgl. Albach 1962, S. 23; Wippermann 2005, S. 18 ff. u. 30) und Finanzierung (vgl. Johansson und Löfgren 1985, S. 138 f.; Tahvonen et al. 2001; Coordes 2014a, S. 173 ff.) sowie Nachhaltigkeitsrestriktionen (vgl. z. B. Tahvonen und Viitala 2006; Möhring und Dieter 2020) auf die optimale Bestandesbehandlung. Unabhängig vom vollkommenen Kapitalmarkt bleibt zudem auch der Einfluss steuerrechtlicher Regelungen (vgl. z. B. Möhring 1994, S. 158 ff.; Hechtner et al. 2015; Möllmann und Möhring 2018) unbeachtet.

Die von der Ausgangsstammzahl unabhängigen Bestandesbegründungskosten unterstellen eine prämissenunabhängige Bestandesbegründung. In Verbindung mit den negativen Deckungsbeiträgen bei geringen Einzelbaumdimensionen (siehe dimensionsabhängig starke DB-Steigerung; Abbildung 23) werden damit bspw. offensichtlich vermeidbare Kosten verursacht. So könnte die stark reduzierte Stammzahl im ersten Zeitschritt (siehe Abbildung 50) bereits durch eine niedrigere Ausgangsstammzahl hergestellt werden. Damit verbunden wären niedrigere Bestandesbegründungskosten (vgl. z. B. Halbritter und Deegen 2015) und gleichzeitig eingesparte negative Deckungsbeiträge im ersten Zeitschritt. Implizit können diese negativen Deckungsbeiträge als (ansonsten im Modell unberücksichtigte) Kulturpflege- bzw. Läuterungskosten angesehen werden. 
Der Einfluss von Erntefixkosten auf die optimale Bestandesbehandlung wird lediglich anhand zweier extremer Varianten aufgezeigt. Es besteht kein Anspruch auf realistische, praxisorientierte Werte, wobei diese i. d. R. zwischen den beiden gewählten Varianten liegen dürften und die abgeleiteten Effekte somit übertragbar sind. Ebenfalls vernachlässigt wird die Abhängigkeit der Erntefixkosten vom Bestandesalter - eine mögliche Reduktion oder Häufung von Vornutzungen in bestimmten Bestandesphasen kann somit nicht analysiert werden.

Produktionsrisiken werden in Abschnitt 2.2.8 ausschließlich in Form von Überlebensrisiken, d. h. den vorzeitigen Ausfall von Bäumen bzw. des Bestandes durch Zwangsnutzungen, eingeführt. Marktrisiken bleiben unbeachtet bzw. sind nur implizit und abhängig von den Überlebensrisiken in Form von relativen Erlösminderungen integriert. Die Einbeziehung der Überlebensrisiken auf der Basis eines Erwartungswertes, als der mit den Eintrittswahrscheinlichkeiten gewichtete Mittelwert einer Zielgröße, unterstellt der rational entscheidenden Person implizit eine neutrale Einstellung ggü. Risiken (vgl. BAYES-Prinzip; Möhring 1994, S. 15). Subjektive und insbesondere auch von der jeweiligen Entscheidungssituation abhängige Risikoeinstellungen (vgl. BERNOULLI-Prinzip; Möhring 1994, S. 15) werden somit vernachlässigt. Ein alternatives Risikomaß stünde bspw. über den sogenannten „Value at Risk“ zur Verfügung (vgl. u. a. Hahn et al. 2014; Härtl et al. 2016; Paul et al. 2019a). Dieser gibt den Wert an, der bei Annahme einer bestimmten Ergebnisverteilung zu einer vorgegebenen Wahrscheinlichkeit mindestens erzielt wird (vgl. u. a. Paul et al. 2019b; Paul und Benfer 2019) und berücksichtigt die häufig risikoaverse Einstellung forstlicher Entscheidungsträgerinnen und Entscheidungsträger (vgl. Mußhoff und Maart-Noelck 2014; Sauter et al. 2016a; Dragicevic et al. 2016). Darüber hinaus stünden für Entscheidungssituationen ohne begründbare Informationen über die Wahrscheinlichkeitsverteilung von Zufallsereignissen bspw. Verfahren der robusten Optimierung (vgl. u. a. Härtl et al. 2018) zur Verfügung.

Die Wechselwirkungen zwischen Bestandesbehandlung und Überlebensrisiken werden in Abschnitt 2.2.9 aus Gründen der Komplexitätsreduktion und Förderung der Interpretierbarkeit auf denkbar einfachste Weise integriert. Die alleinige Abhängigkeit der Überlebensrisikostärke vom Einzelbaumvolumen in einem bestimmten Alter geht dabei von einem risikomindernden Effekt der Einzelbaumstabilität aus und setzt diese in Abhängigkeit zum Einzelbaumvolumen. Ein höheres Volumen bei gleichem Alter (und damit gleicher Baumhöhe) wird mit einem niedrigeren H/D-Verhältnis verknüpft, dessen Einfluss auf die Sturmschadenswahrscheinlichkeit in der Literatur zwar uneinheitlich diskutiert wird (Albrecht 2009, S. 12 f. und 135 f.), insb. aber 
in waldbaulichen Praxisleitlinien als wichtiger Indikator für den Aufbau stabiler Bestände herangezogen wird (vgl. u. a. BaySF 2009). Unterschlagen werden durch den gewählten Ansatz neben standörtlichen und topographischen (vgl. u. a. Mayer et al. 2005; Schmoeckel 2005; Schmidt et al. 2010; Albrecht et al. 2012; Albrecht et al. 2013) auch weitere (nicht einheitlich als signifikant eingestufte) behandlungsabhängige Einflussfaktoren auf die Überlebenswahrscheinlichkeit. Dazu zählen u. a. der zeitliche Abstand und die relative Eingriffsstärke der letzten Vornutzung, die Bestandesdichte oder die Baumkronencharakteristik (vgl. u. a. Lohmander und Helles 1987; Scott und Mitchell 2005; Schütz et al. 2006; Wallentin und Nilsson 2014; Albert et al. 2015; Donis et al. 2018) sowie nicht zuletzt auch Waldschutzmaßnahmen bei der Einbeziehung biotischer Schadfaktoren (vgl. u. a. Grodzki et al. 2006; Faccoli und Stergulc 2008; Seidl et al. 2009; Mezei et al. 2017; Dobor et al. 2020). 


\section{Ertragstafeln - forstliche Wachstums- und Nutzungsmodelle}

Forstliche Ertragstafeln (auch bezeichnet als Normalertragstafeln ${ }^{42}$; vgl. Gadow 2005, S. 134 ff.) bilden anhand ausgewählter ertragskundlicher Kenngrößen die naturale Entwicklung von Waldbeständen als ,gleichzeitig[e] Wachstums- und Nutzungsmodelle“ in Abhängigkeit von der Produktivität eines Standorts und unter Berücksichtigung einer bestimmten Bestandesbehandlung ab (Kramer 1988, S. 96). Pretzsch (2009, S. 437) bezeichnet sie mit Blick auf die mehr als 200-jährige Geschichte als älteste forstwissenschaftliche bzw. forstwirtschaftliche Modelle und Prodan (1965, S. 605) sieht ihre Aufstellung, trotz aller bekannter Schwächen und teils abnehmender Bedeutung, als ,gewaltigste, positive Arbeitsleistung der Forstwissenschaft“ an. Sie dienen der „Prognose der Bestandesentwicklung“ von Reinbeständen ${ }^{43}$ und zeichnen sich mit der tabellarischen Darstellung von u. a. Stammzahl, Höhe, Bestandesgrundfläche, mittlerem Einzelbaumdurchmesser, Bestandesvolumenzuwachs, Vornutzungsvolumen sowie Gesamtwuchsleistung in festen zeitlichen Intervallen durch eine allgemeine Verständlichkeit und leichte Handhabung aus (Gadow 2005, S. 135; vgl. Röhrig et al. 2006, S. 67).

Bevor in Abschnitt 3.3 die ausführliche Analyse einer neuen Generation dynamischer Ertragstafeln erfolgt, geben die beiden vorhergehenden Abschnitte einen allgemeinen Überblick über die Geschichte, Erstellungsmethodik, Struktur und Anwendung forstlicher Ertragstafeln: Abschnitt 3.1.1 skizziert dafür einführend die Entwicklungsgeschichte der Ertragstafeln im deutschsprachigen Raum in enger Anlehnung an Pretzsch (2009, S. 437 ff.) und erläutert klassische Anwendungsgebiete. Abschnitt 3.1.2 erläutert darauf aufbauend das Prinzip der Bonitierung sowie die ertragskundlichen Grundbeziehungen der Ertragstafelerstellung. Abschnitt 3.1.3 zeigt die Struktur forstlicher Ertragstafeln und geht dabei exemplarisch auf die Unterschiede ausgewählter Fichten-Ertragstafelwerke (Wiedemann 1936/42, Assmann und Franz 1963, Wenk et al. 1984) ein. Abschnitt 3.2 zeigt die Ergebnisse einer eigenständig durchgeführten Praxisbefragung zur gegenwärtigen und zukünftigen Verwendung forstlicher Ertragstafeln in der deutschen Forstwirtschaft.

\footnotetext{
${ }^{42}$ Der Zusatz „Normal“ bezieht sich auf den Referenzcharakter der Ertragstafeln, wobei diese Referenz nicht einheitlich definiert ist. Während im deutschsprachigen Raum damit auf eine definierte Bestandesbehandlung verwiesen wird, beschreiben „normal yield tables“ nach Pretzsch (2009, S. 440) im anglo-amerikanischen Raum die ungestörte Bestandesentwicklung (Nullflächen; Anm. d. A.).

${ }^{43}$ Im 20. Jahrhundert ebenfalls erstellte Mischbestandsertragstafeln (vgl. Bonnemann 1939; 1956 und Wiedemann 1942; 1949) wurden laut Gadow 2003, S. 160, Fußnote 3 ,in der Praxis wegen der vielfältigen realen Mischungsverhältnisse kaum verwendet“.
} 


\subsection{Grundlagen}

Aufgrund des in Forschung und Praxis unterschiedlich und teils inkonsistent verwendeten Oberbegriffs der Ertragstafel erfolgen in Anlehnung an Fibich (2019, S. 3) für diese Arbeit folgende begriffliche Festlegungen:

- Als kleinste Einheit bilden Ertragstafeltabellen die standortabhängige Ertragsfähigkeit einer Baumart, getrennt nach absoluten oder relativen Bonitäten, innerhalb einer Ertragstafel ab.

- Die Ertragstafel bildet mit den verschiedenen Ertragstafeltabellen ein spezifisches Wachstums- und Nutzungsmodell, wobei unterschiedliche waldbauliche Behandlungsvarianten (vgl. z. B. Wiedemann 1936/42 und siehe dyn-ET in Abschnitt 3.3) und/oder unterschiedliche Ertragsniveaus (vgl. z. B. Assmann und Franz 1963 oder Bergel 1985 und siehe Abschnitt 3.1.2) bzw. Bonitätssysteme (vgl. z. B. Wenk et al. 1984 und siehe Abschnitt 3.1.2) ausgewiesen werden. Wird also allgemein von Ertragstafeln gesprochen, ist damit das konsistente Modell des Wachstums- und Nutzungsgangs einer Baumart bei festgelegter waldbaulicher Behandlung gemeint.

- Ein Ertragstafelwerk fasst die zusammenhängend veröffentlichten Ertragstafeln einer Baumart zusammen (z. B. Jüttner 1955 mit den Ertragstafeln der mäßigen und starken Durchforstung für die Baumart Eiche).

- Als Ertragstafelsammlung wird schließlich die Zusammenfassung verschiedener Ertragstafelwerke unterschiedlicher Baumarten und/oder Autoren verstanden (vgl. z. B. Schober 1995).

Beispiel: Man findet die Ertragstafeltabelle „I. Ertragsklasse“ der Ertragstafel „,mäßige Durchforstung“ des Fichten-Ertragstafelwerks nach Wiedemann (1936/42) in der Ertragstafelsammlung von Schober (1995).

\subsubsection{Historische Entwicklung und Anwendung}

Als Produkt diverser waldwachstums- bzw. ertragskundlicher Untersuchungen entstanden im deutschsprachigen Raum seit Ende des 18. bzw. Anfang des 19. Jahrhunderts eine Vielzahl forstlicher Ertragstafeln. Sie bilden den Ausgangspunkt einer ganzen Reihe von waldwachstumskundlichen Modellen unterschiedlicher Datengrundlagen, Erstellungsmethoden und Anwendungsgebiete (vgl. Pretzsch 2009, S. 423 ff.). Während sich die Form der tabellarischen Darstellung einer Bestandesentwicklung anhand mittlerer und kumulierter Werte in über 200 Jahren kaum verändert hat, lassen sich die Ertragstafelwerke mit Blick auf die Datengrundlage 
und die Erstellungsmethodik im Wesentlichen drei entwicklungsgeschichtlichen Generationen zuordnen ( Pretzsch 2009, S. 432).

Im 18. Jahrhunderts fand im deutschsprachigen Raum eine allmähliche Abkehr von der zuvor mehr oder weniger ungeregelten Waldnutzung bzw. -rodung zugunsten einer planmäßigen forstlichen Bewirtschaftung statt (Hasel und Schwartz 2006, S. 261 und 375 f.). Auslöser dieser Entwicklung war u. a. die Erkenntnis, dass eine langfristige Versorgung der großen Holzverbraucher, wie etwa des Bergbaus und der nachgelagerten Wirtschaftsbereiche, nur durch den Verzicht auf Raubbau zu gewährleisten war (Hasel und Schwartz 2006, S. 261 und 375 f.). Als Autor des ersten rein forstlichen Buchs stellte der sächsische Berghauptmann Carlowitz 1713 die Forderung, nur so viel Wald (bzw. Holz) zu schlagen, wie wieder nachwächst (Hasel und Schwartz 2006, S. 375). Damit gilt Carlowitz als Begründer der forstlichen Nachhaltigkeit und Grundsteinleger einer mehr als 300-jährigen Entwicklungsgeschichte des vielgestaltigen Begriffs der Nachhaltigkeit (vgl. Carlowitz 1713; 2009 und Sächsische Hans-Carl-von-CarlowitzGesellschaft e. V. 2013). Mit der Etablierung dieses Grundsatzes in der Waldbewirtschaftung entstand das Bedürfnis, die tatsächlichen Zuwächse von Waldbeständen (und damit ihr nachhaltiges Nutzungspotential) zuverlässig und einfach schätzen zu können. Dieses Bedürfnis bewog Paulsen (1795) dazu, eine Reihe von Tabellen zu veröffentlichen, in denen er für verschiedene Reinbestände jeweils Alter, Höhe, Stammzahl, Vorrat und Zuwachs gegenüberstellte. Diese Tabellen gelten heute als älteste deutschsprachige ${ }^{44}$ Ertragstafeln und damit als Grundstein für die erste Ertragstafelgeneration, der Pretzsch (2009, S. 437) u. a. auch die Werke von Cotta (1821) und Hartig (1847) zuordnet.

Auffallend an den Ertragstafeln von Paulsen (1795) sowie nachfolgenden Werken der ersten Ertragstafelgeneration ist ihre äußerliche Ähnlichkeit zu moderneren Ertragstafelwerken. Das Festhalten an der tabellarischen Darstellung sieht Gadow (2005, S. 135) als Anzeichen dafür an, dass Ertragstafelwerke in dieser Form die an sie gestellten Anforderungen über einen langen Zeitraum erfüllen konnten. Abgesehen von diesen strukturellen Ähnlichkeiten bestehen aber kaum Ähnlichkeiten zu den späteren Ertragstafelgenerationen. Pretzsch (2009, S. 437; Übers. d. A.) verweist für die erste Ertragstafelgeneration, die er auch als „Erfahrungstafeln“

\footnotetext{
${ }^{44}$ Pretzsch (2009, S. 437) ordnet die ersten Anweisungen zur Ertragstafelerstellung in Anlehnung an Schwappach (1903, S. 165) dem Werk von Réaumur („Réflexions sur l'état des forêts“; Anm. d. A.) aus dem Jahr $1721 \mathrm{zu}$.
} 
bezeichnet, auf die „unbefriedigende Datengrundlage, regionale Beschränkungen oder Einschränkungen ihrer Gültigkeit und die begrenzte Vergleichbarkeit der jeweils genutzten Erstellungsmethodik“.

Bemerkenswert sind die verschiedenen methodischen Ansätze der ersten Ertragstafelgeneration insbesondere mit Blick auf die Herausforderung der Bonitierung, d. h. der Produktivitätsquantifizierung des Standorts (vgl. Gadow 2003, S. 31 ff.; siehe Abschnitt 3.1.2). Paulsen (1795) und andere zogen für die Bestimmung der „Standortsgüteklasse“ bspw. „Standorts- und Bodenmerkmale“ heran (Kennel 1973, S. 226). Cotta (1821) nutzte hingegen mit dem Vorrat (verbleibendes Bestandesvolumen) im Alter 100 a ein Bestandesmerkmal (Pretzsch 2009, S. 438). Dadurch wurde zwar die Subjektivität der Standortansprache theoretisch umgangen, die Güte der Bonitierung aufgrund der Behandlungsabhängigkeit des Vorrats aber nicht zwangsläufig erhöht (Kennel 1973). Dies wurde insbesondere durch den Übergang zu intensiveren Durchforstungskonzepten und den damit verbundenen, höheren Vornutzungsanteilen an der Gesamtwuchsleistung ab dem 19. Jahrhundert immer bedeutsamer (Assmann 1961, S. 155, Pretzsch 2009, S. 433). Zudem ist bei der Standortsklassifizierung über das Bestandesvolumen ein gewisser Zirkelschluss erkennbar, da die Quantifizierung des verbleibenden Bestandesvolumens ja gerade eine der Aufgaben forstlicher Ertragstafeln ist.

Baur $(1876,1881)$ erkannte die genannten Schwächen der vorangegangenen Bonitierungsansätze und etablierte eine neue Bonitierungsmethodik auf Basis der im jeweiligen Alter erreichten Bestandesmittelhöhe (Kennel 1973; siehe auch Abschnitt 3.1.2). Diese methodische Veränderung fiel zusammen mit dem Bemühen der Deutschen Forstlichen Versuchsanstalten die Ertragstafelerstellung durch eine Richtlinie über deren zukünftige Konstruktion zu vereinheitlichen (Pretzsch 2009, S. 440 nach Ganghofer 1881; siehe auch Pretzsch 2009, S. 101 ff.), was schließlich die zweite Ertragstafelgeneration einleitete. Auf Konferenzen einigte man sich in den 1870er und 1880er Jahren auf Eckpunkte, die das Erscheinungsbild und die dahinterstehende Datengewinnung sowie Konstruktionsmethodik forstlicher Ertragstafeln teilweise bis heute prägen und die Vergleichbarkeit unter den Ertragstafelwerken deutlich erhöhte (vgl. Verein Deutscher Forstlicher Versuchsanstalten 1873; 1902). In Verbindung mit einer immer weiter gewachsenen (überregionalen) Datengrundlage führten die vereinheitlichten Ansätze zur Veröffentlichung einer ganzen Reihe von wegweisenden Ertragstafeln, deren Entwicklung laut Pretzsch (2009, S. 440) im Wesentlichen Weise (1880) zu verdanken ist.

Nach Pretzsch (2009, S. 440) heben sich die Ertragstafeln von Schwappach (1902), Wiedemann (1932, 1936/42, 1949) und Schober (1975) aufgrund der (auch bei späteren Aktualisierungen 
beibehaltenen) einheitlichen konzeptionellen Grundlage von anderen Ertragstafelwerken bzw. Ertragstafelsammlungen der ersten Hälfte des 20. Jahrhunderts (u. a. Guttenberg 1915; Gehrhardt 1909; 1923) ab. Die für den nordwestdeutschen Raum überaus bedeutende und noch heute im Gebrauch befindliche (siehe Abschnitt 3.2) Ertragstafelsammlung von Schober (1975) bzw. Schober (1995) geht dabei u. a. zurück auf eine von Schwappach (1912) veröffentlichte Ertragstafelsammlung, die wiederum von Wiedemann (1949) ergänzt und bearbeitet wurde. Die Überarbeitungen und Neuveröffentlichungen stützen sich dabei insbesondere auf teilweise identische, in der Zwischenzeit weitergeführte, Versuchsflächen der ehemaligen Preußischen Forstlichen Versuchsanstalt (Schober 1995, S. 5).

Gehrhardt (1923) veröffentlichte eine weitere bedeutende Ertragstafelsammlung. Seine für die Hauptbaumarten Eiche, Buche, Tanne, Fichte und Kiefer aufgestellten Ertragstafeln beruhen auf der Zusammenführung bereits existierender Ertragstafeldaten über mathematische Modelle (Pretzsch 2009, S. 442 nach Gehrhardt 1930). Damit handelt es sich bei den Ertragstafeln Gehrhardts um die ersten, die den rein empirischen Ansatz vorangegangener Ertragstafeln um biometrisch-statistische Methoden ergänzten, was als Ausgangspunkt für die folgende, dritte Ertragstafelgeneration gesehen werden kann (Pretzsch 2009, S. 440 ff.).

Die Ertragstafelentwicklung der dritten Generation war in den 1950er und 1960er Jahren gekennzeichnet durch eine zunehmende Bedeutung der EDV-gestützten Auswertung von ertragskundlichen Ausgangsdaten (Pretzsch 2009, S. 442; vgl. u. a. Assmann und Franz 1963; Bradley et al. 1966). Basierend auf mathematisch formalisierten Einzelbaum- und Bestandeswachstumsbeziehungen ermöglichten die biometrisch-statistischen Modelle eine Ableitung der Bestandesentwicklung in Abhängigkeit von der Standortsgüte, der Bestandesbehandlung und dem Ertragsniveau (Pretzsch 2009, S. 442). Das Ertragstafelwerk von Assmann und Franz (1963) gilt zum einen aufgrund der zahlreichen methodisch darin enthaltenden, waldwachstumskundlichen Grundbeziehungen als wichtige Grundlage zahlreicher Ertragstafelwerke im letzten Drittel des 20. Jahrhunderts (Pretzsch 2009, S. 444; vgl. u. a. Lembcke et al. 1975, Wenk et al. 1984 und Bergel 1985). Zum anderen trug die erstmalige Ausweisung unterschiedlicher Ertragsniveaus (siehe Abschnitt 3.1.2) sowie die Bonitierung über die Bestandesoberhöhe (vgl. Pretzsch 2009, S. 433) zu der Bedeutsamkeit des Ertragstafelwerks von Assmann und Franz (1963) bei. Neben den bereits genannten etablierten Standardwerken der wirtschaftlich bedeutenden Hauptbaumarten mit meist relativ großem räumlichem Gültigkeitsanspruch, sei an dieser Stelle auch auf die Erstellung zahlreicher Spezial-Ertragstafeln hingewiesen, auf die in dieser Arbeit 
aber nicht weiter eingegangen wird. Beispielhaft seien etwa die Kiefernertragstafel für wirtschaftlich wichtige Standorteinheiten der Oberpfalz (Schmidt 1971), die Kiefernertragstafel für Kippenstandorte des Lausitzer Braunkohlenreviers (Ertle et al. 2012) oder die Robinienertragstafel von Erteld (1951) genannt.

Parallel zur Entwicklung weiterer Ertragstafeln erfuhren waldwachstumskundlichen Simulationsmodelle ab den 1960er Jahren immer größer werdende Beachtung. Angestoßen durch Arbeiten von u. a. Franz (1968), Hoyer (1975), Hradetzky (1972), Bruce et al. (1977), Curtis et al. (1981) und Curtis (1982) wurden zahlreiche Bestandeswachstumssimulatoren entwickelt, welche die mittlere Bestandesentwicklung auf der Grundlage von Funktionssystemen und Algorithmen sowie in Abhängigkeit von einer Vielzahl an alternativen Bestandesbehandlungen abbilden (Pretzsch 2009, S. 444). Die rasante Entwicklung im Bereich der Waldwachstumssimulation führte bspw. dazu, dass die vorläufige Fichten-Ertragstafel von Assmann und Franz (1963) stets vorläufig blieb - Assmann sah die zukünftige Bedeutung von Ertragstafeln durch die Simulationsmodelle zumindest teilweise in Frage gestellt (Franz und Utschig 1990, S. 10). Die fortlaufende Kapazitätssteigerung im Bereich der elektronischen Datenverarbeitung ermöglichte ab den 1980er Jahren die zunehmende Abkehr von Modellen auf der Basis von Bestandesmittelwerten. Einzelbaumbasierte Wachstumssimulatoren in vielfältiger Ausführung (vgl. Pretzsch 2009, S. 445 ff.) hielten Einzug in die forstwissenschaftliche Forschung, das forstliche Versuchswesen und auch die forstliche Praxis (Pretzsch 2009, S. 444)

Der heutige Stand in Bezug auf der Anwendung von und Forschung zu forstlichen Ertragstafeln ist divers zu sehen. Im Einklang mit Prodan (1965, S. 605), der bereits vor fast 60 Jahren eine abnehmende Bedeutung forstlicher Ertragstafeln prognostizierte, hielten einzelbaumbasierte Waldwachstumssimulatoren auch im deutschsprachigen Raum (z. B. BWINPro, Nagel und Schmidt 2006 oder SILVA, Kahn und Pretzsch 1997) Einzug in Wissenschaft, Versuchswesen und zumindest Teile der großen, öffentlichen Forstbetriebe. Zudem ist die Bedeutung von Betriebsinventuren zur Zustandserfassung im Rahmen der Forstbetriebsplanung (insb. in großen Forstbetrieben) stetig gestiegen (vgl. u. a. Fuchs und Kennel 1994; Nothdurft et al. 2008; Böckmann 2016). Weiterhin existieren in der forstlichen Praxis aber auch zahlreiche Anwendungsgebiete, in denen forstliche Ertragstafeln regelmäßig zum Einsatz kommen bzw. kommen können (vgl. Gerold und Gerold 2013; siehe auch Abschnitt 3.2). Mit der Forsteinrichtung und der Waldbewertung werden im Folgenden zwei bedeutende Anwendungsgebiete exemplarisch und mit besonderem Fokus auf den Einsatz forstlicher Ertragstafeln skizziert. 
Nach Bachmann (1990, S. 421) ist die Forsteinrichtung ,die mittel- und langfristige forstliche Planung auf betrieblicher und überbetrieblicher Ebene, welche unter Berücksichtigung ökologischer, wirtschaftlicher, betrieblicher und gesellschaftlicher Aspekte die Interessen am Wald sicherstellt“،. Kurth et al. (1994, S. 24) definieren sie knapp und dennoch umfassend als ,nachhaltige Regelung des Waldes“. Im Rahmen der Forsteinrichtung kommen forstliche Ertragstafeln einerseits bei der Zustandserfassung und andererseits bei der mittel- und langfristigen Planung zum Einsatz. Die Zustandserfassung beinhaltet dabei, neben einer Reihe anderer Faktoren, die Erhebung der vorliegenden Bestockungsverhältnisse in den einzelnen Waldbeständen eines Forstbetriebes. Dabei werden forstliche Ertragstafeln bzw. auf Ertragstafeldaten basierende Hilfstafeln und Planungssoftware eingesetzt, um u. a. die Bonitäten, Bestockungsgrade, Vorräte und laufenden Volumenzuwächse zu bestimmen. Die Bonität ergibt sich aus der Einordnungsbeziehung der verwendeten Ertragstafel (siehe Abschnitt 3.1.2) und gibt die zum Bestand passende Ertragstafeltabelle vor. Aus dem Verhältnis von Ist-Grundfläche (z. B. über Winkelzählproben nach Bitterlich (1949) ermittelt) zur altersspezifischen Ertragstafelgrundfläche (Soll- oder Referenzgrundfläche) resultiert der Bestockungsgrad (vgl. Speidel 1972, S. 237), über den der Vorrat des Bestandes linear aus dem Ertragstafelvorrat abgeleitet wird. Da abweichend davon keine proportionale Abhängigkeit des Volumenzuwachses von der Bestandesdichte besteht (vgl. z. B. Gadow 2005, S. 149 ff.; Pretzsch 2005a), kommen zur Anpassung der Ertragstafelvolumenzuwächse an die tatsächlichen Bestandesverhältnisse teilweise ${ }^{45}$ dichteabhängige Volumenzuwachskorrekturfaktoren (bzw. Zuwachsreduktionsfaktoren) zum Einsatz, welche in Abhängigkeit vom Bestockungsgrad bspw. in länderspezifischen Hilfstafeln für die Forsteinrichtung zu finden sind (Kramer und Akça 2008, S. 150). Als nachteilig anzusehen sind bei den aktuell verfügbaren dichteabhängigen Volumenzuwachskorrekturfaktoren die Vernachlässigung der Altersabhängigkeit (vgl. z. B. Pretzsch 2005a) sowie (teilweise) das Fehlen von Werten für überbestockte Bestände. Das laufende dyn-ET-Projekt (siehe Abschnitt 3.3) strebt die Ausweisung von Volumenzuwachskorrekturfaktoren an (Spellmann et al. unveröff.) - zum Zeitpunkt der Veröffentlichung dieser Arbeit liegen diese aber noch nicht vor. Sollen die beschriebenen Bestandesdaten für einen Mischbestand geschätzt werden, wird dieser rechnerisch

\footnotetext{
${ }^{45}$ Im Rahmen der durchgeführten Praxisbefragung wurde deutlich, dass die momentan verfügbaren Volumenzuwachskorrekturfaktoren in der forstlichen Praxis häufig keine Anwendung finden und zur Vereinfachung von einer linearen Abhängigkeit zwischen Bestockungsgrad und Volumenzuwachs ausgegangen wird. Kommt Forsteinrichtungssoftware zum Einsatz, sind die Volumenzuwachskorrekturfaktoren darin allerdings häufig berücksichtigt und müssen von der anwendenden Person nicht weiter beachtet werden.
} 
in mehrere Bestandesflächen zerlegt (Speidel 1972, S. 83). Für jede dieser rechnerischen Bestandesflächen erfolgt die Schätzung wie für den Reinbestand beschrieben - lediglich zur Ermittlung der Volumenzuwachskorrekturfaktoren wird nicht der Bestockungsgrad der einzelnen rechnerischen Bestandesflächen, sondern des Gesamtbestandes herangezogen. Durch die Baumartenmischung bedingte Abweichungen vom Volumenzuwachs ggü. dem Wachstum im Reinbestand (vgl. u. a. Pretzsch et al. 2010b; Pretzsch et al. 2013a; Pretzsch et al. 2013b; Pretzsch et al. 2015), sogenannte Mischungseffekte, können ${ }^{46}$ in Abhängigkeit von Mischungsform und -anteilen über Mischbestandsertragstafeln (vgl. nach Gadow 2003, S. 160, Fußnote 3 z. B. Bonnemann 1939; 1956; Wiedemann 1942; 1949) oder Korrekturfaktoren (vgl. z. B. Pretzsch 2016) in die Ertragstafelschätzungen einbezogen werden, weshalb diese bspw. auch im laufenden dyn-ET-Projekt (siehe Abschnitt 3.3) für ausgewählte Mischungsvarianten ausgewiesen werden sollen (Spellmann et al. unveröff.). Liegen mehrere Bestandesschichten vor, werden deren Volumenzuwächse ebenfalls separat geschätzt (vgl. z. B. HMULF 2002). Weiteres Einsatzgebiet forstlicher Ertragstafeln im Rahmen der Forsteinrichtung ist die Ermittlung der mittelfristigen Vor- und Endnutzungsvolumina bei der bestandesweisen Nutzungsplanung (vgl. Speidel 1972, S. 128 ff.). So kann bspw. das Vornutzungsvolumen eines Bestandes ausgehend vom aktuellen Vorrat (gemäß Zustandserfassung; siehe oben) mit Hilfe des geschätzten Volumenzuwachses der Planungsperiode (unter Berücksichtigung von Volumenzuwachskorrekturfaktoren; siehe oben) und des angestrebten Vorrats am Ende der Planungsperiode (in Abhängigkeit vom Ziel-Bestockungsgrad) bestimmt werden. Ergebnis dieser Einzelplanung ist dann u. a. der sogenannte waldbauliche Hiebsatz als Summe der einzelbestandesweisen Nutzungsansätze (Speidel 1972, S. 162). Die betriebliche Gesamtplanung überträgt die bestandesweise Einzelplanung unter Berücksichtigung der betrieblichen Ziele und sonstigen Rahmenbedingungen auf den Gesamtbetrieb - die ,induktive Einzelplanung“ wird „,durch eine deduktive Gesamtplanung ergänzt“" (Speidel 1972, S. 162 nach Schober 1952). Aufgabe sind die Sachziel-, Nachhaltigkeits- und Wirtschaftlichkeitsprüfung (Speidel 1972, S. 162), wobei forstlichen Ertragstafeln insbesondere im Zuge der Berechnung sogenannter Nachhaltsweiser eine wichtige Bedeutung zukommt (Speidel 1972, S. 163 ff.). So dienen Ertragstafeln bspw. im Zuge der Herleitung von Vorrats- und Zuwachsweisern der Berechnung des Normalvorrats. Formelweiser, wie bspw. die Gehrhardt'sche Formel, greifen zudem auf Ertragstafeldaten, wie den SollZuwachs (durchschnittlicher Gesamtvolumenzuwachs bis zur geplanten Umtriebszeit) zurück.

\footnotetext{
${ }^{46}$ Keine der im Rahmen der Praxisbefragung interviewten Personen gab die Nutzung von mischungsabhängigen Volumenzuwachskorrekturfaktoren an.
} 
Wichtige Bedeutung kommt der Forsteinrichtung als anerkanntes Betriebswerk und damit indirekt auch forstlichen Ertragstafeln zudem im Zuge der Ausweisung eines steuerrechtlichen Nutzungssatzes zu. Dieser stellt im Sinne des $§ 34 \mathrm{~b}$ Abs. 3 Nr. 2 EStG in Verbindung mit $§ 68$ EStDV eine Bemessungsgrundlage für außerordentliche Holznutzungen dar und muss nach Einkommenssteuer-Richtlinie R 34b. 4 ,,den Nutzungen entsprechen, die unter Berücksichtigung der vollen jährlichen Ertragsfähigkeit des Waldes in Kubikmetern (Festmetern) objektiv nachhaltig im Betrieb erzielbar sind. Maßgebend für die Bemessung des Nutzungssatzes sind nicht die Nutzungen, die nach dem Willen des Betriebsinhabers in einem Zeitraum von zehn Jahren erzielt werden sollen (subjektiver Hiebsatz), sondern die Nutzungen, die unter Berücksichtigung der vollen Ertragsfähigkeit nachhaltig erzielt werden können (objektive Nutzungsmöglichkeit)“‘. In $§ 68$ EStDV Abs. 2 wird dabei die Bedeutung der Objektivierung des naturalen Wachstums- und Nutzungsmodells (z. B. Ertragstafel) ersichtlich: „Der Festsetzung des Nutzungssatzes ist ein amtlich anerkanntes Betriebsgutachten oder ein Betriebswerk zugrunde zu legen, das auf den Anfang des Wirtschaftsjahres aufzustellen ist, von dem an die Periode von zehn Jahren beginnt. [...]“. Berücksichtigung findet der Nutzungssatz weitergehend bspw. auch bei der Bemessung von Beiträgen zur gesetzlichen Unfallversicherung für Forstbetriebe über 100 ha (vgl. Gutachterlicher Stellungnahme zur Aktualisierung des Beitragsmaßstabs für die Unfallversicherung in der Landwirtschaft, im Forst sowie im Gartenbau; SVLFG 2018).

Nach Mantel (1982, S. 11) befasst sich die Waldbewertung ,mit der Ermittlung von Waldwerten verschiedenster Art, die sich aus der wald- und forstwirtschaftlichen Benützung des Grund und Bodens ergeben“. Eine solche Waldwertermittlung kann u. a. notwendig werden bei einem Eigentumswechsel, im Zuge von Nutzungsbeschränkungen und in Schadensfällen (Mantel 1982, S. 11). Im Rahmen der in der Praxis verwendeten Waldbewertungsmethoden kommen forstliche Ertragstafeln an verschiedenen Stellen zum Einsatz. Beispielhaft sei hier das Alterswertfaktorverfahren zur Ermittlung des sogenannten Bestandeswerts nach Blume (vgl. NMELV 2020) skizziert. Die Wertermittlung geschieht in diesem deterministischen ${ }^{47}$ Verfahren mit Hilfe von Alterswertfaktoren, die den Wert eines Bestandes zu einem bestimmten Zeitpunkt im Verhältnis zu seinem Abtriebswert zum Endnutzungszeitpunkt abbilden und in die Blume-Formel (Blume 1949) eingesetzt werden. Die bundeseinheitlich verfügbaren und zuletzt im Jahr 2019 aktualisierten Alterswertfaktoren für die Baumartengruppen Eiche, Buche, Fichte und

\footnotetext{
${ }^{47}$ Koster (2017) zeigte einen methodischen Weg zur Integration von Überlebensrisiken in das Alterswertfaktorenverfahren.
} 
Kiefer basieren dabei auf von den Bundesländern übermittelten Eingangsdaten (Staupendahl et al. 2020). Länderspezifisch liefern etablierte Ertragstafeln (teilweise in angepasster Form; vgl. z. B. Staupendahl 2018) dafür u. a. die naturale Grundlage (vgl. Haub und Weimann 2000 sowie NLF 2014, Tabelle 1.14 in der Version 04/19). Darüber hinaus dienen forstliche Ertragstafelwerke bzw. darauf basierende Hilfstafeln aber insbesondere der Ermittlung des erntekostenfreien Abtriebswertes im konkreten Bewertungsfall. Bonitätsabhängig werden Vorrat und mittlerer Brusthöhendurchmesser (meist angepasst) des zu bewertenden Bestandes im Alter der planmäßigen Umtriebszeit bestimmt. In der Kombination mit Bestandessortentafeln, Holzerlösen sowie Holzerntekosten lässt sich schließlich der erntekostenfreie Abtriebswert zum Endnutzungszeitpunkt bestimmen (siehe auch Abschnitt 3.3.5), mit dem unter Einbeziehung der Bestandesbegründungskosten und des Alterswertfaktors der Bestandeswert im Bewertungsalter über die Blume-Formel ermittelt wird. Das Verfahren lässt aufgrund der zahlreichen notwendigen Annahmen erkennen, dass es im Rahmen der Waldbewertung weniger um einen im spezifischen Fall subjektiv korrekten, als um einen standardisierten, transparent hergeleiteten und objektivierten Wert geht, der von beiden am Bewertungsfall beteiligten Seiten akzeptiert wird. Ertragstafeln bilden dabei als nachvollziehbare und konsistente Wachstums- und Nutzungsmodelle eine vergleichbare und standardisierte naturale Basis. Bei zusätzlich einheitlichen ökonomischen Kenngrößen erfüllt das Ergebnis der Wertermittlung die Bedürfnisse der Objektivierung. Dies gilt insbesondere vor dem Hintergrund möglicher Alternativen: Die Schätzung auf Basis einer Waldwachstumssimulation wäre zwar im Einzelfall i. d. R. exakter, jedoch für AuBenstehende nicht nachvollziehbar und zudem meist nicht reproduzierbar (vgl. Gerold und Gerold 2013).

\subsubsection{Bonitierung und ertragskundliche Grundbeziehungen}

Ziel der forstlichen Bonitierung ist nach Gadow (2003, S. 31 f.) die „quantitative wie qualitative Einschätzung der produktiven Leistung eines bereits vorhandenen oder eines noch zu begründenden Bestandes“. Sie ist Voraussetzung für die betriebliche Planung und ermöglicht neben der Einschätzung zukünftiger Erträge auch die „,regelmäßige Einschätzung des standörtlichen Leistungspotentials“ im Sinne der Standortnachhaltigkeit (Gadow 2003, S. 53). Die Bonität ist Ausdruck der „Produktivität eines Standorts“ und wird über die Ertragsfähigkeit eines Bestandes, d. h. die altersspezifische Gesamtwuchsleistung einer Baumart, gemessen (Gadow 2003, S. 32). Die Bestimmung der Bonität kann auf direktem und indirektem Weg erfolgen. Methoden der indirekten Bonitierung ziehen dafür Standortsparameter, wie z. B. Bodenzustand, Bodenvegetation und Klima, heran (Gadow 2003, S. 44 ff.) - auf sie wird in dieser Arbeit nicht 
weiter eingegangen. Im Rahmen der direkten Bonitierung dient der Bestand selbst als Bonitätsmaßstab - die Bäume fungieren insoweit als Bioindikatoren (,phytometer“" nach Pretzsch 2009, S. 433).

Da bei der direkten Bonitierung die eigentliche Zielgröße, die altersabhängige Gesamtwuchsleistung, nicht direkt bestimmt werden kann (Gadow 2003, S. 32), greifen forstliche Ertragstafeln dafür, spätestens seit der Etablierung der Bestandeshöhe als Bonitierungsmerkmal durch Baur (1876, 1881), auf drei Grundbeziehungen zurück (Assmann 1961, S. 157 f.):

- Die Grundbeziehung I oder Einordnungsbeziehung,

- die Grundbeziehung II oder Hilfsbeziehung und schließlich

- die Endbeziehung.

Unter der Voraussetzung einer einheitlichen Grundbeziehung I sagt diese aus, dass sich die Höhe $H$ eines Bestandes als standortspezifische Funktion des Alters $t$ ergibt:

$$
H=f(t)
$$

Aus der Vielzahl der möglichen, standortabhängigen Höhenentwicklungen folgt ein sich „füllhornartig“ aufweitender Bonitätsfächer über dem Alter (Assmann 1961, S. 155), wobei zwischen relativen und absoluten Höhenbonitäten unterschieden werden kann ${ }^{48}$. Relative Höhenbonitäten (auch als Ertragsklassen ${ }^{49}$ bezeichnet) werden durch gleichmäßige Aufteilung des gesamten „Streubandes“ an möglichen Höhenentwicklungen erzeugt (Assmann 1961, S. 155; siehe Pretzsch 2009, S. 434, Fig. 11.6b) und weisen die Ertragsfähigkeit somit relativ zueinander aus - Aussagen über die absoluten Leistungsdifferenzen und Vergleiche zwischen den Bonitätsfächern unterschiedlicher Ertragstafeln sind nicht direkt möglich (Gadow 2003, S. 32). Absolute Höhenbonitäten, bspw. verwendet von Assmann und Franz (1963), ermöglichen hingegen die direkte Quantifizierung der Wuchsleistung über die Höhe des Bestandes in einem Bezugsalter (z. B. 100 a, siehe Abschnitt 3.3) und damit auch den Vergleich unterschiedlicher Bonitätsfächer (Gadow 2003, S. 34 f.).

\footnotetext{
${ }^{48}$ Neben den relativen und absoluten Höhenbonitäten verweist Gadow (2003, S. 41) auf die in der forstlichen Praxis teilweise verwendeten, allerdings stark behandlungsabhängigen $d G Z$-Bonitäten. So ist der maximale durchschnittliche Gesamtzuwachs $\left(d G Z_{\max }\right)$ als sogenannte Leistungsklasse (vgl. z. B. Nds. Forstplanungsamt 1987, S. 5) und der durchschnittliche Gesamtzuwachs bis zum Alter 100 a $\left(d G Z_{100}\right)$ als Leistungsstufe (vgl. z. B. Bösch 2002) zu finden. Nach Gadow (2003, S. 41) handelt es sich bei den leicht zu interpretierenden $d G Z$-Bonitäten letztlich aber auch um indirekte Höhenbonitäten.

${ }^{49}$ Die synonyme Verwendung von „Höhenbonität“ und „Ertragsklasse“ bezeichnet Assmann (1961, S. 155) mit Blick auf die im Folgenden noch thematisierte Uneinheitlichkeit der Grundbeziehung II als unberechtigt (vgl. auch Assmann 1959).
} 
Nicht bei allen Ertragstafelwerken wird allerdings von der Einheitlichkeit der Grundbeziehung I ausgegangen. Bspw. verfolgten Wenk et al. (1984) mit der Ausweisung sogenannter Bonitätssysteme ein Konzept zur Vermeidung ,systematische[r] Bonitätsänderungen [...] bei aufeinanderfolgenden Inventuren“(Wenk et al. 1984, S. 3).

Demnach können zwei Bestände die gleiche Höhe im Bezugsalter, und folglich die gleiche Bonität aufweisen, dabei aber unterschiedliche Höhenentwicklungen durchlaufen haben. So kann etwa das Höhenwachstum des einen Bestandes zu einem früheren Zeitpunkt kulminieren als das des anderen. Basierend auf dieser Erkenntnis wiesen Wenk et al. (1984) drei unterschiedliche Bonitätssysteme $(\psi)$ aus, die sie als langsames, mittleres und schnelles System bezeichneten. Die Einordnungsbeziehung ihres Fichten-Ertragstafelwerks lautet demnach:

$$
H=f(t, \psi) \text {. }
$$

Die drei Bonitätssysteme sind durch unterschiedlich rasche Höhenentwicklungen gekennzeichnet, wobei innerhalb einer absoluten Höhenbonität die erreichten Höhen im Bezugsalter (100 Jahre) gleichbleiben. Folglich machen sich die Auswirkungen der unterschiedlichen Bonitätssysteme vor allem in jüngeren Bestandesphasen bemerkbar. Mit zunehmender Annäherung an das Bezugsalter nähern sich die Höhenentwicklungen einander an - nach Erreichen des Bezugsalters kehrt sich ihre Rangfolge um.

Wenk et al. (1984, S. 6 f.) stellen dem Anwender einen Bestimmungsschlüssel zur Ermittlung des Bonitätssystems mit folgenden Eingangsgrößen bereit:

- Ursprung des Bestandes (Naturverjüngung / Saat, Erstaufforstungen, Pflanzung),

- Lage des Bestandes (Tiefland ggü. Kammlagen),

- Standortsmerkmale (Trophie- und Feuchtestufen nach ostdeutschem System) und

- Klimastufe.

Der Bestimmungsschlüssel arbeitet dabei mit einem Rangfolgesystem, bei dem die nachgeordneten Kriterien nur dann berücksichtigt werden müssen, wenn die vorgelagerten Kriterien keine Bestimmung ermöglichen. Inwieweit dieser Bestimmungsschlüssel tatsächlich in der betrieblichen Praxis zur Anwendung kommt, konnte auch im Rahmen einer Praxisbefragung (siehe Abschnitt 3.2) nicht allgemeingültig geklärt werden. Keine der befragten Personen gab allerdings die Verwendung eines anderen als des mittleren Bonitätssystems an. 
Ist die für die konkrete Alters-Höhenkombination passende Einordnungsbeziehung ${ }^{50}$ (und damit die passende Bonität) gefunden, wird über die Grundbeziehung II oder Hilfsbeziehung ein Zusammenhang zwischen der Bestandeshöhe $H$ und der Gesamtwuchsleistung GWL hergestellt. Geht man von einer einheitlichen Beziehung in Anlehnung an das erweiterte Eichhorn'sche Gesetz (vgl. Pretzsch 2009, S. 435 f. nach Eichhorn 1902; 1904 und Gehrhardt 1909; 1923; Gehrhardt 1930) aus, gilt je Bonität:

$$
G W L=f(H) \text {. }
$$

Die Verwendung einer solchen einheitlichen Hilfsbeziehung erleichterte die Aufstellung von Ertragstafeln erheblich - sie kam in zahlreichen Ertragstafelwerken der ersten Hälfte des 20. Jahrhunderts zum Einsatz (z. B. Gehrhardt 1923, Wiedemann 1936/42 oder Wiedemann 1943; 1948).

Assmann aber wies in den 1950er Jahren mehrfach anhand von bayerischen Versuchsflächen nach, dass die Gesamtwuchsleistung von Fichtenbeständen, im Widerspruch zum erweiterten Eichhorn `schen Gesetz, selbst bei völlig altersgleicher Oberhöhe um erhebliche Beträge variierte (Assmann 1961, S. 160 ff.). Basierend auf diesen Untersuchungen prägte Assmann den Begriff des Ertragsniveaus und stellte die Theorie auf, dass zwischen den erreichten Bestandeshöhen und der Gesamtwuchsleistung zweier Bestände ein enger Zusammenhang besteht, solange diese über den gleichen Standort und die gleiche Behandlungsgeschichte verfügen (Franz und Utschig 1990, S. 8). Darauf aufbauend rückten Assmann und Franz (1963) in ihrer Fichten-Ertragstafel (und später bspw. auch Lembcke et al. 1975 und Bergel 1985) von der Verwendung einer einheitlichen Hilfsbeziehung ab und führten das Ertragsniveau $(\varepsilon)$ als weitere Abhängigkeitsvariable in der Grundbeziehung II ein:

$$
G W L=f(H, \varepsilon) \text {. }
$$

Ähnlich wie bei den Bonitätssystemen nach Wenk et al. (1984) liegt die Schwierigkeit bei der Einbeziehung des Ertragsniveaus in dessen praktischer Einschätzung - die Steigerung der Schätzgenauigkeit hängt somit maßgeblich von der Verfüg- und insb. Handhabbarkeit eines praxistauglichen Bestimmungsverfahrens ab (vgl. Wenk et al. 1990, S. 250 nach Schütz 1977). Wenk et al. (1990, S. 251 f.) nennen zwar verschiedene Standortsfaktoren (Klima, Boden, Ve-

\footnotetext{
${ }^{50}$ Die Grundbeziehung I wird auch als Einordnungsbeziehung bezeichnet, da sie der Einordnung eines vorliegenden Bestandes in das Bonitierungsschema einer Ertragstafel dient.
} 
getation), die auf das Ertragsniveau einwirken, und diskutieren Studien zur Ertragsniveaubestimmung über Bestockungsmerkmalen (max. Grundfläche, Oberdurchmesser und Schlankheitsgrad, Kronenschlussgrad, Durchwurzlungstiefe) - die präzise und dabei einfache Bestimmung des Ertragsniveaus bleibt dennoch eine Herausforderung für die forstliche Praxis. Es ist anzunehmen, dass u. a. auch aus diesem Grund oftmals auf die Ausweisung des oberen und unteren Ertragsniveaus verzichtet wird (vgl. z. B. Bay. StMELF 1981).

Durch die Verknüpfung von Einordnungs- und Hilfsbeziehung kann schließlich über die Endbeziehung dem Alter $t$ eine Gesamtwuchsleistung GWL zugeordnet und damit die eigentliche Zielgröße der Bonitierung bestimmt werden (Assmann 1961, S. 157 f.):

$$
G W L=f(t) \text {. }
$$

\subsubsection{Struktur}

Wie bereits erwähnt, hat sich die grundlegende Struktur forstlicher Ertragstafeln seit Paulsen (1795) kaum verändert - sie wird im Folgenden in Anlehnung an die Ertragstafelsammlung von Schober 1995 beschrieben, wobei exemplarisch auf bestehende Unterschiede etablierter Fichten-Ertragstafelwerke ${ }^{51}$ hingewiesen wird.

Getrennt für die einzelnen Bonitätsklassen wird die Bestandesentwicklung über dem Alter typischerweise in 5- oder seltener in 10-Jahresschritten zeilenweise in Ertragstafeltabellen angegeben. In der Regel starten die Tabellen mit, bzw. einen Zeitschritt vor, dem Zeitpunkt der ersten Vornutzung, wobei sich dieser Zeitpunkt mit abnehmender Bonität nach hinten verschiebt. Die Angaben enden baumarten- und bonitätsabhängig in Anlehnung an übliche Umtriebszeiten - so enthält Jüttner (1955, mäßige Durchforstung, I. Ertragsklasse) für die Baumart Eiche Angaben bis zu einem Alter von 200 Jahren, während die Bestandesentwicklung der Baumart Fichte nach Wiedemann (1936/42, mäßige Durchforstung, I. Ertragsklasse) lediglich bis zu einem Alter von 120 Jahren dargelegt wird.

In etablierten Ertragstafelsammlungen (vgl. z. B. Schober 1995) und auch bei der in Abschnitt 3.3 vorgestellten Neuen Generation dynamischer Ertragstafeln beziehen sich die in den

\footnotetext{
${ }^{51}$ Ein ausführlicher methodischer, struktureller, ertragskundlicher und ökonomischer Vergleich der drei Fichten-Ertragstafelwerke (Wiedemann 1936/42; Assmann und Franz 1963; Wenk et al. 1984) und mit einer Aktualisierung für den Landesbetrieb HessenForst von Staupendahl (2018) wurde von Fibich (2019) durchgeführt.
} 
einzelnen Ertragstafeltabellen angegebenen Bestandesdaten spaltenweise auf den verbleibenden, den ausscheidenden sowie den gesamten Bestand (Gesamtbestand). Mit Blick auf die Reihung dieser Spaltenblöcke bestehen allerdings Unterschiede (Abbildung 52). Während Wiedemann (1936/42) sowie Assmann und Franz (1963) mit dem verbleibenden Bestand beginnen, folgen Wenk et al. (1984) gewissermaßen einem üblichen Durchforstungsturnus - die Ertragstafeltabellen beginnen mit Angaben zum Gesamtbestand ganz links, wobei im Gegensatz zu den anderen Autoren auch Vorratswerte angegeben werden. Mittig folgen die Spalten zum ausscheidenden Bestand und erst ganz rechts wird der verbleibende Bestand beschrieben. Die im ausscheidenden Bestand angegebenen Vornutzungen werden von Assmann und Franz (1963) analog zum laufenden Volumenzuwachs (siehe oben) zwischen den Zeilen, also zwischen zwei Zeitpunkten angegeben. Wiedemann (1936/42) und Wenk et al. (1984) verbuchen diese sozusagen in einer gedachten Sekunde rückblickend in der jeweils folgenden Zeile.

Unabhängig von der Spaltenreihung beziehen sich die Angaben zum verbleibenden Bestand als Zeitpunktinformationen auf den Bestandeszustand nach einem periodenbezogenen Zuwachs und nach erfolgter Vornutzung. Typisch sind u. a. Angaben zu

- der Bestandesober- und/oder Bestandesmittelhöhe $[\mathrm{m}]^{52}$,

- der verbleibenden Stammzahl [ha $\left.{ }^{-1}\right]$,

- dem verbleibenden Bestandesvolumen (Vorrat) $\left[\mathrm{Fm} \mathrm{ha}^{-1}\right]^{53}$,

- der Grundfläche des verbleibenden Bestandes $\left[\mathrm{m}^{2} \mathrm{ha}^{-1}\right]$ sowie

- dem mittleren Baumdurchmesser ${ }^{54}$ des verbleibenden Bestandes [cm].

\footnotetext{
${ }^{52}$ Während Assmann und Franz (1963) die Höhe des Grundflächenmittelstammes der 100 stärksten Bäume je Hektar verwenden, nutzen Wenk et al. (1984) die Höhe des Grundflächenmittelstammes der 10 \% stärksten Bäume je Hektar. Bei Wiedemann (1936/42) sind in der Bearbeitung von Schober (1995) die Mittelhöhen der 100 stärksten Bäume je Hektar sowie die Höhe des Grundflächenmittelstammes der $20 \%$ stärksten Bäume des Bestandes (Oberhöhe nach Weise) zu finden.

${ }^{53}$ Während Wiedemann (1936/42) in der Bearbeitung von Schober (1995) ausschließlich Volumenangaben zum Derbholz mit Rinde in Vorratsfestmetern macht, geben Assmann und Franz (1963) auch, bzw. für den ausscheidenden Bestand ausschließlich, Schaftholzvolumina mit Rinde in Vorratsfestmetern an (vgl. Kramer und Akça 2008, S. 35). Darüber hinaus werden „reduzierte Tafelwerte“ in Erntefestmetern Derbholz ohne Rinde aufgezeigt und somit indirekt auch Ausnutzungsgrade angegeben (vgl. Fußnote 78 auf Seite 187). Wenk et al. (1984) verzichten auf Derbholzangaben und zeigen neben Schaftholzvolumina auch Erntevolumina für den ausscheidenden sowie den Gesamtbestand auf.

${ }^{54}$ Der mittlere Baumdurchmesser des verbleibenden und ausscheidenden Bestandes wird i. d. R. über den Durchmesser des Grundflächenmittelstammes (vgl. Kramer und Akça 2008, S. 103) charakterisiert.
} 
Die Angaben zum ausscheidenden Bestand charakterisieren die Vornutzung in der (i. d. R. rückblickend definierten) Periode, $d$. h. sie geben Informationen über die vornutzungsbedingte Veränderung des Bestandes zwischen zwei Zeitpunkten. Typisch sind Angaben zu

- der ausscheidenden Stammzahl $\left[\mathrm{ha}^{-1}\right]$,

- dem ausscheidenden Bestandesvolumen [Fm ha $\left.{ }^{-1}\right]$,

- der Grundfläche des ausscheidenden Bestandes $\left[\mathrm{m}^{2} \mathrm{ha}^{-1}\right]$,

- dem mittleren Baumdurchmesser des ausscheidenden Bestandes ${ }^{55}[\mathrm{~cm}]$ sowie

- der Mittelhöhe des ausscheidenden Bestandes [m].

Weitere Angaben charakterisieren den Gesamtbestand, der sich aus verbleibendem Bestand einschließlich aller erfolgten Vornutzungen (ausscheidender Bestand) bis zum jeweiligen Zeitpunkt zusammensetzt. Typisch sind Angaben zu

- der Gesamtwuchsleistung [Fm ha $\left.{ }^{-1}\right]$,

- dem laufenden Bestandesvolumenzuwachs $\left[\mathrm{Fm} \mathrm{ha}^{-1} \mathrm{a}^{-1}\right]$ und

- dem durchschnittlichen Bestandesvolumenzuwachs $\left[\mathrm{Fm} \mathrm{ha} \mathrm{a}^{-1} \mathrm{a}^{-1}\right]$.

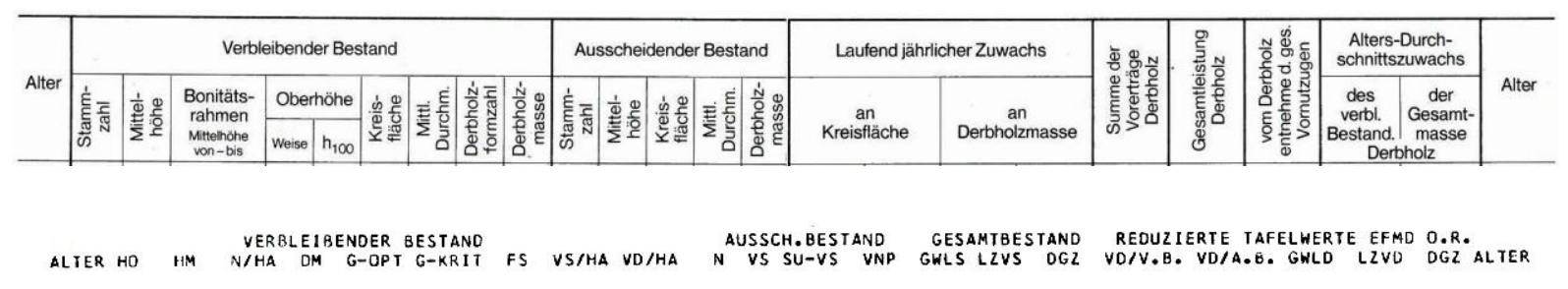

\begin{tabular}{|c|c|c|c|c|c|c|c|c|c|c|c|c|c|c|c|c|c|}
\hline \multicolumn{10}{|c|}{ Geamtbogtand } & \multicolumn{4}{|c|}{ ausbcheidender Bestand } & \multicolumn{4}{|c|}{ verbleibender Bestand } \\
\hline bo & $h \bar{B}$ & $d \bar{g}$ & $\boldsymbol{N}$ & G & vs & $\mathbf{v E}$ & GWIS & zvs & $D G Z S$ & $d \bar{B}$ & $\bar{\nabla}$ & vs & VE SUVS & $d \bar{B}$ & $\mathbf{N}$ & G & vs \\
\hline
\end{tabular}

Abbildung 52: Tabellenköpfe (eingescannt) etablierter Fichten-Ertragstafelwerke (von oben nach unten): Wiedemann (1936/42) in Schober (1995), Assmann und Franz (1963) sowie Wenk et al. (1984).

Die Gesamtwuchsleistung ergibt sich aus der Summe aller Vornutzungen zuzüglich des aktuell verbleibenden Bestandesvolumens und entspricht somit auch der Summe aller Bestandesvolumenzuwächse bis zum jeweiligen Zeitpunkt (Gadow 2003, S. 153). Der laufende Bestandesvolumenzuwachs wird als periodischer Mittelwert zwischen zwei Zeitpunkten angegeben. Uneinheitlich sind dabei die Verbuchungszeitpunkte bzw. die Richtung der Betrachtung. Während Wiedemann (1936/42) und Wenk et al. (1984) den laufenden Volumenzuwachs rückblickend verbuchen, geben Assmann und Franz (1963) den laufenden Volumenzuwachs (wie auch Angaben zum ausscheidenden Bestand) gewissermaßen zwischen den Zeilen, also zwischen zwei Alterszeitpunkten, an. Der durchschnittliche Bestandesvolumenzuwachs wird schließlich als Quotient aus Gesamtwuchsleistung und entsprechendem Alter ermittelt.

\footnotetext{
${ }^{55}$ Bemerkenswert ist, insb. vor dem Hintergrund betriebswirtschaftlicher Kalkulationen, das Fehlen der Angaben zum mittleren Baumdurchmesser des aussch. Bestandes bei Assmann und Franz (1963).
} 


\subsection{Praxisbefragung zur gegenwärtigen und zukünftigen Verwendung}

Zur Erfassung der gegenwärtigen und zukünftigen Verwendung forstlicher Ertragstafeln in der betrieblichen Praxis wurde im Rahmen des Verbundforschungsprojekts „Neue Generation dynamischer Ertragstafeln“ eine Praxisbefragung durchgeführt - Auszüge der Ergebnisse werden im Folgenden vorgestellt.

\subsubsection{Methodik und Charakterisierung der Befragten}

Per Mail (Abbildung 151 im Anhang) wurden 163 Personen aus öffentlichen Behörden, öffentlichen und privaten Forstbetrieben sowie privaten Dienstleistungsunternehmen kontaktiert, bei denen aufgrund ihres Aufgabenschwerpunkts (z. B. Forsteinrichtung oder Waldbewertung) von einer mehr oder weniger intensiven Nutzung forstlicher Ertragstafeln im beruflichen Alltag auszugehen war. Die Auswahl erfolgte dabei über online-verfügbare Organigramme und per Telefon erfragten Informationen zur innerorganisatorischen Zuständigkeit. Zudem wurden (mit Erlaubnis der verantwortlichen Personen) Mailverteiler der Arbeitsgemeinschaft Forsteinrichtung sowie der Abteilung Forstökonomie der Georg-August-Universität Göttingen genutzt. Wie in der Mail zum Erstkontakt ausdrücklich gewünscht, wurde diese darüber hinaus von Adressaten an entsprechend in Frage kommende Personen weitergeleitet.

31 Personen (19\%) meldeten sich zurück und standen für ein leitfragengestütztes Telefoninterview bereit. Bis auf das Saarland, Hamburg und Bremen konnten Interviews mit Personen aus allen Bundesländern durchgeführt werden, wobei der Tätigkeitsschwerpunkt der befragten Personen in den Ländern Niedersachsen, Nordrhein-Westfalen und Hessen lag (Tabelle 3-1). Zudem wurde eine Person der Bundesanstalt für Immobilienaufgaben (Bundesforst) mit bundesweiter Zuständigkeit befragt.

Ein Großteil der befragten Personen (16) ordnete sich auf Nachfrage einer Tätigkeit in öffentlichen Forstbetrieben zu, wobei hier der Schwerpunkt auf Niedersachsen lag (Tabelle 3-1). 10 Personen gaben an, für ein privates, forstliches Dienstleistungsunternehmen zu arbeiten oder mit einem solchen selbstständig zu sein; hier lag ein räumlicher Schwerpunkt auf NordrheinWestfalen. 3 befragte Personen arbeiten für öffentliche Behörden, 2 Personen in privaten Forstbetrieben. 
Tabelle 3-1: Institutionelle und räumliche Tätigkeitszuordnung der 31 per leitfragengestütztem Telefoninterview befragten Anwenderinnen und Anwender forstlicher Ertragstafeln (die Zuordnung erfolgte durch die befragten Personen; bei Mehrfachzuordnung wurden die befragten Personen um eine Schwerpunktsetzung gebeten).

\begin{tabular}{|c|c|c|c|c|}
\hline & $\begin{array}{l}\text { öffentlicher } \\
\text { Forstbetrieb }\end{array}$ & $\begin{array}{r}\text { private } \\
\text { Dienstleister }\end{array}$ & $\begin{array}{r}\text { öffentliche } \\
\text { Behörde }\end{array}$ & $\begin{array}{r}\text { privater } \\
\text { Forstbetrieb }\end{array}$ \\
\hline Baden-Württemberg & - & 1 & - & 1 \\
\hline Bayern & 1 & 1 & - & - \\
\hline Berlin & 1 & - & - & - \\
\hline Brandenburg & 1 & - & - & - \\
\hline Hessen & 3 & 1 & - & - \\
\hline Mecklenburg-Vorp. & 1 & - & - & - \\
\hline Niedersachsen & 4 & - & 1 & 1 \\
\hline Nordrhein-Westfalen & - & 4 & 1 & - \\
\hline Rheinland-Pfalz & 2 & 1 & 1 & - \\
\hline Saarland & - & - & - & - \\
\hline Sachsen & - & 1 & - & - \\
\hline Sachsen-Anhalt & - & 1 & - & - \\
\hline Schleswig-Holstein & 1 & - & - & - \\
\hline Thüringen & 1 & - & - & - \\
\hline Bund & 1 & - & - & - \\
\hline Summe & 16 & 10 & 3 & 2 \\
\hline
\end{tabular}

\subsubsection{Anwendungsgebiete forstlicher Ertragstafeln}

Auf die Frage nach den konkreten Anwendungsgebieten forstlicher Ertragstafeln (Mehrfachnennung waren möglich), gab der überwiegende Teil der Befragten (wie u. a. aufgrund der Auswahl an angeschriebenen Personen zu erwarten) eine Anwendung im Rahmen der Forsteinrichtung und/oder der Waldbewertung an (Abbildung 53). 


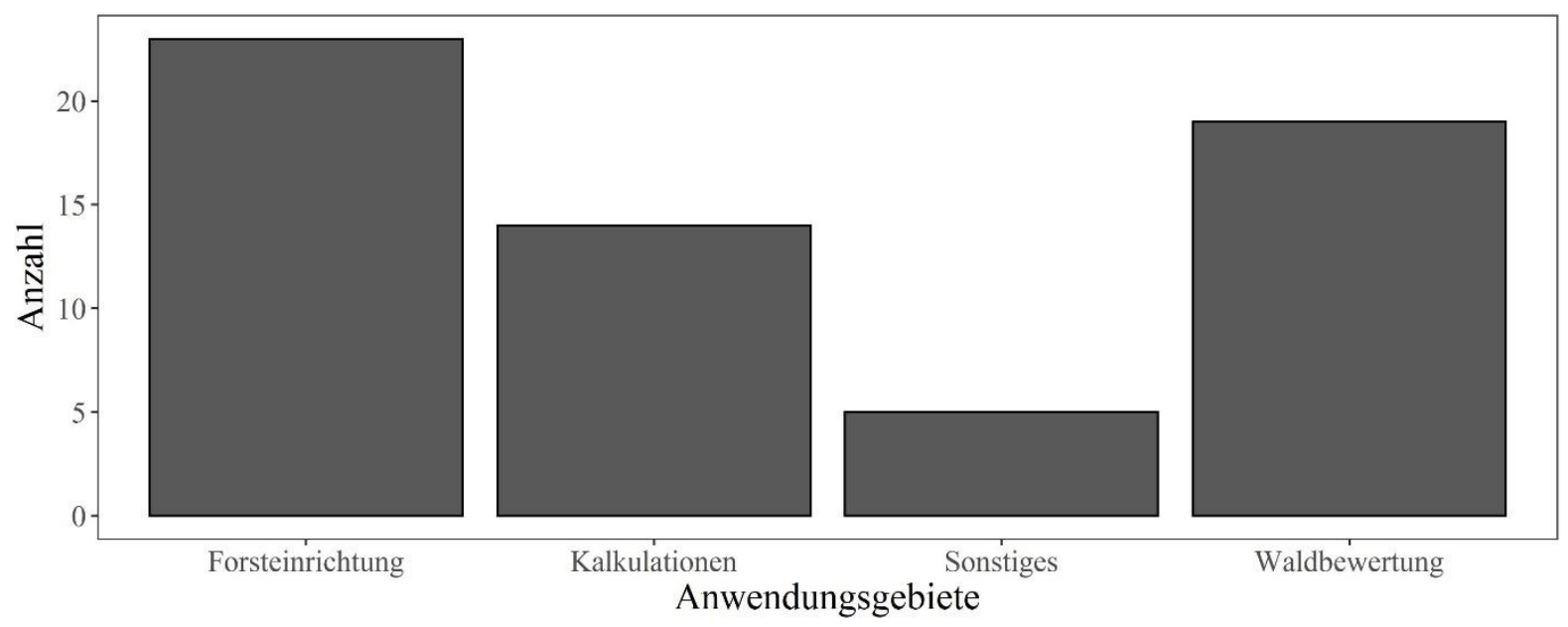

Abbildung 53: Anwendungsgebiete forstlicher Ertragstafeln (Mehrfachnennung möglich) gemäß der 31 per leitfragengestütztem Telefoninterview befragten Anwenderinnen und Anwender.

Weitere genannte Anwendungsgebiete waren betriebliche Kalkulationen ${ }^{56}$, wie z. B. Deckungsbeitragskalkulationen im Rahmen von Holzerntemaßnahmen, und Sonstiges, wie bspw. Wildschadensgutachten.

\subsubsection{Räumliche Einsatzschwerpunkte etablierter Ertragstafelwerke}

Unter Berücksichtigung der teils sehr kleinen Stichproben (vgl. Tabelle 3-1), wird Anhand von Abbildung 54 deutlich, dass in der deutschen Forstpraxis gegenwärtig nur wenige etablierte Ertragstafelwerke mit klarem räumlichen Schwerpunkt zum Einsatz kommen. Während die Anwenderinnen und Anwender aus nordwestdeutschen Bundesländern baumartenübergreifend primär auf die Werke der Ertragstafelsammlung von Schober (1995) zurückgreifen, werden in den ostdeutschen Bundesländern (ohne Berlin) ausschließlich Ertragstafelwerke aus deutlich jüngerer Vergangenheit angewendet, die in der Zeit der ehemaligen Deutschen Demokratischen Republik (DDR) erstellt wurden. In Bayern, Baden-Württemberg und Rheinland-Pfalz kommt für die Baumart Fichte (teilweise) das Ertragstafelwerk von Assmann und Franz (1963) zum Einsatz und für die Baumart Kiefer wurde in Rheinland-Pfalz die Ertragstafel für Buntsandsteingebiete des Pfälzer Waldes von Kern (1958) genannt. In Bayern und Hessen wird die Buche von den befragten Anwenderinnen und Anwendern durch das Ertragstafelwerk von Wiedemann (1931) abgebildet. Inwiefern die räumlichen Einsatzschwerpunkte fachlich begründet sind (z. B. mit den regionalen Spezifika standörtlicher Gegebenheiten) oder auf tradiertes Verhalten zurückgeführt werden kann, wurde im Rahmen der Praxisbefragung nicht untersucht.

\footnotetext{
${ }^{56}$ Unter betrieblichen Kalkulationen werden hier sämtliche ökonomische Analysen auf operativer Ebene eines Forstbetriebs verstanden.
} 

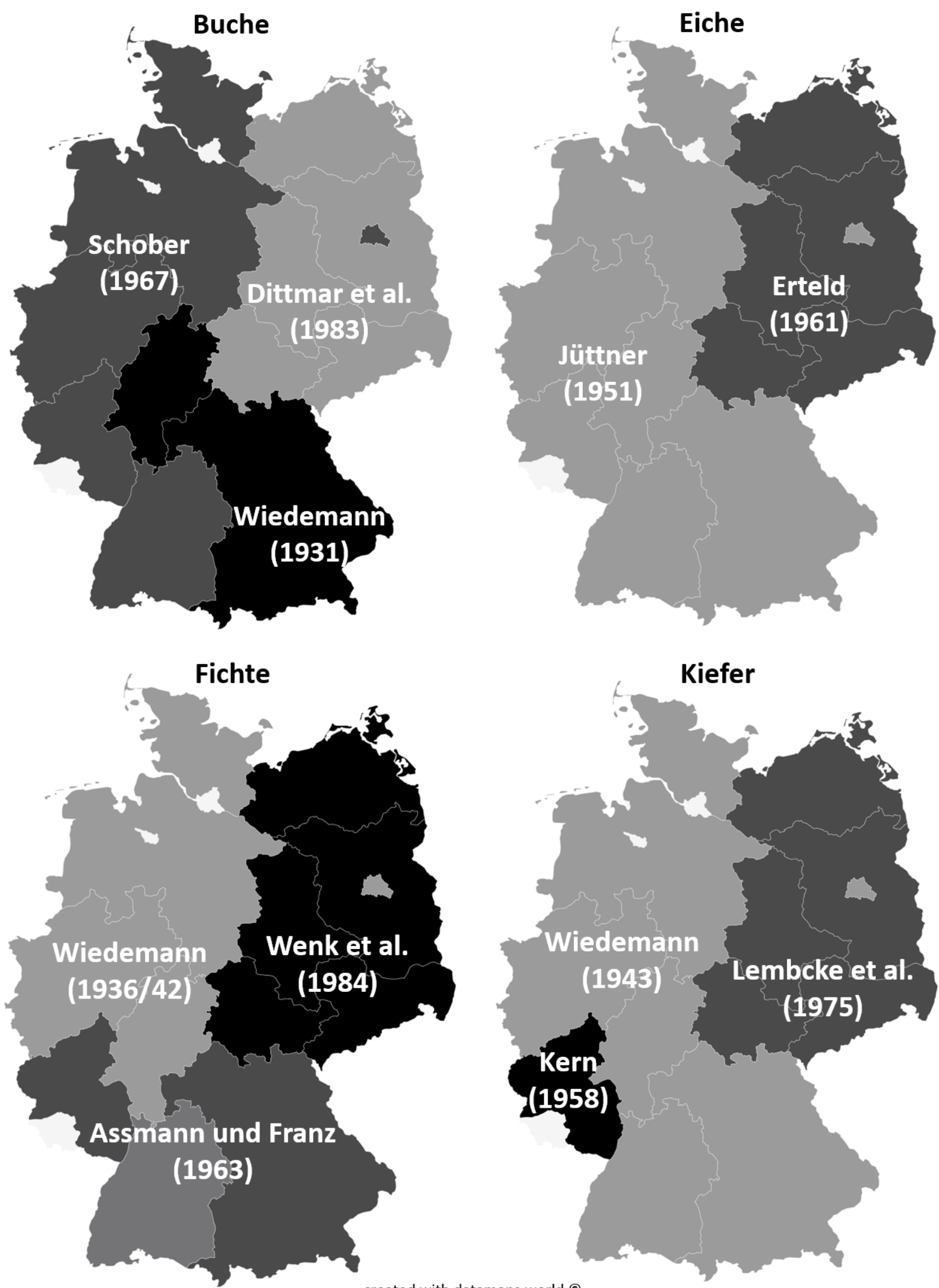

Abbildung 54: Räumliche Zuordnung forstlicher Ertragstafelwerke der Baumarten Buche, Eiche, Fichte und Kiefer gemäß der 31 per leitfragengestütztem Telefoninterview befragten Anwenderinnen und Anwender (in Baden-Württemberg wurden für die Baumart Fichte jeweils einmal die Ertragstafelwerke von Assmann und Franz (1963) und von Wiedemann (1936/42) genannt). 


\subsubsection{Stärken und Schwächen etablierter Ertragstafelwerke}

Auf die bewusst offen gestellte Frage nach Stärken und Schwächen gegenwärtig angewendeter Ertragstafelwerke zeigte sich ein klares Meinungsbild - die Aussagen der befragten Personen (Mehrfachnennungen möglich) konnten zweifelsfrei in vier (Abbildung 55) bzw. fünf (Abbildung 56) im Nachhinein gebildeten Kategorien zusammengefasst werden.

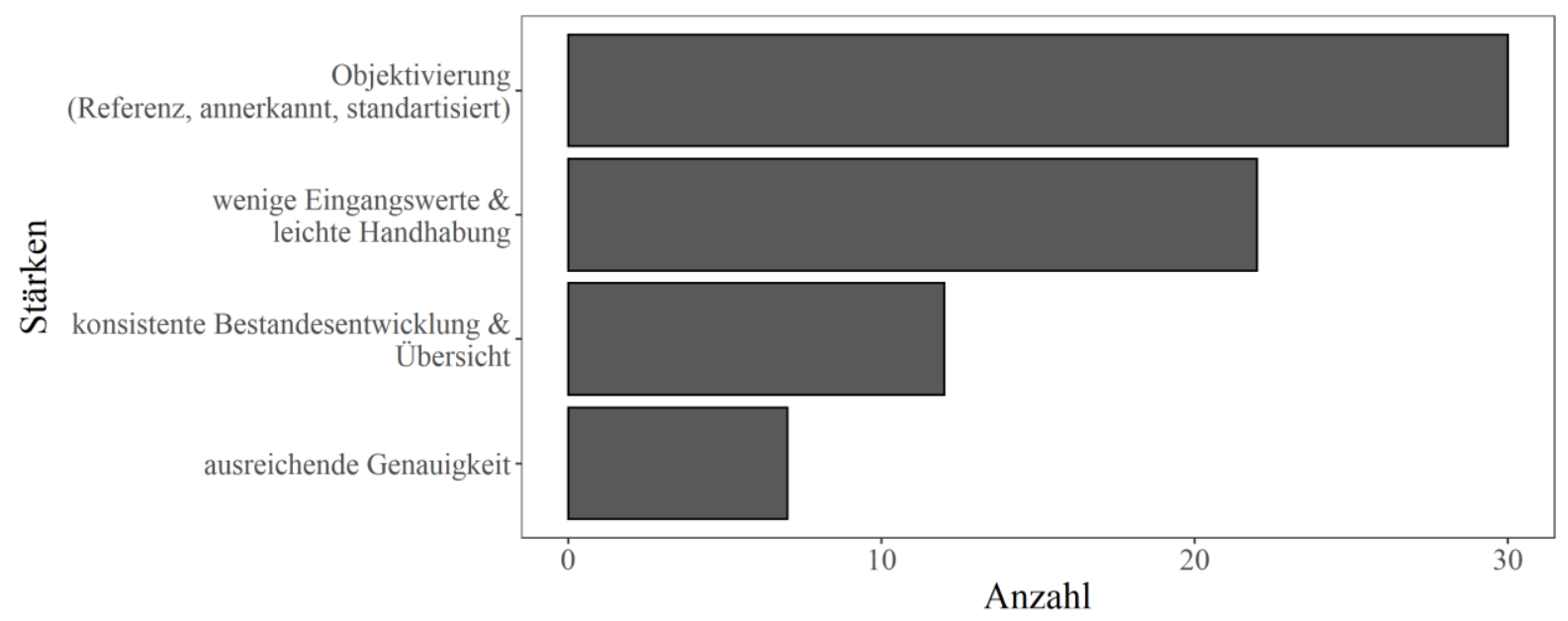

Abbildung 55: Stärken gegenwärtig verwendeter forstlicher Ertragstafelwerke gemäß der 31 per leitfragengestütztem Telefoninterview befragten Anwenderinnen und Anwender (Mehrfachnennungen möglich; zusammengefasst in im Nachhinein gebildeten Kategorien).

30 befragte Personen gaben als Stärke die Objektivierungswirkung von forstlichen Ertragstafeln an - durch die Standardisierung von Wachstums- und Nutzungsmodellen bilden sie eine anerkannte Referenz. Den Befragten geht es vielfach nicht darum, ob ein tatsächlicher Bestand durch die Ertragstafeldaten exakt widergespiegelt wird, sondern vielmehr um ein hinreichend genaues Abbild, auf welches bspw. in Bewertungsverfahren oder bei juristischen Streitfällen zurückgegriffen werden kann. 22 Mal wurden die wenigen benötigten Eingangswerte und die damit verbundene leichte Handhabung als Stärke forstlicher Ertragstafeln genannt. Für eine zügige Vorratsbestimmung oder die grobe Abschätzung von möglichen Hiebsmengen des kommenden Jahrzehnts benötigt der Anwender einer forstlichen Ertragstafel lediglich Alter, Oberhöhe, Grundfläche (und ggf. Volumenzuwachskorrekturfaktoren) eines Bestandes. Selbst für mehrschichtige oder gemischte Bestände genügen mit entsprechenden Verfahren wenige Eckdaten, um eine für die Praxis meist ausreichende Genauigkeit zu erzielen. Diese für viele Anwendungsfälle ausreichende Genauigkeit und damit der bewusste Verzicht auf möglicherweise genauere, aber meist aufwendigere Verfahren (z. B. Stichprobeninventur, Waldwachstumssimulationen) wurde von 7 Anwenderinnen und Anwendern als Vorteil benannt. Schließlich nannten 12 befragte Personen das übersichtliche und konsistente Abbild einer gesamten Be- 
standesentwicklung ${ }^{57}$ von der Begründung bis zur Endnutzung als klare Stärke forstlicher Ertragstafeln, welches Waldwachstumssimulationen in der Regel nicht liefern können. Die etablierten Ertragstafeln sind daher nicht ohne Grund, trotz ihres teils immensen Alters und der bekannten Schwächen, noch immer Grundlage zahlreicher forstpraktischer Anwendungen (siehe Abschnitt 3.1.1) und forstökonomischer Produktionsmodelle (vgl. z. B. Möhring 2010).

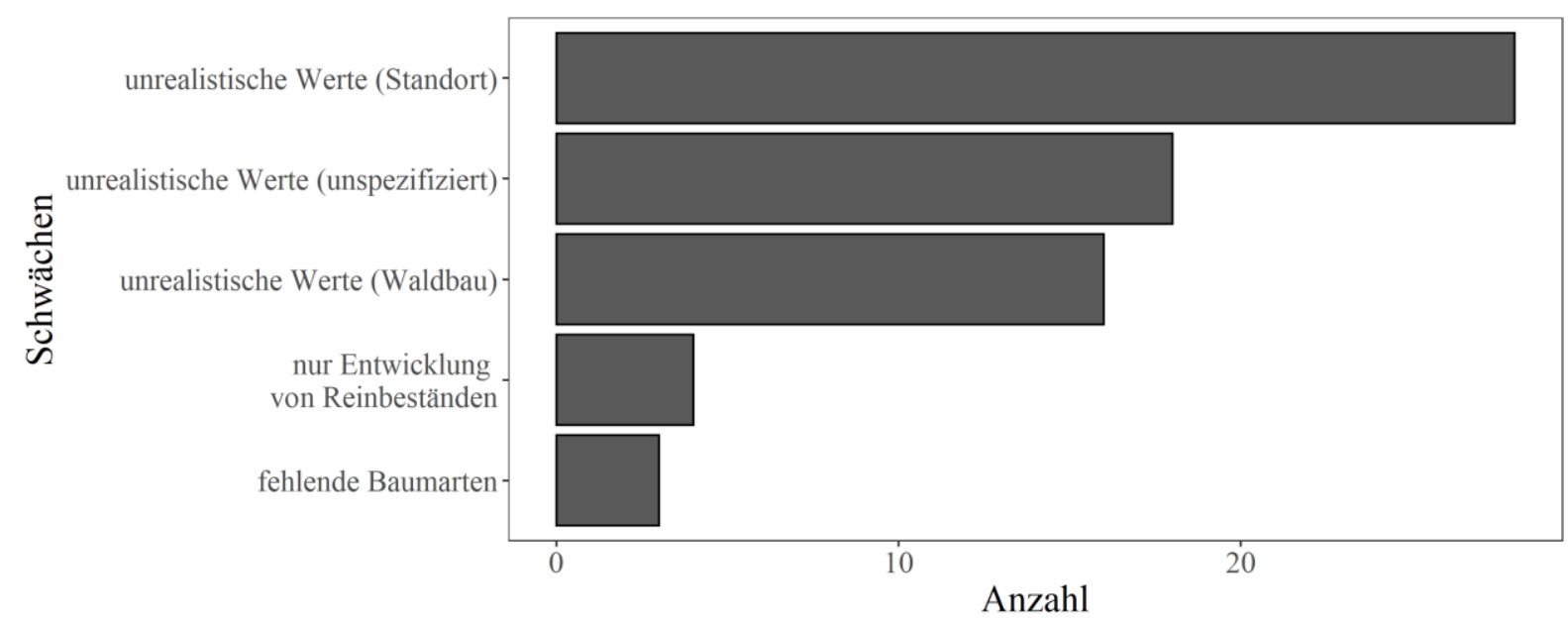

Abbildung 56: Schwächen gegenwärtig verwendeter forstlicher Ertragstafelwerke gemäß der 31 per leitfragengestützten Telefoninterview befragten Anwenderinnen und Anwender (Mehrfachnennung möglich; zusammengefasst in im Nachhinein gebildeten Kategorien).

Die Schwächen forstlicher Ertragstafeln wurden von den 31 befragten Personen ebenfalls sehr eindeutig wiedergegeben. Fast 90 \% (62) der geäußerten Schwächen bezogen sich auf die Tatsache, dass die etablierten Ertragstafeln nicht (mehr) das heutige Niveau der forstlichen Produktion abbilden. Während in 18 Fällen keine Spezifizierung des vermuteten Grundes vorgenommen wurde, führten 28 Personen die Veränderung der Standortsverhältnisse und 16 Personen veränderte waldbauliche Behandlungskonzepte an. Nur wenige Personen nannten die ausschließliche Abbildung von Reinbeständen (4) und das Fehlen bestimmter (Neben-)Baumarten als Schwäche (3).

\subsubsection{Einschätzungen zur zukünftigen Verwendung forstlicher Ertragstafeln}

Den Abschluss der Praxisbefragung bildete eine offen gestellte Frage zur zukünftigen Verwendung und voraussichtlichen Rolle forstlicher Ertragstafeln - auch hier erfolgte eine Zuordnung zu im Nachhinein gebildeten Kategorien. 16 Antworten wurden der Kategorie „,spielen mittelfristig eine wichtige Rolle“ und 6 Antworten der Kategorie „spielen langfristig eine wichtige

\footnotetext{
${ }^{57}$ Einschränkend sei an dieser Stelle darauf hinwegiesen, dass Ertragstafeltabellen i. d. R. konstante Bonitäten unterstellen. Im engeren Sinne ist eine konsistente Beschreibung der gesamten Bestandesentwicklung somit angesichts von sich im Verlauf des Bestandeslebens verändernden Standortsbedingungen niemals gegeben.
} 
Rolle“ zugeordnet. 9 befragte Personen sahen das generelle Konzept forstlicher Ertragstafeln gar als alternativlos an. Häufig wurde dabei allerdings die zukünftige Rolle forstlicher Ertragstafeln an deren (zeitnahe) Aktualisierung geknüpft. Zudem maßen 10 befragte Personen der Ergänzung forstlicher Ertragstafeln durch Inventur- oder Fernerkundungsdaten in der betrieblichen Praxis eine wichtige Rolle zu. Vier Personen sahen in den etablierten Ertragstafeln ein veraltetes Konzept und verwiesen bespielweise vollständig auf Waldwachstumssimulationen oder Betriebsinventuren. Über diese vier Personen hinaus maßen weitere 2 Personen den Waldwachstumssimulationen eine wichtige Bedeutung bei, sehen aber die dringende Notwendigkeit zur Vereinfachung.

Einige ausgewählte Zitate aus den leitfragengestützten Telefoninterviews dienen im Folgenden der ungefilterten Ergänzung des Meinungsbildes:

- ,[Ertragstafeln sind] unabdingbar als Modell bei der jetzigen Konvention zur Berechnung von Waldwerten.“

- „Ertragstafeln bilden das Fundament der Forstwirtschaft.“

- „Wenn die Ertragstafeln sauber weiterentwickelt werden, sind diese auch in Zukunft wichtig."

- „Wenn die Ertragstafeln nicht zeitnah aktualisiert werden, werden diese in der Versenkung verschwinden.“

- „Ertragstafeln, auf die man sich auf breiter Basis stützen kann, sind sehr wichtig.“

- „Wenn nichts gemacht wird, werden Ertragstafeln als Kuriosum auslaufen.“

- „Es ist verwunderlich, dass erst jetzt wieder an diesem Thema gearbeitet wird.“

- „Die Zukunft von überarbeiteten Ertragstafeln liegt in Betrieben mit mehr als 500 ha m. E. nur in Kombination mit einer Betriebsinventur um die Daten statistisch abzusichern."

- „Insbesondere für Arbeiten im Bestand sind Ertragstafeln in Form von direkt verfügbaren Daten auch in der Zukunft wichtig.“

- „Die Bedeutung von Ertragstafeln wird in der Zukunft abnehmen.“

- „Ertragstafeln haben einen hohen betrieblichen Nutzen bei bekannten Schwächen und gutem Preis-Leistungsverhältnis.“

Insgesamt war bei allen befragten Personen ein großes Interesse am Verbundforschungsprojekt „Neue Generation dynamischer Ertragstafeln“ erkennbar. Es herrschte Einigkeit über den Forschungsbedarf, wobei die Dringlichkeit aktualisierter Ertragstafelwerke für die betriebliche Praxis in den westdeutschen deutlich höher als in den ostdeutschen Bundesländern eingeschätzt wurde. 


\subsection{Neue Generation dynamischer Ertragstafeln (dyn-ET)}

Das drittmittelfinanzierte Forschungsprojekt „Neue Generation dynamischer Ertragstafeln (dyn-ET)“, gefördert durch das Bundesministerium für Ernährung und Landwirtschaft über die Fachagentur Nachwachsende Rohstoffe e.V., wird seit dem 01.09.2017 in zwei Teilvorhaben durch die Kooperationspartner Nordwestdeutsche Forstliche Versuchsanstalt (NW-FVA) (Förderkennzeichen: 22027816) und Abteilung Forstökonomie der Georg-August-Universität Göttingen (Förderkennzeichen: 22027916) bearbeitet. Die planmäßige Projektlaufzeit endet nach 3 Jahren am 31.08.2020.

Die Fertigstellung der vorliegenden Dissertation erfolgt vor Projektabschluss, sodass sich die folgenden Analysen auf vorläufige Ertragstafeldaten der Baumarten Buche (Fagus sylvatica L.) und Fichte (Picea abies (L.) H. Karst.) beziehen. Aufbau und Erstellungsmethodik werden für diese vorläufigen Daten beschrieben und es sei an dieser Stelle ausdrücklich darauf hingewiesen, dass sich die Erstellungsmethodik bis zum Projektabschluss weiter verändern wird. Quantitative Abweichungen zwischen der vorliegenden Arbeit und der später veröffentlichten neuen Generation dynamischer Ertragstafeln sind somit unvermeidbar. Dem Charakter von vorläufigen Daten entsprechend, zeigen diese an verschiedenen Stellen zudem schwer interpretierbare bzw. offensichtlich fehlerhafte Werte, was bei der Diskussion aller folgenden Analysen an entsprechender Stelle berücksichtigt wird - auf die methodischen Hintergründe wird in dieser Arbeit aber nicht eingegangen.

\subsubsection{Projektmotivation und -zielsetzung}

Wie bereits in Abschnitt 3.1.1 beschrieben, stammen die meisten der heute verwendeten Ertragstafeln aus ertragskundlichen Untersuchungen auf der Basis von gleichaltrigen Reinbeständen. Diese wurden im 19. und 20. Jahrhundert meist mit hoher Stammzahl begründet und bis zum Kahlschlag oder kurzfristigen Schirmschlag niederdurchforstet (Röhrig et al. 2006, S. 269; Albert et al. 2019 nach Schober 1995). Veränderte waldbauliche Bestandesbehandlungskonzepte (weitere Pflanzverbände, hochdurchforstungsartige Eingriffe, Zielstärkennutzung) sowie meist verbesserte Standortbedingungen (gestiegene Stickstoffeinträge, höhere Kohlenstoffdioxidkonzentration, höhere Temperaturen und verlängerte Vegetationszeiten) führten zu einer immer stärkeren Abweichung der tatsächlich gemessenen Ertragsdaten gegenüber den etablierten Ertragstafelwerten (Albert et al. 2019 i. V. m. Röhle 1995; Spiecker et al. 1996; Pretzsch et al. 2014; siehe u. a. auch Hunter und Schuck 2002; Pretzsch 2005b sowie Bösch 2002 i.V. m. Schöpfer et al. 1994; 1997; Moosmayer et al. 1996). Nach Albert et al. 2019 führt die veränderte waldbauliche Behandlung bei geringeren Stammzahlen zu höheren Mittendurchmesser; 
die verbesserten Standortverhältnisse bedingen in der Regel durch ein gestiegenes Höhenwachstum eine verbesserte Bonität und gleichzeitig steigt bei gegebener Bonität der Volumenzuwachs (höheres Ertragsniveau). In der Forsteinrichtung und Waldbewertung wurden diese Abweichungen bspw. durch inventurbasierte Anpassungen teilweise abgemildert (siehe Abschnitt 3.1.1; vgl. u. a. Wollborn und Böckmann 1998; Staupendahl und Schmidt 2016). Dennoch ist der Ruf der Praxis nach waldbaulich aktualisierten und den heutigen Standortverhältnissen angepassten Ertragstafeln groß (siehe Abschnitt 3.2; vgl. u. a. Gerold und Gerold 2013). Ziel der neuen Generation forstlicher Ertragstafeln ist es daher, auf der Basis von Versuchsflächendaten und einzelbaumbasierten Waldwachstumssimulationen die Bestandesentwicklungen der Hauptbaumarten ${ }^{58}$ im nordwestdeutschen Raum unter heutigen Standortsbedingungen und Nutzungskonzepten herzuleiten (Albert et al. 2019). Es werden dazu anwenderfreundliche, nachvollziehbare und konsistente Tabellenwerke angestrebt. Darauf aufbauend ist es weiteres Ziel, naturale und forstökonomische Plandaten, wie z. B. nachhaltige Hiebssätze und durchschnittliche jährliche Überschussgrößen aus der forstlichen Produktion abzuleiten. Schließlich kennzeichnet der Begriff „dynamisch“ im Projekttitel das Ziel, dass die modellbasierten Ertragstafeldaten verhältnismäßig einfach aktualisiert werden können - eine Neuparametrisierung in regelmäßigen Abständen wird laut Projektantrag angestrebt (Spellmann et al. unveröff.).

Zur Zielerreichung werden die dafür notwendigen Aufgaben gemäß Projektantrag (Spellmann et al. unveröff.) auf fünf Arbeitspakete verteilt: Arbeitspaket 1 leitet die dynamischen Ertragstafeln ab, sorgt für den notwendigen Wissenstransfer und koordiniert das Gesamtprojekt. Arbeitspaket 2 führt Untersuchungen zum Reaktionsvermögen von Einzelbäumen auf Veränderungen ihrer inter- bzw. intraspezifischen Konkurrenz in Abhängigkeit von ihrer sozialen Stellung („Rangerhaltung“) durch. Ziel ist die verbesserte Schätzung des Grundflächenzuwachses von Einzelbäumen, durch die Berücksichtigung der sogenannten Umsetzungsdynamik. Arbeitspaket 3 analysiert die interspezifische Konkurrenz in Mischbeständen, zur verbesserten Ableitung von Volumenzuwachskorrekturfaktoren für die Anwendung der neuen Generation dynamischer Ertragstafeln in Mischbeständen. Arbeitspaket 4 leitet Bestandessortentafeln auf Grundlage der Modellierung sortierungsrelevanter fakultativer und obligater Qualitätsmerk-

${ }^{58}$ Die Ertragstafelsammlung wird bei Projektabschluss voraussichtlich Ertragstafelwerke der fünf Hauptbaumarten Buche (Fagus sylvatica L.), Eiche (Quercus robur L. und Quercus petrae (Mattuschka) Liebl.), Fichte (Picea abies (L.) H. Karst.), Kiefer (Pinus sylvestris L.), Douglasie (Pseudotsuga menziesii (Mirbel) Franco) enthalten. 
male her und berücksichtigt dabei die veränderte Bestandesstruktur und -behandlung. Arbeitspaket 5, bearbeitet durch den Autor dieser Arbeit, führt schließlich die ökonomische Bewertung der Produktionsmodelle durch und analysiert die betrieblichen Implikationen. Dafür wird ein automatisiertes Bewertungsmodell geschaffen, welches die flexible Anpassung der Eingangsdaten (z. B. Sortentafeln, Leitsortimentspreise und Holzerntekosten) an zukünftige Entwicklungen ermöglicht. Auf dieser Grundlage werden einerseits ökonomische Analysen der waldbaulichen Behandlungsvarianten getätigt und andererseits nachhaltige Betriebsmodelle zur Abschätzung von Normalvorräten, nachhaltigen Nutzungsmöglichkeiten und nachhaltigen Reinerträgen geschaffen. Zudem werden die betrieblichen Implikationen einer Umstellung von etablierten auf die neuen Ertragstafelwerke abgebildet.

\subsubsection{Aufbau und Erstellungsmethodik}

Nach bisherigem Projektstand folgt die neue Generation dynamischer Ertragstafeln in Aufbau und Struktur der etablierten Ertragstafelsammlungen von Schober 1995 (siehe Abschnitt 3.1.3). Die verwendeten vorläufigen Daten unterscheiden im Ertragstafelwerk der Baumart Buche zwischen den drei waldbaulichen Behandlungsvarianten mäßige, starke und gestaffelte Hochdurchforstung. Für die Baumart Fichte stehen derzeit lediglich die Ertragstafeln der mäßigen und starken Hochdurchforstung zur Verfügung. Es erfolgt keine Berücksichtigung unterschiedlicher Ertragsniveaus (vgl. z. B. Assmann und Franz 1963) oder Bonitätssysteme (vgl. Wenk et al.

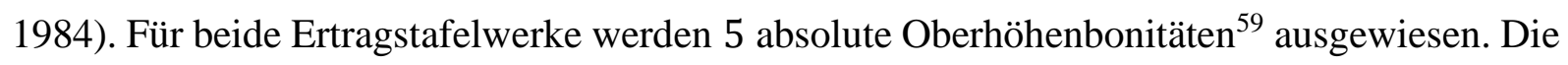
einzelnen Ertragstafeltabellen gliedern sich in Angaben zum Gesamtbestand (Alter, Gesamtwuchsleistung, laufender Bestandesvolumenzuwachs, durchschnittlicher Bestandesvolumenzuwachs), dem verbleibenden Bestand (Stammzahl, Vorrat, Bestandesoberhöhe, Bestandesgrundfläche, Durchmesser des Grundflächenmittelstammes, Durchmesser der 20 \% stärksten Bäume des Bestandes) sowie dem ausscheidenden Bestand (Stammzahl, Vornutzungsvolumen, Bestandesgrundfläche, Durchmesser des Grundflächenmittelstammes).

Nach Albert et al. (2019) folgt die Erstellungsmethodik der neuen Generation dynamischer Ertragstafeln dem Konzept der dritten Ertragstafelgeneration (siehe Abschnitt 3.1.1 und vgl. Pretzsch 2009, S. 442 ff.) und verwendet Funktionsgleichungen zur Ableitung der Ertragstafeldaten. Die Funktionsgleichungen werden dafür an Simulationsdaten des einzelbaumbasierten

\footnotetext{
${ }^{59}$ Unter der Bestandesoberhöhe $H_{100}$ wird hier die Mittelhöhe der 100 stärksten Bäume des Bestandes verstanden (teilweise auch als Bestandesspitzenhöhe bezeichnet, vgl. Kramer und Akça 2008, S. 118). Die Oberhöhenbonität $H_{100}(100$ a) wird über die Oberhöhe im Alter von 100 Jahren definiert.
} 
Waldwachstumsmodells TreeGrOSS (vgl. Hansen und Nagel 2014) angepasst, dessen Wachstumsfunktionen anhand von Versuchsflächenaufnahmen ab 1970 parametrisiert wurden und somit aktuelle Zuwachsniveaus widerspiegeln. Grundlage der Waldwachstumssimulationen bilden Versuchsflächendaten, die entsprechend der gewünschten waldbaulichen Behandlungsvarianten aus dem Versuchsflächennetz der NW-FVA ausgewählt wurden. So wurden für die Baumart Buche 241 und für die Baumart Fichte 278 Versuchsflächenaufnahmen aus hochdurchforstungsartig behandelten Versuchsbeständen ausgewählt (Albert et al. 2019 und dynET 2020). Ein Teil dieser Versuchsbestände bildet auch die Grundlage für die Definition der Grundflächenhaltungen, welche das Nutzungskonzept der neuen Ertragstafeln in Form der drei (Buche) bzw. zwei (Fichte) waldbaulichen Behandlungsvarianten widerspiegeln. Aus Sicht der NW-FVA idealtypisch mäßig und stark hochdurchforstete Parzellen liefern die Ausgangsdaten einer Regressionsanalyse der Grundflächenhaltung (siehe Abb. 2 in Albert et al. 2019). Da für die gestaffelte Hochdurchforstung der Buche nicht genügend Versuchsflächendaten vorliegen, wird die Grundflächenhaltung dieser Behandlungsvariante über die mäßige und die starke Hochdurchforstung definiert: Bis zu einer Oberhöhe von 24 m entspricht die Grundflächenhaltung der starken Hochdurchforstung, anschließend erfolgt in der waldbaulichen Behandlung ein Übergang zur mäßigen Hochdurchforstung (Albert et al. 2019). Während die Z-Baumzahlen und Zielstärken für die Baumart Buche in Abhängigkeit von der Bonität und der waldbaulichen Behandlungsvariante ${ }^{60}$ festgelegt werden (siehe Tab. 1 in Albert et al. 2019) erfolgt die Festlegung bei der Baumart Fichte nur in Abhängigkeit von der Bonität (100 - 250 Z-Bäume je Hektar; Zielstärke $45 \mathrm{~cm}$ bei den Ertragsklassen ${ }^{61}-\mathrm{I}$ und 0, $40 \mathrm{~cm}$ bei den Ertragsklassen I und II sowie $35 \mathrm{~cm}$ bei der Ertragsklasse III). Bei beiden Baumarten werden je Z-Baum 0,5 (mäßige Durchforstung) bzw. 2 (starke und gestaffelte Durchforstung) Bedränger, d. h. Nachbarbäume mit überlappender Krone, pro Vornutzungseingriff entnommen. Reicht diese Entnahme zur Erreichung der Zielgrundfläche im entsprechenden Vornutzungsalter nicht aus, erfolgt eine entsprechende Entnahme von Bäumen in den Zwischenfeldern, d. h. aus dem sogenannten Füllbestand (Albert et al. 2019 und dyn-ET 2020). Auch wenn durchgängig von Hochdurchforstung

\footnotetext{
${ }^{60}$ In späteren Projektversionen werden die Z-Baumzahlen und Zielstärken auch für die Baumart Buche nur noch in Abhängigkeit von der Bonität definiert sein (dyn-ET 2020).

${ }^{61}$ Auch wenn im Rahmen des dyn-ET-Projekts generell absolute Oberhöhenbonitäten verwendet werden, erfolgt an verschiedenen Stellen der Bezug auf Ertragsklassen als relative Bonitätsmaße - bspw. für den Vergleich mit der etablierten Ertragstafelsammlung von Schober (1995) oder wie hier, für die Festlegung von Kriterien der waldbaulichen Behandlungsvarianten (vgl. Albert et al. 2019, Tab. 1).
} 
gesprochen wird, handelt es sich somit eigentlich um eine Kombination aus Z-Baum-orientierter Auslesedurchforstung und einer Hochdurchforstung in den Zwischenfeldern (interne dynET-Projektkommunikation i. V. m. Albert et al. 2019).

Die Startbestände der waldwachstumskundlichen Simulationen werden aus den Durchmesserverteilungen der 241 (Buche) bzw. 278 (Fichte) Versuchsflächenaufnahmen erstellt. Dazu werden bis zur Erreichung der Zielgrundfläche im Startalter zufällig Durchmesser gezogen und es erfolgt ein Ausgleich etwaiger Abweichungen vom gewünschten Nutzungskonzepts über den Durchmesser des Grundflächenmittelstamms $\left(D_{g}\right)$ und den Durchmesser der 100 stärksten Bäume des Bestandes $\left(D_{100}\right)$, wobei die gestaffelte der starken Durchforstung gleich gesetzt wird (Albert et al. 2019). Ergänzt um die entsprechenden Einzelbaumhöhen aus den Versuchsflächendaten sowie über weitere TreeGrOSS-Funktionen (vgl. Hansen und Nagel 2014) abgeleitete Einzelbaumparameter stehen schließlich 241 (Buche) bzw. 278 (Fichte) Startbestände (je 0,4 ha; interne dyn-ET-Projektkommunikation) mit unterschiedlichen Oberhöhenbonitäten für die Simulation zur Verfügung (dyn-ET 2020). Diese erfolgt als Zeitscheibensimulation ${ }^{62}$ in 5-Jahres-Simulationsschritten für je 30 Jahre (Albert et al. 2019).

Die resultierenden laufenden Bestandesvolumenzuwächse (erste zeitliche Ableitung) ermöglichen die Schätzung von Funktionsparametern einer Chapman-Richards-Wachstumsfunktion, welche der Herleitung der Gesamtwuchsleistung (Stammfunktion) über dem Alter dient. Alle weiteren gewünschten Ertragstafelgrößen werden getrennt für die drei Behandlungsvarianten über ein System an Funktionen hergeleitet ${ }^{63}$ (siehe Tabelle 2 in Albert et al. 2019).

\footnotetext{
${ }^{62}$ Als Zeitscheibensimulation wird das Vorgehen bezeichnet, die gesamte Bestandesentwicklung aus einzeln simulierten Zeitscheiben zusammenzusetzen; jede Zeitscheibe wird dabei, ausgehend von versuchsflächenbasierten Startbeständen unterschiedlicher Alter, für je 30 Jahre forstgeschrieben (Albert et al. 2019).

${ }^{63}$ Ebenfalls simulativ hergeleitet (in dieser Arbeit aber nicht verwendet) werden Volumenzuwachskorrekturfaktoren, welche veränderte Volumenzuwächse bei verminderter oder erhöhter Bestandesdichte (bezogen auf die Normbestockung bei einem Bestockungsgrad von 1,0) berücksichtigen (siehe Abschnitt 3.1.1). Dazu werden Startbestände entsprechend dem oben beschriebenen Verfahren mit unterschiedlichen Bestandesdichten (Bestockungsgrad 0,1 bis 1,6 in Bezug zur mäßigen Durchforstung) aufgebaut und über fünf Jahre simuliert. Die Volumenzuwachskorrekturfaktoren ergeben sich als Verhältnis aus den resultierenden Bestandesvolumenzuwächsen und den Bestandesvolumenzuwächsen bei Bestockungsgrad 1,0 der mäßigen Hochdurchforstung (Albert et al. 2019).
} 


\subsubsection{Vorläufige Ertragstafeldaten}

Als Grundlage der ökonomischen Analysen in den Abschnitten 3.3.5 und 3.3.6 werden in diesem Abschnitt ausgewählte ${ }^{64}$ Ertragstafeldaten der Baumarten Buche und Fichte gemäß dynET (unveröff.) analysiert. Es sei an dieser Stelle noch einmal ausdrücklich darauf hingewiesen, dass es sich dabei um vorläufige, unveröffentlichte Ertragstafeldaten aus dem laufenden dynET-Projekt handelt. Bis zur Veröffentlichung der endgültigen Ertragstafelwerke wird die Erstellungsmethodik weiter angepasst - Abweichungen zwischen den Daten dieser Arbeit und den später veröffentlichten Ertragstafeln sind somit unumgänglich. Für beide Baumarten bereits abzusehen ist, dass insbesondere die laufenden Bestandesvolumenzuwächse deutlich geringer ausfallen werden. Folglich überschätzen die hier gezeigten vorläufigen Etragstafeldaten die Ertragsniveausteigerung ggü. etablierten Ertragstafelwerken.

Je Baumart (Buche ab Seite 137, Fichte ab Seite 156) werden folgende Analysen unterschieden:

- Das ertragskundliche Spektrum der mäßigen Hochdurchforstung, als Basis-Behandlungsvariante, wird anhand der Gesamtwuchsleistungen und laufenden Bestandesvolumenzuwächse aller Oberhöhenbonitäten abgebildet. Ergänzend werden die entsprechenden Abbildungen für die starke (Buche und Fichte) und gestaffelte (nur Buche) Hochdurchforstung im Anhang gezeigt. Für eine tabellarische Gegenüberstellung ausgewählter Ertragstafeldaten wird neben den Kulminationszeitpunkten der laufenden und durchschnittlichen Bestandesvolumenzuwächse das einheitliche Alter bzw. die Umtriebszeit 100 a (Buche) und 60 a (Fichte) gewählt, da für diese Alter jeweils Ertragstafeldaten aller Oberhöhenbonitäten zur Verfügung stehen.

- Ein Vergleich zu etablierten Ertragstafeln (Buche: Schober 1995, mäßige Durchforstung; Fichte: Wiedemann 1936/42, mäßige Durchforstung) wird bei vergleichbaren Oberhöhenbonitäten anhand der Gesamtwuchsleistungen, der laufenden Bestandesvolumenzuwächse und verbleibenden Bestandesvolumina sowie den daraus resultierenden Volumenzuwachsraten, dem ausscheidenden Bestandesvolumen, der verbleibenden Stammzahl, den Stückvolumenrelationen, den mittleren Durchmessern des verbleibenden und ausscheidenden Bestandes sowie zwei Kennziffern einer nachhaltigen Betriebsklasse (durchschnittliche Bestandesvolumenzuwächse und Normalvorräte über der Umtriebszeit) gezogen. Die entsprechenden Abbildungen des Vergleichs der starken

\footnotetext{
${ }^{64}$ Die Gesamtheit aller in Abschnitt 3.3 eingegangenen, vorläufigen Daten gemäß dyn-ET unveröff. kann dem Anhang in den Tabellen 8-2 bis 8-26 entnommen werden.
} 
Durchforstungen werden im Anhang gezeigt. Für eine tabellarische Gegenüberstellung ausgewählter Ertragstafeldaten werden neben den Kulminationszeitpunkten der laufenden und durchschnittlichen Bestandesvolumenzuwächse Alter bzw. Umtriebszeiten in Anlehnung an etablierte Umtriebszeiten (vgl. z. B. NLF 2014, Tabelle 1.1) gewählt: 140 a (Buche) und 80 a (Fichte).

- Die ertragskundlichen Auswirkungen der unterschiedlichen waldbaulichen Behandlungsvarianten gemäß dyn-ET (unveröff.) werden schließlich auf Basis der in den Ertragstafeln ausgewiesenen minimalen, mittleren und maximalen Oberhöhenbonitäten anhand der Gesamtwuchsleistungen, der laufenden Bestandesvolumenzuwächse und verbleibenden Bestandesvolumina sowie den daraus resultierenden Volumenzuwachsraten, dem ausscheidenden Bestandesvolumen sowie den mittleren Durchmessern des verbleibenden und ausscheidenden Bestandes aufgezeigt. Die Stückvolumenrelationen dienen zusätzlich der Quantifizierung der Durchforstungsarten über das Verhältnis der Stückvolumina des ausscheidenden und verbleibenden Bestandes ${ }^{65}$. Auch hier wird für die tabellarische Gegenüberstellung ausgewählter Ertragstafeldaten neben den Kulminationszeitpunkten der laufenden und durchschnittlichen Bestandesvolumenzuwächse das Alter bzw. die Umtriebszeit 140 a (Buche) und 80 a (Fichte) gewählt.

\footnotetext{
${ }^{65}$ Bei der Interpretation der mittleren Stückvolumina und Durchmesser sowie der Stückvolumenrelationen ist zu beachten, dass diese aus ertragskundlicher Sicht aufgrund der Kombination aus Z-Baumorientierter Auslesedurchforstung und einer Hochdurchforstung in den Zwischenfeldern kein uneingeschränkt geeignetes Maß zur Charakterisierung der dyn-ET-Durchforstungsarten darstellen (interne dyn-ET-Projektkommunikation i. V. m. Albert et al. 2019). In dieser Arbeit spielen die mittleren Bestandesgrößen aufgrund der Anknüpfungsfähigkeit im Rahmen der ökonomischen Bewertung dennoch eine zentrale Rolle.
} 
Der Bonitätsfächer der Baumart Buche deckt bei mäßiger Hochdurchforstung im Alter $100 \mathrm{a}$ einen Gesamtwuchsleistungsrahmen zwischen 559 und $1.696 \mathrm{Vfm} \mathrm{ha}^{-1}$ ab (Abbildung 57, Tabelle 3-2). Die entsprechenden Gesamtwuchsleistungsfächer der starken (Abbildung 152) und gestaffelten (Abbildung 154) Hochdurchforstung gemäß dyn-ET (unveröff.) können dem Anhang entnommen werden.

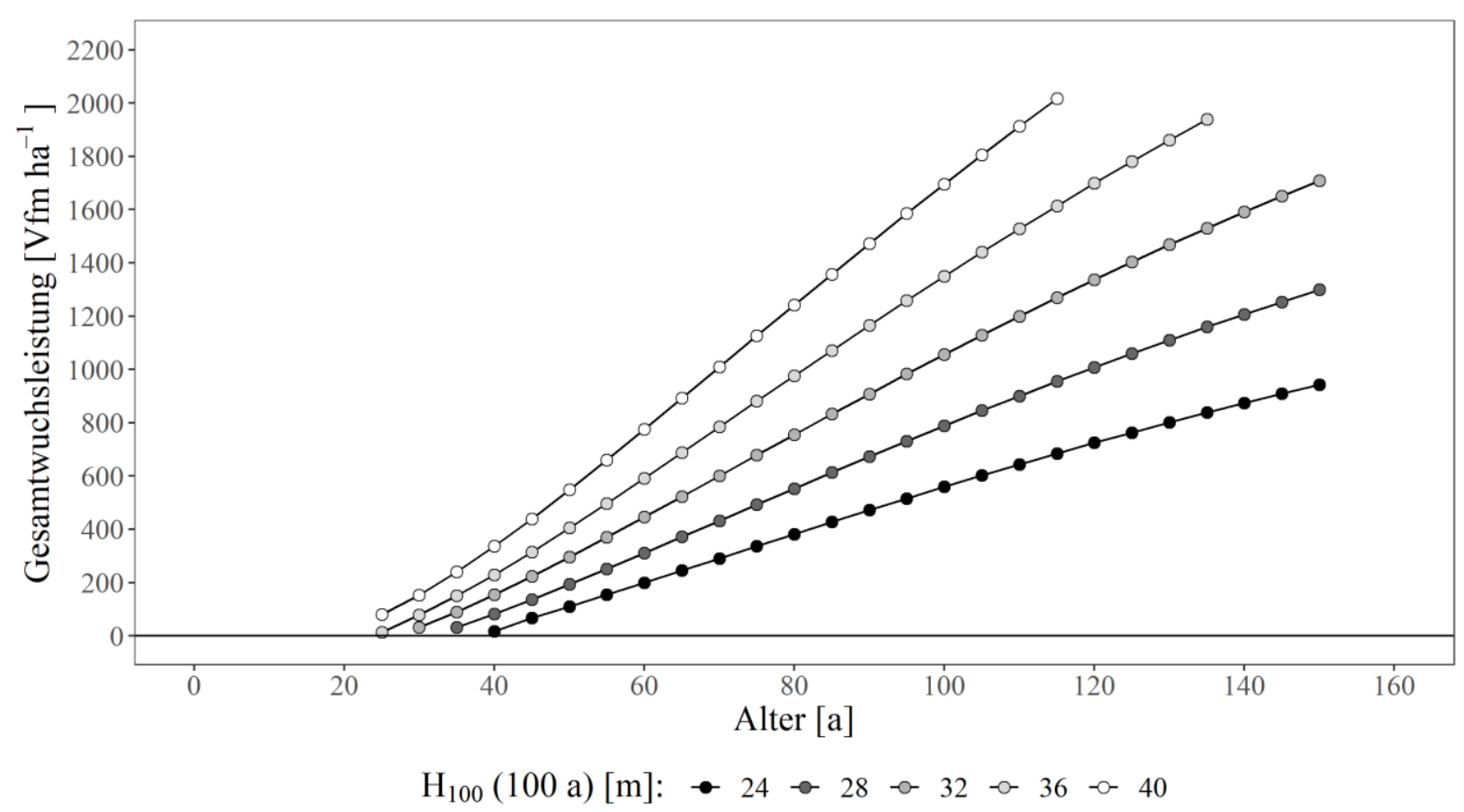

Abbildung 57: Gesamtwuchsleistung über dem Alter; alle Oberhöhenbonitäten der Baumart Buche (mäßige Hochdurchforstung) gemäß dyn-ET (unveröff.).

Mit steigender Standortsgüte nimmt nicht nur das absolute Niveau der Bestandesvolumenzuwächse, sondern auch die Veränderung über dem Alter zu - während die laufenden Bestandesvolumenzuwächse bei einer Oberhöhenbonität von $24 \mathrm{~m}$ altersabhängig nur um 2,3 Vfm ha ${ }^{-1} \mathrm{a}^{-1}$ variieren (Minimum: 6,8 Vfm ha $\mathrm{Vf}^{-1} \mathrm{a}^{-1}$ Maximum $^{66}:$ 9,1 $\mathrm{Vfm} \mathrm{ha}^{-1} \mathrm{a}^{-1}$ ), schwanken sie bei einer Oberhöhenbonität von $40 \mathrm{~m}$ zwischen $14,6 \mathrm{Vfm} \mathrm{ha}^{-1} \mathrm{a}^{-1}$ im Alter 30 a (Minimum) und 23,4 Vfm ha ${ }^{-1} \mathrm{a}^{-1}$ im Alter 75 a (Abbildung 58). Die Kulminationszeitpunkte liegen im Alter 75 a zwischen 9,1 und 23,4 $\mathrm{Vfm} \mathrm{ha}^{-1} \mathrm{a}^{-1}$ (Tabelle 3-2) ${ }^{67}$. Die laufenden Bestandesvolumenzuwächse der starken (Abbildung 153) und gestaffelten (Abbildung 155) Hochdurchforstung gemäß dyn-ET (unveröff.) werden im Anhang gezeigt.

\footnotetext{
${ }^{66}$ Der ersten Datenpunkt der Oberhöhenbonität 24 m (Abbildung 58) wird als modellbedingter AusreiBer der vorläufigen dyn-ET-Daten interpretiert und an dieser Stelle nicht beachtet.

${ }^{67}$ Die einheitlichen Kulminationszeitpunkte werden als modellbedingte Artefakte der vorläufigen dynET-Daten interpretiert - mit abnehmender Oberhöhenbonität wäre eigentlich eine Verschiebung zu höheren Bestandesaltern zu erwarten (siehe Baumart Fichte; interne dyn-ET-Projektkommunikation).
} 
Tabelle 3-2: Vergleich ausgewählter Ertragstafeldaten der Baumart Buche (mäßige Hochdurchforstung) gemäß dyn-ET (unveröff.); alle Oberhöhenbonitäten; in Klammern ist jeweils die relative Differenz zur Oberhöhenbonität $H_{100}(100 \mathrm{a})=32 \mathrm{~m}$ angegeben;

$\boldsymbol{G W L}(\mathbf{1 0 0} \mathrm{a})$ : Gesamtwuchsleistung bis zum Alter $100 \mathrm{a}, \max \boldsymbol{v}_{\boldsymbol{B}}$ : lfd. Bestandesvolumenzuwachs zum Zeitpunkt der Kulmination [...], $\boldsymbol{V}_{\boldsymbol{B}}(\mathbf{1 0 0} \mathbf{a})$ : Vorrat (verbl. Bestand) im Alter $100 \mathrm{a}$, $\boldsymbol{G}_{\boldsymbol{v e r b l}}\left(\mathbf{1 0 0}\right.$ a): Bestandesgrundfläche des verbleibenden Bestandes im Alter $100 \mathrm{a}, \sum_{\mathbf{0}}^{\mathbf{1 0 0}} \boldsymbol{\lambda}$ : kumuliertes ausscheidendes Bestandesvolumen bis zum Alter 100 a, $\boldsymbol{N}_{\text {verbl. }}(\mathbf{1 0 0}$ a): Stammzahl des ver-

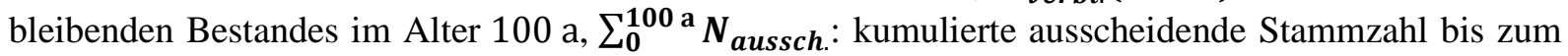
Alter 100 a, $\boldsymbol{D}_{\boldsymbol{g}, \boldsymbol{v e r b l}}$. $(100$ a): Durchmesser des Grundflächenmittelstammes des verbleibenden Bestandes im Alter 100 a, $\boldsymbol{D}_{\boldsymbol{g} \text {,aussch. }}$ (100 a): Durchmesser des Grundflächenmittelstammes des ausscheidenden Bestandes im Alter 100 a, max $\overline{\boldsymbol{v}_{\boldsymbol{B}}}$ : durchschn. Bestandesvolumenzuwachs zum Zeitpunkt der Kulmination [...], $\boldsymbol{V}_{\boldsymbol{\eta}}(\mathbf{1 0 0}$ a): Normalvorrat bei einer Umtriebszeit von 100 a.

\begin{tabular}{|c|c|c|c|c|c|}
\hline \multirow{2}{*}{$\begin{array}{r}\text { Bestandesbehandlung } \\
\qquad H_{100}(100 \text { a) }[\mathrm{m}]\end{array}$} & \multicolumn{5}{|c|}{ mäßige Hochdurchforstung } \\
\hline & 24 & 28 & 32 & 36 & 40 \\
\hline$G W L(100 \mathrm{a})$ & 559 & 789 & 1.056 & 1.350 & 1.696 \\
\hline$\left[\mathrm{Vfm} \mathrm{ha}^{-1}\right]$ & $(-47,1 \%)$ & $(-25,3 \%)$ & & $(+27,8 \%)$ & $(+60,6 \%)$ \\
\hline $\max v_{B}$ & $9,1[75 \mathrm{a}]$ & $12,1[75 \mathrm{a}]$ & $15,5[75 \mathrm{a}]$ & 19,3 [75 a] & $23,4[75 \mathrm{a}]$ \\
\hline$\left[\mathrm{Vfm} \mathrm{ha}^{-1} \mathbf{a}^{-1}\right]$ & $(-41,3 \%)$ & $(-21,9 \%)$ & & $(+24,5 \%)$ & $(+51,0 \%)$ \\
\hline$V_{B}(100 \mathrm{a})$ & 268 & 351 & 437 & 524 & 609 \\
\hline$\left[\mathrm{Vfm} \mathrm{ha}^{-1}\right]$ & $(-38,7 \%)$ & $(-19,7 \%)$ & & $(+19,9 \%)$ & $(+39,4 \%)$ \\
\hline$G_{v e r b l .}(100 \mathrm{a})$ & 24,5 & 26,9 & 28,8 & 30,3 & 31,3 \\
\hline$\left[\mathbf{m}^{2}\right]$ & $(-14,9 \%)$ & $(-6,6 \%)$ & & $(+5,2 \%)$ & $(+8,7 \%)$ \\
\hline$\sum_{0}^{100 \mathrm{a}} \lambda$ & 291 & 438 & 619 & 826 & 1.087 \\
\hline$\left[\mathbf{V f m ~ h a}^{-1}\right]$ & $(-53,0 \%)$ & $(-29,2 \%)$ & & $(+33,4 \%)$ & $(+75,6 \%)$ \\
\hline$N_{\text {verbl. }}(100 \mathrm{a})$ & 416 & 318 & 252 & 205 & 171 \\
\hline$\left[\mathbf{h a}^{-1}\right]$ & $(+65,1 \%)$ & $(+26,2 \%)$ & & $(-18,7 \%)$ & $(-32,1 \%)$ \\
\hline$\sum_{0}^{100 \mathrm{a}} N_{\text {aussch }}$ & 2.459 & 2.596 & 2.946 & 3.622 & 3.015 \\
\hline$\left[\mathrm{ha}^{-1}\right]$ & $(-16,5 \%)$ & $(-11,9 \%)$ & & $(+22,9 \%)$ & $(+2,3 \%)$ \\
\hline$D_{g, v e r b l .}(100$ a $)$ & 27,4 & 32,8 & 38,2 & 43,4 & 48,3 \\
\hline$[\mathbf{c m}]$ & $(-28,3 \%)$ & $(-14,1 \%)$ & & $(+13,6 \%)$ & $(+26,4 \%)$ \\
\hline$D_{g, a u s s c h .}(100$ a) & 27,2 & 34,1 & 40,4 & 46,7 & 54,6 \\
\hline$[\mathbf{c m}]$ & $(-32,7 \%)$ & $(-15,6 \%)$ & & $(+15,6 \%)$ & $(+35,1 \%)$ \\
\hline $\max \overline{v_{B}}$ & $6,3[150 \mathrm{a}]$ & $8,7[150 \mathrm{a}]$ & $11,4[150 \mathrm{a}]$ & $14,4[135 \mathrm{a}]$ & $17,5[115 \mathrm{a}]$ \\
\hline$\left[\mathrm{Vfm} \mathrm{ha}^{-1} \mathbf{a}^{-1}\right]$ & $(-44,7 \%)$ & $(-23,7 \%)$ & & $(+26,3 \%)$ & $(+53,5 \%)$ \\
\hline$V_{\eta}(100 \mathrm{a})$ & 104 & 148 & 198 & 252 & 311 \\
\hline$\left[\mathrm{Vfm} \mathrm{ha}^{-1}\right]$ & $(-47,5 \%)$ & $(-25,3 \%)$ & & $(+27,3 \%)$ & $(+57,1 \%)$ \\
\hline
\end{tabular}




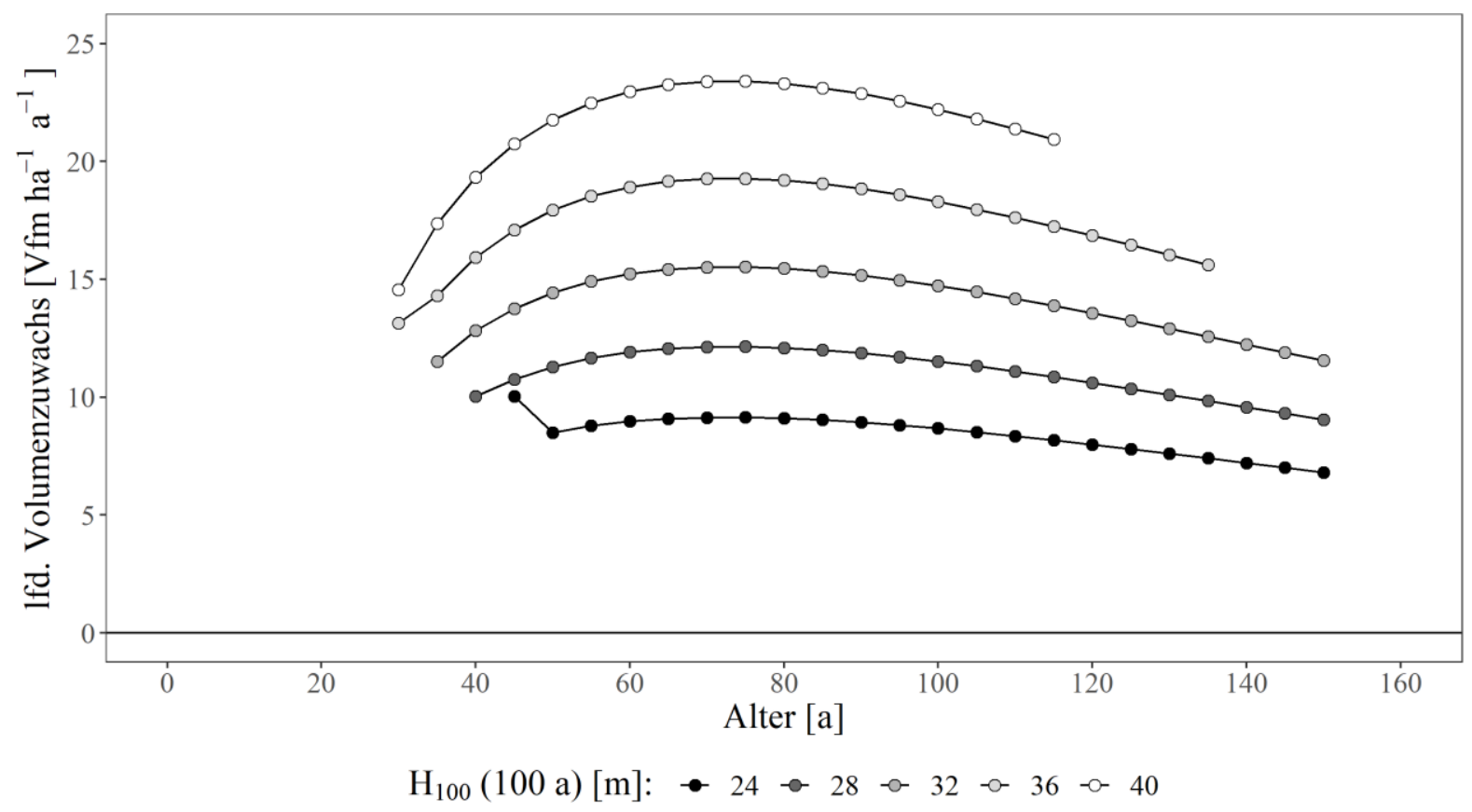

Abbildung 58: Laufender Bestandesvolumenzuwachs über dem Alter; alle Oberhöhenbonitäten der Baumart Buche (mäßige Hochdurchforstung) gemäß dyn-ET (unveröff.).

Gegenüber der etablierten Ertragstafel von Schober (1967, mäßige Durchforstung) kommt es in Anlehnung an Versuchsflächendaten der NW-FVA zu einer Ausweitung und Verschiebung des Bonitätsrahmens - statt der Ertragsklassen IV bis I mit entsprechenden Oberhöhenbonitäten von 20,7 bis 32,4 m bei mäßiger Durchforstung nach Schober (1967), wird gemäß dyn-ET (unveröff.) bei der mäßigen Hochdurchforstung in 5 Stufen ein Oberhöhenbonitätsbereich von 24 bis $40 \mathrm{~m}$ abgedeckt.

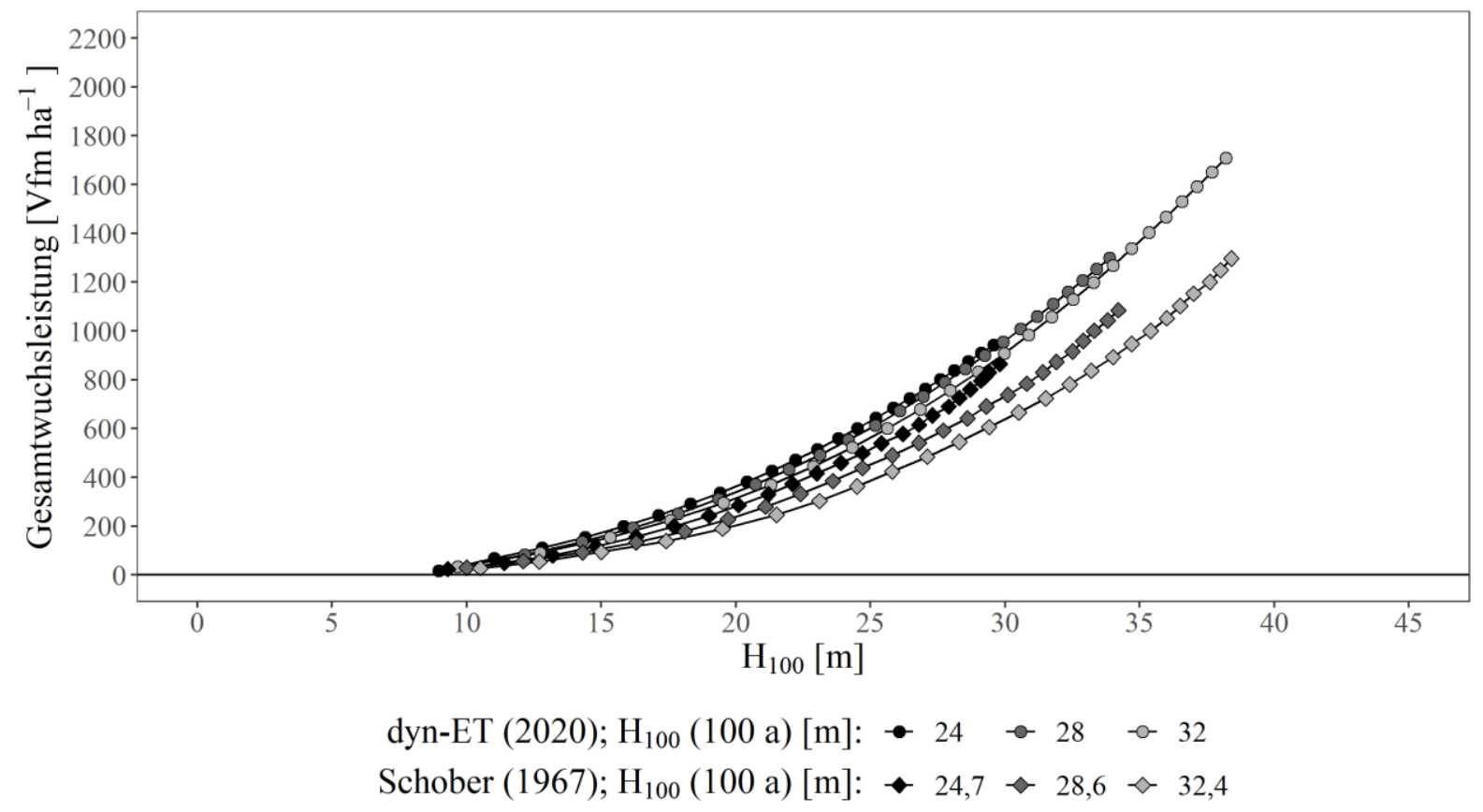

Abbildung 59: Gesamtwuchsleistung über der Oberhöhe; ausgewählte Oberhöhenbonitäten der Baumart Buche (mäßige Durchforstung) gemäß dyn-ET (unveröff.) und Schober (1967). 
Zusätzlich kommt es zu einer Anhebung des Ertragsniveaus - bei gleicher Oberhöhe liegen die Gesamtwuchsleistungen gemäß dyn-ET (unveröff., mäßige Hochdurchforstung) deutlich oberhalb vergleichbarer Oberhöhenbonitäten nach Schober (1967, mäßige Durchforstung) wobei die Abweichung deutlich mit steigender Standortsgüte zunimmt (Abbildung 59). Dieser Effekt zeigt sich in abgeschwächter Form auch bei der starken Durchforstung (Abbildung 156 im Anhang). Ebenfalls in Abbildung 59 bzw. Abbildung 156 erkennbar sind die bei dyn-ET (unveröff.) ggü. Schober (1967) geringer ausfallenden Ertragsniveauunterschiede innerhalb der Ertragstafeln.

Anhand der laufenden Bestandesvolumenzuwächse über dem Alter (Abbildung 61) wird das Ausmaß der Ertragsniveausteigerung ggü. Schober (1967, mäßige Durchforstung) nochmal deutlicher - bei annähernd gleicher Oberhöhenentwicklung über dem Alter (Abbildung 60) liegen die laufenden Volumenzuwächse der Oberhöhenbonität 28 m nach dyn-ET (unveröff., mäBige Hochdurchforstung) auf etwa einem Niveau mit der Volumenzuwachsentwicklung der Oberhöhenbonität 32,4 m nach Schober (1967, mäßige Durchforstung).

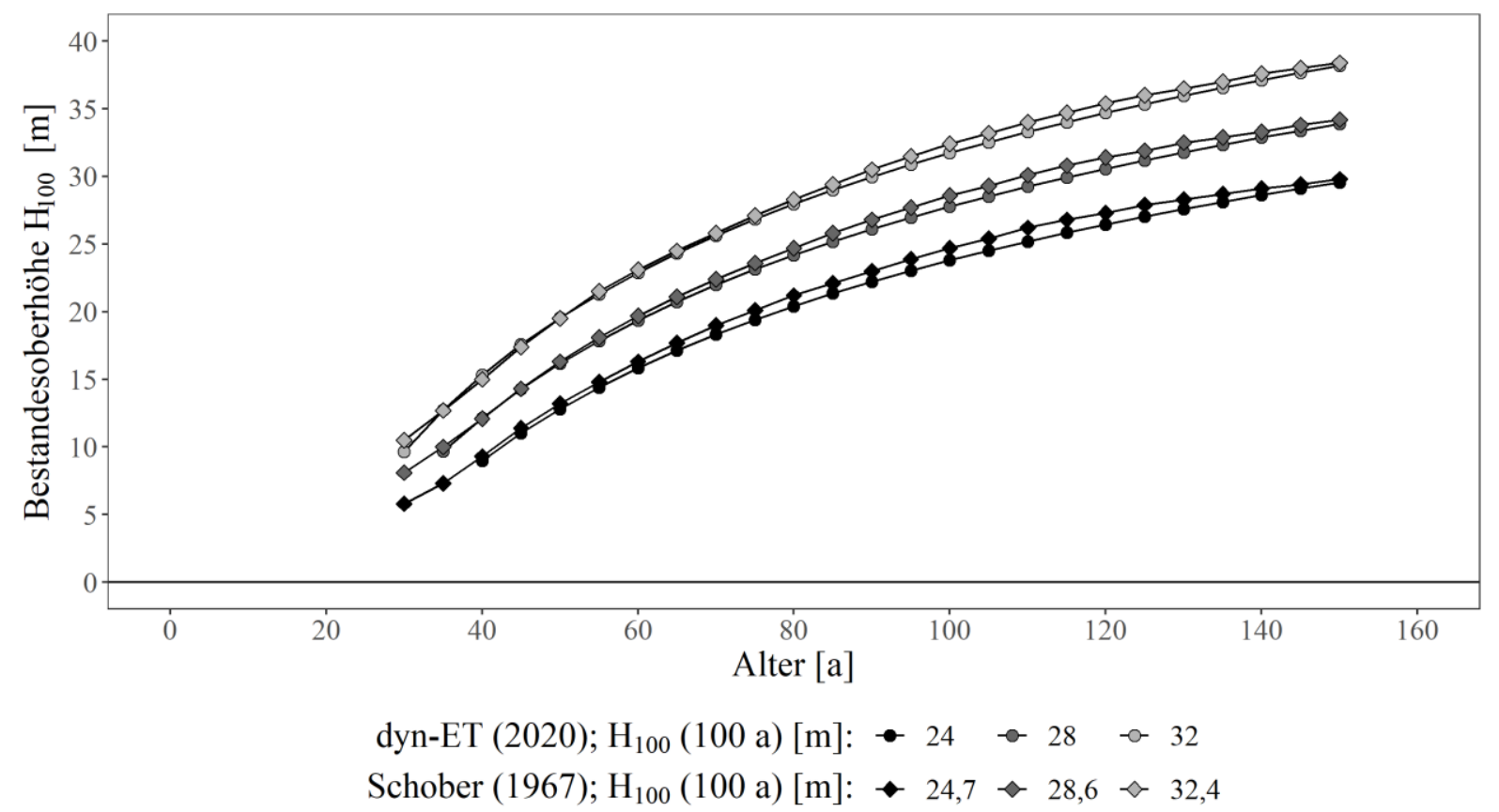

Abbildung 60: Oberhöhe über dem Alter; ausgewählte Oberhöhenbonitäten der Baumart Buche (mäßige Durchforstung) gemäß dyn-ET (unveröff.) und Schober (1967).

Auch dieser Effekt zeigt sich in abgeschwächter Form beim Vergleich der starken Durchforstungen - hier liegen die laufenden Bestandesvolumenzuwächse bei einer Oberhöhenbonität von 24 m gemäß dyn-ET (unveröff.) teilweise sogar unterhalb von Schober (1967), während bei hoher Oberhöhenbonität ein deutlicher Ertragsniveauanstieg erkennbar ist (Abbildungen 157 und 158 im Anhang). 


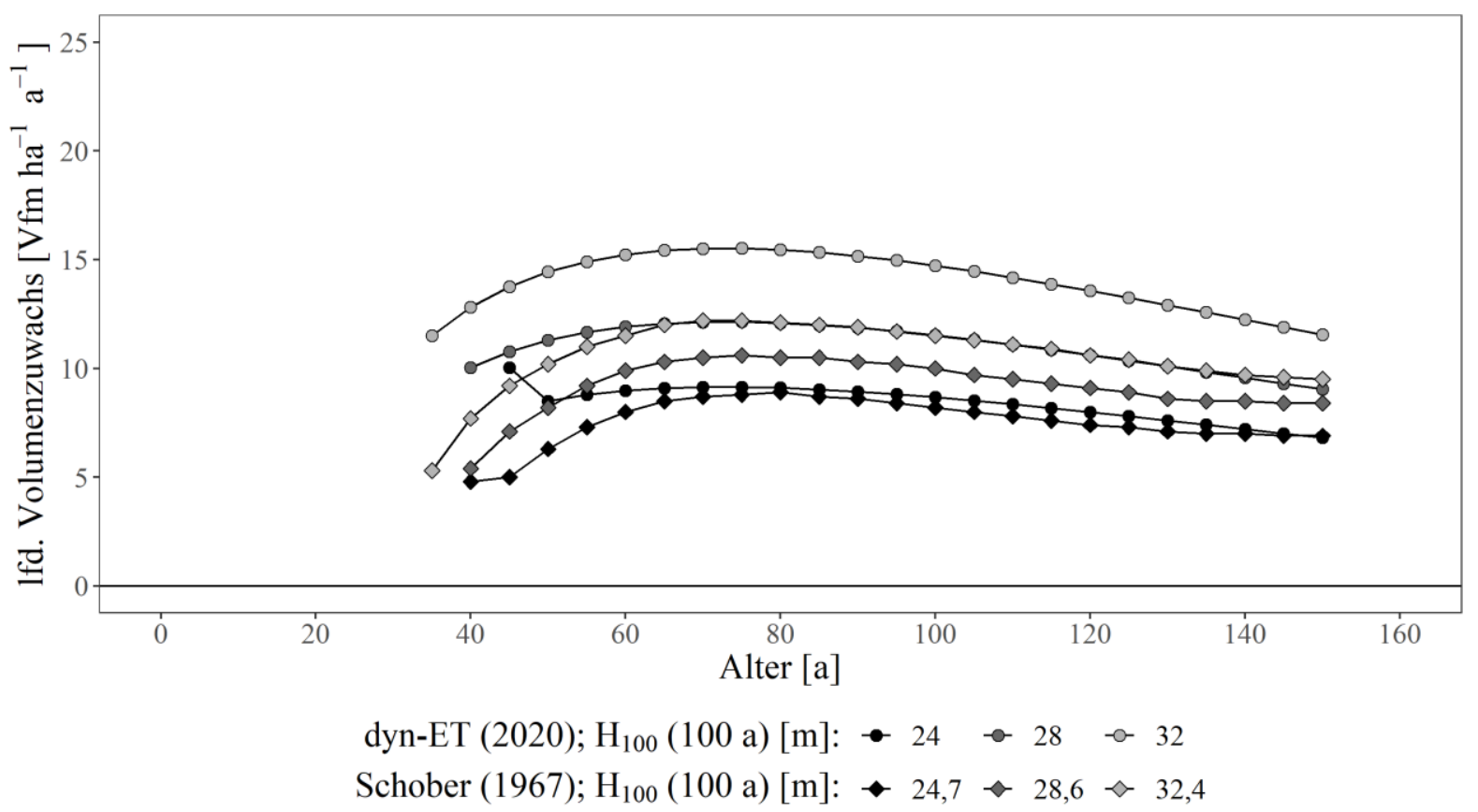

Abbildung 61: Laufender Bestandesvolumenzuwachs über dem Alter; ausgewählte Oberhöhenbonitäten der Baumart Buche (mäßige Durchforstung) gemäß dyn-ET (unveröff.) und Schober (1967).

Gleichzeitig liegen die Vorräte im Alter 140 a bei mäßiger Durchforstung gemäß dyn-ET (unveröff.) um $7 \%$ bis $16 \%$ (Tabelle 3-3; siehe analog dazu auch die gesunkenen Grundflächen des verbleibenden Bestandes) unterhalb der Vorräte gemäß Schober (1967) (Abbildung 62). Abbildung 159 im Anhang zeigt ähnliche Verläufe in erneut abgeschwächter Ausprägung für die starke Durchforstung.

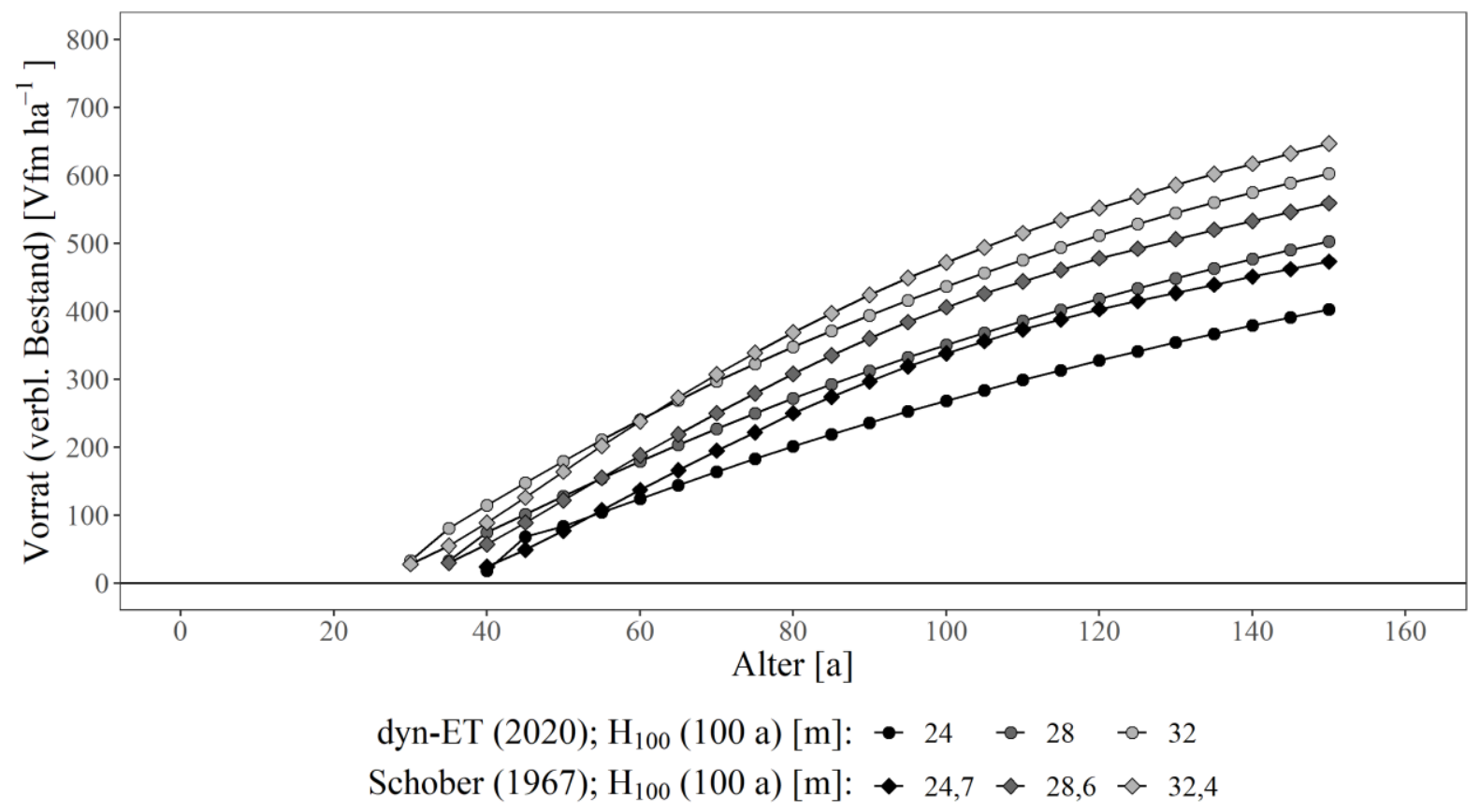

Abbildung 62: Vorrat (verbleibender Bestand) über dem Alter; ausgewählte Oberhöhenbonitäten der Baumart Buche (mäßige Durchforstung) gemäß dyn-ET (unveröff.) und Schober (1967). 
Tabelle 3-3: Vergleich ausgewählter Ertragstafeldaten der Baumart Buche (mäßige Durchforstung); ausgewählte Oberhöhenbonitäten gemäß dyn-ET (unveröff.) und Schober (1967); in Klammern ist jeweils die relative Differenz zur vergleichbaren Oberhöhenbonität nach Schober (1967) angegeben; $\boldsymbol{G W L}\left(140\right.$ a): Gesamtwuchsleistung bis zum Alter $140 \mathrm{a}$, $\max \boldsymbol{v}_{\boldsymbol{B}}$ : lfd. Bestandesvolumenzuwachs zum Zeitpunkt der Kulmination [...], $\boldsymbol{V}_{\boldsymbol{B}}(\mathbf{1 4 0} \mathbf{a})$ : Vorrat (verbl. Bestand) im Alter $140 \mathrm{a}$, $\boldsymbol{G}_{\boldsymbol{v e r b l .}}\left(\mathbf{1 4 0}\right.$ a): Bestandesgrundfläche des verbleibenden Bestandes im Alter $140 \mathrm{a}, \sum_{\mathbf{0}}^{\mathbf{1 4 0}} \boldsymbol{\lambda}$ : kumuliertes ausscheidendes Bestandesvolumen bis zum Alter 140 a, $\boldsymbol{N}_{\text {verbl. }}(140$ a): Stammzahl des verbleibenden Bestandes im Alter 140 a, $\sum_{\mathbf{0}}^{\mathbf{1 4 0}} \boldsymbol{N}_{\boldsymbol{a u s s c h}}$ : kumulierte ausscheidende Stammzahl bis zum Alter 140 a, $\boldsymbol{D}_{\boldsymbol{g}, \boldsymbol{v} \text { erbl. }}(\mathbf{1 4 0}$ a): Durchmesser des Grundflächenmittelstammes des verbleibenden Bestandes im Alter 140 a, $\boldsymbol{D}_{\boldsymbol{g} \text {,aussch. }}$ (140 a): Durchmesser des Grundflächenmittelstammes des ausscheidenden Bestandes im Alter 140 a, max $\overline{\boldsymbol{v}_{B}}$ : durchschn. Bestandesvolumenzuwachs zum Zeitpunkt der Kulmination [...], $\boldsymbol{V}_{\boldsymbol{\eta}}(\mathbf{1 4 0}$ a): Normalvorrat bei einer Umtriebszeit von $140 \mathrm{a}$.

\begin{tabular}{|c|c|c|c|c|c|c|}
\hline \multirow{2}{*}{$\begin{array}{r}\text { Ertragstafel } \\
H_{100}(100 \text { a })[\mathrm{m}]\end{array}$} & \multicolumn{3}{|c|}{ Schober (1967), mäßige Df. } & \multicolumn{3}{|c|}{ dyn-ET (unveröff.), mäßige Df. } \\
\hline & 24,7 & 28,6 & 32,4 & 24 & 28 & 32 \\
\hline$G W L(140$ a) & 796 & 1.001 & 1.201 & 874 & 1.207 & 1.592 \\
\hline$\left[\mathrm{Vfm} \mathrm{ha}^{-1}\right]$ & & & & $(+9,8 \%)$ & $(+20,6 \%)$ & $(+32,6 \%)$ \\
\hline $\max v_{B}$ & $8,9[80 \mathrm{a}]$ & $10,6[75 \mathrm{a}]$ & $12,2[75 \mathrm{a}]$ & $9,1[75 \mathrm{a}]$ & $12,1[75 \mathrm{a}]$ & $15,5[75 \mathrm{a}]$ \\
\hline$\left[\mathrm{Vfm} \mathrm{ha}^{-1} \mathrm{a}^{-1}\right]$ & & & & $(+2,2 \%)$ & $(+14,2 \%)$ & $(+27,0 \%)$ \\
\hline$V_{B}(140 \mathrm{a})$ & 451 & 533 & 617 & 379 & 477 & 575 \\
\hline$\left[\mathrm{Vfm} \mathrm{ha}^{-1}\right]$ & & & & $(-16,0 \%)$ & $(-10,5 \%)$ & $(-6,8 \%)$ \\
\hline$G_{v e r b l .}(140 \mathrm{a})$ & 31,7 & 32,5 & 33,3 & 27,4 & 29,3 & 30,7 \\
\hline$\left[\mathbf{m}^{2}\right]$ & & & & $(-13,6 \%)$ & $(-9,8 \%)$ & $(-7,8 \%)$ \\
\hline$\sum_{0}^{140 \mathrm{a}} \lambda$ & 345 & 468 & 584 & 495 & 730 & 1.017 \\
\hline$\left[\mathrm{Vfm} \mathrm{ha}^{-1}\right]$ & & & & $(+43,5 \%)$ & $(+56,0 \%)$ & $(+74,1 \%)$ \\
\hline$N_{\text {verbl. }}(140 \mathrm{a})$ & 276 & 216 & 168 & 204 & 156 & 124 \\
\hline$\left[\mathbf{h a}^{-1}\right]$ & & & & $(-26,1 \%)$ & $(-27,8 \%)$ & $(-26,2 \%)$ \\
\hline$\sum_{0}^{140 \mathrm{a}} N_{\text {aussch }}$ & 15.326 & 8.736 & 6.235 & 2.671 & 2.758 & 3.074 \\
\hline$\left[\mathrm{ha}^{-1}\right]$ & & & & $(-82,6 \%)$ & $(-68,4 \%)$ & $(-50,7 \%)$ \\
\hline$D_{g, v e r b l .}(140$ a) & 38,2 & 43,7 & 50,2 & 41,3 & 48,9 & 56,2 \\
\hline$[\mathbf{c m}]$ & & & & $(+8,1 \%)$ & $(+11,9 \%)$ & $(+12,0 \%)$ \\
\hline$D_{g, a u s s c h .}(140$ a) & 31,4 & 36,7 & 43,1 & 38,2 & 45,0 & 51,4 \\
\hline$[\mathbf{c m}]$ & & & & $(+21,7 \%)$ & $(+22,6 \%)$ & $(+19,3 \%)$ \\
\hline $\max \overline{v_{B}}$ & $5,8[150 \mathrm{a}]$ & $7,2[150 \mathrm{a}]$ & $8,7[150 \mathrm{a}]$ & $6,3[150 \mathrm{a}]$ & $8,7[150 \mathrm{a}]$ & $11,4[150 \mathrm{a}]$ \\
\hline$\left[\mathrm{Vfm} \mathrm{ha}^{-1} \mathbf{a}^{-1}\right]$ & & & & $(+8,6 \%)$ & $(+20,8 \%)$ & $(+31,0 \%)$ \\
\hline$V_{\eta}(140 \mathrm{a})$ & 202 & 250 & 299 & 171 & 230 & 294 \\
\hline$\left[\mathrm{Vfm} \mathrm{ha}^{-1}\right]$ & & & & $(-15,3 \%)$ & $(-8,2 \%)$ & $(-1,9 \%)$ \\
\hline
\end{tabular}


Bei annähernd gleichen bzw. leicht gesunkenen Vorräten führen die deutlich gestiegenen Bestandesvolumenzuwächse zu (überwiegend) höheren Volumenzuwachsraten, d. h. einem höheren relativem Bestandesvolumenzuwachs in Bezug auf das jeweils vorhandene Bestandesvolumen (Abbildung 63 und analog Abbildung 160 im Anhang für die starke Durchforstung).

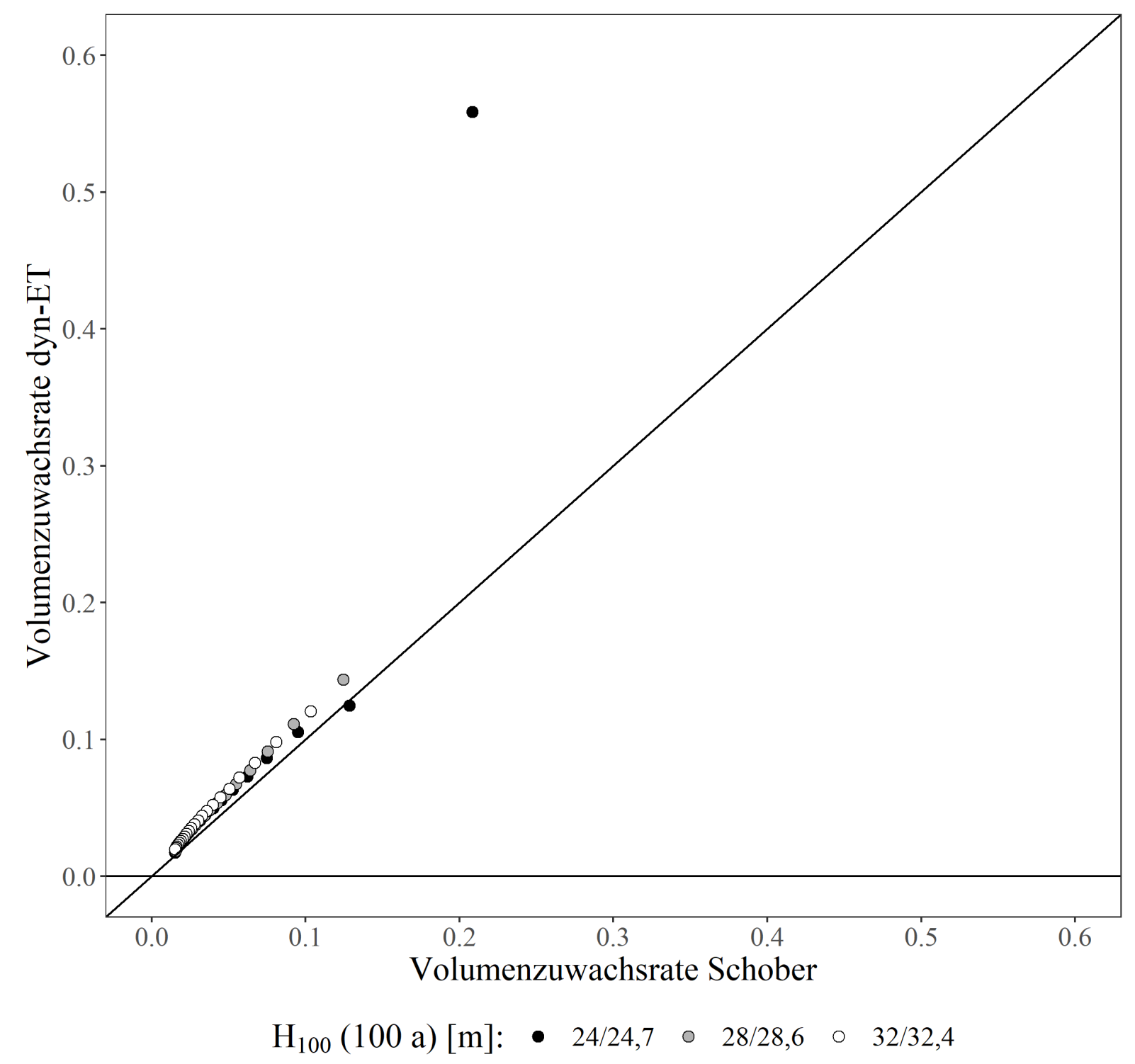

Abbildung 63: Volumenzuwachsraten der Baumart Buche (mäßige Durchforstung, ausgewählte Oberhöhenbonitäten) gemäß dyn-ET (unveröff.) und Schober (1967); es werden nur Vergleichspaare aus Altern dargestellt, in denen für alle Oberhöhenbonitäten Datenpunkte vorliegen; die Winkelhalbierende kennzeichnet gleiche Wertzuwachsraten im gleichen Alter.

Gleichzeitig steigert das gestiegene Ertragsniveau (in Verbidnung mit den veränderten Grundflächenhaltungen) die Vornutzungsvolumina - und zwar im Mittel umso mehr, je höher die Standortsgüte ist (Abbildung 64). Die Summe der Vornutzungsvolumina bis zum Alter $140 \mathrm{a}$ steigt gemäß dyn-ET (unveröff., mäßige Hochdurchforstung) um $44 \%$ bis 74 \% (Tabelle 3-3) 
ggü. Schober (1967, mäßige Durchforstung). Bei starker Durchforstung (Abbildung 161 im Anhang) sind die Unterschiede weniger ausgeprägt und die Vornutzungsvolumina gemäß dyn-ET (unveröff.) bei mittlerer und geringer Standortsgüte in hohen Bestandesaltern sogar niedriger.

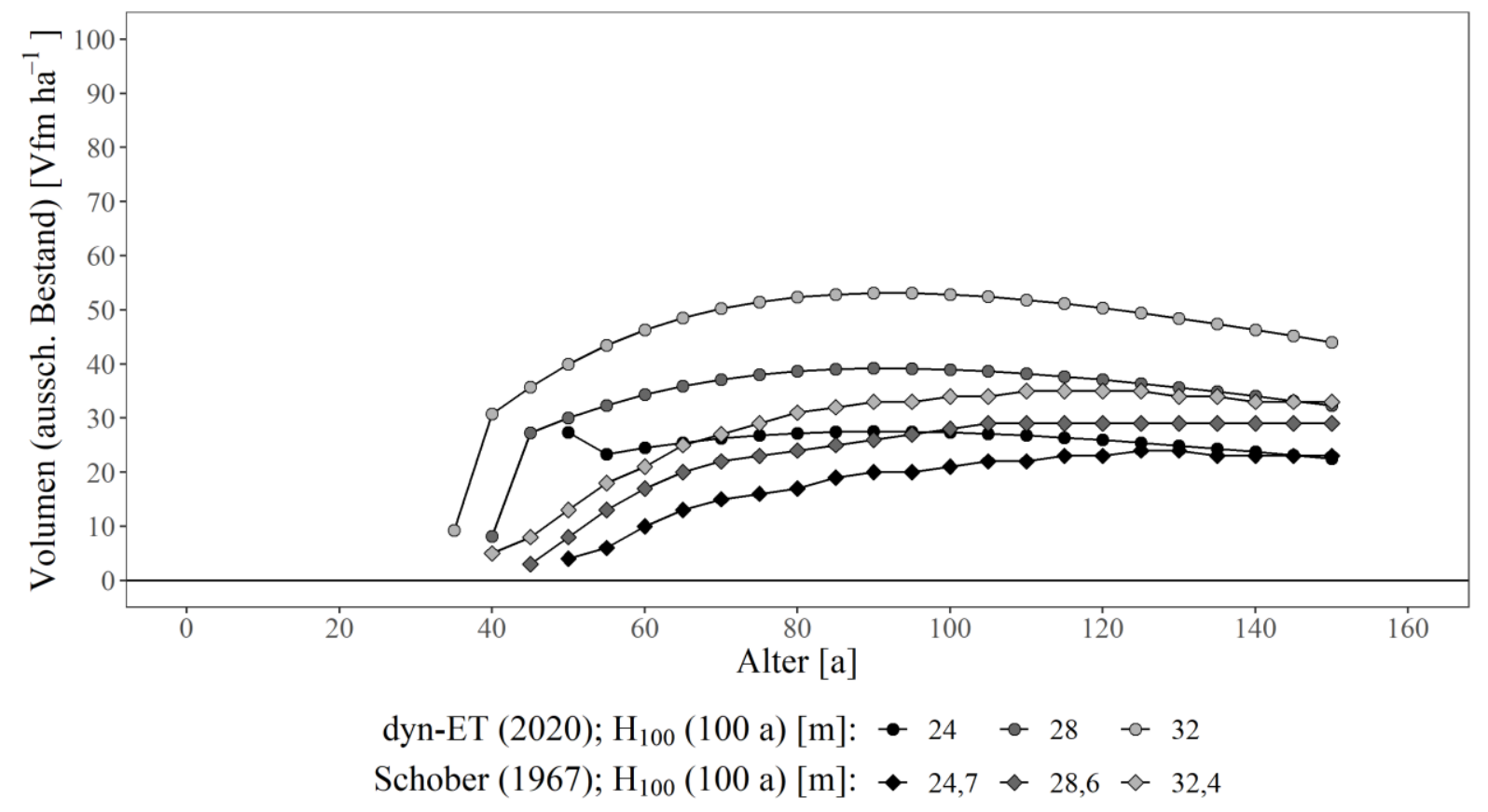

Abbildung 64: Ausscheidendes Bestandesvolumen über dem Alter; ausgewählte Oberhöhenbonitäten der Baumart Buche (mäßige Durchforstung) gemäß dyn-ET (unveröff.) und Schober (1967).

Die quantitativ recht ähnlichen bzw. leicht gesunkenen Vorräte sind aufgrund der veränderten waldbaulichen Behandlung gemäß dyn-ET (unveröff.) aber grundlegend anders aufgebaut.

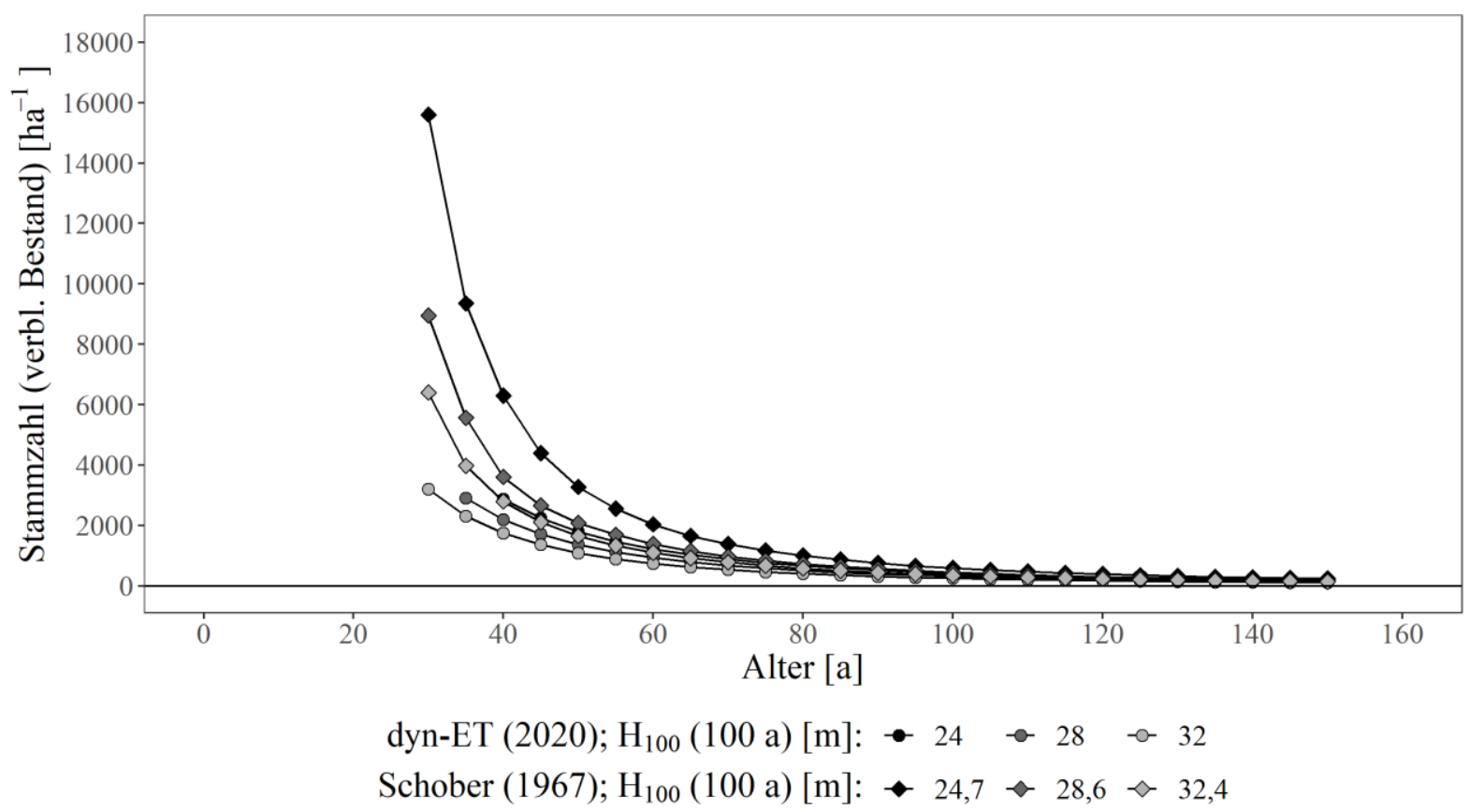

Abbildung 65: Stammzahl (verbleibender Bestand) über dem Alter; ausgewählte Oberhöhenbonitäten der Baumart Buche (mäßige Durchforstung) gemäß dyn-ET (unveröff.) und Schober (1967). 


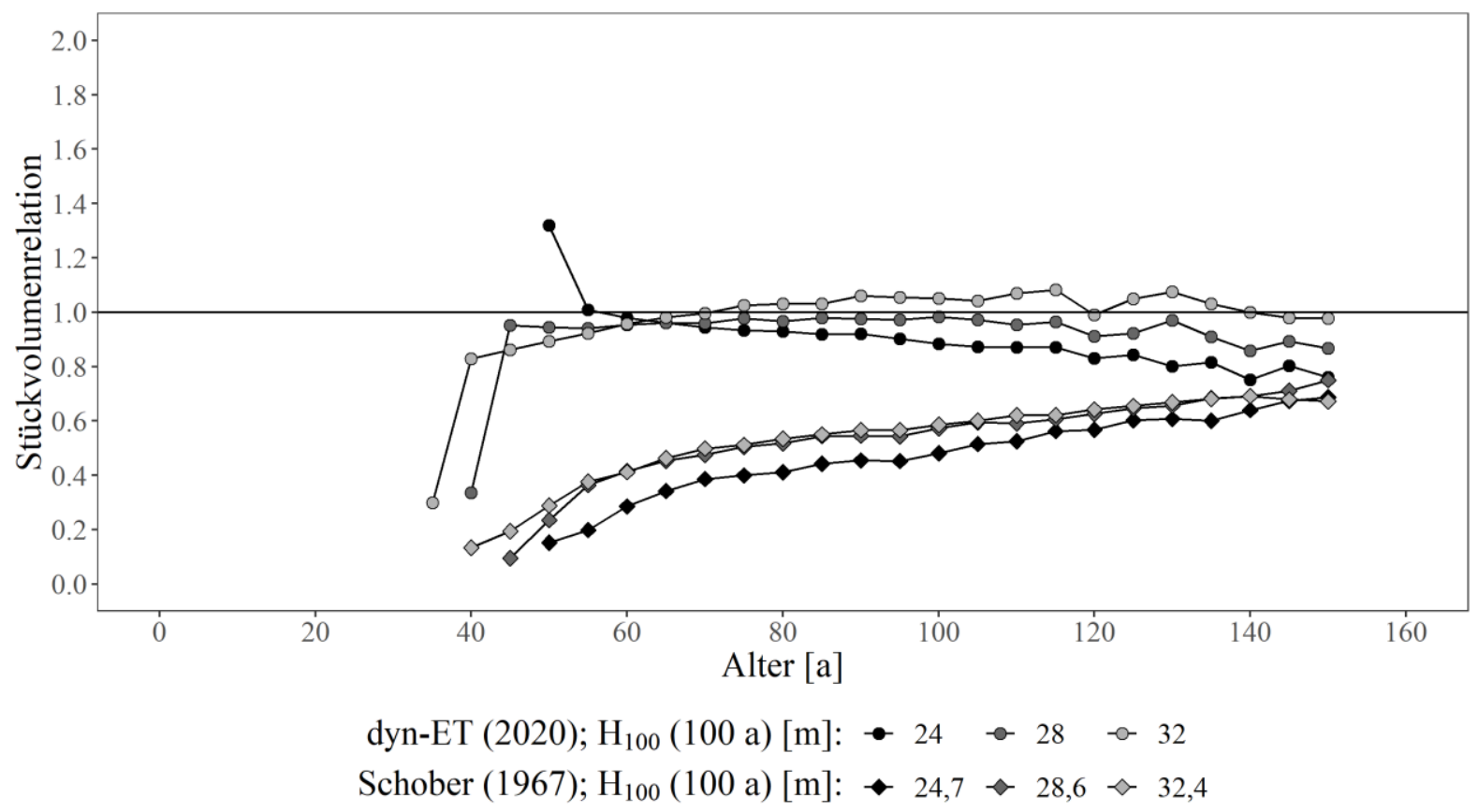

Abbildung 66: Stückvolumenrelationen (ausscheidendes geteilt durch verbleibendes Stückvolumen) über dem Alter; ausgewählte Oberhöhenbonitäten der Baumart Buche (mäßige Durchforstung) gemäß dyn-ET (unveröff.) und Schober (1967).

Deutlich geringere Stammzahlen zu Beginn der forstlichen Produktion (Abbildung 65) führen in Kombination mit den hochdurchforstungsartigeren Eingriffen (Abbildung 66) zu höheren Einzelbaumdimensionen im verbleibenden (Abbildung 67 und Tabelle 3-3) und insbesondere auch im ausscheidenden Bestand (Abbildung 68).

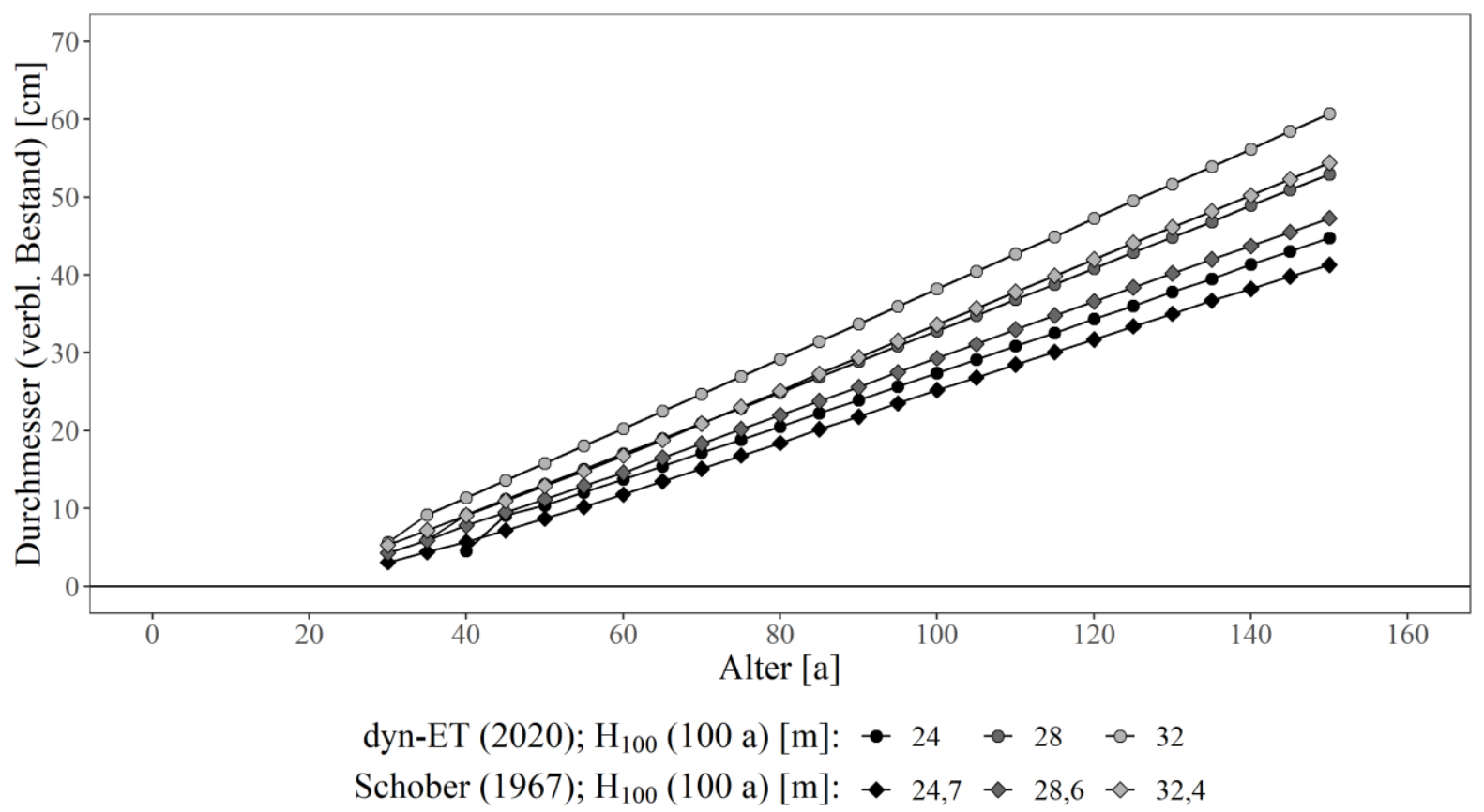

Abbildung 67: Durchmesser des Grundflächenmittelstamms (verbleibender Bestand) über dem Alter; ausgewählte Oberhöhenbonitäten der Baumart Buche (mäßige Durchforstung) gemäß dyn-ET (unveröff.) und Schober (1967). 
Abbildungen 162 bis 165 zeigen im Anhang die Auswirkungen bei starker Durchforstung.

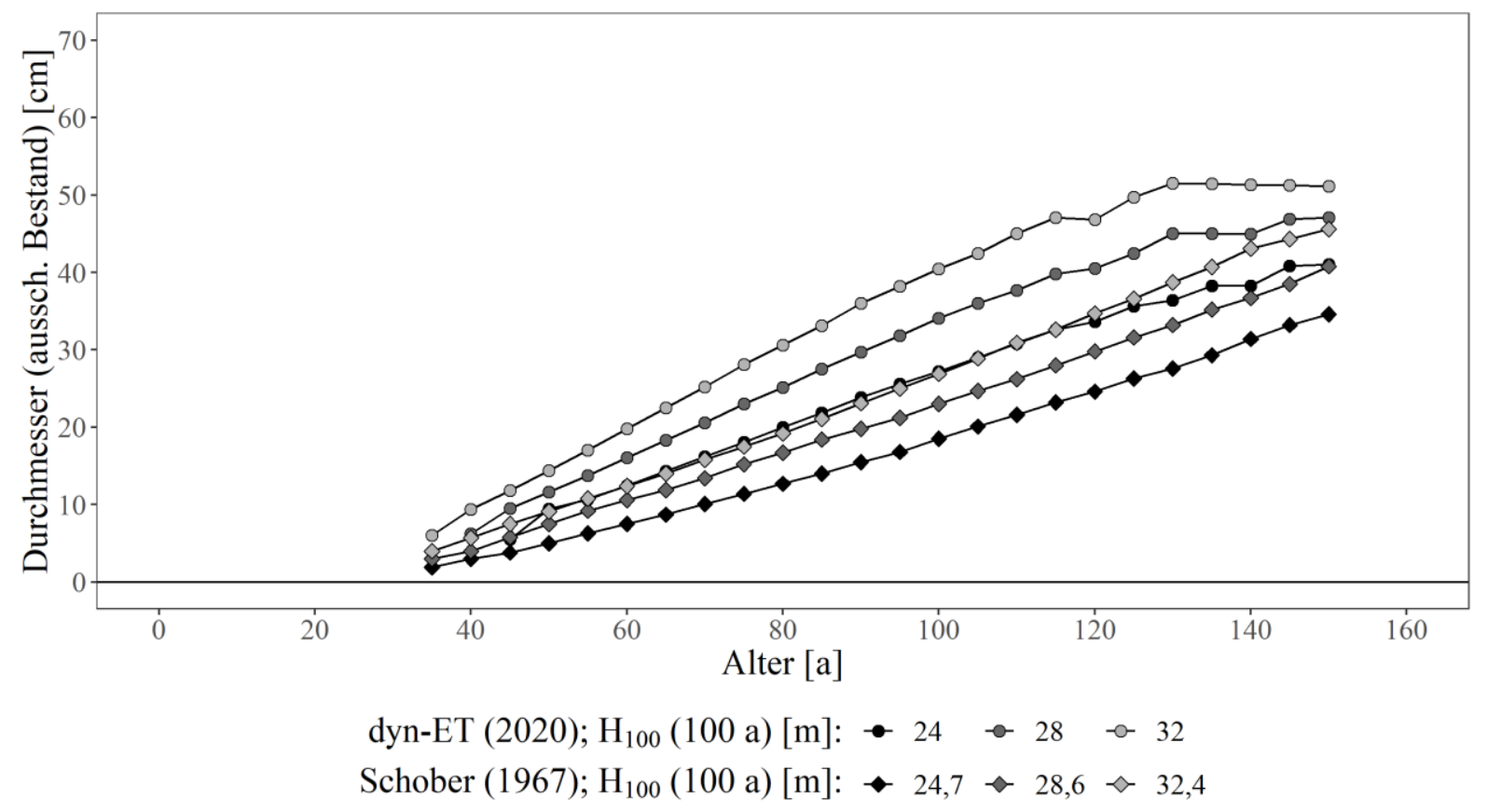

Abbildung 68: Durchmesser des Grundflächenmittelstamms (ausscheidender Bestand) über dem Alter; ausgewählte Oberhöhenbonitäten der Baumart Buche (mäßige Durchforstung) gemäß dyn-ET (unveröff.) und Schober (1967).

Als Kennziffern einer nachhaltigen Betriebsklasse werden im Folgenden noch klassische Elemente der Nachhaltigkeitsprüfung im Rahmen der Forsteinrichtung (vgl. Speidel 1972, S. 163 ff.; siehe Abschnitt 3.2.2) zwischen dyn-ET (unveröff., mäßige Hochdurchforstung) und Schober (1967, mäßige Durchforstung) verglichen. Der durchschnittliche Bestandesvolumenzuwachs, auch als „Alters-Durchschnittszuwachs der Gesamtmasse“ (Schober 1967) oder „durchschnittlicher Gesamtzuwachs“ (Gadow 2003, S. 41) bezeichnet, gibt das durchschnittliche, jährlich nutzbare Holzvolumen eines Bestandes an und geht im Rahmen der Forsteinrichtung bspw. über die Gerhardt'sche Formel in die Bestimmung des Nachhaltshiebsatz auf Grundlage des Normalwaldmodells ein (Gadow 2005, S. 216 f.). Da sich die durchschnittlichen Bestandesvolumenzuwächse als Quotient der Gesamtwuchsleistung und des Alters bzw. der Umtriebszeits ergeben, sind die Vergleichsergebnisse zwischen dyn-ET (unveröff., mäßige Hochdurchforstung) und Schober (1967, mäßige Durchforstung) mit Blick auf die Gesamtwuchsleistungen in Abbildung 59 nicht überraschend. Abbildung 69 verdeutlicht erneut das deutlich gestiegene Ertragsniveau. Das durchschnittliche Nutzungspotential bis zu einer Umtriebszeit von 140 Jahren liegt gemäß dyn-ET (unveröff., mäßige Hochdurchforstung) um 9 \% bis 33 \% höher als bei Schober (1967, mäßige Durchforstung). Die entsprechende Abbildung 166 für die starke Durchforstung kann dem Anhang entnommen werden. 


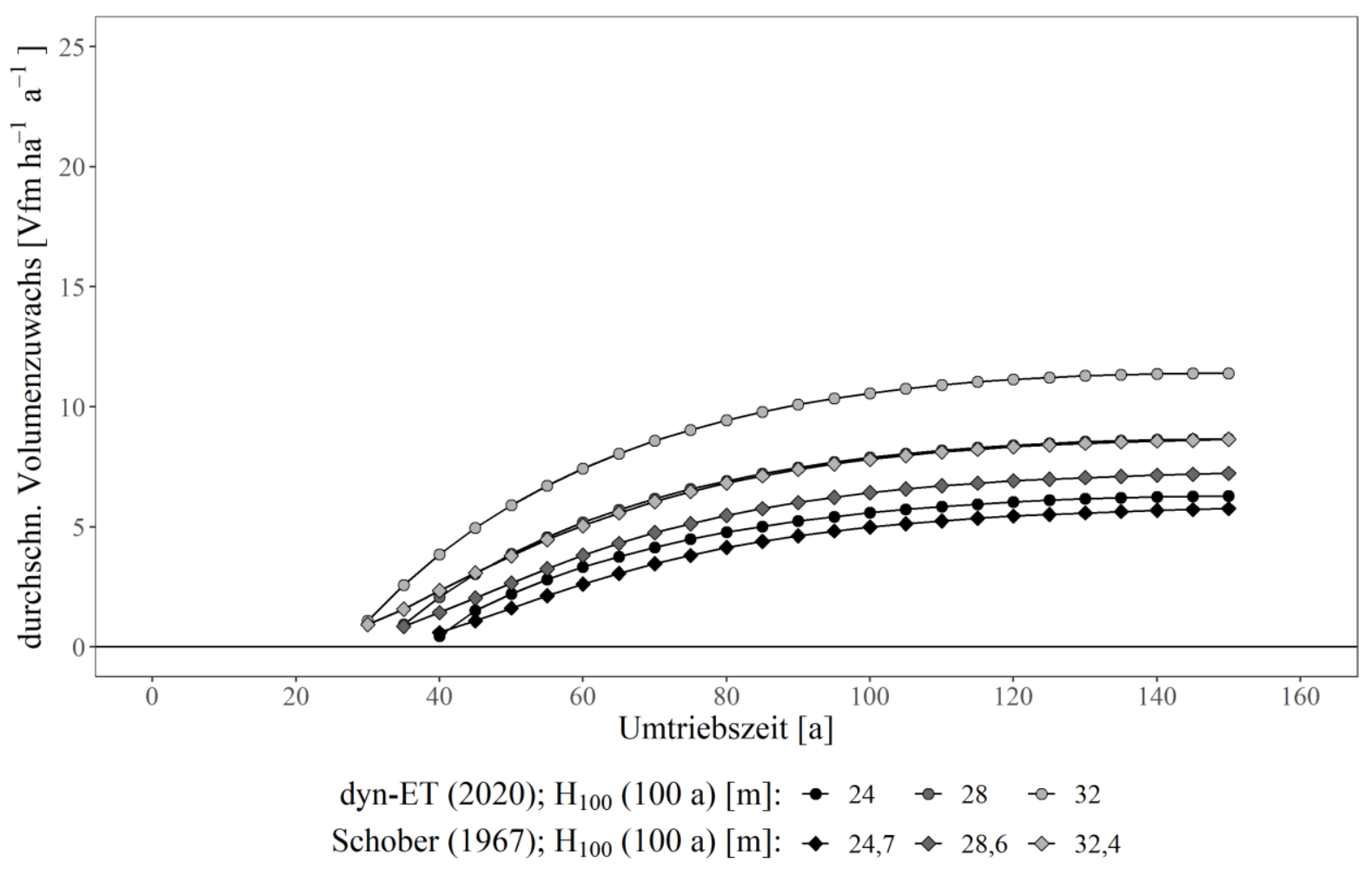

Abbildung 69: Durchschnittlicher Bestandesvolumenzuwachs über der Umtriebszeit; ausgewählte Oberhöhenbonitäten der Baumart Buche (mäßige Durchforstung) gemäß dyn-ET (unveröff.) und Schober (1967).

Während die durchschnittlichen Bestandesvolumenzuwächse direkt aus den Ertragstafeldaten entnommen werden können (siehe Tabellen 8-2 bis 8-26 im Anhang), muss der Normalvorrat über die verbleibenden und ausscheidenden Bestandesvolumina aller Altersstufen eines Normalwaldmodells berechnet werden (vgl. Speidel 1972, S. 101 ff.) - er entspricht somit dem flächendurchschnittlichen Vorrat einer Betriebsklasse in Abhängigkeit von der Umtriebszeit. Die Normalvorräte gemäß dyn-ET (unveröff., mäßige Hochdurchforstung) liegen bei niedrigen Umtriebszeiten zunächst oberhalb der Normalvorräte gemäß Schober (1967, mäßige Durchforstung) und sinken mit steigender Umtriebszeit darunter (Abbildung 70) - bei einer Umtriebszeit von 140 a liegen sie um $2 \%$ bis $15 \%$ niedriger (Tabelle 3-3). Mit steigender Standortsgüte liegt der Punkt gleicher Normalvorräte bei höheren Umtriebszeiten und der Unterschied zwischen den Ertragstafeln verringert sich. Ähnliche Effekte zeigen sich bei starker Durchforstung (Abbildung 167 im Anhang). 


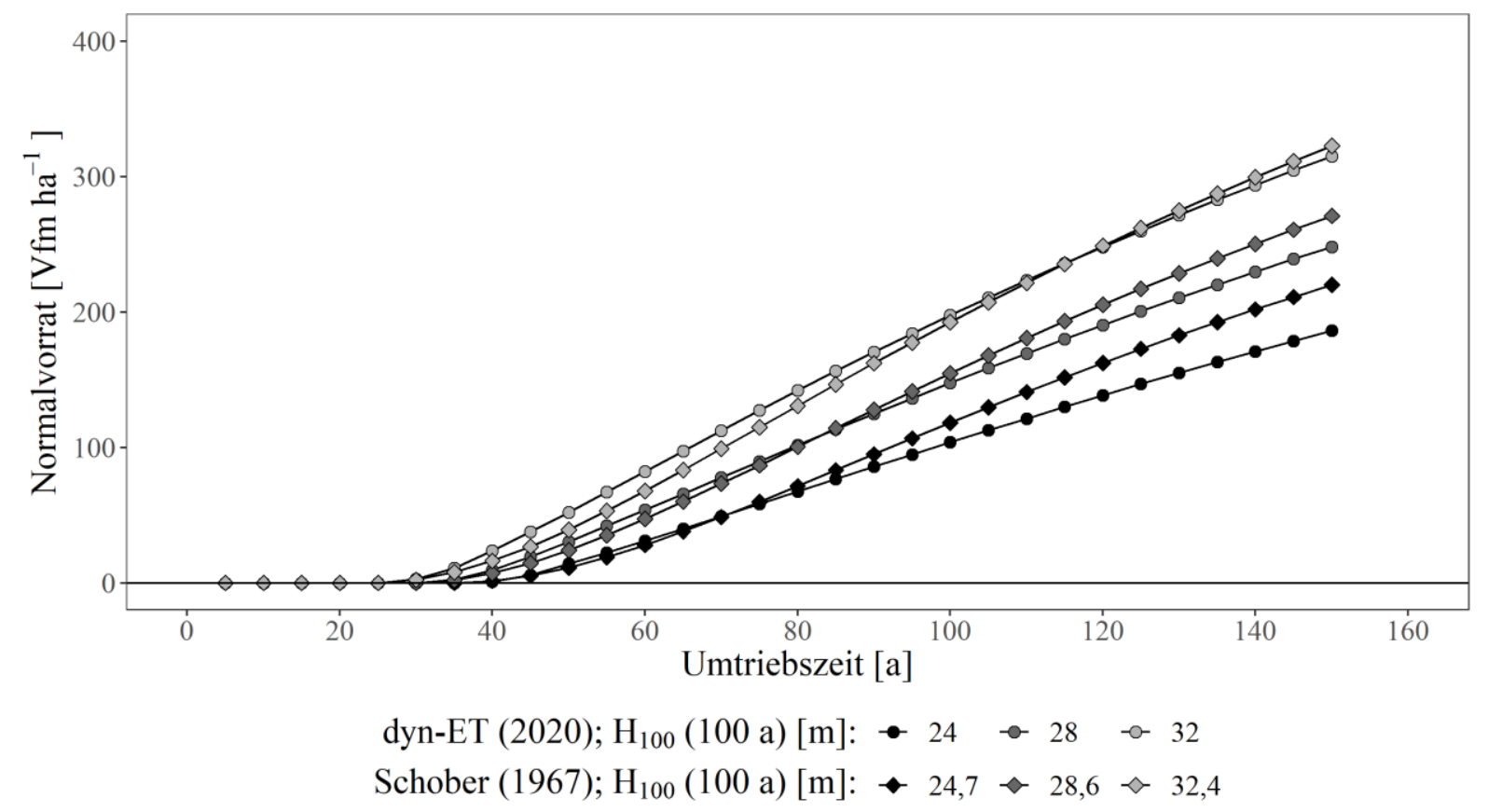

Abbildung 70: Normalvorrat über der Umtriebszeit; ausgewählte Oberhöhenbonitäten der Baumart Buche (mäßige Durchforstung) gemäß dyn-ET (unveröff.) und Schober (1967).

Zwischen den drei waldbaulichen Behandlungsvarianten gemäß dyn-ET (unveröff.) zeigen sich bei gleicher Oberhöhenbonität deutlich differenzierte Gesamtwuchsleistungen (Abbildung 71) und laufende Volumenzuwächse (Abbildung 72).

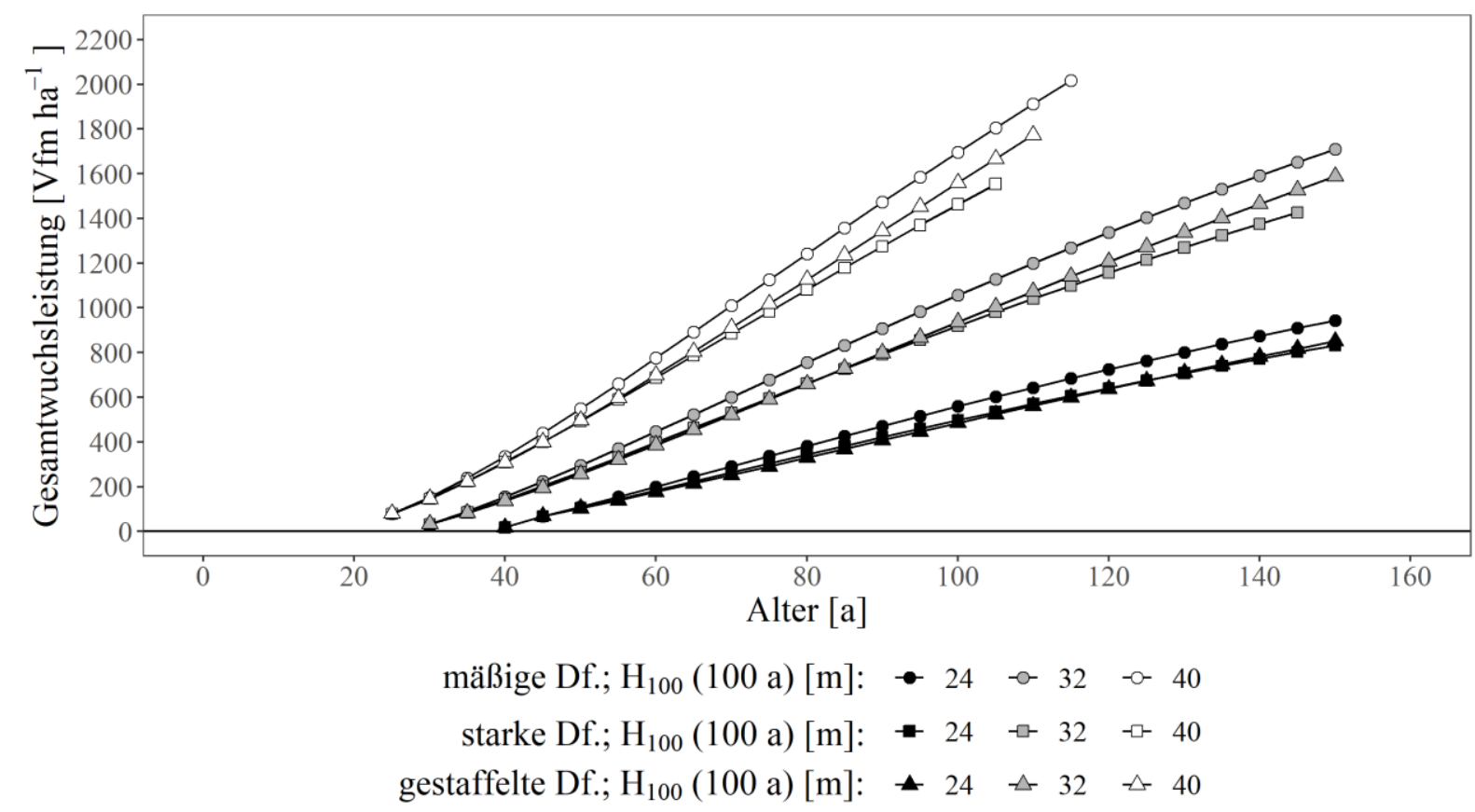

Abbildung 71: Gesamtwuchsleistung über dem Alter; ausgewählte Oberhöhenbonitäten der Baumart Buche getrennt nach Behandlungsvarianten gemäß dyn-ET (unveröff.). 
Tabelle 3-4: Vergleich ausgewählter Ertragstafeldaten der Baumart Buche (Oberhöhenbonität $H_{100}(100 \mathrm{a})=32 \mathrm{~m}$ ) gemäß dyn-ET (unveröff.); unterschiedliche Behandlungsvarianten; in Klammern ist jeweils die relative Differenz zur mäßigen Hochdurchforstung angegeben;

$\boldsymbol{G W L}\left(140\right.$ a): Gesamtwuchsleistung bis zum Alter $140 \mathrm{a}, \max \boldsymbol{v}_{\boldsymbol{B}}$ : lfd. Bestandesvolumenzuwachs zum Zeitpunkt der Kulmination [...], $\boldsymbol{V}_{\boldsymbol{B}}(\mathbf{1 4 0} \mathbf{a})$ : Vorrat (verbl. Bestand) im Alter $140 \mathrm{a}$, $\boldsymbol{G}_{\boldsymbol{v e r b l .}}\left(\mathbf{1 4 0}\right.$ a): Bestandesgrundfläche des verbleibenden Bestandes im Alter $140 \mathrm{a}, \sum_{\mathbf{0}}^{\mathbf{1 4 0}} \boldsymbol{\lambda}$ : kumuliertes ausscheidendes Bestandesvolumen bis zum Alter 140 a, $\boldsymbol{N}_{\text {verbl. }}(\mathbf{1 4 0}$ a): Stammzahl des verbleibenden Bestandes im Alter 140 a, $\sum_{\mathbf{0}}^{\mathbf{1 4 0}} \boldsymbol{N}_{\boldsymbol{a u s s}}$ : kumulierte ausscheidende Stammzahl bis zum Alter 140 a, $\boldsymbol{D}_{\boldsymbol{g}, \boldsymbol{v e r b l}}(\mathbf{1 4 0}$ a): Durchmesser des Grundflächenmittelstammes des verbleibenden Bestandes im Alter 140 a, $\boldsymbol{D}_{\boldsymbol{g} \text {,aussch. }}$ (140 a): Durchmesser des Grundflächenmittelstammes des ausscheidenden Bestandes im Alter 140 a, $\boldsymbol{m a x} \overline{\boldsymbol{v}_{\boldsymbol{B}}}$ : durchschn. Bestandesvolumenzuwachs zum Zeitpunkt der Kulmination [...], $\boldsymbol{V}_{\boldsymbol{\eta}}(\mathbf{1 4 0}$ a): Normalvorrat bei einer Umtriebszeit von 140 a.

\begin{tabular}{|c|c|c|c|}
\hline Bestandesbehandlung & $\begin{array}{r}\text { mäßige } \\
\text { Hochdurchforstung }\end{array}$ & $\begin{array}{r}\text { starke } \\
\text { Hochdurchforstung }\end{array}$ & $\begin{array}{r}\text { gestaffelte } \\
\text { Hochdurchforstung }\end{array}$ \\
\hline$H_{100}(100 \mathrm{a})[\mathrm{m}]$ & 32 & 32 & 32 \\
\hline$G W L(140 \mathrm{a})$ & 1.592 & 1.376 & 1.465 \\
\hline$\left[\mathrm{Vfm} \mathrm{ha}^{-1}\right]$ & & $(-13,6 \%)$ & $(-8,0 \%)$ \\
\hline $\max v_{B}$ & $15,5[75 \mathrm{a}]$ & $13,3[70 \mathrm{a}]$ & 13,9 [85 a] \\
\hline$\left[\mathrm{Vfm} \mathrm{ha}^{-1} \mathrm{a}^{-1}\right]$ & & $(-14,2 \%)$ & $(-10,3 \%)$ \\
\hline$V_{B}(140 \mathrm{a})$ & 575 & 502 & 577 \\
\hline$\left[\mathrm{Vfm} \mathrm{ha}^{-1}\right]$ & & $(-12,7 \%)$ & $(+0,3 \%)$ \\
\hline$G_{v e r b l .}(140 \mathrm{a})$ & 30,7 & 26,4 & 30,7 \\
\hline$\left[\mathbf{m}^{2}\right]$ & & $(-14,0 \%)$ & $(+/-0 \%)$ \\
\hline$\sum_{0}^{140 \mathrm{a}} \lambda$ & 1.017 & 874 & 888 \\
\hline$\left[\mathrm{Vfm} \mathrm{ha}^{-1}\right]$ & & $(-14,1 \%)$ & $(-12,7 \%)$ \\
\hline$N_{\text {verbl. }}(140 \mathrm{a})$ & 124 & 101 & 133 \\
\hline$\left[\mathrm{ha}^{-1}\right]$ & & $(-18,5 \%)$ & $(+7,3 \%)$ \\
\hline$\sum_{0}^{140 \mathrm{a}} N_{\text {aussch }}$ & 3.074 & 2.334 & 1.879 \\
\hline$\left[\mathrm{ha}^{-1}\right]$ & & $(-24,1 \%)$ & $(-38,9 \%)$ \\
\hline$D_{g, v e r b l .}(140$ a $)$ & 56,2 & 57,7 & 54,2 \\
\hline$[\mathbf{c m}]$ & & $(+2,7 \%)$ & $(-3,6 \%)$ \\
\hline$D_{g, a u s s c h .}(140$ a) & 51,4 & 50,1 & 59,4 \\
\hline$[\mathbf{c m}]$ & & $(-2,5 \%)$ & $(+15,6 \%)$ \\
\hline $\max \overline{v_{B}}$ & $11,4[150 \mathrm{a}]$ & $9,8[145 \mathrm{a}]$ & $10,6[150 \mathrm{a}]$ \\
\hline$\left[\mathrm{Vfm} \mathrm{ha}^{-1} \mathbf{a}^{-1}\right]$ & & $(-14,0 \%)$ & $(-7,0 \%)$ \\
\hline$V_{\eta}(140 \mathrm{a})$ & 294 & 248 & 279 \\
\hline$\left[\mathrm{Vfm} \mathrm{ha}^{-1}\right]$ & & $(-15,6 \%)$ & $(-5,0 \%)$ \\
\hline
\end{tabular}


Bonitätsunabhängig liegt die Gesamtwuchsleistung bei mäßiger Hochdurchforstung über den gesamten Altersrahmen hinweg über den Gesamtwuchsleistungen bei starker und gestaffelter Hochdurchforstung. Bei einer mittleren Oberhöhenbonität von $32 \mathrm{~m}$ liegt das insgesamt bis zu einem Alter von 140 Jahren zugewachsene Bestandesvolumen bei gestaffelter Hochdurchforstung um $8 \%$ und bei starker Hochdurchforstung sogar um $14 \%$ unterhalb der Gesamtwuchsleistung bei mäßiger Hochdurchforstung (Tabelle 3-4).

Die laufenden Bestandesvolumenzuwächse der gestaffelten Hochdurchforstung liegen in jungen Bestandesaltern zunächst leicht unterhalb der starken Hochdurchforstung und nähern sich dann mit steigendem Alter der mäßigen Hochdurchforstung an. Bei der mittleren Oberhöhenbonität $32 \mathrm{~m}$ übersteigt der laufende Volumenzuwachs der gestaffelten Hochdurchforstung ab einem Alter von 130 Jahren sogar den Volumenzuwachs der mäßigen Hochdurchforstung (Abbildung 72), wodurch der relative Abstand der Gesamtwuchsleistungen zwischen mäßiger und gestaffelter Hochdurchforstung im hohen Alter zunehmend reduziert wird.

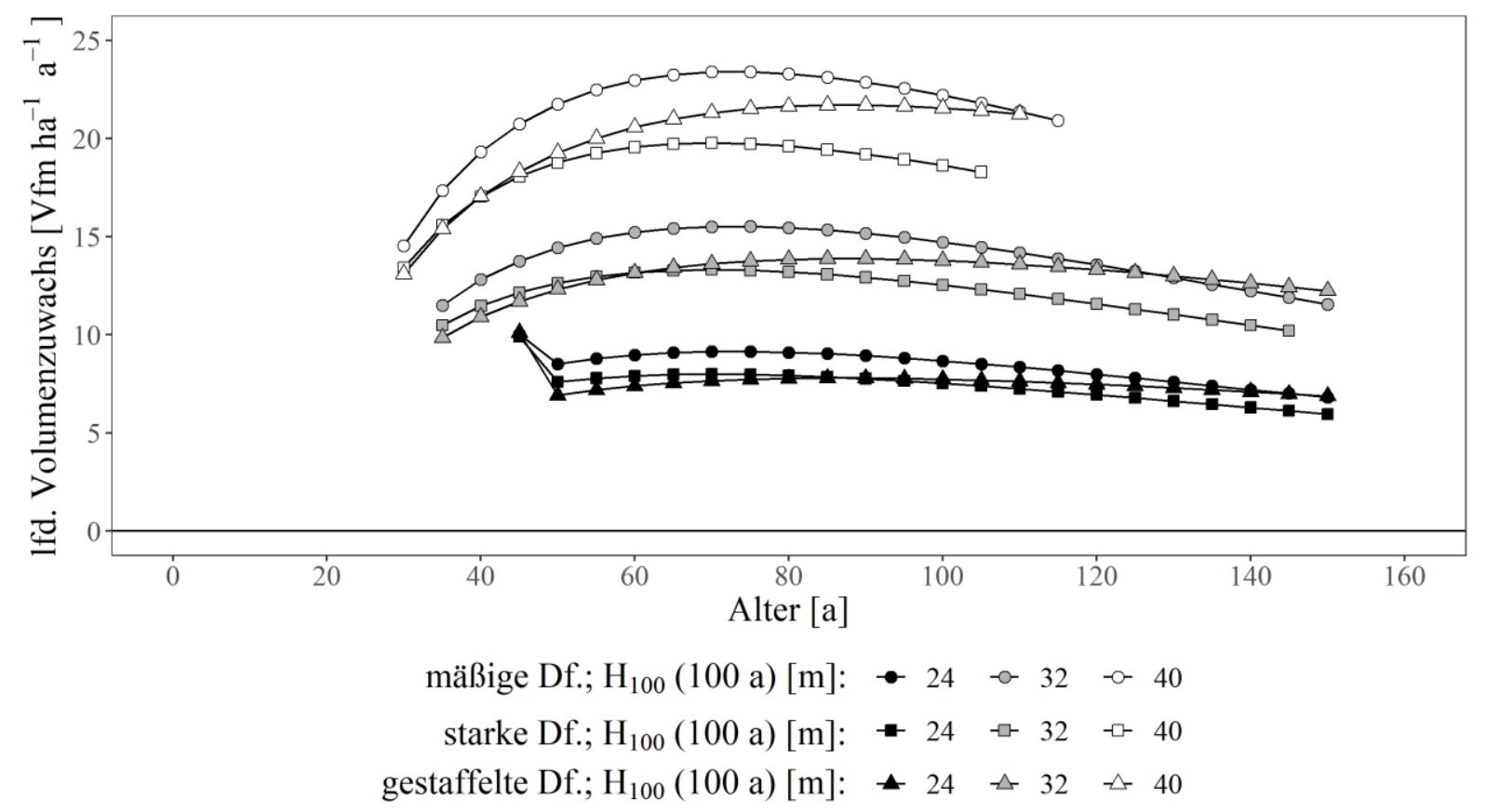

Abbildung 72: Laufender Bestandesvolumenzuwachs über dem Alter; ausgewählte Oberhöhenbonitäten der Baumart Buche getrennt nach Behandlungsvarianten gemäß dyn-ET (unveröff.).

Absolut betragen die laufenden Bestandesvolumenzuwächse im Alter 140 a zwischen 10,5 (starke Hochdurchforstung) und 12,6 $\mathrm{Vfm} \mathrm{ha}^{-1} \mathrm{a}^{-1}$ (gestaffelte Hochdurchforstung; Tabelle 3-4). Während die Kulminationszeitpunkte der starken Hochdurchforstung bonitätsübergreifend in etwa den Kulminationszeitpunkten der mäßigen Hochdurchforstung (70 bzw. 75 a) entsprechen, kulminieren die laufenden Bestandesvolumenzuwächse bei gestaffelter Hochdurchforstung erst in einem Alter von 85 bzw. 90 Jahren. 
Die Unterschiede im Volumenzuwachspotential der Behandlungsvarianten resultieren aus vorgegebenen, an Versuchsflächendaten angelehnten, oberhöhenabhängigen Grundflächenhaltungen (siehe Abschnitt 3.3.2), welche sich auch in den Vorratsentwicklungen widerspiegeln (Abbildung 73). Der für die gestaffelte Hochdurchforstung ab einer Bestandesoberhöhe von $24 \mathrm{~m}$ vorgegebene Grundflächenübergang von der mäßigen zur starken Hochdurchforstung (vgl. Albert et al. 2019) ist deutlich erkennbar. Mit sinkender Standortsgüte wird eine Oberhöhe von 24 m später erreicht und umso länger verläuft auch das Vorratsniveau der gestaffelten auf dem der starken Hochdurchforstung.

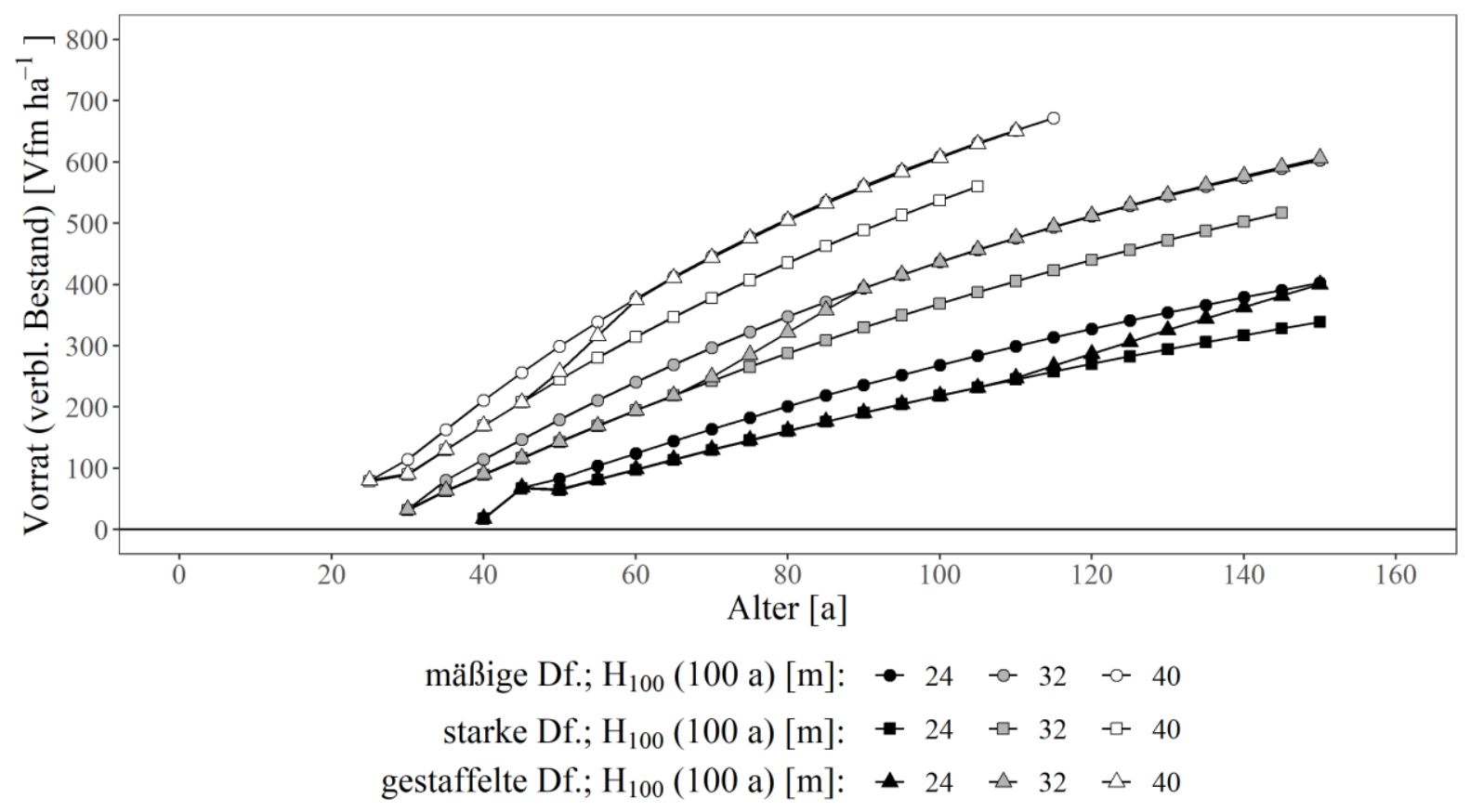

Abbildung 73: Vorrat (verbleibender Bestand) über dem Alter; ausgewählte Oberhöhenbonitäten der Baumart Buche getrennt nach Behandlungsvarianten gemäß dyn-ET (unveröff.).

Die Vorräte liegen bei starker und gestaffelter ggü. der mäßigen Hochdurchforstung insbesondere in jungen Bestandesaltern relativ gesehen niedriger als die laufenden Bestandesvolumenzuwächse, was zu höheren Volumenzuwachsraten führt (Abbildung 74). Die behandlungsabhängigen Unterschiede verschwinden mit steigendem Alter und sind im gleichen Alter umso schwächer, je höher die Standortsgüte ist. 


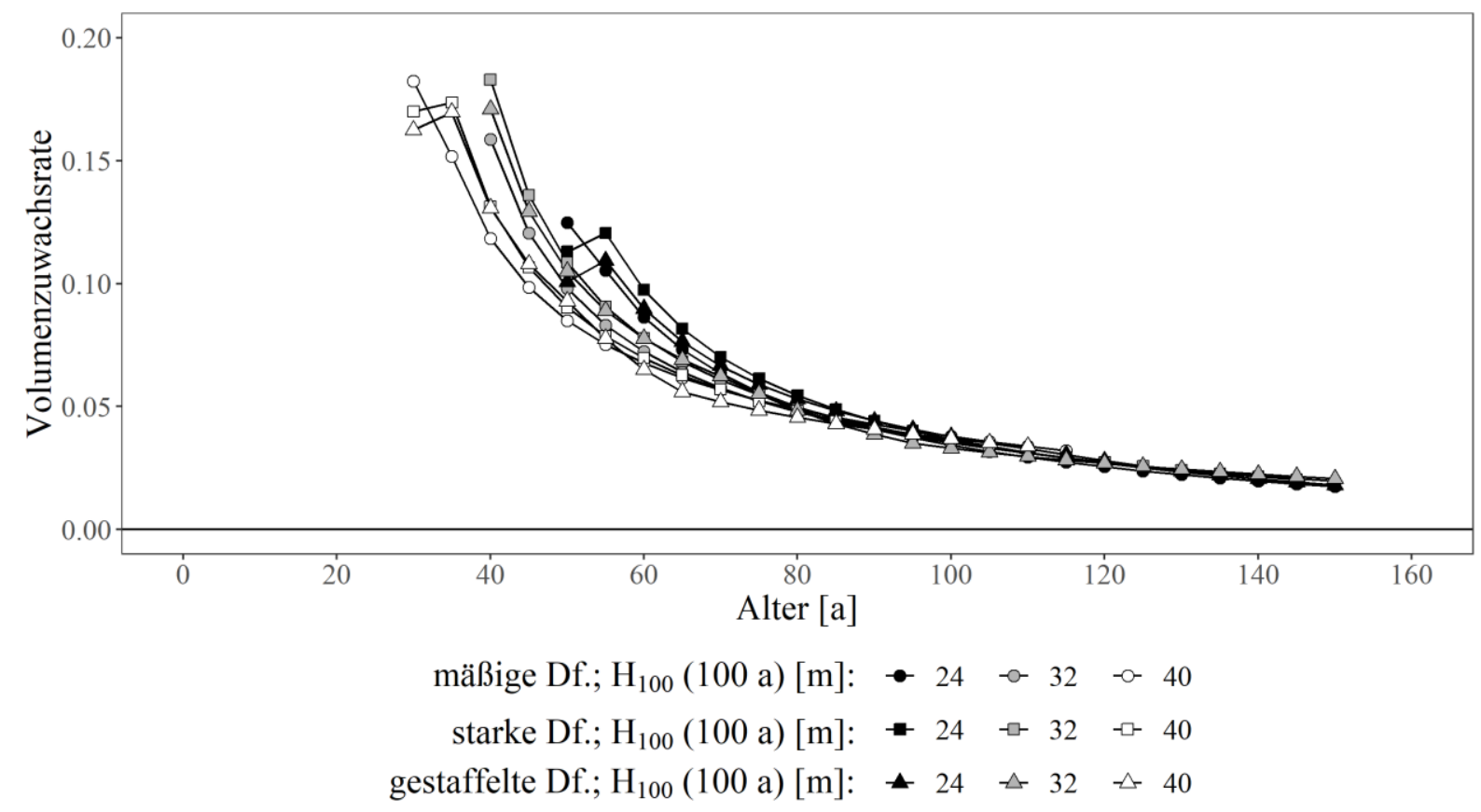

Abbildung 74: Volumenzuwachsraten über dem Alter; ausgewählte Oberhöhenbonitäten der Baumart Buche getrennt nach Behandlungsvarianten gemäß dyn-ET (unveröff.).

Entsprechend der gezeigten Vorräte entwickeln sich die Vornutzungsvolumina, welche das verbleibende Bestandesvolumen u. a. determinieren (Abbildung 75). Erkennbar ist der Übergang in der Grundflächenhaltung bei der gestaffelten Hochdurchforstung durch die kurzzeitig deutlich reduzierten ausscheidenden Bestandesvolumina.

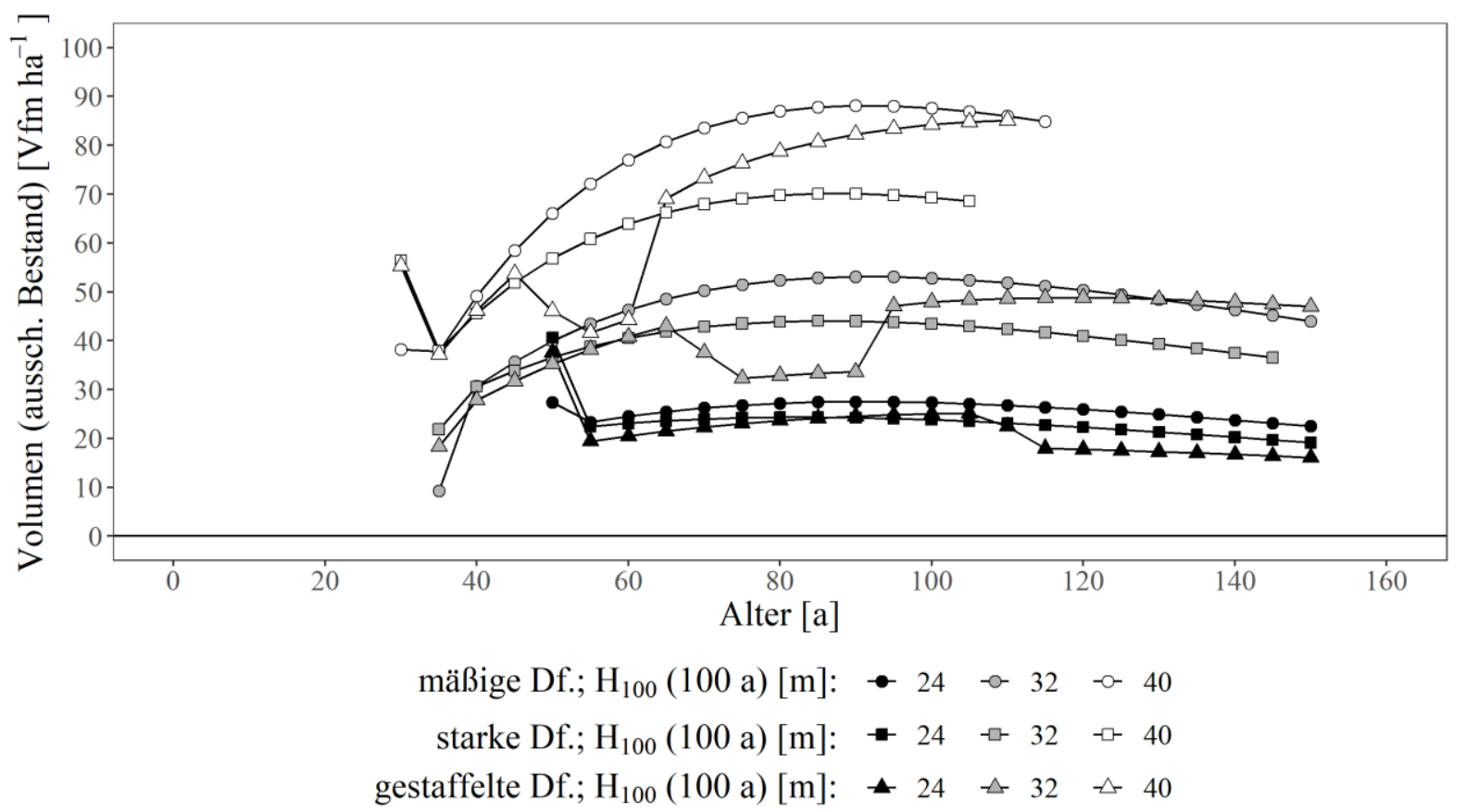

Abbildung 75: Ausscheidendes Bestandesvolumen über dem Alter; ausgewählte Oberhöhenbonitäten der Baumart Buche getrennt nach Behandlungsvarianten gemäß dyn-ET (unveröff.). 
Die mittleren Durchmesser der verbleibenden Bestandesvolumina differenzieren sich bonitätsund altersabhängig sehr unterschiedlich. Im Mittel liegen die mittleren Durchmesser der starken Hochdurchforstung bei mittlerer und hoher Oberhöhenbonität leicht oberhalb der mittleren Durchmesser bei mäßiger Hochdurchforstung -die Differenzierung nimmt mit steigendem Alter zu. Gleichzeitig zeigt sich für die gestaffelte Hochdurchforstung mit steigendem Alter ein Abfall der mittleren Durchmesser unterhalb des Niveaus der mäßigen Hochdurchforstung (Abbildung 76). Bei einer Oberhöhenbonität von $32 \mathrm{~m}$ liegt liegt der Durchmesser des Grundflächenmittelstammes im Alter 140 a bei starker Hochdurchforstung um $3 \%$ höher und bei gestaffelter Hochdurchforstung um $4 \%$ niedriger als bei mäßiger Hochdurchforstung (Tabelle 3-4). Bei niedriger Oberhöhenbonität sind die behandlungsabhängigen Unterschiede minimal.

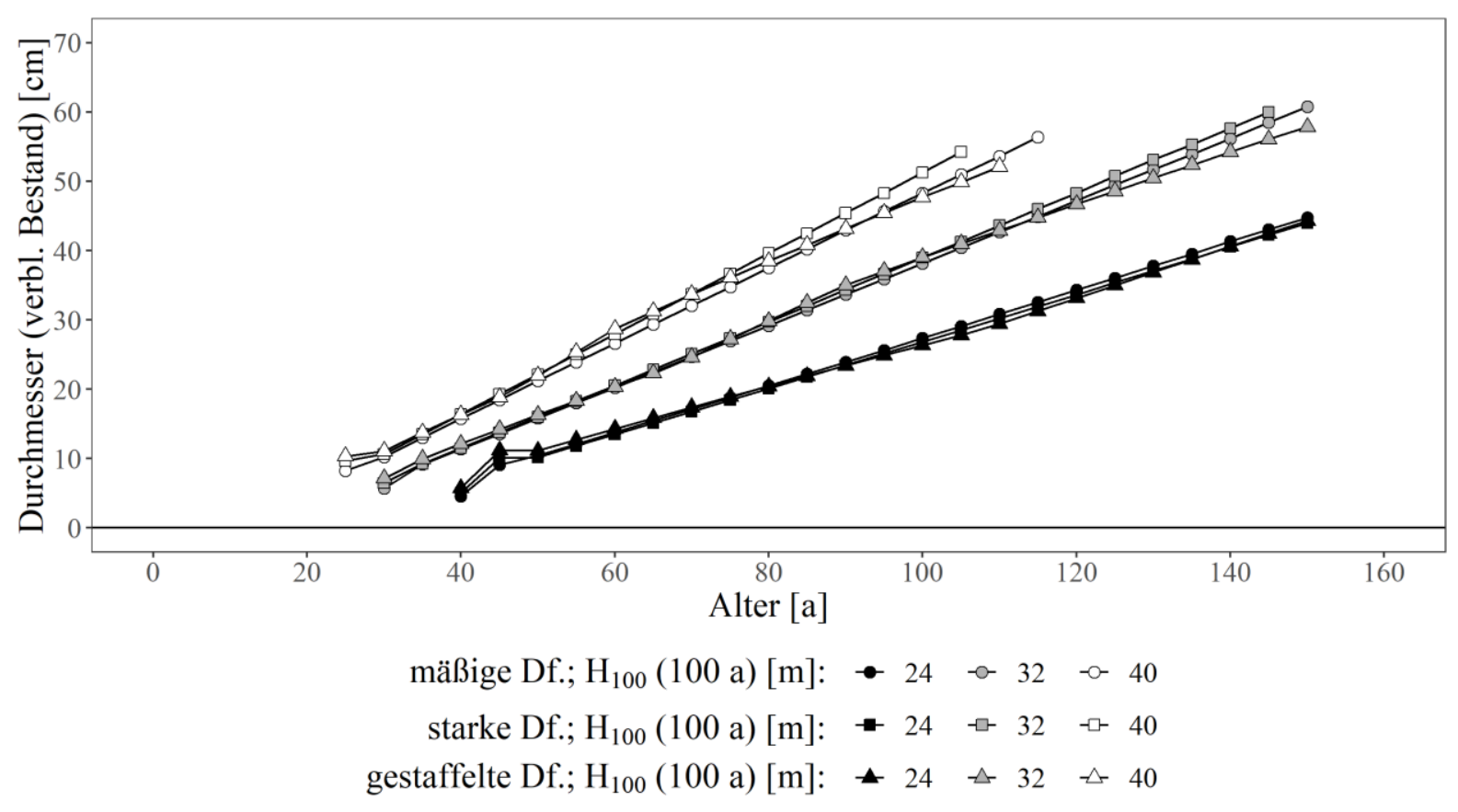

Abbildung 76: Durchmesser des Grundflächenmittelstamms (verbleibender Bestand) über dem Alter; ausgewählte Oberhöhenbonitäten der Baumart Buche getrennt nach Behandlungsvarianten gemäß dynET (unveröff.).

Eine deutlich stärkere behandlungsabhängige Differenzierung ergibt sich bei den mittleren Baumdurchmessern im ausscheidenden Bestand (Abbildung 77). Der Übergang der Grundflächenhaltung bei gestaffelter Durchforstung ist durch kurzzeitig deutlich verminderte mittlere Dimensionen (vgl. niederdurchforstungsartige Eingriffe in Abbildung 78) im ausscheidenden Bestand gekennzeichnet. Anschließend führt die gestaffelte Hochdurchforstung bei mittleren 
und hohen Oberhöhenbonitäten ${ }^{68} \mathrm{zu}$ deutlich erhöhten mittleren Einzelbaumdimensionen im ausscheidenden Bestand - im Alter von 140 a liegt der Durchmesser des Grundflächenmittelstammes bei der Oberhöhenbonität 32 m mit 59,4 cm um 16 \% bzw. $19 \%$ oberhalb des mittleren Durchmessers bei mäßiger bzw. starker Hochdurchforstung.

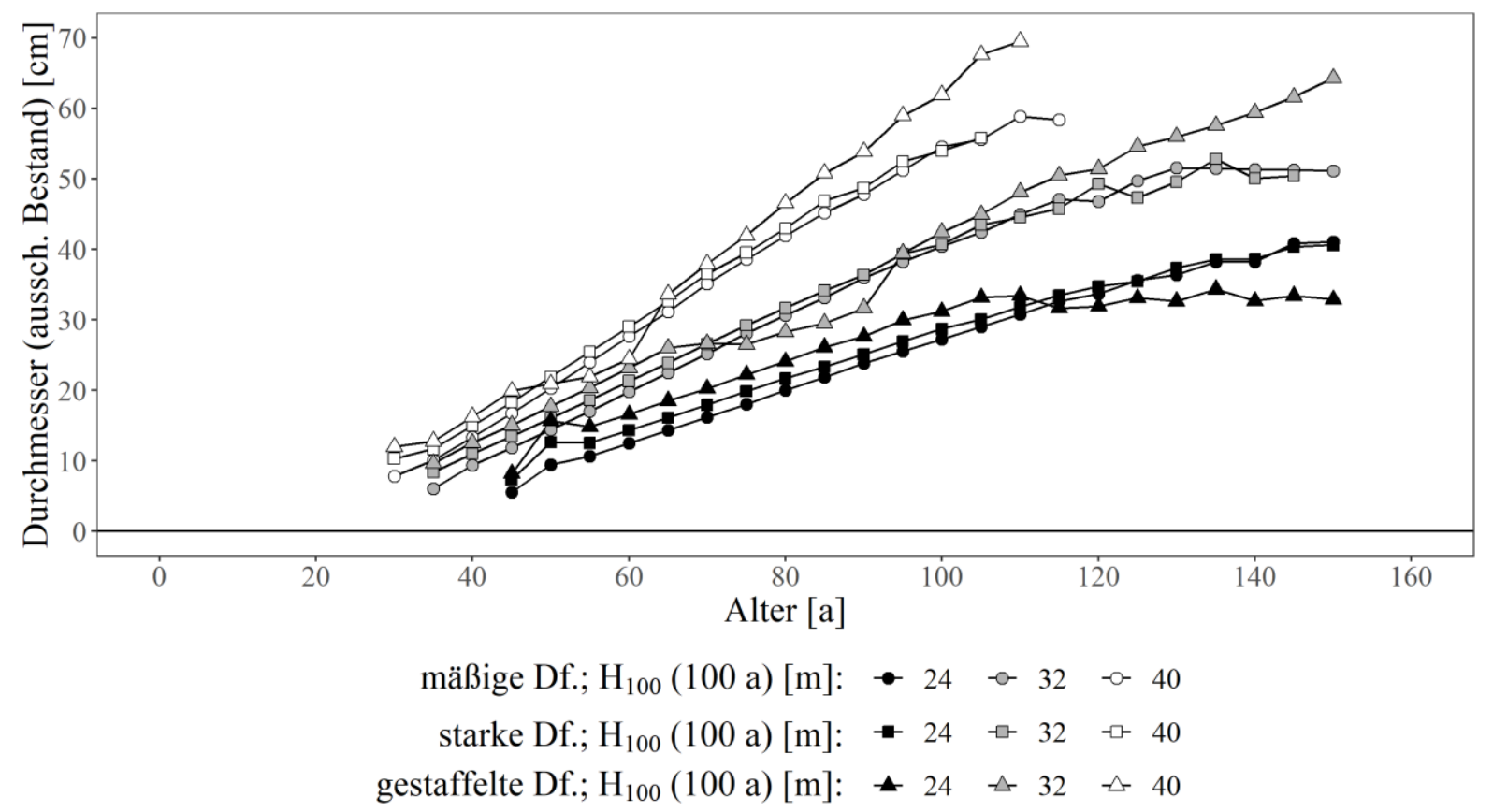

Abbildung 77: Durchmesser des Grundflächenmittelstammes (ausscheidender Bestand) über dem Alter; ausgewählte Oberhöhenbonitäten der Baumart Buche getrennt nach Behandlungsvarianten gemäß dyn-ET (unveröff.).

Die waldbaulichen Unterschiede, die zu den ertragskundlichen Differenzierungen zwischen den Behandlungsvarianten führen, können auch anhand der Stückvolumenrelationen charakterisiert werden (Abbildung 78). Bei der Interpretation der Stückvolumenrelationen zu beachten ist, dass diese aus ertragskundlicher Sicht aufgrund der Kombination aus Z-Baum-orientierter Auslesedurchforstung und einer Hochdurchforstung in den Zwischenfeldern kein uneingeschränkt geeignetes Maß zur Charakterisierung der dyn-ET-Durchforstungsarten darstellen (interne dynET-Projektkommunikation i. V. m. Albert et al. 2019).

Exemplarisch ist anhand der Oberhöhenbonität $32 \mathrm{~m}$ (grau in Abbildung 78) bei mäßiger Hochdurchforstung eine zunächst stärkere Entnahme vom schwachen Ende her erkennbar. Mit steigendem Bestandesalter nähern sich die Eingriffe dann einer schematischen Durchforstung, d. h. gleichen Stückvolumina im ausscheidenden und verbleibenden Bestand, an. Die starke

${ }^{68}$ Die im hohen Bestandesalter deutliche Absenkung des mittleren Durchmessers bei gestaffelter Hochdurchforstung und niedrigster Oberhöhenbonität wird auf modellbedingte Artefakte der vorläufigen Ertragstafeldaten zurückgeführt. 
Hochdurchforstung zeichnet sich durch insgesamt höhere und weniger variierende Stückvolumenrelationen aus. Bei der gestaffelten Hochdurchforstung zeigt sich bei der mittleren und hohen Oberhöhenbonität der Übergang von der Grundflächenhaltung der starken zur mäßigen Hochdurchforstung (siehe oben) anhand eines auffälligen, kurzzeitig niederdurchforstungsartige Eingriffe charakterisierenden, U-Verlaufs im mittleren Bestandesalter. Über alle Behandlungsvarianten hinweg ist mit abnehmender Standortsgüte ein Trend zu niederdurchforstungsartigeren Eingriffen im hohen Bestandesalter zu erkennen.

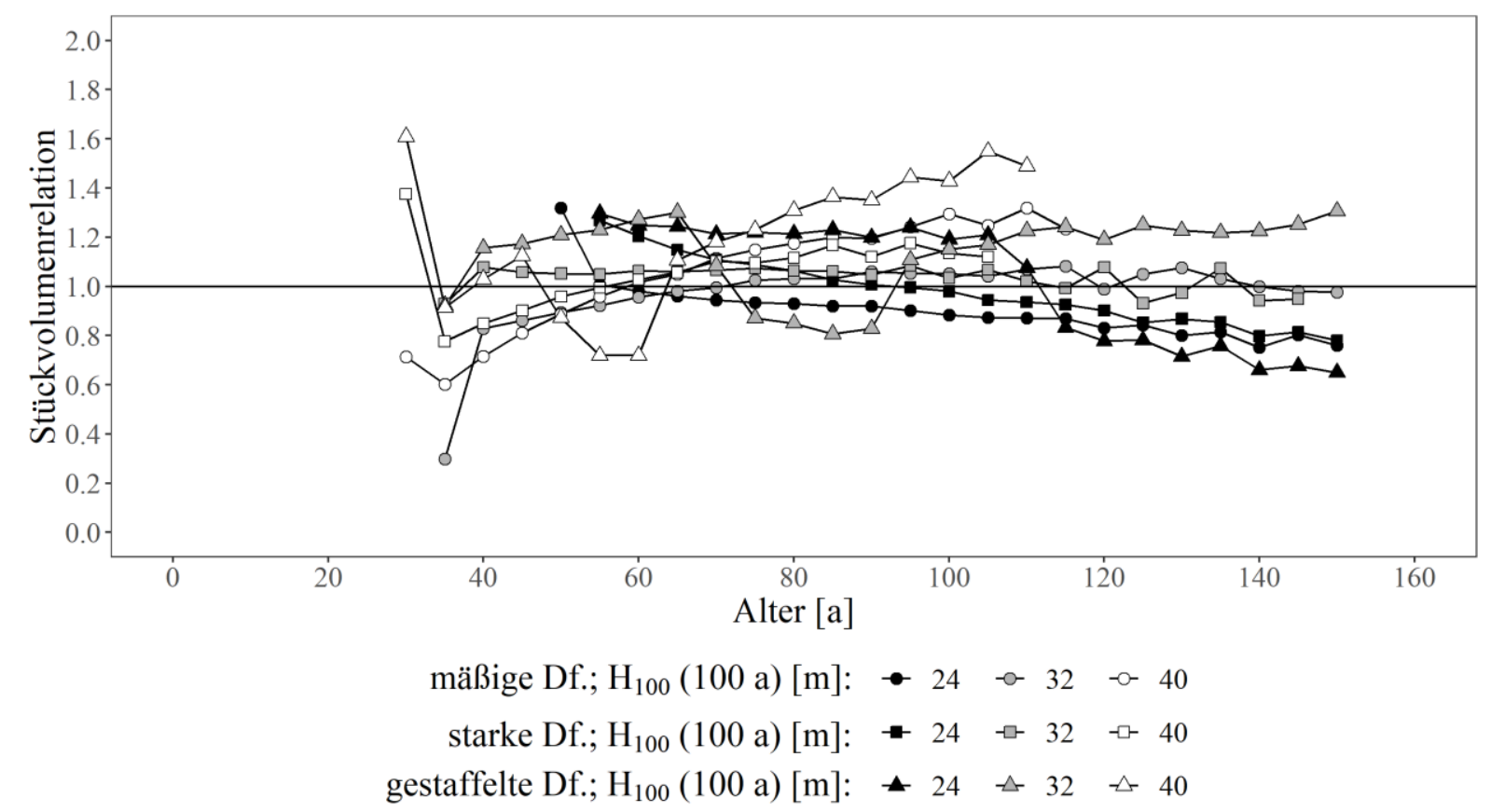

Abbildung 78: Stückvolumenrelationen (ausscheidendes geteilt durch verbleibendes Stückvolumen) über dem Alter; ausgewählte Oberhöhenbonitäten der Baumart Buche getrennt nach Behandlungsvarianten gemäß dyn-ET (unveröff.). 
Der Bonitätsfächer der Baumart Fichte deckt bei mäßiger Hochdurchforstung im Alter 60 a einen Gesamtwuchsleistungsrahmen zwischen 404 und $1.482 \mathrm{Vfm} \mathrm{ha}^{-1}$ ab (Abbildung 79; Tabelle 3-5). Der entsprechenden Gesamtwuchsleistungsfächer der starken Hochdurchforstung gemäß dyn-ET (unveröff.) kann Abbildung 169 im Anhang entnommen werden.

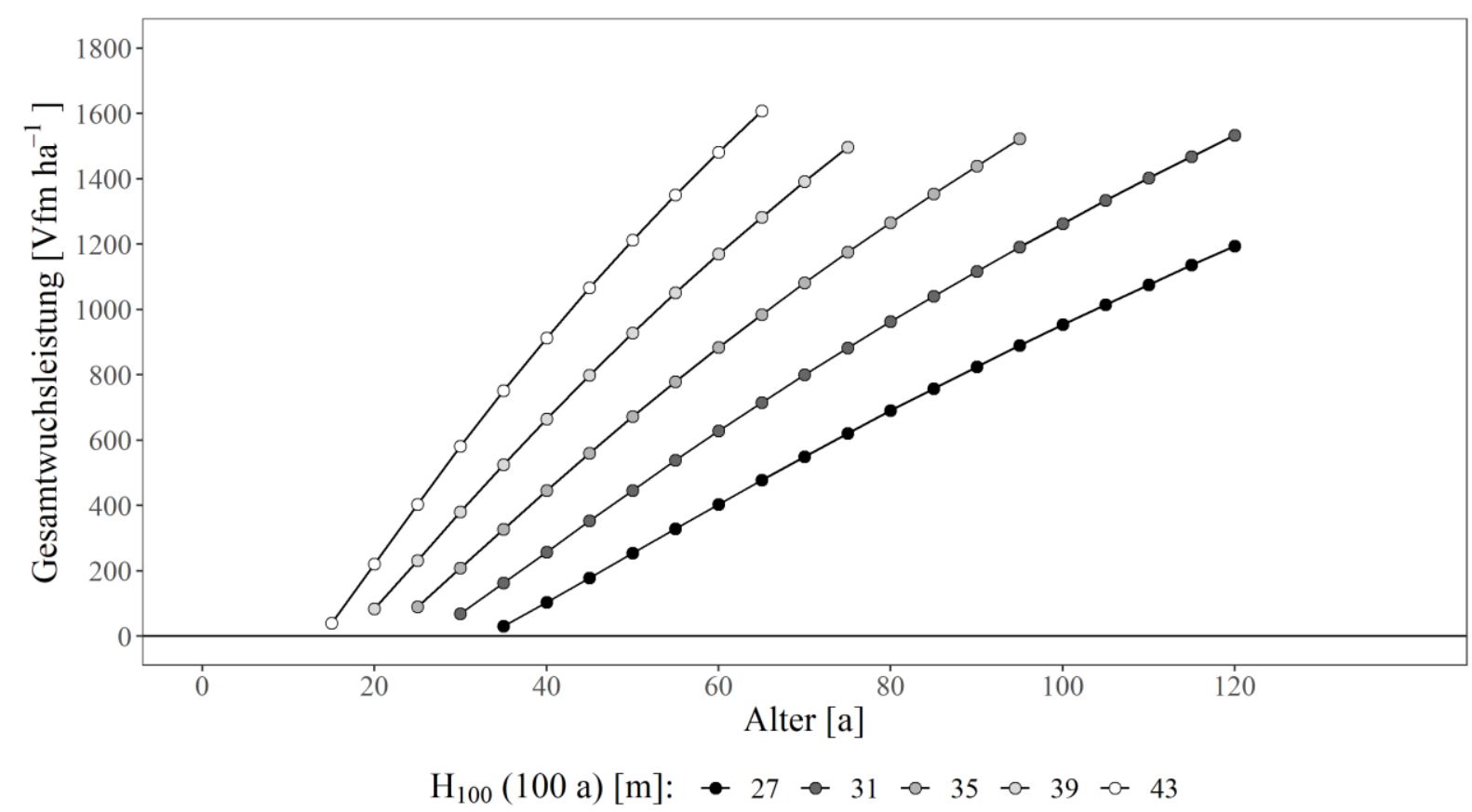

Abbildung 79: Gesamtwuchsleistung über dem Alter; alle Oberhöhenbonitäten der Baumart Fichte (mäßige Hochdurchforstung) gemäß dyn-ET (unveröff.).

Anhand der laufenden Bestandesvolumenzuwächse wird die Bonitätsabhängigkeit der Wuchsverläufe nochmal deutlicher. Mit steigender Standortsgüte nimmt, wie auch schon bei der Baumart Buche, nicht nur das absolute Niveau der Bestandesvolumenzuwächse, sondern auch die Veränderung über dem Alter zu (Abbildung 80; analog dazu für die starke Hochdurchforstung Abbildung 170 im Anhang) - während die laufenden Bestandesvolumenzuwächse bei einer Oberhöhenbonität von $27 \mathrm{~m}$ altersabhängig nur um 3,4 $\mathrm{Vfm} \mathrm{ha}^{-1} \mathrm{a}^{-1}$ variieren (Minimum: 11,7 Vfm ha ${ }^{-1} \mathrm{a}^{-1}$, Maximum: 15,1 Vfm ha $\mathrm{f}^{-1} \mathrm{a}^{-1}$ ), schwanken sie aufgrund eines starken Anstiegs im ersten Zeitschritt bei einer Oberhöhenbonität von $39 \mathrm{~m}$ zwischen 16,9 Vfm ha ${ }^{-1} \mathrm{a}^{-1}$ im Alter 20 a (Minimum) und 29,7 $\mathrm{Vfm} \mathrm{ha}^{-1} \mathrm{a}^{-1}$ im Alter 30 a (Maximum). Die Kulminationszeitpunkte verschieben sich bei der Baumart Fichte, anders als bei der Buche, mit sinkender Oberhöhenbonität deutlich zu höheren Bestandesaltern. Während der laufende Bestandesvolumenzuwachs bei einer Oberhöhenbonität von $43 \mathrm{~m}$ im Alter 25 a mit $36,7 \mathrm{Vfm} \mathrm{ha} \mathrm{a}^{-1} \mathrm{a}^{-1}$ kulminiert, erreicht er mit 15,1 Vfm ha ${ }^{-1} \mathrm{a}^{-1}$ bei einer Oberhöhenbonität von $27 \mathrm{~m}$ sein Maximum erst im Alter von 50 Jahren (Tabelle 3-5). 
Tabelle 3-5: Vergleich ausgewählter Ertragstafeldaten der Baumart Fichte (mäßige Hochdurchforstung) gemäß dyn-ET (unveröff.); alle Oberhöhenbonitäten; in Klammern ist jeweils die relative Differenz zur Oberhöhenbonität $H_{100}(100 \mathrm{a})=35 \mathrm{~m}$ angegeben;

$\boldsymbol{G W L}(60 \mathrm{a})$ : Gesamtwuchsleistung bis zum Alter $60 \mathrm{a}, \max \boldsymbol{v}_{\boldsymbol{B}}$ : lfd. Bestandesvolumenzuwachs zum Zeitpunkt der Kulmination [...], $\boldsymbol{V}_{\boldsymbol{B}}\left(\mathbf{6 0}\right.$ a): Vorrat (verbl. Bestand) im Alter 60 a, $\boldsymbol{G}_{\boldsymbol{v e r b l}}(\mathbf{6 0}$ a): Bestandesgrundfläche des verbleibenden Bestandes im Alter $60 \mathrm{a}, \sum_{\mathbf{0}}^{\mathbf{6 0}} \mathbf{a} \lambda$ : kumuliertes ausscheidendes Bestandesvolumen bis zum Alter 60 a, $\boldsymbol{N}_{\text {verbl. }}(\mathbf{6 0}$ a): Stammzahl des verbleibenden Bestandes im Alter 60 a, $\sum_{\mathbf{0}}^{\mathbf{6 0} \text { a }} \boldsymbol{N}_{\text {aussch. }}$ : kumulierte ausscheidende Stammzahl bis zum Alter 60 a, $\boldsymbol{D}_{\boldsymbol{g}, \boldsymbol{v e r b l}}(\mathbf{6 0}$ a): Durchmesser des Grundflächenmittelstammes des verbleibenden Bestandes im Alter $60 \mathrm{a}$, $\boldsymbol{D}_{\boldsymbol{g}, \boldsymbol{a u s s c h}}(\mathbf{6 0}$ a): Durchmesser des Grundflächenmittelstammes des ausscheidenden Bestandes im Alter $60 \mathrm{a}, \max \overline{\boldsymbol{v}_{\boldsymbol{B}}}$ : durchschn. Bestandesvolumenzuwachs zum Zeitpunkt der Kulmination [...], $\boldsymbol{V}_{\boldsymbol{\eta}}(60 \mathrm{a})$ : Normalvorrat bei einer Umtriebszeit von $60 \mathrm{a}$.

\begin{tabular}{|c|c|c|c|c|c|}
\hline \multirow{2}{*}{$\begin{array}{r}\text { Bestandesbehandlung } \\
H_{100}(100 \text { a })[\mathrm{m}]\end{array}$} & \multicolumn{5}{|c|}{ mäßige Hochdurchforstung } \\
\hline & 27 & 31 & 35 & 39 & 43 \\
\hline$G W L(60$ a $)$ & 404 & 628 & 884 & 1.169 & 1.482 \\
\hline$\left[\mathrm{Vfm} \mathrm{ha}^{-1}\right]$ & $(-54,3 \%)$ & $(-29,0 \%)$ & & $(+32,2 \%)$ & $(+67,6 \%)$ \\
\hline $\max v_{B}$ & $15,1[50 \mathrm{a}]$ & $19,0[40 \mathrm{a}]$ & $23,9[35 a]$ & $29,7[30 \mathrm{a}]$ & $36,7[25 \mathrm{a}]$ \\
\hline$\left[\mathrm{Vfm} \mathrm{ha}^{-1} \mathrm{a}^{-1}\right]$ & $(-36,8 \%)$ & $(-20,5 \%)$ & & $(+24,3 \%)$ & $(+53,6 \%)$ \\
\hline$V_{B}(60 \mathrm{a})$ & 274 & 381 & 490 & 595 & 693 \\
\hline$\left[\mathrm{Vfm} \mathrm{ha}^{-1}\right]$ & $(-44,1 \%)$ & $(-22,2 \%)$ & & $(+21,4 \%)$ & $(+41,4 \%)$ \\
\hline$G_{\text {verbl. }}(60 \mathrm{a})$ & 34,7 & 40,6 & 44,9 & 47,9 & 49,9 \\
\hline$\left[\mathbf{m}^{2}\right]$ & $(-22,7 \%)$ & $(-9,6 \%)$ & & $(+6,7 \%)$ & $(+11,1 \%)$ \\
\hline$\sum_{0}^{60} \mathrm{a} \lambda$ & 130 & 247 & 394 & 574 & 789 \\
\hline$\left[\mathrm{Vfm} \mathrm{ha}^{-1}\right]$ & $(-67,0 \%)$ & $(-37,3 \%)$ & & $(+45,7 \%)$ & $(+100,3 \%)$ \\
\hline$N_{\text {verbl. }}(60 \mathrm{a})$ & 695 & 658 & 627 & 600 & 577 \\
\hline$\left[\mathrm{ha}^{-1}\right]$ & $(+10,8 \%)$ & $(+4,9 \%)$ & & $(-4,3 \%)$ & $(-8,0 \%)$ \\
\hline$\sum_{0}^{60 \mathrm{a}} N_{a u s s c h}$ & 1.805 & 1.842 & 1.873 & 1.900 & 1.923 \\
\hline$\left[\mathrm{ha}^{-1}\right]$ & $(-3,6 \%)$ & $(-1,7 \%)$ & & $(+1,4 \%)$ & $(+2,7 \%)$ \\
\hline$D_{g, v e r b l .}(60$ a $)$ & 25,2 & 28,0 & 30,2 & 31,9 & 33,2 \\
\hline$[\mathbf{c m}]$ & $(-16,6 \%)$ & $(-7,3 \%)$ & & $(+5,6 \%)$ & $(+9,9 \%)$ \\
\hline$D_{g, a u s s c h .}(60$ a) & 28,7 & 30,2 & 32,3 & 34,5 & 36,7 \\
\hline$[\mathrm{cm}]$ & $(-11,1 \%)$ & $(-6,5 \%)$ & & $(+6,8 \%)$ & $(+13,6 \%)$ \\
\hline $\max \overline{v_{B}}$ & $9,9[120 \mathrm{a}]$ & $12,8[120 \mathrm{a}]$ & 16,0 [95 a] & $20,0[75 \mathrm{a}]$ & 24,7 [65 a] \\
\hline$\left[\mathrm{Vfm} \mathrm{ha}^{-1} \mathbf{a}^{-1}\right]$ & $(-38,1 \%)$ & $(-20,0 \%)$ & & $(+25,0 \%)$ & $(+54,4 \%)$ \\
\hline$V_{\eta}(60 \mathrm{a})$ & 65 & 115 & 195 & 274 & 361 \\
\hline$\left[\mathrm{Vfm} \mathrm{ha}^{-1}\right]$ & $(-66,7 \%)$ & $(-41,0 \%)$ & & $(+40,5 \%)$ & $(+85,1 \%)$ \\
\hline
\end{tabular}




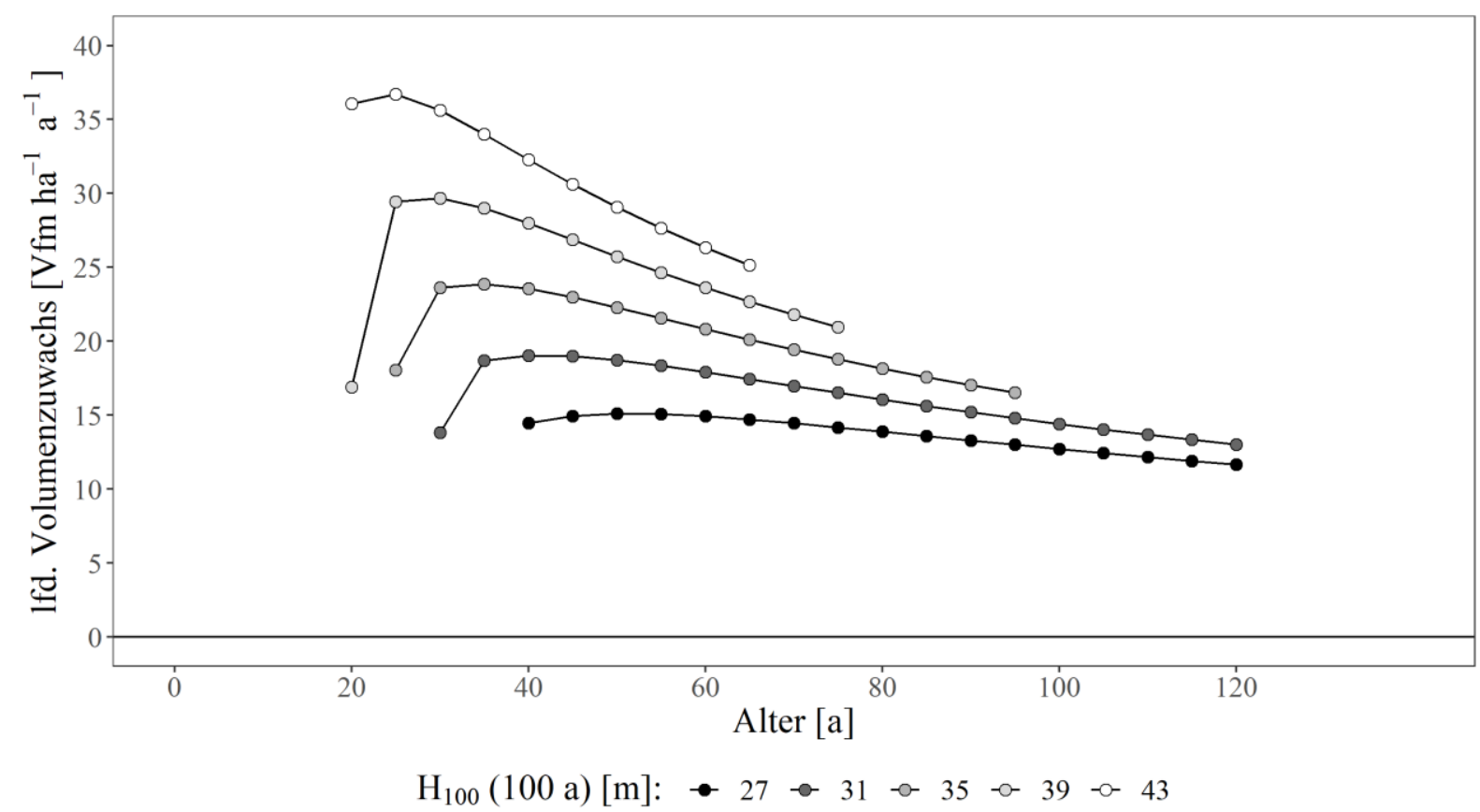

Abbildung 80: Laufender Bestandesvolumenzuwachs über dem Alter; alle Oberhöhenbonitäten der Baumart Fichte (mäßige Hochdurchforstung) gemäß dyn-ET (unveröff.).

Wie auch schon bei der Baumart Buche kommt es gegenüber dem etablierten Ertragstafelwerk von Wiedemann (1936/42) auf Grundlage von Versuchsflächendaten der NW-FVA zu einer Ausweitung und Verschiebung des Bonitätsrahmens - statt den Ertragsklassen V bis I mit entsprechenden Oberhöhenbonitäten von 19,7 bis 35,1 m nach Wiedemann (1936/42) wird gemäß dyn-ET (unveröff.) bei der mäßigen Hochdurchforstung in 5 Stufen nun ein Oberhöhenbonitätsbereich von 27 bis $43 \mathrm{~m}$ abgedeckt.

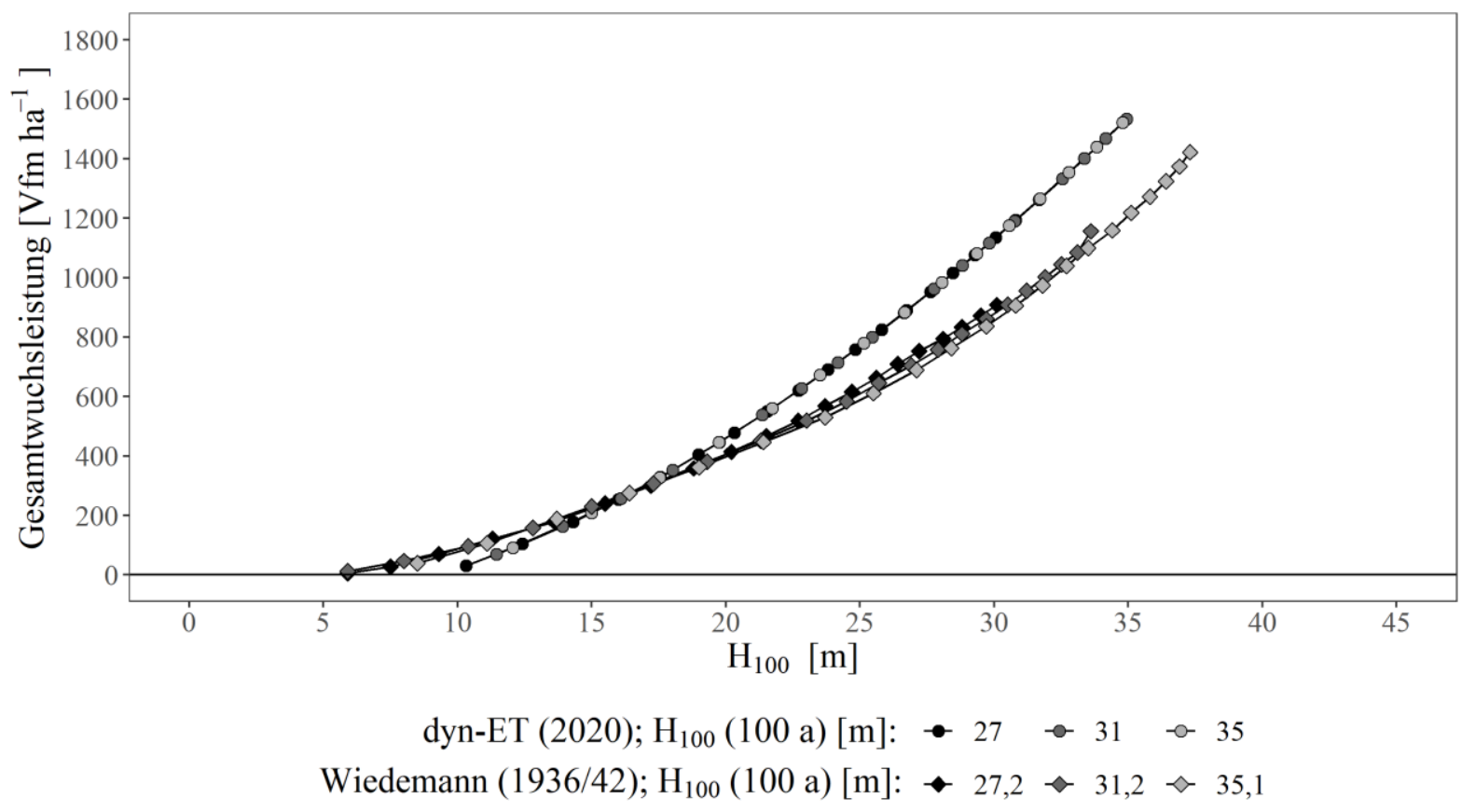

Abbildung 81: Gesamtwuchsleistung über der Oberhöhe; ausgewählte Oberhöhenbonitäten der Baumart Fichte (mäßige Durchforstung) gemäß dyn-ET (unveröff.) und Wiedemann (1936/42). 
Zudem kommt es auch bei der Fichte zu einer Anhebung des Ertragsniveaus - bei gleicher Oberhöhe liegen die Gesamtwuchsleistungen gemäß dyn-ET (unveröff., mäßige Hochdurchforstung) ab einer Oberhöhe von zirka $18 \mathrm{~m}$ oberhalb vergleichbarer Oberhöhenbonitäten nach Wiedemann (1936/42, mäßige Durchforstung $)^{69}$. Ein ähnliches Bild zeigt sich in abgeschwächter Form auch bei starker Durchforstung (Abbildung 171 im Anhang). Anders als bei der Baumart Buche (siehe Abbildung 59) existieren bei der Fichte auch gemäß dem etablierten Ertragstafelwerk von Wiedemann (1936/42) keine bzw. nur minimale Ertragsniveauunterschiede (siehe auch Abschnitt 3.1.2).

Gemäß dyn-ET (unveröff.) hält die Fichte das gesteigerte Zuwachsniveau auch in hohen Altern aufrecht. Bei annähernd gleichem Oberhöhenverlauf (Abbildung 82) liegen die laufenden Bestandesvolumenzuwächse der Oberhöhenbonität 27 m nach dyn-ET (unveröff., mäßige Hochdurchforstung) ab einem Alter von 75 a oberhalb der Volumenzuwachsentwicklung der Oberhöhenbonität 35,1 m gemäß Wiedemann (1936/42, mäßige Durchforstung). Zu den Zeitpunkten der jeweiligen Kulmination liegen die laufenden Volumenzuwächse nach dyn-ET (unveröff., mäßige Hochdurchforstung) um 24 bis 37 \% (Tabelle 3-6) oberhalb vergleichbarer Oberhöhenbonitäten gemäß Wiedemann (1936/42, mäßige Durchforstung).

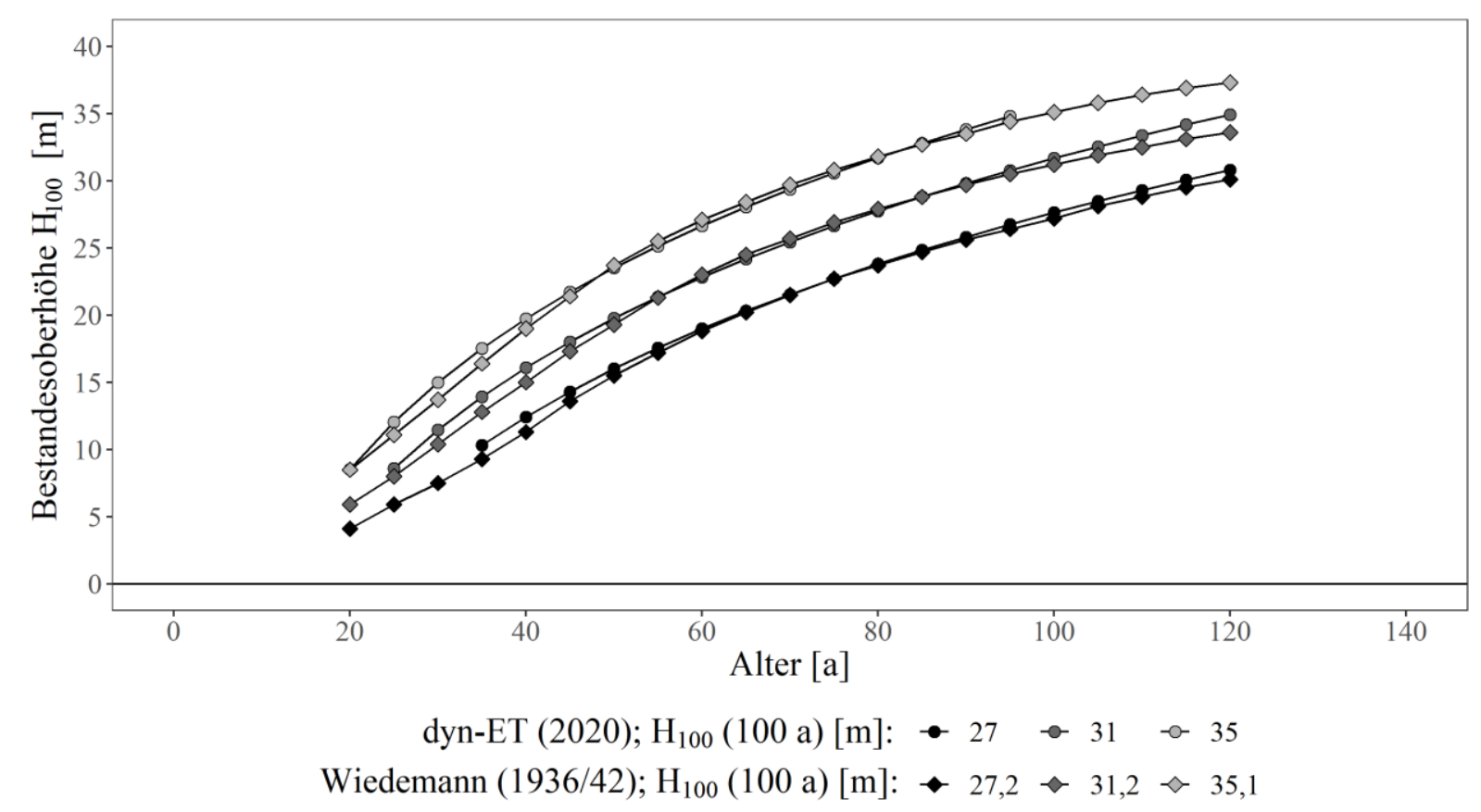

Abbildung 82: Oberhöhe über dem Alter; ausgewählte Oberhöhenbonitäten der Baumart Fichte (mäßige Durchforstung) gemäß dyn-ET (unveröff.) und Wiedemann (1936/42).

\footnotetext{
${ }^{69}$ Wiedemann (1936/42) weist in seinem Ertragstafelwerk Daten für niedrigere Alter als dyn-ET (unveröff.) mit entsprechend geringeren Oberhöhen aus.
} 


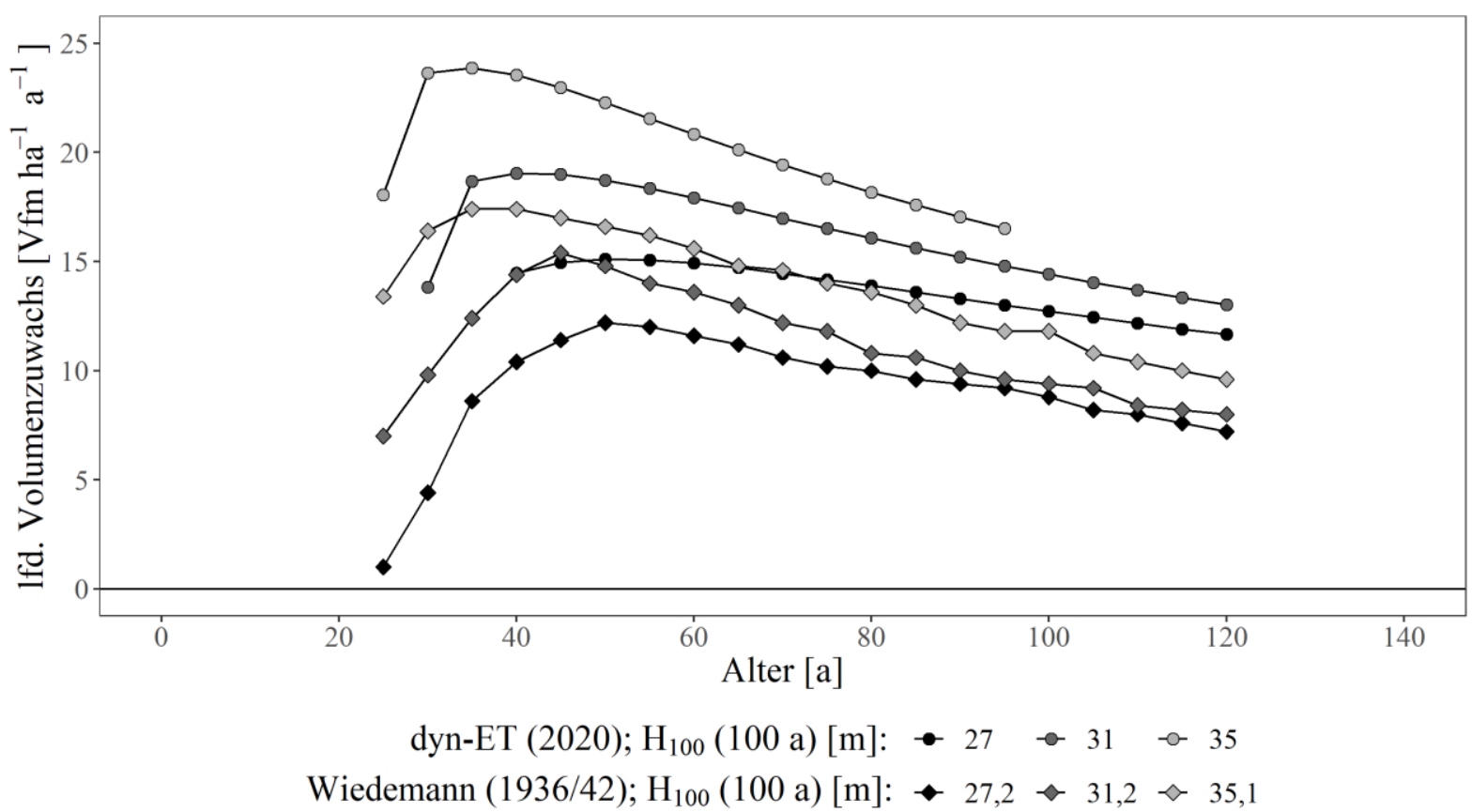

Abbildung 83: Laufender Bestandesvolumenzuwachs über dem Alter; ausgewählte Oberhöhenbonitäten der Baumart Fichte (mäßige Durchforstung) gemäß dyn-ET (unveröff.) und Wiedemann (1936/42).

Gleichzeitig sind die Differenzen bei den Vorräten, d. h. den Volumina des verbleibenden Bestandes, deutlich geringer (Abbildung 62) - im Alter 80 a liegen sie bei der mäßigen Durchforstung gemäß dyn-ET (unveröff.) um $2 \%$ bis 8 \% (Tabelle 3-6) unterhalb der Vorräte gemäß Wiedemann (1936/42).

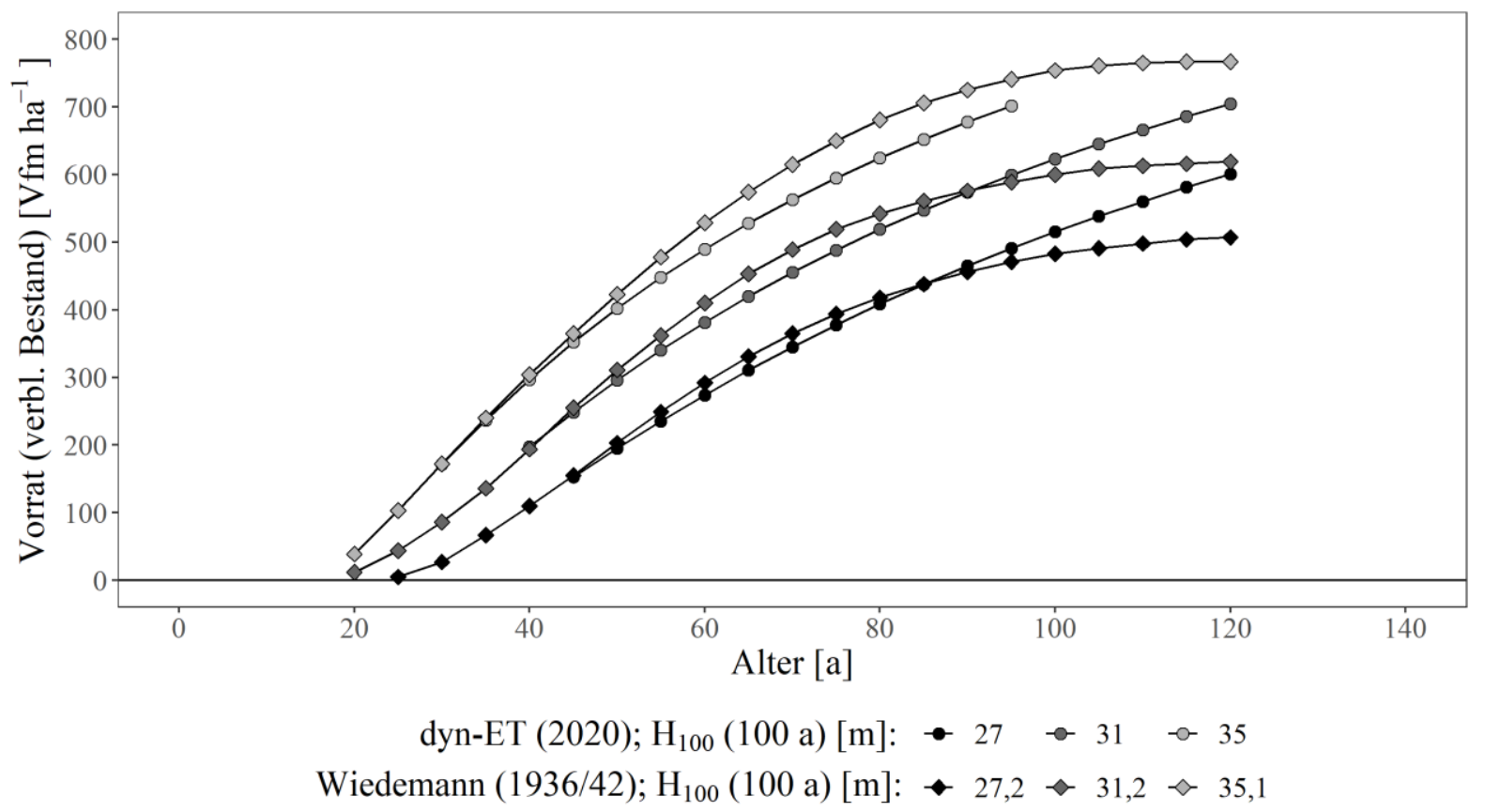

Abbildung 84: Vorrat (verbleibender Bestand) über dem Alter; ausgewählte Oberhöhenbonitäten der Baumart Fichte (mäßige Durchforstung) gemäß dyn-ET (unveröff.) und Wiedemann (1936/42). 
Tabelle 3-6: Vergleich ausgewählter Ertragstafeldaten der Baumart Fichte (mäßige Durchforstung); ausgewählte Oberhöhenbonitäten gemäß dyn-ET (unveröff.) und Wiedemann (1936/42); in Klammern ist jeweils die relative Differenz zur vergleichbaren Oberhöhenbonität nach Wiedemann (1936/42) angegeben;

$\boldsymbol{G W L}(\mathbf{8 0} \mathrm{a})$ : Gesamtwuchsleistung bis zum Alter $80 \mathrm{a}, \boldsymbol{m a x} \boldsymbol{v}_{\boldsymbol{B}}$ : lfd. Bestandesvolumenzuwachs zum Zeitpunkt der Kulmination [...], $\boldsymbol{V}_{\boldsymbol{B}}\left(\mathbf{8 0}\right.$ a): Vorrat (verbl. Bestand) im Alter 80 a, $\boldsymbol{G}_{\boldsymbol{v} \text { erbl. }}(\mathbf{8 0}$ a): Bestandesgrundfläche des verbleibenden Bestandes im Alter $80 \mathrm{a}, \sum_{\mathbf{0}}^{\mathbf{8 0}} \mathbf{a} \boldsymbol{\lambda}$ : kumuliertes ausscheidendes Bestandesvolumen bis zum Alter 80 a, $\boldsymbol{N}_{\text {verbl. }}(\mathbf{8 0}$ a): Stammzahl des verbleibenden Bestandes im Alter 80 a, $\sum_{\mathbf{0}}^{\mathbf{8 0} \text { a }} \boldsymbol{N}_{\text {aussch. }}$ : kumulierte ausscheidende Stammzahl bis zum Alter 80 a, $\boldsymbol{D}_{\boldsymbol{g}, \boldsymbol{v e r b l}}(\mathbf{8 0}$ a): Durchmesser des Grundflächenmittelstammes des verbleibenden Bestandes im Alter $80 \mathrm{a}$, $\boldsymbol{D}_{\text {g,aussch. }}$ (80 a): Durchmesser des Grundflächenmittelstammes des ausscheidenden Bestandes im Alter $80 \mathrm{a}, \max \overline{\boldsymbol{v}_{\boldsymbol{B}}}$ : durchschn. Bestandesvolumenzuwachs zum Zeitpunkt der Kulmination [...], $\boldsymbol{V}_{\boldsymbol{\eta}}(\mathbf{8 0} \mathrm{a})$ : Normalvorrat bei einer Umtriebszeit von $80 \mathrm{a}$.

\begin{tabular}{|c|c|c|c|c|c|c|}
\hline \multirow{2}{*}{$\begin{array}{r}\text { Ertragstafel } \\
H_{100}(100 \mathrm{a})[\mathrm{m}]\end{array}$} & \multicolumn{3}{|c|}{ Wiedemann (1936/42), mäßige Df. } & \multicolumn{3}{|c|}{ dyn-ET (unveröff.), mäßige Df. } \\
\hline & 27,2 & 31,2 & 35,1 & 27 & 31 & 35 \\
\hline$G W L(80$ a $)$ & 568 & 758 & 974 & 690 & 963 & 1.266 \\
\hline$\left[\mathrm{Vfm} \mathrm{ha}^{-1}\right]$ & & & & $(+21,5 \%)$ & $(+27,0 \%)$ & $(+30,0 \%)$ \\
\hline $\max v_{B}$ & $12,2[50 \mathrm{a}]$ & $15,4[45 \mathrm{a}]$ & $17,4[40 \mathrm{a}]$ & $15,1[50 \mathrm{a}]$ & $19,0[40 \mathrm{a}]$ & $23,9[35 \mathrm{a}]$ \\
\hline$\left[\mathrm{Vfm} \mathrm{ha}^{-1} \mathrm{a}^{-1}\right]$ & & & & $(+23,8 \%)$ & $(+23,4 \%)$ & $(+37,4 \%)$ \\
\hline$V_{B}(80 \mathrm{a})$ & 418 & 542 & 681 & 408 & 519 & 625 \\
\hline$\left[\mathrm{Vfm} \mathrm{ha}^{-1}\right]$ & & & & $(-2,4 \%)$ & $(-4,2 \%)$ & $(-8,2 \%)$ \\
\hline$G_{v e r b l .}(80 \mathrm{a})$ & 38,5 & 42,8 & 46,7 & 41,8 & 45,9 & 48,7 \\
\hline$\left[\mathbf{m}^{2}\right]$ & & & & $(+8,6 \%)$ & $(+7,2 \%)$ & $(+4,3 \%)$ \\
\hline$\sum_{0}^{80 a} \lambda$ & 150 & 216 & 293 & 282 & 444 & 641 \\
\hline$\left[\mathrm{Vfm} \mathrm{ha}^{-1}\right]$ & & & & $(+88,0 \%)$ & $(+105,6 \%$ & $(+118,8 \%)$ \\
\hline$N_{\text {verbl. }}(80$ a) & 1.148 & 790 & 631 & 486 & 460 & 438 \\
\hline$\left[\mathbf{h a}^{-1}\right]$ & & & & $(-57,7 \%)$ & $(-41,8 \%)$ & $(-30,6 \%)$ \\
\hline$\sum_{0}^{80 \mathrm{a}} N_{\text {aussch }}$ & 4.769 & 5.127 & 5.286 & 2.014 & 2.040 & 2.062 \\
\hline$\left[\mathrm{ha}^{-1}\right]$ & & & & $(-57,8 \%)$ & $(-60,2 \%)$ & $(-61,0 \%)$ \\
\hline$D_{g, v e r b l .}(80$ a $)$ & 20,7 & 26,3 & 30,7 & 33,1 & 35,6 & 37,6 \\
\hline$[\mathrm{cm}]$ & & & & $(+59,9 \%)$ & $(+35,4 \%)$ & $(+22,5 \%)$ \\
\hline$D_{g, a u s s c h .}(80$ a $)$ & 16,1 & 19,3 & 22,8 & 36,4 & 38,1 & 40,4 \\
\hline$[\mathbf{c m}]$ & & & & $(+126,1 \%)$ & $(+97,4 \%)$ & $(+77,2 \%)$ \\
\hline $\max \overline{v_{B}}$ & $7,6[115 \mathrm{a}]$ & $9,6[120 \mathrm{a}]$ & $12,2[90 \mathrm{a}]$ & $9,9[120 \mathrm{a}]$ & $12,8[120 \mathrm{a}]$ & $16,0[95 \mathrm{a}]$ \\
\hline$\left[\mathrm{Vfm} \mathrm{ha}^{-1} \mathrm{a}^{-1}\right]$ & & & & $(+30,3 \%)$ & $(+33,3 \%)$ & $(+31,1 \%)$ \\
\hline$V_{\eta}(80 \mathrm{a})$ & 155 & 228 & 311 & 140 & 206 & 294 \\
\hline$\left[\mathrm{Vfm} \mathrm{ha}^{-1}\right]$ & & & & $(-9,7 \%)$ & $(-9,6 \%)$ & $(-5,5 \%)$ \\
\hline
\end{tabular}


Bei annähernd gleichen bzw. leicht gesunkenen Vorräten führen die deutlich gestiegenen Bestandesvolumenzuwächse zu höheren Volumenzuwachsraten, d. h. einem höheren relativen Volumenzuwachs in Bezug auf das jeweils vorhandene Bestandesvolumen (Abbildung 85). Abbildungen 172 bis 175 im Anhang zeigen ähnliche Unterschiede zwischen dyn-ET (unveröff., starke Hochdurchforstung) und Wiedemann (1936/42, starke Durchforstung).

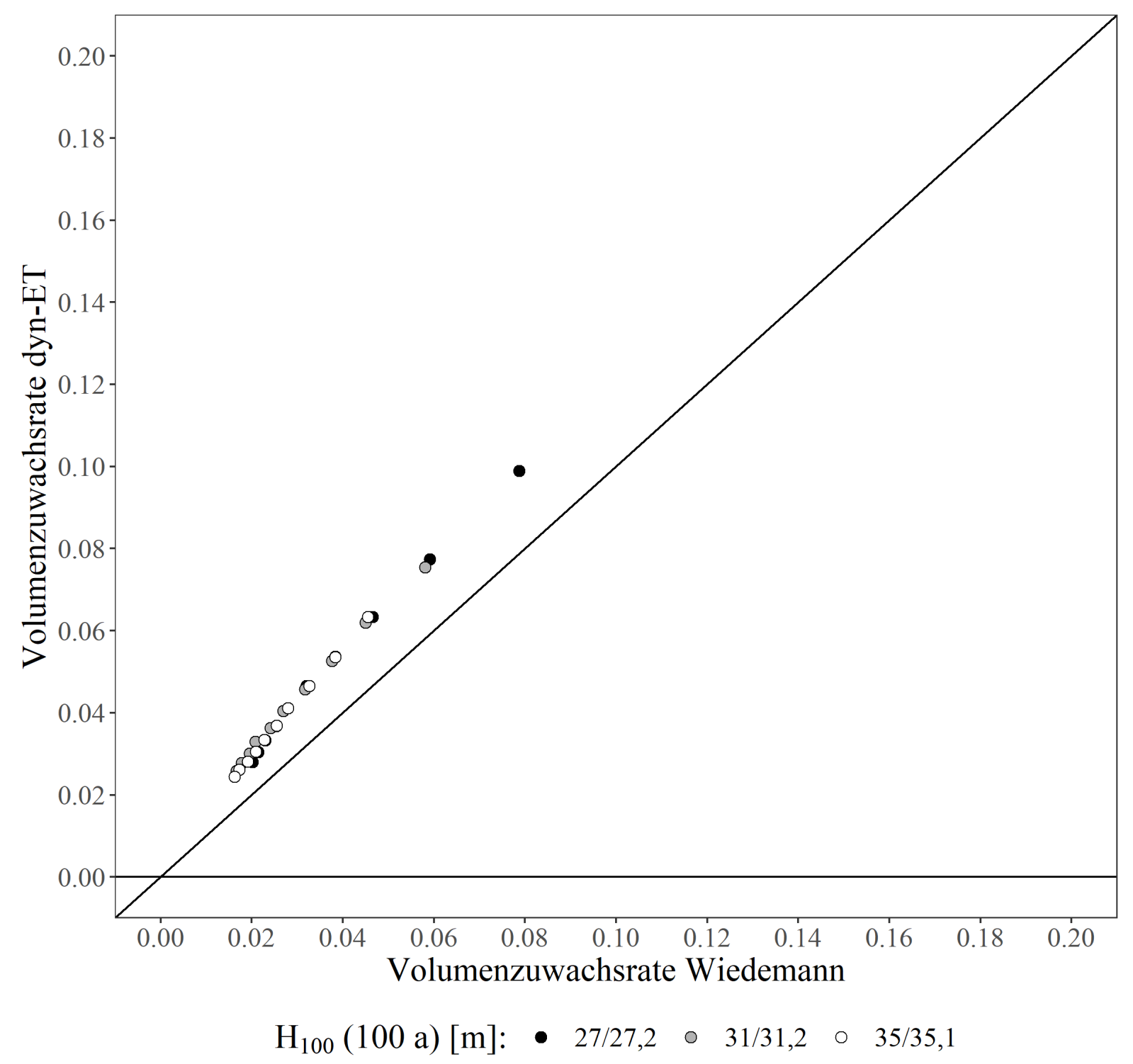

Abbildung 85: Volumenzuwachsraten der Baumart Fichte (mäßige Durchforstung, ausgewählte Oberhöhenbonitäten) gemäß dyn-ET (unveröff.) und Wiedemann (1936/42); es werden nur Vergleichspaare aus Altern dargestellt, in denen für alle Oberhöhenbonitäten Datenpunkte vorliegen; die Winkelhalbierende kennzeichnet gleiche Wertzuwachsraten im gleichen Alter.

Gleichzeitig steigen die Vornutzungsvolumina - und zwar im Mittel umso mehr, je höher die Standortsgüte ist (Abbildung 86). Die Summe der Vornutzungsvolumina bis zum Alter 80 a steigt gemäß dyn-ET (unveröff., mäßige Hochdurchforstung) um 88 bis 119 \% (Tabelle 3-6) 
ggü. Wiedemann (1936/42, mäßige Durchforstung). Bei starker Durchforstung sind die Unterschiede weniger stark ausgeprägt (Abbildung 176 im Anhang).

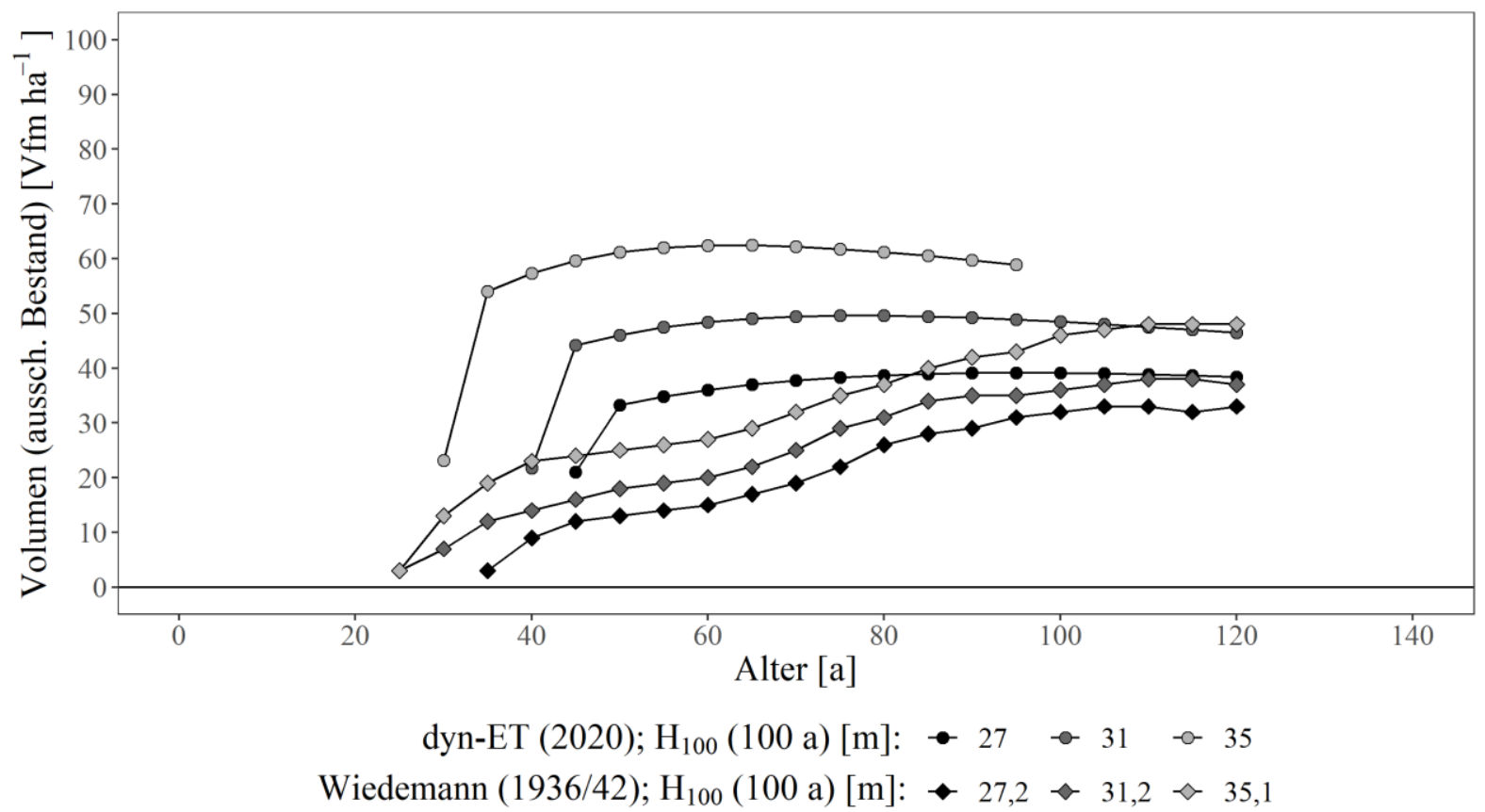

Abbildung 86: Ausscheidendes Bestandesvolumen über dem Alter; ausgewählte Oberhöhenbonitäten der Baumart Fichte (mäßige Durchforstung) gemäß dyn-ET (unveröff.) und Wiedemann (1936/42).

Wie schon bei der Baumart Buche sind die quantitativ recht ähnlichen bzw. leicht gesunkenen Vorräte aufgrund der veränderten waldbaulichen Behandlung gemäß dyn-ET (unveröff.) auch bei der Baumart Fichte grundlegend anders aufgebaut.

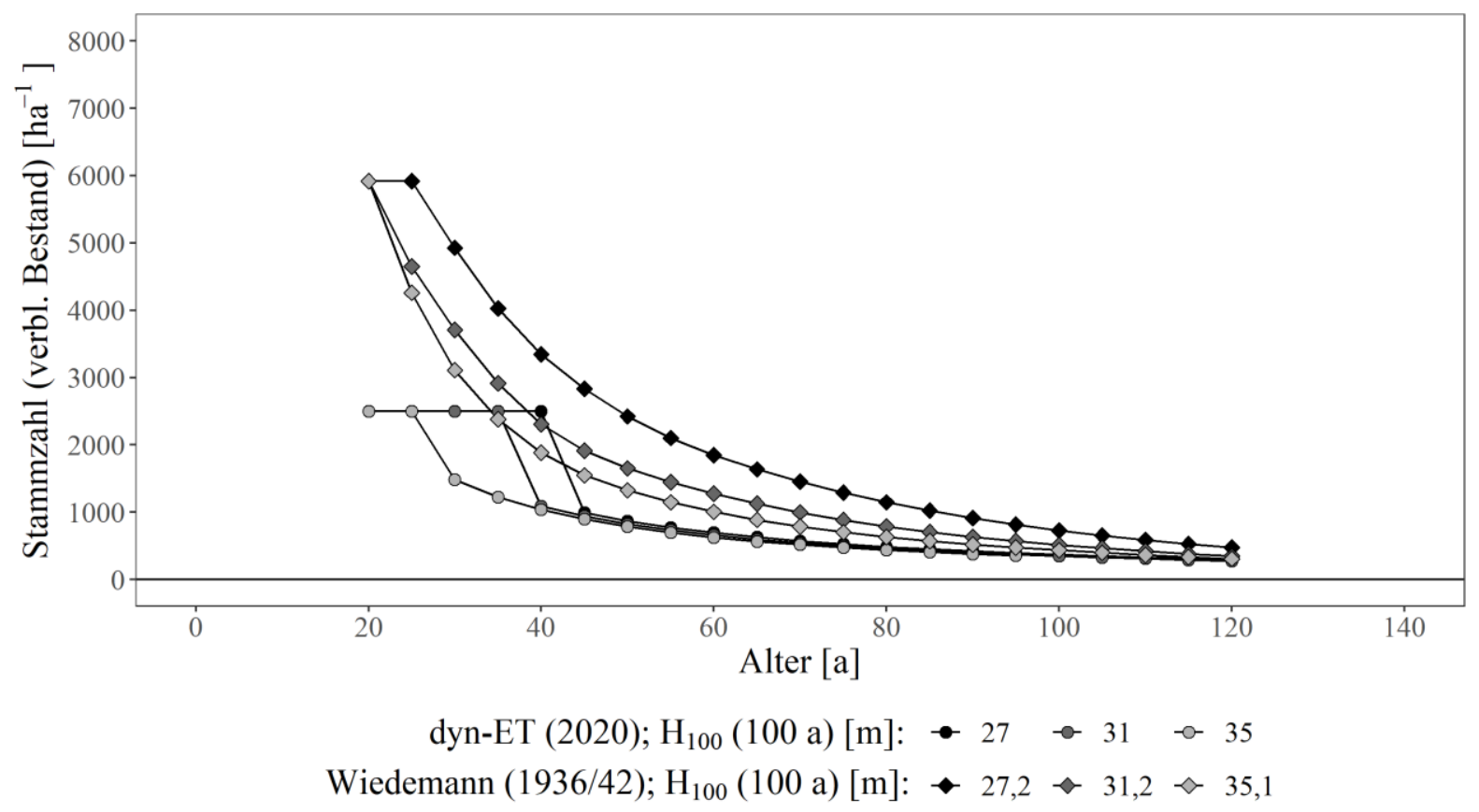

Abbildung 87: Stammzahl (verbleibender Bestand) über dem Alter; ausgewählte Oberhöhenbonitäten der Baumart Fichte (mäßige Durchforstung) gemäß dyn-ET (unveröff.) und Wiedemann (1936/42). 


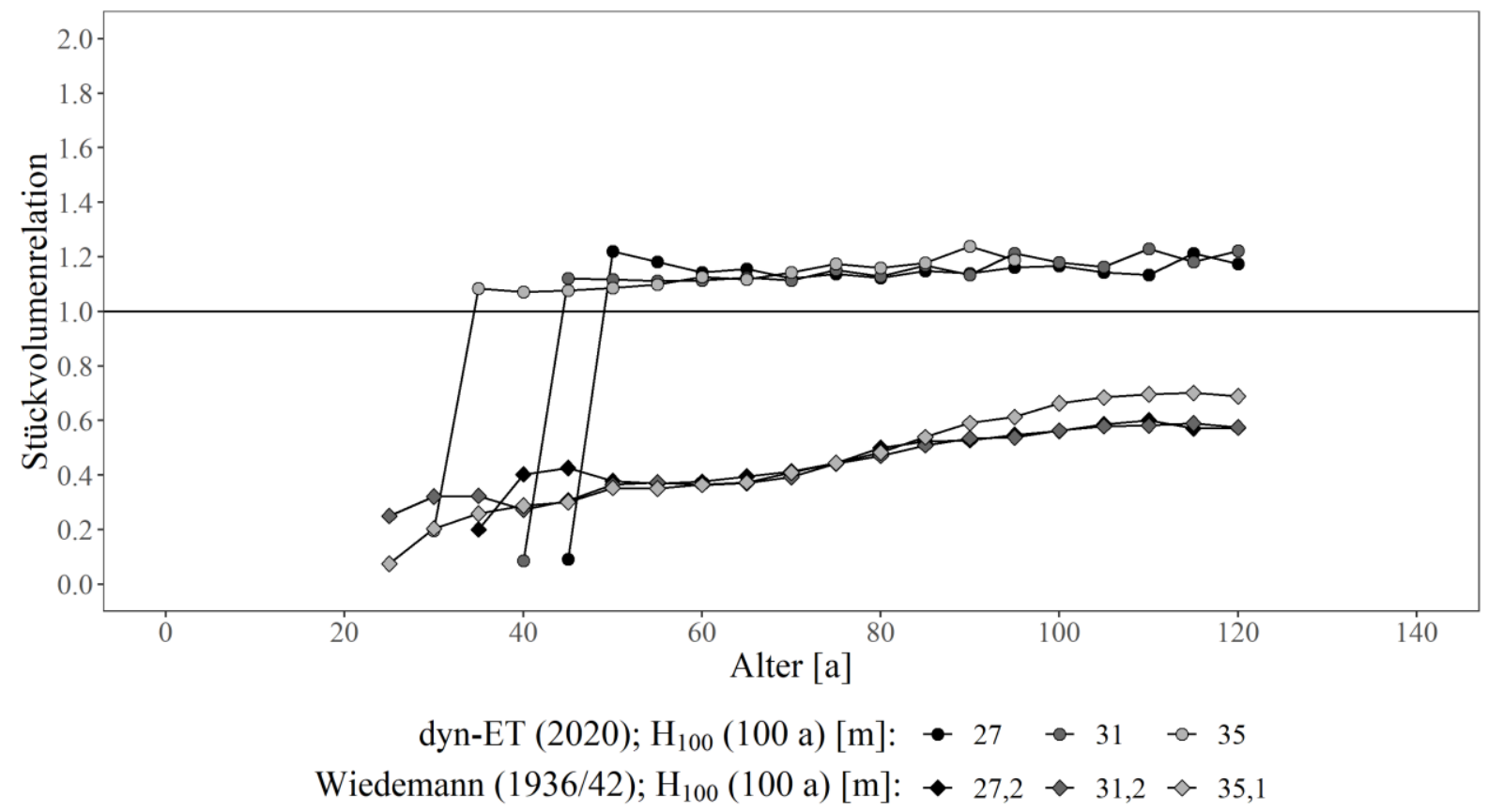

Abbildung 88: Stückvolumenrelationen (ausscheidendes geteilt durch verbleibendes Stückvolumen) über dem Alter; ausgewählte Oberhöhenbonitäten der Baumart Fichte (mäßige Durchforstung) gemäß dyn-ET (unveröff.) und Wiedemann (1936/42).

Deutlich geringere Stammzahlen zu Beginn der forstlichen Produktion (Abbildung 87) führen in Kombination mit den deutlich hochdurchforstungsartigen Eingriffen (Abbildung 88) zu höheren Einzelbaumdurchmessern im verbleibenden (Abbildung 89) und insbesondere auch im ausscheidenden Bestand (Abbildung 90).

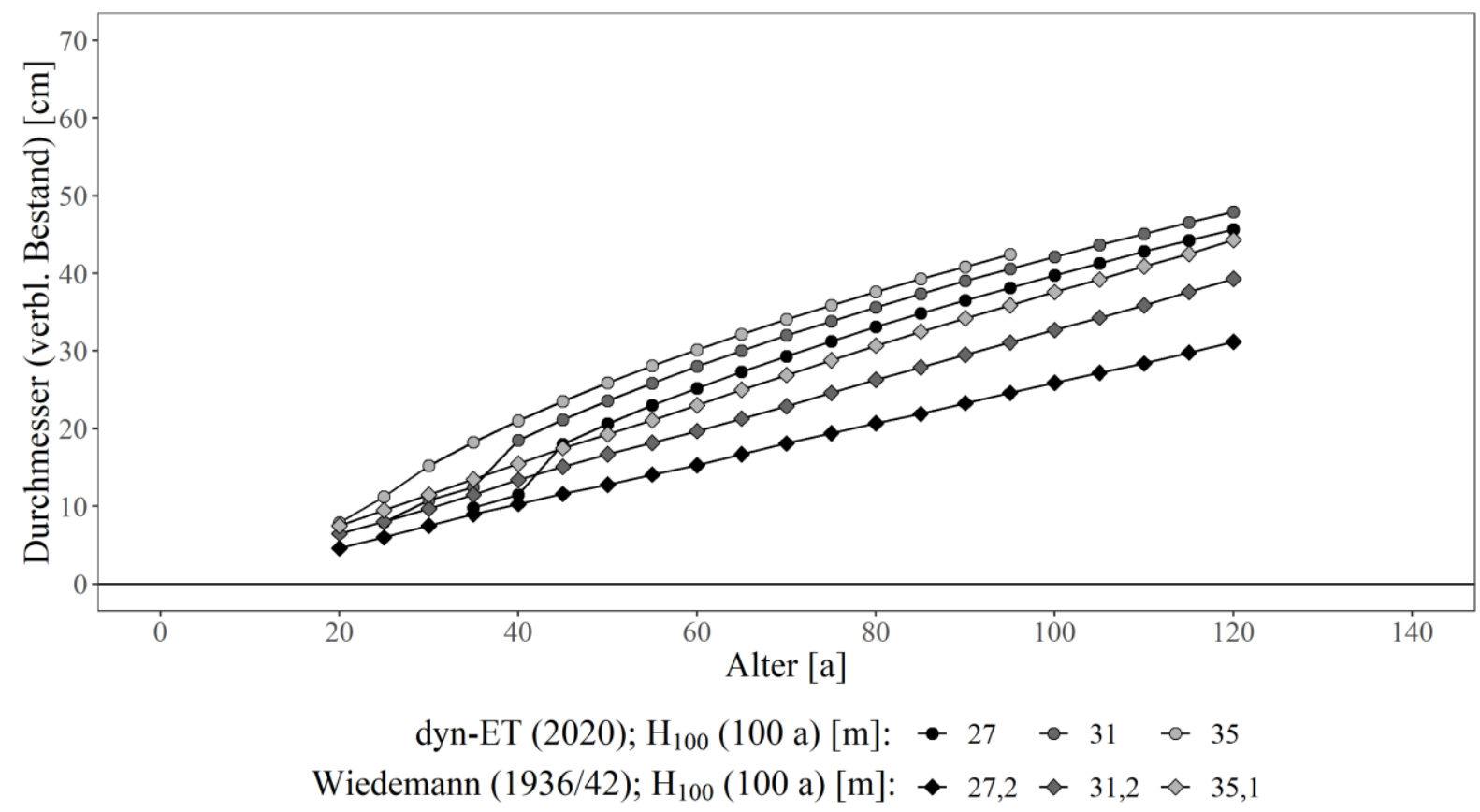

Abbildung 89: Durchmesser des Grundflächenmittelstamms (verbleibender Bestand) über dem Alter; ausgewählte Oberhöhenbonitäten der Baumart Fichte (mäßige Durchforstung) gemäß dyn-ET (unveröff.) und Wiedemann (1936/42). 
Abbildungen 177 bis 180 zeigen im Anhang ähnl. Unterschiede für die starke Durchforstung.

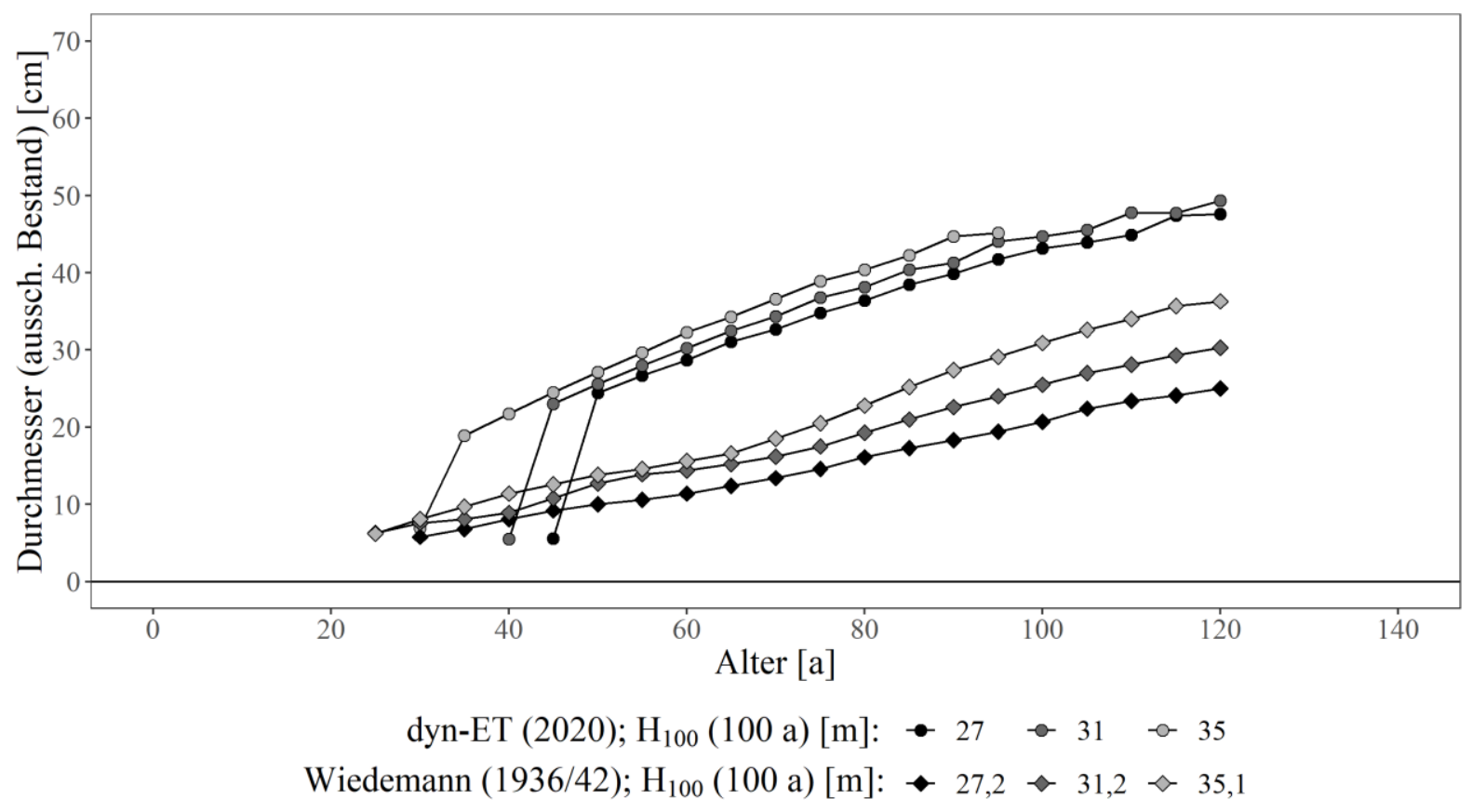

Abbildung 90: Durchmesser des Grundflächenmittelstamms (ausscheidender Bestand) über dem Alter; ausgewählte Oberhöhenbonitäten der Baumart Fichte (mäßige Durchforstung) gemäß dyn-ET (unveröff.) und Wiedemann (1936/42).

Analog zur Baumart Buche werden im Folgenden zwei klassische Elemente der Nachhaltigkeitsprüfung im Rahmen der Forsteinrichtung (vgl. Speidel 1972, S. 163 ff.; siehe Abschnitt 3.2.2) zwischen dyn-ET (unveröff., mäßige Hochdurchforstung) und Wiedemann (1936/42, mäßige Durchforstung) verglichen. Abbildung 91 zeigt den Anstieg der durchschnittlichen Bestandesvolumenzuwächse, welcher nochmals prägnanter ausfällt als bei der Buche. Das durchschnittliche Nutzungspotential liegt bis zum jeweiligen Zeitpunkt der Kulmination demnach gemäß dyn-ET (unveröff., mäßige Hochdurchforstung) um $30 \%$ bis $33 \%$ höher (Tabelle 3-6) als nach Wiedemann (1936/42, mäßige Durchforstung). Die entsprechende Abbildung 181 für die starke Durchforstung kann dem Anhang entnommen werden.

Die Normalvorräte gemäß dyn-ET (unveröff., mäßige Hochdurchforstung) sinken ggü. der etablierten Ertragstafel von Wiedemann (1936/42, mäßige Durchforstung) unabhängig von der Umtriebszeit deutlich ab (Abbildung 70) - bei einer Umtriebszeit von 80 a um $6 \%$ bis $10 \%$ (Tabelle 3-6). Auch bei starker Durchforstung sinken die Normalvorräte ggü. Wiedemann (1936/42) ab (Abbildung 182). 


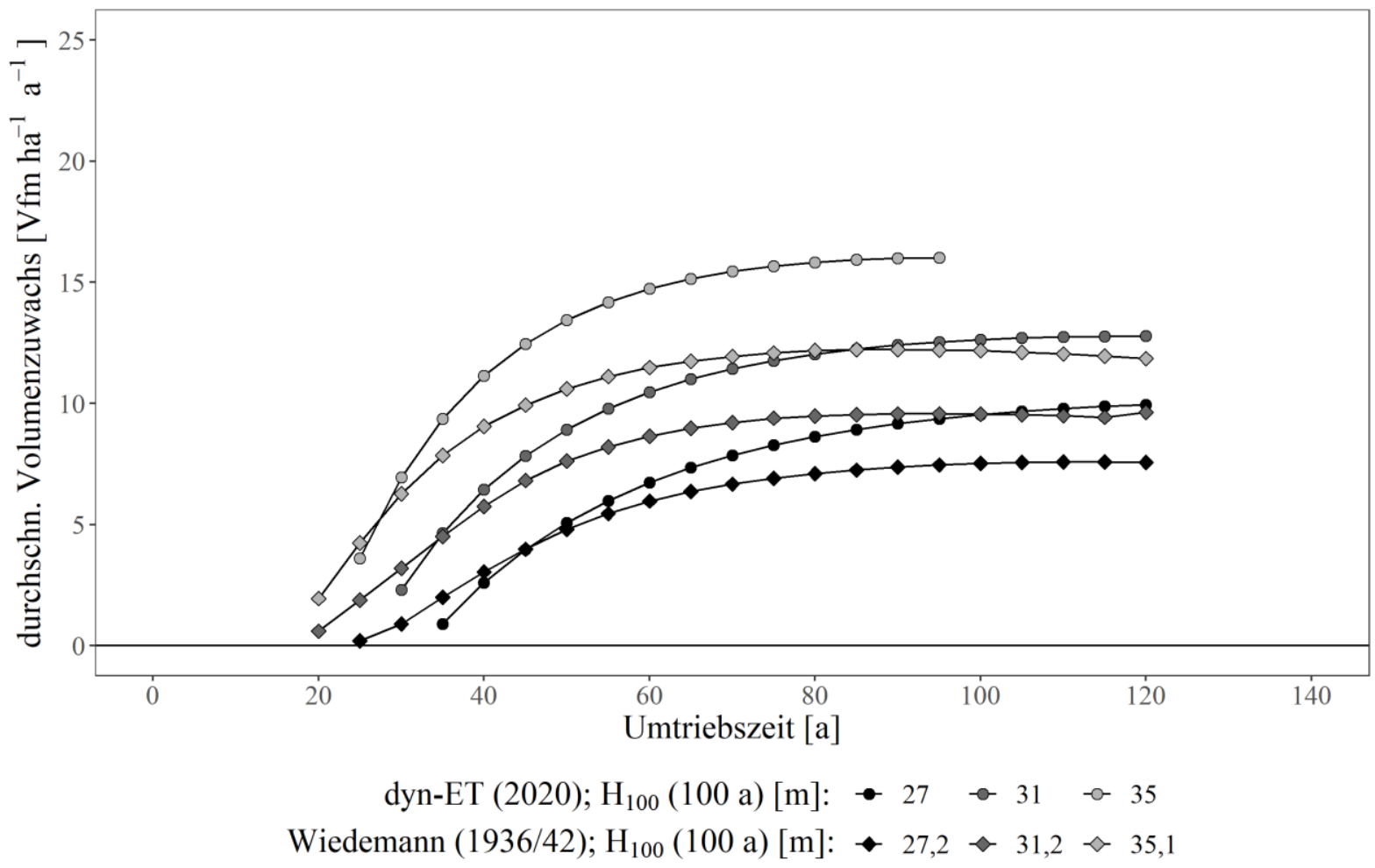

Abbildung 91: Durchschnittlicher Bestandesvolumenzuwachs über der Umtriebszeit; ausgewählte Oberhöhenbonitäten der Baumart Fichte (mäßige Durchforstung) gemäß dyn-ET (unveröff.) und Wiedemann (1936/42).

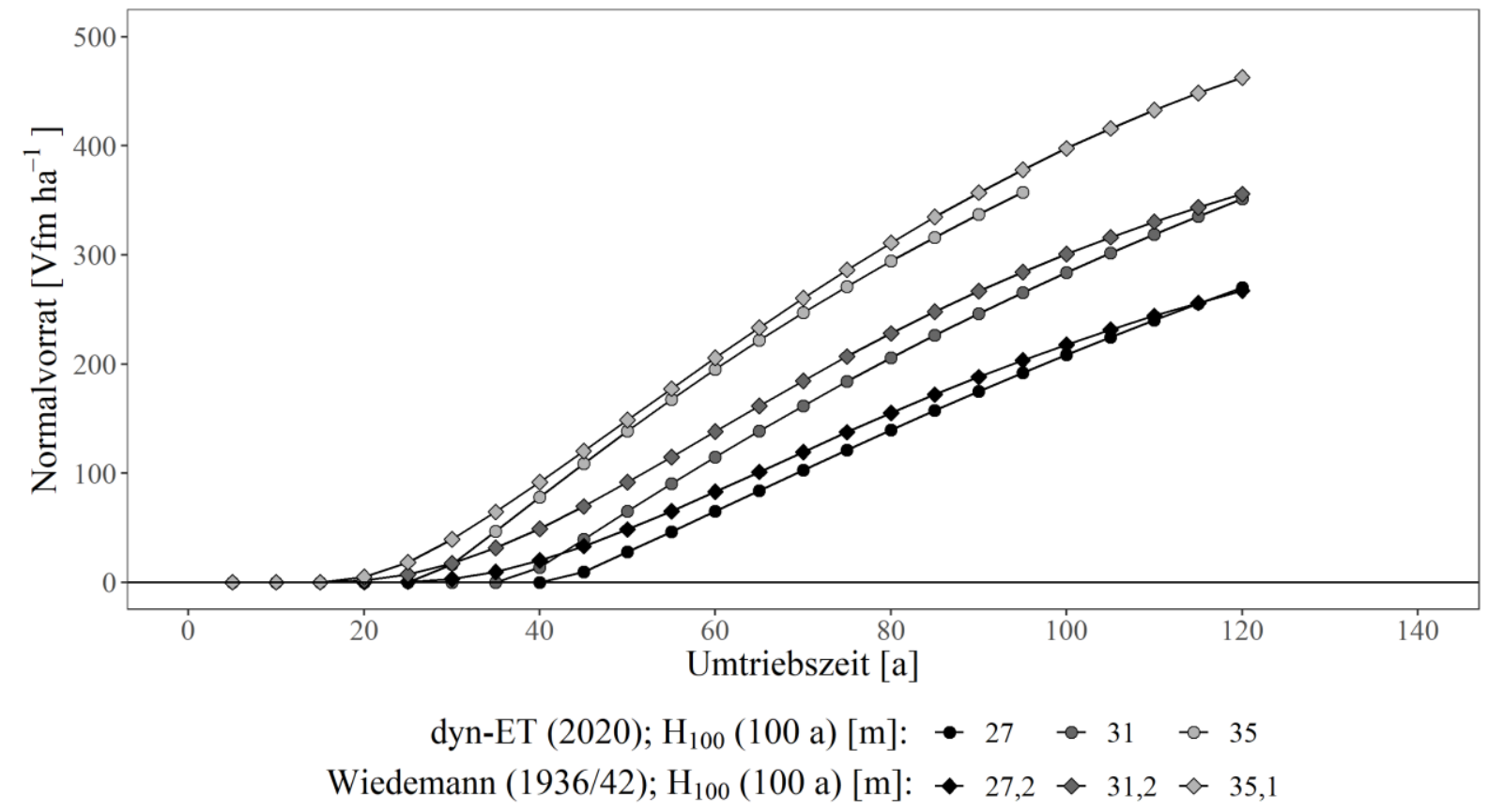

Abbildung 92: Normalvorrat über der Umtriebszeit; ausgewählte Oberhöhenbonitäten der Baumart Fichte (mäßige Durchforstung) gemäß dyn-ET (unveröff.) und Wiedemann (1936/42). 
Zwischen den beiden Behandlungsvarianten der Baumart Fichte gemäß dyn-ET (unveröff.) zeigen sich bei gleicher Oberhöhenbonität deutlich differenzierte Gesamtwuchsleistungen (Abbildung 93) und laufende Volumenzuwächse (Abbildung 94).

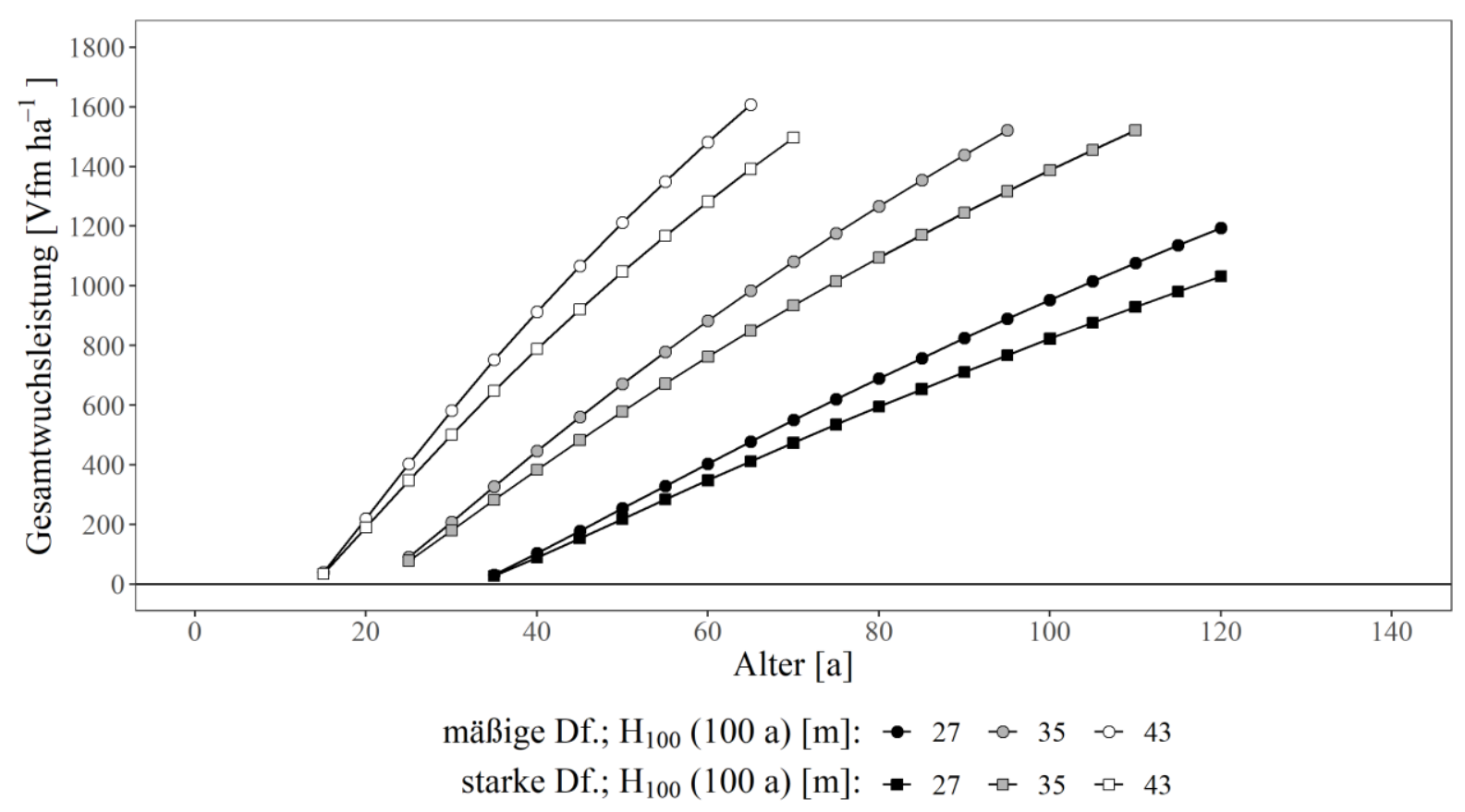

Abbildung 93: Gesamtwuchsleistung über dem Alter; ausgewählte Oberhöhenbonitäten der Baumart Fichte getrennt nach Behandlungsvarianten gemäß dyn-ET (unveröff.).

Bonitätsunabhängig dominiert die Gesamtwuchsleistung der mäßigen ggü. der starken Hochdurchforstung über den gesamten Altersrahmen hinweg und zunehmend mit steigendem Alter.

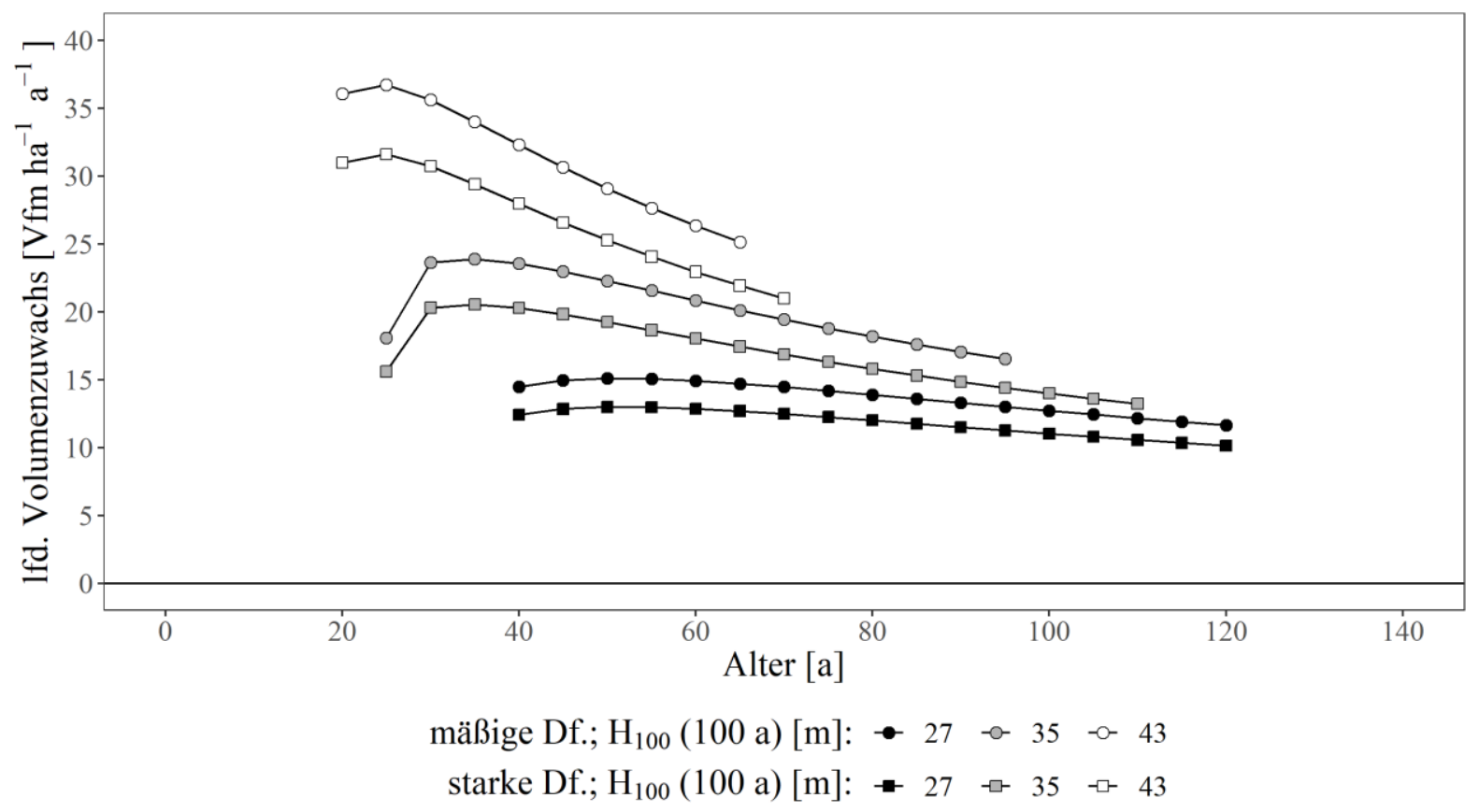

Abbildung 94: Laufender Bestandesvolumenzuwachs über dem Alter; ausgewählte Oberhöhenbonitäten der Baumart Fichte getrennt nach Behandlungsvarianten gemäß dyn-ET (unveröff.). 
Tabelle 3-7: Vergleich ausgewählter Ertragstafeldaten der Baumart Fichte (Oberhöhenbonität $H_{100}(100 \mathrm{a})=35 \mathrm{~m}$ ) gemäß dyn-ET (unveröff.); unterschiedliche Behandlungsvarianten; in Klammern ist jeweils die relative Differenz zur mäßigen Hochdurchforstung angegeben;

$\boldsymbol{G W L}(\mathbf{8 0} \mathrm{a})$ : Gesamtwuchsleistung bis zum Alter $80 \mathrm{a}, \boldsymbol{\operatorname { m a x }} \boldsymbol{v}_{\boldsymbol{B}}$ : lfd. Bestandesvolumenzuwachs zum Zeitpunkt der Kulmination [...], $\boldsymbol{V}_{\boldsymbol{B}}\left(\mathbf{8 0}\right.$ a): Vorrat (verbl. Bestand) im Alter 80 a, $\boldsymbol{G}_{\boldsymbol{v e r b l}}(\mathbf{8 0}$ a): Bestandesgrundfläche des verbleibenden Bestandes im Alter $80 \mathrm{a}, \sum_{\mathbf{0}}^{\mathbf{8 0}} \mathbf{a} \boldsymbol{\lambda}$ : kumuliertes ausscheidendes Bestandesvolumen bis zum Alter 80 a, $\boldsymbol{N}_{\text {verbl. }}$ ( 80 a): Stammzahl des verbleibenden Bestandes im Alter 80 a, $\sum_{\mathbf{0}}^{\mathbf{8 0} a} \boldsymbol{N}_{\text {aussch. }}$ : kumulierte ausscheidende Stammzahl bis zum Alter 80 a, $\boldsymbol{D}_{\boldsymbol{g}, \boldsymbol{v e r b l}}(\mathbf{8 0} \mathbf{a})$ : Durchmesser des Grundflächenmittelstammes des verbleibenden Bestandes im Alter $80 \mathrm{a}$, $\boldsymbol{D}_{\boldsymbol{g}, \boldsymbol{a u s s c h}}(\mathbf{8 0}$ a): Durchmesser des Grundflächenmittelstammes des ausscheidenden Bestandes im Alter $80 \mathrm{a}, \boldsymbol{m a x} \overline{\boldsymbol{v}_{\boldsymbol{B}}}$ : durchschn. Bestandesvolumenzuwachs zum Zeitpunkt der Kulmination [...], $\boldsymbol{V}_{\boldsymbol{\eta}}(\mathbf{8 0} \mathrm{a})$ : Normalvorrat bei einer Umtriebszeit von 80 a.

\begin{tabular}{|c|c|c|}
\hline Bestandesbehandlung & $\begin{array}{r}\text { mäßige } \\
\text { Hochdurchforstung }\end{array}$ & $\begin{array}{r}\text { starke } \\
\text { Hochdurchforstung }\end{array}$ \\
\hline$H_{100}(100 \mathrm{a})[\mathrm{m}]$ & 35 & 35 \\
\hline$G W L(80$ a) & 1.266 & 1.095 \\
\hline$\left[\mathrm{Vfm} \mathrm{ha}^{-1}\right]$ & & $(-13,5 \%)$ \\
\hline $\max v_{B}$ & $23,9[35 \mathrm{a}]$ & 20,5 [35 a] \\
\hline$\left[\mathrm{Vfm} \mathrm{ha}^{-1} \mathrm{a}^{-1}\right]$ & & $(-14,2 \%)$ \\
\hline$V_{B}(80 \mathrm{a})$ & 625 & 511 \\
\hline$\left[\mathrm{Vfm} \mathrm{ha}^{-1}\right]$ & & $(-18,2 \%)$ \\
\hline$G_{\text {verbl. }}(80 \mathrm{a})$ & 48,7 & 39,6 \\
\hline$\left[\mathbf{m}^{2}\right]$ & & $(-18,7 \%)$ \\
\hline$\sum_{0}^{80 \mathrm{a}} \lambda$ & 641 & 584 \\
\hline$\left[\mathrm{Vfm} \mathrm{ha}^{-1}\right]$ & & $(-8,9 \%)$ \\
\hline$N_{\text {verbl. }}(80 \mathrm{a})$ & 438 & 303 \\
\hline$\left[\mathrm{ha}^{-1}\right]$ & & $(-30,8 \%)$ \\
\hline$\sum_{0}^{80 \mathrm{a}} N_{\text {aussch }}$ & 2.062 & 2.197 \\
\hline$\left[\mathbf{h a}^{-1}\right]$ & & $(+6,5 \%)$ \\
\hline$D_{g, v e r b l .}(80$ a $)$ & 37,6 & 40,8 \\
\hline$[\mathbf{c m}]$ & & $(+8,5 \%)$ \\
\hline$D_{g, a u s s c h .}(80 a)$ & 40,4 & 46,9 \\
\hline$[\mathbf{c m}]$ & & $(+16,1 \%)$ \\
\hline $\max \overline{v_{B}}$ & $16,0[95 \mathrm{a}]$ & $13,9[100 \mathrm{a}]$ \\
\hline$\left[\mathrm{Vfm} \mathrm{ha}^{-1} \mathbf{a}^{-1}\right]$ & & $(-13,1 \%)$ \\
\hline$V_{\eta}(80 \mathrm{a})$ & 294 & 244 \\
\hline$\left[\mathrm{Vfm} \mathrm{ha}^{-1}\right]$ & & $(-17,0 \%)$ \\
\hline
\end{tabular}


Bei einer mittleren Oberhöhenbonität von $35 \mathrm{~m}$ liegt das insgesamt bis zu einem Alter von 80 Jahren zugewachsene Holzvolumen bei starker Hochdurchforstung um 14 \% unterhalb der Gesamtwuchsleistung bei mäßiger Hochdurchforstung (Tabelle 3-7). Und auch die laufenden Bestandesvolumenzuwächse der starken Hochdurchforstung liegen bei der mittleren Oberhöhenbonität $35 \mathrm{~m}$ zum einheitlichen Zeitpunkt der Kulmination im Alter $35 \mathrm{a}$ mit 20,5 Vfm ha ${ }^{-1} \mathrm{a}^{-1}$ um $14 \%$ unterhalb der Volumenzuwächse bei mäßiger Hochdurchforstung (23,9 Vfm ha ${ }^{-1} \mathrm{a}^{-1}$; Tabelle 3-7). Bonitätsspezifisch kulminieren die laufenden Bestandesvolumenzuwächse beider Behandlungsvarianten im gleichen Alter, welches sich mit sinkender Oberhöhenbonität nach hinten verschiebt (Abbildung 94).

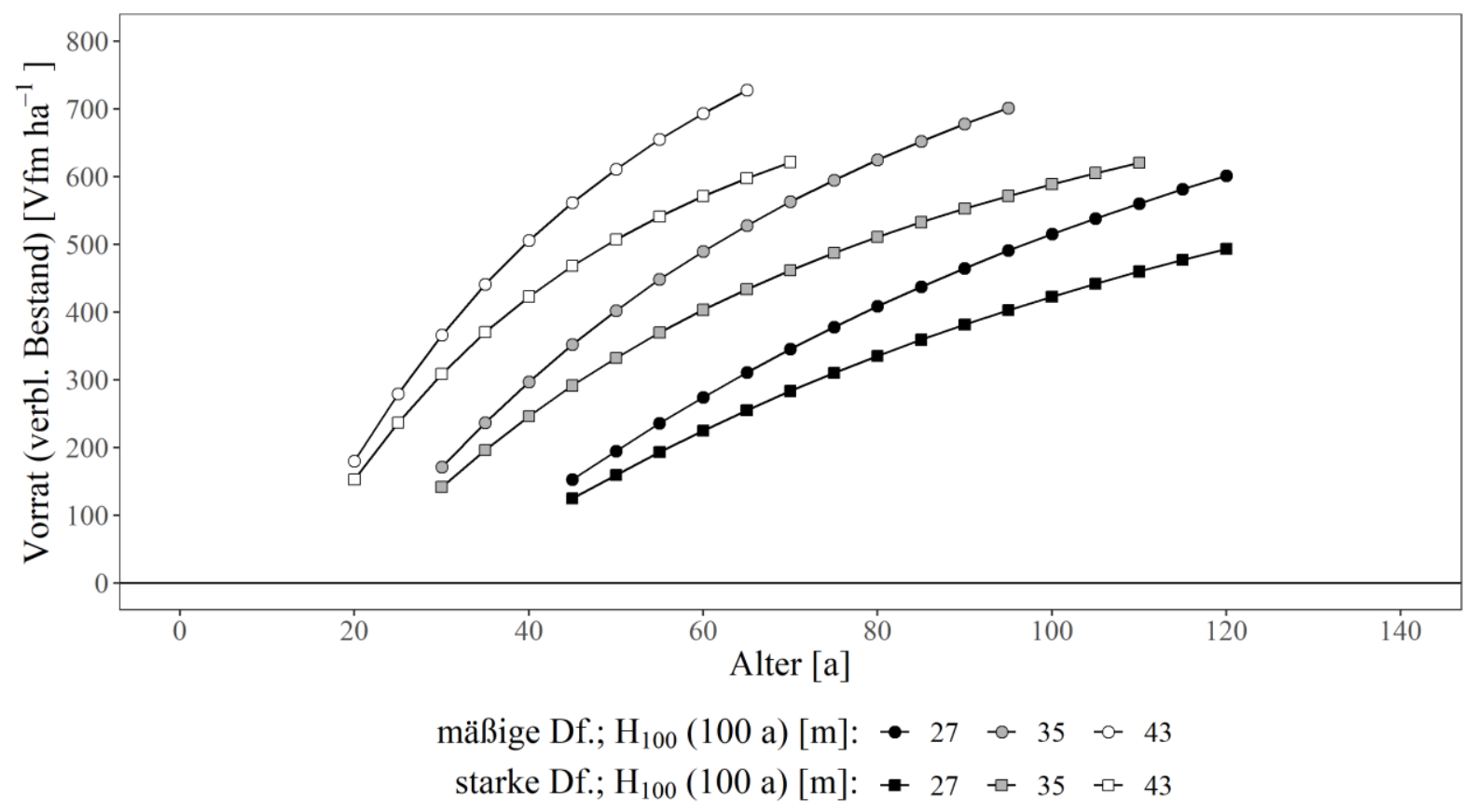

Abbildung 95: Vorrat (verbleibender Bestand) über dem Alter; ausgewählte Oberhöhenbonitäten der Baumart Fichte getrennt nach Behandlungsvarianten gemäß dyn-ET (unveröff.).

Die Vorräte (Abbildung 95) liegen bei starker ggü. der mäßigen Hochdurchforstung insbesondere in jungen Bestandesaltern relativ gesehen niedriger als die laufenden Bestandesvolumenzuwächse, was, ähnlich wie bei der Baumart Buche, zu leicht höheren Volumenzuwachsraten führt (Abbildung 96). 


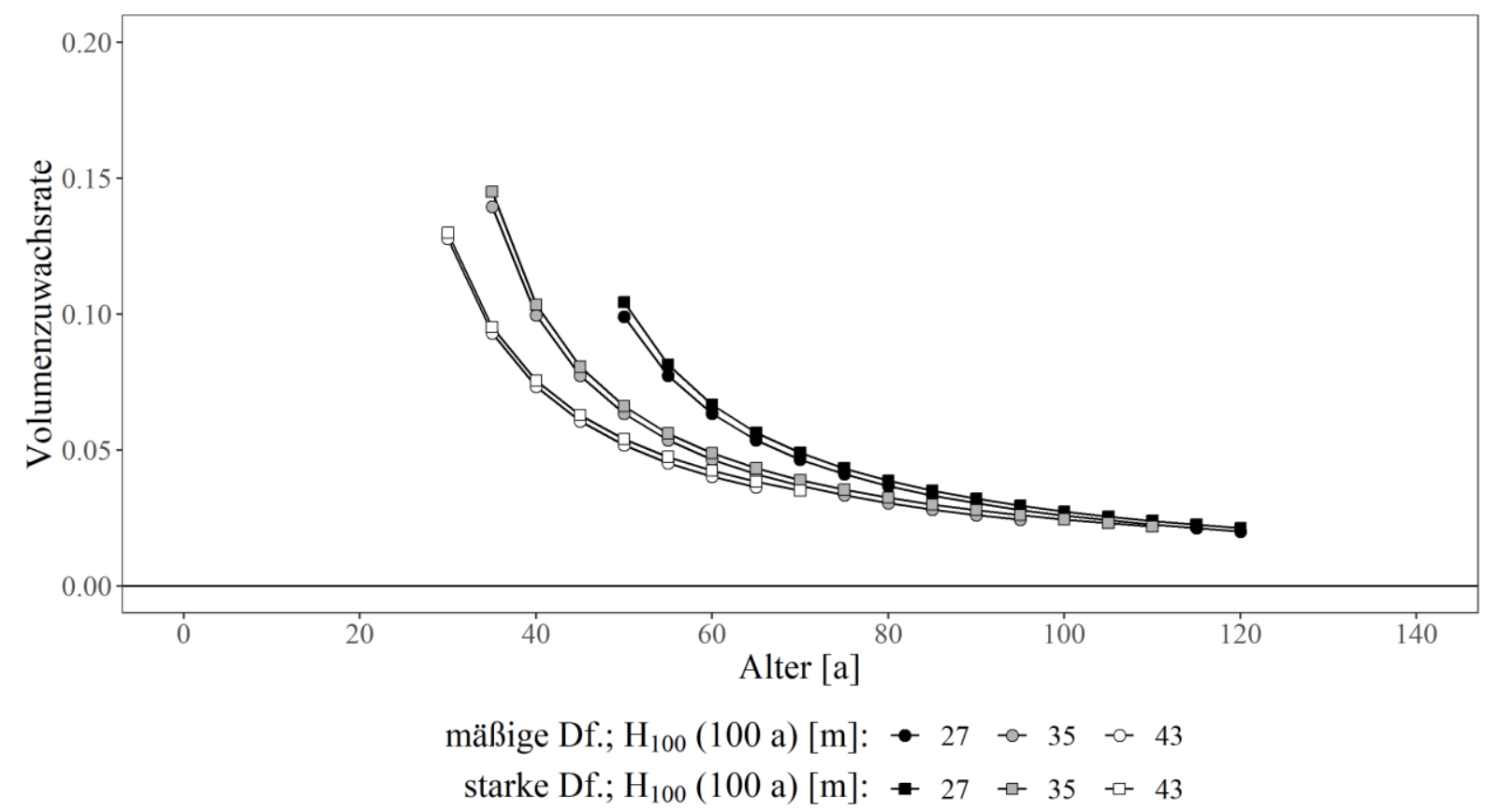

Abbildung 96: Volumenzuwachsraten über dem Alter; ausgewählte Oberhöhenbonitäten der Baumart Fichte getrennt nach Behandlungsvarianten gemäß dyn-ET (unveröff.).

Entsprechend der gezeigten Vorräte entwickeln sich die Vornutzungsvolumina (Abbildung 97), wobei die sehr hohen Vornutzungsmengen bei einer Oberhöhenbonität von 43 m bemerkenswert sind.

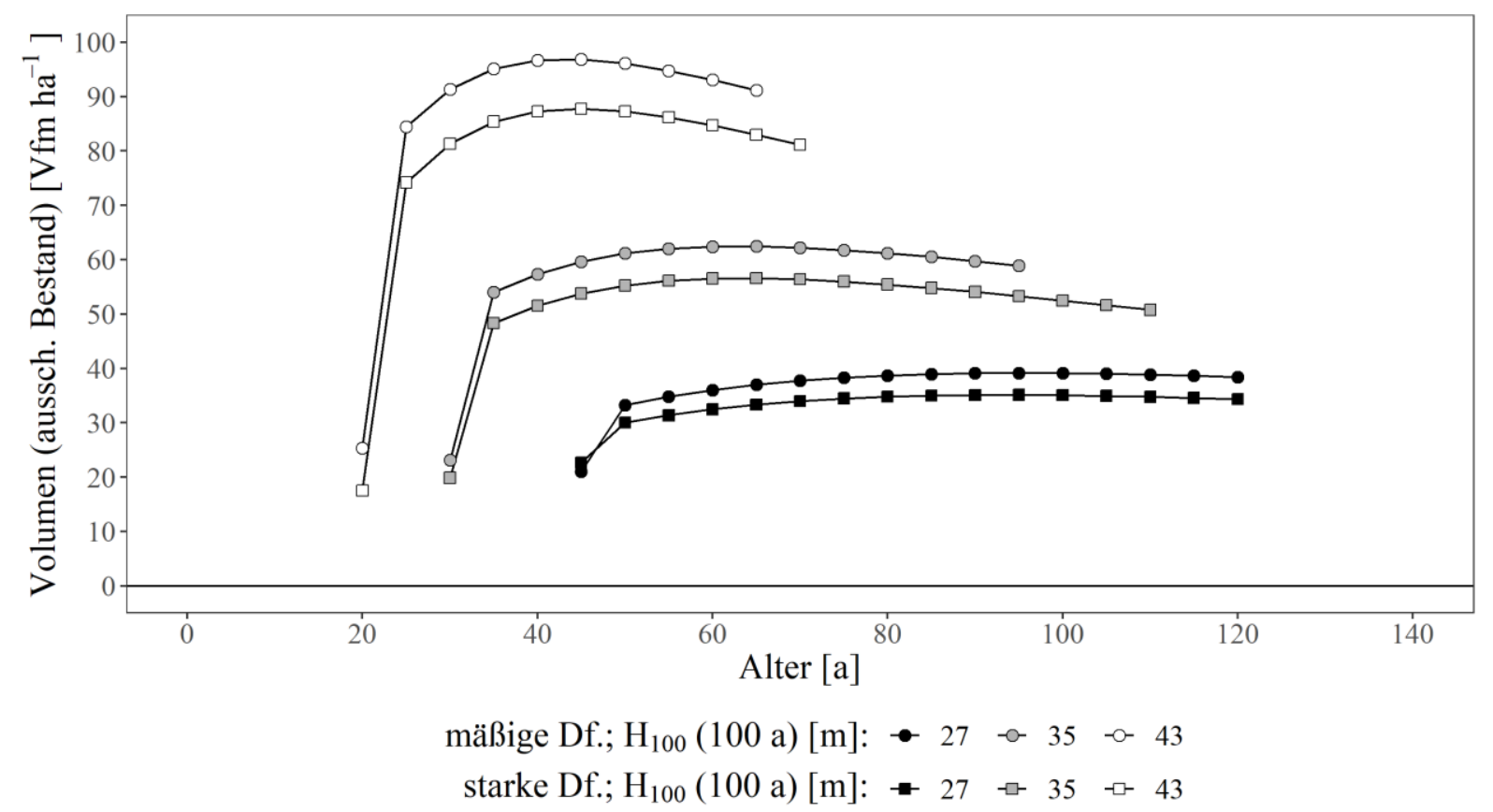

Abbildung 97: Ausscheidendes Bestandesvolumen über dem Alter; ausgewählte Oberhöhenbonitäten der Baumart Fichte getrennt nach Behandlungsvarianten gemäß dyn-ET (unveröff.). 


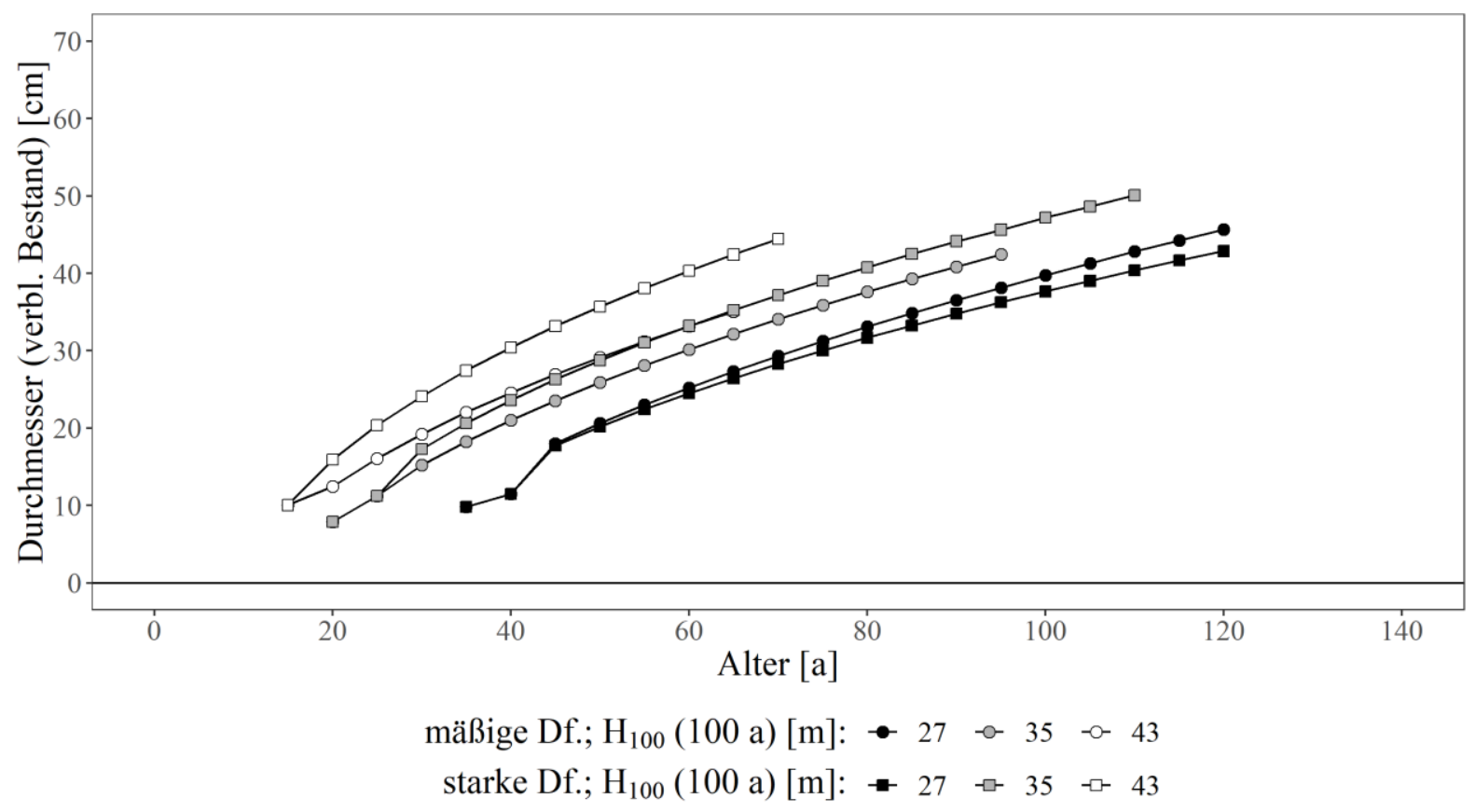

Abbildung 98: Durchmesser des Grundflächenmittelstamms (verbleibender Bestand) über dem Alter; ausgewählte Oberhöhenbonitäten der Baumart Fichte getrennt nach Behandlungsvarianten gemäß dynET (unveröff.).

Die mittleren Durchmesser der verbleibenden Bestandesvolumina differenzieren sich erneut uneinheitlich aber deutlich stärker als bei der Buche. Während die starke Hochdurchforstung bei mittlerer und hoher Oberhöhenbonität deutlich höhere mittlere Einzelbaumdurchmesser aufweist als die mäßige Hochdurchforstung, verhält es sich bei der niedrigsten Oberhöhenbonität genau andersherum (Abbildung 98). Bei der mittleren Oberhöhenbonität $35 \mathrm{~m}$ liegt der Durchmesser des Grundflächenmittelstammes bei starker Hochdurchforstung im Alter von 80 a um $9 \%$ höher als bei mäßiger Hochdurchforstung (Tabelle 3-7). Im gleichen Alter liegt der mittlere Einzelbaumdurchmesser des ausscheidenden Bestandes bei starker Hochdurchforstung sogar um 16 \% höher und es ist auch hier ein deutlicher Trend zu höheren Dimensionsunterschieden bei steigender Oberhöhenbonität erkennbar (Abbildung 99).

Gegenüber der Baumart Buche zeigt der Vergleich der Stückvolumenrelationen bei der Baumart Fichte deutlich einheitlichere Ergebnisse. Bis auf den ersten Vornutzungseingriff, indem hohe Stammzahlen (siehe Abbildung 183 im Anhang) mit relativ geringen ausscheidenden Volumina (siehe Abbildung 97) entnommen werden ${ }^{70}$, erfolgen (fast) alle Durchforstungen klar

\footnotetext{
${ }^{70}$ In Abbildung 100 wird der erste Datenpunkt bei maximaler Oberhöhenbonität und mäßiger Hochdurchforstung auf modellbedingte Artefakte der vorläufigen Ertragstafeldaten gemäß dyn-ET (unveröff.) zurückgeführt.
} 
vom starken Ende her, d. h. die Stückvolumina des ausscheidenden sind größer als die des verbleibenden Bestandes (Abbildung 100). Dieser Effekt ist dabei bonitätsunabhängig und bei der starken Hochdurchforstung stärker ausgeprägt.

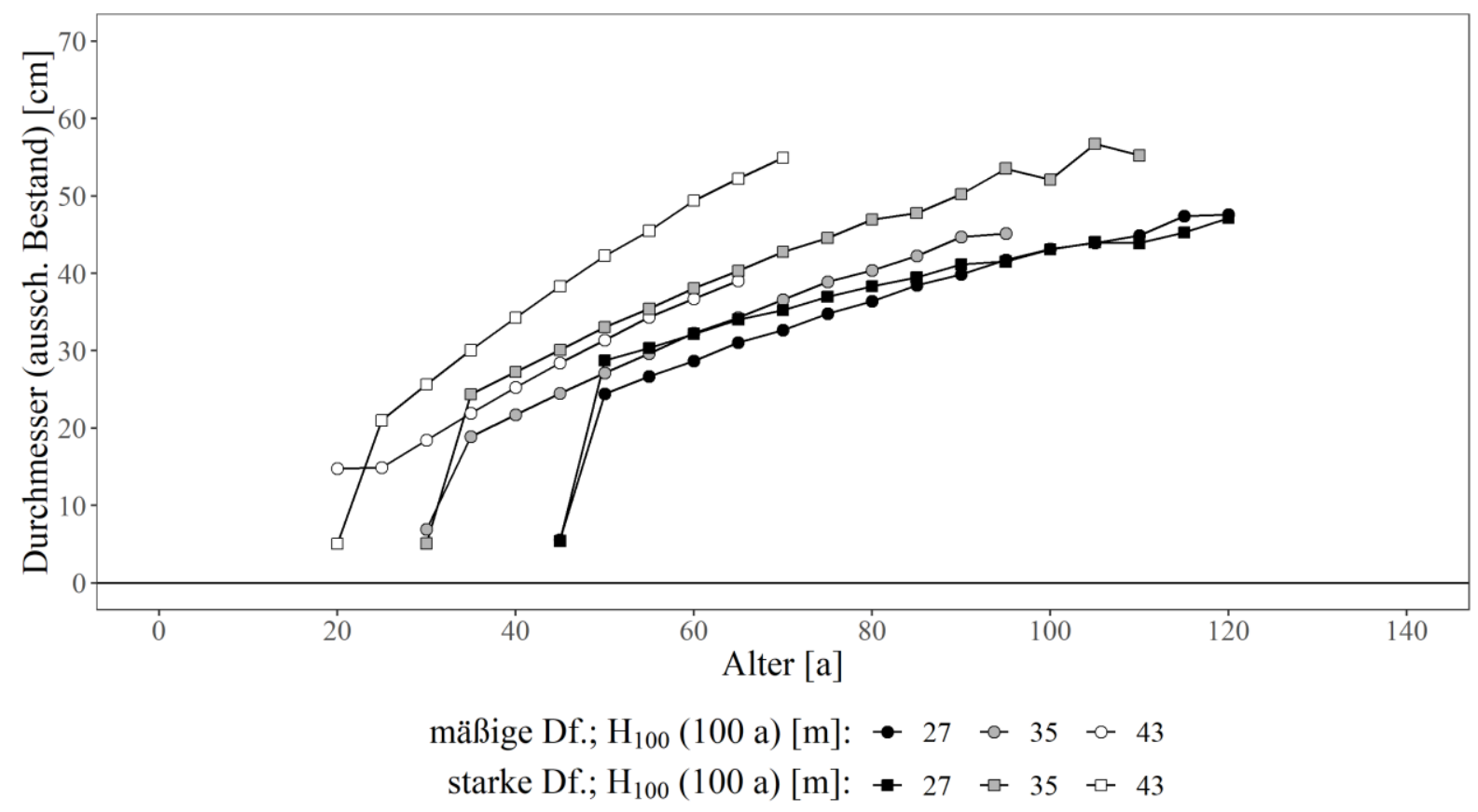

Abbildung 99: Durchmesser des Grundflächenmittelstammes (ausscheidender Bestand) über dem Alter; ausgewählte Oberhöhenbonitäten der Baumart Fichte getrennt nach Behandlungsvarianten gemäß dyn-ET (unveröff.).

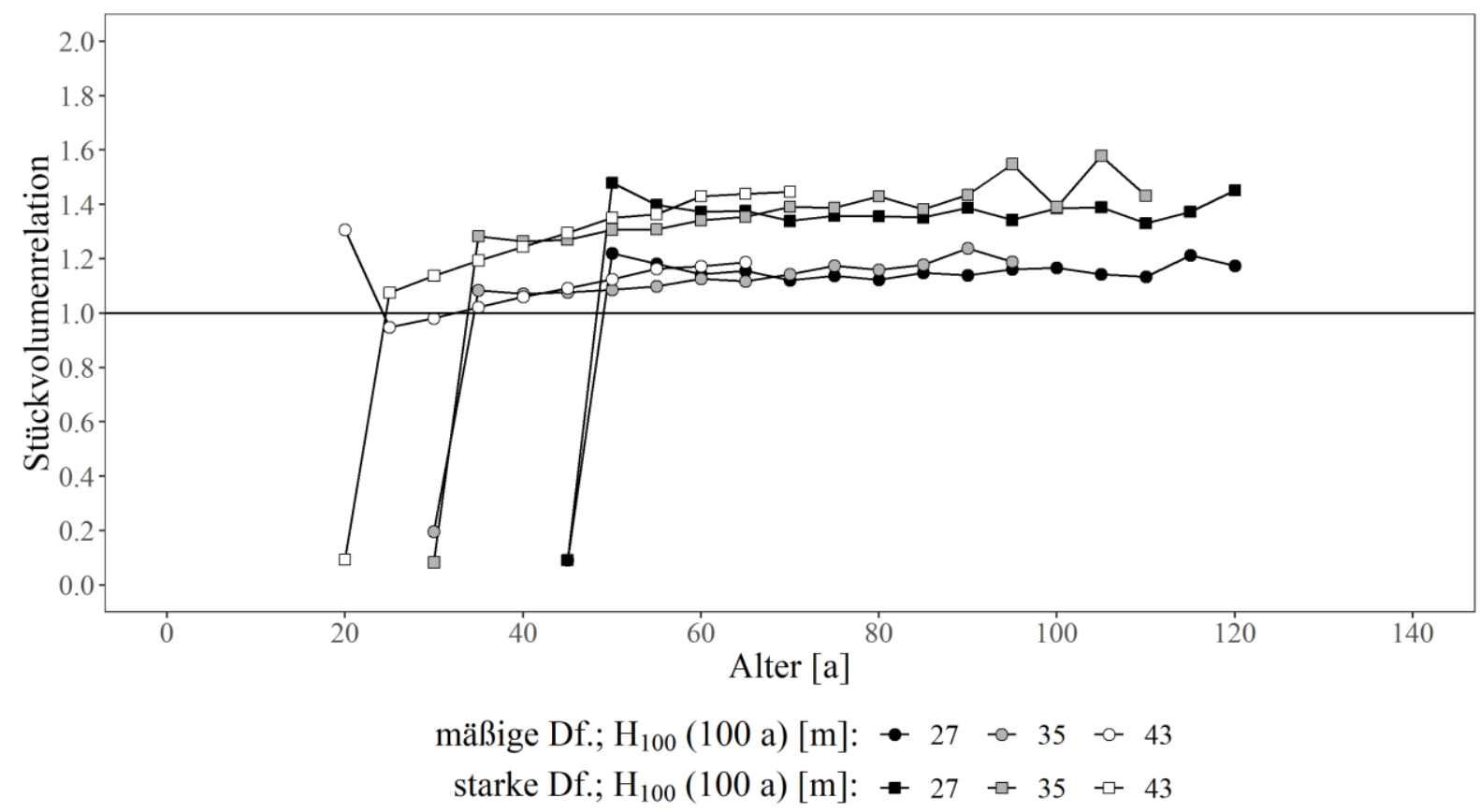

Abbildung 100: Stückvolumenrelationen (ausscheidendes geteilt durch verbleibendes Stückvolumen) über dem Alter; Oberhöhenbonität 35 m der Baumart Fichte getrennt nach Behandlungsvarianten gemäß dyn-ET (unveröff.). 


\subsubsection{Bestandesbehandlungsdiagramme}

Bestandesbehandlungsdiagramme, eher bekannt unter der englischen Bezeichnung Stand Density Management Diagrams (SDMD), bilden die Wachstumsdynamik gleichaltriger Waldbestände grafisch ab (Long und Shaw 2005). Sie zeigen mittlere Bestandeskenngrößen (z. B. Oberhöhe, Stammzahl, Vorrat, Durchmesser) sowie deren Abhängigkeiten im Verlauf der Bestandesentwicklung und ermöglichen damit die zielorientierte Steuerung der Bestandesdichte (Bestandesbehandlung) im Rahmen einer nachhaltigen Forstwirtschaft (Newton 1997; Patrício und Nunes 2017). Barrio Anta und Álvarez González (2005, 210; Übers. d. A.) bezeichnen die Verwendung von SDMDs gar als ,eine der effektivsten Methoden für die Gestaltung, Darstellung und Auswertung alternativer Bestandesbehandlungskonzepte in gleichaltrigen Beständen“. In der vorliegenden Arbeit stellen die Bestandesbehandlungsdiagramme eine alternative Darstellungsform der vorläufigen Ertragstafeldaten gemäß dyn-ET (unveröff.) im Kontrast zu den ertragskundlichen Analysen in Abschnitt 3.3.3 dar. Hierfür werden die klassischen Darstellungsformen (Abbildung 101 für die Buche und Abbildung 103 für die Fichte) in Abbildung 102 (Buche) und Abbildung 104 (Fichte) um die Oberhöhen-Stammzahl-Entwicklungen aller Behandlungsvarianten bei ausgewählten Oberhöhenbonitäten ergänzt - zahlreiche, teilweise bereits in Abschnitt 3.3.3 anhand verschiedener Abbildungen diskutierte, ertragskundliche Zusammenhänge und behandlungsabhängige Effekte sind auf einen Blick erkennbar.

Die Entwicklungsgeschichte der SDMDs und der zugrundeliegenden waldwachstumskundlichen Funktionen reicht bis in die 1950er Jahre zurück (Newton 1997). So basieren die Grundgleichungen über den Zusammenhang zwischen Konkurrenz und Bestandesdichte sowie zwischen Ertrag und Bestandesdichte laut Newton (1997) auf Untersuchungen zur artspezifischen Konkurrenz von u. a. Kira et al. (1953), Hozumi et al. (1956) und Shinozaki und Kira (1956). Ergänzend lieferten Yoda et al. (1963) mit der sogenannten self-thinning rule (,Selbstdifferenzierungs- oder Selbstdurchforstungsregel“; Pretzsch 2000) Erkenntnisse über „den Zusammenhang zwischen mittlerem Gewicht [...] und der Dichte [...] in gleichaltrigen Pflanzenpopulationen bei ungestörter Entwicklung“ (Pretzsch 2000, S. 207) ${ }^{71}$. Ando (1962) leitete schließlich durch die Kombination der genannten Grundgleichungen in einem mathematischen Modell

\footnotetext{
${ }^{71}$ Die Bestandesdichte-Regel (Bestandesdichteindex) von Reineke (1933) kann nach Pretzsch (2000) als Spezialfall der self-thinning rule von Yoda et al. (1963) angesehen werden - beiden liegt das gleiche Allometriegesetz zugrunde, welches zwischen dem Volumen und der Projektionsfläche einer Kugel besteht. Demnach nimmt die Zahl möglicher Kugeln pro Fläche „mit zunehmendem Kugelvolumen mit demselben Gradienten ab, wie die Zahl möglicher Bäume bei Zunahme von Baumvolumen oder Baumbiomasse innerhalb des Bestandeslebens“" (Pretzsch 2000, S. 209).
} 
erstmals ein Bestandesbehandlungsdiagramm für die Japanische Rotkiefer (Pinus densiflora Siebold \& Zucc.) ab (siehe Abb. 1 in Newton 1997). Es folgten in den 1960er, 70er und 80er Jahren zahlreiche Weiterentwicklungen in Japan und Nordamerika. Dabei wurden neben der Anwendung auf weitere Baumarten bspw. die ursprünglichen Grundgleichungen durch empirische Volumen-Dichte-Funktionen ersetzt und zahlreiche unterschiedliche Indizes zur Beschreibung der relativen Bestandesdichte eingeführt (Newton 1997; Barrio Anta und Álvarez González 2005; vgl. z. B. Aiba 1975a, 1975b; Drew und Flewelling 1977, 1979). Darüber hinaus nennt Newton (1997) alternative Ansätze von z. B. McCarter und Long (1986), Long et al. (1988), Smith (1989), Kumar et al. (1995) sowie Newton und Weetman (1993, 1994).

Heute existieren weltweit zahlreiche Varianten von SDMDs für eine Vielzahl von Baumarten in Rein- und Mischbeständen (vgl. u. a. Barrio Anta und Álvarez González 2005; CastañoSantamaría et al. 2013; Menéndez-Miguélez et al. 2016; Patrício und Nunes 2017). Sie charakterisieren die Bestände durch Angaben zum Bestandesvolumen und der mittleren Baumdimension in Abhängigkeit von der Bestandesoberhöhe und der Bestandesdichte (z. B. Stammzahl). Zur Quantifizierung der Bestandesdichte kommen gemäß Barrio Anta und Álvarez González (2005) bspw. der Bestandesdichteindex von Reineke (1933) oder der relative spacing index von Wilson (1946) (vgl. Relativer Baumabstand; Gadow 2003, S. 95) zum Einsatz.

Bei der Erstellung der Bestandesbehandlungsdiagramme auf Basis der vorläufigen Ertragstafeldaten der Baumarten Buche und Fichte gemäß dyn-ET (unveröff.) wird sich an der Methodik von Patrício und Nunes (2017) orientiert. Dabei bilden die Bestandesoberhöhe $H_{100}$ auf der Abszissenachse sowie die Stammzahl $N$ auf der Ordinatenachse (logarithmische Skalierung) den Ausgangspunkt der mathematischen Herleitung aller weiteren Kenngrößen. Diese werden über sogenannte Isolinien, d. h. Linien gleicher Werte, in die Bestandesbehandlungsdiagramme eingezeichnet und umfassen in Anlehnung an Patrício und Nunes (2017) den relativen Baumabstand, das verbleibende Bestandesvolumen sowie den Durchmesser des Grundflächemittelstammes im verbleibenden Bestand.

Der relative Baumabstand RS (engl.: relative spacing) wird als Bestandesdichtemaß rein rechnerisch aus der Bestandesoberhöhe $H_{100}$ und der Stammzahl $N$ bestimmt - ,mit zunehmender Stammzahl bei gleich bleibender Oberhöhe nimmt die Bestandesdichte zu“ und der relative Baumabstand ab (Gadow 2003, S. 95). Der relative Baumabstand ist unabhängig von der Standortsgüte sowie dem Bestandesalter (mit Ausnahme von sehr jungen Beständen) und durch die Einbeziehung der Oberhöhe zudem sehr gut zur Bestimmung von Durchforstungsintervallen 
geeignet (Patrício und Nunes 2017). In Anlehnung an Gadow (2003, Gl. 3-8) wird der relative Baumabstand definiert als:

$$
R S[\%]=\frac{\sqrt{\frac{10000}{N}}}{H_{100}} \cdot 100,
$$

$$
N, H_{100} \in \mathbb{R}^{>0} \text {, }
$$

wobei der Zähler nach Gadow (2003, S. 95) ,ein Schätzer für den durchschnittlichen Abstand zwischen benachbarten Bäumen [ist], dessen Genauigkeit ausreicht, wenn die Bäume auf der Fläche gleichmäßig verteilt sind und die Abstände zwischen benachbarten Bäumen nur gering variieren“(vgl. auch homogenes Bestandesmodell in Kapitel 2).

Für die Herleitung der Vorrats- und Durchmesserisolinien werden die beiden folgenden Funktionen an die vorläufigen Ertragstafeldaten der Baumarten Buche und Fichte gemäß dyn-ET (unveröff.) angepasst:

$$
D_{g}\left(N, H_{100}\right)=b_{0} \cdot N^{b_{1}} \cdot H_{100}{ }^{b_{2}},
$$

$$
b_{0}, b_{1}, b_{2} \in \mathbb{R}
$$

und

$$
\begin{aligned}
& \quad V_{B}\left(D_{g}, N, H_{100}\right)=b_{3} \cdot D_{g}^{b_{4}} \cdot H_{100}{ }^{b_{5}} \cdot N^{b_{6}}, \\
& D_{g} \in \mathbb{R}^{\geq 0}, \\
& b_{3}, b_{4}, b_{5}, b_{6} \in \mathbb{R},
\end{aligned}
$$

mit dem Durchmesser des Grundflächenmittelstammes $D_{g}$, dem Vorrat des verbleibenden Bestandes $V_{B}$, der Bestandesoberhöhe $H_{100}$, der Stammzahl $N$ sowie den Funktionsparametern $b_{0}$ bis $b_{6}$.

Besondere Herausforderung der nichtlinearen regressionsanalytischen Anpassung stellt dabei die Tatsache dar, dass der Durchmesser des Grundflächenmittelstammes $D_{g}$ einerseits abhängige Variable der Funktion [3-7] und andererseits unabhängige Variable der Funktion [3-8] ist. Aufgrund dieser Abhängigkeit besteht bei separater Regressionsanalyse beider Funktionen die Gefahr des sogenannten Simultaneous Equation Bias und damit der systematisch falschen Schätzung der Koeffizienten (Henningsen und Hamann 2007; Patrício und Nunes 2017). Daher erfolgt die Anpassung beider Funktionen in Anlehnung an Patrício und Nunes (2017) simultan, 
wobei auf die im R-Paket systemfit (Henningsen und Hamann 2007) enthaltene nlsystemfitFunktion mit dem 2SLS-Ansatz (Two-Stage Least Squares) zurückgegriffen wird. Die erzielte Anpassungsgüte wird im Folgenden anhand der Wurzel der mittleren Fehlerquadratsumme diskutiert.

Tabelle 3-8: Koeffizienten der Isolinien-Funktionen [3-7] und [3-8] auf Grundlage vorläufiger Ertragstafeldaten der Baumarten Buche und Fichte gemäß dyn-ET (unveröff.).

\begin{tabular}{lrrrrrrr}
\hline Baumart & $b_{0}$ & $b_{1}$ & $b_{2}$ & $b_{3}$ & $b_{4}$ & $b_{5}$ & $b_{6}$ \\
\hline Buche & 86,6820 & $-0,4641$ & 0,4939 & 5,6074 & $-0,2800$ & 1,8584 & $-0,2073$ \\
& & & & & & & \\
Fichte & 88,2758 & $-0,4233$ & 0,4873 & 0,7924 & $-0,5000$ & 2,6033 & $-0,0954$ \\
\hline
\end{tabular}

Die resultierenden Koeffizienten können Tabelle 3-8 entnommen werden und ermöglichen für die Darstellung der Durchmesser- und Vorratsisolinien in den Bestandesbehandlungsdiagrammen die Auflösung der Funktionen [3-7] und [3-8] nach der Stammzahl N (vgl. Barrio Anta und Álvarez González 2005, Gleichungen 4 und 5):

$$
N\left(D_{g}, H_{100}\right)=\left(\frac{D_{g}}{b_{0} \cdot H_{100} b_{2}}\right)^{\left(\frac{1}{b_{1}}\right)}
$$

und

$$
N\left(V_{B}, H_{100}\right)=\left(\frac{V_{B}}{b_{3} \cdot b_{0}{ }^{b_{4} \cdot H_{100}}\left(b_{2} \cdot b_{4}+b_{5}\right)}\right)^{\left(\frac{1}{b_{1} \cdot b_{4}+b_{6}}\right)} .
$$

Entsprechendes Einsetzen der gewünschten Vorrats- bzw. Durchmesserwerte ermöglicht schließlich das Abbilden der Isolinien (Abbildung 101 für die Buche und Abbildung 103 für die Fichte).

Mit diesen Isolinien ermöglichen die Bestandesbehandlungsdiagramme unabhängig von der Oberhöhenbonität und der waldbaulichen Behandlungsvariante eine Quantifizierung der Bestandesdichte sowie eine Schätzung des verbleibenden Bestandesvolumens und des mittleren Baumdurchmessers in Abhängigkeit von der Oberhöhe und Stammzahl des Bestandes. Die SDMDs sind damit als Werkzeug bei der Bewirtschaftung gleichaltriger Reinbestände zu verstehen, welches die tabellarische Darstellungsform forstlicher Ertragstafeln ergänzt - ihre Konstruktion in der vorliegenden Arbeit dient als erster Ansatz für weitergehende Analysen im Rahmen des dyn-ET-Projekts. Neben dem Einsatz in der forstlichen Praxis sieht der Autor auch 
ein hohes Potential für die forstliche Aus- und Fortbildung. Die grafische Darstellung der Zusammenhänge zwischen Oberhöhe, Dichte, Vorrat und mittlerem Einzelbaumdurchmesser dient dabei einem tiefergehenden Verständnis grundlegender waldwachstumskundlicher Beziehungen.

Entscheidend für den ergänzenden Einsatz in der forstlichen Praxis und Lehre wird aber u. a. sein, inwiefern die Isolinien über alle Oberhöhenbonitäten und Behandlungsvarianten hinweg den tatsächlichen Ertragstafeldaten entsprechen, d. h. wie hoch die Anpassungsgüte der oben beschriebenen simultanen, nichtlinearen Regressionsanalyse an die Ausgangsdaten ist.

Als Gütemaß wird in dieser Arbeit die Wurzel der mittleren Fehlerquadratsumme (RMSE; Root Mean Square Error; vgl. Fahrmeir et al. 2013, S. 144 ff.) genutzt. Sie beschreibt die durchschnittliche Abweichung ${ }^{72}$ zwischen Schätzung, d. h. der über die Funktionen [3-7] und [3-8] mit den Koeffizienten der Tabelle 3-8 berechneten Vorrats- und Durchmesserwerte, und den Ertragstafeldaten gemäß dyn-ET (unveröff.). Im Verhältnis zum Mittelwert der Ausgangsdaten ergibt sich die relative Wurzel der mittleren Fehlerquadratsumme (rel.RMSE), wodurch die unterschiedlichen Größenordnungen der beiden Bestandesmerkmale herausgerechnet werden.

Tabelle 3-9: Absolute (RMSE) und relative (rel.RMSE) Wurzel der mittleren Fehlerquadratsumme als Gütemaße für die Regressionsanalyse der Isolinien-Funktionen [3-7] und [3-8] auf Grundlage vorläufiger Ertragstafeldaten der Baumarten Buche und Fichte gemäß dyn-ET (unveröff.).

Vorrat (verbl. Bestand)

Durchmesser (verbl. Bestand)

Baumart

RMSE

rel. RMSE

RMSE

rel. RMSE

\begin{tabular}{|c|c|c|c|c|}
\hline Buche & $29,38 \mathrm{Vfm} \mathrm{ha}^{-1}$ & $9,38 \%$ & $1,43 \mathrm{~cm}$ & $4,69 \%$ \\
\hline Fichte & $69,78 \mathrm{Vfm} \mathrm{ha}^{-1}$ & $18,28 \%$ & $1,61 \mathrm{~cm}$ & $5,42 \%$ \\
\hline
\end{tabular}

Insgesamt wird die Anpassungsgüte mit Blick auf die Anpassungsgüte anderer nichtlinearer Schätzungen forstlicher Zusammenhänge als überwiegend mäßig bis gut eingeschätzt (vgl. z. B. die RMSE in Patrício und Nunes 2017, Tab. 2). Mit 29,4 Vfm ha ${ }^{-1}$ (Buche) bzw.

\footnotetext{
${ }^{72}$ Grafisch interpretiert werden kann diese durchschnittliche Abweichung in Abbildung 102 (Buche) bzw. Abbildung 104 (Fichte) anhand der dort dargestellten Oberhöhen-Stammzahl-Entwicklungen. Es gilt die Frage zu beantworten, wie weit die über die Isolinien dargestellten Prognosewerte im Mittel von den tatsächlichen Werte der entsprechenden Oberhöhen-Stammzahl-Kombinationen abweichen.
} 
69,8 Vfm ha ${ }^{-1}$ (Fichte) weicht die Prognose des Vorrats um 9,4 \% bzw. 18,3 \% vom Mittelwert der vorläufigen Ertragstafeldaten gemäß dyn-ET (unveröff.) ab (Tabelle 3-9) ${ }^{73}$. Die ggü. dem Durchmesser höheren relativen Abweichungen der Vorratsanpassungen werden dabei u. a. auf die bereits beschriebene Abhängigkeit des Vorrats vom Durchmesser zurückgeführt.

Bei der Analyse der resultierenden Bestandesbehandlungsdiagramme zeigt sich baumartenübergreifend, dass bei gleicher Oberhöhe die Stammzahl des verbleibenden Bestandes mit steigender Oberhöhenbonitäten zunimmt, was mit sinkenden relativen Baumabständen einhergeht. Zudem sind die stets höheren Stammzahlen der mäßigen Hochdurchforstung im Vergleich zu den anderen Behandlungsvarianten bei gleicher Oberhöhe ersichtlich, was mit (überwiegend) niedrigeren mittleren Durchmessern im verbleibenden Bestand korrespondiert und bei der Fichte teils deutlich stärker ausgeprägt ist als bei der Buche (vgl. Abbildungen 76 und 98). Während die verbleibenden Bestandesvolumina bei der Buche mit sinkender Oberhöhenbonität leicht steigen (im Uhrzeigersinn gekippte Vorrats-Isolinien in Abbildung 102; vgl. Abbildung 168 im Anhang), charakterisieren die entgegen des Uhrzeigersinns gekippten VolumenIsolinien der Fichte in Abbildung 104 leicht sinkende Vorräte bei sinkender Standortsgüte (vgl. Abbildung 184 im Anhang). An dieser Stelle werden in Abbildung 102 allerdings auch Schwächen der regressionsanalytischen Anpassung ersichtlich. Die insb. bei hohen Oberhöhen stark im Uhrzeigersinn gekippten Vorrats-Isolinien weichen systematisch von dem tatsächlichen Verlauf des Vorrates über den Oberhöhen $\mathrm{ab}^{74}$ und zeigen somit nicht die korrekten Relationen zwischen den waldbaulichen Behandlungsvarianten. Die bei gleicher Oberhöhe relativ höheren

\footnotetext{
${ }^{73}$ Mittels separater, nichtlinearer Regressionsanalyse über die nlxb-Funktion aus dem R-Paket nlmrt Nash (2016) konnte die Wurzel der mittleren Fehlerquadratsumme bspw. für den Vorrat der Baumart Fichte auf 53,77 Vfm ha ${ }^{-1}$ reduziert werden. Dieser leichten Verbesserung der Anpassungsgüte steht aber bspw. die Vernachlässigung des oben beschriebenen Simultaneous Equation Bias und damit bekannter Funktionszusammenhänge gegenüber.

${ }^{74}$ Systematische Abweichungen einzelner Zusammenhänge sind bei simultanen Regressionen nicht ungewöhnlich bzw. Konsequenz des Ziels simultaner Methoden. Da bei der simultanen Anpassung mehrerer Funktionen, die Abweichungsquadratsumme aller Zusammenhänge gleichzeitig minimiert wird, können große Abweichungen eines Zusammenhangs bei relativ kleinen Abweichungen eines anderen Zusammenhangs zu systematischen Abweichungen führen. Dies ist bei der simultanen Regression explizit erwünscht. Das deutliche Abkippen der Vorrats-Isolinien im Uhrzeigersinn kann per separater, nichtlinearer Regressionsanalyse über die nlxb-Funktion aus dem R-Paket nlmrt Nash (2016) für den Vorrat der Baumart Buche zwar verhindert werden $\left(R M S E=28,33 \mathrm{Vfm} \mathrm{ha}^{-1}\right)$, vernachlässigt aber den oben bereits beschriebenen Simultaneous Equation Bias und damit bekannte Funktionszusammenhänge. Insofern sind gelegentliche systematische Abweichungen eine Konsequenz der angewandten Methodik und weisen darauf hin, dass es (auch in diesem Beispiel) tatsächlich abhängige Fehler zwischen den Regressionsfunktionen gibt - es beim separaten Schätzen somit zu einem Simultaneous Equation Bias kommt.
} 
Bestandesvolumina bei starker Hochdurchforstung entsprechen nicht dem Charakter dieser Behandlungsvariante (vgl. Abbildung 168 im Anhang). Demnach wird der Übergang der Grundflächenhaltung ab einer Oberhöhe von $H_{100}=24 \mathrm{~m}$, welcher die gestaffelte Hochdurchforstung definiert, auch nur über die Stammzahlen und nicht über die Vorrats-Isolinien korrekt abgebildet (Abbildung 102). Bis zu dieser Oberhöhe liegen die Stammzahlen der gestaffelten Hochdurchforstung auf etwa einem Niveau mit oder leicht unterhalb von der starken Hochdurchforstung - mit darüber ansteigender Oberhöhe gleichen sich die Stammzahlen bei gestaffelter Hochdurchforstung zunehmend der mäßigen Hochdurchforstung an. 


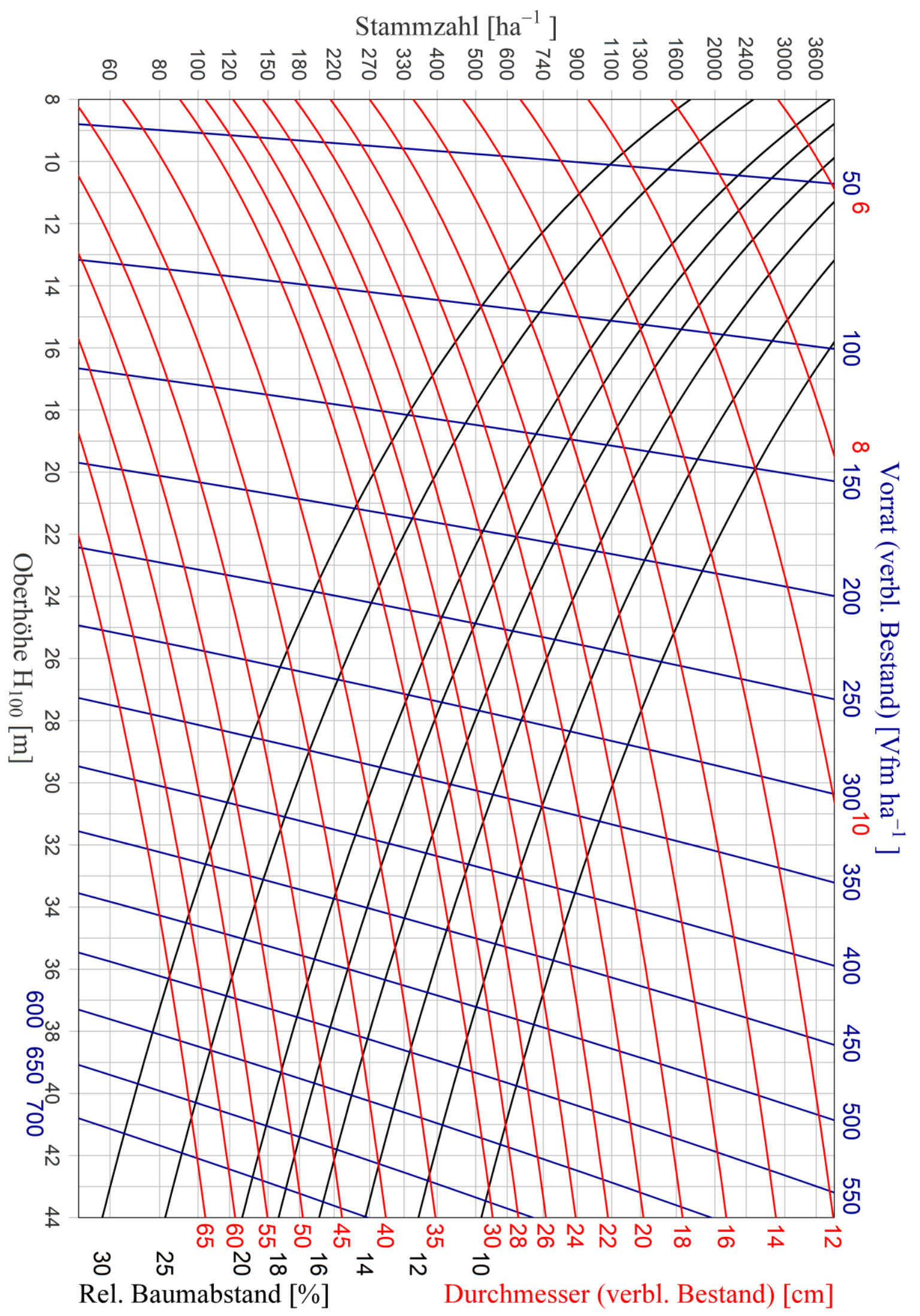

Abbildung 101: Bestandesbehandlungsdiagramm der Baumart Buche; hergeleitet auf Grundlage vorläufiger Ertragstafeldaten nach dyn-ET (unveröff.); relativer Baumabstand, verbleibendes Bestandesvolumen und mittlerer Baumdurchmesser des verbleibenden Bestandes in Abhängigkeit von Oberhöhe und Stammzahl (logarithmische Skalierung). 


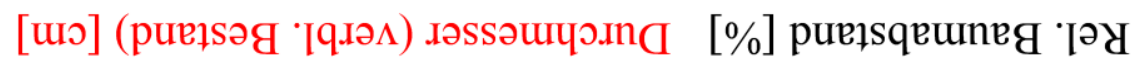

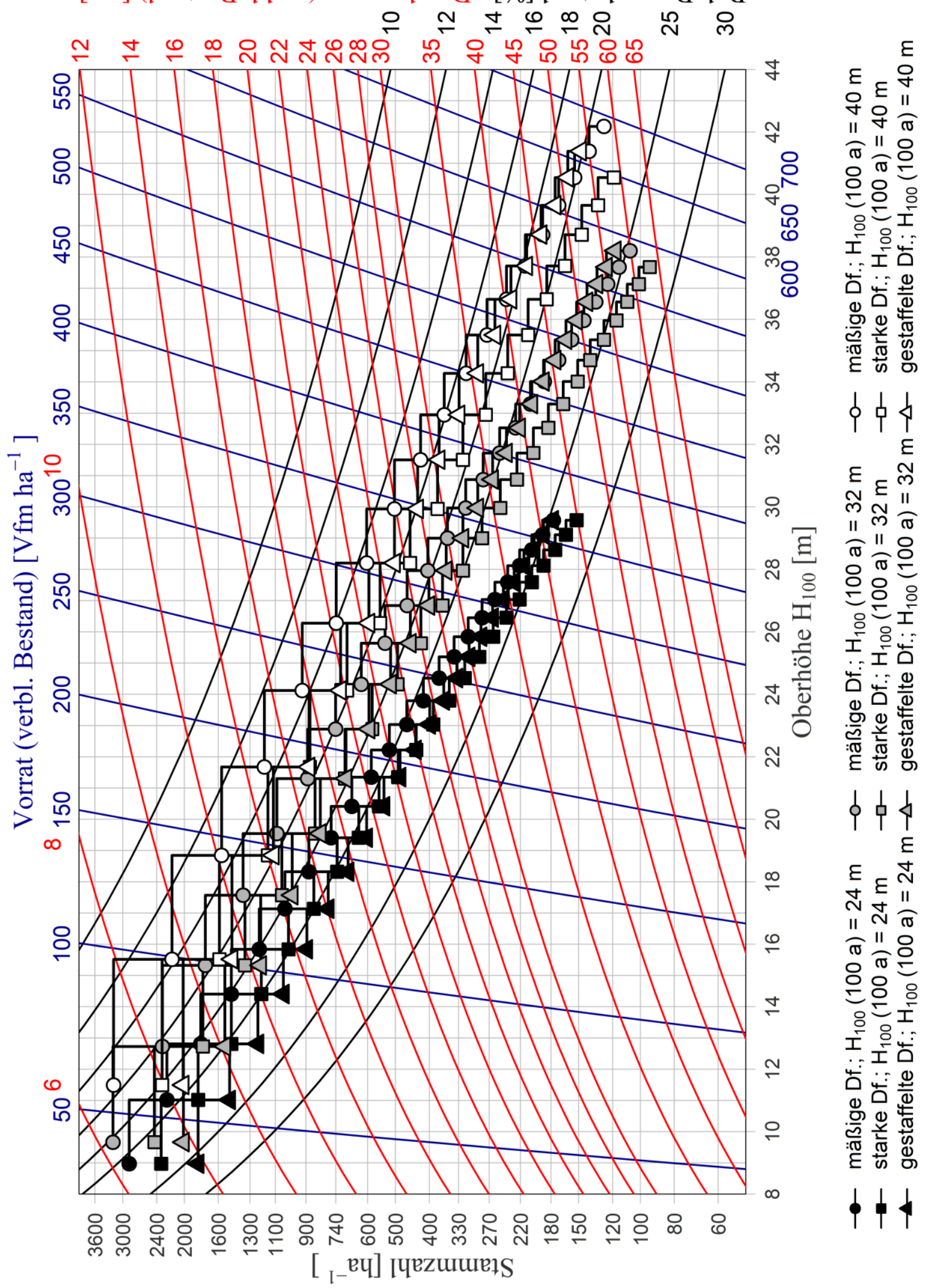

Abbildung 102: Bestandesbehandlungsdiagramm der Baumart Buche; hergeleitet auf Grundlage vorläufiger Ertragstafeldaten nach dyn-ET (unveröff.); relativer Baumabstand, verbleibendes Bestandesvolumen und mittlerer Baumdurchmesser des verbleibenden Bestandes in Abhängigkeit von Oberhöhe und Stammzahl (logarithmische Skalierung); zusätzlich eingezeichnet sind die Oberhöhen-Stammzahl-Entwicklungen aller Behandlungsvarianten bei ausgewählten Oberhöhenbonitäten. 


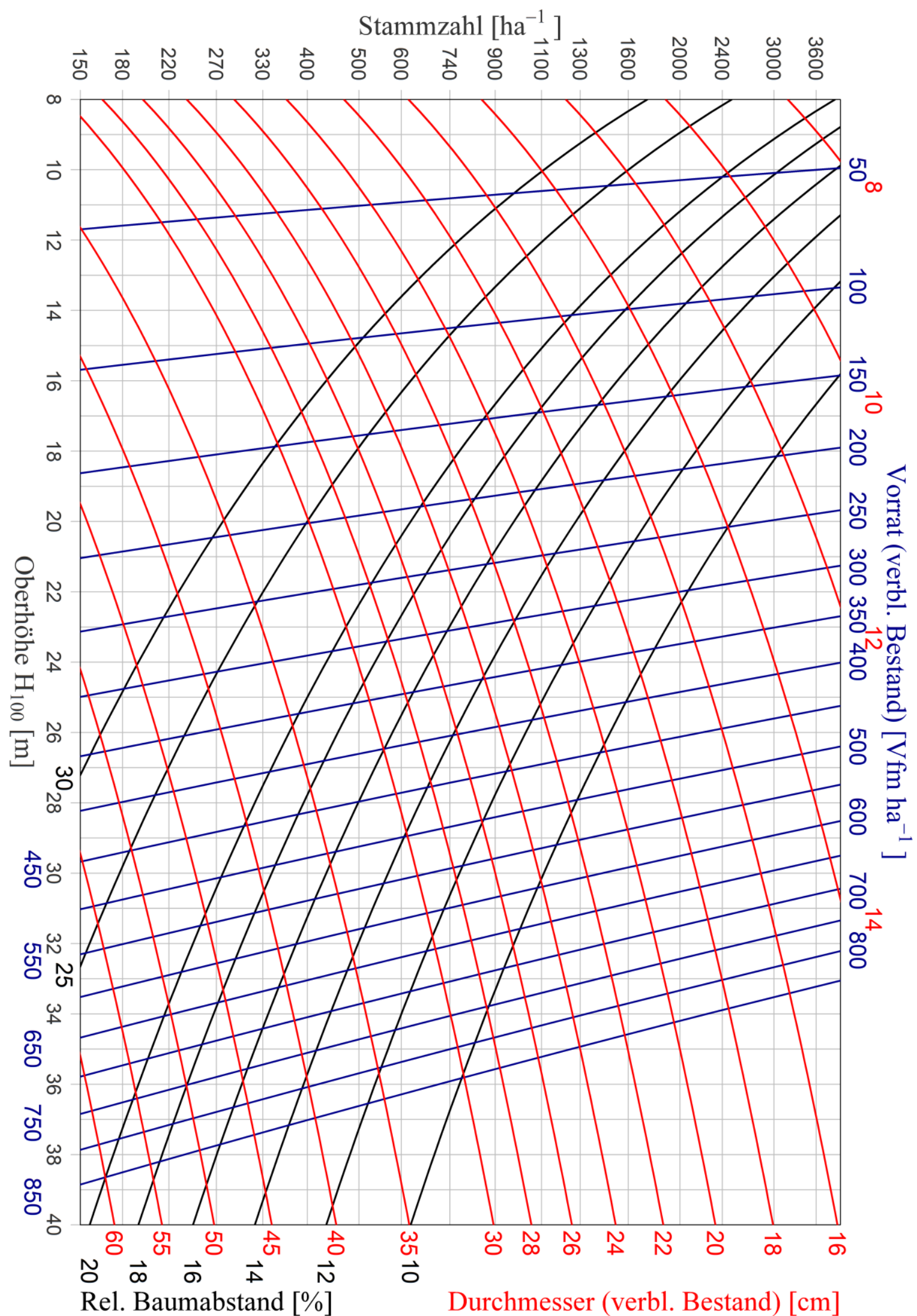

Abbildung 103: Bestandesbehandlungsdiagramm der Baumart Fichte; hergeleitet auf Grundlage vorläufiger Ertragstafeldaten nach dyn-ET (unveröff.); relativer Baumabstand, verbleibendes Bestandesvolumen und mittlerer Baumdurchmesser des verbleibenden Bestandes in Abhängigkeit von Oberhöhe und Stammzahl (logarithmische Skalierung). 


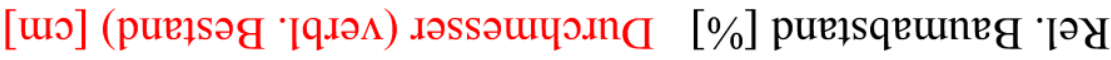

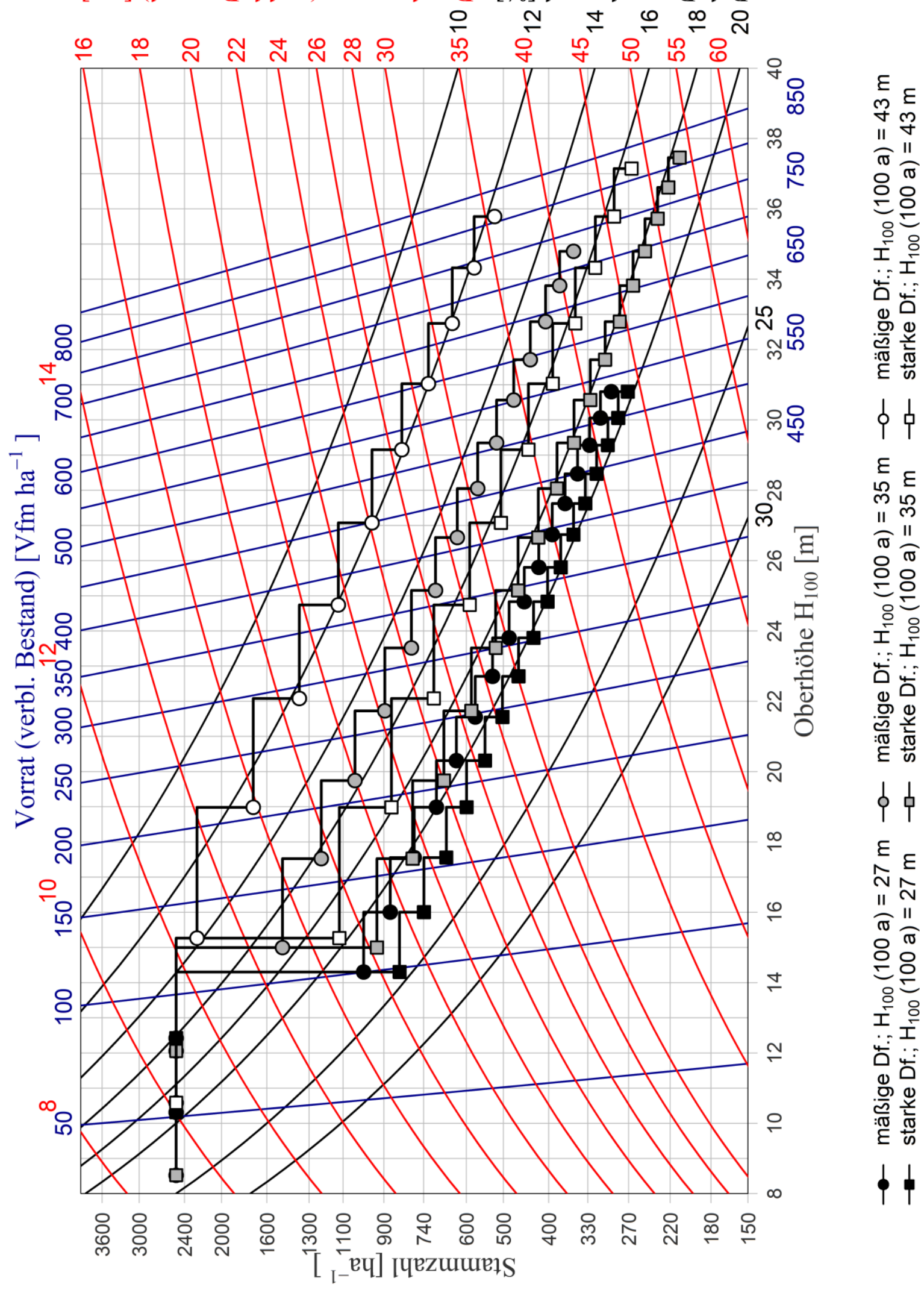

Abbildung 104: Bestandesbehandlungsdiagramm der Baumart Fichte; hergeleitet auf Grundlage vorläufiger Ertragstafeldaten nach dyn-ET (unveröff.); relativer Baumabstand, verbleibendes Bestandesvolumen und mittlerer Baumdurchmesser des verbleibenden Bestandes in Abhängigkeit von Oberhöhe und Stammzahl (logarithmische Skalierung); zusätzlich eingezeichnet sind die Oberhöhen-Stammzahl-Entwicklungen aller Behandlungsvarianten bei ausgewählten Oberhöhenbonitäten. 


\subsection{5 Ökonomische Bewertung}

Aufbauend auf Abschnitt 3.3.3 erfolgt die ökonomische Bewertung ${ }^{75}$ der vorläufigen Ertragstafeldaten nach dyn-ET (unveröff.) mittels modular aufgebautem, automatisiertem R-Modell

(R Core Team 2019).

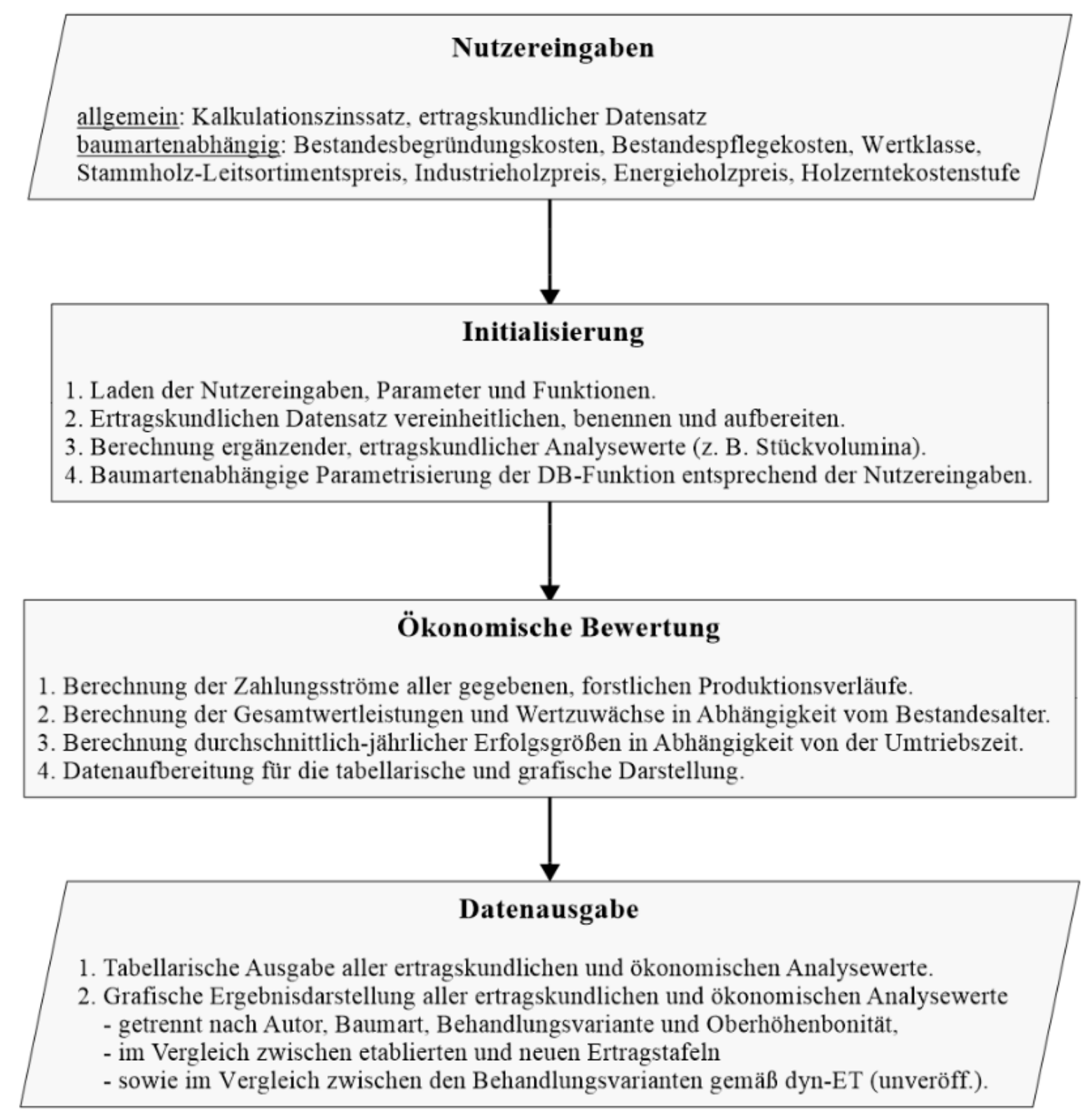

Abbildung 105: Ablaufdiagramm der ökonomischen Bewertung mittels automatisiertem R-Modell in Anlehnung an Groß (2019, S. 28). Parallelogramm: Nutzereingabe und Datenausgabe; Rechteck: Modellschritte.

${ }^{75}$ Die ökonomische Bewertung findet ausschließlich in Bezug auf die forstwirtschaftliche Nutzung des Holzvolumens statt - weitere Waldfunktionen oder Wechselwirkungen, die durch diese entstehen, bleiben unberücksichtigt (siehe Abschnitt 3.3.7). 
Das R-Modell greift auf die Ertragstafeldaten zu, bereitet diese für die weiterführende Anwendung vor und führt schließlich in Verbindung mit allgemeinen und baumartenspezifischen Nutzereingaben die ökonomische Bewertung inkl. Vergleichsanalysen durch (Abbildung 105).

Die Einbindung der dyn-ET-Daten in das R-Modell erfolgt mittels SQLite-Bibliothek. Die Daten der etablierten Ertragstafelwerke für die Baumarten Buche und Fichte aus Schober (1995) wurden von Groß (2019) digitalisiert und werden im CSV-Dateiformat in das R-Modell eingebunden. Es erfolgt für die weitere Verarbeitung eine Vereinheitlichung von Struktur und Benennung der Daten aus dyn-ET (unveröff.) und Schober (1995).

Die allgemeinen und baumartenspezifischen Nutzereingaben können im R-Modell (innerhalb modellbedingter Grenzen) frei gewählt werden und sind in dieser Arbeit so gesetzt, dass eine realistische Bewertung stattfindet, die das ökonomische Potential der Baumarten im langjährigen Mittel ${ }^{76}$ widerspiegelt. Es kommt dabei für die folgenden Analysen weniger auf die absolute Höhe als vielmehr auf die Wertrelationen an.

Es werden Kosten für eine künstliche Bestandesbegründung (Kulturkosten) angenommen, welche sich in Anlehnung an Bodelschwingh (2018, S. 72) baumartenabhängig an der Kulturkostenstufe 5 („Pflanzung mit mittlerem Aufwand“) nach NLF (2014, Tabelle 1.13) orientieren (Tabelle 3-10). Ebenfalls in Anlehnung an Bodelschwingh (2018, S. 73) werden Bestandespflegekosten im Alter von 10 Jahren und bei der Buche zusätzlich im Alter von 20 Jahren angenommen (Tabelle 3-10).

Tabelle 3-10: Baumartenabhängige Bestandesbegründungs- und Bestandespflegekosten in Anlehnung an Bodelschwingh (2018, S. 72 f.).

\begin{tabular}{lrrr}
\hline $\begin{array}{r}\text { Bestandes- } \\
\text { begründungskosten } \\
{\left[€ \mathrm{ha}^{-1}\right]}\end{array}$ & $\begin{array}{r}\text { Pflegekosten } \\
(\text { Alter: 10 Jahre) } \\
{\left[€ \mathrm{ha}^{-1}\right]}\end{array}$ & $\begin{array}{r}\text { Pflegekosten } \\
\text { (Alter: 20 Jahre) } \\
{\left[€ \mathrm{ha}^{-1}\right]}\end{array}$ \\
\hline Buche & 8.800 & 700 & 500 \\
Fichte & 3.400 & 500 & - \\
\hline
\end{tabular}

\footnotetext{
${ }^{76}$ Die stark kalamitätsbeeinflussten Daten der forstwirtschaftlichen Extremjahre 2018 und 2019 werden bewusst nicht berücksichtigt, um bspw. bei der Sensitivitätsanalyse mit Überlebenswahrscheinlichkeiten in Abschnitt 3.3.6 keine Doppelberücksichtigung von kalamitätsbedingten Nachteilen vorzunehmen.
} 
Die Bewertung geernteter Vor- und Endnutzungsvolumina erfolgt über eine Deckungsbeitragsfunktion, welche den Ausnutzungsgrad sowie die Holzerlöse und Holzerntekosten dimensionsabhängig berücksichtigt. Es wird für den dimensionsabhängigen Deckungsbeitrag $P\left(D_{g}\right)$ im Sinne eines holzerntekostenfreien Holzerlöses frei Waldstraße (Deckungsbeitrag) folgende Chapman-Richards-Funktion

$$
\mathrm{P}\left(D_{g}\right)=\delta_{1} \cdot\left(1-e^{\left(-\delta_{2} \cdot D_{g}\right)}\right)^{\delta_{3}}+\delta_{4}
$$

mit dem Durchmesser des Grundflächenmittelstammes $D_{g}$ in cm sowie den baumartenspezifisch hergeleiteten Funktionsparametern $\delta_{1}, \delta_{2}, \delta_{3}$ und $\delta_{4}$ genutzt. Ziel ist eine Deckungsbeitragsfunktion, die einerseits direkt auf die Volumeneinheit der Ertragstafeln, den Vorratsfestmeter, angewendet werden kann und andererseits durch die freie Eingabe von Leitsortiments-, Industrie- und Energieholzpreisen zukünftig an aktuelle Entwicklungen des Holzmarktes angepasst werden kann. Grundlage der Parametrisierung von Funktion [3-11] mittels nlxb-Funktion aus dem R-Paket nImrt (Nash 2016) bilden in dieser Arbeit Sortentafeln nach Offer und Staupendahl (2018), Holzpreismatrizen nach Bodelschwingh (2018, Anhang 3), mittlere Leitsortiment- und Industrieholzpreise nach Schade (2019, S. 118) sowie Holzerntekosten nach Bodelschwingh (2018, S. 64 ff.).

Aus den Sortentafeln nach Offer und Staupendahl (2018) werden baumarten- und dimensionsabhängig in $2 \mathrm{~cm}$-Schritten der Ausnutzungsgrad ${ }^{77}$ sowie die Volumenanteile der Stammholzsortimente (Kombination aus Güte- und Stärkeklasse), des Industrie- und Energieholzes sowie des nicht verwerteten Derbholzes entnommen. Dabei wird als Referenz die Wertklasse 2 (durchschnittliche Qualitätsverhältnisse ${ }^{78}$ ) ohne vorkommende Schäle genutzt und es wird baumartenübergreifend davon ausgegangen, dass die Aufarbeitung bis zu einem Durchmesser des Grundflächenmittelstammes von $D_{g}=40 \mathrm{~cm}$ hochmechanisiert, darüber hinaus motormanuell erfolgt (vgl. Groß 2019, S. 16).

\footnotetext{
${ }^{77}$ Der Ausnutzungsgrad berücksichtigt den Rindenabzug sowie Ernteverluste durch z. B. den Wurzelanlauf, Trennschnitte, Zugaben und das forstliche Abrunden (Englert et al. 2018; Möhring et al. 2018, Abschnitt 5-9). Damit werden die in den Ertragstafeldaten angegebenen stehenden Vorratsfestmeter mit Rinde (Derbholz) auf verwertbares und nicht verwertbares Derbholzvolumen in Erntefestmeter ohne Rinde reduziert.

${ }^{78}$ Die drei von Offer und Staupendahl 2018 angegebenen Wertklassen repräsentieren nach Offer und Staupendahl (2009, S. 18) ,überdurchschnittliche“ (Wertklasse 1), „durchschnittliche“ (Wertklasse 2) und „unterdurchschnittliche“ (Wertklasse 3) Bestandesqualitäten.
} 
Für das von Offer und Staupendahl (2018) angegebene nicht verwertete Derbholzvolumen (vgl. Englert et al. 2018) werden in Anlehnung an Bodelschwingh (2018, S. 60) weder Holzerlöse noch Holzerntekosten angesetzt - eine energetische Nutzung der nicht verwerteten Derbholzvolumina wird somit bspw. nicht angenommen. Für separat ausgewiesenes Energieholz wird im Sinne der Brennholzselbstwerbung ein einheitlicher Brennholzpreis unterstellt - Holzerntekosten fallen nicht an. Die angesetzten Brennholpreise wurden in Anlehnung an Bodelschwingh (2018, ergänzende Auskunft des Autors) hergeleitet und können Tabelle 3-11 entnommen werden. Bei dem von Offer und Staupendahl (2018) ausgewiesenen Industrieholz wird in Anlehnung an Bodelschwingh (2018, S. 60) auf eine Trennung zwischen den Sorten Industrieholzlang (IL) und Industrieholz-kurz/Industrieschichtholz (IS) sowie eine preisliche Differenzierung zwischen Güteklassen verzichtet. Die Industrieholzpreise (Tabelle 3-11) entsprechen den mittleren Industrieholzpreisen (IL und IS bei Nadelhölzern; gerundet) aus Daten des Hessischen Staatswaldes der Jahre 2013 - 2017 nach Schade (2019, S. 124 bzw. 124).

Tabelle 3-11: Preise für das jeweils in Klammern angegebene Stammholzleitsortiment als mittlerer Wert (gerundet) aus Daten des Hessischen Staatswaldes der Jahre 2013 - 2017 nach Schade (2019, S. 118); Industrieholzpreise als mittlerer Wert (IS und IL bei Nadelhölzern; gerundet) aus Daten des Hessischen Staatswaldes der Jahre 2013 - 2017 nach Schade (2019, S. 124); Brennholz- bzw. Energieholzpreise (gerundet) in Anlehnung an Bodelschwingh (2018).

\begin{tabular}{lrrr}
\hline Baumart & $\begin{array}{r}\text { Leitsortimentspreis } \\
{\left[€ \mathrm{Efm}^{-1}\right]}\end{array}$ & $\begin{array}{r}\text { Industrieholzpreis } \\
{\left[€ \mathrm{Efm}^{-1}\right]}\end{array}$ & $\begin{array}{r}\text { Brennholzpreis } \\
{\left[€ \mathrm{Efm}^{-1}\right]}\end{array}$ \\
\hline Buche & $90(\mathrm{~B} 4)$ & 48 & 34 \\
Fichte & $94(\mathrm{~B} 2 \mathrm{~b})$ & 38 & 22 \\
\hline
\end{tabular}

Die preisliche Differenzierung zwischen den Stärke- und Güteklassen des Stammholzvolumens erfolgt über mittlere Leitsortimentspreise (Tabelle 3-11) im Hessischen Staatswald der Jahre 2013 - 2017 (gerundet) nach Schade (2019, S. 118) sowie Wertmatrizen nach Bodelschwingh (2018, Anhang 3 i. V. m. S. 63), welche die Stammholzsortimentspreise in Relation zum baumartenabhängigen Leitsortimentspreis angeben. Es ergeben sich Preismatrizen für die Stammholzsortimente die der Tabelle 8-1 im Anhang entnommen werden können.

Im Summenprodukt mit den Stammholzsortimentsanteilen nach Offer und Staupendahl (2018) ergibt sich der durchschnittliche Stammholzerlös je $2 \mathrm{~cm}$-Schritt des Durchmessers des Grundflächenmittelstammes. Für den Verkaufserlös frei Waldstraße wird der Industrieholzerlös als 
Produkt aus Industrieholzpreis (Tabelle 3-11) und Industrieholzanteil nach Offer und Staupendahl (2018) addiert. Für dieses frei Waldstraße verkaufte Holzvolumen fallen Holzerntekosten an, die nach dem Kostenmodell von Bodelschwingh (2018, S. 65 ff.) hergeleitet werden. Hierbei wird die Kostenstufe 1, d. h. eine „normale Holzernte im befahrbaren Gelände“ mit einer Kombination von motormanuellen und maschinellen Verfahren, angenommen (Bodelschwingh 2018, S. 66).

Die Brennholzerlöse aus Selbstwerbung werden hinzuaddiert und es ergibt sich unter Berücksichtigung der Ausnutzungsgrade und des nicht verwerteten Derbholzes nach Offer und Staupendahl (2018) eine Datenreihe durchschnittlicher erntekostenfreien Holzerlöse in $2 \mathrm{~cm}$ Schritten des Durchmessers des Grundflächenmittelstammes zwischen $10 \mathrm{~cm}$ und $70 \mathrm{~cm}$ (Fichte) bzw. $80 \mathrm{~cm}$ (Buche). Die aus der Funktionsparametrisierung mittels nlxb-Funktion aus dem R-Paket nlmrt (Nash 2016) resultierenden Koeffizienten der Ausgleichsfunktion [3-11] können Tabelle 3-12 entnommen werden und Abbildung 106 zeigt die entsprechenden Funktionsverläufe über den Datenpunkten ${ }^{79}$.

Tabelle 3-12: Koeffizienten der Chapman-Richards-Deckungsbeitragsfunktion [3-11], hergeleitet über Sortentafeln nach Offer und Staupendahl (2018), Holzpreismatrizen nach Bodelschwingh (2018, Anhang 3), mittlere Leitsortiment- und Industrieholzpreise nach Schade (2019, S. 118 bzw. 124), Brennholz- bzw. Energieholzpreise (gerundet) in Anlehnung an Bodelschwingh (2018) sowie Holzerntekosten nach Bodelschwingh (2018, S. 64 ff.).

\begin{tabular}{lrrrr}
\hline Baumart & $\delta_{1}$ & $\delta_{2}$ & $\delta_{3}$ & $\delta_{4}$ \\
\hline Buche & 943,088665 & 0,018691 & 0,036049 & $-887,609469$ \\
Fichte & 160,784217 & 0,088489 & 0,960817 & $-106,027291$ \\
\hline
\end{tabular}

Durch Multiplikation der entsprechenden Deckungsbeiträge mit den Volumina des ausscheidenden Bestandes (Vornutzungswerte) sowie dem verbleibenden Bestandesvolumen (Abtriebswerte) im Alter der Endnutzung entstehen je Ertragstafeltabelle und für alle möglichen Endnutzungszeitpunkte spezifische Zahlungsreihen. Die kumulierte Summe einer Zahlungsreihe ergibt die Gesamtwertleistung und aus dem periodischen Differenzenquotient resultiert der laufende Bestandeswertzuwachs zwischen zwei Zeitpunkten (rückblickend). In Relation zum Abtriebs-

\footnotetext{
${ }^{79}$ Sinkende Deckungsbeiträge bei hohen Einzelbaumdimensionen, bspw. durch Rotkernbildung bei der Buche (vgl. z. B. Bodelschwingh 2018, S. 97) oder reduzierten Absatzmöglichkeiten bei der Fichte (vgl. z. B. Kohnle und Teuffel 2020), werden nicht berücksichtigt.
} 
wert des verbleibenden Bestandes zu Beginn der jeweiligen Periode ergeben sich Wertzuwachsraten. Als jährliche Erfolgsgröße unter der Berücksichtigung von Kapitalknappheit wird die Brutto-Bodenrente genutzt - sie stellt unter der Prämisse des vollkommenen Kapitalmarkts den durchschnittlichen Überschuss der forstlichen Produktion (vgl. Abschnitt 2.2.5) als leicht zu interpretierende ökonomische Entscheidungsgröße dar (vgl. Möhring et al. 2006). Als konstanter Kalkulationszinssatz wird in Anlehnung an Möhring (2001) $r=1,5 \%$ gewählt, wobei dieser nicht zwangsläufig als externer Zinssatz zu verstehen ist, sondern im Sinne eines internen Grenzzinssatzes auch entscheidungsorientiert als Rendite einer innerbetriebliche Investitionsalternative angesehen werden kann (vgl. u. a. Möhring 1994, S. 44 ff.; Möhring und Rüping 2006, S. 6; Möhring 2014; Gerst 2015, S. 46 ff.).

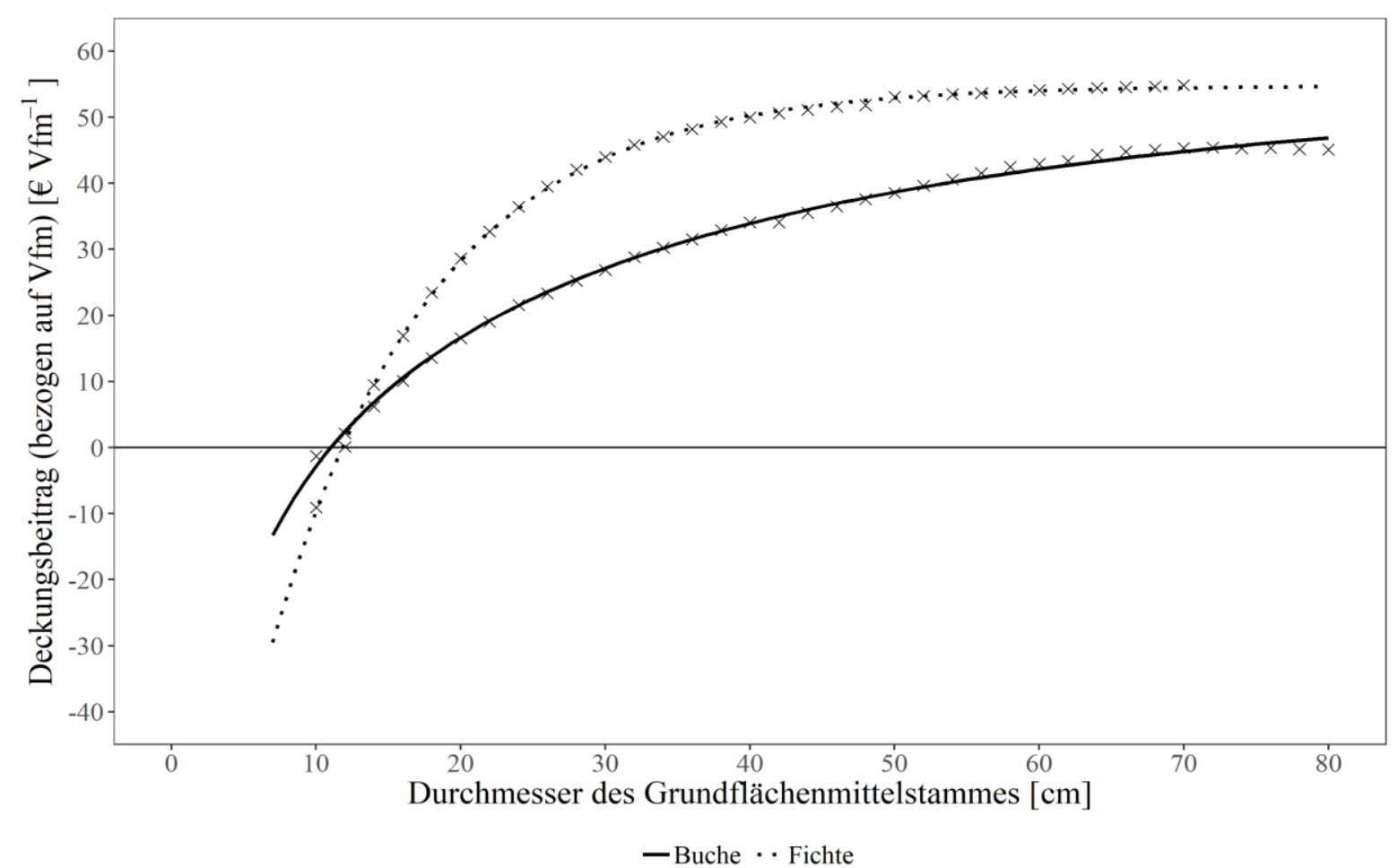

Abbildung 106: Baumartenspezifische, dimensionsabhängige Deckungsbeiträge (erntekostenfreie Holzerlöse) [€ $\mathrm{Vfm}^{-1}$; Datenpunkte in $2 \mathrm{~cm}$-Schritten hergeleitet über Sortentafeln nach Offer und Staupendahl (2018), Holzpreismatrizen nach Bodelschwingh (2018, Anhang 3), mittlere Leitsortiment- und Industrieholzpreise nach Schade (2019, S. 118 bzw. 124), Brennholz- bzw. Energieholzpreise (gerundet) in Anlehnung an Bodelschwingh (2018) sowie Holzerntekosten nach Bodelschwingh (2018, S. 64 ff.); Ausgleichsfunktionen gemäß [3-11] mit Koeffizienten entsprechend Tabelle 3-12. 
Analog zur Analyse der vorläufigen Ertragstafeldaten in Abschnitt 3.3.3 werden die ökonomischen Bewertungsergebnisse im Folgenden für die beiden Baumarten getrennt (Buche ab Seite 192, Fichte ab Seite 206) aufgezeigt:

- Das ökonomische Leistungsspektrum der mäßigen Hochdurchforstung als Basis-Behandlungsvariante wird anhand der Gesamtwertleistungen und laufenden Wertzuwächse aller Oberhöhenbonitäten abgebildet. Ergänzend werden die entsprechenden Abbildungen für die starke und gestaffelte (nur Buche) Hochdurchforstung im Anhang gezeigt. Für eine tabellarische Gegenüberstellung ausgewählter ökonomischer Bewertungsergebnisse wird analog zur Analyse der vorläufigen Ertragstafeldaten neben den Kulminationszeitpunkten der laufenden Bestandeswertzuwächse die Alter bzw. Umtriebszeiten 100 a (Buche) und 60 a (Fichte) gewählt, da für diese Alter jeweils Ertragstafeldaten aller Oberhöhenbonitäten zur Verfügung stehen.

- Ein Vergleich zu etablierten Ertragstafelwerken (Buche: Schober 1995, mäßige Durchforstung; Fichte: Wiedemann 1936/42, mäßige Durchforstung) wird in Anlehnung an die Analyse der vorläufigen Ertragstafeldaten bei vergleichbaren Oberhöhenbonitäten anhand der Gesamtwertleistungen, der laufenden Bestandeswertzuwächse und Abtriebswerte sowie den daraus resultierenden Wertzuwachsraten, zwei Kennziffern einer nachhaltigen Betriebsklasse (durchschnittliche waldbauliche Überschüsse und durchschnittliche Abtriebswerte) sowie den Brutto-Bodenrenten gezogen. Die entsprechenden Abbildungen des Vergleichs der starken Durchforstungen werden im Anhang gezeigt. Für eine tabellarische Gegenüberstellung ausgewählter Bewertungsergebnisse werden analog zur Analyse der vorläufigen Ertragstafeldaten neben den Kulminationszeitpunkten der laufenden Bestandeswertzuwächse Alter bzw. Umtriebszeiten in Anlehnung an etablierte Umtriebszeiten (vgl. z. B. NLF 2014, Tabelle 1.1) gewählt ${ }^{80}-$ 140 a (Buche) und 80 a (Fichte).

\footnotetext{
${ }^{80}$ Es wird sich im Rahmen des Vergleichs ökonomischer Bewertungsergebnisse aus zwei Gründen bewusst für die Verwendung baumartenspezifisch konstanter Umtriebszeiten und somit gegen die jeweilig optimalen Umtriebszeiten (vgl. z. B. Bodelschwingh 2018, S. 121 ff.) entschieden: Erstens, steigen sowohl die durchschnittlichen waldbaulichen Überschüsse als auch die Brutto-Bodenrenten mit den gewählten Eingangsdaten und Modellprämissen nahezu ausnahmslos bis zum höchsten Alter der verfügbaren Ertragstafeldaten an. Es würden also keine optimalen, sondern lediglich modell-maximale Umtriebszeiten verglichen, welche nur über die zur Verfügung stehenden vorläufigen Ertragstafeldaten determiniert und nicht ökonomisch begründet wären. Zweitens, geht es bei den hier getätigten Analysen um den Vergleich gegebener waldbaulicher Behandlungsalternativen. Die Verwendung unterschiedlicher, jeweils optimaler, Umtriebszeiten würde den Erkenntnisgewinn nur steigern, sofern sich diese innerhalb der Modellgrenzen aus ökonomischen Gründen maßgeblich unterscheiden würden.
} 
- Die ökonomischen Auswirkungen der unterschiedlichen waldbaulichen Behandlungsvarianten gemäß dyn-ET (unveröff.) werden schließlich auf Basis der minimalen, mittleren und maximalen Oberhöhenbonitäten anhand der Gesamtwertleistungen, der laufenden Bestandeswertzuwächse und Wertzuwachsraten sowie des durchschnittlichen waldbaulichen Überschusses und der Brutto-Bodenrente analysiert. Auch hier werden für die tabellarische Gegenüberstellung ausgewählter Bewertungsergebnisse die Alter 140 a für die Baumart Buche und 80 a für die Baumart Fichte bzw. die Kulminationszeitpunkte der laufenden Bestandeswertzuwächse gewählt. 
Das ökonomische Leistungsspektrum der Baumart Buche (mäßige Hochdurchforstung) gemäß dyn-ET (unveröff.) wird anhand des Gesamtwertleistungsfächers über dem Alter deutlich (Abbildung 107). Die bis zu einem Alter von 100 Jahren erbrachte Wertleistung deckt einen Rahmen zwischen 10.347 und $52.477 € \mathrm{ha}^{-1}$ ab (Tabelle 3-13). Die entsprechenden Abbildungen der starken (Abbildung 185) und gestaffelten (Abbildung 187) Hochdurchforstung können dem Anhang entnommen werden.

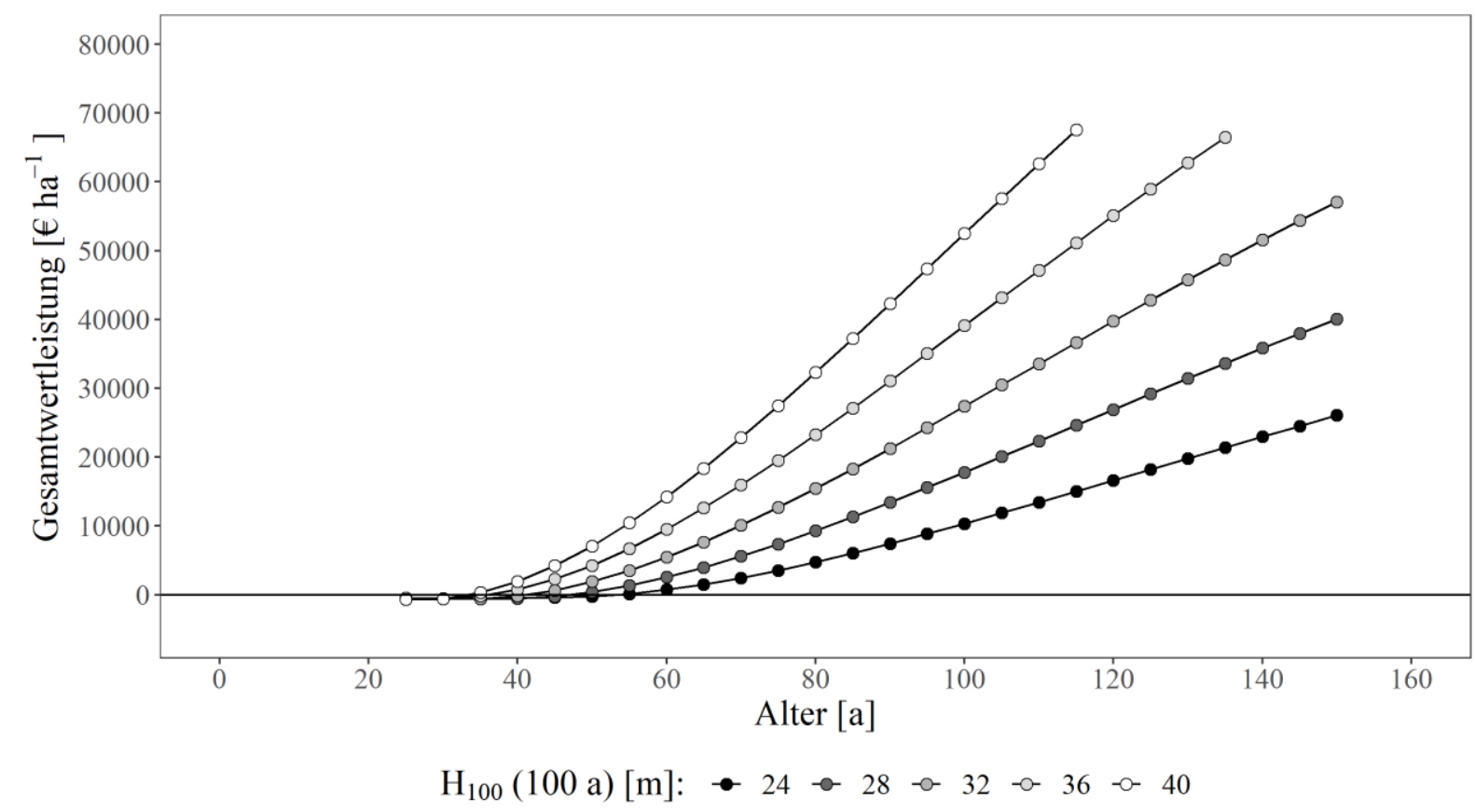

Abbildung 107: Gesamtwertleistung über dem Alter; alle Oberhöhenbonitäten der Baumart Buche (mäßige Hochdurchforstung) gemäß dyn-ET (unveröff.).

Die laufenden Bestandeswertzuwächse der Baumart Buche (mäßige Hochdurchforstung) gemäß dyn-ET (unveröff.) kulminieren umso früher, je höher die Oberhöhenbonität ist (Abbildung 108). Während bei einer Oberhöhenbonität von $24 \mathrm{~m}$ im Alter 125 a ein maximaler laufender Bestandeswertzuwachs von $320 € \mathrm{ha}^{-1} \mathrm{a}^{-1}$ erzielt wird, wächst der Bestandeswert bei einer Oberhöhenbonität von $40 \mathrm{~m}$ im Alter $100 \mathrm{a}$ um $1.021 € \mathrm{ha}^{-1} \mathrm{a}^{-1} \mathrm{zu}$ (Tabelle 3-13). Abbildungen 186 und 188 im Anhang zeigen die entsprechenden Wertzuwachsentwicklungen der starken und gestaffelten Hochdurchforstung.

Die in Abschnitt 3.3.3 beschriebenen, ertragskundlichen Veränderungen ggü. dem etablierten Ertragstafelwerk von Schober (1967) führen zu erheblich veränderten ökonomischen Bewertungsergebnissen (vgl. auch Groß 2019, S. 49 ff.). Für die mäßige Durchforstung führt das erhöhte Ertragsniveau (siehe Abbildung 59) in Verbindung mit den teils stark gestiegenen mittleren Einzelbaumdurchmessern im verbleibenden und ausscheidenden Bestand (siehe Abbildungen 67 und 68) zu einer deutlichen Steigerung der Gesamtwertleistung (Abbildung 109). 
Die Steigerung nimmt mit steigender Oberhöhenbonität zu und beträgt im Alter 140 a bei annähernd gleicher Oberhöhenentwicklung ausgewählter Oberhöhenbonitäten (siehe Abbildung 60) zwischen $18 \%$ und $41 \%$ (Tabelle 3-14).

Tabelle 3-13: Vergleich ausgewählter ökonomischer Bewertungsergebnisse der Baumart Buche (mäßige Hochdurchforstung) gemäß dyn-ET (unveröff.); alle Oberhöhenbonitäten; in Klammern ist jeweils die relative Differenz zur Oberhöhenbonität $H_{100}(100 \mathrm{a})=32 \mathrm{~m}$ angegeben;

GWertL(100 a): Gesamtwertleistung bis zum Alter 100 a, $\max \boldsymbol{w}_{\boldsymbol{B}}$ : lfd. Bestandeswertzuwachs zum Zeitpunkt der Kulmination [...], $\boldsymbol{W}_{\boldsymbol{B}}(\mathbf{1 0 0} \mathbf{a})$ : Abtriebswert (verbl. Bestand) im Alter $100 \mathrm{a}$, $\sum_{\mathbf{0}}^{\mathbf{1 0 0} \text { a }} \lambda \cdot \mathbf{P}\left(\boldsymbol{D}_{\boldsymbol{g}}\right)$ : kumulierte Vornutzungswerte bis zum Alter $100 \mathrm{a}, \boldsymbol{a}_{\boldsymbol{\eta}}(\mathbf{1 0 0} \mathbf{a})$ : durchschn. waldbaulicher Überschuss einer nachhaltigen Betriebsklasse bei einer Umtriebszeit von $100 a, \boldsymbol{W}_{\boldsymbol{\eta}}(\mathbf{1 0 0} \mathbf{a})$ : durchschnittlicher Abtriebswert einer nachhaltigen Betriebsklasse bei einer Umtriebszeit von $100 \mathrm{a}$, $\boldsymbol{a}(\mathbf{1 0 0}$ a): Brutto-Bodenrente bei einer Umtriebszeit von 100 a.

\begin{tabular}{|c|c|c|c|c|c|}
\hline \multirow{2}{*}{$\begin{array}{r}\text { Bestandesbehandlung } \\
\qquad H_{100}(100 \text { a) }[\mathrm{m}]\end{array}$} & \multicolumn{5}{|c|}{ mäßige Hochdurchforstung } \\
\hline & 24 & 28 & 32 & 36 & 40 \\
\hline GWertL $(100$ a $)$ & 10.347 & 17.797 & 27.373 & 39.093 & 52.477 \\
\hline$\left[€ \mathbf{h a}^{-1}\right]$ & $(-62,2 \%)$ & $(-35,0 \%)$ & & $(+42,8 \%)$ & $(+91,7 \%)$ \\
\hline $\max w_{B}$ & 320 [125 a] & 457 [115 a] & $621[110 \mathrm{a}]$ & $809[105 \mathrm{a}]$ & $1.021[100 \mathrm{a}]$ \\
\hline$\left[€ \mathbf{h a}^{-1} \mathbf{a}^{-1}\right]$ & $(-48,5 \%)$ & $(-26,4 \%)$ & & $(+30,3 \%)$ & $(+64,4 \%)$ \\
\hline$W_{B}(100 \mathrm{a})$ & 6.678 & 10.287 & 14.355 & 18.705 & 23.114 \\
\hline$\left[€ \mathbf{h a}^{-1}\right]$ & $(-53,5 \%)$ & $(-28,3 \%)$ & & $(+30,3 \%)$ & $(+61,0 \%)$ \\
\hline$\sum_{0}^{100 a} \lambda \cdot \mathbf{P}\left(D_{g}\right)$ & 3.669 & 7.510 & 13.018 & 20.388 & 29.363 \\
\hline$\left[€ h a^{-1}\right]$ & $(-71,8 \%)$ & $(-42,3 \%)$ & & $(+56,6 \%)$ & $(+125,6 \%)$ \\
\hline$a_{\eta}(100 \mathrm{a})$ & 3 & 78 & 174 & 291 & 424 \\
\hline$\left[€ \mathbf{h a}^{-1} \mathbf{a}^{-1}\right]$ & $(-98,3 \%)$ & $(-52,2 \%)$ & & $(+67,2 \%)$ & $(+143,7 \%)$ \\
\hline$W_{\eta}(100 \mathrm{a})$ & 1.506 & 2.752 & 4.345 & 6.255 & 8.419 \\
\hline$\left[€ \mathbf{h a}^{-\mathbf{1}}\right]$ & $(-65,3 \%)$ & $(-36,7 \%)$ & & $(+44,0 \%)$ & $(+93,8 \%)$ \\
\hline$a(100 a)$ & -141 & -102 & -51 & 15 & 91 \\
\hline$\left[€ \mathbf{h a}^{-1} a^{-1}\right]$ & & & & & \\
\hline
\end{tabular}




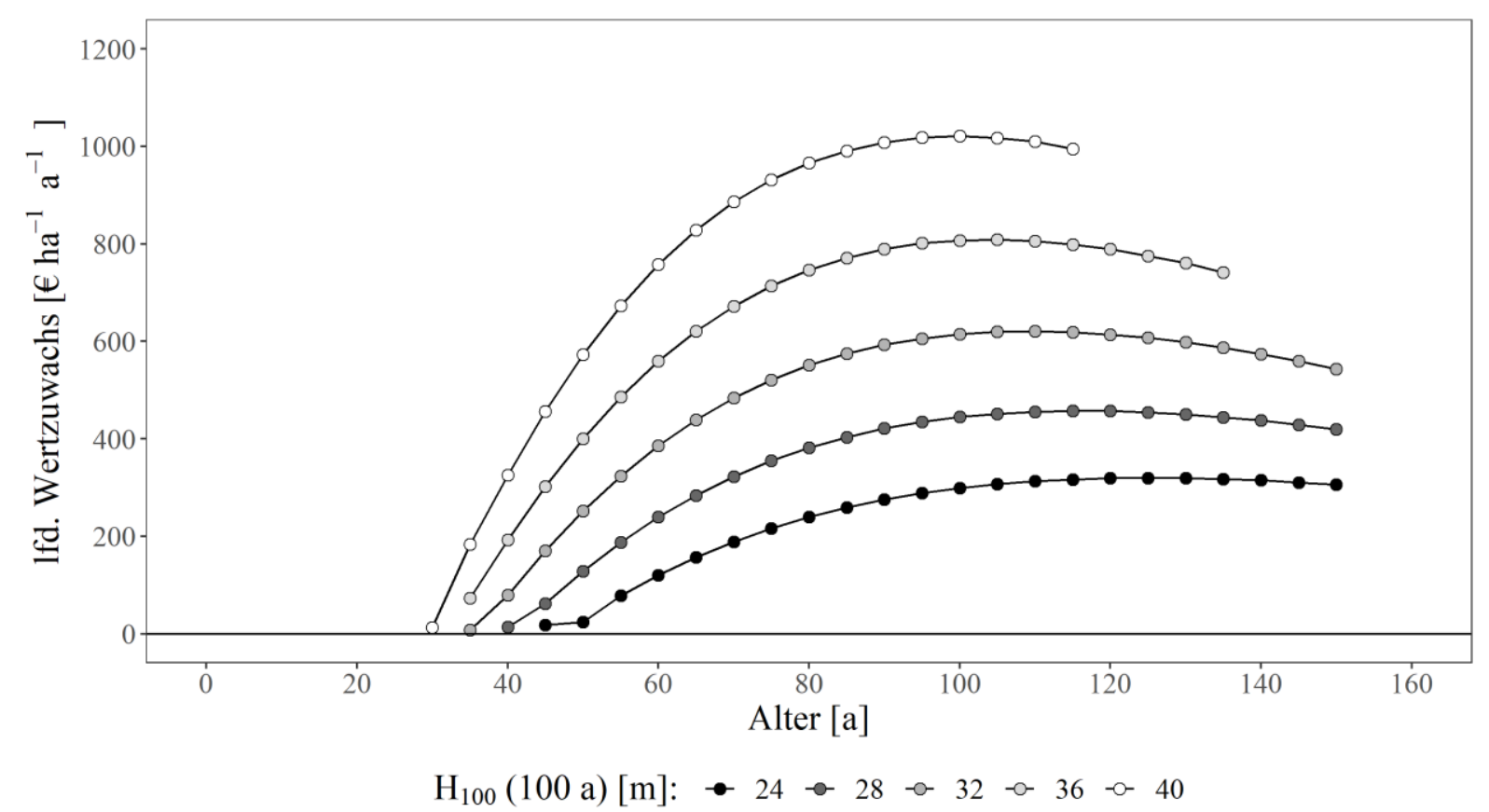

Abbildung 108: Laufender Bestandeswertzuwachs über dem Alter; alle Oberhöhenbonitäten der Baumart Buche (mäßige Hochdurchforstung) gemäß dyn-ET (unveröff.).

Dementsprechend steigen auch die laufenden Bestandeswertzuwächse gemäß dyn-ET (unveröff., mäßige Hochdurchforstung) deutlich ggü. Schober (1967, mäßige Durchforstung), wobei sich die Verlaufsform über dem Alter, bis auf etwas stärker abfallenden Bestandeswertzuwächse gemäß dyn-ET (unveröff.) im hohen Alter, sehr ähneln (Abbildung 110). Die Kulminationszeitpunkte verschieben sich ggü Schober (1967, mäßige Durchforstung) um 15 bis 35 Jahre nach vorne (Tabelle 3-14).

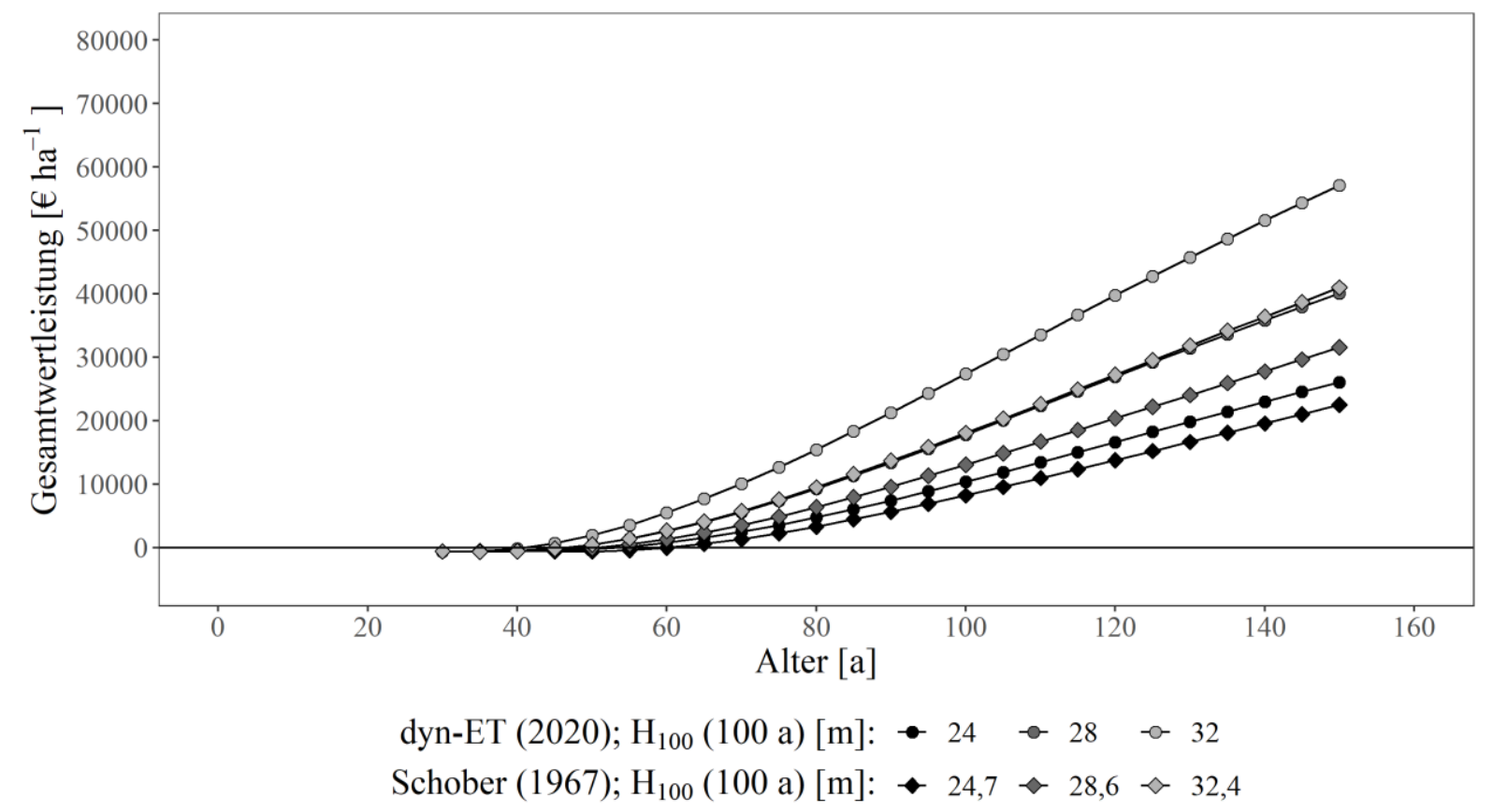

Abbildung 109: Gesamtwertleistung über dem Alter; ausgewählte Oberhöhenbonitäten der Baumart Buche (mäßige Durchforstung) gemäß dyn-ET (unveröff.) und Schober (1967). 
Die entsprechenden Vergleichsgrafiken bei starker Durchforstung können für die Gesamtwertleistungen (Abbildung 189) sowie die laufenden Bestandeswertzuwächse (Abbildung 190) dem Anhang entnommen werden.

Tabelle 3-14: Vergleich ausgewählter ökonomischer Bewertungsergebnisse (mäßige Durchforstung); ausgewählte Oberhöhenbonitäten gemäß dyn-ET (unveröff.) und Schober (1967); in Klammern ist jeweils die relative Differenz zur vergleichbaren Oberhöhenbonität nach Schober (1967) angegeben; GWertL(140 a): Gesamtwertleistung bis zum Alter $140 \mathrm{a}, \boldsymbol{\operatorname { m a x }} \boldsymbol{w}_{\boldsymbol{B}}$ : Ifd. Bestandeswertzuwachs zum Zeitpunkt der Kulmination [...], $\boldsymbol{W}_{\boldsymbol{B}}(\mathbf{1 4 0} \mathbf{a})$ : Abtriebswert (verbl. Bestand) im Alter $140 \mathrm{a}$, $\sum_{\mathbf{0}}^{\mathbf{1 4 0} \text { a }} \boldsymbol{\lambda} \cdot \mathbf{P}\left(\boldsymbol{D}_{\boldsymbol{g}}\right)$ : kumulierte Vornutzungswerte bis zum Alter $140 \mathrm{a}, \boldsymbol{a}_{\boldsymbol{\eta}}(\mathbf{1 4 0} \mathbf{a})$ : durchschn. waldbaulicher Überschuss einer nachhaltigen Betriebsklasse bei einer Umtriebszeit von $140 a, \boldsymbol{W}_{\boldsymbol{\eta}}(\mathbf{1 4 0} \mathbf{a})$ : durchschnittlicher Abtriebswert einer nachhaltigen Betriebsklasse bei einer Umtriebszeit von $140 \mathrm{a}$, $\boldsymbol{a}(140$ a): Brutto-Bodenrente bei einer Umtriebszeit von $140 \mathrm{a}$.

\begin{tabular}{|c|c|c|c|c|c|c|}
\hline \multirow{2}{*}{$\begin{array}{r}\text { Ertragstafel } \\
H_{100}(100 \text { a) }[\mathrm{m}]\end{array}$} & \multicolumn{3}{|c|}{ Schober (1967), mäßige Df. } & \multicolumn{3}{|c|}{ dyn-ET (unveröff.), mäßige Df. } \\
\hline & 24,7 & 28,6 & 32,4 & 24 & 28 & 32 \\
\hline \multirow{2}{*}{$\begin{array}{r}\text { GWertL(140 a) } \\
{\left[€ \mathrm{ha}^{-1}\right]}\end{array}$} & 19.558 & 27.758 & 36.402 & 22.978 & 35.838 & 51.568 \\
\hline & & & & $(+17,5 \%)$ & $(+29,1 \%)$ & $(+41,7 \%)$ \\
\hline $\max w_{B}$ & 294 & 387 & 463 & $320[125 \mathrm{a}]$ & $457[115 \mathrm{a}]$ & $621[110 \mathrm{a}]$ \\
\hline$\left[€ \mathbf{h a}^{-1} \mathbf{a}^{-1}\right]$ & [145 a] & {$[150 \mathrm{a}]$} & {$[135 \mathrm{a}]$} & $(+8,8 \%)$ & $(+18,1 \%)$ & $(+34,1 \%)$ \\
\hline$W_{B}(140 \mathrm{a})$ & 14.830 & 19.114 & 23.910 & 13.133 & 18.234 & 23.536 \\
\hline$\left[€ \mathbf{h a}^{-1}\right]$ & & & & $(-11,4 \%)$ & $(-4,6 \%)$ & $(-1,6 \%)$ \\
\hline \multirow{2}{*}{$\begin{array}{r}\sum_{0}^{140 a} \lambda \cdot P\left(D_{g}\right) \\
{\left[€ h a^{-1}\right]}\end{array}$} & 4.728 & 8.644 & 12.492 & 9.845 & 17.604 & 28.032 \\
\hline & & & & $(+108,2 \%)$ & $(+103,7 \%)$ & $(+124,4 \%)$ \\
\hline$a_{\eta}(140 \mathrm{a})$ & 68 & 127 & 189 & 93 & 184 & 296 \\
\hline$\left[€ \mathbf{h} \mathbf{a}^{-1} \mathbf{a}^{-1}\right]$ & & & & $(+36,8 \%)$ & $(+44,9 \%)$ & $(+56,6 \%)$ \\
\hline$W_{\eta}(140 \mathrm{a})$ & 4.350 & 6.191 & 8.189 & 4.024 & 6.242 & 8.828 \\
\hline$\left[€ \mathbf{h a}^{-1}\right]$ & & & & $(-7,5 \%)$ & $(+0,8 \%)$ & $(+7,8 \%)$ \\
\hline$a(140 a)$ & -123 & -99 & -75 & -105 & -63 & -8 \\
\hline$\left[€ \mathbf{h a}^{-1} a^{-1}\right]$ & & & & & & \\
\hline
\end{tabular}

Aus den ggü. Schober (1967, mäßige Durchforstung) gesunkenen Vorräten (siehe Abbildung 62) resultieren in Verbindung mit den gestiegenen mittleren Einzelbaumdurchmessern des verbleibenden Bestandes (siehe Abbildung 67) ähnliche bzw. im höheren Alter leicht gesunkene (2\% bis $11 \%$ im Alter 140 a) Entwicklungen der Abtriebswerte (Abbildung 111). 
Der Schnittpunkt der Abtriebswertentwicklungen liegt umso später, je höher die Oberhöhenbonität ist - mit steigender Oberhöhenbonität sind die Unterschiede zudem umso geringer.

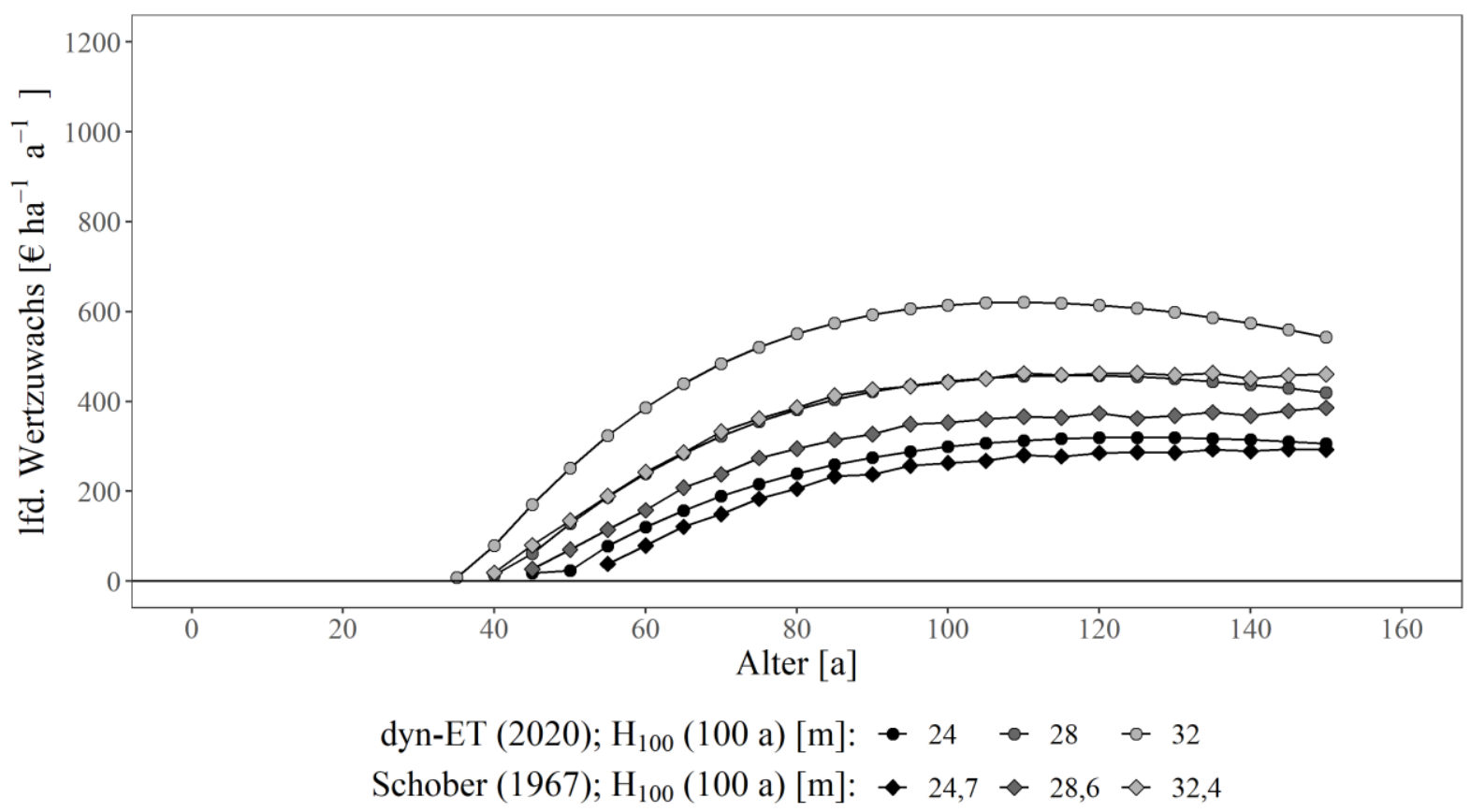

Abbildung 110:Laufender Bestandeswertzuwachs über dem Alter; ausgewählte Oberhöhenbonitäten der Baumart Buche (mäßige Durchforstung) gemäß dyn-ET (unveröff.) und Schober (1967).

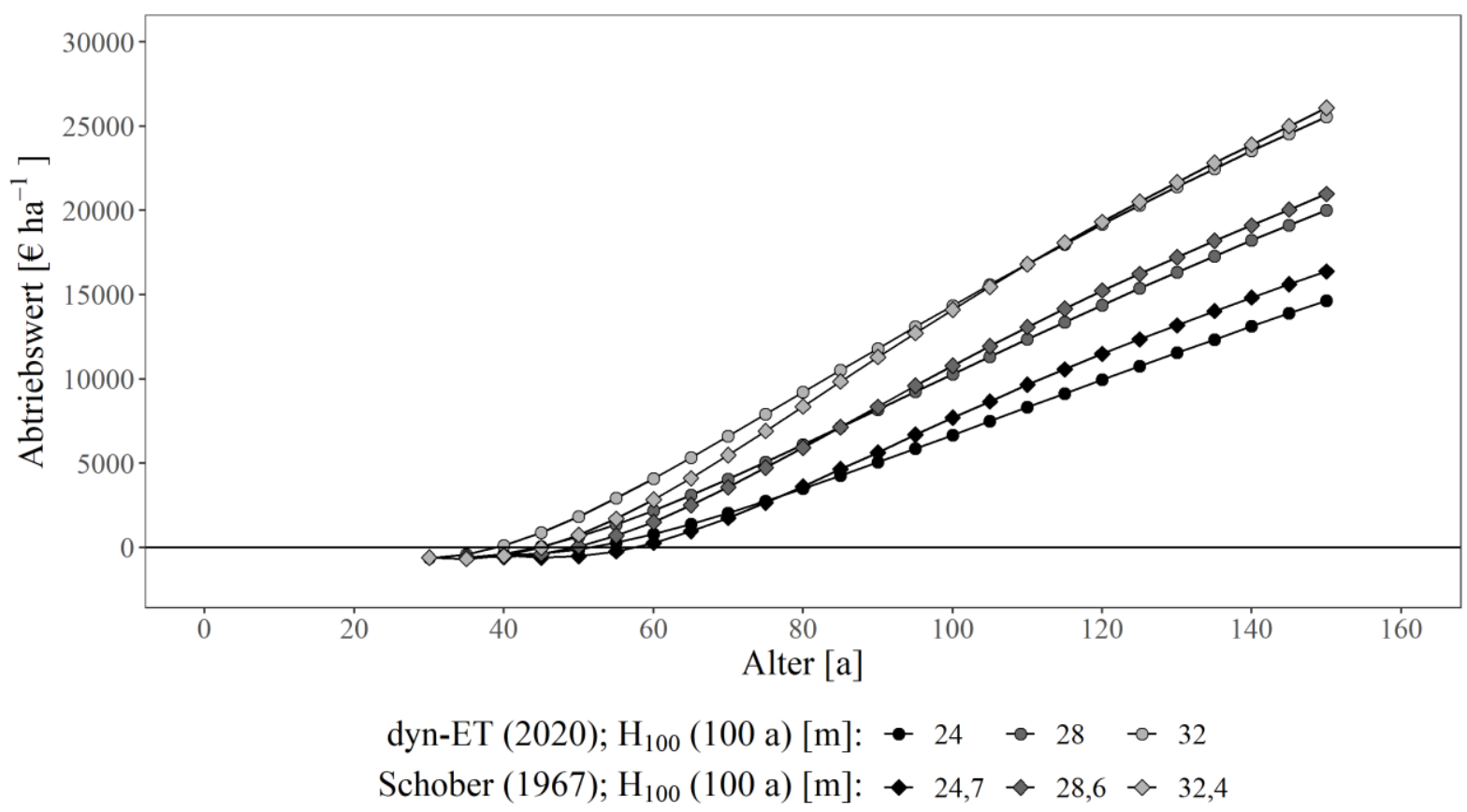

Abbildung 111: Abtriebswert über dem Alter; ausgewählte Oberhöhenbonitäten der Baumart Buche (mäßige Durchforstung) gemäß dyn-ET (unveröff.) und Schober (1967).

Bei den gleichzeitig ggü. Schober (1967, mäßige Durchforstung) gestiegenen laufenden Bestandeswertzuwächsen (Abbildung 110) resultieren überwiegend gestiegene Wertzuwachsraten 
(Abbildung 112). Ähnliche Effekte zeigen sich für den Vergleich der starken Durchforstung in den Abbildungen 191 und 192 im Anhang, wobei die Wertrelationen teilweise ggü. der mäßigen Durchforstung vertauscht sind.

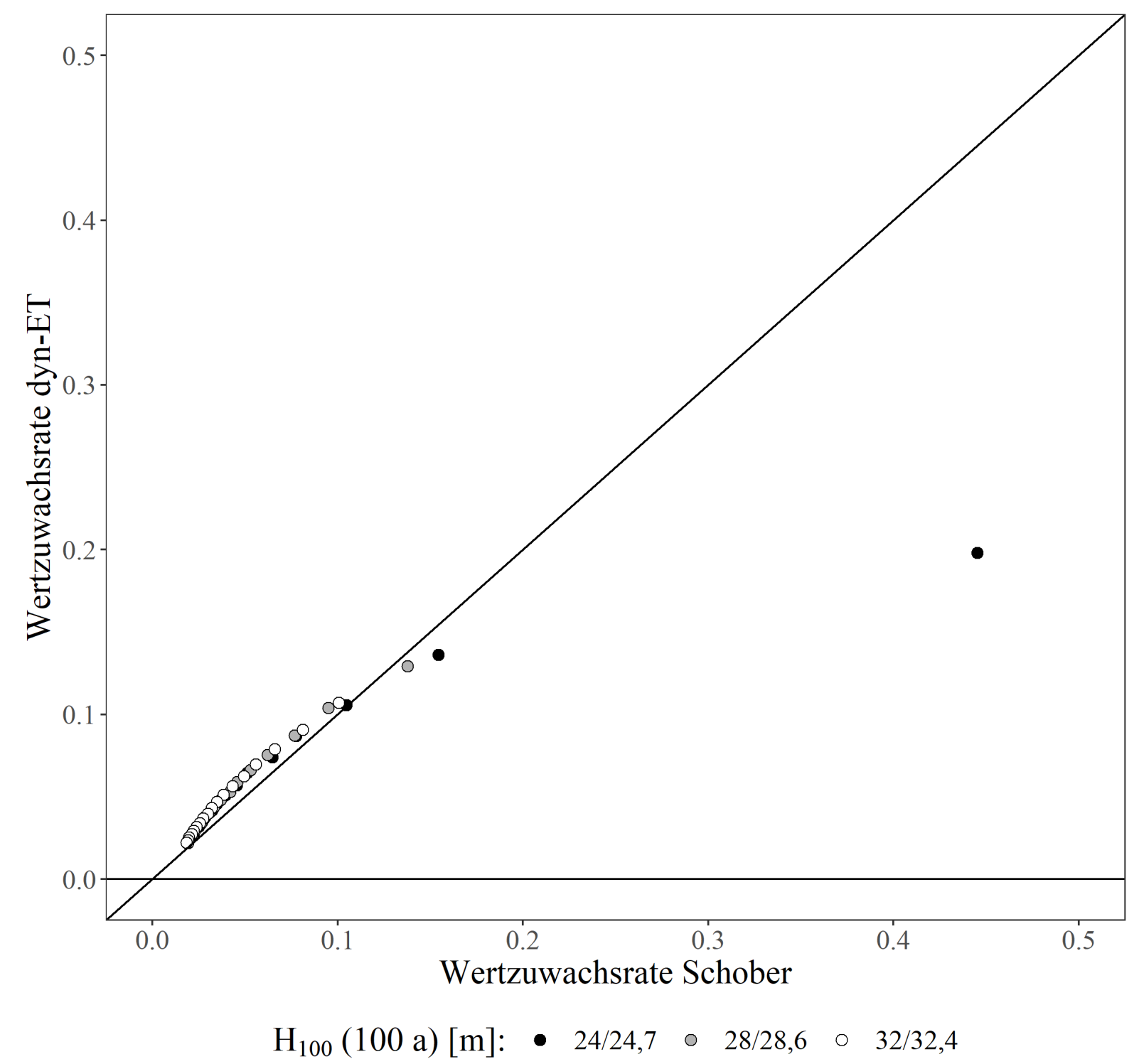

Abbildung 112: Wertzuwachsraten der Baumart Buche (mäßige Durchforstung, ausgewählte Oberhöhenbonitäten) gemäß dyn-ET (unveröff.) und Schober (1967); es werden nur Vergleichspaare aus Altern dargestellt, in denen für alle Oberhöhenbonitäten Datenpunkte vorliegen; die Winkelhalbierende kennzeichnet gleiche Wertzuwachsraten im gleichen Alter.

Als jährliche Erfolgsgröße einer nachhaltigen Betriebsklasse wird der durchschnittliche waldbauliche Überschuss $a_{N}$ im Sinne eines holzernte-, bestandesbegründungs- und bestandespflegekostenfreien Holzerlöses (Deckungsbeitrag II; vgl. Keuffel 1980, S. 57 f.) in Abhängigkeit von der Umtriebszeit im Normalwaldmodell berechnet (Abbildung 113; analog dazu Abbildung 193 im Anhang für die starke Durchforstung). Bei einer Umtriebszeit von 140 a können einer nachhaltigen Betriebsklasse demnach gemäß dyn-ET (unveröff.) jährlich $37 \%$ bis 57 \% 
höhere waldbauliche Überschüsse entnommen werden (Tabelle 3-14). Die korrespondierenden durchschnittlichen Abtriebswerte einer nachhaltigen Betriebsklasse verändern sich ggü. Schober (1967, mäßige Durchforstung) unterschiedlich in Abhängigkeit von der Oberhöhenbonität (Abbildung 114).

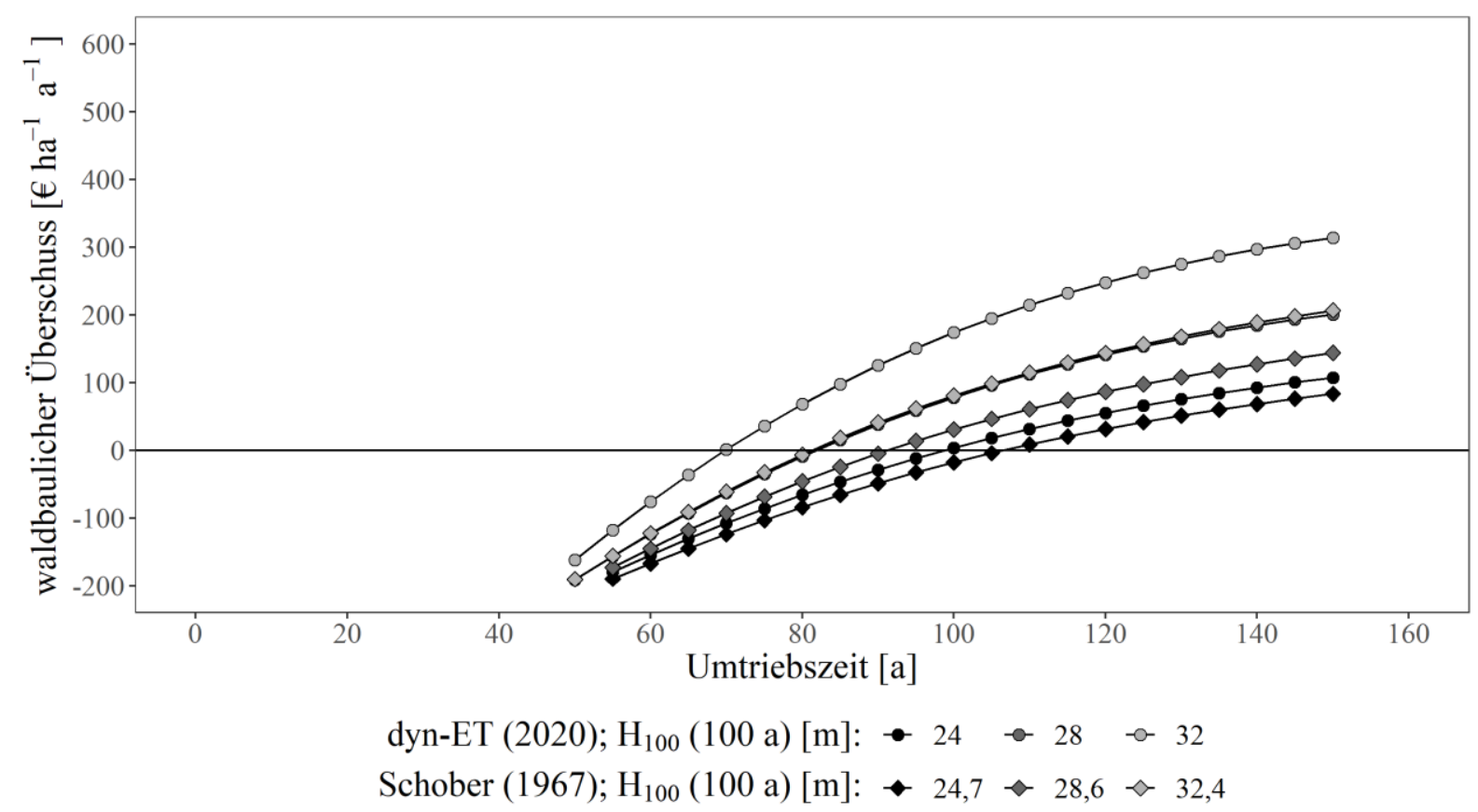

Abbildung 113: Durchschnittlicher waldbaulicher Überschuss der forstlichen Produktion über der Umtriebszeit; ausgewählte Oberhöhenbonitäten der Baumart Buche (mäßige Durchforstung) gemäß dynET (unveröff.) und Schober (1967).

Während sich die durchschnittlichen Abtriebswerte bei einer Oberhöhenbonität von $32 \mathrm{~m}$ ggü. Schober (1967, mäßige Durchforstung) erhöhen (+ 8 \% bei einer Umtriebszeit von 140 a; Tabelle 3-14), kommt es mit sinkender Oberhöhenbonität zu einem zunehmend früheren Kreuzen der Verläufe. Bei 24 m liegen die durchschnittlichen Abtriebswerte bei einer Umtriebszeit von 140 a um $8 \%$ unterhalb der Werte nach Schober (1967, mäßige Durchforstung). Abbildung 194 im Anhang zeigt den Vergleich der durchschnittlichen Abtriebswerte bei starker Durchforstung.

Gegenüber Schober (1967, mäßige Durchforstung) steigen die mit einem Kalkulationszinssatz von $r=1,5 \%$ berechneten Brutto-Bodenrenten an (Abbildung 115 und analog dazu Abbildung 195 im Anhang für die starke Durchforstung). Insbesondere die hohen Oberhöhenbonitäten profitieren von den ggü. Schober (1967, mäßige Durchforstung) höheren Vornutzungsvolumina (siehe Abbildung 64) mit deutlich höheren Einzelbaumdurchmessern (siehe Abbildung 68). Dennoch erreicht unter Berücksichtigung der Kapitalknappheit keine der für den Vergleich ausgewählten Oberhöhenbonitäten mit den genutzten Eingangswerten (siehe Tabellen 3-10 und 3-11) eine positive durchschnittliche Erfolgsgröße (Tabelle 3-14). Die angesetzten 
Bestandesbegründungs- und Bestandespflegekosten können auch mit dem ggü. Schober (1967, mäßige Durchforstung) deutlich erhöhtem Ertragsniveau (siehe Abbildung 59) und den gestiegenen Einzelbaumdurchmessern (siehe Abbildungen 67 und 68) nicht ausgeglichen werden.

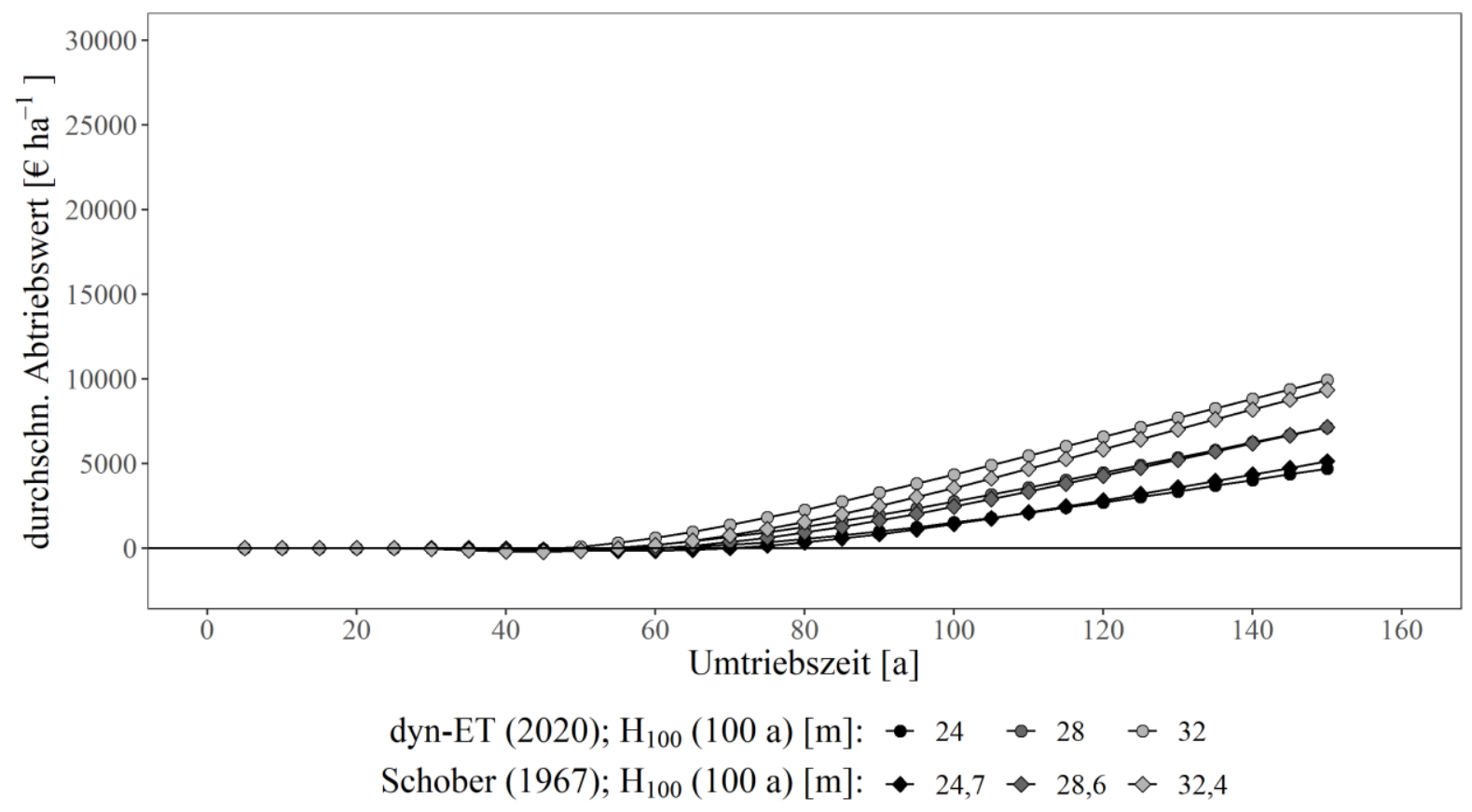

Abbildung 114: Durchschnittlicher Abtriebswert über der Umtriebszeit; ausgewählte Oberhöhenbonitäten der Baumart Buche (mäßige Durchforstung) gemäß dyn-ET (unveröff.) und Schober (1967).

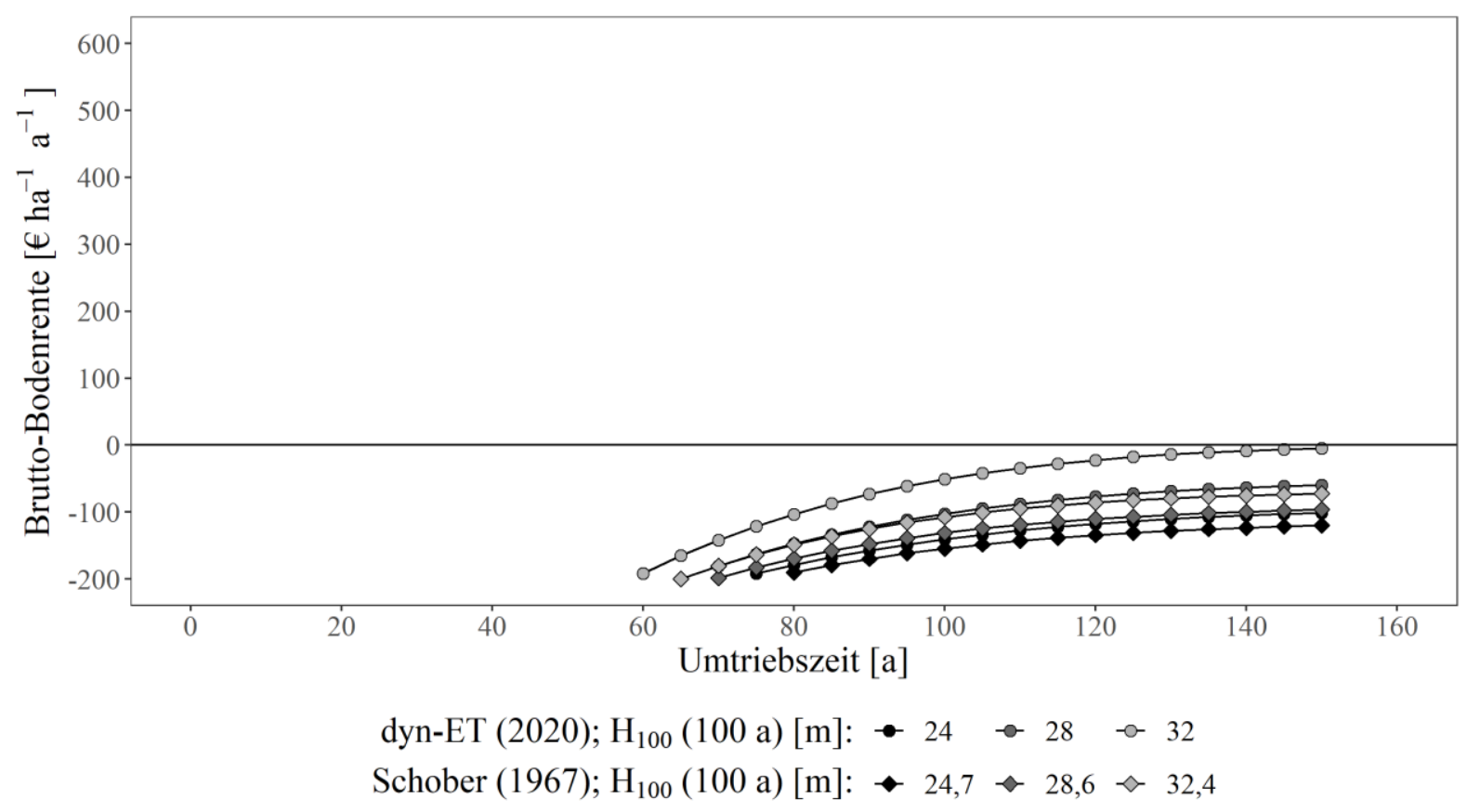

Abbildung 115: Brutto-Bodenrente ( $r=1,5 \%)$ über der Umtriebszeit; ausgewählte Oberhöhenbonitäten der Baumart Buche (mäßige Durchforstung) gemäß dyn-ET (unveröff.) und Schober (1967). 
Die sowieso schon nicht sehr hohen Vor- (Abbildung 116) und Endnutzungswerte (Abbildung 111) werden aufgrund der späten Realisationszeitpunkte durch den Zinseffekt derart reduziert, dass sie in Summe unterhalb der Bestandesbegründungs- und diskontierten Bestandespflegekosten liegen - die Kapitalwerte der forstlichen Produktion und somit auch die daraus abgeleiteten Brutto-Bodenrenten sind negativ (siehe auch Abbildungen 195 und 196 für die starke Durchforstung).

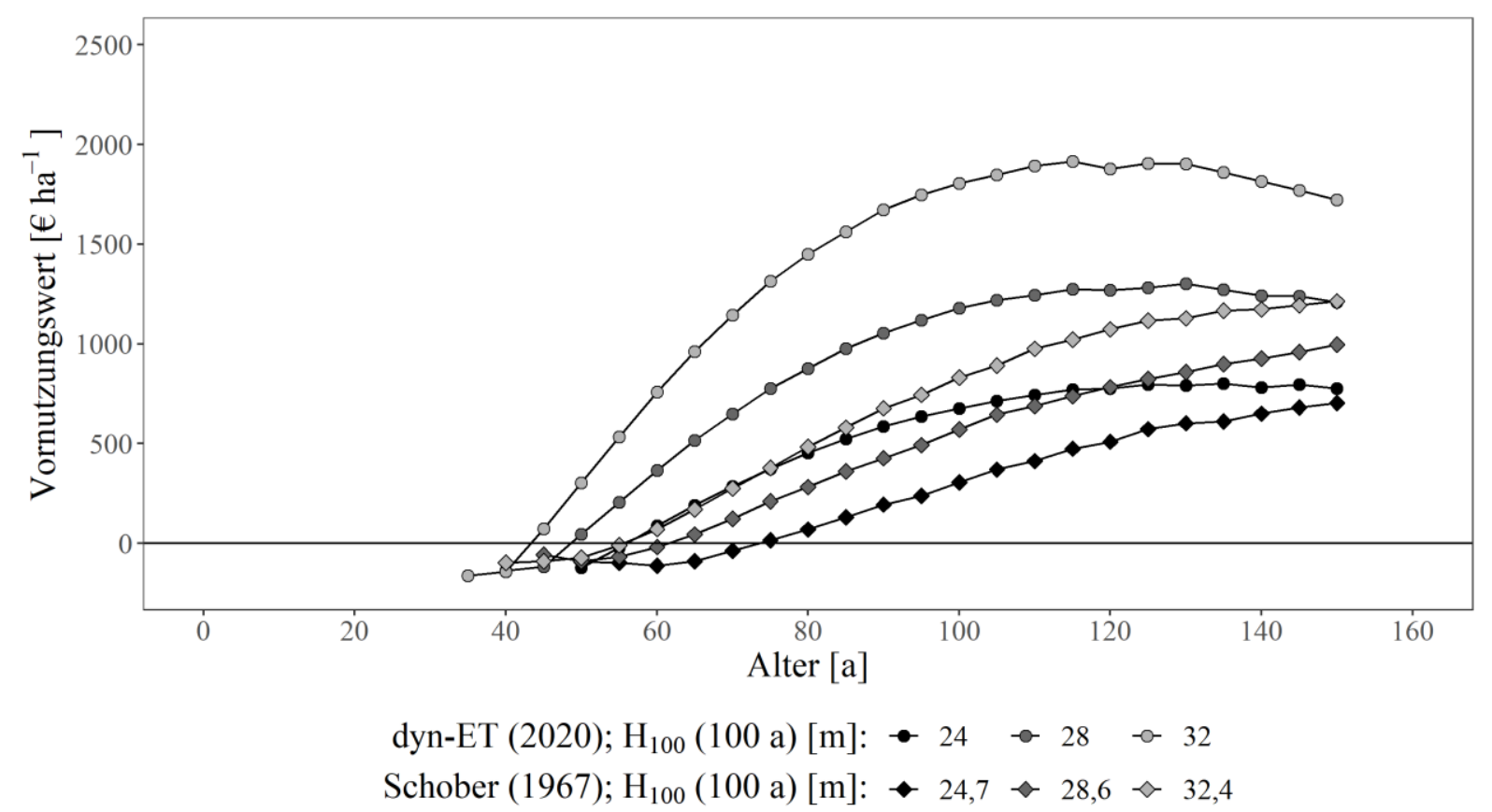

Abbildung 116: Vornutzungswerte über dem Alter; ausgewählte Oberhöhenbonitäten der Baumart Buche (mäßige Durchforstung) gemäß dyn-ET (unveröff.) und Schober (1967).

Im ökonomischen Vergleich der waldbaulichen Behandlungsvarianten gemäß dyn-ET (unveröff.) zeigen sich die Wechselwirkungen der in Abschnitt 3.3.3 analysierten ertragskundlichen Unterschiede. Während die Gesamtwuchsleistung bei mäßige Hochdurchforstung über den gesamten Altersrahmen hinweg eine Vorteilhaftigkeit ggü. der starken und der gestaffelten Hochdurchforstung zeigt (Abbildung 71), liegt die Gesamtwertleistung bei mäßiger Hochdurchforstung bonitätsübergreifend in jungen Bestandesaltern zunächst unterhalb der alternativen Behandlungsvarianten (Abbildung 117). Die höheren mittleren Einzelbaumdurchmesser zu Beginn der forstlichen Produktion (Abbildungen 76 und 77), führen aufgrund der dimensionsabhängigen Deckungsbeitragsfunktion [3-11] zu höheren Werten der ausscheidenden und verbleibenden Bestandesvolumina ${ }^{81}$. Erst mit steigendem Alter wird dieser Effekt durch die deutlich

${ }^{81}$ Die maßgebliche Bedeutung des über die reine Volumenleistung hinausgehenden Effekts der einzelbaumdimensionsabhängigen Deckungsbeitragsfunktion wird in Abschnitt 3.3.6 tiefergehend analysiert. 
höhere Volumenleistung bei mäßiger Hochdurchforstung überlagert - bis zum Alter 140 a liegt die Gesamtwertleistung bei gestaffelter und starker Hochdurchforstung schließlich um $4 \%$ bzw. $13 \%$ unterhalb der Gesamtwertleistung bei mäßiger Hochdurchforstung (Tabelle 3-15). Während sich die ökonomische Vorteilhaftigkeit ggü. der starken Hochdurchforstung mit zunehmendem Alter vergrößert, schließt die Gesamtwertleistung bei gestaffelter Hochdurchforstung im hohen Bestandesalter (mit steigender Oberhöhenbonität früher) zur Gesamtwertleistung bei mäßiger Hochdurchforstung auf.

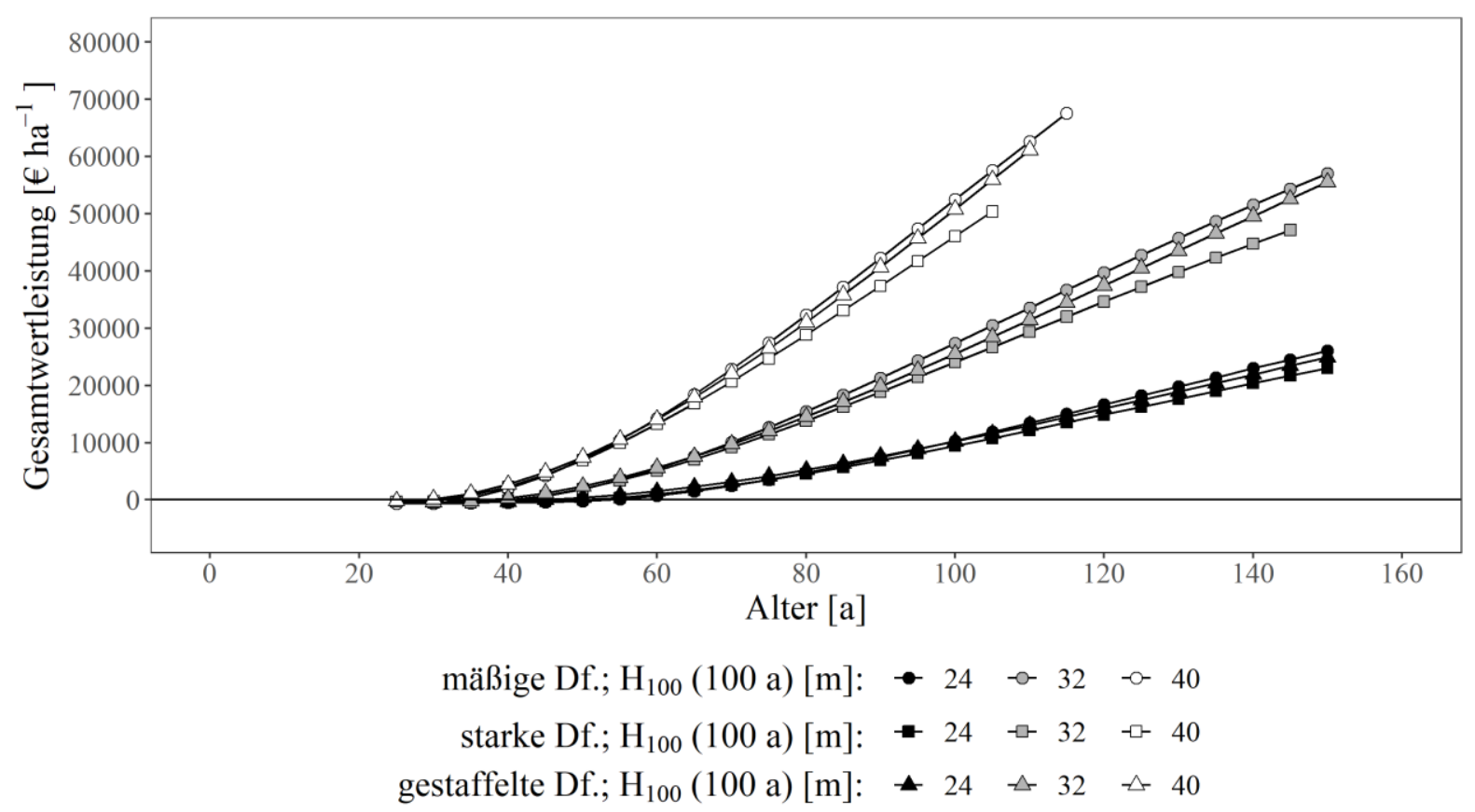

Abbildung 117: Gesamtwertleistung über dem Alter; ausgewählte Oberhöhenbonitäten der Baumart Buche getrennt nach Behandlungsvarianten gemäß dyn-ET (unveröff.).

Nochmal deutlicher wird dieser Effekt der aufholenden Wertleistung anhand der laufenden Bestandeswertzuwächse über dem Alter (Abbildung 118). Mit steigender Standortsgüte schließt die gestaffelte umso früher zur mäßigen Hochdurchforstung auf und übertrifft diese bei mittlerer und hoher Oberhöhenbonität sogar ab einem Alter von 130 bzw. 105 Jahren. Während die laufenden Bestandeswertzuwächse der mittleren Oberhöhenbonität $H_{100}(100 \mathrm{a})=32 \mathrm{~m}$ bei mäßiger und starker Hochdurchforstung zum gleichen Zeitpunkt kulminieren (110 a), erreicht der Wertzuwachs bei gestaffelter Hochdurchforstung mit $607 €$ ha $^{-1} \mathrm{a}^{-1}$ erst bei 125 a sein Maximum.

Bei der Analyse der Wertzuwachsraten zeigen sich überwiegend zu den Volumenzuwachsraten in Abbildung 74 (Abschnitt 3.3.3) vergleichbare Relationen. Insgesamt sind die Wertzuwachsraten im gleichen Alter umso höher und differenzierter, je niedriger die Oberhöhenbonität ist. Bei niedriger und mittlerer Oberhöhenbonität liegen die relativen Wertzuwächse der starken 
und gestaffelten Hochdurchforstung zunächst deutlicher über denen der mäßigen Hochdurchforstung - mit steigendem Alter nähern sie sich aber auch dort den Wertzuwachsraten bei mäBiger Hochdurchforstung an (Abbildung 119). Bei der höchsten Oberhöhenbonität sinkt die Wertzuwachsrate bei gestaffelter Durchforstung ab einem Alter von 55 a deutlich ab und verläuft, wie auch schon die entsprechende Volumenzuwachsrate, kurzzeitig unterhalb der Wertzuwachsraten der anderen Behandlungsvarianten.

Tabelle 3-15: Vergleich ausgewählter ökonomischer Bewertungsergebnisse der Baumart Buche (Oberhöhenbonität $H_{100}(100 \mathrm{a})=32 \mathrm{~m}$ ) gemäß dyn-ET (unveröff.); unterschiedliche Behandlungsvarianten; in Klammern ist jeweils die relative Differenz zur mäßigen Hochdurchforstung angegeben; GWertL(140 a): Gesamtwertleistung bis zum Alter 140 a, $\max \boldsymbol{w}_{B}$ : lfd. Bestandeswertzuwachs zum Zeitpunkt der Kulmination [...], $\boldsymbol{W}_{\boldsymbol{B}}(\mathbf{1 4 0}$ a) : Abtriebswert (verbl. Bestand) im Alter $140 \mathrm{a}$, $\sum_{0}^{140}$ a $\lambda \cdot \mathbf{P}\left(D_{g}\right)$ : kumulierte Vornutzungswerte bis zum Alter 140 a, $\boldsymbol{a}_{\boldsymbol{\eta}}(\mathbf{1 4 0}$ a): durchschn. waldbaulicher Überschuss einer nachhaltigen Betriebsklasse bei einer Umtriebszeit von $140 a, \boldsymbol{W}_{\boldsymbol{\eta}}(\mathbf{1 4 0} \mathbf{a})$ : durchschnittlicher Abtriebswert einer nachhaltigen Betriebsklasse bei einer Umtriebszeit von $140 \mathrm{a}$, $\boldsymbol{a}(140$ a): Brutto-Bodenrente bei einer Umtriebszeit von $140 \mathrm{a}$.

\begin{tabular}{|c|c|c|c|}
\hline Dectondochobondlune & mäßige & starke & gestaffelte \\
\hline$H_{100}(100$ a) $[\mathrm{m}]$ & 32 & 32 & 32 \\
\hline$G W \operatorname{ertL}(140 \mathrm{a})$ & 51.568 & 44.762 & 49.578 \\
\hline$\left[€ \mathbf{h a}^{-1}\right]$ & & $(-13,2 \%)$ & $(-3,9 \%)$ \\
\hline $\max w_{B}$ & 621 [110 a] & 530 [110 a] & 607 [125 a] \\
\hline$\left[€ \mathbf{h a}^{-1} \mathbf{a}^{-1}\right]$ & & $(-14,7 \%)$ & $(-2,3 \%)$ \\
\hline$W_{B}(140 \mathrm{a})$ & 23.536 & 20.823 & 23.250 \\
\hline$\left[€ \mathbf{h a}^{-1}\right]$ & & $(-11,5 \%)$ & $(-1,2 \%)$ \\
\hline$\sum_{0}^{140 a} \lambda \cdot \mathbf{P}\left(D_{g}\right)$ & 28.032 & 23.939 & 26.328 \\
\hline$\left[€ h a^{-1}\right]$ & & $(-14,6 \%)$ & $(-6,1 \%)$ \\
\hline$a_{\eta}(140 \mathrm{a})$ & 296 & 248 & 283 \\
\hline$\left[€ \mathbf{h a}^{-1} \mathbf{a}^{-1}\right]$ & & $(-16,2 \%)$ & $(-4,4 \%)$ \\
\hline$W_{\eta}(140 \mathrm{a})$ & 8.828 & 7.613 & 8.647 \\
\hline$\left[€ \mathbf{h a}^{-1}\right]$ & & $(-13,8 \%)$ & $(-2,1 \%)$ \\
\hline$a(140 \mathrm{a})$ & -8 & -26 & -15 \\
\hline$\left[€ \mathbf{h a}^{-1} a^{-1}\right]$ & & & \\
\hline
\end{tabular}




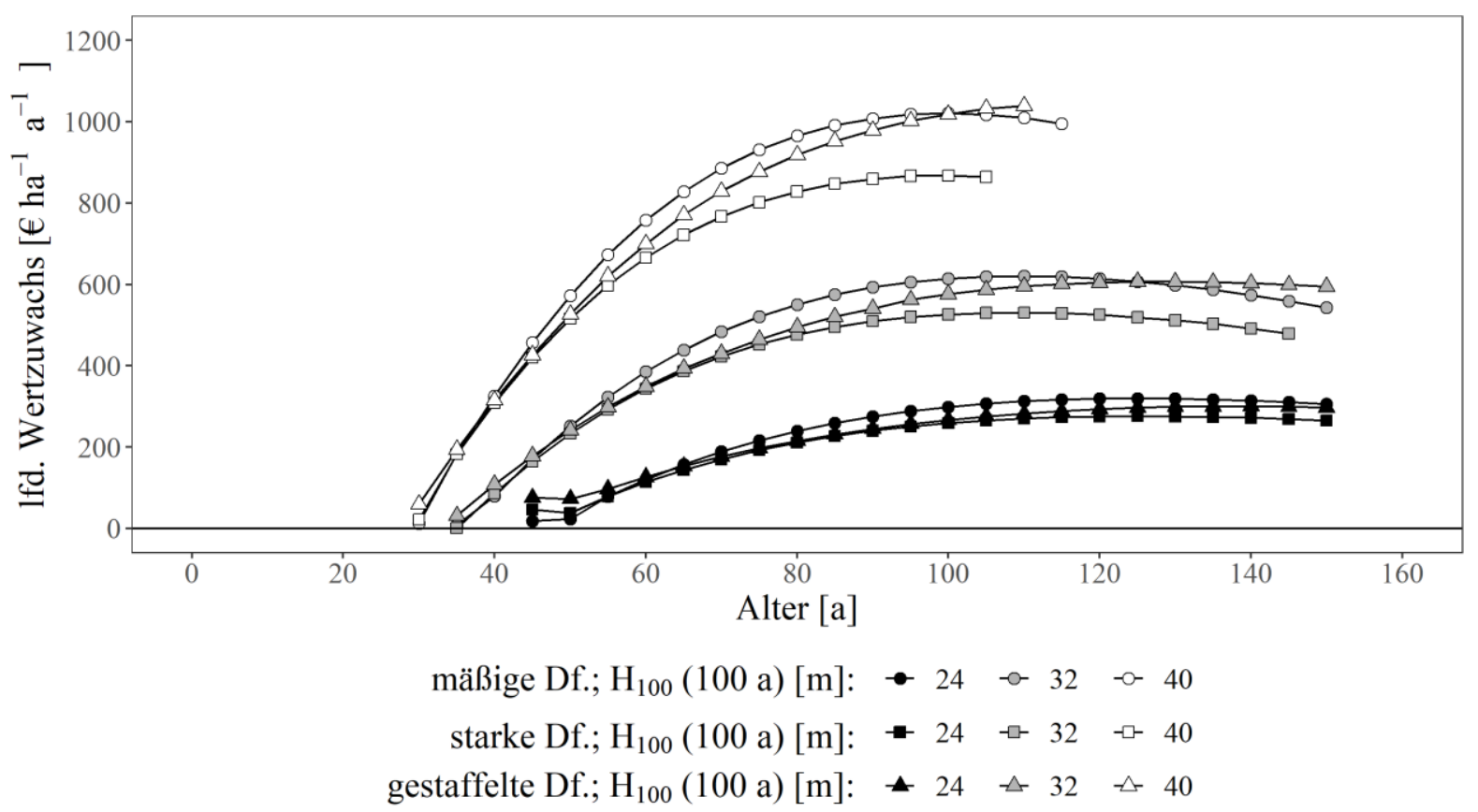

Abbildung 118: Laufender Bestandeswertzuwachs über dem Alter; ausgewählte Oberhöhenbonitäten der Baumart Buche getrennt nach Behandlungsvarianten gemäß dyn-ET (unveröff.).

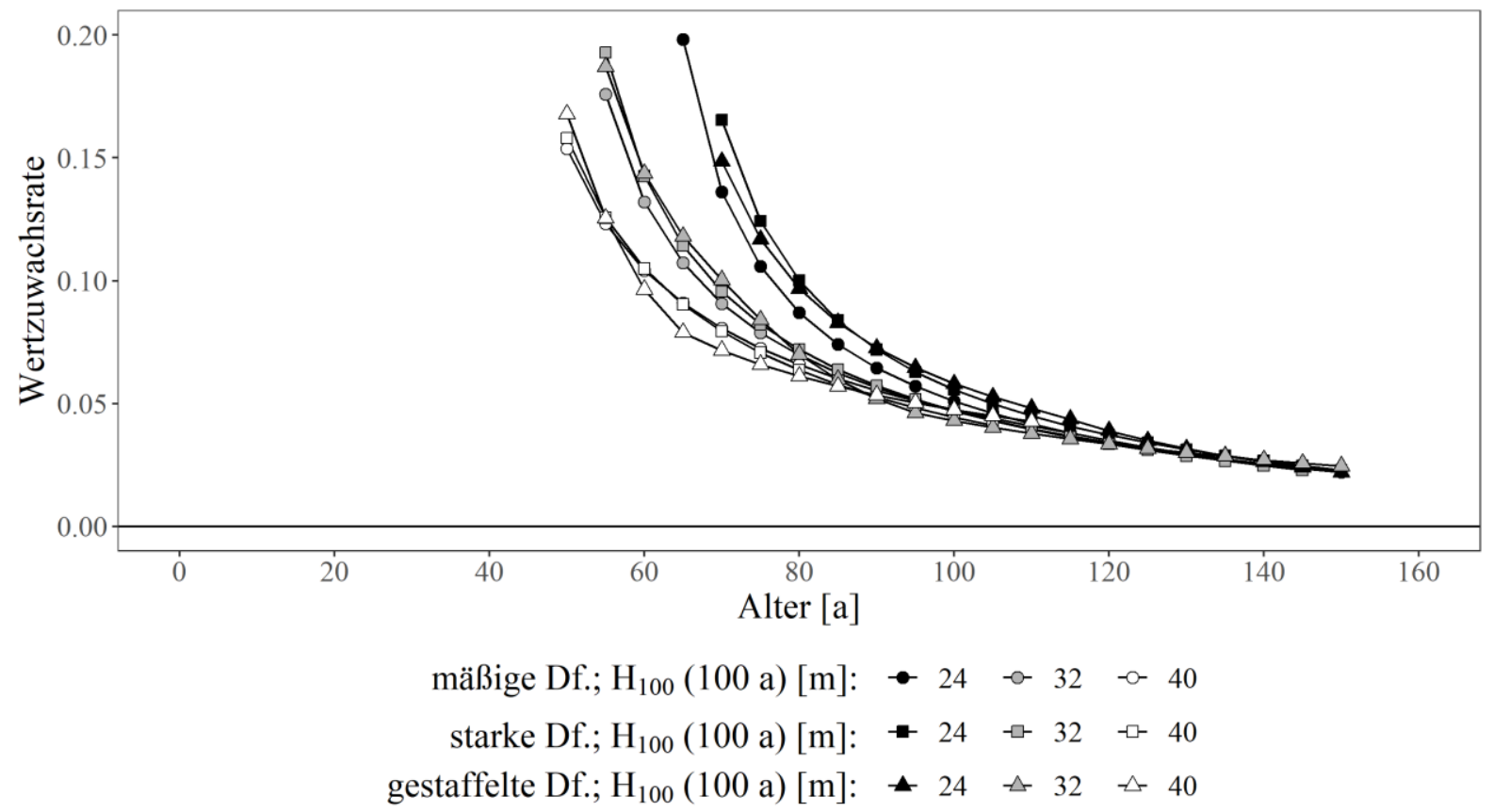

Abbildung 119: Wertzuwachsraten über dem Alter; ausgewählte Oberhöhenbonitäten der Baumart Buche getrennt nach Behandlungsvarianten gemäß dyn-ET (unveröff.). 


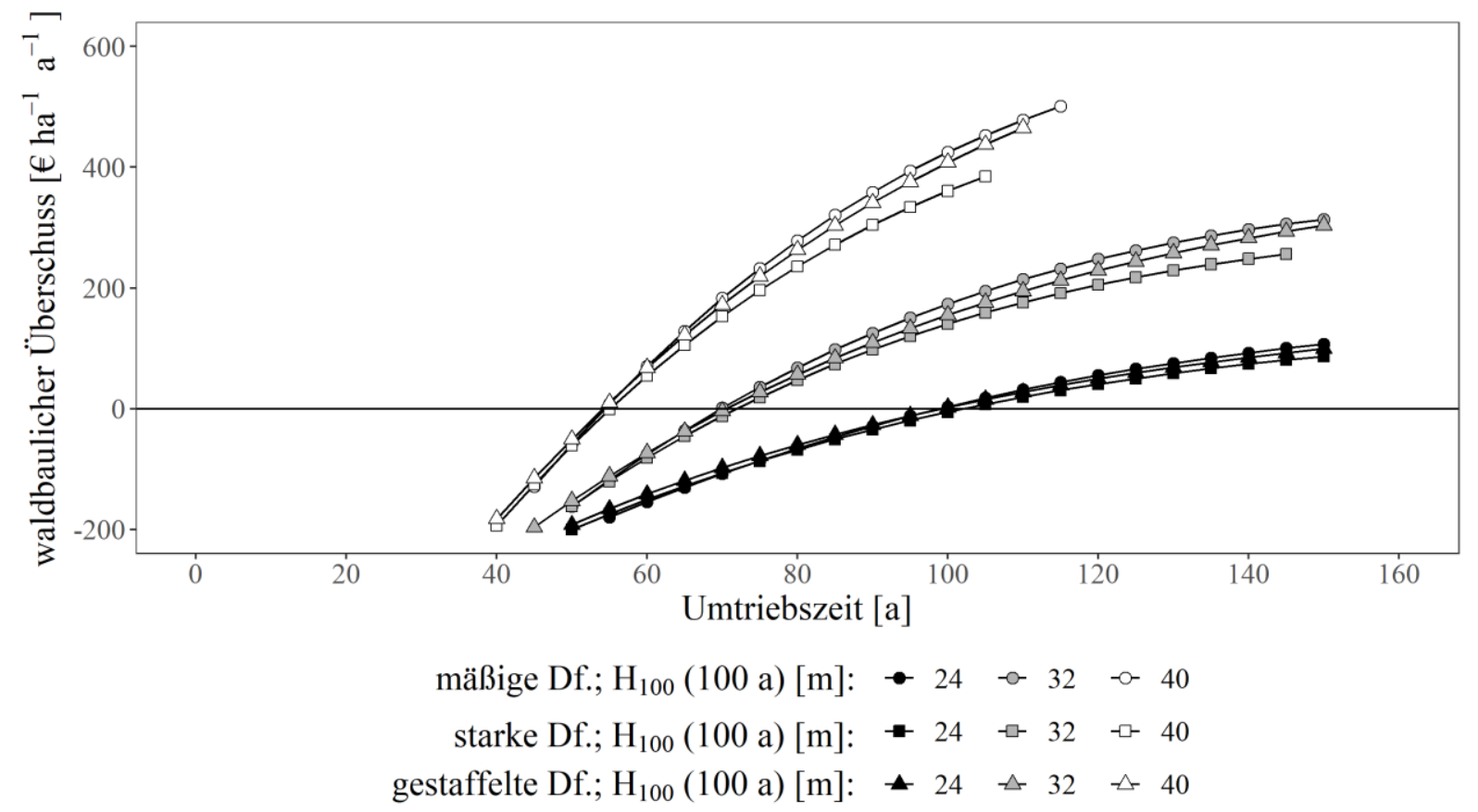

Abbildung 120: Durchschnittlicher waldbaulicher Überschuss der forstlichen Produktion über der Umtriebszeit; ausgewählte Oberhöhenbonitäten der Baumart Buche getrennt nach Behandlungsvarianten gemäß dyn-ET (unveröff.).

Auch bei den für die Analyse gewählten durchschnittlichen Erfolgsgrößen der forstlichen Produktion ist die mäßige Hochdurchforstung im überwiegenden Umtriebszeitbereich vorteilhaft ggü. den alterativen Behandlungsvarianten (Abbildungen 120 und 121). Lediglich bei sehr kurzen Produktionszeiten zeigt sich der bereits diskutierte Effekt einer wertmäßigen Überlegenheit bei gestaffelter und starker Hochdurchforstung aufgrund der höheren mittleren Einzelbaumdurchmesser. Verstärkt wird dies bei der Brutto-Bodenrente durch den Zinseffekt, d. h. die Vorteilhaftigkeit der früheren Realisierung höherer Werte (siehe auch Abschnitt 2.2.5). Mit zunehmender Umtriebszeit schließt die gestaffelte Hochdurchforstung sowohl beim zinsfrei berechneten durchschnittlichen waldbaulichen Überschuss als auch bei der Brutto-Bodenrente aufgrund der zunehmenden Volumenleistung zur mäßigen Hochdurchforstung auf. 


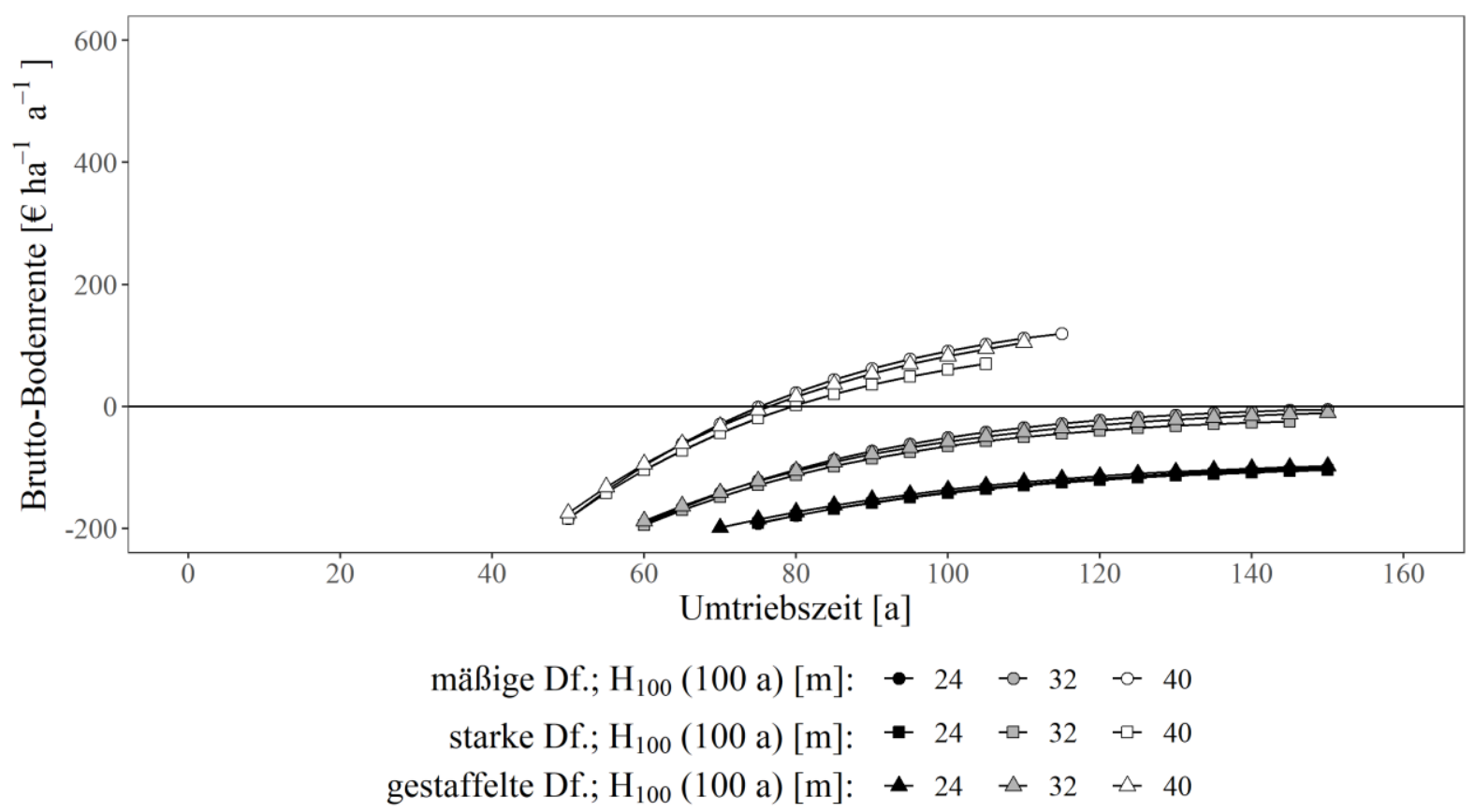

Abbildung 121: Brutto-Bodenrente ( $r=1,5 \%)$ über der Umtriebszeit; ausgewählte Oberhöhenbonitäten der Baumart Buche getrennt nach Behandlungsvarianten gemäß dyn-ET (unveröff.). 
Das ökonomische Leistungsspektrum der Baumart Fichte (mäßige Hochdurchforstung) gemäß dyn-ET (unveröff.) wird anhand des Gesamtwertleistungsfächers über dem Alter ersichtlich (Abbildung 122). Gegenüber der Baumart Buche werden gleiche Gesamtwertleistungen deutlich früher erreicht - bspw. erreicht die Baumart Fichte bei der Oberhöhenbonität $31 \mathrm{~m}$ eine Gesamtwertleistung von $50.000 € \mathrm{ha}^{-1}$ um 50 Jahre früher als die Baumart Buche bei der Oberhöhenbonität $32 \mathrm{~m}$. Die bis zu einem Alter von 60 Jahren erbrachte Wertleistung deckt einen Rahmen zwischen 13.753 und 60.180€ ha ${ }^{-1}$ ab (Tabelle 3-16). Die entsprechenden Gesamtwertleistungsentwicklungen bei starker Hochdurchforstung können Abbildung 197 im Anhang entnommen werden.

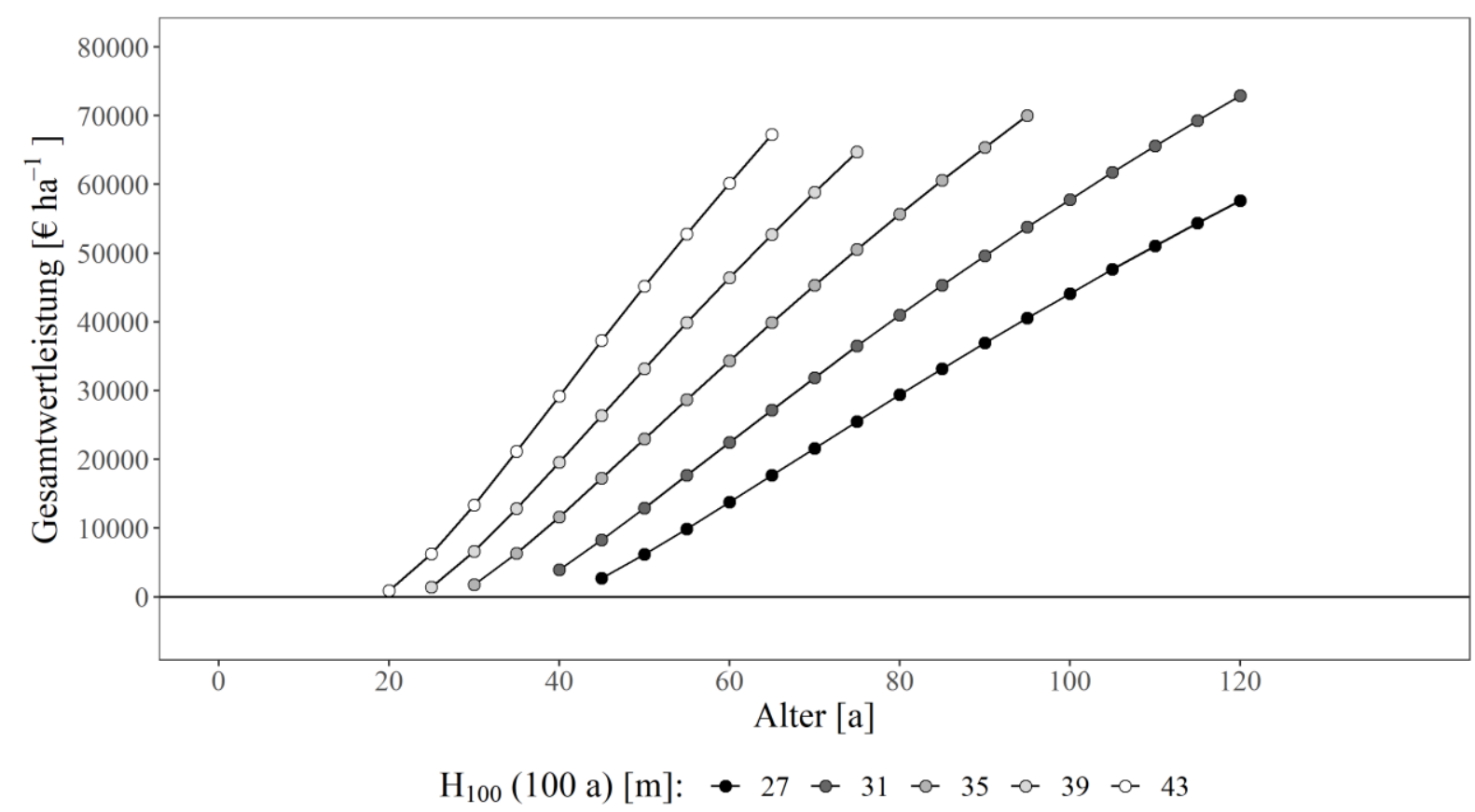

Abbildung 122: Gesamtwertleistung über dem Alter; alle Oberhöhenbonitäten der Baumart Fichte (mäBige Hochdurchforstung) gemäß dyn-ET (unveröff.).

Die laufenden Bestandeswertzuwächse der Baumart Fichte (mäßige Hochdurchforstung) gemäß dyn-ET (unveröff.) kulminieren umso früher, je höher die Oberhöhenbonität ist (Abbildung 123). Während bei einer Oberhöhenbonität von $27 \mathrm{~m}$ im Alter 70 a ein maximaler laufender Bestandeswertzuwachs von $787 € \mathrm{ha}^{-1} \mathrm{a}^{-1}$ erzielt wird, wächst der Bestandeswert bei einer Oberhöhenbonität von $43 \mathrm{~m}$ im Alter $40 \mathrm{a}$ um $1.616 € \mathrm{ha}^{-1} \mathrm{a}^{-1} \mathrm{zu}$. Gleichzeitig nimmt die Veränderung der laufenden Wertzuwächse über dem Alter, wie auch schon bei der Baumart Buche, mit steigender Oberhöhenbonität deutlich zu. Abbildung 198 im Anhang zeigt die entsprechenden Wertzuwachsentwicklungen bei starker Hochdurchforstung. 
Tabelle 3-16: Vergleich ausgewählter ökonomischer Bewertungsergebnisse der Baumart Fichte (mäßige Hochdurchforstung) gemäß dyn-ET (unveröff.); alle Oberhöhenbonitäten; in Klammern ist jeweils die relative Differenz zur Oberhöhenbonität $H_{100}(100 \mathrm{a})=35 \mathrm{~m}$ angegeben;

GWertL(60 a): Gesamtwertleistung bis zum Alter 60 a, $\max \boldsymbol{w}_{\boldsymbol{B}}$ : lfd. Bestandeswertzuwachs zum Zeitpunkt der Kulmination [...], $\boldsymbol{W}_{\boldsymbol{B}}(\mathbf{6 0} \mathbf{a})$ : Abtriebswert (verbl. Bestand) im Alter $60 \mathrm{a}$, $\sum_{\mathbf{0}}^{\mathbf{6 0} \mathbf{a}} \boldsymbol{\lambda} \cdot \mathbf{P}\left(\boldsymbol{D}_{\boldsymbol{g}}\right)$ : kumulierte Vornutzungswerte bis zum Alter $60 \mathrm{a}, \boldsymbol{a}_{\boldsymbol{\eta}}(\mathbf{6 0} \mathrm{a})$ : durchschn. waldbaulicher Überschuss einer nachhaltigen Betriebsklasse bei einer Umtriebszeit von $60 a, \boldsymbol{W}_{\boldsymbol{\eta}}(\mathbf{6 0} \mathbf{a})$ : durchschnittlicher Abtriebswert einer nachhaltigen Betriebsklasse bei einer Umtriebszeit von 60 a, $\boldsymbol{a}(60 \mathrm{a})$ : Brutto-Bodenrente bei einer Umtriebszeit von 60 a.

\begin{tabular}{|c|c|c|c|c|c|}
\hline \multirow{2}{*}{$\begin{array}{l}\text { Bestandesbehandlung } \\
\qquad H_{100}(100 \mathrm{a})[\mathrm{m}]\end{array}$} & \multicolumn{5}{|c|}{ mäßige Hochdurchforstung } \\
\hline & 27 & 31 & 35 & 39 & 43 \\
\hline GWertL $(60$ a $)$ & 13.753 & 22.457 & 34.373 & 46.429 & 60.180 \\
\hline$\left[€ \mathbf{h a}^{-1}\right]$ & $(-60,0 \%)$ & $(-34,7 \%)$ & & $(+35,1 \%)$ & $(+75,1 \%)$ \\
\hline $\max w_{B}$ & $787[70 \mathrm{a}]$ & $956[60 \mathrm{a}]$ & $1.147[55 \mathrm{a}]$ & $1.367[45 \mathrm{a}]$ & $1.616[40 \mathrm{a}]$ \\
\hline$\left[€ \mathbf{h a}^{-1} \mathbf{a}^{-1}\right]$ & $(-31,4 \%)$ & $(-16,7 \%)$ & & $(+19,2 \%)$ & $(+40,9 \%)$ \\
\hline$W_{B}(60 \mathrm{a})$ & 10.457 & 15.937 & 21.575 & 27.090 & 32.279 \\
\hline$\left[€ \mathbf{h a}^{-1}\right]$ & $(-51,5 \%)$ & $(-26,1 \%)$ & & $(+25,6 \%)$ & $(+49,6 \%)$ \\
\hline$\sum_{0}^{60 a} \lambda \cdot \mathbf{P}\left(D_{g}\right)$ & 3.296 & 6.520 & 12.798 & 19.339 & 27.901 \\
\hline$\left[€ h a^{-1}\right]$ & $(-74,2 \%)$ & $(-49,1 \%)$ & & $(+51,1 \%)$ & $(+118,0 \%)$ \\
\hline$a_{\eta}(60 \mathrm{a})$ & 164 & 309 & 508 & 709 & 937 \\
\hline$\left[€ \mathbf{h a}^{-1} \mathbf{a}^{-1}\right]$ & $(-67,7 \%)$ & $(-39,2 \%)$ & & $(+39,6 \%)$ & $(+84,4 \%)$ \\
\hline$W_{\eta}(60 \mathrm{a})$ & 2.029 & 3.964 & 6.784 & 9.794 & 13.070 \\
\hline$\left[€ \mathbf{h a}^{-1}\right]$ & $(-70,1 \%)$ & $(-41,6 \%)$ & & $(+44,4 \%)$ & $(+92,7 \%)$ \\
\hline$a(60 a)$ & 47 & 141 & 281 & 426 & 601 \\
\hline$\left[€ \mathbf{h a}^{-1} a^{-1}\right]$ & $(-83,3 \%)$ & $(-49,8 \%)$ & & $(+51,6 \%)$ & $(+113,9 \%)$ \\
\hline
\end{tabular}




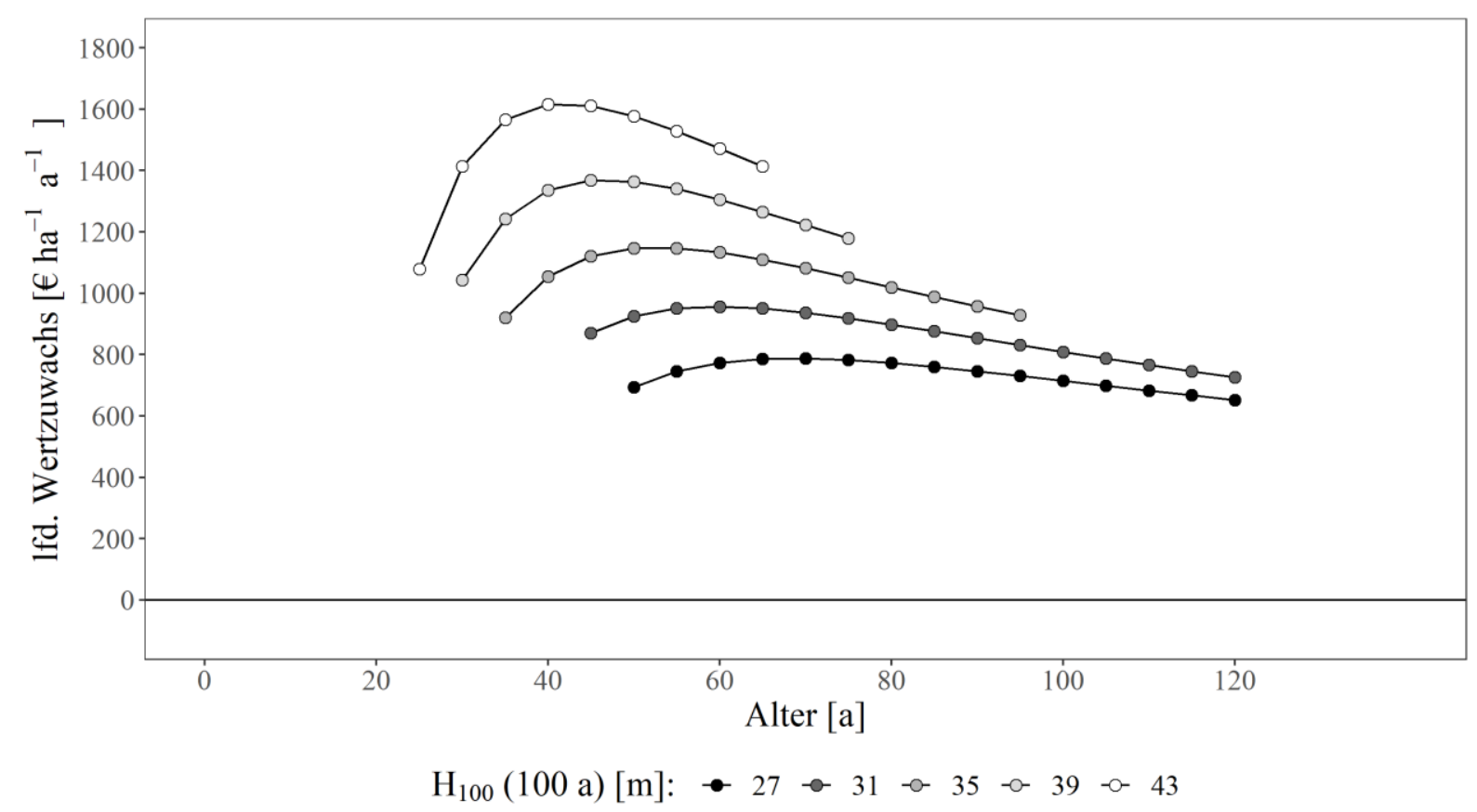

Abbildung 123: Laufender Bestandeswertzuwachs über dem Alter; alle Oberhöhenbonitäten der Baumart Fichte (mäßige Hochdurchforstung) gemäß dyn-ET (unveröff.).

Die in Abschnitt 3.3.3 beschriebenen ertragskundlichen Veränderungen ggü. dem etablierten Ertragstafelwerk von Wiedemann (1936/42) führen auch bei der Fichte zu erheblich veränderten ökonomischen Bewertungsergebnissen. Für die mäßige Durchforstung führt das erhöhte Ertragsniveau (siehe Abbildung 81) in Verbindung mit den stark gestiegenen mittleren Einzelbaumdurchmessern im verbleibenden und ausscheidenden Bestand (siehe Abbildungen 89 und 90) zu einer nochmal deutlicheren Steigerung der Gesamtwertleistung als bei der Baumart Buche - sie beträgt im Alter 80 a bei annähernd gleicher Oberhöhenentwicklung ausgewählter Oberhöhenbonitäten zwischen 62 \% und 131 \% (Tabelle 3-17 und Abbildung 124). Andersherum als bei der Buche (siehe Tabelle 3-14) steigt die relative Zunahme der Gesamtwertleistungen ggü. Wiedemann (1936/42, mäßige Durchforstung) mit sinkender Oberhöhenbonität.

Entsprechend der Gesamtwertleistungen steigen auch die laufenden Bestandeswertzuwächse gemäß dyn-ET (unveröff., mäßige Hochdurchforstung) deutlich an (Abbildung 125). Sie kulminieren ggü. Wiedemann (1936/42, mäßige Durchforstung) früher auf einem um 44 \% und 59 \% höheren Niveau (Tabelle 3-17). Die entsprechenden Vergleichsgrafiken der starken Durchforstung können für die Gesamtwertleistung (Abbildung 199) sowie den laufenden Bestandeswertzuwachs (Abbildung 200) dem Anhang entnommen werden. 


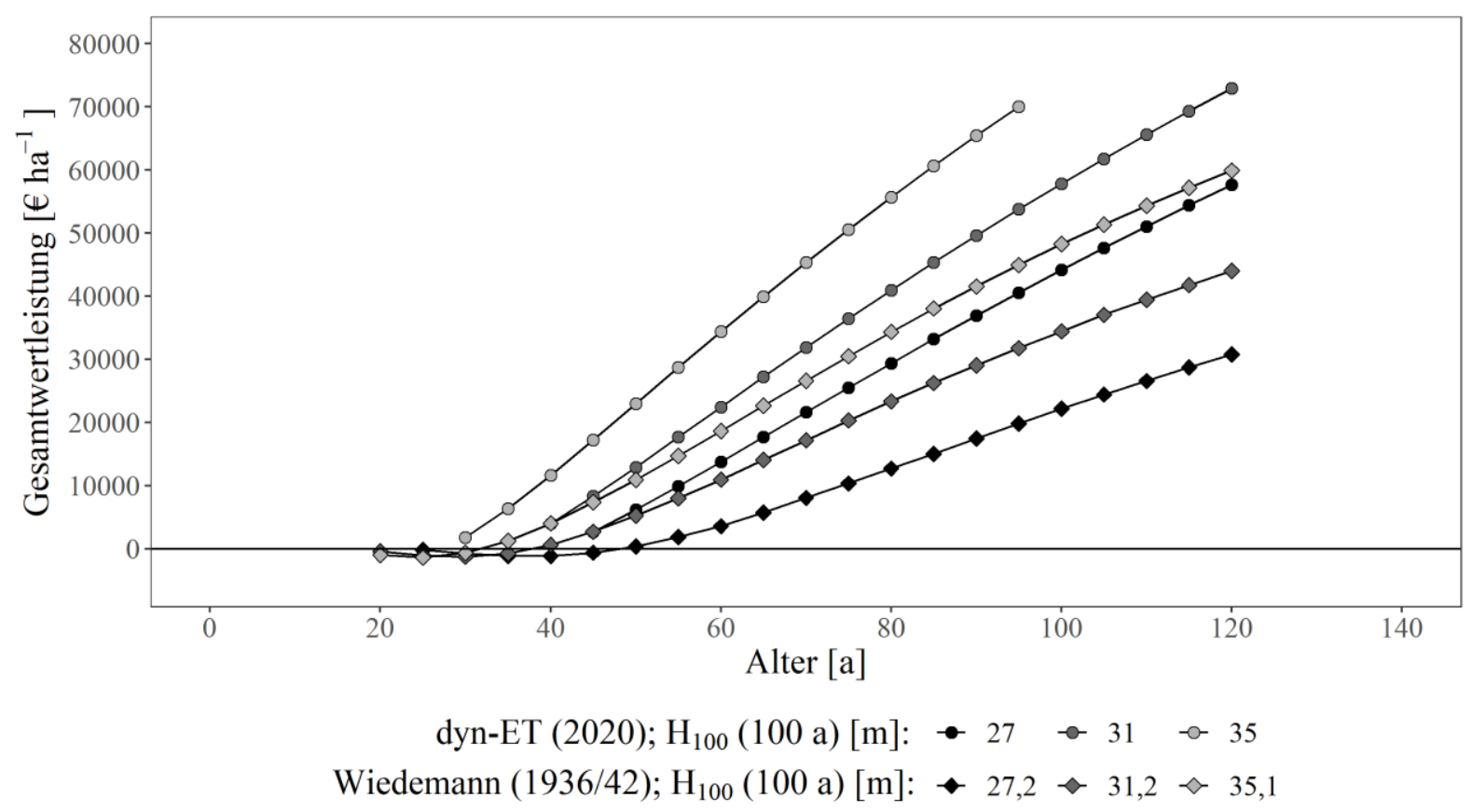

Abbildung 124: Gesamtwertleistung über dem Alter; ausgewählte Oberhöhenbonitäten der Baumart Fichte (mäßige Durchforstung) gemäß dyn-ET (unveröff.) und Wiedemann (1936/42).

Aus den ggü. Wiedemann (1936/42, mäßige Durchforstung) annähernd unveränderten (niedrige Oberhöhenbonität) bzw. leicht gesunkenen (hohe Oberhöhenbonitäten) Vorräten (siehe Abbildung 84) resultieren in Verbindung mit den (bei niedrigen Oberhöhenbonitäten deutlich) gestiegenen mittleren Einzelbaumdurchmessern des verbleibenden Bestandes (siehe Abbildung 89) insb. bei niedrigen Oberhöhenbonitäten gesteigerte Abtriebswerte (Abbildung 126). Im Alter 80 a beträgt die Steigerung $1 \%$ bis $52 \%$ (Tabelle 3-17). Bei den gleichzeitig deutlich höheren laufenden Bestandeswertzuwächsen (Abbildung 125) folgen gestiegene Wertzuwachsraten insbesondere bei mittlerer und hoher Oberhöhenbonität (Abbildung 127). Ähnliche Effekte zeigen sich für den Vergleich bei starker Durchforstung in den Abbildungen 201 und $202 \mathrm{im}$ Anhang. 
Tabelle 3-17: Vergleich ausgewählter ökonomischer Bewertungsergebnisse (mäßige Durchforstung); ausgewählte Oberhöhenbonitäten gemäß dyn-ET (unveröff.) und Wiedemann (1936/42) in Klammern ist jeweils die relative Differenz zur vergleichbaren Oberhöhenbonität nach Wiedemann (1936/42) angegeben;

GWertL(80 a): Gesamtwertleistung bis zum Alter 80 a, $\max \boldsymbol{w}_{\boldsymbol{B}}$ : lfd. Bestandeswertzuwachs zum Zeitpunkt der Kulmination [...], $\boldsymbol{W}_{\boldsymbol{B}}(\mathbf{8 0} \mathrm{a})$ : Abtriebswert (verbl. Bestand) im Alter $80 \mathrm{a}$, $\sum_{0}^{80} \mathbf{a} \cdot \mathbf{P}\left(\boldsymbol{D}_{\boldsymbol{g}}\right)$ : kumulierte Vornutzungswerte bis zum Alter 80 a, $\boldsymbol{a}_{\boldsymbol{\eta}}(\mathbf{8 0} \mathbf{a})$ : durchschn. waldbaulicher Überschuss einer nachhaltigen Betriebsklasse bei einer Umtriebszeit von $80 a, \boldsymbol{W}_{\boldsymbol{\eta}}(\mathbf{8 0}$ a): durchschnittlicher Abtriebswert einer nachhaltigen Betriebsklasse bei einer Umtriebszeit von 80 a, $\boldsymbol{a}(\mathbf{8 0} \mathbf{a})$ : Brutto-Bodenrente bei einer Umtriebszeit von 80 a.

\begin{tabular}{|c|c|c|c|c|c|c|}
\hline \multirow{2}{*}{$\begin{array}{r}\text { Ertragstafel } \\
H_{100}(100 \text { a })[\mathrm{m}]\end{array}$} & \multicolumn{3}{|c|}{ Wiedemann (1936/42), mäßige Df. } & \multicolumn{3}{|c|}{ dyn-ET (unveröff.), mäßige Df. } \\
\hline & 27,2 & 31,2 & 35,1 & 27 & 31 & 35 \\
\hline$G W \operatorname{ert} L(80 \mathrm{a})$ & 12.713 & 23.336 & 34.359 & 29.389 & 40.977 & 55.681 \\
\hline$\left[€ \mathbf{h a}^{-1}\right]$ & & & & $(+131,2 \%$ & $(+75,6 \%)$ & $(+62,1 \%)$ \\
\hline $\max w_{B}$ & 494 & 629 & 799 & $787[70 \mathrm{a}]$ & $956[60 \mathrm{a}]$ & $1.147[55 \mathrm{a}]$ \\
\hline$\left[€ \mathbf{h a}^{-1} \mathbf{a}^{-1}\right]$ & {$[90 \mathrm{a}]$} & {$[75 \mathrm{a}]$} & {$[65 \mathrm{a}]$} & $(+59,3 \%)$ & $(+52,0 \%)$ & $(+43,6 \%)$ \\
\hline$W_{B}(80 \mathbf{a})$ & 12.513 & 21.493 & 30.327 & 18.983 & 24.982 & 30.739 \\
\hline$\left[€ \mathbf{h a}^{-1}\right]$ & & & & $(+51,7 \%)$ & $(+16,2 \%)$ & $(+1,4 \%)$ \\
\hline$\sum_{0}^{80 a} \lambda \cdot \mathbf{P}\left(D_{g}\right)$ & 200 & 1.843 & 4.032 & 10.406 & 15.995 & 24.942 \\
\hline$\left[€ h a^{-1}\right]$ & & & & $(+5.103 \%$ & $(+767,9 \%$ & $(+518,6 \%)$ \\
\hline$a_{\eta}(80 \mathbf{a})$ & 110 & 243 & 381 & 319 & 463 & 647 \\
\hline$\left[€ \mathbf{h a}^{-1} \mathbf{a}^{-1}\right]$ & & & & $(+190,0 \%$ & $(+90,5 \%)$ & $(+69,8 \%)$ \\
\hline$W_{\eta}(80 \mathrm{a})$ & 2.294 & 5.640 & 9.226 & 5.454 & 8.432 & 12.068 \\
\hline$\left[€ \mathbf{h a}^{-1}\right]$ & & & & $(+137,8 \%$ & $(+49,5 \%)$ & $(+30,8 \%)$ \\
\hline$a(80 a)$ & -2 & 68 & 143 & 124 & 212 & 339 \\
\hline$\left[€ \mathbf{h a}^{-1} a^{-1}\right]$ & & & & & $(+212 \%)$ & $(+137 \%)$ \\
\hline
\end{tabular}




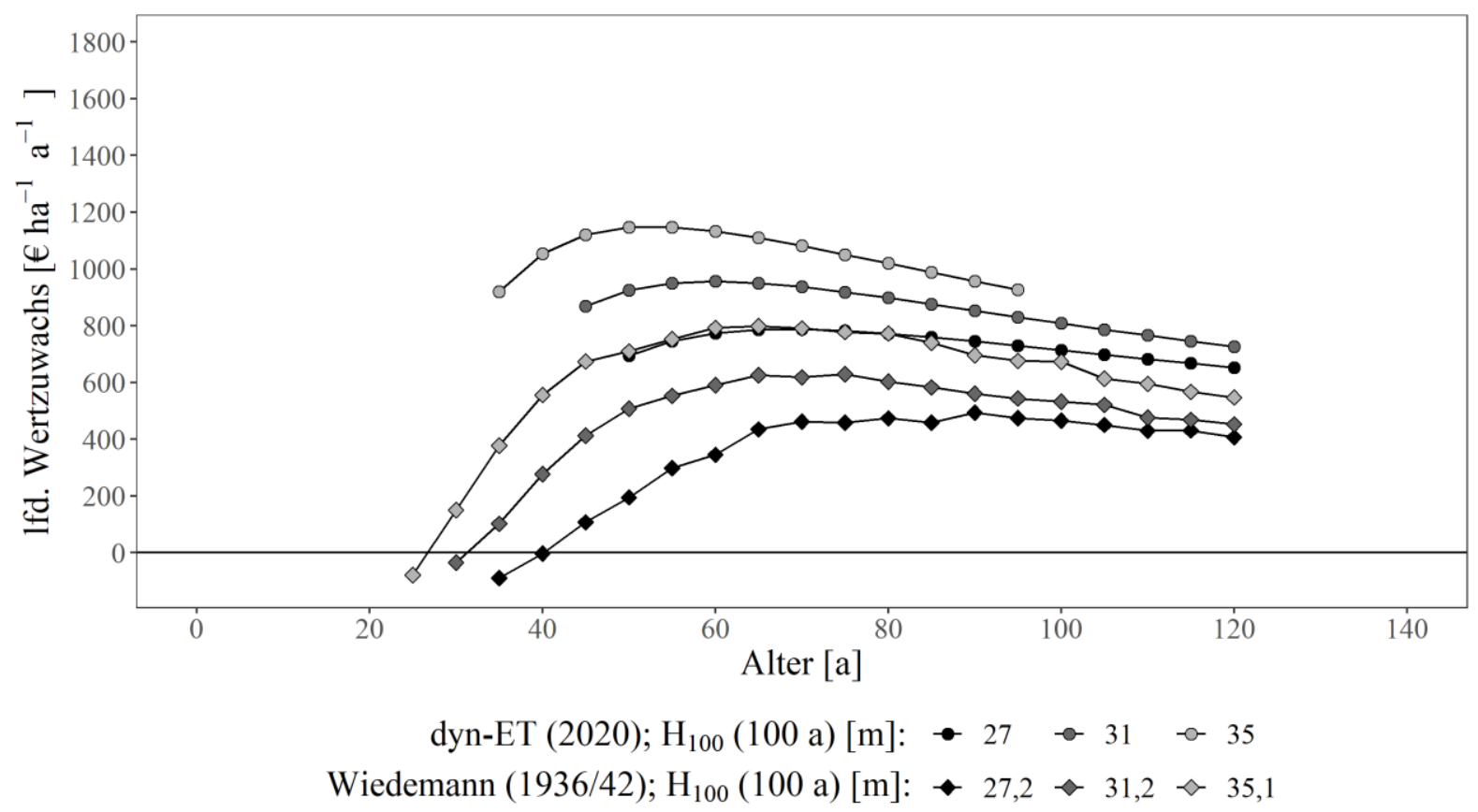

Abbildung 125: Laufender Bestandeswertzuwachs über dem Alter; ausgewählte Oberhöhenbonitäten der Baumart Fichte (mäßige Durchforstung) gemäß dyn-ET (unveröff.) und Wiedemann (1936/42).

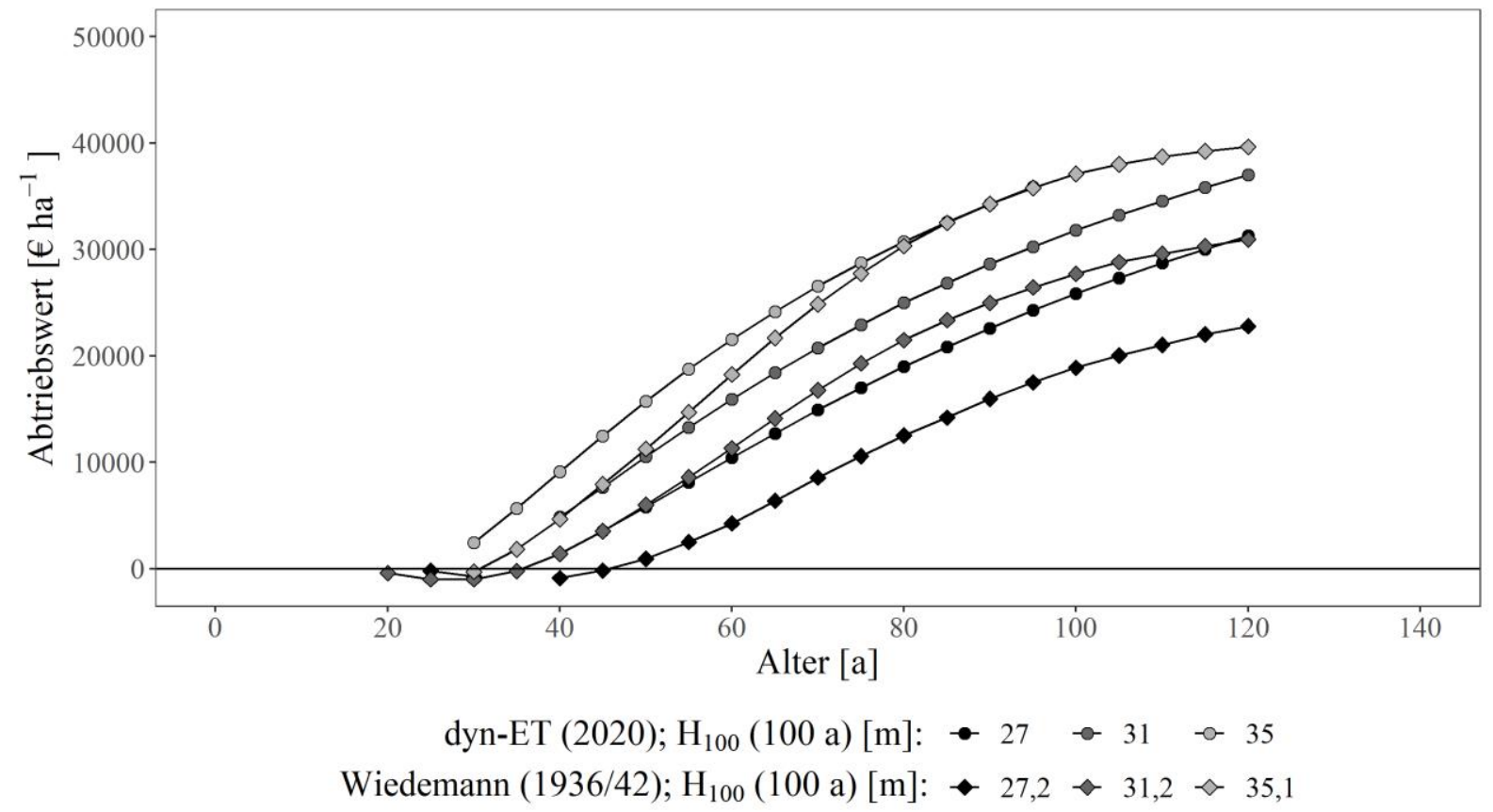

Abbildung 126: Abtriebswert über dem Alter; ausgewählte Oberhöhenbonitäten der Baumart Fichte (mäßige Durchforstung) gemäß dyn-ET (unveröff.) und Wiedemann (1936/42). 


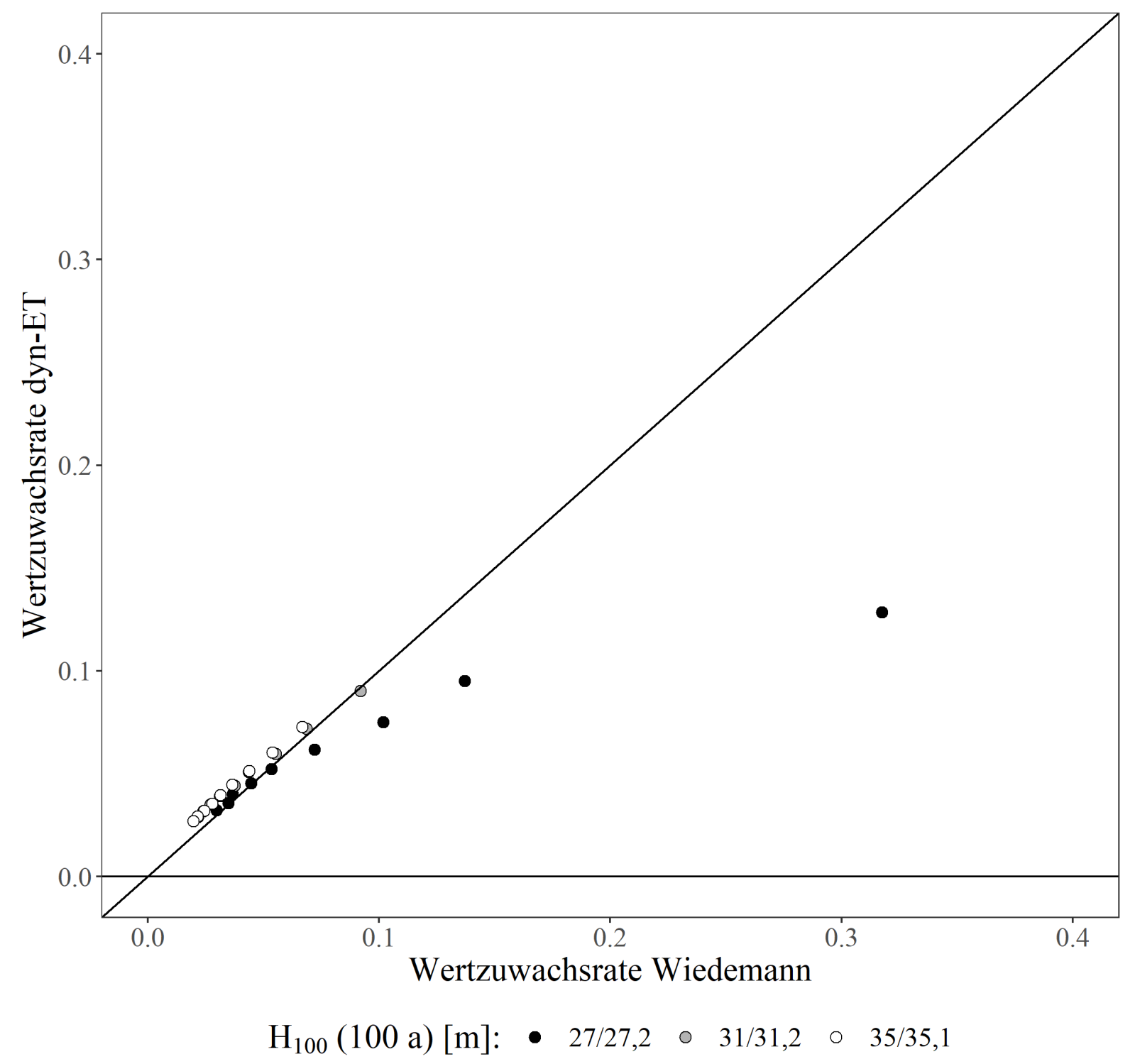

Abbildung 127: Wertzuwachsraten der Baumart Fichte (mäßige Durchforstung, ausgewählte Oberhöhenbonitäten) gemäß dyn-ET (unveröff.) und Wiedemann (1936/42); es werden nur Vergleichspaare aus Altern dargestellt, in denen für alle Oberhöhenbonitäten Datenpunkte vorliegen; die Winkelhalbierende kennzeichnet gleiche Wertzuwachsraten im gleichen Alter.

Bei einer Umtriebszeit von 80 a liegen die durchschnittlichen waldbaulichen Überschüsse gemäß dyn-ET (unveröff., mäßige Hochdurchforstung) um 70 \% (hohe Oberhöhenbonität) bis 190 \% (niedrige Oberhöhenbonität) höher als gemäß Wiedemann (1936/42, mäßige Durchforstung) (Tabelle 3-17 und Abbildung 128 bzw. Abbildung 203 im Anhang für die starke Durchforstung). Die korrespondierenden durchschnittlichen Abtriebswerte einer nachhaltigen Betriebsklasse liegen ggü. Wiedemann (1936/42, mäßige Durchforstung) bonitätsunabhängig höher - bei einer Umtriebszeit von 80 a um $31 \%$ (hohe Oberhöhenbonität) bis 138 \% (niedrige Oberhöhenbonität; Tabelle 3-17 und Abbildung 129). Abbildung 204 zeigt den entsprechende Vergleich der durchschnittlichen Abtriebswerte bei starker Durchforstung. 


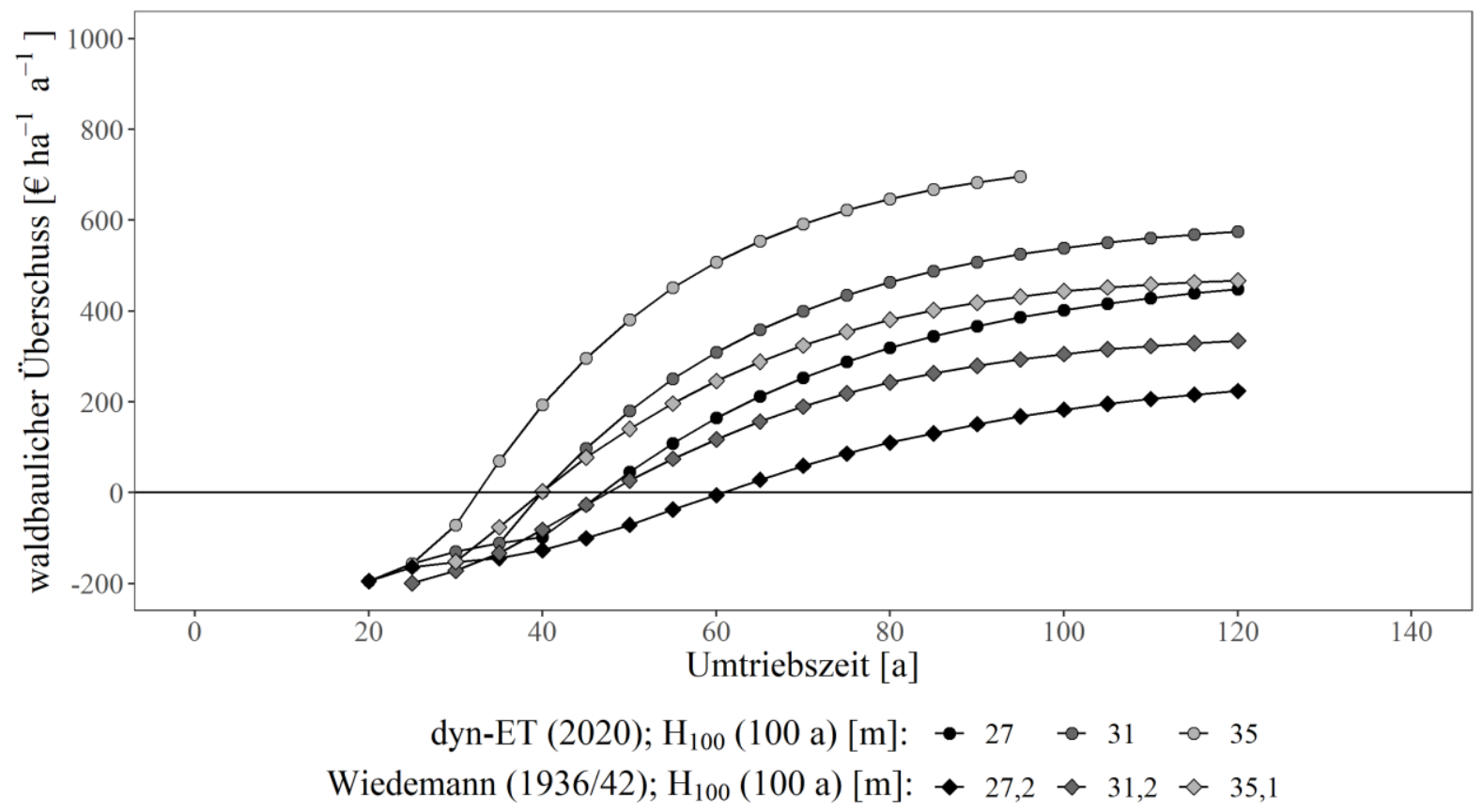

Abbildung 128: Durchschnittlicher waldbaulicher Überschuss der forstlichen Produktion über der Umtriebszeit; ausgewählte Oberhöhenbonitäten der Baumart Fichte (mäßige Durchforstung) gemäß dynET (unveröff.) und Wiedemann (1936/42).

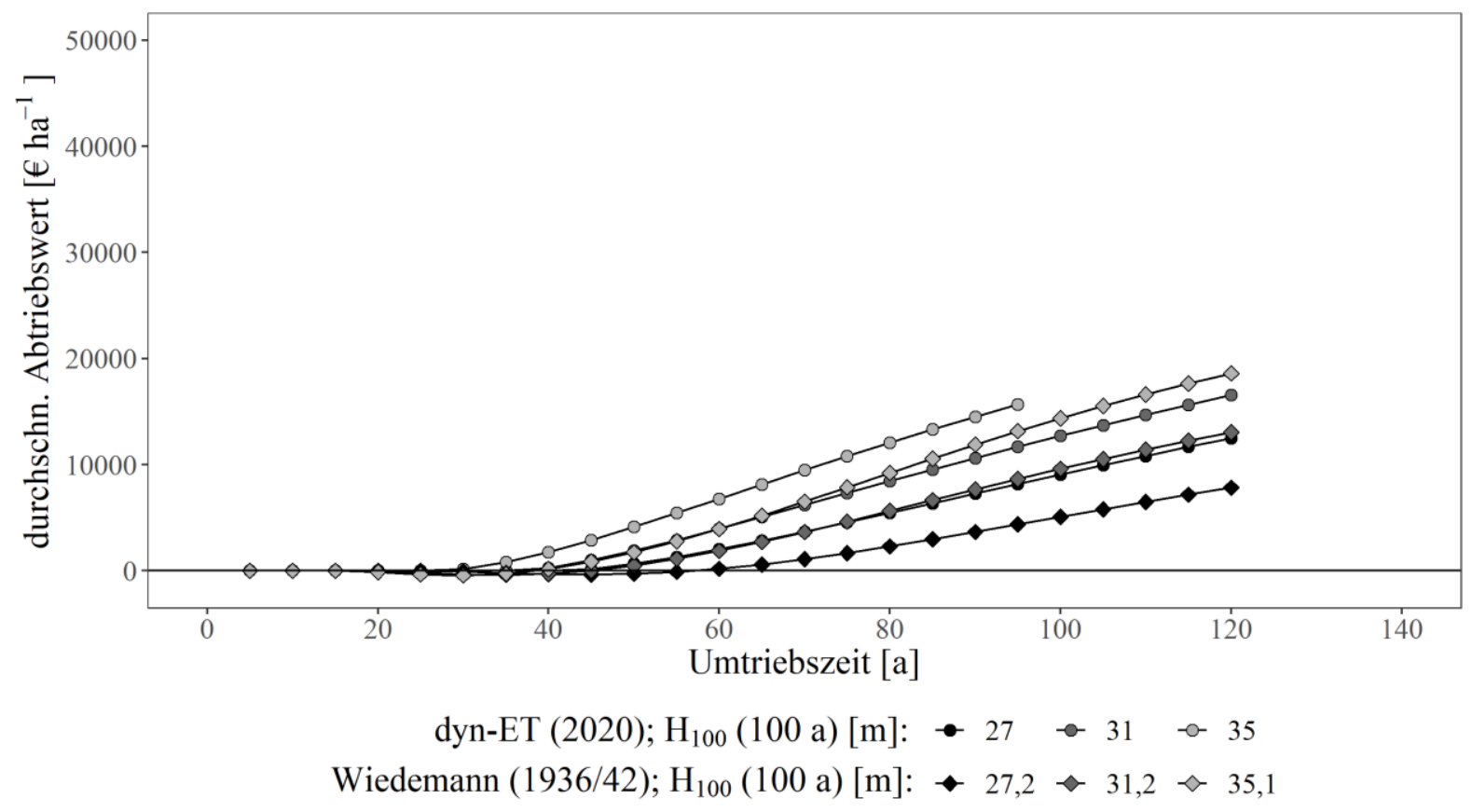

Abbildung 129: Durchschnittlicher Abtriebswert über der Umtriebszeit; ausgewählte Oberhöhenbonitäten der Baumart Fichte (mäßige Durchforstung) gemäß dyn-ET (unveröff.) und Wiedemann (1936/42). 


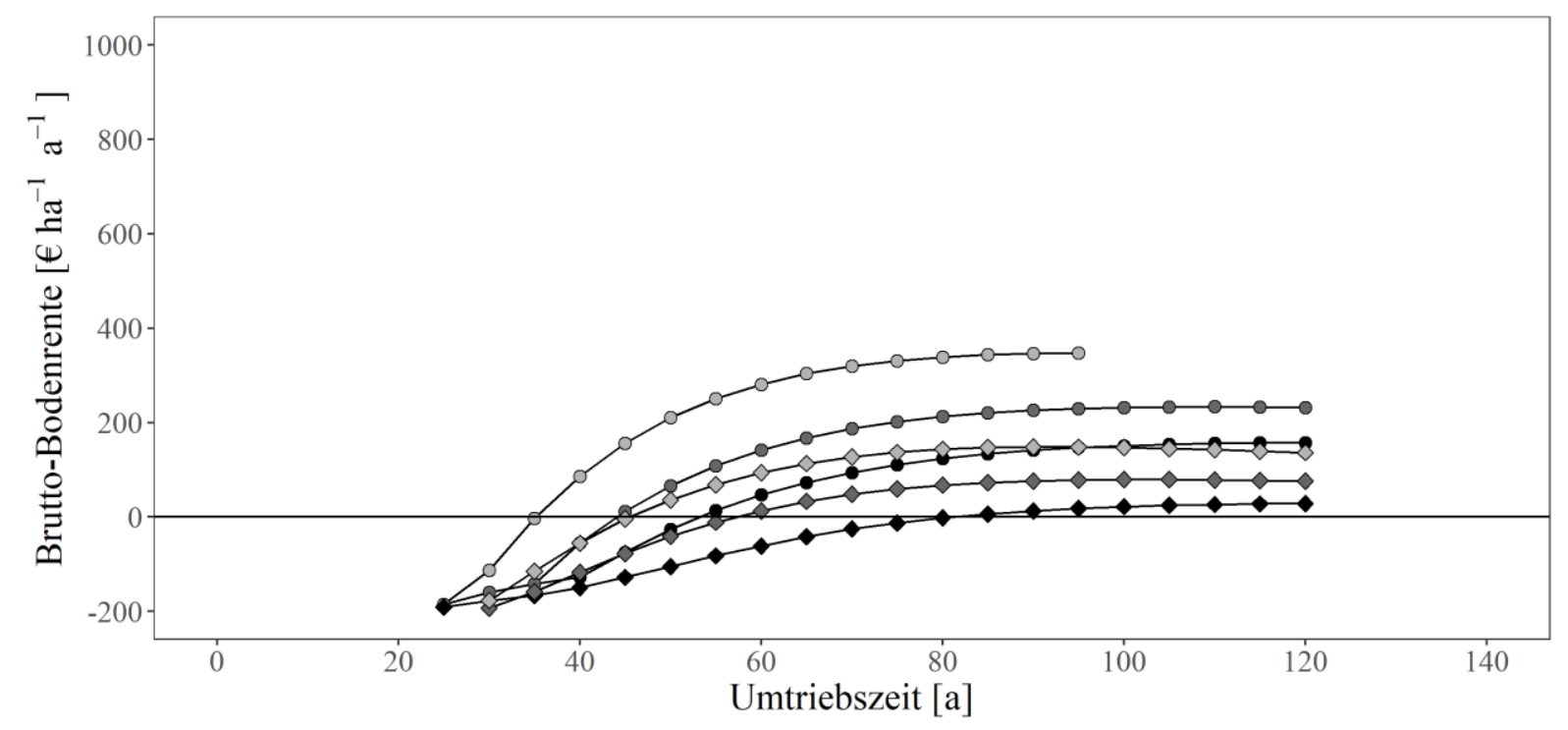

$$
\begin{aligned}
& \text { dyn-ET (2020); } \mathrm{H}_{100}(100 \mathrm{a})[\mathrm{m}]:-27 \quad \rightarrow 31 \quad \text { - } 27 \\
& \text { Wiedemann (1936/42); } \mathrm{H}_{100}(100 \mathrm{a})[\mathrm{m}]: \bullet 27,2 \diamond 31,2 \diamond 35,1
\end{aligned}
$$

Abbildung 130: Brutto-Bodenrente ( $r=1,5 \%)$ über der Umtriebszeit; ausgewählte Oberhöhenbonitäten der Baumart Fichte (mäßige Durchforstung) gemäß dyn-ET (unveröff.) und Wiedemann (1936/42). Gegenüber Wiedemann (1936/42, mäßige Durchforstung) steigen die mit einem Kalkulationszinssatz von $r=1,5 \%$ berechneten Brutto-Bodenrenten bei einer Umtriebszeit von $80 \mathrm{a}$ deutlich an (Tabelle 3-17) - bei Umtriebszeiten über 50 Jahren sind sie selbst bei der niedrigsten Oberhöhenbonität positiv (Abbildung 130; analog dazu Abbildung 205 im Anhang für die starke Durchforstung).

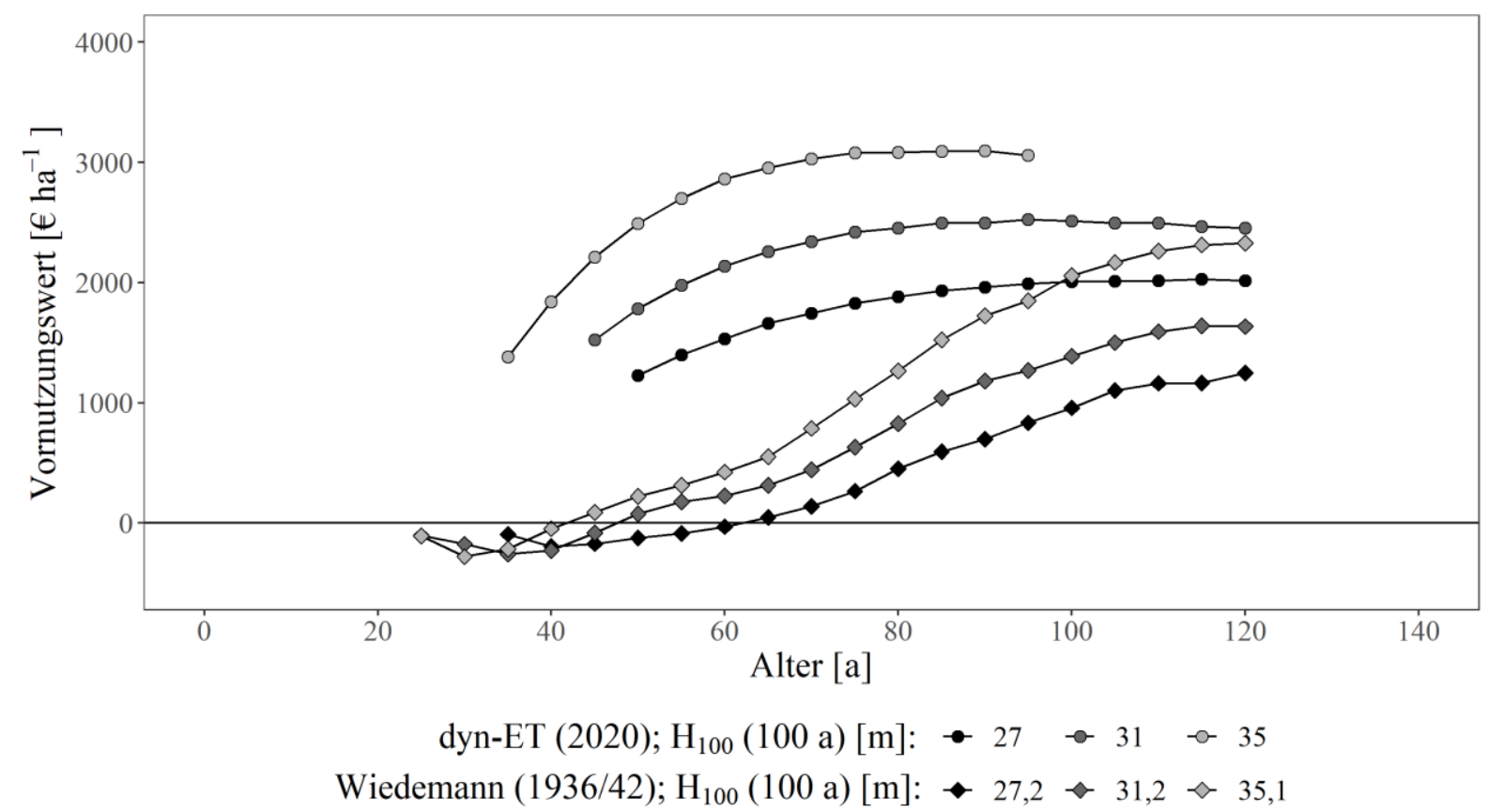

Abbildung 131: Vornutzungswerte über dem Alter; ausgewählte Oberhöhenbonitäten der Baumart Fichte (mäßige Durchforstung) gemäß dyn-ET (unveröff.) und Wiedemann (1936/42). 
Die unter Kapitalknappheit nochmal deutlich höhere Differenz (Tabelle 3-17) zwischen dynET (unveröff., mäßige Hochdurchforstung) und Wiedemann (1936/42, mäßige Durchforstung) lässt sich u. a. anhand der deutlich höheren Vornutzungswerte in niedrigen Bestandesaltern erklären (Abbildung 131 und analog dazu Abbildung 206 im Anhang für die starke Durchforstung). Werte gleichen Niveaus werden nach dyn-ET (unveröff., mäßige Hochdurchforstung) um bis zu 60 Jahre früher realisiert, was unter dem Zinseffekt erhebliche Auswirkungen auf die jährliche Erfolgsgröße hat.

Im ökonomischen Vergleich der waldbaulichen Behandlungsvarianten gemäß dyn-ET (unveröff.) zeigt sich bei der Baumart Fichte im Vergleich zur Analyse der vorläufigen Ertragstafeldaten in Abschnitt 3.3.3 nochmal stärker als bei der Buche der Einfluss der Dimensionssteigerung durch die starke Hochdurchforstung. Während die Gesamtwuchsleistung bei starker Hochdurchforstung bonitätsunabhängig deutlich niedriger als bei mäßiger Hochdurchforstung ist (siehe Abbildung 93), zeigt sich für die Gesamtwertleistung mit zunehmender Oberhöhenbonität eine Reduktion der wertmäßigen Unterschiede (Abbildung 132) bzw. sogar eine ökonomische Vorteilhaftigkeit der starken Hochdurchforstung zu Beginn der forstlichen Produktion. Siehe dazu auch die laufenden Bestandeswertzuwächse in Abbildung 133.

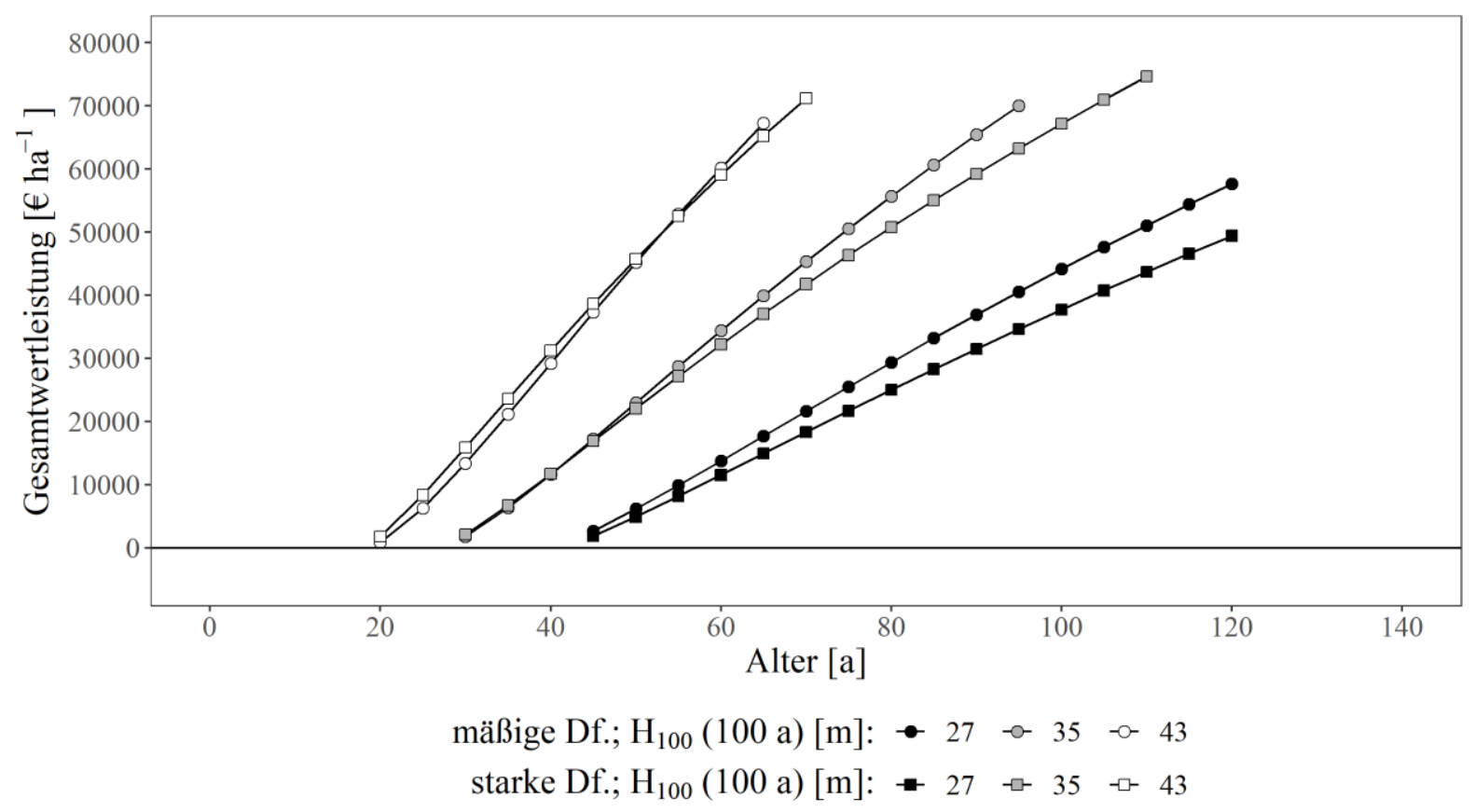

Abbildung 132: Gesamtwertleistung über dem Alter; ausgewählte Oberhöhenbonitäten der Baumart Fichte getrennt nach Behandlungsvarianten gemäß dyn-ET (unveröff.).

Durch die Bewertung der ausscheidenden Bestandesvolumina mit der dimensionsabhängigen Deckungsbeitragsfunktion führen die (insb. bei hohen Oberhöhenbonitäten) deutlich höheren 
mittleren Einzelbaumdurchmesser bei starker Hochdurchforstung (Tabelle 3-7; Abbildungen 98 und 99) zu einer Kompensation der im Vergleich zur mäßigen Hochdurchforstung reduzierten Volumenleistung (Tabelle 3-18; siehe dazu auch die tiefergehenden Sensitivitätsanalysen in Abschnitt 3.3.6).

Tabelle 3-18: Vergleich ausgewählter ökonomischer Bewertungsergebnisse der Baumart Fichte (Oberhöhenbonität $\left.H_{100}(100 \mathrm{a})=35 \mathrm{~m}\right)$ gemäß dyn-ET (unveröff.); unterschiedliche Behandlungsvarianten; in Klammern ist jeweils die relative Differenz zur mäßigen Hochdurchforstung angegeben; GWertL(80 a): Gesamtwertleistung bis zum Alter $80 \mathrm{a}, \boldsymbol{m a x} \boldsymbol{w}_{\boldsymbol{B}}$ : lfd. Bestandeswertzuwachs zum Zeitpunkt der Kulmination [...], $\boldsymbol{W}_{\boldsymbol{B}}(\mathbf{8 0} \mathbf{a})$ : Abtriebswert (verbl. Bestand) im Alter $80 \mathrm{a}$, $\sum_{0}^{80} \mathbf{a} \lambda \cdot \mathbf{P}\left(\boldsymbol{D}_{\boldsymbol{g}}\right)$ : kumulierte Vornutzungswerte bis zum Alter 80 a, $\boldsymbol{a}_{\boldsymbol{\eta}}(\mathbf{8 0} \mathbf{a})$ : durchschn. waldbaulicher Überschuss einer nachhaltigen Betriebsklasse bei einer Umtriebszeit von $80 a, \boldsymbol{W}_{\boldsymbol{\eta}}(\mathbf{8 0} \mathbf{a})$ : durchschnittlicher Abtriebswert einer nachhaltigen Betriebsklasse bei einer Umtriebszeit von 80 a, $\boldsymbol{a}(\mathbf{8 0} \mathbf{a})$ : Brutto-Bodenrente bei einer Umtriebszeit von 80 a.

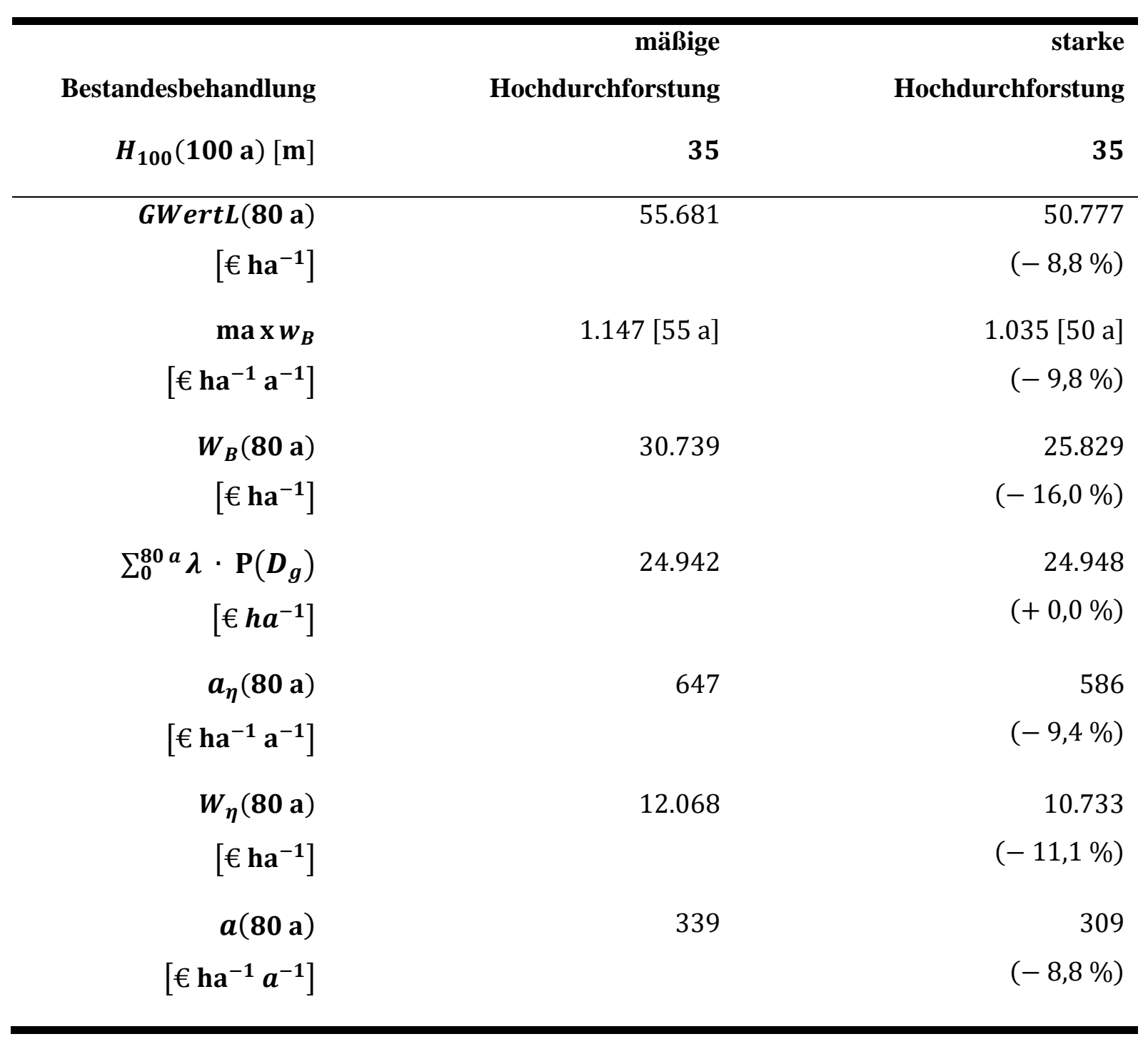


Zum Zeitpunkt der jeweiligen Wertzuwachskulmination liegen die laufenden Bestandeswertzuwächse bei starker aber stets unterhalb der laufenden Bestandeswertzuwächse bei mäßiger Hochdurchforstung - bspw. bei der mittleren Oberhöhenbonität $35 \mathrm{~m}$ mit $1.035 € \mathrm{ha}^{-1} \mathrm{a}^{-1}$ um $10 \%$ (Tabelle 3-18).

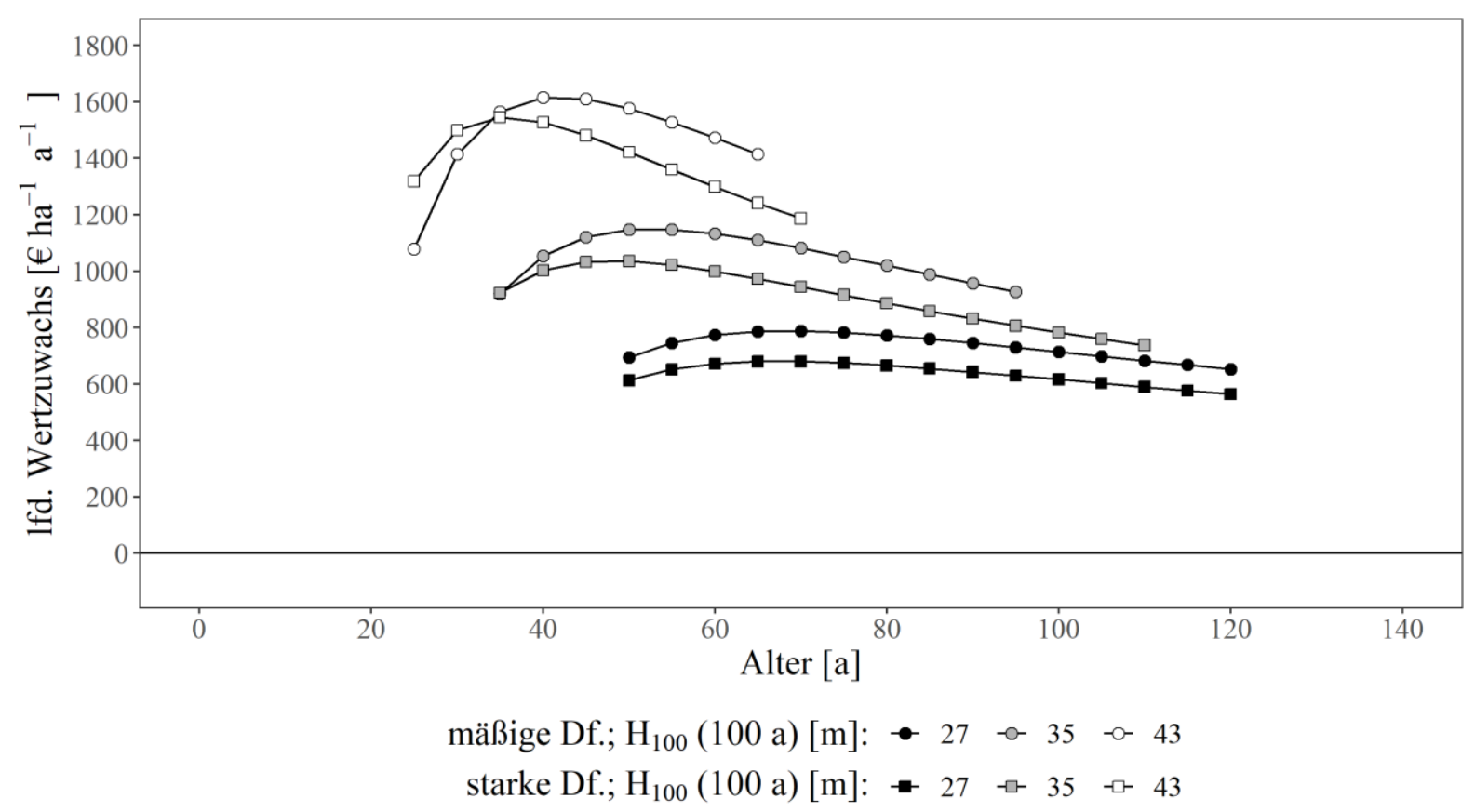

Abbildung 133: Laufender Bestandeswertzuwachs über dem Alter; ausgewählte Oberhöhenbonitäten der Baumart Fichte getrennt nach Behandlungsvarianten gemäß dyn-ET (unveröff.).

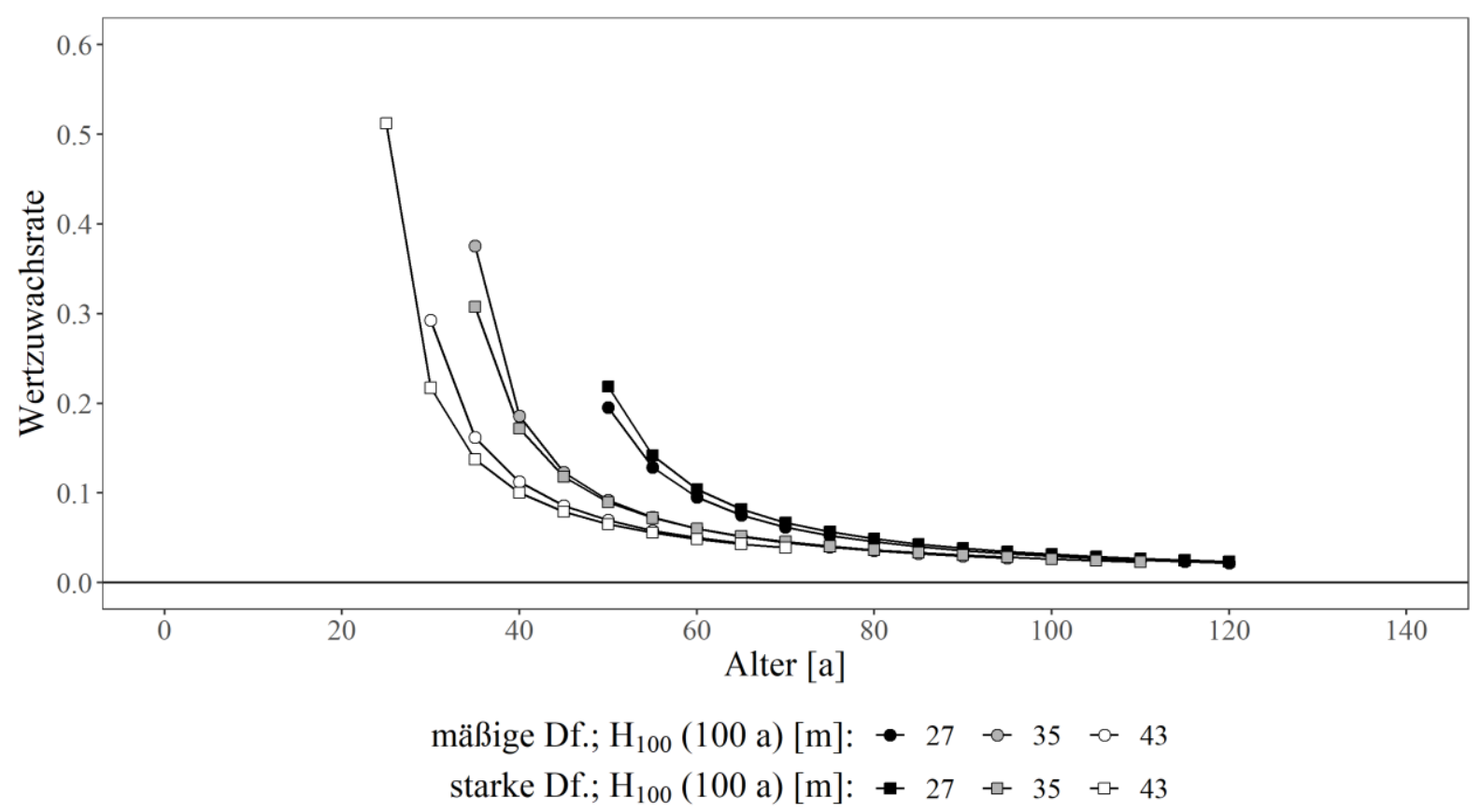

Abbildung 134: Wertzuwachsraten über dem Alter; ausgewählte Oberhöhenbonitäten der Baumart Fichte getrennt nach Behandlungsvarianten gemäß dyn-ET (unveröff.). 
Bei der Analyse der Wertzuwachsraten kehren sich die Verhältnisse zwischen den Behandlungsvarianten im Vergleich zu den Volumenzuwachsraten in Abschnitt 3.3.3 (Abbildung 96) teilweise um. Während die Bestandesvolumenzuwächse bei starker Hochdurchforstung in Relation zum Vorrat stets höher als bei mäßiger Hochdurchforstung sind, liegen die Wertzuwachsraten bei mittlerer und hoher Oberhöhenbonität nun unterhalb der mäßigen Hochdurchforstung (Abbildung 134) - im gleichen Alter liegen die Abtriebswerte der mäßigen Hochdurchforstung dort also in Relation zu den Bestandeswertzuwächsen niedriger als bei starker Hochdurchforstung.

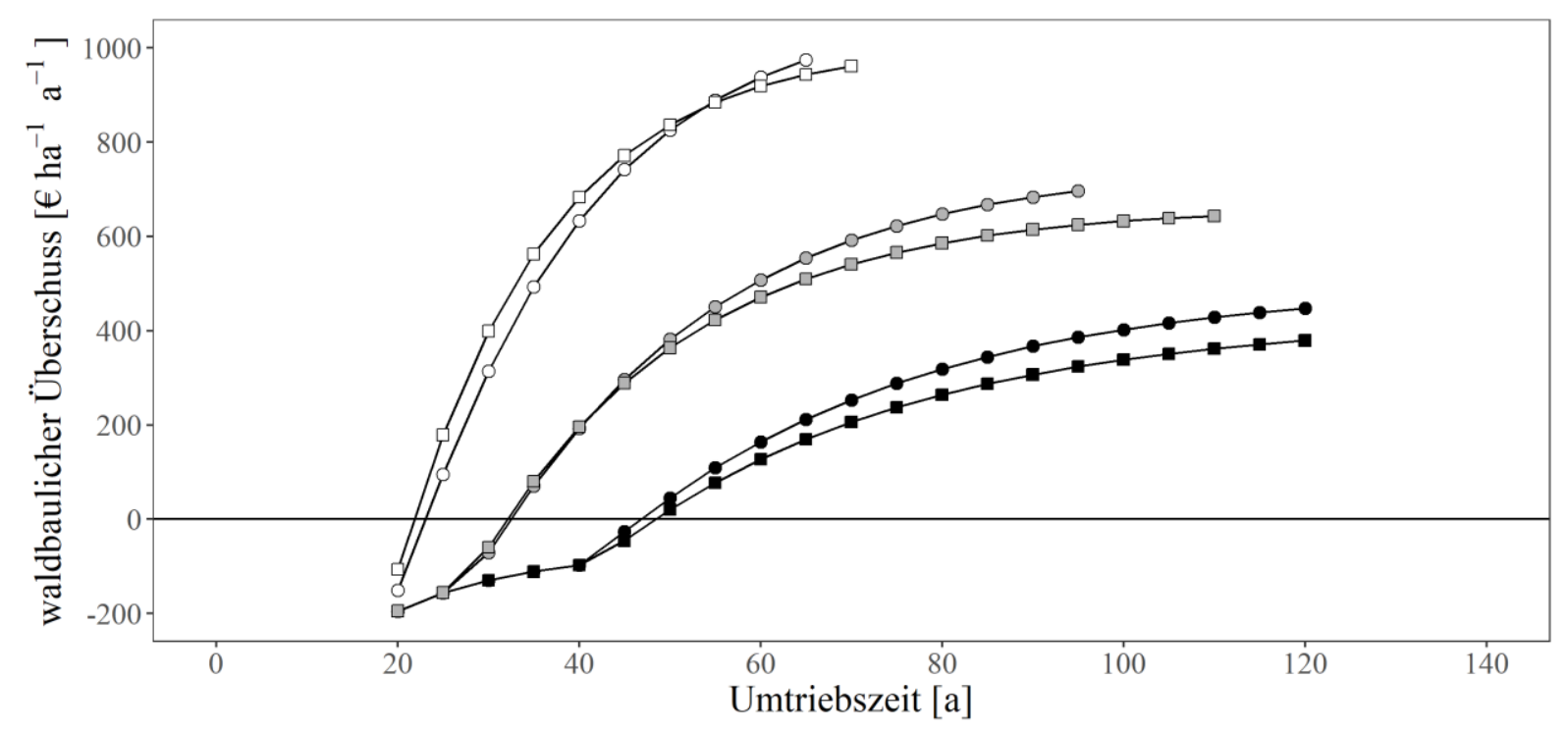

$$
\begin{aligned}
& \text { mäßige Df.; } H_{100} \text { (100 a) [m]: } \bullet 27 \text { - } 35 \text { - } 43 \\
& \text { starke Df.; } H_{100}(100 \mathrm{a})[\mathrm{m}]:-27 \text { 마 } 35 \text { 나 } 43
\end{aligned}
$$

Abbildung 135: Durchschnittlicher waldbaulicher Überschuss der forstlichen Produktion über der Umtriebszeit; ausgewählte Oberhöhenbonitäten der Baumart Fichte getrennt nach Behandlungsvarianten gemäß dyn-ET (unveröff.).

Der durchschnittliche waldbauliche Überschuss der forstlichen Produktion unterstreicht das bereits im Gesamtwuchsleistungs- und Wertzuwachsvergleich erkannte Muster (Abbildung 135). Deutlich vorteilhaft ist die starke Hochdurchforstung allerdings nur bei der höchsten Oberhöhenbonität und lediglich bis zu einer Umtriebszeit von 55 a. Bei der Brutto-Bodenrente profitiert die starke Hochdurchforstung aufgrund der höheren Vornutzungswerte in niedrigen Bestandesaltern nochmal mehr vom Zinseffekt - die Brutto-Bodenrente liegt bei starker Hochdurchforstung und einer Umtriebszeit von 80 a um $9 \%$ niedriger als bei mäßiger Hochdurchforstung (Tabelle 3-18 und Abbildung 136). 


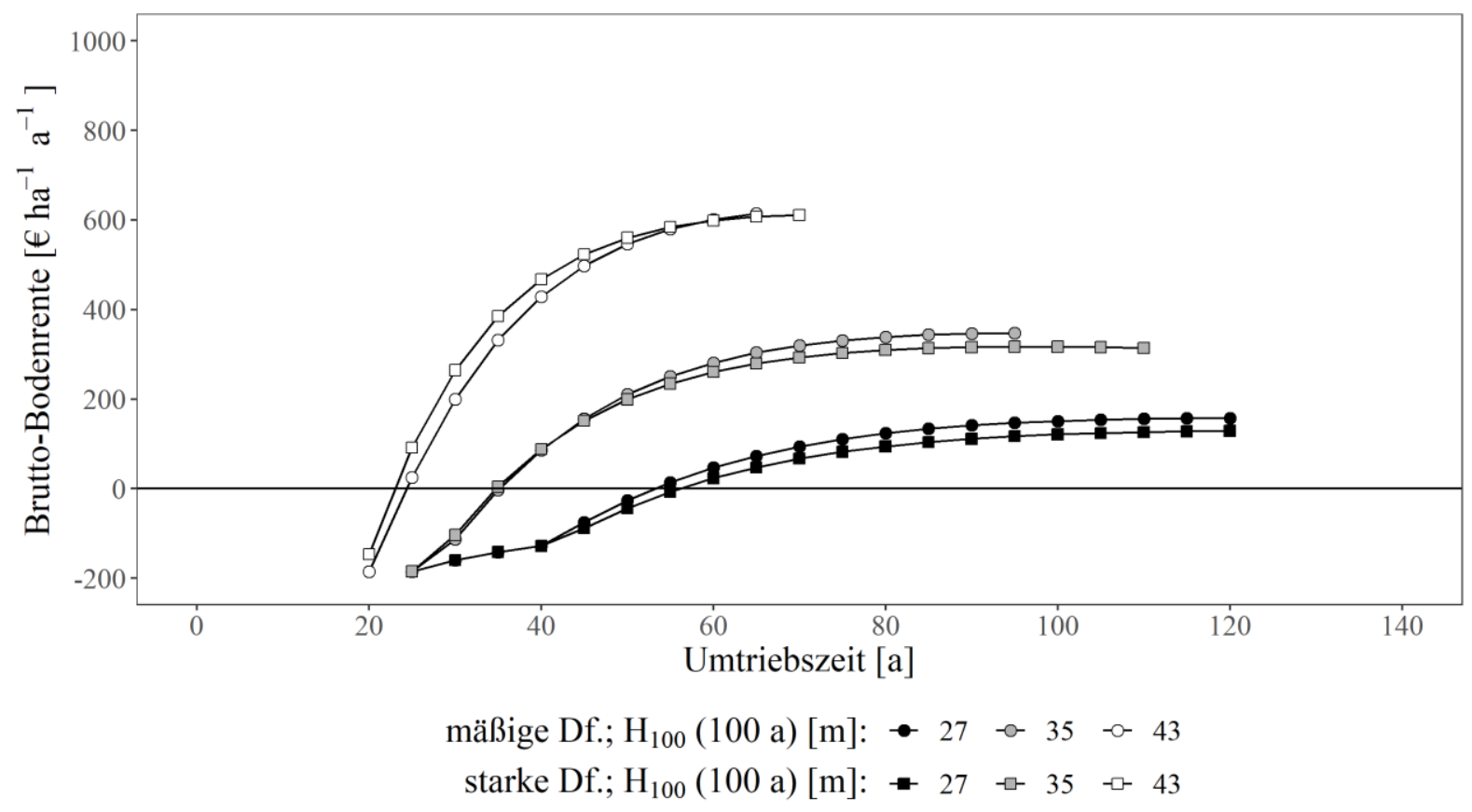

Abbildung 136: Brutto-Bodenrente ( $r=1,5 \%)$ über der Umtriebszeit; ausgewählte Oberhöhenbonitäten der Baumart Fichte getrennt nach Behandlungsvarianten gemäß dyn-ET (unveröff.).

\subsubsection{Sensitivitätsanalysen und Brückenschlag zur forstlichen Praxis}

Die folgenden Sensitivitätsanalysen untersuchen anhand durchschnittlicher Erfolgsgrößen die Wertrelationen zwischen den waldbaulichen Behandlungsvarianten gemäß dyn-ET (unveröff.) unter Veränderung folgender, in Kapitel 2 als besonders relevant identifizierter, Treiber:

- Deckungsbeitragsfunktion,

- Kapitalknappheit und

- Überlebensrisikostärke.

Damit baut dieser Abschnitt auf den naturalen (Abschnitt 3.3.3) und ökonomischen (Abschnitt 3.3.5) Analysen der vorläufigen Ertragstafeldaten auf und dient dem tiefergehenden Verständnis der waldbaulich-ökonomischen Wechselwirkungen bei unterschiedlicher waldbaulicher Behandlung. Ziel ist es, den Einfluss ausgewählter, teilweise bereits in Abschnitt 3.3.5 verwendeter, Eingangsgrößen bzw. Modellprämissen (Treiber) auf die Ergebnisse des ökonomischen Behandlungsvergleichs (Abschnitt 3.3.5) aufzuzeigen.

Es werden die folgenden Fragen beantwortet:

- Welchen Einfluss hat der Verlauf der Deckungsbeitragsfunktion und damit das monetäre Spannungsverhältnis zwischen gering- und hochdimensionierten Sortimente auf die Wertrelationen der waldbaulichen Behandlungsvarianten gemäß dyn-ET (unveröff.) und welche Veränderungen führen zum Wechsel der Rangfolge? 
- Welchen Einfluss hat die Kapitalknappheit auf die Wertrelationen der waldbaulichen Behandlungsvarianten gemäß dyn-ET (unveröff.) und welche Kalkulationszinssätze führen zum Wechsel der Rangfolge?

- Welchen Einfluss hat die Überlebensrisikostärke auf die Wertrelationen der waldbaulichen Behandlungsvarianten gemäß dyn-ET (unveröff.) und welcher behandlungsabhängigen Risikoabsenkung bedarf es zum Wechsel der Rangfolge?

- Welche Wechselwirkungen treten bei Kombination der veränderbaren Treiber auf und welche Schlüsse lassen sich daraus für die forstliche Praxis ziehen?

Durchgeführt werden alle folgenden Sensitivitätsanalysen an den vorläufigen Ertragstafeldaten gemäß dyn-ET (unveröff.) bei der Oberhöhenbonität 32 m (Buche) bzw. 35 m (Fichte) mit Endnutzungszeitpunkten in Anlehnung an etablierte Umtriebszeiten (vgl. z. B. NLF 2014, Tabelle 1.1) von 140 a (Buche) bzw. 80 a (Fichte) $)^{82}$.

Die Sensitivitätsanalysen erfordern treiberpezifische Methoden, die im Folgenden jeweils vor den einzelnen Analyseergebnissen und deren Diskussion beschrieben werden.

Für die Sensitivitätsanalysen des Treibers Deckungsbeitragsfunktion erfolgt die Bewertung geernteter Vor- und Endnutzungsvolumina in Anlehnung an Abschnitt 3.3.5 (Funktion [3-11]) mit folgender Chapman-Richards-Deckungsbeitragsfunktion:

$$
\mathrm{P}\left(D_{g}\right)=\delta_{1} \cdot\left(1-e^{\left(-\delta_{2} \cdot D_{g}\right)}\right)^{\delta_{3}}+\delta_{4}
$$

mit dem Durchmesser des Grundflächenmittelstammes $D_{g}$ in $\mathrm{cm}$ sowie den baumartenspezifisch hergeleiteten Funktionsparametern $\delta_{1}, \delta_{2}, \delta_{3}$ und $\delta_{4}$.

\footnotetext{
${ }^{82}$ Wie schon beim Vergleich der ökonomischen Bewertungsergebnisse wird auch im Rahmen der Sensitivitätsanalysen auf die Verwendung variantenspezifisch optimaler Umtriebszeiten verzichtet. Bis auf wenige Varianten (z. B. hohe Kalkulationszinssätze) liegen die optimalen Umtriebszeiten immer im jeweils maximalen Alter der analysierten Ertragstafeldaten. Ökonomisch begründete Abweichungen zwischen den optimalen Umtriebszeiten der waldbaulichen Behandlungsvarianten von mehr als 10 Jahren bestehen zudem nicht. Bei sehr hohen Kalkulationszinssätzen besteht bei der Verwendung der optimalen Umtriebszeiten außerdem das Problem, dass diese teilweise so gering sind (z. B. 50 a bei Fichte und $r=8 \%$ ), das die eigentlichen Unterschiede der waldbaulichen Behandlungsvarianten, und somit die eigentlichen Analyseobjekte, ggf. nicht mehr adäquat repräsentiert werden. Um dennoch aufzuzeigen, dass die generellen Aussagen der Sensitivitätsanalysen bestehen bleiben, werden ausgewählte Ergebnisse von Sensitivitätsanalysen mit den optimalen Umtriebszeiten im Anhang gezeigt - es wird an entsprechender Stelle darauf verwiesen.
} 
Die Referenzfunktionen der baumartenspezifischen DB-Variantenvergleiche werden dabei von den Deckungsbeitragsfunktionen aus Abschnitt 3.3.5 gebildet, deren Koeffizienten (Tabelle 3-19 für die Baumart Buche und Tabelle 3-20 für die Baumart Fichte) mit den gewählten Eingangsdaten praxisnahe Verläufe im langjährigen Mittel $^{83}$ abbilden.

Neben dieser Referenzfunktion werden DB-Vergleichsfunktionen mittels nlxb-Funktion aus dem R-Paket nlmrt (Nash 2016) derart parametrisiert, dass sie unter Beibehaltung des grundsätzlichen Funktionscharakters einen breiten, frei gewählten, Fächer an dimensionsabhängigen DB-Steigerungen abdecken und mit steigender DB-Variantennummer entgegen des Uhrzeigersinns um einen baumartenspezifischen Referenzpunkt rotieren (Abbildungen 137 a und 138 a). Je höher die DB-Variantennummer, desto stärker ist die DB-Steigerung und damit das Spannungsverhältnis der Deckungsbeiträge geringer und hoher Einzelbaumdurchmesser.

Tabelle 3-19: Koeffizienten der Chapman-Richards-Funktion [3-12] für den DB-Variantenvergleich der Baumart Buche, inkl. Referenz-Deckungsbeitragsfunktion aus Abschnitt 3.3.5.

\begin{tabular}{lrrrr}
\hline & $\delta_{1}$ & $\delta_{2}$ & $\delta_{3}$ & $\delta_{4}$ \\
\hline DB-Variante 1 & 0,00000 & 1,00000 & 1,00000 & 30,17950 \\
DB-Variante 2 & 495,00000 & 0,01869 & 0,02657 & $-455,00000$ \\
Referenz & & & & \\
aus Abschnitt 3.3.5 & 943,08867 & 0,01869 & 0,03605 & $-887,60947$ \\
DB-Variante 4 & $1.900,00000$ & 0,01869 & 0,03522 & $-1.820,00000$ \\
DB-Variante 5 & $3.375,00000$ & 0,01869 & 0,03374 & $-3.260,00000$ \\
DB-Variante 6 & $4.400,00000$ & 0,01869 & 0,03660 & $-4.250,00000$ \\
\hline
\end{tabular}

Die Referenzpunkte, um welche die DB-Varianten entgegen des Uhrzeigersinns rotieren, werden entsprechend der Deckungsbeiträge gewählt, welche die baumartenspezifischen Referenzfunktionen aus Abschnitt 3.3.5 beim mittleren Durchmesser aller in den DB-Variantenvergleich einfließenden Ertragstafeldaten aufweisen. Für die Baumart Buche ist das ein mittlerer Durchmesser von $\overline{D_{g}}=34 \mathrm{~cm}$ mit einem Deckungsbeitrag von $P\left(V_{E}\right)=31 € \mathrm{Vfm}^{-1}$, für die

\footnotetext{
${ }^{83}$ Es wurden in Abschnitt 3.3.5 mittlere Stammholz-Leitsortiments- und Industrieholzpreise aus Daten des Hessischen Staatswaldes der Jahre 2013 - 2017 nach Schade (2019, S. 118) sowie mittlere Brennholz- bzw. Energieholzpreise in Anlehnung an Bodelschwingh (2018) verwendet. Die stark kalamitätsbeeinflussten Daten der forstwirtschaftlichen Extremjahre 2018 und 2019 wurden bewusst nicht berücksichtigt, damit bei der hier durchgeführten Sensitivitätsanalyse mit Überlebenswahrscheinlichkeiten keine Doppelberücksichtigung von kalamitätsbedingten Nachteilen erfolgt.
} 
Baumart Fichte ein mittlerer Durchmesser von $\overline{D_{g}}=31 \mathrm{~cm}$ mit einem Deckungsbeitrag von $P\left(V_{E}\right)=44 € \mathrm{Vfm}^{-1}$ (Abbildungen 137 a und $138 \mathrm{a}$ ).

Tabelle 3-20: Koeffizienten der Chapman-Richards-Funktion [3-12] für den DB-Variantenvergleich der Baumart Fichte, inkl. Referenz-Deckungsbeitragsfunktion aus Abschnitt 3.3.5.

\begin{tabular}{lrrrr}
\hline & $\delta_{1}$ & $\delta_{2}$ & $\delta_{3}$ & $\delta_{4}$ \\
\hline DB-Variante 1 & 0,00000 & 1,00000 & 1,00000 & 43,87794 \\
DB-Variante 2 & 59,00000 & 0,08849 & 1,24547 & $-10,00000$ \\
Referenz & & & & \\
aus Abschnitt 3.3.5 & 160,78422 & 0,08849 & 0,96082 & $-106,02729$ \\
DB-Variante 4 & 200,00000 & 0,08849 & 1,15262 & $-140,00000$ \\
DB-Variante 5 & 370,00000 & 0,08849 & 1,00410 & $-300,00000$ \\
DB-Variante 6 & 620,00000 & 0,08849 & 1,06015 & $-530,00000$ \\
\hline
\end{tabular}

Als ökonomische Vergleichsgröße wird der durchschnittliche waldbauliche Überschuss im Sinne eines holzernte-, bestandesbegründungs- und bestandespflegekostenfreien Holzerlöses (Deckungsbeitrag II; vgl. Keuffel 1980, S. 57 f.) gewählt - Wechselwirkungen unter Berücksichtigung von Kapitalknappheit treten somit zunächst nicht auf.

Für die Baumart Buche zeigen sich bei Bewertung mit der Referenzfunktion für die Behandlungsvarianten zunächst die Wertrelationen entsprechend Abschnitt 3.3.5 (Abbildung 137 b). Mit einem durchschnittlichen waldbaulichen Überschuss von $297 €$ ha $^{-1} \mathrm{a}^{-1}$ liegt die mäßige Hochdurchforstung um $5 \%$ über der gestaffelten (283€ ha-1 $\mathrm{a}^{-1}$ ) bzw. um $19 \%$ über der starken (248€ ha-1 $\mathrm{a}^{-1}$ ) Hochdurchforstung (siehe auch Tabelle 3-15 und Abbildung 120). Mit sinkender DB-Steigerung, d. h. mit zunehmend homogenerer Bewertung eines Festmeters und damit abnehmender Bedeutung der Einzelbaumdimension, verstärkt sich dieser Effekt insb. für die gestaffelte Hochdurchforstung (Abbildung 137 b). So liegt die mäßige Hochdurchforstung bei DB-Variant 2 mit $281 € \mathrm{ha}^{-1} \mathrm{a}^{-1}$ bereits um $9 \%$ über der gestaffelten ( $259 € \mathrm{ha}^{-1} \mathrm{a}^{-1}$ ) bzw. um $20 \%$ über der starken Hochdurchforstung ( $234 € \mathrm{ha}^{-1} \mathrm{a}^{-1}$ ). Bei einem konstanten Deckungsbeitrag (DB-Variante 1), d. h. bei ausschließlicher Bewertung der Volumenleistung (siehe Abschnitt 2.2.2), liegt die mäßige Hochdurchforstung mit $272 € \mathrm{ha}^{-1} \mathrm{a}^{-1}$ schließlich um $12 \%$ über der gestaffelten $\left(244 € \mathrm{ha}^{-1} \mathrm{a}^{-1}\right)$ bzw. um $21 \%$ über der starken (225€ ha ${ }^{-1} \mathrm{a}^{-1}$ ) Hochdurchforstung - die Ergebnisse spiegeln damit die behandlungsabhängigen Relationen der Gesamtwuchsleistungen wider (siehe Tabelle 3-4 und Abbildung 71). Da eine gewisse Dimensionsabhängigkeit der Aufarbeitungskosten forstpraktisch 
immer gegeben sein wird, ist eine zunehmend homogenere Bewertung des Produkts Holz insb. bei der Produktion eines einheitlichen Sortiments ohne dimensionsabhängige Preisunterschiede, z.B. bei ausschließlicher Industrieholzproduktion, zu erwarten. Nimmt hingegen die DB-Steigerung über dem mittleren Einzelbaumdurchmesser zu, werden somit stärkere im Vergleich zu schwächeren Bäumen zunehmend höher bewertet, holt die gestaffelte ggü. der mäßigen Hochdurchforstung auf ( $-1 \%$ bei DB-Variante 4) bzw. überholt diese ab DB-Variante 5. So liegt der durchschnittliche waldbauliche Überschuss der gestaffelten Hochdurchforstung bei DB-Variante 5 bereits um $5 \%$ und schließlich bei DB-Variante 6 um $9 \%$ oberhalb der mäßigen Hochdurchforstung ( $426 € \mathrm{ha}^{-1} \mathrm{a}^{-1}$ ggü. $391 € \mathrm{ha}^{-1} \mathrm{a}^{-1}$ ). Als forstpraktische Interpretation steht der ausschließlichen Industrieholzproduktion damit das Ziel der Säge- und Wertholzproduktion, mit dementsprechend hoch bewerteten starken Sortimenten, bei gleichzeitig deutlicher Abwertung, der als Kuppelprodukt (vgl. Stinglwagner et al. 2005, S. 512) anfallenden, geringdimensionierten Sortimente gegenüber. Wie bereits in Abschnitt 2.3 diskutiert, ist für die Wertrelationen der Behandlungsvarianten dabei nicht die absolute Höhe der Deckungsbeiträge, sondern das Spannungsverhältnis zwischen den Deckungsbeiträgen der gering- und hochdimensionierten Sortimente entscheidend. Das monetäre Spannungsverhältnis der DB-Varianten 5 und 6 reicht aus, damit die höhere Volumenleistung der mäßigen Hochdurchforstung von den monetären Vorteilen höherer Einzelbaumdurchmesser bei gestaffelter Hochdurchforstung kompensiert werden. Die wegen der teils negativen Deckungsbeiträge bei geringen Einzelbaumdurchmessern als Investitionen zu verstehenden stärkeren Vornutzungen in der Bestandesjugend sind aufgrund der sich ergebenden höheren Deckungsbeiträge im hohen Bestandesalter rentabel. Dabei ist im Vergleich zur starken Hochdurchforstung, welche den Wechsel der Wertrelationen selbst bei den DB-Varianten 5 und 6 nicht schafft, entscheidend, dass es bei gestaffelter Hochdurchforstung aufgrund des Übergangs der Grundflächenhaltung zu einer Kombination aus zur mäßigen Hochdurchforstung aufholender Volumenleistung (Abbildung 71) und gleichzeitig teils deutlich gesteigerten mittleren Einzelbaumdurchmessern (Abbildungen 76 und 77) kommt. Die gestaffelte Hochdurchforstung kann mit dieser Kombination anders als die starke Hochdurchforstung auf das steigende DB-Spannungsverhältnis reagieren (siehe Disparitätskurven in den Abbildungen 139 und 140).

Absolut gesehen steigen die durchschnittlichen waldbaulichen Überschüsse aller Behandlungsvarianten mit steigender DB-Steigerung (Abbildung 137 b) - bei zinsfreier Kalkulation tragen die Vor- und Endnutzungsmengen mit einem überdurchschnittlichen mittleren Durchmesser folglich stärker zur durchschnittlichen Erfolgsgröße bei, als die Vor- und Endnutzungsmengen mit einem unterdurchschnittlichen mittleren Einzelbaumdurchmesser. 


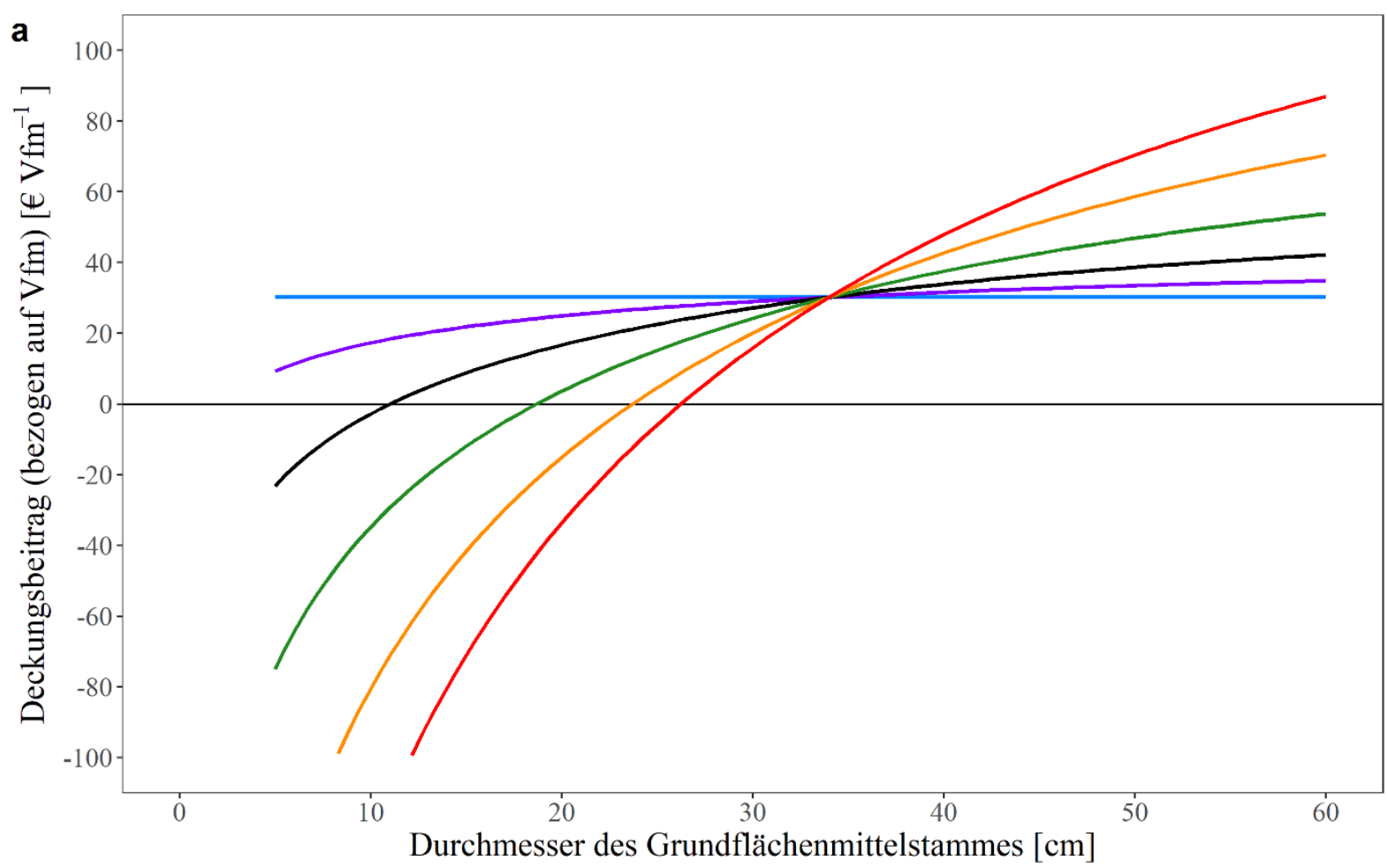

$-1-2-$ Referenz $-4-5-6$

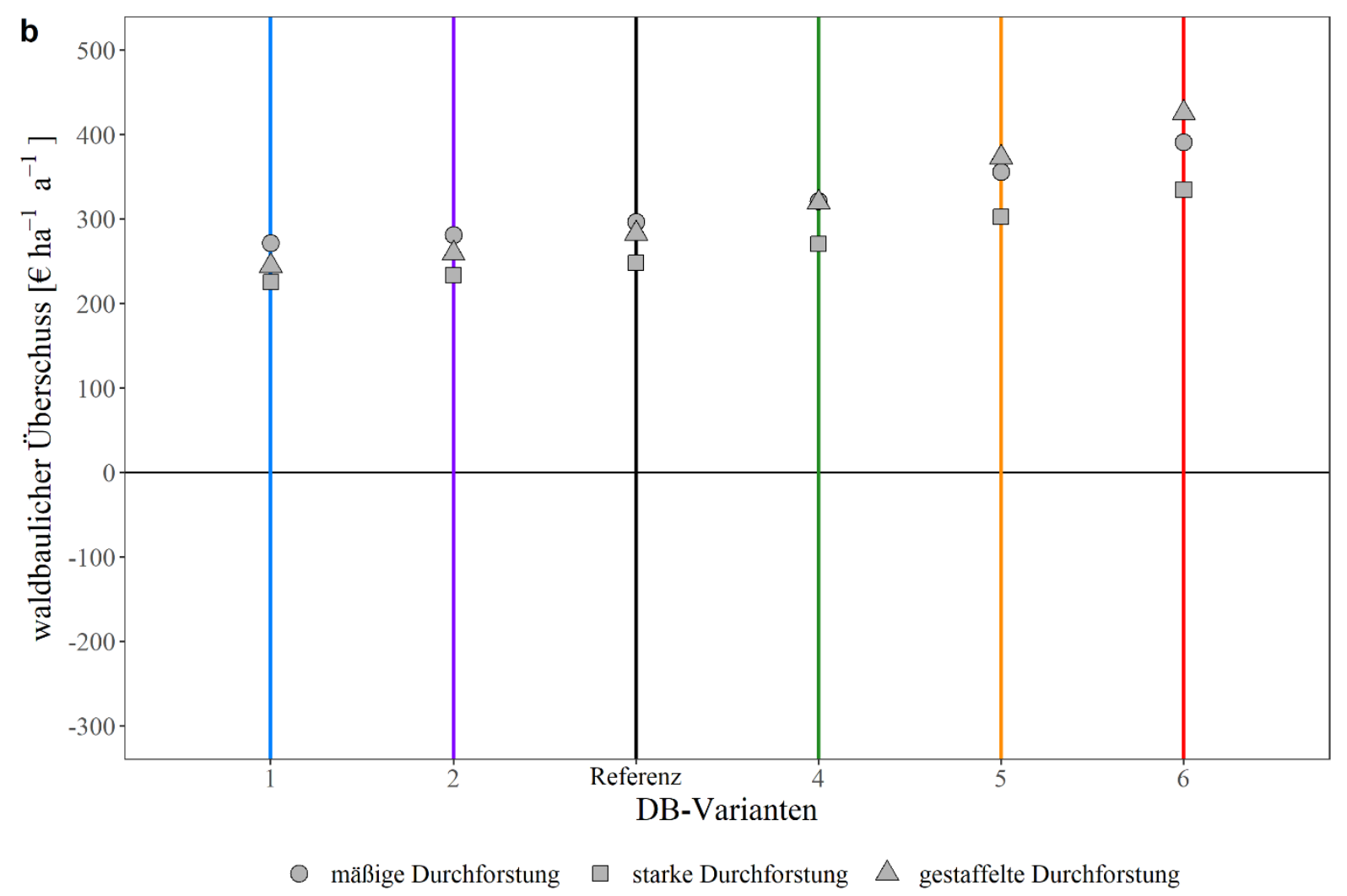

Abbildung 137: Analyse der Auswirkung unterschiedlicher Deckungsbeitragsfunktionen (a) auf den durchschnittlichen waldbaulichen Überschuss (b) der Baumart Buche $\left(H_{100}(100 \mathrm{a})=32 \mathrm{~m}\right) \mathrm{im}$ Vergleich der waldbaulichen Behandlungsvarianten gemäß dyn-ET (unveröff.) bei einer Umtriebszeit von $140 \mathrm{a}$; die schwarze Linie in (a) repräsentiert die Referenz-Deckungsbeitragsfunktion in Anlehnung an die ökonomische Bewertung in Abschnitt 3.3.5. 
Insgesamt zeigen sich bei der Baumart Fichte in Abbildung 138 für die starke Hochdurchforstung ähnliche Effekte wie für die gestaffelte Hochdurchforstung der Baumart Buche ${ }^{84}$. Unter Verwendung der Referenz-Deckungsbeitragsfunktion entsprechen die Wertrelationen der Behandlungsvarianten den Ergebnissen aus Abschnitt 3.3.5 (Abbildung 138 b). Mit einem durchschnittlichen waldbaulichen Überschuss von $647 € \mathrm{ha}^{-1} \mathrm{a}^{-1}$ liegt die mäßige Hochdurchforstung um $10 \%$ über der starken (586€ ha-1 $\mathrm{a}^{-1}$ ) Hochdurchforstung (siehe auch Tabelle 3-18 und Abbildung 135). Mit sinkender DB-Steigerung, d. h. mit zunehmend homogenerer Bewertung eines Festmeters an Holzvolumen, verstärkt sich dieser Effekt. So liegt die mäßige Hochdurchforstung bei DB-Variante 2 mit $643 € \mathrm{ha}^{-1} \mathrm{a}^{-1}$ bereits um $14 \%$ über der starken (564 € ha ${ }^{-1} \mathrm{a}^{-1}$ ) Hochdurchforstung. Bei einem konstanten Deckungsbeitrag (DB-Variante 1) schließlich liegt die mäßige Hochdurchforstung mit $638 €$ ha $^{-1} \mathrm{a}^{-1}$ um $18 \%$ über der starken (542€ ha ${ }^{-1} \mathrm{a}^{-1}$ ) Hochdurchforstung. Die Ergebnisse spiegeln damit auch hier, bei der ausschließlichen Bewertung der Volumenleistung, die Relation der Gesamtwuchsleistungen aus Abschnitt 3.3.3 wider (siehe Tabelle 3-7 und Abbildung 93). Anders als bei der Buche zeigen sich ökonomische Vorteile der starken Hochdurchforstung bei höheren DB-Steigerungen. Bereits bei DB-Variante 4 liegt der durchschnittliche waldbauliche Überschuss bei starker Hochdurchforstung mit $610 € \mathrm{ha}^{-1} \mathrm{a}^{-1}$ um $7 \%$ und bei DB-Variante 5 mit $649 € \mathrm{ha}^{-1} \mathrm{a}^{-1}$ nur noch um $2 \%$ unterhalb der mäßigen Hochdurchforstung. Bei DB-Variante 6 schließlich übertrifft der durchschnittliche waldbauliche Überschuss bei starker $\left(733 € \mathrm{ha}^{-1} \mathrm{a}^{-1}\right)$ den bei mäBiger (681€ ha $\left.\mathrm{h}^{-1} \mathrm{a}^{-1}\right)$ Hochdurchforstung um $8 \%$. Die deutlich gesteigerten mittleren Einzelbaumdurchmesser im verbleibenden (Abbildung 98) und ausscheidenden Bestand (Abbildung 99) bei starker Hochdurchforstung der Fichte vermögen es somit, anders als bei der $\mathrm{Bu}-$ che, die verminderte Volumenleistung (Abbildung 93) zu kompensieren. Die unscharfe Abgrenzung zwischen starker und gestaffelter Hochdurchforstung der zugrundeliegenden FichtenVersuchsflächen (siehe Fußnote 84) trägt sicherlich in nicht unerheblichem Maß dazu bei.

\footnotetext{
${ }^{84}$ Begründend kann laut interner dyn-ET-Projektkommunikation angeführt werden, dass die mit einer starken Hochdurchforstung charakterisierten Fichten-Versuchsflächen der NW-FVA historisch bereits Ansätze eines gestaffelten Behandlungsgangs aufweisen.
} 


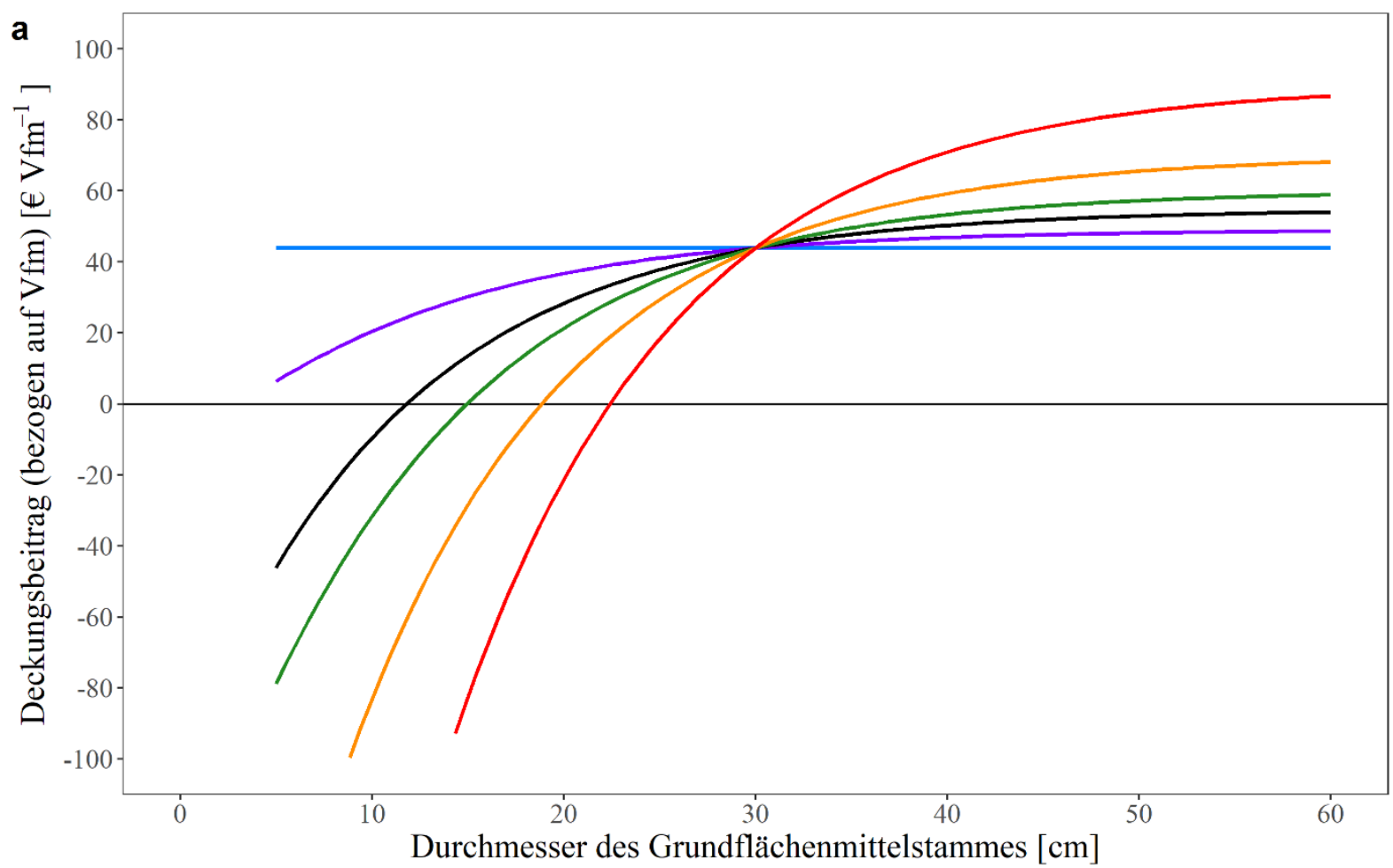

$-1-2-$ Referenz $-4-5-6$

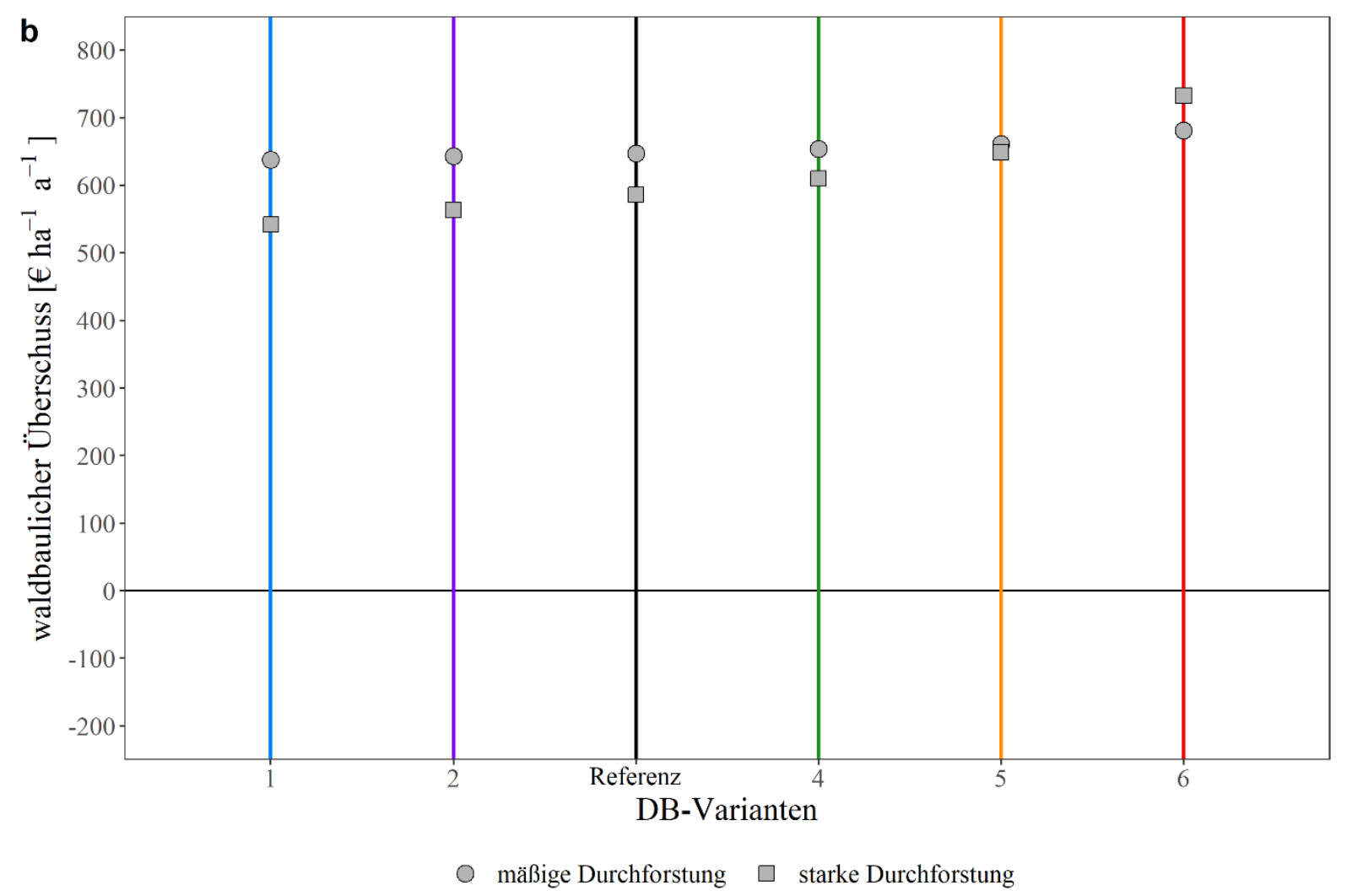

Abbildung 138: Analyse der Auswirkung unterschiedlicher Deckungsbeitragsfunktionen (a) auf den durchschnittlichen waldbaulichen Überschuss (b) der Baumart Fichte $\left(H_{100}(100 \mathrm{a})=35 \mathrm{~m}\right) \mathrm{im}$ Vergleich der waldbaulichen Behandlungsvarianten gemäß dyn-ET (unveröff.) bei einer Umtriebszeit von $80 \mathrm{a}$; die schwarze Linie in (a) repräsentiert die Referenz-Deckungsbeitragsfunktion in Anlehnung an die ökonomische Bewertung in Abschnitt 3.3.5. 
Es zeigt sich anhand der DB-Sensitivitätsanalysen, dass bei der ökonomischen Bewertung mit nichtlinearen, dimensionsabhängigen Deckungsbeitragsfunktionen neben der absoluten Volumenleistung insb. entscheidend ist, mit welchem mittleren Einzelbaumdurchmesser wieviel Bestandesvolumen realisiert wird. Ein Rangfolgenwechsel der relativen Vorteilhaftigkeiten alternativer Behandlungsvarianten ist nur möglich, wenn diese unterschiedlich auf ein verändertes Spannungsverhältnis zwischen Deckungsbeiträgen geringer und hoher Einzelbaumdimension reagieren. Verdeutlicht werden kann dies über Disparitätskurven, welche in Abschnitt 2.2.4 bereits zur Charakterisierung des Spannungsverhältnisses zwischen Deckungsbeiträgen geringund hochdimensionierter Sortimente genutzt wurden. So zeigen sich in den Abbildungen 139 bis 142 einerseits die mit stärker werdender DB-Steigerung zunehmenden Disparitäten, d. h. Abweichungen zwischen den relativen Gesamtwuchs- und Gesamtwertleistungen, für alle waldbaulichen Behandlungsvarianten (vgl. Abschnitt 2.2.4). Andererseits wird in den exemplarischen Abbildungen (jeweils für die Referenz-DB-Variante sowie die DB-Variante 6) aber auch deutlich, dass die Disparitätsverläufe der gestaffelten (Buche) und starken (Fichte) Hochdurchforstung mit stärker werdender DB-Steigerung zunehmend vom Disparitätsverlauf der mäßigen Hochdurchforstung abweichen. Während die DB-Steigerung für die ökonomische Vorteilhaftigkeit zwischen mäßiger und starker Hochdurchforstung bei der Buche kaum eine Rolle spielt (ähnliche Disparitätskurven in Abbildung 140), reagiert die gestaffelte Hochdurchforstung deutlich auf ein steigendes Spannungsverhältnis zwischen den Deckungsbeiträgen geringer und hoher Einzelbaumdimension. Gleiches gilt für die starke Hochdurchforstung bei der Fichte (Abbildung 142), weshalb sich auch dort ein Rangfolgenwechsel ergibt. Tabelle 3-21 zeigt die entsprechenden, das jeweilige Spannungsverhältnis quantifizierenden, normierten Gini-Koeffizienten (vgl. Chen et al. 1982; siehe auch Abschnitt 2.2.3).

Tabelle 3-21: Normierte Gini-Koeffizienten (relative Disparitätsmaße) im Vergleich der waldbaulichen Behandlungsvarianten gemäß dyn-ET (unveröff.) bei einer Umtriebszeit von 140 a (Buche) bzw. 80 a (Fichte) und einer dimensionsabhängiger DB-Steigerung gemäß baumartenspezifischer ReferenzDB-Variante aus Abschnitt 3.3.5 sowie DB-Variante 6.

\begin{tabular}{lrrr}
\hline & Behandlungsvariante & Buche & Fichte \\
\hline \multirow{2}{*}{ Referenz-DB-Variante } & mäßige Durchforstung & 0,230 & 0,156 \\
& starke Durchforstung & 0,233 & 0,113 \\
& gestaffelte Durchforstung & 0,205 & - \\
\hline \multirow{2}{*}{ DB-Variante 6 } & mäßige Durchforstung & 0,650 & 0,480 \\
& starke Durchforstung & 0,665 & 0,309 \\
& gestaffelte Durchforstung & 0,567 & - \\
\hline
\end{tabular}




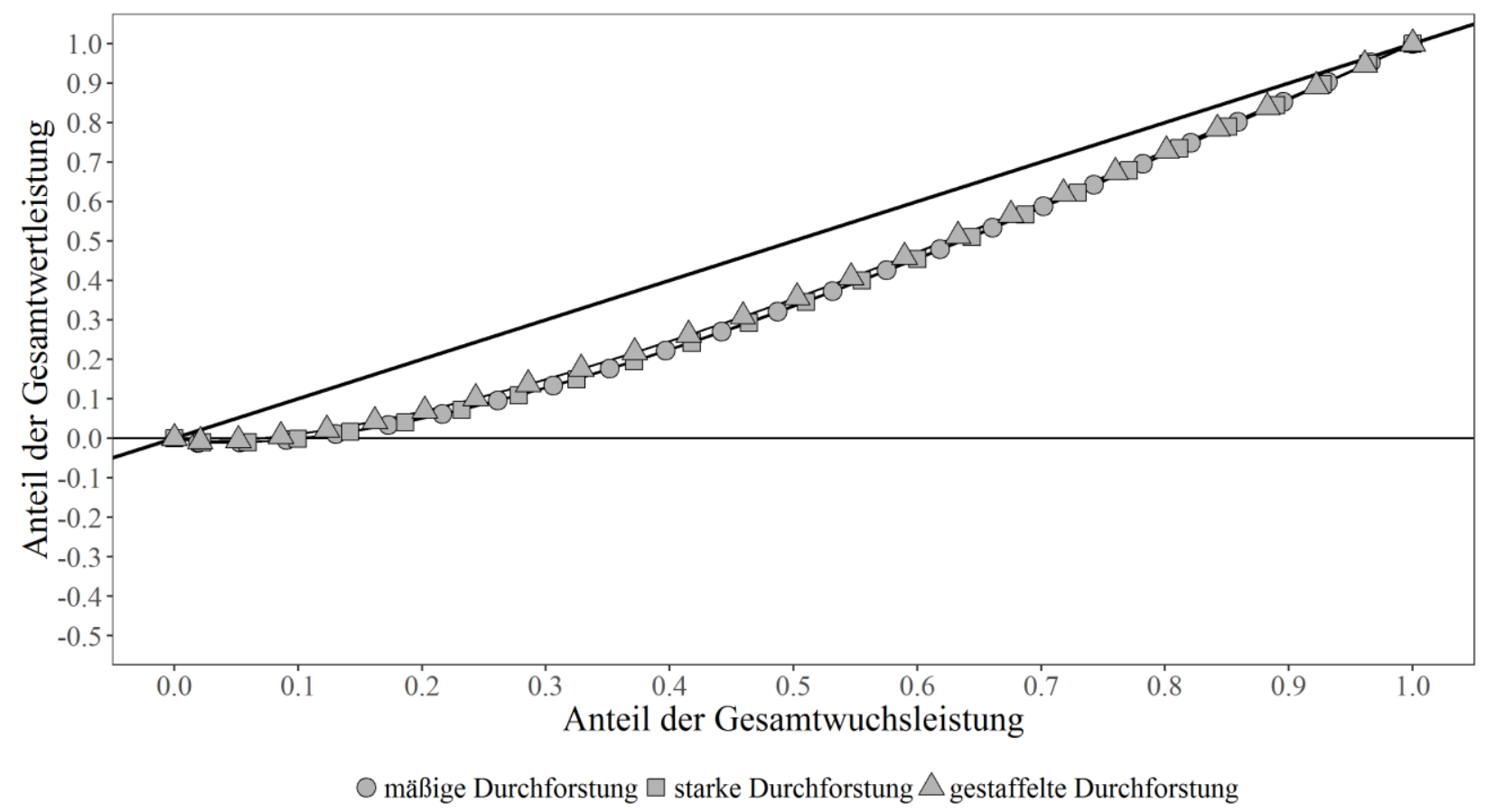

Abbildung 139: Disparitätskurven der relativen Gesamtwuchs- und Gesamtwertleistung der Baumart Buche $\left(H_{100}(100 \mathrm{a})=32 \mathrm{~m}\right)$ im Vergleich der waldbaulichen Behandlungsvarianten gemäß dyn-ET (unveröff.) bei einer Umtriebszeit von 140 a und einer dimensionsabhängiger DB-Steigerung gemäß der Referenz-DB-Variante aus Abschnitt 3.3.5; die Diagonale stellt die Deckungsgleichheit von relativer Volumen- und Wertleistung bei der Bewertung mit einem konstanten Deckungsbeitrag dar.

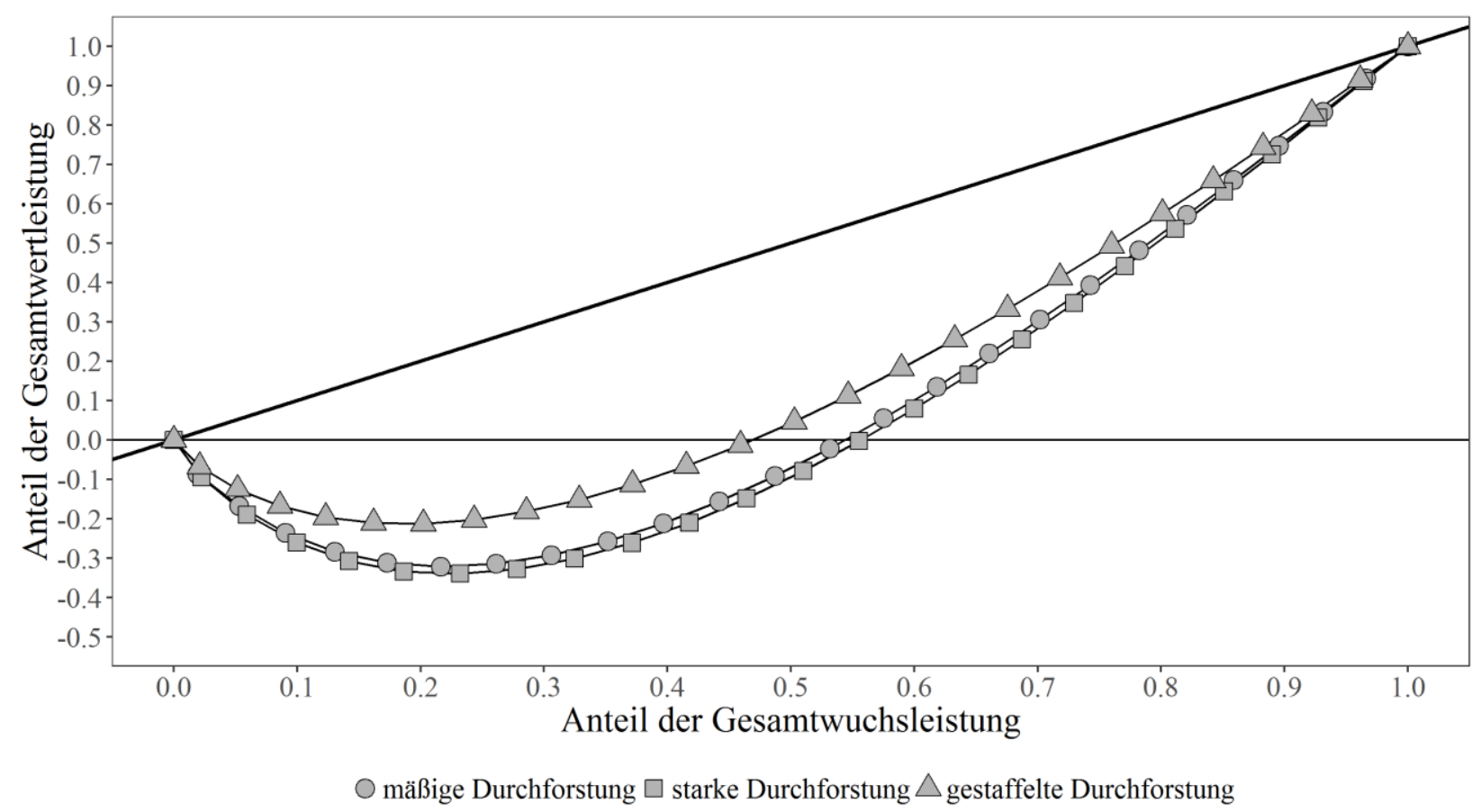

Abbildung 140:Disparitätskurven der relativen Gesamtwuchs- und Gesamtwertleistung der Baumart Buche $\left(H_{100}(100 \mathrm{a})=32 \mathrm{~m}\right)$ im Vergleich der waldbaulichen Behandlungsvarianten gemäß dyn-ET (unveröff.) bei einer Umtriebszeit von 140 a und einer dimensionsabhängiger DB-Steigerung gemäß der DB-Variante 6 (siehe Abbildung 137a); die Diagonale stellt die Deckungsgleichheit von relativer Volumen- und Wertleistung bei der Bewertung mit einem konstanten Deckungsbeitrag dar. 


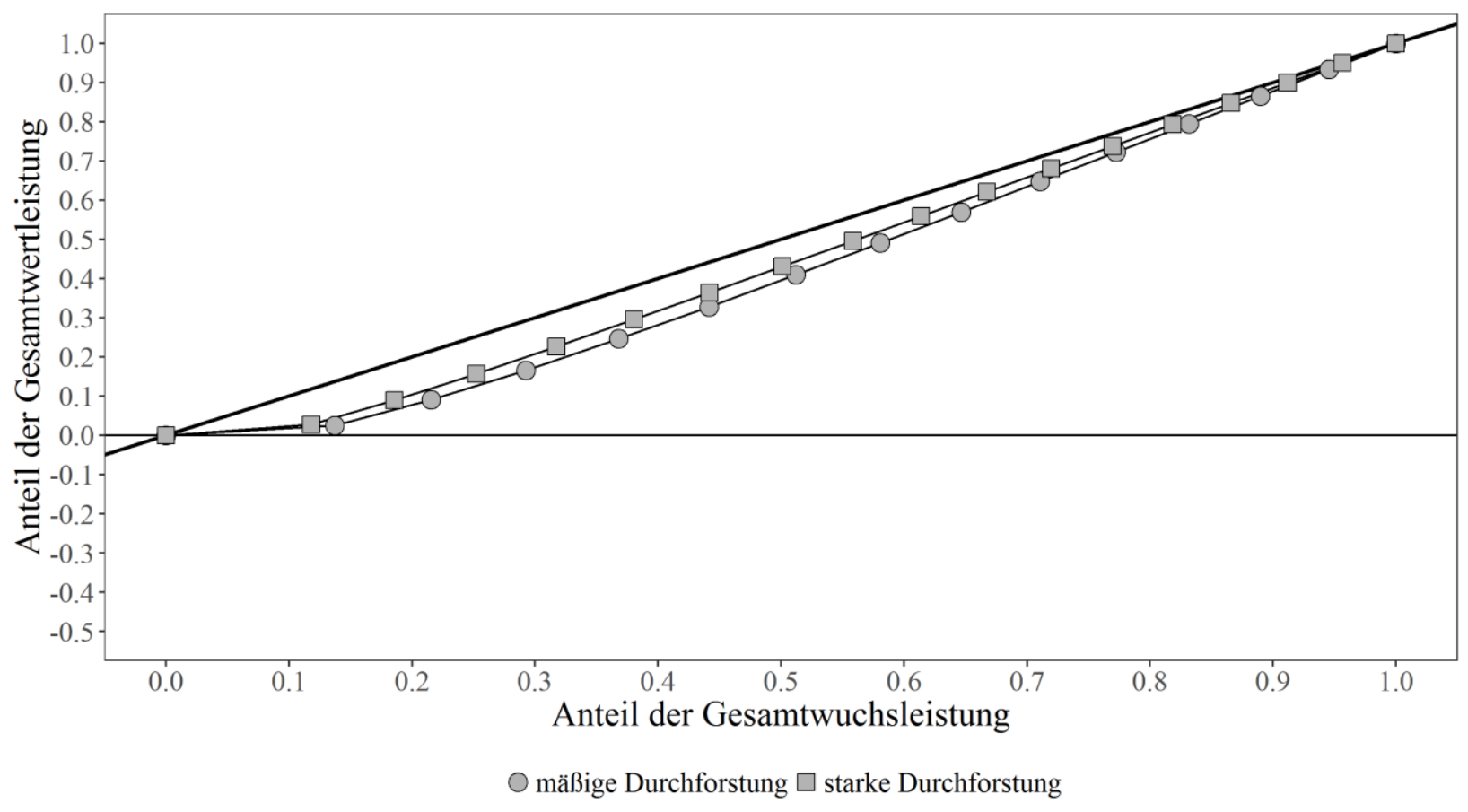

Abbildung 141: Disparitätskurven der relativen Gesamtwuchs- und Gesamtwertleistung der Baumart Fichte $\left(H_{100}(100 \mathrm{a})=35 \mathrm{~m}\right)$ im Vergleich der waldbaulichen Behandlungsvarianten gemäß dyn-ET (unveröff.) bei einer Umtriebszeit von 80 a und einer dimensionsabhängiger DB-Steigerung gemäß der Referenz-DB-Variante aus Abschnitt 3.3.5; die Diagonale stellt die Deckungsgleichheit von relativer Volumen- und Wertleistung bei der Bewertung mit einem konstanten Deckungsbeitrag dar.

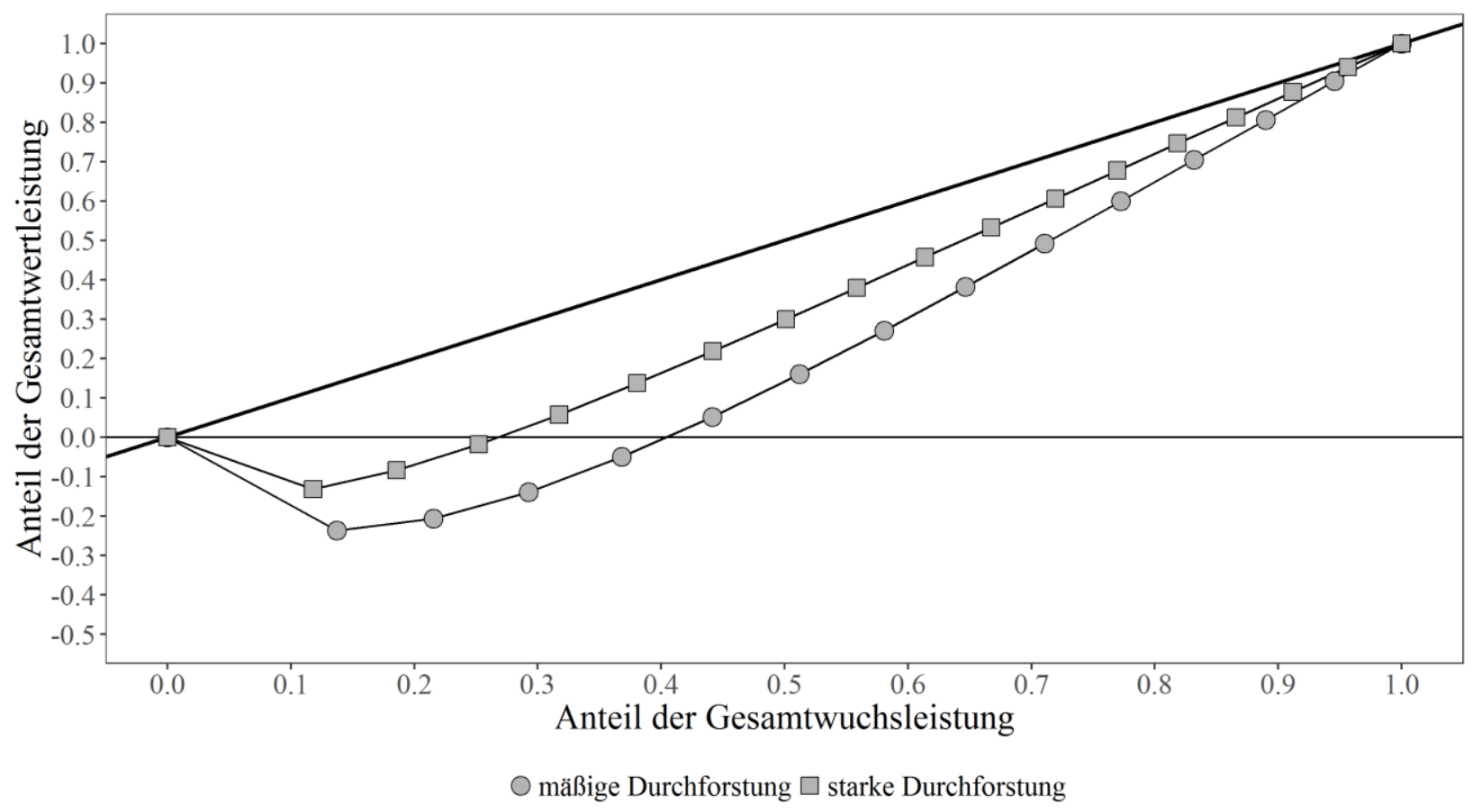

Abbildung 142: Disparitätskurven der relativen Gesamtwuchs- und Gesamtwertleistung der Baumart Fichte $\left(H_{100}(100 \mathrm{a})=35 \mathrm{~m}\right)$ im Vergleich der waldbaulichen Behandlungsvarianten gemäß dyn-ET (unveröff.) bei einer Umtriebszeit von 80 a und einer dimensionsabhängiger DB-Steigerung gemäß der DB-Variante 6 (siehe Abbildung 138a); die Diagonale stellt die Deckungsgleichheit von relativer Volumen- und Wertleistung bei der Bewertung mit einem konstanten Deckungsbeitrag dar. 
Werden die durchschnittlichen Überschüsse anstatt zur baumartenspezifisch konstanten zu den DB-variantenabhängig optimalen bzw. modell-maximalen Umtriebszeiten analysiert, bleiben die gezeigten Effekte bestehen und werden in ihrer Ausprägung eher verstärkt (Abbildungen 207 und 208 im Anhang). Bei der Buche liegt der durchschnittliche Überschuss bei gestaffelter Hochdurchforstung nun schon bei DB-Variante 4 knapp oberhalb der jährlichen Erfolgsgröße bei mäßiger Hochdurchforstung $\left(+1 \% ; T_{\text {mäßig }}=150 \mathrm{a} ; T_{\text {stark }}=145 \mathrm{a}\right.$; $T_{\text {gestaffelt }}=150 \mathrm{a}$ ). Auch bei der Fichte wechselt die Vorteilhaftigkeit zwischen den waldbaulichen Behandlungsvarianten früher - der durchschnittliche Überschuss bei starker Hochdurchforstung liegt bei DB-Variante 5 nun mit $755 €$ ha $^{-1} \mathrm{a}^{-1}$ knapp oberhalb der jährlichen Erfolgsgröße bei mäßiger Hochdurchforstung $\left(754 € \mathrm{ha}^{-1} \mathrm{a}^{-1} ; T_{\text {mäßig }}=95 \mathrm{a}\right.$; $\left.T_{\text {stark }}=110 \mathrm{a}\right)$.

Für die Sensitivitätsanalyse des Treibers Kapitalknappheit wird unter Verwendung steigender Kalkulationszinssätze $(0,5 \%$ bis $10 \%$ in 0,5 er-Schritten) aus dem durchschnittlichen waldbaulichen Überschuss der forstlichen Produktion eine Brutto-Bodenrente (Annuität; siehe Abschnitt 2.2.5).

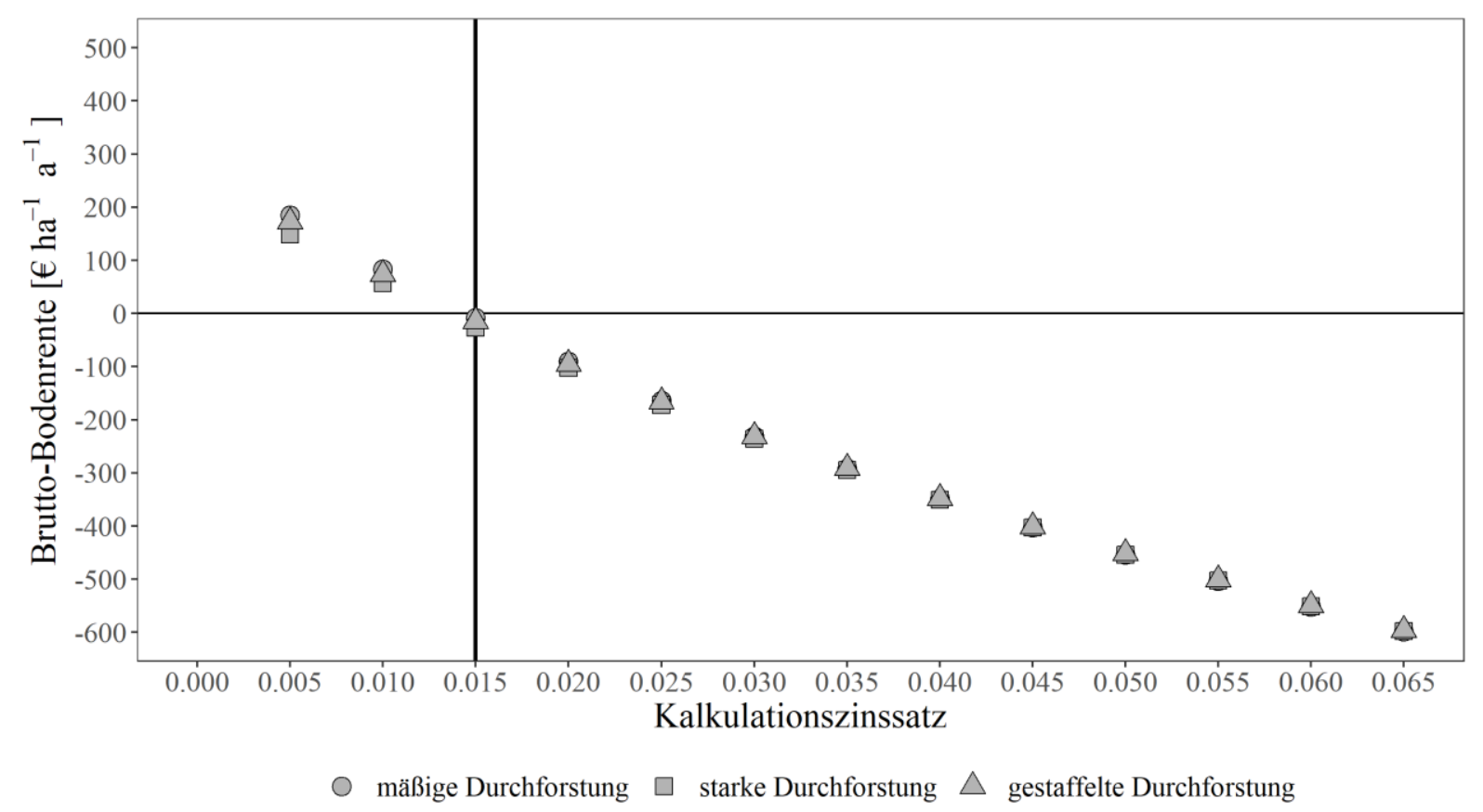

Abbildung 143: Analyse der Auswirkung verschiedener Kalkulationszinssätze auf die Brutto-Bodenrente der Baumart Buche $\left(H_{100}(100 \mathrm{a})=32 \mathrm{~m}\right)$ im Vergleich der waldbaulichen Behandlungsvarianten gemäß dyn-ET (unveröff.) bei einer Umtriebszeit von $140 \mathrm{a}$; die schwarze Linie repräsentiert als Referenz den in Abschnitt 3.3.5 verwendeten Kalkulationszinssatz von $r=1,5 \%$.

Dabei werden alle sonstigen Eingangsdaten entsprechend des Abschnitts 3.3.5 gewählt - die Deckungsbeitragsfunktionen entsprechen also den schwarzen Referenz-DB-Varianten in Abbildung 137 a (Buche) bzw. Abbildung 138 a (Fichte). Aufgrund der stark sinkenden Brutto- 
Bodenrenten und dem dämpfenden Zinseffekt (vgl. Abschnitt 3.3.5) fällt die grafische Analyse bei steigendem Kalkulationszinssatz zunehmend schwer (Abbildungen 143 und 144).

Wie auch schon in Abschnitt 3.3.5 gesehen, sind die Brutto-Bodenrenten bei der Baumart Buche bereits bei einem Kalkulationszinssatz von $r=1,5 \%$ negativ (siehe Tabelle 3-15 und schwarze, senkrechte Linie in Abbildung 143). Die mäßige Hochdurchforstung ist aber relativ gesehen vorteilhaft, da sie mit $-8 € \mathrm{ha}^{-1} \mathrm{a}^{-1}$ eine weniger negative Brutto-Bodenrente hat, als die gestaffelte $\left(-15 € \mathrm{ha}^{-1} \mathrm{a}^{-1}\right)$ und die starke Hochdurchforstung $\left(-26 € \mathrm{ha}^{-1} \mathrm{a}^{-1}\right)$. Mit steigendem Kalkulationszinssatz büßt die mäßige Hochdurchforstung diese relative Vorteilhaftigkeit ggü. den anderen Behandlungsvarianten ein - bei $r=3,5 \%$ sinkt ihre Brutto-Bodenrente $\left(-292 € \mathrm{ha}^{-1} \mathrm{a}^{-1}\right)$ unter die der gestaffelten $\left(-291 € \mathrm{ha}^{-1} \mathrm{a}^{-1}\right)$ und bei $r=5,0 \%$ schließlich auch unter die der starken Hochdurchforstung $\left(-454,0 € \mathrm{ha}^{-1} \mathrm{a}^{-1}\right.$ ggü. - 453,8 € ha $\mathrm{h}^{-1} \mathrm{a}^{-1}$ ). Die Unterschiede sind allerdings minimal - bei einem Kalkulationszinssatz von $r=10 \%$ beträgt die maximale Differenz zwischen den Behandlungsvarianten lediglich $1,27 € \mathrm{ha}^{-1} \mathrm{a}^{-1}$ bei einem absoluten mittleren Niveau von $-913 € \mathrm{ha}^{-1} \mathrm{a}^{-1}$.

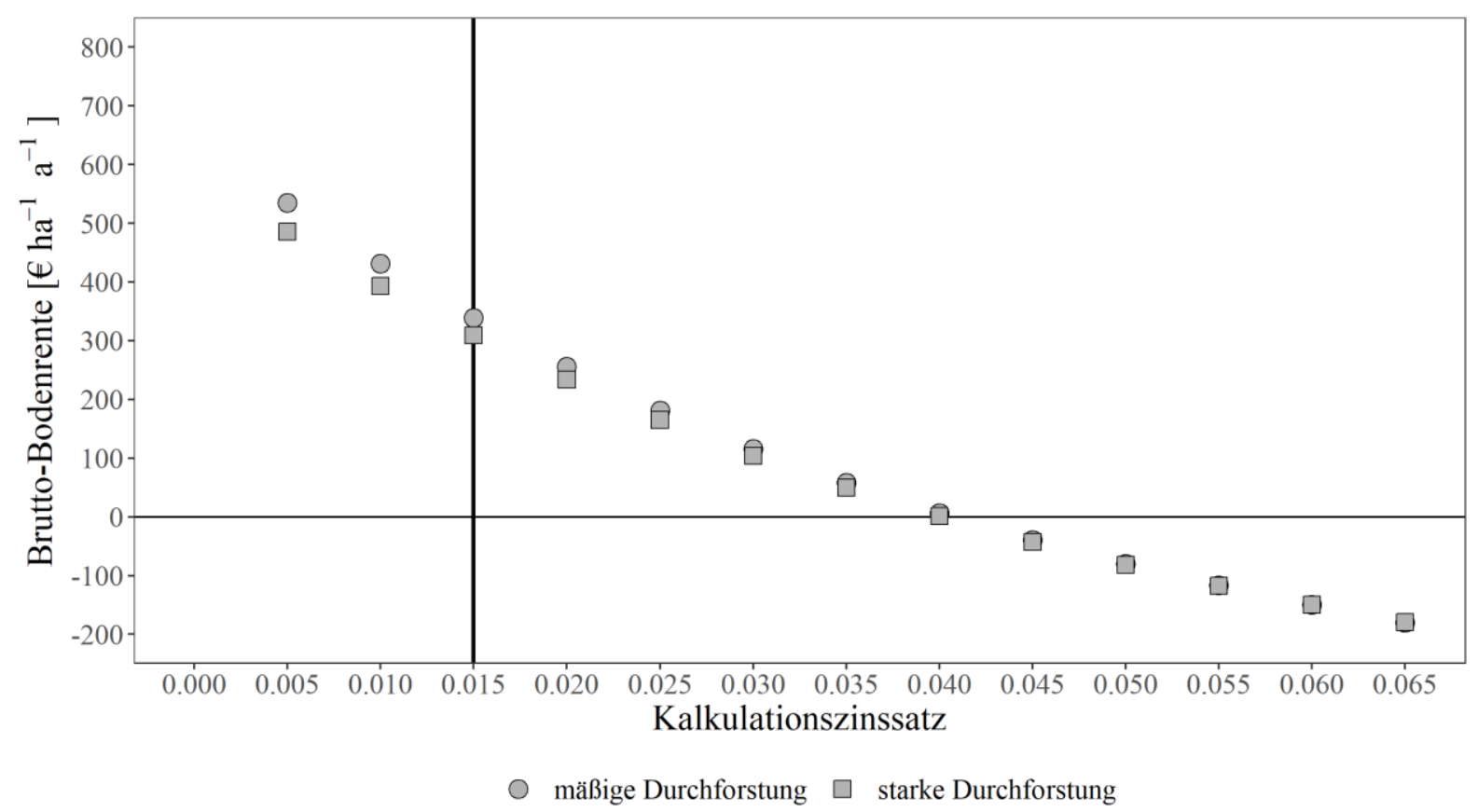

Abbildung 144: Analyse der Auswirkung verschiedener Kalkulationszinssätze auf die Brutto-Bodenrente der Baumart Fichte $\left(H_{100}(100 \mathrm{a})=35 \mathrm{~m}\right)$ im Vergleich der waldbaulichen Behandlungsvarianten gemäß dyn-ET (unveröff.) bei einer Umtriebszeit von 80 a; die schwarze Linie repräsentiert als Referenz den in Abschnitt 3.3.5 verwendeten Kalkulationszinssatz von $r=1,5 \%$.

Auch bei der Fichte (Abbildung 144) liegt die Brutto-Bodenrente der mäßigen Hochdurchforstung beim Referenz-Kalkulationszinssatz von $r=1,5 \%$ mit $339 € \mathrm{ha}^{-1} \mathrm{a}^{-1}$ um $10 \%$ oberhalb der starken (309€ ha ${ }^{-1} \mathrm{a}^{-1}$ ) Hochdurchforstung (siehe auch Tabelle 3-18 und Abbil- 
dung 136). Erst bei einem Kalkulationszinssatz von $r=4,5 \%$ werden die Brutto-Bodenrenten der Fichte negativ - auch hier ist die mäßige der starken Hochdurchforstung noch überlegen. $\mathrm{Ab}$ einem Kalkulationszinssatz von $r=6,5 \%$ liegt die Brutto-Bodenrente bei mäßiger schließlich unter der bei starker Hochdurchforstung $\left(-179,9 €\right.$ ha $^{-1} \mathrm{a}^{-1}$ ggü. - 179,5€ $\mathrm{ha}^{-1} \mathrm{a}^{-1}$ ). Selbst bei $r=10 \%$ beträgt die Differenz allerdings lediglich $0,8 € \mathrm{ha}^{-1} \mathrm{a}^{-1}$ bei einem absoluten mittleren Niveau von $-344 € \mathrm{ha}^{-1} \mathrm{a}^{-1}$.

Werden die Brutto-Bodenrenten anstatt zur baumartenspezifisch konstanten zu den kalkulationszinssatzspezifisch optimalen bzw. modell-maximalen Umtriebszeiten analysiert, zeigt sich ein baumartenabhängig differenziertes Bild. Bei der Buche bleiben die Effekte gleich - wie schon bei der konstanten Umtriebszeit 140 a büßt die mäßige Hochdurchforstung ihre relative Vorteilhaftigkeit ggü. den anderen Behandlungsvarianten ein - bei $r=3,5 \%$ $\left(T_{\text {mäßig }}=140 \mathrm{a} ; T_{\text {stark }}=145 \mathrm{a} ; T_{\text {gestaffelt }}=145 \mathrm{a}\right)$ sinkt ihre Brutto-Bodenrente unter die der gestaffelten und bei $r=5,0 \% \quad\left(T_{\text {mäßig }}=125 \mathrm{a} ; T_{\text {stark }}=130 \mathrm{a}\right.$; $T_{\text {gestaffelt }}=125$ a) schließlich auch unter die der starken Hochdurchforstung. Bei der Fichte führt ein steigender Kalkulationszinssatz nun nicht mehr zum Wechsel der Vorteilhaftigkeit selbst bei einem Kalkulationszinssatz von $r=10 \%\left(T_{\text {mäßig }}=T_{\text {stark }}=50 \mathrm{a}\right)$ ist die mäBige Hochdurchforstung, wenn auch minimal, relativ gesehen vorteilhaft. Die nun stark verkürzte Umtriebszeit verhindert die Ausnutzung der mit den höheren Einzelbaumdurchmessern verbundenen monetären Vorteilen bei starker Hochdurchforstung. Bemerkenswert ist, dass die Brutto-Bodenrenten bei beiden Baumarten trotz der variantenspezifisch optimalen Umtriebszeiten nahezu unverändert ggü. der Sensitivitätsanalyse mit konstanten Umtriebszeiten bleiben (Abbildungen 209 und 210 im Anhang) - über der Umtriebszeit verlaufen die Entwicklungen der Brutto-Bodenrenten entsprechend flach (vgl. Abbildungen 121 und 136).

Im Bereich forstlich realistischer Kalkulationszinssätze, bspw. in Anlehnung an tatsächlich von Entscheidungsträgerinnen und Entscheidungsträgern realisierte interne Grenzzinssätze $(1,3 \%$ bis 2,0 \% nach Möhring 2001 1,6 \% bis 3,1 \% nach Gerst 2015, S. 107) ist die mäßige Hochdurchforstung bei Verwendung der Referenz-Deckungsbeitragsfunktion aus Abschnitt 3.3.5 somit den alternativen Behandlungsvarianten überlegen. Die durch den Zinseffekt positiv wirkenden höheren Vornutzungswerte in früheren Jahren vermögen es bei starker Hochdurchforstung alleine nicht, die höhere Volumenleistung bei mäßiger Hochdurchforstung zu kompensieren. 
Für die Sensitivitätsanalyse des Treibers Überlebensrisikostärke werden die Erwartungswerte der durchschnittlichen waldbaulichen Überschüsse der forstlichen Produktion entsprechend der Methodik in Abschnitt 2.2.8 mit unterschiedlichen Überlebensfunktionsparametern berechnet. Analog zum DB-Variantenvergleich wird auf die Berücksichtigung von Kapitalknappheit zunächst verzichtet, um Wechselwirkungen mit dem Zinseffekt zu vermeiden. Alle sonstigen Eingangsdaten werden entsprechend des Abschnitts 3.3.5 gewählt - die genutzten Deckungsbeitragsfunktionen entsprechen also den schwarzen Referenz-DB-Varianten in Abbildung 137 a (Buche) bzw. Abbildung 138 a (Fichte), die relativen und absoluten Deckungsbeitragsminderungen bei Zwangsnutzung bleiben gleich. Bei einer an empirisch hergeleiteten Werten nach Brandl et al. (2020) ${ }^{85}$ orientierten Überlebensrisikoart, d. h. $\alpha$-Parameter der Überlebensfunktion [2-49] von $\alpha=1,11$ (Buche) bzw. $\alpha=1,29$ (Fichte), wird die Überlebensrisikostärke zwischen $S_{100}=0,05$ (ein Bestand erreicht nur mit $5 \%$ iger Wahrscheinlichkeit das Alter 100 a) und $S_{100}=1,0$ (risikofrei) in 0,05er-Schritten variiert (eine Ausnahme bilden jeweils die Referenz-Überlebensrisikostärken). Die farbigen Linien in Abbildung 145 b (Buche) bzw. Abbildung 146 b (Fichte) repräsentieren ausgewählte Überlebensrisikostärken, wobei die Referenz-Überlebensfunktionen (schwarz) die Koeffizienten nach Brandl et al. (2020) abbilden: $S_{100}=0,797$ (Buche) und $S_{100}=0,515$ (Fichte).

Baumartenübergreifend sinken die Erwartungswerte der durchschnittlichen waldbaulichen Überschüsse bei steigender Überlebensrisikostärke (sinkendes $S_{100}$ ) ab, wodurch sich auch die absoluten Differenzen zwischen den waldbaulichen Varianten verkleinern (Abbildungen 145 a und 146 b). Während sich bei der Baumart Buche allerdings der relative Abstand zwischen den Erfolgsgrößen der Behandlungsvarianten vergrößert, sinkt dieser bei der Fichte mit steigender Überlebensrisikostärke leicht ab. Zu einem risikobedingten Rangfolgenwechsel kommt es bei beiden Baumarten nicht.

\footnotetext{
${ }^{85}$ Die Schätzung der Überlebenswahrscheinlichkeiten von Brandl et al. (2020) basiert auf einem Accelerated Failure Time Modell mit einer Weibullverteilung. Als Datengrundlage dient die bundesweite Waldzustandserhebung mit den europäischen Level I und Level II Daten. Das Modell ermöglicht u. a. die Implementierung von durchschnittlichen Klimadaten (jährl. Durchschnittstemperatur, durchschn. Temperatur des wärmsten Quartals, max. Temperatur des wärmsten Monats, min. Temperatur des kältesten Monats, Summe der jährlichen Niederschläge, Summe der Niederschläge des wärmsten Quartals) zur Schätzung des Klimaeinflusses auf die Überlebenswahrscheinlichkeit. Für die in dieser Arbeit verwendeten baumartenspezifischen Koeffizienten der Überlebensfunktion wurden durchschnittliche Klimadaten der Jahre 1960 - 1990 der Region Solling (Hijmans et al. 2005) verwendet.
} 


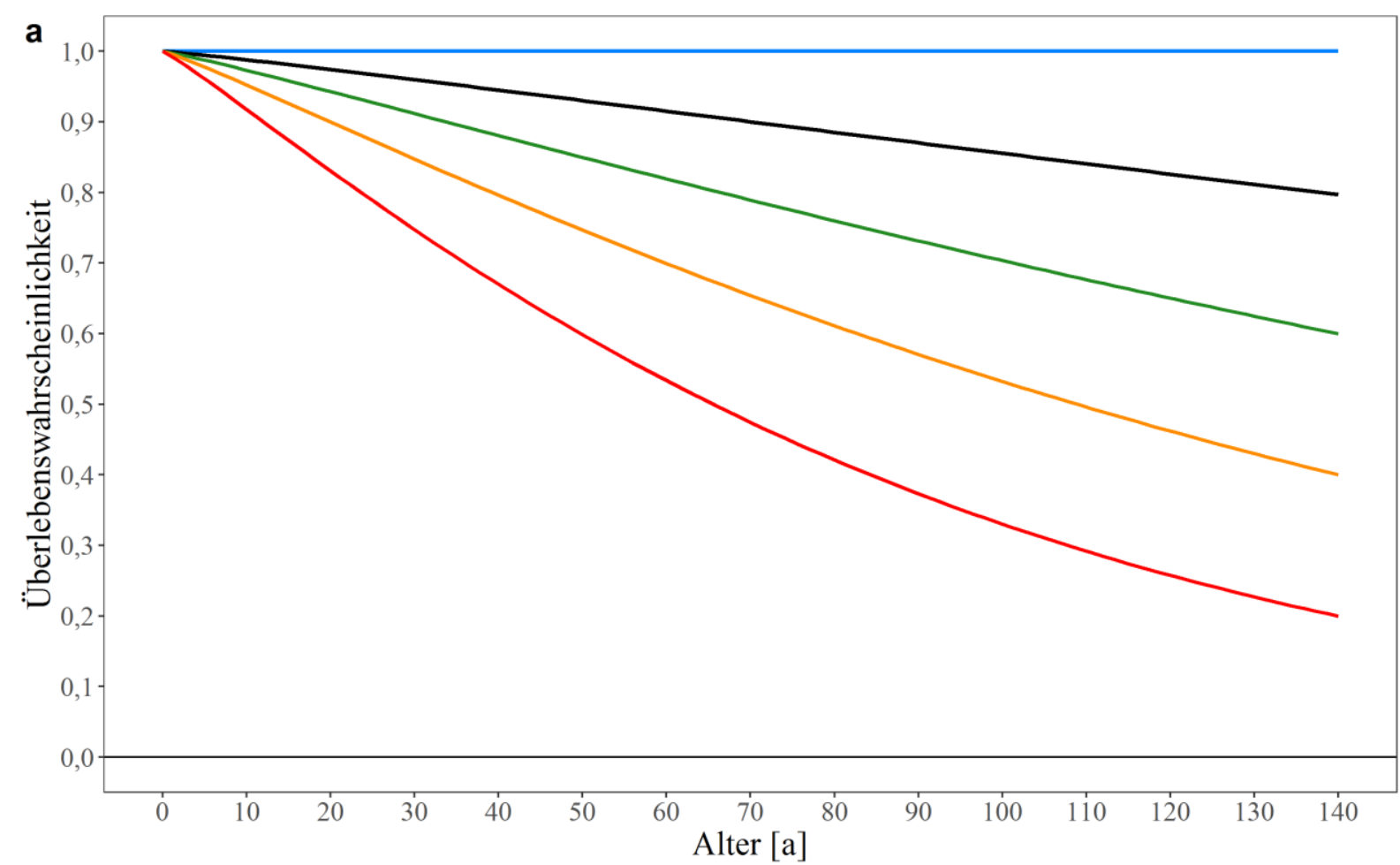

Überlebensrisikostärke $S_{100}:-0,2-0,4-0,6-0,797-1,0$

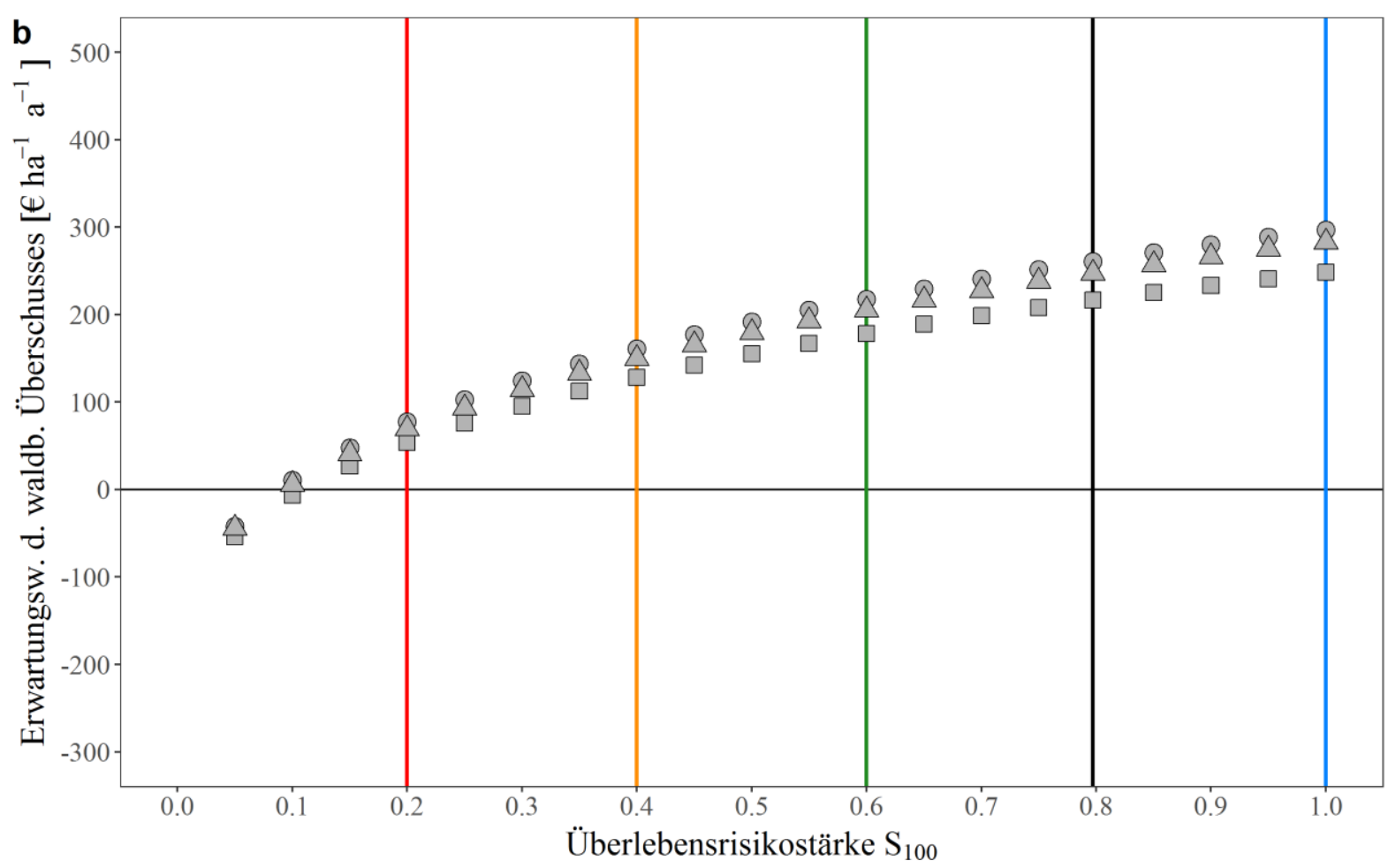

$\bigcirc$ mäßige Durchforstung $\square$ starke Durchforstung $\triangle$ gestaffelte Durchforstung

Abbildung 145: Analyse der Auswirkung unterschiedlicher Überlebensrisikostärken (a) auf den Erwartungswert des durchschnittlichen waldbaulichen Überschusses (b) der Baumart Buche $\left(H_{100}(100 \mathrm{a})=32 \mathrm{~m}\right)$ im Vergleich der waldbaulichen Behandlungsvarianten gemäß dyn-ET (unveröff.) bei einer Umtriebszeit von $140 \mathrm{a}$; die schwarze Linie in (a) repräsentiert eine Überlebensfunktion mit Koeffizienten $\left(S_{100}=0,797, \alpha=1,11\right)$ nach Brandl et al. (2020). 


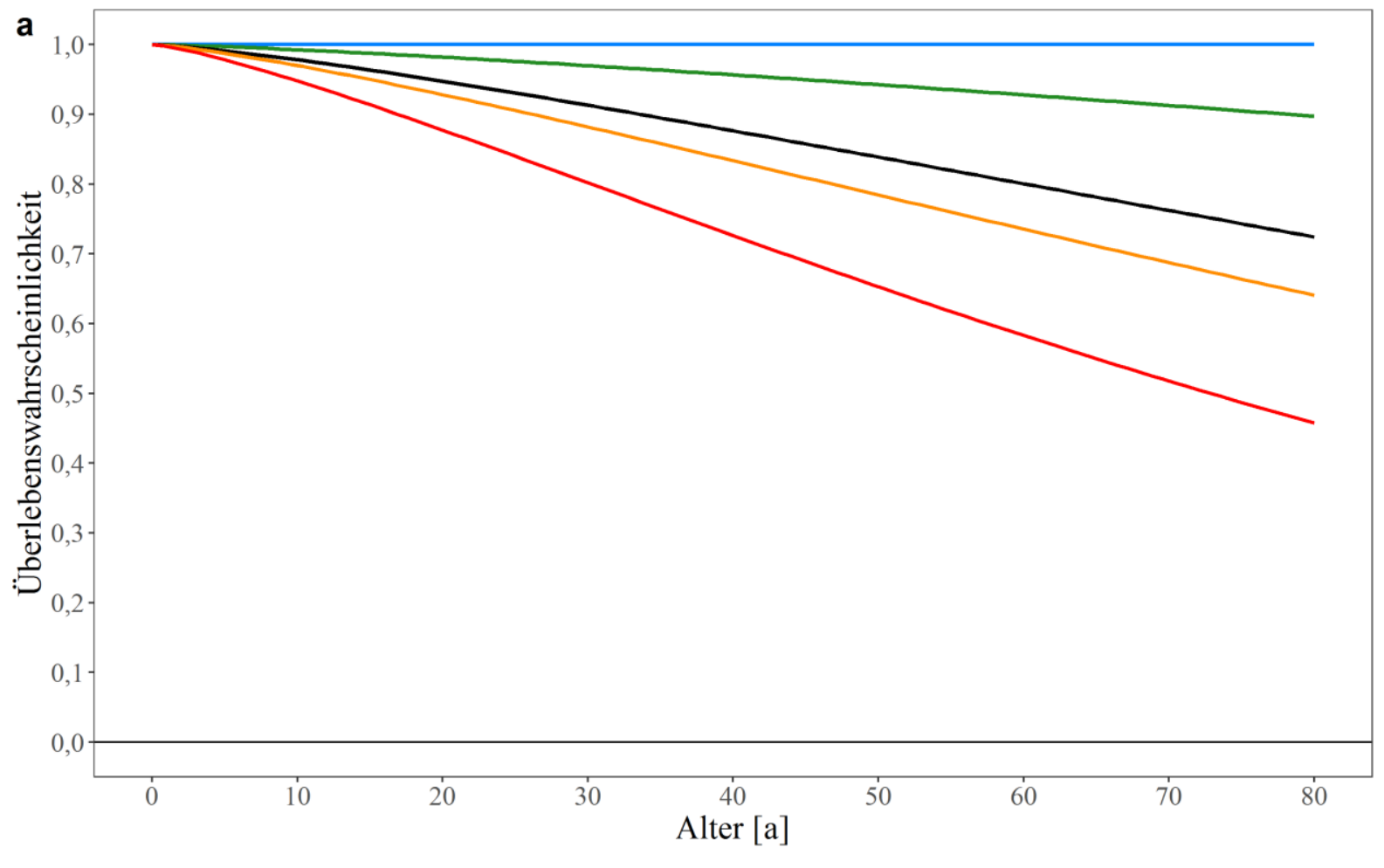

Überlebensrisikostärke $\mathrm{S}_{100}:-0,2-0,4-0,515-0,8-1,0$

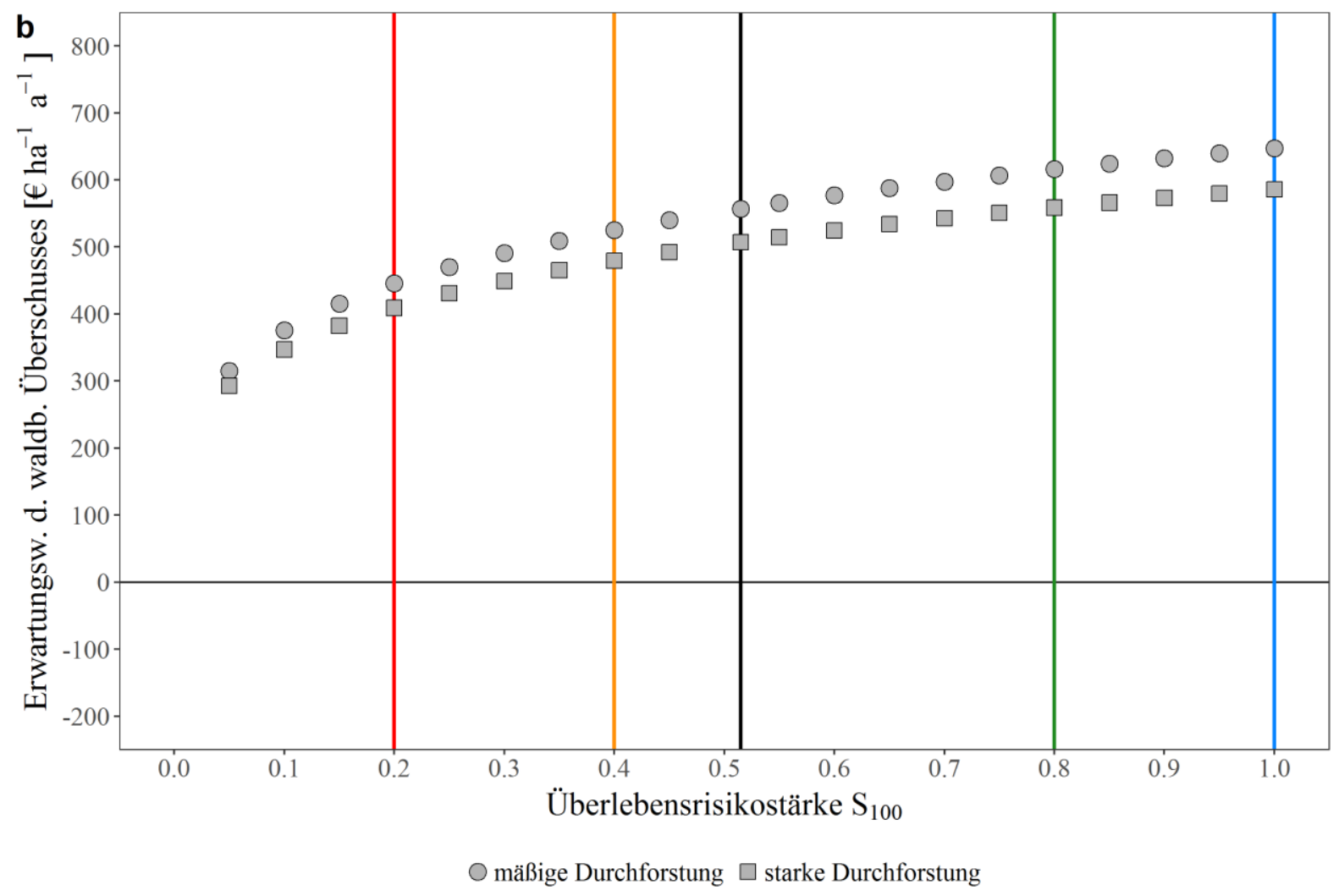

Abbildung 146: Analyse der Auswirkung unterschiedlicher Überlebensrisikostärken (a) auf den Erwartungswert des durchschnittlichen waldbaulichen Überschusses (b) der Baumart Fichte $\left(H_{100}(100 \mathrm{a})=35 \mathrm{~m}\right)$ im Vergleich der waldbaulichen Behandlungsvarianten gemäß dyn-ET (unveröff.) bei einer Umtriebszeit von $80 \mathrm{a}$; die schwarze Linie in (a) repräsentiert eine Überlebensfunktion mit Koeffizienten $\left(S_{100}=0,515, \alpha=1,29\right)$ nach Brandl et al. (2020). 
Bei der Referenz-Überlebensrisikostärke der Baumart Buche (schwarze Linie in Abbildung 145 a) liegt der Erwartungswert des durchschnittlichen waldbaulichen Überschusses der Baumart Buche bei mäßiger Hochdurchforstung mit $261 €$ ha $^{-1} \mathrm{a}^{-1}$ um $6 \%$ oberhalb der Erfolgsgröße bei gestaffelter ( $247 € \mathrm{ha}^{-1} \mathrm{a}^{-1}$ ) bzw. um $21 \%$ höher als bei starker (216 € ha ${ }^{-1} \mathrm{a}^{-1}$ ) Hochdurchforstung (Abbildung $145 \mathrm{~b}$ ). Bei der letzten noch positive Erwartungswerte generierenden Überlebensrisikostärke von $S_{100}=0,15$ liegt die mäßige Hochdurchforstung mit $48 € \mathrm{ha}^{-1} \mathrm{a}^{-1}$ um $17 \%$ oberhalb der Erfolgsgröße bei gestaffelter (41€ ha-1 $\mathrm{a}^{-1}$ ) bzw. um 77 \% höher als bei starker $\left(27 € \mathrm{ha}^{-1} \mathrm{a}^{-1}\right.$ ) Hochdurchforstung. Bei der Fichte schrumpft die Differenz zwischen den Erwartungswerten der durchschnittlichen waldbaulichen Überschüsse von $10 \%$ (557 € ha-1 $\mathrm{a}^{-1} \mathrm{zu} 507 € \mathrm{ha}^{-1} \mathrm{a}^{-1}$ ) bei der ReferenzÜberlebensrisikostärke (schwarze Linie in Abbildung 146 a) auf $8 \%$ (315€ ha $\mathrm{ha}^{-1} \mathrm{a}^{-1} \mathrm{zu}$ $293 € \mathrm{ha}^{-1} \mathrm{a}^{-1}$ ) bei einer Überlebensrisikostärke von $S_{100}=0,05$ ein (Abbildung $146 \mathrm{~b}$ ).

Auch ohne den risikobedingten Wechsel der Wertrelationen ermöglicht diese Sensitivitätsanalyse aber die Abschätzung der ökonomischen Auswirkungen einer Risikominderung durch waldbauliche Handlungen. Anders als bei den veränderten Deckungsbeitragsfunktionen ${ }^{86}$ und den variierenden Kalkulationszinssätzen kann es aufgrund der Behandlungsabhängigkeit der Überlebensrisikostärke (siehe Abschnitt 2.2.9) nämlich zu unterschiedlichen Überlebensrisikostärken der verschiedenen Behandlungsvarianten kommen (siehe Abschnitt 2.2.9). Mehr als die Wertrelation bei gleicher Überlebensrisikostärke steht hier folglich die Frage im Vordergrund, wie hoch eine behandlungsbedingte Risikominderung sein muss, damit es zu einem Wechsel der Rangfolge kommt.

Geht man in Anlehnung an Albrecht (2009, S. 140 und 145) und die bereits in den Abschnitten 2.2.9 und 2.3 getätigten Diskussionen bspw. von einer Risikominderung bei gestaffelter Hochdurchforstung aus, reicht bei der Buche ausgehend von der Referenz-Überlebensrisikostärke $\left(S_{100}=0,797\right)$ eine $9 \%$ ige behandlungsabhängige Senkung der Überlebensrisikostärke auf $S_{100}=0,870$ aus, um das Referenzniveau des Erwartungswertes des durchschnittlichen waldbaulichen Überschusses bei mäßiger Hochdurchforstung ( $261 € \mathrm{ha}^{-1} \mathrm{a}^{-1}$ ) zu erreichen und somit einen Rangfolgenwechsel herbeizuführen. Bei starke Hochdurchforstung wird solch eine Rangfolgenwechsel selbst bei Risikofreiheit nicht erreicht (Abbildung 145 b).

\footnotetext{
${ }^{86}$ Eine Behandlungsabhängigkeit der Deckungsbeitragsfunktion wird in dieser Arbeit nicht beachtet, wäre bei der Einbeziehung von behandlungsabhängigen Qualitätsentwicklungen aber natürlich gegeben.
} 
Aufgrund der in den vorläufigen Fichten-Ertragstafeldaten gemäß dyn-ET (unveröff.) nicht ausgewiesenen gestaffelten Hochdurchforstung sowie der unscharfen Abgrenzung zur starken Hochdurchforstung (siehe Fußnote 85 auf Seite 225) fällt die Interpretation der Ergebnisse an dieser Stelle schwer. Dieser Umstand ist bedauerlich, da die gestaffelte Hochdurchforstung insb. bei der Fichte, auch mit Blick auf zahlreiche Waldbaurichtlinien bzw. -empfehlungen (vgl. z. B. ForstBW 2014, S. 55 ff.), als risikomindernd angesehen wird. Es bleibt abzuwarten, welche Erkenntnisse die endgültigen Ertragstafeldaten des dyn-ET-Projekts diesbezüglich liefern werden. Die starke Hochdurchforstung der Fichte müsste in ihrer jetzigen Form ausgehend von der Referenz-Überlebensrisikostärke $\left(S_{100}=0,515\right)$ zu einer 53 \%igen Senkung der Überlebensrisikostärke auf $S_{100}=0,790$ führen, um das Referenzniveau des Erwartungswertes des durchschnittlichen waldbaulichen Überschusses bei mäßiger Hochdurchforstung zu erreichen (557€ ha $\mathrm{h}^{-1} \mathrm{a}^{-1}$ ) und somit einen Rangfolgenwechsel herbeizuführen (Abbildung $146 \mathrm{~b}$ ).

Die Analyse der Erwartungswerte der durchschnittlichen waldbaulichen Überschüsse zu den DB-variantenabhängig optimalen Umtriebszeiten (immer die modellmaximalen Umtriebszeiten) zeigt keine markanten Veränderungen ggü. der Analyse bei baumartenspezifisch konstanten Umtriebszeiten - auf eine entsprechende Darstellung im Anhang wird verzichtet.

Unabhängig von den tatsächlich zu realisierenden Überlebensrisikoabsenkungen verdeutlichen die starken Absenkungen der jährlichen Erfolgsgrößen das Potential von investiven, waldbaulichen Maßnahmen zur Stabililisierung von Waldbeständen. Dabei sind aber stets die damit verbundenen sonstigen Effekte, bspw. einer reduzierten Volumenleistung, zu beachten. Wie in Kapitel 2 ausführlich diskutiert, müssen die Grenznutzen einer Maßnahme (z. B. höhere Einzelbaumdimension mit damit verbundenen höheren Deckungsbeiträgen und verringerten Überlebensrisiken) stets im Gleichgewicht mit den damit verbundenen Grenzkosten (z. B. verringerte Gesamtwuchsleistung) stehen. 
Wie in den vorhergehenden Analysen gezeigt, reichen realitätsnahe Veränderungen der analysierten Treiber einzelnd überwiegend nicht aus, um die deutlich höhere Volumenleistung der mäßigen Hochdurchforstung (Abbildungen 71 und 93) durch die alternativen waldbaulichen Behandlungsvarianten ökonomisch zu kompensieren. Aufbauend auf den singulären Faktorveränderungen werden die Treiber im Folgenden somit kombiniert und deren Wechselwirkungen analysiert.

Abbildung 147 (Buche) und Abbildung 148 (Fichte) zeigen dafür zunächst die Auswirkungen unterschiedlicher DB-Funktionen auf die Brutto-Bodenrente der waldbaulichen Behandlungsalternativen. Hierfür werden die dimensionsabhängigen DB-Steigerungen entsprechend der Funktionskoeffizienten aus Tabelle 3-19 (Buche) und Tabelle 3-20 (Fichte) sowie der ReferenzKalkulationszinssatz $r=1,5 \%$ aus Abschnitt 3.3.5 gewählt. Diese Kombination der Treiber führt ggü. der zinsfreien Analyse unterschiedlicher Deckungsbeitragsfunktionen bereits bei deutlich geringeren DB-Steigerungen zu einem Rangfolgenwechsel.

Mit einer Brutto-Bodenrente von $-8 € \mathrm{ha}^{-1} \mathrm{a}^{-1}$ liegt die mäßige Hochdurchforstung der Baumart Buche bei der Referenz-Deckungsbeitragsfunktion zunächst noch über der gestaffelten $\left(-15 € \mathrm{ha}^{-1} \mathrm{a}^{-1}\right)$ bzw. der starken $\left(-26 € \mathrm{ha}^{-1} \mathrm{a}^{-1}\right)$ Hochdurchforstung (siehe auch Tabelle 3-15 und Abbildung 121). Mit sinkender DB-Steigerung verstärkt sich auch unter Kapitalknappheit der Effekt der ökonomischen Überlegenheit bei mäßiger Hochdurchforstung diese erzielt bei einem konstanten Deckungsbeitrag (DB-Variante 1) mit $17 € \mathrm{ha}^{-1} \mathrm{a}^{-1}$ schließlich eine positive Brutto-Bodenrente und liegt damit deutlich über den Brutto-Bodenrenten bei gestaffelter $\left(-2,6 € \mathrm{ha}^{-1} \mathrm{a}^{-1}\right)$ bzw. starker $\left(-3,5 € \mathrm{ha}^{-1} \mathrm{a}^{-1}\right)$ Hochdurchforstung. Nimmt die DB-Steigerung hingegen zu, kommt es ggü. der zinsfreien Analyse zu einem früheren Aufholen der gestaffelten Hochdurchforstung. Bereits bei DB-Variante 4 (grüne Linien in Abbildung 147) ist sie bei insgesamt negativen Brutto-Bodenrenten mit - $27 € \mathrm{ha}^{-1} \mathrm{a}^{-1}$ relativ vorteilhaft ggü. der mäßigen ( $\left.-33 € \mathrm{ha}^{-1} \mathrm{a}^{-1}\right)$ und starken $\left(-49 € \mathrm{ha}^{-1} \mathrm{a}^{-1}\right)$ Hochdurchforstung. Die höheren Vornutzungsmengen in jungen Bestandesaltern (Zinseffekt) in Kombination mit den insgesamt gesteigerten mittleren Einzelbaumdimensionen (Dimensionseffekt) kompensieren die höhere Volumenleistung bei mäßiger Hochdurchforstung. Die starke Hochdurchforstung der Buche schafft dies nicht - über alle DB-Varianten hinweg bleiben die BruttoBodenrenten deutlich hinter denen der alternativen Behandlungsvarianten zurück.

Anders sieht dies bei der Baumart Fichte aus - die bereits bei der zinsfreien Analyse ersichtlich gewordene Kompensation der höheren Volumenleistung bei mäßiger Hochdurchforstung durch 
die ertragskundlichen Auswirkungen bei starke Hochdurchforstung zeigt sich unter Kapitalknappheit bei nochmal geringeren DB-Steigerungen. Während die Brutto-Bodenrente bei mäßiger Hochdurchforstung und Bewertung mittels Referenz-DB-Funktion (schwarze Linien in Abbildung 148) mit $339 € \mathrm{ha}^{-1} \mathrm{a}^{-1}$ noch $10 \%$ über der Brutto-Bodenrente bei starker Hochdurchforstung (309€ ha $\mathrm{a}^{-1} \mathrm{a}^{-1}$ ) liegt, dreht sich das Wertverhältnis nun schon bei DBVariante 5 (gelbe Linien in Abbildung 148) um. Mit $325 € \mathrm{ha}^{-1} \mathrm{a}^{-1}$ liegt die Brutto-Bodenrente bei starker Hochdurchforstung dann um $4 \%$ oberhalb der Brutto-Bodenrente bei mäßiger Hochdurchforstung ( $314 € \mathrm{ha}^{-1} \mathrm{a}^{-1}$ ). Die dimensionseffektbedingte Kompensation der verminderten Volumenleistung (siehe ausschließliche DB-Variation) wird mit dem Zinseffekt durch die höheren Vornutzungswerte in jungen Bestandesaltern verstärkt. Gleichzeitig ist unter Kapitalknappheit nochmal mehr davon auszugehen, dass eine deutlich ausgeprägte, gestaffelte Hochdurchforstung der Fichte bereits bei deutlich geringeren DB-Steigerungen eine ökonomische Vorteilhaftigkeit ggü. der mäßigen Hochdurchforstung zeigen würde.

Werden die Brutto-Bodenrenten anstatt zur baumartenspezifisch konstanten zu den DB-variantenabhängig optimalen bzw. modell-maximalen Umtriebszeiten analysiert, ergeben sich für beide Baumarten leicht veränderte Wertrelationen, aber keine veränderten Rangfolgenwechsel - auf eine entsprechende Darstellung im Anhang wird verzichtet.

Werden zuletzt die Wechselwirkungen aller Treiber beachtet und anstatt der Referenz-DB-Varianten die DB-Varianten 4 (grüne Linien in den Abbildungen 147 und 148) verwendet, ist die gestaffelte Hochdurchforstung der Buche bereits bei Überlebensrisikostärken entsprechend der Referenz-Überlebensfunktion mit $\alpha=1,11$ und $S_{100}=0,797$ relativ gesehen vorteilhaft (Abbildung 149 b). Bei diesem DB-Spannungsverhältnis reicht der starken Hochdurchforstung der Buche eine behandlungsabhängige Absenkung der Überlebensrisikostärke auf $S_{100}=0,950(+19 \%)$, dass auch sie vorteilhaft ggü. der mäßigen Hochdurchforstung wird. Bei der Baumart Fichte genügt nun ausgehend von der Referenz-Überlebensfunktion mit $\alpha=1,29$ und $S_{100}=0,515$ eine behandlungsabhängige Absenkung der Überlebensrisikostärke um $13 \%$ auf $S_{100}=0,583$ (Abbildung 150 b), dass auch dort ein Rangfolgenwechsel der jährlichen Erfolgsgrößen stattfindet. 


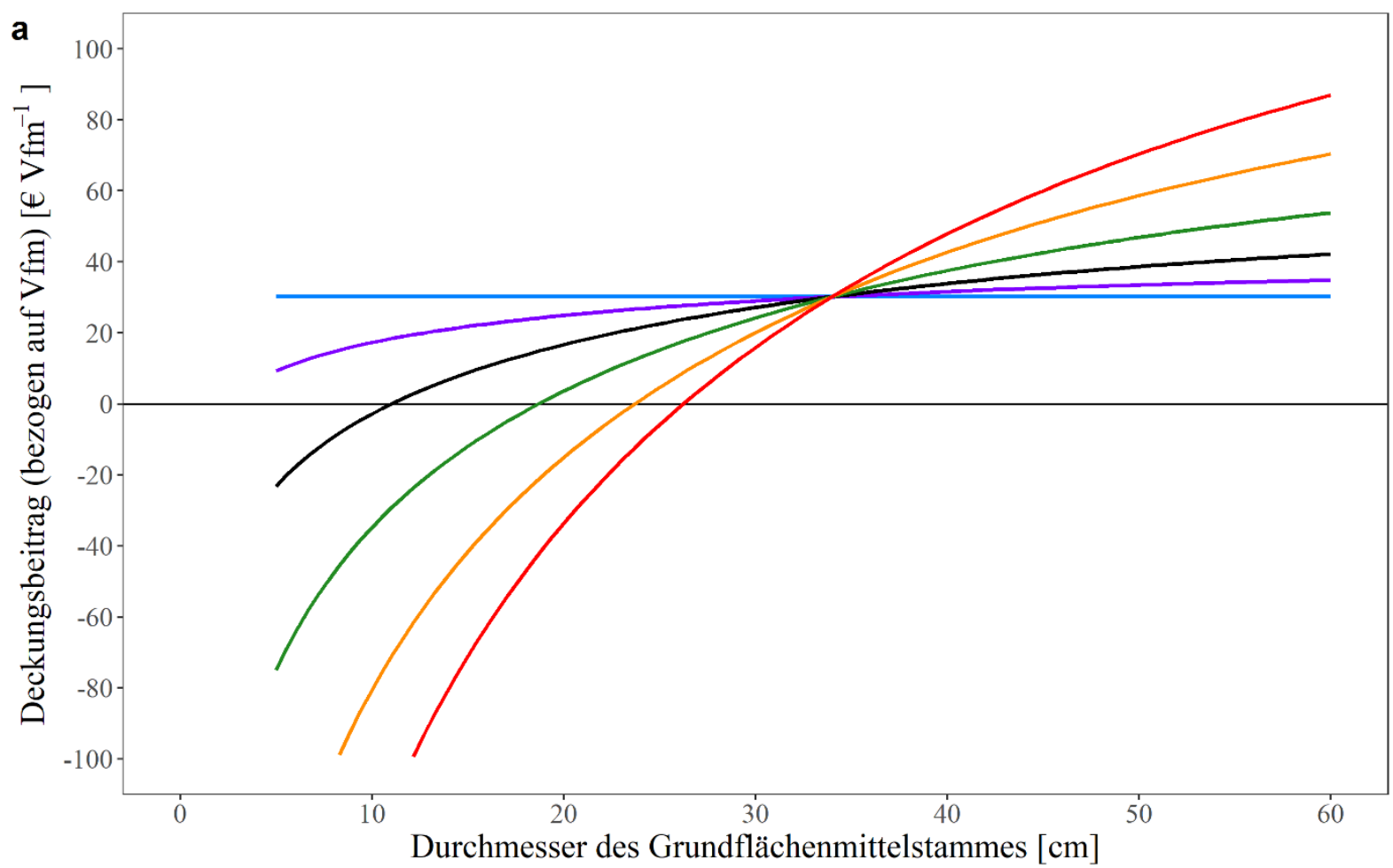

$-1-2-$ Referenz $-4-5-6$

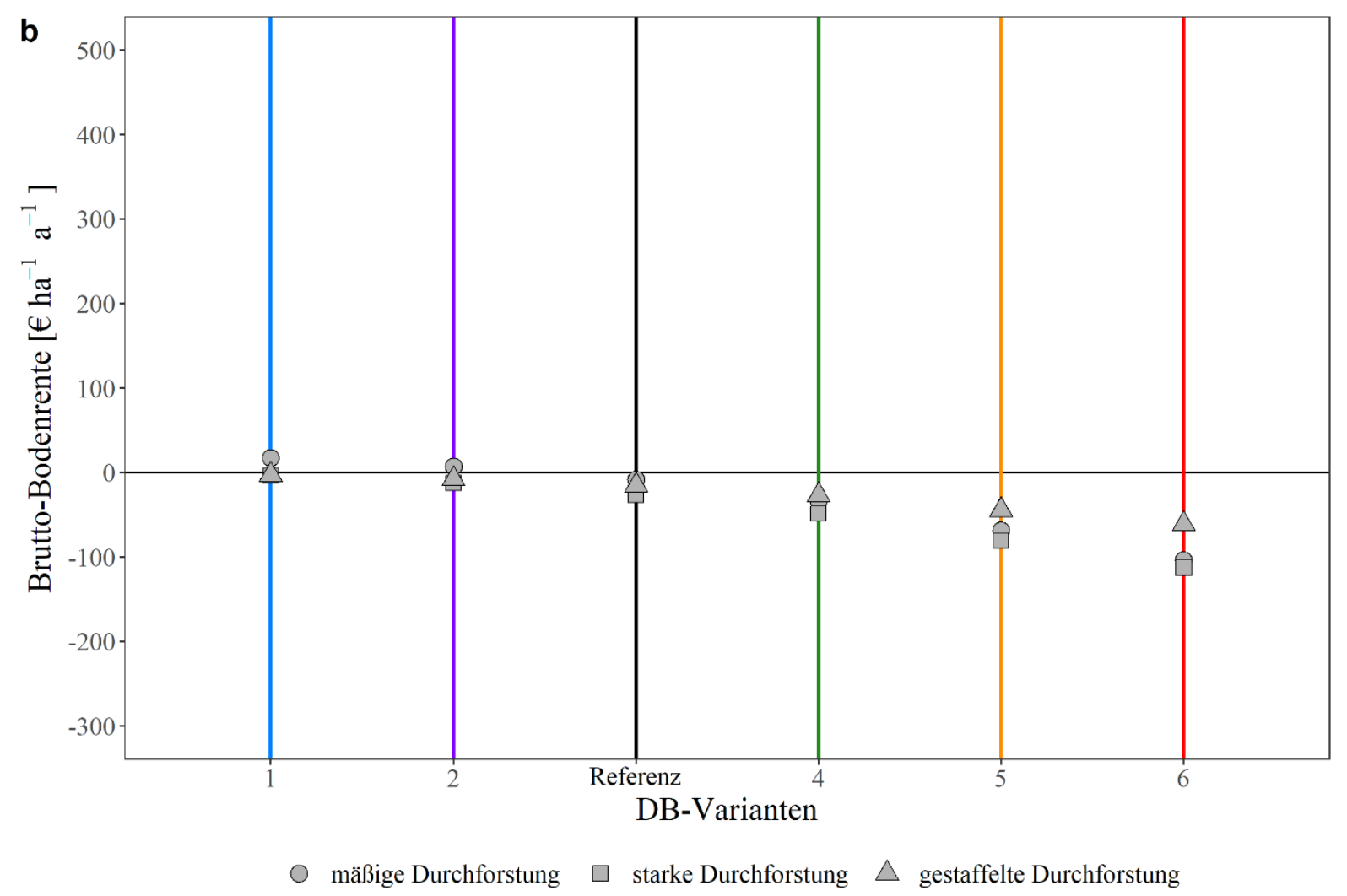

Abbildung 147: Analyse der Auswirkung unterschiedlicher Deckungsbeitragsfunktionen (a) auf die Brutto-Bodenrente $(\mathrm{b})$ der Baumart Buche $\left(H_{100}(100 \mathrm{a})=32 \mathrm{~m}\right)$ im Vergleich der waldbaulichen Behandlungsvarianten gemäß dyn-ET (unveröff.) bei einer Umtriebszeit von 140 a und einem Kalkulationszinssatz von $r=1,5 \%$. 


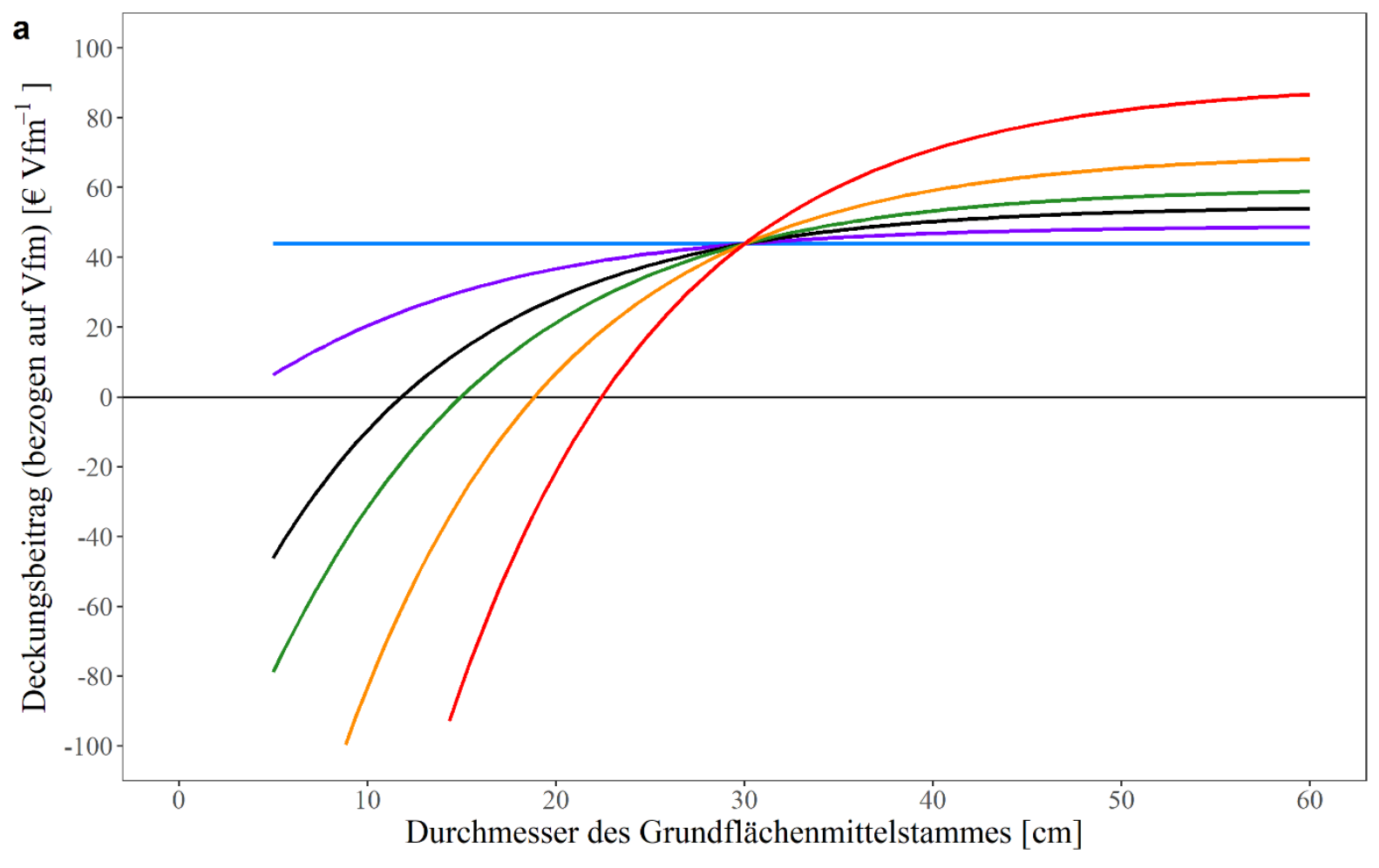

$-1-2-$ Referenz $-4-5-6$

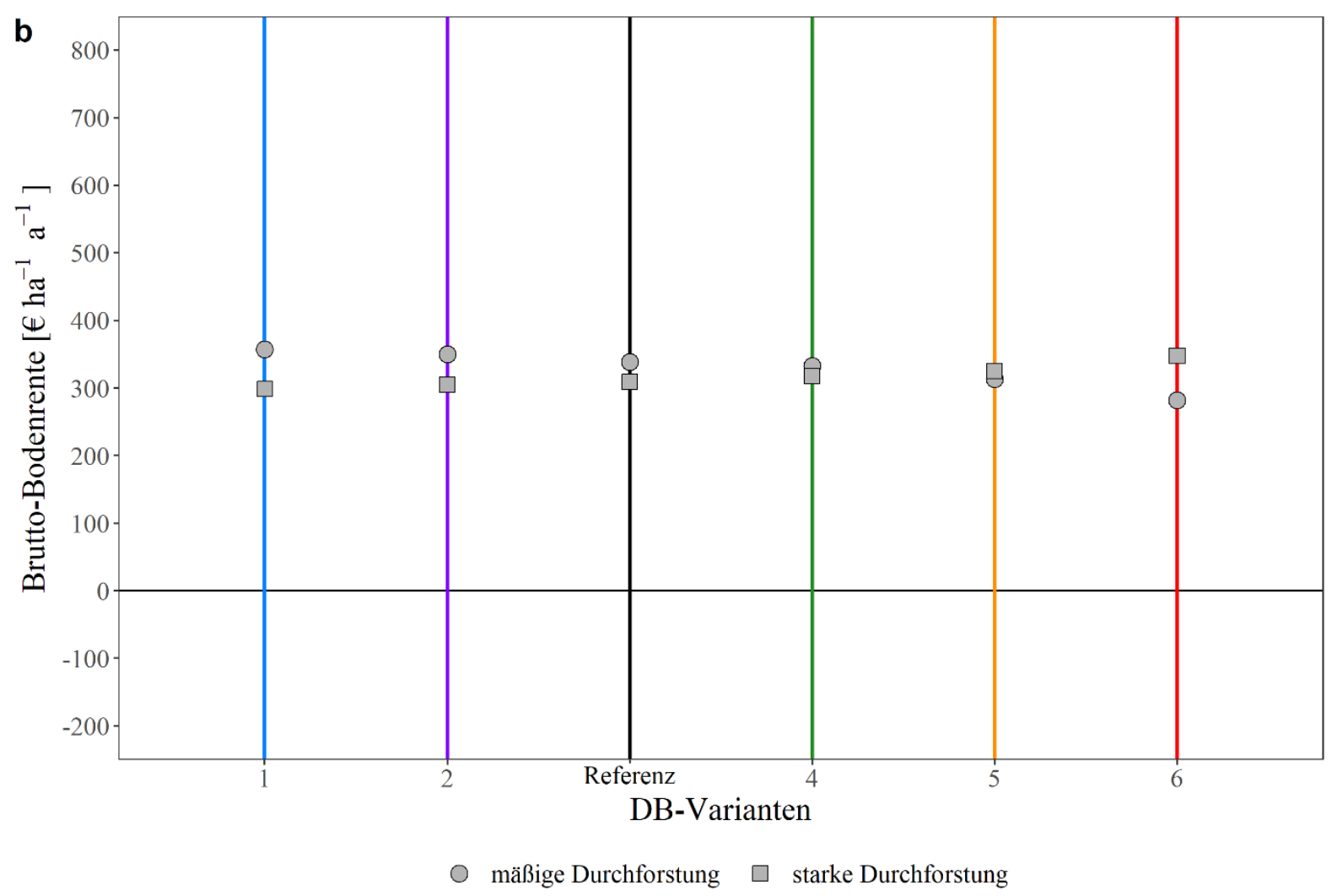

Abbildung 148: Analyse der Auswirkung unterschiedlicher Deckungsbeitragsfunktionen (a) auf die Brutto-Bodenrente (b) der Baumart Fichte $\left(H_{100}(100 \mathrm{a})=35 \mathrm{~m}\right)$ im Vergleich der waldbaulichen Behandlungsvarianten gemäß dyn-ET (unveröff.) bei einer Umtriebszeit von 80 a und einem Kalkulationszinssatz von $r=1,5 \%$. 


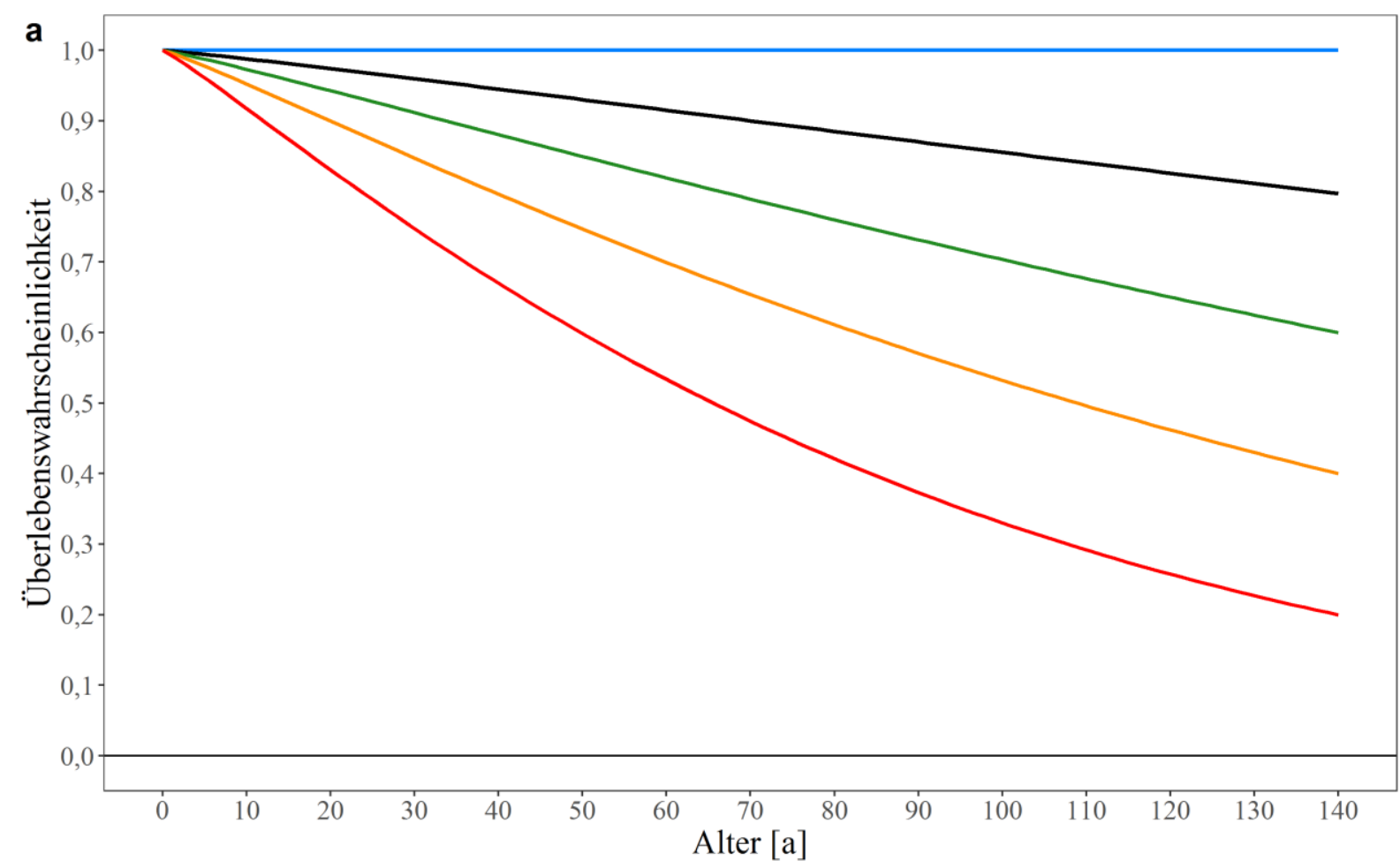

Überlebensrisikostärke $S_{100}:-0,2-0,4-0,6-0.797-1,0$

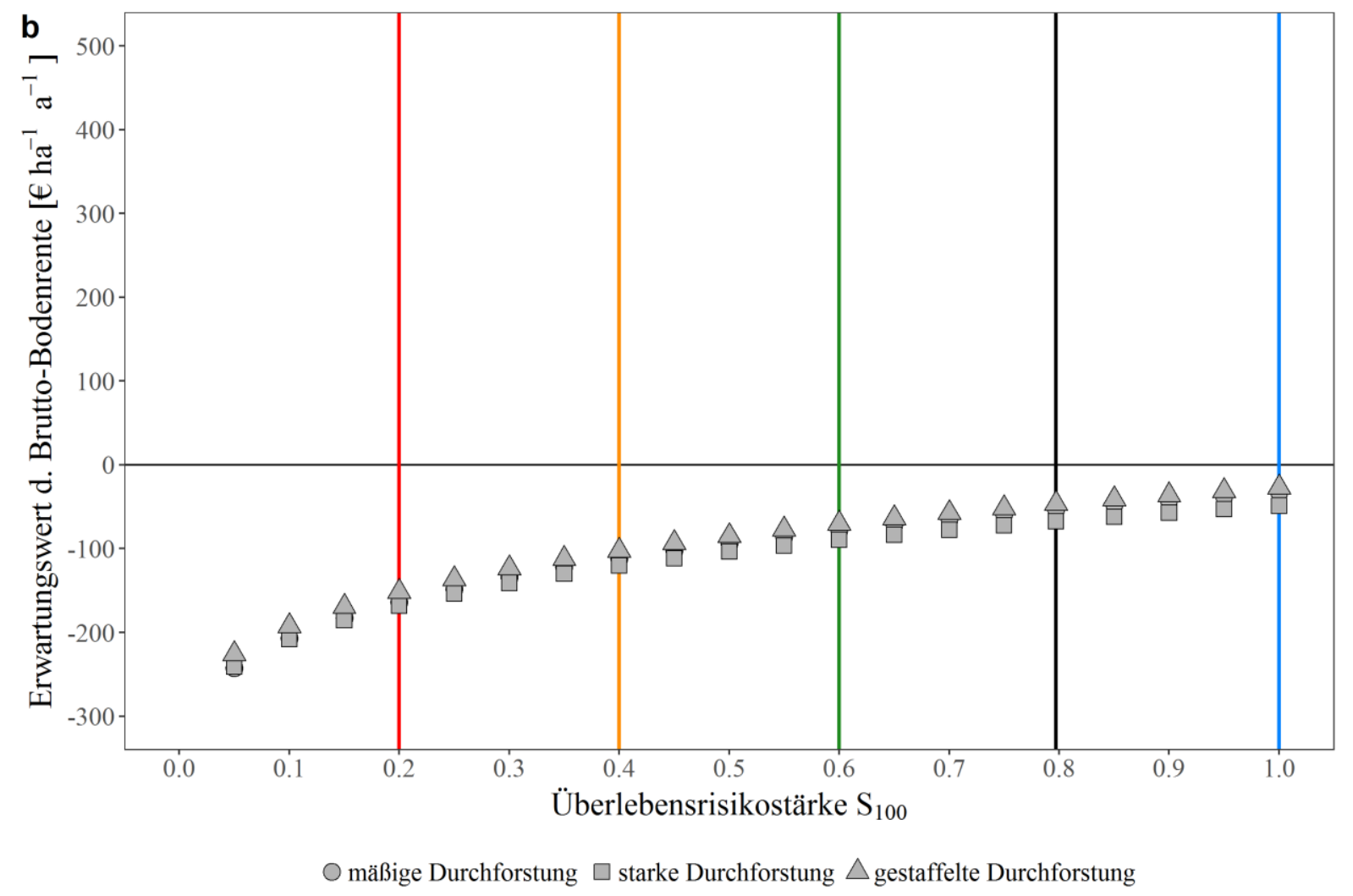

Abbildung 149: Analyse der Auswirkung unterschiedlicher Überlebensrisikostärken (a) auf den Erwartungswert der Brutto-Bodenrente $(\mathrm{b})$ der Baumart Buche $\left(H_{100}(100 \mathrm{a})=32 \mathrm{~m}\right)$ im Vergleich der waldbaulichen Behandlungsvarianten gemäß dyn-ET (unveröff.) bei einer Umtriebszeit von 140 a und einer Deckungsbeitragsfunktion entsprechend der DB-Variante 4 (siehe grüne Linie in Abbildung 147); die schwarze Linie in (a) repräsentiert eine Überlebensfunktion mit Koeffizienten $\left(S_{100}=0,797\right.$, $\alpha=1,11)$ nach Brandl et al. (2020). 

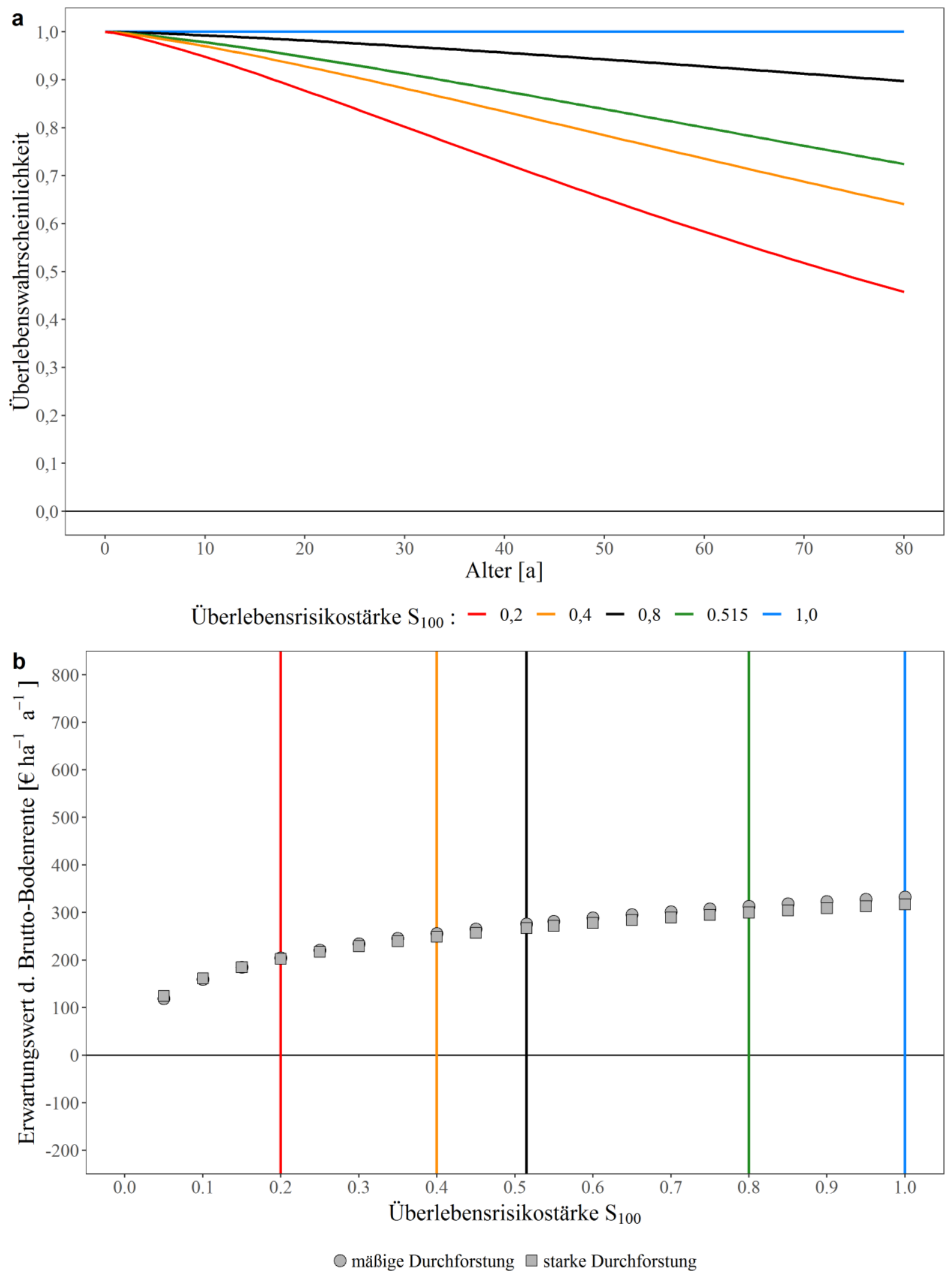

Abbildung 150: Analyse der Auswirkung unterschiedlicher Überlebensrisikostärken (a) auf den Erwartungswert der Brutto-Bodenrente $(\mathrm{b})$ der Baumart Fichte $\left(H_{100}(100 \mathrm{a})=35 \mathrm{~m}\right)$ im Vergleich der waldbaulichen Behandlungsvarianten gemäß dyn-ET (unveröff.) bei einer Umtriebszeit von 80 a und einer Deckungsbeitragsfunktion entsprechend der DB-Variante 4 (siehe grüne Linie in Abbildung 148); die schwarze Linie in (a) repräsentiert eine Überlebensfunktion mit Koeffizienten $\left(S_{100}=0,515\right.$, $\alpha=1,29)$ nach Brandl et al. (2020). 
Als Fazit aus den getätigten Sensitivitätsanalysen und gleichzeitiger Brückenschlag zur forstlichen Praxis lässt sich anhand der vorläufigen Ertragstafeldaten gemäß dyn-ET (unveröff.) festhalten, dass alle drei variierten Treiber zu einer Kompensation der volumenleistungsbedingten Vorteilhaftigkeit bei mäßiger Hochdurchforstung beitragen können. Insbesondere die gestaffelte Hochdurchforstung der Buche profitiert aufgrund der nur leicht verminderten Gesamtwuchsleistung, den gleichzeitig (überwiegend) gestiegenen mittleren Einzelbaumdurchmessern und den höheren Vornutzungswerten zu früheren Zeitpunkten von stärkeren DB-Steigerungen und höheren Kalkulationszinssätzen. Es genügen für einen Rangfolgenwechsel der jährlichen Erfolgsgrößen zudem geringfügige behandlungsabhängige Absenkungen der Überlebensrisikostärke. Die Kombination aller Treiber führt bei der Buche schließlich zu einem Rangfolgenwechsel innerhalb realistischer Wertebereiche. Bei an empirische Untersuchungen angelehnten Überlebenswahrscheinlichkeiten (vgl. Brandl et al. 2020) und einem forstlich realistischen Kalkulationszinssatz (vgl. Möhring 2014 und Gerst 2015, S. 46 ff.) bedarf es im Vergleich zu der in Abschnitt 3.3.5 verwendeten Deckungsbeitragsfunktion nur einer leichten Erhöhung der DBSteigerung, damit der Erwartungswert der Brutto-Bodenrente bei gestaffelter Hochdurchforstung relativ vorteilhaft wird (Abbildung 149). Geht die waldbewirtschaftende Person somit von einem leicht höheren als in Abschnitt 3.3.5 angenommenen monetären Spannungsverhältnis zwischen gering- und hochdimensionierten Sortimenten aus, ist eine Bestandesbehandlung mit zunächst starken und mit zunehmender Oberhöhe abschwächenden Vornutzungseingriffen sinnvoll. Die Lenkung des Zuwachses auf relativ wenige Einzelbäume durch geringe Stammzahlen in der Bestandesjugend bei gleichzeitig aufholender Volumenleistung in höheren Altern kombiniert die ökonomischen Erfolgsfaktoren und sorgt für eine optimale Allokation von Fläche und Kapital. Da die genannten Effekte für die Fichte auch bei starker Hochdurchforstung (mit Ansätzen einer gestaffelten Hochdurchforstung; siehe Fußnote 84 auf Seite 225) erkennbar sind, ist davon auszugehen, dass die Kompensation der volumenleistungsbedingten Vorteilhaftigkeit der mäßigen durch eine deutlich gestaffelte Hochdurchforstung nochmal stärker ausfallen würde. Dies hätte besondere Bedeutung, da insb. bei der Fichte von einer Absenkung der Überlebensrisikostärke durch eine gestaffelte ggü. einer mäßigen Hochdurchforstung ausgegangen wird (vgl. Röhrig et al. 2006, S. 282 f.; Albrecht 2009, S. 140 und 145; ForstBW 2014, S. 55 ff.). 


\subsubsection{Kritische Methoden- und Modellwürdigung}

Zunächst sei an dieser Stelle nochmals darauf hingewiesen, dass es sich bei den analysierten Ertragstafeldaten des Abschnitts 3.3 um vorläufige Projektdaten gemäß dyn-ET (unveröff.) handelt. Anstehende Änderungen der Erstellungsmethodik werden bis zum Projektende bzw. der Veröffentlichung der neuen Generation dynamischer Ertragstafeln zwangsläufig zu veränderten Ertragstafeldaten führen. Die absoluten Ergebniswerte der getätigten Analyse dürfen folglich nicht mit letzter Konsequenz interpretiert werden. Vielmehr stehen im gesamten Abschnitt die Wertrelationen im Vordergrund - es wird davon ausgegangen, dass sich diese im Vergleich zu den endgültigen Ertragstafeldaten grundsätzlich nicht unterscheiden. Umso bedauernswerter ist das Fehlen der gestaffelten Hochdurchforstung bei der Fichte zum Zeitpunkt der Abgabe dieser Arbeit. Diese hätte einige der aufgezeigten Effekte höchstwahrscheinlich nochmal stärker betont. Neben diesem vorläufigen Charakter der verwendeten Daten wird ebenfalls darauf hingewiesen, dass die Analysen des Abschnitts 3.3 lediglich eine Auswahl der innerhalb des dyn-ET-Projekts zur Verfügung stehenden ertragskundlichen Daten einbeziehen. Die Auswahl erfolgte dabei mit starkem Fokus auf die Analyse der naturalen und ökonomischen Unterschiede zu etablierten Ertragstafelwerken sowie den ökonomisch relevanten Unterschieden der waldbaulichen Behandlungsvarianten.

Für die Anpassung der Isolinien-Funktionen an die vorläufigen Ertragstafeldaten gemäß dynET (unveröff.) wurden im Rahmen der Erstellung baumartenspezifischer Bestandesbehandlungsdiagramme (SDMDs; Abschnitt 3.3.4) simultane nichtlineare Regressionsanalysen genutzt. Alternative Ansätze der separaten nichtlinearen Regressionsanalyse, mit teils geringeren mittleren Abweichungen von den Ausgangsdaten, wurden getestet und mit den verbundenen Schwächen entsprechend diskutiert. Trotz der insgesamt zufriedenstellenden Ergebnisse ist Abschnitt 3.3.4 insbesondere als methodischer Grundstein einer andauernden Methodendiskussion innerhalb des dyn-ET-Projekts zu verstehen. Auf Grundlage der endgültigen dyn-ET-Daten müssen die Anpassungsgüten zukünftiger Bestandesbehandlungsdiagramme erneut baumartenspezifisch getestet und diskutiert werden - die Entscheidung über eine zielführende Anpassungsmethode kann nur auf dieser Grundlage erfolgen.

Wie auch schon in Kapitel 2, beziehen sich auch die getätigten Analysen in Abschnitt 3.3 ausschließlich auf die gewinnorientierte Rohholzerzeugung und vernachlässigt somit sämtliche andere Funktionen und Leistungen des Ökosystems Wald (vgl. z. B. Samuelson 1976 und MEA 
2005, S. 26 ff.). Die Auswirkungen der waldbaulichen Behandlungsvarianten auf andere Leistungen abseits des Holzertrags (z. B. Biodiversität, Emissionsschutz oder Trinkwasserbereitstellung) werden nicht berïcksichtigt.

Die verwendeten Eingangsdaten für die ökonomische Bewertung spiegeln mit mittleren, überwiegend am hessischen Staatswald orientierten, Werten der Jahre 2013 bis 2017 realitätsnahe, baumartenspezifische Verhältnisse wider. Auch dabei geht es weniger um die absolute Höhe der Bewertungsergebnisse, als um die Wertrelationen zwischen den waldbaulichen Behandlungsvarianten. Die zur Funktionalisierung verwendete Chapman-Richards-Funktion ist geeignet, die nichtlinearen, dimensionsabhängigen Wertentwicklungen der Baumarten Buche und Fichte auf Basis der gewählten Eingangsdaten abzubilden. Bei veränderten Eingangsdaten und für jede andere Baumart muss die Eignung der Funktion sowie die Güte der Funktionalisierung, trotz der automatisierten Parametrisierung im Bewertungsmodell, stets aufs Neue überprüft werden - eine grafische Analyse, wie in Abbildung 106, wir dazu in der Regel ausreichen. Die aus den verwendeten Eingangsdaten in Verbindung mit den Ertragstafeldaten resultierenden immer weiter steigenden, jährlichen Erfolgsgrößen stehen teilweise im Widerspruch zu Analysen von z. B. Bodelschwingh (2018, S. 122) oder Möhring (2010). Tests mit im hohen Alter deutlich absinkenden DB-Funktionen ergaben innerhalb der Modellgrenzen dennoch keine Kulminationspunkte der jährlichen Erfolgsgrößen, weshalb auf die Integration von wertmindernden Effekten (z. B. Rotkernbildung bei der Buche) verzichtet wurde.

Mit der Verwendung eines einheitlichen Kalkulationszinssatzes ( $r=1,5 \%)$ wird, wie auch schon in Kapitel 2, bei der finanzmathematisch korrekten Berechnung durchschnittlicher Erfolgsgrößen (Brutto-Bodenrente) in Anlehnung an die Prämissen des FPO-Theorems ein vollkommener Kapitalmarkt und damit u. a. die unbegrenzte Möglichkeit zur Kapitalaufnahme und -anlage zu einem einheitlichen Zinssatz sowie rationales Entscheiden auf Grundlage vollständiger Informationen unterstellt (vgl. Samuelson 1976; Johansson und Löfgren 1985, S. 74; Wippermann 2005, S. 28 f.; Hering 2017, S. 33 ff.; siehe auch Abschnitt 2.4).

Die in Abschnitt 3.3.6 durchgeführte Sensitivitätsanalysen untersuchen die Auswirkungen ausgewählter Treiber auf durchschnittliche Erfolgsgrößen mittels Variantenstudium. Dabei geht es weniger um die „Empfindlichkeit“ (Thommen und Achleitner 2012, S. 688) der Bewertungsergebnisse im engeren Sinne einer Sensitivitätsanalyse, als um die Wertrelationen zwischen den waldbaulichen Behandlungsalternativen gemäß dyn-ET (unveröff.) bei Veränderung der Eingangsdaten. Die in diesem Zuge frei gewählten DB-Varianten rotieren jeweils um einen festen, baumartenspezifischen Punkt - eine höhere Bewertung stärkerer Einzelbaumdimensionen geht 
somit immer mit einer Abwertung geringdimensionierter Sortimente einher. Wie an verschiedenen Stellen diskutiert, geht es dabei aufgrund des nichtlinearen Funktionscharakters stets um das monetäre Spannungsverhältnis zwischen gering- und hochdimensionierten Sortimenten. Die getroffenen Aussagen zum Einfluss der DB-Steigerungen auf die Wertrelationen der waldbaulichen Behandlungsalternativen beziehen sich somit auf diese spezifischen Funktionsverläufe - zahlreiche andere Formen der dimensionsabhängigen DB-Variation, mit ggf. anderem Einfluss auf die Wertrelationen, sind vorstellbar, werden hier aber nicht diskutiert.

Wie auch schon in Abschnitt 2.2.8 werden Produktionsrisiken bei der Sensitivitätsanalyse ausschließlich in Form von Überlebensrisiken, d. h. dem vorzeitigen, unplanmäßigen Ausfall von Bäumen bzw. des Bestandes, eingeführt. Die Einbeziehung der Überlebensrisiken auf der Basis eines Erwartungswertes, als der mit den Eintrittswahrscheinlichkeiten gewichtete Mittelwert einer Zielgröße, unterstellt der rational entscheidenden Person implizit eine neutrale Einstellung ggü. Risiken (vgl. BAYES-Prinzip; Möhring 1994, S. 15). Subjektive und insbesondere auch von der jeweiligen Entscheidungssituation abhängige Risikoeinstellungen (vgl. BERNOULLIPrinzip; Möhring 1994, S. 15) werden vernachlässigt.

Trotz der in die Sensitivitätsanalyse einbezogenen Wechselwirkungen bleibt es lediglich bei einer (kleinen) Auswahl an analysierten Treibern. Andere, für eine gesamtheitliche Beurteilung forstwirtschaftlichen Handelns wichtige Aspekte, wie z. B. die qualitativen Auswirkungen der unterschiedlichen Behandlungsvarianten, bleiben unberücksichtigt. 



\author{
"Am besten hat's die Forstpartie - \\ denn der Wald wächst auch ohne sie." \\ (Sprichwort; Ursprung unbekannt)
}

\title{
4. Schlussfolgerungen
}

Die Ergebnisse dieser Arbeit stehen im klaren Widerspruch zum oft zitierten, teilweise Wilhelm Busch zugesprochenen, Sprichwort des für sich alleine wachsenden Waldes und der sich darauf ausruhenden Forstpartie. Natürlich wächst ein Waldbestand auch ohne die menschliche Einflussnahme - das hat dann aber nichts mit Forstwirtschaft zu tun, sofern man darunter die „Bereitstellung materieller und immaterieller Leistungen“ des Waldes für die Gesellschaft nach dem „Prinzip der Wirtschaftlichkeit“ (ökonomisches Prinzip) versteht (Stinglwagner et al. 2005, S. 314 i. V. m. S. 972). Konzentriert man sich unter allen Aufgaben der Forstwirtschaft auf die Produktion des nachwachsenden Rohstoffs Holz, bedeutet dies, dass die Bewirtschaftung eines Waldbestandes mehr ist, als das Wachsenlassen von Bäumen und die Ernte von möglichst viel Holzvolumen. Mit dem Ziel einer effizienten Holzproduktion und vor dem Hintergrund bestehender Prämissen, ist die optimale Allokation der knappen Ressourcen Fläche und Kapital essentielle und niemals abgeschlossene Aufgabe einer waldbewirtschaftenden Person. Es geht darum, die Komplexität der optimalen forstlichen Bestandesbehandlung vor dem Hintergrund einer spezifischen Entscheidungssituation zu beherrschen. Modelle, wie sie bspw. in dieser Arbeit über zwei unterschiedliche Ansätze zum Einsatz kommen, schaffen im Idealfall ein angemessenes Verhältnis zwischen Realitätsnähe und Realitätsvereinfachung, helfen bei der Reduktion der Komplexität und unterstützen somit die Entscheidungsfindung.

In diesem Sinne tragen forstliche Ertragstafeln als Wachstums- und Nutzungsmodelle seit über 200 Jahren zur nachhaltigen Bewirtschaftung unserer Wälder bei - eine Tatsache, die in der mitteleuropäischen Forstwirtschaft allzu oft als Selbstverständlichkeit angesehen wird und die gleichzeitig nicht hoch genug gewürdigt werden kann. Trotz der zurecht steigenden Bedeutung von Waldwachstumssimulationen und Betriebsinventuren (bspw. zur Einschätzung ertragskundlicher Kenngrößen von ungleichaltrigen Mischbeständen) besteht weiterhin Bedarf nach einfach handhabbaren (Kleinprivatwald), konsistenten und langfristigen (Betriebskalkulationen, Waldbewertung) sowie transparenten und objektivierten (Steuerrecht, Waldbewertung, Lehre) Wachstums- und Nutzungsmodellen. Um den Bedürfnissen der forstlichen Wirklichkeit aber auch zukünftig gerecht werden zu können und gleichzeitig auf Stoffeinträge, veränderte 
klimatische Verhältnisse und sich wandelnde Bestandesbehandlungskonzepte einzugehen, bedarf es nach Ansicht des Autors einer verstärkten ertragskundlichen Forschung (vgl. Möhring et al. 2019).

Gleichzeitig sollten die Schnittstellen zwischen Waldwachstumskunde, Waldbau, Ertragskunde und Forstökonomie ausgebaut und dabei insb. Rückkopplungen einbezogen werden. Die Forstökonomie sollte eben nicht nur als Monetarisierung gegebener naturaler Modelle verstanden werden, sondern zur effizienten Gestaltung der Waldbewirtschaftung genutzt werden. Für die zukünftige Erstellung forstlicher Erftragstafeln würde das bspw. bedeuten, dass neben den an der waldbaulichen Zielvorstellung der erstellenden Personen orientierten Behandlungsvariante auch eine Bestandesbehandlung der effizienten Flächen- und Kapitalallokation (,ökonomische Durchforstung“") ausgewiesen würde. Denn die Analysen der vorliegenden Arbeit verdeutlichen, dass zwischen den gegebenen waldbaulichen Behandlungsvarianten forstlicher Ertragstafeln diesbezüglich teils massive Unterschiede bestehen und es bleibt die Frage nach der nicht nur relativ, sondern absolut vorteilhaften Bestandesbehandlung.

Grundsätzlich böte die im dyn-ET-Projekt verwendete Erstellungsmethodik über einzelbaumbasierte Waldwachstumssimulationen die Möglichkeit zur Ausweisung einer solchen ökonomischen Behandlungsvariante, da alternative Zielvorstellungen implementierbar und vielfältige Nutzungskonzepte umsetzbar sind. Nötig wären allerdings zahlreiche Erweiterungen der verfügbaren Simulationsmodelle, wobei hier in erster Linie die Verknüpfung mit einem geeigneten Optimierungsalgorithmus zu nennen ist. Darüber hinaus bliebe natürlich stets zu klären, mit Blick auf welche Zielvorstellung und unter Einbeziehung welcher Prämissen das ökonomische Optimum definiert würde. Wie in den Analysen dieser Arbeit gezeigt, hat bspw. die verwendete Deckungsbeitragsfunktion und damit die unterstellte Entwicklung zukünftiger Holzerlöse und Holzerntekosten bedeutenden Einfluss auf die Bestandesbehandlung. Für das relevante Spannungsverhältnis zwischen den Deckungsbeiträgen niedrig- und hochdimensionierter Sortimente ist neben dem Produktionsziel der waldbewirtschaftenden Person somit auch die zukünftige Holzmarktentwicklung entscheidend. Darüber hinaus müssten Behandlungsvarianten unterschiedlicher Kapitalknappheit ausgegeben werden, da der Kalkulationszinssatz stets Ausdruck der individuellen alternativen Kapitalverwendungsmöglichkeiten der bewirtschaftenden Person ist. Nicht zuletzt bliebe mit Blick auf eine ökonomische Behandlungsvariante zu klären, in welcher Form welche Risiken einzubeziehen und welche Wechselwirkungen mit der Bestandesbehandlung zu berücksichtigen wären. 
Zur Darstellung einer Vielzahl von ziel- und prämissenspezifischen Behandlungsvarianten wären bspw. Bestandesbehandlungsdiagramme (SDMDs), wie sie in dieser Arbeit vorgestellt wurden, mit einem eingegrenzten Behandlungskorridor denkbar. Dieser könnte denkbare Extreme, bspw. eines sehr hohen Kalkulationszinssatzes ggü. einer zinsfreien Variante oder zweier unterschiedlich starker DB-Steigerungen, abbilden. Die waldbewirtschaftende Person könnte dann je nach Zielsetzung und berücksichtigten Prämissen einen individuellen Pfad innerhalb dieses Korridors wählen. 



\section{Zusammenfassung}

Die ökonomische Analyse forstlicher Bestandesbehandlung dient dem Verständnis einer effizienten Produktion des nachwachsenden Rohstoffs Holz in einer Welt knapper Ressourcen die vorliegende Arbeit strebt dafür in Anlehnung an Möhring (2009) einen Brückenschlag zwischen ökonomischer Theorie und forstlicher Praxis an. Im Sinne der normativen Entscheidungstheorie ist es übergeordnetes Ziel dieser Arbeit, rationale Entscheidungen im Rahmen der forstlichen Bestandesbehandlung sowie die ökonomischen Konsequenzen ausgewählter waldbaulicher Handlungsalternativen aufzuzeigen. Der resultierenden Komplexität wird mit zwei unterschiedlichen Ansätzen der Modellbildung begegnet, anhand derer die Teilziele der Arbeit in zwei unabhängigen, sich methodisch ergänzenden, Kapiteln bearbeitet werden.

Kapitel 2 analysiert die Ökonomie der forstlichen Bestandesbehandlung anhand eines theoretischen Analyserahmens. Ziel dieses Kapitels ist die numersiche Bestimmung der jeweils optimalen Bestandesbehandlung vor dem Hintergrund unterschiedlicher Modellprämissen. Grundlage bildet dabei ein stark vereinfachtes forstliches Produktionsmodell (Abschnitt 2.1), welches die numerische Bestimmung optimaler Bestandesbehandlungspfade ermöglicht. Ausgehend vom Fall der maximalen Volumenleistung (Abschnitt 2.2.1) wird die Modellkomplexität durch schrittweise Einführung von Prämissen erhöht und damit eine systematische Analyse der resultierenden Veränderungen von Zielfunktion und Optimalitätsbedingung ermöglicht. Es werden zunächst konstante (Abschnitt 2.2.2), dann zeit- (Abschnitt 2.2.3) und schließlich dimensionsabhängige (Abschnitt 2.2.4) Deckungsbeiträge zur Bewertung der Vor- und Endnutzungsvolumina eingeführt. Während konstante Deckungsbeiträge lediglich die Zielgröße, nicht aber die Bestandesbehandlung verändern, führen zeit- und dimensionsabhängige Deckungsbeiträge im Optimum zum Verzicht auf Bestandesvolumenzuwachs. Über Zeitpunkt und Ausprägung der Einbußen entscheidet dabei der spezifische Verlauf der Deckungsbeitragsfunktion. Ein mit der Zeit konstant steigender Deckungsbeitrag kann als negativer Kalkulationszinssatz interpretiert werden und führt zur Realisierung möglichst hoher Bestandesvolumina zu möglichst späten Zeitpunkten. Demgegenüber führen dimensionsabhängig steigende Deckungsbeiträge zur Förderung der mittleren Einzelbaumdimension durch verringerte Bestandesdichten in der Bestandesjugend - je höher das monetäre Spannungsverhältnis zwischen gering- und hochdimensionierten Sortimenten, desto geringer die Bestandesdichte und damit stärker die $\mathrm{Zu}$ wachslenkung auf weniger Einzelbäume. Gleichzeitig werden die Vorteile der höheren Einzelbaumdimensionen durch verlängerte Produktionszeiten umso länger ausgenutzt, je stärker die Deckungsbeitragssteigerung über der Einzelbaumdimension ausfällt. Die Berücksichtigung 
von Kapitalknappheit (Abschnitt 2.2.5) verwandelt die Zielgröße des durchschnittlichen Überschusses finanzmathematisch in eine Annuität und verkürzt die forstliche Produktion umso mehr, je höher der Kalkulationszinssatz angesetzt ist. Mit steigenden Kalkulationszinssätzen verringert sich bei dimensionsabhängigen Deckungsbeiträgen zudem die Förderung der Einzelbaumdimension und es werden mit zunehmendem Alter höhere Vornutzungsvolumina realisiert. Erntefixkosten (Abschnitt 2.2.6) verringern mit steigender Höhe zunehmend die Anzahl forstlicher Eingriffe und erhöhen dementsprechend die jeweiligen Vornutzungsvolumina. Mit der Einführung von Bestandesbegründungskosten (Abschnitt 2.2.7) wird die Zielgröße schließlich zur Brutto-Bodenrente und die Endnutzung erfolgt umso später, je höher die Kosten der Begründung eines Folgebestandes sind. Bei der Einbeziehung von Überlebensrisiken (Abschnitt 2.2.8) auf der Basis eines Erwartungswertes sinkt dieser umso mehr, je geringer die Überlebenswahrscheinlichkeit des Bestandes ist. Gleichzeitig wird mit dem Alter steigenden Ausfallraten (Altersrisiken) mit stärkeren Vornutzungen in hohen Bestandesaltern und früheren Endnutzungszeitpunkten begegnet. Etwas abgemildert wird dieser Risikoeffekt schließlich, wenn die Überlebensrisikostärke behandlungsabhängig definiert wird (Abschnitt 2.2.9). Dabei wird von einer stabilisierenden Wirkung höherer Einzelbaumvolumina ausgegangen - im Optimum werden diese durch verringerte Bestandesdichten in der Jugend erzielt.

Kapitel 3 analysiert forstliche Ertragstafeln, welche die Entwicklung von gleichaltrigen Waldbeständen als praxisnahe Wachstums- und Nutzungsmodelle abbilden. Ziel dieses Kapitels ist die ökonomische Bewertung sowie der Vergleich der in forstlichen Ertragstafeln ausgewiesenen waldbaulichen Behandlungsvarianten. Nach einem einleitenden Überblick über die Entstehungsgeschichte sowie die ertragskundlichen Grundbeziehungen und Anwendungsgebiete (Abschnitt 3.1) zeigt Abschnitt 3.2 die Ergebnisse einer Praxisbefragung zur gegenwärtigen und zukünftigen Nutzung forstlicher Ertragstafeln. Dem anhaltenden Bedarf der forstlichen Praxis nach konsistenten und langfristigen sowie transparenten und objektivierten Wachstumsund Nutzungsmodellen stehen quantitativ oftmals überholte aber zugleich etablierte Ertragstafelwerke gegenüber. Mit diesen Ergebnissen wird zur Motivation und Zielsetzung für das laufende Forschungsprojekt „Neue Generation dynamischer Ertragstafeln (dyn-ET)“ übergeleitet (Abschnitt 3.3.1). Die zum Abgabezeitpunkt dieser Arbeit zur Verfügung stehenden vorläufigen Ertragstafeldaten der Baumarten Buche (Fagus sylvatica L.) und Fichte (Picea abies (L.) H. Karst.) werden auf naturaler (Abschnitte 3.3.3 und 3.3.4) und ökonomisch bewerteter (Abschnitt 3.3.5) Ebene analysiert. Neben dem Vergleich zu etablierten Ertragstafelwerken liegt der Fokus insbesondere auf den Unterschieden zwischen den waldbaulichen Behandlungsvarianten (mäßige, starke und gestaffelte Hochdurchforstung). Es zeigen sich deutlich gesteigerte 
Ertragsniveaus ggü. den etablierten Ertragstafelwerken von Schober (1967) und Wiedemann (1936/42). Bei annähernd unveränderten Vorräten führen die gestiegenen Volumenzuwächse insbesondere zu höheren Vornutzungsvolumina, die aufgrund verringerter Stammzahlen zudem über teils deutlich gesteigerte mittlere Einzelbaumdurchmesser verfügen. In der Summe führen diese ertragskundlichen Veränderungen bei der Verwendung dimensionsabhängiger Deckungsbeiträge zu deutlich höheren monetären Werten. Im Vergleich der waldbaulichen Behandlungsvarianten zeichnet sich die mäßige Hochdurchforstung durch die höchste Gesamtwuchsleistung aus. Dafür steigen die mittleren Einzelbaumdurchmesser bei starker Hochdurchforstung aufgrund der verringerten Bestandesdichte in jungen Bestandesaltern und der damit verbundenen höheren Einzelbaumzuwächse. Die gestaffelte Hochdurchforstung der Buche kombiniert die Vorteile beider Behandlungsvarianten - sie erreicht einerseits ein relativ hohes Gesamtwuchsleistungsniveau und steigert andererseits die mittleren Einzelbaumdurchmesser insbesondere im ausscheidenden Bestand. Im Zuge der ökonomischen Bewertung profitiert die gestaffelte Hochdurchforstung von dieser Kombination, vermag es aber mit den gewählten Eingangsdaten nicht, die volumenleistungsbedingte Vorteilhaftigkeit der mäßigen Hochdurchforstung abzuwenden. Die Sensitivitätsanalysen in Abschnitt 3.3.6 zeigen darauf aufbauend, dass die in Kapitel 2 als relevant identifizierten Treiber (Deckungsbeitragsfunktion, Kalkulationszinssatz und Überlebensrisikostärke) die Wertrelationen zwischen den waldbaulichen Behandlungsvarianten verändern können - durch ausgewählte Kombination der Treiber kommt es zum Rangfolgenwechsel innerhalb realistischer Wertebereiche.

Die in dieser Arbeit durchgeführten ökonomischen Analysen verdeutlichen die Komplexität der forstlichen Bestandesbehandlung vor dem Hintergrund einer effizienten Produktion des nachwachsenden Rohstoffs Holz. Gleichzeitig zeigen sie aber auch, dass die Komplexität reduzierbar ist - Modelle können die Entscheidungsfindung prämissenspezifisch unterstützen und zur Zielerreichung beitragen. Es existiert dabei nicht die eine „richtige“ Bestandesbehandlung vielmehr sind alternative Bestandesbehandlungskonzepte in Abhängigkeit von der Zielsetzung und den bestehenden Prämissen stets individuell zu bewerten. Die vorliegende Arbeit liefert daher keinen optimalen Bestandesbehandlungspfad, sondern trägt zum Verständnis waldbaulich-ökonomischer Wechselwirkungen bei und verhilft waldbewirtschaftenden Personen somit zur adäquaten und zielorientierten Modellbildung. 



\section{Summary}

\section{Economic Analysis of Forest Stand Management}

The economic analysis of forest stand management serves the understanding of an efficient timber production in a world of scarce resources. Following Möhring (2009), the present study aims to build a bridge between economic theory and forest practice. In regard to normative decision theory, the overall aim of this study is to demonstrate rational decision making in forest management and highlight the economic consequences of selected silvicultural alternatives. The resulting complexity is met by two different approaches of modelling, where the sub-objectives of the thesis are dealt with in two independent, methodologically complementary, chapters.

Chapter 2 analyses the economics of forest stand management using a theoretical framework. The objective of this chapter is to numerically determine the optimal stand treatment considering different model assumptions. The analysis is based on a simplified forest production model (Section 2.1), which allows numerical determination of optimal stand treatments. Starting from the case of maximum total volume production (Section 2.2.1), the complexity of the model is increased by progressively adding assumptions, which enables a systematic analysis of the resulting changes in the objective function and optimal condition. Constant (Section 2.2.2), time-dependent (Section 2.2.3) and finally dimension-dependent (Section 2.2.4) net timber revenues are introduced to evaluate timber volumes from thinning and final harvest. While constant net timber revenues only change the target value, but not forest stand treatment, time- and dimension-dependent net timber revenues lead to abandoning stand volume increment at the optimum level. The specific characteristic of the net timber revenue function determines the time and extent of the losses. Net timber revenues that constantly increase over time can be interpreted as a negative interest rate and leads to achieving the highest possible stand volume at the latest possible point in time. On the other hand, dimension-dependent increasing net timber revenues lead to increased mean single-tree dimensions through reduced stand densities during youth. At the same time, the benefits from greater single-tree dimensions due to longer production periods are extended depending on the increase of the net timber revenues per single-tree dimension. The consideration of capital scarcity (Section 2.2.5) transforms the average net income into an annuity and shortens the forest production time the higher the interest rate is. With rising interest rates, the promotion of single-tree dimensions is also reduced in the case of dimension-dependent net timber revenues, and higher thinning volumes are harvested with increasing age. An increase in fixed harvesting costs (Section 2.2.6) reduces the number of 
thinnings while thinning volumes are increased respectively. By introducing planting costs (Section 2.2.7), the target value finally becomes the land rent and the higher the planting costs of a subsequent stand, the later the final-harvest takes place. If survival risks (Section 2.2.8) are included in the form of an expectation value, this value decreases as the probability of survival declines. At the same time, hazard rates that increase with age (old-age risks) are countered with stronger thinnings in older stands and an earlier final-harvest. This risk effect is somewhat mitigated if the survival risk level is defined as treatment-dependent (Section 2.2.9). In this context, a stabilizing effect of higher single-tree volumes is assumed - at the optimum, this is achieved by reducing stand densities in young stands.

Chapter 3 analyzes forest yield tables which illustrate the development of even-aged forest stands as practical growth and harvest models. The objective of this chapter is the economic evaluation and comparison of the silvicultural treatment alternatives shown in forest yield tables. Following an overview of the history of origin as well as the fundamental relationships and fields of application (Section 3.1), Section 3.2 presents the results of a survey on the current and future use of forest yield tables. The continued need in forest practice for consistent, long-term, transparent and objective growth and harvest models is contrasted by quantitatively outdated but at the same time established yield tables. These results reflect the motivation and objectives of the current research project "New generation of dynamic yield tables (dyn-ET)" (Section 3.3.1). The preliminary yield table data of the tree species European beech (Fagus sylvatica L.) and Norway spruce (Picea abies (L.) H. Karst.) are analyzed and evaluated on a natural (Sections 3.3.3 and 3.3.4) and monetary (Section 3.3.5) level. Besides comparing the results to established yield tables, the focus lies particularly on the differences between silvicultural treatment alternatives (moderate, heavy and graduated thinning from above). The results show a clear increase in yield levels compared to the established yield tables of Schober (1967) and Wiedemann (1936/42). With practically unchanged growing stocks, the increased volume increment leads to higher thinning volumes, which, due to a reduction in the number of stems, also partially show a significant increase in average single-tree diameters. All in all, these changes in the use of dimension-dependent net timber revenues lead to significantly higher monetary values. In comparing silvicultural treatment alternatives, moderate thinning from above is characterized by the highest total volume production. On the other hand, the mean single-tree diameters increase with heavy thinnings from above due to reduced stand density at a young stand age and the associated increased mean single-tree volume increment. Graduated thinning from above of European beech combines the advantages of both types of treatment on the one hand, a relatively high total volume production level is achieved, while on the other 
hand, the mean single-tree diameters are increased, especially in regard to the thinned timber. In the course of the economic evaluation, graduated thinning from above benefits from this combination, but with the selected input data it is not able to beat the advantages of moderate thinning from above due to the overall higher volume production. Based on this, the sensitivity analyses in Section 3.3.6 show that the relevant drivers identified in Chapter 2 (net timber revenue function, interest rate and survival risk level) can change the relations in value between the silvicultural treatment alternatives - a selected combination of drivers change the order of value within realistic ranges.

The economic analyses carried out in this thesis illustrate the complexity of forest stand management in the context of efficient timber production. At the same time, however, they also show that this complexity can be reduced - models can support decision making under varying assumptions and contribute to the achievement of objectives. There is not only one "right" way to treat a stand - alternative stand treatment concepts must always be evaluated individually depending on the objectives and existing assumptions. The present study therefore does not provide an instruction for optimal stand treatments, but contributes to the understanding of silvicultural-economic interactions and therefore helps forest managers to develop adequate and objective-oriented models. 



\section{Literaturverzeichnis}

Abetz, Karl (1929): Gedanken zu Theorie und Praxis der forstlichen Rentabilitätslehre. In: Allg. Forst- u. J.-Ztg. (Allgemeine Forst- und Jagdzeitung) 105, S. 287-298.

Adams, Darius M.; Ek, Alan R. (1974): Optimizing the Management of Uneven-aged Forest Stands. In: Can. J. For. Res. 4 (3), S. 274-287. DOI: 10.1139/x74-041.

Aiba, Yoshinori (1975a): Effects of cultural system on the stand growth of Sugi-plantation (Cryptomeria japonica) (II). In: J. Jpn. For. Soc. 57 (2), S. 39-44. DOI: 10.11519/jjfs1953.57.2_39.

Aiba, Yoshinori (1975b): Effects of cultural system on the stand growth of Sugi-plantation (Cryptomeria japonica) (III). In: J. Jpn. For. Soc. 57 (3), S. 67-73. DOI: 10.11519/jjfs1953.57.3_67.

Albach, Horst (1962): Investition und Liquidität: die Planung des optimalen Investitionsbudgets. Wiesbaden: Betriebswirtschaftllicher Verlag Dr. Th. Gabler.

Albert, M.; Schmidt, M. (2010): Climate-sensitive modelling of site-productivity relationships for Norway spruce (Picea abies (L.) Karst.) and common beech (Fagus sylvatica L.). In: For. Ecol. Manage. 259 (4), S. 739-749. DOI: 10.1016/j.foreco.2009.04.039.

Albert, Matthias; Hansen, Jan; Nagel, Jürgen; Schmidt, Matthias; Spellmann, Hermann (2015): Assessing risks and uncertainties in forest dynamics under different management scenarios and climate change. In: Forest Ecosystems 2 (14). DOI: 10.1186/s40663-015-0036-5.

Albert, Matthias; Nagel, Jürgen; Nagel, Ralf-Volker; Spellmann, Hermann (2019): Eine neue Generation dynamischer Ertragstafeln für Nordwestdeutschland - erste Ergebnisse für die Baumart Buche. In: DVFFA (Hg.): Jahrestagung 2019. Stralsund. Deutscher Verband Forstlicher Versuchs- uns Forschungsanstalten, S. 21-30.

Albert, Matthias; Nagel, Ralf-Volker; Nuske, Robert S.; Sutmöller, Johannes; Spellmann, Hermann (2017): Tree Species Selection in the Face of Drought Risk-Uncertainty in Forest Planning. In: Forests 8 (10), S. 363. DOI: 10.3390/f8100363.

Albrecht, Axel (2009): Sturmschadensanalyse langfristiger waldwachstumskundlicher Versuchsflächendaten in Baden-Württemberg. Inaugural-Dissertation zur Erlangung der Doktorwürde der Fakultät für Forst- und Umweltwissenschaften der Albert-Ludwigs-Universität Freiburg i. Brsg., zuletzt geprüft am 16.05.2018.

Albrecht, Axel; Hanewinkel, Marc; Bauhus, Jürgen; Kohnle, Ulrich (2012): How does silviculture affect storm damage in forests of south-western Germany? Results from empirical modeling based on long-term observations. In: European Journal of Forest Research 131 (1), S. 229-247. DOI: 10.1007/s10342-010-0432-x.

Albrecht, Axel; Kohnle, Ulrich; Hanewinkel, Marc; Bauhus, Jürgen (2013): Storm damage of Douglas-fir unexpectedly high compared to Norway spruce. In: Ann. For. Sci. 70 (2), S. 195207. 
Albrecht, Axel; Lenk, E.; Rose, Bernd; Kohnle, Ulrich (2017): Effekte von Jungbestandspflege in baumzahlreichen Fichtenverjüngungen. In: Forstarchiv 88, S. 79-90.

Amacher, Gregory S.; Ollikainen, Markku; Koskela, Erkki (2009): Economics of Forest Resources. Cambridge, Massachusetts: The MIT Press.

Ammer, Christian (2016): Starkholz-wertvolles Instrument der waldbaulichen Steuerung. In: Allgemeine Forstzeitschrift/Der Wald 71 (19), S. 33-36.

Ammer, Christian; Puettmann, Klaus J. (2009): Waldbau, quo vadis? - Waldbewirtschaftung zwischen Funktionenorientierung und Multifunktionalität. In: Forstarchiv 80, S. 90-96. Online verfügbar unter https://www.researchgate.net/publication/255881142_Waldbau_quo_vadis_-_Waldbewirtschaftung_zwischen_Funktionenorientierung_und_Multifunktionalitat.

Ammer, Christian; Schall, Peter; Gossner, Martin M.; Boch, Steffen; Prati, Daniel; Blaser, Stefan; Fischer, Markus (2017): Waldbewirtschaftung und Biodiversität: Vielfalt ist gefragt. In: AFZ-DerWald (17), S. 20-25. DOI: 10.7892/BORIS.106387.

Ando, T. (1962): Growth analysis on the natural stands of Japanese red pine (Pinus densiflora Sieb. et. Zucc.), II : analysis of stand density and growth. In: Bulletin of the forestry experimental station of Japan 147.

Assmann, Ernst (1959): Höhenbonität und wirkliche Ertragsleistung. In: Forstwiss. Centralbl. 78 (1/2), S. 1-64, zuletzt geprüft am 06.10.18.

Assmann, Ernst (1961): Waldertragskunde. Organische Produktion, Struktur, Zuwachs und Ertrag von Waldbeständen. München: BLV Verlagsgesellschaft.

Assmann, Ernst; Franz, Friedrich (1963): Vorläufige Fichten-Ertragstafel für Bayern. Autoren-Referat: Institut für Ertragskunde der Forstlichen Versuchsanstalt München.

Audet, Charles; Le Digabel, Sébastien; Tribes, Christophe (2009): NOMAD User Guide: Technical Report G-2009-37. Hg. v. Les cahiers du Gerard. Gerard. Montreal.

Bachmann, Peter (1990): Forsteinrichtung als Mittel der Betriebsführung. In: Schweiz. Z. Forstwes. 141 (6), S. 415-430.

Bamberg, Günter; Coenenberg, Adolf Gerhard; Krapp, Michael (2019): Betriebswirtschaftliche Entscheidungslehre. 16. Auflage. München: Vahlen Franz (Vahlens Kurzlehrbücher).

Barrio Anta, M.; Álvarez González, J. G. (2005): Development of a stand density management diagram for even-aged pedunculate oak stands and its use in designing thinning schedules. In: Forestry (Lond) 78 (3), S. 209-216. DOI: 10.1093/forestry/cpi033.

Baur, Franz von (1876): Die Fichte in Bezug auf Ertrag, Zuwachs und Form. Berlin.

Baur, Franz von (1881): Die Rotbuche in Bezug auf Ertrag, Zuwachs und Form. Berlin.

Bay. StMELF (1981): Hilfstafeln für die Forsteinrichtung. Zusammengestellt für den Gebrauch in der Bayerischen Staatsforstverwaltung. München: Staatsministerium für Ernährung, Landwirtschaft und Forsten. 
BaySF (2009): Richtlinie „Bewirtschaftung von Fichten- und Fichtenmischbeständen im Bayerischen Staatswald“. Unter Mitarbeit von Rudolf Habereder, Markus Neufanger, Reinhard Schröpfer, Heinz Utschig, Thomas Zanker und Daniel Zippert. Hg. v. Bayerische Staatsforsten. Regensburg (Waldbauhandbuch Bayerische Staatsforsten). Online verfügbar unter https://www.baysf.de/de/wald-verstehen/waldbau.html, zuletzt geprüft am 24.03.2020.

Beinhofer, Bernhard (2009): Zur Anwendung der Portfoliotheorie in der Forstwissenschaft. Finanzielle Optimierungsansätze zur Bewertung von Diversifikationseffekten. Dissertation. Technische Universität München, München. Fachgebiet für Waldinventur und nachhaltige Nutzung.

Bergel, Dieter (1985): Ertragstafel für die Baumart Douglasie (mäßige und starke Durchforstung). In: Schober, R. (Hg.): Ertragstafeln wichtiger Baumarten bei verschiedener Durchforstung. 4. Aufl. Frankfurt am Main: Sauerländer 1995.

Bergen, Volker; Löwenstein, Wilhelm; Olschewski, Roland (2013): Forstökonomie. Volkswirtschaftliche Ansätze für eine vernünftige Umwelt- und Landnutzung. 2., überarb. und erw. Aufl. München: Verlag Franz Vahlen (Vahlens Handbücher der Wirtschafts- und Sozialwissenschaften).

Betters, David R.; Steinkamp, Eric A.; Turner, Matthew T. (1991): Singular path solutions and optimal rates for thinning even-aged forest stands. In: For. Sci. 37 (6), S. 1632-1640.

Bitterlich, Walter (1949): Die Winkelzählprobe. Dissertation, Wien.

Blume (1949): Taschenhandbuch der Forstwirtschaft. Hannover.

Böckmann, Thomas (2016): Warum sind Betriebsinventuren für die forstliche Praxis wichtig? In: Forstarchiv 87, S. 31-37.

Bodelschwingh, Hilmar von (2018): Ökonomische Potentiale von Waldbeständen. Konzeption und Abschätzung im Rahmen einer Fallstudie in hessischen Staatswaldflächen. Bad Orb: J.D. Sauerländer's Verlag (Schriften zur Forst- und Umweltökonomie, 47).

Bolte, Andreas; Ammer, Christian; Löf, Magnus; Madsen, Palle; Nabuurs, Gert-Jan; Schall, Peter et al. (2009): Adaptive forest management in central Europe: Climate change impacts, strategies and integrative concept. In: Scandinavian Journal of Forest Research 24 (6), S. 473-482. DOI: 10.1080/02827580903418224.

Bonnemann, Alfred (1939): Der gleichaltrige Mischbestand von Kiefer und Buche. In: Mitteilungen aus der Forstwirtschaft und Forstwissenschaft (5), S. 439-483.

Bonnemann, Alfred (1956): Eichen-Buchen-Mischbestände. In: Allg. Forst- u. J.-Ztg. (Allgemeine Forst- und Jagdzeitung) 127, S. 118-126.

Borchert, Herbert (2002): The economically optimal amount of timber cut in forests. An approach by control theory. Zugl.: München, Techn. Univ., Diss. Frankfurt am Main: J.D. Sauerländer's Verlag (Schriften zur Forstökonomie, 24).

Bösch, Bernhard (2002): Neue Bonitierungs- und Zuwachshilfen. In: Schriftenreihe Freiburger Forstliche Forschung 18. Online verfügbar unter https://www.waldwissen.net/technik/inventur/fva_schaetzhilfen/fva_schaetzhilfen_1.pdf, zuletzt geprüft am 27.02.2020. 
Bradley, R. T.; Christie, J. M.; Johnston, D. R. (1966): Forest management tabels. London (Forestry Commission booklet/Großbritannien /Forestry Commission).

Brandl, Susanne; Falk, Wolfgang (2019): Mortalität der Fichte und Buche. Einfluss von Klima und Mischung. In: AFZ-DerWald 74 (2), S. 10-13.

Brandl, Susanne; Paul, Carola; Knoke, Thomas; Falk, Wolfgang (2020): The influence of climate and management on survival probability for Germany's most important tree species. In: Forest Ecology and Management 458, S. 117652. DOI: 10.1016/j.foreco.2019.117652.

Bruce, David; DeMars, Donald J.; Reukema, Donald L. (1977): Douglas-fir Managed Yield Simulator: DFIT User's Guide. USDA Forest and Range Exp Station. Portland (General Technical Report PNW-57).

BWaldG (1975): Gesetz zur Erhaltung des Waldes und zur Förderung der Forstwirtschaft. Bundeswaldgesetz vom 2. Mai 1975 (BGBl. I S. 1037), das zuletzt durch Artikel 1 des Gesetzes vom 17. Januar 2017 (BGB1. I S. 75) geändert worden ist.

Cao, Tianjian; Hyytiäinen, Kari; Tahvonen, Olli; Valsta, Lauri (2006): Effects of initial stand states on optimal thinning regime and rotation of Picea abies stands. In: Scand. J. For. Res. (Scandinavian Journal of Forest Research) 21 (5), S. 388-398. DOI:

10.1080/02827580600951915.

Carlowitz, Hanns Carl von (1713; 2009): Sylvicultura Oeconomica oder haußwirthliche Nachricht und Naturmäßige Anweisung zur Wilden Baum-Zucht. Reprint der zweiten Auflage 1732. Leipzig: Verlag Kessel.

Castaño-Santamaría, J.; Barrio-Anta, M.; Álvarez-Álvarez, P. (2013): Regional-scale stand density management diagrams for Pyrenean oak (Quercus pyrenaica Willd.) stands in northwest Spain. In: iForest 6 (3), S. 113-122. DOI: 10.3832/ifor0880-006.

Cawrse, David C.; Betters, David R.; Kent, Brian M. (1984): A variational solution technique for determining optimal thinning and rotational schedules. In: For. Sci. 30 (3), S. 793-802.

Chang, Sun Joseph (1981): Determination of the Optimal Growing Stock and Cutting Cycle for an Uneven-Aged Stand. In: for sci 27 (4), S. 739-744. DOI: 10.1093/forestscience/27.4.739.

Chang, Sun Joseph (1983): Rotation age, management intensity, and the economic factors of timber production. Do changes in stumpage price, interest rate, regeneration cost, and forest taxation matter? In: For. Sci. 29 (2), S. 267-277.

Chang, Sun Joseph (1984): Determination of the optimal rotation age: A theoretical analysis. In: For. Ecol. Manage. 8 (2), S. 137-147. DOI: 10.1016/0378-1127(84)90031-8.

Chang, Sun Joseph (1998): A generalized Faustmann model for the determination of optimal harvest age. In: Can. J. For. Res. 28 (5), S. 652-659. DOI: 10.1139/x98-017.

Chang, Sun Joseph; Deegen, Peter (2011): Pressler's indicator rate formula as a guide for forest management. In: Journal of Forest Economics 17 (3), S. 258-266. DOI:

10.1016/j.jfe.2011.04.002. 
Chen, Chau-Nan; Tsaur, Tien-Wang; Rhai, Tong-Shieng (1982): The Gini Coefficient and Negative Income. In: Oxford Economic Papers 34 (3), S. 473-478. Online verfügbar unter www.jstor.org/stable/2662589.

Clark, Colin W.; Pree, John D. de (1979): A simple linear model for the optimal exploitation of renewable resources. In: Appl. Math. Optim. (Applied Mathematics and Optimization) 5 (1), S. 181-196. DOI: 10.1007/BF01442553.

Clark, Colin Whitcomb (2010): Mathematical Bioeconomics. The Mathematics of Conservation. 3. ed. Hoboken, NJ: Wiley (Pure and applied mathematics, 91).

Clarke, Harry R.; Reed, William J. (1989): The tree-cutting problem in a stochastic environment: The case of age-dependent growth. In: Journal of Economic Dynamics and Control 13 (4), S. 569-595. DOI: 10.1016/0165-1889(89)90004-3.

Coordes, Renke (2013): Influence of planting density and rotation age on the profitability of timber production for Norway spruce in Central Europe. In: Eur. J. For. Res. 132 (2), S. $297-$ 311. DOI: $10.1007 / \mathrm{s} 10342-012-0675-9$.

Coordes, Renke (2014a): Optimal Thinning within the Faustmann Approach. Wiesbaden: Springer-Verlag GmbH.

Coordes, Renke (2014b): Thinnings as Unequal Harvest Ages in Even-Aged Forest Stands. In: For. Sci. 60 (4), S. 677-690. DOI: 10.5849/forsci.13-004.

Coordes, Renke (2014c): Waldbau und die Faustmann-Theorie. In: Schweiz. Z. Forstwes. 165 (10), S. 321-328. DOI: 10.3188/szf.2014.0321.

Coordes, Renke (2016): The emergence of forest age structures as determined by uneven-aged stands and age class forests. In: J. For. Econ. 25, S. 160-179. DOI: 10.1016/j.jfe.2016.09.003. Cotta, Heinrich (1821): Hülfstafeln für Forstwirthe und Forsttaxatoren. Dresden.

Curtis, R. O.; Clendenen, G. W.; Demars, D. J. (1981): A new stand simulator for coast Douglas-fir: DFSIM user's guide. USDA Forest and Range Exp Station. Portland (General Technical Report PNW-128).

Curtis, Robert O. (1982): A Simple Index of Stand Density for Douglas-fir. In: for sci 28 (1), S. 92-94. DOI: 10.1093/forestscience/28.1.92.

Deegen, Peter (1997): Forstökonomie kennenlernen. Eine Einführung in die Ressourcenökonomie für das Ökosystem Wald. Dresden: Taupitz Bogenschützen Verl.

Deegen, Peter; Matolepszy, Kai (2015): Economic balancing of forest management under storm risk, the case of the Ore Mountains (Germany). In: J. For. Econ. 21 (1), S. 1-13. DOI: 10.1016/j.jfe.2014.10.005.

Degenhardt, Annett (2011): Durchforstung nach Zahlen. Optimale Behandlungsstrategien auf Basis heuristischer Verfahren. In: Deutscher Verband Forstlicher Versuchsanstalten, Sektion Ertragskunde (Hg.): Tagungsband zur Jahrestagung, S. 11-20.

Detten, Roderich von (2018): Forstwirtschaft oder die Strategie des «muddling-through» (Essay). In: Schweiz. Z. Forstwes. 169 (4), S. 189-193. DOI: 10.3188/szf.2018.0189. 
Dichtl, Erwin; Issing, Otmar (Hg.) (1987): Vahlens großes Wirtschaftslexikon. In 4 Bänden. München: Beck (dtv, 59006).

Dieter, Matthias (2001): Land expectation values for spruce and beech calculated with Monte Carlo modelling techniques. In: For. Policy Econ. 2 (2), S. 157-166. DOI: 10.1016/S13899341(01)00045-4.

Dobor, Laura; Hlásny, Tomáš; Rammer, Werner; Zimová, Soňa; Barka, Ivan; Seidl, Rupert (2020): Is salvage logging effectively dampening bark beetle outbreaks and preserving forest carbon stocks? In: Journal of Applied Ecology 57 (1), S. 67-76.

Donis, Janis; Kitenberga, Mara; Snepsts, Guntars; Dubrovskis, Edgars; Jansons, Aris (2018): Factors affecting windstorm damage at the stand level in hemiboreal forests in Latvia: case study of 2005 winter storm. In: Silva Fennica 52 (4).

Dorfman, Robert (1979): A Formula for the Gini Coefficient. In: The Review of Economics and Statistics 61 (1), S. 146. DOI: 10.2307/1924845.

Dragicevic, Arnaud; Lobianco, Antonello; Lebois, Antoine (2016): Forest planning and productivity-risk trade-off through the Markowitz mean-variance model. In: For. Policy Econ. 64, S. 25-34. DOI: 10.1016/j.forpol.2015.12.010.

Drew, T. John; Flewelling, James W. (1977): Some Recent Japanese Theories of Yield-Density Relationships and their Application to Monterey Pine Plantations. In: for sci 23 (4), S. 517-534. DOI: 10.1093/forestscience/23.4.517.

Drew, T. John; Flewelling, James W. (1979): Stand Density Management: an Alternative Approach and Its Application to Douglas-fir Plantations. In: for sci 25 (3), S. 518-532. DOI: 10.1093/forestscience/25.3.518.

dyn-ET (unveröff.): Neue Generation dynamischer Ertragstafeln: Vorläufige Projektdaten der Nordwestdeutschen Forstlichen Versuchsanstalt (NW-FVA) für die Baumarten Buche und Fichte. Göttingen.

dyn-ET (2020): Neue generation dynamischer Ertragstafeln: Projektzwischenbericht 2019. Nordwestdeutsche Forstliche Versuchsanstalt (NW-FVA); Abteilung Forstökonomie der Georg-August-Universität Göttingen. Göttingen.

Eichhorn, Fritz (1902): Ertragstafeln für die Weißtanne. Berlin.

Eichhorn, Fritz (1904): Beziehungen zwischen Bestandshöhe und Bestandsmasse. In: Allg. Forst- u. J.-Ztg. (Allgemeine Forst- und Jagdzeitung) 80, S. 45-49.

Englert, Hermann; Rosenkranz, Lydia; Seintsch, Björn (2018): Abschätzung des nicht verwerteten Derbholzes. In: AFZ-DerWald (23), S. 14-16.

Eriksson, Ljusk Ola (1999): The Faustmann rotation with thinning and economies of scale. In: Scandinavian Forest Economics, S. 6.

Erteld, Werner (1951): Die Robinie und ihr Holz. Berlin: Deutscher Bauernverlag. 
Ertle, Christoph; Knoche, Dirk; Wenk, Günter (2012): Ertragstafel der Gemeinen Kiefer (Pinus sylvestris L.) für Kippenstandorte des Lausitzer Braunkohlenreviers. 1. Aufl. Finsterwalde: Forschungsinstitut für Bergbaufolgelandschaften (Schriftenreihe des Forschungsinstituts für Bergbaufolgelandschaften).

Faccoli, Massimo; Stergulc, Fabio (2008): Damage reduction and performance of mass trapping devices for forest protection against the spruce bark beetle, Ips typographus (Coleoptera Curculionidae Scolytinae). In: Ann. For. Sci. 65 (3), S. 309. DOI: 10.1051/forest:2008010.

Fahrmeir, Ludwig; Kneib, Thomas; Lang, Stefan; Marx, Brian (2013): Regression. Models, Methods and Applications. Dordrecht: Springer-Verlag GmbH.

Fahrmeir, Ludwig; Künstler, Rita; Pigeot, Iris; Tutz, Gerhard; Heumann, Ludwig (2011): Statistik. Der Weg zur Datenanalyse. 7. Auflage, korrigierter Nachdruck. Berlin, Heidelberg: Springer-Verlag GmbH (Springer-Lehrbuch).

Faustmann, Martin (1849; 1995): Berechnung des Werthes, welchen Waldboden, sowie noch nicht haubare Holzbestände für die Waldwirthschaft besitzen. In: Allg. Forst- Jagdztg. 15, 441-455. Translated by: Gane, M. and Linnard, W. (1995). Calculation of the Value which Forest Land and Immature Stands Possess for Forestry. Journal of Forest Economics, 1: 7-44.

Fibich, Jonathan (2019): Vergleich verschiedener Fichtenertragstafeln anhand ertragskundlicher und ökonomischer Kenngrößen. Bachelorarbeit. Georg-August-Universität, Fakultät für Forstwissenschaften und Waldökologie, Abteilung Forstökonomie, Göttingen.

ForstBW (2014): Richtlinie landesweiter Waldentwicklungstypen. Unter Mitarbeit von Gabriele Wicht-Lückge, Gudrun Biewald, Christoph Göckel, Armin Jacob, Michael Kilian, Ulrich Kohnle et al. Hg. v. Landesbetrieb Forst Baden-Württemberg, Ministerium für Ländlichen Raum und Verbraucherschutz Baden-Württemberg. Stuttgart (ForstBW Praxis). Online verfügbar unter www.forstbw.de, zuletzt geprüft am 24.03.2020.

Forster, Otto (2013): Analysis 1. 11. Aufl. Wiesbaden: Springer-Verlag GmbH.

Franz, Friedrich (1968): Das EDV-Programm STAOET - zur Herleitung mehrgliedirger Standort-Leistungstafeln. Unveröffentlichtes Manuskript. München.

Franz, Friedrich; Utschig, Heinz (Hg.) (1990): Ernst Assmanns Wirken in München. Ein Rückblick zu seinem zehnten Todestag. Gedenksymposium anlässlich des zehnten Todestages von Professor Ernst Assmann. München. Lehrstuhl für Wachstumskunde der Technischen Universität München.

Fuchs, Alfred; Kennel, Eckhard (1994): Erste Wiederholungsaufnahme einer permanenten Betriebsinventur im bayerischen Staatswald. In: Forstw. Cbl. 113, S. 224-235.

Fuchs, Jasper (2018): Ökonomie der Einzelbaumentnahme. Masterarbeit. Georg-August-Universität Göttingen, Göttingen. Fakultät für Forstwissenschaften und Waldökologie, zuletzt geprüft am 07.09.2018.

Gadow, Klaus von (2003): Waldstruktur und Waldwachstum. Beilage zur Vorlesung im Wintersemester 2003/2004. Universität Göttingen, zuletzt geprüft am 18.10.2018. 
Gadow, Klaus von (2005): Forsteinrichtung. Analyse und Entwurf der Waldentwicklung. Göttingen: Universitätsdrucke Göttingen, zuletzt geprüft am 22.05.2018.

Ganghofer, August von (Hg.) (1881): Das Forstliche Versuchswesen. Augsburg: Schmid'sche Buchhandlung.

Gastwirth, Joseph L. (1971): A General Definition of the Lorenz Curve. In: Econometrica 39 (6), S. 1037. DOI: 10.2307/1909675.

Gehrhardt, Ernst (1909): Ueber Bestandes-Wachstumsgesetze und ihre Anwendung zur Aufstellung von Ertragstafeln. In: Allg. Forst- u. J.-Ztg. (Allgemeine Forst- und Jagdzeitung) (85), S. 117-128.

Gehrhardt, Ernst (1923): Ertragstafeln für Eiche, Buche, Tanne, Fichte und Kiefer. Berlin: Springer.

Gehrhardt, Ernst (1930): Ertragstafeln für reine und gleichartige Hochwaldbestände von Eiche, Buche, Tanne, Fichte, Kiefer, grüner Douglafie und Lärche. Berlin: Springer-Verlag $\mathrm{GmbH}$.

Gerold, Denie; Gerold, Dorothea (2013): Ertragstafeln und/oder Wachstumsmodelle. Chancen und Risiken für den Forstsachverständigen. In: Wertermittlungsforum (3), 90-101.

Gerst, Johannes (2015): Der Grenzzins als Entscheidungskriterium in der Forstwirtschaft. Methodisches Konzept und empirische Evidenz. Zugl.: Göttingen, Univ., Diss., 2015. Bad Orb:

J.D. Sauerländer's Verlag (Schriften zur Forst- und Umweltökonomie, 41).

Getz, Wayne M.; Haight, Robert G. (1989): Population harvesting: demographic models of fish, forest, and animal resources: Princeton University Press.

Griess, Verena C.; Knoke, Thomas (2013): Bioeconomic modeling of mixed Norway spruce-European beech stands. Economic consequences of considering ecological effects. In: European Journal of Forest Research 132 (3), S. 511-522. DOI: 10.1007/s10342-0130692-3.

Grodzki, Wojciech; Jakuš, Rastislav; Lajzová, Eva; Sitková, Zuzana; Maczka, Tomasz; Škvarenina, Jaroslav (2006): Effects of intensive versus no management strategies during an outbreak of the bark beetle Ips typographus (L.) (Col.: Curculionidae, Scolytinae) in the Tatra Mts. in Poland and Slovakia. In: Ann. For. Sci. 63 (1), S. 55-61. DOI: 10.1051/forest:2005097.

Groß, Volker von (2019): Auswirkungen der Anwendung einer neuen Generation forstlicher Ertragstafeln auf betriebliche Planungs- und Entscheidungsgrößen. Masterarbeit. Georg-August-Universität Göttingen, Göttingen. Fakultät für Forstwissenschaften und Waldökologie.

Guttenberg, Adolf Ritter von (1915): Wachstum und Ertrag der Fichte im Hochgebirge. Wien, Leipzig: Deuticke.

Haarsma, Reindert J.; Hazeleger, Wilco; Severijns, Camiel; Vries, Hylke de; Sterl, Andreas; Bintanja, Richard et al. (2013): More hurricanes to hit western Europe due to global warming. In: Geophys. Res. Lett. 40 (9), S. 1783-1788. DOI: 10.1002/grl.50360. 
Hahn, W. Andreas; Härtl, Fabian; Irland, Lloyd C.; Kohler, Christoph; Moshammer, Ralf; Knoke, Thomas (2014): Financially optimized management planning under risk aversion results in even-flow sustained timber yield. In: For. Policy Econ. 42, S. 30-41. DOI: 10.1016/j.forpol.2014.02.002.

Haight, Robert G. (1985): A Comparison of Dynamic and Static Economic Models of Uneven-Aged Stand Management. In: for sci 31 (4), S. 957-974. DOI: 10.1093/forestscience/31.4.957.

Haight, Robert G.; Monserud, Robert A. (1990): Optimizing Any-Aged Management of Mixed-Species Stands: II. Effects of Decision Criteria. In: for sci 36 (1), S. 125-144. DOI: 10.1093/forestscience/36.1.125.

Halbritter, Andreas (2015): An economic analysis of double-cohort forest resources. In: Journal of Forest Economics 21 (1), S. 14-31. DOI: 10.1016/j.jfe.2014.11.001.

Halbritter, Andreas (2020): An economic analysis of thinning intensity and thinning type of a two-tiered even-aged Forest stand. In: For. Policy Econ. 111, S. 102054. DOI: 10.1016/j.forpol.2019.102054.

Halbritter, Andreas; Deegen, Peter (2015): A combined economic analysis of optimal planting density, thinning and rotation for an even-aged forest stand. In: For. Policy Econ. 51, S. 3846. DOI: 10.1016/j.forpol.2014.10.006.

Hanewinkel, M. (2002): Comparative economic investigations of even-aged and uneven-aged silvicultural systems: a critical analysis of different methods. In: Forestry 75 (4), S. 473-481. DOI: $10.1093 /$ forestry/75.4.473.

Hanewinkel, Marc; Cullmann, Dominik A.; Schelhaas, Mart-Jan; Nabuurs, Gert-Jan; Zimmermann, Niklaus E. (2013): Climate change may cause severe loss in the economic value of European forest land. In: Nat. Clim. Change 3 (3), S. 203. DOI: 10.1038/nclimate1687.

Hansen, Jan; Nagel, Jürgen (2014): Waldwachstumskundliche Softwaresysteme auf Basis von TreeGrOSS. Anwendung und theoretische Grundlagen. Göttingen: Universitätsverlag Göttingen (Beiträge aus der Nordwestdeutschen Forstlichen Versuchsanstalt, 11).

Hartig, Theodor (1847): Vergleichende Untersuchungen über den Ertrag der Rotbuche im Hoch- und Pflanz-Walde, im Mittel- und Niederwald-Betriebe. nebst Anleit. z. vergleichenden Ertragsforschungen. Berlin: Verlag von Albert Förstner.

Härtl, Fabian; Barka, Ivan; Hahn, W. Andreas; Hlásny, Tomáš; Irauschek, Florian; Knoke, Thomas et al. (2016): Multifunctionality in European mountain forests - an optimization under changing climatic conditions. In: Can. J. For. Res. 46 (2), S. 163-171. DOI: 10.1139/cjfr2015-0264.

Härtl, Fabian; Hahn, W. Andreas; Knoke, Thomas (2010): Integrating neighbourhood effects in the calculation of optimal final tree diameters. In: Journal of Forest Economics 16 (3), S. 179-193. DOI: 10.1016/j.jfe.2009.11.003.

Härtl, Fabian; Langhammer, Peter; Knoke, Thomas (2018): Strategien zur Minimierung von Opportunitätskosten der Totholzbereitstellung. In: Schweiz. Z. Forstwes. 169 (1), S. 9-17. DOI: $10.3188 /$ szf.2018.0009. 
Hartman, Richard (1976): The Harvesting Decision when a standing Forest has Value. In: Economic Inquiry 14 (1), S. 52-58.

Hasel, Karl; Schwartz, Ekkehard (2006): Forstgeschichte. Ein Grundriß für Studium und Praxis. 3., erw. und verb. Aufl. Remagen: Kessel.

Haub, Hartmut; Weimann, Hans-Joachim (2000): Neue Alterswertfaktoren der Bewertungsrichtlinien. In: AFZ-DerWald (22), S. 1194-1198.

Hechtner, F.; Kruschwitz, Lutz; Löffler, Andreas; Möllmann, Torsten Bernhard (2015): Faustmann und der Einfluss von Steuern. In: Allg. Forst- u. J.-Ztg. (Allgemeine Forst- und Jagdzeitung) 186 (5/6), S. 116-124.

Henningsen, Arne; Hamann, Jeff D. (2007): systemfit: A Package for Estimating Systems of Simultaneous Equations in R. In: Journal of Statistical Software 23 (4), S. 1-40, zuletzt geprüft am 29.11.2019.

Hering, Thomas (2017): Investitionstheorie. 5., überarbeitete und aktualisierte Auflage. Berlin, Boston: De Gruyter (Lehr- und Handbücher der Wirtschaftswissenschaft).

Heshmatol Vaezin, S. M.; Peyron, Jean-Luc; Lecocq, Franck (2009): A simple generalization of the Faustmann formula to tree level. In: Can. J. For. Res. 39 (4), S. 699-711. DOI:

10.1139/X08-202.

Hickler, Thomas; Bolte, Andreas; Beierkuhnlein, Carl; Blaschke, Markus; Blick, Theo; Brüggemann, Wolfgang et al. (2014): Folgen des Klimawandels für die Biodiversität in Wald und Forst. In: Volker Mosbrugger, Guy Brasseur, Michaela Schaller und Bernhard Stribrny (Hg.): Klimawandel und Biodiversität - Folgen für Deutschland. 2., unveränd. Aufl. Darmstadt: WBG Wiss. Buchges, S. 164-221.

Hijmans, Robert J.; Cameron, Susan E.; Parra, Juan L.; Jones, Peter G.; Jarvis, Andy (2005): Very high resolution interpolated climate surfaces for global land areas. In: Int. J. Climatol. 25 (15), S. 1965-1978. DOI: 10.1002/joc.1276.

Hilf, Hubert Hugo (1928): Die Arbeitsleistung im Hauungsbetrieb. Unveröffentlichte Akte. Insitut für forstliche Arbeitswissenschaft. Eberswalde.

Hilf, Hubert Hugo (1941): Die Erforschung und Verbesserung der Waldarbeit. Zwei Vorträge an der Forstlichen Hochschule Stockholm. Hannover: M. u. H. Schaper.

HMUKLV (2018): Richtlinie für die Bewirtschaftung des Staatswaldes. RiBeS 2018. Hg. v. Hessisches Ministerium für Umwelt, Klimaschutz, Landwirtschaft und Verbraucherschutz. Wiesbaden. Online verfügbar unter www.hessen-forst.de, zuletzt geprüft am 03.04.2020.

HMULF (2002): Hessische Anweisung für Forsteinrichtungsarbeiten, HAFEA, vom 21.08.2002. In: Staatsanzeiger für das Land Hessen (Hessisches Miniterium für Umwelt, Landwirtschaft und Forsten).

Höwler, Kirsten; Annighöfer, Peter; Ammer, Christian; Seidel, Dominik (2017): Competition improves quality-related external stem characteristics of Fagus sylvatica. In: Can. J. For. Res. 47 (12), S. 1603-1613. DOI: 10.1139/cjfr-2017-0262. 
Hoyer, Gerald E. (1975): Measuring and interpreting Douglas-fir management practices. State of Washington, Dept. of Natural Resources. Olympia.

Hozumi, K.; Asabira, T.; Kira, T. (1956): Intraspecific competition among higher plants. VI. Effect of some growth factors on the process of competition. In: J Inst Polytech Osaka City Univ D 7, S. 15-34.

Hradetzky, Joachim (1972): Modell eines integrierten Ertragstafel-Systems in modularer Form. Doktorarbeit. Albert-Ludwigs-Universität Freiburg, Freiburg.

Hultkrantz, Lars (1991): A Note on the Optimal Rotation Period in a Synchronized Normal Forest. In: Forest Science 37 (4), S. 1201-1206. DOI: 10.1093/forestscience/37.4.1201.

Hunter, Ian; Schuck, Annette (2002): Increasing forest growth in europe - possible causes and implications for sustainable forest management. In: Plant Biosystems - An International Journal Dealing with all Aspects of Plant Biology 136 (2), S. 133-141. DOI: 10.1080/11263500212331351039.

Hyytiäinen, Kari; Tahvonen, O. (2002): Economics of Forest Thinnings and Rotation Periods for Finnish Conifer Cultures. In: Scand. J. For. Res. (Scandinavian Journal of Forest Research) 17 (3), S. 274-288. DOI: 10.1080/028275802753742945.

Jacobsen, Jette Bredahl; Jensen, Frank; Thorsen, Bo Jellesmark (2018): Forest Value and Optimal Rotations in Continuous Cover Forestry. In: Environ Resource Econ 69 (4), S. 713-732. DOI: 10.1007/s10640-016-0098-z.

Johansson, Per-Olov; Löfgren, Karl-Gustaf (1985): The Economics of Forestry and Natural Resources. Oxford: Basil Blackwell.

Jüttner, Otto (1955): Ertragstafel für die Baumart Eiche (mäßige und starke Durchforstung). In: Schober, R. (Hg.): Ertragstafeln wichtiger Baumarten bei verschiedener Durchforstung. 4. Aufl. Frankfurt am Main: Sauerländer 1995.

Kahn, Markus; Pretzsch, Hans (1997): Das Wuchsmodell SILVA - Parametrisierung der Vers ion 2.1 für Rein- und Mischbestände aus Fichte und Buche. In: Allg. Forst- u. J.-Ztg. (Allgemeine Forst- und Jagdzeitung) 168 (6-7), S. 115-123, zuletzt geprüft am 08.01.2019.

Kennel, R. (1973): Die Bestimmung des Ertragsniveaus bei der Buche. In: Forstwiss. Centralbl. 92 (1), S. 226-234. DOI: 10.1007/BF02736053.

Kern, Karl Guenter (1958): Untersuchungen über den Wachstumsgang der Kiefer im Buntsandsteingebiet des Pfälzer Waldes: Ein Vergleich mit d. Wiedemann'schen Ertragstafel 1948 - Mäßige Durchforstung (Schriftenreihe der Bad.-Württ. Forstlichen Versuchs- und Forschungsanstalt, Abt. Ertragskunde / Baden-Württembergische Forstliche Versuchs- und Forschungsanstalt).

Keuffel, Walter (1980): Programmierte Deckungsbeitragskalkulation als betriebswirtschaftliche Entscheidungshilfe. Dargestellt an Beispielen aus der Niedersächsischen Landesforstverwaltung. Inauguraldissertation zur Erlangung der Doktorwürde. Albert-Ludwigs-Universität Freiburg, Freiburg i. Br. Institut für Forsteinrichtung. 
Kira, T.; Ogawa, H.; Sakazaki, N. (1953): Intraspecific competition among higher plants I. Competition-yield-density interrelationship in regularly dispersed population. In: J. Inst. Poly. Osaka City Univ. D 4, S. 1-16.

Klemperer, W. David (1996): Forest Resource Economics and Finance. Singapore: McGrawHill Book Company.

Knoke, Thomas (2002): Value of complete information on red heartwood formation in beech (Fagus sylvatica). In: Silva Fennica (36 (4)), S. 841-851.

Knoke, Thomas; Stang, Sebastian; Remler, Norbert; Seifert, Thomas (2006): Ranking the importance of quality variables for the price of high quality beech timber (Fagus sylvatica L.). In: Ann. For. Sci. 63 (4), S. 399-413. DOI: 10.1051/forest:2006020.

Kohnle, Ulrich; Teuffel, Konstantin von (2020): Probleme mit zu viel Geld, zu wertvollen Beständen? In: AFZ-DerWald (3), S. 28-34.

Kolström, Marja; Lindner, Marcus; Vilén, Terhi; Maroschek, Michael; Seidl, Rupert; Lexer, Manfred J. et al. (2011): Reviewing the Science and Implementation of Climate Change Adaptation Measures in European Forestry. In: Forests 2 (4), S. 961-982. DOI: 10.3390/f2040961.

Koster, Roman (2017): Implementierung von Überlebensrisiken in die Bestandesbewertung und Berechnung von Alterswertfaktoren. Masterarbeit. Georg-August-Universität Göttingen, Fakultät für Forstwissenschaften und Waldökologie, Göttingen.

Kramer, Horst (1988): Waldwachstumslehre. Ökologische und anthropogene Einflüsse auf das Wachstum des Waldes, seine Massen- und Wertleistung und die Bestandessicherheit. Hamburg und Berlin: Paul Parey Zeitschriftenverlag GmbH.

Kramer, Horst; Akça, Alparslan (2008): Leitfaden zur Waldmesslehre. 5., überarb. Aufl. Frankfurt am Main: J.D. Sauerländer's Verlag.

Kumar, B. Mohan; Long, James N.; Kumar, Prasoon (1995): A density management diagram for teak plantations of Kerala in peninsular India. In: For. Ecol. Manage. 74 (1-3), S. 125131.

Kurth, Horst; Gerold, Denie; Ulbricht, Rolf (1994): Forsteinrichtung. Nachhaltige Regelung des Waldes. Berlin: Dt. Landwirtschaftsverl.

Kuusela, Kullervo (1994): Forest Resources in Europe 1950 - 1990. Cambridge: Cambrige University Press.

Lembcke, Gunter; Knapp, Eugen; Dittmar, Otto (1975): Die neue DDR-Kiefernertragstafel. In: Beiträge für die Forstwirtschaft 15 (2), S. 55-64.

Lindner, Marcus; Maroschek, Michael; Netherer, Sigrid; Kremer, Antoine; Barbati, Anna; Garcia-Gonzalo, Jordi et al. (2010): Climate change impacts, adaptive capacity, and vulnerability of European forest ecosystems. In: For. Ecol. Manage. 259 (4), S. 698-709. DOI: 10.1016/j.foreco.2009.09.023. 
Lohmander, Peter; Helles, Finn (1987): Windthrow probability as a function of stand characteristics and shelter. In: Scand. J. For. Res. (Scandinavian Journal of Forest Research) 2 (14), S. 227-238. DOI: 10.1080/02827588709382460.

Loisel, Patrice (2014): Impact of storm risk on Faustmann rotation. In: For. Policy Econ. 38, S. 191-198. DOI: 10.1016/j.forpol.2013.08.002.

Long, James N. (1985): A Practical Approach to Density Management. In: The Forestry Chronicle 61 (1), S. 23-27. DOI: 10.5558/tfc61023-1.

Long, James N.; McCarter, James B.; Jack, Steven B. (1988): A Modified Density Management Diagram for Coastal Douglas-Fir. In: west j appl for 3 (3), S. 88-89. DOI: 10.1093/wjaf/3.3.88.

Long, James N.; Shaw, John D. (2005): A Density Management Diagram for Even-aged Ponderosa Pine Stands. In: Western Journal of Applied Forestry 20 (4), S. 205-215. DOI: 10.1093/wjaf/20.4.205.

LWF (2019): Klima - Boden - Baumartenwahl. Praxishilfe. Unter Mitarbeit von Olaf Schmidt, Manuela Forster, Wolfgang Falk, Birgit Reger, Markus Blaschke, Paul Dimke et al. Hg. v. Bayerische Landesanstalt für Wald und Forstwirtschaft (LWF). Freising. Online verfügbar unter www.lwf.bayern.de, zuletzt geprüft am 03.04.2020.

Mantel, Wilhelm (1982): Waldbewertung. Einführung und Anleitung. 6., neubearb. u. erw. Aufl. München: BLV Verlagsgesellschaft.

Mayer, Philipp; Brang, Peter; Dobbertin, Matthias; Hallenbarter, Dionys; Renaud, JeanPierre; Walthert, Lorenz; Zimmermann, Stefan (2005): Forest storm damage is more frequent on acidic soils. In: Ann. For. Sci. 62 (4), S. 303-311. DOI: 10.1051/forest:2005025.

McCarter, James B.; Long, James N. (1986): A Lodgepole Pine Density Management Diagram. In: Western Journal of Applied Forestry 1 (1), S. 6-11. DOI: 10.1093/wjaf/1.1.6.

MCPFE (2003): Improved Pan-European Indicators for Sustainable Forest Management. Hg. v. Fourth Ministerial Conference on the Protection of Forests in Europe. Living Forest Summit. Vienna.

MEA (2005): Ecosystems and Human Well-being. Volume 1: Current State \& Trends. Hg. v. Millennium Ecosystem Assessment. Washington (Global Assessment Reports). Online verfügbar unter https://www.millenniumassessment.org/en/Global.html, zuletzt geprüft am 25.03.2020.

Meilby, Henrik; Nord-Larsen, Thomas (2012): Spatially explicit determination of individual tree target diameters in beech. In: For. Ecol. Manage. 270, S. 291-301. DOI: 10.1016/j.foreco.2011.08.037.

Menéndez-Miguélez, María; Álvarez-Álvarez, Pedro; Majada, Juan; Canga, Elena (2016): Management tools for Castanea sativa coppice stands in northwestern Spain. In: Bosque (Valdivia) 37 (1), S. 119-133. DOI: 10.4067/S0717-92002016000100012. 
Messerer, Katharina; Kacprowski, Tim; Kolo, Horst; Baumbach, Jan; Knoke, Thomas (2020): Importance of considering the growth response after partial harvesting and economic risk of discounted net revenues when optimizing uneven-aged forest management. In: Can. J. For. Res. DOI: 10.1139/cjfr-2018-0546.

Mezei, Pavel; Blaženec, Miroslav; Grodzki, Wojciech; Škvarenina, Jaroslav; Jakuš, Rastislav (2017): Influence of different forest protection strategies on spruce tree mortality during a bark beetle outbreak. In: Ann. For. Sci. 74 (4), S. 660. DOI: 10.1007/s13595-017-0663-9.

Möhring, Bernhard (1994): Über ökonomische Kalküle für forstliche Nutzungsentscheidungen. Ein Beitrag zur Förderung des entscheidungsorientierten Ansatzes der forstlichen Betriebswirtschaftslehre. Frankfurt a. M.: J.D. Sauerländer's Verlag (Schriften zur Forstökonomie, 7).

Möhring, Bernhard (2001): Nachhaltige Forstwirtschaft und Rentabilitätsrechnung - ein Widerspruch? In: Allg. Forst- u. J.-Ztg. (Allgemeine Forst- und Jagdzeitung) 172 (04), S. 61-66.

Möhring, Bernhard (2009): Wie gelingt der Brückenschlag zwischen Theorie und Praxis bei den ökonomischen Hiebsreifekriterien? In: Forstarchiv 80 (4), S. 109-118.

Möhring, Bernhard (2010): Optimierung forstlicher Produktion unter Beachtung von finanziellen Restriktionen. In: Schweiz. Z. Forstwes. 161 (9), S. 346-354. DOI:

10.3188/szf.2010.0346.

Möhring, Bernhard (2014): Gibt es einen 'richtigen' Zinssatz bei der forstlichen Wertermittlung? In: Wertermittlungsforum (32), S. 72-78.

Möhring, Bernhard; Dieter, Matthias (2020): Zins - Fluch oder Segen in der forstlichen Planung? In: Allg. Forst- u. J.-Ztg. (Allgemeine Forst- und Jagdzeitung) 190 (5-6), S. 101-120.

Möhring, Bernhard; Husmann, Kai; Koster, Roman (2019): Doppelter BHD - dreifacher Zuwachs: Beeindruckend!? Ein Diskussionsbeitrag mit Bezug zum Klimaschutz-Projekt „Speicher-Wald“. In: AFZ-DerWald 74 (14), S. 47-51.

Möhring, Bernhard; Paul, Carola; Bodelschwingh, Hilmar von; Dög, Markus; Gerst, Johannes; Kleinschmit, Chrisitan et al. (2018): Arbeitsmaterialien zur Betriebswirtschaftlichen Kalkulation. Hg. v. Abteilung für Forstökonomie und Forsteinrichtung des Burckhardt-Instituts. Fakultät für Forstwissenschaften und Waldökologie der Georg-August-Universität Göttingen. Möhring, Bernhard; Rüping, Ursula (2006): Bewertungskonzept für forstliche Nutzungsbeschränkungen. Frankfurt am Main: J.D. Sauerländer's Verlag (Schriften zur Forstökonomie, 32).

Möhring, Bernhard; Rüping, Ursula; Leefken, Georg; Ziegeler, M. (2006): Die Annuität - ein 'missing link' der Forstökonomie? In: Allg. Forst- u. J.-Ztg. (Allgemeine Forst- und Jagdzeitung) 177 (2), S. 21-29.

Möllmann, Torsten Bernhard; Möhring, Bernhard (2017): A practical way to integrate risk in forest management decisions. In: Ann. For. Sci. 74 (4), S. 75-86. DOI: 10.1007/s13595-0170670-x. 
Möllmann, Torsten Bernhard; Möhring, Bernhard (2018): Zur Bedeutung und ökonomischen Wirkung verminderter Steuersätze bei außerordentlicher Holznutzung gemäß des § 34b EStG. In: Landbauforschung - Applied Agricultural and Forestry Research 67 (3/4), S. 155-165.

Moosmayer, Hans-Ulrich; Schöpfer, Walter; Mühlhäuser, G.; Kublin, Edgar; Burger, H. U. (1996): Schätzfunktionen zur Ermittlung der standortstypischen Wuchsleistung von Fichte und Buche. In: Allg. Forst- u. J.-Ztg. (Allgemeine Forst- und Jagdzeitung) (167), S. 95-103.

Morin, Xavier; Fahse, Lorenz; Scherer-Lorenzen, Michael; Bugmann, Harald (2011): Tree species richness promotes productivity in temperate forests through strong complementarity between species. In: Ecology Letters 14 (12), S. 1211-1219. DOI: 10.1111/j.14610248.2011.01691.x.

Mußhoff, Oliver; Maart-Noelck, Syster Christin (2014): An experimental analysis of the behavior of forestry decision-makers - The example of timing in sales decisions. In: For. Policy Econ. (41), S. 31-39. DOI: 10.1016/j.forpol.2013.12.006.

Nabuurs, Gert-Jan; Lindner, Marcus; Verkerk, Pieter Johannes; Gunia, Katja; Deda, Paola; Michalak, Roman; Grassi, Giacomo (2013): First signs of carbon sink saturation in European forest biomass. In: Nature Clim Change 3 (9), S. 792-796. DOI: 10.1038/nclimate1853.

Nagel, Jürgen; Schmidt, Matthias (2006): The silvicultural decision support system BWINPro. In: Sustainable Forest Management: Springer-Verlag GmbH, S. 59-63.

Nash, John C. (2016): Functions for Nonlinear Least Squares Solutions [R package nlmrt]. Version 2016.3.2: R Foundation for Statistical Computing.

Näslund, Bertil (1969): Optimal rotation and thinning. In: For. Sci. 15 (4), S. 446-451.

Nds. Forstplanungsamt (1987): Hilfstafeln für die Forsteinrichtung. Zusammengestellt für den Gebrauch in den Niedersächsischen Landesforsten: Niedersächsisches Forstplanungsamt.

Newman, D. H. (2002): Forestry's golden rule and the development of the optimal forest rotation literature. In: Journal of Forest Economics 8 (1), S. 5-27. DOI: 10.1078/1104-689900002 .

Newton, P. F. (1997): Stand density management diagrams: Review of their development and utility in stand-level management planning. In: For. Ecol. Manage. 98 (3), S. 251-265. DOI: 10.1016/S0378-1127(97)00086-8.

Newton, P. F.; Weetman, G. F. (1993): Stand density management diagrams and their development and utility in black spruce management. In: The Forestry Chronicle 69 (4), S. 421430. DOI: $10.5558 / \mathrm{tfc} 69421-4$.

Newton, P. F.; Weetman, G. F. (1994): Stand density management diagram for managed black spruce stands. In: The Forestry Chronicle 70 (1), S. 65-74. DOI: 10.5558/tfc70065-1.

NLF (2014): Tabellen, Vordrucke und Hinweise zu den Niedersächsischen Waldbewertungsrichtlinien (WBR 2014). Niedersächsische Landesforsten. Online verfügbar unter www.landesforsten.de, zuletzt geprüft am 12.04.2020. 
NLF (2019): Klimaangepasste Baumartenwahl in den Niedersächsischen Landesforsten. Unter Mitarbeit von Thomas Böckmann, Jan Hansen, Karen Hauskeller-Bullerjahn, Thomas Jensen, Jürgen Nagel, Ralf-Volker Nagel et al. In: Aus dem Walde - Schriftenreihe Waldentwicklung in Niedersachsen, Bd. 61. Online verfügbar unter www.ml.niedersachsen.de, zuletzt geprüft am 03.04.2020.

NMELV (2020): Waldbewertungsrichtlinien (WBR 2020). Anlage zum Runderlass des ML vom 4.12.2019 - Nds. MB1. Nr. 48/2019, S. 1774.

Nothdurft, Arne; Borchers, Jens; Niggemeyer, Peter; Saborowski, Joachim; Kändler, Gerald (2008): Erste Folgeaufnahme einer Betriebsinventur als zweiphasige Stichprobe zur Stratifizierung. In: Allg. Forst- u. J.-Ztg. (Allgemeine Forst- und Jagdzeitung) 180 (7/8), S. 133-140.

Oesten, Gerhard (1986): Über die forstliche Betriebswirtschaftslehre als angewandte Sozialwissenschaft: ein Beitrag zu den methodologischen Grundlagen der Disziplin unter besonderer Berücksichtigung verhaltenswissenschaftlicher Forschungsansätze. Habilitationsschrift. Albert-Ludwigs-Universität Freiburg. Forstwissenschaftliche Fakultät.

Oesten, Gerhard; Roeder, Axel (2012a): Management von Forstbetrieben. Band I - Grundlagen, Betriebspolitik. 3., überarb. Aufl. Freiburg. Online verfügbar unter www.freidok.uni-freiburg.de, zuletzt geprüft am 04.04.2020.

Oesten, Gerhard; Roeder, Axel (2012b): Management von Forstbetrieben. Band III - Leistungssystem, Zusammenfassung und Ausblick. 1. Aufl. Freiburg. Online verfügbar unter www.freidok.uni-freiburg.de, zuletzt geprüft am 04.04.2020.

Offer, Armin; Staupendahl, Kai (2009): Neue Bestandessortentafeln für die Waldbewertung und ihr Einsatz in der Bewertungspraxis. In: Forst und Holz 64 (5), S. 16-25.

Offer, Armin; Staupendahl, Kai (2018): Holzwerbungskosten- und Bestandessortentafeln. Kassel: HessenForst (Hrsg.).

Ohlin, B. (1921; 1995): Till frågan om skogarnas omloppstid. In: Ekonomisk Tidskrift 22, 89114. Translated by: Hudson, C. (1995). Concerning the Question of the Rotation Period in Forestry. Journal of Forest Economics, 1: 89-114.

Patrício, M. S.; Nunes, L. (2017): Density management diagrams for sweet chestnut high-forest stands in Portugal. In: iForest 10 (6), S. 865-870. DOI: 10.3832/ifor2411-010.

Paul, Carola; Benfer, Nils (2019): Risiko und Risikomanagement in Theorie und Praxis. In: AFZ-DerWald 74 (22), S. 31-34.

Paul, Carola; Brandl, Susanne; Friedrich, Stefan; Falk, Wolfgang; Härtl, Fabian; Knoke, Thomas (2019a): Climate change and mixed forests: how do altered survival probabilities impact economically desirable species proportions of Norway spruce and European beech? In: Ann. For. Sci. 76 (1), S. 363. DOI: 10.1007/s13595-018-0793-8.

Paul, Carola; Brandl, Susanne; Friedrich, Stefan; Härtl, Fabian; Falk, Wolfgang; Knoke, Thomas (2019b): Der Einfluss des Klimawandels auf die Baumartenwahl. In: AFZ-DerWald 74 (2), S. 21-23.

Paulsen, Johann Christian (1795): Praktische Anweisung zum Forstwesen. Detmold. 
Pearse, P. H. (1967): The optimum forest rotation. In: The Forestry Chronicle 43 (2), S. $178-$ 195. DOI: $10.5558 / \mathrm{tfc} 43178-2$.

Pertz, Klaus (1983): Grenzen einer marktwirtschaftlichen Interpretation der Forstwirtschaft. In: Forstarchiv 54 (4), S. 147-152.

Pressler, Max (1860; 1995): Zur Verständigung über den Reinertragswaldbau und sein Betriebsideal. Zweiter Artikel. Aus der Holzzuwachslehre. In: Allg. Forst- u. J.-Ztg. (Allgemeine Forst- und Jagdzeitung) 36, 173-191. Translated by: Löwenstein, W.; Wirkner, J. R. (1995). For the Comprehension of Net Revenue Silviculture and the Management Objectives Derived Thereof. Part Two. Increment Theory. Journal of Forest Economics, 1: 45-87.

Pretzsch, Hans (2000): Die Regeln von REINEKE, YODA und das Gesetz der räumlichen Allometrie. In: Allg. Forst- u. J.-Ztg. (Allgemeine Forst- und Jagdzeitung) 171 (11), S. 205-210.

Pretzsch, Hans (2005a): Stand density and growth of Norway spruce (Picea abies (L.) Karst.) and European beech (Fagus sylvatica L.): evidence from long-term experimental plots. In: Eur. J. For. Res. 124 (3), S. 193-205. DOI: 10.1007/s10342-005-0068-4.

Pretzsch, Hans (2005b): Wachstum von Rein- und Mischbestäden bei veränderten Umweltbedingungen. In: AFZ-DerWald (9), S. 465-468.

Pretzsch, Hans (2009): Forest dynamics, growth and yield. From Measurement to Model. Berlin Heidelberg: Springer-Verlag GmbH.

Pretzsch, Hans (2010a): Zur Verteilung des Zuwachses zwischen den Bäumen eines Bestandes und Abhängigkeit des Verteilungsschlüssels von den Standortbedingungen. In: Allg.

Forst- u. J.-Ztg. (Allgemeine Forst- und Jagdzeitung) 181 (1/2), S. 4-13.

Pretzsch, Hans (2016): Korrekturfaktoren für Umwelt- und Mischungseffekte bei der Anwendung von Ertragstafeln auf gleichaltrige Rein -und Mischbestände. In: AFZ-DerWald 71 (14), S. 47-50.

Pretzsch, Hans; Biber, Peter; Schütze, Gerhard; Uhl, Enno; Rötzer, Thomas (2014): Forest stand growth dynamics in Central Europe have accelerated since 1870. In: Nature communications 5, S. 4967. DOI: 10.1038/ncomms5967.

Pretzsch, Hans; Bielak, Kamil; Block, Joachim; Bruchwald, Arkadiusz; Dieler, Jochen; Ehrhart, Hans-Peter et al. (2013a): Productivity of mixed versus pure stands of oak (Quercus petraea (Matt.) Liebl. and Quercus robur L.) and European beech (Fagus sylvatica L.) along an ecological gradient. In: European Journal of Forest Research 132 (2), S. 263-280. DOI: 10.1007/s10342-012-0673-y.

Pretzsch, Hans; Bielak, Kamil; Bruchwald, Arkadiusz; Dieler, Jochen; Dudzińska, M.; Ehrhart, Hans-Peter et al. (2013b): Mischung und Produktivität von Waldbeständen. Ergebnisse langfristiger ertragskundlicher Versuche. (Species mixing and productivity of forests. Results from long-term experiment). In: Allg. Forst- u. J.-Ztg. (Allgemeine Forst- und Jagdzeitung) 184 (7-8), S. 177-195. 
Pretzsch, Hans; Block, Joachim; Dieler, Jochen; Dong, P. H.; Kohnle, Ulrich; Nagel, Jürgen et al. (2010b): Comparison between the productivity of pure and mixed stands of Norway spruce and European beech along an ecological gradient. In: Ann. For. Sci. 67 (7), S. 712. DOI: $10.1051 /$ forest/2010037.

Pretzsch, Hans; del Río, M.; Ammer, Christian; Avdagic, A.; Barbeito, Ignacio; Bielak, Kamil et al. (2015): Growth and yield of mixed versus pure stands of Scots pine (Pinus sylvestris L.) and European beech (Fagus sylvatica L.) analysed along a productivity gradient through Europe. In: European Journal of Forest Research 134 (5), S. 927-947. DOI: 10.1007/s10342015-0900-4.

Prodan, Michail (1965): Holzmeßlehre. Frankfurt am Main: Sauerländer,s Verlag. R Core Team (2019): R: A Language and Environment for Statistical Computing. Version 3.6.0. Wien: R Foundation for Statistical Computing: R Core Team.

Racine, J. S.; Nie, Zhenghua; Ripley, Brian D. (2018): Categorical Regression Splines [R package crs]. Version 0.15-31: R Foundation for Statistical Computing.

Rämö, Janne; Tahvonen, Olli (2017): Optimizing the Harvest Timing in Continuous Cover Forestry. In: Environ Resource Econ 67 (4), S. 853-868. DOI: 10.1007/s10640-016-0008-4.

Reineke, Lester Henry (1933): Perfecting a Stand-Density Index for Even-Aged Forests. In: Journal of Agricultural Research 46 (7), S. 627-638.

Robbins, Lionel Charles (1932): An Essay on the Nature and Significance of Economic Science. London: Macmillan.

Roessiger, Joerg; Ficko, Andrej; Clasen, Christian; Griess, Verena C.; Knoke, Thomas (2016): Variability in growth of trees in uneven-aged stands displays the need for optimizing diversified harvest diameters. In: Eur J Forest Res 135 (2), S. 283-295. DOI: 10.1007/s10342-015-0935-6.

Röhle, Heinz (1995): Zum Wachstum der Fichte auf Hochleistungsstandorten in Südbayern. Ertragskundliche Auswertung langfristig beobachteter Versuchsreihen unter besonderer Berücksichtigung von Trendänderungen im Wuchsverhalten. Mitteilungen aus der Staatsforstverwaltung Bayerns. 48: Bayerisches Staatsministerium für Ernährung, Landwirtschaft und Forsten, Referat Forstliche Aus- und Fortbildung.

Röhrig, Ernst; Bartsch, Norbert; Lüpke, Burghard von; Dengler, Alfred (2006): Waldbau auf ökologischer Grundlage: Eugen Ulmer KG.

Sächsische Hans-Carl-von-Carlowitz-Gesellschaft e. V. (Hg.) (2013): Die Erfindung der Nachhaltigkeit. Leben, Werk und Wirkung des Hans Carl von Carlowitz. München: oekomVerl.

Samuelson, Paul A. (1976): Economics of Forestry in an Evolving Society. In: Economic Inquiry 14 (4), S. 466-492. DOI: 10.1111/j.1465-7295.1976.tb00437.x. 
Sauter, Philipp A.; Möllmann, Torsten Bernhard; Anastassiadis, Friederike; Mußhoff, Oliver; Möhring, Bernhard (2016a): To insure or not to insure? Analysis of foresters' willingness-topay for fire and storm insurance. In: For. Policy Econ. (73), S. 78-89. DOI: 10.1016/j.forpol.2016.08.005.

Sauter, Udo Hans; Brüchert, Franka; Breinig, Lorenz (2016b): Qualität von Starkholz - Erwartungen und Wirklichkeit. In: AFZ-DerWald (19), S. 26-28.

Schade, V. (2019): HMI - Marktbilanz Forst und Holz 2019 Deutschland. Hg. v. HMIHolzmarktinfo. Bonn.

Schaefer, Milner B. (1954): Some aspects of the dynamics of populations important to the management of the commercial marine fisheries. In: Inter-American Tropical Tuna Commission Bulletin 1 (2), S. 23-56.

Schall, Peter; Ammer, Christian (2013): How to quantify forest management intensity in Central European forests. In: Eur J Forest Res 132 (2), S. 379-396. DOI: 10.1007/s10342-0130681-6.

Schelhaas, Mart-Jan; Nabuurs, Gert-Jan; Schuck, Andreas (2003): Natural disturbances in the European forests in the $19^{\text {th }}$ and $20^{\text {th }}$ centuries. In: Glob. Change Biol. 9 (11), S. 1620-1633. DOI: $10.1046 /$ j.1365-2486.2003.00684.x.

Schmidt, Anton (1971): Wachstum und Ertrag der Kiefer auf wirtschaftlich wichtigen Standorteinheiten der Oberpfalz: mit Leistungstaf. München: Forstl. Forschungsanst. (Forschungsberichte / Forstliche Forschungsanstalt).

Schmidt, Matthias; Hanewinkel, Marc; Kändler, Gerald; Kublin, Edgar; Kohnle, Ulrich (2010): An inventory-based approach for modeling single-tree storm damage - experiences with the winter storm of 1999 in southwestern Germany. In: Can. J. For. Res. 40 (8), S. 1636-1652. DOI: 10.1139/X10-099.

Schmidt, Matthias; Nowack, Stefan; Riebeling, Rüdiger (2008): Methodische Ansätze und Ergebnisse zur Quantifizierung des Buchen-Rotkerns in Hessen. Methods and results of quantifying beech red heartwood in Hesse. In: Beiträge aus der NW-FVA, Band 3, S. 267-290.

Schmoeckel, Julia (2005): Orographischer Einfluss auf die Strömung abgeleitet aus Sturmschäden im Schwarzwald während des Orkans „Lothar“. Dissertation. Universität Karlsruhe, Karlsruhe. Fakultät für Physik.

Schober, Reinhard (1952): Einzelplanung und Gesamtplanung der Forsteinrichtung und Betriebsvollzug - Einheit oder Gegensatz, Theorie und Wirklichkeit. In: Vorträge der Hochschulwoche Hann. Münden 1951. Bd. 2 Schriftenreihe der Forstlichen Fakultät der Universität Göttingen, Hg. R. Schober. Frankfurt am Main: J.D. Sauerländer's Verlag, S. 51-54.

Schober, Reinhard (1967): Ertragstafel für die Baumart Buche (mäßige und starke Durchforstung). In: Schober, R. (Hg.): Ertragstafeln wichtiger Baumarten bei verschiedener Durchforstung. 4. Aufl., Frankfurt am Main: J. D. Sauerländer,s Verlag, 1995.

Schober, Reinhard (1975): Ertragstafeln wichtiger Baumarten bei verschiedener Durchforstung. Neubearb. Frankfurt a. M: J.D. Sauerländer's Verlag. 
Schober, Reinhard (1995): Ertragstafeln wichtiger Baumarten bei verschiedener Durchforstung. 4. Aufl. Frankfurt am Main: J.D. Sauerländer's Verlag.

Schöpfer, W.; Hradetzky, J.; Kublin, E. (1994): Wachstumsänderungen der Fichte in BadenWürttemberg. In: Forst und Holz 49 (21), S. 633-644.

Schöpfer, W.; Hradetzky, J.; Kublin, E. (1997): Wachstumsvergleiche von Fichte und Tanne in Baden-Württemberg. In: Forst und Holz 52 (16), S. 443-448.

Schou, Erik; Thorsen, Bo Jellesmark; Jacobsen, Jette Bredahl (2015): Regeneration decisions in forestry under climate change related uncertainties and risks: Effects of three different aspects of uncertainty. In: For. Policy Econ. 50, S. 11-19. DOI: 10.1016/j.forpol.2014.09.006.

Schreuder, Gerard F. (1971): The Simultaneous Determination of Optimal Thinning Schedule and Rotation for an Even-Aged Forest. In: For. Sci. 17 (3), S. 333-339. DOI: 10.1093/forestscience/17.3.333.

Schulze, Ernst-Detlef; Ammer, Christian (2015): Spannungsfeld Forstwirtschaft und Naturschutz. In: Biologie in unserer Zeit 45 (5), S. 304-314. DOI: 10.1002/biuz.201510574.

Schütz (1977): Beitrag. In: Deutscher Verband Forstlicher Versuchsanstalten, Sektion Ertragskunde (Hg.): Referate und Diskussionen. Gießen.

Schütz, Jean-Philippe; Götz, Michael; Schmid, Willi; Mandallaz, Daniel (2006): Vulnerability of spruce (Picea abies) and beech (Fagus sylvatica) forest stands to storms and consequences for silviculture. In: European Journal of Forest Research 125 (3), S. 291-302. DOI:

10.1007/s10342-006-0111-0.

Schwappach, Adam (1902): Wachstum und Ertrag normaler Fichtenbestände in Preussen unter besonderer Berücksichtigung des Einflusses verschiedener wirtschaftlicher Behandlungsweisen. In: Mitt. Forstl. Versuchswesens Preussens, S. 44-119.

Schwappach, Adam (1903): Leitfaden der Holzmeßkunde. 2. Aufl.: Verlag Julius Springer. Schwappach, Adam (1912): Ertragstafeln der wichtigsten Holzarten : in tabellarischer und graphischer Form. Neudamm: Neumann.

Scott, Robyn E.; Mitchell, Stephen J. (2005): Empirical modelling of windthrow risk in partially harvested stands using tree, neighbourhood, and stand attributes. In: For. Ecol. Manage. 218 (1-3), S. 193-209. DOI: 10.1016/j.foreco.2005.07.012.

Seidl, Rupert; Schelhaas, Mart-Jan; Lexer, Manfred J. (2011): Unraveling the drivers of intensifying forest disturbance regimes in Europe. In: Glob. Change Biol. 17 (9), S. 2842-2852. DOI: $10.1111 /$ j.1365-2486.2011.02452.x.

Seidl, Rupert; Schelhaas, Mart-Jan; Lindner, Marcus; Lexer, Manfred J. (2009): Modelling bark beetle disturbances in a large scale forest scenario model to assess climate change impacts and evaluate adaptive management strategies. In: Reg. Environ. Change 9 (2), S. 101119. DOI: $10.1007 / \mathrm{s} 10113-008-0068-2$.

Seidl, Rupert; Schelhaas, Mart-Jan; Rammer, Werner; Verkerk, Pieter Johannes (2014): Increasing forest disturbances in Europe and their impact on carbon storage. In: Nat. Clim. Change 4 (9), S. 806-810. DOI: 10.1038/nclimate2318. 
Seidl, Rupert; Thom, Dominik; Kautz, Markus; Martin-Benito, Dario; Peltoniemi, Mikko; Vacchiano, Giorgio et al. (2017): Forest disturbances under climate change. In: Nat. Clim. Change 7 (6), S. 395-402. DOI: 10.1038/nclimate3303.

Shinozaki, K.; Kira, T. (1956): Intraspecific competition among higher plants. VII. Logistic theory of the C-D effect. In: J Inst Polytech Osaka City Univ Ser 12, S. 69-82.

Sieben, Günter.; Schildbach, Thomas. (1990): Betriebswirtschaftliche Entscheidungstheorie. 3. Auflage. Düsseldorf: Werner-Verlag.

Skovsgaard, J. P.; Vanclay, J. K. (2013): Forest site productivity: a review of spatial and temporal variability in natural site conditions. In: Forestry 86 (3), S. 305-315. DOI: 10.1093/forestry/cpt010.

Smith, N. J. (1989): A stand-density control diagram for western red cedar, Thuja plicata. In: Forest Ecology and Management 27 (3), S. 235-244. DOI: 10.1016/0378-1127(89)90109-6.

Speidel, Gerhard (1972): Planung im Forstbetrieb: Paul Parey Zeitschriftenverlag GmbH.

Speidel, Gerhard (1984): Forstliche Betriebswirtschaftslehre. 2. Aufl. Hamburg, Berlin: Paul Parey Zeitschriftenverlag GmbH.

Spellmann, Hermann (2010): Nachhaltige Waldbewirtschaftung auf ökologischen Grundlagen. In: Otto Depenheuer und Bernhard Möhring (Hg.): Waldeigentum. Dimensionen und Perspektiven, Bd. 8. Berlin, Heidelberg: Springer Berlin Heidelberg (Bibliothek des Eigentums, Im Auftrag der Deutschen Stiftung Eigentum), S. 99-116.

Spellmann, Hermann; Möhring, Bernhard; Nagel, Jürgen; Nagel, Ralf-Volker; Albert, Matthias (unveröff.): Projektantrag: Neue Generation dynamischer Ertragstafeln. Nordwestdeutsche Forstliche Versuchsanstalt (NW-FVA); Abteilung für Forstökonomie der Georg-August-Universität Göttingen. Göttingen, zuletzt geprüft am 09.12.2019.

Spiecker, Heinrich; Mielikäinen, Kari; Köhl, Michael; Skovsgaard, J. P. (1996): Growth Trends in European Forests. Studies from 12 Countries. Berlin, Heidelberg: Springer-Verlag $\mathrm{GmbH}$.

Staupendahl, Kai (2011): Modellierung der Überlebenswahrscheinlichkeit von Waldbeständen mithilfe der neu parametrisierten Weibull-Funktion. In: Forstarchiv 82 (1), S. 10-19.

Staupendahl, Kai (2018): Aktualisierung der Ertragstafeln für den Landesbetrieb HessenForst. Erstellt von Dr. Kai Staupendahl, ARGUS Forstplanung, im Auftrag des Landes Hessen, vertreten durch HessenForst, Landesbetriebsleitung - Abt. Waldentwicklung und Umwelt, Gießen. Worpswede und Gießen, zuletzt geprüft am 10.07.2018.

Staupendahl, Kai; Möhring, Bernhard (2011): Integrating natural risks into silvicultural decision models: A survival function approach. In: For. Policy Econ. 13 (6), S. 496-502. DOI: 10.1016/j.forpol.2011.05.007.

Staupendahl, Kai; Rummel, Dirk; Raschka, Wolfgang; Köhler, Jürgen K. (2020): Herleitung der neuen Alterswertfaktoren. In: AFZ-DerWald (10), S. 24-28. 
Staupendahl, Kai; Schmidt, Matthias (2016): Kalibrierung der Ertragstafeln für die Hauptbaumarten in Hessen mithilfe der Daten der Bundeswaldinventur. In: Allg. Forst- u. J.-Ztg. (Allgemeine Forst- und Jagdzeitung) 187 (9/10), S. 197-216.

Staupendahl, Kai; Zucchini, Walter (2011): Schätzung von Überlebensfunktionen der Hauptbaumarten auf der Basis von Zeitreihen der Rheinland-Pfälzischen Waldzustandserhebung. In: Allg. Forst- u. J.-Ztg. (Allgemeine Forst- und Jagdzeitung) 182 (7/8), S. 129-145.

Stinglwagner, G.; Haseder, I.; Erlbeck, R. (2005): Das Kosmos Wald- und Forstlexikon. 3. Aufl., [vollkommen aktualisierte und erw. Ausg.]. Stuttgart: Kosmos.

SVLFG (2018): Aktualisierung des Beitragsmaßstabs für die Unfallversicherung in der Landwirtschaft, im Forst sowie im Gartenbau. - Gutachterliche Stellungnahme -. Unter Mitarbeit von E. Prof.Dr. Bahrs. Hg. v. Sozialversicherung für Landwirtschaft, Forsten und Gartenbau (SVLFG). Online verfügbar unter http://www.svlfg.de/50-vmb/vmb02_neu/index.html, zuletzt geprüft am 15.03.2019.

Tahvonen, Olli (2004): Optimal harvesting of forest age classes: a survey of some recent results. In: Mathematical Population Studies 11 (3-4), S. 205-232. DOI: 10.1080/08898480490513616.

Tahvonen, Olli (2009): Optimal choice between even- and uneven-aged forestry. In: Natural Resource Modeling 22 (2), S. 289-321. DOI: 10.1111/j.1939-7445.2008.00037.x.

Tahvonen, Olli (2016): Economics of rotation and thinning revisited: the optimality of clearcuts versus continuous cover forestry. In: For. Policy Econ. 62, S. 88-94. DOI: 10.1016/j.forpol.2015.08.013.

Tahvonen, Olli; Pihlainen, Sampo; Niinimäki, Sami (2013): On the economics of optimal timber production in boreal Scots pine stands. In: Can. J. For. Res. 43 (8), S. 719-730. DOI: 10.1139/cjfr-2012-0494.

Tahvonen, Olli; Rämö, Janne (2016): Optimality of continuous cover vs. clear-cut regimes in managing forest resources. In: Can. J. For. Res. 46 (7), S. 891-901. DOI: 10.1139/cjfr-20150474.

Tahvonen, Olli; Salo, Seppo; Kuuluvainen, Jari (2001): Optimal forest rotation and land values under a borrowing constraint. In: Journal of Economic Dynamics and Control 25 (10), S. 1595-1627. DOI: 10.1016/S0165-1889(99)00065-2.

Tahvonen, Olli; Viitala, Esa-Jussi (2006): Does Faustmann Rotation Apply to Fully Regulated Forests? In: Forest Science 52 (1), S. 23-30. DOI: 10.1093/forestscience/52.1.23.

Thommen, Jean-Paul; Achleitner, Ann-Kristin (2012): Allgemeine Betriebswirtschaftslehre. Umfassende Einführung aus managementorientierter Sicht. 7., vollst. überarb. Aufl. 2012. Wiesbaden: Imprint Gabler Verlag.

Utschig, Heinz (2002): Analyse der Standraumökonomie von Einzelbäumen auf langfristig beobachteten Versuchsflächen. Methoden, Programmentwicklung und erste Ergebnisse. In: Forstwiss. Centralbl. 121 (6), S. 335-348. 
Verein Deutscher Forstlicher Versuchsanstalten (1873): Anleitung für Durchforstungsversuche. In: Ganghofer, August von (1881) Das Forstliche Versuchswesen. Augsburg: Schmid'sche Buchhandlung, S. 247-253.

Verein Deutscher Forstlicher Versuchsanstalten (1902): Beratungen der vom Vereine Deutscher Forstlicher Versuchsanstalten eingesetzten Kommission zur Feststellung des neuen Arbeitsplanes für Durchforstungs- und Lichtungsversuche. In: Allg. Forst- u. J.-Ztg. (Allgemeine Forst- und Jagdzeitung) 78, S. 180-184, zuletzt geprüft am 11.07.2018.

Wallentin, Cristofer; Nilsson, Urban (2014): Storm and snow damage in a Norway spruce thinning experiment in southern Sweden. In: Forestry 87 (2), S. 229-238.

Weise, Wilhelm (1880): Ertragstafeln für die Kiefer in Deutschland. Berlin: Springer-Verlag $\mathrm{GmbH}$.

Wenk, Günter; Antanaītis, Vaīdotas; Šmelko, Štefan (1990): Waldertragslehre. 1. Aufl. Berlin: Deutscher Landwirtschaftsverlag.

Wenk, Günter; Römisch, Klaus; Gerold, Dorothea (1984): DDR-Fichtenertragstafel.

Wiedemann, Eilhard (1931): Ertragstafel für die Baumart Buche. In: Wiedemann (Hg.): Ertragstafeln für Buche 1931, Fichte (1936), Douglasie (1937). Preußische Versuchsanstalt für Waldwirtschaft, Hannover: Schaper 1938.

Wiedemann, Eilhard (1932): Die Rotbuche 1931. In: Mitteilungen aus der Forstwirtschaft und Forstwissenschaft 3 (1), S. 189.

Wiedemann, Eilhard (1935): Zur Klärung der Durchforstungsbegriffe. In: Zeitschrift für Forst- und Jagdwesen 67, S. 56-64.

Wiedemann, Eilhard (1936/42): Ertragstafel für die Baumart Fichte (mäßige, starke und gestaffelte Durchforstung). In: Schober, R. (Hg.): Ertragstafeln wichtiger Baumarten bei verschiedener Durchforstung. 4. Aufl., Frankfurt am Main: J. D. Sauerländer,s Verlag, 1995.

Wiedemann, Eilhard (1937): Die Fichte. Teil 2: Die Durchforstung und Lichtung, die neuen Ertragstafeln für verschiedene Durchforstung, die standörtlichen Untersuchungen, Zusammenfassung. In: Mitteilungen aus der Forstwirtschaft und Forstwissenschaft (8), S. 103-248.

Wiedemann, Eilhard (1942): Der gleichaltrige Buchen-Fichten-Mischbestand. In: Mitteilungen aus der Forstwirtschaft und Forstwissenschaft 13, S. 1-88.

Wiedemann, Eilhard (1943): Ertragstafel für die Baumart Kiefer (mäßige, starke und gestaffelte Durchforstung). In: Schober, R. (Hg.): Ertragstafeln wichtiger Baumarten bei verschiedener Durchforstung. 4. Aufl. Frankfurt am Main: Sauerländer 1995.

Wiedemann, Eilhard (1948): Die Kiefer 1948 : waldbauliche und ertragskundliche Untersuchungen. Hannover: Schaper.

Wiedemann, Eilhard (1949): Ertragstafeln der wichtigen Holzarten bei verschiedener Durchforstung. Hannover: Verlag M \& H Schaper.

Wiedemann, Eilhard (1951): Ertragskundliche und waldbauliche Grundlagen der Forstwirtschaft. 2. Aufl. Frankfurt (Main): J.D. Sauerländer's Verlag. 
Wilson, F. G. (1946): Numerical Expression of Stocking in Terms of Height. In: j for 44 (10), S. 758-761. DOI: 10.1093/jof/44.10.758.

Wippermann, C. (2005): Ökonomische Optimierung von Durchforstungen und Umtriebszeit. Eine modellgestützte Analyse am Beispiel der Kiefer. Zugl.:Göttingen, Univ., Diss., 2005. Frankfurt am Main: J.D. Sauerländer's Verlag (Schriften zur Forstökonomie, 30).

Wollborn, Peter; Böckmann, Thomas (1998): Ein praktikables Modell zur Strukturierung des Vorrates aus Ertragstafelschätzung (A Practicable Model for Diamter Structures of Stand Volumes estimated with Yield Tables). In: Forst und Holz 53 (18), S. 547-550.

Yoda, K.; Kira, T.; Ogawa, H.; Hozumi, K. (1963): Self-thinning in overcrowded pure stands under cultivated and natural conditions (Intraspecific competition among higher plants. XI). In: J. Inst. Polytech. Osaka City Univ. Ser. D. 14, S. 107-129.

Yousefpour, Rasoul; Hanewinkel, Marc (2016): Climate Change and Decision-Making Under Uncertainty. In: Curr Forestry Rep 2 (2), S. 143-149. DOI: 10.1007/s40725-016-0035-y.

Zingg, Andreas (2005): Ertragskunde - Waldbau auf wissenschaftlicher Grundlage: Plädoyer für einen akademisch abgestützten Waldbau. In: Schweizerische Zeitschrift fur Forstwesen 156 (12), S. 510-515. DOI: 10.3188/szf.2005.0510. 


\section{Anhang}

Sehr geehrte Damen und Herren,

die Abteilung Forstökonomie (Prof. Möhring) der Universität Göttingen führt im

Rahmen des FNR-Verbundvorhabens „Neue Generation dynamischer Ertragstafeln (dyn$E T)^{\text {“ }}$ eine Befragung zur Nutzung forstlicher Ertragstafeln durch.

Ziel ist es, die bisherige Anwendungspraxis von Ertragstafeln in privaten und öffentlichen Forstbetrieben, Verwaltungen sowie bei forstlichen Dienstleistern, Sachverständigen und Beratungsunternehmen zu analysieren.

Die gewonnenen Erkenntnisse sollen bei der Konzeption einer neuen Generation dynamischer Ertragstafeln berücksichtigt werden.

Ihre Expertise im Bereich der Anwendung von Ertragstafeln möchten wir gerne im Zuge eines ca. 15-minütigen Telefonats (strukturiertes Interview) nutzen.

Folgende Themenfelder würden Sie dabei erwarten:

- Anwendungsgebiete forstlicher Ertragstafeln in Ihrem Betrieb/Unternehmen

- Verwendete Ertragstafeln (Hauptbaumarten) in Ihrem Betrieb/Unternehmen

- Rechnerische Überarbeitungen der Ertragstafeln

- Anpassungen an Bestandesdichte, Baumartenmischung und Bestandesschichten

- Kombination mit Sortentafeln und Erlös-/Kostendaten für z. B. betriebliche Kalkulationen

- Stärken/Vorteile und Schwächen/Nachteile etablierter Ertragstafeln

- Einschätzung der zukünftigen Verwendung forstlicher Ertragstafeln

Wir würden uns freuen, wenn Sie uns als Antwort auf diese Mail einen passenden Termin sowie Ihre Telefonnummer für ein strukturiertes Interview nennen - wir rufen Sie entsprechend an.

Sollten Sie persönlich nicht zur Verfügung stehen, freuen wir uns über die Nennung geeigneter Kontaktpersonen.

Schon jetzt möchten wir uns ganz herzlich für Ihre Unterstützung bedanken! Für Rückfragen und Anmerkungen stehen wir Ihnen gerne zur Verfügung.

Mit freundlichen Grüßen

Roman Koster

Abbildung 151: Anschreiben der Mailanfrage an 163 gezielt ausgewählte Personen für die Durchführung eines leitfadengestützten Telefoninterviews zur Praxisanwendung forstlicher Ertragstafeln. 


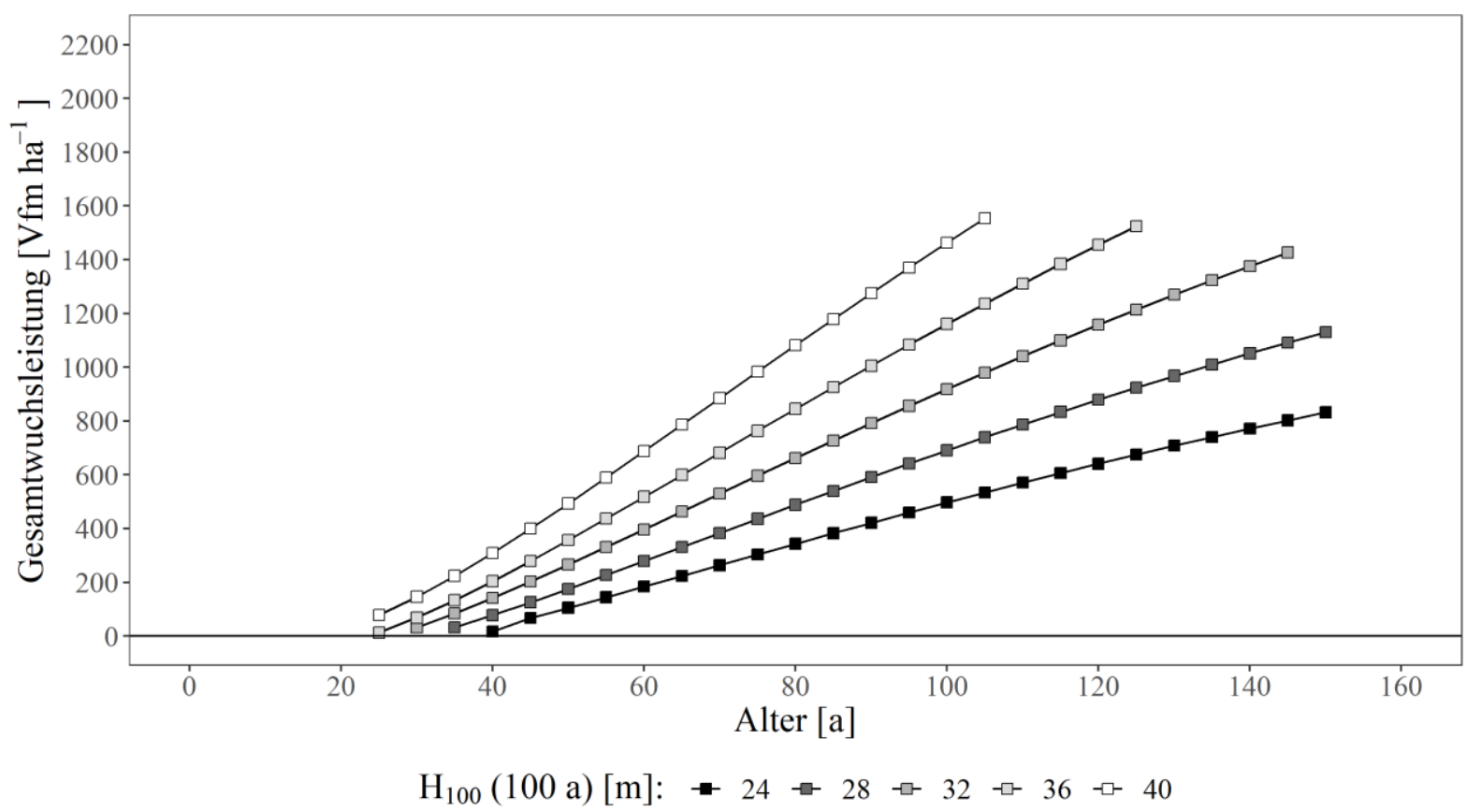

Abbildung 152: Gesamtwuchsleistung über dem Alter; alle Oberhöhenbonitäten der Baumart Buche (starke Hochdurchforstung) gemäß dyn-ET (unveröff.).

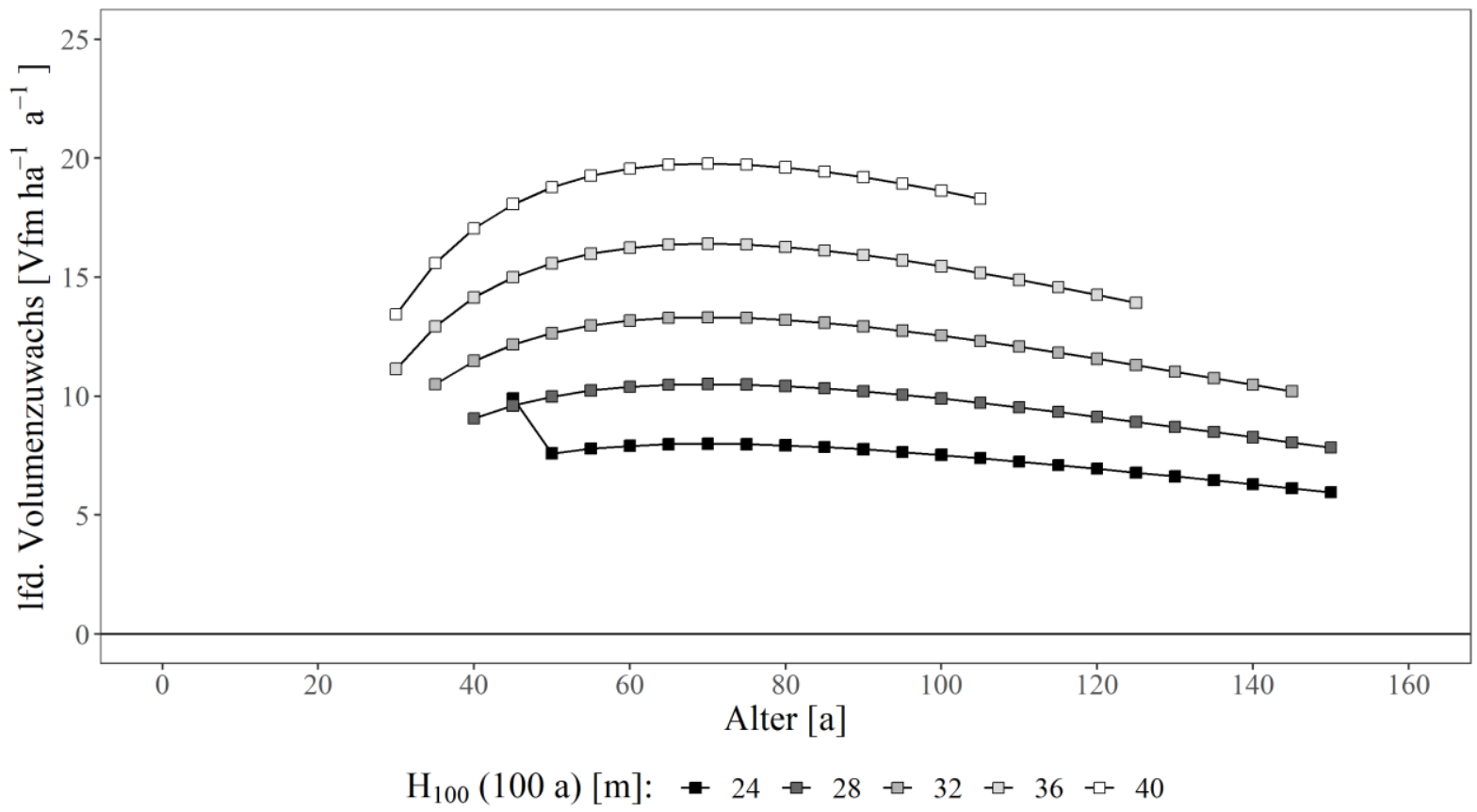

Abbildung 153:Laufender Bestandesvolumenzuwachs über dem Alter; alle Oberhöhenbonitäten der Baumart Buche (starke Hochdurchforstung) gemäß dyn-ET (unveröff.). 


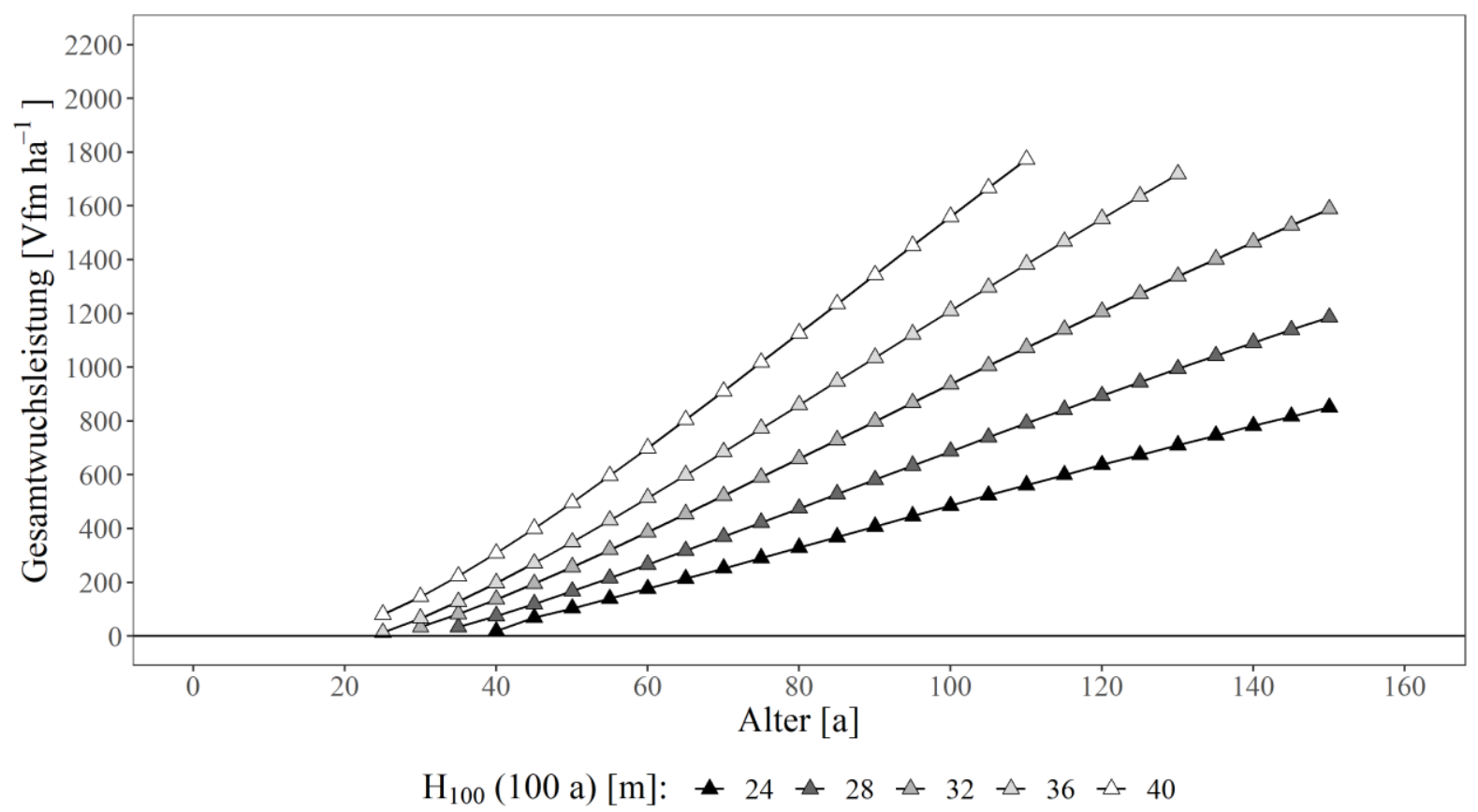

Abbildung 154: Gesamtwuchsleistung über dem Alter; alle Oberhöhenbonitäten der Baumart Buche (gestaffelte Hochdurchforstung) gemäß dyn-ET (unveröff.).

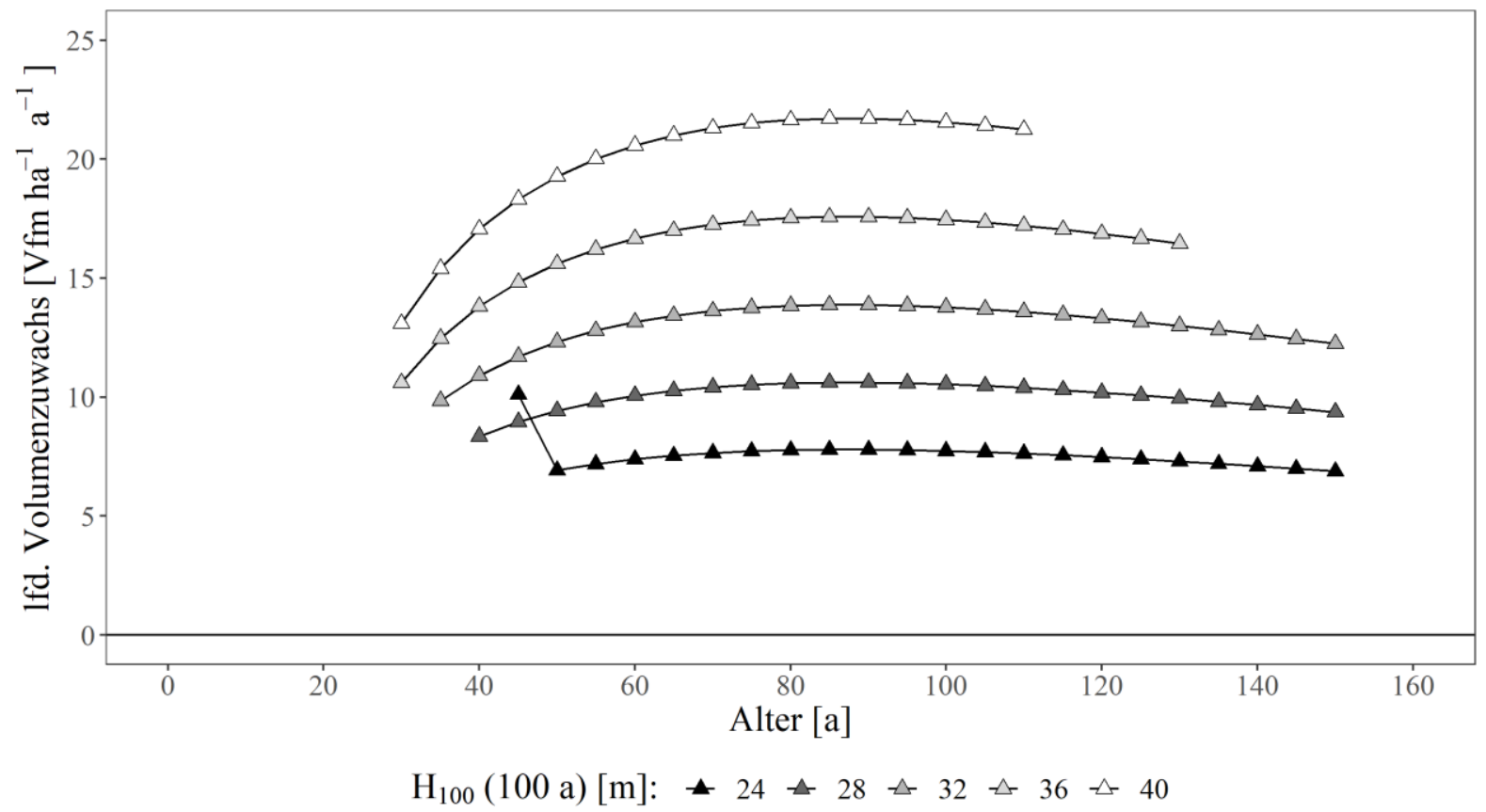

Abbildung 155: Laufender Bestandesvolumenzuwachs über dem Alter; alle Oberhöhenbonitäten der Baumart Buche (gestaffelte Hochdurchforstung) gemäß dyn-ET (unveröff.). 


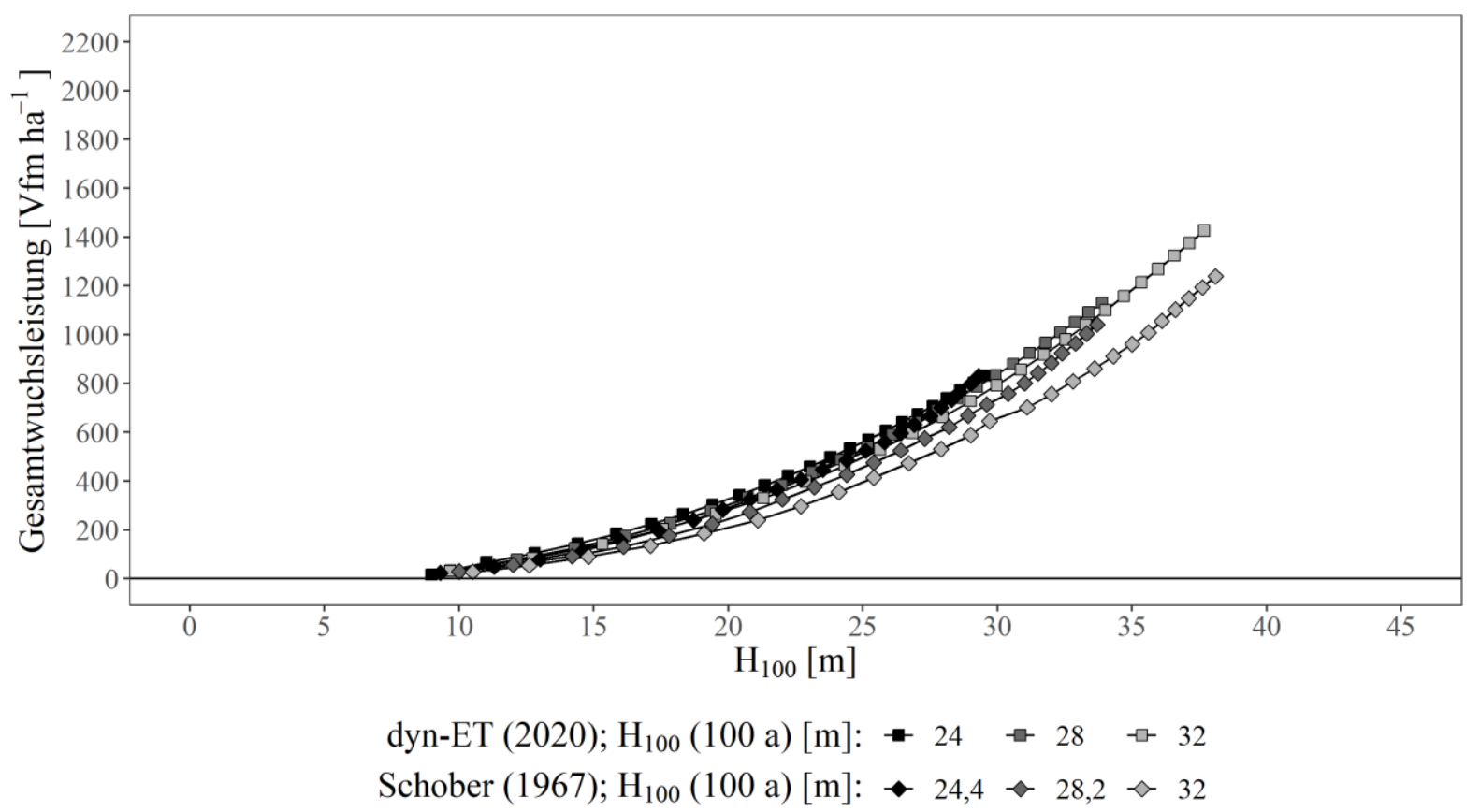

Abbildung 156: Gesamtwuchsleistung über der Oberhöhe; ausgewählte Oberhöhenbonitäten der Baumart Buche (starke Durchforstung) gemäß dyn-ET (unveröff.) und Schober (1967).

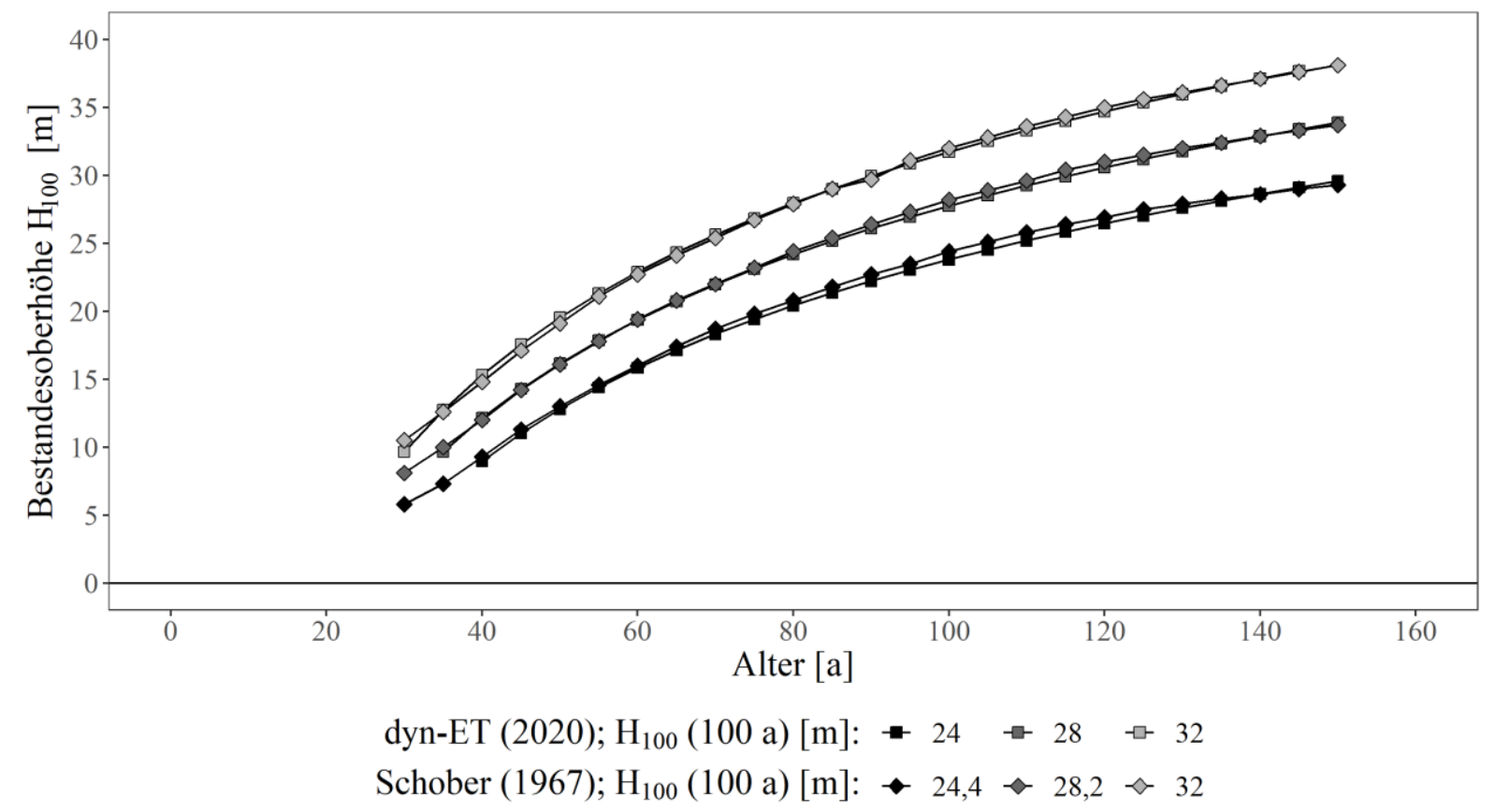

Abbildung 157: Oberhöhe über dem Alter; ausgewählte Oberhöhenbonitäten der Baumart Buche (starke Durchforstung) gemäß dyn-ET (unveröff.) und Schober (1967). 


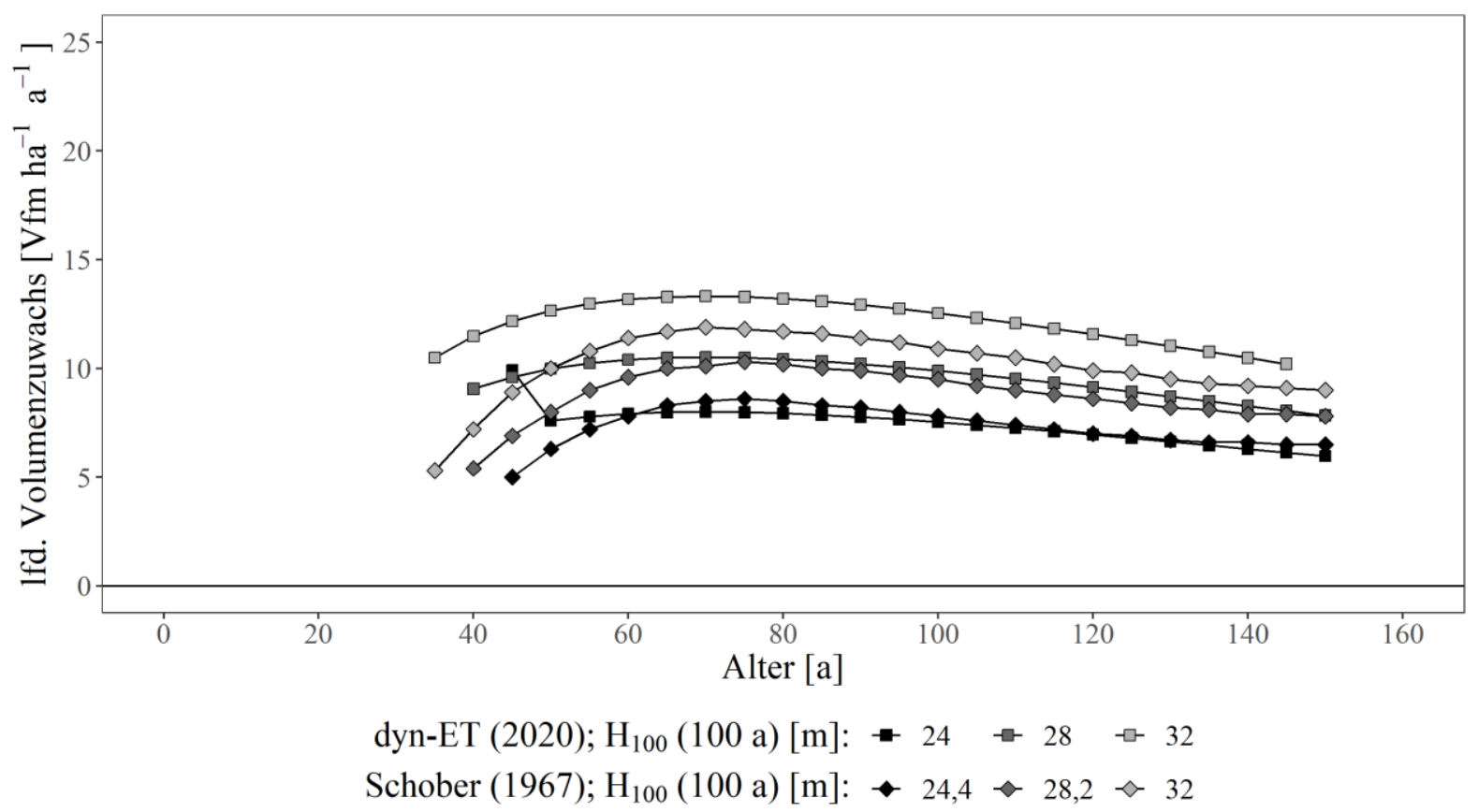

Abbildung 158: Laufender Bestandesvolumenzuwachs über dem Alter; ausgewählte Oberhöhenbonitäten der Baumart Buche (starke Durchforstung) gemäß dyn-ET (unveröff.) und Schober (1967).

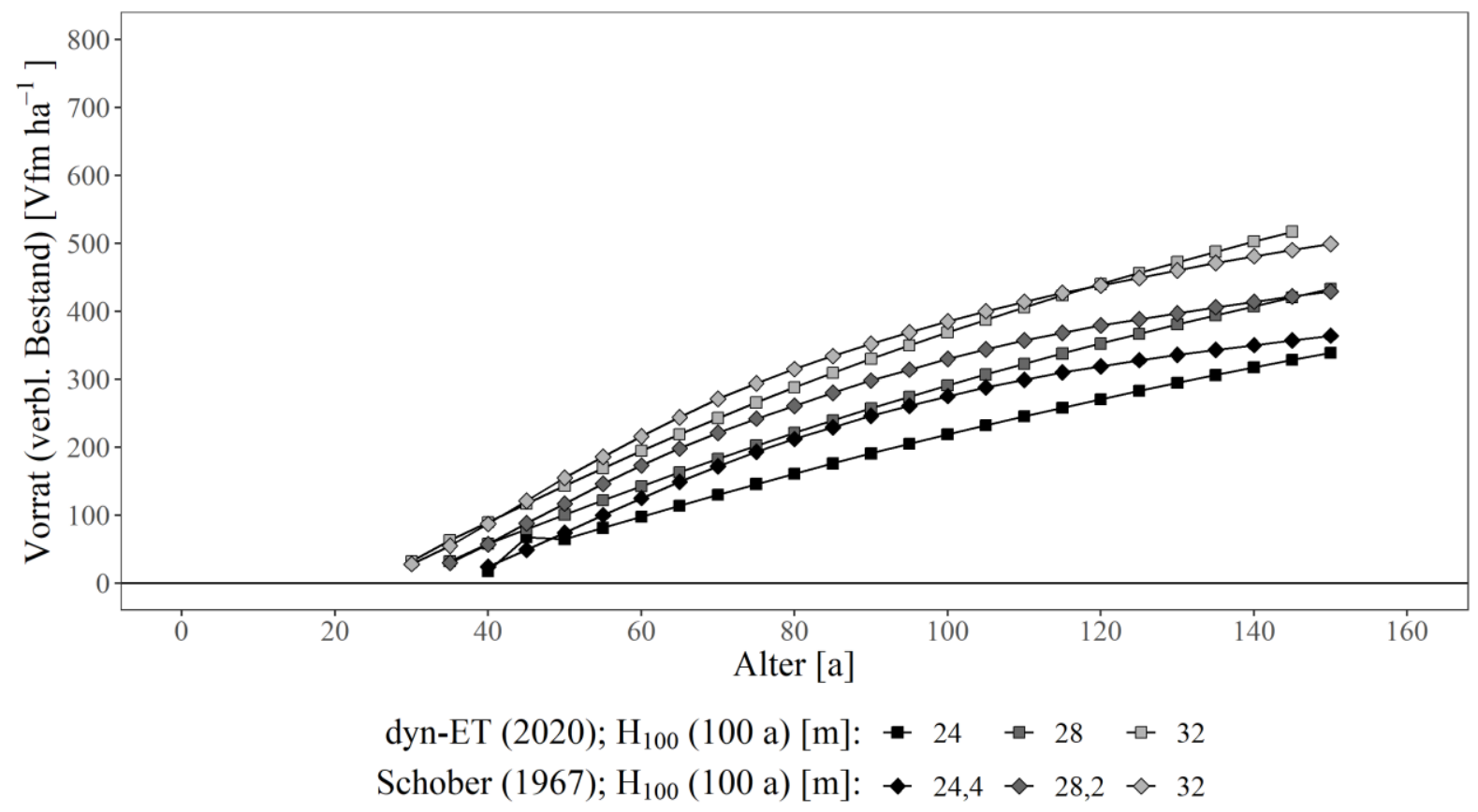

Abbildung 159: Vorrat (verbleibender Bestand) über dem Alter; ausgewählte Oberhöhenbonitäten der Baumart Buche (starke Durchforstung) gemäß dyn-ET (unveröff.) und Schober (1967). 


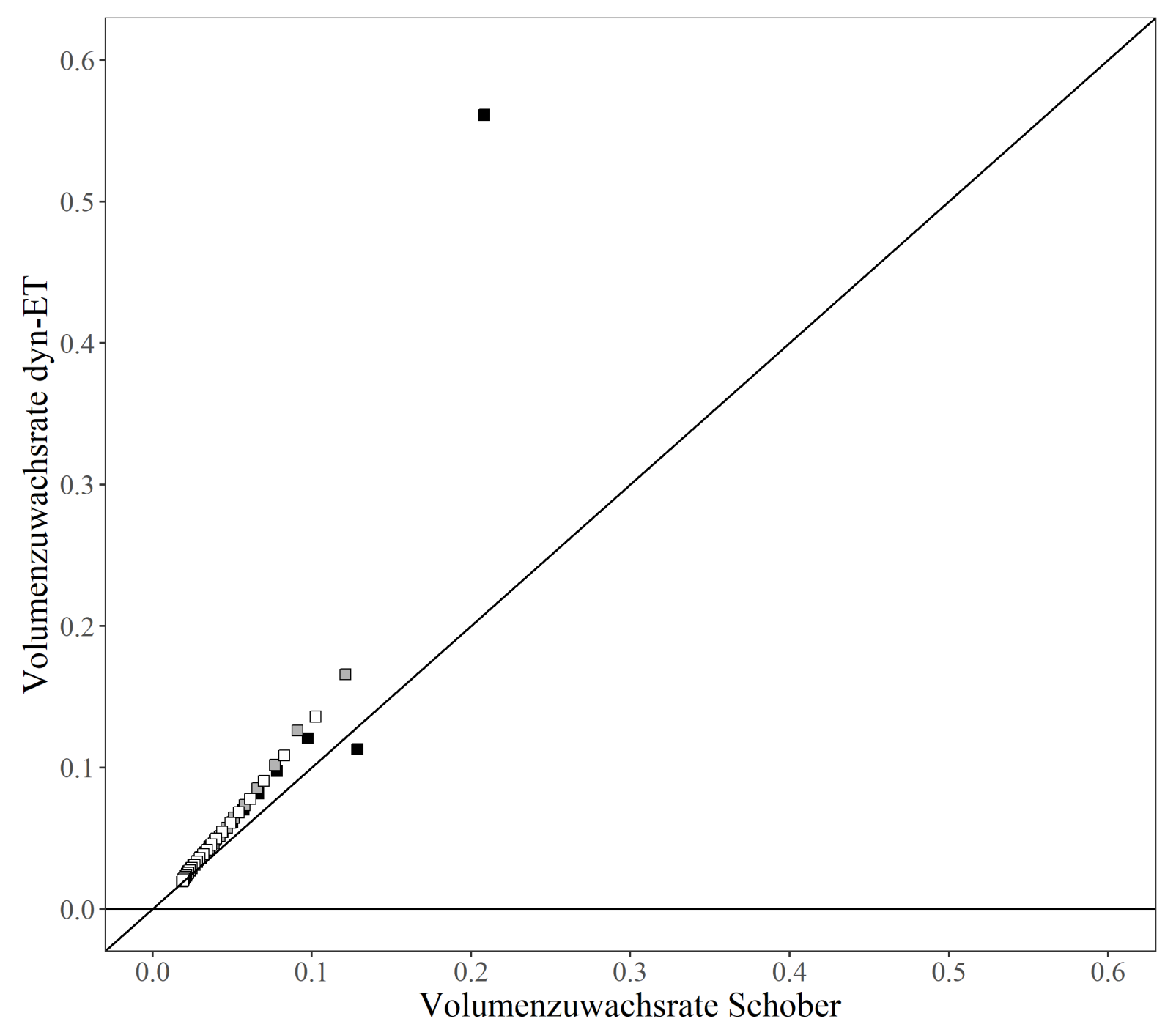

$\mathrm{H}_{100}(100 \mathrm{a})[\mathrm{m}]: \quad$ - 24/24,4 $\square \quad 28 / 28,2 \quad \square \quad 32 / 32$

Abbildung 160: Volumenzuwachsraten der Baumart Buche (starke Durchforstung, ausgewählte Oberhöhenbonitäten) gemäß dyn-ET (unveröff.) und Schober (1967); es werden nur Vergleichspaare aus Altern dargestellt, in denen für alle Oberhöhenbonitäten Datenpunkte vorliegen; die Winkelhalbierende kennzeichnet gleiche Wertzuwachsraten im gleichen Alter. 


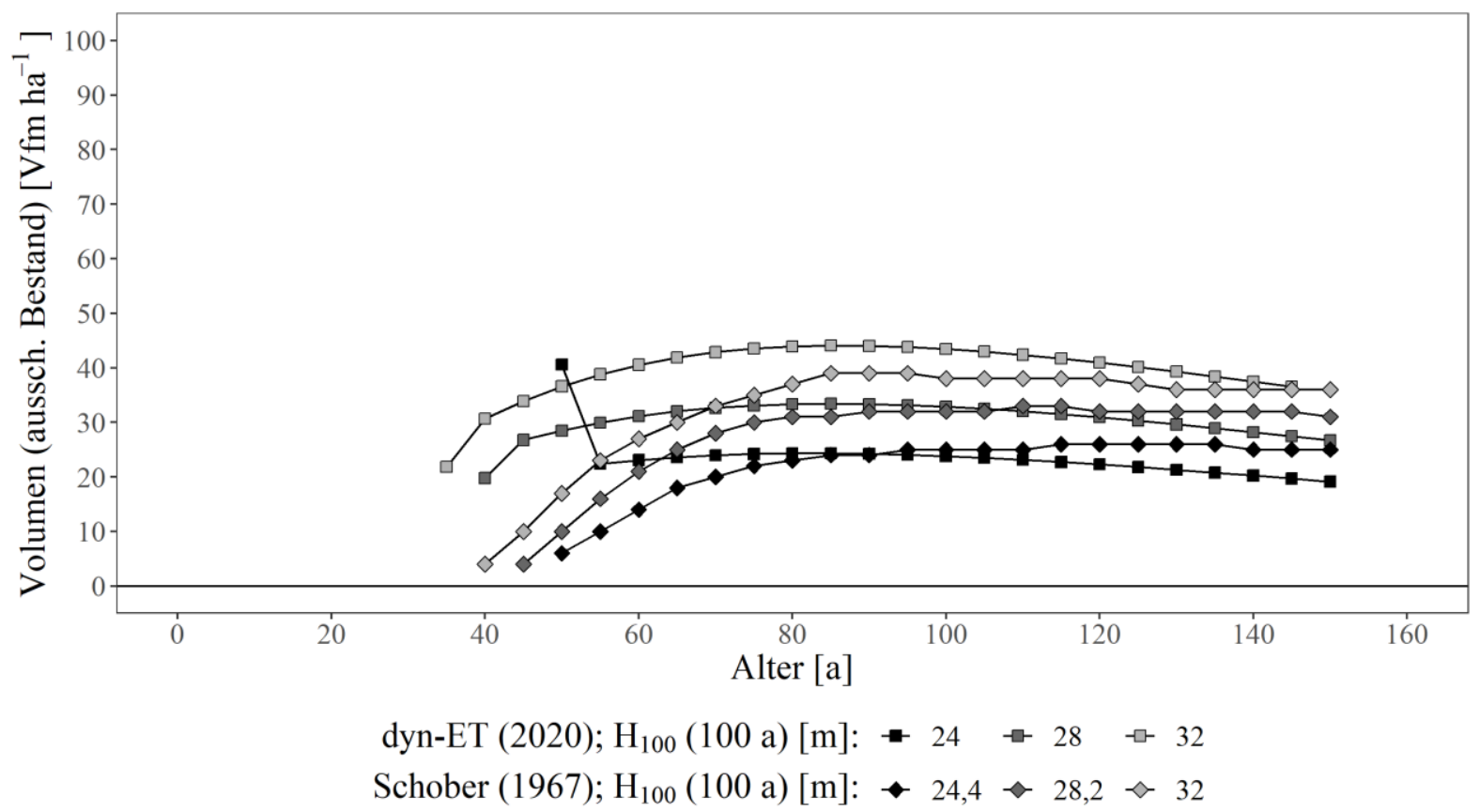

Abbildung 161: Ausscheidendes Bestandesvolumen über dem Alter; ausgewählte Oberhöhenbonitäten der Baumart Buche (starke Durchforstung) gemäß dyn-ET (unveröff.) und Schober (1967).

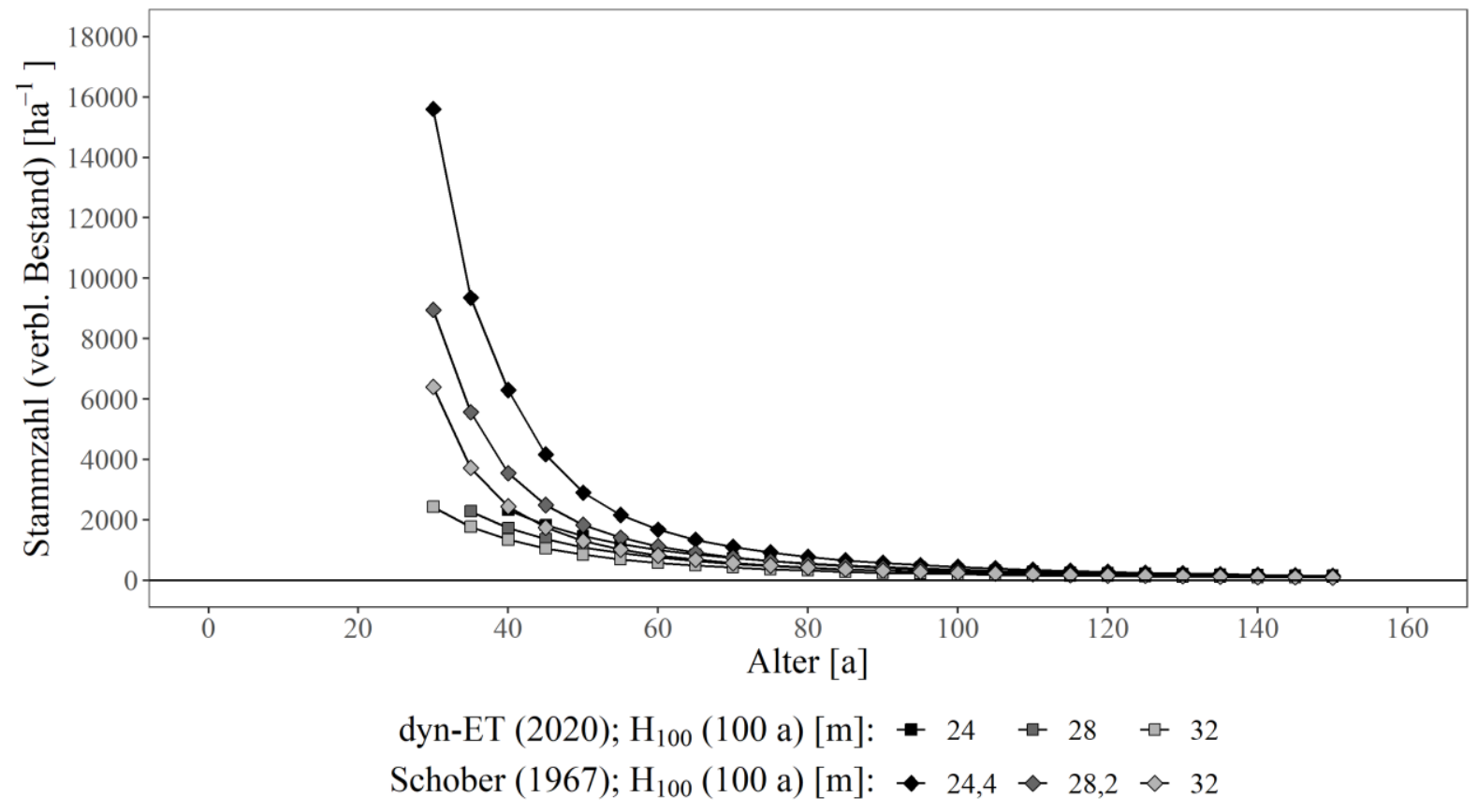

Abbildung 162: Stammzahl (verbleibender Bestand) über dem Alter; ausgewählte Oberhöhenbonitäten der Baumart Buche (starke Durchforstung) gemäß dyn-ET (unveröff.) und Schober (1967). 


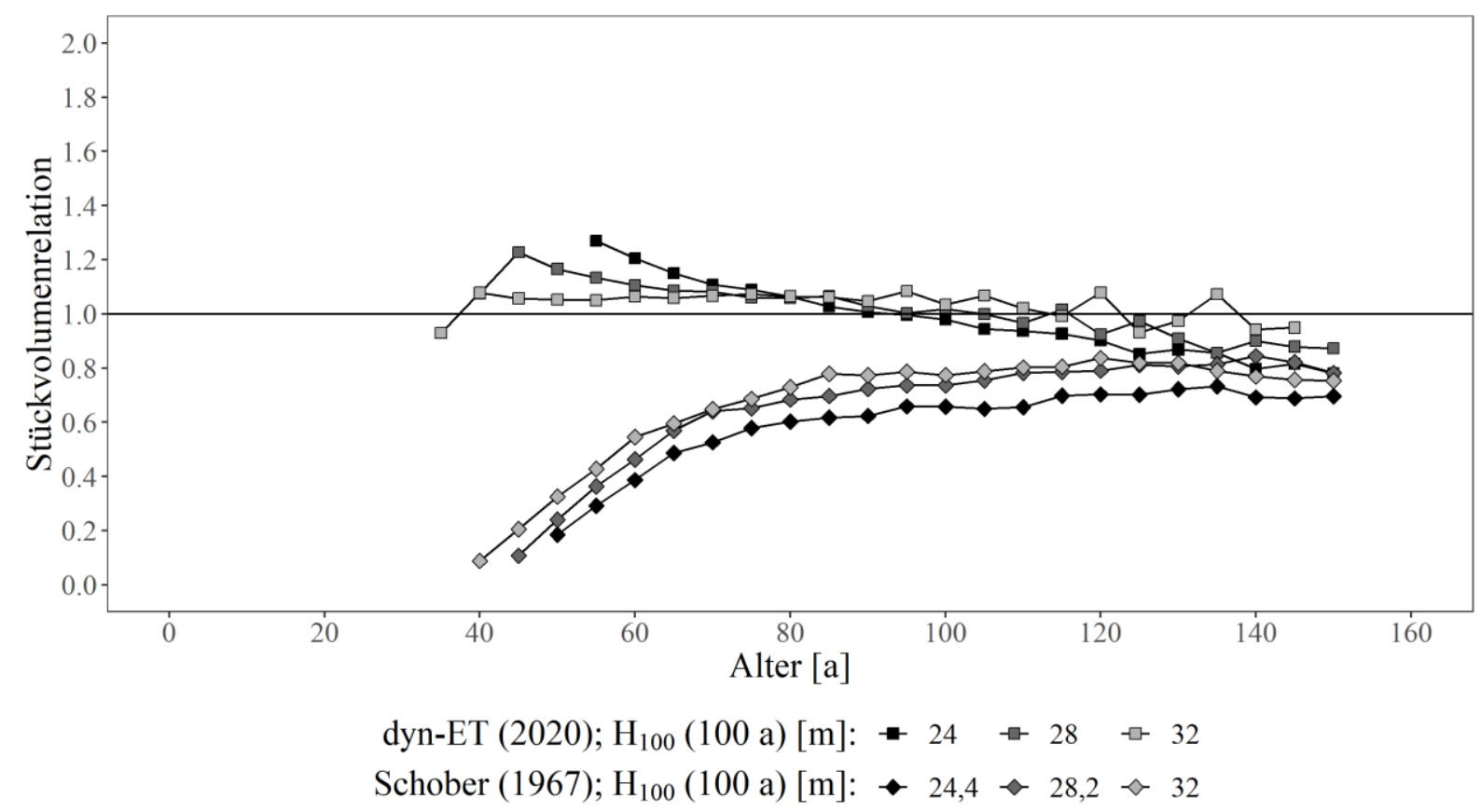

Abbildung 163: Stückvolumenrelationen (ausscheidendes geteilt durch verbleibendes Stückvolumen) über dem Alter; ausgewählte Oberhöhenbonitäten der Baumart Buche (starke Durchforstung) gemäß dyn-ET (unveröff.) und Schober (1967).

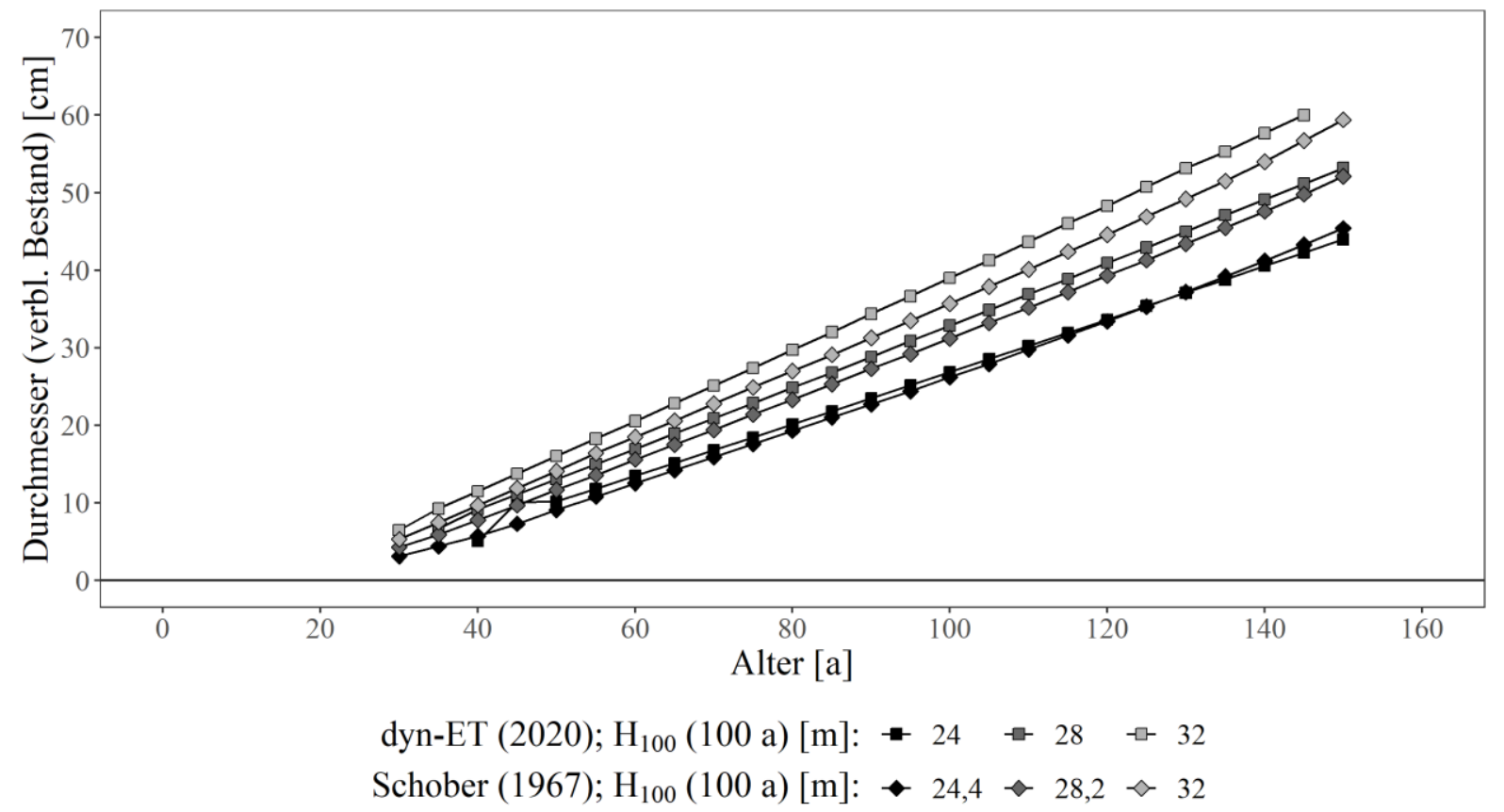

Abbildung 164: Durchmesser des Grundflächenmittelstamms (verbleibender Bestand) über dem Alter; ausgewählte Oberhöhenbonitäten der Baumart Buche (starke Durchforstung) gemäß dyn-ET (unveröff.) und Schober (1967). 


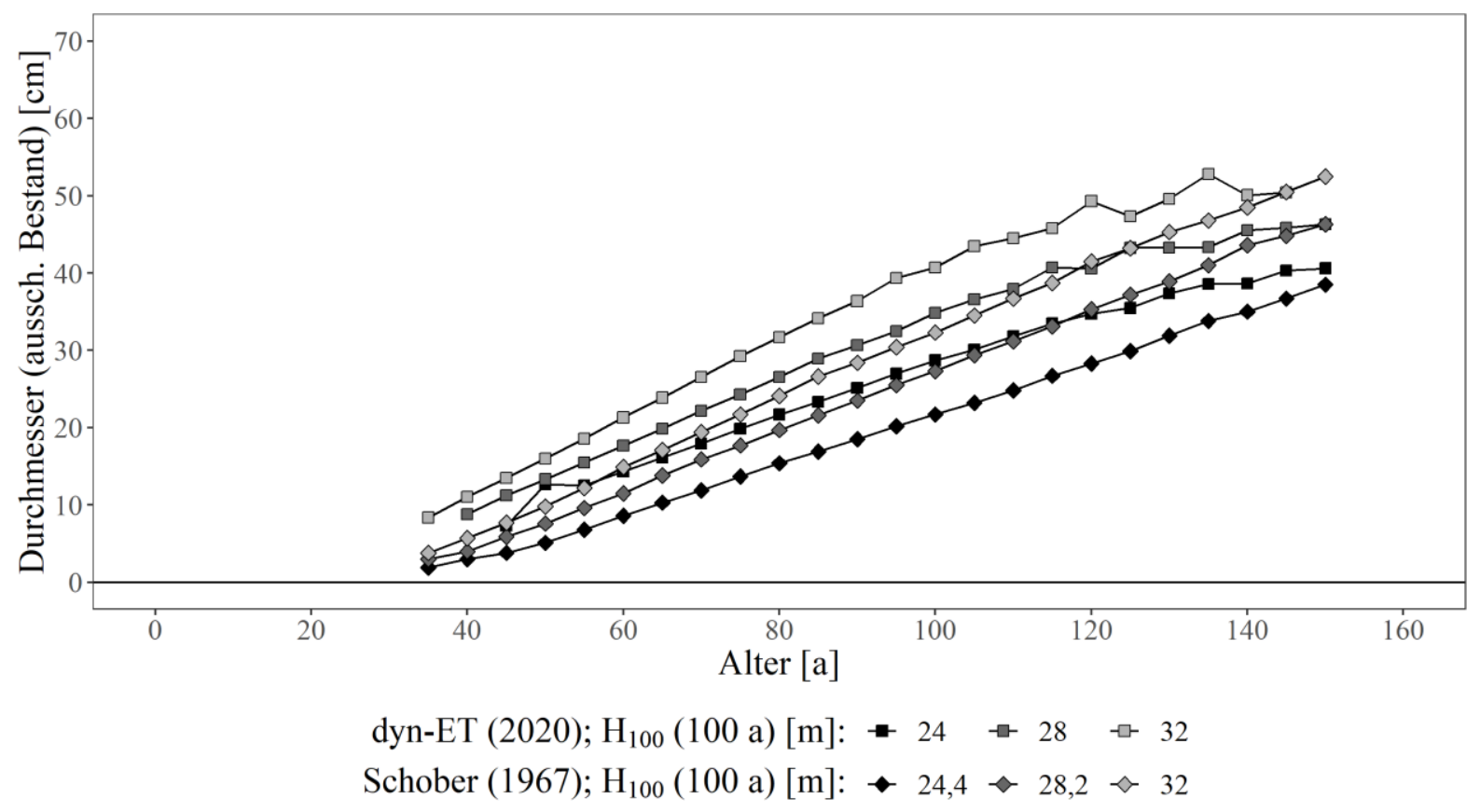

Abbildung 165: Durchmesser des Grundflächenmittelstamms (ausscheidender Bestand) über dem Alter; ausgewählte Oberhöhenbonitäten der Baumart Buche (starke Durchforstung) gemäß dyn-ET (unveröff.) und Schober (1967).

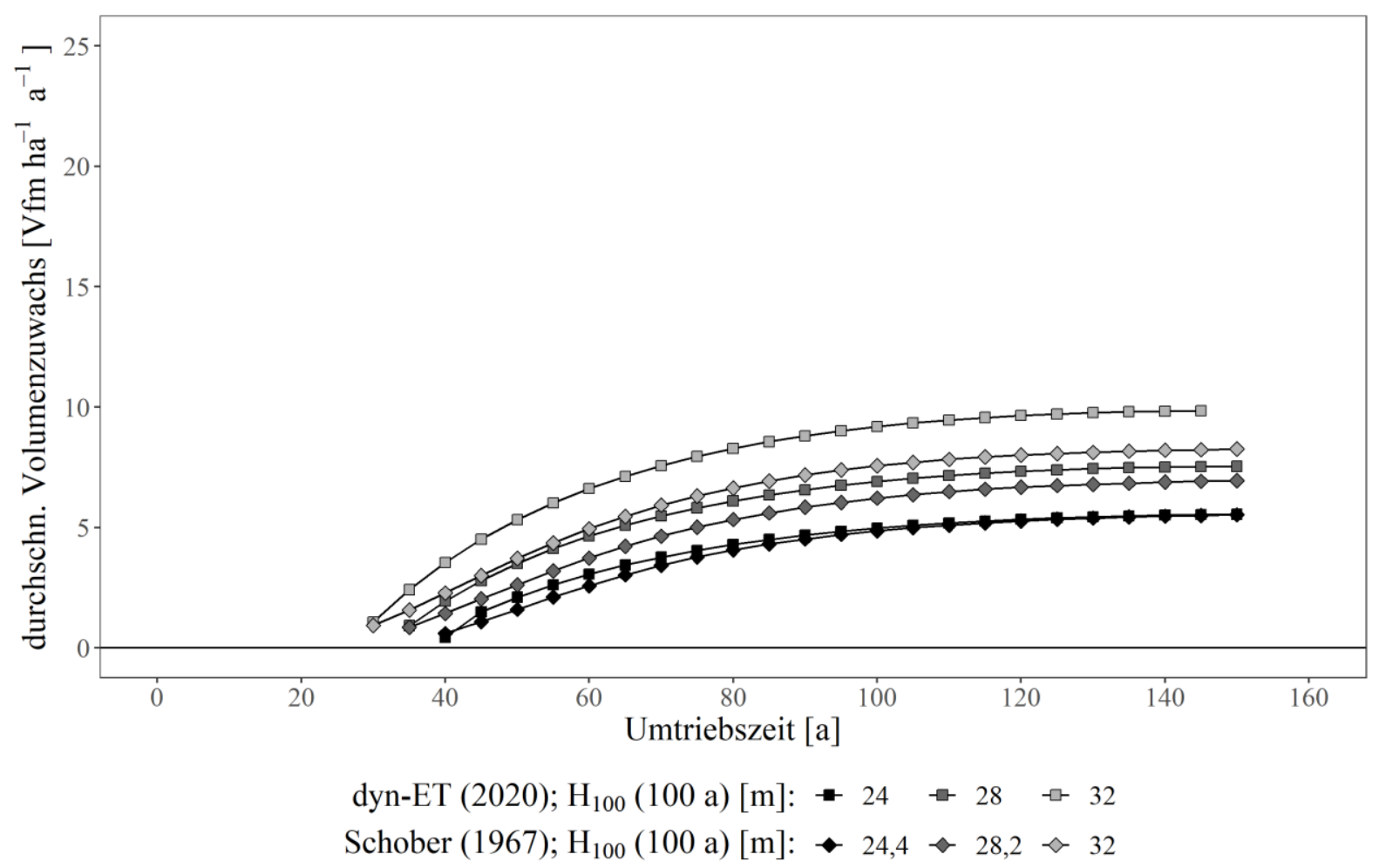

Abbildung 166: Durchschnittlicher Bestandesvolumenzuwachs über der Umtriebszeit; ausgewählte Oberhöhenbonitäten der Baumart Buche (starke Durchforstung) gemäß dyn-ET (unveröff.) und Schober (1967). 


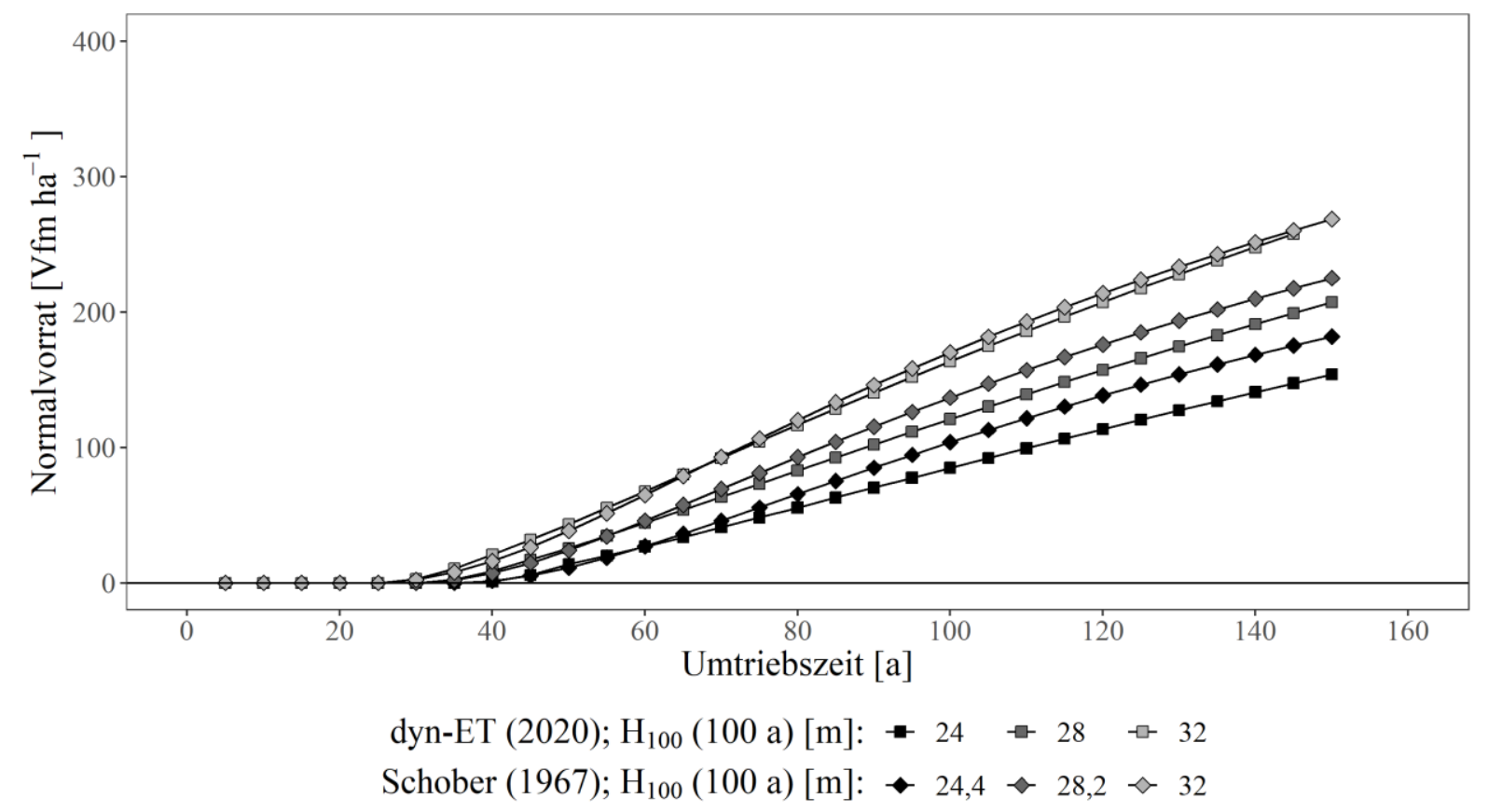

Abbildung 167: Normalvorrat über der Umtriebszeit; ausgewählte Oberhöhenbonitäten der Baumart Buche (starke Durchforstung) gemäß dyn-ET (unveröff.) und Schober (1967).

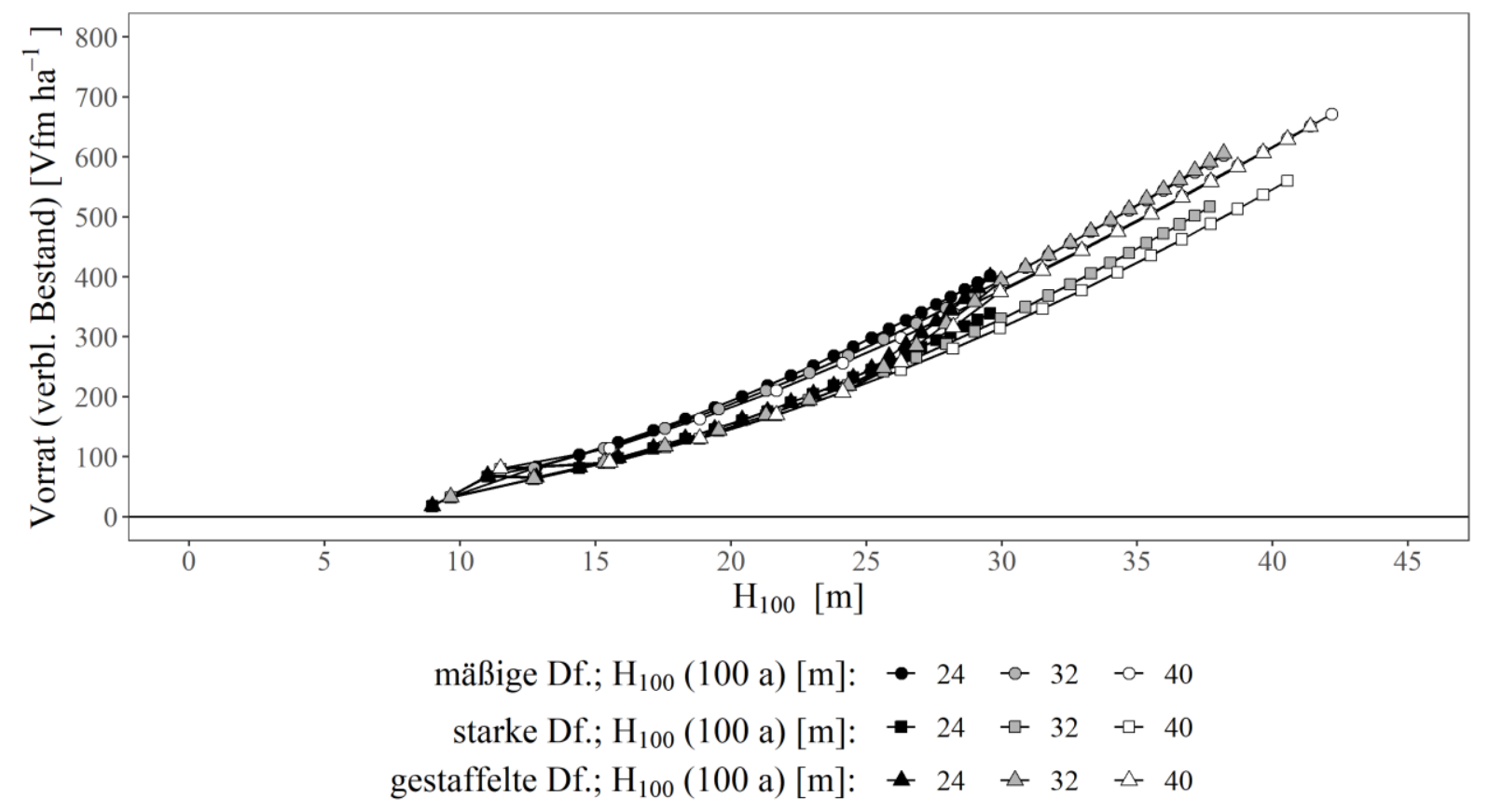

Abbildung 168: Vorrat (verbl. Bestand) über der Oberhöhe; ausgewählte Oberhöhenbonitäten der Baumart Buche getrennt nach Behandlungsvarianten gemäß dyn-ET (unveröff.). 


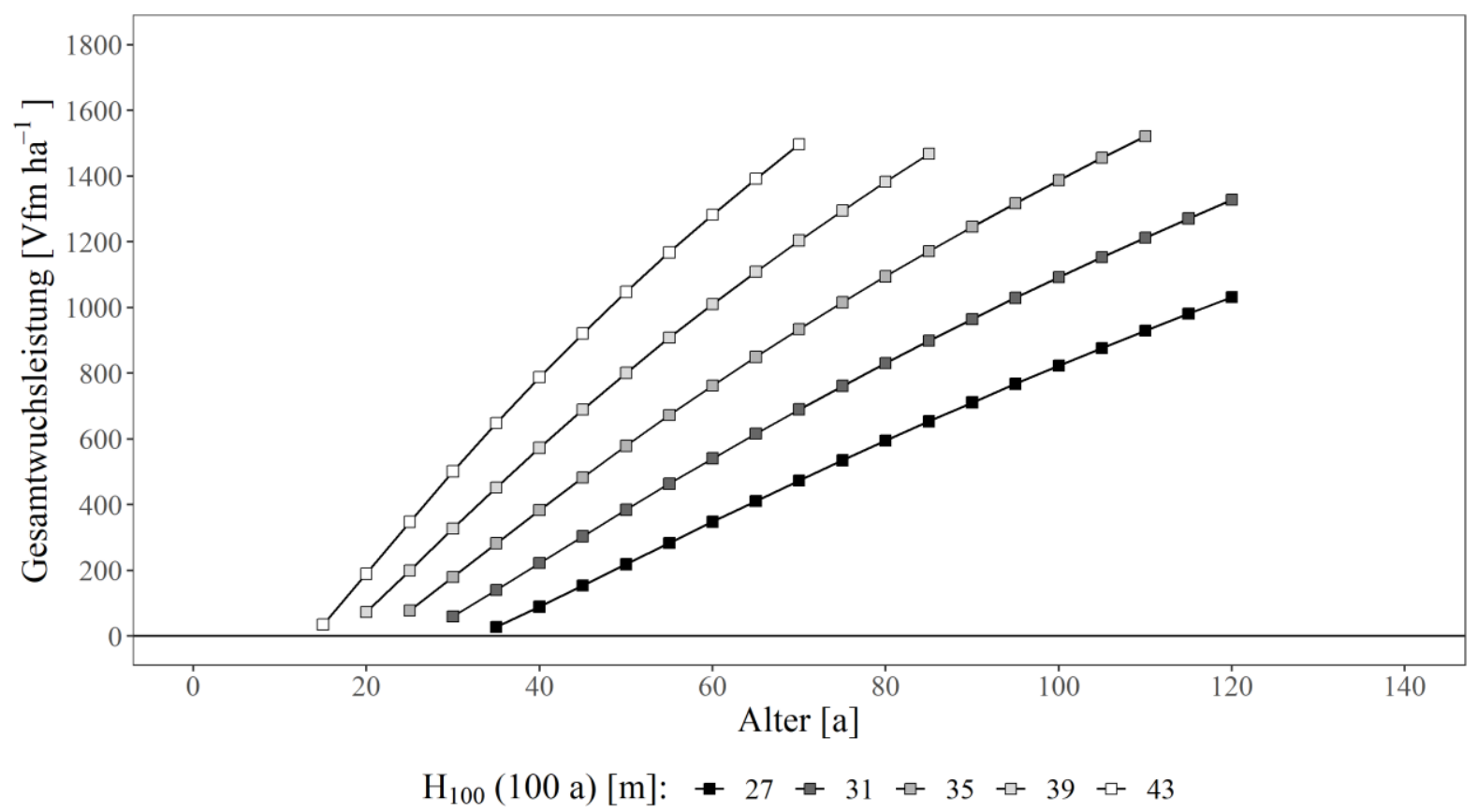

Abbildung 169: Gesamtwuchsleistung über dem Alter; alle Oberhöhenbonitäten der Baumart Fichte (starke Hochdurchforstung) gemäß dyn-ET (unveröff.).

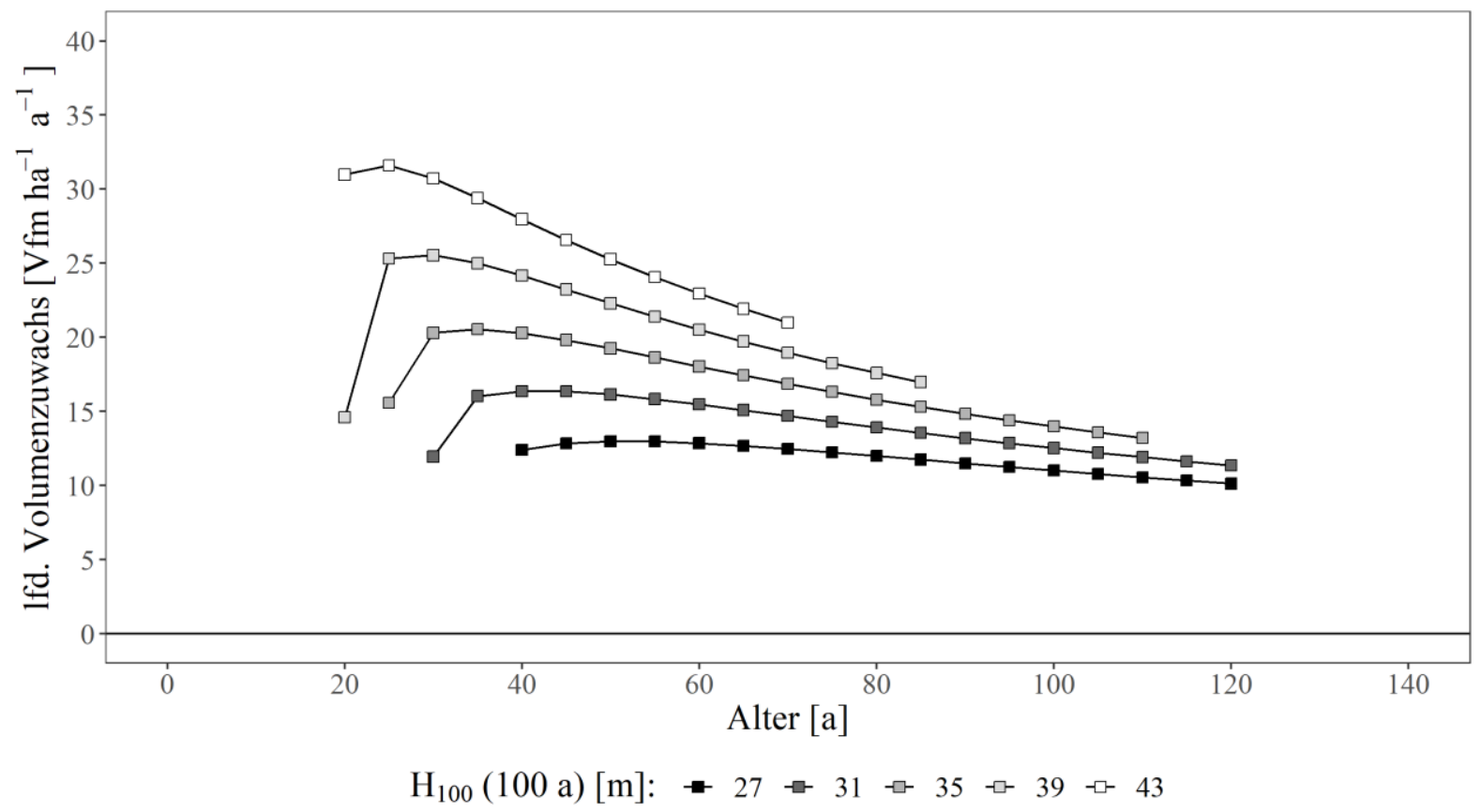

Abbildung 170: Laufender Bestandesvolumenzuwachs über dem Alter; alle Oberhöhenbonitäten der Baumart Fichte (starke Hochdurchforstung) gemäß dyn-ET (unveröff.). 


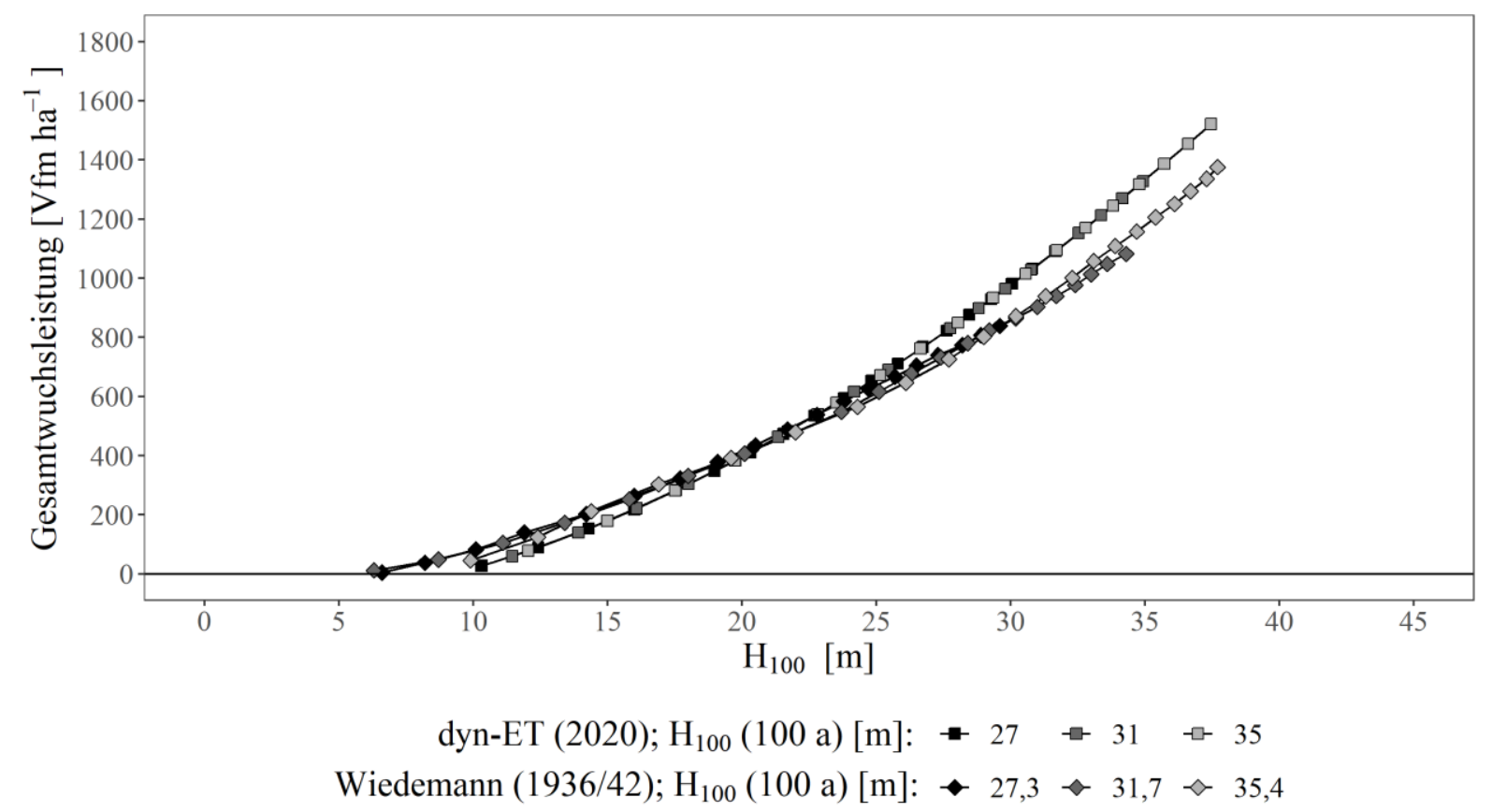

Abbildung 171: Gesamtwuchsleistung über der Oberhöhe; ausgewählte Oberhöhenbonitäten der Baumart Fichte (starke Durchforstung) gemäß dyn-ET (unveröff.) und Wiedemann (1936/42).

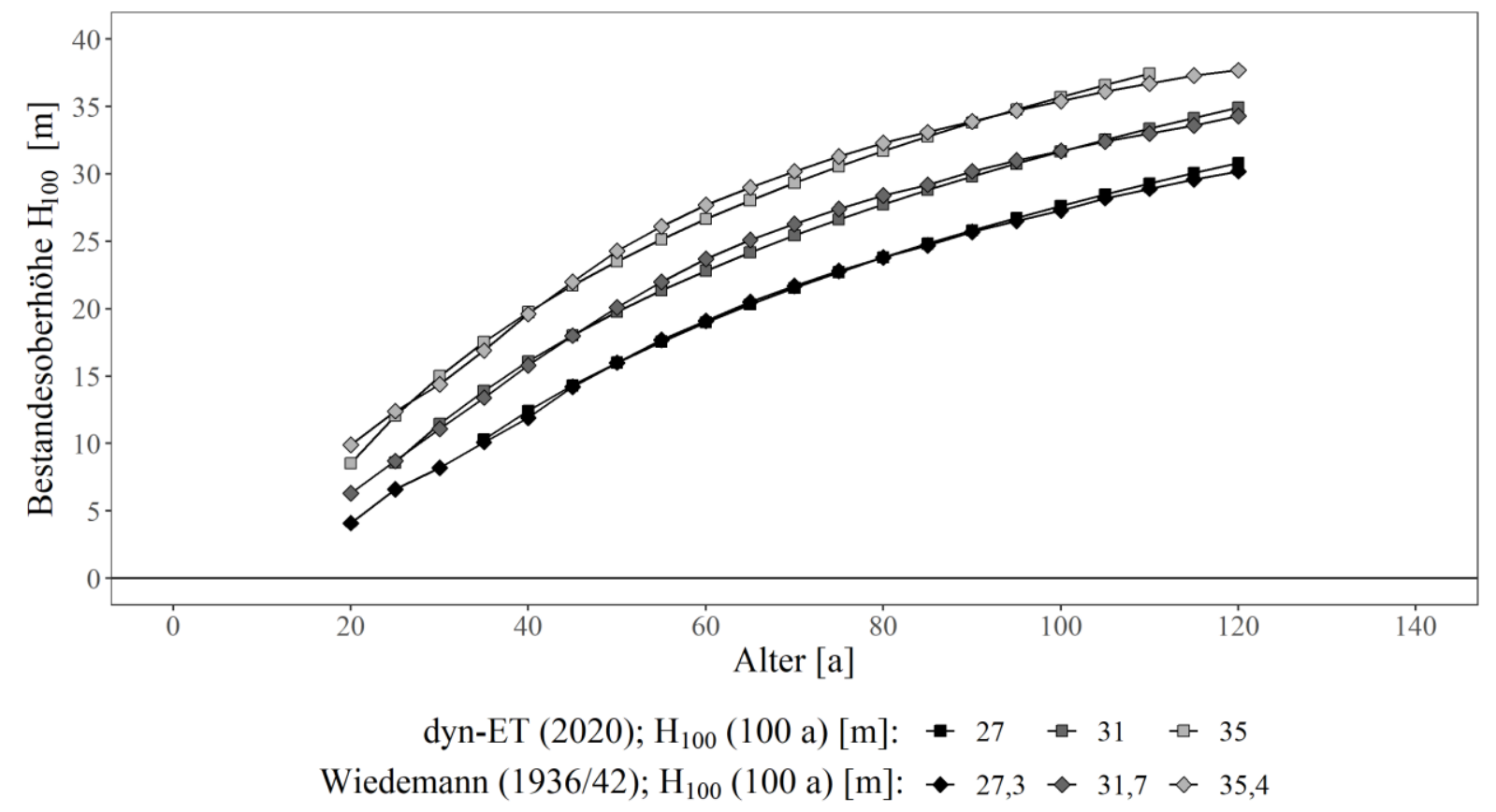

Abbildung 172: Oberhöhe über dem Alter; ausgewählte Oberhöhenbonitäten der Baumart Fichte (starke Durchforstung) gemäß dyn-ET (unveröff.) und Wiedemann (1936/42). 


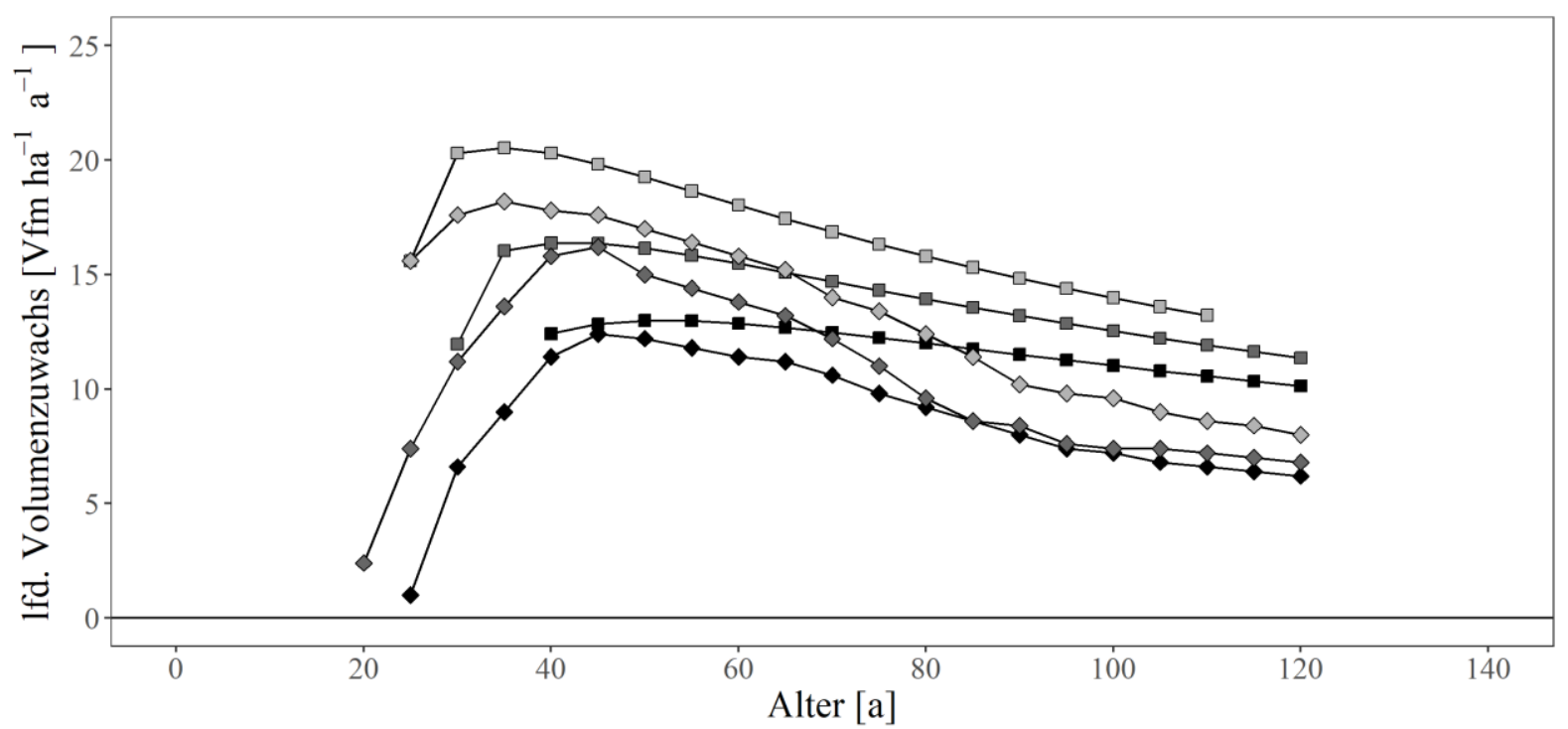

dyn-ET (2020); $\mathrm{H}_{100}(100 \mathrm{a})[\mathrm{m}]:-27 \rightarrow 31 \rightarrow 35$

Wiedemann (1936/42); $\mathrm{H}_{100}(100 \mathrm{a})[\mathrm{m}]: \bullet 27,3 \diamond 31,7 \diamond 35,4$

Abbildung 173: Laufender Bestandesvolumenzuwachs über dem Alter; ausgewählte Oberhöhenbonitäten der Baumart Fichte (starke Durchforstung) gemäß dyn-ET (unveröff.) und Wiedemann (1936/42).

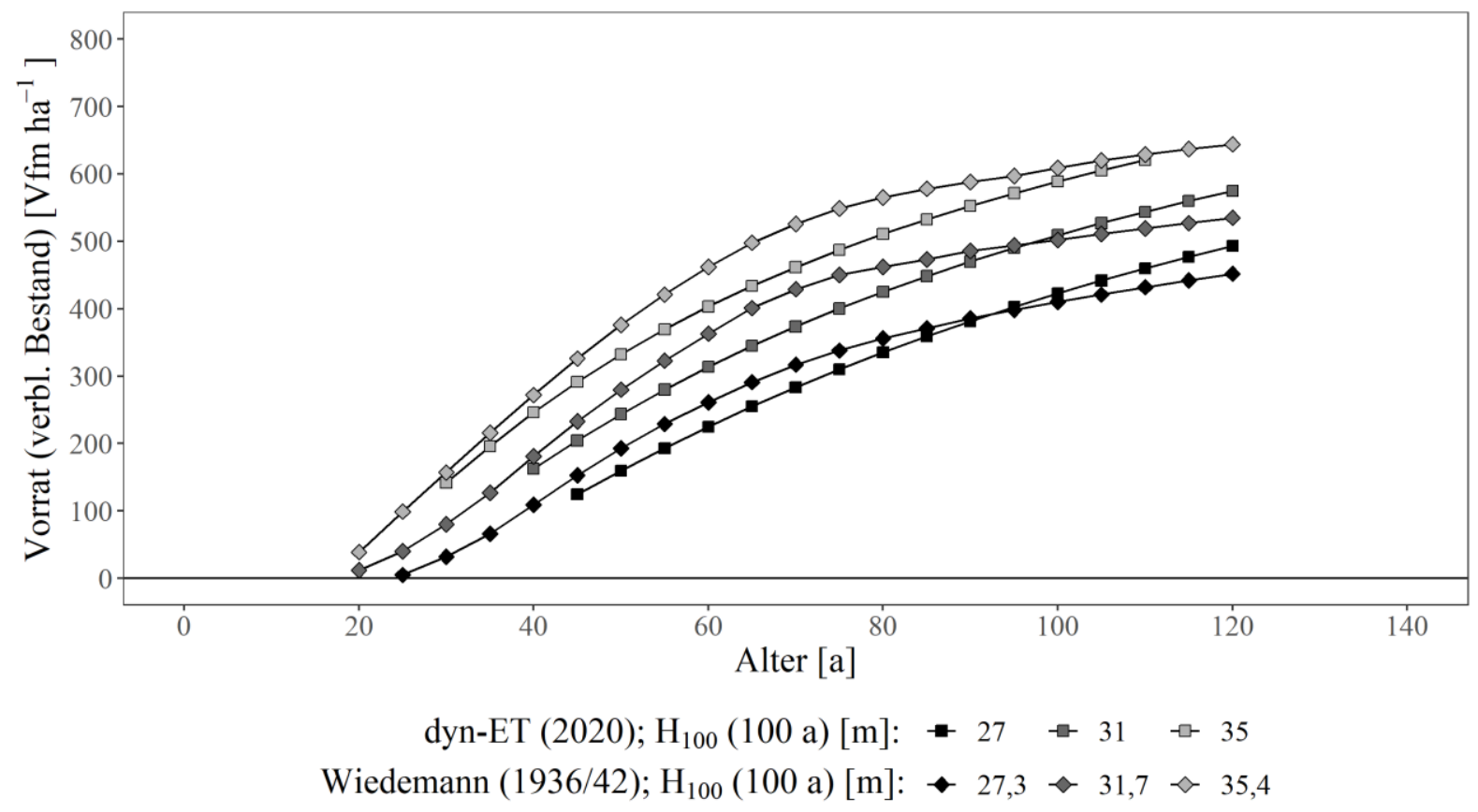

Abbildung 174: Vorrat (verbleibender Bestand) über dem Alter; ausgewählte Oberhöhenbonitäten der Baumart Fichte (starke Durchforstung) gemäß dyn-ET (unveröff.) und Wiedemann (1936/42). 


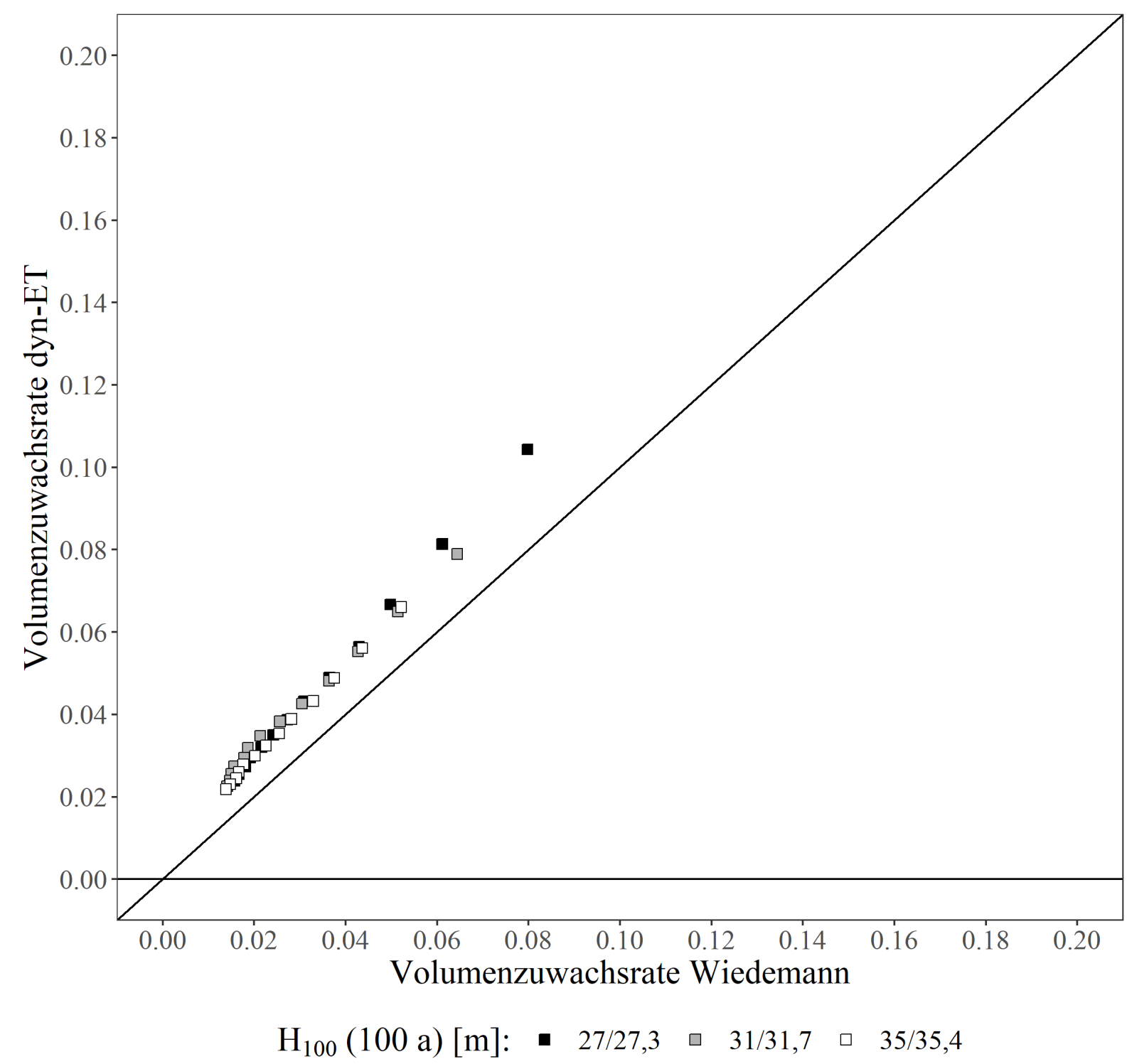

Abbildung 175: Volumenzuwachsraten der Baumart Fichte (starke Durchforstung, ausgewählte Oberhöhenbonitäten) gemäß dyn-ET (unveröff.) und Wiedemann (1936/42); es werden nur Vergleichspaare aus Altern dargestellt, in denen für alle Oberhöhenbonitäten Datenpunkte vorliegen; die Winkelhalbierende kennzeichnet gleiche Wertzuwachsraten im gleichen Alter. 


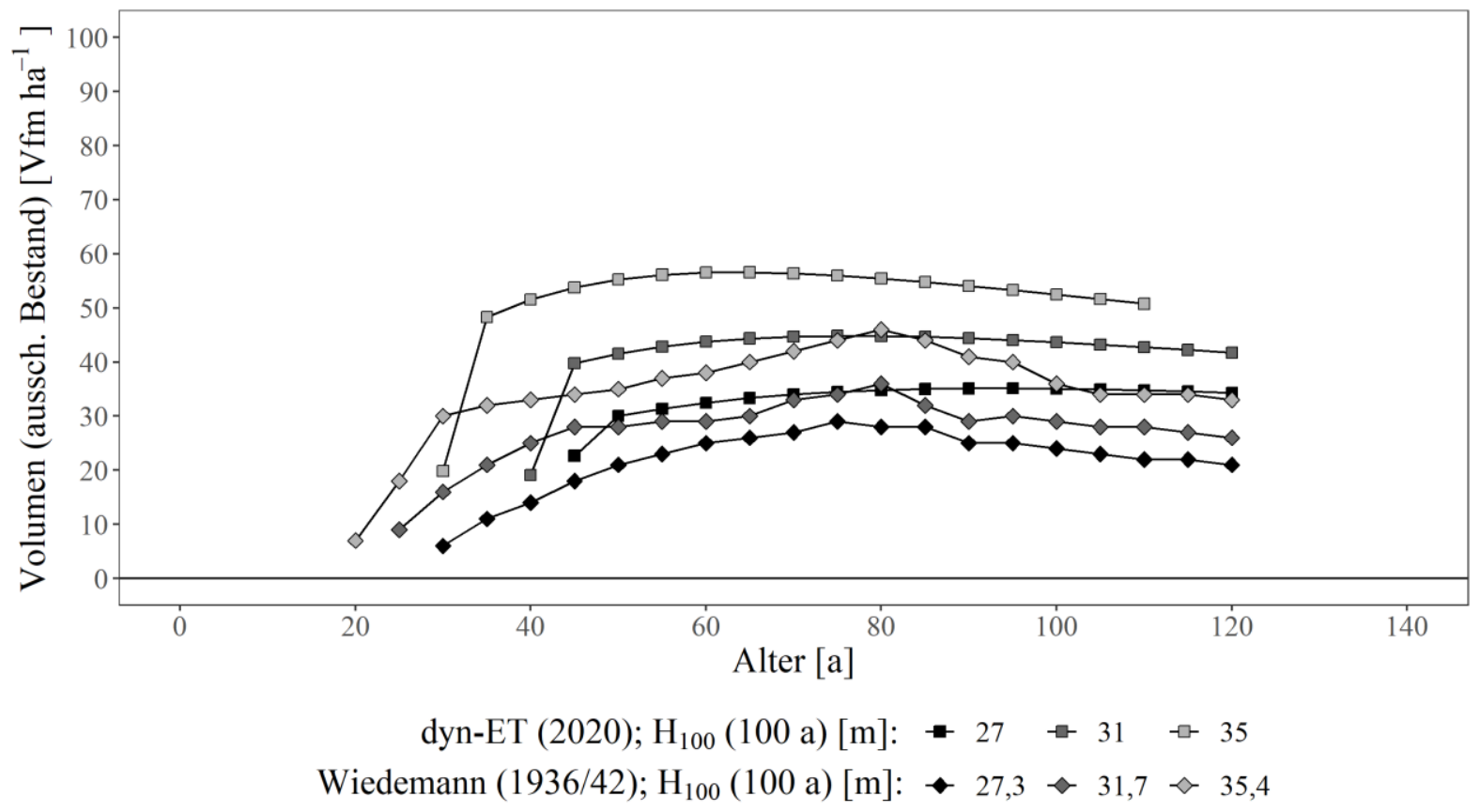

Abbildung 176: Ausscheidendes Bestandesvolumen über dem Alter; ausgewählte Oberhöhenbonitäten der Baumart Fichte (starke Durchforstung) gemäß dyn-ET (unveröff.) und Wiedemann (1936/42).

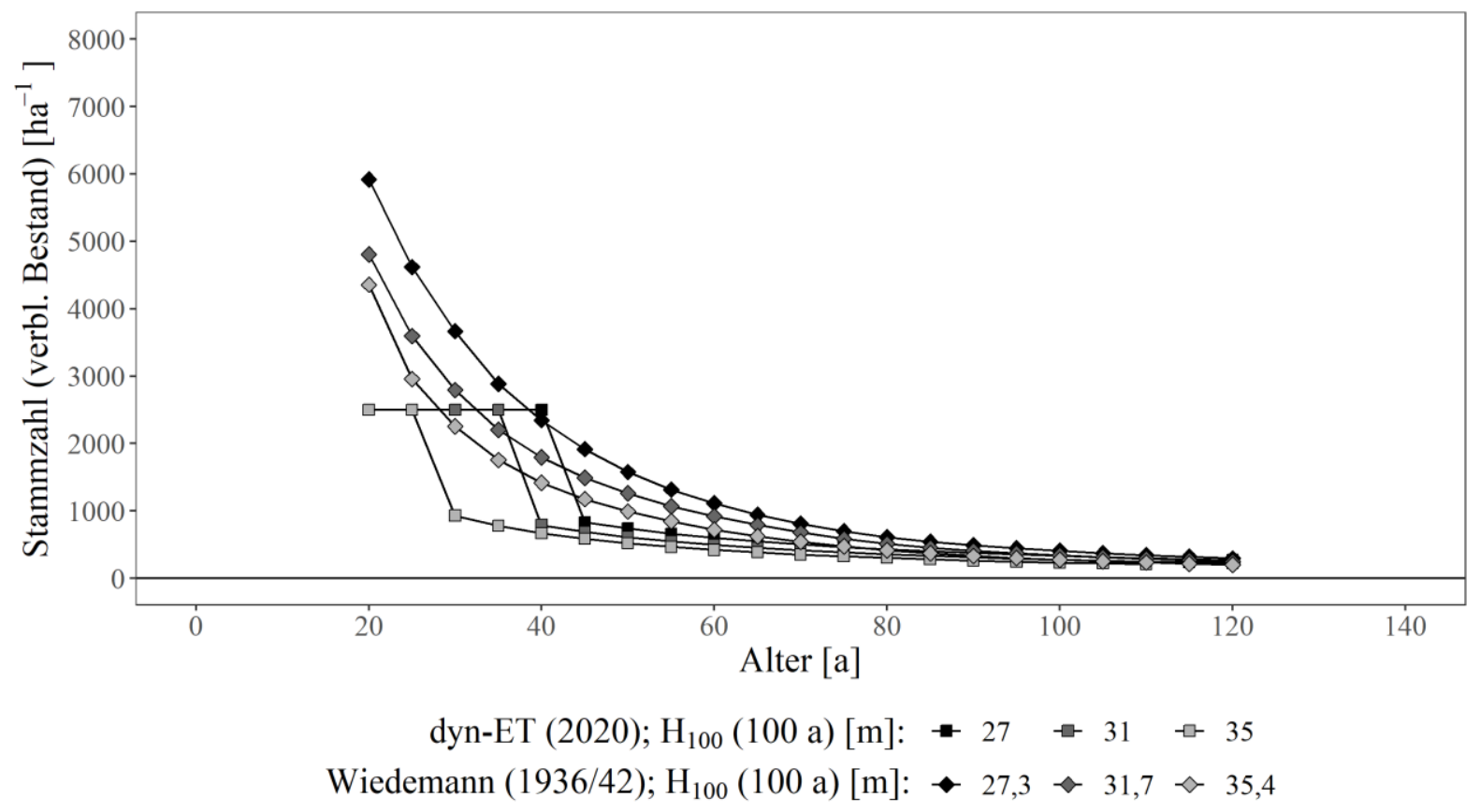

Abbildung 177: Stammzahl (verbleibender Bestand) über dem Alter; ausgewählte Oberhöhenbonitäten der Baumart Fichte (starke Durchforstung) gemäß dyn-ET (unveröff.) und Wiedemann (1936/42). 


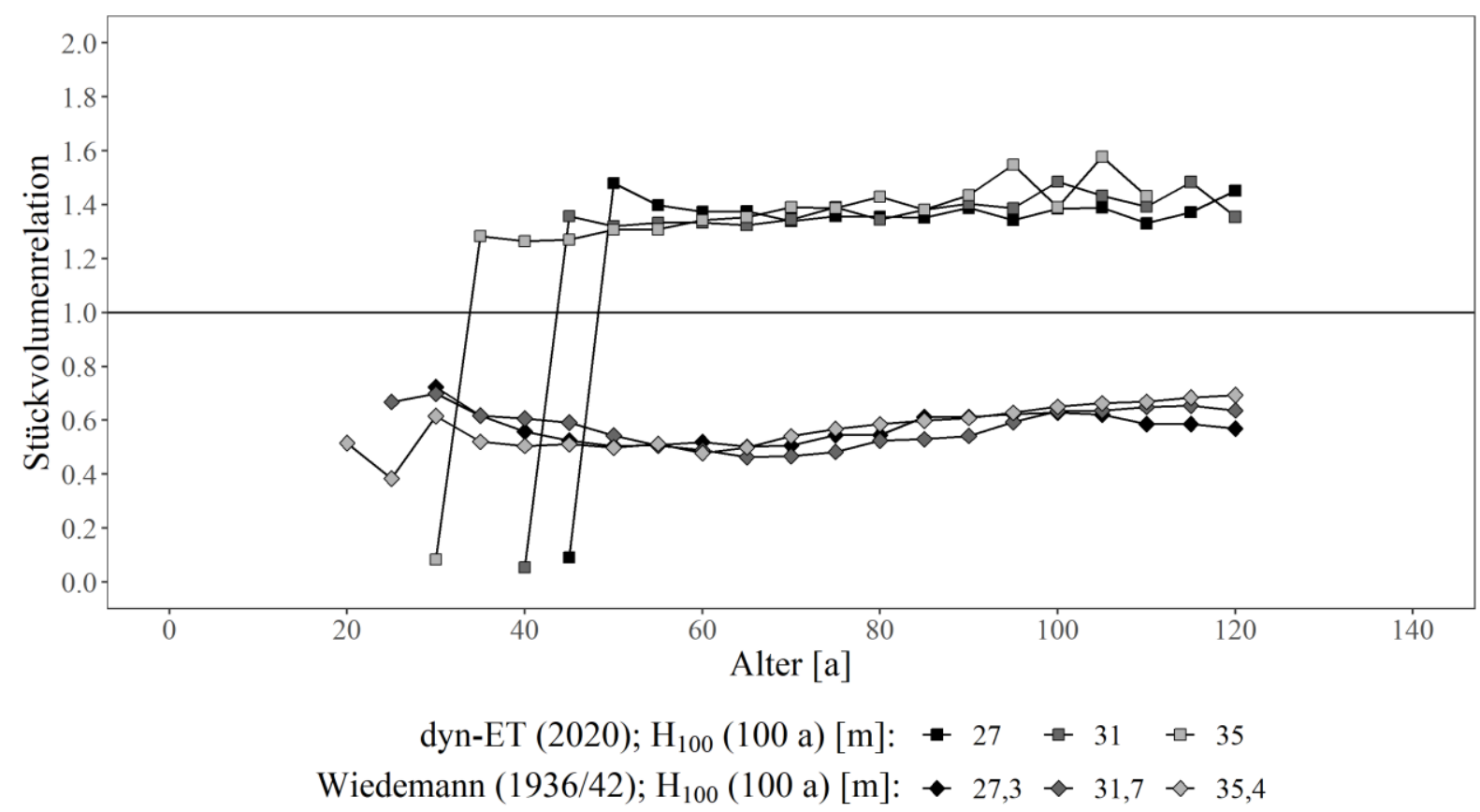

Abbildung 178: Stückvolumenrelationen (ausscheidendes geteilt durch verbleibendes Stückvolumen) über dem Alter; ausgewählte Oberhöhenbonitäten der Baumart Fichte (starke Durchforstung) gemäß dyn-ET (unveröff.) und Wiedemann (1936/42).

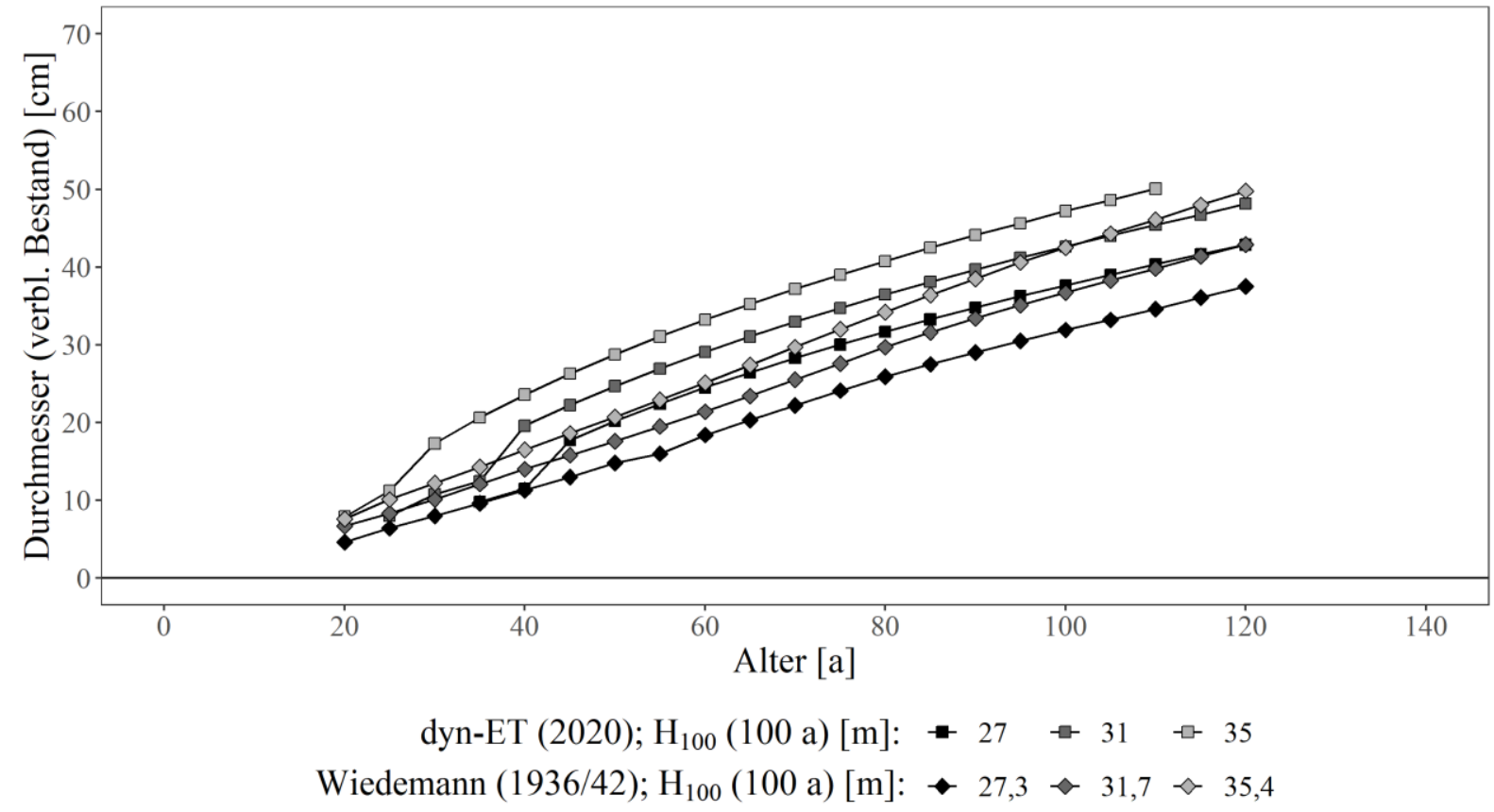

Abbildung 179:Durchmesser des Grundflächenmittelstamms (verbleibender Bestand) über dem Alter; ausgewählte Oberhöhenbonitäten der Baumart Fichte (starke Durchforstung) gemäß dyn-ET (unveröff.) und Wiedemann (1936/42). 


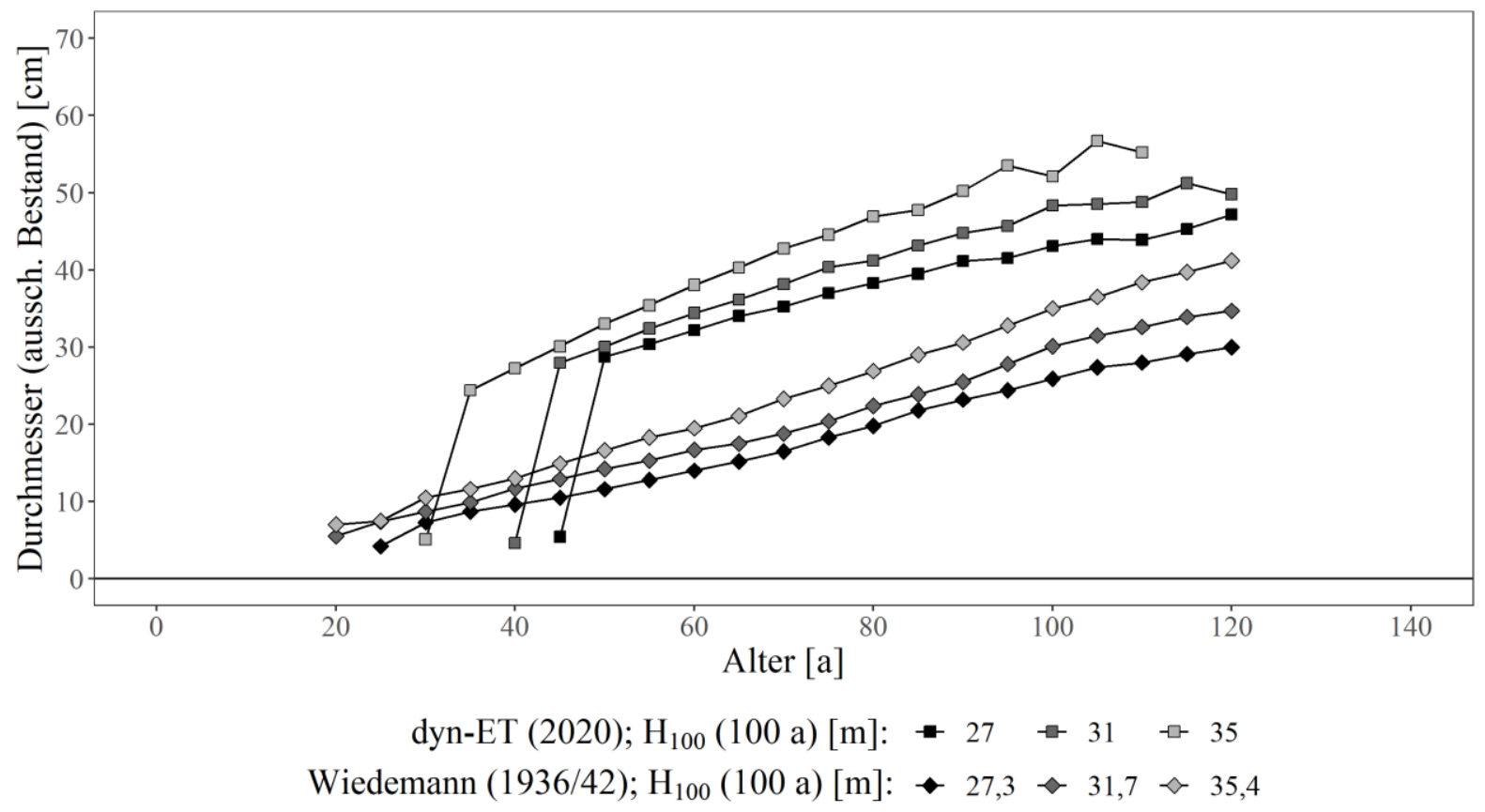

Abbildung 180: Durchmesser des Grundflächenmittelstamms (ausscheidender Bestand) über dem Alter; ausgewählte Oberhöhenbonitäten der Baumart Fichte (starke Durchforstung) gemäß dyn-ET (unveröff.) und Wiedemann (1936/42).

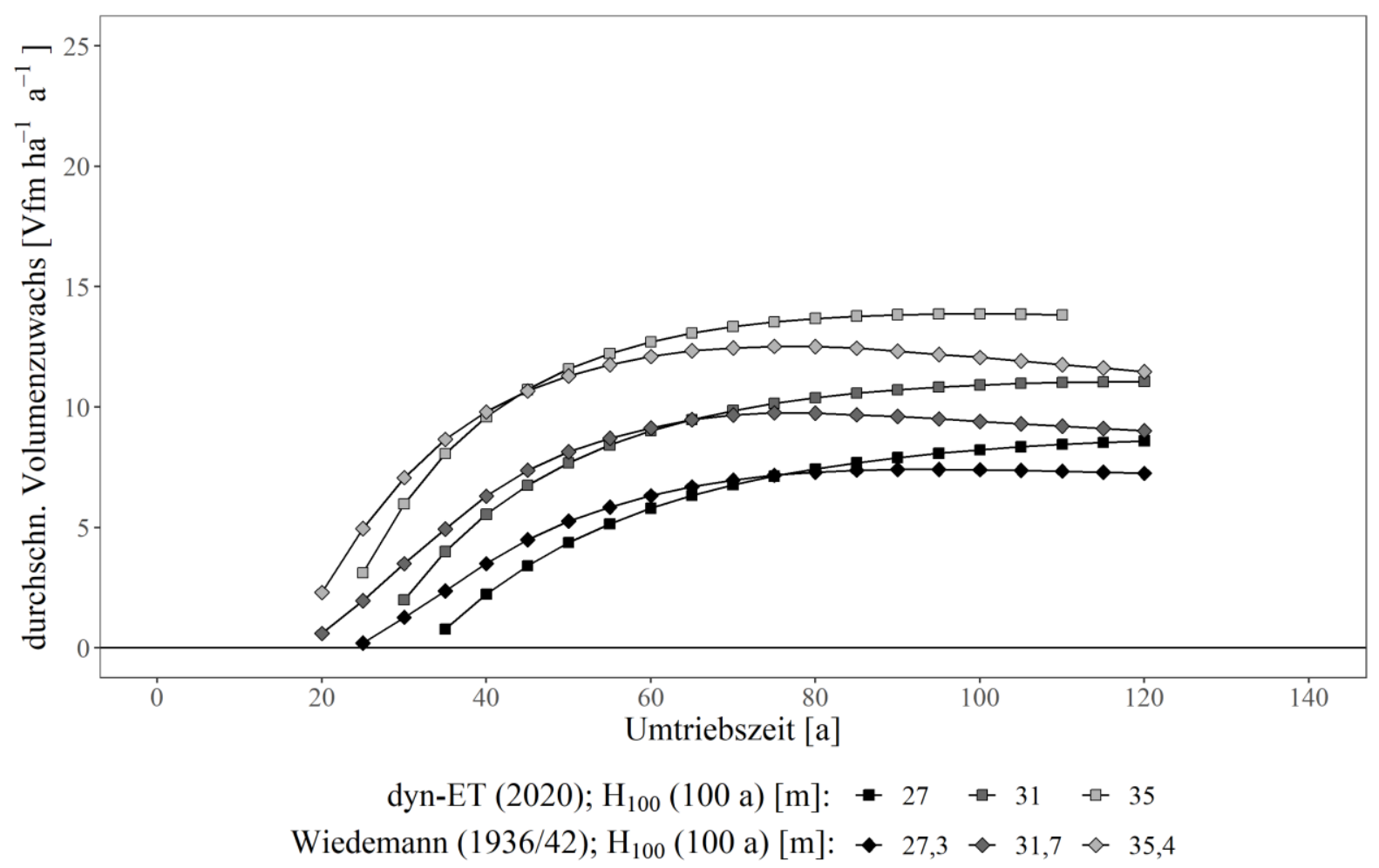

Abbildung 181:Durchschnittlicher Bestandesvolumenzuwachs über der Umtriebszeit; ausgewählte Oberhöhenbonitäten der Baumart Fichte (starke Durchforstung) gemäß dyn-ET (unveröff.) und Wiedemann (1936/42). 


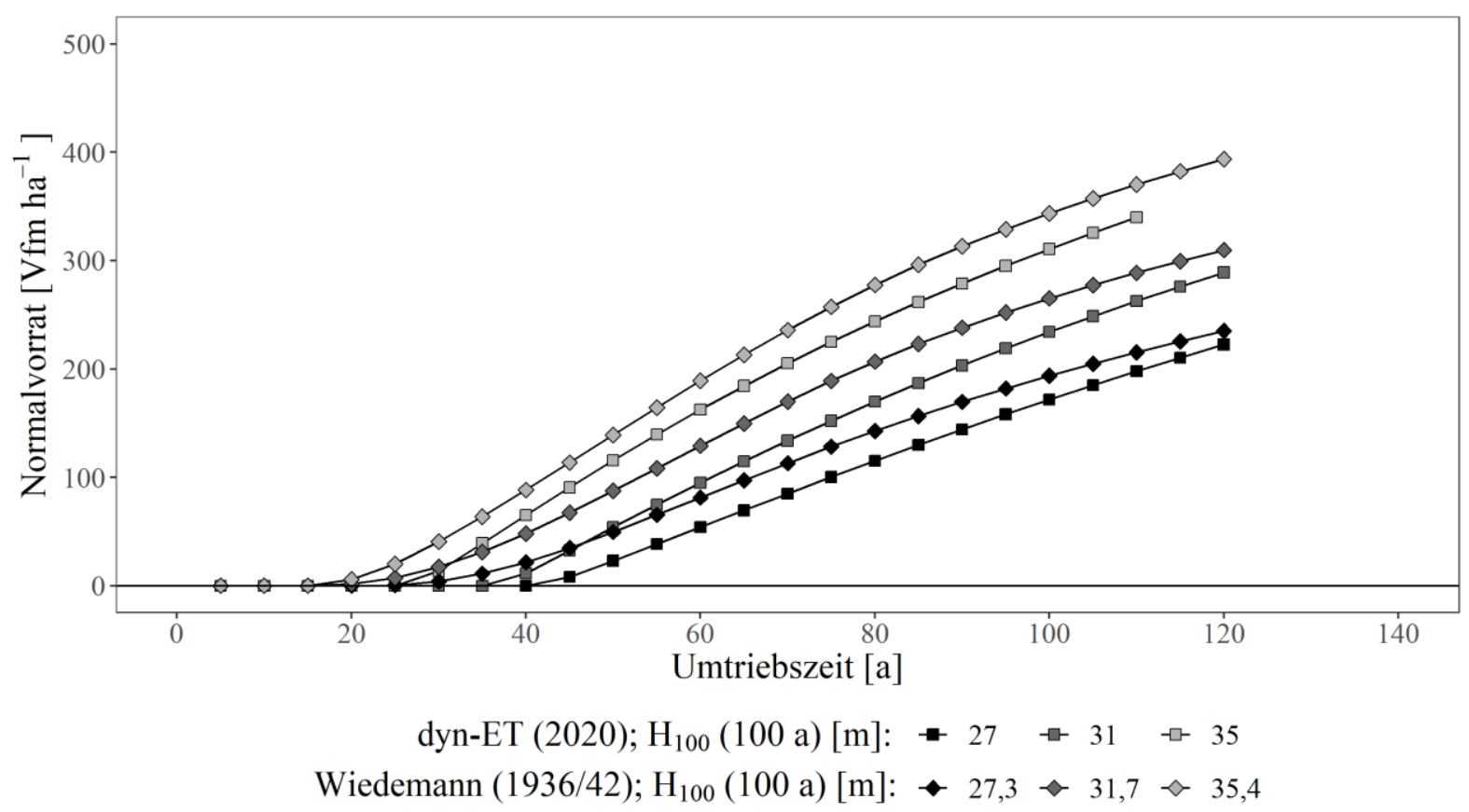

Abbildung 182: Normalvorrat über der Umtriebszeit; ausgewählte Oberhöhenbonitäten der Baumart Fichte (starke Durchforstung) gemäß dyn-ET (unveröff.) und Wiedemann (1936/42).

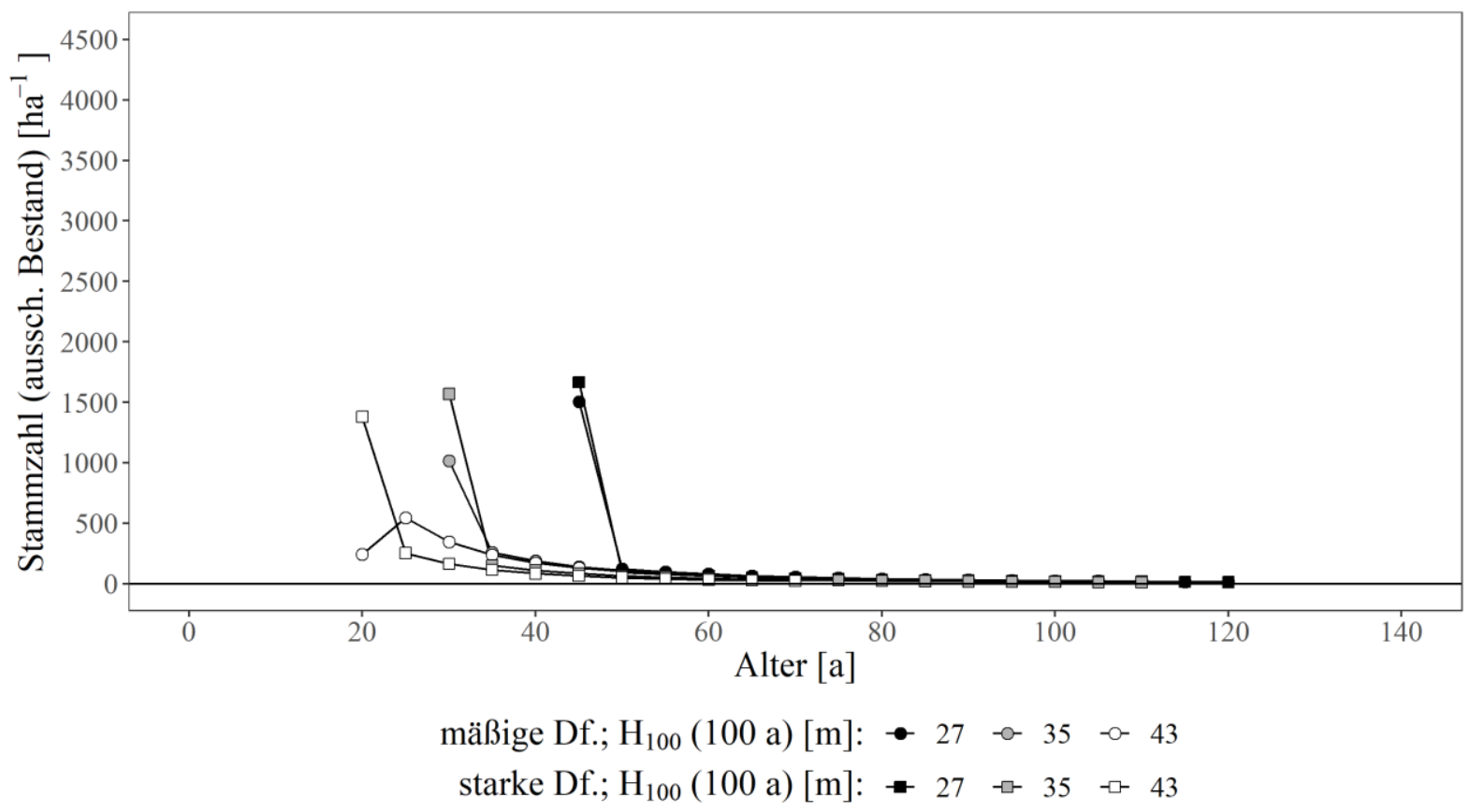

Abbildung 183: Stammzahl (ausscheidender Bestand) über dem Alter; ausgewählte Oberhöhenbonitäten der Baumart Fichte (mäßige Durchforstung) gemäß dyn-ET (unveröff.) und Wiedemann (1936/42). 


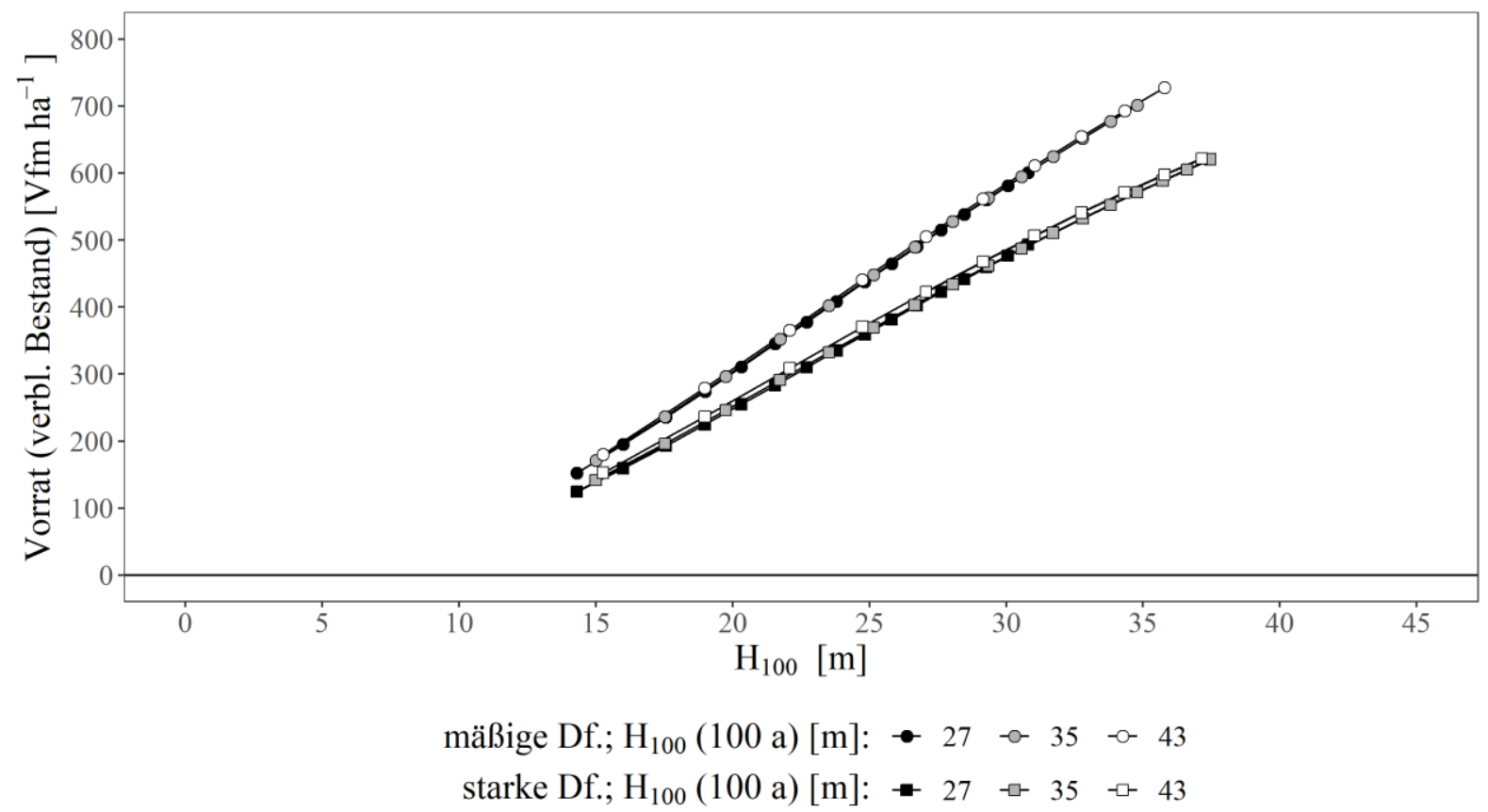

Abbildung 184: Vorrat (verbl. Bestand) über der Oberhöhe; ausgewählte Oberhöhenbonitäten der Baumart Fichte getrennt nach Behandlungsvarianten gemäß dyn-ET (unveröff.).

Tabelle 8-1: Preismatrizen der Stammholzsortimente ${ }^{87}$ als Kombination aus Wertmatrizen nach Bodelschwingh (2018, Anhang 3 i.V.m. S. 63) und mittleren Leitsortimentspreisen nach Schade (2019, S. 118); die baumartenspezifischen Leitsortimente sind fett abgedruckt.

\section{Stärkeklasse}

\begin{tabular}{|c|c|c|c|c|c|c|c|c|c|c|}
\hline 矛 & $\begin{array}{l}\text { Güte- } \\
\text { klasse }\end{array}$ & $1 a$ & $1 b$ & $2 a$ & $2 b$ & $3 a$ & $3 b$ & 4 & 5 & 6 \\
\hline \multirow{4}{*}{$\frac{\mathscr{q}}{0}$} & $\bar{A}$ & - & - & - & - & - & 91,80 & 146,70 & 174,60 & 190,80 \\
\hline & B & - & - & 63,00 & 66,60 & 69,30 & 74,70 & 90,00 & 149,40 & 110,70 \\
\hline & $\mathrm{C}$ & - & - & 63,90 & 65,70 & 69,30 & 72,00 & 81,90 & 88,20 & 90,00 \\
\hline & $\mathrm{D}$ & - & - & - & 63,90 & 64,80 & 66,60 & 71,10 & 72,00 & 72,00 \\
\hline \multirow{5}{*}{$\begin{array}{l}\frac{0}{0} \\
\substack{0 \\
i}\end{array}$} & $\mathrm{~A}$ & - & - & - & - & - & 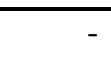 & 137,24 & 148,52 & 183,30 \\
\hline & B & 58,28 & 77,08 & 88,36 & 94,00 & 94,00 & 94,00 & 94,00 & 94,00 & 95,88 \\
\hline & $\mathrm{C}$ & 50,76 & 68,62 & 78,96 & 85,54 & 87,42 & 88,36 & 89,30 & 90,24 & 90,24 \\
\hline & CGW & - & 63,92 & 73,32 & 79,90 & 80,84 & 80,84 & 79,90 & 78,96 & 77,08 \\
\hline & $\mathrm{D}$ & - & - & - & 61,10 & 61,10 & 61,10 & 60,16 & 59,22 & 59,22 \\
\hline
\end{tabular}

${ }^{87}$ Bei der Baumart Fichte wird in Anlehnung an Bodelschwingh (2018, S. 61) die Sorte CGW (,geringwertiges C“ für z. B. frisches Käferholz) separat ausgewiesen. 


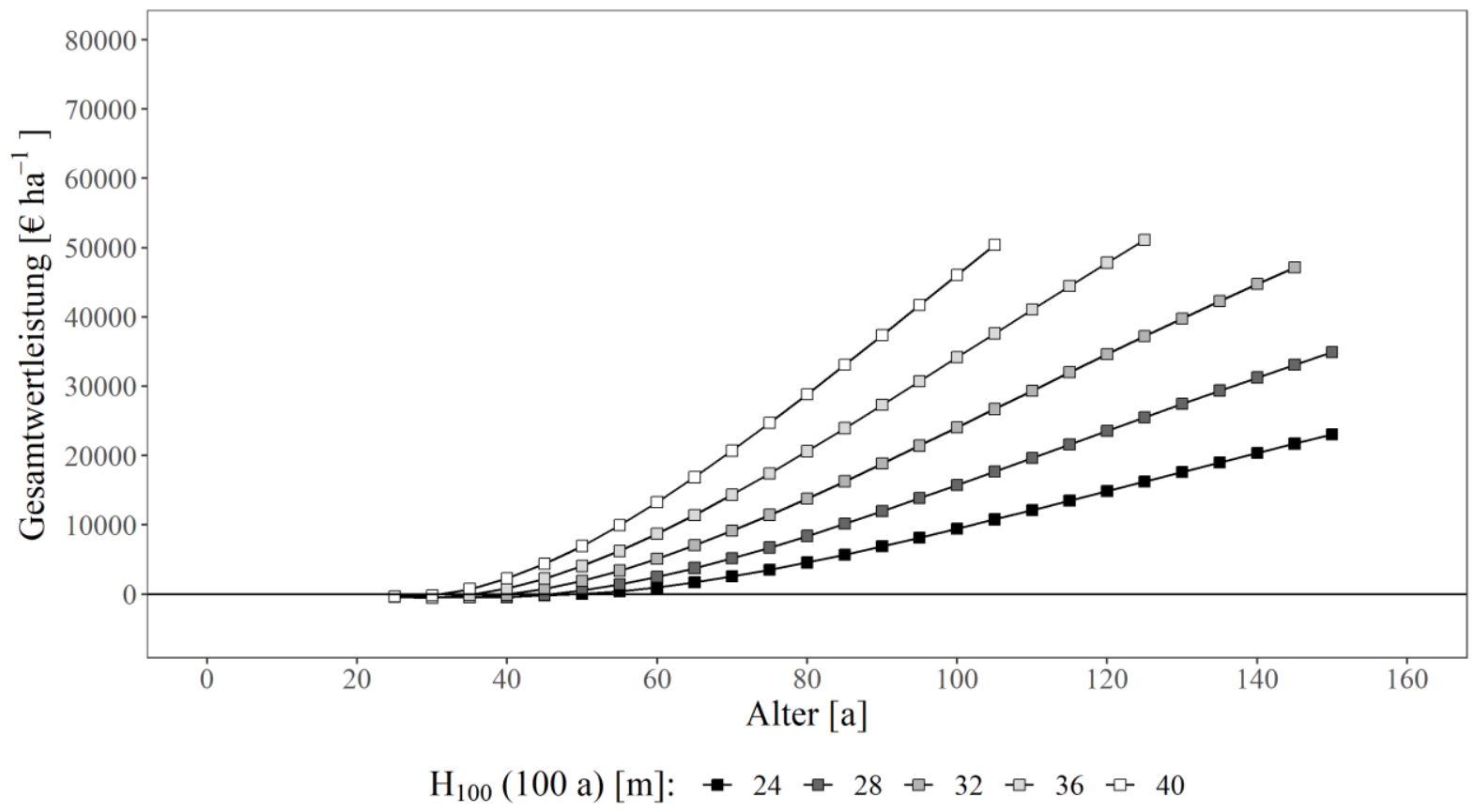

Abbildung 185:Gesamtwertleistung über dem Alter; alle Oberhöhenbonitäten der Baumart Buche (starke Hochdurchforstung) gemäß dyn-ET (unveröff.).

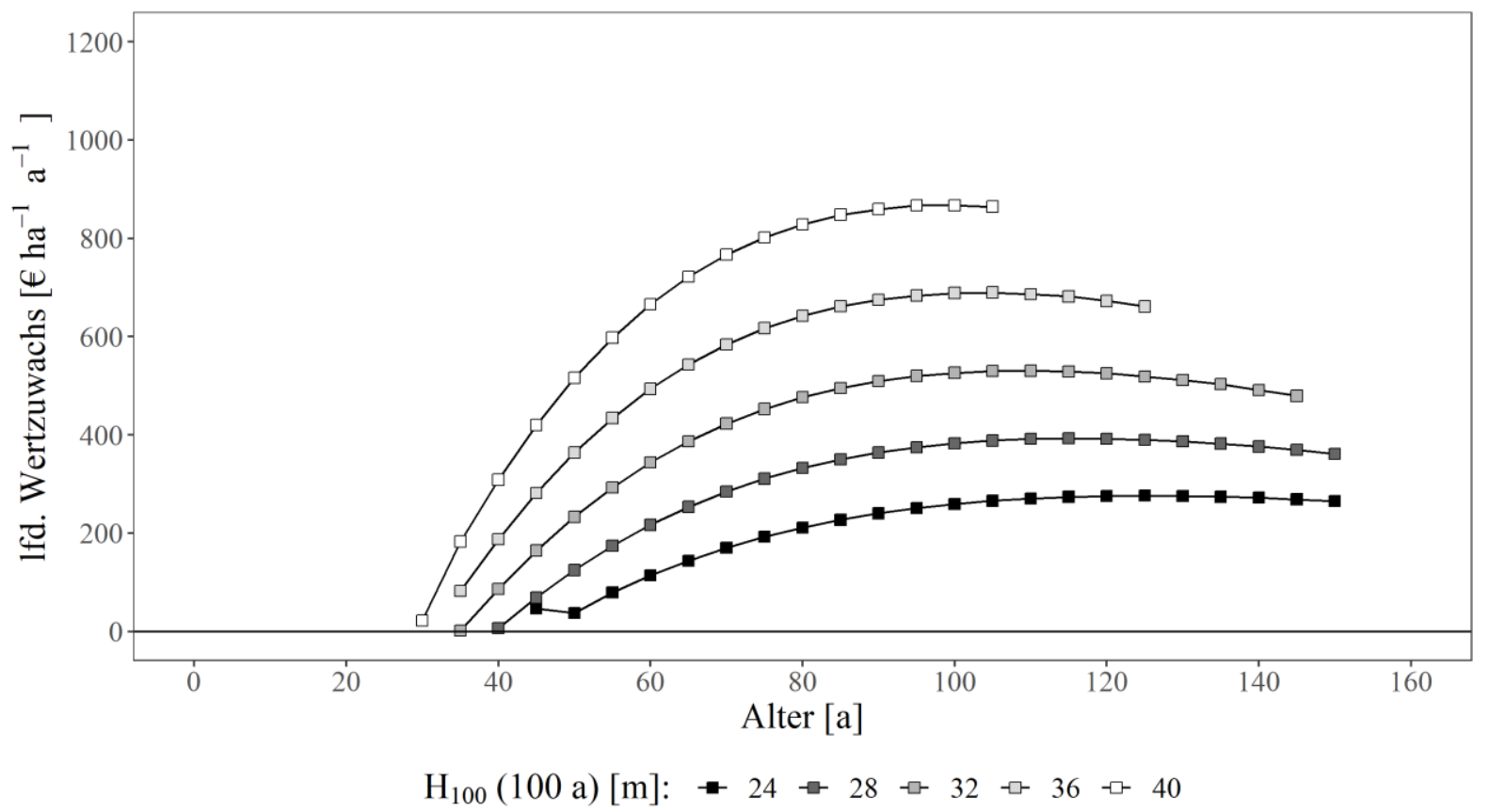

Abbildung 186: Laufender Bestandeswertzuwachs über dem Alter; alle Oberhöhenbonitäten der Baumart Buche (starke Hochdurchforstung) gemäß dyn-ET (unveröff.). 


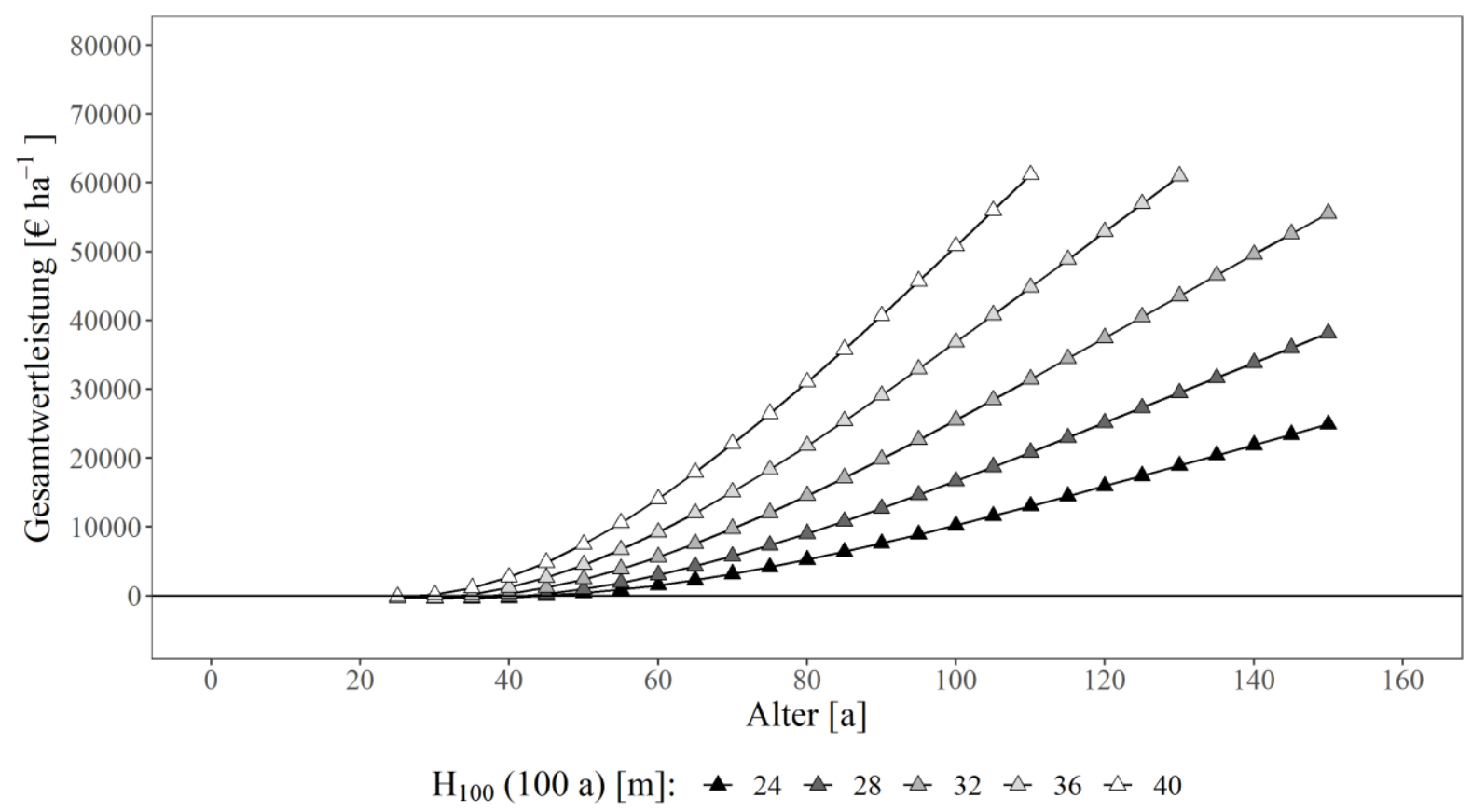

Abbildung 187: Gesamtwertleistung über dem Alter; alle Oberhöhenbonitäten der Baumart Buche (gestaffelte Hochdurchforstung) gemäß dyn-ET (unveröff.).

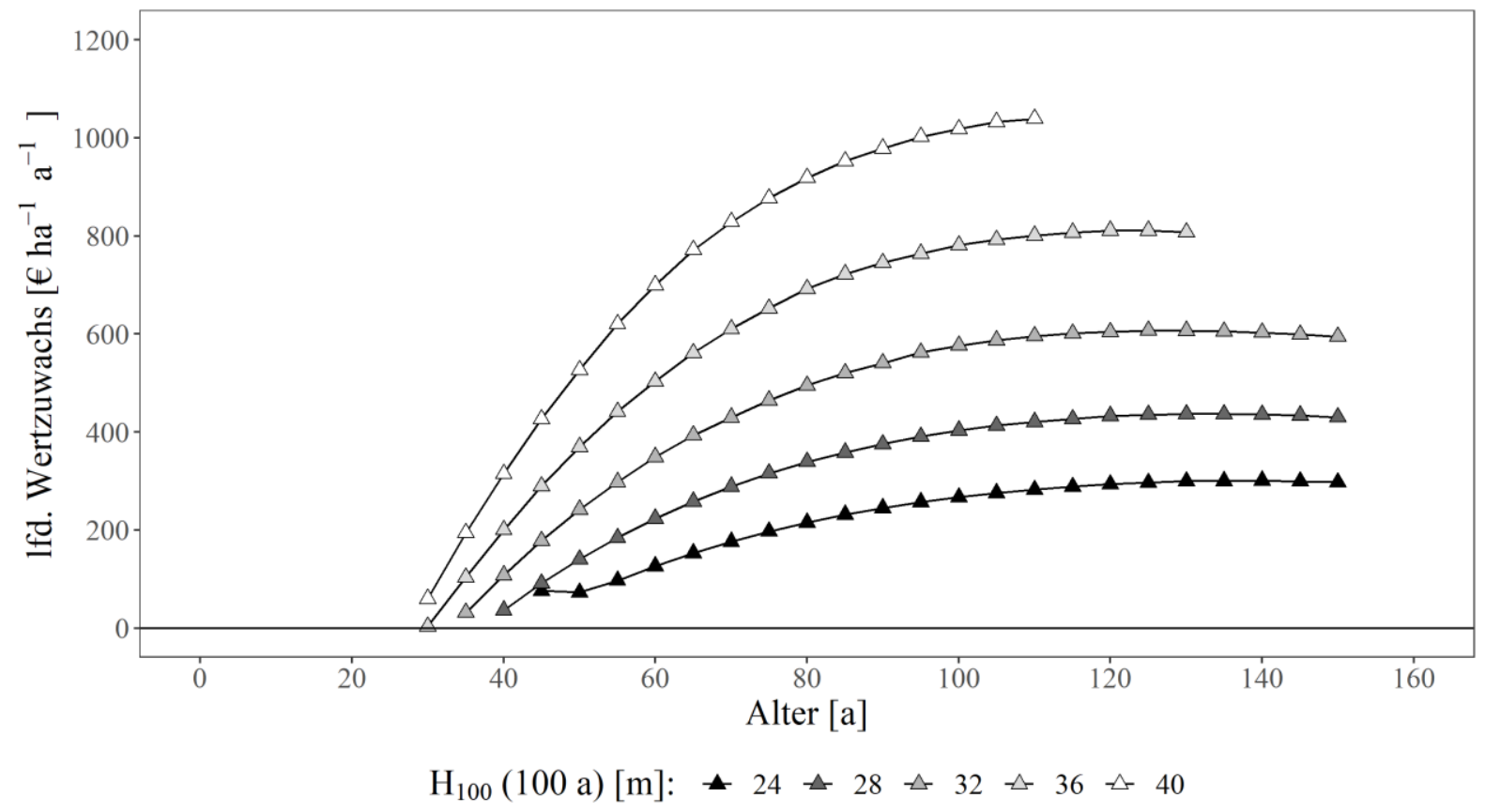

Abbildung 188: Laufender Bestandeswertzuwachs über dem Alter; alle Oberhöhenbonitäten der Baumart Buche (gestaffelte Hochdurchforstung) gemäß dyn-ET (unveröff.). 


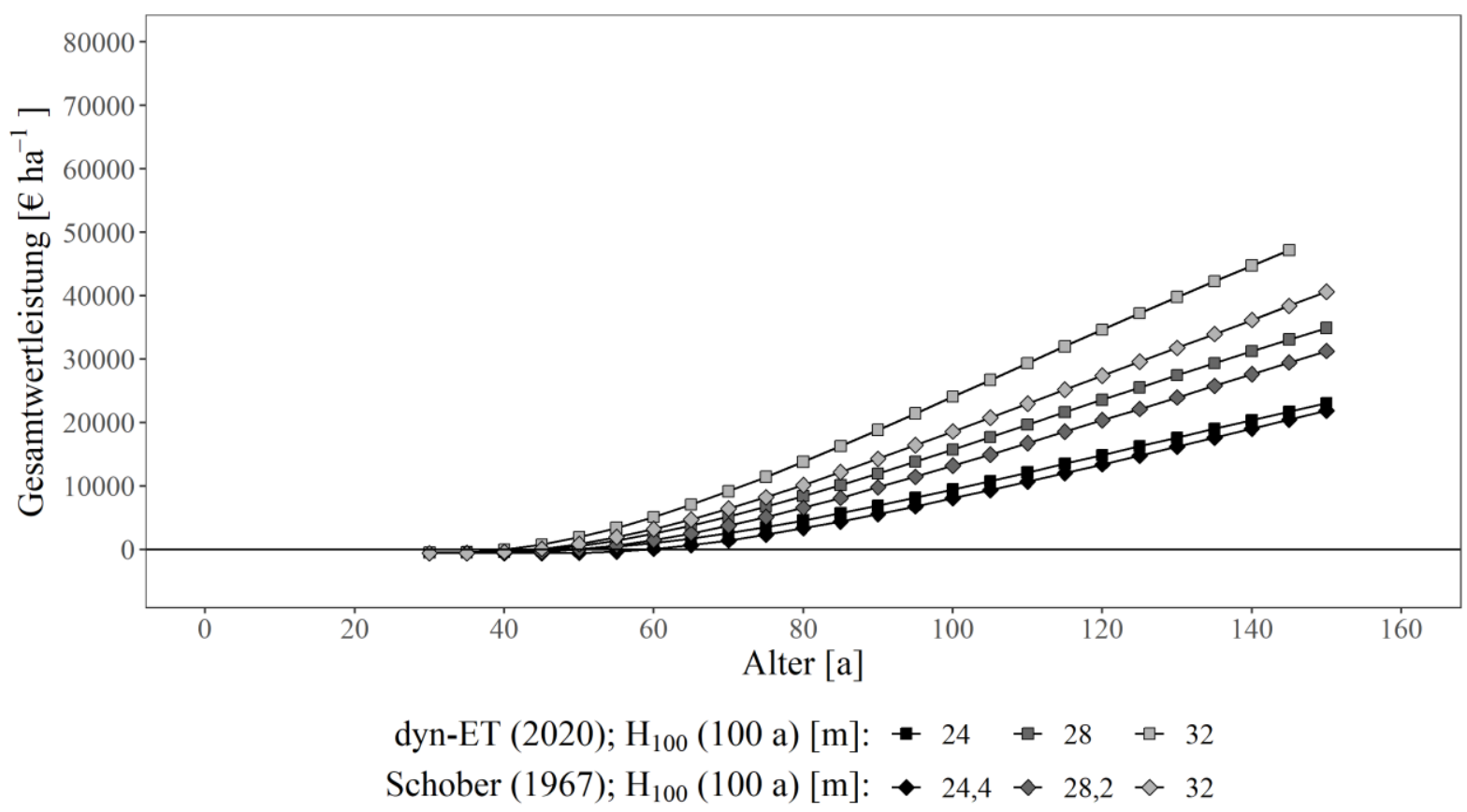

Abbildung 189: Gesamtwertleistung über dem Alter; ausgewählte Oberhöhenbonitäten der Baumart Buche (starke Durchforstung) gemäß dyn-ET (unveröff.) und Schober (1967).

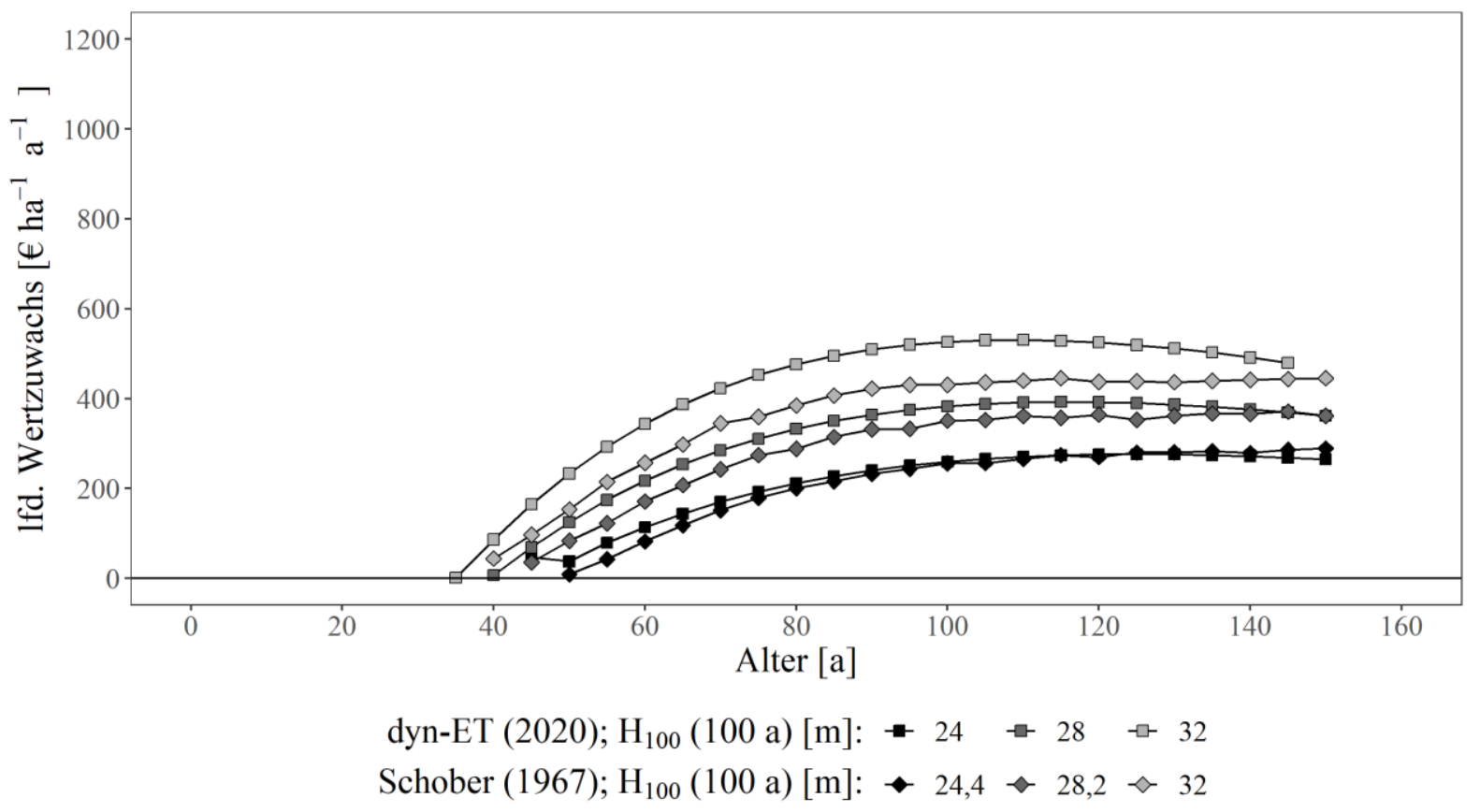

Abbildung 190:Laufender Bestandeswertzuwachs über dem Alter; ausgewählte Oberhöhenbonitäten der Baumart Buche (starke Durchforstung) gemäß dyn-ET (unveröff.) und Schober (1967). 


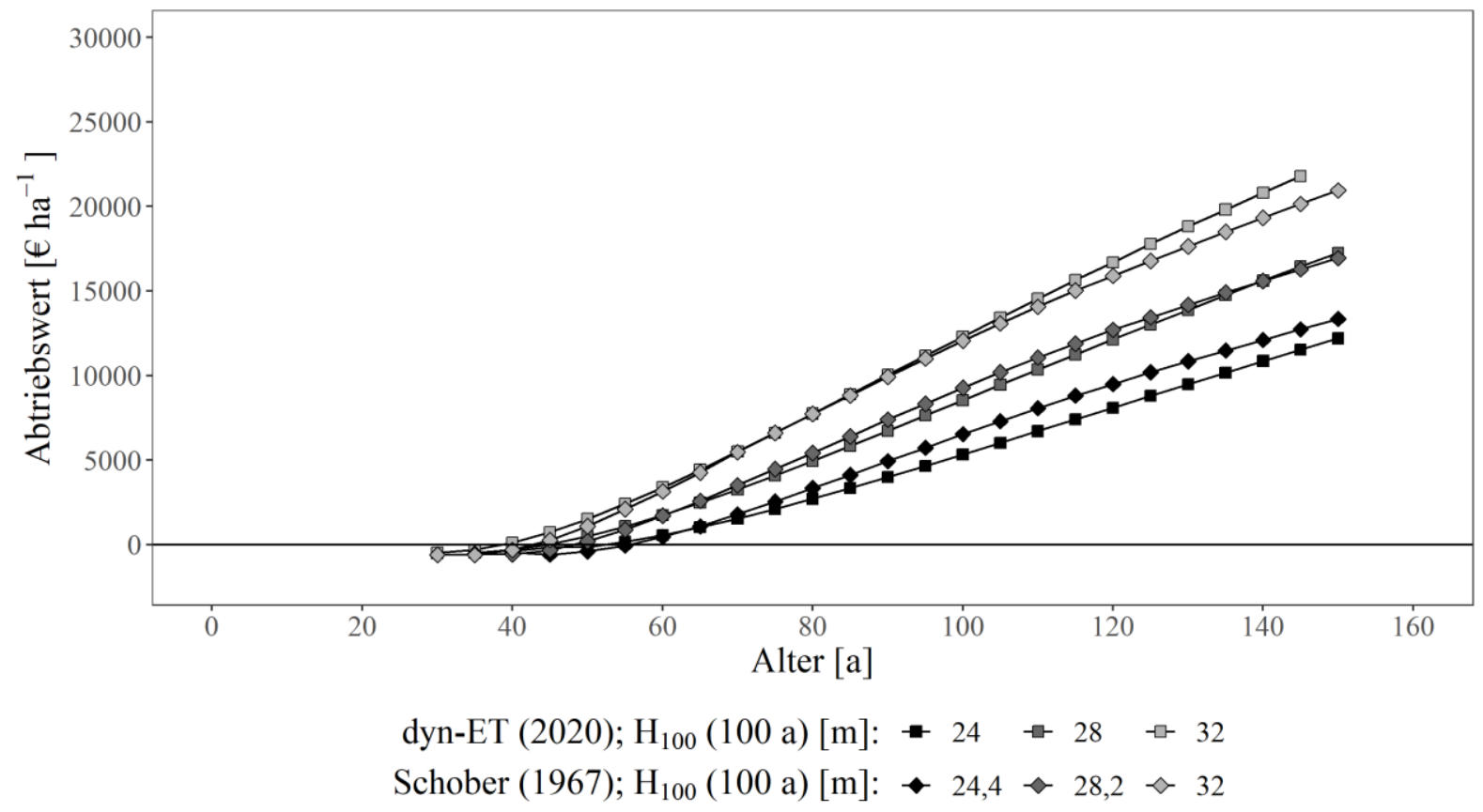

Abbildung 191: Abtriebswert über dem Alter; ausgewählte Oberhöhenbonitäten der Baumart Buche (starke Durchforstung) gemäß dyn-ET (unveröff.) und Schober (1967). 


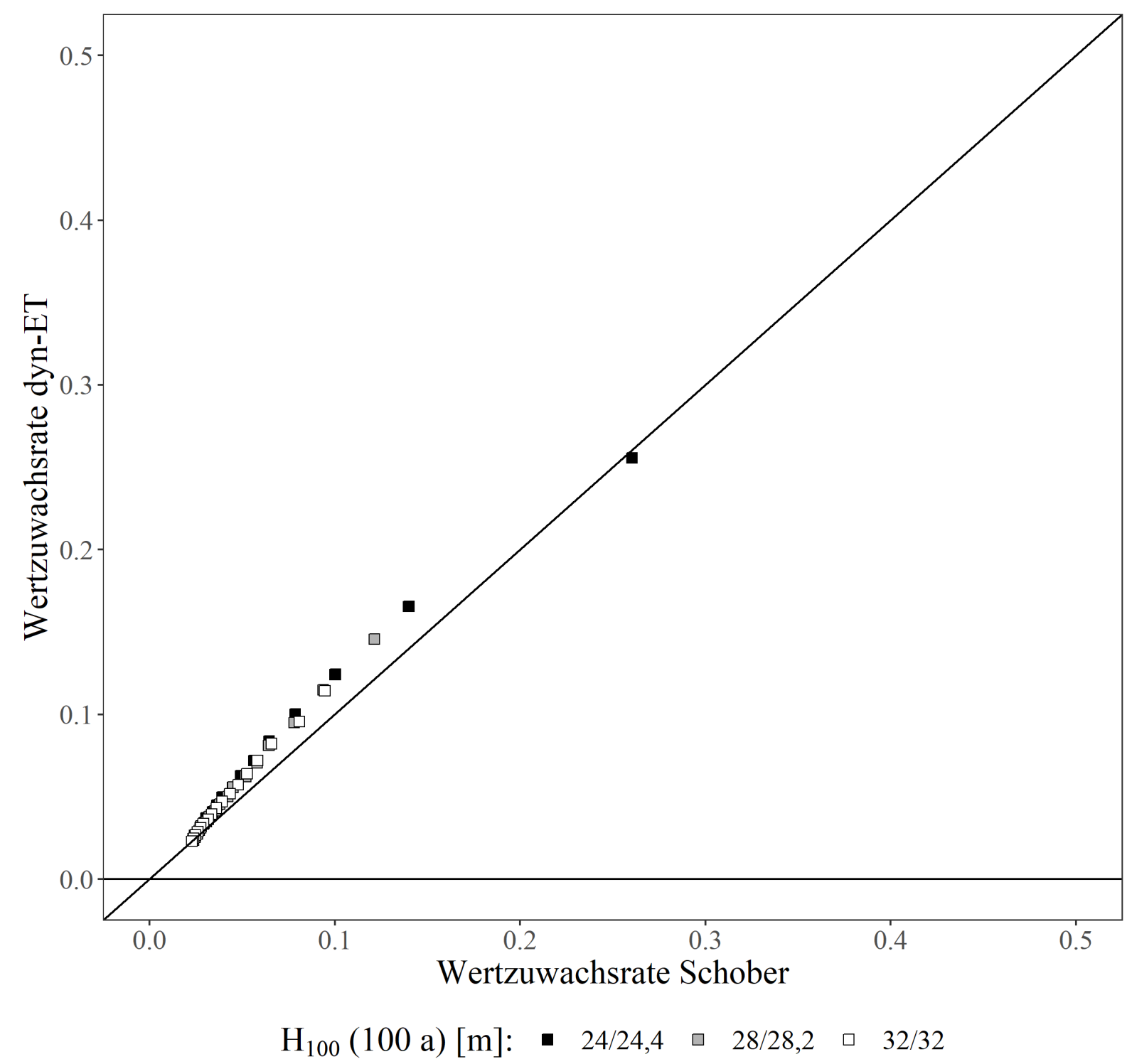

Abbildung 192: Wertzuwachsraten der Baumart Buche (starke Durchforstung, ausgewählte Oberhöhenbonitäten) gemäß dyn-ET (unveröff.) und Schober (1967); es werden nur Vergleichspaare aus Altern dargestellt, in denen für alle Oberhöhenbonitäten Datenpunkte vorliegen; die Winkelhalbierende kennzeichnet gleiche Wertzuwachsraten im gleichen Alter. 


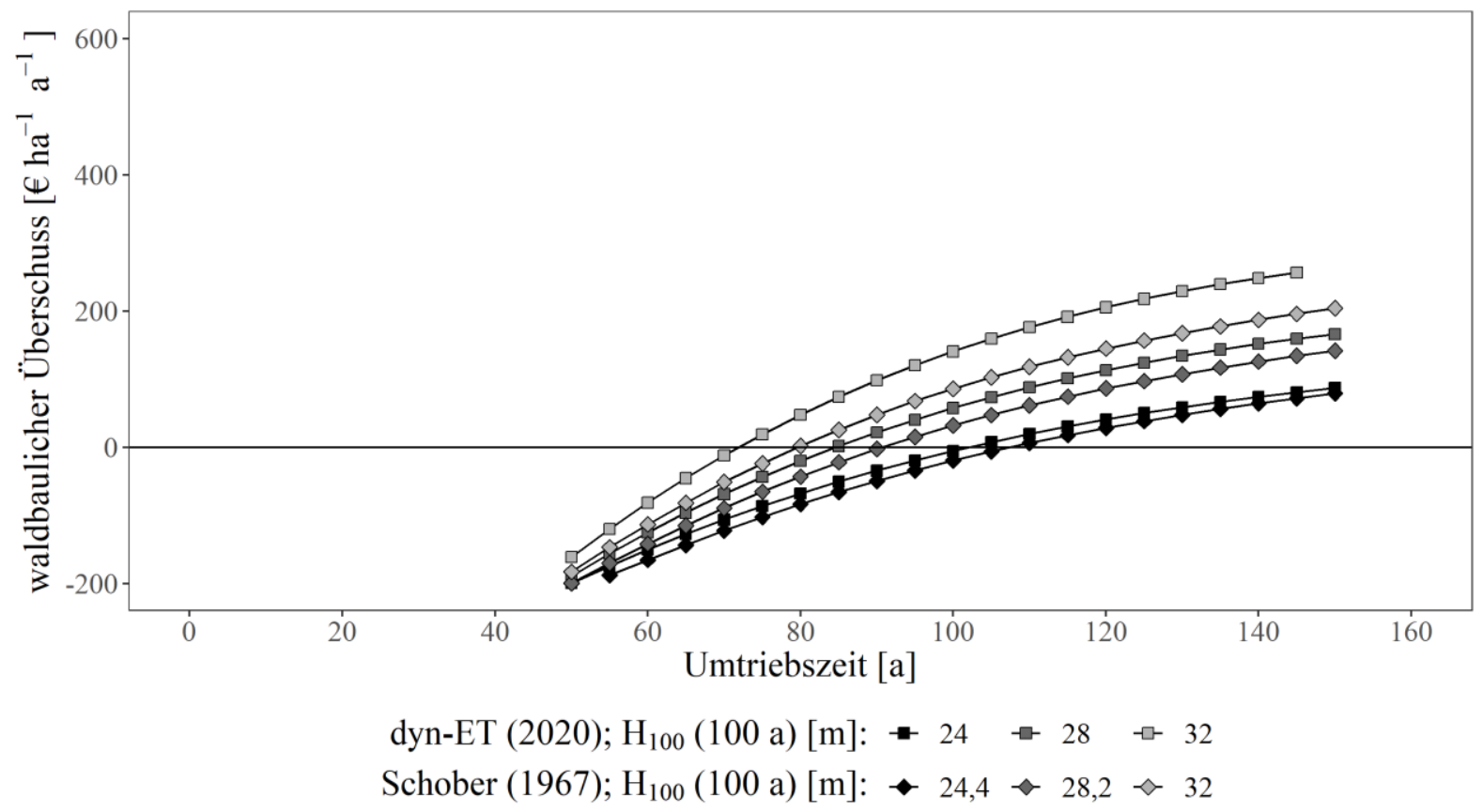

Abbildung 193: Durchschnittlicher waldbaulicher Überschuss über der Umtriebszeit; ausgewählte Oberhöhenbonitäten der Baumart Buche (starke Durchforstung) gemäß dyn-ET (unveröff.) und Schober (1967).

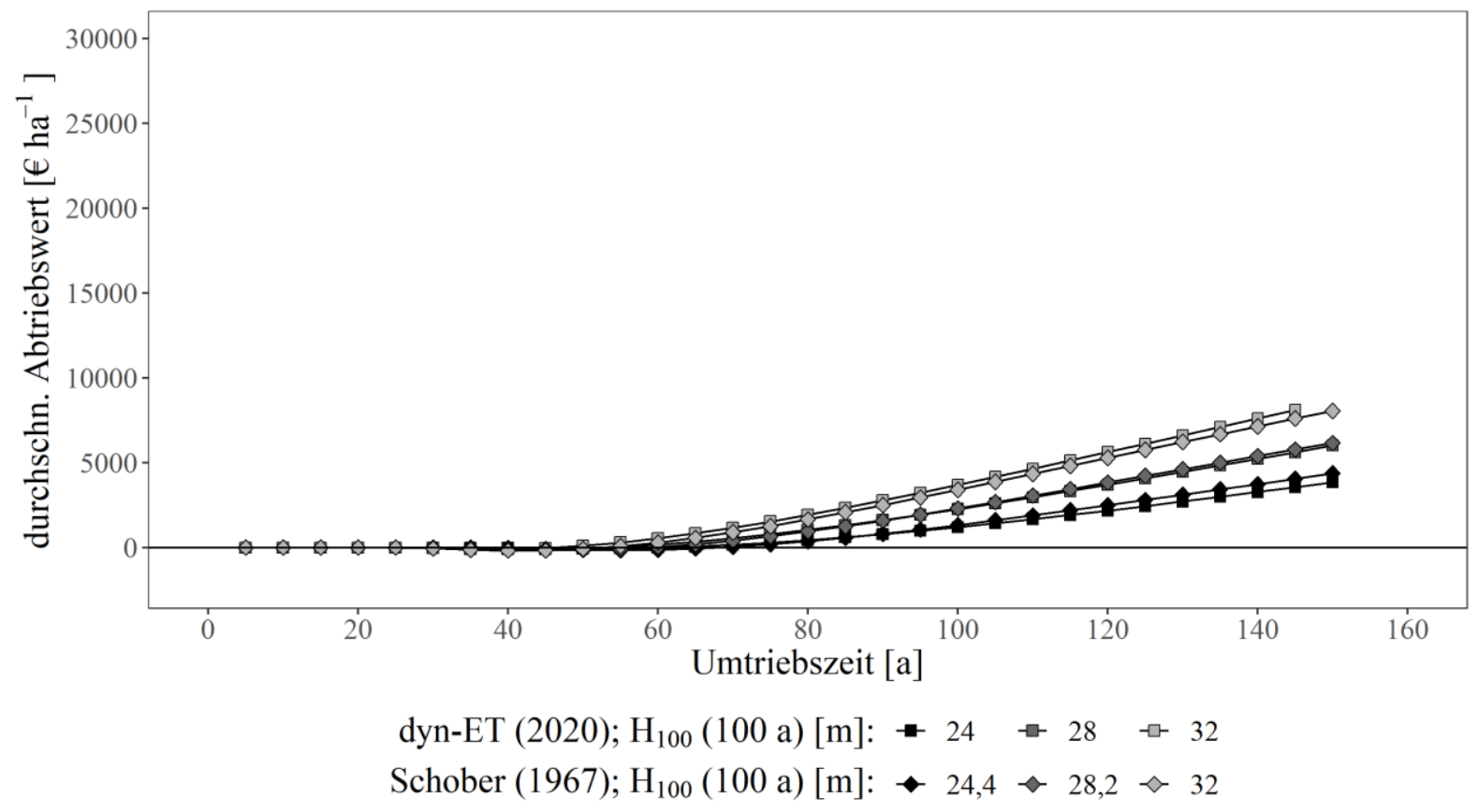

Abbildung 194: Durchschnittlicher Abtriebswert über der Umtriebszeit; ausgewählte Oberhöhenbonitäten der Baumart Buche (starke Durchforstung) gemäß dyn-ET (unveröff.) und Schober (1967). 


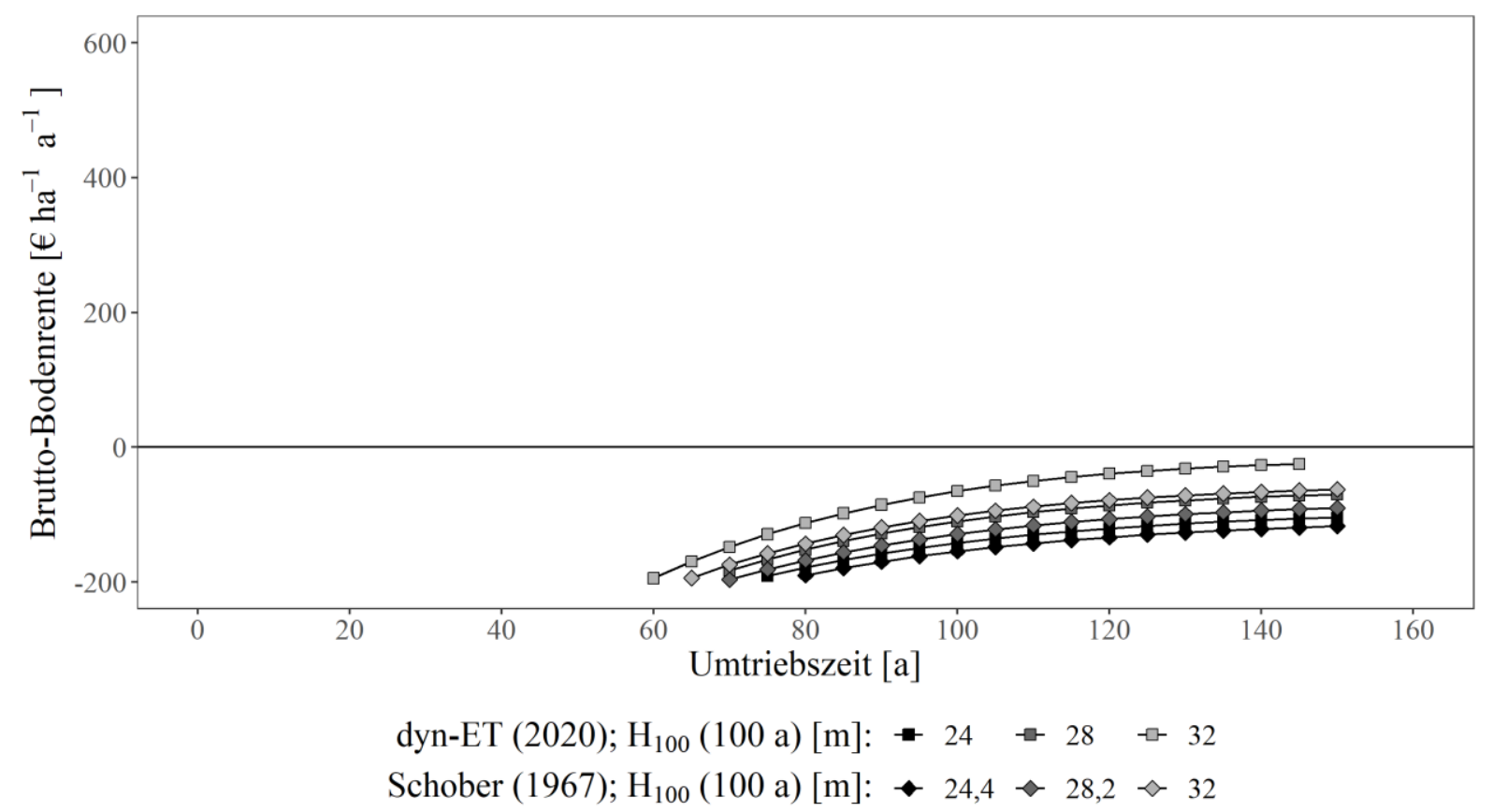

Abbildung 195: Brutto-Bodenrente über dem Alter; ausgewählte Oberhöhenbonitäten der Baumart Buche (starke Durchforstung) gemäß dyn-ET (unveröff.) und Schober (1967).

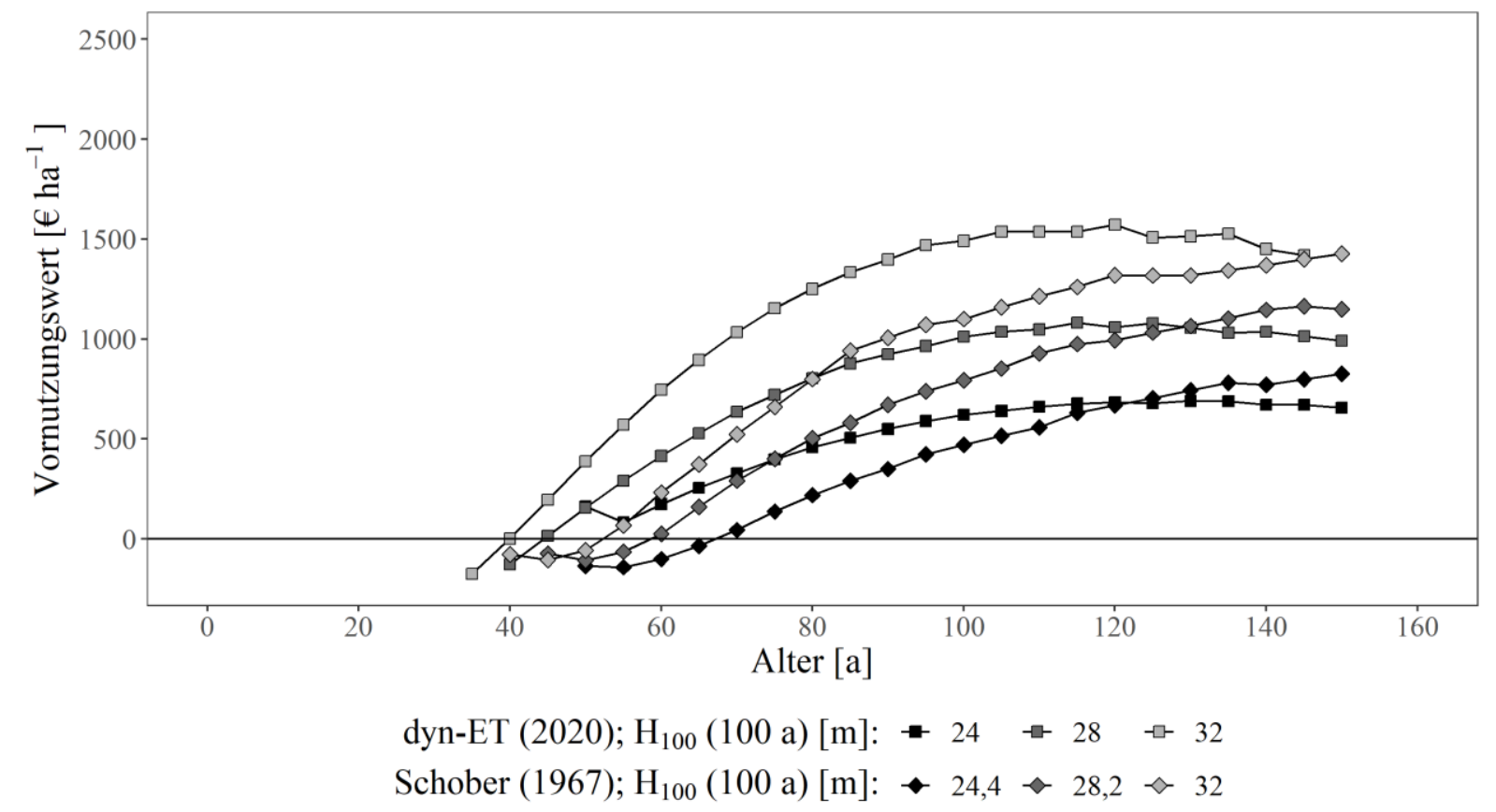

Abbildung 196: Vornutzungswerte über dem Alter; ausgewählte Oberhöhenbonitäten der Baumart Buche (starke Durchforstung) gemäß dyn-ET (unveröff.) und Schober (1967). 


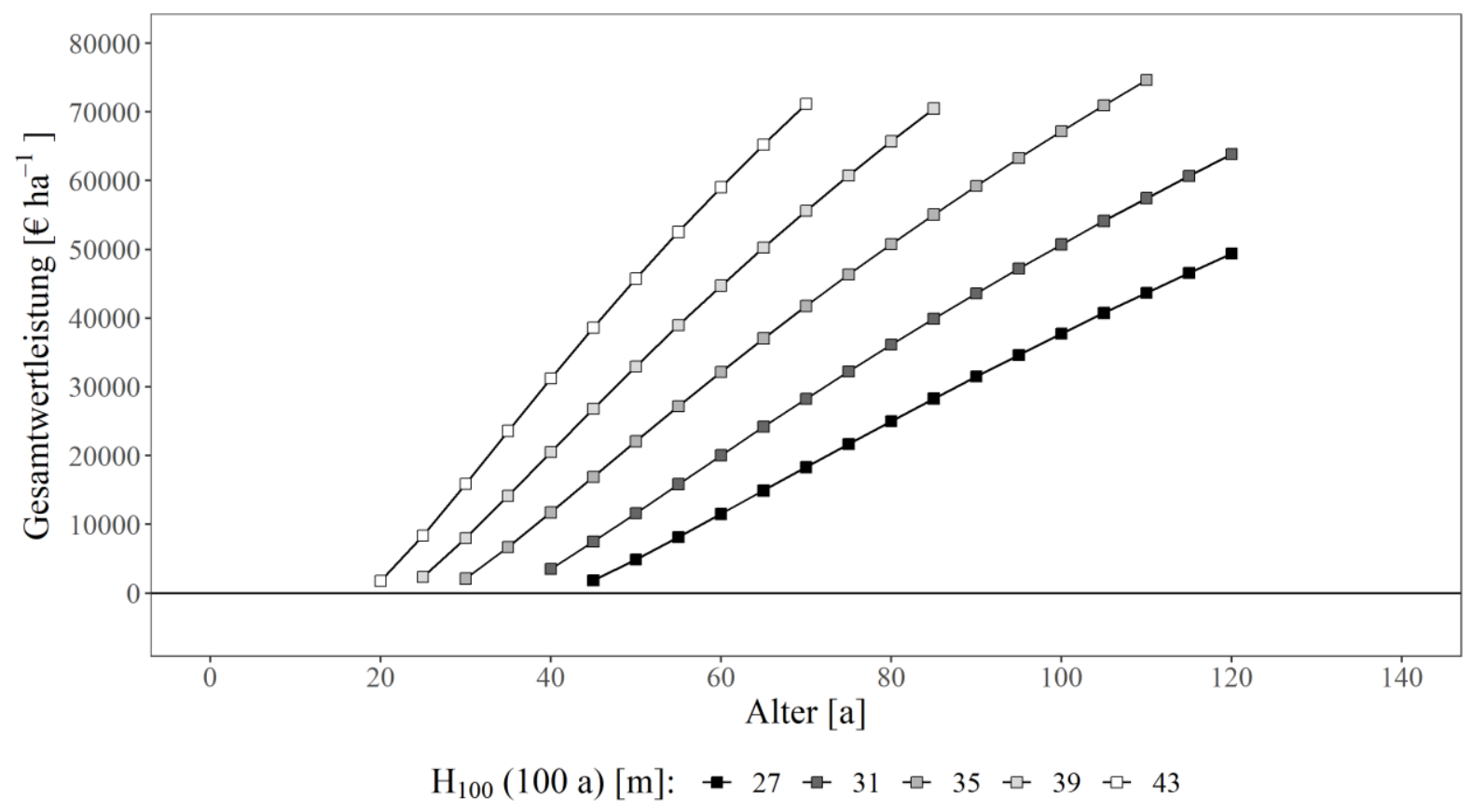

Abbildung 197: Gesamtwertleistung über dem Alter; alle Oberhöhenbonitäten der Baumart Fichte (starke Hochdurchforstung) gemäß dyn-ET (unveröff.).

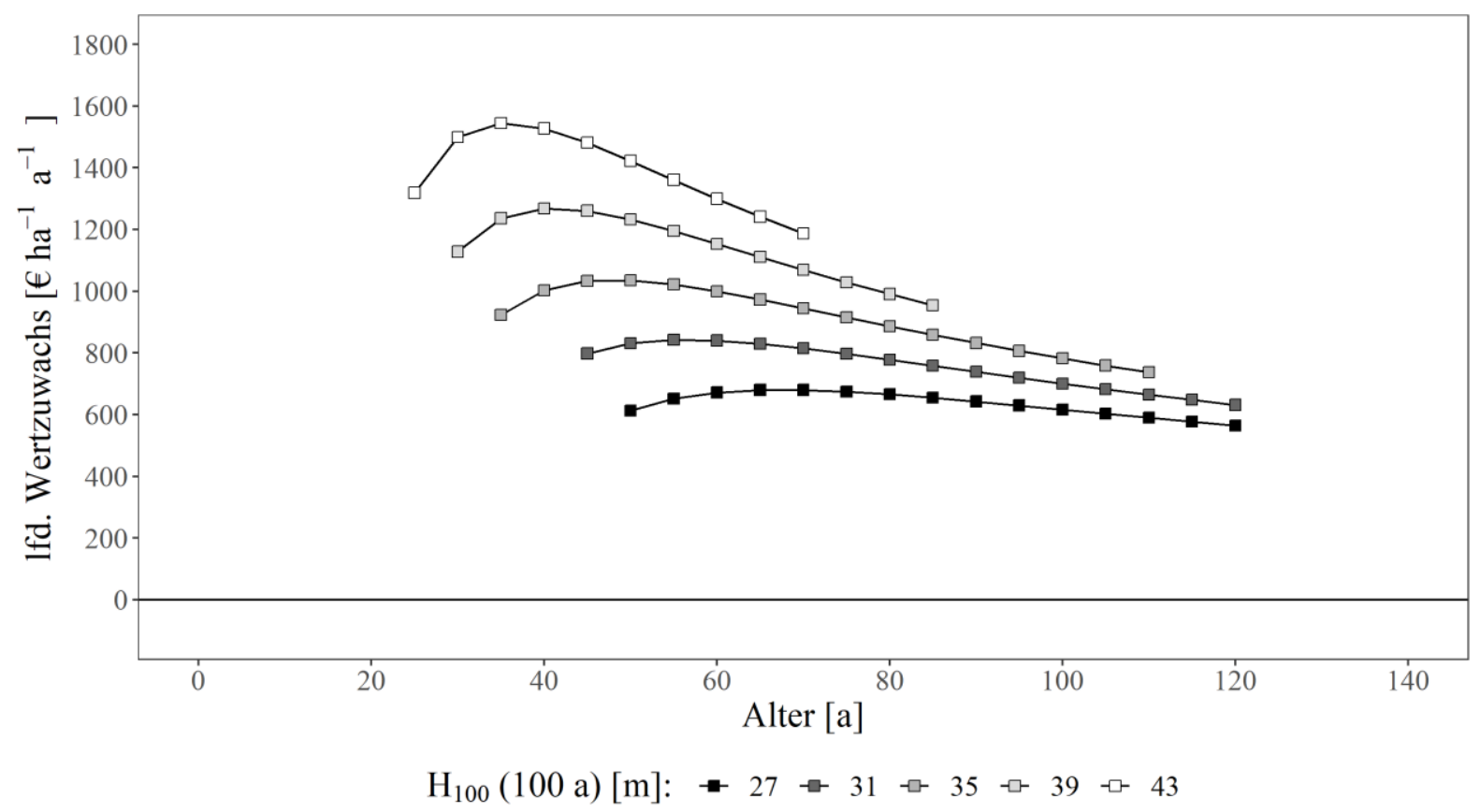

Abbildung 198: Laufender Bestandeswertzuwachs über dem Alter; alle Oberhöhenbonitäten der Baumart Fichte (starke Hochdurchforstung) gemäß dyn-ET (unveröff.). 


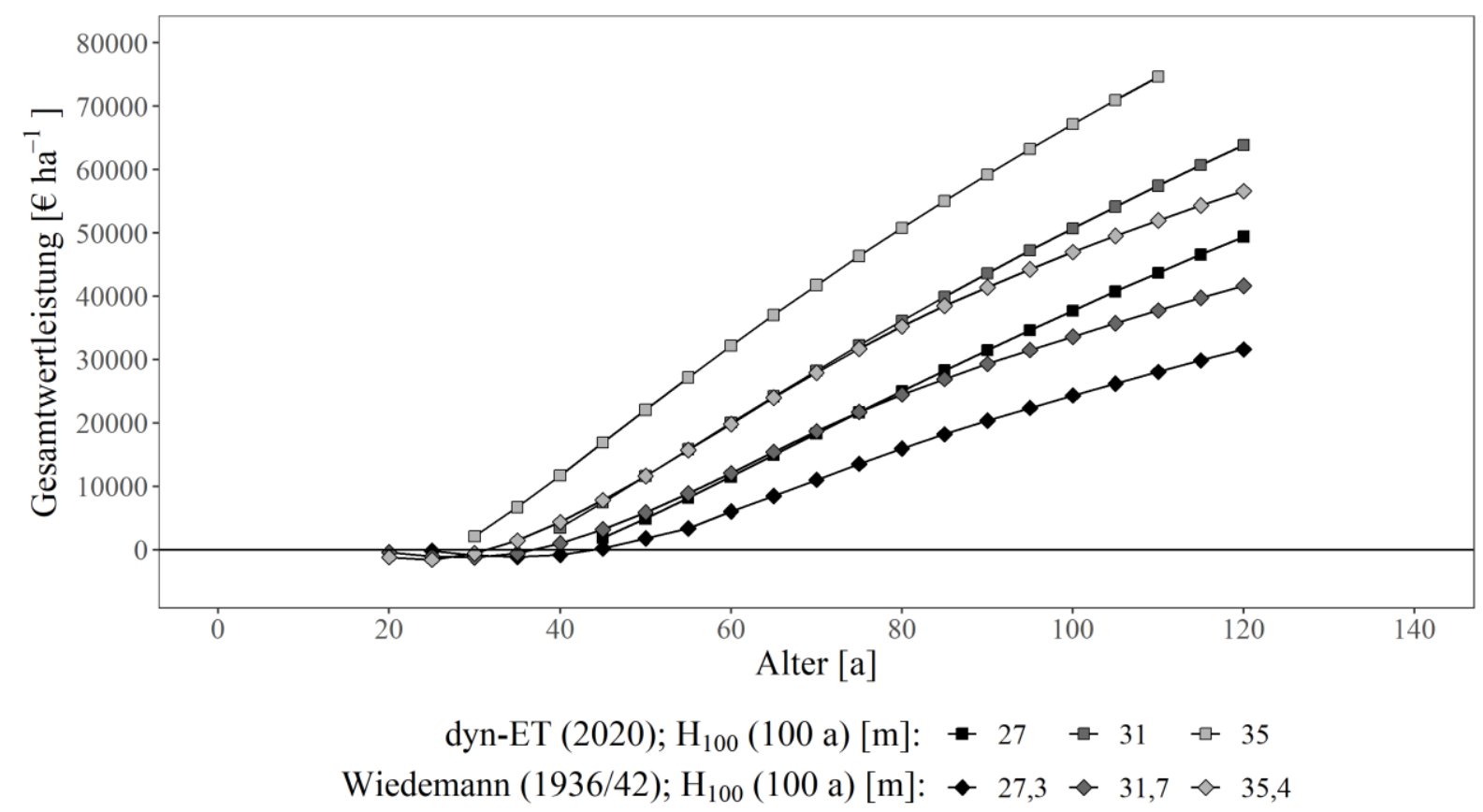

Abbildung 199: Gesamtwertleistung über dem Alter; ausgewählte Oberhöhenbonitäten der Baumart Fichte (starke Durchforstung) gemäß dyn-ET (unveröff.) und Wiedemann (1936/42).

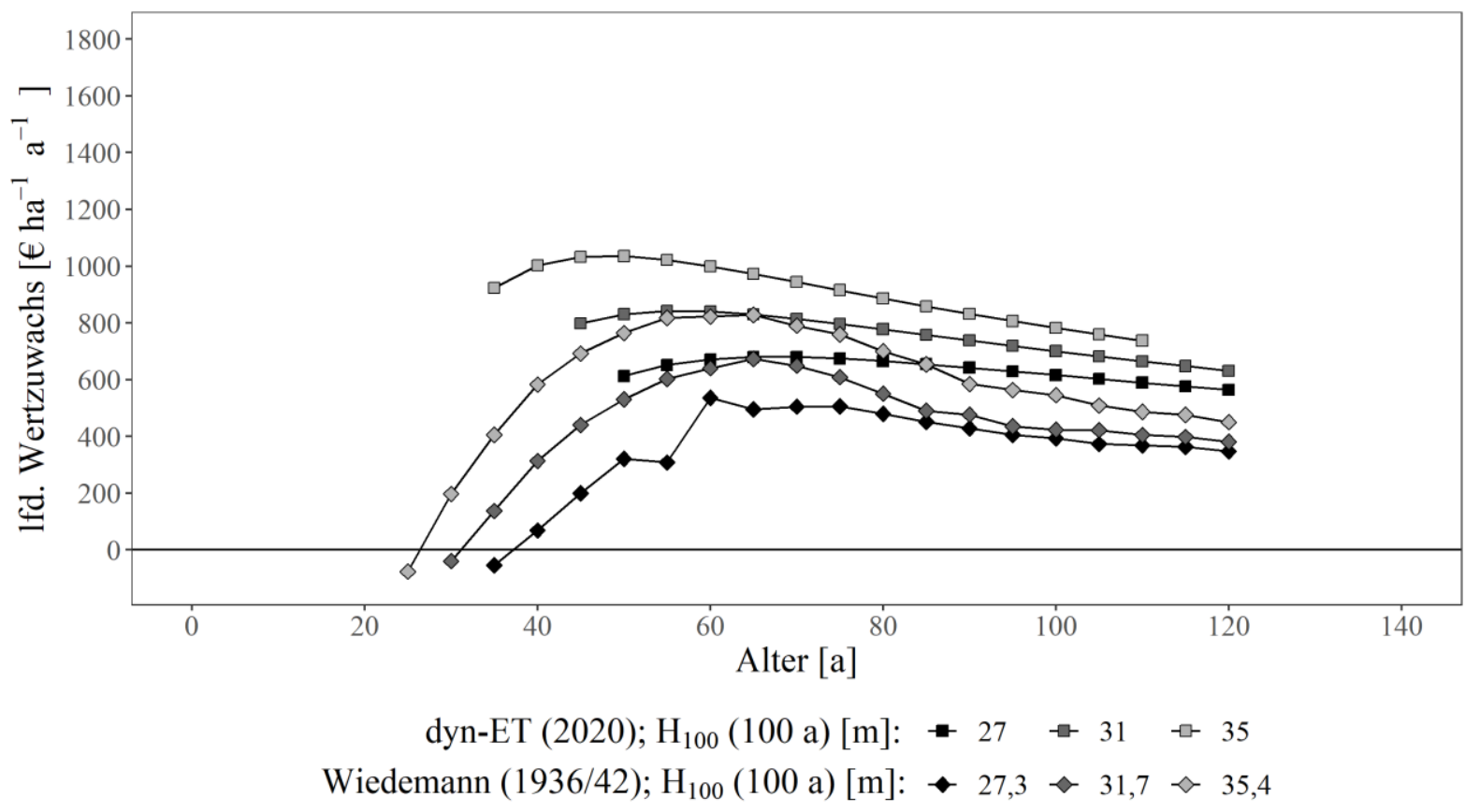

Abbildung 200:Laufender Bestandeswertzuwachs über dem Alter; ausgewählte Oberhöhenbonitäten der Baumart Fichte (starke Durchforstung) gemäß dyn-ET (unveröff.) und Wiedemann (1936/42). 


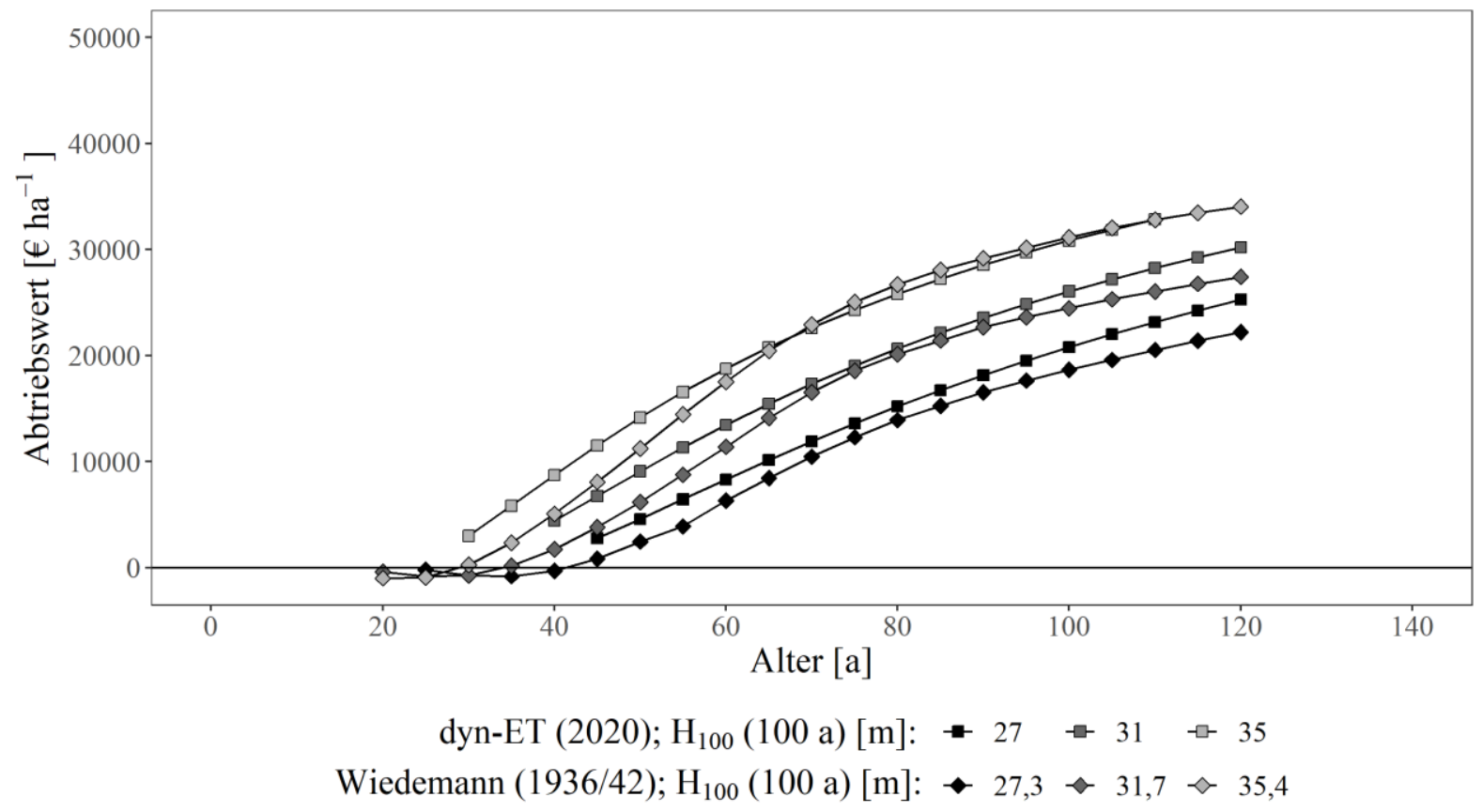

Abbildung 201: Abtriebswert über dem Alter; ausgewählte Oberhöhenbonitäten der Baumart Fichte (starke Durchforstung) gemäß dyn-ET (unveröff.) und Wiedemann (1936/42). 


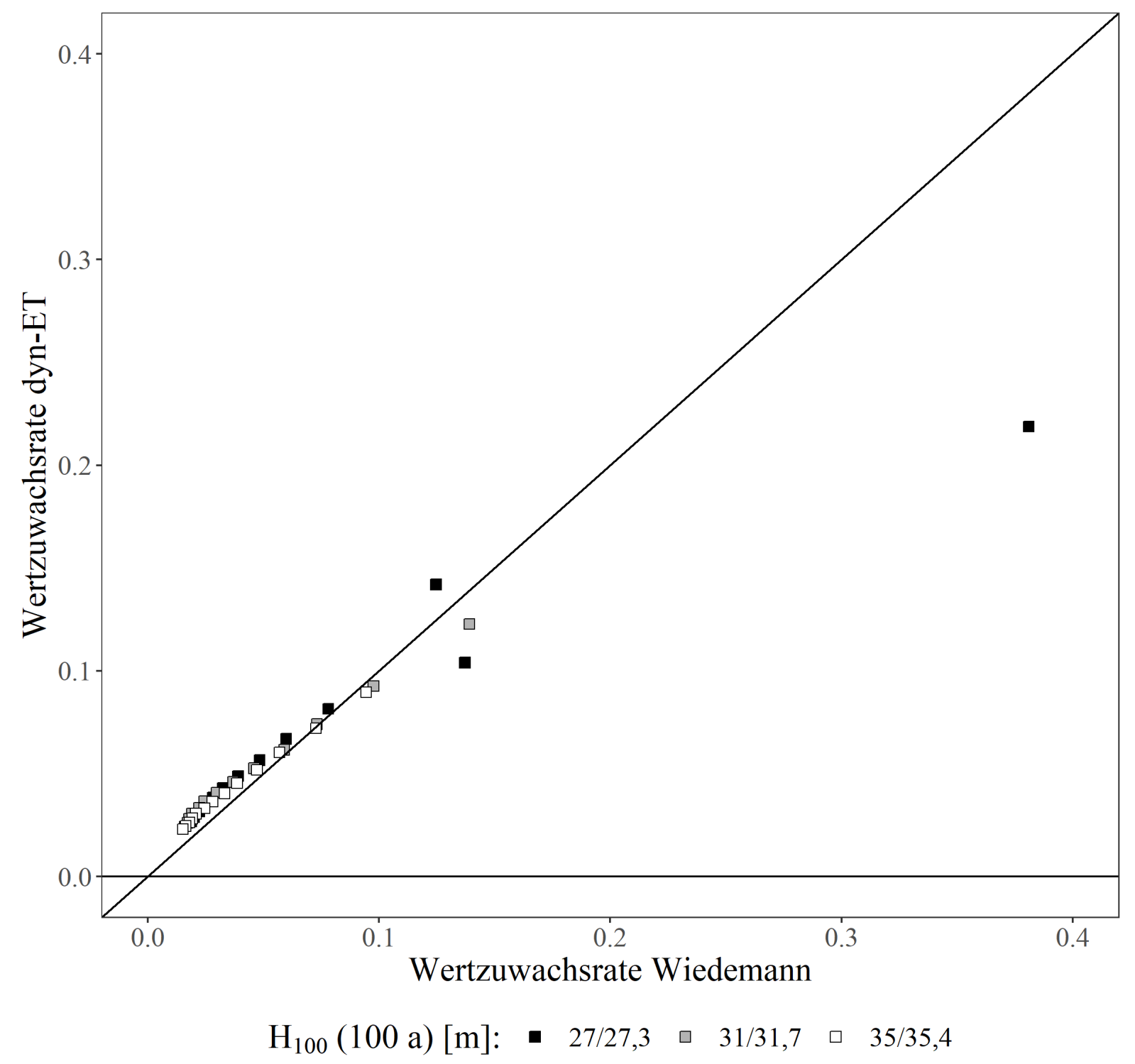

Abbildung 202: Wertzuwachsraten der Baumart Fichte (starke Durchforstung, ausgewählte Oberhöhenbonitäten) gemäß dyn-ET (unveröff.) und Wiedemann (1936/42); es werden nur Vergleichspaare aus Altern dargestellt, in denen für alle Oberhöhenbonitäten Datenpunkte vorliegen; die Winkelhalbierende kennzeichnet gleiche Wertzuwachsraten im gleichen Alter. 


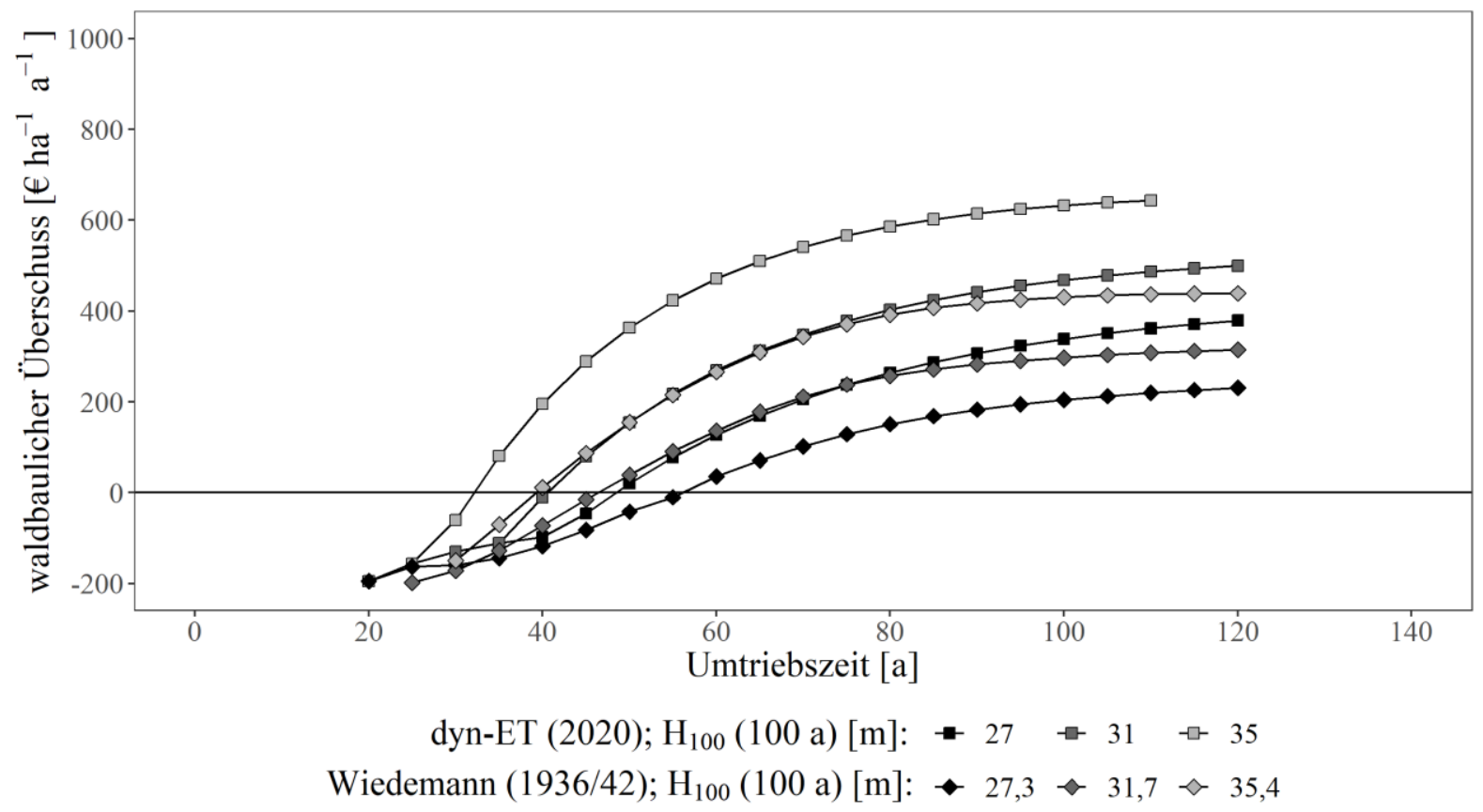

Abbildung 203: Durchschnittlicher waldbaulicher Überschuss über der Umrtriebszeit; ausgewählte Oberhöhenbonitäten der Baumart Fichte (starke Durchforstung) gemäß dyn-ET (unveröff.) und Wiedemann (1936/42).

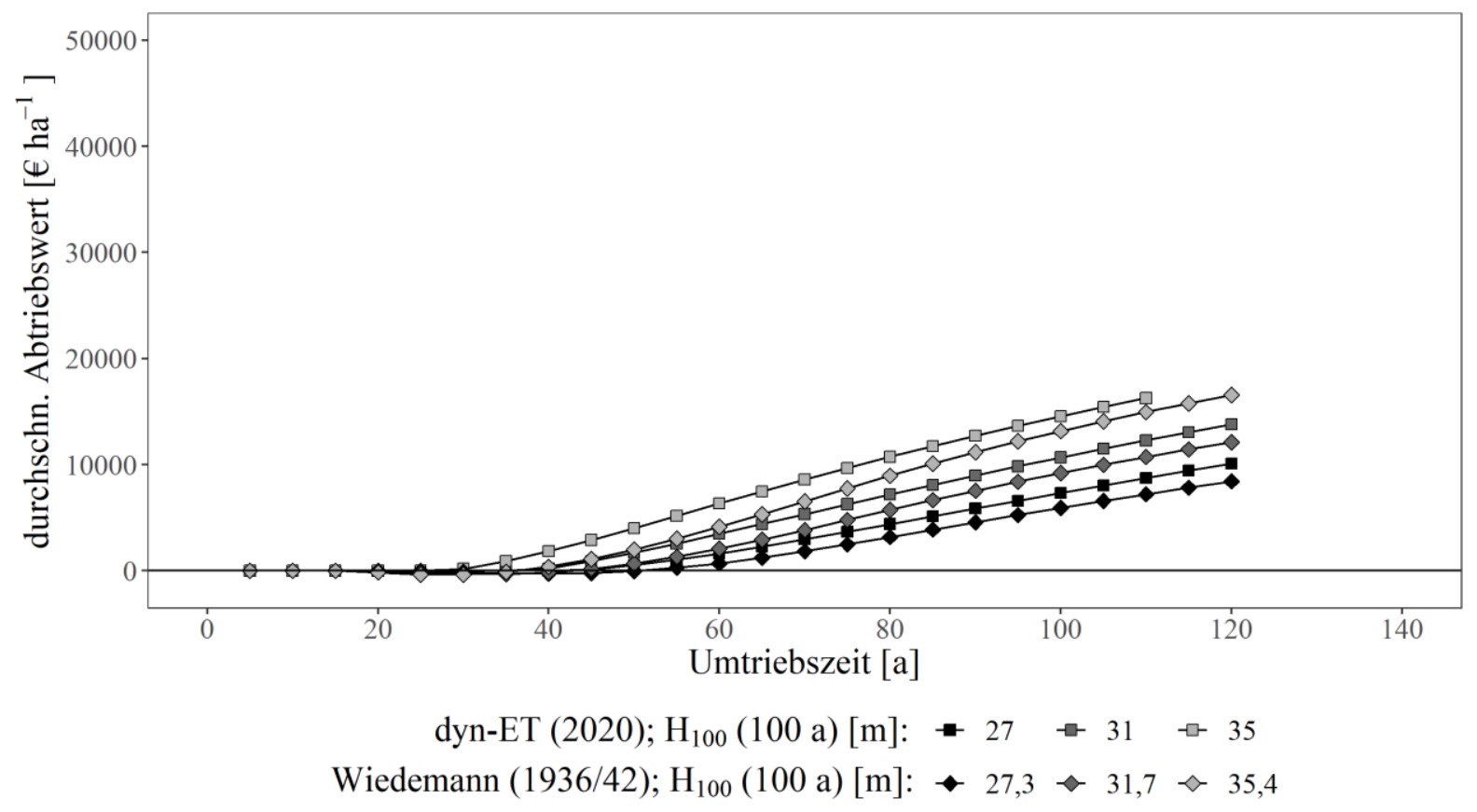

Abbildung 204: Durchschnittlicher Abtriebswert über der Umtriebszeit; ausgewählte Oberhöhenbonitäten der Baumart Fichte (starke Durchforstung) gemäß dyn-ET (unveröff.) und Wiedemann (1936/42). 


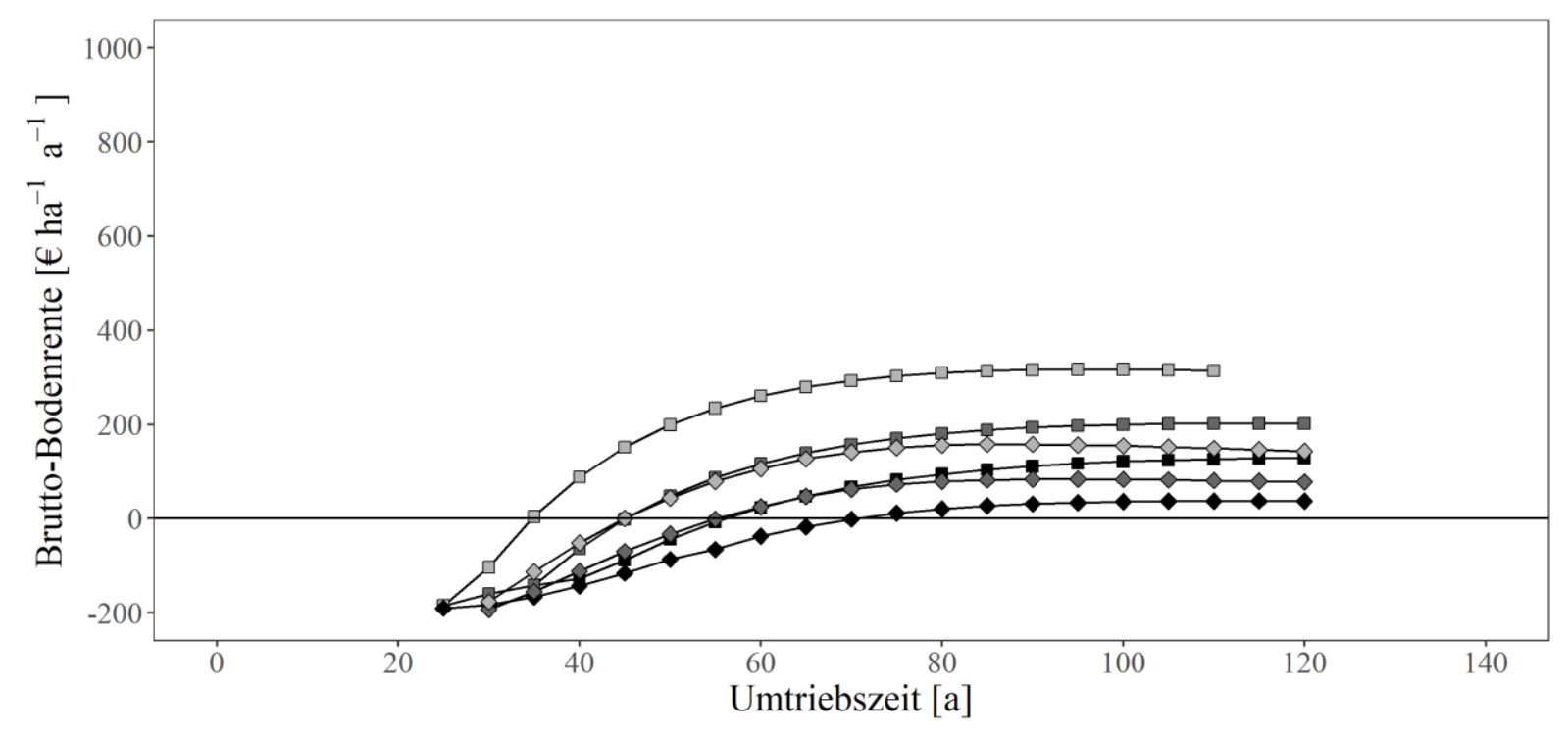

$$
\begin{aligned}
& \text { dyn-ET (2020); } \mathrm{H}_{100}(100 \mathrm{a})[\mathrm{m}]:-27 \rightarrow 31 \rightarrow 35 \\
& \text { Wiedemann (1936/42); } \mathrm{H}_{100}(100 \mathrm{a})[\mathrm{m}]: \diamond 27,3 \diamond 31,7 \diamond 35,4
\end{aligned}
$$

Abbildung 205: Brutto-Bodenrente über dem Alter; ausgewählte Oberhöhenbonitäten der Baumart Fichte (starke Durchforstung) gemäß dyn-ET (unveröff.) und Wiedemann (1936/42).

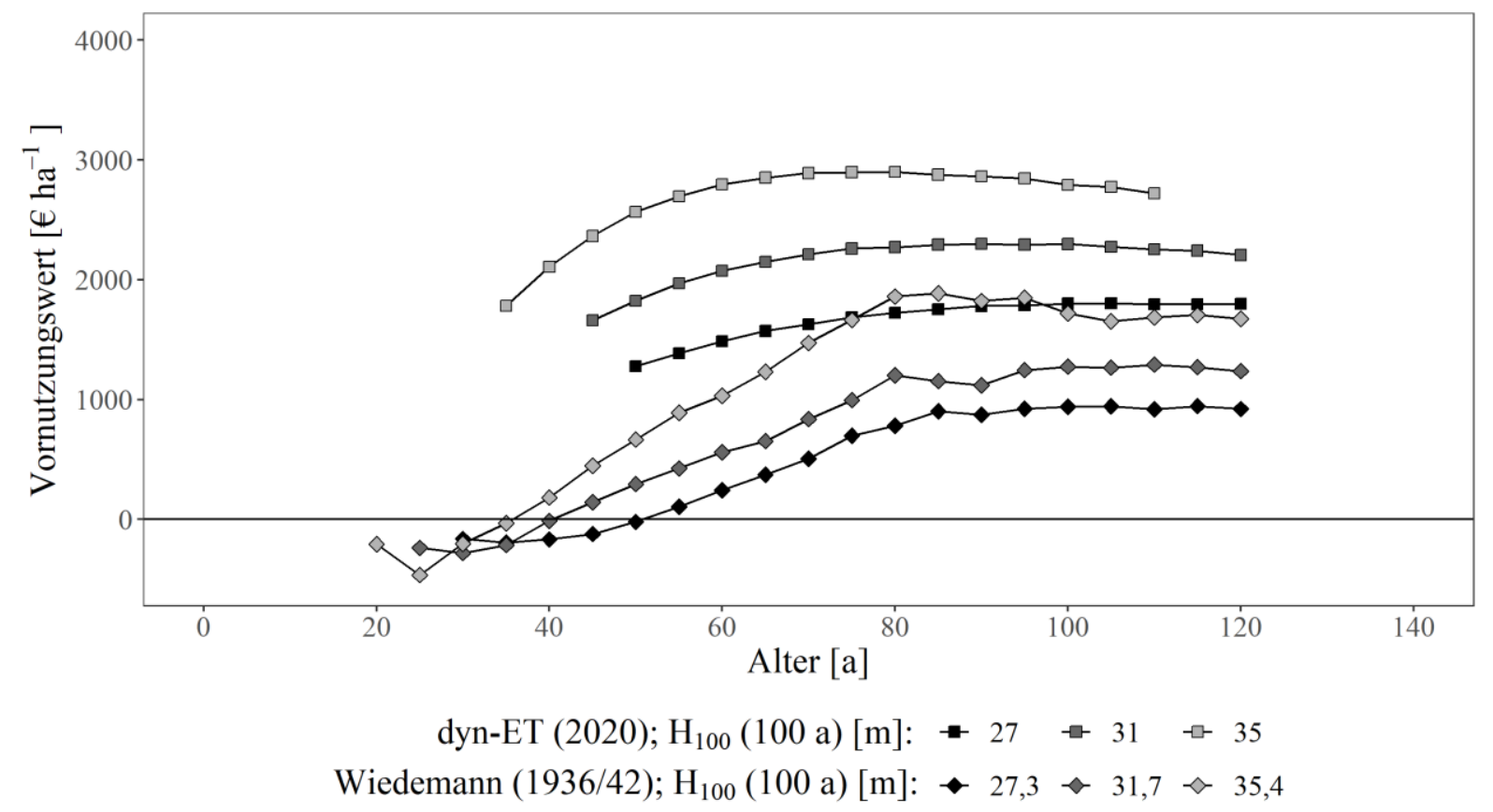

Abbildung 206: Vornutzungswerte über dem Alter; ausgewählte Oberhöhenbonitäten der Baumart Fichte (starke Durchforstung) gemäß dyn-ET (unveröff.) und Wiedemann (1936/42). 


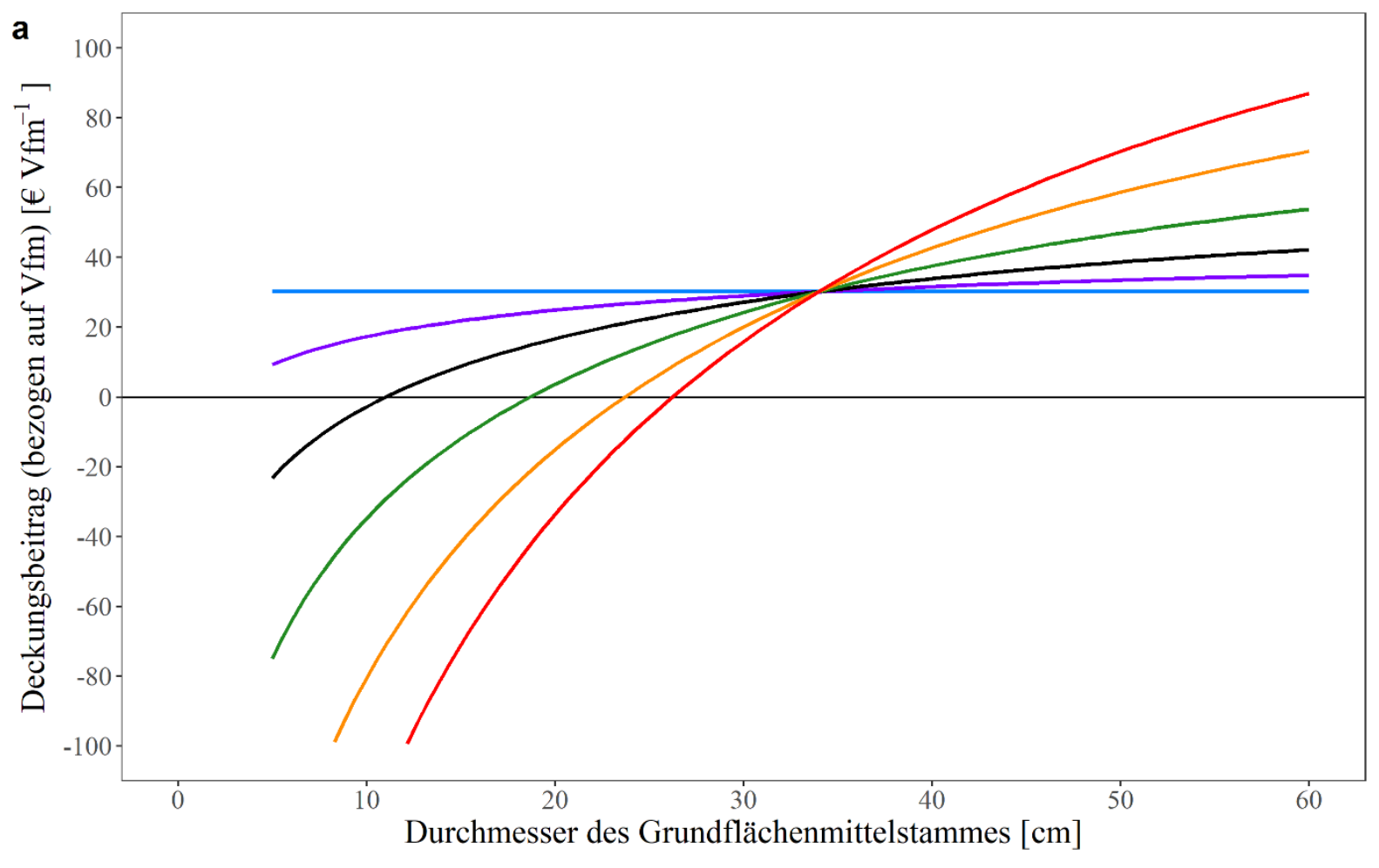

$-1-2-$ Referenz $-4-5-6$

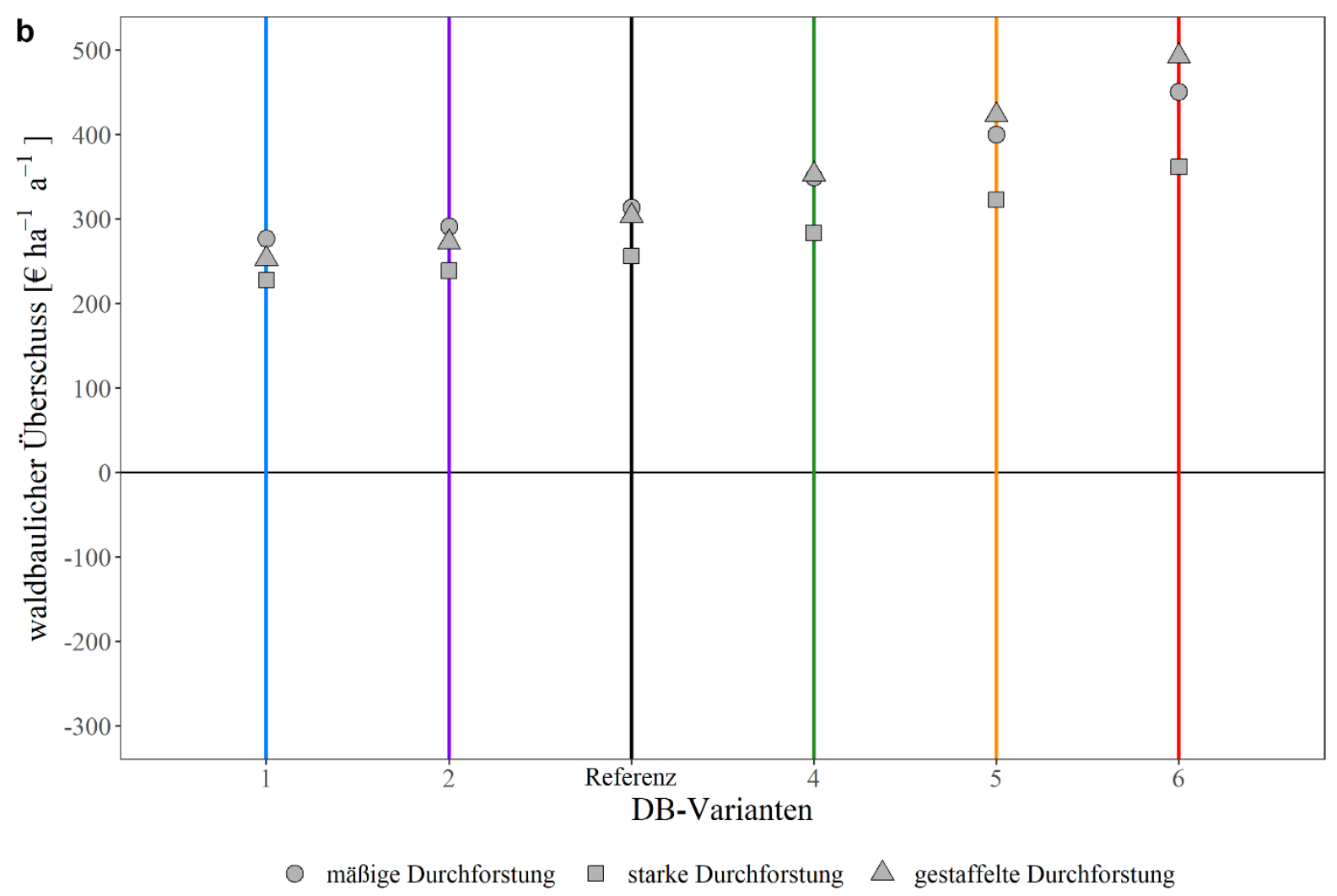

Abbildung 207: Analyse der Auswirkung unterschiedlicher Deckungsbeitragsfunktionen (a) auf den durchschnittlichen waldbaulichen Überschuss (b) der Baumart Buche $\left(H_{100}(100 \mathrm{a})=32 \mathrm{~m}\right)$ im Vergleich der waldbaulichen Behandlungsvarianten gemäß dyn-ET (unveröff.) bei den DB-variantenabhängig optimalen Umtriebszeiten; die schwarze Linie in (a) repräsentiert die Referenz-Deckungsbeitragsfunktion in Anlehnung an die ökonomische Bewertung in Abschnitt 3.3.5. 


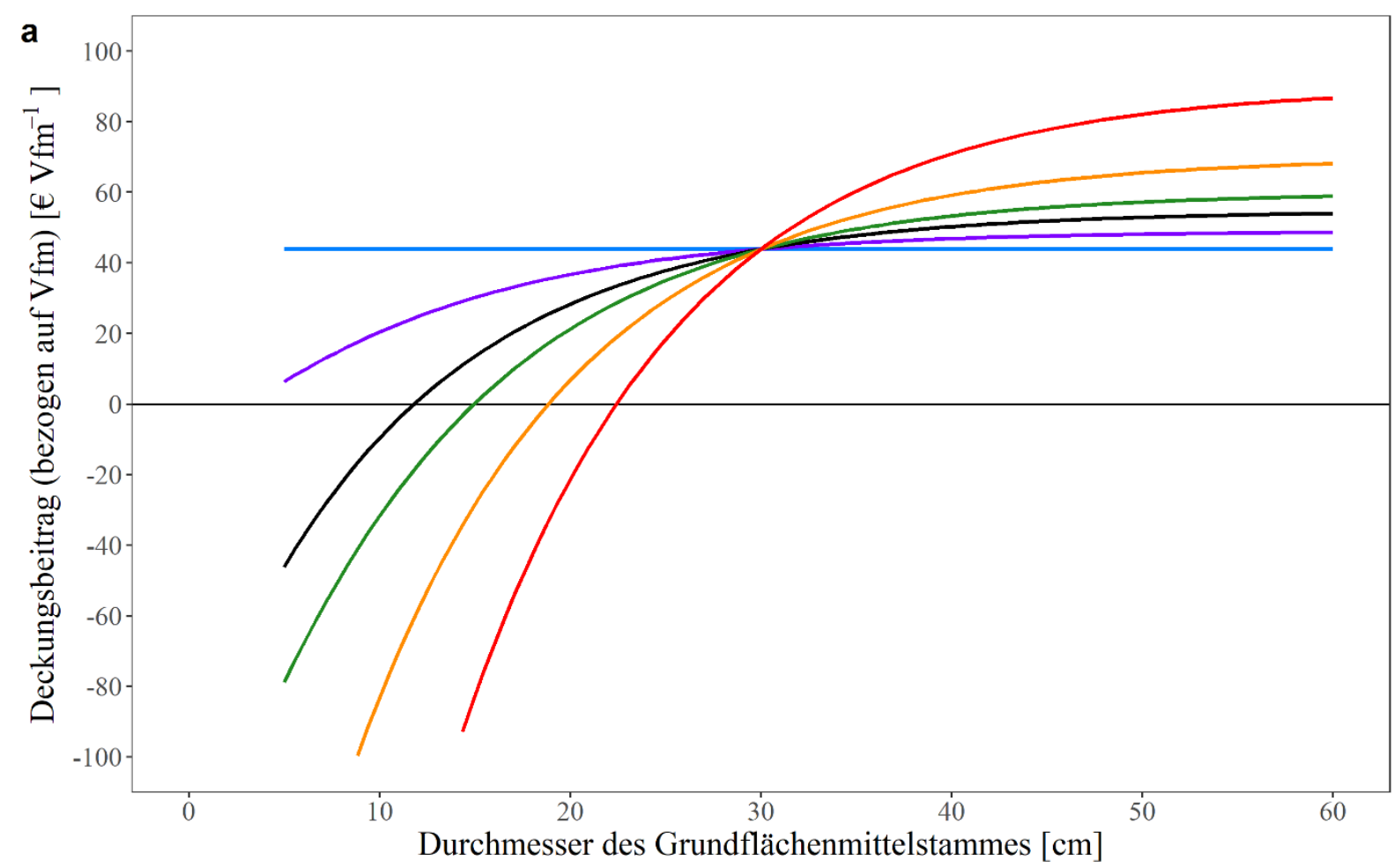

$-1-2-$ Referenz $-4-5-6$

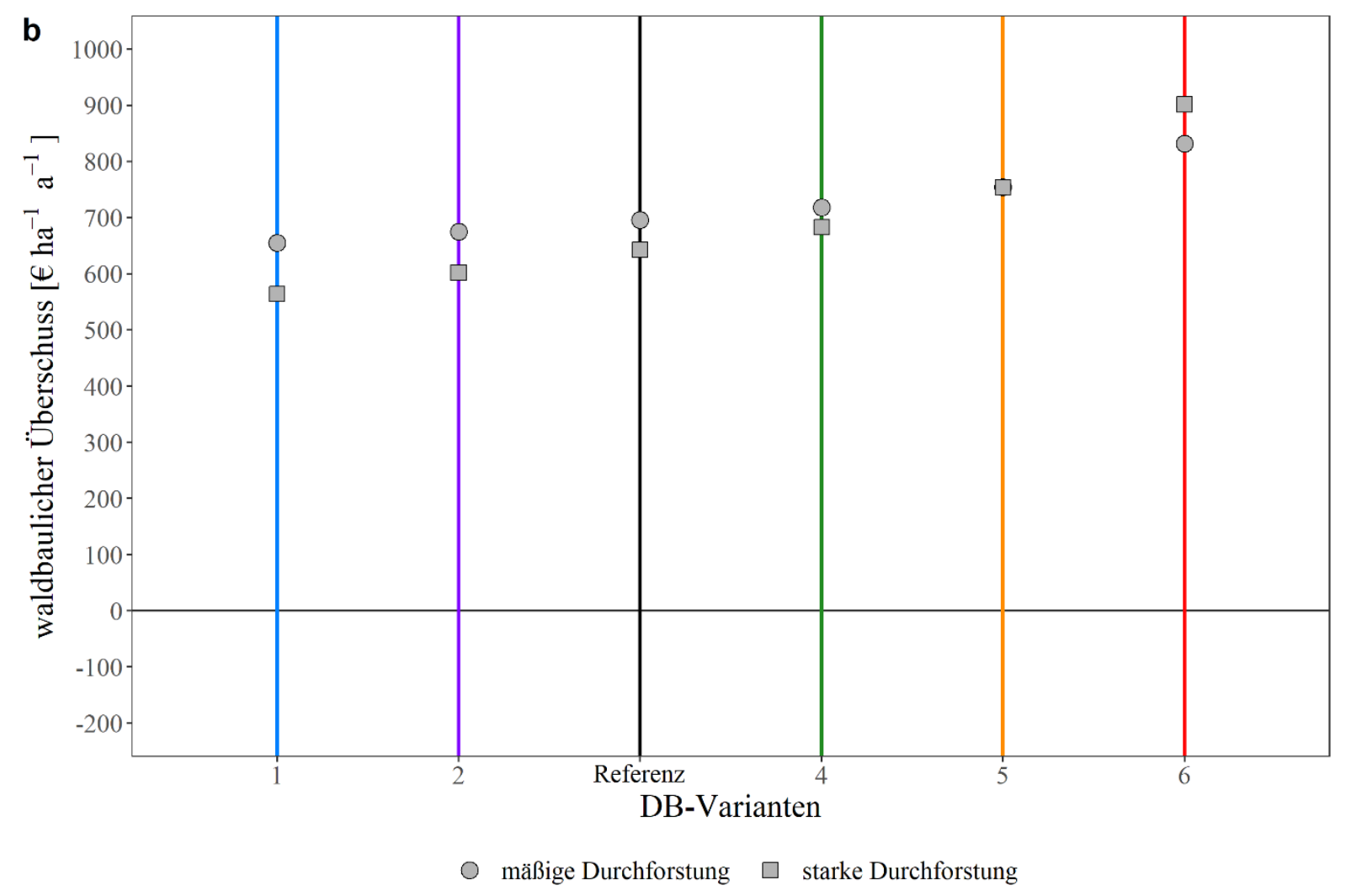

Abbildung 208: Analyse der Auswirkung unterschiedlicher Deckungsbeitragsfunktionen (a) auf den durchschnittlichen waldbaulichen Überschuss (b) der Baumart Fichte $\left(H_{100}(100 \mathrm{a})=35 \mathrm{~m}\right) \mathrm{im}$ Vergleich der waldbaulichen Behandlungsvarianten gemäß dyn-ET (unveröff.) bei den DB-variantenabhängig optimalen Umtriebszeiten; die schwarze Linie in (a) repräsentiert die Referenz-Deckungsbeitragsfunktion in Anlehnung an die ökonomische Bewertung in Abschnitt 3.3.5. 


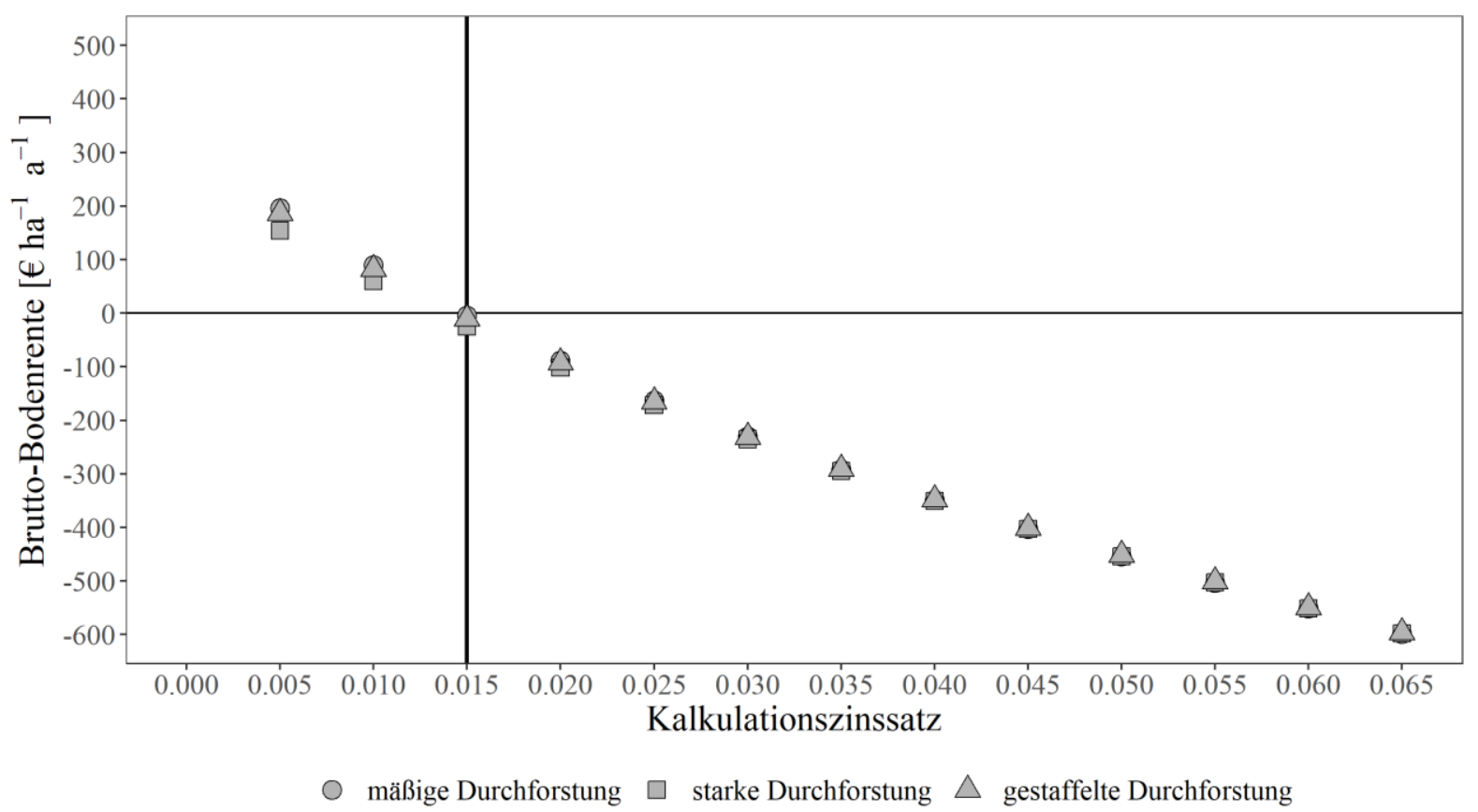

Abbildung 209: Analyse der Auswirkung verschiedener Kalkulationszinssätze auf die Brutto-Bodenrente der Baumart Buche $\left(H_{100}(100 \mathrm{a})=32 \mathrm{~m}\right)$ im Vergleich der waldbaulichen Behandlungsvarianten gemäß dyn-ET (unveröff.) bei den kalkulationszinssatzspezifisch optimalen Umtriebszeiten; die schwarze Linie repräsentiert als Referenz den in Abschnitt 3.3.5 verwendeten Kalkulationszinssatz von $r=1,5 \%$.

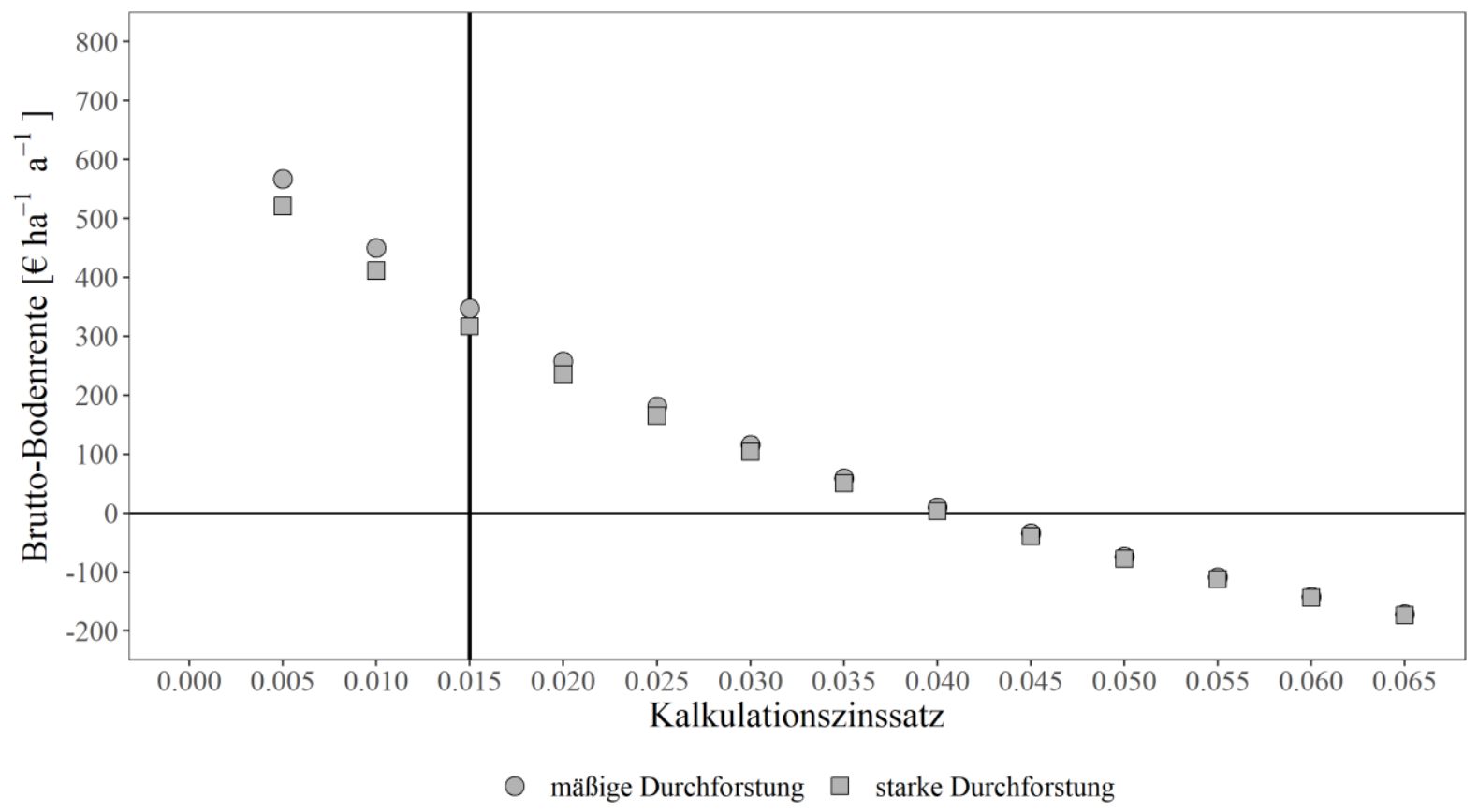

Abbildung 210: Analyse der Auswirkung verschiedener Kalkulationszinssätze auf die Brutto-Bodenrente der Baumart Fichte $\left(H_{100}(100 \mathrm{a})=35 \mathrm{~m}\right)$ im Vergleich der waldbaulichen Behandlungsvarianten gemäß dyn-ET (unveröff.) bei den kalkulationszinssatzspezifisch optimalen Umtriebszeiten; die schwarze Linie repräsentiert als Referenz den in Abschnitt 3.3.5 verwendeten Kalkulationszinssatz von $r=1,5 \%$. 
Tabelle 8-2: Ausgewählte Daten der vorläufigen Ertragstafeltabelle Buche, mäßige Hochdurchforstung, Oberhöhenbonität $H_{100}(100 \mathrm{a})=24 \mathrm{~m}$ gemäß dyn-ET (unveröff.):

$\boldsymbol{t}$ : Alter [a], $\boldsymbol{H}_{\mathbf{1 0 0}}$ : Oberhöhe [m], $\boldsymbol{N}$ : Stammzahl [ha $\left.{ }^{-1}\right], \boldsymbol{V}_{\boldsymbol{B}}$ : Bestandesvolumen [Vfm ha ${ }^{-1}$ ], $\boldsymbol{D}_{\boldsymbol{g}}$ : Durchmesser des Grundflächenmittelstammes [ $\left.\mathrm{cm}\right], \boldsymbol{G}_{\boldsymbol{B}}$ : Bestandesgrundfläche $\left[\mathrm{m}^{2} \mathrm{ha}^{-1}\right]$, $\lambda$ : Vornutzungsvolumen [Vfm ha $\left.{ }^{-1}\right], \boldsymbol{G W} \boldsymbol{L}$ : Gesamtwuchsleistung [Vfm ha $\left.{ }^{-1}\right], \boldsymbol{v}_{\boldsymbol{B}}$ : lfd. Bestandesvolumenzuwachs [Vfm ha ${ }^{-1} \mathrm{a}^{-1}$ ], $\overline{\boldsymbol{v}_{\boldsymbol{B}}}$ : durchsch..-jährl. Bestandesvolumenzuw. [Vfm ha ${ }^{-1} \mathrm{a}^{-1}$ ].

\begin{tabular}{|c|c|c|c|c|c|c|c|c|c|c|c|c|}
\hline \multirow{2}{*}{\multicolumn{2}{|c|}{$t \quad H_{100}$}} & \multicolumn{4}{|c|}{ verbleibender Bestand } & \multicolumn{4}{|c|}{ ausscheidender Bestand } & \multicolumn{3}{|c|}{ Gesamtbestand } \\
\hline & & $N$ & $V_{B}$ & $G_{B}$ & $D_{g}$ & $N$ & $\lambda$ & $\overline{G_{B}}$ & $D_{g}$ & $G W L$ & $v_{B}$ & $\overline{v_{B}}$ \\
\hline \multicolumn{13}{|l|}{25} \\
\hline \multicolumn{13}{|l|}{30} \\
\hline \multicolumn{13}{|l|}{35} \\
\hline 40 & 9,0 & 2.875 & 18 & 4,7 & 4,6 & 0 & 0 & 0,3 & 0,0 & 18 & 0,0 & 0,4 \\
\hline 45 & 11,0 & 2.243 & 68 & 14,6 & 9,1 & 632 & 0 & 1,5 & 5,5 & 68 & 10,0 & 1,5 \\
\hline 50 & 12,8 & 1.796 & 83 & 15,3 & 10,4 & 447 & 27 & 3,1 & 9,4 & 111 & 8,5 & 2,2 \\
\hline 55 & 14,4 & 1.469 & 104 & 16,9 & 12,1 & 327 & 23 & 2,9 & 10,7 & 155 & 8,8 & 2,8 \\
\hline 60 & 15,8 & 1.223 & 124 & 18,2 & 13,8 & 246 & 24 & 3,0 & 12,5 & 199 & 9,0 & 3,3 \\
\hline 65 & 17,1 & 1.033 & 144 & 19,4 & 15,4 & 190 & 25 & 3,1 & 14,3 & 245 & 9,1 & 3,8 \\
\hline 70 & 18,3 & 883 & 164 & 20,4 & 17,1 & 150 & 26 & 3,1 & 16,2 & 291 & 9,1 & 4,2 \\
\hline 75 & 19,4 & 763 & 183 & 21,3 & 18,8 & 120 & 27 & 3,1 & 18,1 & 336 & 9,1 & 4,5 \\
\hline 80 & 20,4 & 666 & 201 & 22,0 & 20,5 & 97 & 27 & 3,0 & 20,0 & 382 & 9,1 & 4,8 \\
\hline 85 & 21,3 & 586 & 219 & 22,8 & 22,2 & 80 & 27 & 3,0 & 21,9 & 427 & 9,0 & 5,0 \\
\hline 90 & 22,2 & 520 & 236 & 23,4 & 23,9 & 66 & 28 & 2,9 & 23,8 & 472 & 8,9 & 5,2 \\
\hline 95 & 23,0 & 464 & 252 & 24,0 & 25,6 & 56 & 27 & 2,9 & 25,6 & 516 & 8,8 & 5,4 \\
\hline 100 & 23,8 & 416 & 268 & 24,5 & 27,4 & 48 & 27 & 2,8 & 27,2 & 559 & 8,7 & 5,6 \\
\hline 105 & 24,5 & 375 & 284 & 25,0 & 29,1 & 41 & 27 & 2,7 & 29,0 & 602 & 8,5 & 5,7 \\
\hline 110 & 25,2 & 340 & 299 & 25,4 & 30,8 & 35 & 27 & 2,6 & 30,8 & 643 & 8,4 & 5,8 \\
\hline 115 & 25,8 & 310 & 313 & 25,8 & 32,5 & 30 & 26 & 2,5 & 32,6 & 684 & 8,2 & 6,0 \\
\hline 120 & 26,5 & 283 & 327 & 26,2 & 34,3 & 27 & 26 & 2,4 & 33,7 & 724 & 8,0 & 6,0 \\
\hline 125 & 27,0 & 260 & 341 & 26,5 & 36,0 & 23 & 25 & 2,3 & 35,6 & 763 & 7,8 & 6,1 \\
\hline 130 & 27,6 & 239 & 354 & 26,8 & 37,8 & 21 & 25 & 2,2 & 36,4 & 801 & 7,6 & 6,2 \\
\hline 135 & 28,1 & 221 & 367 & 27,1 & 39,5 & 18 & 24 & 2,1 & 38,2 & 838 & 7,4 & 6,2 \\
\hline 140 & 28,6 & 204 & 379 & 27,4 & 41,3 & 17 & 24 & 2,0 & 38,2 & 874 & 7,2 & 6,2 \\
\hline 145 & 29,1 & 190 & 391 & 27,6 & 43,0 & 14 & 23 & 1,8 & 40,9 & 909 & 7,0 & 6,3 \\
\hline 150 & 29,6 & 177 & 403 & 27,9 & 44,8 & 13 & 22 & 1,7 & 41,0 & 944 & 6,8 & 6,3 \\
\hline
\end{tabular}


Tabelle 8-3: Ausgewählte Daten der vorläufigen Ertragstafeltabelle Buche, mäßige Hochdurchforstung, Oberhöhenbonität $H_{100}(100 \mathrm{a})=28 \mathrm{~m}$ gemäß dyn-ET (unveröff.):

$\boldsymbol{t}$ : Alter [a], $\boldsymbol{H}_{\mathbf{1 0 0}}$ : Oberhöhe [m], $\boldsymbol{N}$ : Stammzahl [ha $\left.{ }^{-1}\right], \boldsymbol{V}_{\boldsymbol{B}}$ : Bestandesvolumen [Vfm ha ${ }^{-1}$ ], $\boldsymbol{D}_{\boldsymbol{g}}$ : Durchmesser des Grundflächenmittelstammes $[\mathrm{cm}], \boldsymbol{G}_{\boldsymbol{B}}$ : Bestandesgrundfläche $\left[\mathrm{m}^{2} \mathrm{ha}^{-1}\right]$, $\boldsymbol{\lambda}$ : Vornutzungsvolumen [Vfm ha $\left.{ }^{-1}\right], \boldsymbol{G W} \boldsymbol{L}$ : Gesamtwuchsleistung [Vfm ha $\left.{ }^{-1}\right], \boldsymbol{v}_{\boldsymbol{B}}$ : lfd. Bestandesvolumenzuwachs [Vfm ha ${ }^{-1} \mathrm{a}^{-1}$ ], $\overline{\boldsymbol{v}_{\boldsymbol{B}}}$ : durchsch..-jährl. Bestandesvolumenzuw. [Vfm ha ${ }^{-1} \mathrm{a}^{-1}$ ].

\begin{tabular}{|c|c|c|c|c|c|c|c|c|c|c|c|c|}
\hline \multirow[b]{2}{*}{$\boldsymbol{t}$} & \multirow[b]{2}{*}{$H_{100}$} & \multicolumn{4}{|c|}{ verbleibender Bestand } & \multicolumn{4}{|c|}{ ausscheidender Bestand } & \multicolumn{3}{|c|}{ Gesamtbestand } \\
\hline & & $N$ & $V_{B}$ & $G_{B}$ & $D_{g}$ & $N$ & $\lambda$ & $G_{B}$ & $D_{g}$ & $G W L$ & $v_{B}$ & $\overline{\overline{v_{B}}}$ \\
\hline \multicolumn{13}{|l|}{25} \\
\hline \multicolumn{13}{|l|}{30} \\
\hline 35 & 9,7 & 2.914 & 33 & 8,1 & 5,9 & 0 & 0 & 1,0 & 0,0 & 33 & 0,0 & 0,9 \\
\hline 40 & 12,1 & 2.199 & 75 & 14,6 & 9,2 & 715 & 8 & 2,2 & 6,2 & 83 & 10,0 & 2,1 \\
\hline 45 & 14,3 & 1.715 & 101 & 16,8 & 11,2 & 484 & 27 & 3,4 & 9,5 & 137 & 10,8 & 3,0 \\
\hline 50 & 16,2 & 1.374 & 128 & 18,5 & 13,1 & 341 & 30 & 3,6 & 11,6 & 193 & 11,3 & 3,9 \\
\hline 55 & 17,9 & 1.123 & 154 & 20,0 & 15,0 & 251 & 32 & 3,7 & 13,8 & 252 & 11,7 & 4,6 \\
\hline 60 & 19,4 & 935 & 179 & 21,2 & 17,0 & 188 & 34 & 3,8 & 16,1 & 311 & 11,9 & 5,2 \\
\hline 65 & 20,7 & 790 & 203 & 22,3 & 19,0 & 145 & 36 & 3,8 & 18,3 & 371 & 12,1 & 5,7 \\
\hline 70 & 22,0 & 675 & 227 & 23,2 & 20,9 & 115 & 37 & 3,8 & 20,6 & 432 & 12,1 & 6,2 \\
\hline 75 & 23,1 & 584 & 250 & 24,0 & 22,9 & 91 & 38 & 3,8 & 23,0 & 493 & 12,1 & 6,6 \\
\hline 80 & 24,2 & 509 & 272 & 24,7 & 24,9 & 75 & 39 & 3,7 & 25,1 & 553 & 12,1 & 6,9 \\
\hline 85 & 25,2 & 448 & 292 & 25,4 & 26,9 & 61 & 39 & 3,6 & 27,5 & 613 & 12,0 & 7,2 \\
\hline 90 & 26,1 & 397 & 313 & 25,9 & 28,8 & 51 & 39 & 3,5 & 29,7 & 673 & 11,9 & 7,5 \\
\hline 95 & 27,0 & 354 & 332 & 26,5 & 30,8 & 43 & 39 & 3,4 & 31,8 & 731 & 11,7 & 7,7 \\
\hline 100 & 27,8 & 318 & 351 & 26,9 & 32,8 & 36 & 39 & 3,3 & 34,1 & 789 & 11,5 & 7,9 \\
\hline 105 & 28,5 & 287 & 369 & 27,3 & 34,8 & 31 & 39 & 3,2 & 36,0 & 845 & 11,3 & 8,1 \\
\hline 110 & 29,2 & 260 & 386 & 27,7 & 36,8 & 27 & 38 & 3,0 & 37,7 & 901 & 11,1 & 8,2 \\
\hline 115 & 29,9 & 237 & 402 & 28,0 & 38,8 & 23 & 38 & 2,9 & 39,8 & 955 & 10,9 & 8,3 \\
\hline 120 & 30,6 & 216 & 418 & 28,3 & 40,9 & 21 & 37 & 2,7 & 40,5 & 1.008 & 10,6 & 8,4 \\
\hline 125 & 31,2 & 198 & 434 & 28,6 & 42,9 & 18 & 36 & 2,5 & 42,5 & 1.060 & 10,4 & 8,5 \\
\hline 130 & 31,8 & 183 & 449 & 28,9 & 44,8 & 15 & 36 & 2,4 & 45,0 & 1.110 & 10,1 & 8,5 \\
\hline 135 & 32,3 & 169 & 463 & 29,1 & 46,8 & 14 & 35 & 2,2 & 45,0 & 1.160 & 9,8 & 8,6 \\
\hline 140 & 32,9 & 156 & 477 & 29,3 & 48,9 & 13 & 34 & 2,1 & 45,0 & 1.207 & 9,6 & 8,6 \\
\hline 145 & 33,4 & 145 & 490 & 29,5 & 50,9 & 11 & 33 & 1,9 & 46,9 & 1.254 & 9,3 & 8,6 \\
\hline 150 & 33,9 & 135 & 503 & 29,7 & 52,9 & 10 & 32 & 1,7 & 47,1 & 1.299 & 9,0 & 8,7 \\
\hline
\end{tabular}


Tabelle 8-4: Ausgewählte Daten der vorläufigen Ertragstafeltabelle Buche, mäßige Hochdurchforstung, Oberhöhenbonität $H_{100}(100 \mathrm{a})=32 \mathrm{~m}$ gemäß dyn-ET (unveröff.):

$\boldsymbol{t}$ : Alter [a], $\boldsymbol{H}_{\mathbf{1 0 0}}$ : Oberhöhe [m], $\boldsymbol{N}$ : Stammzahl [ha $\left.{ }^{-1}\right], \boldsymbol{V}_{\boldsymbol{B}}$ : Bestandesvolumen [Vfm ha ${ }^{-1}$ ], $\boldsymbol{D}_{\boldsymbol{g}}$ : Durchmesser des Grundflächenmittelstammes $[\mathrm{cm}], \boldsymbol{G}_{\boldsymbol{B}}$ : Bestandesgrundfläche $\left[\mathrm{m}^{2} \mathrm{ha}^{-1}\right]$, $\boldsymbol{\lambda}$ : Vornutzungsvolumen [Vfm ha $\left.{ }^{-1}\right], \boldsymbol{G W} \boldsymbol{L}$ : Gesamtwuchsleistung [Vfm ha $\left.{ }^{-1}\right], \boldsymbol{v}_{\boldsymbol{B}}$ : lfd. Bestandesvolumenzuwachs [Vfm ha ${ }^{-1} \mathrm{a}^{-1}$ ], $\overline{\boldsymbol{v}_{\boldsymbol{B}}}$ : durchsch..-jährl. Bestandesvolumenzuw. [Vfm ha ${ }^{-1} \mathrm{a}^{-1}$ ].

\begin{tabular}{|c|c|c|c|c|c|c|c|c|c|c|c|c|}
\hline \multirow[b]{2}{*}{$t$} & \multirow[b]{2}{*}{$H_{100}$} & \multicolumn{4}{|c|}{ verbleibender Bestand } & \multicolumn{4}{|c|}{ ausscheidender Bestand } & \multicolumn{3}{|c|}{ Gesamtbestand } \\
\hline & & $N$ & $V_{B}$ & $G_{B}$ & $D_{g}$ & $N$ & $\lambda$ & $G_{B}$ & $D_{g}$ & $G W L$ & $v_{B}$ & $\overline{\bar{v}_{B}}$ \\
\hline \multicolumn{13}{|l|}{25} \\
\hline 30 & 9,7 & 3.198 & 33 & 8,1 & 5,7 & 0 & 0 & 1,2 & 0,0 & 33 & 0,0 & 1,1 \\
\hline 35 & 12,7 & 2.310 & 81 & 15,2 & 9,2 & 888 & 9 & 2,5 & 6,0 & 90 & 11,5 & 2,6 \\
\hline 40 & 15,3 & 1.743 & 114 & 17,7 & 11,4 & 567 & 31 & 3,9 & 9,4 & 154 & 12,8 & 3,9 \\
\hline 45 & 17,6 & 1.360 & 147 & 19,7 & 13,6 & 383 & 36 & 4,2 & 11,8 & 223 & 13,8 & 5,0 \\
\hline 50 & 19,5 & 1.089 & 180 & 21,4 & 15,8 & 271 & 40 & 4,4 & 14,4 & 295 & 14,4 & 5,9 \\
\hline 55 & 21,3 & 890 & 211 & 22,7 & 18,0 & 199 & 43 & 4,5 & 17,0 & 370 & 14,9 & 6,7 \\
\hline 60 & 22,9 & 741 & 241 & 23,9 & 20,2 & 149 & 46 & 4,6 & 19,8 & 446 & 15,2 & 7,4 \\
\hline 65 & 24,3 & 626 & 269 & 24,8 & 22,5 & 115 & 49 & 4,6 & 22,5 & 523 & 15,4 & 8,0 \\
\hline 70 & 25,6 & 535 & 297 & 25,7 & 24,7 & 91 & 50 & 4,5 & 25,2 & 601 & 15,5 & 8,6 \\
\hline 75 & 26,8 & 463 & 323 & 26,4 & 26,9 & 72 & 51 & 4,5 & 28,1 & 678 & 15,5 & 9,0 \\
\hline 80 & 28,0 & 404 & 348 & 27,0 & 29,2 & 59 & 52 & 4,3 & 30,6 & 756 & 15,5 & 9,4 \\
\hline 85 & 29,0 & 355 & 371 & 27,6 & 31,4 & 49 & 53 & 4,2 & 33,1 & 832 & 15,3 & 9,8 \\
\hline 90 & 30,0 & 315 & 394 & 28,0 & 33,7 & 40 & 53 & 4,1 & 36,0 & 908 & 15,2 & 10,1 \\
\hline 95 & 30,9 & 281 & 416 & 28,5 & 35,9 & 34 & 53 & 3,9 & 38,2 & 983 & 15,0 & 10,3 \\
\hline 100 & 31,7 & 252 & 437 & 28,8 & 38,2 & 29 & 53 & 3,7 & 40,4 & 1.056 & 14,7 & 10,6 \\
\hline 105 & 32,5 & 227 & 457 & 29,2 & 40,5 & 25 & 52 & 3,5 & 42,4 & 1.129 & 14,5 & 10,8 \\
\hline 110 & 33,3 & 206 & 476 & 29,5 & 42,7 & 21 & 52 & 3,3 & 45,0 & 1.200 & 14,2 & 10,9 \\
\hline 115 & 34,0 & 188 & 494 & 29,8 & 44,9 & 18 & 51 & 3,1 & 47,1 & 1.269 & 13,9 & 11,0 \\
\hline 120 & 34,7 & 171 & 511 & 30,0 & 47,3 & 17 & 50 & 2,9 & 46,8 & 1.337 & 13,6 & 11,1 \\
\hline 125 & 35,3 & 157 & 528 & 30,2 & 49,5 & 14 & 49 & 2,7 & 49,7 & 1.403 & 13,2 & 11,2 \\
\hline 130 & 36,0 & 145 & 544 & 30,4 & 51,7 & 12 & 48 & 2,5 & 51,5 & 1.468 & 12,9 & 11,3 \\
\hline 135 & 36,6 & 134 & 560 & 30,6 & 53,9 & 11 & 47 & 2,3 & 51,5 & 1.531 & 12,6 & 11,3 \\
\hline 140 & 37,1 & 124 & 575 & 30,7 & 56,2 & 10 & 46 & 2,1 & 51,4 & 1.592 & 12,2 & 11,4 \\
\hline 145 & 37,7 & 115 & 589 & 30,9 & 58,5 & 9 & 45 & 1,9 & 51,2 & 1.651 & 11,9 & 11,4 \\
\hline 150 & 38,2 & 107 & 603 & 31,0 & 60,7 & 8 & 44 & 1,6 & 51,1 & 1.709 & 11,6 & 11,4 \\
\hline
\end{tabular}


Tabelle 8-5: Ausgewählte Daten der vorläufigen Ertragstafeltabelle Buche, mäßige Hochdurchforstung, Oberhöhenbonität $H_{100}(100$ a) $=36 \mathrm{~m}$ gemäß dyn-ET (unveröff.):

$\boldsymbol{t}$ : Alter [a], $\boldsymbol{H}_{\mathbf{1 0 0}}$ : Oberhöhe [m], $\boldsymbol{N}$ : Stammzahl $\left[\mathrm{ha}^{-1}\right], \boldsymbol{V}_{\boldsymbol{B}}$ : Bestandesvolumen $\left[\mathrm{Vfm} \mathrm{ha} \mathrm{h}^{-1}\right]$, $\boldsymbol{D}_{\boldsymbol{g}}$ : Durchmesser des Grundflächenmittelstammes $[\mathrm{cm}], \boldsymbol{G}_{\boldsymbol{B}}$ : Bestandesgrundfläche $\left[\mathrm{m}^{2} \mathrm{ha}^{-1}\right]$, $\lambda$ : Vornutzungsvolumen [Vfm ha $\left.{ }^{-1}\right], \boldsymbol{G W L}$ : Gesamtwuchsleistung [Vfm ha $\left.{ }^{-1}\right], \boldsymbol{v}_{\boldsymbol{B}}$ : lfd. Bestandesvolumenzuwachs [Vfm ha ${ }^{-1} \mathrm{a}^{-1}$ ], $\overline{\boldsymbol{v}_{\boldsymbol{B}}}$ : durchsch..-jährl. Bestandesvolumenzuw. [Vfm ha ${ }^{-1} \mathrm{a}^{-1}$ ].

\begin{tabular}{|c|c|c|c|c|c|c|c|c|c|c|c|c|}
\hline \multirow[b]{2}{*}{$t$} & \multirow[b]{2}{*}{$H_{100}$} & \multicolumn{4}{|c|}{ verbleibender Bestand } & \multicolumn{4}{|c|}{ ausscheidender Bestand } & \multicolumn{3}{|c|}{ Gesamtbestand } \\
\hline & & $N$ & $V_{B}$ & $G_{B}$ & $D_{g}$ & $N$ & $\lambda$ & $G_{B}$ & $D_{g}$ & $G W L$ & $v_{B}$ & $\overline{v_{B}}$ \\
\hline 25 & 8,7 & 3.827 & 13 & 3,5 & 3,4 & 0 & 0 & 0,9 & 0,0 & 13 & 0,0 & 0,5 \\
\hline 30 & 12,6 & 2.606 & 79 & 15,1 & 8,6 & 1.221 & 0 & 2,2 & 4,8 & 79 & 13,1 & 2,6 \\
\hline 35 & 15,8 & 1.882 & 119 & 18,2 & 11,1 & 724 & 31 & 4,2 & 8,6 & 150 & 14,3 & 4,3 \\
\hline 40 & 18,5 & 1.420 & 160 & 20,5 & 13,6 & 462 & 39 & 4,7 & 11,3 & 230 & 15,9 & 5,7 \\
\hline 45 & 20,8 & 1.108 & 199 & 22,4 & 16,0 & 312 & 46 & 5,0 & 14,3 & 315 & 17,1 & 7,0 \\
\hline 50 & 22,9 & 887 & 237 & 23,9 & 18,5 & 221 & 52 & 5,2 & 17,3 & 405 & 17,9 & 8,1 \\
\hline 55 & 24,8 & 725 & 273 & 25,1 & 21,0 & 162 & 57 & 5,3 & 20,4 & 497 & 18,5 & 9,0 \\
\hline 60 & 26,4 & 604 & 307 & 26,1 & 23,5 & 121 & 60 & 5,3 & 23,7 & 592 & 18,9 & 9,9 \\
\hline 65 & 27,9 & 510 & 339 & 27,0 & 26,0 & 94 & 63 & 5,3 & 26,8 & 688 & 19,2 & 10,6 \\
\hline 70 & 29,3 & 436 & 370 & 27,7 & 28,4 & 74 & 66 & 5,2 & 30,0 & 784 & 19,3 & 11,2 \\
\hline 75 & 30,6 & 377 & 399 & 28,3 & 30,9 & 59 & 67 & 5,1 & 33,2 & 880 & 19,3 & 11,7 \\
\hline 80 & 31,7 & 329 & 427 & 28,9 & 33,4 & 48 & 68 & 4,9 & 36,2 & 976 & 19,2 & 12,2 \\
\hline 85 & 32,8 & 289 & 453 & 29,3 & 35,9 & 40 & 69 & 4,8 & 39,0 & 1.072 & 19,0 & 12,6 \\
\hline 90 & 33,8 & 256 & 478 & 29,7 & 38,4 & 33 & 69 & 4,6 & 42,0 & 1.166 & 18,8 & 13,0 \\
\hline 95 & 34,8 & 229 & 501 & 30,0 & 40,9 & 27 & 69 & 4,3 & 45,3 & 1.259 & 18,6 & 13,3 \\
\hline 100 & 35,7 & 205 & 524 & 30,3 & 43,4 & 24 & 69 & 4,1 & 46,7 & 1.350 & 18,3 & 13,5 \\
\hline 105 & 36,5 & 185 & 545 & 30,6 & 45,9 & 20 & 68 & 3,9 & 49,6 & 1.440 & 18,0 & 13,7 \\
\hline 110 & 37,3 & 168 & 565 & 30,8 & 48,3 & 17 & 68 & 3,6 & 51,9 & 1.528 & 17,6 & 13,9 \\
\hline 115 & 38,1 & 153 & 585 & 31,0 & 50,8 & 15 & 67 & 3,3 & 53,2 & 1.614 & 17,2 & 14,0 \\
\hline 120 & 38,8 & 140 & 603 & 31,1 & 53,2 & 13 & 66 & 3,1 & 54,9 & 1.699 & 16,8 & 14,2 \\
\hline 125 & 39,5 & 128 & 621 & 31,3 & 55,8 & 12 & 65 & 2,8 & 54,5 & 1.781 & 16,4 & 14,2 \\
\hline 130 & 40,2 & 118 & 638 & 31,4 & 58,2 & 10 & 63 & 2,5 & 56,7 & 1.861 & 16,0 & 14,3 \\
\hline 135 & 40,8 & 109 & 654 & 31,5 & 60,7 & 9 & 62 & 2,3 & 56,5 & 1.939 & 15,6 & 14,4 \\
\hline 140 & & & & & & & & & & & & \\
\hline 145 & & & & & & & & & & & & \\
\hline 150 & & & & & & & & & & & & \\
\hline
\end{tabular}


Tabelle 8-6: Ausgewählte Daten der vorläufigen Ertragstafeltabelle Buche, mäßige Hochdurchforstung, Oberhöhenbonität $H_{100}(100 \mathrm{a})=40 \mathrm{~m}$ gemäß dyn-ET (unveröff.):

$\boldsymbol{t}$ : Alter [a], $\boldsymbol{H}_{\mathbf{1 0 0}}$ : Oberhöhe [m], $\boldsymbol{N}$ : Stammzahl [ha $\left.{ }^{-1}\right], \boldsymbol{V}_{\boldsymbol{B}}$ : Bestandesvolumen [Vfm ha ${ }^{-1}$ ], $\boldsymbol{D}_{\boldsymbol{g}}$ : Durchmesser des Grundflächenmittelstammes [ $\left.\mathrm{cm}\right], \boldsymbol{G}_{\boldsymbol{B}}$ : Bestandesgrundfläche $\left[\mathrm{m}^{2} \mathrm{ha}^{-1}\right.$ ], $\lambda$ : Vornutzungsvolumen [Vfm ha $\left.{ }^{-1}\right], \boldsymbol{G W} \boldsymbol{L}$ : Gesamtwuchsleistung [Vfm ha $\left.{ }^{-1}\right], \boldsymbol{v}_{\boldsymbol{B}}$ : lfd. Bestandesvolumenzuwachs [Vfm ha $\left.{ }^{-1} \mathrm{a}^{-1}\right], \overline{\boldsymbol{v}_{\boldsymbol{B}}}$ : durchsch..-jährl. Bestandesvolumenzuw. [Vfm ha ${ }^{-1} \mathrm{a}^{-1}$ ].

\begin{tabular}{|c|c|c|c|c|c|c|c|c|c|c|c|c|}
\hline \multirow[b]{2}{*}{$t$} & \multirow[b]{2}{*}{$H_{100}$} & \multicolumn{4}{|c|}{ verbleibender Bestand } & \multicolumn{4}{|c|}{ ausscheidender Bestand } & \multicolumn{3}{|c|}{ Gesamtbestand } \\
\hline & & $N$ & $V_{B}$ & $G_{B}$ & $D_{g}$ & $N$ & $\lambda$ & $G_{B}$ & $D_{g}$ & GWL & $v_{B}$ & $\overline{\bar{v}_{B}}$ \\
\hline 25 & 11,5 & 3.186 & 80 & 16,9 & 8,2 & 0 & 0 & 2,9 & 0,0 & 80 & 0,0 & 3,2 \\
\hline 30 & 15,5 & 2.169 & 114 & 17,9 & 10,3 & 1.017 & 38 & 4,9 & 7,9 & 153 & 14,6 & 5,1 \\
\hline 35 & 18,8 & 1.567 & 163 & 20,8 & 13,0 & 602 & 38 & 4,8 & 10,1 & 239 & 17,4 & 6,8 \\
\hline 40 & 21,7 & 1.182 & 211 & 23,0 & 15,7 & 385 & 49 & 5,4 & 13,4 & 336 & 19,3 & 8,4 \\
\hline 45 & 24,1 & 922 & 256 & 24,7 & 18,5 & 260 & 58 & 5,7 & 16,8 & 440 & 20,7 & 9,8 \\
\hline 50 & 26,3 & 738 & 299 & 26,1 & 21,2 & 184 & 66 & 6,0 & 20,3 & 549 & 21,8 & 11,0 \\
\hline 55 & 28,2 & 604 & 339 & 27,1 & 23,9 & 134 & 72 & 6,1 & 24,0 & 661 & 22,5 & 12,0 \\
\hline 60 & 29,9 & 503 & 377 & 28,0 & 26,6 & 101 & 77 & 6,1 & 27,7 & 776 & 23,0 & 12,9 \\
\hline 65 & 31,5 & 424 & 412 & 28,8 & 29,4 & 79 & 81 & 6,0 & 31,1 & 892 & 23,3 & 13,7 \\
\hline 70 & 32,9 & 363 & 446 & 29,4 & 32,1 & 61 & 84 & 5,9 & 35,1 & 1.009 & 23,4 & 14,4 \\
\hline 75 & 34,3 & 314 & 477 & 29,8 & 34,8 & 49 & 86 & 5,7 & 38,6 & 1.126 & 23,4 & 15,0 \\
\hline 80 & 35,5 & 274 & 507 & 30,3 & 37,5 & 40 & 87 & 5,5 & 41,9 & 1.242 & 23,3 & 15,5 \\
\hline 85 & 36,6 & 241 & 535 & 30,6 & 40,2 & 33 & 88 & 5,3 & 45,2 & 1.358 & 23,1 & 16,0 \\
\hline 90 & 37,7 & 213 & 561 & 30,9 & 43,0 & 28 & 88 & 5,0 & 47,8 & 1.472 & 22,9 & 16,4 \\
\hline 95 & 38,7 & 190 & 586 & 31,1 & 45,7 & 23 & 88 & 4,7 & 51,2 & 1.585 & 22,6 & 16,7 \\
\hline 100 & 39,7 & 171 & 609 & 31,3 & 48,3 & 19 & 88 & 4,4 & 54,6 & 1.696 & 22,2 & 17,0 \\
\hline 105 & 40,5 & 154 & 631 & 31,5 & 51,0 & 17 & 87 & 4,1 & 55,6 & 1.805 & 21,8 & 17,2 \\
\hline 110 & 41,4 & 140 & 652 & 31,6 & 53,6 & 14 & 86 & 3,8 & 58,9 & 1.912 & 21,4 & 17,4 \\
\hline 115 & 42,2 & 127 & 672 & 31,7 & 56,4 & 13 & 85 & 3,5 & 58,4 & 2.017 & 20,9 & 17,5 \\
\hline 120 & & & & & & & & & & & & \\
\hline 125 & & & & & & & & & & & & \\
\hline 130 & & & & & & & & & & & & \\
\hline 135 & & & & & & & & & & & & \\
\hline 140 & & & & & & & & & & & & \\
\hline 145 & & & & & & & & & & & & \\
\hline 150 & & & & & & & & & & & & \\
\hline
\end{tabular}


Tabelle 8-7: Ausgewählte Daten der vorläufigen Ertragstafeltabelle Buche, starke Hochdurchforstung, Oberhöhenbonität $H_{100}(100$ a) $=24$ m gemäß dyn-ET (unveröff.):

$\boldsymbol{t}$ : Alter [a], $\boldsymbol{H}_{\mathbf{1 0 0}}$ : Oberhöhe [m], $\boldsymbol{N}$ : Stammzahl $\left[\mathrm{ha}^{-1}\right], \boldsymbol{V}_{\boldsymbol{B}}$ : Bestandesvolumen $\left[\mathrm{Vfm} \mathrm{ha} \mathrm{h}^{-1}\right]$, $\boldsymbol{D}_{\boldsymbol{g}}$ : Durchmesser des Grundflächenmittelstammes $[\mathrm{cm}], \boldsymbol{G}_{\boldsymbol{B}}$ : Bestandesgrundfläche $\left[\mathrm{m}^{2} \mathrm{ha}^{-1}\right]$, $\lambda$ : Vornutzungsvolumen [Vfm ha $\left.{ }^{-1}\right], \boldsymbol{G W L}$ : Gesamtwuchsleistung [Vfm ha $\left.{ }^{-1}\right], \boldsymbol{v}_{\boldsymbol{B}}$ : lfd. Bestandesvolumenzuwachs [Vfm ha ${ }^{-1} \mathrm{a}^{-1}$ ], $\overline{\boldsymbol{v}_{\boldsymbol{B}}}$ : durchsch..-jährl. Bestandesvolumenzuw. [Vfm ha ${ }^{-1} \mathrm{a}^{-1}$ ].

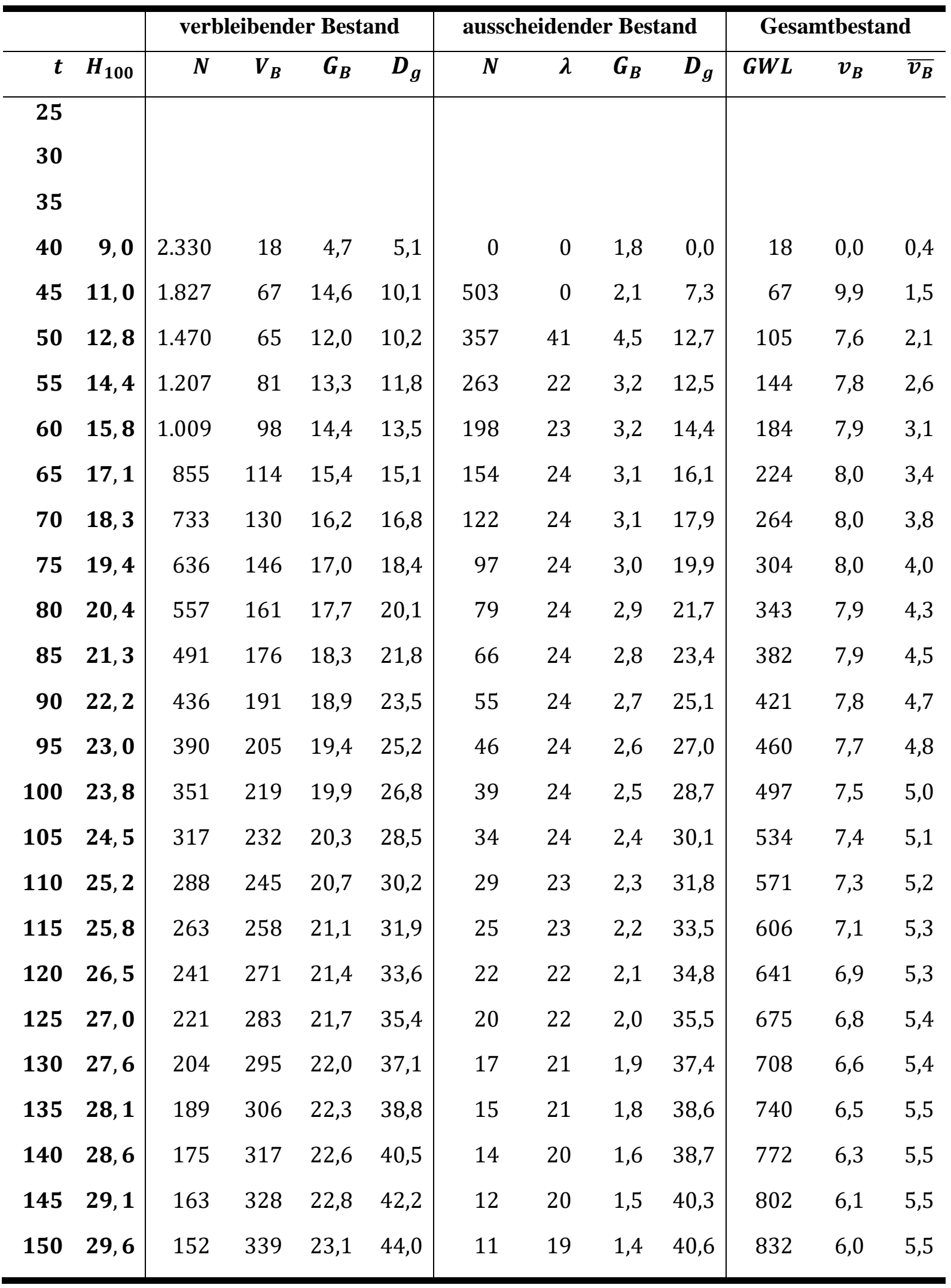


Tabelle 8-8: Ausgewählte Daten der vorläufigen Ertragstafeltabelle Buche, starke Hochdurchforstung, Oberhöhenbonität $H_{100}(100 \mathrm{a})=28 \mathrm{~m}$ gemäß dyn-ET (unveröff.):

$\boldsymbol{t}$ : Alter [a], $\boldsymbol{H}_{\mathbf{1 0 0}}$ : Oberhöhe [m], $\boldsymbol{N}$ : Stammzahl [ha $\left.{ }^{-1}\right], \boldsymbol{V}_{\boldsymbol{B}}$ : Bestandesvolumen [Vfm ha ${ }^{-1}$ ], $\boldsymbol{D}_{\boldsymbol{g}}$ : Durchmesser des Grundflächenmittelstammes [ $\left.\mathrm{cm}\right], \boldsymbol{G}_{\boldsymbol{B}}$ : Bestandesgrundfläche $\left[\mathrm{m}^{2} \mathrm{ha}^{-1}\right]$, $\lambda$ : Vornutzungsvolumen [Vfm ha $\left.{ }^{-1}\right], \boldsymbol{G W} \boldsymbol{L}$ : Gesamtwuchsleistung [Vfm ha $\left.{ }^{-1}\right], \boldsymbol{v}_{\boldsymbol{B}}$ : lfd. Bestandesvolumenzuwachs [Vfm ha ${ }^{-1} \mathrm{a}^{-1}$ ], $\overline{\boldsymbol{v}_{\boldsymbol{B}}}$ : durchsch..-jährl. Bestandesvolumenzuw. [Vfm ha ${ }^{-1} \mathrm{a}^{-1}$ ].

\begin{tabular}{|c|c|c|c|c|c|c|c|c|c|c|c|c|}
\hline \multirow{2}{*}{\multicolumn{2}{|c|}{$t \quad H_{100}$}} & \multicolumn{4}{|c|}{ verbleibender Bestand } & \multicolumn{4}{|c|}{ ausscheidender Bestand } & \multicolumn{3}{|c|}{ Gesamtbestand } \\
\hline & & $N$ & $V_{B}$ & $G_{B}$ & $D_{g}$ & $N$ & $\lambda$ & $G_{B}$ & $D_{g}$ & GWL & $v_{B}$ & $\overline{\bar{v}_{B}}$ \\
\hline \multicolumn{13}{|l|}{25} \\
\hline \multicolumn{13}{|l|}{30} \\
\hline 35 & 9,7 & 2.286 & 32 & 8,1 & 6,7 & 0 & 0 & 2,1 & 0,0 & 32 & 0,0 & 0,9 \\
\hline 40 & 12,1 & 1.735 & 58 & 11,5 & 9,2 & 551 & 20 & 3,4 & 8,8 & 78 & 9,1 & 1,9 \\
\hline 45 & 14,3 & 1.360 & 79 & 13,2 & 11,1 & 375 & 27 & 3,7 & 11,2 & 126 & 9,6 & 2,8 \\
\hline 50 & 16,2 & 1.094 & 101 & 14,6 & 13,1 & 266 & 28 & 3,7 & 13,3 & 176 & 10,0 & 3,5 \\
\hline 55 & 17,9 & 899 & 122 & 15,9 & 15,0 & 195 & 30 & 3,7 & 15,5 & 227 & 10,2 & 4,1 \\
\hline 60 & 19,4 & 751 & 143 & 17,0 & 17,0 & 148 & 31 & 3,6 & 17,7 & 279 & 10,4 & 4,6 \\
\hline 65 & 20,7 & 636 & 163 & 17,9 & 18,9 & 115 & 32 & 3,6 & 19,9 & 331 & 10,5 & 5,1 \\
\hline 70 & 22,0 & 546 & 183 & 18,7 & 20,9 & 90 & 33 & 3,5 & 22,2 & 384 & 10,5 & 5,5 \\
\hline 75 & 23,1 & 473 & 202 & 19,4 & 22,9 & 73 & 33 & 3,4 & 24,3 & 436 & 10,5 & 5,8 \\
\hline 80 & 24,2 & 414 & 221 & 20,1 & 24,9 & 59 & 33 & 3,3 & 26,6 & 488 & 10,4 & 6,1 \\
\hline 85 & 25,2 & 366 & 239 & 20,7 & 26,8 & 48 & 33 & 3,2 & 28,9 & 540 & 10,3 & 6,4 \\
\hline 90 & 26,1 & 325 & 257 & 21,2 & 28,8 & 41 & 33 & 3,0 & 30,7 & 591 & 10,2 & 6,6 \\
\hline 95 & 27,0 & 290 & 274 & 21,7 & 30,9 & 35 & 33 & 2,9 & 32,5 & 641 & 10,1 & 6,8 \\
\hline 100 & 27,8 & 261 & 291 & 22,1 & 32,9 & 29 & 33 & 2,8 & 34,8 & 691 & 9,9 & 6,9 \\
\hline 105 & 28,5 & 236 & 307 & 22,5 & 34,9 & 25 & 32 & 2,6 & 36,6 & 739 & 9,7 & 7,0 \\
\hline 110 & 29,2 & 214 & 322 & 22,9 & 36,9 & 22 & 32 & 2,5 & 37,9 & 787 & 9,5 & 7,2 \\
\hline 115 & 29,9 & 196 & 338 & 23,3 & 38,9 & 18 & 32 & 2,3 & 40,7 & 834 & 9,3 & 7,3 \\
\hline 120 & 30,6 & 179 & 352 & 23,6 & 41,0 & 17 & 31 & 2,2 & 40,6 & 879 & 9,1 & 7,3 \\
\hline 125 & 31,2 & 165 & 367 & 23,9 & 42,9 & 14 & 30 & 2,1 & 43,3 & 924 & 8,9 & 7,4 \\
\hline 130 & 31,8 & 152 & 381 & 24,2 & 45,0 & 13 & 30 & 1,9 & 43,3 & 968 & 8,7 & 7,4 \\
\hline 135 & 32,3 & 140 & 394 & 24,4 & 47,1 & 12 & 29 & 1,8 & 43,4 & 1010 & 8,5 & 7,5 \\
\hline 140 & 32,9 & 130 & 407 & 24,7 & 49,1 & 10 & 28 & 1,6 & 45,5 & 1051 & 8,3 & 7,5 \\
\hline 145 & 33,4 & 121 & 420 & 24,9 & 51,2 & 9 & 27 & 1,5 & 45,9 & 1092 & 8,1 & 7,5 \\
\hline 150 & 33,9 & 113 & 433 & 25,1 & 53,2 & 8 & 27 & 1,3 & 46,3 & 1131 & 7,8 & 7,5 \\
\hline
\end{tabular}


Tabelle 8-9: Ausgewählte Daten der vorläufigen Ertragstafeltabelle Buche, starke Hochdurchforstung, Oberhöhenbonität $H_{100}(100 \mathrm{a})=32 \mathrm{~m}$ gemäß dyn-ET (unveröff.):

$\boldsymbol{t}$ : Alter [a], $\boldsymbol{H}_{\mathbf{1 0 0}}$ : Oberhöhe [m], $\boldsymbol{N}$ : Stammzahl [ha $\left.{ }^{-1}\right], \boldsymbol{V}_{\boldsymbol{B}}$ : Bestandesvolumen [Vfm ha ${ }^{-1}$ ], $\boldsymbol{D}_{\boldsymbol{g}}$ : Durchmesser des Grundflächenmittelstammes $[\mathrm{cm}], \boldsymbol{G}_{\boldsymbol{B}}$ : Bestandesgrundfläche $\left[\mathrm{m}^{2} \mathrm{ha}^{-1}\right]$, $\boldsymbol{\lambda}$ : Vornutzungsvolumen [Vfm ha $\left.{ }^{-1}\right], \boldsymbol{G W} \boldsymbol{L}$ : Gesamtwuchsleistung [Vfm ha $\left.{ }^{-1}\right], \boldsymbol{v}_{\boldsymbol{B}}$ : lfd. Bestandesvolumenzuwachs [Vfm ha ${ }^{-1} \mathrm{a}^{-1}$ ], $\overline{\boldsymbol{v}_{\boldsymbol{B}}}$ : durchsch..-jährl. Bestandesvolumenzuw. [Vfm ha ${ }^{-1} \mathrm{a}^{-1}$ ].

\begin{tabular}{|c|c|c|c|c|c|c|c|c|c|c|c|c|}
\hline & & \multicolumn{4}{|c|}{ verbleibender Bestand } & \multicolumn{4}{|c|}{ ausscheidender Bestand } & \multicolumn{3}{|c|}{ Gesamtbestand } \\
\hline$t$ & $H_{100}$ & $N$ & $V_{B}$ & $G_{B}$ & $D_{g}$ & $N$ & $\lambda$ & $\boldsymbol{G}_{\boldsymbol{B}}$ & $D_{g}$ & $G W L$ & $v_{B}$ & $\overline{v_{B}}$ \\
\hline \multicolumn{13}{|l|}{25} \\
\hline 30 & 9,7 & 2.435 & 32 & 8,1 & 6,5 & 0 & 0 & 2,3 & 0,0 & 32 & 0,0 & 1,1 \\
\hline 35 & 12,7 & 1.771 & 63 & 11,9 & 9,3 & 664 & 22 & 3,7 & 8,4 & 85 & 10,5 & 2,4 \\
\hline 40 & 15,3 & 1.344 & 89 & 14,0 & 11,5 & 427 & 31 & 4,1 & 11,0 & 142 & 11,5 & 3,5 \\
\hline 45 & 17,6 & 1.054 & 116 & 15,7 & 13,8 & 290 & 34 & 4,1 & 13,5 & 203 & 12,2 & 4,5 \\
\hline 50 & 19,5 & 848 & 143 & 17,1 & 16,0 & 206 & 37 & 4,2 & 16,0 & 266 & 12,6 & 5,3 \\
\hline 55 & 21,3 & 696 & 169 & 18,3 & 18,3 & 152 & 39 & 4,1 & 18,6 & 331 & 13,0 & 6,0 \\
\hline 60 & 22,9 & 582 & 194 & 19,3 & 20,5 & 114 & 41 & 4,1 & 21,3 & 397 & 13,2 & 6,6 \\
\hline 65 & 24,3 & 493 & 219 & 20,2 & 22,8 & 89 & 42 & 4,0 & 23,9 & 463 & 13,3 & 7,1 \\
\hline 70 & 25,6 & 423 & 243 & 21,0 & 25,1 & 70 & 43 & 3,9 & 26,6 & 530 & 13,3 & 7,6 \\
\hline 75 & 26,8 & 367 & 266 & 21,6 & 27,4 & 56 & 44 & 3,8 & 29,2 & 596 & 13,3 & 7,9 \\
\hline 80 & 28,0 & 321 & 288 & 22,2 & 29,7 & 46 & 44 & 3,6 & 31,7 & 662 & 13,2 & 8,3 \\
\hline 85 & 29,0 & 283 & 309 & 22,8 & 32,0 & 38 & 44 & 3,5 & 34,2 & 728 & 13,1 & 8,6 \\
\hline 90 & 30,0 & 251 & 330 & 23,3 & 34,4 & 32 & 44 & 3,3 & 36,4 & 792 & 12,9 & 8,8 \\
\hline 95 & 30,9 & 225 & 350 & 23,7 & 36,6 & 26 & 44 & 3,2 & 39,4 & 856 & 12,7 & 9,0 \\
\hline 100 & 31,7 & 202 & 369 & 24,1 & 39,0 & 23 & 43 & 3,0 & 40,7 & 919 & 12,5 & 9,2 \\
\hline 105 & 32,5 & 183 & 388 & 24,5 & 41,3 & 19 & 43 & 2,8 & 43,5 & 980 & 12,3 & 9,3 \\
\hline 110 & 33,3 & 166 & 406 & 24,8 & 43,6 & 17 & 42 & 2,6 & 44,5 & 1.041 & 12,1 & 9,5 \\
\hline 115 & 34,0 & 151 & 423 & 25,1 & 46,0 & 15 & 42 & 2,5 & 45,8 & 1.100 & 11,8 & 9,6 \\
\hline 120 & 34,7 & 139 & 440 & 25,4 & 48,3 & 12 & 41 & 2,3 & 49,3 & 1.158 & 11,6 & 9,6 \\
\hline 125 & 35,3 & 127 & 456 & 25,7 & 50,8 & 12 & 40 & 2,1 & 47,3 & 1.214 & 11,3 & 9,7 \\
\hline 130 & 36,0 & 117 & 472 & 25,9 & 53,1 & 10 & 39 & 1,9 & 49,6 & 1.270 & 11,0 & 9,8 \\
\hline 135 & 36,6 & 109 & 488 & 26,2 & 55,3 & 8 & 38 & 1,8 & 52,8 & 1.323 & 10,8 & 9,8 \\
\hline 140 & 37,1 & 101 & 502 & 26,4 & 57,7 & 8 & 37 & 1,6 & 50,1 & 1.376 & 10,5 & 9,8 \\
\hline 145 & 37,7 & 94 & 517 & 26,6 & 60,0 & 7 & 37 & 1,4 & 50,4 & 1.427 & 10,2 & 9,8 \\
\hline 150 & & & & & & & & & & & & \\
\hline
\end{tabular}


Tabelle 8-10: Ausgewählte Daten der vorläufigen Ertragstafeltabelle Buche, starke Hochdurchforstung, Oberhöhenbonität $H_{100}(100 \mathrm{a})=36 \mathrm{~m}$ gemäß dyn-ET (unveröff.):

$\boldsymbol{t}$ : Alter [a], $\boldsymbol{H}_{\mathbf{1 0 0}}$ : Oberhöhe [m], $\boldsymbol{N}$ : Stammzahl [ha $\left.{ }^{-1}\right], \boldsymbol{V}_{\boldsymbol{B}}$ : Bestandesvolumen [Vfm ha ${ }^{-1}$ ], $\boldsymbol{D}_{\boldsymbol{g}}$ : Durchmesser des Grundflächenmittelstammes [ $\left.\mathrm{cm}\right], \boldsymbol{G}_{\boldsymbol{B}}$ : Bestandesgrundfläche $\left[\mathrm{m}^{2} \mathrm{ha}^{-1}\right.$ ], $\boldsymbol{\lambda}$ : Vornutzungsvolumen [Vfm ha $\left.{ }^{-1}\right], \boldsymbol{G W} \boldsymbol{L}$ : Gesamtwuchsleistung [Vfm ha $\left.{ }^{-1}\right], \boldsymbol{v}_{\boldsymbol{B}}$ : lfd. Bestandesvolumenzuwachs [Vfm ha ${ }^{-1} \mathrm{a}^{-1}$ ], $\overline{\boldsymbol{v}_{\boldsymbol{B}}}$ : durchsch..-jährl. Bestandesvolumenzuw. [Vfm ha ${ }^{-1} \mathrm{a}^{-1}$ ].

\begin{tabular}{|c|c|c|c|c|c|c|c|c|c|c|c|c|}
\hline \multirow[b]{2}{*}{$t$} & \multirow[b]{2}{*}{$H_{100}$} & \multicolumn{4}{|c|}{ verbleibender Bestand } & \multicolumn{4}{|c|}{ ausscheidender Bestand } & \multicolumn{3}{|c|}{ Gesamtbestand } \\
\hline & & $N$ & $V_{B}$ & $G_{B}$ & $D_{g}$ & $N$ & $\lambda$ & $G_{B}$ & $D_{g}$ & GWL & $v_{B}$ & $\overline{v_{B}}$ \\
\hline 25 & 8,7 & 2.832 & 13 & 3,5 & 4,0 & 0 & 0 & 2,3 & 0,0 & 13 & 0,0 & 0,5 \\
\hline 30 & 12,6 & 1.943 & 61 & 11,8 & 8,8 & 889 & 7 & 2,9 & 6,5 & 68 & 11,1 & 2,3 \\
\hline 35 & 15,8 & 1.414 & 94 & 14,3 & 11,4 & 529 & 32 & 4,3 & 10,2 & 133 & 12,9 & 3,8 \\
\hline 40 & 18,5 & 1.073 & 127 & 16,3 & 13,9 & 341 & 38 & 4,5 & 12,9 & 204 & 14,1 & 5,1 \\
\hline 45 & 20,8 & 841 & 160 & 18,0 & 16,5 & 232 & 42 & 4,6 & 15,8 & 279 & 15,0 & 6,2 \\
\hline 50 & 22,9 & 677 & 192 & 19,3 & 19,1 & 164 & 46 & 4,6 & 18,9 & 357 & 15,6 & 7,1 \\
\hline 55 & 24,8 & 556 & 222 & 20,4 & 21,6 & 121 & 49 & 4,6 & 21,9 & 437 & 16,0 & 7,9 \\
\hline 60 & 26,4 & 464 & 252 & 21,4 & 24,2 & 92 & 51 & 4,5 & 25,0 & 518 & 16,2 & 8,6 \\
\hline 65 & 27,9 & 393 & 281 & 22,2 & 26,8 & 71 & 53 & 4,4 & 28,1 & 600 & 16,4 & 9,2 \\
\hline 70 & 29,3 & 337 & 308 & 22,9 & 29,4 & 56 & 55 & 4,3 & 31,2 & 682 & 16,4 & 9,7 \\
\hline 75 & 30,6 & 293 & 334 & 23,6 & 32,0 & 44 & 55 & 4,1 & 34,6 & 763 & 16,4 & 10,2 \\
\hline 80 & 31,7 & 256 & 360 & 24,1 & 34,6 & 37 & 56 & 4,0 & 37,0 & 845 & 16,3 & 10,6 \\
\hline 85 & 32,8 & 226 & 384 & 24,6 & 37,3 & 30 & 56 & 3,8 & 40,2 & 925 & 16,1 & 10,9 \\
\hline 90 & 33,8 & 201 & 408 & 25,1 & 39,9 & 25 & 56 & 3,6 & 42,9 & 1.005 & 15,9 & 11,2 \\
\hline 95 & 34,8 & 179 & 430 & 25,5 & 42,6 & 22 & 56 & 3,4 & 44,5 & 1.084 & 15,7 & 11,4 \\
\hline 100 & 35,7 & 161 & 452 & 25,8 & 45,2 & 18 & 56 & 3,2 & 47,7 & 1.161 & 15,5 & 11,6 \\
\hline 105 & 36,5 & 146 & 473 & 26,2 & 47,8 & 15 & 55 & 3,0 & 50,5 & 1.237 & 15,2 & 11,8 \\
\hline 110 & 37,3 & 132 & 493 & 26,5 & 50,5 & 14 & 54 & 2,8 & 50,4 & 1.311 & 14,9 & 11,9 \\
\hline 115 & 38,1 & 121 & 512 & 26,7 & 53,0 & 11 & 53 & 2,6 & 54,6 & 1.384 & 14,6 & 12,0 \\
\hline 120 & 38,8 & 111 & 531 & 27,0 & 55,6 & 10 & 53 & 2,4 & 54,8 & 1.455 & 14,3 & 12,1 \\
\hline 125 & 39,5 & 102 & 549 & 27,2 & 58,3 & 9 & 51 & 2,1 & 55,0 & 1.525 & 13,9 & 12,2 \\
\hline 130 & & & & & & & & & & & & \\
\hline 135 & & & & & & & & & & & & \\
\hline 140 & & & & & & & & & & & & \\
\hline 145 & & & & & & & & & & & & \\
\hline 150 & & & & & & & & & & & & \\
\hline
\end{tabular}


Tabelle 8-11: Ausgewählte Daten der vorläufigen Ertragstafeltabelle Buche, starke Hochdurchforstung, Oberhöhenbonität $H_{100}(100 \mathrm{a})=40 \mathrm{~m}$ gemäß dyn-ET (unveröff.):

$\boldsymbol{t}$ : Alter [a], $\boldsymbol{H}_{\mathbf{1 0 0}}$ : Oberhöhe [m], $\boldsymbol{N}$ : Stammzahl [ha-1 ], $\boldsymbol{V}_{\boldsymbol{B}}$ : Bestandesvolumen [Vfm ha $\left.{ }^{-1}\right]$, $\boldsymbol{D}_{\boldsymbol{g}}$ : Durchmesser des Grundflächenmittelstammes [ $\left.\mathrm{cm}\right], \boldsymbol{G}_{\boldsymbol{B}}$ : Bestandesgrundfläche $\left[\mathrm{m}^{2} \mathrm{ha}^{-1}\right]$, $\lambda$ : Vornutzungsvolumen [Vfm ha $\left.{ }^{-1}\right], \boldsymbol{G W L}$ : Gesamtwuchsleistung [Vfm ha $\left.{ }^{-1}\right], \boldsymbol{v}_{\boldsymbol{B}}$ : lfd. Bestandesvolumenzuwachs [Vfm ha ${ }^{-1} \mathrm{a}^{-1}$ ], $\overline{\boldsymbol{v}_{\boldsymbol{B}}}$ : durchsch..-jährl. Bestandesvolumenzuw. [Vfm ha ${ }^{-1} \mathrm{a}^{-1}$ ].

\begin{tabular}{|c|c|c|c|c|c|c|c|c|c|c|c|c|}
\hline \multirow[b]{2}{*}{$t$} & \multirow[b]{2}{*}{$H_{100}$} & \multicolumn{4}{|c|}{ verbleibender Bestand } & \multicolumn{4}{|c|}{ ausscheidender Bestand } & \multicolumn{3}{|c|}{ Gesamtbestand } \\
\hline & & $N$ & $V_{B}$ & $\boldsymbol{G}_{\boldsymbol{B}}$ & $D_{g}$ & $N$ & $\lambda$ & $\boldsymbol{G}_{\boldsymbol{B}}$ & $D_{g}$ & $G W L$ & $v_{B}$ & $\overline{\overline{v_{B}}}$ \\
\hline 25 & 11,5 & 2.315 & 79 & 16,9 & 9,7 & 0 & 0 & 2,9 & 0,0 & 79 & 0,0 & 3,2 \\
\hline 30 & 15,5 & 1.589 & 90 & 14,1 & 10,6 & 726 & 56 & 6,1 & 10,3 & 146 & 13,4 & 4,9 \\
\hline 35 & 18,8 & 1.155 & 130 & 16,6 & 13,5 & 434 & 38 & 4,7 & 11,7 & 224 & 15,6 & 6,4 \\
\hline 40 & 21,7 & 877 & 169 & 18,5 & 16,4 & 278 & 46 & 4,9 & 15,0 & 309 & 17,0 & 7,7 \\
\hline 45 & 24,1 & 687 & 208 & 20,1 & 19,3 & 190 & 52 & 5,0 & 18,3 & 400 & 18,1 & 8,9 \\
\hline 50 & 26,3 & 553 & 245 & 21,3 & 22,2 & 134 & 57 & 5,1 & 21,9 & 494 & 18,8 & 9,9 \\
\hline 55 & 28,2 & 454 & 280 & 22,4 & 25,1 & 99 & 61 & 5,0 & 25,5 & 590 & 19,3 & 10,7 \\
\hline 60 & 29,9 & 379 & 314 & 23,3 & 28,0 & 75 & 64 & 5,0 & 29,1 & 688 & 19,6 & 11,5 \\
\hline 65 & 31,5 & 321 & 347 & 24,0 & 30,9 & 58 & 66 & 4,9 & 32,7 & 786 & 19,7 & 12,1 \\
\hline 70 & 32,9 & 276 & 378 & 24,7 & 33,7 & 45 & 68 & 4,7 & 36,5 & 885 & 19,8 & 12,6 \\
\hline 75 & 34,3 & 239 & 407 & 25,3 & 36,7 & 37 & 69 & 4,5 & 39,5 & 984 & 19,7 & 13,1 \\
\hline 80 & 35,5 & 209 & 436 & 25,8 & 39,6 & 30 & 70 & 4,3 & 43,0 & 1.082 & 19,6 & 13,5 \\
\hline 85 & 36,6 & 185 & 463 & 26,2 & 42,5 & 24 & 70 & 4,1 & 46,9 & 1.179 & 19,4 & 13,9 \\
\hline 90 & 37,7 & 164 & 489 & 26,6 & 45,4 & 21 & 70 & 3,9 & 48,7 & 1.275 & 19,2 & 14,2 \\
\hline 95 & 38,7 & 147 & 513 & 26,9 & 48,3 & 17 & 70 & 3,7 & 52,5 & 1.370 & 18,9 & 14,4 \\
\hline 100 & 39,7 & 132 & 537 & 27,2 & 51,3 & 15 & 69 & 3,4 & 54,0 & 1.463 & 18,6 & 14,6 \\
\hline 105 & 40,5 & 119 & 560 & 27,5 & 54,3 & 13 & 69 & 3,2 & 55,8 & 1.555 & 18,3 & 14,8 \\
\hline 110 & & & & & & & & & & & & \\
\hline 115 & & & & & & & & & & & & \\
\hline 120 & & & & & & & & & & & & \\
\hline 125 & & & & & & & & & & & & \\
\hline 130 & & & & & & & & & & & & \\
\hline 135 & & & & & & & & & & & & \\
\hline 140 & & & & & & & & & & & & \\
\hline 145 & & & & & & & & & & & & \\
\hline 150 & & & & & & & & & & & & \\
\hline
\end{tabular}


Tabelle 8-12: Ausgewählte Daten der vorläufigen Ertragstafeltabelle Buche, gestaffelte Hochdurchforstung, Oberhöhenbonität $H_{100}(100 \mathrm{a})=24 \mathrm{~m}$ gemäß dyn-ET (unveröff.):

$\boldsymbol{t}$ : Alter [a], $\boldsymbol{H}_{\mathbf{1 0 0}}$ : Oberhöhe [m], $\boldsymbol{N}$ : Stammzahl $\left[\mathrm{ha}^{-1}\right], \boldsymbol{V}_{\boldsymbol{B}}$ : Bestandesvolumen [Vfm ha ${ }^{-1}$ ], $\boldsymbol{D}_{\boldsymbol{g}}$ : Durchmesser des Grundflächenmittelstammes [ $\left.\mathrm{cm}\right], \boldsymbol{G}_{\boldsymbol{B}}$ : Bestandesgrundfläche $\left[\mathrm{m}^{2} \mathrm{ha}^{-1}\right]$, $\lambda$ : Vornutzungsvolumen [Vfm ha $\left.{ }^{-1}\right], \boldsymbol{G W} \boldsymbol{L}$ : Gesamtwuchsleistung [Vfm ha $\left.{ }^{-1}\right], \boldsymbol{v}_{\boldsymbol{B}}$ : lfd. Bestandesvolumenzuwachs [Vfm ha ${ }^{-1} \mathrm{a}^{-1}$ ], $\overline{\boldsymbol{v}_{\boldsymbol{B}}}$ : durchsch..-jährl. Bestandesvolumenzuw. [Vfm ha ${ }^{-1} \mathrm{a}^{-1}$ ].

\begin{tabular}{|c|c|c|c|c|c|c|c|c|c|c|c|c|}
\hline \multirow{2}{*}{\multicolumn{2}{|c|}{$\begin{array}{ll}t \quad H_{100}\end{array}$}} & \multicolumn{4}{|c|}{ verbleibender Bestand } & \multicolumn{4}{|c|}{ ausscheidender Bestand } & \multicolumn{3}{|c|}{ Gesamtbestand } \\
\hline & & $N$ & $V_{B}$ & $G_{B}$ & $D_{g}$ & $N$ & $\lambda$ & $\overline{G_{B}}$ & $D_{g}$ & $G W L$ & $v_{B}$ & $\overline{v_{B}}$ \\
\hline \multicolumn{13}{|l|}{25} \\
\hline \multicolumn{13}{|l|}{30} \\
\hline \multicolumn{13}{|l|}{35} \\
\hline 40 & 9,0 & 1.828 & 18 & 4,7 & 5,7 & 0 & 0 & 2,6 & 0,0 & 18 & 0,0 & 0,5 \\
\hline 45 & 11,0 & 1.486 & 69 & 14,6 & 11,2 & 342 & 0 & 1,8 & 8,2 & 69 & 10,1 & 1,5 \\
\hline 50 & 12,8 & 1.234 & 66 & 12,0 & 11,1 & 252 & 38 & 4,9 & 15,7 & 103 & 6,9 & 2,1 \\
\hline 55 & 14,4 & 1.044 & 82 & 13,3 & 12,7 & 190 & 19 & 3,3 & 14,8 & 139 & 7,2 & 2,5 \\
\hline 60 & 15,8 & 895 & 99 & 14,4 & 14,3 & 149 & 21 & 3,2 & 16,6 & 176 & 7,4 & 2,9 \\
\hline 65 & 17,1 & 778 & 115 & 15,4 & 15,9 & 117 & 21 & 3,2 & 18,5 & 214 & 7,5 & 3,3 \\
\hline 70 & 18,3 & 682 & 131 & 16,2 & 17,4 & 96 & 22 & 3,1 & 20,3 & 252 & 7,7 & 3,6 \\
\hline 75 & 19,4 & 604 & 146 & 17,0 & 18,9 & 78 & 23 & 3,0 & 22,2 & 291 & 7,7 & 3,9 \\
\hline 80 & 20,4 & 539 & 162 & 17,7 & 20,4 & 65 & 24 & 3,0 & 24,1 & 330 & 7,8 & 4,1 \\
\hline 85 & 21,3 & 485 & 176 & 18,3 & 21,9 & 54 & 24 & 2,9 & 26,1 & 369 & 7,8 & 4,3 \\
\hline 90 & 22,2 & 438 & 191 & 18,9 & 23,4 & 47 & 25 & 2,8 & 27,7 & 408 & 7,8 & 4,5 \\
\hline 95 & 23,0 & 399 & 205 & 19,4 & 24,9 & 39 & 25 & 2,7 & 30,0 & 446 & 7,8 & 4,7 \\
\hline 100 & 23,8 & 364 & 219 & 19,9 & 26,4 & 35 & 25 & 2,7 & 31,2 & 485 & 7,7 & 4,9 \\
\hline 105 & 24,5 & 334 & 232 & 20,3 & 27,8 & 30 & 25 & 2,6 & 33,2 & 524 & 7,7 & 5,0 \\
\hline 110 & 25,2 & 308 & 247 & 20,9 & 29,4 & 26 & 22 & 2,3 & 33,4 & 562 & 7,6 & 5,1 \\
\hline 115 & 25,8 & 285 & 267 & 21,9 & 31,3 & 23 & 18 & 1,8 & 31,7 & 600 & 7,6 & 5,2 \\
\hline 120 & 26,5 & 264 & 287 & 22,8 & 33,2 & 21 & 18 & 1,7 & 31,9 & 637 & 7,5 & 5,3 \\
\hline 125 & 27,0 & 246 & 306 & 23,7 & 35,0 & 18 & 18 & 1,5 & 33,1 & 674 & 7,4 & 5,4 \\
\hline 130 & 27,6 & 229 & 326 & 24,5 & 36,9 & 17 & 17 & 1,4 & 32,6 & 710 & 7,3 & 5,5 \\
\hline 135 & 28,1 & 215 & 345 & 25,3 & 38,7 & 14 & 17 & 1,3 & 34,3 & 746 & 7,2 & 5,5 \\
\hline 140 & 28,6 & 201 & 363 & 26,0 & 40,6 & 14 & 17 & 1,2 & 32,7 & 782 & 7,1 & 5,6 \\
\hline 145 & 29,1 & 189 & 382 & 26,8 & 42,5 & 12 & 16 & 1,1 & 33,4 & 817 & 7,0 & 5,6 \\
\hline 150 & 29,6 & 178 & 400 & 27,4 & 44,3 & 11 & 16 & 0,9 & 32,9 & 851 & 6,9 & 5,7 \\
\hline
\end{tabular}


Tabelle 8-13: Ausgewählte Daten der vorläufigen Ertragstafeltabelle Buche, gestaffelte Hochdurchforstung, Oberhöhenbonität $H_{100}(100$ a) $=28 \mathrm{~m}$ gemäß dyn-ET (unveröff.):

$\boldsymbol{t}$ : Alter [a], $\boldsymbol{H}_{\mathbf{1 0 0}}$ : Oberhöhe [m], $\boldsymbol{N}$ : Stammzahl [ha ${ }^{-1}$ ], $\boldsymbol{V}_{\boldsymbol{B}}$ : Bestandesvolumen [Vfm ha ${ }^{-1}$ ], $\boldsymbol{D}_{\boldsymbol{g}}$ : Durchmesser des Grundflächenmittelstammes $[\mathrm{cm}], \boldsymbol{G}_{\boldsymbol{B}}$ : Bestandesgrundfläche $\left[\mathrm{m}^{2} \mathrm{ha}^{-1}\right]$, $\boldsymbol{\lambda}$ : Vornutzungsvolumen [Vfm ha $\left.{ }^{-1}\right], \boldsymbol{G W} \boldsymbol{L}$ : Gesamtwuchsleistung [Vfm ha $\left.{ }^{-1}\right], \boldsymbol{v}_{\boldsymbol{B}}$ : lfd. Bestandesvolumenzuwachs [Vfm ha ${ }^{-1} \mathrm{a}^{-1}$ ], $\overline{\boldsymbol{v}_{\boldsymbol{B}}}$ : durchsch..-jährl. Bestandesvolumenzuw. [Vfm ha ${ }^{-1} \mathrm{a}^{-1}$ ].

\begin{tabular}{|c|c|c|c|c|c|c|c|c|c|c|c|c|}
\hline \multirow[b]{2}{*}{$t$} & \multirow[b]{2}{*}{$H_{100}$} & \multicolumn{4}{|c|}{ verbleibender Bestand } & \multicolumn{4}{|c|}{ ausscheidender Bestand } & \multicolumn{3}{|c|}{ Gesamtbestand } \\
\hline & & $N$ & $V_{B}$ & $G_{B}$ & $D_{g}$ & $N$ & $\lambda$ & $G_{B}$ & $\overline{D_{g}}$ & $G W L$ & $v_{B}$ & $\overline{\overline{v_{B}}}$ \\
\hline \multicolumn{13}{|l|}{25} \\
\hline \multicolumn{13}{|l|}{30} \\
\hline 35 & 9,7 & 1.856 & 33 & 8,1 & 7,4 & 0 & 0 & 2,4 & 0,0 & 33 & 0,0 & 0,9 \\
\hline 40 & 12,1 & 1.467 & 59 & 11,5 & 10,0 & 389 & 16 & 3,3 & 10,4 & 75 & 8,3 & 1,9 \\
\hline 45 & 14,3 & 1.192 & 80 & 13,2 & 11,9 & 275 & 24 & 3,7 & 13,1 & 120 & 9,0 & 2,7 \\
\hline 50 & 16,2 & 990 & 102 & 14,6 & 13,7 & 202 & 26 & 3,7 & 15,2 & 167 & 9,4 & 3,3 \\
\hline 55 & 17,9 & 837 & 123 & 15,9 & 15,5 & 153 & 28 & 3,6 & 17,4 & 216 & 9,8 & 3,9 \\
\hline 60 & 19,4 & 718 & 143 & 17,0 & 17,3 & 119 & 30 & 3,6 & 19,7 & 266 & 10,1 & 4,4 \\
\hline 65 & 20,7 & 624 & 164 & 17,9 & 19,1 & 94 & 31 & 3,6 & 22,0 & 317 & 10,3 & 4,9 \\
\hline 70 & 22,0 & 548 & 183 & 18,7 & 20,8 & 76 & 32 & 3,5 & 24,4 & 370 & 10,4 & 5,3 \\
\hline 75 & 23,1 & 485 & 202 & 19,4 & 22,6 & 63 & 34 & 3,5 & 26,6 & 422 & 10,5 & 5,6 \\
\hline 80 & 24,2 & 433 & 221 & 20,1 & 24,3 & 52 & 34 & 3,4 & 29,0 & 475 & 10,6 & 5,9 \\
\hline 85 & 25,2 & 389 & 241 & 20,9 & 26,1 & 44 & 33 & 3,2 & 30,2 & 528 & 10,6 & 6,2 \\
\hline 90 & 26,1 & 352 & 269 & 22,3 & 28,4 & 37 & 26 & 2,4 & 28,9 & 581 & 10,6 & 6,5 \\
\hline 95 & 27,0 & 320 & 296 & 23,5 & 30,6 & 32 & 26 & 2,3 & 30,0 & 634 & 10,6 & 6,7 \\
\hline 100 & 27,8 & 292 & 323 & 24,7 & 32,8 & 28 & 26 & 2,1 & 30,9 & 687 & 10,5 & 6,9 \\
\hline 105 & 28,5 & 268 & 350 & 25,9 & 35,1 & 24 & 26 & 1,9 & 32,2 & 739 & 10,5 & 7,0 \\
\hline 110 & 29,2 & 247 & 377 & 27,0 & 37,3 & 21 & 25 & 1,8 & 33,0 & 791 & 10,4 & 7,2 \\
\hline 115 & 29,9 & 228 & 403 & 28,0 & 39,5 & 19 & 25 & 1,6 & 33,2 & 843 & 10,3 & 7,3 \\
\hline 120 & 30,6 & 212 & 420 & 28,3 & 41,3 & 16 & 34 & 2,2 & 42,2 & 894 & 10,2 & 7,4 \\
\hline 125 & 31,2 & 197 & 436 & 28,6 & 43,0 & 15 & 35 & 2,2 & 43,4 & 944 & 10,1 & 7,6 \\
\hline 130 & 31,8 & 184 & 451 & 28,9 & 44,7 & 13 & 35 & 2,1 & 45,6 & 994 & 9,9 & 7,6 \\
\hline 135 & 32,3 & 172 & 465 & 29,1 & 46,4 & 12 & 34 & 2,0 & 46,5 & 1.043 & 9,8 & 7,7 \\
\hline 140 & 32,9 & 161 & 480 & 29,3 & 48,2 & 11 & 34 & 1,9 & 47,5 & 1.091 & 9,7 & 7,8 \\
\hline 145 & 33,4 & 152 & 493 & 29,5 & 49,7 & 9 & 34 & 1,9 & 51,2 & 1.139 & 9,5 & 7,9 \\
\hline 150 & 33,9 & 143 & 507 & 29,7 & 51,4 & 9 & 34 & 1,8 & 49,9 & 1.186 & 9,4 & 7,9 \\
\hline
\end{tabular}


Tabelle 8-14: Ausgewählte Daten der vorläufigen Ertragstafeltabelle Buche, gestaffelte Hochdurchforstung, Oberhöhenbonität $H_{100}(100 \mathrm{a})=32 \mathrm{~m}$ gemäß dyn-ET (unveröff.):

$\boldsymbol{t}$ : Alter [a], $\boldsymbol{H}_{\mathbf{1 0 0}}$ : Oberhöhe [m], $\boldsymbol{N}$ : Stammzahl [ha ${ }^{-1}$ ], $\boldsymbol{V}_{\boldsymbol{B}}$ : Bestandesvolumen [Vfm ha ${ }^{-1}$ ], $\boldsymbol{D}_{\boldsymbol{g}}$ : Durchmesser des Grundflächenmittelstammes $[\mathrm{cm}], \boldsymbol{G}_{\boldsymbol{B}}$ : Bestandesgrundfläche $\left[\mathrm{m}^{2} \mathrm{ha}^{-1}\right]$, $\lambda$ : Vornutzungsvolumen [Vfm ha $\left.{ }^{-1}\right], \boldsymbol{G W} \boldsymbol{L}$ : Gesamtwuchsleistung [Vfm ha $\left.{ }^{-1}\right], \boldsymbol{v}_{\boldsymbol{B}}$ : lfd. Bestandesvolumenzuwachs [Vfm ha ${ }^{-1} \mathrm{a}^{-1}$ ], $\overline{\boldsymbol{v}_{\boldsymbol{B}}}$ : durchsch..-jährl. Bestandesvolumenzuw. [Vfm ha-1 $\mathrm{a}^{-1}$ ].

\begin{tabular}{|c|c|c|c|c|c|c|c|c|c|c|c|c|}
\hline \multirow{2}{*}{\multicolumn{2}{|c|}{$\begin{array}{ll}H_{100}\end{array}$}} & \multicolumn{4}{|c|}{ verbleibender Bestand } & \multicolumn{4}{|c|}{ ausscheidender Bestand } & \multicolumn{3}{|c|}{ Gesamtbestand } \\
\hline & & $N$ & $V_{B}$ & $G_{B}$ & $D_{g}$ & $N$ & $\lambda$ & $G_{B}$ & $D_{g}$ & GWL & $v_{B}$ & $\overline{\bar{v}_{B}}$ \\
\hline \multicolumn{13}{|l|}{25} \\
\hline 30 & 9,7 & 2.012 & 33 & 8,1 & 7,1 & 0 & 0 & 2,4 & 0,0 & 33 & 0,0 & 1,1 \\
\hline 35 & 12,7 & 1.534 & 64 & 11,9 & 10,0 & 478 & 18 & 3,5 & 9,7 & 82 & 9,8 & 2,3 \\
\hline 40 & 15,3 & 1.212 & 91 & 14,0 & 12,1 & 322 & 28 & 4,0 & 12,5 & 137 & 10,9 & 3,4 \\
\hline 45 & 17,6 & 985 & 117 & 15,7 & 14,2 & 227 & 32 & 4,0 & 15,1 & 195 & 11,7 & 4,3 \\
\hline 50 & 19,5 & 819 & 144 & 17,1 & 16,3 & 166 & 35 & 4,1 & 17,7 & 257 & 12,3 & 5,1 \\
\hline 55 & 21,3 & 692 & 169 & 18,3 & 18,3 & 127 & 38 & 4,1 & 20,4 & 321 & 12,8 & 5,8 \\
\hline 60 & 22,9 & 594 & 194 & 19,3 & 20,3 & 98 & 41 & 4,1 & 23,2 & 387 & 13,2 & 6,4 \\
\hline 65 & 24,3 & 516 & 219 & 20,2 & 22,3 & 78 & 43 & 4,1 & 26,0 & 454 & 13,4 & 7,0 \\
\hline 70 & 25,6 & 453 & 249 & 21,6 & 24,6 & 63 & 38 & 3,5 & 26,7 & 522 & 13,6 & 7,5 \\
\hline 75 & 26,8 & 401 & 286 & 23,4 & 27,2 & 52 & 32 & 2,9 & 26,5 & 591 & 13,8 & 7,9 \\
\hline 80 & 28,0 & 358 & 322 & 25,0 & 29,8 & 43 & 33 & 2,7 & 28,3 & 660 & 13,8 & 8,2 \\
\hline 85 & 29,0 & 321 & 358 & 26,6 & 32,5 & 37 & 33 & 2,5 & 29,5 & 729 & 13,9 & 8,6 \\
\hline 90 & 30,0 & 291 & 394 & 28,0 & 35,0 & 30 & 34 & 2,4 & 31,7 & 799 & 13,9 & 8,9 \\
\hline 95 & 30,9 & 264 & 416 & 28,5 & 37,1 & 27 & 47 & 3,3 & 39,4 & 868 & 13,8 & 9,1 \\
\hline 100 & 31,7 & 241 & 437 & 28,8 & 39,0 & 23 & 48 & 3,2 & 42,4 & 937 & 13,8 & 9,4 \\
\hline 105 & 32,5 & 221 & 457 & 29,2 & 41,0 & 20 & 48 & 3,2 & 44,9 & 1005 & 13,7 & 9,6 \\
\hline 110 & 33,3 & 204 & 476 & 29,5 & 42,9 & 17 & 49 & 3,1 & 48,1 & 1073 & 13,6 & 9,8 \\
\hline 115 & 34,0 & 189 & 495 & 29,8 & 44,8 & 15 & 49 & 3,0 & 50,5 & 1140 & 13,5 & 9,9 \\
\hline 120 & 34,7 & 175 & 512 & 30,0 & 46,7 & 14 & 49 & 2,9 & 51,4 & 1207 & 13,3 & 10,1 \\
\hline 125 & 35,3 & 163 & 530 & 30,2 & 48,6 & 12 & 49 & 2,8 & 54,6 & 1273 & 13,2 & 10,2 \\
\hline 130 & 36,0 & 152 & 546 & 30,4 & 50,5 & 11 & 49 & 2,7 & 56,0 & 1338 & 13,0 & 10,3 \\
\hline 135 & 36,6 & 142 & 562 & 30,6 & 52,4 & 10 & 48 & 2,6 & 57,6 & 1402 & 12,8 & 10,4 \\
\hline 140 & 37,1 & 133 & 577 & 30,7 & 54,2 & 9 & 48 & 2,5 & 59,4 & 1465 & 12,6 & 10,5 \\
\hline 145 & 37,7 & 125 & 592 & 30,9 & 56,1 & 8 & 47 & 2,4 & 61,6 & 1527 & 12,4 & 10,5 \\
\hline 150 & 38,2 & 118 & 606 & 31,0 & 57,8 & 7 & 47 & 2,3 & 64,3 & 1588 & 12,2 & 10,6 \\
\hline
\end{tabular}


Tabelle 8-15: Ausgewählte Daten der vorläufigen Ertragstafeltabelle Buche, gestaffelte Hochdurchforstung, Oberhöhenbonität $H_{100}(100 \mathrm{a})=36 \mathrm{~m}$ gemäß dyn-ET (unveröff.):

$\boldsymbol{t}$ : Alter [a], $\boldsymbol{H}_{\mathbf{1 0 0}}$ : Oberhöhe [m], $\boldsymbol{N}$ : Stammzahl $\left[\mathrm{ha}^{-1}\right], \boldsymbol{V}_{\boldsymbol{B}}$ : Bestandesvolumen $\left[\mathrm{Vfm} \mathrm{ha}^{-1}\right]$, $\boldsymbol{D}_{\boldsymbol{g}}$ : Durchmesser des Grundflächenmittelstammes $[\mathrm{cm}], \boldsymbol{G}_{\boldsymbol{B}}$ : Bestandesgrundfläche $\left[\mathrm{m}^{2} \mathrm{ha}^{-1}\right]$, $\boldsymbol{\lambda}$ : Vornutzungsvolumen [Vfm ha $\left.{ }^{-1}\right], \boldsymbol{G W} \boldsymbol{L}$ : Gesamtwuchsleistung $\left[\mathrm{Vfm} \mathrm{ha}^{-1}\right], \boldsymbol{v}_{\boldsymbol{B}}$ : Ifd. Bestandesvolumenzuwachs [Vfm ha ${ }^{-1} \mathrm{a}^{-1}$ ], $\overline{\boldsymbol{v}_{\boldsymbol{B}}}$ : durchsch..-jährl. Bestandesvolumenzuw. [Vfm ha ${ }^{-1} \mathrm{a}^{-1}$ ].

\begin{tabular}{|c|c|c|c|c|c|c|c|c|c|c|c|c|}
\hline \multirow[b]{2}{*}{$\boldsymbol{t}$} & \multirow[b]{2}{*}{$H_{100}$} & \multicolumn{4}{|c|}{ verbleibender Bestand } & \multicolumn{4}{|c|}{ ausscheidender Bestand } & \multicolumn{3}{|c|}{ Gesamtbestand } \\
\hline & & $N$ & $V_{B}$ & $\boldsymbol{G}_{\boldsymbol{B}}$ & $D_{g}$ & $N$ & $\lambda$ & $G_{B}$ & $D_{g}$ & $G W L$ & $v_{B}$ & $\overline{\overline{v_{B}}}$ \\
\hline 25 & 8,7 & 2.344 & 13 & 3,5 & 4,4 & 0 & 0 & 2,8 & 0,0 & 13 & 0,0 & 0,5 \\
\hline 30 & 12,6 & 1.701 & 62 & 11,8 & 9,4 & 643 & 4 & 2,5 & 7,0 & 66 & 10,6 & 2,2 \\
\hline 35 & 15,8 & 1.296 & 95 & 14,3 & 11,9 & 405 & 30 & 4,1 & 11,4 & 128 & 12,5 & 3,7 \\
\hline 40 & 18,5 & 1.025 & 128 & 16,3 & 14,2 & 271 & 36 & 4,4 & 14,3 & 197 & 13,8 & 4,9 \\
\hline 45 & 20,8 & 833 & 160 & 18,0 & 16,6 & 192 & 42 & 4,5 & 17,3 & 272 & 14,8 & 6,0 \\
\hline 50 & 22,9 & 692 & 191 & 19,3 & 18,8 & 141 & 47 & 4,7 & 20,5 & 350 & 15,6 & 7,0 \\
\hline 55 & 24,8 & 585 & 222 & 20,4 & 21,1 & 107 & 51 & 4,8 & 23,8 & 431 & 16,2 & 7,8 \\
\hline 60 & 26,4 & 502 & 267 & 22,7 & 24,0 & 83 & 38 & 3,5 & 23,2 & 514 & 16,7 & 8,6 \\
\hline 65 & 27,9 & 436 & 313 & 25,0 & 27,0 & 66 & 38 & 3,2 & 24,8 & 599 & 17,0 & 9,2 \\
\hline 70 & 29,3 & 383 & 360 & 27,0 & 30,0 & 53 & 40 & 3,0 & 27,0 & 685 & 17,3 & 9,8 \\
\hline 75 & 30,6 & 339 & 398 & 28,3 & 32,6 & 44 & 49 & 3,5 & 32,1 & 772 & 17,4 & 10,3 \\
\hline 80 & 31,7 & 302 & 426 & 28,9 & 34,9 & 37 & 60 & 4,3 & 38,3 & 860 & 17,5 & 10,7 \\
\hline 85 & 32,8 & 272 & 452 & 29,3 & 37,0 & 30 & 62 & 4,2 & 42,4 & 948 & 17,6 & 11,1 \\
\hline 90 & 33,8 & 246 & 477 & 29,7 & 39,2 & 26 & 63 & 4,2 & 45,4 & 1.036 & 17,6 & 11,5 \\
\hline 95 & 34,8 & 223 & 500 & 30,0 & 41,4 & 23 & 64 & 4,1 & 47,9 & 1.123 & 17,5 & 11,8 \\
\hline 100 & 35,7 & 204 & 523 & 30,3 & 43,5 & 19 & 65 & 4,1 & 52,3 & 1.210 & 17,4 & 12,1 \\
\hline 105 & 36,5 & 187 & 545 & 30,6 & 45,6 & 17 & 65 & 4,0 & 54,7 & 1.297 & 17,3 & 12,4 \\
\hline 110 & 37,3 & 172 & 565 & 30,8 & 47,7 & 15 & 65 & 3,9 & 57,6 & 1.383 & 17,2 & 12,6 \\
\hline 115 & 38,1 & 159 & 585 & 31,0 & 49,8 & 13 & 66 & 3,8 & 61,1 & 1.468 & 17,0 & 12,8 \\
\hline 120 & 38,8 & 148 & 604 & 31,1 & 51,8 & 11 & 66 & 3,7 & 65,5 & 1.553 & 16,9 & 12,9 \\
\hline 125 & 39,5 & 138 & 622 & 31,3 & 53,7 & 10 & 65 & 3,6 & 67,6 & 1.636 & 16,7 & 13,1 \\
\hline 130 & 40,2 & 128 & 639 & 31,4 & 55,9 & 10 & 65 & 3,5 & 66,5 & 1.718 & 16,5 & 13,2 \\
\hline 135 & & & & & & & & & & & & \\
\hline 140 & & & & & & & & & & & & \\
\hline 145 & & & & & & & & & & & & \\
\hline 150 & & & & & & & & & & & & \\
\hline
\end{tabular}


Tabelle 8-16: Ausgewählte Daten der vorläufigen Ertragstafeltabelle Buche, gestaffelte Hochdurchforstung, Oberhöhenbonität $H_{100}(100 \mathrm{a})=40 \mathrm{~m}$ gemäß dyn-ET (unveröff.):

$\boldsymbol{t}$ : Alter [a], $\boldsymbol{H}_{\mathbf{1 0 0}}$ : Oberhöhe [m], $\boldsymbol{N}$ : Stammzahl [ha ${ }^{-1}$ ], $\boldsymbol{V}_{\boldsymbol{B}}$ : Bestandesvolumen [Vfm ha ${ }^{-1}$ ], $\boldsymbol{D}_{\boldsymbol{g}}$ : Durchmesser des Grundflächenmittelstammes [ $\left.\mathrm{cm}\right], \boldsymbol{G}_{\boldsymbol{B}}$ : Bestandesgrundfläche $\left[\mathrm{m}^{2} \mathrm{ha}^{-1}\right]$, $\boldsymbol{\lambda}$ : Vornutzungsvolumen [Vfm ha $\left.{ }^{-1}\right], \boldsymbol{G W} \boldsymbol{L}$ : Gesamtwuchsleistung [Vfm ha $\left.{ }^{-1}\right], \boldsymbol{v}_{\boldsymbol{B}}$ : lfd. Bestandesvolumenzuwachs [Vfm ha $\left.{ }^{-1} \mathrm{a}^{-1}\right], \overline{\boldsymbol{v}_{\boldsymbol{B}}}$ : durchsch..-jährl. Bestandesvolumenzuw. [Vfm ha ${ }^{-1} \mathrm{a}^{-1}$ ].

\begin{tabular}{|c|c|c|c|c|c|c|c|c|c|c|c|c|}
\hline \multirow[b]{2}{*}{$t$} & \multirow[b]{2}{*}{$H_{100}$} & \multicolumn{4}{|c|}{ verbleibender Bestand } & \multicolumn{4}{|c|}{ ausscheidender Bestand } & \multicolumn{3}{|c|}{ Gesamtbestand } \\
\hline & & $N$ & $V_{B}$ & $G_{B}$ & $D_{g}$ & $N$ & $\lambda$ & $G_{B}$ & $D_{g}$ & GWL & $v_{B}$ & $\overline{\bar{v}_{B}}$ \\
\hline 25 & 11,5 & 2.017 & 81 & 16,9 & 10,3 & 0 & 0 & 1,9 & 0,0 & 81 & 0,0 & 3,2 \\
\hline 30 & 15,5 & 1.463 & 91 & 14,1 & 11,1 & 554 & 55 & 6,2 & 12,0 & 146 & 13,1 & 4,9 \\
\hline 35 & 18,8 & 1.115 & 131 & 16,6 & 13,8 & 348 & 37 & 4,5 & 12,8 & 223 & 15,4 & 6,4 \\
\hline 40 & 21,7 & 882 & 170 & 18,5 & 16,3 & 233 & 46 & 4,8 & 16,3 & 308 & 17,1 & 7,7 \\
\hline 45 & 24,1 & 717 & 208 & 20,1 & 18,9 & 165 & 54 & 5,1 & 19,9 & 400 & 18,3 & 8,9 \\
\hline 50 & 26,3 & 595 & 258 & 22,5 & 22,0 & 122 & 46 & 4,2 & 20,9 & 496 & 19,3 & 9,9 \\
\hline 55 & 28,2 & 503 & 316 & 25,4 & 25,4 & 92 & 42 & 3,5 & 21,9 & 596 & 20,0 & 10,8 \\
\hline 60 & 29,9 & 432 & 375 & 28,0 & 28,7 & 71 & 44 & 3,3 & 24,5 & 699 & 20,6 & 11,7 \\
\hline 65 & 31,5 & 375 & 411 & 28,8 & 31,2 & 57 & 69 & 5,1 & 33,6 & 804 & 21,0 & 12,4 \\
\hline 70 & 32,9 & 329 & 444 & 29,4 & 33,7 & 46 & 73 & 5,2 & 38,0 & 911 & 21,3 & 13,0 \\
\hline 75 & 34,3 & 291 & 475 & 29,8 & 36,1 & 38 & 76 & 5,3 & 42,0 & 1.018 & 21,5 & 13,6 \\
\hline 80 & 35,5 & 260 & 505 & 30,3 & 38,5 & 31 & 79 & 5,3 & 46,6 & 1.127 & 21,6 & 14,1 \\
\hline 85 & 36,6 & 234 & 532 & 30,6 & 40,8 & 26 & 81 & 5,3 & 50,8 & 1.235 & 21,7 & 14,5 \\
\hline 90 & 37,7 & 211 & 559 & 30,9 & 43,2 & 23 & 82 & 5,2 & 53,9 & 1.344 & 21,7 & 14,9 \\
\hline 95 & 38,7 & 192 & 584 & 31,1 & 45,4 & 19 & 83 & 5,2 & 59,0 & 1.452 & 21,6 & 15,3 \\
\hline 100 & 39,7 & 175 & 607 & 31,3 & 47,7 & 17 & 84 & 5,1 & 61,9 & 1.560 & 21,6 & 15,6 \\
\hline 105 & 40,5 & 161 & 629 & 31,5 & 49,9 & 14 & 85 & 5,0 & 67,6 & 1.667 & 21,4 & 15,9 \\
\hline 110 & 41,4 & 148 & 651 & 31,6 & 52,1 & 13 & 85 & 4,9 & 69,5 & 1.773 & 21,2 & 16,1 \\
\hline 115 & & & & & & & & & & & & \\
\hline 120 & & & & & & & & & & & & \\
\hline 125 & & & & & & & & & & & & \\
\hline 130 & & & & & & & & & & & & \\
\hline 135 & & & & & & & & & & & & \\
\hline 140 & & & & & & & & & & & & \\
\hline 145 & & & & & & & & & & & & \\
\hline 150 & & & & & & & & & & & & \\
\hline
\end{tabular}


Tabelle 8-17: Ausgewählte Daten der vorläufigen Ertragstafeltabelle Fichte, mäßige Hochdurchforstung, Oberhöhenbonität $H_{100}(100 \mathrm{a})=27 \mathrm{~m}$ gemäß dyn-ET (unveröff.):

$\boldsymbol{t}$ : Alter [a], $\boldsymbol{H}_{\mathbf{1 0 0}}$ : Oberhöhe [m], $\boldsymbol{N}$ : Stammzahl $\left[\mathrm{ha}^{-1}\right], \boldsymbol{V}_{\boldsymbol{B}}$ : Bestandesvolumen $\left[\mathrm{Vfm} \mathrm{ha} \mathrm{h}^{-1}\right]$, $\boldsymbol{D}_{\boldsymbol{g}}$ : Durchmesser des Grundflächenmittelstammes $[\mathrm{cm}], \boldsymbol{G}_{\boldsymbol{B}}$ : Bestandesgrundfläche $\left[\mathrm{m}^{2} \mathrm{ha}^{-1}\right]$, $\lambda$ : Vornutzungsvolumen [Vfm ha $\left.{ }^{-1}\right], \boldsymbol{G W}$ : Gesamtwuchsleistung [Vfm ha $\left.{ }^{-1}\right], \boldsymbol{v}_{\boldsymbol{B}}$ : lfd. Bestandesvolumenzuwachs [Vfm ha ${ }^{-1} \mathrm{a}^{-1}$ ], $\overline{\boldsymbol{v}_{\boldsymbol{B}}}$ : durchsch..-jährl. Bestandesvolumenzuw. [Vfm ha ${ }^{-1} \mathrm{a}^{-1}$ ].

\begin{tabular}{|c|c|c|c|c|c|c|c|c|c|c|c|c|}
\hline \multirow[b]{2}{*}{$t$} & \multirow[b]{2}{*}{$H_{100}$} & \multicolumn{4}{|c|}{ verbleibender Bestand } & \multicolumn{4}{|c|}{ Ausscheidender Bestand } & \multicolumn{3}{|c|}{ Gesamtbestand } \\
\hline & & $N$ & $V_{B}$ & $\boldsymbol{G}_{\boldsymbol{B}}$ & $\overline{D_{g}}$ & $N$ & $\lambda$ & $\overline{G_{B}}$ & $D_{g}$ & $G W L$ & $v_{B}$ & $\overline{\overline{v_{B}}}$ \\
\hline \multicolumn{13}{|l|}{15} \\
\hline \multicolumn{13}{|l|}{20} \\
\hline \multicolumn{13}{|l|}{25} \\
\hline \multicolumn{13}{|l|}{30} \\
\hline 35 & 10,3 & 2.500 & 0 & 18,9 & 9,8 & 0 & 0 & 0,0 & 0,0 & 31 & 0,0 & 0,9 \\
\hline 40 & 12,4 & 2.500 & 0 & 26,0 & 11,5 & 0 & 0 & 0,0 & 0,0 & 104 & 14,5 & 2,6 \\
\hline 45 & 14,3 & 994 & 153 & 25,4 & 18,0 & 1.506 & 21 & 3,7 & 5,6 & 178 & 14,9 & 4,0 \\
\hline 50 & 16,0 & 872 & 195 & 29,1 & 20,6 & 122 & 33 & 5,7 & 24,4 & 254 & 15,1 & 5,1 \\
\hline 55 & 17,6 & 775 & 235 & 32,2 & 23,0 & 97 & 35 & 5,4 & 26,7 & 329 & 15,1 & 6,0 \\
\hline 60 & 19,0 & 695 & 274 & 34,7 & 25,2 & 80 & 36 & 5,2 & 28,7 & 404 & 14,9 & 6,7 \\
\hline 65 & 20,3 & 630 & 311 & 36,9 & 27,3 & 65 & 37 & 4,9 & 31,1 & 478 & 14,7 & 7,3 \\
\hline 70 & 21,5 & 574 & 345 & 38,8 & 29,3 & 56 & 38 & 4,7 & 32,7 & 550 & 14,5 & 7,9 \\
\hline 75 & 22,7 & 527 & 378 & 40,4 & 31,2 & 47 & 38 & 4,5 & 34,8 & 621 & 14,2 & 8,3 \\
\hline 80 & 23,8 & 486 & 408 & 41,8 & 33,1 & 41 & 39 & 4,3 & 36,4 & 690 & 13,9 & 8,6 \\
\hline 85 & 24,8 & 451 & 437 & 43,0 & 34,8 & 35 & 39 & 4,1 & 38,5 & 758 & 13,6 & 8,9 \\
\hline 90 & 25,8 & 420 & 465 & 44,0 & 36,5 & 31 & 39 & 3,9 & 39,9 & 825 & 13,3 & 9,2 \\
\hline 95 & 26,7 & 393 & 491 & 45,0 & 38,2 & 27 & 39 & 3,7 & 41,7 & 890 & 13,0 & 9,4 \\
\hline 100 & 27,6 & 369 & 515 & 45,8 & 39,7 & 24 & 39 & 3,5 & 43,2 & 953 & 12,7 & 9,5 \\
\hline 105 & 28,5 & 347 & 538 & 46,5 & 41,3 & 22 & 39 & 3,3 & 43,9 & 1.015 & 12,4 & 9,7 \\
\hline 110 & 29,3 & 327 & 560 & 47,1 & 42,8 & 20 & 39 & 3,2 & 44,9 & 1.076 & 12,2 & 9,8 \\
\hline 115 & 30,1 & 310 & 581 & 47,6 & 44,2 & 17 & 39 & 3,0 & 47,4 & 1.136 & 11,9 & 9,9 \\
\hline 120 & 30,8 & 294 & 601 & 48,1 & 45,7 & 16 & 38 & 2,8 & 47,6 & 1.194 & 11,7 & 9,9 \\
\hline
\end{tabular}


Tabelle 8-18: Ausgewählte Daten der vorläufigen Ertragstafeltabelle Fichte, mäßige Hochdurchforstung, Oberhöhenbonität $H_{100}(100$ a) $=31 \mathrm{~m}$ gemäß dyn-ET (unveröff.):

$\boldsymbol{t}$ : Alter [a], $\boldsymbol{H}_{\mathbf{1 0 0}}$ : Oberhöhe [m], $\boldsymbol{N}$ : Stammzahl [ha $\left.{ }^{-1}\right], \boldsymbol{V}_{\boldsymbol{B}}$ : Bestandesvolumen [Vfm ha ${ }^{-1}$ ], $\boldsymbol{D}_{\boldsymbol{g}}$ : Durchmesser des Grundflächenmittelstammes [ $\left.\mathrm{cm}\right], \boldsymbol{G}_{\boldsymbol{B}}$ : Bestandesgrundfläche $\left[\mathrm{m}^{2} \mathrm{ha}^{-1}\right]$, $\lambda$ : Vornutzungsvolumen [Vfm ha $\left.{ }^{-1}\right], \boldsymbol{G W} \boldsymbol{L}$ : Gesamtwuchsleistung [Vfm ha $\left.{ }^{-1}\right], \boldsymbol{v}_{\boldsymbol{B}}$ : lfd. Bestandesvolumenzuwachs [Vfm ha ${ }^{-1} \mathrm{a}^{-1}$ ], $\overline{\boldsymbol{v}_{\boldsymbol{B}}}$ : durchsch..-jährl. Bestandesvolumenzuw. [Vfm ha ${ }^{-1} \mathrm{a}^{-1}$ ].

\begin{tabular}{|c|c|c|c|c|c|c|c|c|c|c|c|c|}
\hline \multirow[b]{2}{*}{$t$} & \multirow[b]{2}{*}{$H_{100}$} & \multicolumn{4}{|c|}{ verbleibender Bestand } & \multicolumn{4}{|c|}{ ausscheidender Bestand } & \multicolumn{3}{|c|}{ Gesamtbestand } \\
\hline & & $N$ & $V_{B}$ & $G_{B}$ & $D_{g}$ & $N$ & $\lambda$ & $G_{B}$ & $D_{g}$ & $G W L$ & $v_{B}$ & $\overline{\bar{v}_{B}}$ \\
\hline \multicolumn{13}{|l|}{15} \\
\hline \multicolumn{13}{|l|}{20} \\
\hline 25 & 8,6 & 2.500 & 0 & 12,5 & 8,0 & 0 & 0 & 0,0 & 0,0 & 0 & 0,0 & 0,0 \\
\hline 30 & 11,5 & 2.500 & 0 & 22,8 & 10,8 & 0 & 0 & 0,0 & 0,0 & 69 & 13,8 & 2,3 \\
\hline 35 & 13,9 & 2.500 & 0 & 30,6 & 12,5 & 0 & 0 & 0,0 & 0,0 & 162 & 18,7 & 4,6 \\
\hline 40 & 16,1 & 1.089 & 198 & 29,2 & 18,5 & 1.411 & 22 & 3,4 & 5,5 & 258 & 19,0 & 6,4 \\
\hline 45 & 18,0 & 940 & 248 & 33,0 & 21,1 & 149 & 44 & 6,2 & 23,0 & 353 & 19,0 & 7,8 \\
\hline 50 & 19,8 & 825 & 296 & 36,0 & 23,6 & 115 & 46 & 5,9 & 25,6 & 446 & 18,7 & 8,9 \\
\hline 55 & 21,4 & 733 & 340 & 38,5 & 25,9 & 92 & 47 & 5,6 & 27,9 & 538 & 18,4 & 9,8 \\
\hline 60 & 22,8 & 658 & 381 & 40,6 & 28,0 & 75 & 48 & 5,4 & 30,2 & 628 & 17,9 & 10,5 \\
\hline 65 & 24,2 & 596 & 420 & 42,2 & 30,0 & 62 & 49 & 5,1 & 32,5 & 715 & 17,5 & 11,0 \\
\hline 70 & 25,4 & 543 & 455 & 43,7 & 32,0 & 53 & 49 & 4,9 & 34,3 & 800 & 17,0 & 11,4 \\
\hline 75 & 26,6 & 499 & 488 & 44,9 & 33,8 & 44 & 50 & 4,7 & 36,8 & 882 & 16,5 & 11,8 \\
\hline 80 & 27,8 & 460 & 519 & 45,9 & 35,6 & 39 & 50 & 4,4 & 38,1 & 963 & 16,1 & 12,0 \\
\hline 85 & 28,8 & 427 & 548 & 46,7 & 37,3 & 33 & 49 & 4,2 & 40,4 & 1.041 & 15,6 & 12,2 \\
\hline 90 & 29,8 & 397 & 574 & 47,5 & 39,0 & 30 & 49 & 4,0 & 41,3 & 1.117 & 15,2 & 12,4 \\
\hline 95 & 30,8 & 372 & 599 & 48,1 & 40,6 & 25 & 49 & 3,8 & 44,1 & 1.191 & 14,8 & 12,5 \\
\hline 100 & 31,7 & 349 & 623 & 48,6 & 42,1 & 23 & 48 & 3,6 & 44,7 & 1.263 & 14,4 & 12,6 \\
\hline 105 & 32,5 & 328 & 645 & 49,1 & 43,7 & 21 & 48 & 3,4 & 45,5 & 1.333 & 14,0 & 12,7 \\
\hline 110 & 33,4 & 310 & 666 & 49,5 & 45,1 & 18 & 48 & 3,2 & 47,8 & 1.402 & 13,7 & 12,7 \\
\hline 115 & 34,2 & 293 & 686 & 49,8 & 46,5 & 17 & 47 & 3,0 & 47,8 & 1.468 & 13,4 & 12,8 \\
\hline 120 & 34,9 & 278 & 705 & 50,1 & 47,9 & 15 & 46 & 2,9 & 49,3 & 1.534 & 13,0 & 12,8 \\
\hline
\end{tabular}


Tabelle 8-19: Ausgewählte Daten der vorläufigen Ertragstafeltabelle Fichte, mäßige Hochdurchforstung, Oberhöhenbonität $H_{100}(100 \mathrm{a})=35 \mathrm{~m}$ gemäß dyn-ET (unveröff.):

$\boldsymbol{t}$ : Alter [a], $\boldsymbol{H}_{\mathbf{1 0 0}}$ : Oberhöhe [m], $\boldsymbol{N}$ : Stammzahl [ha $\left.{ }^{-1}\right], \boldsymbol{V}_{\boldsymbol{B}}$ : Bestandesvolumen [Vfm ha $\left.{ }^{-1}\right]$, $\boldsymbol{D}_{\boldsymbol{g}}$ : Durchmesser des Grundflächenmittelstammes [ $\left.\mathrm{cm}\right], \boldsymbol{G}_{\boldsymbol{B}}$ : Bestandesgrundfläche $\left[\mathrm{m}^{2} \mathrm{ha}^{-1}\right]$, $\lambda$ : Vornutzungsvolumen [Vfm ha $\left.{ }^{-1}\right], \boldsymbol{G W L}$ : Gesamtwuchsleistung [Vfm ha $\left.{ }^{-1}\right], \boldsymbol{v}_{\boldsymbol{B}}$ : lfd. Bestandesvolumenzuwachs [Vfm ha ${ }^{-1} \mathrm{a}^{-1}$ ], $\overline{\boldsymbol{v}_{\boldsymbol{B}}}$ : durchsch..-jährl. Bestandesvolumenzuw. [Vfm ha ${ }^{-1} \mathrm{a}^{-1}$ ].

\begin{tabular}{|c|c|c|c|c|c|c|c|c|c|c|c|c|}
\hline \multirow[b]{2}{*}{$t$} & \multirow[b]{2}{*}{$H_{100}$} & \multicolumn{4}{|c|}{ verbleibender Bestand } & \multicolumn{4}{|c|}{ ausscheidender Bestand } & \multicolumn{3}{|c|}{ Gesamtbestand } \\
\hline & & $N$ & $V_{B}$ & $G_{B}$ & $\overline{D_{g}}$ & $N$ & $\lambda$ & $G_{B}$ & $D_{g}$ & $G W L$ & $v_{B}$ & $\overline{\overline{v_{B}}}$ \\
\hline 15 & & & & & & & & & & & & \\
\hline 20 & 8,5 & 2.500 & 0 & 12,2 & 7,9 & 0 & 0 & 0,0 & 0,0 & 0 & 0,0 & 0,0 \\
\hline 25 & 12,1 & 2.500 & 0 & 24,8 & 11,2 & 0 & 0 & 0,0 & 0,0 & 90 & 18,1 & 3,6 \\
\hline 30 & 15,0 & 1.482 & 171 & 26,9 & 15,2 & 1.018 & 23 & 3,9 & 7,0 & 208 & 23,6 & 6,9 \\
\hline 35 & 17,5 & 1.224 & 236 & 32,1 & 18,3 & 258 & 54 & 7,2 & 18,8 & 328 & 23,9 & 9,4 \\
\hline 40 & 19,7 & 1.037 & 297 & 36,0 & 21,0 & 187 & 57 & 6,9 & 21,7 & 446 & 23,5 & 11,1 \\
\hline 45 & 21,7 & 896 & 352 & 39,1 & 23,6 & 141 & 60 & 6,6 & 24,5 & 560 & 23,0 & 12,5 \\
\hline 50 & 23,5 & 786 & 402 & 41,4 & 25,9 & 110 & 61 & 6,4 & 27,1 & 672 & 22,3 & 13,4 \\
\hline 55 & 25,2 & 698 & 448 & 43,3 & 28,1 & 88 & 62 & 6,1 & 29,7 & 780 & 21,6 & 14,2 \\
\hline 60 & 26,7 & 627 & 490 & 44,9 & 30,2 & 71 & 62 & 5,8 & 32,3 & 884 & 20,8 & 14,7 \\
\hline 65 & 28,0 & 567 & 528 & 46,1 & 32,2 & 60 & 62 & 5,5 & 34,3 & 984 & 20,1 & 15,1 \\
\hline 70 & 29,3 & 517 & 563 & 47,1 & 34,1 & 50 & 62 & 5,3 & 36,6 & 1.081 & 19,4 & 15,4 \\
\hline 75 & 30,6 & 475 & 595 & 48,0 & 35,9 & 42 & 62 & 5,0 & 38,9 & 1.175 & 18,8 & 15,7 \\
\hline 80 & 31,7 & 438 & 625 & 48,7 & 37,6 & 37 & 61 & 4,7 & 40,4 & 1.266 & 18,2 & 15,8 \\
\hline 85 & 32,8 & 406 & 652 & 49,2 & 39,3 & 32 & 61 & 4,5 & 42,2 & 1.354 & 17,6 & 15,9 \\
\hline 90 & 33,8 & 379 & 678 & 49,7 & 40,9 & 27 & 60 & 4,2 & 44,7 & 1.439 & 17,0 & 16,0 \\
\hline 95 & 34,8 & 354 & 701 & 50,1 & 42,4 & 25 & 59 & 4,0 & 45,1 & 1.522 & 16,5 & 16,0 \\
\hline 100 & & & & & & & & & & & & \\
\hline 105 & & & & & & & & & & & & \\
\hline 110 & & & & & & & & & & & & \\
\hline 115 & & & & & & & & & & & & \\
\hline 120 & & & & & & & & & & & & \\
\hline
\end{tabular}


Tabelle 8-20: Ausgewählte Daten der vorläufigen Ertragstafeltabelle Fichte, mäßige Hochdurchforstung, Oberhöhenbonität $H_{100}(100$ a) $=39 \mathrm{~m}$ gemäß dyn-ET (unveröff.):

$\boldsymbol{t}$ : Alter [a], $\boldsymbol{H}_{\mathbf{1 0 0}}$ : Oberhöhe [m], $\boldsymbol{N}$ : Stammzahl [ha $\left.{ }^{-1}\right], \boldsymbol{V}_{\boldsymbol{B}}$ : Bestandesvolumen [Vfm ha ${ }^{-1}$ ], $\boldsymbol{D}_{\boldsymbol{g}}$ : Durchmesser des Grundflächenmittelstammes [ $\left.\mathrm{cm}\right], \boldsymbol{G}_{\boldsymbol{B}}$ : Bestandesgrundfläche $\left[\mathrm{m}^{2} \mathrm{ha}^{-1}\right.$ ], $\boldsymbol{\lambda}$ : Vornutzungsvolumen [Vfm ha $\left.{ }^{-1}\right], \boldsymbol{G W} \boldsymbol{L}$ : Gesamtwuchsleistung [Vfm ha $\left.{ }^{-1}\right], \boldsymbol{v}_{\boldsymbol{B}}$ : lfd. Bestandesvolumenzuwachs [Vfm ha ${ }^{-1} \mathrm{a}^{-1}$ ], $\overline{\boldsymbol{v}_{\boldsymbol{B}}}$ : durchsch..-jährl. Bestandesvolumenzuw. [Vfm ha ${ }^{-1} \mathrm{a}^{-1}$ ].

\begin{tabular}{|c|c|c|c|c|c|c|c|c|c|c|c|c|}
\hline \multirow[b]{2}{*}{$t$} & \multirow[b]{2}{*}{$H_{100}$} & \multicolumn{4}{|c|}{ verbleibender Bestand } & \multicolumn{4}{|c|}{ ausscheidender Bestand } & \multicolumn{3}{|c|}{ Gesamtbestand } \\
\hline & & $N$ & $V_{B}$ & $G_{B}$ & $D_{g}$ & $N$ & $\lambda$ & $G_{B}$ & $D_{g}$ & GWL & $v_{B}$ & $\overline{v_{B}}$ \\
\hline 15 & 7,3 & 2.500 & 0 & 7,4 & 6,2 & 0 & 0 & 0,0 & 0,0 & 0 & 0,0 & 0,0 \\
\hline 20 & 11,9 & 2.500 & 0 & 24,3 & 11,1 & 0 & 0 & 0,0 & 0,0 & 85 & 16,9 & 4,2 \\
\hline 25 & 15,5 & 1.779 & 185 & 28,1 & 14,2 & 721 & 24 & 3,9 & 8,3 & 232 & 29,5 & 9,3 \\
\hline 30 & 18,5 & 1.419 & 265 & 34,0 & 17,5 & 360 & 68 & 8,2 & 17,1 & 380 & 29,7 & 12,7 \\
\hline 35 & 21,1 & 1.172 & 337 & 38,2 & 20,4 & 247 & 73 & 8,0 & 20,3 & 525 & 29,0 & 15,0 \\
\hline 40 & 23,4 & 993 & 401 & 41,3 & 23,0 & 179 & 76 & 7,7 & 23,4 & 665 & 28,0 & 16,6 \\
\hline 45 & 25,4 & 857 & 458 & 43,7 & 25,5 & 136 & 77 & 7,4 & 26,2 & 799 & 26,9 & 17,8 \\
\hline 50 & 27,3 & 752 & 509 & 45,4 & 27,7 & 105 & 78 & 7,0 & 29,2 & 928 & 25,7 & 18,6 \\
\hline 55 & 28,9 & 668 & 554 & 46,8 & 29,9 & 84 & 78 & 6,7 & 31,9 & 1.051 & 24,7 & 19,1 \\
\hline 60 & 30,5 & 600 & 595 & 47,9 & 31,9 & 68 & 77 & 6,4 & 34,5 & 1.169 & 23,6 & 19,5 \\
\hline 65 & 31,9 & 543 & 631 & 48,8 & 33,8 & 57 & 77 & 6,0 & 36,7 & 1.283 & 22,7 & 19,7 \\
\hline 70 & 33,2 & 495 & 665 & 49,5 & 35,7 & 48 & 76 & 5,7 & 38,9 & 1.392 & 21,8 & 19,9 \\
\hline 75 & 34,5 & 455 & 695 & 50,0 & 37,4 & 40 & 74 & 5,4 & 41,4 & 1.496 & 21,0 & 20,0 \\
\hline 80 & & & & & & & & & & & & \\
\hline 85 & & & & & & & & & & & & \\
\hline 90 & & & & & & & & & & & & \\
\hline 95 & & & & & & & & & & & & \\
\hline 100 & & & & & & & & & & & & \\
\hline 105 & & & & & & & & & & & & \\
\hline 110 & & & & & & & & & & & & \\
\hline 115 & & & & & & & & & & & & \\
\hline 120 & & & & & & & & & & & & \\
\hline
\end{tabular}


Tabelle 8-21: Ausgewählte Daten der vorläufigen Ertragstafeltabelle Fichte, mäßige Hochdurchforstung, Oberhöhenbonität $H_{100}(100$ a) $=43 \mathrm{~m}$ gemäß dyn-ET (unveröff.):

$\boldsymbol{t}$ : Alter [a], $\boldsymbol{H}_{\mathbf{1 0 0}}$ : Oberhöhe [m], $\boldsymbol{N}$ : Stammzahl $\left[\mathrm{ha}^{-1}\right], \boldsymbol{V}_{\boldsymbol{B}}$ : Bestandesvolumen $\left[\mathrm{Vfm} \mathrm{ha} \mathrm{h}^{-1}\right]$, $\boldsymbol{D}_{\boldsymbol{g}}$ : Durchmesser des Grundflächenmittelstammes $[\mathrm{cm}], \boldsymbol{G}_{\boldsymbol{B}}$ : Bestandesgrundfläche $\left[\mathrm{m}^{2} \mathrm{ha}^{-1}\right]$, $\lambda$ : Vornutzungsvolumen [Vfm ha $\left.{ }^{-1}\right], \boldsymbol{G W L}$ : Gesamtwuchsleistung [Vfm ha $\left.{ }^{-1}\right], \boldsymbol{v}_{\boldsymbol{B}}$ : lfd. Bestandesvolumenzuwachs [Vfm ha ${ }^{-1} \mathrm{a}^{-1}$ ], $\overline{\boldsymbol{v}_{\boldsymbol{B}}}$ : durchsch..-jährl. Bestandesvolumenzuw. [Vfm ha ${ }^{-1} \mathrm{a}^{-1}$ ].

\begin{tabular}{|c|c|c|c|c|c|c|c|c|c|c|c|c|}
\hline \multirow[b]{2}{*}{$t$} & \multirow[b]{2}{*}{$H_{100}$} & \multicolumn{4}{|c|}{ verbleibender Bestand } & \multicolumn{4}{|c|}{ ausscheidender Bestand } & \multicolumn{3}{|c|}{ Gesamtbestand } \\
\hline & & $N$ & $V_{B}$ & $G_{B}$ & $D_{g}$ & $N$ & $\lambda$ & $G_{B}$ & $D_{g}$ & $G W L$ & $v_{B}$ & $\overline{\overline{v_{B}}}$ \\
\hline 15 & 10,6 & 2.500 & 0 & 19,8 & 10,1 & 0 & 0 & 0,0 & 0,0 & 40 & 0,0 & 2,7 \\
\hline 20 & 15,3 & 2.257 & 180 & 27,5 & 12,5 & 243 & 25 & 4,2 & 14,8 & 220 & 36,1 & 11,0 \\
\hline 25 & 19,0 & 1.711 & 279 & 34,7 & 16,1 & 546 & 84 & 9,5 & 14,9 & 404 & 36,7 & 16,2 \\
\hline 30 & 22,1 & 1.364 & 366 & 39,6 & 19,2 & 347 & 91 & 9,3 & 18,5 & 582 & 35,6 & 19,4 \\
\hline 35 & 24,7 & 1.126 & 441 & 42,9 & 22,0 & 238 & 95 & 9,0 & 21,9 & 752 & 34,0 & 21,5 \\
\hline 40 & 27,1 & 954 & 505 & 45,3 & 24,6 & 172 & 97 & 8,6 & 25,3 & 913 & 32,3 & 22,8 \\
\hline 45 & 29,2 & 824 & 562 & 47,0 & 26,9 & 130 & 97 & 8,2 & 28,4 & 1.066 & 30,6 & 23,7 \\
\hline 50 & 31,0 & 723 & 611 & 48,3 & 29,2 & 101 & 96 & 7,8 & 31,4 & 1.212 & 29,1 & 24,2 \\
\hline 55 & 32,7 & 643 & 655 & 49,2 & 31,2 & 80 & 95 & 7,4 & 34,3 & 1.350 & 27,7 & 24,5 \\
\hline 60 & 34,3 & 577 & 693 & 49,9 & 33,2 & 66 & 93 & 7,0 & 36,7 & 1.482 & 26,3 & 24,7 \\
\hline 65 & 35,8 & 522 & 728 & 50,4 & 35,1 & 55 & 91 & 6,6 & 39,0 & 1.608 & 25,1 & 24,7 \\
\hline 70 & & & & & & & & & & & & \\
\hline 75 & & & & & & & & & & & & \\
\hline 80 & & & & & & & & & & & & \\
\hline 85 & & & & & & & & & & & & \\
\hline 90 & & & & & & & & & & & & \\
\hline 95 & & & & & & & & & & & & \\
\hline 100 & & & & & & & & & & & & \\
\hline 105 & & & & & & & & & & & & \\
\hline 110 & & & & & & & & & & & & \\
\hline 115 & & & & & & & & & & & & \\
\hline 120 & & & & & & & & & & & & \\
\hline
\end{tabular}


Tabelle 8-22: Ausgewählte Daten der vorläufigen Ertragstafeltabelle Fichte, starke Hochdurchforstung, Oberhöhenbonität $H_{100}(100 \mathrm{a})=27 \mathrm{~m}$ gemäß dyn-ET (unveröff.):

$\boldsymbol{t}$ : Alter [a], $\boldsymbol{H}_{\mathbf{1 0 0}}$ : Oberhöhe [m], $\boldsymbol{N}$ : Stammzahl [ha $\left.{ }^{-1}\right], \boldsymbol{V}_{\boldsymbol{B}}$ : Bestandesvolumen [Vfm ha ${ }^{-1}$ ], $\boldsymbol{D}_{\boldsymbol{g}}$ : Durchmesser des Grundflächenmittelstammes $[\mathrm{cm}], \boldsymbol{G}_{\boldsymbol{B}}$ : Bestandesgrundfläche $\left[\mathrm{m}^{2} \mathrm{ha}^{-1}\right]$, $\lambda$ : Vornutzungsvolumen [Vfm ha $\left.{ }^{-1}\right], \boldsymbol{G W}$ : Gesamtwuchsleistung [Vfm ha $\left.{ }^{-1}\right], \boldsymbol{v}_{\boldsymbol{B}}$ : lfd. Bestandesvolumenzuwachs [Vfm ha ${ }^{-1} \mathrm{a}^{-1}$ ], $\overline{\boldsymbol{v}_{\boldsymbol{B}}}$ : durchsch..-jährl. Bestandesvolumenzuw. [Vfm ha ${ }^{-1} \mathrm{a}^{-1}$ ].

\begin{tabular}{|c|c|c|c|c|c|c|c|c|c|c|c|c|}
\hline \multirow[b]{2}{*}{$t$} & \multirow[b]{2}{*}{$H_{100}$} & \multicolumn{4}{|c|}{ verbleibender Bestand } & \multicolumn{4}{|c|}{ ausscheidender Bestand } & \multicolumn{3}{|c|}{ Gesamtbestand } \\
\hline & & $N$ & $V_{B}$ & $G_{B}$ & $D_{g}$ & $N$ & $\lambda$ & $G_{B}$ & $D_{g}$ & $G W L$ & $v_{B}$ & $\overline{v_{B}}$ \\
\hline \multicolumn{13}{|l|}{15} \\
\hline \multicolumn{13}{|l|}{20} \\
\hline \multicolumn{13}{|l|}{25} \\
\hline \multicolumn{13}{|l|}{30} \\
\hline 35 & 10,3 & 2.500 & 0 & 18,9 & 9,8 & 0 & 0 & 0,0 & 0,0 & 27 & 0,0 & 0,8 \\
\hline 40 & 12,4 & 2.500 & 0 & 26,0 & 11,5 & 0 & 0 & 0,0 & 0,0 & 89 & 12,4 & 2,2 \\
\hline 45 & 14,3 & 833 & 125 & 20,6 & 17,7 & 1.667 & 23 & 3,8 & 5,4 & 154 & 12,8 & 3,4 \\
\hline 50 & 16,0 & 739 & 159 & 23,6 & 20,2 & 94 & 30 & 6,1 & 28,7 & 219 & 13,0 & 4,4 \\
\hline 55 & 17,6 & 662 & 193 & 26,1 & 22,4 & 77 & 31 & 5,6 & 30,4 & 284 & 13,0 & 5,2 \\
\hline 60 & 19,0 & 599 & 225 & 28,2 & 24,5 & 63 & 32 & 5,1 & 32,2 & 348 & 12,9 & 5,8 \\
\hline 65 & 20,3 & 547 & 255 & 30,0 & 26,4 & 52 & 33 & 4,7 & 34,0 & 411 & 12,7 & 6,3 \\
\hline 70 & 21,5 & 502 & 283 & 31,5 & 28,3 & 45 & 34 & 4,4 & 35,2 & 474 & 12,5 & 6,8 \\
\hline 75 & 22,7 & 464 & 310 & 32,8 & 30,0 & 38 & 34 & 4,1 & 37,0 & 535 & 12,2 & 7,1 \\
\hline 80 & 23,8 & 431 & 335 & 34,0 & 31,7 & 33 & 35 & 3,8 & 38,3 & 595 & 12,0 & 7,4 \\
\hline 85 & 24,8 & 402 & 359 & 34,9 & 33,3 & 29 & 35 & 3,6 & 39,5 & 654 & 11,8 & 7,7 \\
\hline 90 & 25,8 & 377 & 381 & 35,8 & 34,8 & 25 & 35 & 3,3 & 41,1 & 711 & 11,5 & 7,9 \\
\hline 95 & 26,7 & 354 & 403 & 36,5 & 36,3 & 23 & 35 & 3,1 & 41,5 & 768 & 11,3 & 8,1 \\
\hline 100 & 27,6 & 334 & 423 & 37,2 & 37,7 & 20 & 35 & 2,9 & 43,1 & 823 & 11,0 & 8,2 \\
\hline 105 & 28,5 & 316 & 442 & 37,8 & 39,0 & 18 & 35 & 2,7 & 44,0 & 877 & 10,8 & 8,3 \\
\hline 110 & 29,3 & 299 & 460 & 38,3 & 40,4 & 17 & 35 & 2,6 & 43,9 & 929 & 10,6 & 8,4 \\
\hline 115 & 30,1 & 284 & 477 & 38,7 & 41,7 & 15 & 35 & 2,4 & 45,3 & 981 & 10,3 & 8,5 \\
\hline 120 & 30,8 & 271 & 493 & 39,1 & 42,9 & 13 & 34 & 2,3 & 47,2 & 1.032 & 10,1 & 8,6 \\
\hline
\end{tabular}


Tabelle 8-23: Ausgewählte Daten der vorläufigen Ertragstafeltabelle Fichte, starke Hochdurchforstung, Oberhöhenbonität $H_{100}(100$ a) $=31 \mathrm{~m}$ gemäß dyn-ET (unveröff.):

$\boldsymbol{t}$ : Alter [a], $\boldsymbol{H}_{\mathbf{1 0 0}}$ : Oberhöhe [m], $\boldsymbol{N}$ : Stammzahl $\left[\mathrm{ha}^{-1}\right], \boldsymbol{V}_{\boldsymbol{B}}$ : Bestandesvolumen $\left[\mathrm{Vfm} \mathrm{ha} \mathrm{h}^{-1}\right]$, $\boldsymbol{D}_{\boldsymbol{g}}$ : Durchmesser des Grundflächenmittelstammes $[\mathrm{cm}], \boldsymbol{G}_{\boldsymbol{B}}$ : Bestandesgrundfläche $\left[\mathrm{m}^{2} \mathrm{ha}^{-1}\right]$, $\lambda$ : Vornutzungsvolumen [Vfm ha $\left.{ }^{-1}\right], \boldsymbol{G W L}$ : Gesamtwuchsleistung [Vfm ha $\left.{ }^{-1}\right], \boldsymbol{v}_{\boldsymbol{B}}$ : lfd. Bestandesvolumenzuwachs [Vfm ha ${ }^{-1} \mathrm{a}^{-1}$ ], $\overline{\boldsymbol{v}_{\boldsymbol{B}}}$ : durchsch..-jährl. Bestandesvolumenzuw. [Vfm ha ${ }^{-1} \mathrm{a}^{-1}$ ].

\begin{tabular}{|c|c|c|c|c|c|c|c|c|c|c|c|c|}
\hline \multirow[b]{2}{*}{$\boldsymbol{t}$} & \multirow[b]{2}{*}{$H_{100}$} & \multicolumn{4}{|c|}{ verbleibender Bestand } & \multicolumn{4}{|c|}{ ausscheidender Bestand } & \multicolumn{3}{|c|}{ Gesamtbestand } \\
\hline & & $N$ & $V_{B}$ & $G_{B}$ & $D_{g}$ & $N$ & $\lambda$ & $G_{B}$ & $D_{g}$ & $G W L$ & $v_{B}$ & $\overline{v_{B}}$ \\
\hline \multicolumn{13}{|l|}{15} \\
\hline \multicolumn{13}{|l|}{20} \\
\hline 25 & 8,6 & 2.500 & 0 & 12,5 & 8,0 & 0 & 0 & 0,0 & 0,0 & 0 & 0,0 & 0,0 \\
\hline 30 & 11,5 & 2.500 & 0 & 22,8 & 10,8 & 0 & 0 & 0,0 & 0,0 & 60 & 12,0 & 2,0 \\
\hline 35 & 13,9 & 2.500 & 0 & 30,6 & 12,5 & 0 & 0 & 0,0 & 0,0 & 140 & 16,0 & 4,0 \\
\hline 40 & 16,1 & 790 & 162 & 23,8 & 19,6 & 1.710 & 19 & 2,9 & 4,6 & 222 & 16,4 & 5,5 \\
\hline 45 & 18,0 & 691 & 204 & 26,8 & 22,2 & 99 & 40 & 6,1 & 28,0 & 304 & 16,4 & 6,7 \\
\hline 50 & 19,8 & 612 & 244 & 29,3 & 24,7 & 79 & 42 & 5,6 & 30,1 & 384 & 16,1 & 7,7 \\
\hline 55 & 21,4 & 549 & 280 & 31,3 & 26,9 & 63 & 43 & 5,2 & 32,4 & 464 & 15,8 & 8,4 \\
\hline 60 & 22,8 & 497 & 314 & 33,0 & 29,1 & 52 & 44 & 4,8 & 34,4 & 541 & 15,5 & 9,0 \\
\hline 65 & 24,2 & 453 & 345 & 34,3 & 31,1 & 44 & 44 & 4,5 & 36,2 & 616 & 15,1 & 9,5 \\
\hline 70 & 25,4 & 416 & 374 & 35,5 & 33,0 & 37 & 45 & 4,2 & 38,2 & 690 & 14,7 & 9,9 \\
\hline 75 & 26,6 & 385 & 400 & 36,5 & 34,7 & 31 & 45 & 4,0 & 40,4 & 761 & 14,3 & 10,2 \\
\hline 80 & 27,8 & 357 & 425 & 37,3 & 36,5 & 28 & 45 & 3,7 & 41,2 & 831 & 13,9 & 10,4 \\
\hline 85 & 28,8 & 333 & 448 & 38,0 & 38,1 & 24 & 45 & 3,5 & 43,2 & 899 & 13,6 & 10,6 \\
\hline 90 & 29,8 & 312 & 470 & 38,6 & 39,7 & 21 & 44 & 3,3 & 44,8 & 965 & 13,2 & 10,7 \\
\hline 95 & 30,8 & 293 & 490 & 39,1 & 41,2 & 19 & 44 & 3,1 & 45,7 & 1.029 & 12,9 & 10,8 \\
\hline 100 & 31,7 & 277 & 509 & 39,5 & 42,6 & 16 & 44 & 2,9 & 48,4 & 1.092 & 12,5 & 10,9 \\
\hline 105 & 32,5 & 262 & 527 & 39,9 & 44,0 & 15 & 43 & 2,8 & 48,5 & 1.153 & 12,2 & 11,0 \\
\hline 110 & 33,4 & 248 & 544 & 40,2 & 45,5 & 14 & 43 & 2,6 & 48,8 & 1.212 & 11,9 & 11,0 \\
\hline 115 & 34,2 & 236 & 560 & 40,5 & 46,8 & 12 & 42 & 2,5 & 51,2 & 1.271 & 11,6 & 11,0 \\
\hline 120 & 34,9 & 224 & 575 & 40,8 & 48,1 & 12 & 42 & 2,3 & 49,8 & 1.327 & 11,4 & 11,1 \\
\hline
\end{tabular}


Tabelle 8-24: Ausgewählte Daten der vorläufigen Ertragstafeltabelle Fichte, starke Hochdurchforstung, Oberhöhenbonität $H_{100}(100 \mathrm{a})=35 \mathrm{~m}$ gemäß dyn-ET (unveröff.):

$\boldsymbol{t}$ : Alter [a], $\boldsymbol{H}_{\mathbf{1 0 0}}$ : Oberhöhe [m], $\boldsymbol{N}$ : Stammzahl [ha $\left.{ }^{-1}\right], \boldsymbol{V}_{\boldsymbol{B}}$ : Bestandesvolumen [Vfm ha ${ }^{-1}$ ], $\boldsymbol{D}_{\boldsymbol{g}}$ : Durchmesser des Grundflächenmittelstammes $[\mathrm{cm}], \boldsymbol{G}_{\boldsymbol{B}}$ : Bestandesgrundfläche $\left[\mathrm{m}^{2} \mathrm{ha}^{-1}\right]$, $\lambda$ : Vornutzungsvolumen [Vfm ha $\left.{ }^{-1}\right], \boldsymbol{G W} \boldsymbol{L}$ : Gesamtwuchsleistung [Vfm ha $\left.{ }^{-1}\right], \boldsymbol{v}_{\boldsymbol{B}}$ : lfd. Bestandesvolumenzuwachs [Vfm ha ${ }^{-1} \mathrm{a}^{-1}$ ], $\overline{\boldsymbol{v}_{\boldsymbol{B}}}$ : durchsch..-jährl. Bestandesvolumenzuw. [Vfm ha-1 $\mathrm{a}^{-1}$ ].

\begin{tabular}{|c|c|c|c|c|c|c|c|c|c|c|c|c|}
\hline \multirow{2}{*}{\multicolumn{2}{|c|}{$\begin{array}{ll}t \quad H_{100}\end{array}$}} & \multicolumn{4}{|c|}{ verbleibender Bestand } & \multicolumn{4}{|c|}{ ausscheidender Bestand } & \multicolumn{3}{|c|}{ Gesamtbestand } \\
\hline & & $N$ & $V_{B}$ & $G_{B}$ & $D_{g}$ & $N$ & $\lambda$ & $G_{B}$ & $D_{g}$ & $G W L$ & $v_{B}$ & $\overline{v_{B}}$ \\
\hline \multicolumn{13}{|l|}{15} \\
\hline 20 & 8,5 & 2.500 & 0 & 12,2 & 7,9 & 0 & 0 & 0,0 & 0,0 & 0 & 0,0 & 0,0 \\
\hline 25 & 12,1 & 2.500 & 0 & 24,8 & 11,2 & 0 & 0 & 0,0 & 0,0 & 78 & 15,6 & 3,1 \\
\hline 30 & 15,0 & 931 & 142 & 21,9 & 17,3 & 1.569 & 20 & 3,2 & 5,1 & 179 & 20,3 & 6,0 \\
\hline 35 & 17,5 & 781 & 196 & 26,1 & 20,6 & 150 & 48 & 7,0 & 24,4 & 282 & 20,5 & 8,1 \\
\hline 40 & 19,7 & 670 & 246 & 29,3 & 23,6 & 111 & 52 & 6,5 & 27,3 & 384 & 20,3 & 9,6 \\
\hline 45 & 21,7 & 585 & 291 & 31,7 & 26,3 & 85 & 54 & 6,0 & 30,1 & 483 & 19,8 & 10,7 \\
\hline 50 & 23,5 & 519 & 332 & 33,7 & 28,7 & 66 & 55 & 5,7 & 33,0 & 579 & 19,3 & 11,6 \\
\hline 55 & 25,2 & 465 & 369 & 35,2 & 31,1 & 54 & 56 & 5,3 & 35,4 & 672 & 18,6 & 12,2 \\
\hline 60 & 26,7 & 421 & 403 & 36,5 & 33,2 & 44 & 57 & 5,0 & 38,1 & 762 & 18,0 & 12,7 \\
\hline 65 & 28,0 & 384 & 434 & 37,5 & 35,3 & 37 & 57 & 4,7 & 40,3 & 850 & 17,4 & 13,1 \\
\hline 70 & 29,3 & 353 & 462 & 38,3 & 37,2 & 31 & 56 & 4,5 & 42,8 & 934 & 16,9 & 13,3 \\
\hline 75 & 30,6 & 326 & 487 & 39,0 & 39,0 & 27 & 56 & 4,2 & 44,6 & 1.016 & 16,3 & 13,5 \\
\hline 80 & 31,7 & 303 & 511 & 39,6 & 40,8 & 23 & 55 & 4,0 & 46,9 & 1.095 & 15,8 & 13,7 \\
\hline 85 & 32,8 & 282 & 533 & 40,0 & 42,5 & 21 & 55 & 3,8 & 47,8 & 1.171 & 15,3 & 13,8 \\
\hline 90 & 33,8 & 264 & 553 & 40,4 & 44,1 & 18 & 54 & 3,6 & 50,2 & 1.245 & 14,8 & 13,8 \\
\hline 95 & 34,8 & 249 & 571 & 40,7 & 45,6 & 15 & 53 & 3,4 & 53,5 & 1.317 & 14,4 & 13,9 \\
\hline 100 & 35,7 & 234 & 589 & 41,0 & 47,2 & 15 & 52 & 3,2 & 52,1 & 1.387 & 14,0 & 13,9 \\
\hline 105 & 36,6 & 222 & 605 & 41,2 & 48,6 & 12 & 52 & 3,0 & 56,7 & 1.455 & 13,6 & 13,9 \\
\hline 110 & 37,5 & 210 & 621 & 41,4 & 50,1 & 12 & 51 & 2,9 & 55,2 & 1.521 & 13,2 & 13,8 \\
\hline \multicolumn{13}{|l|}{115} \\
\hline 120 & & & & & & & & & & & & \\
\hline
\end{tabular}


Tabelle 8-25: Ausgewählte Daten der vorläufigen Ertragstafeltabelle Fichte, starke Hochdurchforstung, Oberhöhenbonität $H_{100}(100$ a) $=39 \mathrm{~m}$ gemäß dyn-ET (unveröff.):

$\boldsymbol{t}$ : Alter [a], $\boldsymbol{H}_{\mathbf{1 0 0}}$ : Oberhöhe [m], $\boldsymbol{N}$ : Stammzahl $\left[\mathrm{ha}^{-1}\right], \boldsymbol{V}_{\boldsymbol{B}}$ : Bestandesvolumen $\left[\mathrm{Vfm} \mathrm{ha} \mathrm{h}^{-1}\right]$, $\boldsymbol{D}_{\boldsymbol{g}}$ : Durchmesser des Grundflächenmittelstammes $[\mathrm{cm}], \boldsymbol{G}_{\boldsymbol{B}}$ : Bestandesgrundfläche $\left[\mathrm{m}^{2} \mathrm{ha}^{-1}\right]$, $\lambda$ : Vornutzungsvolumen [Vfm ha $\left.{ }^{-1}\right], \boldsymbol{G W L}$ : Gesamtwuchsleistung [Vfm ha $\left.{ }^{-1}\right], \boldsymbol{v}_{\boldsymbol{B}}$ : lfd. Bestandesvolumenzuwachs [Vfm ha ${ }^{-1} \mathrm{a}^{-1}$ ], $\overline{\boldsymbol{v}_{\boldsymbol{B}}}$ : durchsch..-jährl. Bestandesvolumenzuw. [Vfm ha ${ }^{-1} \mathrm{a}^{-1}$ ].

\begin{tabular}{|c|c|c|c|c|c|c|c|c|c|c|c|c|}
\hline \multirow[b]{2}{*}{$t$} & \multirow[b]{2}{*}{$H_{100}$} & \multicolumn{4}{|c|}{ verbleibender Bestand } & \multicolumn{4}{|c|}{ ausscheidender Bestand } & \multicolumn{3}{|c|}{ Gesamtbestand } \\
\hline & & $N$ & $V_{B}$ & $G_{B}$ & $D_{g}$ & $N$ & $\lambda$ & $G_{B}$ & $D_{g}$ & $G W L$ & $v_{B}$ & $\overline{\overline{v_{B}}}$ \\
\hline 15 & 7,3 & 2.500 & 0 & 7,4 & 6,2 & 0 & 0 & 0,0 & 0,0 & 0 & 0,0 & 0,0 \\
\hline 20 & 11,9 & 2.500 & 0 & 24,3 & 11,1 & 0 & 0 & 0,0 & 0,0 & 73 & 14,6 & 3,7 \\
\hline 25 & 15,5 & 990 & 155 & 22,8 & 17,1 & 1.510 & 18 & 2,8 & 4,9 & 200 & 25,3 & 8,0 \\
\hline 30 & 18,5 & 804 & 222 & 27,6 & 20,9 & 186 & 61 & 7,7 & 23,0 & 327 & 25,5 & 10,9 \\
\hline 35 & 21,1 & 674 & 282 & 31,0 & 24,2 & 130 & 65 & 7,3 & 26,7 & 452 & 25,0 & 12,9 \\
\hline 40 & 23,4 & 578 & 334 & 33,6 & 27,2 & 96 & 68 & 6,9 & 30,2 & 573 & 24,2 & 14,3 \\
\hline 45 & 25,4 & 505 & 380 & 35,5 & 29,9 & 73 & 70 & 6,5 & 33,7 & 689 & 23,2 & 15,3 \\
\hline 50 & 27,3 & 448 & 421 & 36,9 & 32,4 & 57 & 71 & 6,2 & 37,1 & 801 & 22,3 & 16,0 \\
\hline 55 & 28,9 & 401 & 457 & 38,1 & 34,8 & 47 & 71 & 5,8 & 39,8 & 908 & 21,4 & 16,5 \\
\hline 60 & 30,5 & 363 & 490 & 39,0 & 37,0 & 38 & 70 & 5,5 & 43,1 & 1.010 & 20,5 & 16,8 \\
\hline 65 & 31,9 & 331 & 519 & 39,6 & 39,1 & 32 & 70 & 5,2 & 45,7 & 1.109 & 19,7 & 17,1 \\
\hline 70 & 33,2 & 304 & 545 & 40,2 & 41,0 & 27 & 69 & 5,0 & 48,4 & 1.204 & 19,0 & 17,2 \\
\hline 75 & 34,5 & 281 & 569 & 40,6 & 42,9 & 23 & 68 & 4,7 & 51,1 & 1.295 & 18,3 & 17,3 \\
\hline 80 & 35,7 & 261 & 590 & 41,0 & 44,7 & 20 & 66 & 4,5 & 53,3 & 1.383 & 17,6 & 17,3 \\
\hline 85 & 36,8 & 244 & 610 & 41,2 & 46,4 & 17 & 65 & 4,2 & 56,3 & 1.468 & 17,0 & 17,3 \\
\hline 90 & & & & & & & & & & & & \\
\hline 95 & & & & & & & & & & & & \\
\hline 100 & & & & & & & & & & & & \\
\hline 105 & & & & & & & & & & & & \\
\hline 110 & & & & & & & & & & & & \\
\hline 115 & & & & & & & & & & & & \\
\hline 120 & & & & & & & & & & & & \\
\hline
\end{tabular}


Tabelle 8-26: Ausgewählte Daten der vorläufigen Ertragstafeltabelle Fichte, starke Hochdurchforstung, Oberhöhenbonität $H_{100}(100 \mathrm{a})=43 \mathrm{~m}$ gemäß dyn-ET (unveröff.):

$\boldsymbol{t}$ : Alter [a], $\boldsymbol{H}_{\mathbf{1 0 0}}$ : Oberhöhe [m], $\boldsymbol{N}$ : Stammzahl [ha $\left.{ }^{-1}\right], \boldsymbol{V}_{\boldsymbol{B}}$ : Bestandesvolumen [Vfm ha ${ }^{-1}$ ], $\boldsymbol{D}_{\boldsymbol{g}}$ : Durchmesser des Grundflächenmittelstammes [ $\left.\mathrm{cm}\right], \boldsymbol{G}_{\boldsymbol{B}}$ : Bestandesgrundfläche $\left[\mathrm{m}^{2} \mathrm{ha}^{-1}\right.$ ], $\boldsymbol{\lambda}$ : Vornutzungsvolumen [Vfm ha $\left.{ }^{-1}\right], \boldsymbol{G W} \boldsymbol{L}$ : Gesamtwuchsleistung [Vfm ha $\left.{ }^{-1}\right], \boldsymbol{v}_{\boldsymbol{B}}$ : lfd. Bestandesvolumenzuwachs [Vfm ha $\left.{ }^{-1} \mathrm{a}^{-1}\right], \overline{\boldsymbol{v}_{\boldsymbol{B}}}$ : durchsch..-jährl. Bestandesvolumenzuw. [Vfm ha ${ }^{-1} \mathrm{a}^{-1}$ ].

\begin{tabular}{|c|c|c|c|c|c|c|c|c|c|c|c|c|}
\hline \multirow[b]{2}{*}{$\boldsymbol{t}$} & \multirow[b]{2}{*}{$H_{100}$} & \multicolumn{4}{|c|}{ verbleibender Bestand } & \multicolumn{4}{|c|}{ ausscheidender Bestand } & \multicolumn{3}{|c|}{ Gesamtbestand } \\
\hline & & $N$ & $V_{B}$ & $G_{B}$ & $D_{g}$ & $N$ & $\lambda$ & $G_{B}$ & $D_{g}$ & $G W L$ & $v_{B}$ & $\overline{v_{B}}$ \\
\hline 15 & 10,6 & 2.500 & 0 & 19,8 & 10,1 & 0 & 0 & 0,0 & 0,0 & 35 & 0,0 & 2,3 \\
\hline 20 & 15,3 & 1.120 & 153 & 22,4 & 15,9 & 1.380 & 18 & 2,8 & 5,1 & 190 & 31,0 & 9,5 \\
\hline 25 & 19,0 & 867 & 236 & 28,2 & 20,4 & 253 & 74 & 8,8 & 21,0 & 348 & 31,6 & 13,9 \\
\hline 30 & 22,1 & 704 & 309 & 32,1 & 24,1 & 163 & 81 & 8,4 & 25,7 & 501 & 30,7 & 16,7 \\
\hline 35 & 24,7 & 590 & 370 & 34,9 & 27,4 & 114 & 85 & 8,1 & 30,1 & 648 & 29,4 & 18,5 \\
\hline 40 & 27,1 & 506 & 423 & 36,8 & 30,4 & 84 & 87 & 7,7 & 34,3 & 788 & 28,0 & 19,7 \\
\hline 45 & 29,2 & 442 & 468 & 38,2 & 33,2 & 64 & 88 & 7,4 & 38,3 & 921 & 26,6 & 20,5 \\
\hline 50 & 31,0 & 392 & 507 & 39,2 & 35,7 & 50 & 87 & 7,0 & 42,3 & 1.047 & 25,3 & 20,9 \\
\hline 55 & 32,7 & 351 & 541 & 40,0 & 38,1 & 41 & 86 & 6,7 & 45,5 & 1.168 & 24,1 & 21,2 \\
\hline 60 & 34,3 & 318 & 571 & 40,6 & 40,3 & 33 & 85 & 6,3 & 49,4 & 1.282 & 22,9 & 21,4 \\
\hline 65 & 35,8 & 290 & 598 & 41,0 & 42,4 & 28 & 83 & 6,0 & 52,2 & 1.392 & 21,9 & 21,4 \\
\hline 70 & 37,1 & 266 & 621 & 41,3 & 44,5 & 24 & 81 & 5,7 & 54,9 & 1.497 & 21,0 & 21,4 \\
\hline 75 & & & & & & & & & & & & \\
\hline 80 & & & & & & & & & & & & \\
\hline 85 & & & & & & & & & & & & \\
\hline 90 & & & & & & & & & & & & \\
\hline 95 & & & & & & & & & & & & \\
\hline 100 & & & & & & & & & & & & \\
\hline 105 & & & & & & & & & & & & \\
\hline 110 & & & & & & & & & & & & \\
\hline 115 & & & & & & & & & & & & \\
\hline 120 & & & & & & & & & & & & \\
\hline
\end{tabular}




\section{Eigenständigkeitserklärung}

Hiermit erkläre ich, dass die vorliegende Arbeit ohne unzulässige Hilfe und ohne Benutzung anderer als der angegebenen Hilfsmittel selbstständig angefertigt wurde und dass die aus fremden Quellen direkt oder indirekt übernommenen Gedanken in der Arbeit als solche kenntlich gemacht worden sind. Alle genutzten fremden Quellen sind im Literaturverzeichnis gelistet.

Roman Koster

Göttingen, Oktober 2020 